Retention:

Permanent
Key Words:

Part B-1

Viscosity

Calorimetry

\title{
PHYSICAL CHARACTERIZATION FOR HANFORD TANK WASTE SAMPLES AN-102, AN-103, AND AZ-102
}

\section{Scott W. Rosencrance \\ William D. King \\ Charles Nash}

APRIL 2002

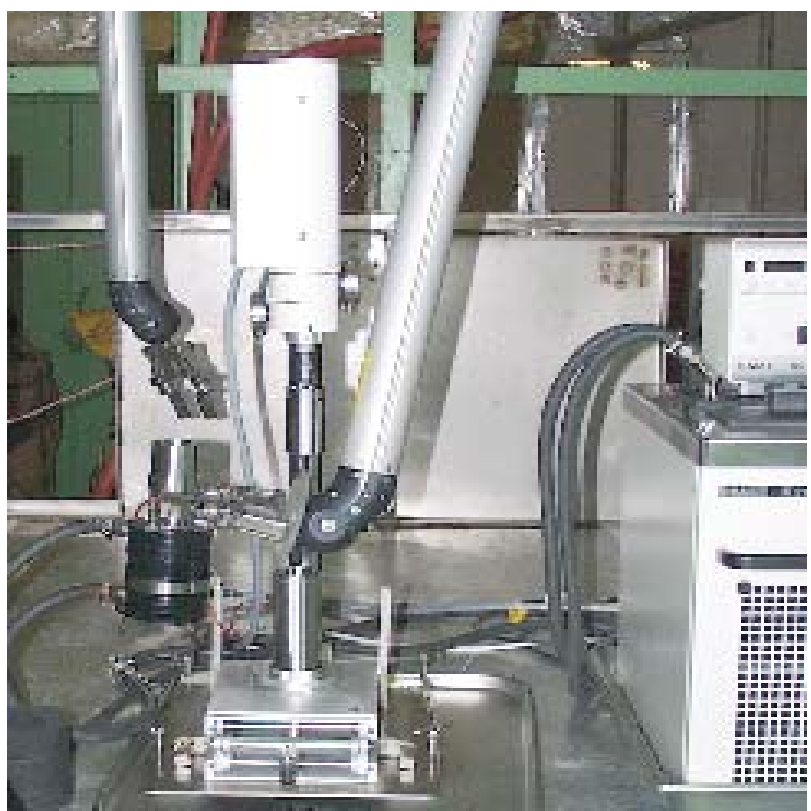

DOES NOT CONTAIN

UNCLASSIFIED CONTROLLED

NUCLEAR INFORMATION

ADC \&

Reviewing Official:

Date:

Westinghouse Savannah River Company

Savannah River Site

Aiken, SC 29808 
This document was prepared in conjunction with work accomplished under Contract No. DEAC09-96SR18500 with the U.S. Department of Energy.

\section{DISCLAIMER}

This report was prepared as an account of work sponsored by an agency of the United States Government. Neither the United States Government nor any agency thereof, nor any of their employees, makes any warranty, express or implied, or assumes any legal liability or responsibility for the accuracy, completeness, or usefulness of any information, apparatus, product or process disclosed, or represents that its use would not infringe privately owned rights. Reference herein to any specific commercial product, process or service by trade name, trademark, manufacturer, or otherwise does not necessarily constitute or imply its endorsement, recommendation, or favoring by the United States Government or any agency thereof. The views and opinions of authors expressed herein do not necessarily state or reflect those of the United States Government or any agency thereof.

This report has been reproduced directly from the best available copy.

Available for sale to the public, in paper, from: U.S. Department of Commerce, National Technical Information Service, 5285 Port Royal Road, Springfield, VA 22161

phone: (800) 553-6847

fax: (703) 605-6900

email: orders@ntis.fedworld.gov

online ordering: http://www.ntis.gov/support/index.html

Available electronically at http://www.osti.gov/bridge

Available for a processing fee to U.S. Department of Energy and its contractors, in paper, from: U.S. Department of Energy, Office of Scientific and Technical Information, P.O. Box 62, Oak Ridge, TN 37831-0062

phone: (865)576-8401

fax: (865)576-5728

email: reports@adonis.osti.gov 
WSRC-TR-2000-00352

SRT-RPP-2000-00026

Key Words:

Part B-1

Viscosity

Calorimetry

Retention:

Permanent

\title{
PHYSICAL CHARACTERIZATION FOR HANFORD TANK
}

WASTE SAMPLES AN-102, AN-103, AND AZ-102

\author{
Scott W. Rosencrance \\ William D. King \\ Charles Nash
}

APRIL 2002

Westinghouse Savannah River Company

Savannah River Site

Aiken, SC 29808

Prepared for the U.S. Department of Energy Under

Contract Number DE-AC09-96SR18500

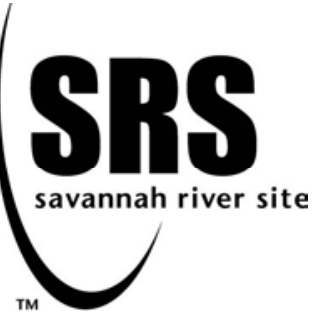




\section{REVIEWS AND APPROVALS}

Scott W. Rosencrance, Author, Waste Processing Technology

Date

William D. King, Author, Waste Processing Technology

Date

Charles Nash, Author, Waste Processing Technology

Date

James Marra, Manager, Waste Processing Technology External Programs

Date

David Koopman, Immobilization Technology, Technical Reviewer

Date

River Protection Project, Customer

Date 
WSRC-TR-2000-00352

SRT-RPP-2000-00026

\section{TABLE OF CONTENTS}

LIST OF FIGURES .............................................................................................................. $\mathrm{v}$

LIST OF TABLES ............................................................................................................................ $\mathrm{v}$

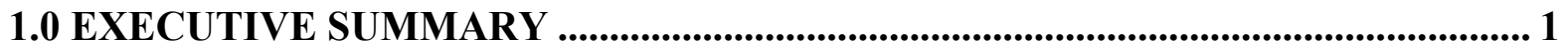

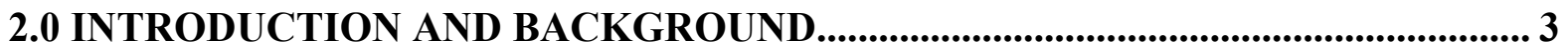

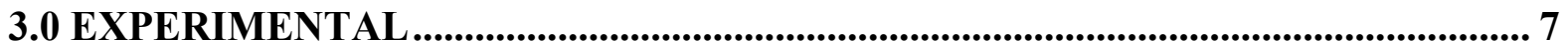

3.1 RHEOLOGY .................................................................................................................... 7

3.2 CALORIMETRY ........................................................................................................ 10

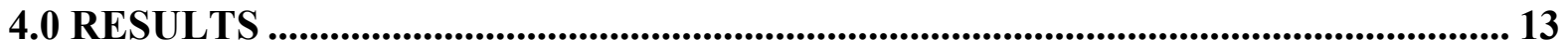

4.1 RHEOLOGY OF AS-RECEIVED AND DILUTED SUPERNATES ...................... 13

4.1.1 Envelope A As-Received .................................................................................... 13

4.1.2 Envelope A Diluted....................................................................................................... 13

4.1.3 Envelope B As-received ............................................................................................... 13

4.1.4 Envelope C As-received ......................................................................................... 15

4.1.5 Envelope C Diluted............................................................................................................... 15

4.2 Rheology of Envelope C Sr/TRU Precipitate Slurries ................................................... 15

4.2.1 Envelope C 2 Wt \% Sr/TRU Precipitate Slurry...................................................... 16

4.2.2 Envelope C 10 Wt \% Sr/TRU Precipitate Slurry................................................... 16

4.2.3 Envelope C 13 Wt \% Sr/TRU Precipitate Slurry...................................................... 16

4.2.4 Envelope C 16 Wt \% Sr/TRU Precipitate Slurry...................................................... 16

4.3 Rheology of Pre-treated Samples and Concentrates..................................................... 17

4.3.1 Envelope B Evaporator Concentrate........................................................................... 17

4.3.2 Envelope C Pretreated Liquid ............................................................................... 17

4.4 Calorimetry …...................................................................................................................... 18

4.4.1 Calorimetry of Envelope C (AN-102) Diluted Supernate..................................... 18

4.4.2 Calorimetry of Envelope C (AN-102) Sr/TRU Precipitate Slurries .................. 19

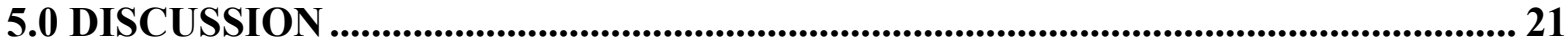

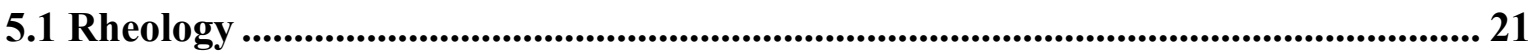

5.1.1 Rheology of Sr/TRU Precipitate Slurry Samples ................................................... 21

5.2 Calorimetry ......................................................................................................................... 23

5.2.1 Calorimetry of Envelope C (AN-102) Diluted Supernate...................................... 23

5.2.2 Calorimetry of Envelope C (AN-102) Sr/TRU Precipitate Slurries .................. 25

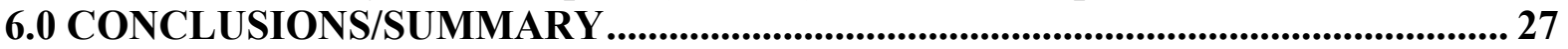

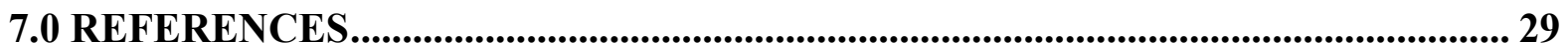




\section{APPENDICES}

Appendix A Instrument Mockup and Calibration 35

Part 1 Photographs of Rheometer Remoting Efforts 37

Part 2 Rheometer Calibration Prior to Shielded Cells Installation with 51 the $95 \mathrm{cP}$ Standard

Part 3 Rheometer Pre-Calibration in the Shielded Cells with the Low 55 Viscosity Standard

Part 4 Rheometer Post-Calibration in the Shielded Cells with the Low 75 Viscosity Standard

Part 5 Temperature Dependence of Viscosity for the Low Viscosity Standard Oil

$\begin{array}{lll}\text { Appendix B Envelope A Rheology Data } & 97\end{array}$

$\begin{array}{lll}\text { Appendix C Envelope B Rheology Data } & 119\end{array}$

Appendix D Envelope C Rheology Data 141

Appendix E Envelope C SR/TRU Precipitate Slurry Rheology Data 173

Appendix F Envelope A Rheograms 239

Appendix G Envelope B Rheograms 269

Appendix H Envelope C Rheograms 297

Part 1 Envelope C Rheograms As Received and Diluted 299

Part 2 Envelope C Rheograms Pretreated 329

$\begin{array}{lll}\text { Appendix I } & \text { SR/TRU Precipitate Slurry Rheograms } & 345\end{array}$

$\begin{array}{lll}\text { Part } 1 & \text { SR/TRU Precipitate Slurry Rheograms at } 2 \mathrm{wt} \% & 347\end{array}$

Part 2 SR/TRU Precipitate Slurry Rheograms at $10 \mathrm{wt} \% \quad 371$

Part 3 SR/TRU Precipitate Slurry Rheograms at $13 \mathrm{wt} \%$

Part 4 SR/TRU Precipitate Slurry Rheograms at $16 \mathrm{wt} \%$

Appendix J Envelope A Rheology Summaries 447

Appendix K Envelope B Rheology Summaries 455

Appendix L Envelope C Rheology Summaries 463

Appendix M SR/TRU Precipitate Slurry Rheology Summaries 479

Appendix N Envelope C As-Received Calorimetry Data 501

$\begin{array}{lll}\text { Appendix O SR/TRU Precipitate Slurry Calorimetry Data } & 509\end{array}$ 


\section{LIST OF FIGURES}

Figure 1. Plot of Actual AN-102 Sr/TRU Precipitate Slurry Viscosity Data Versus Wt \%

Solids for Each Analysis Temperature. 22

Figure 2. Temperature-dependent Average Apparent Viscosities for the AN-102 Supernate

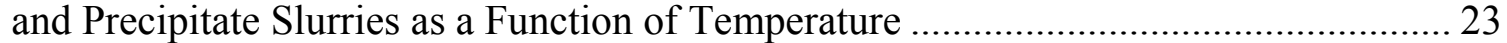

Figure 3. Heat Capacity Versus Temperature Data for the Actual 241-AN-102 Diluted Supernate Samples 24

Figure 4. Heat Capacity Versus Temperature Data for the Actual 241-AN-102 Sr/TRU Precipitate Slurries

\section{LIST OF TABLES}

Table 1. Viscosity and Heat Capacity Testing on LAW Supernates ................................... 4

Table 2. Summary of Rheology Testing Results .......................................................... 14

Table 3. Calorimetry Data for Diluted AN-102 Supernate Samples ................................. 18

Table 4. Calorimetry Data for AN-102 Sr/TRU Precipitate Slurries................................... 19

Table 5. Linear Regression Analysis Results for the Sr/TRU Precipitate Slurry Viscosity Data as a Function of Wt \% Solids 
WSRC-TR-2000-00352

SRT-RPP-2000-00026

This page intentionally left blank. 


\subsection{EXECUTI VE SUMMARY}

Waste in the various storage tanks at the Hanford reservation will be vitrified. Prior to the vitrification process, a series of pretreatment operations will be performed. Among the key pretreatment processes will be the removal of strontium and transuranic elements from some of the tank wastes (i.e. 241-AN-102 and 241-AN-107). The goal of this pretreatment step is to prepare a product stream that is filterable and decontaminated of both strontium and transuranic elements. In addition, all tanks will undergo a decontamination operation to remove cesium and technetium using ion exchange technology. Decontaminated solutions will then be evaporated as necessary to accommodate the necessary glass formulation for vitrification.

The transfer of supernate and slurries at various stages in this complex processing strategy is a necessity. Rheological data are thus key to plant design. Likewise, the solutions will undergo heating at various stages in the processing, so knowledge of heat capacities is important. In order to properly design the facility to accommodate the materials of interest during the processing, a series of physical property measurements have been made on actual tank waste. Both a rheometer and a differential scanning calorimeter were installed in the Shielded Cells Facility of the Savannah River Technology Center and were utilized to obtain the pertinent rheological and calorimetric information requested by the customer. This work was conducted for CH2M Hill Hanford Group, Inc. to satisfy the requirements of the Part B-1 contract. ${ }^{1}$

Note: After installation of the rheometer in the shielded cells facility, the instrumentation demonstrated severe bias in measuring low viscosity samples. Variation in the measured viscosity for repeated tests with the same sample was frequently as large as $50 \%$. Although the sensitivity of the Haake system procured by a former SRTC researcher was already limited for low viscosity samples, the observed errors were even larger than would be normally expected when using this instrumentation in the range (viscosity) of limiting operation that was pertinent to the Part B-1 samples.

In order to utilize the flawed device and attempt to supply rheological information for Part B-1, an empirical method was developed to extract viscosity data. This method was not based on first principles or theoretical foundation with regard to the mathematical understanding and complexity surrounding the discipline of rheology. The developed strategy is rather an attempt to develop an innovative empirical method to supply data for the Part B-1 samples and thus utilize an instrument which had an inherent bias. The alternative to not attempting to develop this empirical method would have been determination of no rheological properties during Part B-1 given the time constraints. 
The following list summarizes the tests conducted and the major conclusions for the conditions evaluated.

- Rheograms were obtained for both as-received and diluted Envelope A (Tank 241-AN103) and C (Tank 241-AN-102) samples and an as-received Envelope B (Tank 241-AZ102) sample.

- Rheograms were obtained for the pretreated Envelope B (Tank 241-AZ-102) evaporator concentrate.

- Rheograms were obtained for the Envelope C sample after full pretreatment (strontium/transuranics precipitation, crossflow filtration, and cesium and technetium ion exchange processing) but prior to evaporation.

- Rheograms were obtained for Envelope C strontium/transuranics precipitate slurries of 2, 10, 13, and 16 weight percent insoluble solids.

- Rheograms were collected for a series of temperatures ranging between 10 and $50{ }^{\circ} \mathrm{C}$.

- All rheograms were described by a Bingham plastic model.

- The temperature dependence of viscosity was described by an Arrhenius-type exponential expression.

- Precipitate sample apparent viscosity data for the Envelope C supernate and Sr/TRU precipitate slurries was incorporated into the temperature-dependant Arrhenius model to produce a modified unified model describing all Envelope $\mathrm{C}$ precipitate apparent viscosity data as a function of temperature and weight percent solids.

- Calorimetric data was obtained for various Envelope $\mathrm{C}$ supernates and precipitates.

- The specific heat was calculated for the various samples tested. 


\subsection{INTRODU CTION AND BACKGROUND}

The Hanford River Protection Project Waste Treatment Plant (RPP-WTP) pretreatment and immobilization processes being developed by the DOE Office of River Protection will decontaminate Low-Activity Waste (LAW) Envelopes A and B supernates using crossflow filtration followed by cesium and technetium ion exchange. Prior to filtration and ion exchange, Envelope $\mathrm{C}$ will undergo strontium/transuranics (Sr/TRU) precipitation to remove radioactive strontium and chelated actinides. The decontaminated supernates will be concentrated in the LAW Melter Feed Evaporator. The concentrated LAW Melter Feed will be mixed with glass-forming minerals and chemicals in the LAW Melter Feed Preparation Tank. The resulting slurry will then be transferred to a Melter Feed Tank from which it will be fed to one of the joule-heated, refractory-lined melters.

The objective of this work was to characterize the physical properties (rheology and calorimetry) of actual tank wastes at various stages of the River Protection Project treatment process. The transfer of supernate and slurries at various stages in this complex processing strategy is a necessity. Likewise, the solution undergoes heating at various stages in the processing and heat capacity data is important. In order to properly design the facility to accommodate the materials of interest during the processing, physical property measurements have been made on actual tank waste. Both a rheometer and a differential scanning calorimeter were installed in the Shielded Cells Facility of the Savannah River Technology Center (SRTC) and were utilized to obtain the pertinent rheological and calorimetric information.

This work was requested by the customer ${ }^{2}$ and the experimental details and Quality Assurance requirements were specified in a Task Plan. ${ }^{3}$ A list of the viscosity and heat capacity tests requested by the customer on LAW supernate samples is provided in Table I along with the status of each test. As indicated in the table, some of the planned tests were not performed because the samples were used for other tests or were composited for return to Hanford, WA in order to satisfy the deadlines for return imposed by the Hanford Waste Treatability Study. 
WSRC-TR-2000-00352

SRT-RPP-2000-00026

Table 1. Viscosity and Heat Capacity Testing on LAW Supernates

\begin{tabular}{|c|c|c|}
\hline Sample & Physical Property & Status \\
\hline \multicolumn{3}{|l|}{ Envelope A } \\
\hline AN-103 (as-received) & Rheology & Completed \\
\hline $\mathrm{AN}-103$ (as-received) & Specific Heat & $\begin{array}{l}\text { Not Completed, sample was } \\
\text { returned to meet treatability } \\
\text { study deadline }\end{array}$ \\
\hline AN-103 (diluted) & Rheology & Completed \\
\hline AN-103 (pretreated, before evaporation) & Rheology and Specific Heat & $\begin{array}{l}\text { Not Completed, all material } \\
\text { utilized for other testing }\end{array}$ \\
\hline \multicolumn{3}{|l|}{ Envelope B } \\
\hline AZ-102 (as-received) & Rheology & Completed \\
\hline AZ-102 (as-received) & Specific Heat & $\begin{array}{l}\text { Not Completed, sample was } \\
\text { returned to meet treatability } \\
\text { study deadline }\end{array}$ \\
\hline AZ-102 (pretreated, prior to evaporation) & Rheology and Specific Heat & $\begin{array}{l}\text { Not Completed, all material } \\
\text { utilized for other testing }\end{array}$ \\
\hline AZ-102 (pretreated and concentrated) & Rheology & Completed \\
\hline \multicolumn{3}{|l|}{ Envelope C } \\
\hline AN-102 (as-received) & Rheology & Completed \\
\hline AN-102 (as-received) & Specific Heat & $\begin{array}{l}\text { Not Completed, sample was } \\
\text { returned to meet treatability } \\
\text { study deadline }\end{array}$ \\
\hline AN-102 (diluted) & Rheology & Completed \\
\hline AN-102 (diluted) & Specific Heat & Completed \\
\hline AN-102 Sr/TRU Precipitate Slurries & Rheology & Completed \\
\hline AN-102 Sr/TRU Precipitate Slurries & Specific Heat & Completed \\
\hline AN-102 (pretreated, prior to evaporation) & Rheology & Completed \\
\hline AN-102 (pretreated, prior to evaporation) & Specific Heat & $\begin{array}{l}\text { Not Completed, sample was } \\
\text { returned to meet treatability } \\
\text { study deadline }\end{array}$ \\
\hline $\begin{array}{l}\text { All work performed under a WFO } \\
\text { agreement with BNFL }\end{array}$ & & \\
\hline
\end{tabular}


Additional evaporation and physical property data (some of which was listed in the Task $\mathrm{Plan}^{2}$ ) was collected on a variety of other sample types under the Part B-1 contract. The samples are listed below with references provided to the reports describing the results.

- As-received and diluted supernate samples (density measurements) ${ }^{4,5,6}$

- Simulant LAW evaporation ${ }^{7,8,9,10}$

- Actual Envelope C (AN-102) pretreated LAW evaporation ${ }^{11,12}$

- Simulant LAW melter feed ${ }^{13}$

- Simulant LAW melter feed with glass formers ${ }^{12}$

- Simulant Sr/TRU precipitate slurry ${ }^{14}$

- Simulant Cs eluate evaporation ${ }^{15}$

- Actual AZ-102 Tc eluate evaporation ${ }^{16}$

The following tests were canceled or modified as indicated.

- There were some differences between the letter from the customer and the task plan regarding the temperatures at which sample viscosities were to be measured. All changes were verbally approved by the customer prior to analysis.

- Particle size, volume \%, and solids solubility tests on the AN-103 and AN-102 samples were canceled due to the fact that insufficient solids were available for analysis.

- Simulant Tc eluate evaporation was canceled. ${ }^{17}$

- Rheology testing was canceled on the HLW melter feed and the feed with glass formers because the AZ-102 sample did not contain sufficient solids and the C-106 sludge sample that was received was not considered representative due to sample and tank histories. ${ }^{18}$

- Tc eluate evaporation was not conducted for actual samples other than AZ-102 because the decision was made not to include the Tc eluates in the HLW melter feed. ${ }^{17}$

- The AA sample was canceled from the test program at SRTC.

Test results for the following samples will be reported in future documents. The likely authors of the reports are indicated.

- Actual Env. C, pretreated LAW melter feed and melter feed with glass formers Zamecnik, J.; Crawford, C.; Koopman, D. (A status report has been issued. ${ }^{19}$ )

- Actual Env. B, pretreated LAW melter feed with glass formers - Schumacher, R.; Crawford, C.; Koopman, D. (Rheology testing was delayed until the final glass former recipe is developed. A status report has been issued. ${ }^{20}$ )

- Actual Cs eluate evaporation - Schumacher, Crawford, Koopman (A status report has been issued. ${ }^{21}$ ) 
WSRC-TR-2000-00352

SRT-RPP-2000-00026

This page intentionally left blank. 


\subsection{EXPERIM ENTAL}

\subsection{RHEOLOGY}

The decision to use the Haake M5 head for the measurement of actual Hanford supernate samples and Sr/TRU precipitate slurries was made by a former researcher in SRTC (Bao Ha). Ha assisted with Part B-1 work and had previous experience in rheological characterization of samples from the Savannah River Site. Ha initiated procurement, mock-up, and remote operation of the rheometer in the SRTC shielded cells facility. He left the company shortly after initiating this work. The authors inherited the instrumentation and the responsibility of modifying, installing, and operating the rheometer for the Part B-1 contract. Appendix A, Part 1 contains figures detailing various aspects of the mock-up efforts. The captions on each figure describe the series of innovative tactics utilized to allow for remoted operation of the rheometer. An important facet of the mock-up was the design and acquisition of a remoted temperature control unit from Haake that allowed for temperature stability of approximately $\pm 0.1{ }^{\circ} \mathrm{C}$. Because the rheological properties of these types of samples are generally observed to be highly temperature-dependent, acquisition of the temperature control unit was deemed to be an important step in the mock-up procedure.

After the initial strategy was developed to allow for remoted operation of the equipment a $95 \mathrm{cP}\left(\right.$ at $\left.25^{\circ} \mathrm{C}\right)$ Newtonian standard oil from Cannon Instrument Company (2139 High Tech Road, State College, PA 16803) was analyzed prior to installation of the unit into the shielded cells. The results are shown in Appendix A, Part 2. The data was accurately described by a Newtonian relation and the fit yielded an apparent viscosity of $98.2 \mathrm{cP}$, which corresponds to $\sim 3 \%$ error from the known value. After this successful demonstration of the equipment's performance, the entire experimental apparatus was installed into the shielded cells.

Initial attempts to characterize lower viscosity samples, after installation of the equipment into the shielded cells, consistently yielded extremely poor reproducibility. Variation in the measured viscosity for repeated tests with the same sample was frequently as large as $50 \%$. Although the sensitivity of the Haake system procured by Ha is already limited for low viscosity samples, the observed errors were even larger than would be normally expected for this instrumentation in this range (viscosity) of limiting operation that is pertinent to the Part B-1 samples. Given that the mock-up using a 95cP oil was successful, this instrumental problem/bias only appeared to be significant for lower viscosity samples. An effort was undertaken to reduce this instrumental bias/error and develop a more reproducible method for the determination of viscosity of the Part B-1 samples. The authors would like to emphasize that removal of the instrument for inspection and repair at this point was prohibitive with respect to both cost and time because the unit had been installed in a radioactive environment. Given the importance of this data in coordination with the schedule and costs, a decision was made to proceed with the development of a method for use with the installed equipment. 
Specifically, the goal was to develop a method to obtain results with high precision and accuracy for a Newtonian standard oil in the range of viscosities expected for the Hanford samples of interest. A $14.8 \mathrm{cP}$ (at $25^{\circ} \mathrm{C}$ ) Newtonian oil was obtained from Cannon Instrument Company for this purpose. The developed method could then be used to obtain the requested viscosity information for Part B-1 samples and eliminate a lengthy and costly delay which would have extended beyond August 2000 and the end of the contract.

Further testing revealed that the instrumental problem was also apparent when the rotor was operated while suspended in air, which has a viscosity under ambient conditions of about $0.02 \mathrm{cP}$ and no yield stress. The rheometer consistently demonstrated a shear rate dependence of increasing shear stress with a non-zero intercept and well-defined slope. The exact functional form of this instrumental bias or offset, as we refer to it, was variable from day to day and ultimately was realized to be the root of the observed lack of accuracy for the low viscosity samples, such as those of interest in this work. Once this realization was made, the authors developed an empirical method to characterize the offset of the rotor suspended in air. Theoretically, there would be a zero response if the instrument was operating in an unbiased manner. This blank was measured prior to each sample and used for background correction/subtraction for the data collected on various unknown samples. Specifically, the instrument was operated in the absence of sample (rotor suspended in air) for the blank run. This operation was from $0-2700 \mathrm{~s}^{-1}$ using a linear ramp over three minutes. The rheometer was held at $2700 \mathrm{~s}^{-1}$ for 30 seconds before a linear ramp back to $0 \mathrm{~s}^{-1}$ was introduced over the final three minutes of the run. This sequence was performed multiple times for the same sample (typically nine) to obtain replicates. The data from $300-1500 \mathrm{~s}^{-1}$ was used for subsequent evaluations (the instrumental bias being corrected for by this method did not allow evaluation below $300 \mathrm{~s}^{-1}$ because of low feedback). This correction method then took the statistical average (mean) of the slope for the blank run and subtracted this signal from the statistical average (mean) of the run containing the actual sample. This correction was used for all subsequent standards and samples.

The results of this strategy are shown in Appendix A, Part 3 as a pre-calibration performed on the standard oil before the Hanford samples were run. This was a validation of the empirical model that was developed to correct for the instrumental bias. Namely, the calculated viscosity using this novel empirical method was $16.7 \mathrm{cP}$ (uncorrected response would have been $34 \mathrm{cP}$ ) at $25^{\circ} \mathrm{C}$ with a known value of $14.8 \mathrm{cP}$. This result translates to a viscosity about $13 \%$ more than the known value for the standard and a $95 \%$ confidence interval of less than $5 \%$ with regard to precision. It is important to note that the observed accuracy is for a sample of about $15 \mathrm{cP}$ and would be expected to decrease as the viscosity of the sample decreases.

The actual Hanford samples were analyzed using this same method. The authors would like to emphasize that this method is not based on first principles or theoretical foundation with regard to the mathematical understanding and complexity surrounding the discipline of rheology. The developed strategy is rather an attempt to develop an innovative empirical method to supply more accurate and precise viscosity data for the Part B-1 samples and thus utilize an instrument which had an inherent bias. 
A post-calibration was conducted following the use of the rheometer for the Part B-1 samples. These results are shown in Appendix A, Part 4 and are interpreted using the same empirical background correction. The post-calibration performed on the Newtonian standard oil at $15^{\circ} \mathrm{C}$ gave a viscosity of $25.9 \mathrm{cP}$. Shown in Appendix A, Part 5 is the temperaturedependent viscosity data supplied by the vendor for the Newtonian standard oil used in the post calibration. This data is well described by an Arrhenious-type relation. A plot of the natural $\log$ of the viscosity versus the value of $1 / \mathrm{T}$ (where $\mathrm{T}$ is the temperature of the sample in Kelvin) is used to linearize the data and provide a convenient format to calculate the viscosity at other temperatures of interest. The resulting evaluation of this data yields a viscosity of $22.6 \mathrm{cP}$ at $15{ }^{\circ} \mathrm{C}$. This result supplied a value that was within $14 \%$ of the known value and agrees well with the pre-calibration data using the designed method.

The authors would like to emphasize that the replicate tests in all cases involved multiple runs of the rheometer using the same aliquot of material in the rheometer cell for evaluation. In addition, the replicate tests were conducted through continuous cycling of the instrument until the desired number of replicates was obtained. In other words, the ramp down in shear rate of a given cycle was immediately followed by a shear ramp up for the following cycle. This was necessary for two reasons. The first is that evaluations such as the remoted operation of a rheometer are extremely tedious and time consuming. Secondly, there is a limited amount of actual Hanford tank waste available. As a result some sample results demonstrate small, time-dependent alterations in viscosity, which are likely the result of a shear-induced modification of the sample. All data for a given sample were averaged which includes the previously discussed time dependent facet of the data. Further work of a more extensive fashion could be performed in the future to better characterize this phenomenon for the Hanford waste.

The customer requested the determination of viscosity for a number of actual Hanford samples with relatively low viscosities. The empirical method was designed as a strategy to overcome the instrumental bias and provide meaningful viscosity data for these samples of interest to the customer. Since the yield stress data was not requested, a corresponding empirical method for determination of yield stress data using the biased instrument was not developed. Yield stress data is provided for some samples, but is of unknown accuracy and was often observed to vary significantly for a given sample. If yield stress data or improved viscosity data is needed by the customer in future work, this will require development of a new empirical test method (if possible) or more preferably procurement, mock-up, and installation of a new Haake M5 head.

Samples for rheological testing were obtained after various stages of processing. All samples were shipped from Hanford and stored at SRTC for various times between tests under ambient conditions. Sample histories are briefly described in the results section below with references to individual reports which provide more detailed sample information. All measurements were performed using the Haake RV30/M5 controller/head combination and the NV sensor. For each sample, a representative aliquot of $9 \mathrm{ml}$ of material was placed in the thermally-jacketed sample cup and allowed to equilibrate for 30 minutes at each temperature of analysis. 
The stress-strain curves were plotted for each replicate analysis over the shear rate range $300-1500 \mathrm{~s}^{-1}$ and fitted by least squares linear regression analysis. Each plot included data acquired under increasing and decreasing shear rate conditions. In every case, the shear stress vs. shear rate curves for both the blanks and the actual samples were well described by the simple linear Bingham Model equation ${ }^{22}$ shown below.

$\sigma=\mathrm{M} \gamma+\mathrm{B}$

where:

$\sigma=$ shear stress $(\mathrm{Pa})$

$\mathrm{M}=$ plastic viscosity (Pa-s)

$\gamma=$ shear rate $(1 / \mathrm{s})$

$\mathrm{B}=$ yield stress $(\mathrm{Pa})$

The slope (M), intercept (B), and residual explanation $\left(\mathrm{R}^{2}\right)$ determined by regression are provided in the appendices. $R^{2}$ values for the blank tests generally were not as high as those observed for the samples, because small differences in the increasing and decreasing shear rate portions of the analysis cycle are more significant when the shear stress feedback is low. The mean, standard deviation (denoted as "sigma"), and 95\% confidence limits (precision) for $\mathrm{M}$ and $\mathrm{B}$ are also provided in the tables. The $95 \%$ confidence limits were calculated from the mean and standard deviation of each series of replicates assuming $\mathrm{N}-1$ degrees of freedom (DF; where $\mathrm{N}=$ number of replicates) and are an indication of precision. Sample viscosities in $\mathrm{cP}$ were calculated from the slope for each replicate test. The average viscosity and the associated $95 \%$ confidence limit for precision are also provided in the associated appendices.

\subsection{CALORIME TRY}

An Instrument Specialists differential scanning calorimeter (Model DSC550E) was remotely installed in the SRTC shielded cells. 20-60 mg of actual tank waste was introduced into a $0.11-\mathrm{mL}$ sealable stainless steel crucible and weighed. All analysis was performed in at least triplicate over the range from 10 to $100{ }^{\circ} \mathrm{C}$ at a heating rate of $10{ }^{\circ} \mathrm{C} / \mathrm{min}$. The reference material used for heat capacity determination was sapphire (mass: $63 \mathrm{mg}$ ). For each experiment, initial runs were conducted with two empty crucibles of equal weight to determine the blank or background. Secondly, a known mass of sapphire standard was placed into the sample disk and compared against the remaining empty crucible. Finally, a third series of replicates was performed after replacing the crucible containing the standard with the weighed tank sample . 
The data were analyzed by simple ratio analysis of the instrument response for the standard and the sample adjusting for the known heat capacity of the standard at each analysis temperature as shown below. Sample and standard data at each temperature were background corrected.

$\mathrm{HC}_{\text {sample }}=\left[\left(\mathrm{mW}_{\text {sample }}-\mathrm{mW}_{\text {empty }}\right) /\left(\mathrm{mW}_{\text {standard }}-\mathrm{mW}_{\text {empty }}\right)\right]^{*}\left[\mathrm{M}_{\text {standard }} / \mathrm{M}_{\text {sample }}\right]^{*} \mathrm{HC}_{\text {standard }}$ where:

$\mathrm{HC}=$ heat capacity

$\mathrm{mW}=$ measured heat flow in milliWatts 
WSRC-TR-2000-00352

SRT-RPP-2000-00026

This page intentionally left blank.

Page 12 of 514 


\subsection{RESULTS}

\subsection{RHEOLOG Y OF AS-RECEIVED AND DILUTED SUPERNATES}

Rheograms were obtained for as-received and diluted supernate samples prior to filtration.

\subsubsection{Envelope A As-Received}

Rheograms were obtained on actual Tank 241-AN-103 as-received sample, which was taken from the as-received archive from compositing and characterization work by Hay. The asreceived sample was 11.7 molar in sodium and contained visible solids $(24.7 \mathrm{wt} \%$ insoluble solids). A summary of the resulting data for the blank and the Envelope A sample at $25^{\circ} \mathrm{C}$ and at $50{ }^{\circ} \mathrm{C}$ are shown in Appendix B. The data for the actual sample are well described by the linear Bingham model as reflected by the high residual explanation $\left(\mathrm{R}^{2}\right)$. The blank experiments also exhibited a linear response. The average apparent viscosity for the Envelope A sample was calculated to be $23.1 \mathrm{cP}$ at $25^{\circ} \mathrm{C}$ and $6.2 \mathrm{cP}$ at $50{ }^{\circ} \mathrm{C}$, respectively, with the $95 \%$ confidence limits indicated in Table 2 .

\subsubsection{Envelope A Diluted}

Rheograms were obtained on the actual AN-103 diluted tank sample. The sample had been diluted to 5.3 molar sodium as described by Hay, causing nearly complete dissolution of the solids. The solids content of the diluted Tank 241-AN-103 sample was $0.1 \mathrm{wt} \%$. A summary of the resulting data for the blank and the diluted Envelope A sample at $25{ }^{\circ} \mathrm{C}$ and $50{ }^{\circ} \mathrm{C}$ are shown in Appendix B. The data for the actual sample are well described by the linear Bingham model as reflected by the high residual explanation $\left(\mathrm{R}^{2}\right)$. The blank experiments also exhibited a linear response. The average apparent viscosity for this sample was calculated to be $3.2 \mathrm{cP}$ at $25^{\circ} \mathrm{C}$ and $2.5 \mathrm{cP}$ at $50^{\circ} \mathrm{C}$ with the confidence intervals indicated in Table 2 .

\subsubsection{Envelope B A s-received}

Rheograms were obtained on actual Tank 241-AZ-102 as-received sample taken from the asreceived archive from compositing and characterization work by Hay. The as-received sample was 2.8 molar in sodium in the supernate fraction and contained visible sludge solids ( 0.48 to $1.2 \mathrm{wt} \%$ insoluble solids). A summary of the resulting data for the blank and the asreceived Envelope B sample at $25^{\circ} \mathrm{C}$ and $50^{\circ} \mathrm{C}$ are shown in Appendix C. The data for the actual sample are well described by the linear Bingham model as reflected by the high residual explanation $\left(\mathrm{R}^{2}\right)$. The blank experiments also exhibited a linear response. The average apparent viscosity for this sample was calculated to be $3.7 \mathrm{cP}$ at $25^{\circ} \mathrm{C}$ and $2.4 \mathrm{cP}$ at $50{ }^{\circ} \mathrm{C}$, with the confidence intervals indicated in Table 2 . 
WSRC-TR-2000-00352

SRT-RPP-2000-00026

Table 2. Summary of Rheology Testing Results

\begin{tabular}{|c|c|c|c|c|c|}
\hline Sample ID & {$[\mathrm{Na}](\mathrm{M})$} & $\begin{array}{l}\text { Wt\% } \\
\text { Solids }\end{array}$ & $\mathbf{T}\left({ }^{\circ} \mathrm{C}\right)$ & $\begin{array}{c}\text { Viscosity } \\
\text { (cP) }\end{array}$ & $95 \% \mathrm{CL}$ \\
\hline \multicolumn{6}{|l|}{ Envelope A (AN-103) } \\
\hline As-received & 11.7 & 24.7 & 25 & 23.1 & 0.6 \\
\hline As-received & 11.7 & --- & 50 & 6.2 & 0.4 \\
\hline Diluted & 5.3 & 0.1 & 25 & 3.2 & 0.5 \\
\hline Diluted & 5.3 & --- & 50 & 2.5 & 0.5 \\
\hline \multicolumn{6}{|l|}{ Envelope B (AZ-102) } \\
\hline As-received & 2.8 & $0.48-1.2$ & 25 & 3.7 & 0.7 \\
\hline As-received & 2.8 & --- & 50 & 2.4 & 0.6 \\
\hline Evap. Conc. & 4.1 & --- & 25 & 2.1 & 0.3 \\
\hline Evap. Conc. & 4.1 & --- & 50 & 0.7 & 0.4 \\
\hline \multicolumn{6}{|l|}{ Envelope C (AN-102) } \\
\hline As-received & 10.2 & $<0.1$ & 25 & 6.7 & 0.6 \\
\hline As-received & 10.2 & --- & 50 & 0.8 & 0.5 \\
\hline Diluted & 6.4 & --- & 25 & 2.7 & 0.5 \\
\hline Diluted & 6.4 & --- & 50 & 2.0 & 0.6 \\
\hline Pretreated & 4.9 & --- & 25 & 4.2 & 0.8 \\
\hline Pretreated & 4.9 & --- & 50 & 2.8 & 0.7 \\
\hline \multicolumn{6}{|c|}{ Envelope C Sr/Tru Precipitate Slurries } \\
\hline 2 wt.\% Sr/TRU Precipitate Slurry & 6 & --- & 10 & 4.4 & 0.3 \\
\hline 2 wt.\% Sr/TRU Precipitate Slurry & 6 & --- & 15 & 4.0 & 0.3 \\
\hline 2 wt.\% Sr/TRU Precipitate Slurry & 6 & 2 & 25 & 3.5 & 0.5 \\
\hline 2 wt.\% Sr/TRU Precipitate Slurry & 6 & --- & 50 & 4.0 & 0.5 \\
\hline 10 wt.\% Sr/TRU Precipitate Slurry & 6 & 10 & 25 & 11.8 & 0.5 \\
\hline 10 wt.\% Sr/TRU Precipitate Slurry & 6 & --- & 50 & 5.5 & 0.5 \\
\hline 13 wt.\% Sr/TRU Precipitate Slurry & 6 & --- & 10 & 21.6 & 0.8 \\
\hline 13 wt.\% Sr/TRU Precipitate Slurry & 6 & --- & 15 & 18.6 & 1 \\
\hline 13 wt.\% Sr/TRU Precipitate Slurry & 6 & 13 & 25 & 14.6 & 1.2 \\
\hline 13 wt.\% Sr/TRU Precipitate Slurry & 6 & --- & 50 & 8.8 & 0.7 \\
\hline 16 wt.\% Sr/TRU Precipitate Slurry & 6 & --- & 10 & 27.5 & 0.4 \\
\hline 16 wt.\% Sr/TRU Precipitate Slurry & 6 & --- & 15 & 23.4 & 0.4 \\
\hline 16 wt.\% Sr/TRU Precipitate Slurry & 6 & 16 & 25 & 18.0 & 0.4 \\
\hline 16 wt.\% Sr/TRU Precipitate Slurry & 6 & --- & 50 & 10.2 & 0.4 \\
\hline
\end{tabular}




\subsubsection{Envelope C As-received}

Rheograms were obtained on actual Tank 241-AN-102 as-received sample, which was taken from the as-received archive from compositing and characterization work by Hay. The asreceived composite sample was 10.2 molar in sodium and contained small amounts of visible solids $(<0.1 \mathrm{wt} \%$ insoluble solids). A summary of the resulting data for the blank and the Envelope $\mathrm{C}$ sample at $25^{\circ} \mathrm{C}$ and at $50^{\circ} \mathrm{C}$ are shown in Appendix D. The data for the actual sample are well described by the linear Bingham model as reflected by the high residual explanation $\left(\mathrm{R}^{2}\right)$. The blank experiments also exhibited a linear response. The average apparent viscosity for this sample was calculated to be $6.7 \mathrm{cP}$ at $25^{\circ} \mathrm{C}$ and $0.8 \mathrm{cP}$ at $50{ }^{\circ} \mathrm{C}$, with the confidence intervals indicated in Table 2.

\subsubsection{Envelope C Diluted}

Rheograms were obtained on actual Tank 241-AN-102 diluted sample which was 6.4 molar in sodium. A summary of the resulting data for the blank and the diluted Envelope C sample at $25^{\circ} \mathrm{C}$ and $50^{\circ} \mathrm{C}$ are shown in Appendix D. The data for the actual sample are well described by the linear Bingham model as reflected by the high residual explanation $\left(\mathrm{R}^{2}\right)$. The blank rheograms also exhibited a linear response. The average apparent viscosity for this sample was calculated to be $2.7 \mathrm{cP}$ at $25^{\circ} \mathrm{C}$ and $2.0 \mathrm{cP}$ at $50{ }^{\circ} \mathrm{C}$, with the confidence intervals indicated in Table 2.

\subsection{RHEOLOGY OF ENVELOPE C SR/TRU PRECIPITATE SLURRIES}

The diluted Tank 241-AN-102 sample was treated to precipitate Sr-90 and transuranic elements by the addition of sodium hydroxide, strontium nitrate, and sodium permanganate reagents at $50{ }^{\circ} \mathrm{C}^{23} \mathrm{~A}$ sample of the resulting slurry, containing both the entrained solids and the $\mathrm{Sr} / \mathrm{TRU}$ precipitate was obtained for rheology measurements. This sample and other slurry precipitate samples with higher wt $\%$ solids loadings were obtained from the Large C Cells Filter campaign. ${ }^{23}$ The Sr/TRU precipitate slurry was processed as a series of seven batches with $\mathrm{Na}^{+}$molarities varying from 5.8 to $6.4 \mathrm{M}$. The sodium concentrations of the concentrate samples isolated for physical property determinations were not measured but are expected to be near $6 \mathrm{M}$ based on the measured sodium levels in the various batches. The slurries were not washed prior to analysis. Weight percent solids in this manuscript refers to total insoluble solids at $25^{\circ} \mathrm{C}$. Fitting of the rheograms was again performed by using a linear fit. Samples containing solids tend to have more complex rheology and thus often require more complex mathematical description. This manuscript recognizes this issue and for simplification has used linear fitting routines. This choice is based on the empirical nature of the linear correction method employed and the desire not to apply the rigor typically associated with unbiased instrumentation. 


\subsubsection{Envelope C 2 Wt \% Sr/TRU Precipitate Slurry}

Rheograms were obtained on the actual Envelope C (241-AN-102) precipitated tank sample slurry prior to crossflow filtration. A summary of the resulting data for the blank and the precipitate slurry at $10,15,25$, and $50^{\circ} \mathrm{C}$ are shown in Appendix E. The data are well described by the linear Bingham model as reflected by the high residual explanation $\left(\mathrm{R}^{2}\right)$. The average apparent viscosities for this sample at $10,15,25$, and $50{ }^{\circ} \mathrm{C}$ were calculated to be 4.4, 4.0, 3.5, and $4.0 \mathrm{cP}$, respectively, with the confidence intervals indicated in Table 2. Given the confidence intervals, it is apparent from the data that the viscosity does not change significantly across the temperature range studied.

\subsubsection{Envelope C 10 Wt \% Sr/TRU Precipitate Slurry}

Rheograms were obtained on actual Envelope $\mathrm{C} \mathrm{Sr}$ /TRU precipitate slurry concentrated to $10 \mathrm{wt} \%$ by crossflow filtration. A summary of the resulting data for the blank and the precipitate slurry at $25{ }^{\circ} \mathrm{C}$ and $50{ }^{\circ} \mathrm{C}$ are shown in Appendix E. The data are well described by the linear Bingham model as reflected by the high residual explanation $\left(\mathrm{R}^{2}\right)$. The average apparent viscosity for this sample was calculated to be $11.8 \mathrm{cP}$ at $25{ }^{\circ} \mathrm{C}$ and $5.5 \mathrm{cP}$ at $50{ }^{\circ} \mathrm{C}$, with the confidence intervals indicated in Table 2.

\subsubsection{Envelope C 13 Wt \% Sr/TRU Precipitate Slurry}

Rheograms were obtained on the actual Envelope C Sr/TRU precipitate slurry concentrated to $13 \mathrm{wt} \%$ by crossflow filtration. A summary of the resulting data for the blank and the precipitate slurry at $10,15,25$ and $50^{\circ} \mathrm{C}$ are shown in Appendix E. The data are well described by the linear Bingham model as reflected by the high residual explanation $\left(\mathrm{R}^{2}\right)$. The average apparent viscosities for this sample at $10,15,25$, and $50^{\circ} \mathrm{C}$ were calculated to be $21.6,18.6,14.6$, and $8.8 \mathrm{cP}$, respectively, with the confidence intervals indicated in Table 2.

\subsubsection{Envelope C 16 Wt \% Sr/TRU Precipitate Slurry}

Rheograms were obtained on actual Envelope $\mathrm{C} \mathrm{Sr}$ /TRU precipitate slurry concentrated to $16 \mathrm{wt} \%$ by a combination of crossflow and deadend filtration. A summary of the resulting data for the blank and the precipitate slurry at $10,15,25$, and $50{ }^{\circ} \mathrm{C}$ is shown in Appendix E. The data are described by the linear Bingham model as reflected by the high residual explanation $\left(\mathrm{R}^{2}\right)$. The average apparent viscosities for this sample at $10,15,25$, and $50{ }^{\circ} \mathrm{C}$ were calculated to be $27.5,23.4,18.0$, and $10.2 \mathrm{cP}$, respectively, with the confidence intervals indicated in Table 2. 


\subsection{RHEOLOG Y OF PRE-TREATED SAMPLES AND CONCENTRATES}

Two samples were tested after all pretreatment steps had been completed. The complete pretreatment process includes $\mathrm{Sr} / \mathrm{TRU}$ precipitation (only for Envelope $\mathrm{C}$ samples), filtration, and cesium and technetium ion exchange. The Envelope B (241-AZ-102) sample was pretreated and concentrated by evaporation prior to rheology testing. Rheology testing was also conducted on an Envelope C sample (241-AN-102) after pretreatment but before evaporation. The Envelope $\mathrm{C}$ sample was pretreated in two portions. A smaller portion of the sample $(\sim 0.5 \mathrm{~L})$ was treated first, in order to confirm that the Sr/TRU process would sufficiently decontaminate the sample without interfering with other downstream processes (e.g. ion exchange). The remainder of the sample $(\sim 17 \mathrm{~L})$ was treated after the small sample had been successfully processed.

Rheograms were obtained using a sample from the $17 \mathrm{~L}$ run of the Envelope $\mathrm{C}$ sample after full pretreatment, but prior to evaporation.

\subsubsection{Envelope B E vaporator Concentrate}

Rheograms were obtained on the actual Envelope B (241-AZ-102) evaporator concentrate. The sample history for the evaporator concentrate included (sequentially) deadend filtration, ${ }^{5}$ cesium ion exchange, ${ }^{24}$ technetium ion exchange, ${ }^{24}$ and evaporation to 4.1 molar sodium in preparation for subsequent vitrification testing. It should be noted that the Env. B sample was diluted during ion exchange processing to $2.2 \mathrm{M} \mathrm{Na}^{+}(\sim 20 \%)$ with $\mathrm{NaOH}$ solutions $(0.25-1.0 \mathrm{M})$ used to pretreat the ion exchange columns (see reference 8 ). A summary of the resulting data for the blank and the evaporator concentrate at $25^{\circ} \mathrm{C}$ and $50{ }^{\circ} \mathrm{C}$ are shown in Appendix C. The data are well described by the linear Bingham model as reflected by the high residual explanation $\left(\mathrm{R}^{2}\right)$. The average apparent viscosities for this sample at $25^{\circ} \mathrm{C}$ and $50^{\circ} \mathrm{C}$ were calculated to be $2.1 \mathrm{cP}$ and $0.7 \mathrm{cP}$, respectively, with the confidence intervals indicated in Table 2.

\subsubsection{Envelope C Pretreated Liquid}

Rheograms were obtained on the actual 241-AN-102 Envelope C product after Sr/TRU precipitation and crossflow filtration, ${ }^{7}$ and cesium and technetium ion exchange processing ${ }^{23}$. The pretreated liquid was 4.9 molar in sodium. The liquid sample had not been processed in an evaporator. Characterization of this product is reported in the ion exchange report. A summary of the resulting data for the blank and the pretreated Envelope $\mathrm{C}$ sample at $25^{\circ} \mathrm{C}$ and $50^{\circ} \mathrm{C}$ are shown in Appendix D. The data for the actual sample are well described by the linear Bingham model as reflected by the high residual explanation $\left(\mathrm{R}^{2}\right)$. The blank experiments also exhibited a linear response. The average apparent viscosities for this sample at $25^{\circ} \mathrm{C}$ and $50{ }^{\circ} \mathrm{C}$ were calculated to be $4.2 \mathrm{cP}$ and $2.8 \mathrm{cP}$, respectively, with the confidence intervals indicated in Table 2 . 


\subsection{CALORIME TRY}

\subsubsection{Calorimetry of Envelope C (AN-102) Diluted Supernate}

Three dilutions of the as-received AN-102 sample (10.2 $\left.\mathrm{M} \mathrm{Na}^{+}\right)$were conducted to give separate solutions containing 5, 7, and $9 \mathrm{M} \mathrm{Na}^{+}$. Calorimetry experiments were conducted on each sample. Table 3 shows the averaged heat flow data observed for the experiments with the empty crucible, the standard, and the actual sample. The calculated heat capacity data are also provided along with an estimate of the error associated with each. Propagation of errors analysis was conducted for each data point using the standard deviations calculated from replicate analyses to determine the error. Sigma $(\sigma)$ corresponds to the calculated best guess as to the standard deviation of the derived heat capacity values. The coefficient of variation $(\mathrm{CV})$ is the ratio of $\sigma$ to the calculated heat capacity.

Table 3. Calorimetry Data for Diluted AN-102 Supernate Samples

\begin{tabular}{|c|c|c|c|c|c|c|}
\hline $\mathbf{T}\left({ }^{\circ} \mathbf{C}\right)$ & $\begin{array}{c}\text { Sample } \\
\text { (mW) }\end{array}$ & $\begin{array}{c}\text { Standard } \\
(\mathrm{mW})\end{array}$ & $\begin{array}{l}\text { Empty } \\
\text { (mW) }\end{array}$ & $\begin{array}{c}\mathrm{HC} \\
\text { Cal./g }{ }^{\circ} \mathrm{C}\end{array}$ & $\begin{array}{c}\sigma \\
\text { Cal../g }{ }^{\circ} \mathbf{C}\end{array}$ & CV \\
\hline \multicolumn{7}{|c|}{$5 \mathrm{M} \mathrm{Na}^{+} \mathrm{AN}-102$} \\
\hline 40 & -30.28 & -13.04 & -1.70 & 0.70 & 0.07 & $10.2 \%$ \\
\hline 50 & -30.16 & -13.21 & -2.03 & 0.71 & 0.07 & $10.0 \%$ \\
\hline 60 & -29.91 & -13.48 & -2.34 & 0.72 & 0.06 & $8.9 \%$ \\
\hline 70 & $-29-66$ & -13.47 & -2.60 & 0.74 & 0.06 & $8.6 \%$ \\
\hline 80 & -29.45 & -13.61 & -2.88 & 0.75 & 0.07 & $9.3 \%$ \\
\hline 90 & -29.10 & -13.75 & -3.10 & 0.75 & 0.07 & $9.8 \%$ \\
\hline \multicolumn{7}{|c|}{$7 \mathrm{M} \mathrm{Na}^{+} \mathrm{AN}-102$} \\
\hline 40 & -38.45 & -13.04 & -1.70 & 0.65 & 0.07 & $10.8 \%$ \\
\hline 50 & -37.84 & -13.21 & -2.03 & 0.65 & 0.07 & $10.6 \%$ \\
\hline 60 & -37.32 & -13.48 & -2.34 & 0.65 & 0.06 & $9.7 \%$ \\
\hline 70 & -36.89 & -13.47 & -2.60 & 0.67 & 0.06 & $9.6 \%$ \\
\hline 80 & -36.33 & -13.61 & -2.88 & 0.67 & 0.07 & $10.3 \%$ \\
\hline 90 & -35.81 & -13.75 & -3.10 & 0.68 & 0.07 & $10.8 \%$ \\
\hline \multicolumn{7}{|c|}{$9 \mathrm{M} \mathrm{Na}^{+} \mathrm{AN}-102$} \\
\hline 40 & -37.14 & -13.04 & -1.70 & 0.68 & 0.07 & $10.7 \%$ \\
\hline 50 & -36.48 & -13.21 & -2.03 & 0.68 & 0.07 & $10.5 \%$ \\
\hline 60 & -35.57 & -13.48 & -2.34 & 0.68 & 0.06 & $9.6 \%$ \\
\hline 70 & -34.70 & -13.47 & -2.60 & 0.68 & 0.06 & $9.4 \%$ \\
\hline 80 & -33.96 & -13.61 & -2.88 & 0.68 & 0.07 & $10.0 \%$ \\
\hline 90 & -33.18 & -13.75 & -3.10 & 0.68 & 0.07 & $10.5 \%$ \\
\hline
\end{tabular}




\subsubsection{Calorimetry of Envelope C (AN-102) Sr/TRU Precipitate Slurries}

Calorimetry experiments were conducted on AN-102 Sr/TRU precipitate slurries containing 13 and $15 \mathrm{wt} \%$ insoluble solids. Table 4 shows the averaged heat flow data observed for the experiments with the empty crucible, the standard, and the actual sample. The calculated heat capacity data are also provided along with an estimate of the error associated with each.

Table 4. Calorimetry Data for AN-102 Sr/TRU Precipitate Slurries

\begin{tabular}{|c|c|c|c|c|c|c|}
\hline $\mathbf{T}\left({ }^{\circ} \mathbf{C}\right)$ & $\begin{array}{c}\text { Sample } \\
(\mathrm{mW})\end{array}$ & $\begin{array}{c}\text { Standard } \\
(\mathrm{mW})\end{array}$ & $\begin{array}{c}\text { Empty } \\
\text { (mW) }\end{array}$ & $\begin{array}{c}\mathbf{H C} \\
\text { Cal./g }{ }^{\circ} \mathrm{C}\end{array}$ & $\begin{array}{c}\sigma \\
\text { Cal. } / \mathbf{g}^{\circ} \mathbf{C}\end{array}$ & CV \\
\hline \multicolumn{7}{|l|}{13 wt. $\%$} \\
\hline 35 & -26.72 & -28.67 & 7.28 & 0.49 & 0.03 & $5.9 \%$ \\
\hline 45 & -26.44 & -29.02 & 7.70 & 0.49 & 0.03 & $5.3 \%$ \\
\hline 55 & -26.03 & -29.44 & 8.09 & 0.49 & 0.02 & $4.4 \%$ \\
\hline 65 & -25.74 & -29.73 & 8.40 & 0.50 & 0.02 & $4.1 \%$ \\
\hline 75 & -25.62 & -29.80 & 8.57 & 0.50 & 0.02 & $4.1 \%$ \\
\hline 85 & -25.57 & -30.07 & 8.70 & 0.51 & 0.02 & $4.1 \%$ \\
\hline \multicolumn{7}{|l|}{15 wt. $\%$} \\
\hline 35 & -72.57 & -28.67 & 7.28 & 0.68 & 0.03 & $4.8 \%$ \\
\hline 45 & -72.03 & -29.02 & 7.70 & 0.68 & 0.04 & $5.1 \%$ \\
\hline 55 & -71.51 & -29.44 & 8.09 & 0.68 & 0.03 & $5.1 \%$ \\
\hline 65 & -71.00 & -29.73 & 8.40 & 0.68 & 0.03 & $4.8 \%$ \\
\hline 75 & -70.62 & -29.80 & 8.57 & 0.69 & 0.03 & $4.6 \%$ \\
\hline 85 & -70.82 & -30.07 & 8.70 & 0.70 & 0.04 & $5.4 \%$ \\
\hline
\end{tabular}


WSRC-TR-2000-00352

SRT-RPP-2000-00026

This page intentionally left blank. 


\subsection{DISCUSSI ON}

\subsection{RHEOLOG Y}

\subsubsection{Rheology of Sr/TRU Precipitate Slurry Samples}

The viscosity of the Sr/TRU precipitate slurries is directly related to wt \% solids. The slurry viscosities exhibit a linear relationship with wt \% solids as shown in Table 5 and Figure 1. Least squares linear regression analysis of the data at each temperature reveals that the slope gradually decreases from 1.6 to 0.45 across the temperature range 10 to $50{ }^{\circ} \mathrm{C}$. The fact that the viscosity data remains linear as a function of wt $\%$ solids at each analysis temperature suggests that either the wt \% solids does not change across the temperature range analyzed for each sample or the wt \% solids changes by the same amount at a given temperature for every sample.

Table 5. Linear Regression Analysis Results for the Sr/TRU Precipitate Slurry Viscosity Data as a Function of Wt \% Solids

\begin{tabular}{|c|c|c|}
\hline $\mathbf{T}\left({ }^{\circ} \mathbf{C}\right)$ & Regression Fit & $\mathbf{R}^{\mathbf{2}}$ \\
\hline 10 & $\mathrm{y}=1.6267 \mathrm{x}+1.0242$ & 1.00 \\
\hline 15 & $\mathrm{y}=1.3699 \mathrm{x}+1.1773$ & 1.00 \\
\hline 25 & $\mathrm{y}=1.0283 \mathrm{x}+1.4352$ & 1.00 \\
\hline 50 & $\mathrm{y}=1.4457 \mathrm{x}+2.5561$ & 0.88 \\
\hline
\end{tabular}

Shown in the Figure 2 are the temperature-dependant average apparent viscosities as determined for the AN-102 supernate and precipitate slurries as a function of temperature. As a simplification tool an empirical model was developed to relate the apparent viscosity to temperature and weight percent solids for these samples. Because all samples were described by a Bingham fit, the apparent viscosity can be explained as a function of weight percent insoluble solids and temperature. The apparent viscosity is independent of the shear rate. An Arrhenius expression was used to fit each individual Envelope $\mathrm{C}$ sample as a function of temperature. A plot of the natural log of the apparent viscosity versus the reciprocal of the temperature in Kelvin yielded the parameters of interest. The y-intercept is defined as the natural log of the pre-exponential term and the slope of the fit is equal to the energy of flow activation divided by the gas constant $\mathrm{R}$. The pre-exponential and slope was determined for each sample. Each of these terms was then linearized as a function of weight percent solids. 


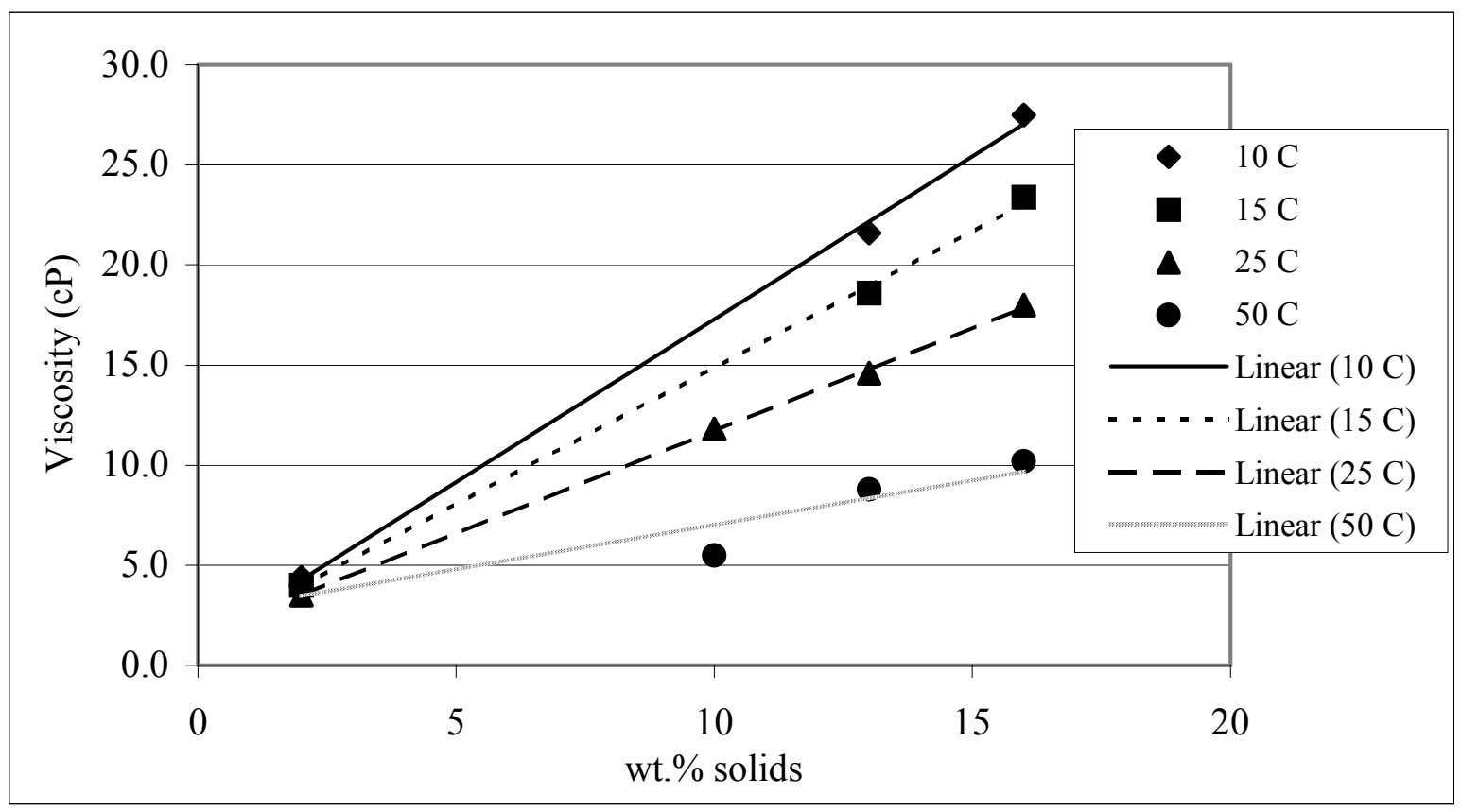

\section{Figure 1. Plot of Actual AN-102 Sr/TRU Precipitate Slurry Viscosity Data Versus Wt \% Solids for Each Analysis Temperature}

Because the weight percent solids are known at $25^{\circ} \mathrm{C}$ as well as the temperature dependence from the Arhenious fit the unified relation could be established. The assumption is that coincident temperature effects on weight percent solids only change the amount of solids and not the type or characteristics with respect to rheology for the limited range evaluated. The unification of the model was performed at $25^{\circ} \mathrm{C}$ where the solids were known. The model assumes the user knows the $\mathrm{wt} \%$ solids and the temperature for the system of interest and predicts the viscosity. The model does not predict weight percent solid or temperature.

The final unified and empirical model that resulted was an Arrhenius-type exponential with the linearized functional fits determined for the effects of weight percent solids embedded into the Arrhenius expression. The end result was a single empirical function relating the apparent viscosity to shear rate, temperature, and insoluble solids. 
Env. C Tank 241-AN-102 Rheology

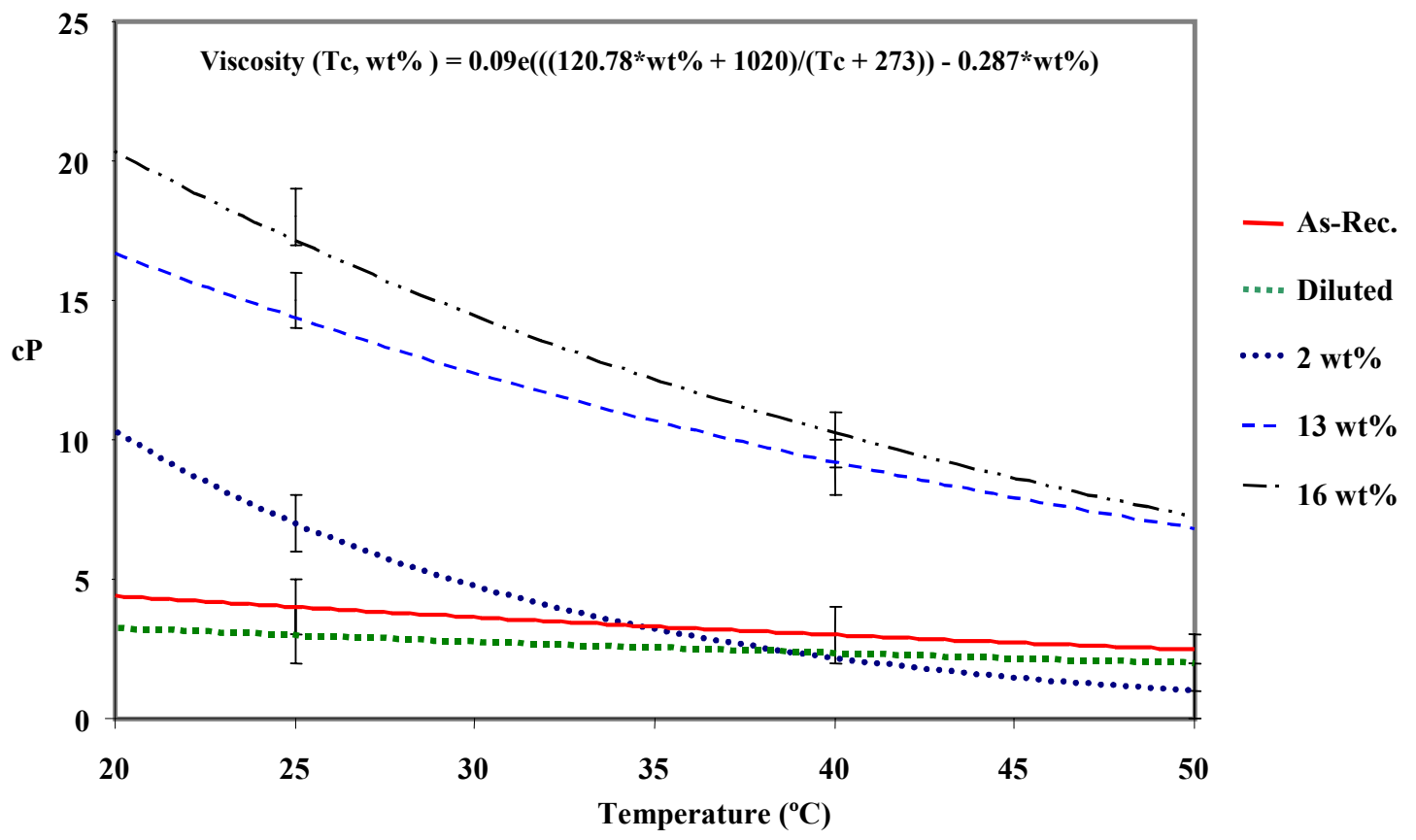

Figure 2. Temperature-dependent Average Apparent Viscosities for the AN-102 Supernate and Precipitate Slurries as a Function of Temperature

[The equation at the top of the figure relates apparent viscosity to temperature and $\mathrm{wt} \%$ solids for all $\mathrm{AN}-102$ samples. $\left.\mathrm{Tc}=\mathrm{T}\left({ }^{\circ} \mathrm{C}\right).\right]$

\subsection{CALORIME TRY}

\subsubsection{Calorimetry of Envelope C (AN-102) Diluted Supernate}

A plot of the heat capacity values observed for the diluted AN-102 supernate samples as a function of temperature $\left(40-90^{\circ} \mathrm{C}\right)$ is shown in Figure 3. The heat capacity values obtained at each sodium concentration and temperature are not distinguishable, based on the propagation of error analysis reported in Table 3 . 
WSRC-TR-2000-00352

SRT-RPP-2000-00026

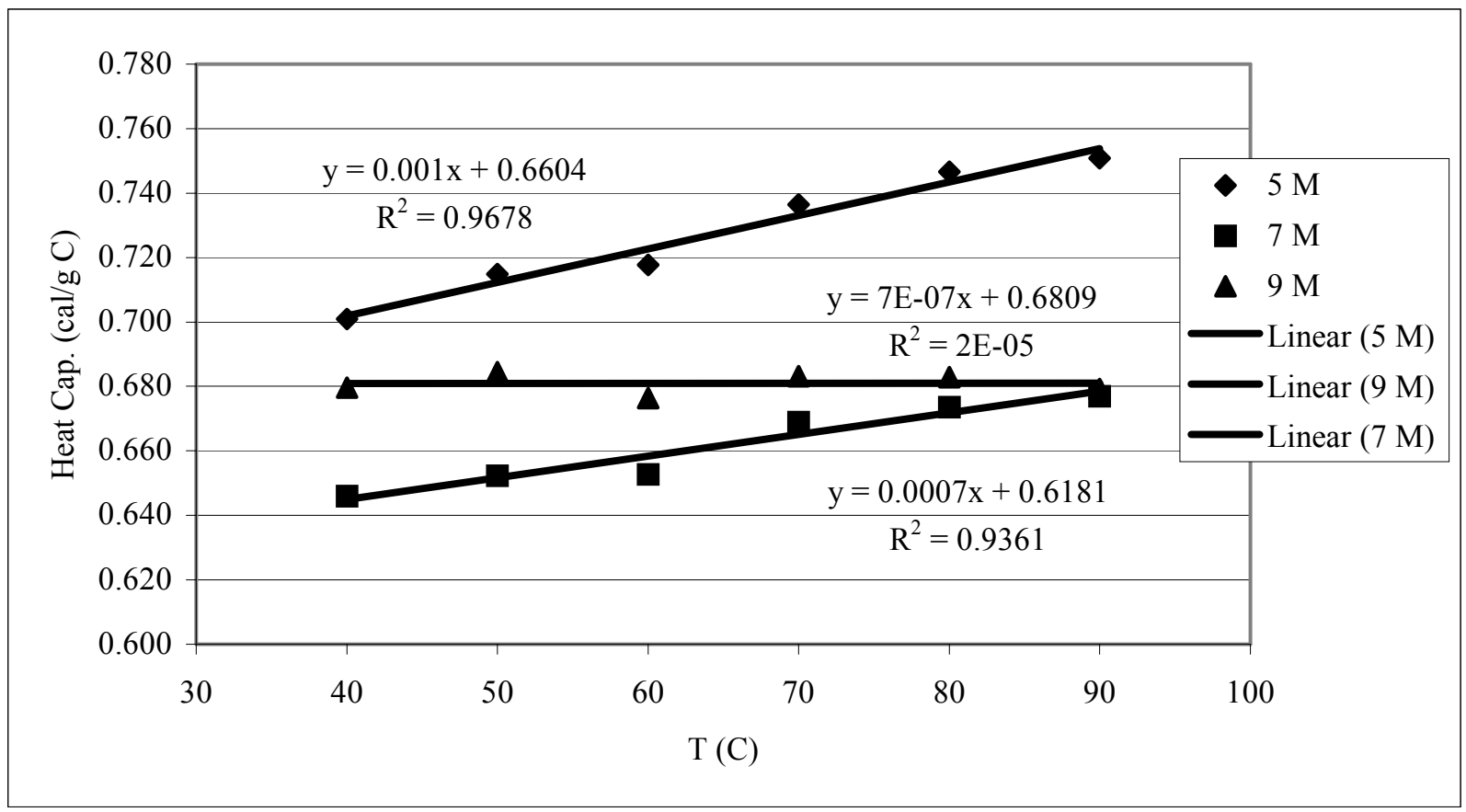

Figure 3. Heat Capacity Versus Temperature Data for the Actual 241-AN-102 Diluted Supernate Samples 


\subsubsection{Calorimetry of Envelope C (AN-102) Sr/TRU Precipitate Slurries}

A plot of the heat capacity values observed for the AN-102 Sr/TRU precipitate slurries as a function of temperature $\left(35-85^{\circ} \mathrm{C}\right)$ is shown in Figure 4. Propagation of error analysis (Table 4) indicates that the results obtained for the two samples are statistically different with respect to $\mathrm{wt} \%$ solids. The analysis of error also strongly suggests that there is no temperature dependence of heat capacity for either sample for the temperature range reported. The decrease in heat capacity observed in going from 13 to $15 \mathrm{wt} \%$ solids seems large and could possibly be attributed to differences in composition (perhaps total solids). Further evaluation would be necessary in order to more definitively understand this observation.

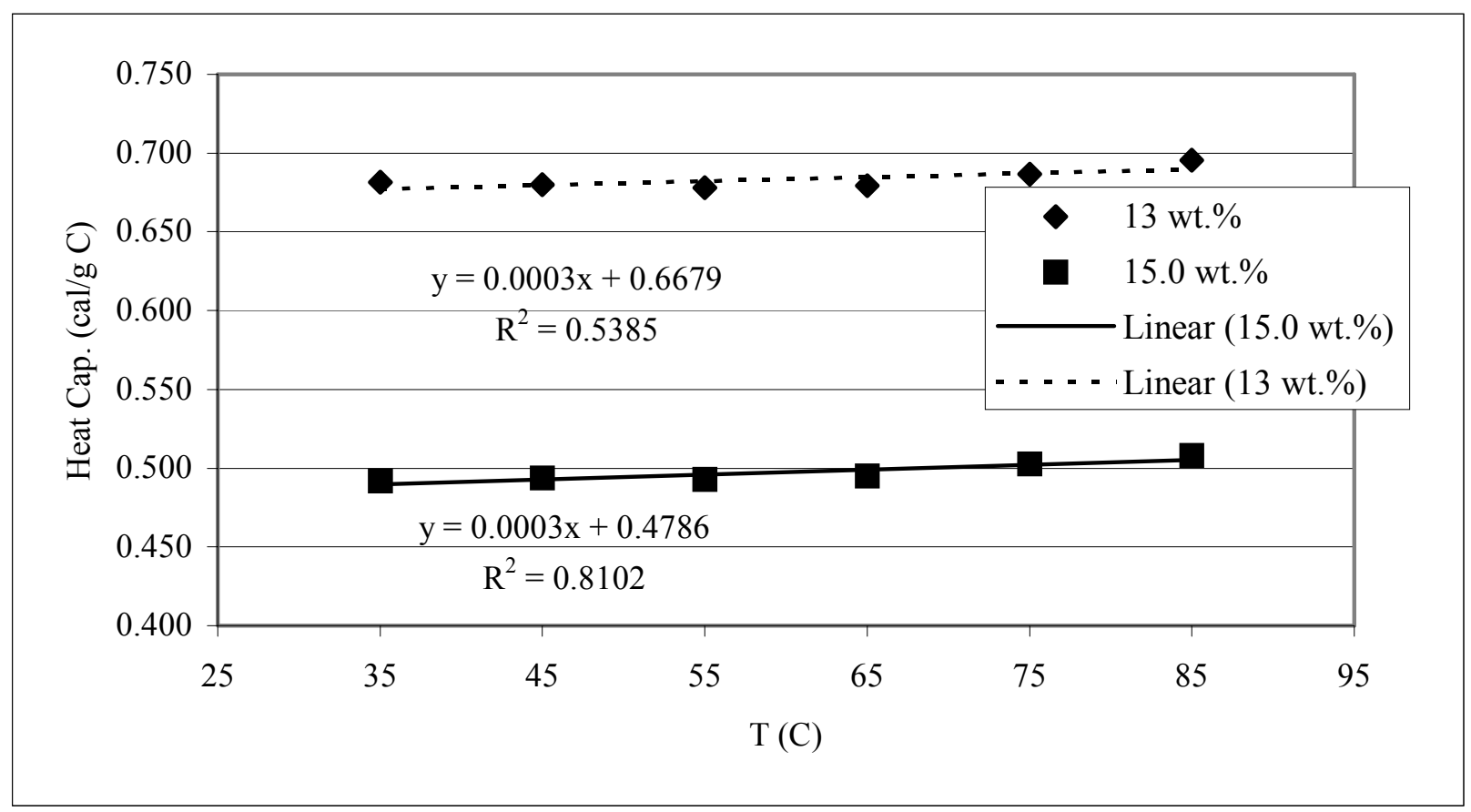

Figure 4. Heat Capacity Versus Temperature Data for the Actual 241-AN-102 Sr/TRU Precipitate Slurries 
WSRC-TR-2000-00352

SRT-RPP-2000-00026

This page intentionally left blank. 


\subsection{CONCLUS IONS/SUMMARY}

Rheograms were obtained for a number of actual Hanford sample supernates, both asreceived and diluted, and for two samples which had been subjected to the full pretreatment process. At $25^{\circ} \mathrm{C}$, the as-received Envelope A sample exhibited the highest observed viscosity of all supernate samples tested $(23.1 \mathrm{cP})$. The high viscosity is most likely due to the high wt \% solids observed for this sample. All other supernate viscosities were below 10 $\mathrm{cP}$ at all temperatures measured. In general, the supernate viscosities appear to be intermediate between the viscosities of sodium hydroxide and sodium nitrate solutions at similar concentrations (viscosities of $6 \mathrm{M}$ solutions at $25^{\circ} \mathrm{C}: \mathrm{NaOH}-4.6 \mathrm{cP} ; \mathrm{NaNO}_{3}-2.2$ $\mathrm{cP})$. Rheograms obtained for the $\mathrm{Sr} / \mathrm{TRU}$ precipitate slurries indicate, as expected, increase in viscosity with wt \% insoluble solids. The viscosity of the $\mathrm{AN}-102$ samples at $25{ }^{\circ} \mathrm{C}$ was observed to increase from near $3 \mathrm{cP}$ at $0 \mathrm{wt} \%$ solids to $18 \mathrm{cP}$ at $16 \mathrm{wt} \%$ solids.

Heat capacity data were obtained for the diluted AN-102 sample at various concentrations. Error analysis indicates that the heat capacities of supernate samples at 5, 7, and $9 \mathrm{M} \mathrm{Na}^{+}$ were not significantly different. The average heat capacity observed for the diluted supernate samples at $40^{\circ} \mathrm{C}$ was 0.68 cal. $/ g$ ' $\mathrm{C}$. Heat capacity data obtained for AN-102 SR/TRU precipitate slurries was lower than was observed for the $\mathrm{AN}-102$ supernate. At $35^{\circ} \mathrm{C}$ the average heat capacity observed for the 13 and $15 \mathrm{wt} \%$ slurries was $0.59 \mathrm{cal} . / \mathrm{g}{ }^{\circ} \mathrm{C}$. 
WSRC-TR-2000-00352

SRT-RPP-2000-00026

This page intentionally left blank. 


\subsection{REFEREN CES}

${ }^{1}$ TWRS Privatization, Contract No. DE-RP06-96RL13308, Modification No. A005.

${ }^{2}$ Letter number 000115 dated October 1, 1998 from M. E. Johnson, BNFL Inc. to S. Wach, Westinghouse Savannah River Company, "TWRS-P Contract No. DE-AC06-96RL13308 W375 - Physical Properties Measurements for Process Solutions - Action Item 09/17/9821."

3 Rosencrance, S.; Nash, C. "Task Technical and Quality Assurance Plan for Physical Characterization,” BNF-003-98-0037, Rev. 0, January 12, 1999.

${ }^{4}$ Hay, M. "Chemical Characterization of an Envelope A Sample from Hanford Tank 241AN-103,” BNF-003-98-248, Rev. 0, July 31, 2000.

${ }^{5}$ Hay, M. "Chemical Characterization of an Envelope B/D Sample from Hanford Tank 241AN-103,” BNF-003-98-249, Rev. 0, July 31, 2000.

${ }^{6}$ Hay, M. "Chemical Characterization of an Envelope C Sample from Hanford Tank 241AN-102," BNF-003-98-250, Rev. 0, July 31, 2000.

${ }^{7}$ Monson, P. R. "Env. A, B and C Shaker/Bath Evaporation Saturation Studies," BNFL-00398-0189, SRT-PTD-99-0062, Rev.0, January 4, 2000.

${ }^{8}$ Calloway, Jr., T. B. "Evaporation of Hanford Env. A Simulant (AN-105)," WSRC-TR2000-00300, SRT-RPP-2000-00010, Rev. 0, August, 2000.

${ }^{9}$ Calloway, Jr., T. B.; Choi, A. S.; Monson, P. R. "Evaporation of Hanford Env. B Simulant (AZ-101) Preliminary Report,” BNF-003- 98-0166, Rev. 1, January 6, 2001.

${ }^{10}$ Saito, H. H.; Calloway, Jr., T. B.; Ferrara, D. M.; Choi, A. S.; White, T. L.; Gibson, Jr., L. V.; Burdette, M. A. "AN-107 (C) Simulant Bench-Scale LAW Evaporation with Organic Regulatory Analysis," WSRC-TR-2000-00486, SRT-RPP-2000-00047, Rev. 0, February, 2001.

${ }^{11}$ Hay, M., Coleman, C., Hassan, N. M., McCabe, D. J., King, W. D., Nash, C., Saito, H. H., Siler, J., Calloway, B., Crawford, C. "Sulfate Removal for the River Protection Project B1 (U),”WSRC-TR-2000-489, SRT-RPP-2000-49, Rev. 0, January, 2001.

${ }^{12}$ Crowder, M. L.; Crawford, C. L.; Saito, H. H.; Calloway, T. B. "Bench-Scale Evaporation of a Large Hanford Envelope C Sample (Tank 241-AN-102)," SRT-RPP-2000-00043, Rev. 0. 
${ }^{13}$ Hansen, E. K.; Calloway, Jr.,T. B. "Characterization of Simulant LAW Env. A, B, and C with Glass Formers,” WSRC-TR-2000-00298, SRT-RPP-2000-00004, Rev. 0, Aug, 24, 2000 .

${ }^{14}$ Duignan, M. R. "Final Report: Pilot-scale Cross-flow Ultrafiltration Test Using a Hanford Site Tank 241-AN-107 Waste Simulant - Envelope C + Entrained Solids + SrontiumTransuranic Precipitation,” BNF-003-98-0226, Rev. 0, March 24, 2000.

${ }^{15}$ Baich, M. A. "Cesium Eluate Physical Properties Determination,” WSRC-TR-2000-00306, SRT-RPP-2000-00012, Rev. 0, April 24, 2000.

16 King, W. D.; Calloway, Jr., T. B. "Tank 241-AZ-102 SuperLig 639 Technetium Ion Exchange Eluate Evaporation Study," WSRC-TR-2000-00424, SRT-RPP-2000-00024, Rev. 0, November 30, 2000.

17 Johnson, M. E. "Eluate Physical Properties and Evaporation Test Specification," TSPW375-99-00008, Rev. 3, December 6, 1999.

${ }^{18}$ Letter number 005554 dated August, 19, 1999 from M. E. Johnson, BNFL Inc. to S. Wach, Westinghouse Savannah River Company, "C-106 to Replace AZ-102 Solids for High-Level Waste Tests."

${ }^{19}$ Hutson, N. D.; et.al. "Vitrification of Pretreated Large C (AN-102) Sample - Preliminary Status Report”, SRT-RPP-2001-00009, January 15, 2001.

${ }^{20}$ Crawford, C. L.; Ferrara, D. M.; Schumacher, R. F.; Bibler; N. E. "Status Report for Crucible Scale Vitrification of Waste Envelope B (AZ-102)," SRT-RPP-2001-00004, Rev. 0.

21 Schumacher, R.F.; Crawford, C.L.; Bibler, N.E. "Status Report for Crucible Scale Vitrification of Waste Envelope D (C-106)," SRT-RPP-2001-00003, Rev. 0, January 2001.

${ }^{22}$ Bird, R. B.; Stewart, W. E.; Lightfoot, E. N. Transport Phenomena, John Wiley and Sons, New York, 1960.

${ }^{23}$ Nash, C.; Saito, H. H. "Strontium-Transuranic Precipitation and Crossflow Filtration of 241-AN-102 Large C,” SRT-RPP-2001-00006, Rev. 0, January, 2000.

${ }^{24}$ King, W. D.; McCabe, D. J.; Hassan, N. M. "Intermediate-Scale Ion Exchange Removal of Cesium and Technetium from Hanford Tank 241-AN-102," BNF-003-98-0244, Rev. 0, January 17, 2001. 
WSRC-TR-2000-00352

SRT-RPP-2000-00026

This page intentionally left blank. 
WSRC-TR-2000-00352

SRT-RPP-2000-00026

This page intentionally left blank. 
WSRC-TR-2000-00352

SRT-RPP-2000-00026

This page intentionally left blank.

Page 33 of 514 
WSRC-TR-2000-00352

SRT-RPP-2000-00026

This page intentionally left blank.

Page 34 of 514 


\title{
APPENDIX A
}

\author{
PART 1 Page 37 \\ PHOTOGRAPHS OF RHEOMETER REMOTING EFFORTS
}

\author{
PART 2 Page 51 \\ RHEOMETER CALIBRATION PRIOR TO SHIELDED CELLS INSTALLATION \\ WITH THE 95 cP STANDARD
}

\author{
PART 3 Page 55 \\ RHEOMETER PRE-CALIBRATION IN THE SHIELDED CELLS \\ WITH THE LOW VISCOSITY STANDARD \\ PART 4 Page 75 \\ RHEOMETER POST-CALIBRATION IN THE SHIELDED CELLS \\ WITH THE LOW VISCOSITY STANDARD
}

PART 5 Page 93

TEMPERATURE DEPENDENCE OF VISCOSITY

FOR THE LOW VISCOSITY STANDARD OIL 
This page intentionally left blank. 


\section{APPENDIX A - PART 1}

\section{PHOTOGRAPHS OF RHEOMETER REMOTING EFFORTS}

Figure 1. Front view of all components of customized remoted unit in mockup

Figure 2. Front view of specially designed remoted Haake DC5 temperature control unit........................40

Figure 3. Connectors positioned on top of KAPL plug for DC5 temperature control unit ........................41

Figure 4. Top view of cup for the NV sensor system resting inside of temperature control jacket...........42

Figure 5. Front view of cup for the NV sensor system resting inside of temperature control jacket ........43

Figure 6. Front view of cut for the system in mockup .........................................................................4

Figure 7. Front view of special jack to accommodate sample system remotely for use in shielded cell...45

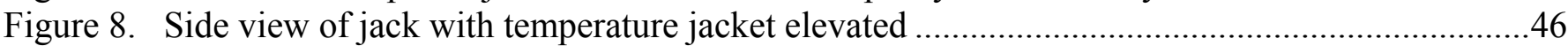

Figure 9. Front view prior to raising jack to position rotor inside sample cup ..........................................47

Figure 10. Side view of manipulator turning crank to raise jack and place rotor inside cup......................48

Figure 11. Side view of properly positioned sample cup following elevation of jack...............................49 


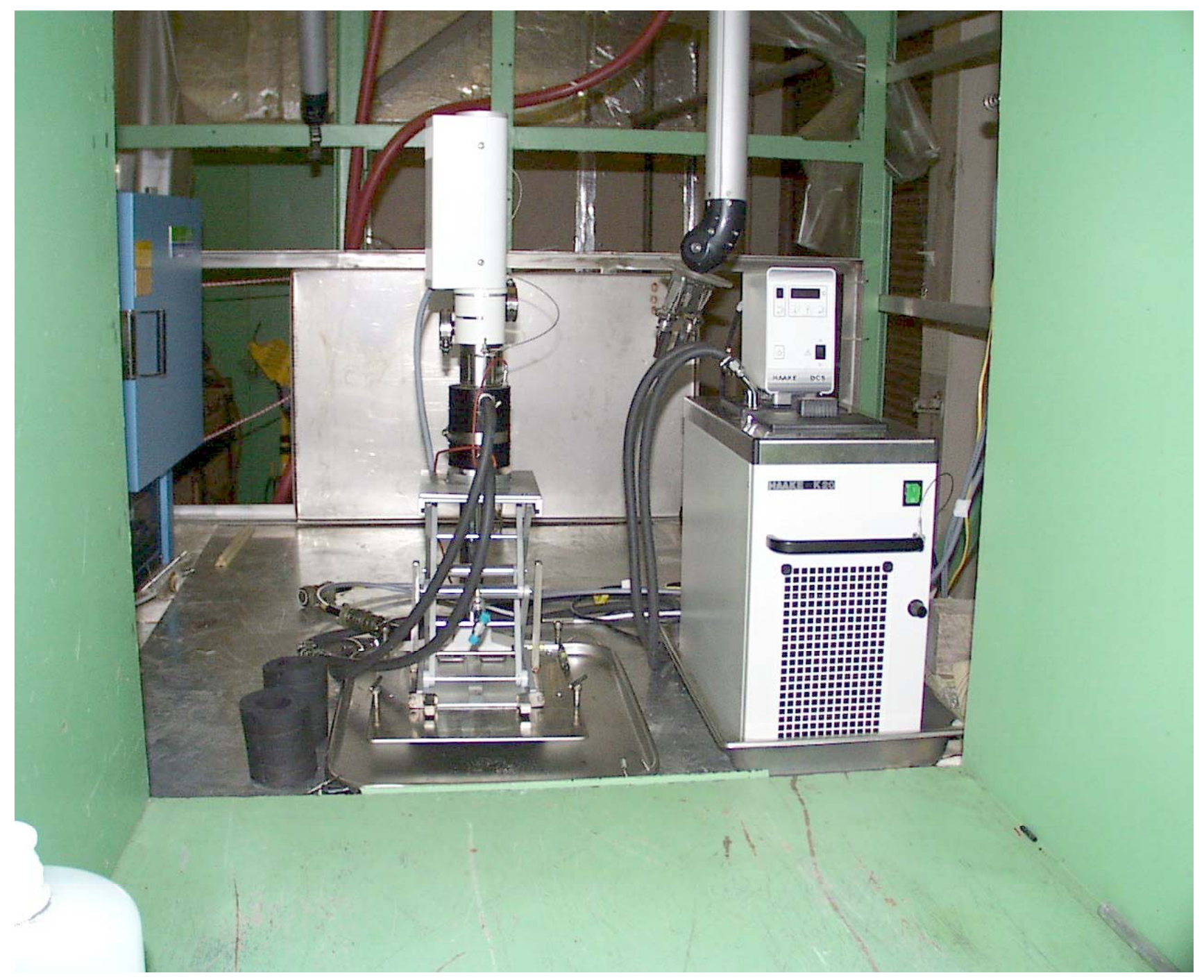

Figure 1. Front view of all components of customized remoted unit in mockup 


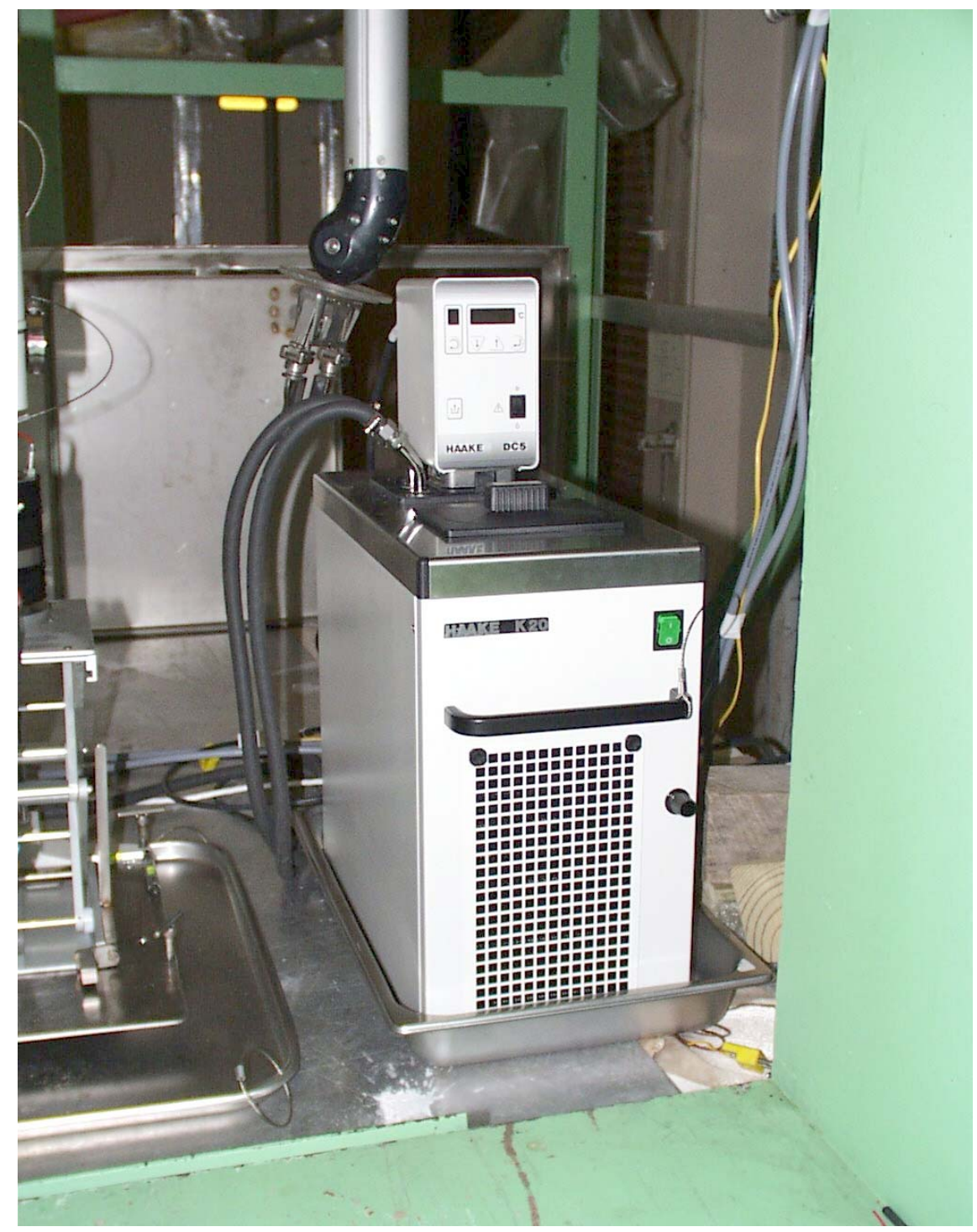

Figure 2. Front view of specially designed remoted Haake DC5 temperature control unit 


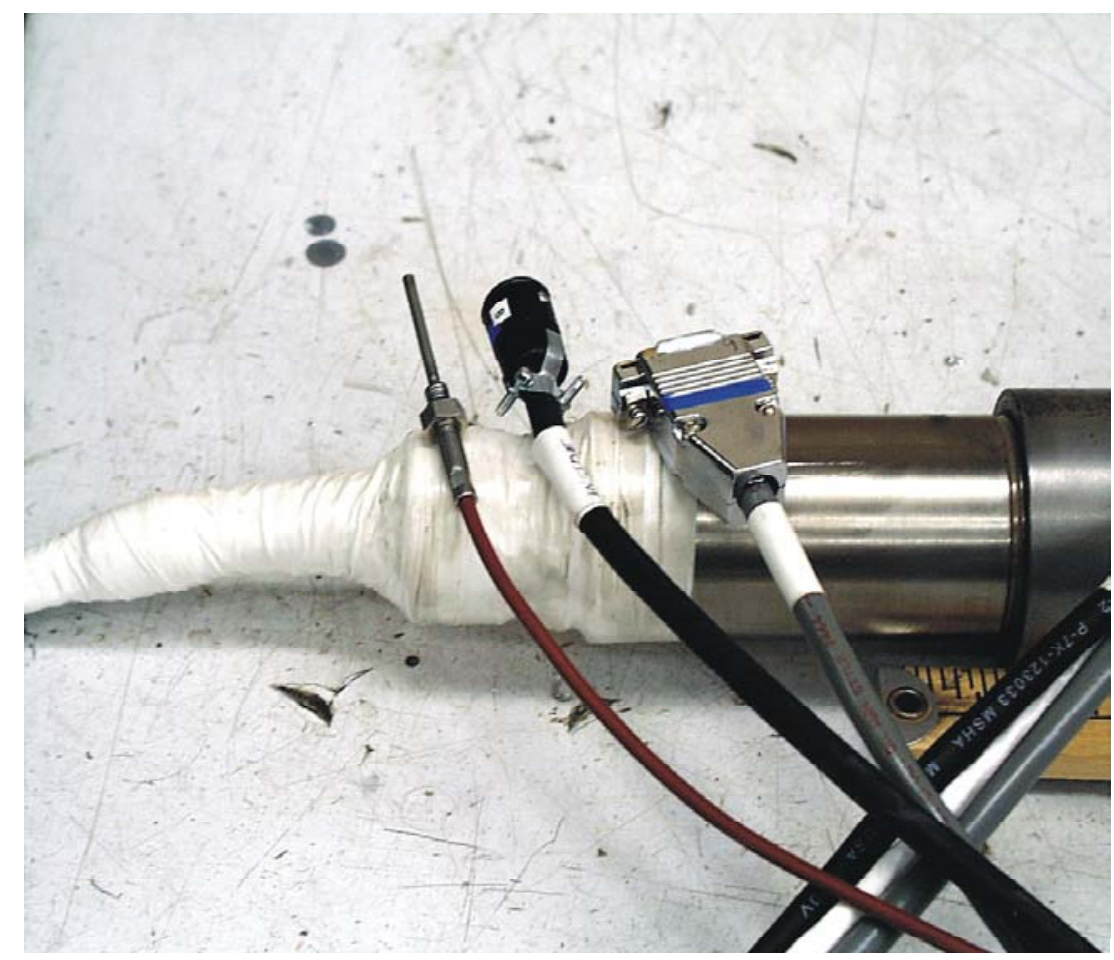

Figure 3. Connectors positioned on top of KAPL plug for DC5 temperature control unit

From left to right are the PT100 thermocouple for insertion into the cup temperature jacket, and two control cables for feedback from DC5 control unit positioned exterior to shielded cell. 


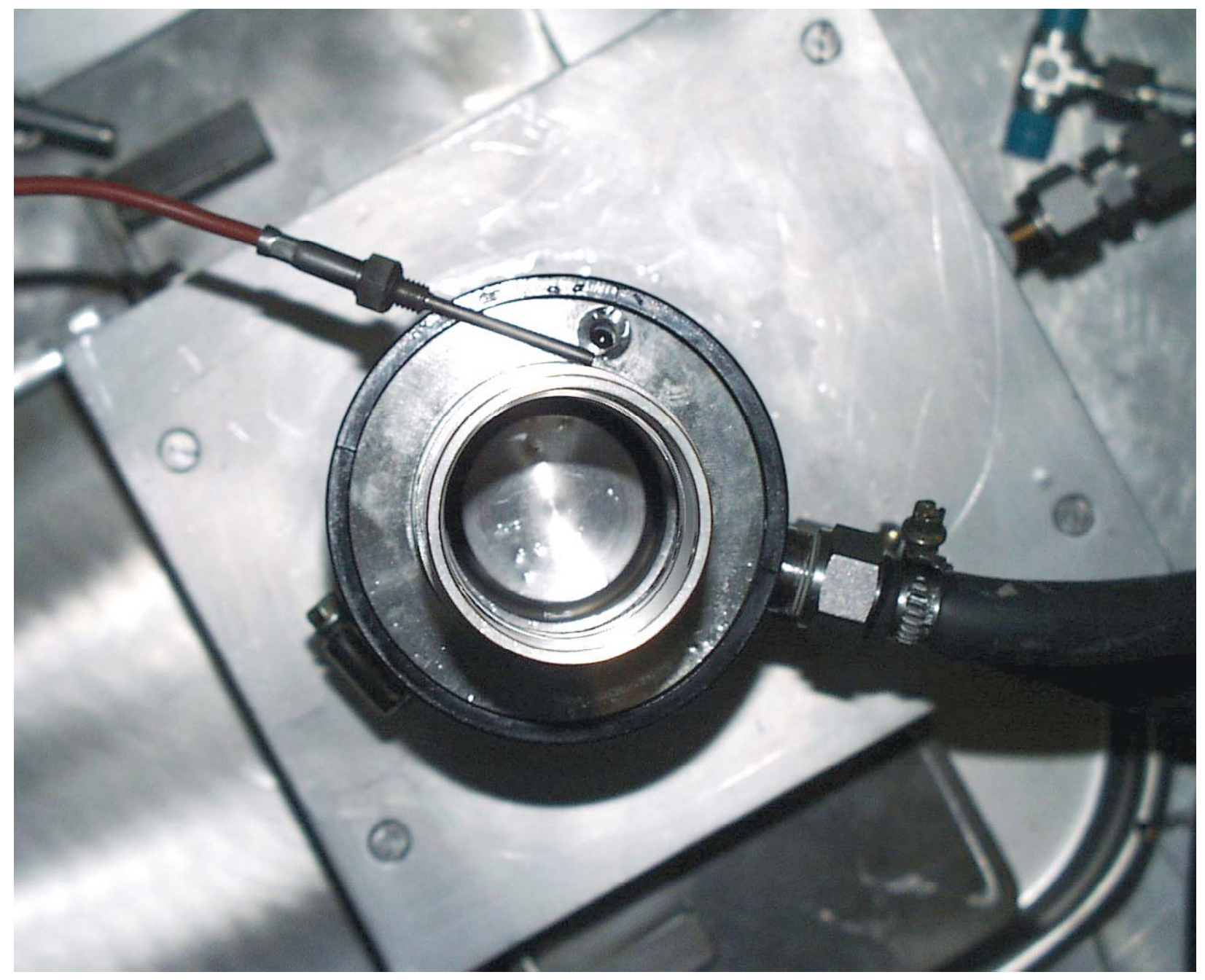

Figure 4. Top view of cup for the NV sensor system resting inside of temperature control jacket

Notice the fluid circulation cables from the DC5 bath exiting at the right. The PT100 temperature sensor is resting adjacent to the entrance hole in the temperature jacket. 


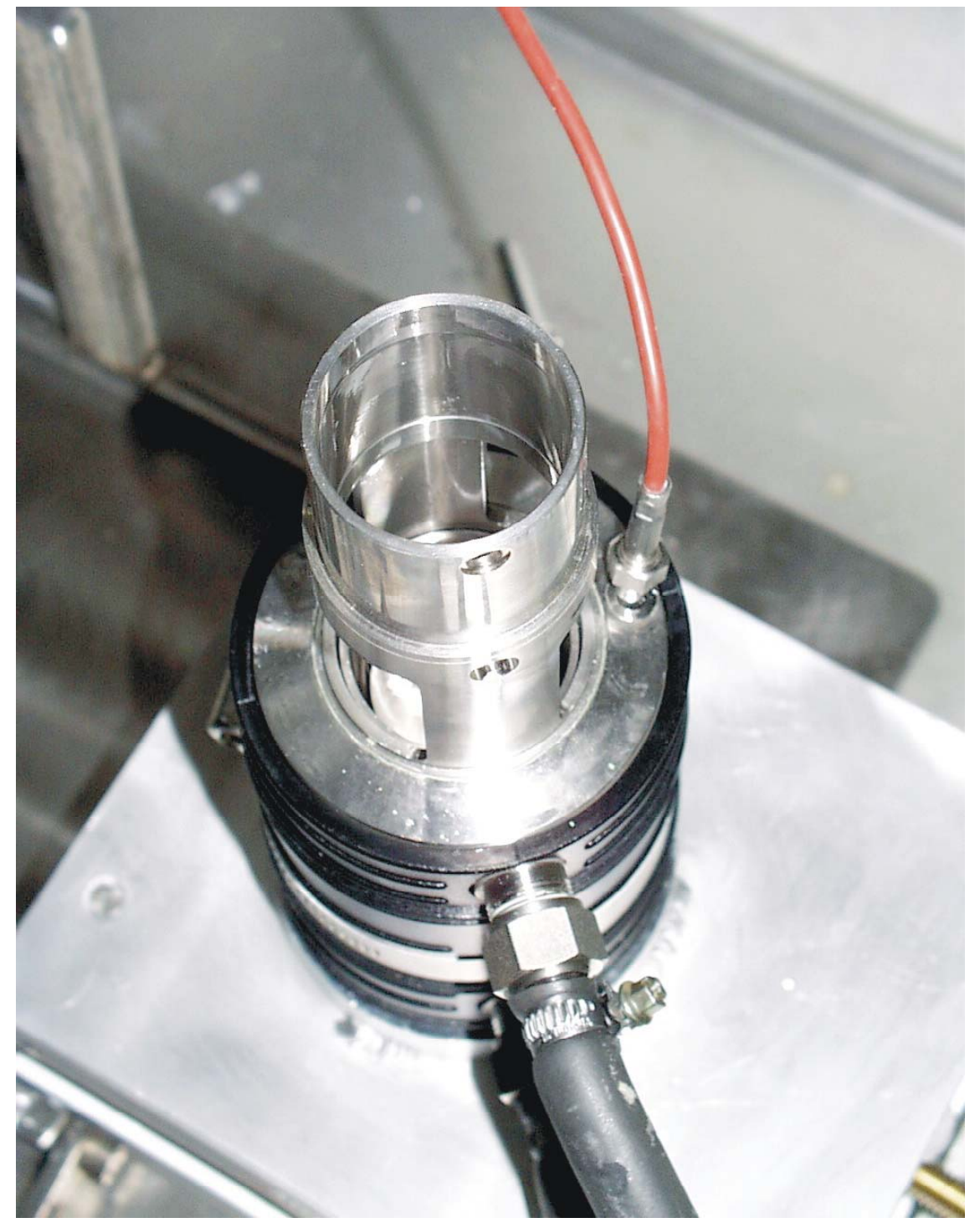

Figure 5. Front view of cup for the NV sensor system resting inside of temperature control jacket

Notice the fluid circulation cables from the DC5 bath exiting at the bottom. The PT temperature sensor is inserted into the jacket. 


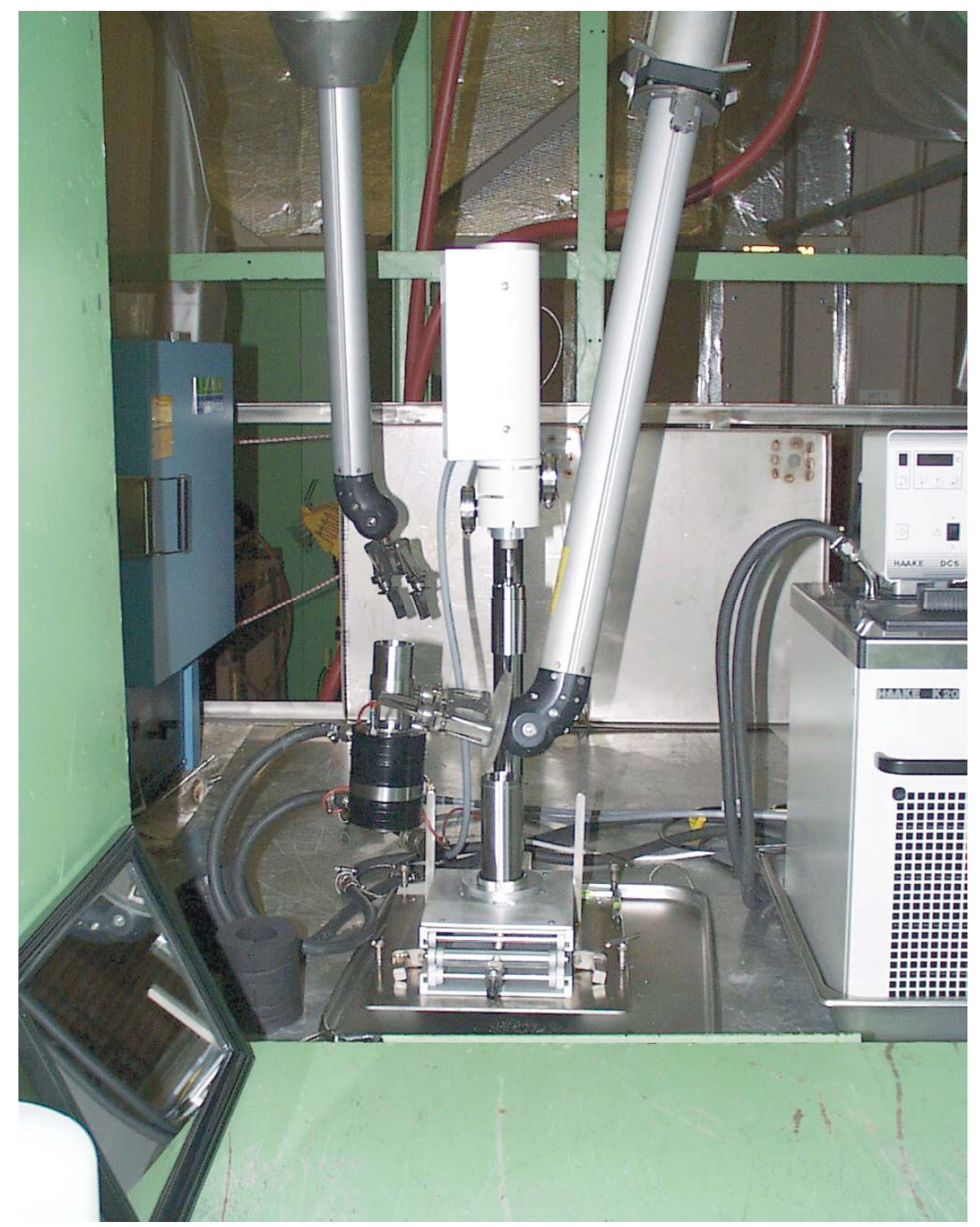

Figure 6. Front view of cut for the system in mockup

Note the sample cup positioned below the rotor and the thermal jacket to the left prior to positioning over the sample cup. A special jack was designed to accommodate the sample system and allow for remoted operations. 


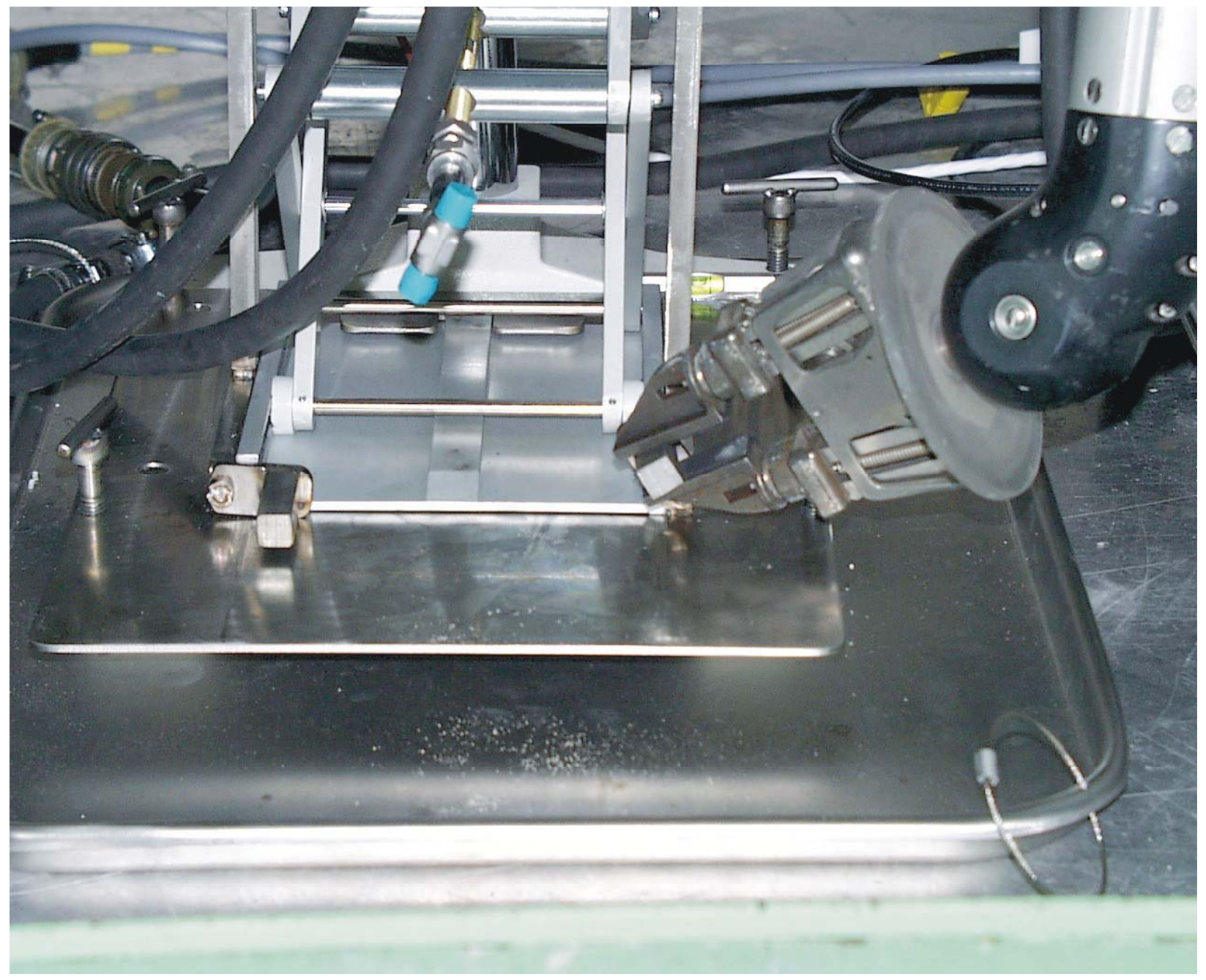

Figure 7. Front view of special jack to accommodate sample system remotely for use in shielded cell

Guide tracks are placed at the left and right and allow the jack to slide into the support base which is attached to the secondary container. Leveling screws are placed on the base to allow for leveling. A stop is placed at the rear to properly define positioning with regard to front and back. Locking keys which rotate (manipulator touching the right key) ensure proper alignment of jack with regard to the rheometer head. 


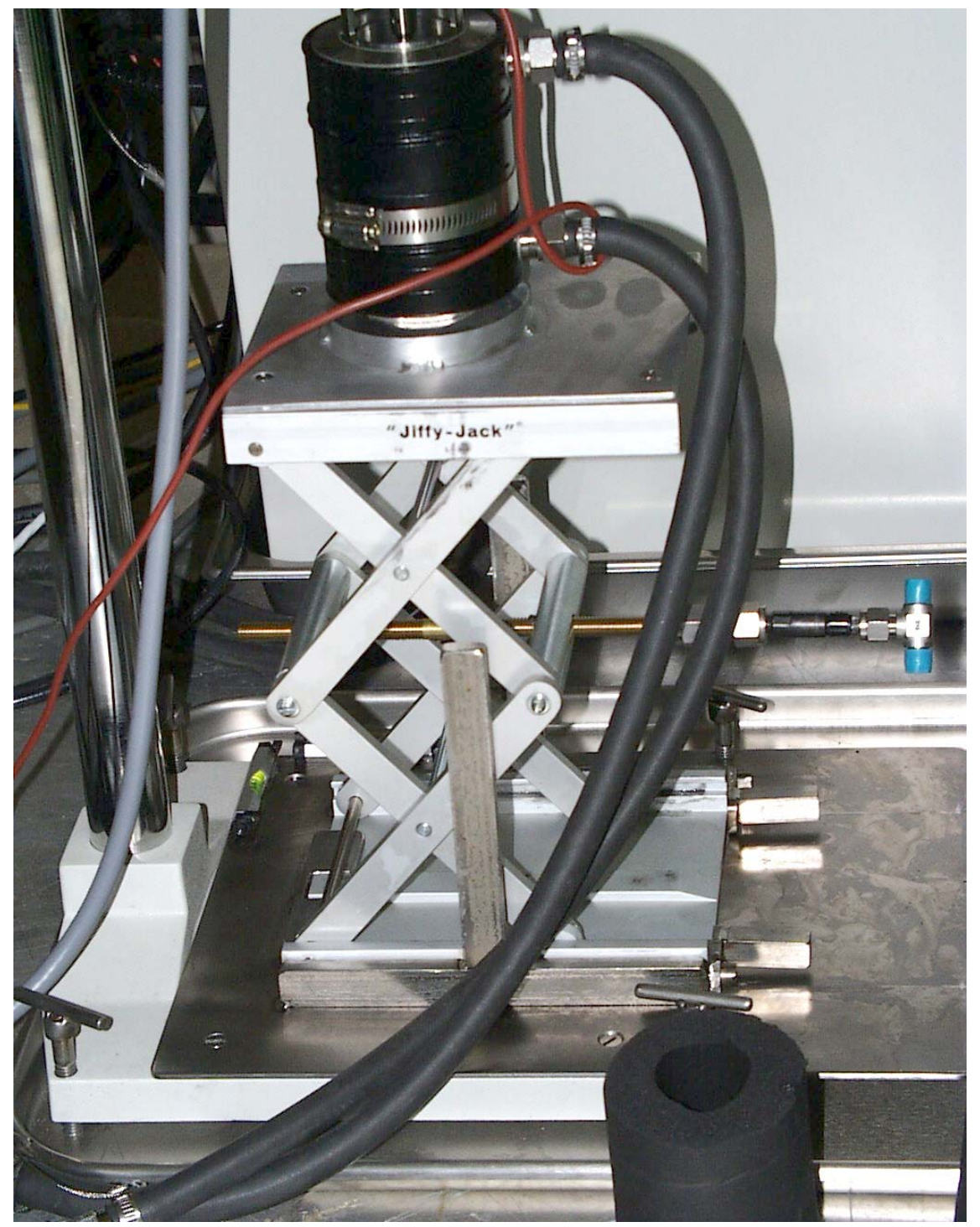

Figure 8. Side view of jack with temperature jacket elevated

Note the leveling system, locking keys, and side supports. The side supports are necessary to allow reproducible lateral positioning. This is necessary when remotely placing the rotor into the cup using the jack. 


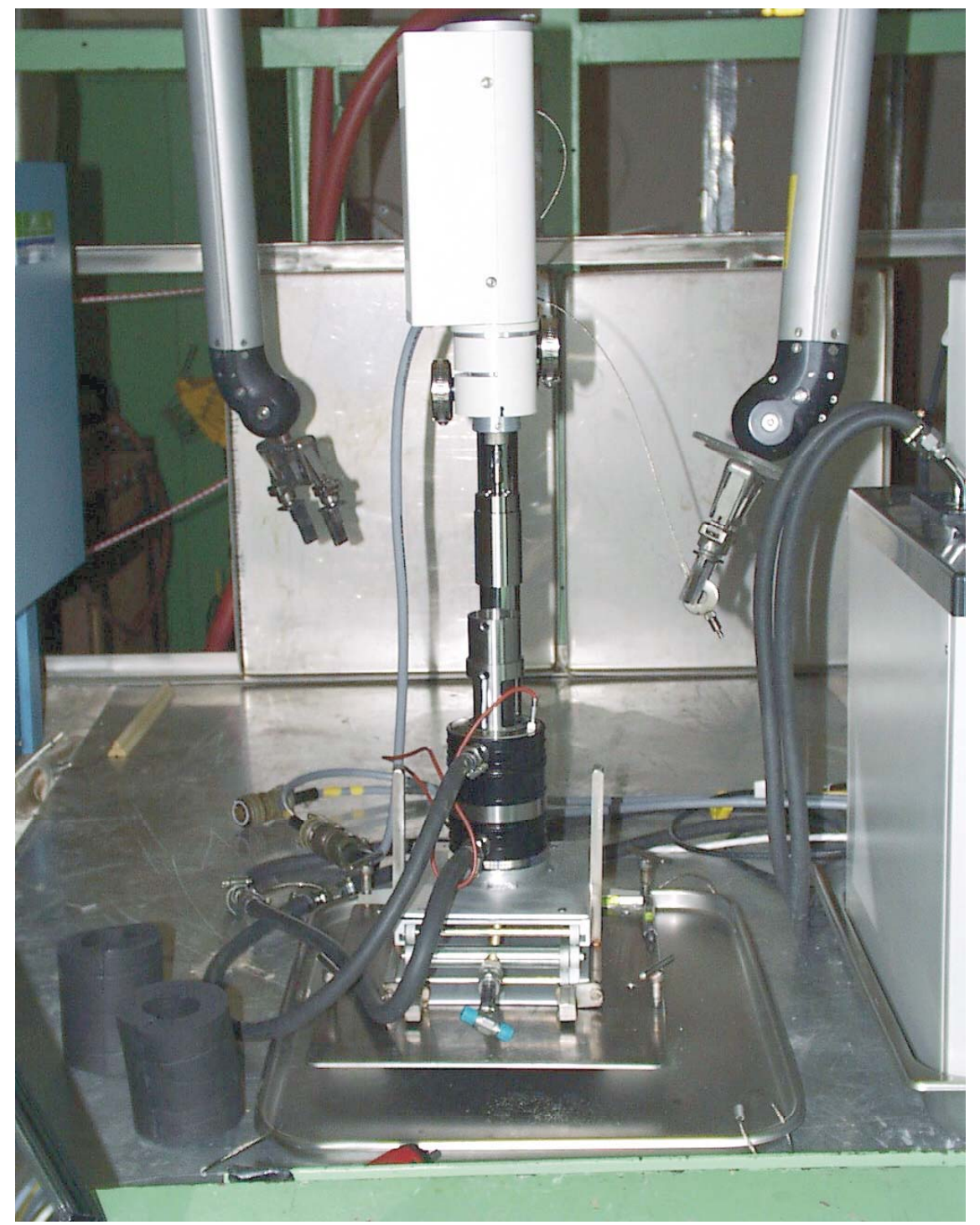

Figure 9. Front view prior to raising jack to position rotor inside sample cup 


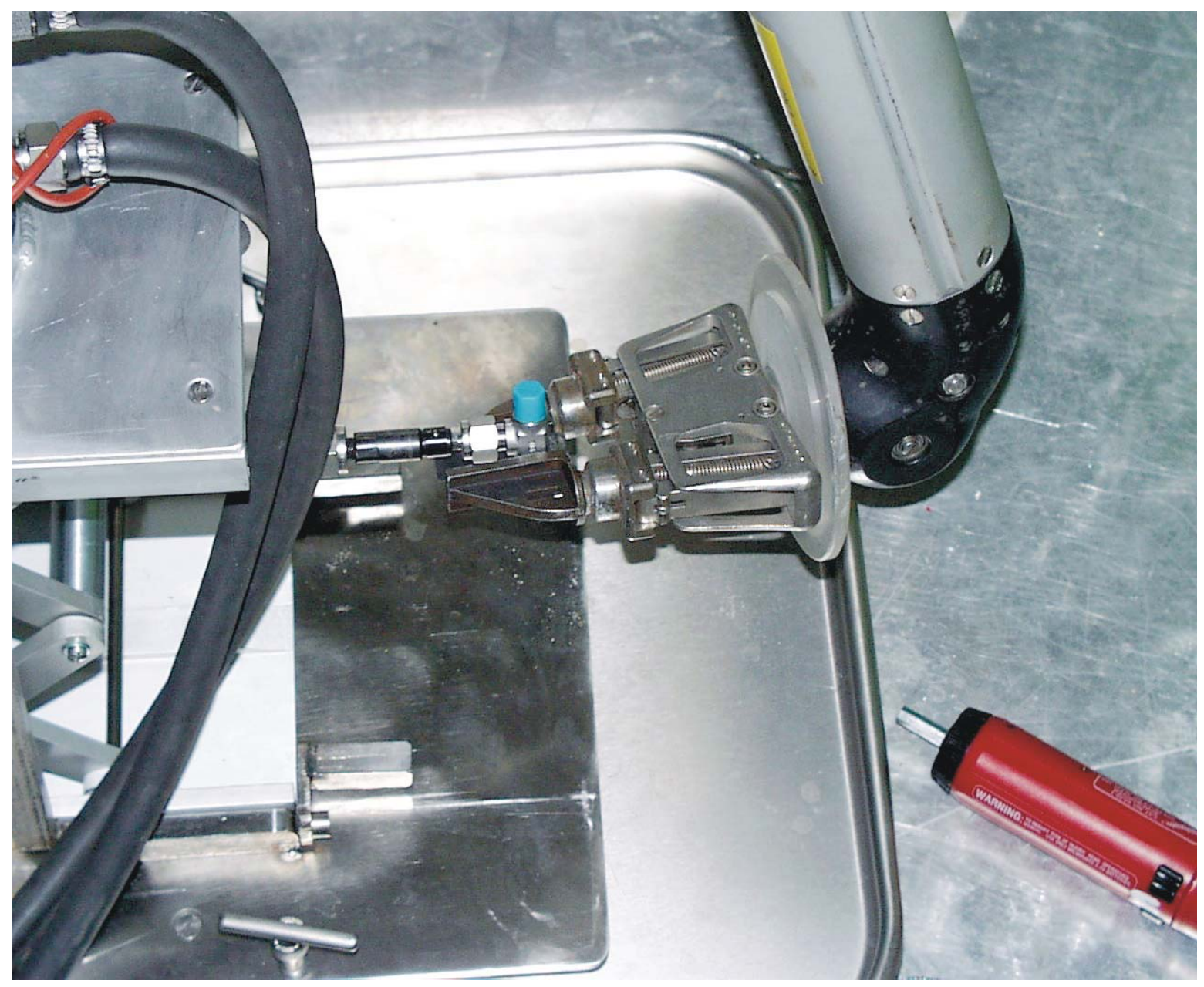

Figure 10. Side view of manipulator turning crank to raise jack and place rotor inside cup 


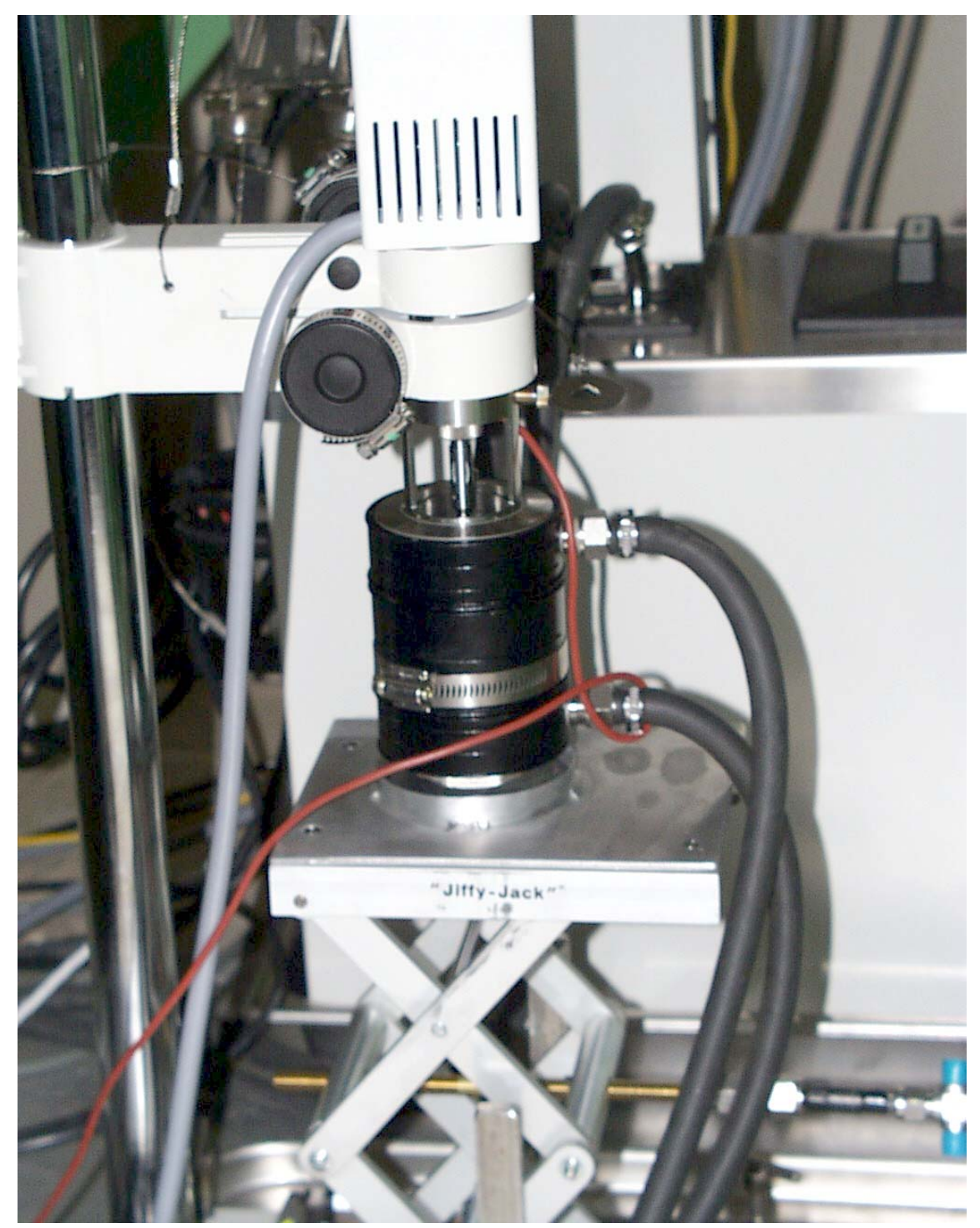

Figure 11. Side view of properly positioned sample cup following elevation of jack 


\section{APPENDIX A - PART 2}

\section{RHEOMETER CALIBRATION PRIOR TO SHIELDED CELLS INSTALLATION WITH THE 95 cP STANDARD}

Table 1. 95 cP Standard Rheology Data

Figure 1. $95 \mathrm{cP}$ Standard Rheogram 


APPENDIX A - PART 2
RHEOMETER CALIBRATION PRIOR TO SHIELDED CELLS INSTALLATION

WITH THE 95 cP STANDARD
WSRC-TR-2000-00352

SRT-RPP-2000-00026

Page 52 of 514

This page intentionally left blank. 
Table 1. 95 cP Standard Rheology Data

\begin{tabular}{|c|c|c|c|c|c|c|c|}
\hline [Pa] & {$[1 / \mathrm{s}]$} & [Pa] & {$[1 / \mathrm{s}]$} & {$[\mathrm{Pa}]$} & {$[1 / \mathrm{s}]$} & [Pa] & [1/s] \\
\hline 46.14 & 506.8 & 73.66 & 763.7 & 99.82 & 1006 & 73.66 & 759.7 \\
\hline 48.12 & 518.2 & 74.76 & 771.2 & 98.99 & 999 & 72.39 & 747.8 \\
\hline 48.63 & 528.4 & 75.64 & 780.9 & 98.25 & 991.1 & 71.83 & 740.3 \\
\hline 49.99 & 538.1 & 76.53 & 787.5 & 97.31 & 982.3 & 70.55 & 731.5 \\
\hline 50.73 & 546.4 & 77.24 & 795.4 & 96.75 & 976.1 & 69.87 & 724.5 \\
\hline 51.32 & 552.2 & 77.86 & 802 & 95.86 & 968.2 & 69.31 & 718.3 \\
\hline 51.91 & 559.7 & 78.72 & 809.1 & 95.12 & 961.1 & 68.66 & 710.8 \\
\hline 53.54 & 571.6 & 80 & 822.3 & 94.41 & 953.6 & 67.54 & 702.4 \\
\hline 54.28 & 578.6 & 80.79 & 828.9 & 92.99 & 942.6 & 66.83 & 695 \\
\hline 54.96 & 587.4 & 81.5 & 837.3 & 92.42 & 935.6 & 65.46 & 683.1 \\
\hline 55.7 & 594.5 & 82.1 & 843.9 & 91.45 & 927.2 & 64.78 & 675.6 \\
\hline 56.47 & 602.4 & 83.1 & 851.4 & 90.74 & 920.1 & 64.07 & 668.1 \\
\hline 57.24 & 608.6 & 83.69 & 858 & 89.91 & 913.5 & 62.98 & 658.8 \\
\hline 57.86 & 615.6 & 85.14 & 869.9 & 89.14 & 906 & 62.3 & 652.7 \\
\hline 59.4 & 628 & 85.83 & 877.4 & 87.78 & 892.8 & 61.29 & 644.3 \\
\hline 60.14 & 634.6 & 86.8 & 884.5 & 86.95 & 885.8 & 60.49 & 637.7 \\
\hline 60.82 & 642.5 & 87.42 & 892.4 & 86.18 & 877.4 & 59.87 & 628.4 \\
\hline 61.97 & 652.2 & 88.55 & 902.5 & 85.26 & 870.3 & 59.1 & 621.4 \\
\hline 62.8 & 658.8 & 89.32 & 909.6 & 84.73 & 865.1 & 58.33 & 613.4 \\
\hline 63.42 & 665.4 & 90.06 & 916.6 & 83.64 & 853.2 & 56.85 & 602.9 \\
\hline 64.31 & 673.4 & 90.74 & 924.1 & 82.78 & 845.7 & 56.08 & 595.8 \\
\hline 65.49 & 683.9 & 91.69 & 931.6 & 82.07 & 837.7 & 55.34 & 587.4 \\
\hline 66.38 & 691.4 & 92.51 & 940.4 & 81.15 & 830.7 & 54.48 & 580.8 \\
\hline 67.03 & 699.4 & 93.22 & 947.5 & 80.35 & 821.9 & 53.69 & 571.6 \\
\hline 67.68 & 706.4 & 94.59 & 958.5 & 79.52 & 815.3 & 52.92 & 564.1 \\
\hline 68.93 & 715.2 & 95.27 & 966 & 78.25 & 802.9 & 52.15 & 557 \\
\hline 69.64 & 722.3 & 96.27 & 975.2 & 77.66 & 796.8 & 51.44 & 549.5 \\
\hline 70.2 & 729.3 & 97.07 & 982.3 & 76.65 & 787.9 & 49.87 & 537.6 \\
\hline 71.24 & 737.3 & 97.87 & 988.9 & 75.97 & 780.9 & 49.6 & 533.2 \\
\hline 72.36 & 749.2 & 98.43 & 995.9 & 75.35 & 774.3 & 48.77 & 525.3 \\
\hline 73.04 & 756.2 & 99.35 & 1003 & 74.67 & 767.7 & 47.74 & 516 \\
\hline
\end{tabular}




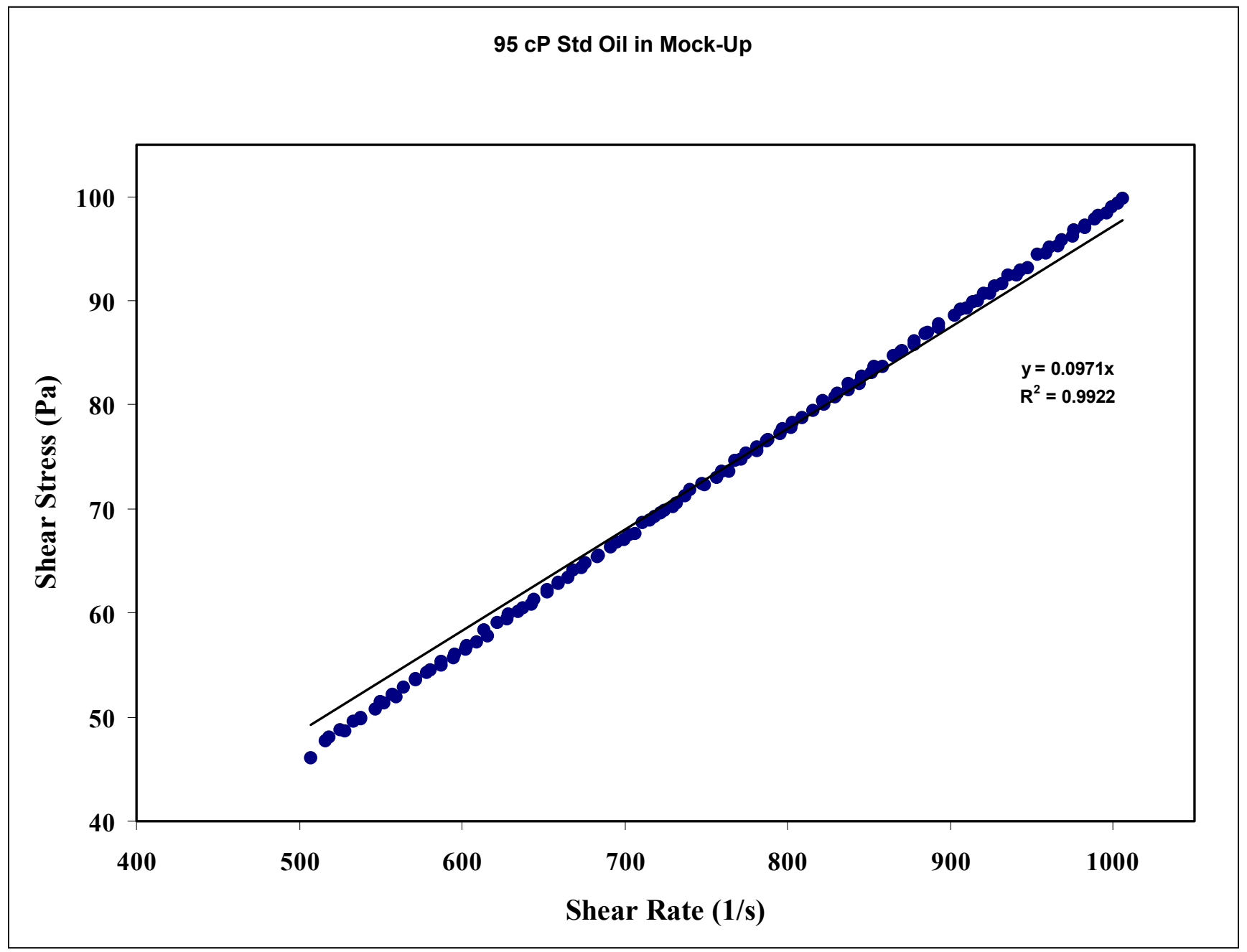

Figure 1. 95 cP Standard Rheogram 


\begin{tabular}{|ll|}
\hline APPENDIX A - PART 3 & WSRC-TR-2000-00352 \\
RHEOMETER PRE-CALIBRATION IN THE SHIELDED CELLS & SRT-RPP-2000-00026 \\
WITH THE LOW VISCOSITY STANDARD & Page 55 of 514 \\
\hline
\end{tabular}

\section{APPENDIX A - PART 3 \\ RHEOMETER PRE-CALIBRATION IN THE SHIELDED CELLS WITH THE LOW VISCOSITY STANDARD}

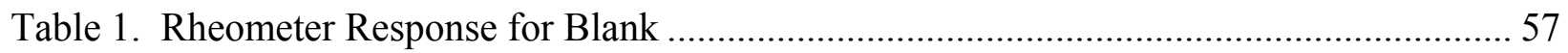

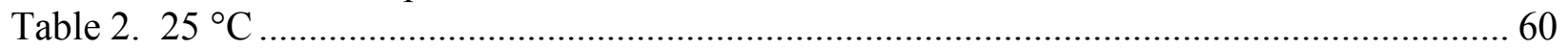

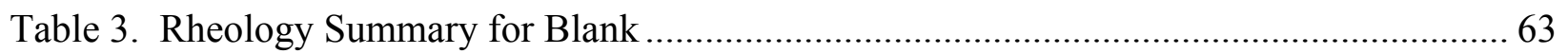

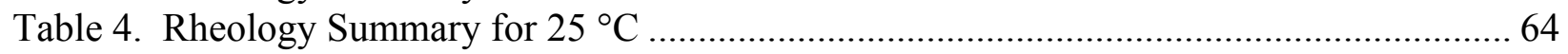

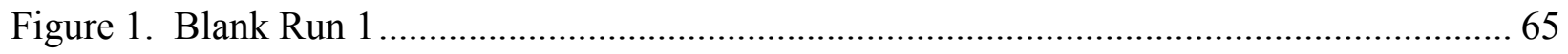

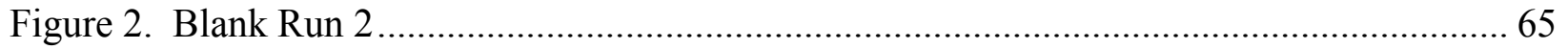

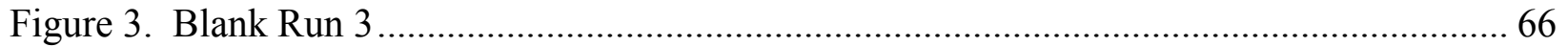

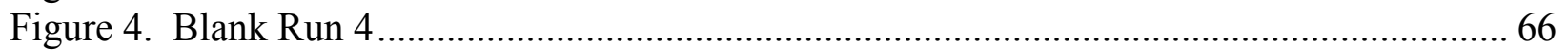

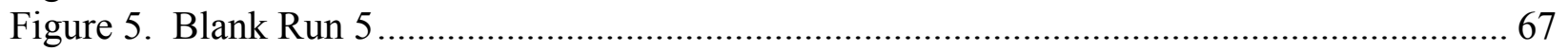

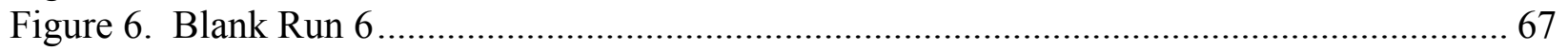

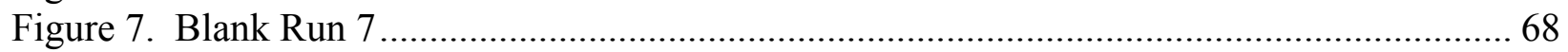

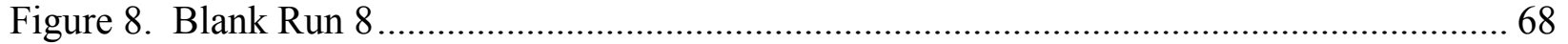

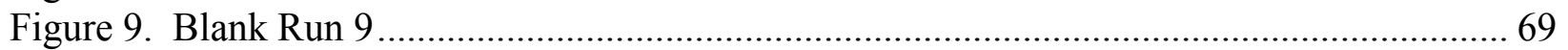

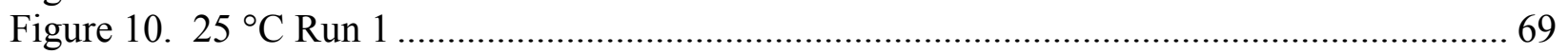

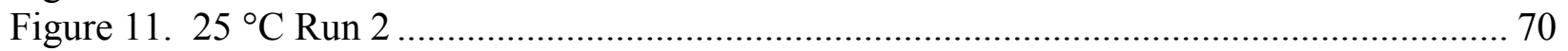

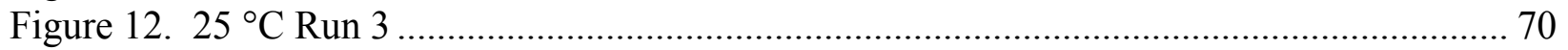

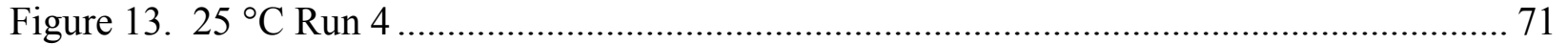

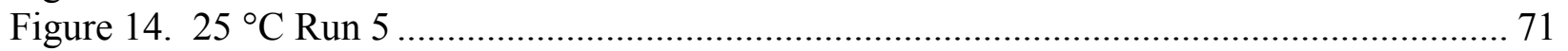

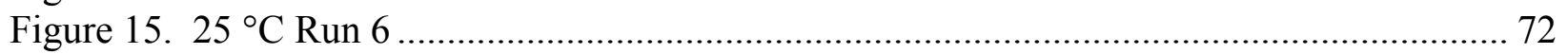

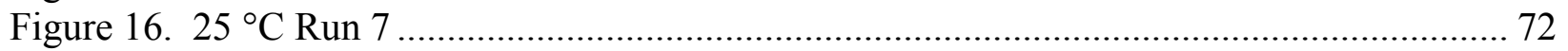

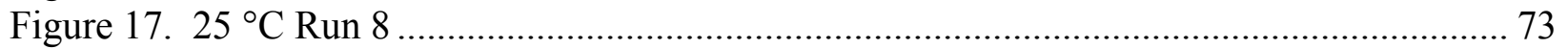

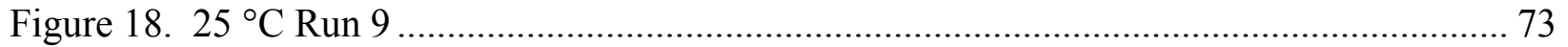




\begin{tabular}{|ll|}
\hline APPENDIX A - PART 3 & WRC-TR-2000-00352 \\
RHEOMETER PRE-CALIBRATION IN THE SHIELDED CELLS & SRT-RPP-2000-00026 \\
WITH THE LOW VISCOSITY STANDARD & Page 56 of 514 \\
\hline
\end{tabular}

This page intentionally left blank. 
Table 1. Rheometer Response for Blank

\begin{tabular}{|c|c|c|c|c|c|c|c|c|c|c|c|c|c|c|c|c|c|}
\hline run1 & & run2 & & run3 & & run4 & & run5 & & run6 & & run7 & & run8 & & run9 & \\
\hline$[\mathrm{Pa}]$ & {$[1 / \mathrm{s}]$} & {$[\mathrm{Pa}]$} & {$[1 / \mathrm{s}]$} & {$[\mathrm{Pa}]$} & {$[1 / \mathrm{s}]$} & {$[\mathrm{Pa}]$} & {$[1 / \mathrm{s}]$} & {$[\mathrm{Pa}]$} & {$[1 / \mathrm{s}]$} & {$[\mathrm{Pa}]$} & {$[1 / \mathrm{s}]$} & [Pa] & {$[1 / \mathrm{s}]$} & {$[\mathrm{Pa}]$} & {$[1 / \mathrm{s}]$} & {$[\mathrm{Pa}]$} & {$[1 / \mathrm{s}]$} \\
\hline 5.345 & 305.4 & 5.961 & 325.2 & 5.689 & 327 & 4.499 & 301.4 & 6.138 & 311.1 & 5.274 & 307.6 & 5.286 & 303.6 & 4.753 & 301.4 & 5.248 & 308.9 \\
\hline 5.993 & 322.1 & 6.473 & 344.6 & 6.278 & 341.1 & 4.7 & 319.1 & 6.621 & 324.8 & 5.973 & 320.4 & 5.97 & 322.6 & 6.02 & 323 & 5.982 & 323.5 \\
\hline 6.458 & 339.3 & 6.958 & 375 & 6.751 & 358.3 & 5.937 & 327.9 & 7.124 & 348.6 & 6.482 & 338 & 6.494 & 346.4 & 6.059 & 331 & 6.491 & 341.5 \\
\hline 6.92 & 368.4 & 7.47 & 400.6 & 7.331 & 389.6 & 6.076 & 366.2 & 7.55 & 376.3 & 7.041 & 368 & 7.014 & 371.5 & 7.068 & 371.1 & 6.923 & 373.3 \\
\hline 7.399 & 394 & 7.843 & 422.6 & 7.757 & 413.8 & 7.017 & 396.6 & 7.97 & 399.7 & 7.55 & 389.6 & 7.506 & 394.9 & 7.512 & 393.5 & 7.381 & 392.6 \\
\hline 7.876 & 419.5 & 8.246 & 444.6 & 8.246 & 438.5 & 7.485 & 420 & 8.358 & 421.7 & 8 & 422.2 & 7.967 & 422.2 & 7.914 & 423.1 & 7.805 & 420.9 \\
\hline 8.263 & 441.6 & 8.695 & 470.2 & 9.039 & 476.8 & 7.92 & 445.5 & 9.225 & 445.5 & 8.509 & 447.7 & 8.479 & 448.2 & 8.376 & 445.5 & 8.278 & 442.9 \\
\hline 8.974 & 479 & 9.583 & 509.9 & 9.432 & 501.1 & 8.411 & 469.3 & 9.672 & 485.2 & 8.941 & 473.3 & 8.944 & 472.9 & 8.846 & 473.7 & 9.045 & 481.2 \\
\hline 9.358 & 508.5 & 10.02 & 534.1 & 9.894 & 524.4 & 8.811 & 509 & 10.14 & 508.5 & 9.403 & 499.7 & 9.364 & 498 & 9.287 & 496.6 & 9.489 & 505.9 \\
\hline 9.817 & 531 & 10.41 & 558.8 & 10.29 & 553.1 & 9.607 & 517.4 & 10.66 & 536.8 & 9.856 & 525.7 & 10.21 & 535.9 & 10.09 & 536.3 & 9.909 & 530.1 \\
\hline 10.25 & 557 & 10.87 & 591 & 10.79 & 576.9 & 9.675 & 542.9 & 11.15 & 561.9 & 10.35 & 549.1 & 10.63 & 562.3 & 10.54 & 561.4 & 10.41 & 562.8 \\
\hline 10.71 & 580.4 & 11.33 & 614.3 & 11.14 & 598.4 & 10.15 & 583.5 & 11.59 & 594.5 & 10.73 & 573.8 & 11.13 & 583.9 & 10.95 & 589.2 & 10.8 & 587 \\
\hline 11.07 & 605.1 & 11.78 & 637.7 & 11.56 & 623.6 & 10.98 & 616.1 & 12.03 & 619.2 & 11.62 & 614.8 & 11.62 & 619.2 & 11.43 & 615.2 & 11.34 & 616.1 \\
\hline 11.55 & 629.3 & 12.52 & 675.6 & 12.13 & 651.3 & 11.46 & 637.7 & 12.41 & 642.5 & 12.1 & 639.4 & 12.06 & 642.1 & 11.85 & 638.1 & 11.83 & 642.1 \\
\hline 12.43 & 672.5 & 12.93 & 697.6 & 12.66 & 681.3 & 11.94 & 661.9 & 12.88 & 665.9 & 12.52 & 669.8 & 12.45 & 665.4 & 12.29 & 661.5 & 12.36 & 671.6 \\
\hline 12.84 & 696.3 & 13.34 & 720.5 & 13.47 & 721 & 12.46 & 691.4 & 13.68 & 690.1 & 13 & 693.6 & 13.2 & 703.3 & 12.74 & 690.1 & 12.9 & 700.2 \\
\hline 13.27 & 722.7 & 13.87 & 752.7 & 13.88 & 744.3 & 13.01 & 724.5 & 14.09 & 729.8 & 13.39 & 717.4 & 13.62 & 725.8 & 13.22 & 717 & 13.31 & 721.4 \\
\hline 13.7 & 754.4 & 14.25 & 774.3 & 14.32 & 772.5 & 13.5 & 746.1 & 14.56 & 751.4 & 13.84 & 739 & 13.66 & 735.1 & 13.73 & 743 & 13.73 & 745.6 \\
\hline 14.18 & 777.8 & 14.58 & 797.6 & 14.82 & 799.8 & 13.91 & 769.4 & 15.03 & 783.5 & 14.23 & 765.5 & 14.3 & 765 & 14.19 & 769.9 & 14.51 & 784 \\
\hline 14.63 & 805.1 & 15.45 & 838.6 & 15.23 & 821 & 14.29 & 795.4 & 15.43 & 809.5 & 15.16 & 805.1 & 15.2 & 813.9 & 14.67 & 797.6 & 14.88 & 807.8 \\
\hline 15.03 & 825.4 & 15.89 & 866.8 & 15.93 & 858 & 14.79 & 824.1 & 15.91 & 832.9 & 15.5 & 829.4 & 15.27 & 821 & 15.21 & 824.1 & 15.38 & 832.9 \\
\hline 15.44 & 849.2 & 16.27 & 891.9 & 16.06 & 865.9 & 15.3 & 849.2 & 16.51 & 858.9 & 15.97 & 858 & 15.77 & 849.2 & 15.61 & 851.8 & 15.91 & 865.1 \\
\hline 16.26 & 891.9 & 16.79 & 914.4 & 16.86 & 910 & 15.69 & 889.3 & 16.99 & 888.4 & 16.38 & 881.4 & 16.71 & 891.5 & 16.52 & 891.9 & 16.2 & 888 \\
\hline 16.62 & 913.1 & 17.06 & 940 & 17.42 & 937.8 & 16.53 & 923.7 & 17.33 & 914.4 & 16.88 & 909.6 & 17.12 & 914.4 & 16.97 & 917.1 & 16.66 & 909.6 \\
\hline 17.03 & 943.9 & 17.51 & 964.7 & 17.71 & 965.1 & 17.06 & 929.4 & 17.8 & 940.9 & 17.3 & 935.1 & 17.51 & 946.6 & 17.42 & 945.3 & 16.97 & 933.8 \\
\hline 17.45 & 969.1 & 18.28 & 1002 & 18.3 & 990.2 & 17 & 967.7 & 18.63 & 962.9 & 17.74 & 962 & 17.98 & 973 & 17.92 & 973.5 & 17.42 & 961.1 \\
\hline 17.86 & 995.5 & 18.66 & 1029 & 18.78 & 1015 & 17.95 & 992.9 & 18.96 & 1004 & 18.19 & 987.1 & 18.42 & 995.5 & 18.39 & 999.9 & 18.28 & 1002 \\
\hline 18.33 & 1021 & 19.13 & 1053 & 19.16 & 1046 & 18.36 & 1027 & 19.46 & 1027 & 18.75 & 1012 & 19.07 & 1025 & 18.87 & 1025 & 18.78 & 1025 \\
\hline 18.84 & 1047 & 19.61 & 1077 & 19.64 & 1068 & 18.9 & 1052 & 20.02 & 1055 & 19.49 & 1050 & 19.52 & 1052 & 19.37 & 1055 & 19.19 & 1050 \\
\hline 19.16 & 1070 & 20.11 & 1110 & 19.99 & 1095 & 19.28 & 1077 & 20.49 & 1086 & 19.96 & 1080 & 19.93 & 1077 & 19.78 & 1078 & 19.61 & 1081 \\
\hline 19.7 & 1095 & 20.49 & 1133 & 20.52 & 1120 & 19.78 & 1100 & 20.85 & 1111 & 20.44 & 1103 & 20.44 & 1109 & 20.23 & 1104 & 20.02 & 1107 \\
\hline
\end{tabular}


Table 1. Rheometer Response for Blank - continued

\begin{tabular}{|c|c|c|c|c|c|c|c|c|c|c|c|c|c|c|c|c|c|}
\hline run1 & & run2 & & run3 & & run4 & & run5 & & run6 & & run7 & & run8 & & run9 & \\
\hline$[\mathrm{Pa}]$ & {$[1 / \mathrm{s}]$} & [Pa] & {$[1 / \mathrm{s}]$} & [Pa] & {$[1 / \mathrm{s}]$} & [Pa] & {$[1 / \mathrm{s}]$} & [Pa] & {$[1 / \mathrm{s}]$} & [Pa] & {$[1 / s]$} & [Pa] & {$[1 / \mathrm{s}]$} & [Pa] & {$[1 / \mathrm{s}]$} & [Pa] & {$[1 / \mathrm{s}]$} \\
\hline 20.08 & 1119 & 20.85 & 1154 & 20.94 & 1145 & 20.17 & 1126 & 21.23 & 1134 & 20.91 & 1129 & 20.91 & 1136 & 20.73 & 1129 & 20.44 & 1133 \\
\hline 20.88 & 1160 & 21.62 & 1191 & 21.38 & 1171 & 20.67 & 1149 & 21.71 & 1158 & 21.47 & 1161 & 21.32 & 1160 & 21.18 & 1162 & 20.97 & 1160 \\
\hline 21.32 & 1188 & 22.09 & 1218 & 21.94 & 1198 & 21 & 1174 & 22.24 & 1185 & 21.83 & 1187 & 21.74 & 1186 & 21.59 & 1186 & 21.44 & 1185 \\
\hline 21.8 & 1216 & 22.45 & 1241 & 22.33 & 1222 & 21.44 & 1215 & 22.95 & 1207 & 22.27 & 1214 & 22.18 & 1212 & 22.03 & 1209 & 21.83 & 1211 \\
\hline 22.21 & 1243 & 22.98 & 1268 & 22.83 & 1249 & 22.27 & 1240 & 23.48 & 1249 & 22.68 & 1237 & 22.71 & 1239 & 22.45 & 1236 & 22.36 & 1236 \\
\hline 22.6 & 1263 & 23.39 & 1296 & 23.75 & 1294 & 22.77 & 1265 & 23.87 & 1274 & 23.16 & 1263 & 23.16 & 1263 & 22.95 & 1262 & 22.77 & 1263 \\
\hline 22.98 & 1288 & 23.78 & 1318 & 24.16 & 1318 & 23.16 & 1296 & 24.37 & 1300 & 23.6 & 1286 & 23.6 & 1286 & 23.37 & 1287 & 23.6 & 1303 \\
\hline 23.42 & 1314 & 24.52 & 1357 & 24.61 & 1341 & 23.66 & 1320 & 24.73 & 1330 & 23.99 & 1311 & 24.4 & 1329 & 24.19 & 1327 & 23.99 & 1327 \\
\hline 23.99 & 1342 & 24.93 & 1381 & 25.02 & 1373 & 24.08 & 1345 & 25.23 & 1354 & 24.9 & 1352 & 24.87 & 1352 & 24.7 & 1352 & 24.58 & 1353 \\
\hline 24.37 & 1367 & 25.32 & 1404 & 25.5 & 1397 & 24.49 & 1382 & 25.61 & 1378 & 25.29 & 1377 & 25.29 & 1382 & 25.11 & 1379 & 24.9 & 1376 \\
\hline 25.17 & 1404 & 25.91 & 1429 & 25.82 & 1419 & 25.26 & 1404 & 26.12 & 1406 & 25.76 & 1406 & 25.7 & 1405 & 25.5 & 1410 & 25.38 & 1410 \\
\hline 25.58 & 1433 & 26.3 & 1463 & 26.27 & 1446 & 25.67 & 1429 & 26.59 & 1431 & 26.24 & 1432 & 26.09 & 1429 & 25.91 & 1426 & 25.79 & 1430 \\
\hline 25.97 & 1458 & 26.68 & 1483 & 28.07 & 1579 & 26.12 & 1460 & 27.01 & 1455 & 26.65 & 1453 & 26.59 & 1458 & 26.44 & 1455 & 26.27 & 1456 \\
\hline 26.41 & 1483 & 27.06 & 1507 & 27.57 & 1553 & 26.59 & 1481 & 27.83 & 1481 & 26.98 & 1474 & 27.86 & 1569 & 27.48 & 1571 & 26.71 & 1486 \\
\hline 26.8 & 1508 & 27.83 & 1547 & 27.09 & 1528 & 26.89 & 1560 & 27.75 & 1522 & 27.69 & 1512 & 27.36 & 1547 & 27.06 & 1542 & 27.54 & 1524 \\
\hline 27.27 & 1553 & 26.98 & 1526 & 26.62 & 1503 & 27.54 & 1504 & 26.89 & 1556 & 26.65 & 1537 & 27.04 & 1521 & 26.5 & 1512 & 26.77 & 1541 \\
\hline 26.47 & 1517 & 26.89 & 1506 & 26.27 & 1479 & 26.38 & 1492 & 26.47 & 1517 & 26.12 & 1499 & 26.09 & 1482 & 26.06 & 1488 & 26.32 & 1515 \\
\hline 26.09 & 1491 & 25.67 & 1446 & 25.44 & 1439 & 26.32 & 1466 & 26.06 & 1492 & 26.03 & 1482 & 25.67 & 1452 & 25.64 & 1464 & 25.82 & 1488 \\
\hline 25.67 & 1463 & 25.58 & 1436 & 24.96 & 1414 & 25.85 & 1457 & 25.56 & 1467 & 25.61 & 1454 & 25.2 & 1428 & 25.26 & 1438 & 25.44 & 1462 \\
\hline 25.23 & 1438 & 24.31 & 1403 & 24.55 & 1389 & 25.79 & 1424 & 25.08 & 1438 & 25.53 & 1443 & 24.7 & 1404 & 24.85 & 1416 & 24.93 & 1430 \\
\hline 24.79 & 1415 & 24.05 & 1347 & 24.08 & 1358 & 24.93 & 1384 & 24.61 & 1406 & 25.11 & 1414 & 24.34 & 1377 & 24.43 & 1392 & 24.58 & 1408 \\
\hline 24.34 & 1392 & 22.83 & 1319 & 23.57 & 1333 & 24.43 & 1366 & 24.22 & 1382 & 24.73 & 1387 & 23.78 & 1347 & 23.51 & 1352 & 24.13 & 1382 \\
\hline 23.63 & 1352 & 22.68 & 1291 & 23.19 & 1310 & 24.25 & 1329 & 23.78 & 1359 & 24.25 & 1360 & 23.37 & 1319 & 23.16 & 1321 & 23.69 & 1355 \\
\hline 23.22 & 1328 & 21.29 & 1226 & 22.74 & 1283 & 23.16 & 1316 & 23.39 & 1334 & 23.75 & 1335 & 22.86 & 1295 & 22.63 & 1295 & 23.25 & 1332 \\
\hline 22.68 & 1301 & 21.2 & 1208 & 22.27 & 1259 & 23.04 & 1280 & 22.57 & 1309 & 23.25 & 1310 & 22.45 & 1268 & 22.24 & 1269 & 22.74 & 1306 \\
\hline 22.21 & 1272 & 20.67 & 1177 & 21.86 & 1235 & 22.57 & 1255 & 22.06 & 1269 & 22.42 & 1268 & 21.92 & 1242 & 21.77 & 1244 & 22 & 1265 \\
\hline 21.74 & 1244 & 20.61 & 1170 & 21.12 & 1194 & 22.21 & 1218 & 21.65 & 1245 & 22.03 & 1246 & 21.41 & 1211 & 21.26 & 1217 & 21.53 & 1241 \\
\hline 21.2 & 1218 & 20.23 & 1148 & 20.58 & 1167 & 21.47 & 1195 & 21.15 & 1218 & 21.62 & 1215 & 21 & 1188 & 20.79 & 1192 & 21.09 & 1215 \\
\hline 20.79 & 1185 & 20.14 & 1140 & 20.11 & 1143 & 21.09 & 1163 & 20.67 & 1191 & 21.18 & 1190 & 20.55 & 1166 & 20.44 & 1164 & 20.61 & 1185 \\
\hline 20.29 & 1159 & 19.73 & 1122 & 19.64 & 1111 & 20.64 & 1137 & 20.2 & 1159 & 20.73 & 1167 & 20.14 & 1140 & 19.96 & 1141 & 20.23 & 1163 \\
\hline 19.84 & 1135 & 19.7 & 1115 & 19.19 & 1087 & 20.14 & 1114 & 19.78 & 1134 & 20.26 & 1138 & 19.31 & 1099 & 19.13 & 1103 & 19.81 & 1139 \\
\hline
\end{tabular}


Table 1. Rheometer Response for Blank - continued

\begin{tabular}{|c|c|c|c|c|c|c|c|c|c|c|c|c|c|c|c|c|c|}
\hline run1 & & run2 & & run3 & & run4 & & run5 & & run6 & & run7 & & run8 & & run9 & \\
\hline [Pa] & {$[1 / \mathrm{s}]$} & {$[\mathrm{Pa}]$} & {$[1 / \mathrm{s}]$} & [Pa] & {$[1 / \mathrm{s}]$} & {$[\mathrm{Pa}]$} & {$[1 / \mathrm{s}]$} & {$[\mathrm{Pa}]$} & [1/s] & {$[\mathrm{Pa}]$} & {$[1 / \mathrm{s}]$} & [Pa] & {$[1 / \mathrm{s}]$} & {$[\mathrm{Pa}]$} & {$[1 / \mathrm{s}]$} & [Pa] & {$[1 / \mathrm{s}]$} \\
\hline 19.37 & 1112 & 19.31 & 1092 & 18.84 & 1063 & 19.73 & 1088 & 19.31 & 1110 & 19.75 & 1114 & 18.84 & 1073 & 18.75 & 1081 & 19.34 & 1115 \\
\hline 18.84 & 1080 & 18.57 & 1052 & 18.33 & 1036 & 19.28 & 1060 & 18.9 & 1085 & 19.37 & 1089 & 18.42 & 1048 & 18.36 & 1055 & 18.6 & 1077 \\
\hline 18.45 & 1055 & 18.04 & 1030 & 17.89 & 1013 & 18.81 & 1037 & 18.42 & 1060 & 18.93 & 1064 & 17.95 & 1022 & 17.83 & 1022 & 18.13 & 1051 \\
\hline 18.01 & 1027 & 17.65 & 1001 & 17.45 & 987.6 & 18.36 & 995.9 & 18.04 & 1036 & 18.39 & 1036 & 17.48 & 994.2 & 17.36 & 1001 & 17.68 & 1021 \\
\hline 17.59 & 1003 & 17.3 & 976.1 & 16.74 & 949.7 & 17.54 & 988 & 17.15 & 1012 & 17.95 & 1011 & 16.97 & 966 & 16.77 & 971.3 & 17.3 & 995.1 \\
\hline 17.09 & 978.3 & 16.82 & 953.2 & 16.2 & 921.5 & 17.51 & 944.4 & 16.77 & 969.9 & 17.09 & 969.1 & 16.56 & 938.7 & 16.32 & 945.3 & 16.88 & 971.7 \\
\hline 16.35 & 940.9 & 16.41 & 931.2 & 15.85 & 898.1 & 16.62 & 914 & 16.32 & 946.6 & 16.74 & 946.1 & 16.09 & 915.3 & 15.88 & 920.1 & 16.53 & 950.6 \\
\hline 15.97 & 917.5 & 16 & 903.4 & 15.37 & 866.8 & 16.17 & 891.9 & 15.88 & 917.9 & 16.2 & 916.2 & 15.64 & 889.7 & 15.43 & 889.3 & 15.73 & 905.6 \\
\hline 15.46 & 893.3 & 15.11 & 859.8 & 14.87 & 839.1 & 15.76 & 867.7 & 15.4 & 893.7 & 15.82 & 892.8 & 15.2 & 865.9 & 15.08 & 864.6 & 15.23 & 884.9 \\
\hline 15.05 & 862.4 & 14.72 & 839.1 & 14.34 & 818.3 & 15.37 & 842.1 & 14.93 & 868.1 & 15.35 & 869.5 & 14.46 & 827.2 & 14.58 & 840.4 & 14.84 & 853.6 \\
\hline 14.66 & 840.8 & 14.22 & 810.9 & 13.87 & 786.6 & 14.93 & 818.8 & 14.4 & 840.4 & 14.93 & 843.5 & 13.98 & 798.1 & 13.84 & 803.8 & 14.43 & 829.8 \\
\hline 14.25 & 815.3 & 13.75 & 784.4 & 13.36 & 758.9 & 14.4 & 776.5 & 13.95 & 810.4 & 14.43 & 818.8 & 13.48 & 773.8 & 13.48 & 780.4 & 13.95 & 804.2 \\
\hline 13.45 & 776.5 & 13.3 & 754 & 12.92 & 734.6 & 13.63 & 753.1 & 13.57 & 787.9 & 13.63 & 780 & 13.04 & 748.7 & 13.04 & 752.7 & 13.45 & 780.4 \\
\hline 12.98 & 752.2 & 12.89 & 730.2 & 12.53 & 710.4 & 13.24 & 728.9 & 12.74 & 764.6 & 13.18 & 750.9 & 12.56 & 717.9 & 12.62 & 727.1 & 13.04 & 756.2 \\
\hline 12.53 & 721 & 12.47 & 706.9 & 12.09 & 685.7 & 12.77 & 698 & 12.3 & 722.3 & 12.71 & 724.5 & 12.11 & 691 & 12.22 & 703.3 & 12.74 & 730.7 \\
\hline 12.24 & 698 & 12.1 & 683.1 & 11.7 & 662.3 & 12.39 & 671.6 & 11.89 & 699.4 & 12.12 & 694.1 & 11.64 & 665.9 & 11.84 & 678.7 & 12.28 & 703.3 \\
\hline 11.76 & 672.9 & 11.65 & 659.3 & 11.28 & 637.2 & 11.94 & 644.3 & 11.38 & 670.7 & 12.15 & 687.5 & 11.21 & 639 & 11.36 & 651.8 & 11.71 & 676.9 \\
\hline 11.36 & 649.1 & 10.76 & 615.2 & 10.38 & 598.9 & 11.4 & 619.2 & 10.93 & 643.4 & 11.24 & 643 & 10.8 & 615.2 & 10.5 & 613.9 & 11.25 & 653.1 \\
\hline 10.89 & 625.3 & 10.33 & 589.2 & 9.998 & 566.3 & 10.93 & 594.5 & 10.45 & 617.8 & 10.72 & 614.8 & 10.3 & 589.2 & 10.1 & 590.1 & 10.46 & 611.2 \\
\hline 10.14 & 588.3 & 9.853 & 565 & 9.512 & 543.8 & 10.51 & 568.5 & 9.977 & 591.8 & 10.32 & 590.5 & 9.912 & 565 & 9.616 & 562.8 & 10.03 & 588.8 \\
\hline 9.728 & 564.1 & 9.376 & 535.9 & 8.98 & 510.8 & 10.02 & 545.6 & 9.568 & 565.8 & 9.906 & 567.2 & 9.376 & 540.3 & 9.222 & 532.8 & 9.574 & 556.1 \\
\hline 9.293 & 533.2 & 8.956 & 509.9 & 8.53 & 486.1 & 9.545 & 520 & 9.187 & 541.2 & 9.412 & 534.5 & 8.562 & 498.4 & 8.772 & 507.7 & 9.163 & 534.1 \\
\hline 8.811 & 509.4 & 8.494 & 481.7 & 8.035 & 458.8 & 9.157 & 479.5 & 8.684 & 516 & 8.944 & 511.6 & 8.183 & 475.5 & 8.195 & 479.5 & 8.42 & 498.9 \\
\hline 8.408 & 486.5 & 8.068 & 457.9 & 7.535 & 437.6 & 8.281 & 453.5 & 8.148 & 490.5 & 8.533 & 486.5 & 7.707 & 448.2 & 7.728 & 452.6 & 8.03 & 475.1 \\
\hline 8.006 & 463.2 & 7.547 & 435.4 & 7.011 & 403.2 & 7.816 & 428.8 & 7.313 & 465.4 & 8.056 & 462.3 & 7.325 & 421.7 & 7.222 & 423.9 & 7.6 & 445.5 \\
\hline 7.624 & 438.5 & 6.855 & 397.1 & 6.556 & 376.8 & 7.411 & 399.3 & 6.926 & 424.4 & 7.583 & 435.4 & 6.787 & 394.4 & 6.795 & 400.6 & 7.245 & 423.1 \\
\hline 6.721 & 395.7 & 6.42 & 373.3 & 6.103 & 353.9 & 7.003 & 375.9 & 6.426 & 397.5 & 7.053 & 408.5 & 6.319 & 371.1 & 6.378 & 375.5 & 6.795 & 395.7 \\
\hline 6.343 & 373.3 & 5.961 & 350.8 & 5.632 & 329.2 & 6.541 & 349.9 & 6.011 & 376.8 & 6.612 & 383.4 & 5.878 & 346.4 & 5.869 & 349 & 6.289 & 372.4 \\
\hline 5.845 & 343.7 & 5.481 & 317.7 & 4.827 & 290.4 & 6.124 & 325.7 & 5.49 & 344.2 & 5.733 & 341.5 & 5.372 & 317.7 & 5.357 & 324.3 & 5.801 & 345.1 \\
\hline 5.481 & 321.7 & 5.112 & 293.9 & & & 5.621 & 285.1 & 5.073 & 319.1 & 5.322 & 317.3 & 4.931 & 292.2 & 4.543 & 275 & 5.375 & 321.3 \\
\hline 5.008 & 294.8 & 4.644 & 271.5 & & & 4.771 & 258.7 & 4.576 & 293.9 & 4.872 & 292.2 & & & & & 4.898 & 294.8 \\
\hline 4.537 & 269.7 & 4.218 & 246.3 & & & 4.318 & & & 269.3 & 4.437 & 268.4 & & & & & 4.455 & 271.5 \\
\hline
\end{tabular}


Table 2. $25^{\circ} \mathrm{C}$

\begin{tabular}{|c|c|c|c|c|c|c|c|c|c|c|c|c|c|c|c|c|c|}
\hline run1 & & run2 & & run3 & & run4 & & run5 & & run6 & & run7 & & run8 & & run9 & \\
\hline [Pa] & {$[1 / \mathrm{s}]$} & {$[\mathrm{Pa}]$} & {$[1 / \mathrm{s}]$} & {$[\mathrm{Pa}]$} & {$[1 / \mathrm{s}]$} & {$[\mathrm{Pa}]$} & {$[1 / \mathrm{s}]$} & {$[\mathrm{Pa}]$} & {$[1 / \mathrm{s}]$} & {$[\mathrm{Pa}]$} & {$[1 / \mathrm{s}]$} & {$[\mathrm{Pa}]$} & {$[1 / \mathrm{s}]$} & {$[\mathrm{Pa}]$} & {$[1 / \mathrm{s}]$} & {$[\mathrm{Pa}]$} & {$[1 / \mathrm{s}]$} \\
\hline 8.197 & 309.4 & 8.893 & 319.9 & 8.395 & 305.4 & 9.185 & 316.4 & 10.23 & 327.4 & 8.292 & 309.8 & 10.17 & 323.5 & 8.964 & 320.8 & 9.097 & 328.3 \\
\hline 10.36 & 312.9 & .274 & 321.3 & 10.59 & 323.5 & 9.943 & 327.9 & 10.54 & 345.5 & 9.431 & 312 & 10.83 & 347.3 & 9.896 & 327 & 9.434 & 327.4 \\
\hline 10.98 & 335.8 & 11.09 & 335.8 & 10.73 & 342.4 & 11.52 & 347.7 & 11.85 & 371.1 & 10.82 & 343.3 & 12.49 & 376.8 & 10.63 & 343.7 & 11.18 & 344.2 \\
\hline 12.24 & 369.7 & 11.29 & 358.3 & 11.65 & 370.6 & 11.7 & 369.7 & 13.34 & 396.6 & 11.66 & 370.6 & 13.58 & 402.8 & 12.05 & 365.3 & 12.08 & 369.3 \\
\hline 13.12 & 400.1 & 12.21 & 382.5 & 13.2 & 394.9 & 12.8 & 402.3 & 13.96 & 420.9 & 13.25 & 392.2 & 13.6 & 419.1 & 12.67 & 391.3 & 12.49 & 393.5 \\
\hline 14.76 & 420.4 & 13.63 & 422.6 & 13.47 & 414.2 & 13.79 & 429.7 & 14.86 & 450.4 & 13.98 & 417.3 & 15.05 & 454.3 & 14.44 & 431.4 & 13.48 & 417.8 \\
\hline 15.98 & 465.4 & 14.81 & 446.4 & 15.27 & 451.7 & 15.23 & 453.9 & 15.46 & 474.6 & 14.39 & 441.1 & 15.86 & 480.3 & 15.37 & 457.4 & 14.82 & 445.1 \\
\hline 15.88 & 475.9 & 15.77 & 475.5 & 16.35 & 482.1 & 16.34 & 480.8 & 17.2 & 509 & 15.26 & 464.9 & 16.81 & 507.7 & 15.79 & 465.8 & 15.82 & 482.5 \\
\hline 17 & 490.9 & 16.89 & 498 & 16.73 & 503.3 & 17.1 & 503.3 & 18.48 & 540.3 & 16.23 & 490.5 & 17.8 & 532.8 & 17.12 & 509.4 & 16.93 & 506.3 \\
\hline 18.45 & 530.1 & 17.75 & 527.5 & 17.32 & 526.6 & 17.87 & 527.5 & 19.3 & 564.5 & 17.2 & 518.2 & 18.83 & 563.2 & 17.62 & 532.3 & 17.9 & 529.7 \\
\hline 18.65 & 550.4 & 18.71 & 550.9 & 18.12 & 549.1 & 18.45 & 550 & 20.07 & 587.4 & 19.3 & 563.6 & 19.87 & 588.8 & 19.3 & 566.3 & 18.45 & 560.1 \\
\hline 20.37 & 582.1 & 19.54 & 582.6 & 19.04 & 574.7 & 19.9 & 594 & 20.93 & 613 & 19.04 & 570.7 & 20.93 & 613 & 20.22 & 590.1 & 19.39 & 585.2 \\
\hline 20.72 & 595.8 & 20.43 & 607.7 & 20.64 & 618.7 & 21.14 & 619.6 & 21.58 & 638.5 & 20.1 & 596.2 & 21.91 & 637.2 & 20.93 & 618.3 & 20.25 & 609.5 \\
\hline 22.56 & 655.7 & 21.91 & 639.4 & 21.79 & 643.4 & 21.91 & 642.5 & 22.2 & 663.7 & 21.23 & 622.2 & 22.94 & 674.2 & 21.64 & 642.1 & 21.35 & 634.1 \\
\hline 22.26 & 655.7 & 22.62 & 660.6 & 22.97 & 671.2 & 22.77 & 676.9 & 23.89 & 701.6 & 22.97 & 667.2 & 23.83 & 696.7 & 22.44 & 667.2 & 22.59 & 660.1 \\
\hline & & 23.42 & 694.1 & 23.54 & 697.6 & 23.89 & 702.4 & 25.05 & 731.5 & 23.8 & 696.7 & 24.81 & 725.8 & 23.71 & 693.6 & 23.18 & 686.1 \\
\hline & & 24.45 & 717.9 & 24.25 & 719.2 & 24.96 & 728.9 & 25.73 & 756.7 & 24.39 & 721 & 25.49 & 749.6 & 24.72 & 718.8 & 24.66 & 728 \\
\hline & & 25.16 & 749.2 & 25.55 & 751.4 & 25.61 & 750.9 & 26.44 & 780.4 & 25.55 & 745.2 & 26.58 & 771.6 & 25.31 & 746.5 & 25.4 & 750.9 \\
\hline & & 26.29 & 771.6 & 26.05 & 774.3 & 26.17 & 774.7 & 27.65 & 805.1 & 26.32 & 770.3 & 27.32 & 797.2 & 26.47 & 773.8 & 26.7 & 776.9 \\
\hline & & 27.15 & 795 & 26.82 & 796.3 & 27.41 & 799 & 28.24 & 832 & 26.91 & 792.8 & 28.33 & 827.2 & 27.56 & 802 & 27.77 & 810 \\
\hline & & 27.53 & 817.5 & 28.45 & 833.3 & 28.51 & 839.5 & 29.37 & 856.7 & 28.18 & 816.6 & 29.16 & 851 & 28.98 & 838.6 & 28.24 & 835.1 \\
\hline & & 29.28 & 860.7 & 29.16 & 860.7 & 29.69 & 865.5 & 30.4 & 895 & 29.34 & 857.1 & 30.14 & 877 & 29.57 & 862.9 & 29.48 & 859.3 \\
\hline 29.75 & 857.1 & 29.84 & 882.7 & 30.08 & 89 & 30.43 & 893.3 & 31.64 & 919.7 & 30.52 & 883.1 & 31.67 & 918.8 & 30.64 & 892.4 & 29.87 & 884 \\
\hline 34.87 & 978.3 & 30.82 & 914.4 & 30.96 & & 31.35 & 922.3 & 32.33 & 946.1 & 31.38 & 914.9 & 32.71 & 948.3 & 31.64 & 917.1 & 31.05 & 907.8 \\
\hline 36.88 & 1055 & 31.91 & 938.2 & 31.97 & 942.2 & 32.18 & 947 & 32.92 & 967.7 & 32 & 934.2 & 33.57 & 976.6 & 32.15 & 940 & 31.94 & 937.8 \\
\hline 37.53 & 1085 & 32.56 & 961.6 & 32.59 & 964.2 & 33.18 & 970.8 & 33.78 & 988.9 & 33.12 & 967.7 & 34.19 & 999.5 & 33.24 & 971.7 & 32.77 & 962.5 \\
\hline 39.07 & 1120 & 33.27 & 985.8 & 33.51 & 993.7 & 33.89 & 995.5 & 35.4 & 1031 & 33.95 & 994.2 & 35.17 & 1027 & 34.04 & 995.1 & 33.51 & 986.7 \\
\hline 39.37 & 1141 & 34.87 & 1022 & 34.46 & 1018 & 34.75 & 1022 & 36.14 & 1057 & 34.81 & 1020 & 36.14 & 1047 & 34.99 & 1021 & 34.99 & 1025 \\
\hline 40.79 & 1170 & 35.73 & 1048 & 35.4 & 1043 & 36 & 1059 & 37.06 & 1082 & 35.82 & 1048 & 36.91 & 1074 & 35.76 & 1042 & 35.88 & 1050 \\
\hline 41.03 & 1190 & 36.5 & 1076 & 36.79 & 1083 & 36.79 & 1085 & 37.8 & 1107 & 36.59 & 1071 & 37.8 & 1098 & 36.65 & 1072 & 36.56 & 1080 \\
\hline 42 & 1222 & 37.45 & 1102 & 37.71 & 1109 & 37.71 & 1108 & 38.75 & 1137 & 37.56 & 1095 & 38.6 & 1125 & 37.62 & 1098 & 37.33 & 1105 \\
\hline
\end{tabular}


Table 2. $25^{\circ} \mathrm{C}$ - continued

\begin{tabular}{|c|c|c|c|c|c|c|c|c|c|c|c|c|c|c|c|c|c|}
\hline run1 & & run2 & & run3 & & run4 & & run5 & & run6 & & run7 & & run8 & & run9 & \\
\hline$[\mathrm{Pa}]$ & {$[1 / \mathrm{s}]$} & {$[\mathrm{Pa}]$} & {$[1 / \mathrm{s}]$} & {$[\mathrm{Pa}]$} & {$[1 / \mathrm{s}]$} & {$[\mathrm{Pa}]$} & {$[1 / \mathrm{s}]$} & {$[\mathrm{Pa}]$} & {$[1 / \mathrm{s}]$} & {$[\mathrm{Pa}]$} & {$[1 / \mathrm{s}]$} & {$[\mathrm{Pa}]$} & {$[1 / \mathrm{s}]$} & {$[\mathrm{Pa}]$} & {$[1 / \mathrm{s}]$} & {$[\mathrm{Pa}]$} & {$[1 / \mathrm{s}]$} \\
\hline 42.56 & 1238 & 38.16 & 1130 & 38.48 & 1134 & 38.69 & 1137 & 39.69 & 1164 & 38.36 & 1118 & 40.26 & 1170 & 39.01 & 1139 & 38.36 & 1129 \\
\hline 43.51 & 1265 & 38.95 & 1153 & 39.22 & 1161 & 39.25 & 1159 & 40.49 & 1188 & 39.66 & 1159 & 40.2 & 1178 & 39.72 & 1162 & 39.07 & 1157 \\
\hline 44.07 & 1286 & 39.75 & 1178 & 40.11 & 1183 & 40.02 & 1181 & 41.44 & 1219 & 40.52 & 1184 & 41.91 & 1221 & 40.88 & 1194 & 40.2 & 1182 \\
\hline 45.11 & 1311 & 40.73 & 1202 & 40.85 & 1211 & 40.88 & 1205 & 42.48 & 1246 & 41.47 & 1209 & 42.65 & 1244 & 41.68 & 1220 & 41.03 & 1210 \\
\hline 45.82 & 1331 & 41.8 & 1239 & 41.88 & 1237 & 41.8 & 1233 & 43.33 & 1268 & 42.39 & 1242 & 43.48 & 1269 & 42.51 & 1241 & 41.83 & 1236 \\
\hline 46.53 & 1353 & 42.65 & 1265 & 42.54 & 1264 & 42.8 & 1261 & 44.13 & 1299 & 43.25 & 1267 & 44.58 & 1304 & 43.22 & 1267 & 42.71 & 1262 \\
\hline 47.06 & 1376 & 43.39 & 1289 & 44.28 & 1306 & 44.28 & 1305 & 45.08 & 1323 & 44.19 & 1292 & 45.38 & 1329 & 44.07 & 1291 & 44.22 & 1304 \\
\hline 48.4 & 1408 & 44.25 & 1315 & 44.9 & 1327 & 44.96 & 1327 & 45.76 & 1349 & 44.99 & 1320 & 46.15 & 1353 & 44.9 & 1313 & 45.05 & 1328 \\
\hline 48.45 & 1423 & 45.11 & 1343 & 45.67 & 1353 & 46 & 1352 & 46.47 & 1372 & 45.94 & 1343 & 47.06 & 1378 & 46.12 & 1351 & 45.88 & 1353 \\
\hline 49.79 & 1452 & 46.18 & 1369 & 46.53 & 1378 & 46.94 & 1385 & 47.98 & 1408 & 46.59 & 1367 & 47.89 & 1404 & 47.06 & 1381 & 46.74 & 1379 \\
\hline 50.14 & 1473 & 46.8 & 1394 & 47.36 & 1403 & 47.57 & 1409 & 48.93 & 1440 & 48.1 & 1408 & 48.81 & 1428 & 47.92 & 1408 & 47.68 & 1409 \\
\hline 51.3 & 1498 & 47.74 & 1421 & 48.45 & 1435 & 48.51 & 1435 & 49.67 & 1462 & 48.84 & 1431 & 49.4 & 1452 & 48.87 & 1433 & 48.42 & 1431 \\
\hline 51.68 & 1519 & 48.75 & 1449 & 49.11 & 1457 & 49.25 & 1458 & 50.58 & 1489 & 49.67 & 1455 & 50.91 & 1492 & 49.67 & 1460 & 49.16 & 1454 \\
\hline 52.95 & 1552 & 49.99 & 1485 & 49.79 & 1479 & 50.02 & 1485 & 51.56 & 1519 & 50.53 & 1482 & 51.77 & 1516 & 50.56 & 1488 & 49.96 & 1481 \\
\hline 53.28 & 1568 & 50.79 & 1511 & 51.06 & 1516 & 51.41 & 1523 & 52.45 & 1543 & 51.44 & 1512 & 52.72 & 1547 & 51.38 & 1514 & 50.91 & 1507 \\
\hline 50.67 & 1547 & 46.92 & 1417 & 46.26 & 1400 & 46.86 & 1405 & 47.09 & 1407 & 49.19 & 1403 & 45.2 & 1400 & 47.15 & 1412 & 46.47 & 1413 \\
\hline 50.05 & 1522 & 45.88 & 1389 & 45.49 & 1377 & 45.97 & 1382 & 46.21 & 1385 & 48.42 & 1393 & 44.34 & 1376 & 45.55 & 1370 & 45.85 & 1390 \\
\hline 49.08 & 1493 & 45.26 & 1364 & 44.7 & 1348 & 45.2 & 1356 & 45.2 & 1357 & 46.83 & 1351 & 43.54 & 1352 & 44.61 & 1345 & 44.7 & 1355 \\
\hline 48.25 & 1470 & 44.19 & 1340 & 43.87 & 1326 & 444.4 & 13 & 44.19 & 1323 & 46.89 & 1327 & 42.68 & 1326 & 43.99 & 1323 & 43.66 & 1325 \\
\hline 47.51 & 1445 & 43.39 & 1311 & 43.13 & 1303 & 43.57 & 1308 & 43.42 & 1301 & 45.23 & 1301 & 41.11 & 1303 & 42.89 & 1289 & 42.89 & 1301 \\
\hline 46.68 & 1422 & 41.91 & 1272 & 41.85 & 1265 & 42.54 & 1278 & 42.06 & 1264 & 44.46 & 1271 & 40.4 & 1278 & 42.12 & 1264 & 42.06 & 1278 \\
\hline 45.23 & 1380 & 41.03 & 1249 & 40.97 & 1239 & 41.71 & 1255 & 41.38 & 1241 & 43.33 & 1245 & 39.64 & 1237 & 41.35 & 1241 & 41.32 & 1255 \\
\hline 44.58 & 1355 & 40.23 & 1218 & 40.08 & 1212 & 40.2 & 1211 & 40.52 & 1212 & 42.56 & 1220 & 38.42 & 1214 & 40.26 & 1211 & 39.78 & 1215 \\
\hline 43.69 & 1329 & 39.55 & 1195 & 39.07 & 1189 & 39.43 & 1188 & 39.72 & 1191 & 41.65 & 1194 & 37.56 & 1188 & 39.37 & 1184 & 39.55 & 1205 \\
\hline 42.83 & 1306 & 38.72 & 1172 & 38.24 & 1163 & 38.54 & 1155 & 38.54 & 1159 & 40.79 & 1170 & 36.88 & 1155 & 38.45 & 1155 & 38.16 & 1163 \\
\hline 42.18 & 1281 & 37.95 & 1148 & 37.47 & 1131 & 37.68 & 1130 & 37.8 & 1135 & 40.11 & 1131 & 36.14 & 1129 & 37.56 & 1134 & 37.36 & 1139 \\
\hline 41.26 & 1257 & 37.21 & 1122 & 36.73 & 1105 & 36.82 & & 37.06 & 111 & 39.22 & 1109 & 34.63 & 1109 & 36.44 & 1099 & 36.35 & 1110 \\
\hline 40.46 & 1231 & 35.55 & & 35.64 & & 35.79 & & 36.26 & & 37.71 & 1080 & 33.72 & 1084 & 35.64 & 1077 & 35.64 & 1085 \\
\hline 38.84 & 1190 & 34.81 & 1058 & 34.75 & 1057 & 35.11 & 1059 & 34.93 & 1048 & 36.88 & 1054 & 32.92 & 1044 & 34.93 & 1053 & 34.78 & 1059 \\
\hline 38.04 & 1167 & 33.95 & 1026 & 34.16 & & 34.49 & & 33.95 & 1025 & 36.08 & 1027 & 32.09 & 1021 & 34.1 & 1027 & 33.83 & 1037 \\
\hline 37.24 & 1135 & 32.95 & 003 & 32.53 & 0.2 & 33.04 & 994.6 & 33.42 & 1003 & 35.2 & 1003 & 30.76 & 995.9 & 32.62 & 991.1 & 32.95 & 1012 \\
\hline 36.5 & 1112 & 32.24 & 980.1 & 31.85 & 966.4 & 31.97 & 969.5 & 32.03 & 969.5 & 34.28 & 979.6 & 30.16 & 969.1 & 31.91 & 966.9 & 32.09 & 984.9 \\
\hline
\end{tabular}


Table 2. $25^{\circ} \mathrm{C}$ - continued

\begin{tabular}{|c|c|c|c|c|c|c|c|c|c|c|c|c|c|c|c|c|c|}
\hline run1 & & run2 & & run3 & & run4 & & run5 & & run6 & & run7 & & run8 & & run9 & \\
\hline$[\mathrm{Pa}]$ & {$[1 / \mathrm{s}]$} & {$[\mathrm{Pa}]$} & {$[1 / \mathrm{s}]$} & {$[\mathrm{Pa}]$} & {$[1 / \mathrm{s}]$} & {$[\mathrm{Pa}]$} & {$[1 / \mathrm{s}]$} & {$[\mathrm{Pa}]$} & {$[1 / \mathrm{s}]$} & {$[\mathrm{Pa}]$} & {$[1 / \mathrm{s}]$} & {$[\mathrm{Pa}]$} & {$[1 / \mathrm{s}]$} & {$[\mathrm{Pa}]$} & {$[1 / \mathrm{s}]$} & {$[\mathrm{Pa}]$} & {$[1 / \mathrm{s}]$} \\
\hline 35.58 & 1092 & 31.44 & 955.8 & 30.88 & 943.5 & 30.96 & 938.7 & 31.44 & 943.9 & 33.21 & 956.3 & 29.07 & 935.1 & 30.82 & 941.7 & 31.53 & 960.2 \\
\hline 34.84 & 1067 & 30.4 & 929.8 & 29.78 & 911.3 & 30.08 & 913.5 & 30.49 & 919.7 & 32.83 & 929.4 & 28.57 & 908.7 & 30.34 & 918.4 & 29.87 & 916.2 \\
\hline 34.31 & 1043 & 28.98 & 888.9 & 29.4 & 889.7 & 29.37 & 889.7 & 29.63 & 896.3 & 31.64 & 888.9 & 27.41 & 885.3 & 28.89 & 883.1 & 28.86 & 891.9 \\
\hline 32.65 & 998.6 & 28.24 & 863.3 & 28.24 & 863.7 & 28.54 & 864.6 & 28.92 & 870.3 & 30.76 & 865.1 & 26.73 & 861.5 & 28.18 & 856.2 & 28.18 & 869.5 \\
\hline 31.85 & 974.3 & 27.12 & 834.7 & 27.71 & 838.2 & 27.59 & 841.3 & 28.09 & 847.9 & 29.54 & 832 & 25.22 & 837.3 & 26.88 & 829.8 & 27.06 & 836.4 \\
\hline 30.82 & 949.7 & 26.38 & 811.7 & 26.41 & 811.7 & 26.17 & 796.8 & 26.35 & 803.4 & 28.57 & 806.4 & 24.78 & 810 & 25.81 & 792.3 & 26.47 & 811.7 \\
\hline 30.16 & 923.2 & 25.96 & 789.3 & 25.9 & 784.4 & 25.84 & 789.7 & 25.76 & 778.7 & 27.59 & 781.3 & 23.59 & 769.9 & 25.7 & 782.7 & 25.31 & 787.9 \\
\hline 29.28 & 893.3 & 24.84 & 765 & 24.54 & 758 & 24.54 & 747.4 & 24.69 & 754.4 & 26.58 & 755.8 & 22.74 & 761.9 & 24.39 & 756.7 & 24.63 & 762.4 \\
\hline 28.03 & 868.6 & 23.3 & 721.8 & 23.27 & 720.5 & 23.8 & 723.2 & 23.8 & 720.5 & 25.96 & 731.5 & 21.55 & 717.9 & 23.51 & 727.1 & 23.06 & 725.4 \\
\hline 27.56 & 847.4 & 23.42 & 713 & 22.26 & 697.6 & 22.5 & 694.1 & 22.97 & 697.2 & 24.75 & 707.3 & 20.4 & 693.6 & 21.61 & 685.7 & 22.5 & 702 \\
\hline 26.7 & 821.9 & 21.76 & 669.4 & 21.79 & 664.1 & 21.61 & 673.4 & 21.94 & 673.4 & 23.95 & 681.7 & 19.51 & 666.8 & 21.46 & 673.8 & 21.76 & 674.2 \\
\hline 24.99 & 780 & 21.05 & 647.8 & 20.75 & 638.5 & 21.05 & 641.2 & 20.96 & 650 & 23.39 & 642.5 & 18.86 & 637.2 & 20.99 & 654 & 20.52 & 647.8 \\
\hline 24.39 & 758.9 & 20.07 & 621.4 & 19.98 & 615.2 & 20.13 & 620 & 19.98 & 622.2 & 22.47 & 617.4 & 18.15 & 616.1 & 20.25 & 638.1 & 19.39 & 617.8 \\
\hline 23.45 & 728 & 18.56 & 589.6 & 18.8 & 587 & 19.45 & 595.4 & 19.22 & 596.2 & 21.08 & 577.7 & 16.85 & 593.6 & 18.71 & 589.6 & 18.53 & 597.1 \\
\hline 22.44 & 704.7 & 17.79 & 566.3 & 17.71 & 560.5 & 17.44 & 556.6 & 18.59 & 567.6 & 20.28 & 549.1 & 16.08 & 562.8 & 17.94 & 574.2 & 17.85 & 568.9 \\
\hline 21.61 & 682.2 & 17.2 & 543.4 & 17.32 & 537.6 & 16.67 & 530.1 & 17.47 & 543.4 & 19.04 & 533.2 & 14.89 & 525.3 & 17.03 & 545.1 & 16.85 & 546 \\
\hline 20.93 & 657.9 & 15.57 & 507.2 & 16.55 & 512.5 & 15.66 & 503.3 & 16.14 & 504.1 & 17.17 & 506.3 & 13.98 & 501.1 & 15.31 & 503.3 & 16.08 & 521.8 \\
\hline 19.48 & 617.8 & 15.1 & 485.6 & 14.66 & 475.9 & 15.25 & 479.5 & 15.4 & 477.3 & 17.29 & 479 & 12.7 & 479.5 & 14.98 & 490 & 14.89 & 480.8 \\
\hline 18.48 & 591.8 & 13.89 & 452.1 & 14.07 & 447.3 & 14.57 & 471.1 & 14.36 & 451.7 & 15.81 & 478.1 & 12.52 & 444.6 & 13.89 & 457.4 & 13.74 & 453 \\
\hline 17.59 & 568 & 13.56 & 425.7 & 13.56 & 424.8 & 12.76 & 424.4 & 13.33 & 420 & 15.13 & 455.2 & 11.6 & 421.7 & 13.68 & 431.9 & 12.59 & 426.1 \\
\hline 16.82 & 535.9 & 12.73 & 401 & 12.11 & 402.3 & 12.57 & 409.4 & 12.06 & 397.9 & 15.16 & 397.5 & 10.06 & 396.6 & 12.25 & 391.3 & 11.83 & 397.1 \\
\hline 15.81 & 512.5 & 11.11 & 369.3 & 10.7 & 365.3 & 11.34 & 372.8 & 12.12 & 375 & 14.36 & 381.2 & 10.08 & 372.8 & 11.18 & 366.2 & 11.52 & 373.7 \\
\hline 15.19 & 489.6 & 10.51 & 341.1 & 10.64 & 339.8 & 10.17 & 327 & 10.67 & 348.6 & 12.26 & 359.6 & 8.327 & 346.8 & 9.591 & 334.5 & 9.99 & 343.3 \\
\hline 14.57 & 463.2 & 10.13 & 327.4 & 9.174 & 316.4 & 8.691 & 291.3 & 9.733 & 323.5 & 12.23 & 344.2 & 8.383 & 323 & 9.621 & 310.2 & 9.872 & 322.6 \\
\hline 13.95 & 437.6 & 7.993 & 284.2 & 8.745 & 289.1 & & & 9.183 & 300.5 & 10.41 & 290.4 & 6.507 & 294.8 & 7.981 & 285.6 & 8.576 & 289.5 \\
\hline 12.61 & 397.9 & 7.88 & 273.7 & 7.587 & 260.9 & & & 7.037 & 260.9 & 10.38 & 278.5 & & & 7.945 & 260 & 7.706 & 266.2 \\
\hline 11.14 & 375.5 & & & 7.738 & 253 & & & 7.081 & 253 & 8.875 & 244.6 & & & & & 6.489 & 242.4 \\
\hline 10.24 & 346.8 & & & & & & & & & 8.446 & 157.9 & & & & & & \\
\hline 10.04 & 323.9 & & & & & & & & & 6.558 & 162.1 & & & & & & \\
\hline 8.611 & 300.5 & & & & & & & & & & & & & & & & \\
\hline 8.046 & 261.8 & & & & & & & & & & & & & & & & \\
\hline 7.549 & 254.3 & & & & & & & & & & & & & & & & \\
\hline
\end{tabular}


Table 3. Rheology Summary for Blank

\begin{tabular}{|c|c|c|c|}
\hline Run \# & $\begin{array}{c}\text { M*1000 } \\
(\mathbf{P a - s})\end{array}$ & $\begin{array}{c}\mathbf{B} \\
(\mathbf{P a})\end{array}$ & $\mathbf{R}^{\mathbf{2}}$ \\
\hline 1 & 17.6 & 0.1561 & \\
\hline 2 & 17.8 & 0.0832 & \\
\hline 3 & 17.9 & 0.1271 & \\
\hline 4 & 17.9 & 0.1214 & \\
\hline 5 & 17.9 & 0.1771 & \\
\hline 6 & 18.0 & 0.0835 & \\
\hline 7 & 17.9 & 0.1080 & \\
\hline 8 & 17.8 & 0.0579 & \\
\hline 9 & 17.7 & 0.1030 & \\
\hline & & & \\
\hline$\delta \cong \mathrm{S}$ & $\mathrm{Z}= \pm 1.96 \delta$ & $\mathrm{N}=9$ & $95 \% \mathrm{CL}= \pm 0.1$ \\
\hline & & Sigma $=0.1$ & \\
\hline Statistics for $\mathrm{M}$ & $\mathrm{Mean}=17.8$ & $\mathrm{Sigma}=0.0373$ & \\
\hline Statistics for $\mathrm{B}$ & $\mathrm{Mean}=0.1130$ & & \\
\hline
\end{tabular}


Table 4. Rheology Summary for $25^{\circ} \mathrm{C}$

\begin{tabular}{|c|c|c|c|c|}
\hline Run \# & $\begin{array}{c}\mathbf{M}^{*} \mathbf{1 0 0 0} \\
\text { (Pa-s) }\end{array}$ & & $\mathbf{R}^{\mathbf{2}}$ & $\begin{array}{c}\text { Viscosity } \\
\text { (cP) }\end{array}$ \\
\hline 1 & 34.3 & & 0.9953 & 16.5 \\
\hline 2 & 34.2 & & 0.9980 & 16.4 \\
\hline 3 & 34.3 & & 0.9983 & 16.5 \\
\hline 4 & 34.4 & & 0.9983 & 16.6 \\
\hline 5 & 34.6 & & 0.9985 & 16.8 \\
\hline 6 & 34.4 & & 0.9965 & 16.6 \\
\hline 7 & 35.1 & & 0.9860 & 17.3 \\
\hline 8 & 34.8 & & 0.9973 & 17.0 \\
\hline 9 & 34.5 & & 0.9972 & 16.7 \\
\hline & & & & \\
\hline$\delta \cong \mathrm{s}$ & $\mathrm{Z}= \pm 1.96 \delta$ & $\mathrm{N}=9$ & & \\
\hline & & & & \\
\hline Statistics for M & $\mathrm{Mean}=34.5$ & Sigma $=0.3$ & $95 \% \mathrm{CL}= \pm 0.2$ & $(13 \%)$ High \\
\hline
\end{tabular}




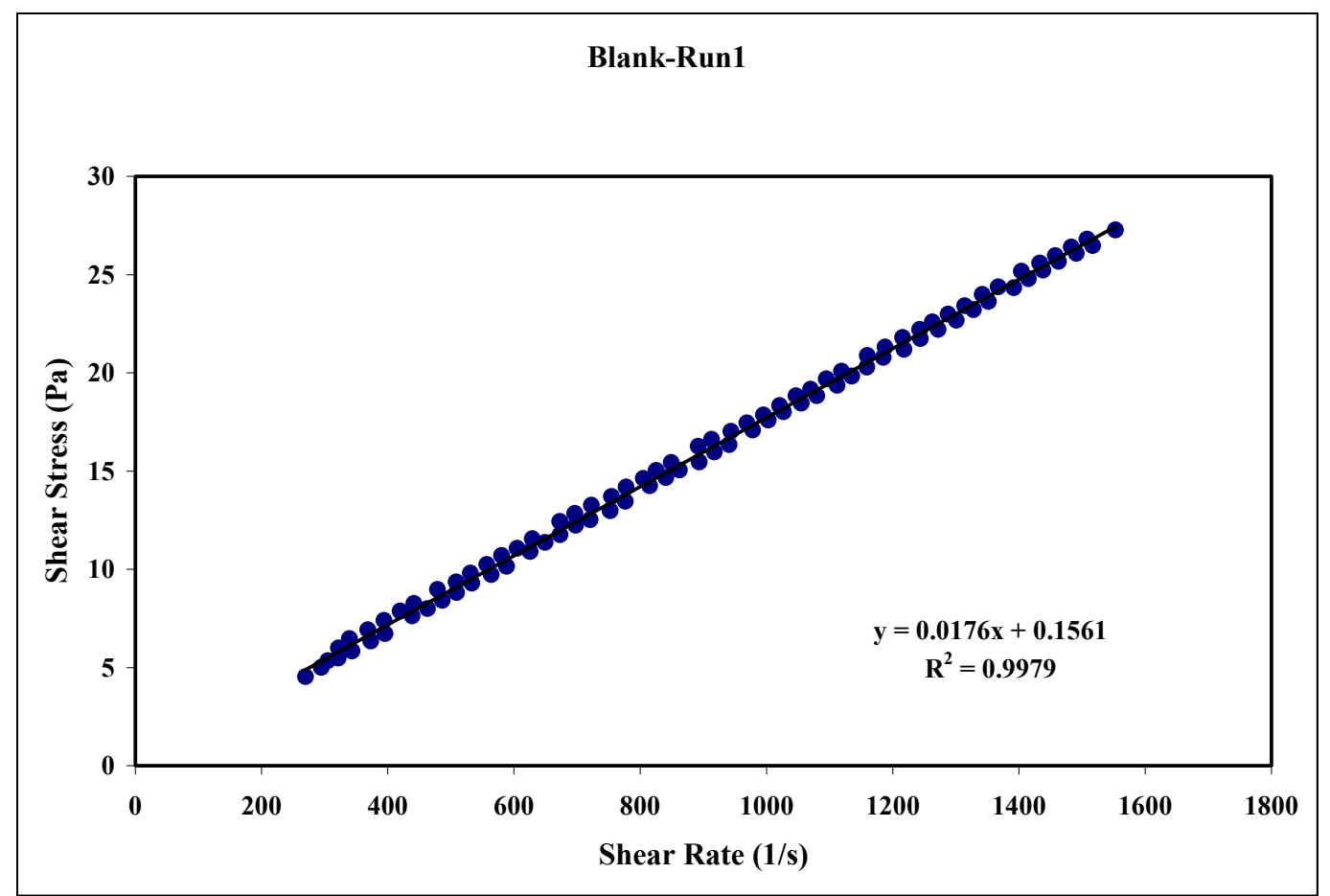

Figure 1. Blank Run 1

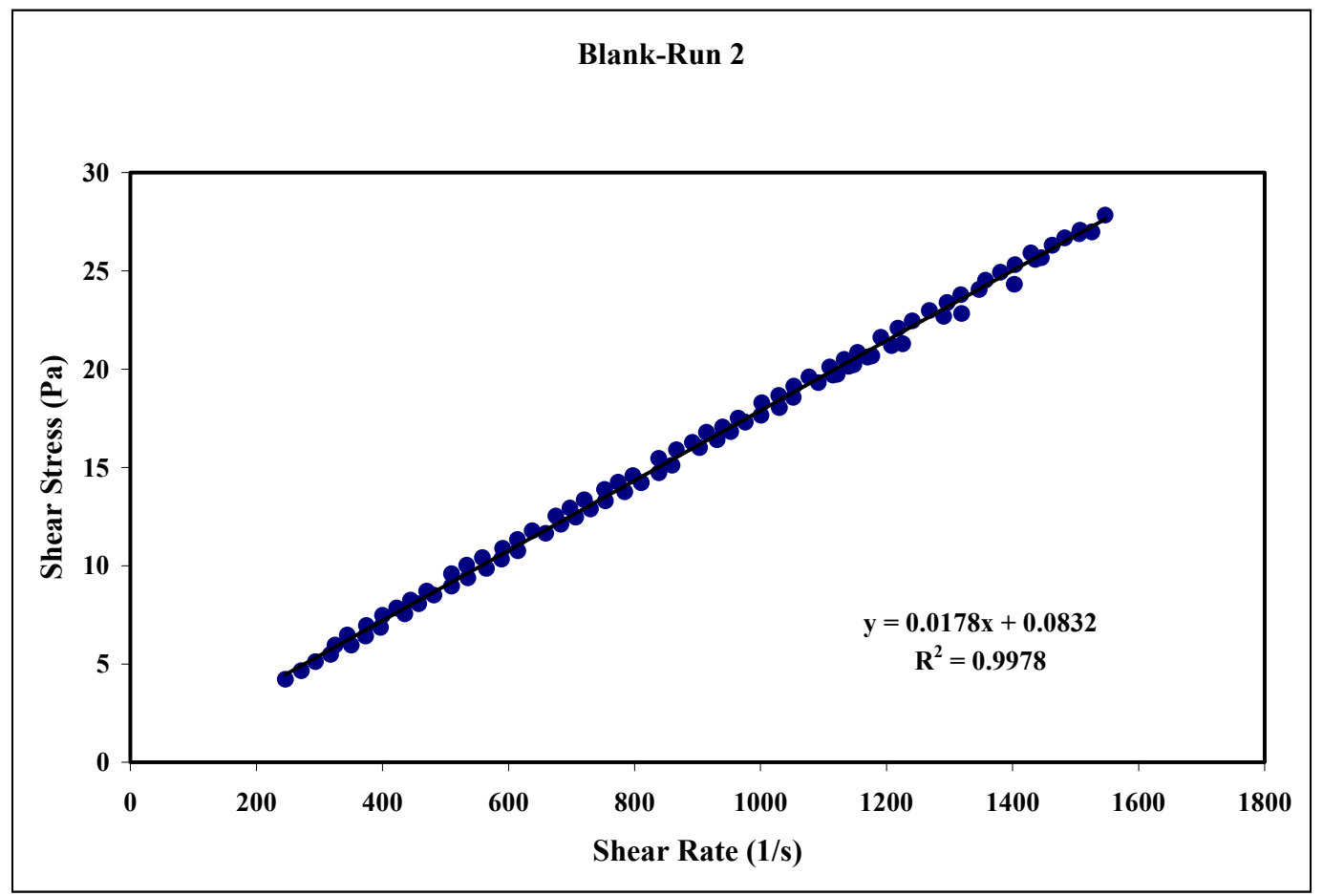

Figure 2. Blank Run 2 


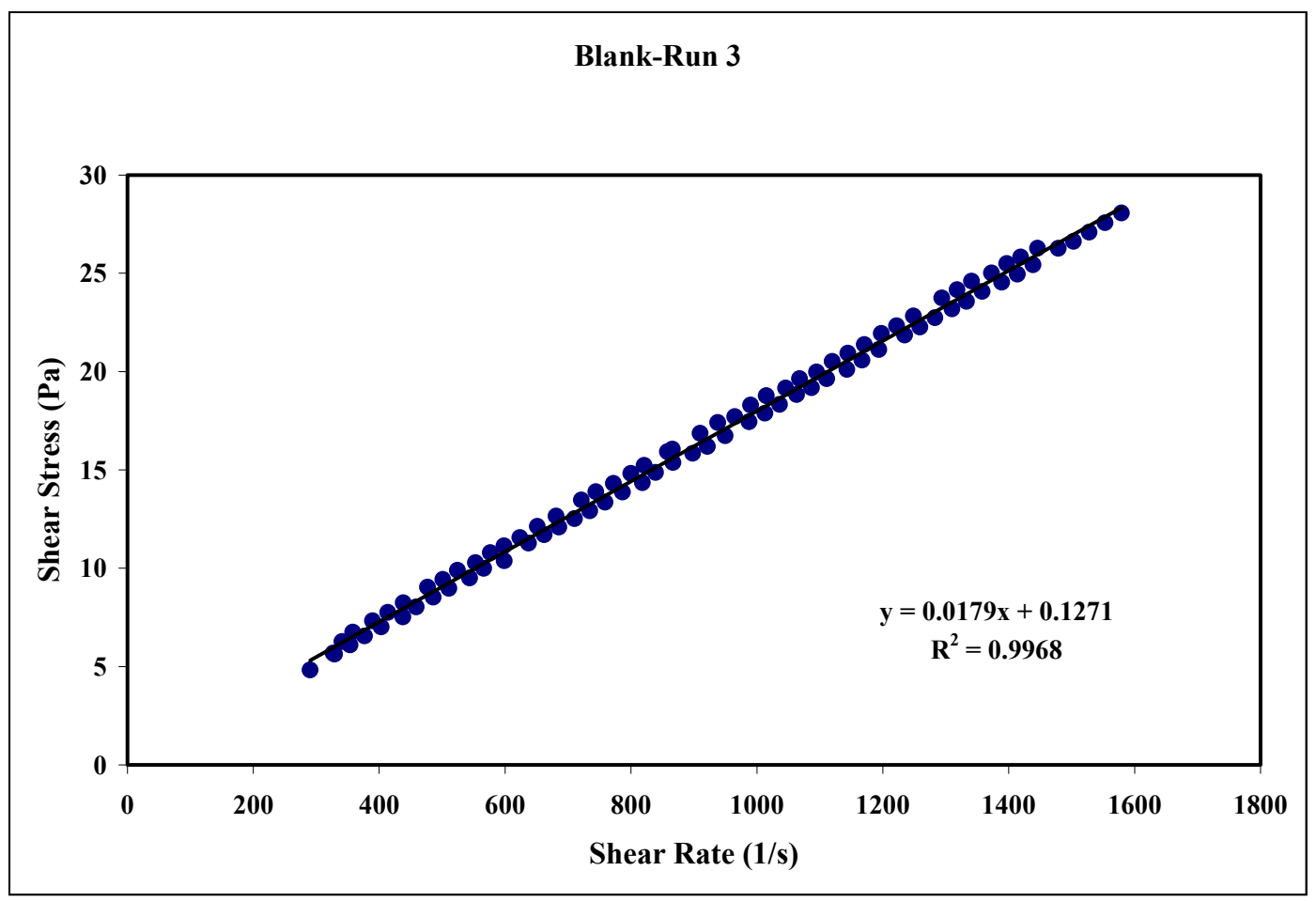

Figure 3. Blank Run 3

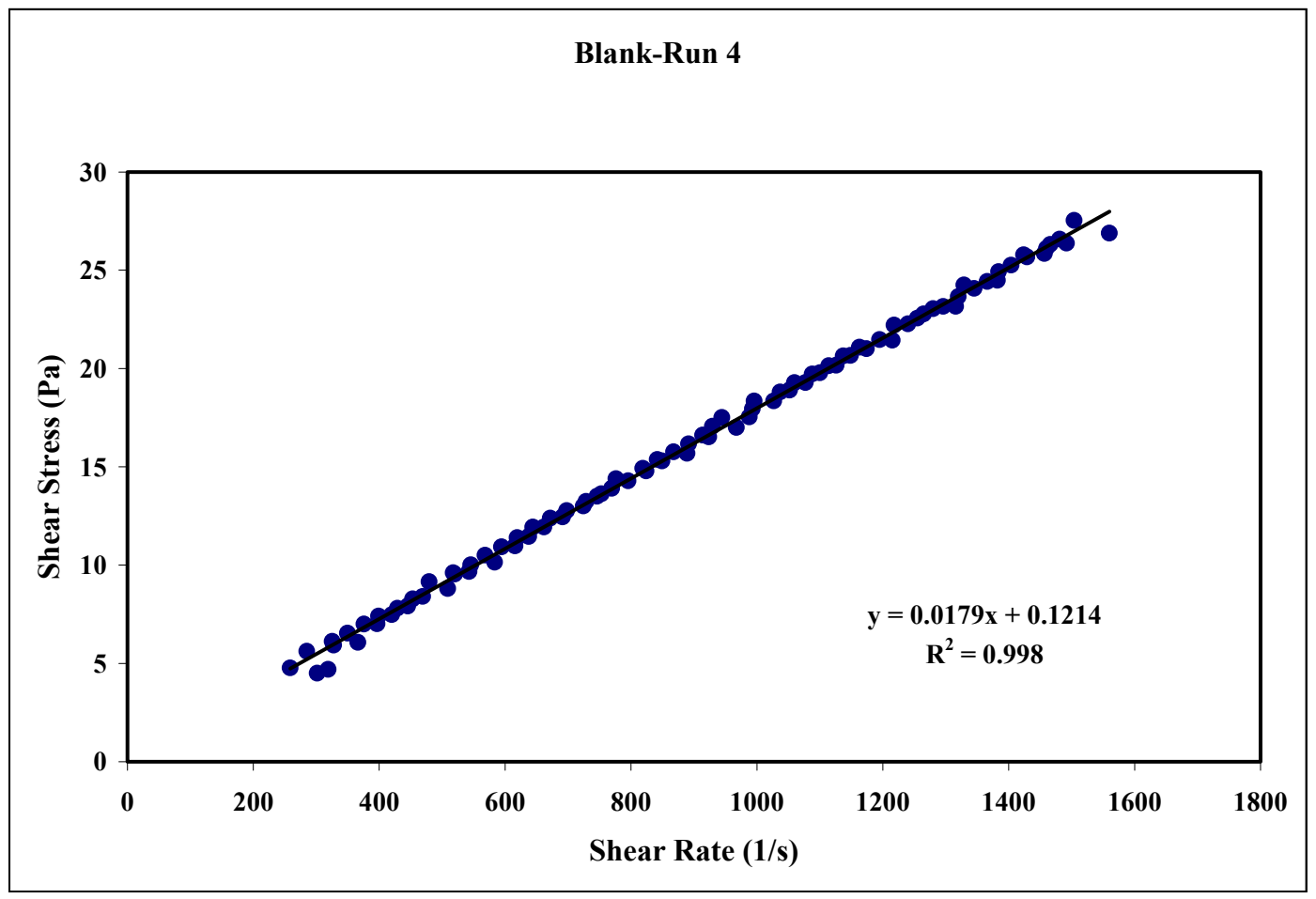

Figure 4. Blank Run 4 


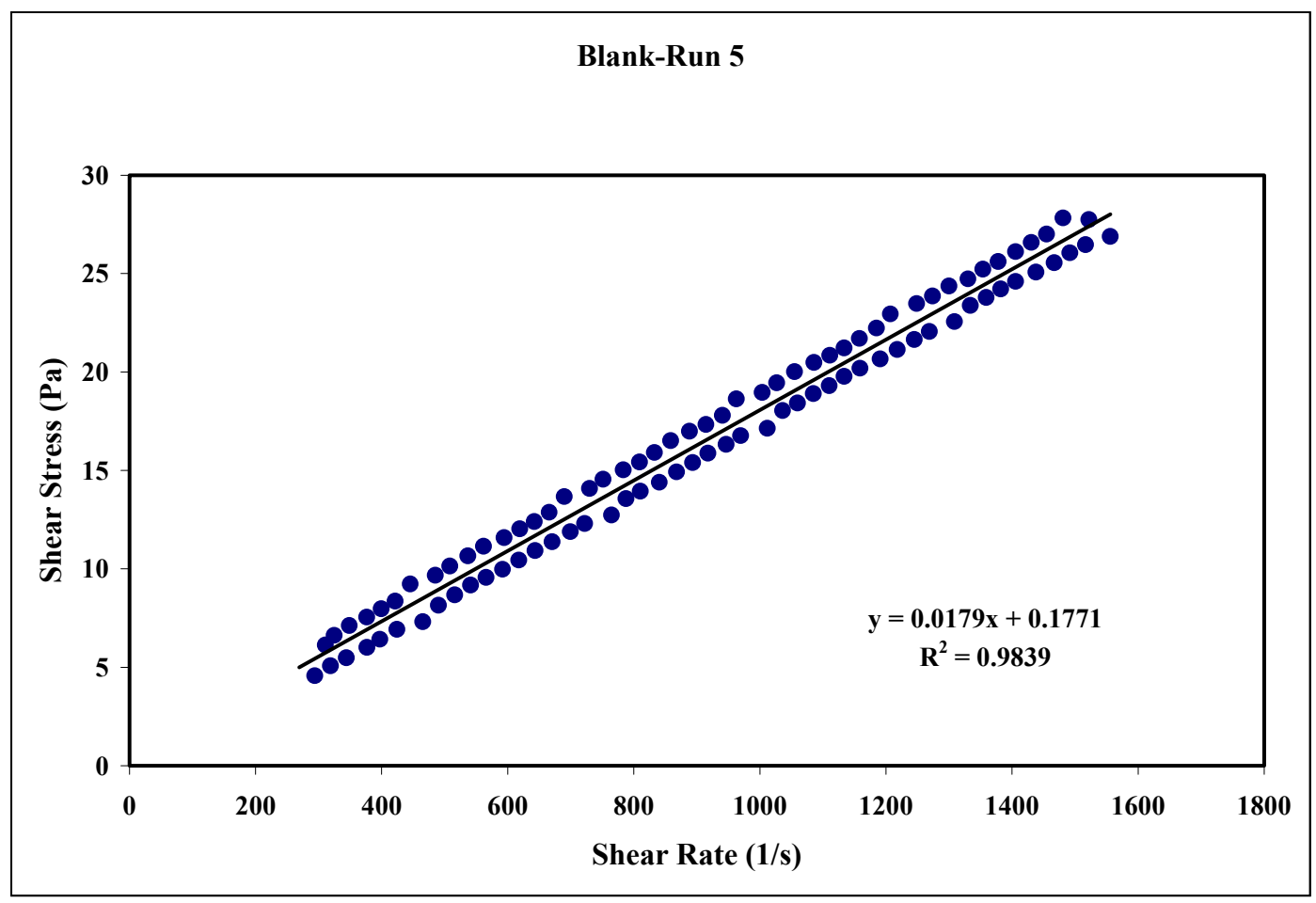

Figure 5. Blank Run 5

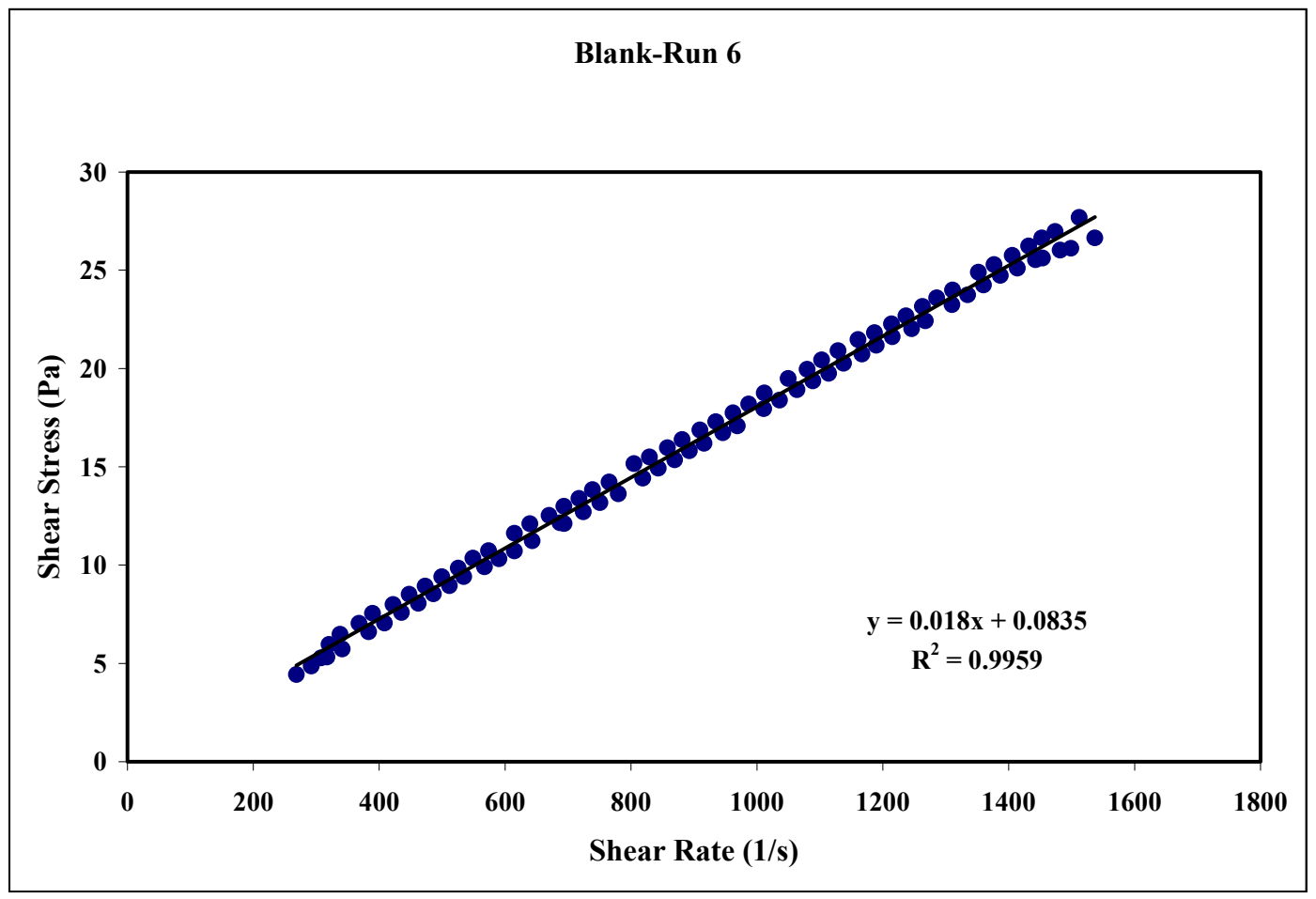

Figure 6. Blank Run 6 


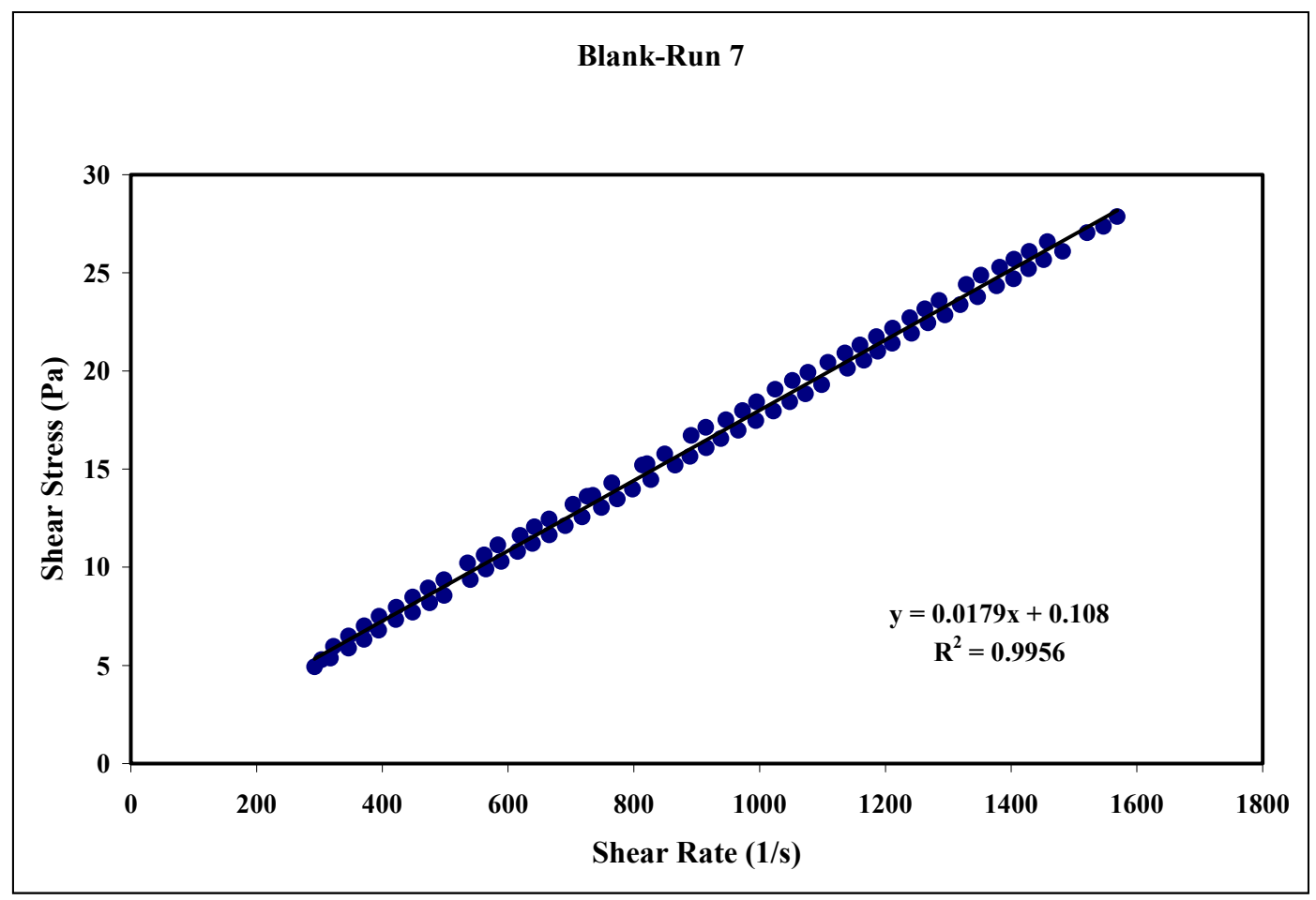

Figure 7. Blank Run 7

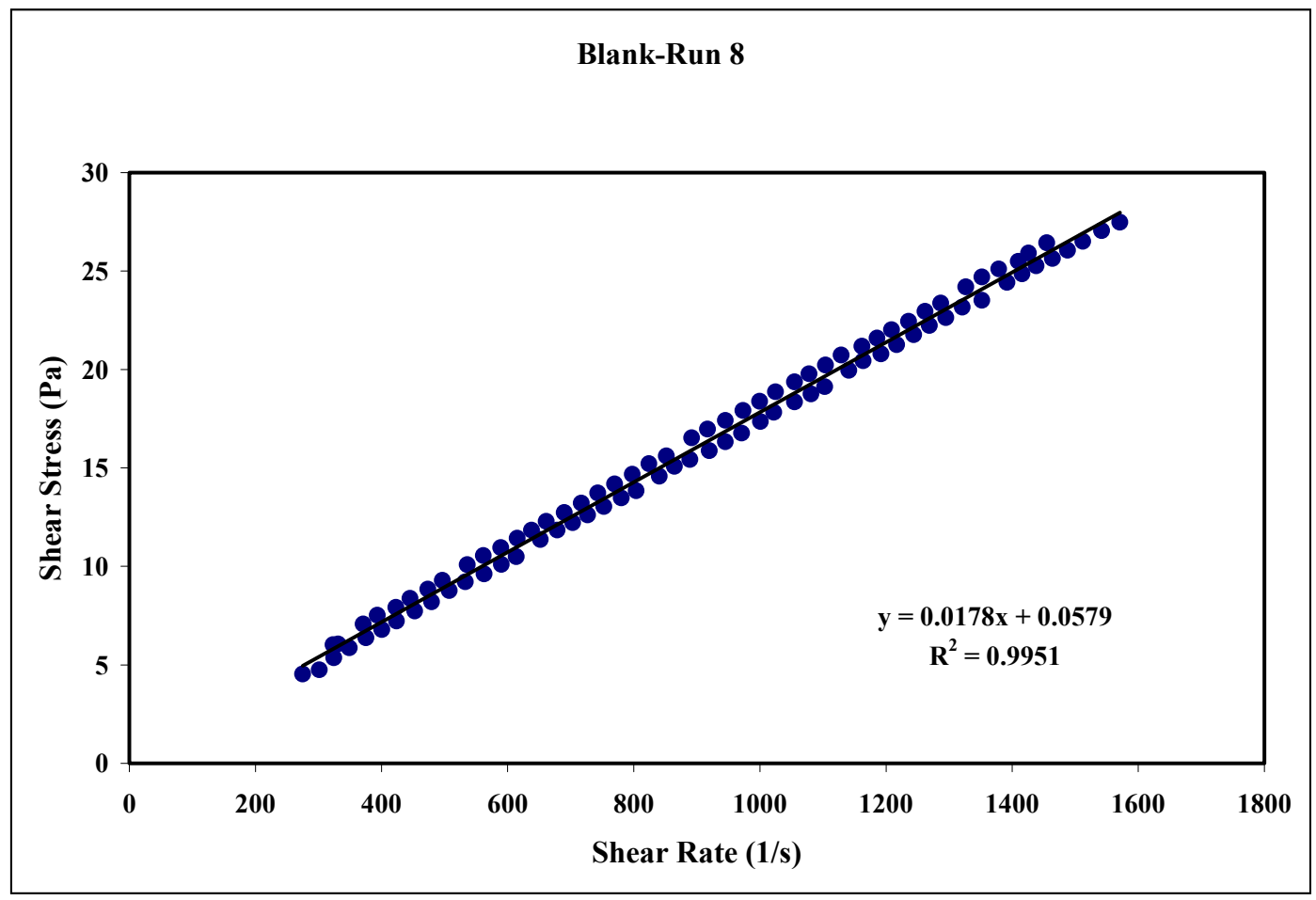

Figure 8. Blank Run 8 


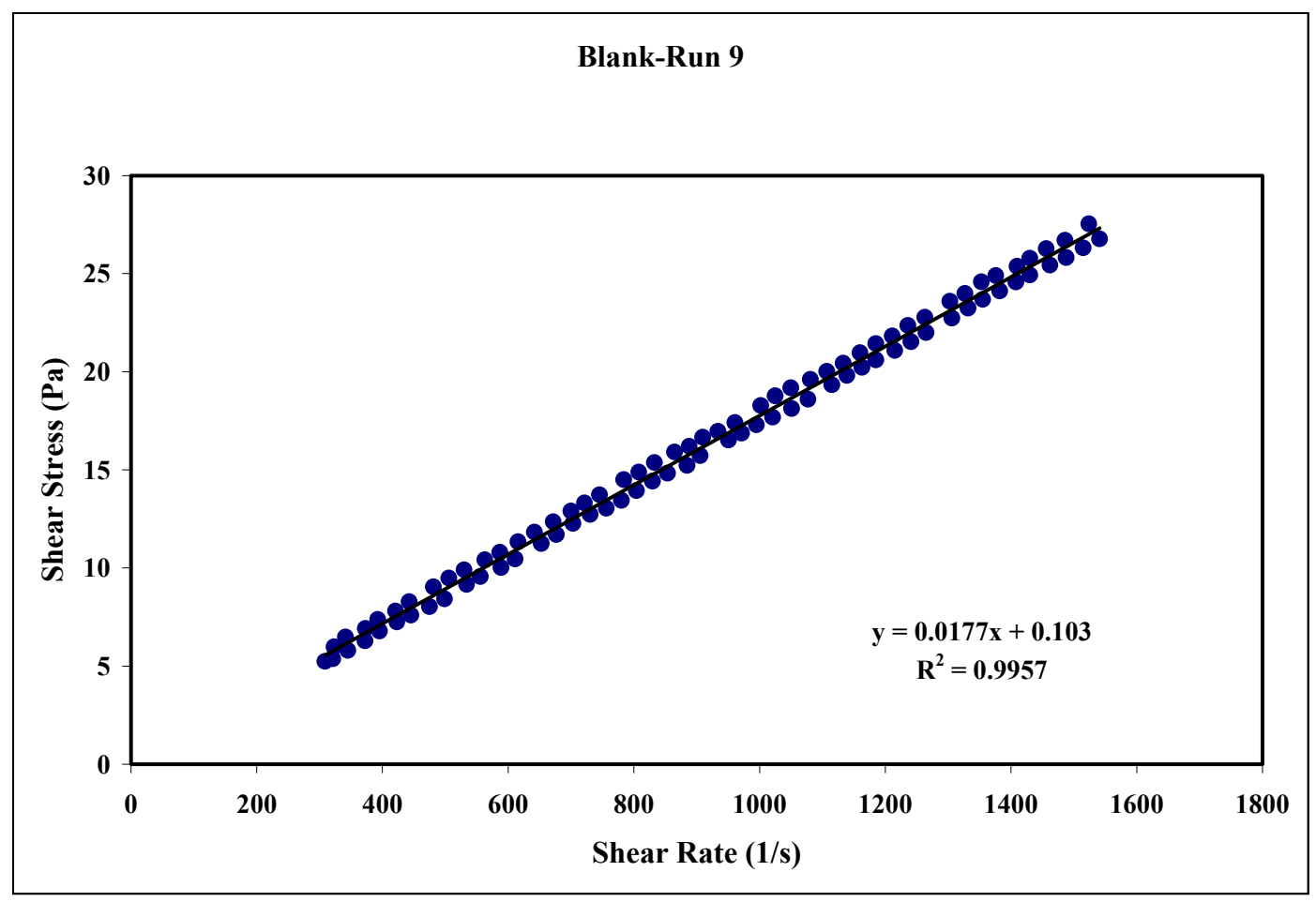

Figure 9. Blank Run 9

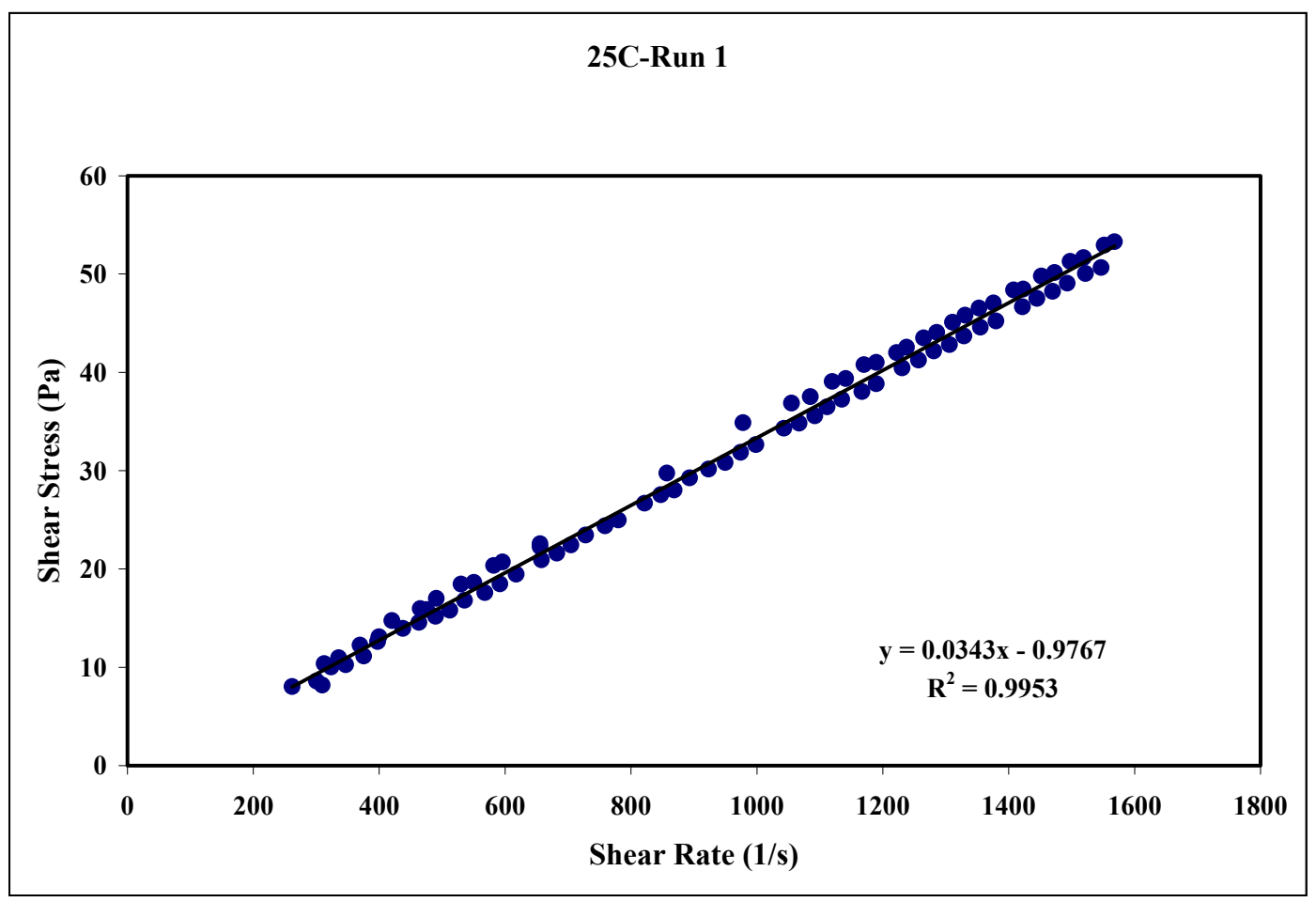

Figure 10. $25^{\circ} \mathrm{C}$ Run 1 


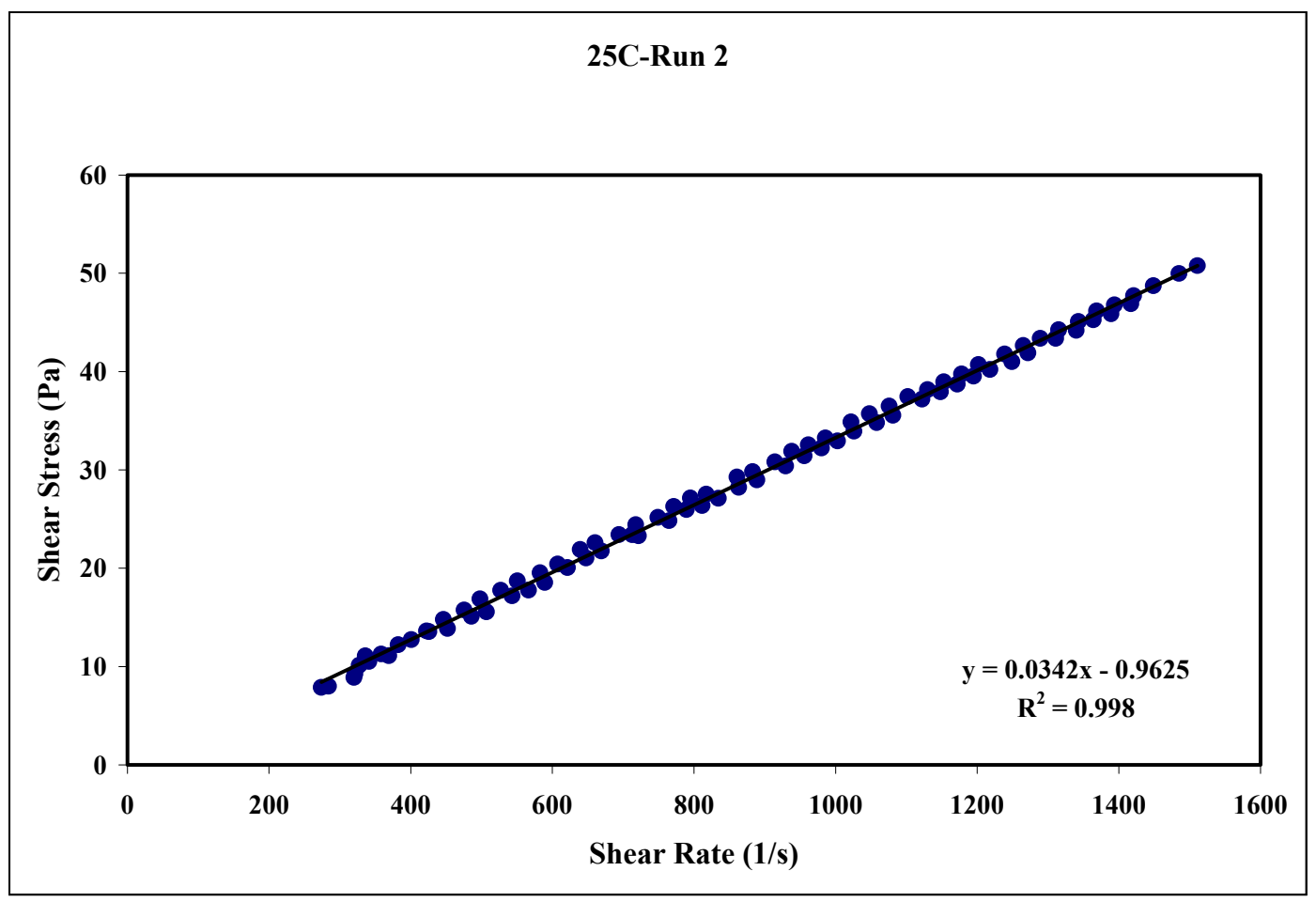

Figure 11. $25^{\circ} \mathrm{C}$ Run 2

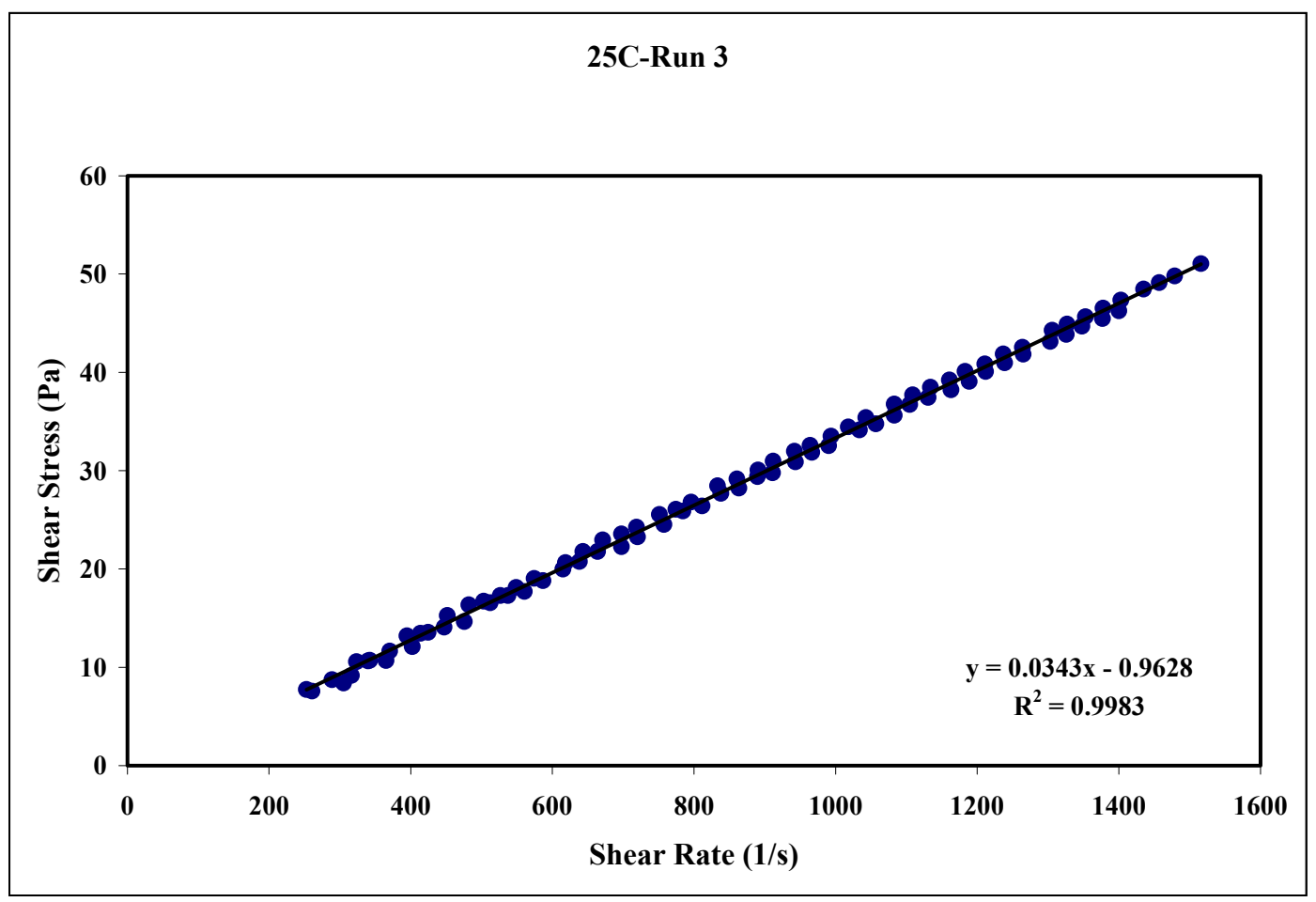

Figure 12. $25^{\circ} \mathrm{C}$ Run 3 


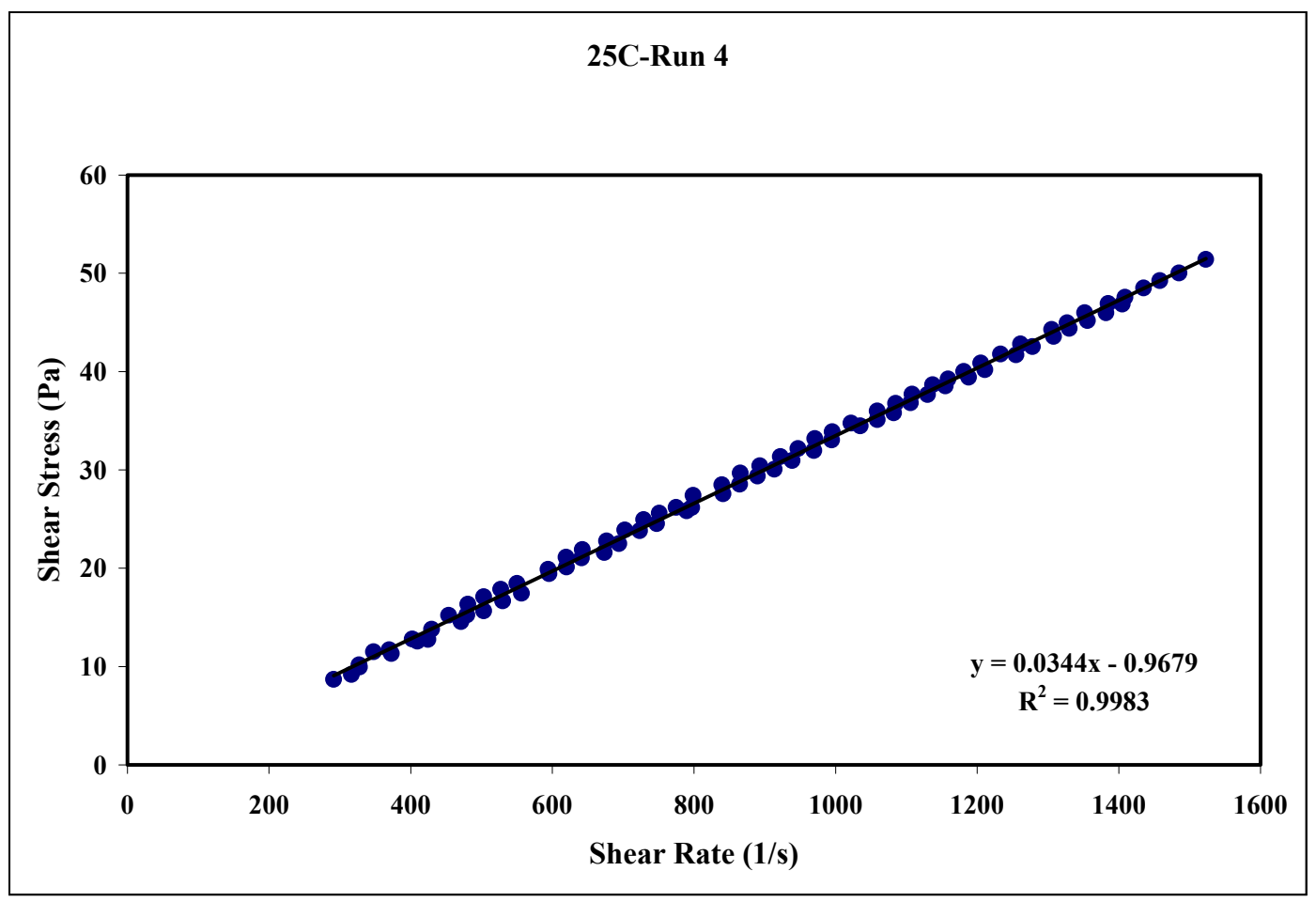

Figure 13. $25^{\circ} \mathrm{C}$ Run 4

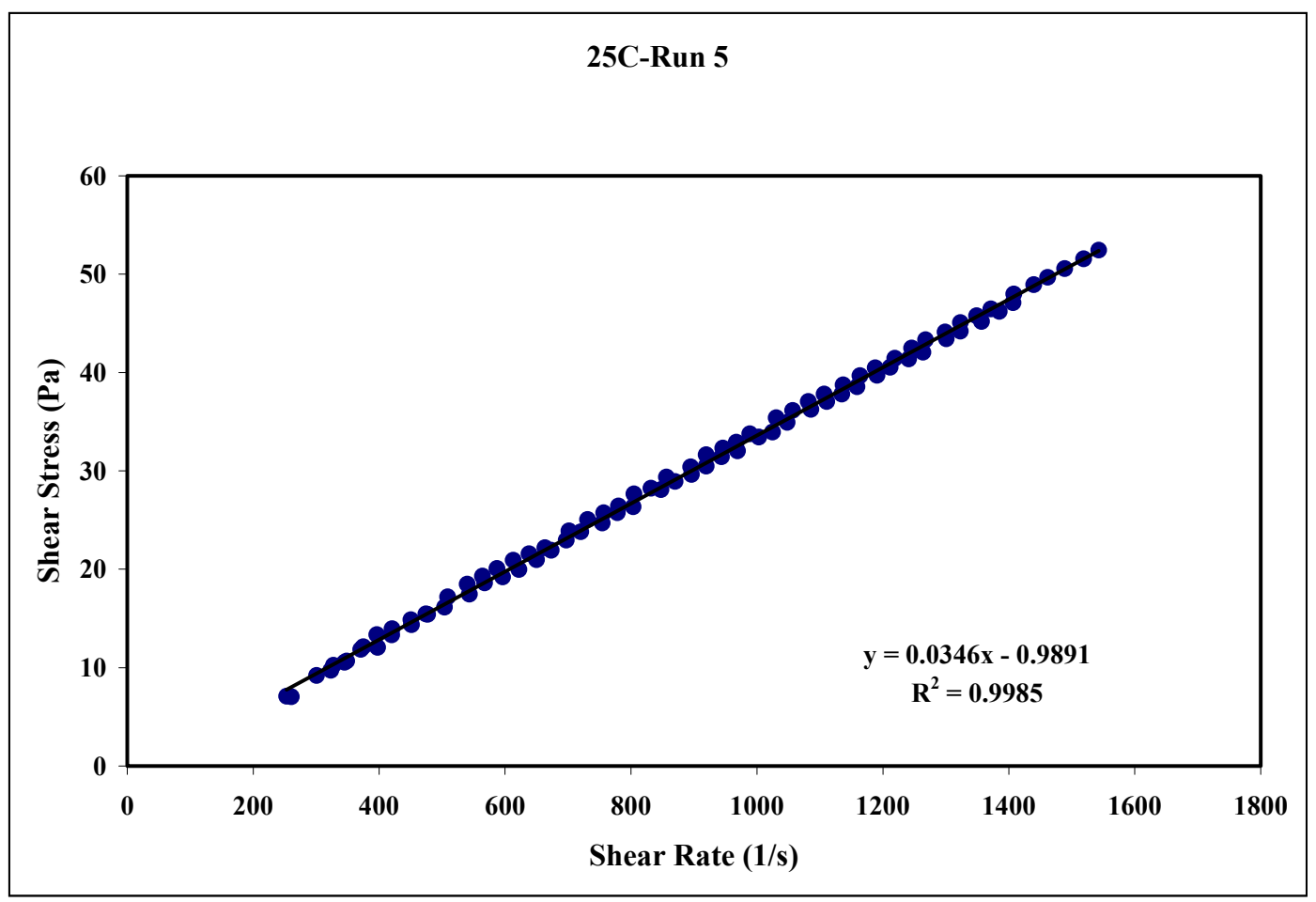

Figure 14. $25^{\circ} \mathrm{C}$ Run 5 


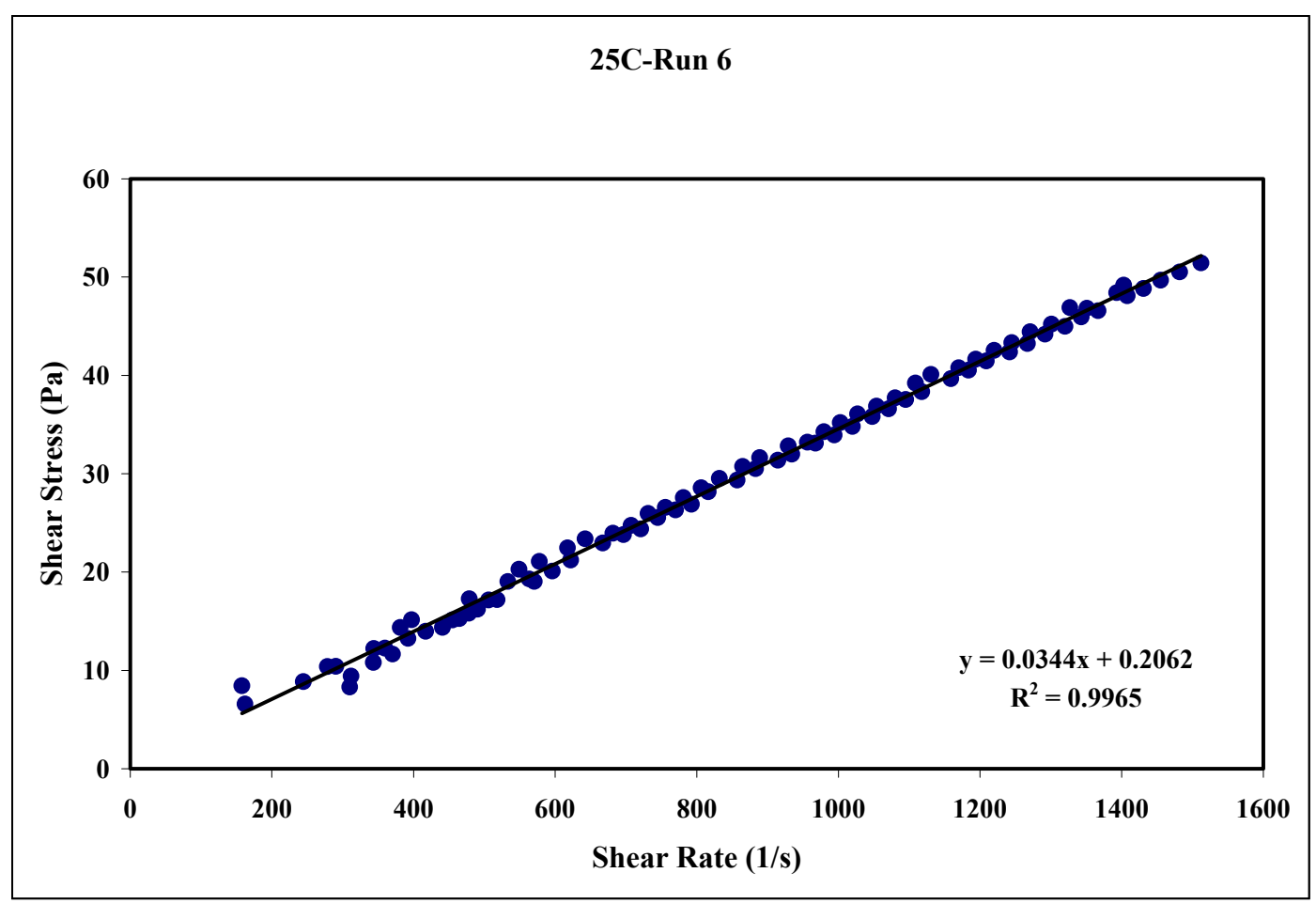

Figure 15. $25^{\circ} \mathrm{C}$ Run 6

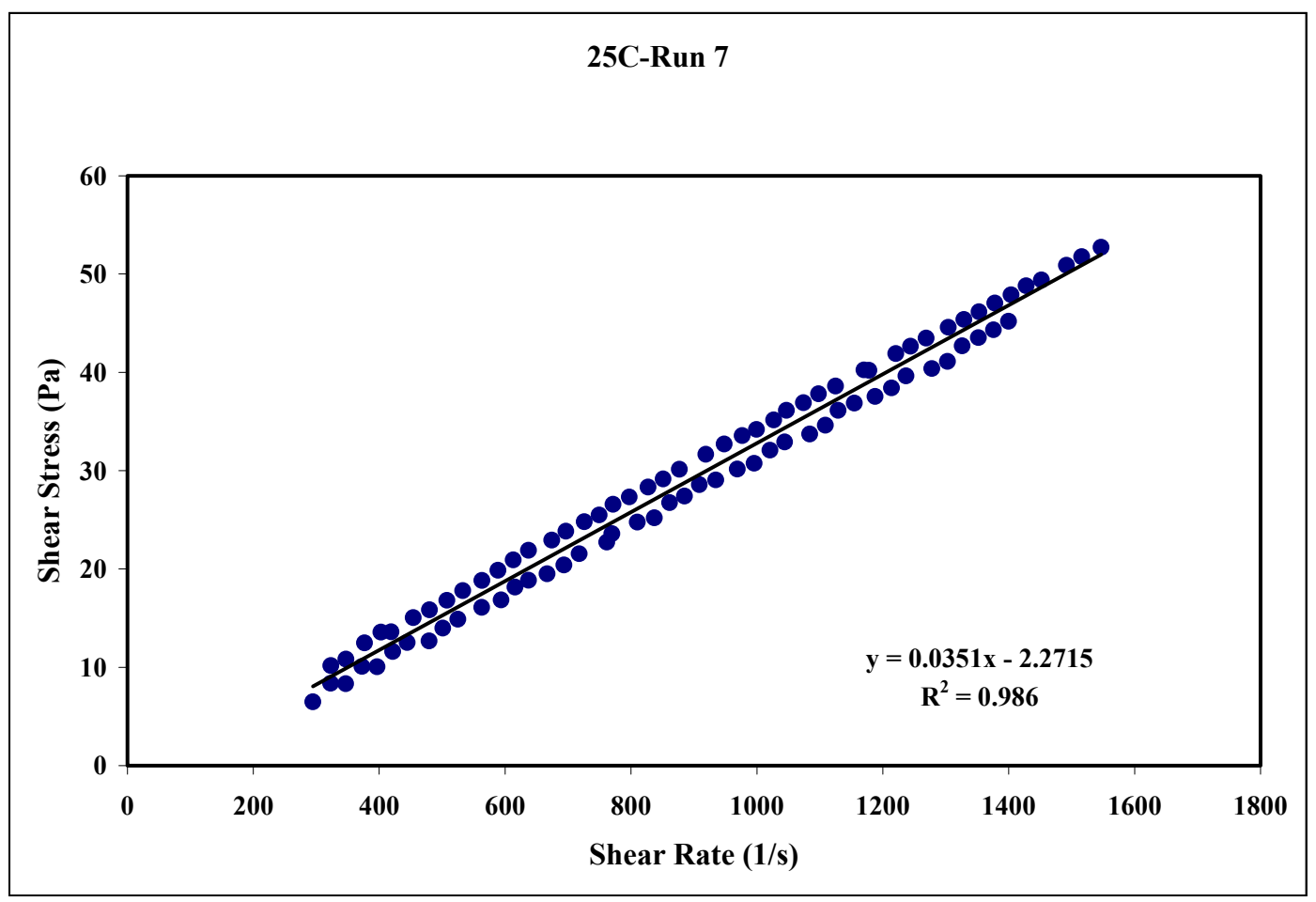

Figure 16. $25^{\circ} \mathrm{C}$ Run 7 


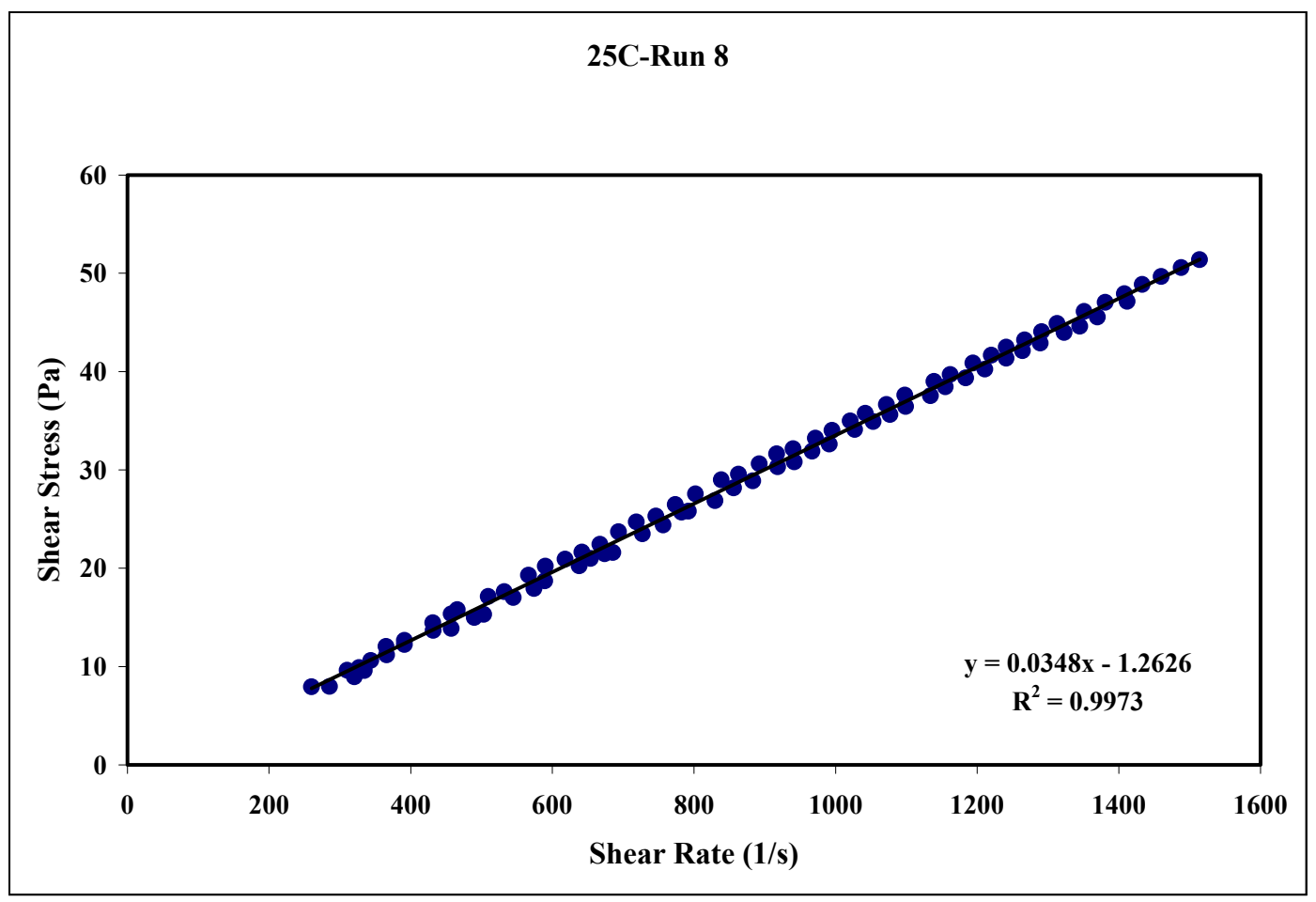

Figure 17. $25^{\circ} \mathrm{C}$ Run 8

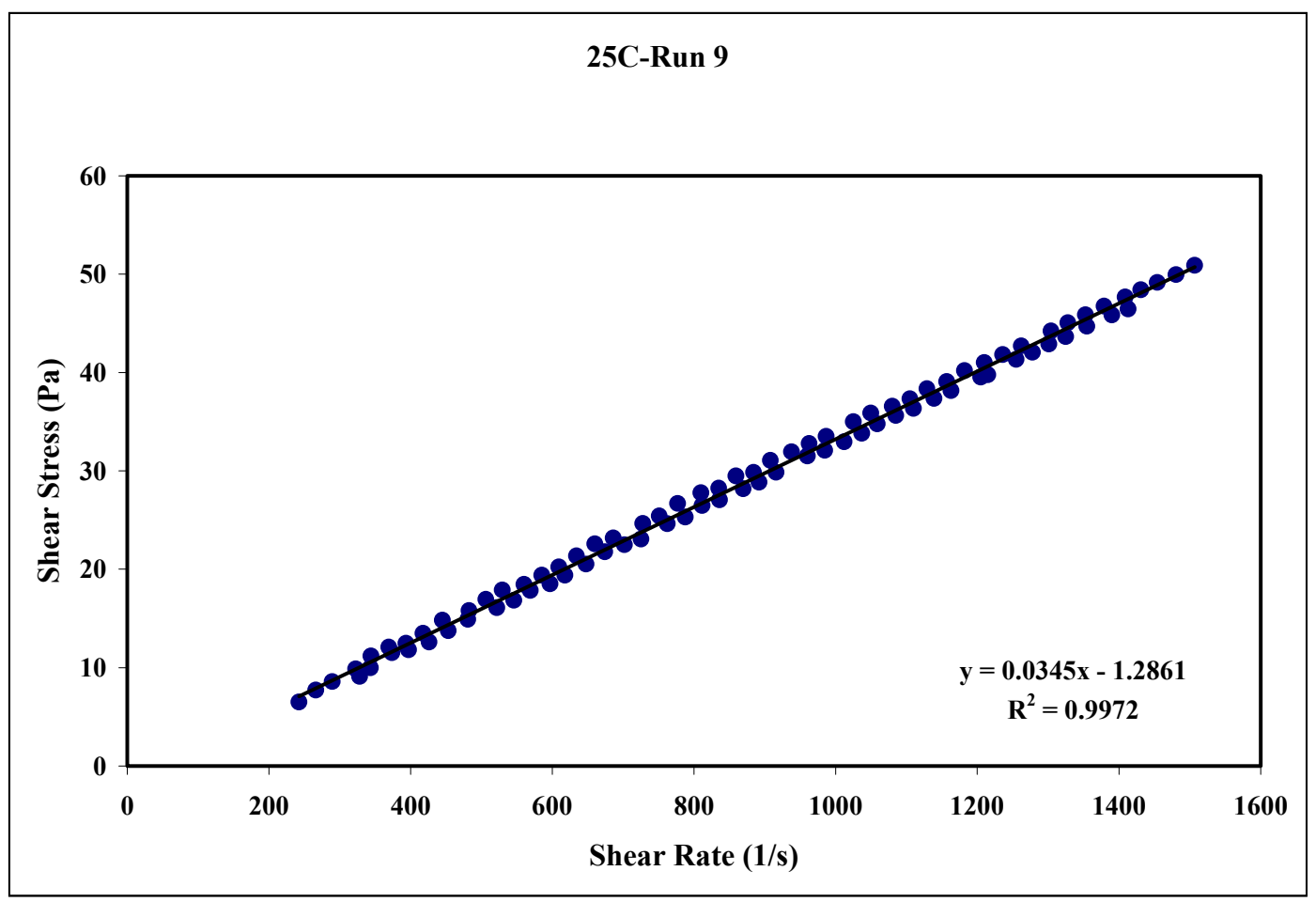

Figure 18. $25^{\circ} \mathrm{C}$ Run 9 
This page intentionally left blank. 


\begin{tabular}{|ll|}
\hline APPENDIX A - PART 4 & WSRC-TR-2000-00352 \\
RHEOMETER POST-CALIBRATION IN THE SHIELDED CELLS & SRT-RPP-2000-00026 \\
WITH THE LOW VISCOSITY STANDARD & Page 75 of 514 \\
\hline
\end{tabular}

\section{APPENDIX A - PART 4 \\ RHEOMETER POST-CALIBRATION IN THE SHIELDED CELLS WITH THE LOW VISCOSITY STANDARD}

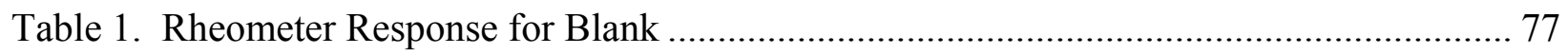

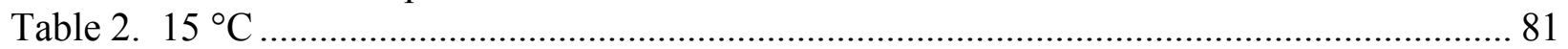

Table 3. Rheology Summary for Blank .......................................................................... 84

Table 4. Rheology Summary for $15{ }^{\circ} \mathrm{C}$.......................................................................... 85

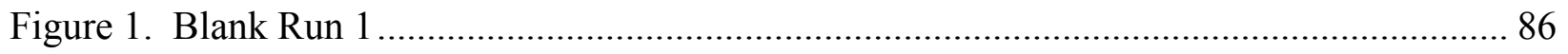

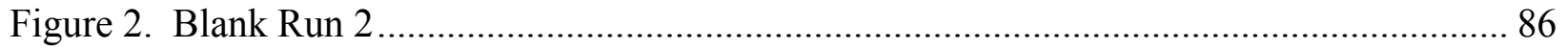

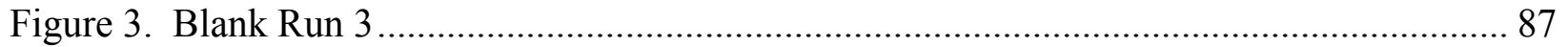

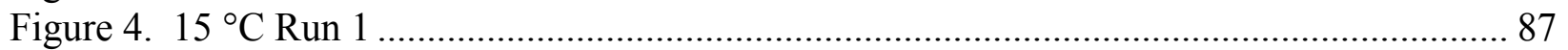

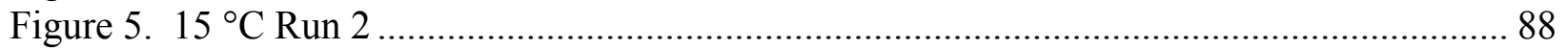

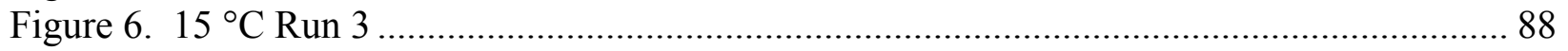

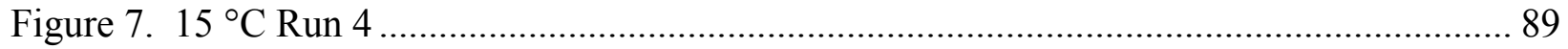

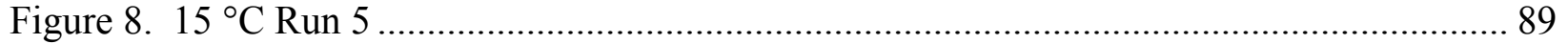

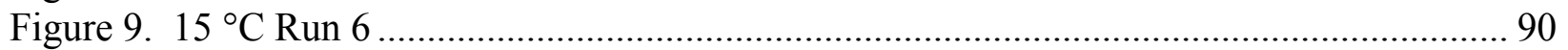

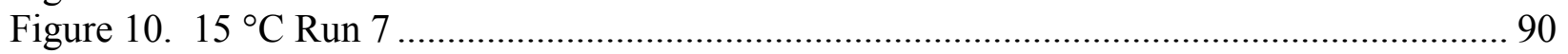

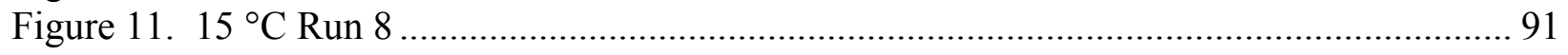

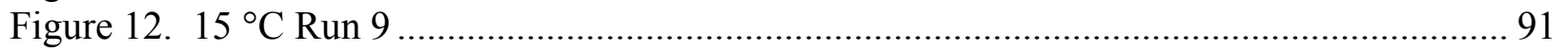


APPENDIX A - PART 4
RHEOMETER POST-CALIBRATION IN THE SHIELDED CELLS

WITH THE LOW VISCOSITY STANDARD
WSRC-TR-2000-00352

SRT-RPP-2000-00026

Page 76 of 514 


\section{RHEOMETER POST-CALIBRATION IN THE SHIELDED CELLS WITH THE LOW VISCOSITY STANDARD}

\section{Table 1. Rheometer Response for Blank}

\begin{tabular}{|c|c|c|c|c|c|c|c|c|c|c|c|c|c|c|c|c|c|}
\hline run1 & & run2 & & run3 & & run4 & & run5 & & run6 & & run7 & & run8 & & run9 & \\
\hline [Pa] & {$[1 / \mathrm{s}]$} & {$[\mathrm{Pa}]$} & {$[1 / \mathrm{s}]$} & [Pa] & {$[1 / \mathrm{s}]$} & [Pa] & {$[1 / \mathrm{s}]$} & [Pa] & {$[1 / \mathrm{s}]$} & [Pa] & {$[1 / \mathrm{s}]$} & [Pa] & {$[1 / \mathrm{s}]$} & [Pa] & {$[1 / \mathrm{s}]$} & [Pa] & {$[1 / \mathrm{s}]$} \\
\hline 4.764 & 261.8 & 4.945 & 273.7 & 4.504 & 271 & & & & & & & & & & & & \\
\hline 5.252 & 279 & 5.463 & 289.1 & 5.323 & 280.7 & & & & & & & & & & & & \\
\hline 6.081 & 316 & 6.081 & 321.3 & 5.853 & 308.5 & & & & & & & & & & & & \\
\hline 6.537 & 343.7 & 6.584 & 349.5 & 6.344 & 337.1 & & & & & & & & & & & & \\
\hline 7.105 & 374.1 & 7.025 & 373.7 & 6.924 & 365.3 & & & & & & & & & & & & \\
\hline 7.516 & 396.6 & 7.519 & 402.8 & 7.371 & 397.1 & & & & & & & & & & & & \\
\hline 8.093 & 428.3 & 7.901 & 424.8 & 7.883 & 424.8 & & & & & & & & & & & & \\
\hline 8.552 & 454.8 & 8.472 & 457.4 & 8.395 & 456.5 & & & & & & & & & & & & \\
\hline 8.937 & 477.7 & 8.922 & 484.8 & 8.901 & 483.4 & & & & & & & & & & & & \\
\hline 9.591 & 509.4 & 9.328 & 511.2 & 9.437 & 513.8 & & & & & & & & & & & & \\
\hline 9.976 & 535.4 & 9.837 & 535.4 & 9.937 & 543.4 & & & & & & & & & & & & \\
\hline 10.42 & 560.5 & 10.42 & 567.6 & 10.48 & 571.6 & & & & & & & & & & & & \\
\hline 10.91 & 585.2 & 10.89 & 594 & 10.51 & 583 & & & & & & & & & & & & \\
\hline 11.54 & 615.2 & 11.31 & 616.5 & 10.94 & 602 & & & & & & & & & & & & \\
\hline 11.95 & 642.5 & 11.95 & 650 & 11.49 & 633.3 & & & & & & & & & & & & \\
\hline 12.54 & 674.2 & 12.31 & 670.7 & 12.01 & 663.7 & & & & & & & & & & & & \\
\hline 12.98 & 698 & 13.01 & 703.3 & 12.47 & 691 & & & & & & & & & & & & \\
\hline 13.49 & 726.7 & 13.41 & 731.5 & 13.02 & 721 & & & & & & & & & & & & \\
\hline 13.91 & 753.6 & 13.79 & 754.9 & 13.58 & 751.4 & & & & & & & & & & & & \\
\hline 14.33 & 778.7 & 14.18 & 780 & 13.89 & 774.7 & & & & & & & & & & & & \\
\hline 14.89 & 806.4 & 14.78 & 809.1 & 14.43 & 809.1 & & & & & & & & & & & & \\
\hline 15.34 & 831.6 & 15.36 & 839.5 & 14.97 & 836.9 & & & & & & & & & & & & \\
\hline 15.92 & 862 & 15.81 & 865.5 & 15.49 & 867.7 & & & & & & & & & & & & \\
\hline 16.38 & 884.5 & 16.25 & 884.5 & 16.01 & 896.8 & & & & & & & & & & & & \\
\hline 16.92 & 917.9 & 17.08 & 931.2 & 16.55 & 925.4 & & & & & & & & & & & & \\
\hline 17.29 & 940.9 & 17.08 & 941.3 & 16.6 & 936 & & & & & & & & & & & & \\
\hline 17.94 & 970.8 & 17.11 & 951.9 & 17.04 & 957.2 & & & & & & & & & & & & \\
\hline 18.32 & 996.8 & 18.05 & 990.2 & 17.55 & 986.2 & & & & & & & & & & & & \\
\hline 18.82 & 1023 & 18.14 & 1010 & 18.08 & 1017 & & & & & & & & & & & & \\
\hline 19.48 & 1057 & 19.15 & 1057 & 18.44 & 1047 & & & & & & & & & & & & \\
\hline 19.86 & 1079 & 19.53 & 1083 & 19.06 & 1074 & & & & & & & & & & & & \\
\hline
\end{tabular}




\section{RHEOMETER POST-CALIBRATION IN THE SHIELDED CELLS WITH THE LOW VISCOSITY STANDARD}

Table 1. Rheometer Response for Blank - continued

\begin{tabular}{|c|c|c|c|c|c|c|c|c|c|c|c|c|c|c|c|c|c|}
\hline run1 & & run2 & & run3 & & run4 & & run5 & & run6 & & run7 & & run8 & & run9 & \\
\hline$[\mathrm{Pa}]$ & {$[1 / \mathrm{s}]$} & {$[\mathrm{Pa}]$} & {$[1 / \mathrm{s}]$} & [Pa] & {$[1 / \mathrm{s}]$} & [Pa] & {$[1 / \mathrm{s}]$} & [Pa] & {$[1 / \mathrm{s}]$} & [Pa] & {$[1 / \mathrm{s}]$} & [Pa] & {$[1 / \mathrm{s}]$} & [Pa] & {$[1 / \mathrm{s}]$} & {$[\mathrm{Pa}]$} & [1/s] \\
\hline 20.36 & 1106 & 20.01 & 1112 & 19.59 & 1105 & & & & & & & & & & & & \\
\hline 20.93 & 1136 & 20.48 & 1137 & 20.19 & 1133 & & & & & & & & & & & & \\
\hline 21.31 & 1161 & 21.1 & 1168 & 20.54 & 1164 & & & & & & & & & & & & \\
\hline 21.96 & 1192 & 21.55 & 1193 & 21.1 & 1192 & & & & & & & & & & & & \\
\hline 22.41 & 1215 & 22.05 & 1222 & 21.64 & 1223 & & & & & & & & & & & & \\
\hline 22.88 & 1244 & 22.43 & 1245 & 22.14 & 1252 & & & & & & & & & & & & \\
\hline 23.32 & 1268 & 23.03 & 1277 & 22.7 & 1280 & & & & & & & & & & & & \\
\hline 23.94 & 1297 & 23.47 & 1302 & 23.09 & 1309 & & & & & & & & & & & & \\
\hline 24.39 & 1324 & 24.06 & 1333 & 23.62 & 1337 & & & & & & & & & & & & \\
\hline 24.89 & 1348 & 24.42 & 1358 & 23.65 & 1345 & & & & & & & & & & & & \\
\hline 25.42 & 1380 & 24.89 & 1382 & 24.09 & 1374 & & & & & & & & & & & & \\
\hline 25.87 & 1407 & 25.51 & 1413 & 24.6 & 1394 & & & & & & & & & & & & \\
\hline 26.34 & 1430 & 26.02 & 1440 & 25.19 & 1425 & & & & & & & & & & & & \\
\hline 26.96 & 1462 & 26.37 & 1467 & 25.72 & 1452 & & & & & & & & & & & & \\
\hline 27.5 & 1490 & 26.96 & 1495 & 26.22 & 1484 & & & & & & & & & & & & \\
\hline 27.85 & 1512 & 27.35 & 1521 & 26.73 & 1513 & & & & & & & & & & & & \\
\hline 27.58 & 1539 & 26.49 & 1536 & 19.89 & 1184 & & & & & & & & & & & & \\
\hline 26.9 & 1507 & 26.13 & 1512 & 19.45 & 1158 & & & & & & & & & & & & \\
\hline 26.34 & 1479 & 25.48 & 1481 & 18.94 & 1134 & & & & & & & & & & & & \\
\hline 25.93 & 1453 & 25.04 & 1452 & 18.71 & 1112 & & & & & & & & & & & & \\
\hline 25.48 & 1430 & 24.65 & 1429 & 18.08 & 1083 & & & & & & & & & & & & \\
\hline 24.83 & 1399 & 24.03 & 1400 & 17.73 & 1059 & & & & & & & & & & & & \\
\hline 24.3 & 1373 & 23.59 & 1374 & 17.2 & 1033 & & & & & & & & & & & & \\
\hline 23.91 & 1345 & 23.17 & 1350 & 16.72 & 999.5 & & & & & & & & & & & & \\
\hline 23.29 & 1315 & 22.52 & 1315 & 15.98 & 962.5 & & & & & & & & & & & & \\
\hline 22.88 & 1290 & 22.14 & 1292 & 15.63 & 938.7 & & & & & & & & & & & & \\
\hline 22.46 & 1262 & 21.64 & 1263 & 15.21 & 914.9 & & & & & & & & & & & & \\
\hline 21.96 & 1239 & 21.01 & 1232 & 14.8 & 892.4 & & & & & & & & & & & & \\
\hline 21.4 & 1207 & 20.6 & 1209 & 14.44 & 868.6 & & & & & & & & & & & & \\
\hline 20.96 & 1183 & 20.27 & 1183 & 13.91 & 835.1 & & & & & & & & & & & & \\
\hline 20.36 & 1153 & 19.65 & 1153 & 13.53 & 811.3 & & & & & & & & & & & & \\
\hline
\end{tabular}




\section{RHEOMETER POST-CALIBRATION IN THE SHIELDED CELLS WITH THE LOW VISCOSITY STANDARD}

Table 1. Rheometer Response for Blank - continued

\begin{tabular}{|c|c|c|c|c|c|c|c|c|c|c|c|c|c|c|c|c|c|}
\hline run1 & & run2 & & run3 & & run4 & & run5 & & run6 & & run7 & & run8 & & run9 & \\
\hline$[\mathrm{Pa}]$ & {$[1 / \mathrm{s}]$} & {$[\mathrm{Pa}]$} & {$[1 / \mathrm{s}]$} & [Pa] & {$[1 / \mathrm{s}]$} & [Pa] & {$[1 / \mathrm{s}]$} & [Pa] & {$[1 / \mathrm{s}]$} & [Pa] & {$[1 / \mathrm{s}]$} & [Pa] & {$[1 / \mathrm{s}]$} & [Pa] & {$[1 / \mathrm{s}]$} & {$[\mathrm{Pa}]$} & [1/s] \\
\hline 19.95 & 1129 & 19.24 & 1126 & 12.82 & 775.2 & & & & & & & & & & & & \\
\hline 19.33 & 1098 & 18.79 & 1102 & 12.31 & 750 & & & & & & & & & & & & \\
\hline 18.91 & 1074 & 18.38 & 1074 & 12.01 & 725.8 & & & & & & & & & & & & \\
\hline 18.5 & 1051 & 17.91 & 1042 & 11.59 & 701.6 & & & & & & & & & & & & \\
\hline 17.88 & 1017 & 17.37 & 1015 & 11.29 & 679.1 & & & & & & & & & & & & \\
\hline 17.4 & 991.5 & 16.99 & 994.2 & 10.75 & 644.3 & & & & & & & & & & & & \\
\hline 16.99 & 965.5 & 16.43 & 962.5 & 10.34 & 618.7 & & & & & & & & & & & & \\
\hline 16.52 & 937.3 & 16.07 & 938.2 & 9.878 & 594.5 & & & & & & & & & & & & \\
\hline 15.89 & 908.7 & 15.45 & 909.6 & 9.227 & 558.3 & & & & & & & & & & & & \\
\hline 15.51 & 881.4 & 15.04 & 881.8 & 8.821 & 534.1 & & & & & & & & & & & & \\
\hline 15.07 & 857.1 & 14.53 & 851.4 & 8.44 & 510.3 & & & & & & & & & & & & \\
\hline 14.41 & 824.5 & 13.97 & 825.8 & 7.978 & 487.4 & & & & & & & & & & & & \\
\hline 13.85 & 798.1 & 13.82 & 810.4 & 7.653 & 463.2 & & & & & & & & & & & & \\
\hline 13.38 & 773 & 13.29 & 790.6 & 7.117 & 427.5 & & & & & & & & & & & & \\
\hline 12.91 & 744.3 & 12.88 & 761.5 & 6.415 & 392.6 & & & & & & & & & & & & \\
\hline 12.49 & 716.1 & 12.37 & 731.5 & 5.998 & 367.5 & & & & & & & & & & & & \\
\hline 12.1 & 691.4 & 11.93 & 702 & 5.575 & 342.9 & & & & & & & & & & & & \\
\hline 11.56 & 663.2 & 11.45 & 673.4 & 5.134 & 319.5 & & & & & & & & & & & & \\
\hline 11.13 & 637.2 & 10.9 & 646.5 & 4.808 & 296.6 & & & & & & & & & & & & \\
\hline 10.53 & 605.5 & 10.41 & 616.1 & 4.305 & 262.2 & & & & & & & & & & & & \\
\hline 10.03 & 579.5 & 9.934 & 587.9 & 3.941 & 238.9 & & & & & & & & & & & & \\
\hline 9.541 & 550.9 & 9.452 & 561 & & & & & & & & & & & & & & \\
\hline 9.218 & 532.3 & 9.014 & 532.8 & & & & & & & & & & & & & & \\
\hline 8.623 & 498.4 & 8.552 & 504.6 & & & & & & & & & & & & & & \\
\hline 8.088 & 469.8 & 8.002 & 477.7 & & & & & & & & & & & & & & \\
\hline 7.629 & 443.8 & 7.525 & 447.3 & & & & & & & & & & & & & & \\
\hline 7.206 & 418.2 & 7.061 & 420.4 & & & & & & & & & & & & & & \\
\hline 6.646 & 388.2 & 6.555 & 391.8 & & & & & & & & & & & & & & \\
\hline 6.282 & 363.6 & 6.051 & 362.7 & & & & & & & & & & & & & & \\
\hline 5.779 & 334.9 & 5.696 & 340.6 & & & & & & & & & & & & & & \\
\hline 5.3 & 310.2 & 5.143 & 311.1 & & & & & & & & & & & & & & \\
\hline & & & & & & & & & & & & & & & & & \\
\hline
\end{tabular}




\section{Table 1. Rheometer Response for Blank - continued}

\begin{tabular}{|c|c|c|c|c|c|c|c|c|c|c|c|c|c|c|c|c|c|}
\hline run1 & & run2 & & run3 & & run4 & & run5 & & run6 & & run7 & & run8 & & run9 & \\
\hline$[\mathrm{Pa}]$ & [1/s] & {$[\mathrm{Pa}]$} & {$[1 / \mathrm{s}]$} & {$[\mathrm{Pa}]$} & {$[1 / \mathrm{s}]$} & {$[\mathrm{Pa}]$} & {$[1 / \mathrm{s}]$} & {$[\mathrm{Pa}]$} & [1/s] & {$[\mathrm{Pa}]$} & {$[1 / \mathrm{s}]$} & {$[\mathrm{Pa}]$} & {$[1 / \mathrm{s}]$} & {$[\mathrm{Pa}]$} & [1/s] & {$[\mathrm{Pa}]$} & {$[1 / \mathrm{s}]$} \\
\hline 4.871 & 285.6 & 4.666 & 281.2 & & & & & & & & & & & & & & \\
\hline 4.231 & 253.8 & 4.178 & 251.6 & & & & & & & & & & & & & & \\
\hline 4.006 & 237.5 & 4.137 & 242.4 & & & & & & & & & & & & & & \\
\hline 0 & 188.5 & 0 & 169.6 & & & & & & & & & & & & & & \\
\hline 0 & 172.7 & 0 & 189.7 & & & & & & & & & & & & & & \\
\hline 0 & 144.7 & 0 & 158.3 & & & & & & & & & & & & & & \\
\hline 0 & 117.2 & 0 & 128.8 & & & & & & & & & & & & & & \\
\hline 0 & 89.81 & 0 & 102.2 & & & & & & & & & & & & & & \\
\hline 0 & 65.57 & 0 & 69.72 & & & & & & & & & & & & & & \\
\hline 0 & 40.06 & 0 & 42.13 & & & & & & & & & & & & & & \\
\hline 0 & 16.37 & 0 & 30.32 & & & & & & & & & & & & & & \\
\hline & & & & & & & & & & & & & & & & & \\
\hline
\end{tabular}


Table 2. $15^{\circ} \mathrm{C}$

\begin{tabular}{|c|c|c|c|c|c|c|c|c|c|c|c|c|c|c|c|c|c|}
\hline run1 & & run2 & & run3 & & run4 & & run5 & & run6 & & run7 & & run8 & & run9 & \\
\hline$[\mathrm{Pa}]$ & {$[1 / \mathrm{s}]$} & {$[\mathrm{Pa}]$} & {$[1 / \mathrm{s}]$} & {$[\mathrm{Pa}]$} & {$[1 / \mathrm{s}]$} & [Pa] & {$[1 / \mathrm{s}]$} & {$[\mathrm{Pa}]$} & {$[1 / \mathrm{s}]$} & [Pa] & {$[1 / \mathrm{s}]$} & {$[\mathrm{Pa}]$} & {$[1 / \mathrm{s}]$} & {$[\mathrm{Pa}]$} & {$[1 / \mathrm{s}]$} & {$[\mathrm{Pa}]$} & {$[1 / \mathrm{s}]$} \\
\hline 8.871 & 275 & 9.374 & 273.2 & 9.249 & 274.5 & 8.936 & 275.9 & 9.436 & 283.4 & 9.791 & 276.3 & 9.767 & 275.4 & 9.501 & 278.5 & 9.356 & 268.4 \\
\hline 10.64 & 297.9 & 0.61 & 294.8 & 10.48 & 293.9 & 10.76 & 297.5 & 10.39 & 293.1 & 10.63 & 291.7 & 10.64 & 294.8 & 10.41 & 291.7 & 10.38 & 287.8 \\
\hline 11.58 & 320.4 & 11.83 & 323.9 & 11.42 & 316 & 11.63 & 319.1 & 11.52 & 316.4 & 11.69 & 317.3 & 11.63 & 325.7 & 11.84 & 321.3 & 11.44 & 319.5 \\
\hline 12.48 & 346.4 & 11.9 & 333.2 & 12.43 & 339.3 & 12.73 & 349 & 12.64 & 349.5 & 12.62 & 347.7 & 12.62 & 349 & 12.55 & 344.2 & 12.61 & 346.8 \\
\hline 14.14 & 376.8 & 13.2 & 360.5 & 13.86 & 375 & 13.87 & 376.3 & 13.43 & 370.2 & 13.9 & 375 & 13.91 & 376.8 & 13.82 & 374.6 & 13.71 & 375 \\
\hline 15.02 & 404.5 & 14.14 & 381.2 & 14.96 & 401.9 & 15.03 & 406.3 & 14.87 & 404.5 & 14.85 & 404.5 & 15.17 & 408.5 & 14.76 & 401.9 & 14.72 & 402.8 \\
\hline 16.38 & 428.3 & 15.61 & 419.1 & 15.99 & 427 & 16.27 & 431 & 15.92 & 427 & 16.16 & 431.4 & 16.02 & 430.5 & 15.95 & 428.3 & 15.75 & 425.7 \\
\hline 17.72 & 457.4 & 16.48 & 438.9 & 16.95 & 453 & 17.05 & 453.5 & 17.27 & 458.8 & 16.88 & 453.5 & 17.18 & 457.4 & 16.74 & 452.6 & 17.03 & 454.8 \\
\hline 18.54 & 483.9 & 17.63 & 458.8 & 18.31 & 483.9 & 18.28 & 485.6 & 18.07 & 481.7 & 18.13 & 483.4 & 17.92 & 482.1 & 18.28 & 487.8 & 18.01 & 483.4 \\
\hline 19.73 & 509 & 19.4 & 507.7 & 19.49 & 513 & 19.4 & 510.3 & 19.43 & 513 & 19.23 & 511.6 & 19.34 & 513.4 & 18.99 & 503.3 & 18.78 & 505 \\
\hline 20.97 & 533.2 & 20.85 & 537.2 & 20.73 & 539.4 & 20.71 & 540.7 & 20.53 & 537.6 & 20.23 & 536.3 & 20.59 & 540.7 & 20.26 & 538.5 & 19.85 & 530.1 \\
\hline 22.72 & 568.9 & 21.92 & 563.2 & 21.86 & 565 & 21.65 & 564.1 & 21.59 & 561.9 & 21.89 & 568 & 21.62 & 565.4 & 21.27 & 563.2 & 21.36 & 565.4 \\
\hline 23.81 & 593.2 & 23.58 & 597.1 & 22.98 & 589.6 & 23.25 & 595.8 & 23.25 & 594.9 & 22.95 & 593.2 & 22.78 & 594.9 & 22.84 & 596.2 & 22.36 & 589.2 \\
\hline 24.88 & 619.6 & 24.67 & 621.4 & 24.32 & 617 & 24.23 & 618.7 & 24.05 & 615.6 & 23.9 & 618.7 & 23.87 & 621.4 & 23.69 & 617.8 & 23.34 & 617 \\
\hline 25.97 & 644.3 & 26 & 651.3 & 25.65 & 647.8 & 25.5 & 648.7 & 25.09 & 640.8 & 25.2 & 647.8 & 25.03 & 646.9 & 24.7 & 643 & 24.79 & 647.4 \\
\hline 27.57 & 675.1 & 27.13 & 678.2 & 26.77 & 675.6 & 26.65 & 675.6 & 26.54 & 675.1 & 26.21 & 670.3 & 26.15 & 673.4 & 26.06 & 675.6 & 25.65 & 670.7 \\
\hline 28.81 & 705.5 & 28.4 & 704.2 & 28.13 & 703.8 & 27.87 & 704.2 & 27.78 & 700.7 & 27.63 & 702 & 27.66 & 706 & 27.33 & 702 & 26.95 & 698 \\
\hline 30.18 & 730.7 & 29.47 & 730.2 & 29.26 & 728.9 & 29.17 & 735.1 & 28.81 & 727.1 & 28.7 & 728.4 & 28.64 & 732.9 & 28.22 & 727.1 & 28.19 & 728 \\
\hline 31.51 & 760.2 & 30.56 & 754 & 30.09 & 748.3 & 30.38 & 758 & 30.35 & 759.3 & 30.03 & 757.5 & 29.94 & 761.5 & 29.58 & 756.2 & 29.35 & 754.4 \\
\hline 32.75 & 784.9 & 32.13 & 786.6 & 31.74 & 784 & 31.48 & 785.3 & 31.42 & 785.7 & 30.92 & 780.4 & 30.62 & 776.9 & 30.8 & 784.4 & 30.18 & 778.2 \\
\hline 33.96 & 810.4 & 33.28 & 813.1 & 32.84 & 810.4 & 32.37 & 809.1 & 32.34 & 808.2 & 32.04 & 805.1 & 32.1 & 813.1 & 31.89 & 810.4 & 31.66 & 810.4 \\
\hline 35.5 & 843 & 34.59 & 841.7 & 34.17 & 841.3 & 33.9 & 841.3 & 33.79 & 840.4 & 33.46 & 839.5 & 33.25 & 839.1 & 33.08 & 836.9 & 32.63 & 833.3 \\
\hline 36.6 & 67.3 & .68 & 868.1 & 35.21 & 865.9 & 34.97 & 865.9 & 34.97 & 867.7 & 34.53 & 860.7 & 34.56 & 866.8 & 34.17 & 865.1 & 33.99 & 865.5 \\
\hline 37.93 & 893.7 & & 894.1 & .63 & 895 & 36.3 & 895 & 36.15 & 892.4 & 35.92 & 892.8 & 35.65 & 894.6 & 35.56 & 893.7 & 35.09 & 891.1 \\
\hline 39.08 & 921 & 37.99 & 917.9 & 37.51 & 915.7 & 37.34 & 920.1 & 37.57 & 923.7 & 37.07 & 921 & 36.92 & 921.9 & 36.57 & 918.8 & 36.12 & 914.4 \\
\hline 40.71 & 53.6 & 39.53 & 951.9 & 38.97 & 947.9 & 38.64 & 948.3 & 38.64 & 947.9 & 38.08 & 942.6 & 38.28 & 953.2 & 37.54 & 943.5 & 37.49 & 949.2 \\
\hline 41.81 & 978.3 & 40.68 & 976.6 & 40 & 971.7 & 39.97 & 979.2 & 39.68 & 970.4 & 39.53 & 974.3 & 39.41 & 977.4 & 39.08 & 976.1 & 38.55 & 972.6 \\
\hline 43.02 & 1003 & 41.72 & 1003 & 41.45 & 1004 & 41.07 & 1002 & 41.18 & 1006 & 40.74 & 1002 & 40.68 & 1006 & 40.12 & 1002 & 39.65 & 999.5 \\
\hline 44.38 & 1031 & 42.78 & 1025 & 42.49 & 1029 & 42.25 & 1030 & 42.25 & 1029 & 41.84 & 1027 & 41.95 & 1034 & 41.24 & 1028 & 40.71 & 1023 \\
\hline 45.68 & 1060 & 44.44 & 1061 & 44.06 & 1062 & 43.32 & 1054 & 43.58 & 1059 & 43.02 & 1055 & 43.11 & 1061 & 42.49 & 1055 & 42.19 & 1056 \\
\hline 47.01 & 1088 & 45.54 & 1085 & 44.38 & 1071 & 44.68 & 1085 & 44.97 & 1087 & 44.29 & 1083 & 44.08 & 1085 & 43.52 & 1079 & 43.32 & 1083 \\
\hline
\end{tabular}


Table 2. $15{ }^{\circ} \mathrm{C}$ - continued

\begin{tabular}{|c|c|c|c|c|c|c|c|c|c|c|c|c|c|c|c|c|c|}
\hline run1 & & run2 & & run3 & & run4 & & run5 & & run6 & & run7 & & run8 & & run9 & \\
\hline$[\mathrm{Pa}]$ & {$[1 / \mathrm{s}]$} & {$[\mathrm{Pa}]$} & {$[1 / \mathrm{s}]$} & {$[\mathrm{Pa}]$} & {$[1 / \mathrm{s}]$} & {$[\mathrm{Pa}]$} & {$[1 / \mathrm{s}]$} & {$[\mathrm{Pa}]$} & {$[1 / \mathrm{s}]$} & {$[\mathrm{Pa}]$} & {$[1 / \mathrm{s}]$} & {$[\mathrm{Pa}]$} & {$[1 / \mathrm{s}]$} & {$[\mathrm{Pa}]$} & {$[1 / \mathrm{s}]$} & {$[\mathrm{Pa}]$} & {$[1 / \mathrm{s}]$} \\
\hline 48.14 & 1112 & 46.9 & 1114 & 45.65 & 1096 & 45.8 & 1111 & 45.95 & 1114 & 45.27 & 1107 & 45.24 & 1111 & 44.97 & 1113 & 44.26 & 1107 \\
\hline 49.59 & 1142 & 48.08 & 1141 & 46.93 & 1123 & 47.16 & 1140 & 47.04 & 1137 & 46.87 & 1141 & 46.63 & 1142 & 46.19 & 1141 & 45.59 & 1137 \\
\hline 50.77 & 1169 & 49.29 & 1168 & 47.99 & 1150 & 48.38 & 1166 & 48.61 & 1170 & 47.81 & 1164 & 47.7 & 1168 & 47.31 & 1166 & 46.72 & 1164 \\
\hline 52.08 & 1196 & 50.36 & 1192 & 49.74 & 1189 & 49.44 & 1193 & 49.77 & 1195 & 49.09 & 1191 & 49.06 & 1198 & 48.29 & 1190 & 47.81 & 1189 \\
\hline 53.41 & 1224 & 51.42 & 1216 & 50.42 & 1206 & 50.77 & 1223 & 50.83 & 1217 & 50 & 1215 & 50.18 & 1221 & 49.56 & 1218 & 49 & 1216 \\
\hline 54.47 & 1248 & 53.08 & 1250 & 52.19 & 1244 & 52.02 & 1248 & 52.28 & 1248 & 51.63 & 1249 & 51.28 & 1248 & 50.8 & 1248 & 50.15 & 1244 \\
\hline 55.86 & 1278 & 54.24 & 1279 & 53.32 & 1270 & 53.17 & 1277 & 53.32 & 1275 & 52.58 & 1272 & 52.34 & 1274 & 52.13 & 1276 & 51.31 & 1271 \\
\hline 57.05 & 1304 & 55.36 & 1302 & 54.83 & 1304 & 54.12 & 1300 & 54.59 & 1302 & 54.06 & 1304 & 53.38 & 1297 & 53.35 & 1304 & 52.61 & 1302 \\
\hline 58.17 & 1328 & 56.72 & 1332 & 56.07 & 1332 & 55.6 & 1333 & 55.66 & 1327 & 55.09 & 1329 & 54.98 & 1335 & 54.44 & 1330 & 53.67 & 1326 \\
\hline 59.68 & 1360 & 57.88 & 1357 & 57.34 & 1359 & 56.75 & 1358 & 57.14 & 1358 & 56.16 & 1352 & 56.13 & 1361 & 55.45 & 1355 & 54.83 & 1355 \\
\hline 61.01 & 1387 & 59 & 1382 & 58.38 & 1384 & 57.85 & 1382 & 58.23 & 1382 & 57.55 & 1384 & 57.31 & 1387 & 56.46 & 1380 & 55.98 & 1379 \\
\hline 62.2 & 1411 & 60.6 & 1417 & 59.59 & 1411 & 58.91 & 1406 & 59.36 & 1408 & 58.73 & 1411 & 58.47 & 1412 & 57.96 & 1412 & 57.43 & 1413 \\
\hline 63.62 & 1441 & 61.69 & 1440 & 60.78 & 1438 & 60.13 & 1433 & 60.95 & 1440 & 59.83 & 1435 & 59.71 & 1440 & 58.94 & 1435 & 58.47 & 1437 \\
\hline 64.8 & 1469 & 62.91 & 1469 & 62.02 & 1464 & 61.6 & 1469 & 62.17 & 1468 & 61.22 & 1469 & 60.66 & 1464 & 60.3 & 1467 & 59.65 & 1466 \\
\hline 66.1 & 1497 & 64.21 & 1496 & 63.03 & 1489 & 62.88 & 1495 & 63.41 & 1494 & 62.26 & 1491 & 62.08 & 1497 & 61.43 & 1493 & 60.72 & 1493 \\
\hline 67.55 & 1525 & 65.3 & 1519 & 64.56 & 1521 & 63.85 & 1519 & 64.39 & 1516 & 63.59 & 1523 & 63.05 & 1520 & 62.55 & 1518 & 61.87 & 1519 \\
\hline 66.25 & 1542 & 63.74 & 1507 & 64.51 & 1543 & 63.97 & 1537 & 63.82 & 1543 & 63.11 & 1535 & 62.88 & 1539 & 63.14 & 1551 & 61.78 & 1535 \\
\hline 65.07 & 1515 & 62.49 & 1479 & 63 & 1508 & 62.85 & 1512 & 62.43 & 1512 & 62.14 & 1512 & 61.81 & 1514 & 61.31 & 1513 & 60.72 & 1508 \\
\hline 63.5 & 1483 & 61.07 & 1450 & 61.78 & 1482 & 61.55 & 1484 & 61.37 & 1484 & 60.63 & 1479 & 60.66 & 1488 & 59.89 & 1480 & 59.36 & 1479 \\
\hline 62.23 & 1456 & 60.13 & 1425 & 60.66 & 1455 & 60.42 & 1454 & 59.92 & 1453 & 59.77 & 1457 & 59.27 & 1456 & 58.67 & 1452 & 58.38 & 1452 \\
\hline 60.95 & 1427 & 59 & 1402 & 59.53 & 1430 & 59.3 & 1430 & 58.79 & 1427 & 58.41 & 1428 & 58.05 & 1426 & 57.52 & 1428 & 57.34 & 1430 \\
\hline 59.86 & 1404 & 57.37 & 1367 & 58.29 & 1403 & 57.91 & 1399 & 57.79 & 1404 & 57.43 & 1403 & 56.87 & 1401 & 56.37 & 1399 & 55.89 & 1397 \\
\hline 58.79 & 1381 & 56.28 & 1342 & 57.2 & 1376 & 56.75 & 1374 & 56.31 & 1372 & 55.86 & 1370 & 55.66 & 1373 & 55.15 & 1371 & 54.8 & 1370 \\
\hline 57.34 & 1348 & 55.03 & 1316 & 56.1 & 1352 & 55.63 & 1348 & 55.15 & 1345 & 54.83 & 1345 & 54.59 & 1348 & 54 & 1343 & 53.79 & 1347 \\
\hline 56.13 & 1322 & 53.97 & 1290 & 54.86 & 1325 & 54.29 & 1319 & 54.18 & 1319 & 53.47 & 1314 & 53.41 & 1322 & 52.82 & 1320 & 52.49 & 1318 \\
\hline 54.8 & 1294 & 52.58 & 1259 & 53.29 & 1291 & 53.14 & 1292 & 53.11 & 1296 & 52.49 & 1292 & 52.19 & 1293 & 51.69 & 1291 & 51.31 & 1290 \\
\hline 53.47 & 1264 & 51.48 & 1235 & 52.19 & 1266 & 51.93 & 1264 & 51.66 & 1263 & 51.1 & 1261 & 50.92 & 1263 & 50.8 & 1268 & 50.18 & 1264 \\
\hline 52.34 & 1241 & 50.33 & 1210 & 50.95 & 1237 & 50.74 & 1240 & 50.48 & 1235 & 50.03 & 1235 & 49.89 & 1240 & 49.62 & 1242 & 49.03 & 1236 \\
\hline 51.07 & 1211 & 48.91 & 1179 & 49.97 & 1215 & 49.53 & 1212 & 49.35 & 1209 & 48.76 & 1206 & 48.49 & 1209 & 48.05 & 1206 & 47.93 & 1210 \\
\hline 49.94 & 1189 & 47.64 & 1152 & 48.82 & 1189 & 48.49 & 1187 & 48.02 & 1179 & 47.75 & 1182 & 47.37 & 1183 & 47.1 & 1182 & 46.54 & 1180 \\
\hline 48.46 & 1154 & 46.54 & 1126 & 47.22 & 1155 & 47.1 & 1156 & 46.96 & 1157 & 46.3 & 1153 & 46.16 & 1154 & 46.04 & 1158 & 45.54 & 1155 \\
\hline
\end{tabular}


Table 2. $15^{\circ} \mathrm{C}$ - continued

\begin{tabular}{|c|c|c|c|c|c|c|c|c|c|c|c|c|c|c|c|c|c|}
\hline run1 & & run2 & & run3 & & run4 & & run5 & & run6 & & run7 & & run8 & & run9 & \\
\hline$[\mathrm{Pa}]$ & {$[1 / \mathrm{s}]$} & {$[\mathrm{Pa}]$} & {$[1 / \mathrm{s}]$} & {$[\mathrm{Pa}]$} & {$[1 / \mathrm{s}]$} & {$[\mathrm{Pa}]$} & {$[1 / \mathrm{s}]$} & {$[\mathrm{Pa}]$} & {$[1 / \mathrm{s}]$} & {$[\mathrm{Pa}]$} & {$[1 / \mathrm{s}]$} & {$[\mathrm{Pa}]$} & {$[1 / \mathrm{s}]$} & {$[\mathrm{Pa}]$} & {$[1 / \mathrm{s}]$} & {$[\mathrm{Pa}]$} & {$[1 / \mathrm{s}]$} \\
\hline 47.43 & 1133 & 45.36 & 1100 & 45.95 & 1126 & 45.95 & 1131 & 45.74 & 1128 & 45.3 & 1127 & 45.15 & 1129 & 44.88 & 1131 & 44.23 & 1124 \\
\hline 45.98 & 1101 & 43.88 & 1069 & 44.82 & 1099 & 44.62 & 1100 & 44.44 & 1097 & 44.32 & 1104 & 43.88 & 1101 & 43.85 & 1106 & 43.23 & 1099 \\
\hline 44.88 & 1077 & 42.72 & 1042 & 43.67 & 1074 & 43.37 & 1071 & 43.32 & 1072 & 42.87 & 1071 & 42.81 & 1074 & 42.28 & 1072 & 42.28 & 1077 \\
\hline 43.49 & 1047 & 41.36 & 1012 & 42.63 & 1050 & 42.34 & 1046 & 42.1 & 1044 & 41.66 & 1043 & 41.51 & 1044 & 41.33 & 1047 & 41.1 & 1051 \\
\hline 42.46 & 022 & .36 & 988 & 41.27 & 1020 & 41.13 & 1021 & 40.98 & 1020 & 40.62 & 1018 & 40.71 & 1025 & 0.24 & 1022 & 40.42 & 1034 \\
\hline 41.3 & 98.1 & 39.14 & 962.5 & 40.24 & 994.2 & 39.97 & 993.7 & 40 & 995.5 & 39.35 & 988.9 & 39.14 & 991.5 & 88.76 & 988.9 & 38.99 & 1001 \\
\hline 39.79 & 65.5 & 38.05 & 937.3 & 39.02 & 967.7 & 38.85 & 968.2 & 38.49 & 963.3 & 38.23 & 963.3 & 38.14 & 965.5 & 37.75 & 964.7 & 38.05 & 976.1 \\
\hline 38.55 & 937.8 & 36.63 & 904.3 & 37.78 & 940 & 37.46 & 936 & 37.25 & 933.8 & 36.98 & 933.4 & 37.19 & 943.9 & 36.57 & 936.5 & 36.39 & 938.2 \\
\hline 37.6 & 914 & 35.53 & 878.7 & 36.3 & 906.9 & 36.3 & 909.6 & 36.18 & 909.6 & 35.95 & 908.7 & 35.65 & 908.7 & 35.5 & 912.2 & 35.59 & 921.9 \\
\hline 36.04 & 882.2 & 34.32 & 854 & 35.18 & 881.8 & 35.03 & 879.6 & 35.18 & 882.7 & 34.56 & 877.8 & 34.67 & 885.3 & 34.11 & 878.7 & 34.08 & 883.1 \\
\hline 34.85 & 854.9 & 33.16 & 827.6 & 33.96 & 854 & 33.9 & 853.6 & 34.05 & 857.1 & 33.58 & 855.8 & 33.58 & 860.7 & 33.13 & 853.6 & 32.84 & 852.7 \\
\hline 33.82 & 831.6 & 31.68 & 793.7 & 33.02 & 830.2 & 32.84 & 829.8 & 32.81 & 829.8 & 32.45 & 830.2 & 32.28 & 828 & 32.01 & 828.9 & 31.51 & 825 \\
\hline 32.22 & 799.8 & 30.65 & 772.1 & 31.63 & 801.6 & 31.45 & 801.2 & 31.39 & 799.4 & 31.15 & 797.2 & 31 & 801.6 & 30.68 & 797.6 & 30.71 & 801.2 \\
\hline 31 & 773.4 & 29.44 & 745.6 & 30.59 & 776 & 30.32 & 773.8 & 30.21 & 772.1 & 30.12 & 774.7 & 29.82 & 772.5 & 29.7 & 775.2 & 29.2 & 769.4 \\
\hline 29.94 & 747 & 28.04 & 715.2 & 29.52 & 751.4 & 29.08 & 744.3 & 29.23 & 748.7 & 28.81 & 745.6 & 28.84 & 749.2 & 28.52 & 744.8 & 28.13 & 743 \\
\hline 28.52 & 717.9 & 26.68 & 683.5 & 28.04 & 718.3 & 28.1 & 722.7 & 27.66 & 715.2 & 27.63 & 719.2 & 27.57 & 718.8 & 27.42 & 721.4 & 27.22 & 719.6 \\
\hline 27.42 & 691.4 & 26.12 & 667.6 & 26.92 & 693.6 & 26.71 & 688.8 & 26.59 & 688.3 & 26.45 & 689.7 & 26.57 & 696.3 & 26.51 & 695.4 & 26 & 691.9 \\
\hline 26.15 & 664.5 & 24.4 & 631.1 & 25.97 & 667.6 & 25.44 & 663.2 & 25.8 & 668.1 & 25.41 & 666.3 & 25.14 & 663.2 & 24.97 & 661.9 & 24.76 & 662.3 \\
\hline 25.09 & 9.9 & 23.19 & 604.2 & 24.52 & 636.8 & 24.43 & .5 & 24.26 & 634 & 24.02 & 634.1 & 23.99 & 635 & 23.78 & 636.8 & 23.72 & 637.2 \\
\hline 23.69 & 0.3 & 22.24 & 577.7 & 23.43 & 610.8 & 23.28 & 610.8 & 23.1 & 607.3 & 23.04 & 610.8 & 22.87 & 09.5 & 22.75 & 609.5 & 22.84 & 613.4 \\
\hline 22.54 & 581.7 & 21.21 & 553.9 & 22.3 & 585.7 & 22.04 & 583.5 & 22.01 & 584.3 & 21.8 & 579.5 & 21.83 & 584.8 & 21.74 & 583 & 21.36 & 578.2 \\
\hline 21.47 & 560.5 & 19.7 & 520.9 & 21.39 & 562.3 & 21.09 & 559.7 & 20.94 & 554.8 & 20.76 & 553.9 & 20.71 & 555.3 & 20.73 & 559.2 & 20.23 & 554.8 \\
\hline 20.38 & 534.1 & 18.78 & 499.3 & 20.05 & 534.1 & 19.64 & 523.5 & 19.88 & 528.8 & 19.43 & 524.9 & 19.64 & 528.8 & 19.55 & 530.6 & 19.2 & 527.5 \\
\hline 18.9 & 502.4 & 17.45 & 471.1 & 18.6 & 502.4 & 18.6 & 499.7 & 18.72 & 503.7 & 18.34 & 498 & 18.52 & 503.3 & 17.98 & 497.1 & 18.22 & 503.7 \\
\hline 17.83 & 477.3 & 16.33 & 445.5 & 17.45 & 472.9 & 17.3 & 472 & 17.54 & 477.3 & 17.48 & 474.6 & 17.33 & 476.4 & 17.12 & 471.5 & 16.77 & 472 \\
\hline 17.01 & 453 & 15.44 & 420.9 & 16.09 & 444.2 & 16.5 & 449.5 & 16.06 & 442.9 & 16.15 & 442.9 & 16.47 & 452.1 & 16.12 & 446.9 & 15.82 & 442.9 \\
\hline 15.56 & 425.7 & 13.93 & 386 & 15.32 & 419.5 & 15.32 & 421.7 & 15.26 & 419.5 & 14.9 & 418.6 & 15.17 & 418.6 & 14.79 & 420 & 14.85 & 421.7 \\
\hline 13.99 & 391.3 & 2.86 & 360.5 & 13.75 & 390 & 14.22 & 396.6 & 13.99 & 393.1 & 4.02 & 390.4 & 13.81 & 388.7 & 14.08 & 395.7 & 13.54 & 389.6 \\
\hline 13.25 & 368.9 & 11.53 & 331 & 13.01 & 363.1 & 12.92 & 363.6 & 12.83 & 361.8 & 12.92 & 365.3 & 12.83 & 362.2 & 12.42 & 361.4 & 12.6 & 366.6 \\
\hline 11.89 & 36.2 & .57 & 308 & & 9.8 & 11.77 & 335.4 & 12 & 339.8 & 11.68 & 335.4 & 11.75 & 337.1 & 11.74 & 335.8 & 11.93 & 339.8 \\
\hline 10.58 & 309.4 & 9.637 & 282.9 & 10.96 & 315.5 & 10.78 & 312 & 10.71 & 309.8 & 10.83 & 307.2 & 10.79 & 312 & 10.75 & 307.6 & 10.57 & 308.9 \\
\hline 9.676 & 284.2 & 8.486 & 255.2 & 9.581 & 283.8 & 9.486 & 282 & 9.835 & 281.6 & 9.676 & 278.5 & 9.741 & 280.3 & 9.785 & 282.9 & 9.397 & 279.8 \\
\hline
\end{tabular}


Table 3. Rheology Summary for Blank

\begin{tabular}{|c|c|c|c|}
\hline Run \# & $\begin{array}{c}\text { M*1000 } \\
(\mathbf{P a}-\mathbf{s})\end{array}$ & $\begin{array}{c}\mathbf{B} \\
\mathbf{( P a )}\end{array}$ & $\mathbf{R}^{\mathbf{2}}$ \\
\hline 1 & 18.1 & -0.0669 & 0.9964 \\
\hline 2 & 17.5 & 0.0603 & 0.9939 \\
\hline 3 & 17.5 & -0.1379 & 0.9935 \\
\hline & & & \\
\hline & $\mathrm{t}= \pm 3.96 \delta$ & $\mathrm{N}=3$ & \\
\hline Statistics for M & & & \\
\hline Statistics for B & Mean $=17.7$ & Sigma $=0.8$ & $95 \% \mathrm{CL}= \pm 0.1$ \\
\hline
\end{tabular}


Table 4. Rheology Summary for $15^{\circ} \mathrm{C}$

\begin{tabular}{|c|c|c|c|c|}
\hline Run \# & $\begin{array}{c}\mathrm{M}^{* 1000} \\
(\mathrm{~Pa}-\mathrm{s})\end{array}$ & & $\mathbf{R}^{2}$ & $\begin{array}{c}\text { Viscosity } \\
\text { (cP) }\end{array}$ \\
\hline 1 & 45.9 & & 0.9983 & 28.2 \\
\hline 2 & 44.6 & & 0.9994 & 26.9 \\
\hline 3 & 44.0 & & 0.9994 & 26.3 \\
\hline 4 & 43.7 & & 0.9995 & 26.0 \\
\hline 5 & 43.8 & & 0.9991 & 26.1 \\
\hline 6 & 43.1 & & 0.9993 & 25.4 \\
\hline 7 & 42.8 & & 0.9993 & 25.1 \\
\hline 8 & 42.5 & & 0.9992 & 24.8 \\
\hline 9 & 42.1 & & 0.9993 & 24.4 \\
\hline$\delta \cong \mathrm{S}$ & $Z= \pm 1.96 \delta$ & $\mathrm{N}=9$ & & \\
\hline Statistics for M & Mean $=43.6$ & Sigma $=1.2$ & $95 \% \mathrm{CL}= \pm 0.8$ & $\begin{array}{c}\text { Avg }=25.9 \pm 1.6 \\
(13 \%) \text { High }\end{array}$ \\
\hline & & & Calc $\mathrm{cP}=22.6$ & $15 \%$ High \\
\hline
\end{tabular}




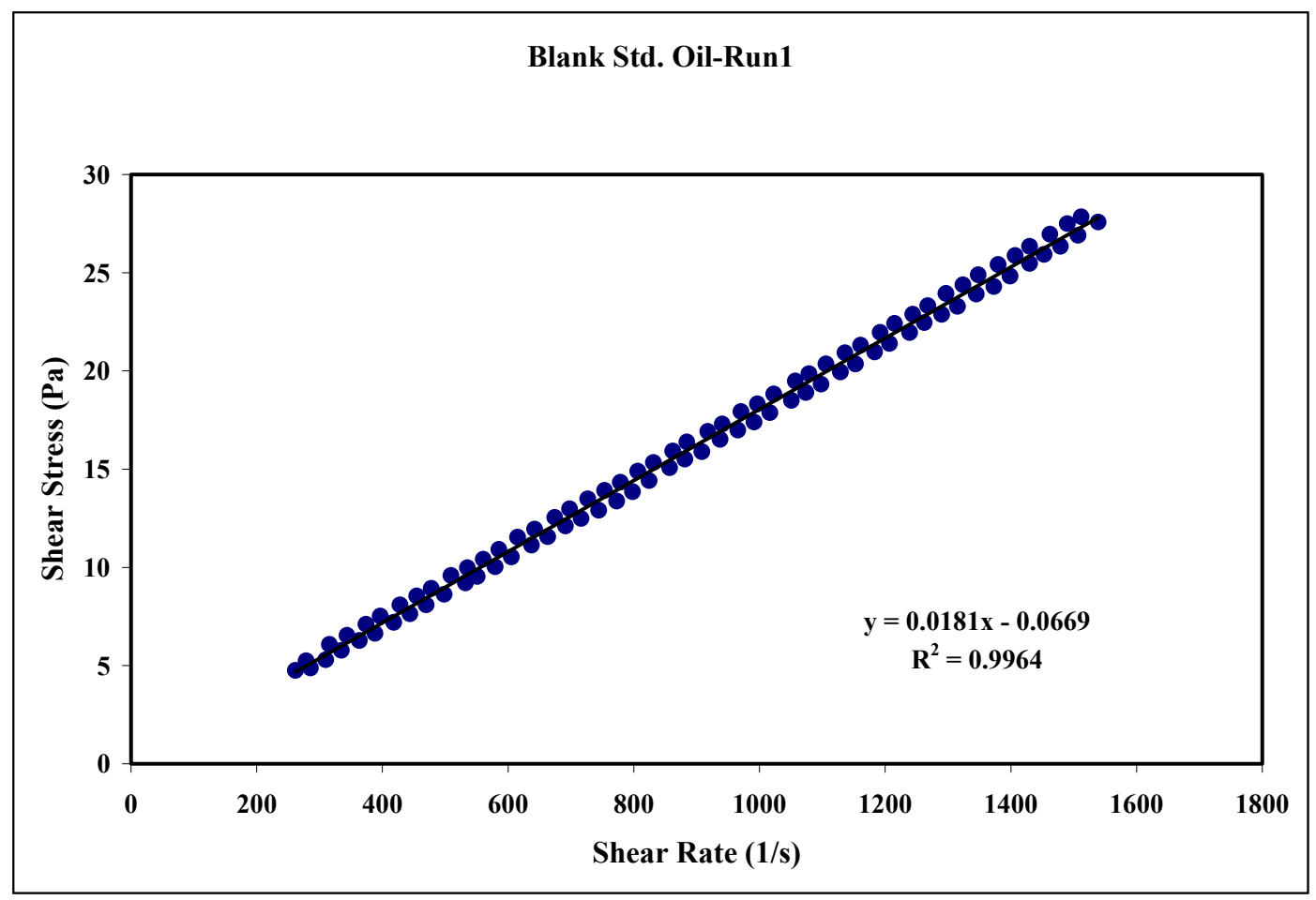

Figure 1. Blank Run 1

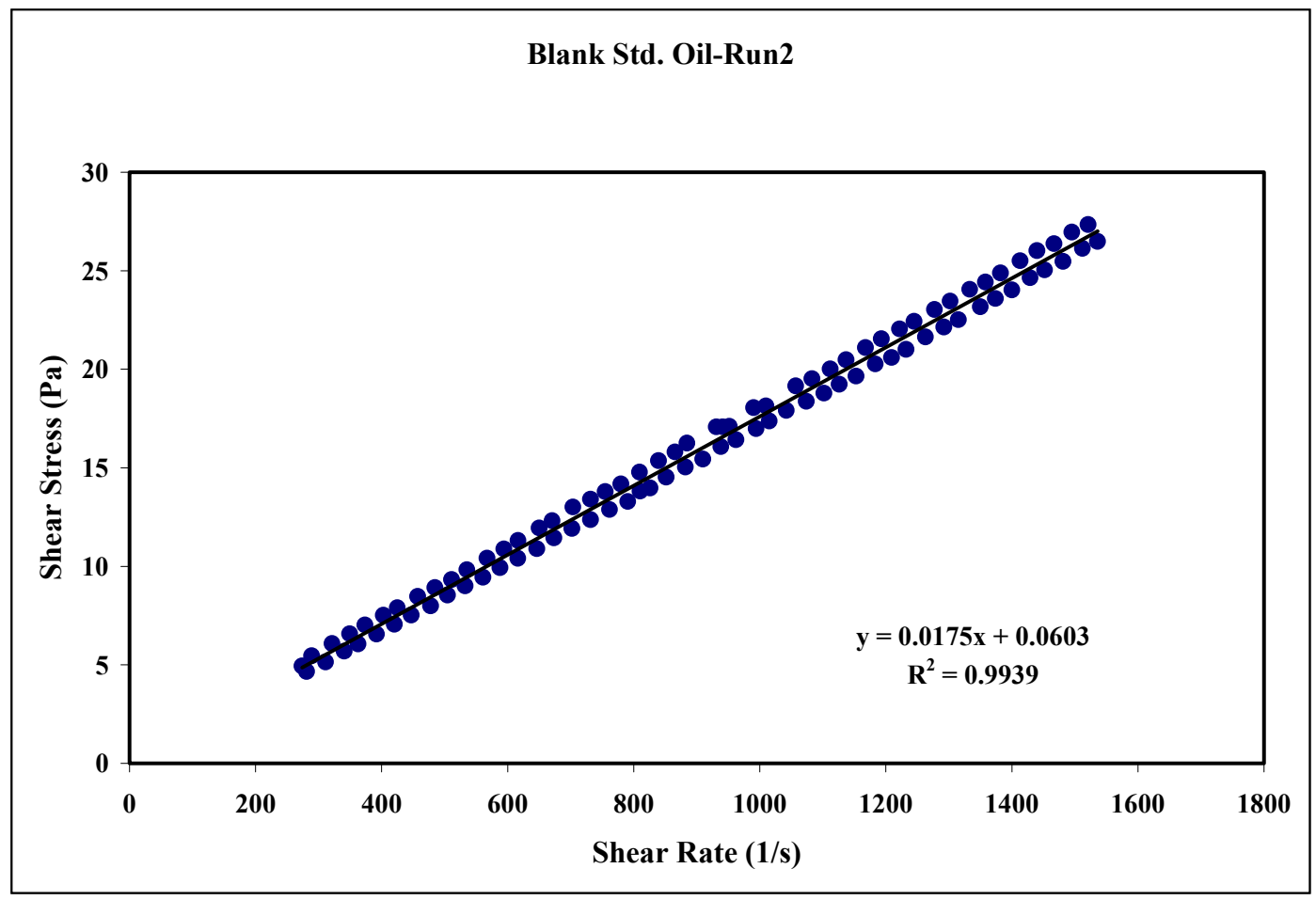

Figure 2. Blank Run 2 


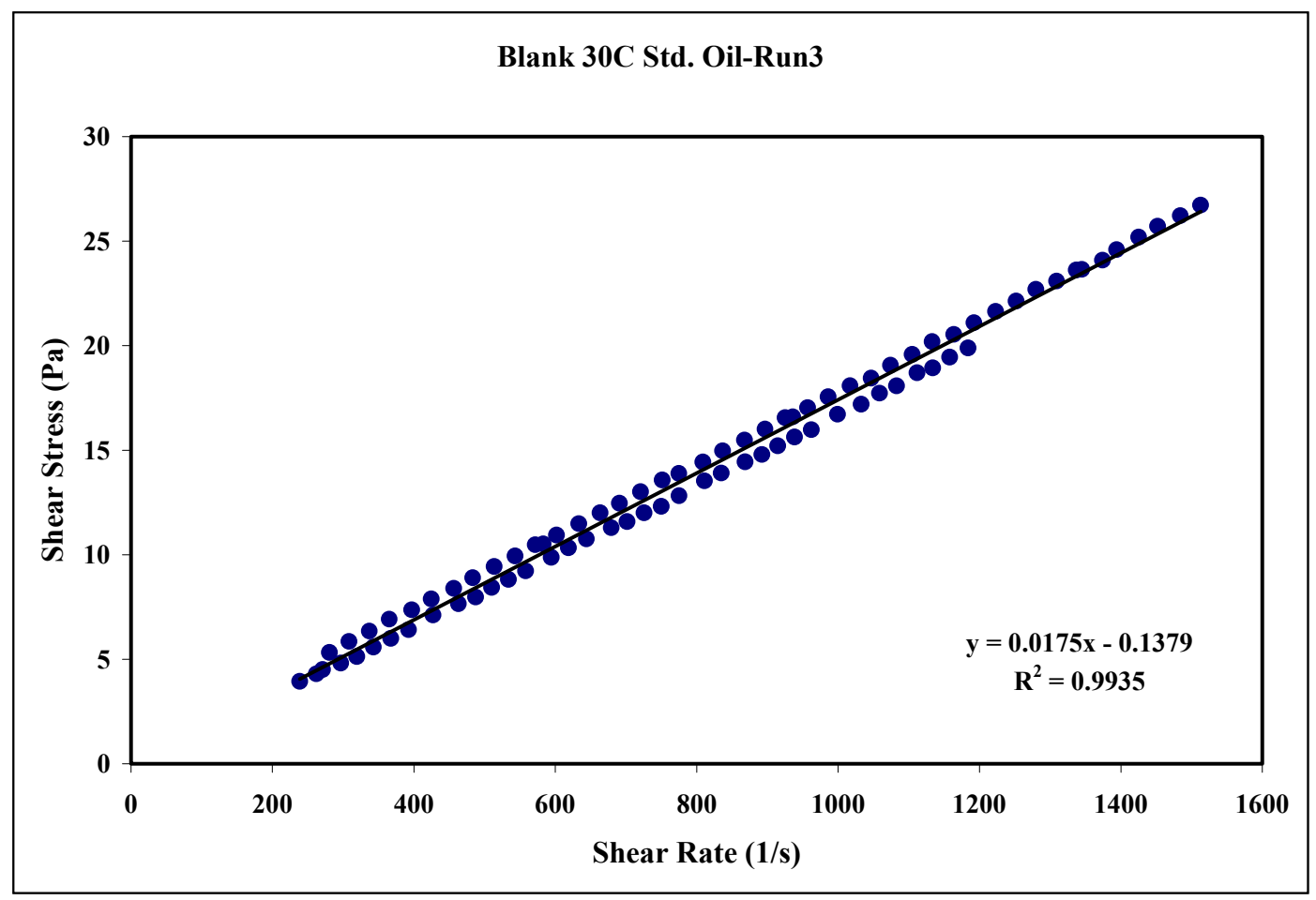

Figure 3. Blank Run 3

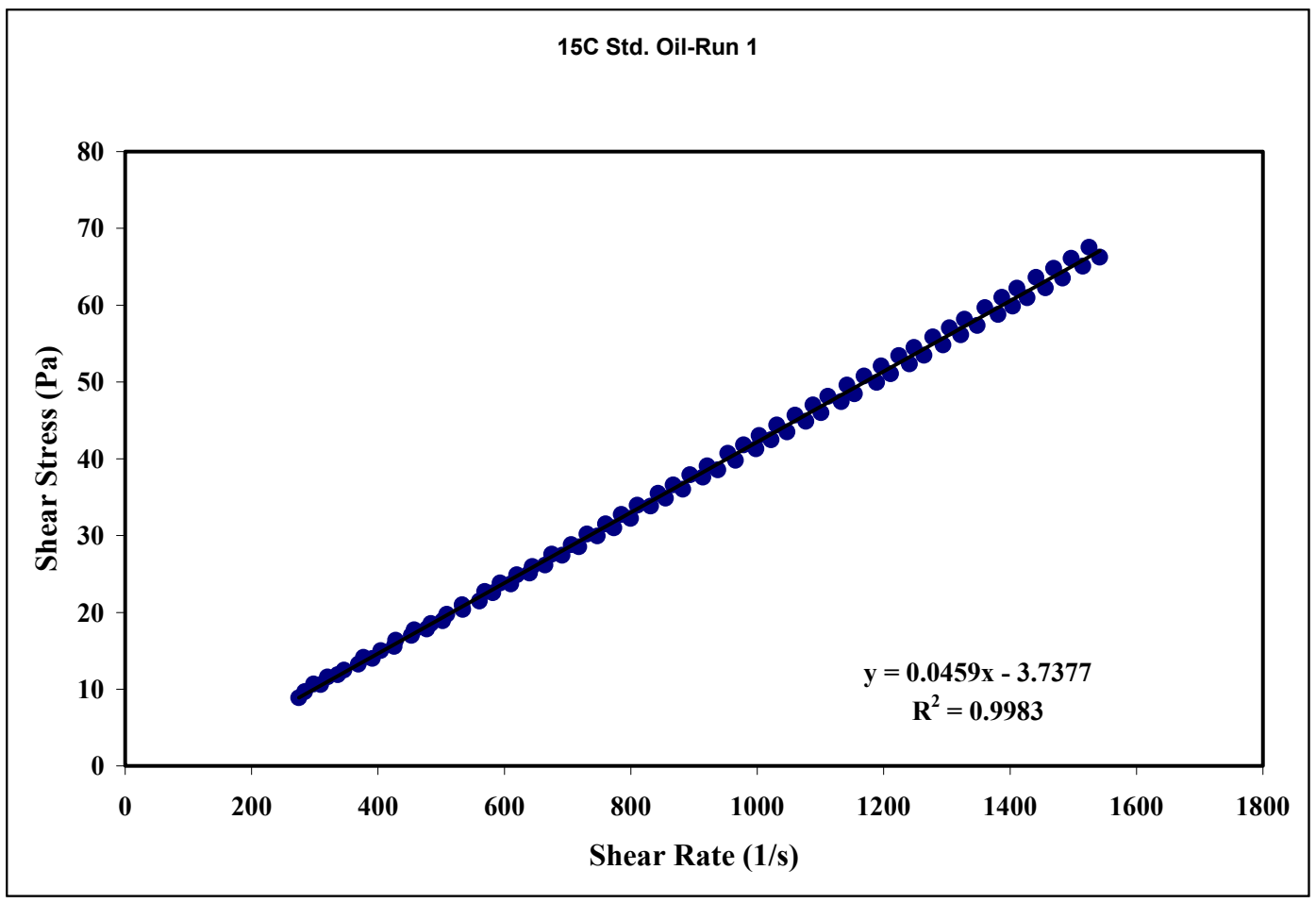

Figure 4. $15^{\circ} \mathrm{C}$ Run 1 


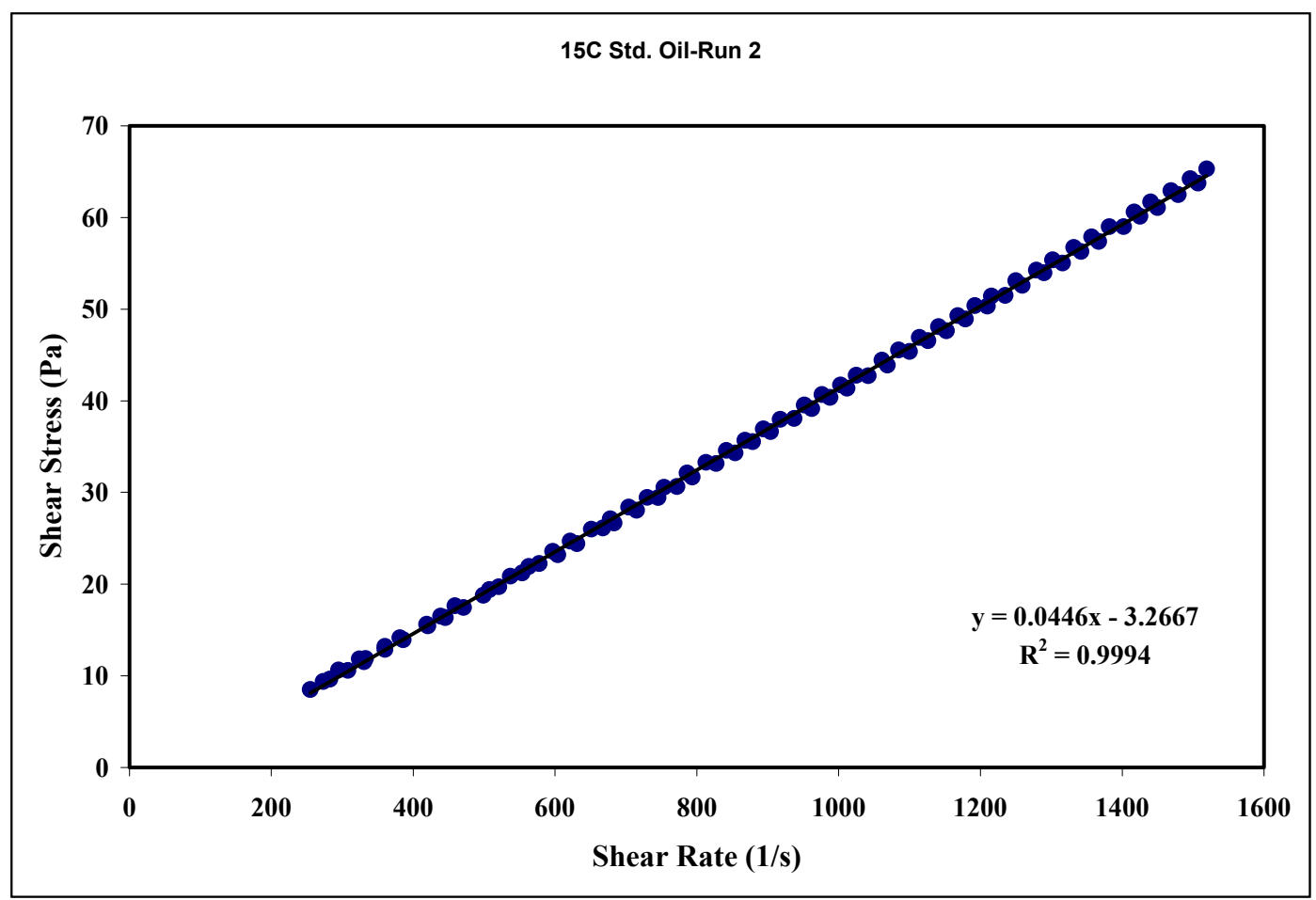

Figure 5. $15^{\circ} \mathrm{C}$ Run 2

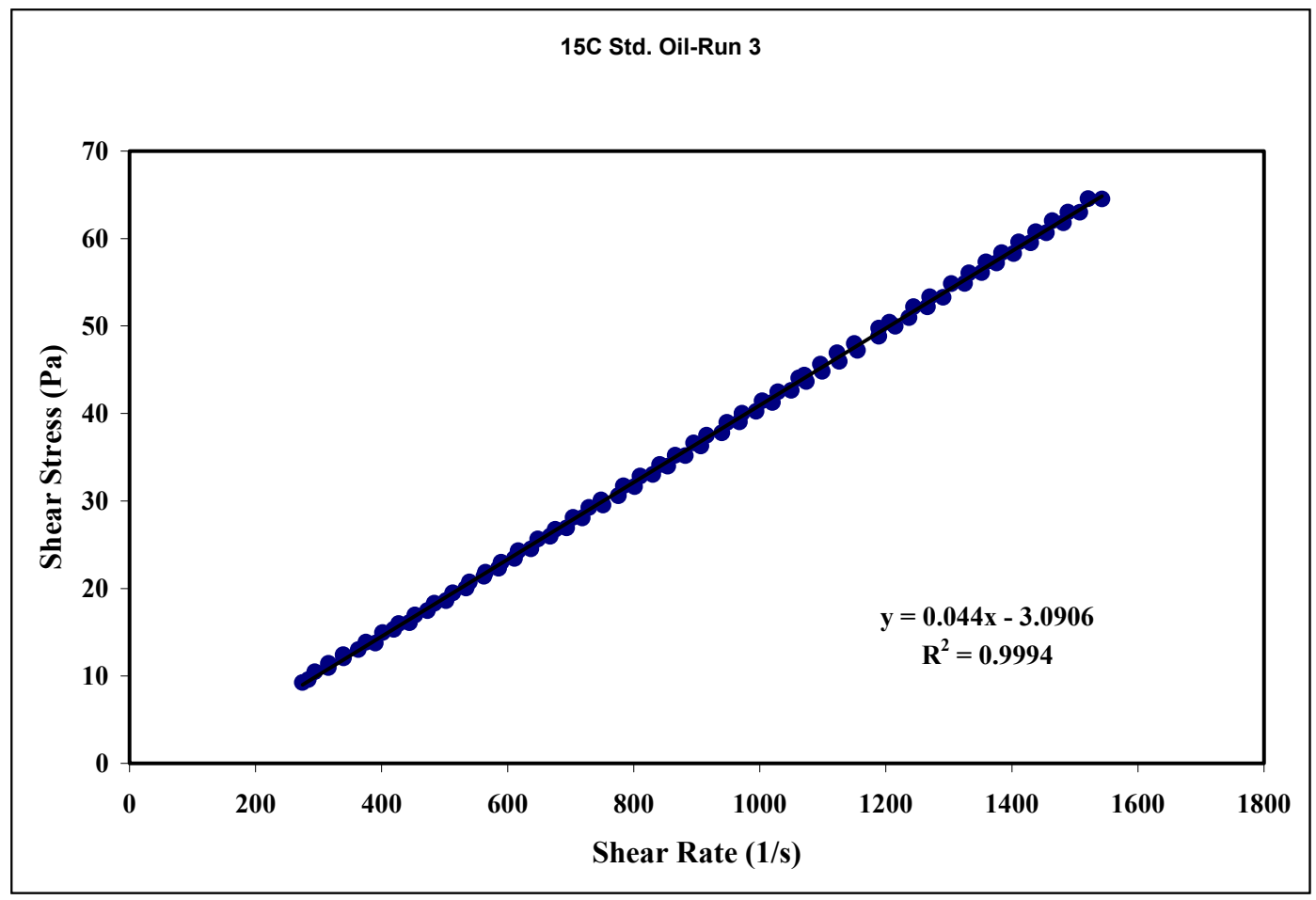

Figure 6. $15^{\circ} \mathrm{C}$ Run 3 


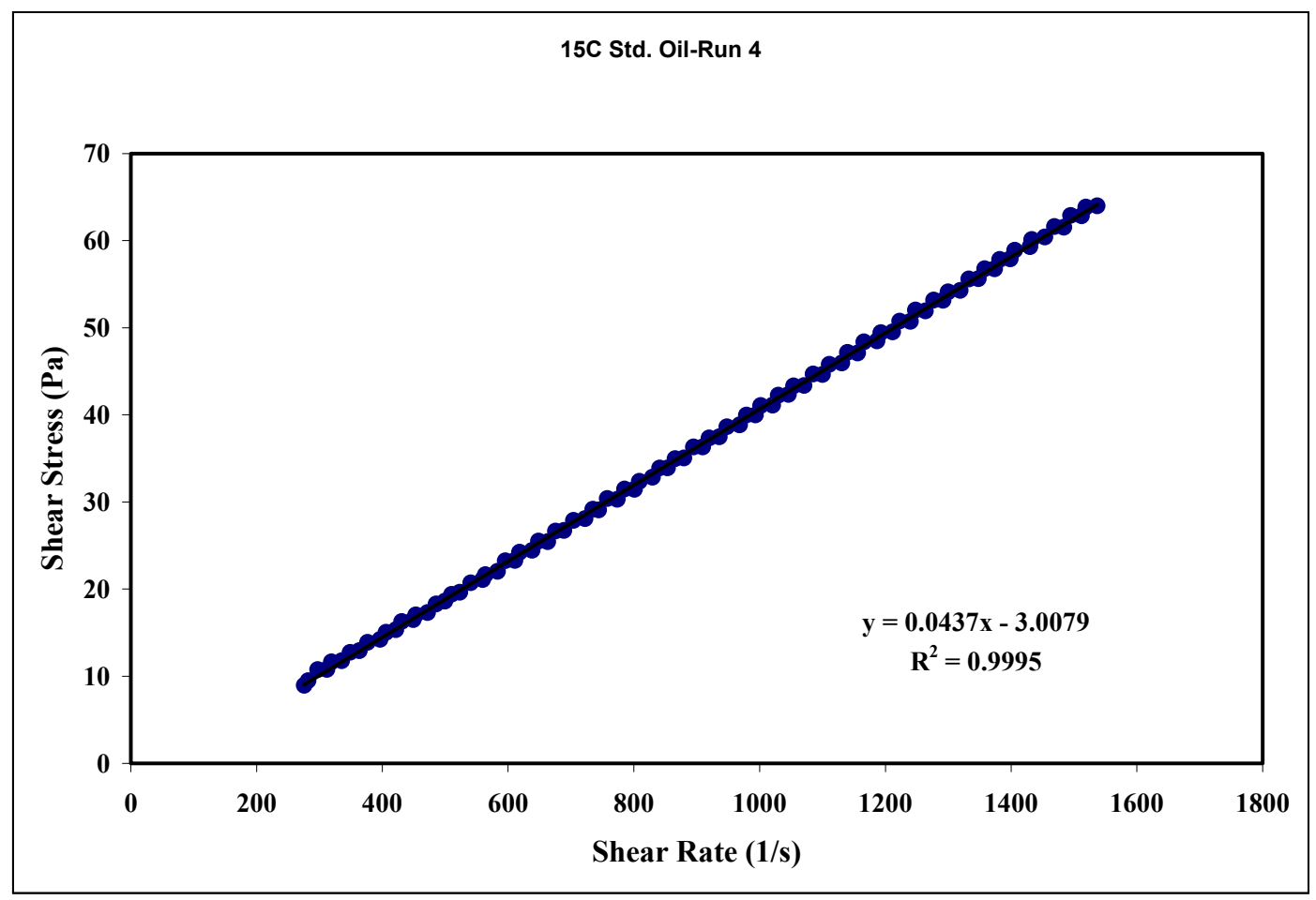

Figure 7. $15^{\circ} \mathrm{C}$ Run 4

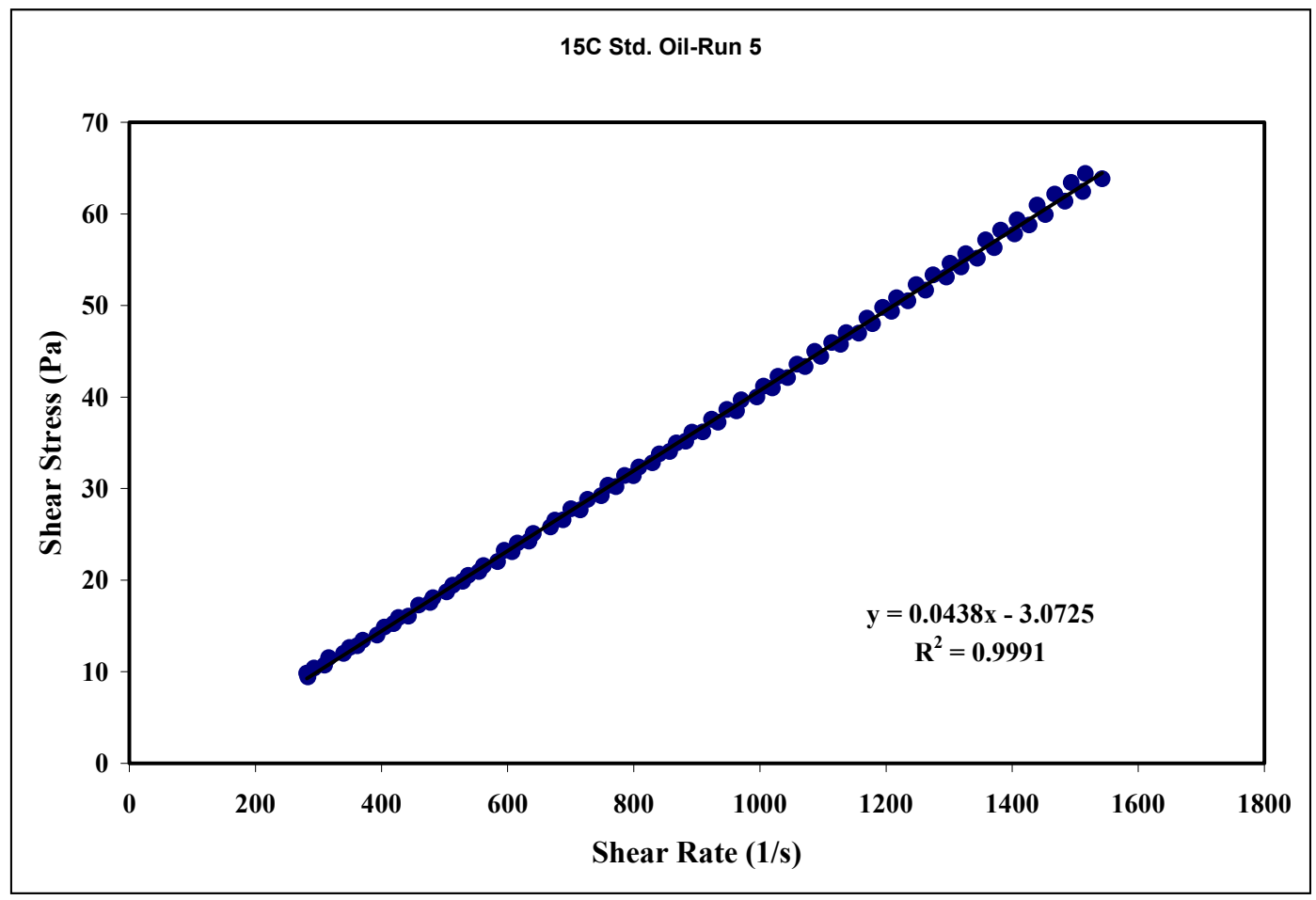

Figure 8. $15^{\circ} \mathrm{C}$ Run 5 


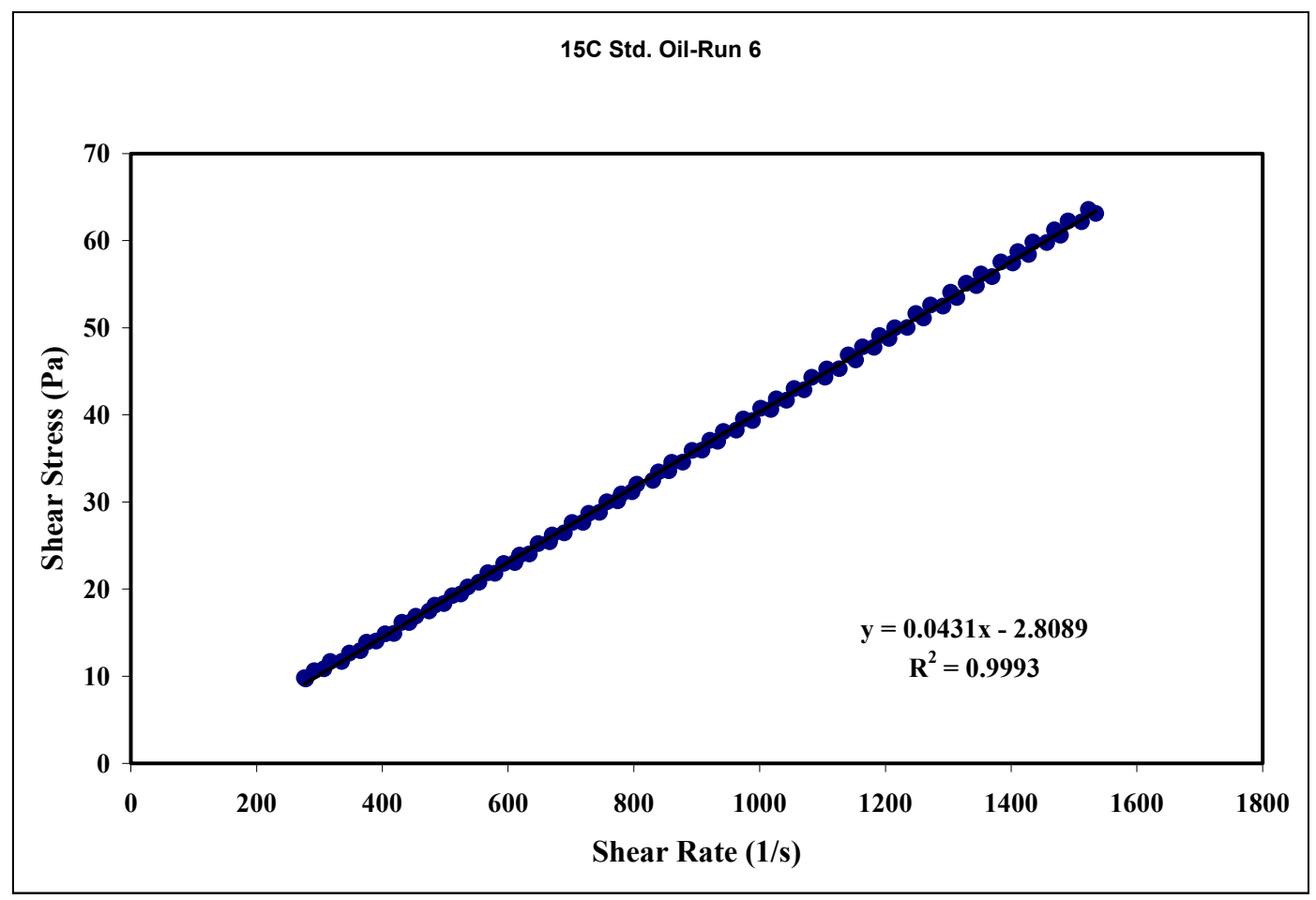

Figure 9. $15^{\circ} \mathrm{C}$ Run 6

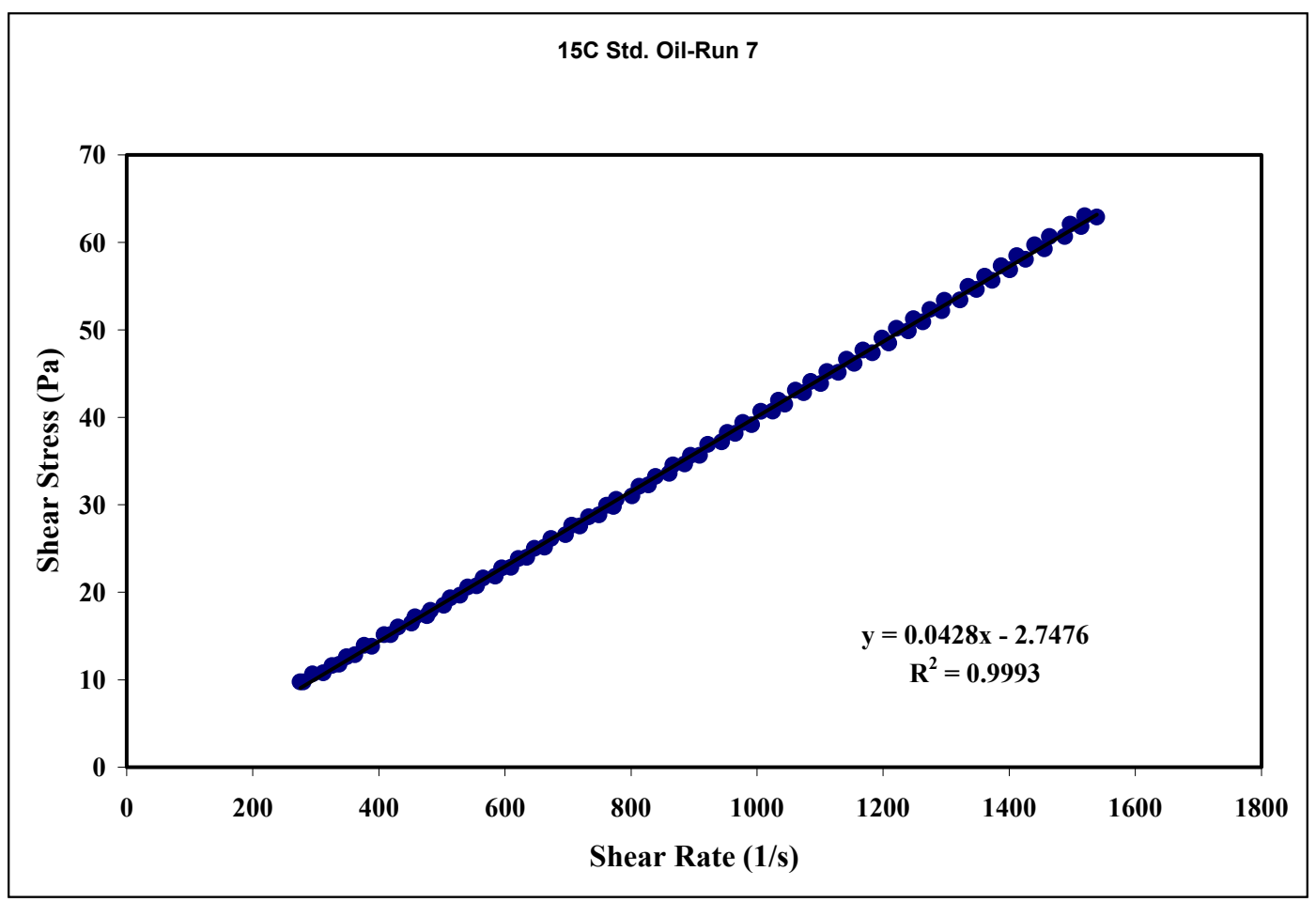

Figure 10. $15^{\circ} \mathrm{C}$ Run 7 


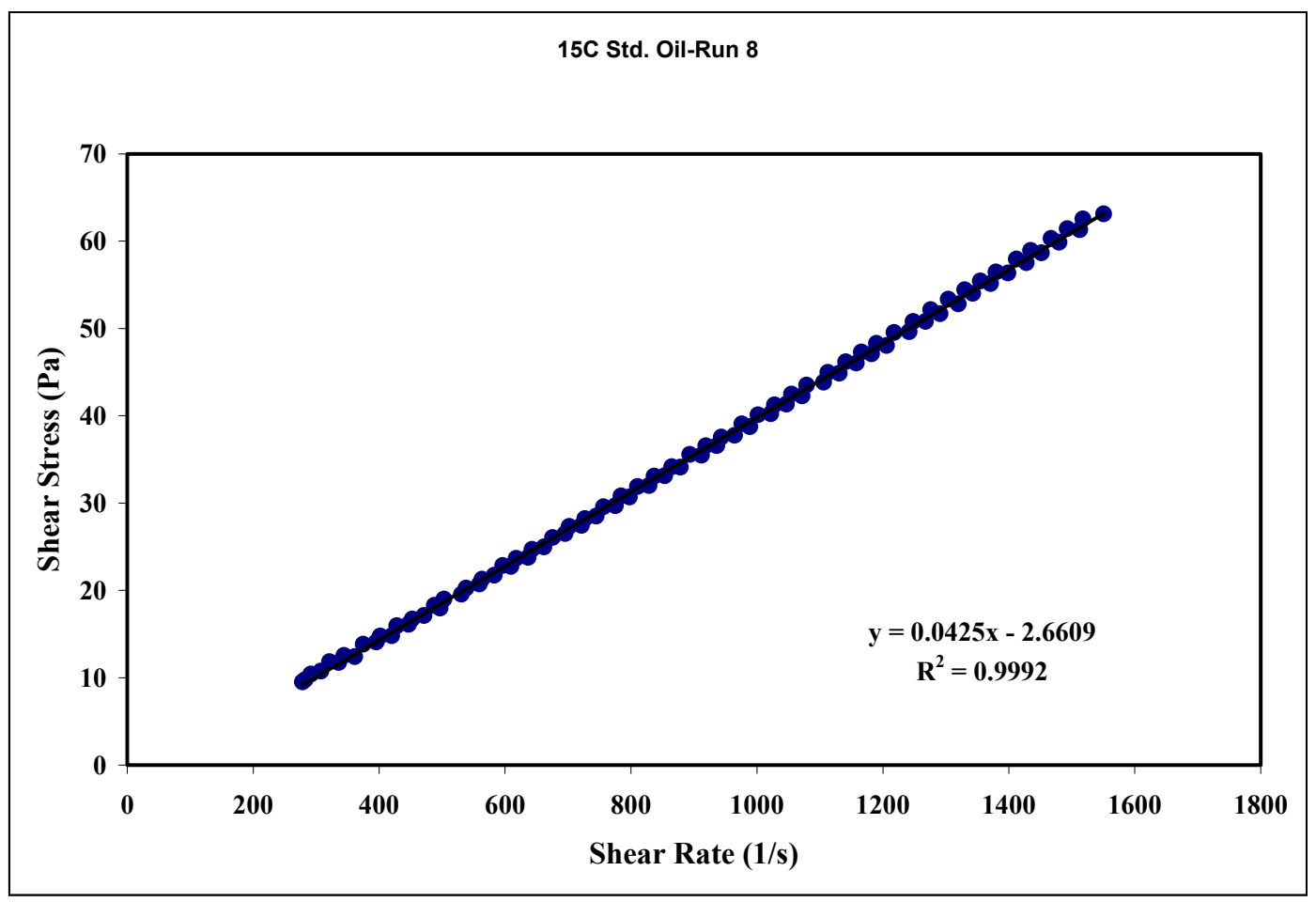

Figure 11. $15^{\circ} \mathrm{C}$ Run 8

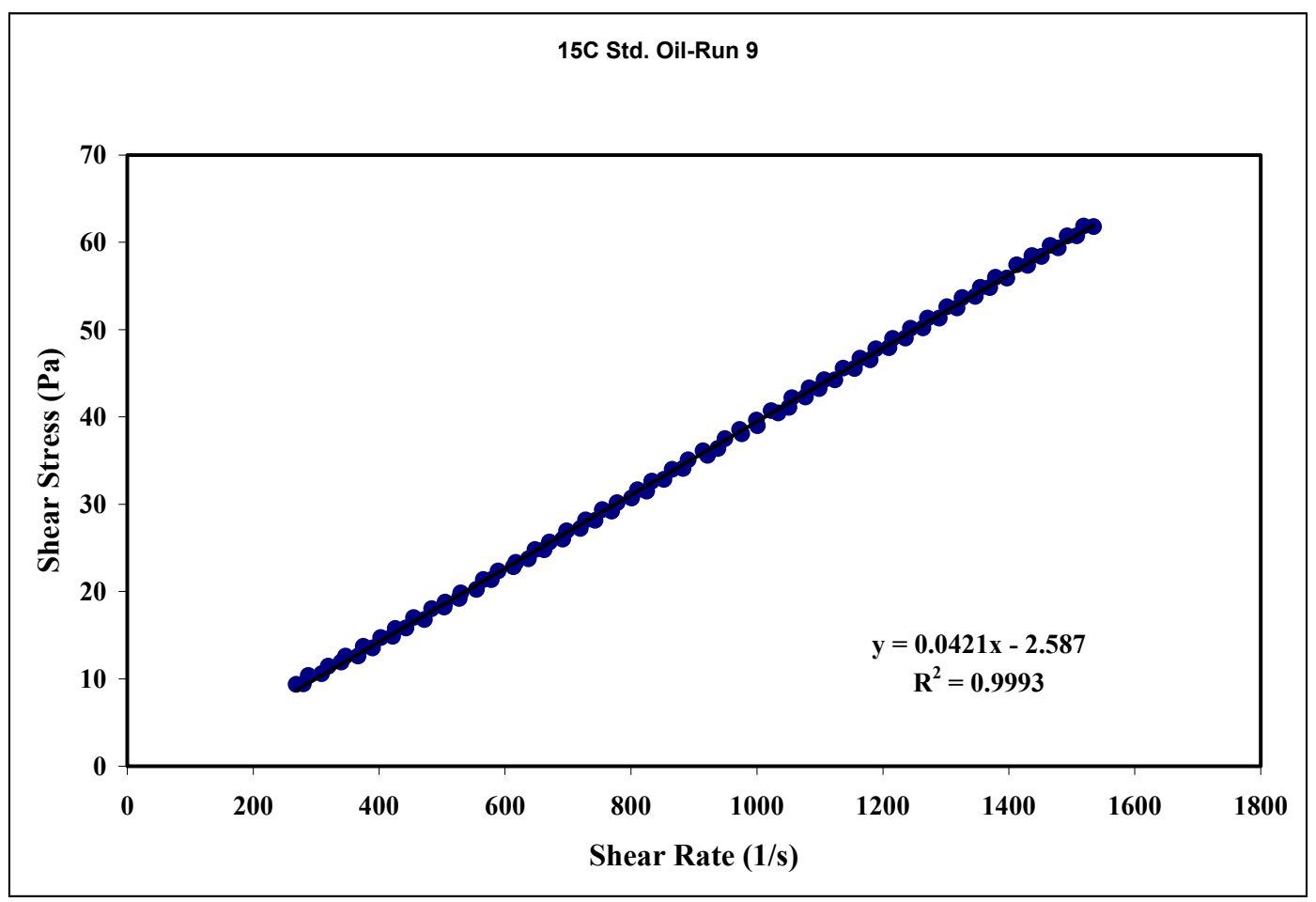

Figure 12. $15^{\circ} \mathrm{C}$ Run 9 
This page intentionally left blank. 


\section{APPENDIX A - PART 5}

\section{TEMPERATURE DEPENDENCE OF VISCOSITY FOR THE LOW VISCOSITY STANDARD OIL}

Table 1. Known Temperature-Dependant Viscosity Data for Low-Viscosity Standard .........................95

Table 2. Calculated viscosity data at $15{ }^{\circ} \mathrm{C}$ based on Arrhenius fit

Figure 1. Arrhenius Plot of Temperature-Dependence of Viscosity for the Low Viscosity Standard ......96 
This page intentionally left blank. 
Table 1. Known Temperature-Dependant Viscosity Data for Low-Viscosity Standard

\begin{tabular}{|c|c|c|c|}
\hline $\mathbf{T}(\mathbf{C})$ & $\mathbf{1 / T}(\mathbf{K})$ & Viscosity (cP) & In (viscosity) \\
\hline 20.00 & $3.411 \mathrm{E}-03$ & 18.38 & $2.911 \mathrm{E}+00$ \\
\hline 25.00 & $3.354 \mathrm{E}-03$ & 14.78 & $2.693 \mathrm{E}+00$ \\
\hline 37.78 & $3.216 \mathrm{E}-03$ & 9.089 & $2.207 \mathrm{E}+00$ \\
\hline 40.00 & $3.193 \mathrm{E}-03$ & 8.429 & $2.132 \mathrm{E}+00$ \\
\hline 98.89 & $2.688 \mathrm{E}-03$ & 2.095 & $7.396 \mathrm{E}-01$ \\
\hline 100.00 & $2.680 \mathrm{E}-03$ & 2.056 & $7.208 \mathrm{E}-01$ \\
\hline
\end{tabular}

Table 2. Calculated viscosity data at $15^{\circ} \mathrm{C}$ based on Arrhenius fit

\begin{tabular}{|c|c|c|c|}
\hline $\mathbf{T}(\mathbf{C})$ & $\mathbf{1 / T}(\mathrm{K})$ & Viscosity (cP) & In (viscosity) \\
\hline 15.00 & $3.470 \mathrm{E}-03$ & 22.6 & \\
\hline
\end{tabular}




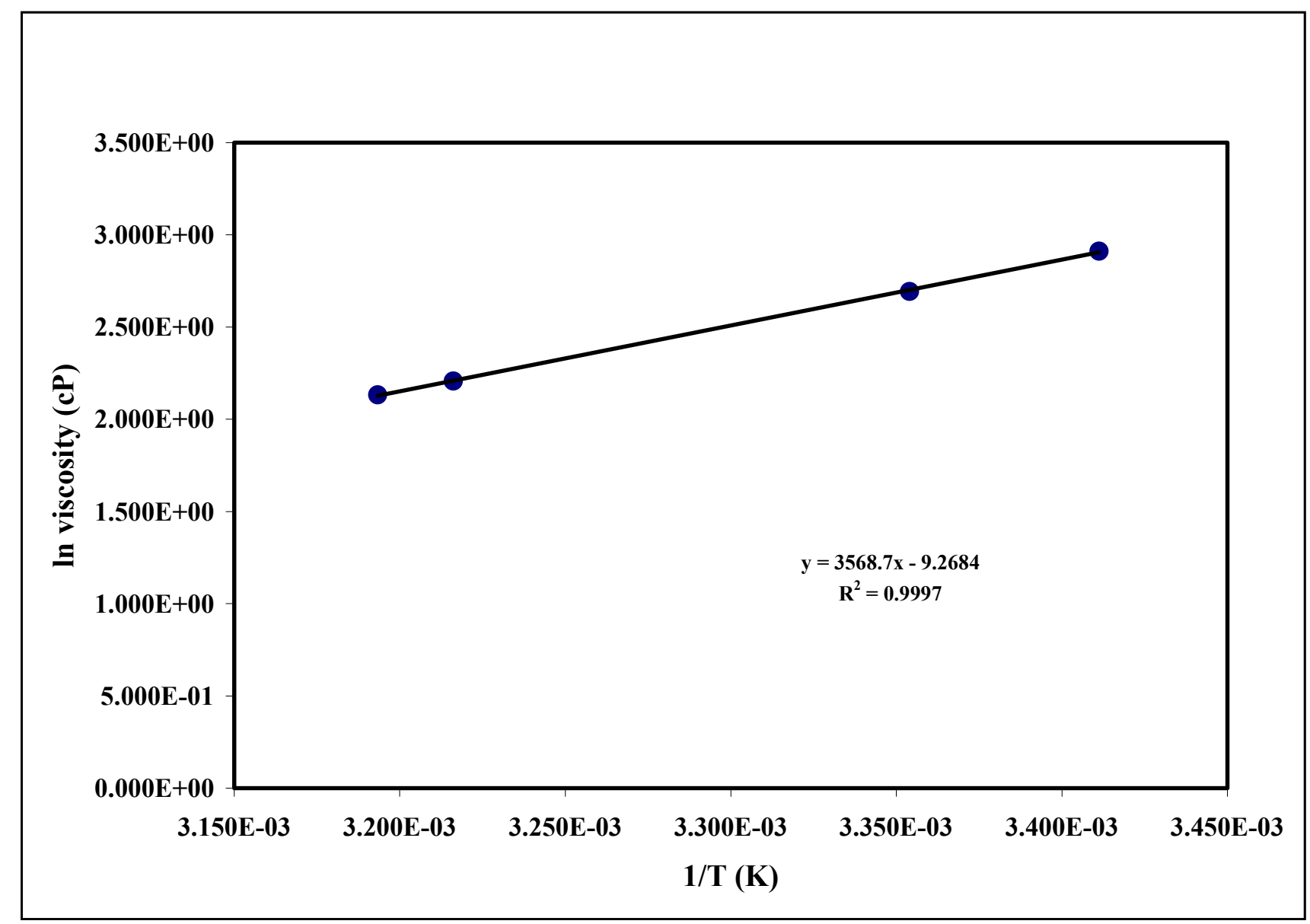

Figure 1. Arrhenius Plot of Temperature-Dependence of Viscosity for the Low Viscosity Standard 
\begin{tabular}{ll}
\hline APPENDIX B & WSRC-TR-2000-00352
\end{tabular}

ENVELOPE A RHEOLOGY DATA

\section{APPENDIX B}

\section{ENVELOPE A RHEOLOGY DATA}

Table 1. Rheometer Response for Blank - As Received ................................................... 99

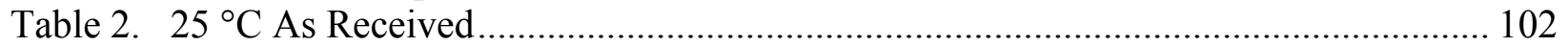

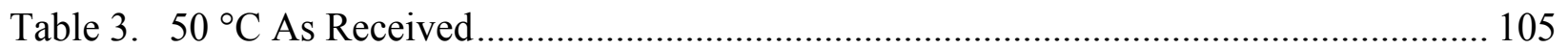

Table 4. Rheometer Response for Blank - Diluted ........................................................... 108

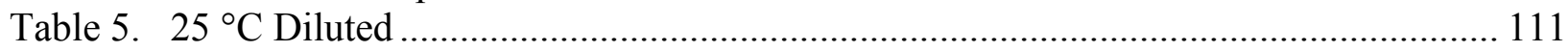

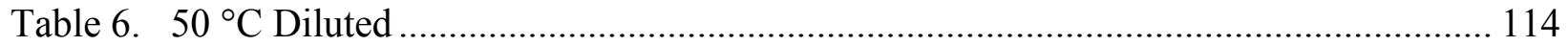


This page intentionally left blank. 
Table 1. Rheometer Response for Blank - As Received

\begin{tabular}{|c|c|c|c|c|c|c|c|c|c|c|c|c|c|c|c|c|c|}
\hline run1 & & run2 & & run3 & & run4 & & run5 & & run6 & & run7 & & run8 & & run9 & \\
\hline [Pa] & {$[1 / s]$} & [Pa] & [1/s] & [Pa] & {$[1 / s]$} & [Pa] & {$[1 / s]$} & [Pa] & [1/s] & [Pa] & [1/s] & [Pa] & [1/s] & [Pa] & {$[1 / s]$} & [Pa] & {$[1 / s]$} \\
\hline 3.068 & 323.9 & 2.613 & 306.3 & 2.092 & 302.3 & 1.861 & 305.8 & 1.876 & 304.5 & 1.985 & 312 & 1.828 & 325.2 & 2.157 & 306.3 & 1.882 & 326.5 \\
\hline 3.48 & 327.9 & .906 & 324.3 & .432 & 308 & 2.545 & 319.9 & 2.257 & 312.4 & 2.379 & 318.6 & 2.281 & 323.5 & 2.175 & 309.8 & 2.423 & 325.2 \\
\hline 3.79 & 346.8 & 133 & 346.4 & 648 & 328.7 & 2.74 & 341.5 & 2.802 & 347.3 & 2.601 & 341.1 & 2.574 & 342.4 & 2.666 & 346.4 & 2.601 & 344.2 \\
\hline 3.938 & 372.4 & 3.338 & 371.9 & 3.113 & 365.3 & 2.95 & 361.8 & 2.968 & 371.9 & 2.861 & 365.8 & 2.959 & 378.1 & 2.861 & 368.4 & 2.846 & 368.9 \\
\hline 4.075 & 402.3 & 3.48 & 401 & .228 & 388.7 & 3.113 & 389.6 & 3.054 & 402.3 & 3.287 & 402.3 & 3.089 & 401.5 & 3.027 & 391.8 & 3.057 & 393.1 \\
\hline 4.214 & 426.1 & 3.625 & 424.4 & .364 & 421.3 & 3.361 & 420.9 & 3.264 & 427.9 & 3.382 & 428.8 & 3.287 & 424.8 & 3.169 & 424.4 & 3.255 & 416.4 \\
\hline 4.391 & 453 & 3.835 & 450.8 & .512 & 445.1 & 3.533 & 446.9 & 3.423 & 452.1 & 3.586 & 452.6 & 3.412 & 455.2 & 3.347 & 451.7 & 3.426 & 442.9 \\
\hline 4.595 & 472.4 & 4.083 & 475.5 & 3.713 & 468 & 3.642 & 475.1 & 3.554 & 478.6 & 3.737 & 484.8 & 3.503 & 479.9 & 3.527 & 479.5 & 3.604 & 468.4 \\
\hline 4.702 & 499.7 & 4.202 & 507.2 & 832 & 492.7 & .909 & 498 & 3.716 & 505 & 3.873 & 510.3 & 3.631 & 502.8 & 3.728 & 504.1 & 3.971 & 507.7 \\
\hline 5.096 & 536.8 & 4.314 & 531.5 & & 518.7 & 4.021 & 527.5 & 3.924 & 529.3 & & 536.3 & 4.042 & 541.2 & 3.87 & 528.8 & 4.154 & 533.2 \\
\hline 5.241 & 561.4 & 4.459 & 556.6 & 4.184 & 542 & 4.122 & 552.2 & 4.075 & 553.1 & 4.252 & 561.9 & 3.935 & 553.5 & 4.012 & 554.8 & 4.32 & 558.8 \\
\hline 5.391 & 591.4 & 4.64 & 581.7 & & 569.8 & & 577.7 & 4.225 & 580.4 & & 587.9 & & 595.4 & & 578.2 & 4.456 & 582.6 \\
\hline 5.578 & 612.5 & 4.814 & 607.3 & 4.533 & 596.2 & 4.459 & 602.4 & 4.631 & 620 & 4.589 & 611.2 & 4.575 & 621.4 & 4.311 & 602.9 & 4.581 & 614.8 \\
\hline 5.898 & 651.3 & 5.202 & 646.9 & 4.894 & 639.9 & 4.619 & 625.3 & 4.737 & 644.7 & 382 & 650.4 & 4.77 & 646.5 & 4.666 & 640.8 & 4.773 & 641.2 \\
\hline 6.093 & 674.2 & 5.344 & 672.5 & 4.865 & 649.1 & 4.743 & 651.3 & 4.874 & 674.2 & 5.084 & 682.2 & 4.862 & 671.2 & 4.838 & 665 & 4.9 & 667.6 \\
\hline 6.232 & 699.8 & 5.548 & 701.1 & 5.291 & 689.7 & 4.992 & 676.9 & 5.022 & 698 & 5.258 & 702 & 5.057 & 699.8 & 4.903 & 695.8 & 5.078 & 692.3 \\
\hline 6.427 & 728.4 & 5.69 & 728 & & 716.1 & & 717.9 & 5.184 & 719.2 & & 725.4 & & 725.4 & & 720.1 & 5.273 & 719.2 \\
\hline 6.56 & 753.1 & 5.871 & 749.2 & 5.575 & 744.3 & 5.415 & 746.5 & 5.3 & 747.8 & 5.415 & 750.9 & 5.448 & 753.1 & 5.377 & 754.4 & 5.442 & 743.9 \\
\hline 6.726 & 776 & & 776 & & 773 & 5.584 & 770.3 & 5.468 & 772.1 & & 786.2 & 5.563 & 784.4 & & 783.1 & 5.563 & 769.4 \\
\hline 6.972 & 795 & 6.211 & 796.3 & 5.986 & 797.2 & 5.708 & 794.6 & 5.821 & 812.2 & 5.753 & 812.6 & 5.64 & 810 & & 800.7 & 5.667 & 792.8 \\
\hline 7.226 & 834.2 & 6.584 & 837.7 & 6.146 & 823.2 & 5.88 & 817.9 & 5.747 & 822.3 & 5.877 & 839.9 & 5.865 & 833.3 & 5.797 & 835.1 & 6.063 & 832.4 \\
\hline 7.318 & 859.3 & 6.812 & 860.7 & 6.247 & 854.9 & 5.966 & 843 & 6.007 & 849.2 & & 865.5 & 07 & 857.6 & 5.942 & 859.8 & 6.229 & 855.8 \\
\hline 7.546 & 884 & 6.901 & 890.2 & 6.415 & 880 & 6.27 & 867.7 & 6.359 & 891.1 & 6.238 & 888.9 & 6.324 & 898.6 & 6.128 & 884.5 & 6.265 & 886.7 \\
\hline 7.726 & 910.9 & 7.037 & 913.5 & 6.605 & 903 & 6.575 & 908.7 & 6.495 & 917.1 & 386 & 914 & 6.528 & 923.7 & 6.217 & 905.6 & 6.436 & 911.8 \\
\hline 7.957 & 932.9 & 7.203 & 936.9 & 6.759 & 930.7 & 6.726 & 931.2 & 6.679 & 941.7 & 6.549 & 941.3 & 6.688 & 950.6 & 6.584 & 949.2 & 6.596 & 940 \\
\hline 8.108 & 960.2 & 7.605 & 975.7 & 6.954 & 955.8 & 6.791 & 963.3 & 6.824 & 967.3 & & 971.7 & 6.827 & 973.5 & 6.492 & 957.2 & 6.904 & 959.4 \\
\hline 8.573 & 1005 & 7.756 & 999.5 & 7.105 & 983.2 & 6.996 & 988.9 & 6.93 & 997.3 & 6.924 & 996.4 & 6.91 & 1003 & 6.922 & 997.7 & 6.963 & 993.3 \\
\hline 8.715 & 1029 & 7.857 & 1029 & 7.274 & 1008 & 7.102 & 1013 & 7.087 & 1022 & 7.072 & 1022 & 7.081 & 1026 & 6.945 & 1021 & 7.155 & 1019 \\
\hline 8.904 & 1059 & 7.993 & 1051 & 7.422 & 1032 & 7.318 & 1038 & 7.232 & 1045 & 7.206 & 1046 & 7.22 & 1049 & 7.129 & 1052 & 7.286 & 1045 \\
\hline 8.99 & 1081 & 8.114 & 1075 & 7.576 & 1059 & 7.442 & 1064 & 7.502 & 1070 & & 1086 & 7.3 & 1080 & 7.297 & 1077 & 7.422 & 1067 \\
\hline 9.114 & 1104 & 8.194 & 1098 & 7.963 & 1097 & 7.581 & 1087 & 7.682 & 1098 & 7.679 & 1116 & 7.596 & 1107 & 7.365 & 1099 & 7.608 & 1092 \\
\hline
\end{tabular}


Table 1. Rheometer Response for Blank - As Received - continued

\begin{tabular}{|c|c|c|c|c|c|c|c|c|c|c|c|c|c|c|c|c|c|}
\hline run1 & & run2 & & run3 & & run4 & & run5 & & run6 & & run7 & & run8 & & run9 & \\
\hline [Pa] & [1/s] & [Pa] & [1/s] & [Pa] & [1/s] & [Pa] & {$[1 / s]$} & [Pa] & [1/s] & [Pa] & [1/s] & [Pa] & [1/s] & [Pa] & [1/s] & [Pa] & {$[1 / s]$} \\
\hline 9.331 & 1127 & 8.431 & 1126 & 8.179 & 1125 & 7.75 & 1115 & 8.099 & 1138 & 7.786 & 1140 & 7.706 & 1137 & 7.564 & 1122 & 7.786 & 1117 \\
\hline 9.748 & 1166 & 8.745 & 1166 & 8.375 & 1151 & 8.156 & 1157 & 8.182 & 1161 & 7.966 & 1164 & 7.88 & 1163 & 7.871 & 1161 & 7.922 & 1148 \\
\hline 9.964 & 1191 & 8.827 & 1193 & 8.543 & 1179 & 8.289 & 1181 & 8.262 & 1192 & 8.07 & 1187 & 8.052 & 1187 & 8.031 & 1191 & 8.357 & 1187 \\
\hline 10.11 & 1223 & 9.038 & 1216 & 8.567 & 1210 & 8.508 & 1203 & 8.466 & 1218 & 8.389 & 1227 & 8.194 & 1211 & 8.126 & 1211 & 8.502 & 1215 \\
\hline 10.34 & 1241 & 9.251 & 1240 & 8.739 & 1233 & 8.561 & 1237 & 8.665 & 1239 & 8.351 & 1235 & 8.576 & 1250 & 8.295 & 1234 & 8.407 & 1228 \\
\hline 10.5 & 1275 & 9.357 & 1263 & 8.842 & 1256 & 8.75 & 1260 & 8.783 & 1266 & 8.747 & 1277 & 8.703 & 1272 & 8.62 & 1274 & 8.709 & 1253 \\
\hline 10.68 & 1296 & 9.532 & 1289 & 9.224 & 1293 & 8.89 & 1284 & 8.895 & 1288 & 8.928 & 1302 & 8.83 & 1304 & 8.774 & 1296 & 9.1 & 1297 \\
\hline 10.77 & 1320 & 9.878 & 1330 & 9.39 & 1315 & 9.029 & 1308 & 9.271 & 1329 & 9.067 & 1327 & 8.981 & 1328 & 9.011 & 1319 & 9.032 & 1305 \\
\hline 11.02 & 1345 & 10.05 & 1352 & 9.434 & 1345 & 9.203 & 1334 & 9.44 & 1352 & 9.262 & 1353 & 9.109 & 1351 & 9.123 & 1351 & 9.372 & 1352 \\
\hline 11.46 & 1383 & 10.19 & 1384 & 9.641 & 1369 & 9.354 & 1360 & 9.621 & 1378 & 9.437 & 1379 & 9.221 & 1375 & 9.227 & 1376 & 9.476 & 1375 \\
\hline 11.59 & 1415 & 10.37 & 1406 & 9.825 & 1394 & 9.763 & 1404 & 9.78 & 1404 & 9.594 & 1413 & 9.402 & 1399 & 9.399 & 1397 & 9.65 & 1401 \\
\hline 11.93 & 1436 & 10.54 & 1434 & 10.01 & 1418 & 9.653 & 1413 & 9.911 & 1436 & 9.68 & 1438 & 9.813 & 1439 & 9.697 & 1437 & 9.875 & 1427 \\
\hline 11.99 & 1464 & 10.72 & 1456 & 10.31 & 1458 & 10.14 & 1456 & 9.976 & 1462 & 9.837 & 1466 & 9.976 & 1464 & 9.869 & 1460 & 9.952 & 1455 \\
\hline 12.24 & 1487 & & 1479 & 10.46 & 1482 & & 1462 & 10.12 & 1486 & 9.993 & 1489 & 10.18 & 1496 & 10.06 & 1483 & 10.22 & 1487 \\
\hline 12.37 & 1521 & 11.34 & 1521 & 10.61 & 1506 & 10.53 & 1508 & 10.32 & 1512 & 10.2 & 1513 & 10.24 & 1523 & 10.19 & 1513 & 10.47 & 1510 \\
\hline 10.1 & 1524 & 8.872 & 1427 & 9.647 & 1553 & 8.449 & 1393 & 9.236 & 1525 & 8.558 & 1414 & 9.067 & 1511 & 9.251 & 1517 & 9.502 & 1545 \\
\hline 9.742 & 1483 & 8.694 & 1404 & 9.257 & 1511 & 8.357 & 1365 & 9.097 & 1497 & 8.339 & 1387 & 8.913 & 1488 & 9.088 & 1490 & 9.357 & 1524 \\
\hline 9.532 & 1462 & 8.268 & 1364 & 9.038 & 1489 & 8.206 & 1334 & 8.875 & 1472 & 8.153 & 1362 & 8.78 & 1457 & 8.966 & 1469 & 9.017 & 1486 \\
\hline 9.396 & 1427 & 8.099 & 1339 & 8.969 & 1456 & 8.028 & 1309 & 8.662 & 1449 & 8.025 & 1336 & 8.558 & 1430 & 8.576 & 1426 & 8.827 & 1464 \\
\hline 9.177 & 1404 & 7.931 & 1309 & 8.774 & 1432 & 7.806 & 1285 & 8.327 & 1405 & 7.789 & 1312 & 8.392 & 1407 & 8.6 & 1419 & 8.682 & 1441 \\
\hline 8.996 & 1380 & 7.821 & 1284 & 8.605 & 1410 & 7.685 & 1257 & 8.138 & 1380 & 7.638 & 1287 & 8.253 & 1382 & 8.2 & 1374 & 8.517 & 1414 \\
\hline 8.842 & 1355 & 7.493 & 1259 & 8.457 & 1382 & 7.362 & 1230 & 7.919 & 1355 & 7.203 & 1239 & 7.954 & 1344 & 7.99 & 1352 & 8.46 & 1388 \\
\hline 8.6 & 1332 & 7.357 & 1234 & 8.244 & 1357 & 7.244 & 1208 & 7.759 & 1330 & 7.096 & 1217 & 7.67 & 1323 & 7.866 & 1320 & 8.182 & 1350 \\
\hline 8.298 & 1291 & 7.191 & 1210 & 8.037 & 1330 & 7.117 & 1182 & 7.51 & 1305 & 6.833 & 1191 & 7.596 & 1292 & 7.738 & 1297 & 8.049 & 1329 \\
\hline 8.058 & 1269 & 6.975 & 1181 & 7.608 & 1292 & 6.759 & 1141 & 7.478 & 1274 & 6.67 & 1163 & 7.428 & 1268 & 7.54 & 1273 & 7.863 & 1299 \\
\hline 7.907 & 1238 & 6.812 & 1157 & 7.472 & 1268 & 6.782 & 1132 & 7.312 & 1248 & 6.448 & 1138 & 7.3 & 1242 & 7.413 & 1249 & 7.715 & 1275 \\
\hline 7.718 & 1213 & 6.448 & 1118 & 7.351 & 1238 & 6.291 & 1087 & 7.072 & 1225 & 6.386 & 1106 & 7.132 & 1221 & 7.2 & 1222 & 7.528 & 1250 \\
\hline 7.487 & 1191 & 6.282 & 1088 & 7.226 & 1214 & 6.143 & 1061 & 6.951 & 1199 & 6.211 & 1081 & 6.951 & 1195 & 6.993 & 1198 & 7.135 & 1214 \\
\hline 7.374 & 1165 & 6.17 & 1067 & 7.019 & 1190 & 5.96 & 1031 & 6.797 & 1173 & 6.054 & 1055 & 6.785 & 1171 & 6.812 & 1173 & 7.007 & 1191 \\
\hline 7.25 & 1140 & 5.975 & 1048 & 6.782 & 1162 & 5.812 & 1007 & 6.572 & 1148 & 5.803 & 1039 & 6.54 & 1143 & 6.626 & 1144 & 6.812 & 1165 \\
\hline 7.078 & 1113 & 5.667 & 1018 & 6.534 & 1134 & 5.634 & 982.3 & 6.188 & 1107 & 5.599 & 1006 & 6.176 & 1101 & 6.285 & 1104 & 6.637 & 1135 \\
\hline
\end{tabular}


Table 1. Rheometer Response for Blank - As Received - continued

\begin{tabular}{|c|c|c|c|c|c|c|c|c|c|c|c|c|c|c|c|c|c|}
\hline run1 & & run2 & & run3 & & run4 & & run5 & & run6 & & run7 & & run8 & & run9 & \\
\hline [Pa] & [1/s] & [Pa] & {$[1 / s]$} & [Pa] & [1/s] & [Pa] & {$[1 / s]$} & [Pa] & [1/s] & [Pa] & [1/s] & [Pa] & [1/s] & [Pa] & [1/s] & [Pa] & {$[1 / s]$} \\
\hline 6.839 & 1087 & 5.575 & 981 & 6.427 & 1109 & 5.477 & 957.2 & 6.037 & 1082 & 5.338 & 975.7 & 5.977 & 1077 & 6.131 & 1080 & 6.581 & 1108 \\
\hline 6.611 & 1061 & 5.445 & 955.8 & 6.276 & 1086 & 5.255 & 932 & 5.835 & 1057 & 5.193 & 945.7 & 5.933 & 1047 & 6.007 & 1051 & 6.35 & 1081 \\
\hline 6.262 & 1020 & 5.229 & 929 & 5.874 & 1048 & 5.084 & 910 & 5.667 & 1032 & 5.072 & 919.7 & 5.747 & 1024 & 5.753 & 1028 & 6.031 & 1061 \\
\hline 6.004 & 995.9 & 5.022 & 903 & 5.756 & 1023 & 4.746 & 869 & 5.545 & 1000 & 4.951 & 895.5 & 5.616 & 999 & 5.581 & 999.5 & 5.844 & 1034 \\
\hline 5.918 & 971.7 & 4.85 & 878.3 & 5.628 & 993.7 & 4.581 & 844.3 & 5.374 & 975.2 & 4.735 & 872.6 & 5.433 & 975.7 & 5.46 & 968.2 & 5.741 & 999.5 \\
\hline 5.699 & 945.3 & 4.711 & 854.9 & 5.46 & 971.7 & 4.365 & 819.7 & 5.143 & 947 & 4.388 & 834.2 & 5.202 & 947.5 & 5.294 & 946.1 & 5.551 & 972.6 \\
\hline 5.492 & 911.3 & 4.273 & 816.6 & 5.27 & 948.3 & 4.282 & 788.8 & 5.013 & 916.2 & 4.282 & 805.1 & 5.125 & 923.2 & 5.193 & 921.5 & 5.43 & 946.6 \\
\hline 5.362 & 885.8 & 4.172 & 794.6 & 4.933 & 909.1 & 4.063 & 765.9 & 4.808 & 891.1 & 4.169 & 782.7 & 4.723 & 887.1 & 5.004 & 900.3 & 5.161 & 921.5 \\
\hline 5.143 & 861.5 & 4.039 & 771.2 & 4.826 & 887.5 & 3.935 & 739.5 & 4.782 & 866.4 & 4.021 & 759.3 & 4.575 & 860.7 & 4.726 & 853.6 & 5.004 & 895.5 \\
\hline 4.962 & 836.9 & 3.891 & 738.1 & 4.545 & 857.1 & 3.779 & 716.1 & 4.554 & 842.6 & 3.811 & 734.2 & 4.365 & 833.8 & 4.752 & 846.6 & 4.874 & 871.7 \\
\hline 4.823 & 812.6 & 3.776 & 714.3 & 4.468 & 832.4 & 3.429 & 677.3 & 4.394 & 819.7 & 3.48 & 695 & 4.193 & 799.4 & 4.294 & 804.2 & 4.761 & 843.9 \\
\hline 4.409 & 773.8 & 3.607 & 690.5 & 4.184 & 808.7 & 3.323 & 655.7 & 4.012 & 781.3 & 3.406 & 667.6 & 3.968 & 780.9 & 4.181 & 780.4 & 4.477 & 817.5 \\
\hline 4.252 & 748.7 & 3.225 & 653.5 & 4.033 & 779.1 & 3.187 & 624.4 & 3.882 & 758 & 3.204 & 641.6 & 3.832 & 753.6 & 3.977 & 754.9 & 4.116 & 778.7 \\
\hline 4.146 & 721.4 & 3.151 & 623.6 & 3.941 & 745.2 & 3.062 & 602.4 & 3.663 & 731.1 & 3.015 & 618.7 & 3.776 & 719.6 & 3.853 & 721.8 & 3.971 & 755.3 \\
\hline 3.903 & 697.2 & 3.015 & 599.3 & 3.779 & 723.2 & 2.867 & 579.1 & 3.604 & 697.2 & 2.965 & 584.3 & 3.619 & 698.9 & 3.622 & 699.8 & 3.829 & 724 \\
\hline 3.663 & 674.2 & 2.858 & 576.4 & 3.657 & 700.2 & 2.559 & 538.5 & 3.4 & 672.9 & 2.687 & 563.6 & 3.521 & 672.9 & 3.5 & 669.8 & 3.734 & 698.5 \\
\hline 3.586 & 639.9 & 2.613 & 547.3 & 3.48 & 673.4 & 2.417 & 515.6 & 3.267 & 649.1 & 2.459 & 540.3 & 3.11 & 635 & 3.21 & 646 & 3.601 & 675.1 \\
\hline 3.379 & 618.7 & 2.471 & 526.2 & 3.293 & 650.4 & 2.314 & 486.1 & 3.145 & 624.9 & 2.373 & 506.8 & 3.204 & 624 & 3.11 & 620.9 & 3.4 & 651.8 \\
\hline 3.243 & 593.6 & 2.343 & 501.5 & 3.024 & 608.1 & 2.121 & 459.2 & 2.968 & 597.1 & 2.254 & 477.7 & 2.749 & 584.3 & 3.024 & 586.1 & 3.252 & 626.7 \\
\hline 3.074 & 569.8 & 1.964 & 462.3 & 2.829 & 583.9 & 2.033 & 434.5 & 2.808 & 573.8 & 2.006 & 453 & 2.817 & 569.4 & 2.826 & 560.1 & 2.894 & 586.5 \\
\hline 2.713 & 530.1 & 1.787 & 438 & 2.639 & 560.1 & 1.846 & 408.1 & 2.444 & 533.7 & 1.879 & 425.3 & 2.545 & 540.7 & 2.654 & 534.5 & 2.731 & 563.6 \\
\hline 2.536 & 503.7 & 1.743 & 408.5 & 2.524 & 529.3 & 1.6 & 383.8 & 2.34 & 505.9 & 1.754 & 402.3 & 2.397 & 514.3 & 2.521 & 512.1 & 2.684 & 534.1 \\
\hline 2.287 & 476.8 & 1.565 & 385.2 & 2.417 & 503.3 & 1.497 & 356.1 & 2.169 & 484.3 & 1.574 & 378.1 & 2.254 & 490.9 & 2.417 & 485.2 & 2.506 & 513.4 \\
\hline 2.107 & 449.5 & 1.37 & 356.1 & 2.207 & 478.1 & 1.316 & 329.2 & 2.035 & 453.9 & 1.222 & 341.5 & 1.908 & 448.2 & 2.181 & 461.8 & 2.426 & 486.5 \\
\hline 1.994 & 416.4 & 1.207 & 331 & 2.033 & 452.6 & 1.145 & 305 & 1.97 & 427 & 1.112 & 316 & 1.748 & 423.9 & 2.077 & 435 & 2.216 & 461.4 \\
\hline 1.748 & 395.3 & 1.077 & 307.6 & 1.926 & 429.7 & 0.795 & 267.9 & 1.754 & 402.3 & 0.908 & 290 & 1.669 & 394.4 & 1.701 & 394.9 & 2.098 & 435 \\
\hline 1.651 & 365.3 & 0.92 & 285.1 & 1.79 & 402.8 & 0.621 & 242.8 & 1.532 & 375.5 & 0.79 & 264.9 & 1.521 & 368.9 & 1.568 & 370.2 & 1.899 & 409 \\
\hline 1.482 & 337.6 & 0.816 & 260.4 & 1.618 & 375.5 & 0.695 & 234 & 1.417 & 351.2 & 0.606 & 241.9 & 1.384 & 342.4 & 1.438 & 338.9 & 1.754 & 383.8 \\
\hline & & & & & & & & & & & & & & & & & \\
\hline & & & & & & & & & & & & & & & & & \\
\hline & & & & & & & & & & & & & & & & & \\
\hline
\end{tabular}


Table 2. $25^{\circ} \mathrm{C}$ As Received

\begin{tabular}{|c|c|c|c|c|c|c|c|c|c|c|c|c|c|c|c|c|c|}
\hline run1 & & run2 & & run3 & & run4 & & run5 & & run6 & & run7 & & run8 & & run9 & \\
\hline [Pa] & [1/s] & [Pa] & [1/s] & [Pa] & [1/s] & [Pa] & [1/s] & [Pa] & [1/s] & [Pa] & [1/s] & [Pa] & [1/s] & [Pa] & {$[1 / s]$} & [Pa] & {$[1 / s]$} \\
\hline 7.172 & 313.3 & 6.405 & 314.2 & 6.846 & 305.4 & 6.293 & 324.3 & 6.106 & 331 & 6.124 & 317.3 & 6.471 & 308.9 & 6.521 & 304.1 & 6.065 & 323.5 \\
\hline 7.409 & 16.9 & 787 & 316.9 & 7.625 & 321.3 & 6.861 & 319.1 & 7.509 & 323.5 & 7.429 & 319.1 & 7.722 & 319.9 & 7.571 & 316.4 & 7.358 & 318.2 \\
\hline 8.672 & 46.4 & 125 & 335.8 & 7.968 & 341.1 & 8.001 & 334 & 8.075 & 344.6 & 8.344 & 342.9 & 8.11 & 339.8 & 8.335 & 342.9 & 7.787 & 338.9 \\
\hline 9.753 & 368.4 & .921 & 359.2 & 9.042 & 365.3 & 8.746 & 361.4 & 8.732 & 366.6 & 8.581 & 362.7 & 8.826 & 364 & 8.616 & 364.9 & 8.933 & 365.3 \\
\hline 10.3 & 390.4 & 892 & 383.4 & 9.708 & 394.4 & 9.152 & 386.9 & 9.687 & 390 & 9.522 & 386.5 & 9.753 & 393.1 & 9.545 & 389.1 & 9.427 & 387.4 \\
\hline 11.09 & 423.5 & 0.37 & 408.1 & 10.05 & 418.6 & 9.992 & 411.6 & 10.3 & 412.9 & 10.37 & 412 & 10.55 & 417.3 & 10.27 & 414.2 & 9.853 & 411.2 \\
\hline 11.59 & 445.5 & 1.67 & 443.3 & 10.79 & 441.6 & 10.95 & 435 & 10.75 & 438.5 & 11.43 & 449.5 & 11.14 & 444.2 & 11.45 & 452.1 & 10.77 & 435 \\
\hline 12.63 & 477.3 & 12.26 & 469.3 & 11.75 & 464 & 11.6 & 460.1 & 12.4 & 479.5 & 12.24 & 474.2 & 11.94 & 471.5 & 12.29 & 478.1 & 11.77 & 475.5 \\
\hline 13.29 & 501.5 & 3.18 & 499.3 & 12.75 & 505 & 12.75 & 499.7 & 13.03 & 503.3 & 13 & 505.5 & 12.59 & 496.6 & 13.06 & 505 & 12.49 & 497.1 \\
\hline 14.1 & 524.9 & 13.78 & 522.6 & 13.55 & 529.7 & 13.47 & 527.1 & 3.93 & 533.2 & 13.72 & 527.9 & 13.98 & 532.8 & 13.84 & 532.3 & 13.4 & 529.3 \\
\hline 14.96 & 544.7 & 4.45 & 546 & 14.4 & 559.7 & 14.2 & 552.2 & 4.69 & 556.1 & 14.41 & 550.9 & 14.74 & 557 & 14.57 & 556.1 & 14.27 & 557.5 \\
\hline 15.7 & 569.8 & 15.9 & 583.5 & & 582.6 & & 579.1 & & 579.9 & & 574.2 & & 580.8 & & 587 & & 582.1 \\
\hline 17.29 & 613.9 & 16.55 & 608.1 & 15.95 & 608.1 & 15.81 & 603.7 & 16.09 & 613 & 16.01 & 611.7 & 16.12 & 612.5 & 15.86 & 610.8 & 15.76 & 606.4 \\
\hline 17.95 & 638.1 & 17.2 & 631.1 & 16.75 & 639 & 16.58 & 629.3 & 16.76 & 638.1 & 17.07 & 641.6 & 16.78 & 637.7 & 16.55 & 636.3 & 16.43 & 629.3 \\
\hline 18.66 & 668.5 & 18.15 & 662.8 & 17.21 & 663.7 & 17.38 & 663.2 & 17.41 & 661 & 17.71 & 665 & 17.62 & 665 & 17.33 & 660.6 & 17.2 & 654.4 \\
\hline 19.57 & 691.4 & 18.89 & 686.6 & 18.01 & 685.7 & 18.04 & 685.3 & 18.27 & 684.8 & 18.27 & 690.5 & 18.33 & 690.1 & 18.18 & 687.5 & 18.33 & 695 \\
\hline 20.4 & 713.5 & & 708.6 & 3.86 & 710.8 & & 709.9 & & 723.6 & & 713 & & 716.1 & & 711.7 & 18.95 & 717.4 \\
\hline 20.97 & 738.6 & 20.08 & 731.1 & 20.2 & 751.4 & 19.66 & 735.5 & 20.31 & 746.5 & 20.14 & 750.9 & 20.14 & 744.3 & 19.63 & 738.6 & 19.6 & 743.4 \\
\hline 22.5 & 782.7 & 0.97 & 761.1 & 20.79 & 773.8 & & 765.5 & & 776.9 & & 778.7 & 64 & 769.9 & 20.37 & 765.9 & 20.46 & 773 \\
\hline 22.65 & 789.3 & 21.68 & 784.9 & 21.53 & 798.5 & 21.29 & 791.9 & 21.76 & 801.2 & & 801.6 & 65 & 796.8 & 21.26 & 790.6 & 21.11 & 797.6 \\
\hline 24.34 & 835.1 & 23.01 & 825.8 & 22.36 & 830.7 & 22.06 & 818.3 & 22.24 & 821.9 & & 825.8 & 22.24 & 820.1 & 22.53 & 828.9 & 22 & 823.6 \\
\hline 24.99 & 858 & 23.78 & 852.7 & 23.21 & 854 & 22.8 & 843.9 & 23.42 & 860.2 & 23.07 & 848.3 & 22.98 & 844.8 & 23.16 & 856.7 & 22.86 & 851 \\
\hline 25.88 & 883.1 & 24.67 & 876.1 & 23.9 & 879.6 & 23.72 & 870.3 & 24.22 & 884 & 23.84 & 873 & 23.75 & 873.9 & 23.95 & 884.9 & 23.39 & 874.8 \\
\hline 26.56 & 909.1 & 25.55 & 908.2 & 24.61 & 905.2 & 24.96 & 912.2 & 25.05 & 914.4 & 24.49 & 898.6 & 25.11 & 913.1 & 24.84 & 909.1 & 24.16 & 896.8 \\
\hline 27.42 & 940 & 26.14 & 932.5 & 25.49 & 929.8 & 25.05 & 921.9 & 25.73 & 937.3 & 25.88 & 937.8 & 25.73 & 936.9 & 25.76 & 937.3 & 24.93 & 924.6 \\
\hline 28.25 & 965.1 & 27.03 & 962.5 & 26.56 & 967.7 & 26 & 947 & 26.35 & 960.7 & 26.74 & 965.5 & 26.5 & 965.1 & 26.62 & 969.1 & 26.23 & 966 \\
\hline 29.07 & 985.8 & 27.74 & 986.2 & 27.39 & 992.9 & 27.48 & 989.3 & 27.68 & 999 & 27.51 & 992.4 & 27.21 & 985.8 & 27.21 & 989.8 & 27.03 & 990.7 \\
\hline 29.7 & 1011 & 28.66 & 1011 & 28.31 & 1025 & 27.92 & 1010 & 28.42 & 1023 & 28.42 & 1024 & 27.92 & 1013 & 28.22 & 1022 & 27.83 & 1018 \\
\hline 31 & 1047 & 29.46 & 1043 & 29.1 & 1051 & 29.1 & 1046 & 29.28 & 1054 & 29.22 & 1046 & 28.75 & 1039 & 28.72 & 1045 & 28.69 & 1045 \\
\hline 31.77 & 1075 & 30.23 & 1066 & 30.02 & 1079 & 29.84 & 1073 & 29.99 & 1077 & 29.93 & 1071 & 30.05 & 1078 & 29.49 & 1067 & 29.43 & 1070 \\
\hline 32.54 & 1099 & 31.15 & 1096 & 30.64 & 1098 & 30.61 & 1097 & 30.61 & 1100 & 30.52 & 1094 & 30.76 & 1104 & 30.23 & 1089 & 30.2 & 1101 \\
\hline
\end{tabular}


Table 2. $25^{\circ} \mathrm{C}$ As Received - continued

\begin{tabular}{|c|c|c|c|c|c|c|c|c|c|c|c|c|c|c|c|c|c|}
\hline run1 & & run2 & & run3 & & run4 & & run5 & & run6 & & run7 & & run8 & & run9 & \\
\hline [Pa] & [1/s] & [Pa] & {$[1 / s]$} & [Pa] & [1/s] & [Pa] & [1/s] & [Pa] & [1/s] & [Pa] & {$[1 / s]$} & [Pa] & [1/s] & [Pa] & [1/s] & [Pa] & {$[1 / s]$} \\
\hline 33.31 & 1122 & 31.86 & 1118 & 31.62 & 1129 & 31.35 & 1121 & 31.38 & 1124 & 31.89 & 1133 & 31.56 & 1126 & 31.03 & 1120 & 30.94 & 1125 \\
\hline 34.55 & 1160 & 33.04 & 1154 & 32.36 & 1155 & 32.06 & 1147 & 32.39 & 1157 & 32.69 & 1158 & 32.48 & 1160 & 31.92 & 1145 & 32.12 & 1160 \\
\hline 35.41 & 1185 & 33.72 & 1177 & 33.1 & 1178 & 32.92 & 1174 & 33.19 & 1182 & 33.34 & 1183 & 33.31 & 1185 & 33.25 & 1185 & 32.95 & 1184 \\
\hline 36.35 & 1215 & 34.67 & 1210 & 33.81 & 1204 & 33.69 & 1199 & 34.11 & 1213 & 34.16 & 1213 & 34.08 & 1212 & 33.96 & 1208 & 33.75 & 1213 \\
\hline 37.09 & 1239 & 35.29 & 1228 & 34.99 & 1241 & 34.49 & 1226 & 34.82 & 1236 & 34.99 & 1239 & 34.88 & 1240 & 34.82 & 1241 & 34.46 & 1237 \\
\hline 37.86 & 1263 & 36.27 & 1256 & 35.79 & 1265 & 35.29 & 1253 & 35.47 & 1259 & 35.79 & 1264 & 35.73 & 1269 & 35.56 & 1266 & 35.29 & 1268 \\
\hline 38.72 & 1288 & 37.07 & 1284 & 36.62 & 1296 & 36.21 & 1280 & 36.62 & 1295 & 36.41 & 1289 & 36.5 & 1294 & 36.18 & 1289 & 35.97 & 1288 \\
\hline 39.58 & 1311 & 37.98 & 1315 & 37.42 & 1321 & 37.42 & 1319 & 37.45 & 1324 & 37.33 & 1315 & 37.24 & 1319 & 36.92 & 1312 & 36.86 & 1320 \\
\hline 40.85 & 1350 & 38.72 & 1341 & 38.01 & 1342 & 38.25 & 1345 & 38.16 & 1348 & 38.04 & 1339 & 37.95 & 1343 & 38.16 & 1350 & 37.42 & 1342 \\
\hline 41.56 & 1373 & 39.49 & 1364 & 38.78 & 1367 & 38.93 & 1367 & 38.93 & 1373 & 38.84 & 1365 & 39.08 & 1377 & 39.08 & 1380 & 38.19 & 1364 \\
\hline 42.48 & 1402 & 40.29 & 1389 & 40.08 & 1407 & 39.7 & 1397 & 39.64 & 1397 & 39.61 & 1392 & 39.94 & 1408 & 39.76 & 1401 & 39.43 & 1404 \\
\hline 43.19 & 1427 & 41.56 & 1427 & 40.97 & 1433 & 40.47 & 1419 & 40.5 & 1424 & 41 & 1432 & 40.73 & 1434 & 40.53 & 1427 & 40.11 & 1427 \\
\hline 44.08 & 1452 & 42.21 & 1448 & 41.74 & 1462 & 41.27 & 1443 & 41.3 & 1451 & 41.77 & 1460 & 41.59 & 1458 & 41.39 & 1453 & 41 & 1458 \\
\hline 44.88 & 1478 & 43.01 & 1476 & 42.36 & 1484 & 42.48 & 1482 & 42.21 & 1478 & 42.48 & 1482 & 42.13 & 1482 & 42.13 & 1482 & 41.71 & 1483 \\
\hline 45.65 & 1502 & 43.99 & 1508 & 43.1 & 1507 & 43.34 & 1508 & 43.13 & 1506 & 43.34 & 1512 & 42.92 & 1504 & 43.01 & 1512 & 42.48 & 1510 \\
\hline 43.1 & 1511 & 43.22 & 1530 & 42.07 & 1510 & 41.42 & 1490 & 41.5 & 1497 & 41.53 & 1497 & 41.95 & 1517 & 41.53 & 1508 & 42.24 & 1534 \\
\hline 42.24 & 1485 & 42.18 & 1504 & 41.3 & 1485 & 40.65 & 1465 & 40.82 & 1472 & 40.56 & 1463 & 41.68 & 1504 & .79 & 1480 & 40.32 & 1482 \\
\hline 41.62 & 1463 & 41.65 & 1481 & 40.44 & 1458 & 39.91 & 1440 & 39.79 & 1440 & 39.82 & 1439 & 40.08 & 1460 & 40.05 & 1460 & 39.97 & 1466 \\
\hline 40.82 & 1439 & 40.05 & 1443 & 39.64 & 1434 & 38.84 & 1408 & 39.2 & 1417 & 39.23 & 1418 & 39.76 & 1447 & .11 & 1427 & 39.2 & 1441 \\
\hline 40.08 & 1412 & 39.02 & 1412 & 38.9 & 1406 & 38.13 & 1381 & 38.46 & 1393 & 37.89 & 1381 & 37.6 & 1376 & 38.31 & 1401 & 38.22 & 1405 \\
\hline 39.17 & 1386 & 38.31 & 1389 & 37.98 & 1380 & 37.3 & 1355 & 37.72 & 1370 & 37.09 & 1354 & 37.39 & 1363 & 37.72 & 1382 & 37.51 & 1382 \\
\hline 38.43 & 1360 & 37.54 & 1366 & 37.27 & 1356 & 36.47 & 1332 & 36.98 & 1346 & 36.35 & 1327 & 36.5 & 1341 & 36.89 & 1352 & 36.53 & 1351 \\
\hline 37.66 & 1337 & 36.86 & 1344 & 36.53 & 1329 & 35.79 & 1308 & 35.44 & 1303 & 35.7 & 1302 & 36 & 1320 & 36.06 & 1326 & 35.82 & 1326 \\
\hline 36.21 & 1295 & 35.53 & 1303 & 35.56 & 1300 & 35.02 & 1282 & 35.23 & 1291 & 34.96 & 1278 & 34.67 & 1289 & 35.29 & 1301 & 34.93 & 1298 \\
\hline 35.38 & 1270 & 34.76 & 1279 & 34.64 & 1273 & 34.22 & 1254 & 34.37 & 1260 & 34.22 & 1255 & 34.49 & 1274 & 34.58 & 1278 & 34.19 & 1271 \\
\hline 34.58 & 1242 & 34.02 & 1249 & 34.08 & 1247 & 33.43 & 1230 & 32.92 & 1225 & 33.34 & 1230 & 33.34 & 1237 & 33.25 & 1237 & 33.4 & 1246 \\
\hline 33.75 & 1213 & 33.22 & 1225 & 33.13 & 1219 & 32.03 & 1188 & 32.57 & 1210 & 32.66 & 1203 & 33.13 & 1218 & 32.45 & 1212 & 32.51 & 1219 \\
\hline 32.98 & 1189 & 32.45 & 1201 & 31.5 & 1176 & 31.32 & 1165 & 31.8 & 1181 & 31.77 & 1177 & 31.62 & 1188 & 31.68 & 1189 & 31.74 & 1194 \\
\hline 32.18 & 1156 & 31.77 & 1178 & 31.15 & 1158 & 30.5 & 1134 & 31.06 & 1154 & 30.47 & 1136 & 31.59 & 1177 & 30.97 & 1160 & 30.5 & 1155 \\
\hline 30.64 & 1128 & 31.12 & 1155 & 30.38 & 1134 & 29.81 & 1111 & 30.67 & 1135 & 29.76 & 1112 & 30.58 & 1143 & 29.99 & 1128 & 29.78 & 1128 \\
\hline 30.38 & 1112 & 29.73 & 1114 & 29.81 & 1104 & 28.99 & 1083 & 29.4 & 1111 & 28.99 & 1084 & 29.78 & 1115 & 29.13 & 1104 & 29.02 & 1108 \\
\hline
\end{tabular}


Table 2. $25^{\circ} \mathrm{C}$ As Received - continued

\begin{tabular}{|c|c|c|c|c|c|c|c|c|c|c|c|c|c|c|c|c|c|}
\hline run1 & & run2 & & run3 & & run4 & & run5 & & run6 & & run7 & & run8 & & run9 & \\
\hline$[\mathrm{Pa}]$ & {$[1 / s]$} & {$[\mathrm{Pa}]$} & {$[1 / \mathrm{s}]$} & {$[\mathrm{Pa}]$} & {$[1 / \mathrm{s}]$} & {$[\mathrm{Pa}]$} & {$[1 / \mathrm{s}]$} & {$[\mathrm{Pa}]$} & {$[1 / \mathrm{s}]$} & {$[\mathrm{Pa}]$} & {$[1 / \mathrm{s}]$} & {$[\mathrm{Pa}]$} & {$[1 / \mathrm{s}]$} & {$[\mathrm{Pa}]$} & {$[1 / \mathrm{s}]$} & {$[\mathrm{Pa}]$} & {$[1 / \mathrm{s}]$} \\
\hline 29.4 & 1074 & 28.84 & 1088 & 29.76 & 1103 & 28.25 & 1060 & 28.28 & 1066 & 28.16 & 1059 & 29.02 & 1090 & 28.36 & 1074 & 28.25 & 1077 \\
\hline 28.96 & 1054 & 28.28 & 1063 & 28.33 & 1074 & 27.21 & 1025 & 27.89 & 1043 & 27.36 & 1033 & 28.19 & 1067 & 27.65 & 1051 & 27.54 & 1048 \\
\hline 27.77 & 1035 & 27.45 & 1035 & 28.04 & 1059 & 26.38 & 999.5 & 26.71 & 1023 & 26.41 & 999.9 & 26.86 & 1026 & 26.94 & 1026 & 26.68 & 1022 \\
\hline 27.45 & 1019 & 26.83 & 1014 & 26.32 & 1007 & 25.73 & 974.3 & 25.82 & 982.7 & 25.76 & 974.8 & 26.14 & 999.5 & 26.23 & 1003 & 25.85 & 996.8 \\
\hline 26 & 957.6 & 26.06 & 987.6 & 25.88 & 992.4 & 24.87 & 951.4 & 24.93 & 953.2 & 24.84 & 949.7 & 25.32 & 971.3 & 25.43 & 977.9 & 25.05 & 970.4 \\
\hline 25.94 & 956.7 & 24.93 & 951.9 & 24.19 & 923.2 & 24.4 & 928.1 & 24.07 & 920.6 & 24.13 & 925.9 & 24.69 & 946.6 & 24.34 & 939.5 & 24.43 & 946.1 \\
\hline 24.58 & 928.1 & 24.13 & 926.3 & 23.87 & 910.9 & 23.07 & 891.5 & 23.19 & 893.7 & 23.51 & 900.8 & 23.81 & 921 & 23.3 & 907.4 & 23.63 & 919.3 \\
\hline 24.22 & 905.2 & 23.33 & 895.9 & 22.62 & 887.1 & 22.39 & 863.7 & 22.65 & 869 & 22.68 & 875.6 & 22.98 & 891.5 & 22.53 & 884.5 & 22.77 & 893.7 \\
\hline 22.48 & 861.1 & 22.42 & 868.6 & 22 & 866.4 & 21.47 & 834.2 & 21.76 & 845.2 & 21.79 & 846.1 & 22.39 & 865.1 & 21.71 & 853.2 & 22.21 & 869 \\
\hline 22.15 & 843.5 & 21.82 & 843.9 & 21.17 & 835.1 & 20.82 & 809.5 & 21.17 & 821.9 & 20.49 & 806.4 & 21.56 & 839.5 & 21.17 & 831.1 & 21.32 & 842.1 \\
\hline 21.26 & 817.5 & 20.88 & 818.3 & 21.05 & 818.8 & 20.02 & 786.2 & 19.96 & 782.2 & 19.93 & 782.7 & 20.94 & 816.1 & 20.34 & 803.4 & 20.67 & 815.7 \\
\hline 20.37 & 777.8 & 20.29 & 793.7 & 20.11 & 788.4 & 19.43 & 761.1 & 19.25 & 759.7 & 19.34 & 759.3 & 19.99 & 791.9 & 19.69 & 776 & 19.25 & 776 \\
\hline 19.63 & 750.9 & 19.46 & 766.8 & 19.28 & 759.3 & 18.45 & 729.8 & 18.48 & 732.4 & 18.36 & 726.7 & 19.46 & 765 & 18.84 & 750 & 19.16 & 765 \\
\hline 19.04 & 725.4 & 18.72 & 744.3 & 18.66 & 733.7 & 17.8 & 706 & 17.8 & 705.1 & 17.74 & 704.2 & 18.24 & 723.2 & 18.12 & 725.4 & 17.8 & 720.5 \\
\hline 18.27 & 701.1 & 17.41 & 703.8 & 17.3 & 691 & 17.15 & 679.1 & 16.88 & 681.3 & 17.15 & 680.4 & 17.5 & 700.7 & 17.5 & 701.6 & 17.06 & 696.7 \\
\hline 17.33 & 675.6 & 16.79 & 682.6 & 16.59 & 666.8 & 16.2 & 652.7 & 16.08 & 653.5 & 16.35 & 654.4 & 16.62 & 674.7 & 16.91 & 677.3 & 16.32 & 666.8 \\
\hline 16.59 & 651.8 & 16.23 & 650 & 15.76 & 643 & 15.37 & 626.2 & 15.46 & 626.2 & 15.46 & 630.2 & 15.88 & 651.3 & 16.02 & 649.1 & 15.7 & 645.2 \\
\hline 15.46 & 610.8 & 15.55 & 626.7 & 14.96 & 612.5 & 14.63 & 599.8 & 14.66 & 593.6 & 14.48 & 587.4 & 15.28 & 616.5 & 14.87 & 609 & 14.9 & 614.8 \\
\hline 14.69 & 585.7 & 14.22 & 588.3 & 14.34 & 587.4 & 13.6 & 560.1 & 14.1 & 571.1 & 13.8 & 566.3 & 14.51 & 589.6 & 14.16 & 582.1 & 14.1 & 590.1 \\
\hline 13.95 & 559.2 & 13.54 & 566.7 & 13.69 & 562.3 & 12.92 & 535.4 & 13.45 & 549.5 & 13.09 & 541.2 & 13.77 & 564.5 & 13.36 & 557 & 13.12 & 556.1 \\
\hline 13.06 & 531 & 12.86 & 539.8 & 12.83 & 539.4 & 12.36 & 509.4 & 12.11 & 509 & 12.33 & 507.7 & 13.03 & 539.4 & 12.5 & 525.3 & 12.47 & 529.7 \\
\hline 12.4 & 489.6 & 12.06 & 513 & 12.26 & 514.7 & 11.62 & 483 & 11.82 & 488.3 & 11.73 & 486.1 & 12.52 & 514.7 & 11.99 & 500.6 & 11.7 & 503.7 \\
\hline 11.73 & 479 & 11.44 & 488.7 & 11.29 & 472.4 & 10.87 & 456.5 & 11.04 & 457 & 10.2 & 438.9 & 11.84 & 490.5 & 11.43 & 476.4 & 11.37 & 481.7 \\
\hline 10.96 & 455.2 & 10.55 & 461.4 & 11.03 & 464.5 & 9.96 & 427.5 & 10.31 & 434.1 & 10.18 & 427 & 11 & 464.5 & 10.65 & 452.1 & 10.79 & 454.8 \\
\hline 10.39 & 423.5 & 10.12 & 434.5 & 9.519 & 421.3 & 9.288 & 404.1 & 9.462 & 406.3 & 10.08 & 421.3 & 10.13 & 440.2 & 9.794 & 426.6 & 9.933 & 423.5 \\
\hline 9.61 & 394 & 9.539 & 403.7 & 8.974 & 392.6 & 8.959 & 381.2 & 9.096 & 386.9 & 8.362 & 371.5 & 9.359 & 408.5 & 9.329 & 403.2 & 9.172 & 394 \\
\hline 8.581 & 370.2 & 8.652 & 381.6 & 8.658 & 369.3 & 7.906 & 344.2 & 7.971 & 345.9 & 8.196 & 362.7 & 8.527 & 375.5 & 8.178 & 363.1 & 8.329 & 375.9 \\
\hline 8.092 & 337.6 & 7.784 & 350.8 & 7.61 & 345.9 & 6.864 & 313.3 & 7.264 & 323.5 & 6.888 & 316 & 8.086 & 348.6 & 7.693 & 339.3 & 7.631 & 341.1 \\
\hline 7.462 & 314.6 & 7.645 & 327.9 & 7.379 & 320.4 & 6.624 & 290.4 & 6.417 & 292.2 & 6.408 & 286.4 & 7.012 & 321.7 & 6.63 & 307.6 & 7.021 & 316 \\
\hline 6.846 & 290.9 & 6.624 & 299.2 & 6.438 & 295.3 & 5.538 & 264 & 6.142 & 268.8 & 6.086 & 279 & 6.846 & 297.5 & 6.518 & 283.8 & 6.536 & 293.9 \\
\hline 6.154 & 267.5 & 6.059 & 272.3 & 5.396 & 256.5 & 5.523 & 241.1 & 5.58 & 246.3 & 5.819 & 251.6 & 5.819 & 274.5 & 5.438 & 260.9 & 5.885 & 267.5 \\
\hline
\end{tabular}


Table 3. $50{ }^{\circ} \mathrm{C}$ As Received

\begin{tabular}{|c|c|c|c|c|c|c|c|c|c|c|c|c|c|c|c|c|c|}
\hline run1 & & run2 & & run3 & & run4 & & run5 & & run6 & & run7 & & run8 & & run9 & \\
\hline [Pa] & {$[1 / \mathrm{s}]$} & {$[\mathrm{Pa}]$} & {$[1 / \mathbf{s}]$} & {$[\mathrm{Pa}]$} & {$[1 / \mathrm{s}]$} & [Pa] & {$[1 / s]$} & {$[\mathrm{Pa}]$} & {$[1 / s]$} & {$[\mathrm{Pa}]$} & {$[1 / \mathrm{s}]$} & {$[\mathrm{Pa}]$} & {$[1 / \mathrm{s}]$} & {$[\mathrm{Pa}]$} & {$[1 / \mathrm{s}]$} & {$[\mathrm{Pa}]$} & {$[1 / \mathrm{s}]$} \\
\hline 3.982 & 316.4 & 3.615 & 306.7 & 4.145 & 315.1 & 3.846 & 305 & 3.506 & 305.4 & 3.799 & 312.9 & 3.636 & 310.7 & 3.805 & 308 & 3.355 & 336.2 \\
\hline 4.367 & 317.3 & 4.399 & 321.7 & 4.311 & 325.2 & 4.402 & 322.6 & 3.932 & 313.3 & 3.953 & 321.7 & 3.802 & 314.2 & 4.219 & 321.3 & 4.13 & 330.1 \\
\hline 4.832 & 338.9 & 4.909 & 343.3 & 4.849 & 346.4 & 4.781 & 343.3 & 4.447 & 330.1 & 4.441 & 341.5 & 4.343 & 330.5 & 4.293 & 347.7 & 4.293 & 347.3 \\
\hline 4.814 & 360.5 & 4.98 & 365.8 & 4.882 & 369.7 & 4.746 & 365.8 & 4.521 & 357 & 4.722 & 362.2 & 4.456 & 355.2 & 4.728 & 368.4 & 4.624 & 368.4 \\
\hline 5.16 & 382.5 & 5.13 & 392.6 & 5.157 & 398.8 & 5.172 & 389.1 & 4.84 & 383.4 & 4.959 & 393.1 & 4.66 & 378.5 & 5.009 & 400.1 & 4.959 & 391.3 \\
\hline 5.557 & 422.6 & 5.583 & 416.9 & 5.45 & 423.9 & 5.503 & 427.9 & 5.273 & 423.1 & 5.11 & 417.3 & 5.042 & 405 & 5.296 & 427 & 5.332 & 429.7 \\
\hline 5.983 & 446 & 5.873 & 449.1 & 5.746 & 452.1 & 5.9 & 453 & 5.592 & 449.1 & 5.426 & 443.3 & 5.358 & 431 & 5.332 & 450.8 & 5.716 & 454.3 \\
\hline 6.32 & 472.9 & 6.122 & 475.9 & 6.006 & 478.6 & 6.231 & 480.3 & 5.968 & 472 & 5.826 & 467.1 & 5.725 & 471.5 & 5.586 & 478.6 & 5.912 & 480.8 \\
\hline 6.649 & 496.6 & 6.394 & 501.1 & 6.379 & 505 & 6.459 & 504.6 & 6.104 & 504.1 & 6.004 & 494 & 6.083 & 494.9 & 5.808 & 501.9 & 6.092 & 503.7 \\
\hline 6.874 & 527.1 & 6.737 & 524.4 & 6.681 & 527.1 & 6.755 & 531.5 & 6.397 & 529.3 & 6.338 & 517.8 & 6.19 & 526.2 & 6.539 & 541.2 & 6.462 & 537.6 \\
\hline 7.22 & 550 & 6.924 & 546.9 & 7.001 & 566.3 & 7.025 & 558.3 & 6.72 & 550.9 & 6.584 & 549.5 & 6.43 & 549.5 & 6.755 & 568 & 6.681 & 560.1 \\
\hline 7.386 & 576 & 7.306 & 574.7 & 7.326 & 589.6 & 7.264 & 589.2 & 7.063 & 586.5 & 6.806 & 577.7 & 6.634 & 576.4 & 7.084 & 589.6 & 6.915 & 586.5 \\
\hline 7.608 & 600.7 & 7.779 & 614.3 & 7.649 & 620.5 & 7.534 & 612.1 & 7.35 & 609.9 & 7.554 & 614.3 & 6.989 & 598.4 & 7.149 & 620.5 & 7.187 & 611.2 \\
\hline 8.35 & 644.3 & 8.205 & 641.2 & 8.051 & 644.7 & 8.007 & 646 & 7.767 & 637.2 & 7.844 & 638.1 & 7.658 & 638.1 & 7.418 & 642.1 & 7.394 & 635.9 \\
\hline 8.253 & 651.3 & 8.38 & 672.5 & 8.191 & 670.3 & 8.247 & 669.8 & 7.966 & 666.3 & 7.98 & 669.4 & 7.847 & 660.1 & 7.676 & 666.3 & 7.8 & 664.1 \\
\hline 9.061 & 695 & 8.741 & 696.7 & 8.596 & 695.4 & 8.439 & 694.1 & 8.347 & 694.1 & 8.495 & 691.4 & 8.078 & 685.3 & 8.131 & 694.1 & 8.223 & 687.5 \\
\hline 9.454 & 717 & 9.055 & 717.4 & 9.01 & 720.5 & 8.779 & 716.6 & 8.768 & 717.9 & 8.865 & 717.4 & 8.688 & 711.3 & 8.412 & 721.4 & 8.327 & 715.2 \\
\hline 9.584 & 749.2 & 9.513 & 751.4 & 9.65 & 758.4 & 9.223 & 744.3 & 9.046 & 740.3 & 9.01 & 747.8 & 8.865 & 739.5 & 8.898 & 749.6 & 9.049 & 752.7 \\
\hline 10.05 & 774.3 & 9.957 & 775.2 & 9.809 & 782.2 & 9.579 & 770.3 & 9.212 & 763.7 & 9.389 & 770.3 & 9.288 & 771.2 & 9.146 & 772.1 & 9.283 & 778.2 \\
\hline 10.29 & 798.1 & 10.13 & 804.7 & 10.21 & 805.6 & 10.16 & 809.1 & 9.937 & 803.8 & 9.614 & 794.6 & 9.528 & 790.1 & 9.496 & 799.4 & 9.623 & 810 \\
\hline 10.75 & 822.8 & 10.5 & 831.6 & 10.49 & 832.4 & 10.54 & 834.2 & 10.24 & 832.9 & 10 & 819.7 & 9.969 & 818.8 & 10.18 & 839.5 & 10.06 & 835.5 \\
\hline 10.98 & 847.4 & 10.95 & 858.9 & 10.95 & 860.7 & 10.85 & 864.6 & 10.59 & 863.7 & 10.23 & 844.3 & 10.37 & 851 & 10.38 & 865.5 & 10.32 & 861.1 \\
\hline 11.47 & 872.1 & 11.16 & 881.8 & 11. & 884.9 & 11.23 & 890.6 & 10.99 & 885.8 & 10.71 & 872.6 & 10.63 & 873 & 10.73 & 893.3 & 10.72 & 885.8 \\
\hline 12.01 & 909.6 & 11.55 & 905.2 & 11.52 & 909.1 & 11.53 & 910.9 & 11.35 & 915.7 & 11.42 & 914.4 & 10.96 & 900.3 & 11.11 & 917.1 & 10.98 & 906.5 \\
\hline 12.44 & 934.2 & 12.22 & 944.8 & 11.87 & 940.9 & 12.02 & 937.3 & 11.58 & 942.2 & 11.69 & 943.5 & 11.35 & 926.8 & 11.3 & 938.2 & 11.3 & 931.6 \\
\hline 12.83 & 967.3 & 12.53 & 970.4 & 12.35 & 966.9 & 12.34 & 969.1 & 12.14 & 969.9 & 12.04 & 964.7 & 11.67 & 952.3 & 11.69 & 965.1 & 11.93 & 975.2 \\
\hline 13.22 & 993.3 & 12.92 & 993.7 & 12.73 & 990.7 & 12.64 & 994.2 & 12.5 & 991.1 & 12.4 & 993.7 & 11.92 & 976.6 & 12.11 & 988.5 & 12.25 & 997.7 \\
\hline 13.54 & 1016 & 12.87 & 1006 & 12.97 & 1016 & 12.93 & 1021 & 12.8 & 1022 & 12.83 & 1018 & 12.6 & 1020 & 12.75 & 1032 & 12.62 & 1022 \\
\hline 13.94 & 1043 & 13.69 & 1048 & 13.76 & 1060 & 13.26 & 1043 & 13.12 & 1046 & 13.05 & 1048 & 13.02 & 1047 & 13.13 & 1058 & 12.96 & 1048 \\
\hline 14.24 & 1067 & 13.99 & 1075 & 13.99 & 1083 & 13.9 & 1082 & 13.4 & 1069 & 13.51 & 1070 & 13.45 & 1077 & 13.48 & 1082 & 13.33 & 1081 \\
\hline 14.94 & 1103 & 14.4 & 1107 & 14.27 & 1107 & 14.3 & 1109 & 13.78 & 1095 & 13.75 & 1099 & 13.36 & 1085 & 13.73 & 1107 & 13.67 & 1104 \\
\hline
\end{tabular}


Table 3. $50^{\circ} \mathrm{C}$ As Received - continued

\begin{tabular}{|c|c|c|c|c|c|c|c|c|c|c|c|c|c|c|c|c|c|}
\hline run1 & & run2 & & run3 & & run4 & & run5 & & run6 & & run7 & & run8 & & run9 & \\
\hline [Pa] & {$[1 / \mathbf{s}]$} & {$[\mathrm{Pa}]$} & {$[1 / \mathbf{s}]$} & [Pa] & {$[1 / \mathrm{s}]$} & {$[\mathrm{Pa}]$} & {$[1 / \mathrm{s}]$} & {$[\mathrm{Pa}]$} & {$[1 / \mathrm{s}]$} & {$[\mathrm{Pa}]$} & {$[1 / \mathbf{s}]$} & {$[\mathrm{Pa}]$} & {$[1 / \mathrm{s}]$} & {$[\mathrm{Pa}]$} & {$[1 / \mathrm{s}]$} & {$[\mathrm{Pa}]$} & {$[1 / \mathbf{s}]$} \\
\hline 15.35 & 1129 & 14.78 & 1131 & 14.65 & 1139 & 14.54 & 1133 & 14.09 & 1120 & 14.17 & 1120 & 13.75 & 1111 & 14.03 & 1136 & 13.94 & 1127 \\
\hline 15.72 & 1159 & 15.12 & 1158 & 15.05 & 1162 & 14.99 & 1165 & 14.79 & 1159 & 14.75 & 1161 & 14.5 & 1155 & 14.34 & 1159 & 14.24 & 1152 \\
\hline 16.1 & 1182 & 15.47 & 1185 & 15.39 & 1186 & 15.31 & 1189 & 15.04 & 1182 & 14.76 & 1170 & 14.86 & 1178 & 14.71 & 1181 & 14.93 & 1192 \\
\hline 16.33 & 1207 & 15.81 & 1211 & 15.63 & 1212 & 15.54 & 1212 & 15.35 & 1211 & 15.4 & 1213 & 15.12 & 1204 & 15.24 & 1221 & 15.21 & 1215 \\
\hline 16.68 & 1232 & 16.22 & 1236 & 16.07 & 1237 & 15.85 & 1236 & 15.77 & 1237 & 15.7 & 1236 & 15.44 & 1237 & 15.7 & 1248 & 15.63 & 1244 \\
\hline 17.03 & 1257 & 16.49 & 1259 & 16.39 & 1266 & 16.47 & 1273 & 16.23 & 1267 & 16.09 & 1267 & 15.91 & 1256 & 15.96 & 1276 & 15.94 & 1267 \\
\hline 17.68 & 1296 & 17.22 & 1304 & 17.01 & 1303 & 16.84 & 1301 & 16.53 & 1297 & 16.44 & 1291 & 16.13 & 1287 & 16.34 & 1298 & 16.21 & 1299 \\
\hline 17.94 & 1323 & 17.22 & 1310 & 17.36 & 1331 & 17.39 & 1328 & 16.82 & 1322 & 16.71 & 1317 & 16.44 & 1311 & 16.67 & 1330 & 16.56 & 1323 \\
\hline 18.32 & 1346 & 17.88 & 1352 & 17.79 & 1357 & 17.65 & 1351 & 17.19 & 1346 & 17.02 & 1342 & 16.73 & 1333 & 17 & 1353 & 16.88 & 1348 \\
\hline 18.56 & 1376 & 18.2 & 1377 & 17.76 & 1366 & 17.82 & 1377 & 17.46 & 1367 & 17.36 & 1368 & 17.32 & 1369 & 17.27 & 1374 & 17.26 & 1371 \\
\hline 18.97 & 1399 & 18.5 & 1403 & 18.44 & 1409 & 18.2 & 1404 & 17.7 & 1391 & 17.7 & 1394 & 17.58 & 1401 & 17.7 & 1411 & 17.4 & 1397 \\
\hline 19.18 & 1424 & 18.85 & 1426 & 18.85 & 1434 & 18.47 & 1434 & 18.35 & 1433 & 18 & 1424 & 17.91 & 1426 & 17.97 & 1435 & 18.05 & 1435 \\
\hline 19.5 & 1448 & 19.27 & 1456 & 19.06 & 1459 & 18.79 & 1456 & 18.59 & 1455 & 18.41 & 1451 & 18.2 & 1448 & 18.47 & 1461 & 18.47 & 1464 \\
\hline 19.95 & 1474 & 19.5 & 1480 & 19.42 & 1492 & 19.21 & 1482 & 19.03 & 1482 & 18.71 & 1475 & 18.62 & 1479 & 18.73 & 1491 & 18.85 & 1493 \\
\hline 20.63 & 1514 & 20.13 & 1518 & 19.89 & 1516 & 19.5 & 1504 & 19.36 & 1510 & 19.09 & 1501 & 18.97 & 1503 & 19.03 & 1519 & 19.18 & 1521 \\
\hline 19.53 & 1549 & 19 & 1520 & 18.85 & 1528 & 18.94 & 1552 & 18.62 & 1542 & 18.38 & 1524 & 18.2 & 1523 & 18.02 & 1521 & 17.28 & 1475 \\
\hline 19.03 & 1523 & 18.65 & 1493 & 18.5 & 1502 & 18.5 & 1526 & 18.32 & 1519 & 18 & 1496 & 17.49 & 1482 & 17.76 & 1492 & 16.63 & 1422 \\
\hline 18.76 & 1498 & 18.23 & 1468 & 17.88 & 1463 & 18.17 & 1498 & 17.97 & 1489 & 17.64 & 1472 & 17.52 & 1473 & 17.31 & 1467 & 16.69 & 1420 \\
\hline 18.08 & 1458 & 17.91 & 1438 & 17.43 & 1439 & 17.58 & 1457 & 17.67 & 1469 & 16.96 & 1432 & 17.2 & 1434 & 16.96 & 1443 & 15.86 & 1399 \\
\hline 17.79 & 1437 & 17.37 & 1412 & 17.05 & 1408 & 17.05 & 1430 & 17.31 & 1447 & 16.69 & 1408 & 17.14 & 1433 & 16.22 & 1400 & 15.89 & 1383 \\
\hline 17.49 & 1405 & 17.08 & 1388 & 16.66 & 1381 & 16.75 & 1405 & 17.02 & 1420 & 16.34 & 1383 & 16.19 & 1402 & 15.98 & 1372 & 15.57 & 1357 \\
\hline 17.14 & 1386 & 16.72 & 1361 & 16.25 & 1355 & 16.43 & 1382 & 16.63 & 1391 & 15.95 & 1352 & 15.92 & 1345 & 15.63 & 1351 & 15.54 & 1350 \\
\hline 16.81 & 1360 & & 1340 & 15.92 & 1331 & 16.07 & 1352 & 15.95 & 1351 & 15.63 & 1331 & 15.95 & 1344 & 15.21 & 1323 & 15.24 & 1331 \\
\hline 16.52 & 1335 & 15.63 & 1299 & 15.51 & 1304 & 15.72 & 1325 & 15.66 & 1327 & 15.15 & 1295 & 15.01 & 1319 & 14.86 & 1294 & 14.86 & 1304 \\
\hline 15.78 & 1294 & 15.27 & 1275 & 15.18 & 1274 & 15.3 & 1299 & 15.24 & 1300 & 14.74 & 1273 & 14.98 & 1305 & 14.53 & 1271 & 14.53 & 1273 \\
\hline 15.39 & 1268 & 15.01 & 1244 & 14.89 & 1250 & 14.98 & 1273 & 14.98 & 1274 & 14.53 & 1241 & 14.24 & 1255 & 14.27 & 1248 & 14.27 & 1249 \\
\hline 15.04 & 1242 & 14.62 & 1218 & 14.53 & 1229 & 14.59 & 1246 & 14.53 & 1244 & 14.12 & 1219 & 14.09 & 1236 & 13.97 & 1225 & 13.94 & 1222 \\
\hline 14.86 & 1219 & 14.33 & 1196 & 14.03 & 1190 & 14.27 & 1221 & 13.76 & 1193 & 13.76 & 1195 & 13.76 & 1205 & 13.35 & 1186 & 13.53 & 1196 \\
\hline 14.44 & 1194 & 14.12 & 1173 & 13.62 & 1165 & 13.91 & 1196 & 13.7 & 1182 & 13.5 & 1169 & 12.93 & 1153 & 13.11 & 1161 & 13.2 & 1173 \\
\hline 14.09 & 1167 & 13.67 & 1147 & 13.32 & 1133 & 13.59 & 1170 & 13.7 & 1174 & 13.2 & 1146 & 13.03 & 1141 & 12.73 & 1138 & 12.69 & 1133 \\
\hline 13.7 & 1144 & 13.08 & 1111 & 12.96 & 1095 & 13.29 & 1144 & 12.7 & 1122 & 12.69 & 1103 & 12.61 & 1114 & 12.45 & 1106 & 12.32 & 1111 \\
\hline
\end{tabular}


Table 3. $50{ }^{\circ} \mathrm{C}$ As Received - continued

\begin{tabular}{|c|c|c|c|c|c|c|c|c|c|c|c|c|c|c|c|c|c|}
\hline run1 & & run2 & & run3 & & run4 & & run5 & & run6 & & run7 & & run8 & & run9 & \\
\hline$[\mathrm{Pa}]$ & {$[1 / s]$} & {$[\mathrm{Pa}]$} & {$[1 / s]$} & {$[\mathrm{Pa}]$} & {$[1 / \mathrm{s}]$} & {$[\mathrm{Pa}]$} & {$[1 / \mathrm{s}]$} & {$[\mathrm{Pa}]$} & {$[1 / s]$} & {$[\mathrm{Pa}]$} & {$[1 / \mathrm{s}]$} & {$[\mathrm{Pa}]$} & {$[1 / \mathrm{s}]$} & {$[\mathrm{Pa}]$} & {$[1 / s]$} & {$[\mathrm{Pa}]$} & {$[1 / s]$} \\
\hline 13.11 & 1109 & 12.79 & 1085 & 13 & 1094 & 12.69 & 1105 & 12.49 & 1107 & 12.37 & 1079 & 12.11 & 1073 & 12.24 & 1081 & 11.99 & 1082 \\
\hline 12.76 & 1078 & 12.47 & 1050 & 12.04 & 1067 & 12.43 & 1074 & 12.27 & 1086 & 12.06 & 1049 & 11.81 & 1046 & 11.9 & 1054 & 11.72 & 1051 \\
\hline 12.85 & 1056 & 12.1 & 1028 & 12.03 & 1029 & 12.11 & 1048 & 12.04 & 1043 & 11.72 & 1023 & 11.56 & 1021 & 11.55 & 1030 & 11.38 & 1029 \\
\hline 12.8 & 1055 & 11.8 & 1005 & 10.95 & 988 & 11.73 & 1023 & 12.02 & 1031 & 11.35 & 1000 & 11.21 & 998.1 & 11.21 & 1004 & 11.04 & 1002 \\
\hline 11.69 & 1031 & 11.39 & 980.1 & 11.04 & 975.7 & 11.33 & 998.6 & 11.06 & 1011 & 11.02 & 974.8 & 10.86 & 972.6 & 10.6 & 966 & 10.68 & 978.3 \\
\hline 11.6 & 1013 & 1.2 & 56.7 & 55 & 910.5 & 11.03 & 971.7 & 11.05 & 985.8 & 10.75 & 950.1 & 52 & 946.6 & 10.51 & 957.6 & 10.47 & 955 \\
\hline 10.71 & 7.2 & .52 & 917.5 & 10.54 & 909.1 & 10.64 & 942.2 & 0.14 & 933.4 & 10.37 & 926.8 & 9.981 & 907.4 & 854 & 913.1 & 9.795 & 913.1 \\
\hline 10.82 & 42.2 & 0.25 & 892.4 & 9.744 & 881.8 & 10.26 & 914 & 10.18 & 921.5 & 10.15 & 901.6 & 9.644 & 885.3 & 599 & 882.7 & 9.505 & 887.5 \\
\hline 10.46 & 08.7 & 9.951 & 862.9 & 9.67 & 870.8 & 9.913 & 890.6 & 9.907 & 896.3 & 9.759 & 875.2 & .404 & 854 & 351 & 858 & 9.505 & 879.6 \\
\hline 10.27 & 864.6 & 9.567 & 839.1 & 9.28 & 819.2 & 9.738 & 867.7 & 9.496 & 865.5 & 9.058 & 832 & 8.978 & 829.4 & 8.957 & 833.8 & 9.075 & 851 \\
\hline 10.18 & 863.7 & 9.327 & 813.9 & 9.398 & 818.3 & 9.268 & 843.9 & 9.22 & 839.9 & 8.839 & 802.5 & 8.732 & 807.8 & 8.661 & 807.3 & 8.507 & 806.4 \\
\hline 9.25 & 828 & 8.853 & 787.9 & 8.46 & 792.3 & 8.723 & 802.5 & 8.85 & 811.3 & 8.489 & 779.1 & 8.404 & 780 & 8.244 & 780.4 & 8.122 & 777.8 \\
\hline 9.019 & 773.4 & 8.617 & 762.8 & 8.368 & 780.9 & 8.51 & 778.7 & 8.62 & 787.9 & 8.137 & 755.3 & & 755.3 & 8.04 & 756.7 & 7.98 & 754 \\
\hline 8.898 & 770.8 & 8.22 & 735.9 & 8.137 & 741.7 & 8.099 & 753.1 & 8.019 & 754.4 & 7.738 & 725.4 & 7.812 & 727.1 & 7.738 & 730.7 & 7.741 & 732.9 \\
\hline 7.261 & 735.1 & 818 & 709.9 & 7.542 & 699.4 & 7.889 & 723.6 & 915 & 723.6 & 7.285 & 700.2 & 7.329 & 702.9 & 7.276 & 704.7 & 7.433 & 707.7 \\
\hline 7.264 & 707.7 & 508 & 685.3 & 7.3 & 675.6 & 7.504 & 697.2 & 7.634 & 700.7 & 7.004 & 675.1 & 6.965 & 664.1 & 7.027 & 677.3 & 6.909 & 670.3 \\
\hline 7.039 & 665 & 12 & 644.3 & 6.853 & 643.4 & 7.098 & 673.4 & 7.232 & 678.2 & 6.794 & 646.9 & 6.563 & 642.1 & 749 & 651.3 & 6.655 & 647.8 \\
\hline 6.933 & 650.9 & 6.604 & 619.6 & 6.566 & 620.5 & 6.817 & 648.2 & 6.918 & 653.5 & 6.326 & 615.2 & 6.205 & 612.5 & 6.089 & 612.1 & 6.196 & 614.8 \\
\hline 6.791 & 625.8 & 6.524 & 590.5 & 6.299 & 597.1 & 6.711 & 620.5 & 6.359 & 612.5 & 6.057 & 588.8 & 5.986 & 584.3 & 5.77 & 589.2 & 5.894 & 592.7 \\
\hline 6.489 & 590.5 & 6.086 & 570.7 & 5.962 & 571.1 & 6.27 & 593.6 & 6.341 & 603.3 & 5.776 & 563.6 & 5.595 & 562.8 & 5.669 & 557.5 & 5.645 & 567.2 \\
\hline & 0.4 & & 540.7 & 28 & 546 & & 568.5 & & 562.8 & 45 & 539.8 & & 539.4 & & 5.9 & 5.281 & 540.3 \\
\hline 5.675 & 534.5 & 45 & 513.8 & 5.273 & 506.3 & & 528.8 & 503 & 538.1 & 5.246 & 513.4 & & 513.4 & .083 & 505.9 & 5.012 & 516.5 \\
\hline 5.426 & 506.8 & 889 & 492.2 & 4.906 & 479.5 & 5.036 & 505.9 & 5.225 & 508.1 & 4.994 & 488.7 & 4.598 & 472.9 & .639 & 480.3 & 4.843 & 490.5 \\
\hline 5.213 & 482.5 & 766 & 454.3 & 4.524 & 452.6 & 4.903 & 478.6 & 4.985 & 483 & 4.766 & 461.8 & 4.207 & 447.3 & 305 & 452.6 & 4.157 & 451.7 \\
\hline 4.953 & 453.5 & 4.465 & 425.3 & 4.204 & 430.1 & 4.627 & 455.2 & 4.761 & 456.5 & 4.482 & 433.6 & 3.979 & 418.2 & 4.047 & 430.1 & 3.976 & 425.7 \\
\hline 4.53 & 427 & 4.183 & 406.3 & 4.012 & 396.2 & 4.414 & 424.8 & 4.45 & 432.8 & 3.905 & 394.4 & 3.858 & 396.2 & 3.947 & 400.6 & 3.84 & 400.6 \\
\hline 4.175 & 404.5 & 4 & 381.6 & 3.935 & 372.8 & 3.932 & 400.1 & 3.994 & 407.6 & 3.799 & 385.2 & 3.485 & 374.6 & 3.515 & 377.7 & 3.384 & 373.7 \\
\hline 3.686 & 368 & 3.754 & 345.9 & 3.426 & 350.3 & 3.793 & 375.5 & 3.411 & 371.5 & 3.097 & 338.9 & 3.284 & 348.6 & 3.017 & 341.1 & 3.145 & 342 \\
\hline 3.654 & 342.4 & 3.201 & 318.6 & 3.077 & 311.6 & 3.355 & 337.6 & 3.426 & 341.5 & 3.118 & 314.6 & 3.0 & 313.8 & 2.946 & 310.2 & 3.177 & 317.3 \\
\hline 3.228 & 311.6 & 3.213 & 290 & 2.955 & 288.2 & 2.991 & 315.5 & 3.201 & 319.1 & 2.719 & 283.4 & 2.639 & 284.2 & 2.727 & 287.8 & 2.636 & 294.8 \\
\hline 3.139 & 287.3 & 2.674 & 264 & 2.656 & 266.2 & 2.745 & 287.3 & 3 & 295.7 & 2.701 & 260 & 2.511 & 260.9 & 2.559 & 261.3 & 2.721 & 267.9 \\
\hline 2.748 & 263.5 & 2.721 & 239.3 & 2.544 & 256.9 & 2.6 & 263.5 & 2.739 & 270.6 & 2.257 & 234.4 & 2.411 & 251.2 & 0 & 162.2 & 2.278 & 244.1 \\
\hline
\end{tabular}


Table 4. Rheometer Response for Blank - Diluted

\begin{tabular}{|c|c|c|c|c|c|c|c|c|c|c|c|c|c|c|c|c|c|}
\hline run1 & & run2 & & run3 & & run4 & & run5 & & run6 & & run7 & & run8 & & run9 & \\
\hline [Pa] & {$[1 / s]$} & [Pa] & [1/s] & [Pa] & {$[1 / s]$} & [Pa] & {$[1 / s]$} & [Pa] & [1/s] & [Pa] & [1/s] & [Pa] & [1/s] & [Pa] & {$[1 / s]$} & [Pa] & {$[1 / s]$} \\
\hline 2.674 & 375.9 & 2.824 & 389.6 & 2.233 & 327.4 & 2.372 & 344.6 & 2.573 & 371.5 & 2.458 & 365.8 & 2.073 & 312.9 & 2.185 & 324.8 & 1.969 & 319.9 \\
\hline 2.783 & 396.2 & .863 & 414.2 & 2.381 & 342.4 & 2.475 & 369.7 & 2.552 & 403.2 & 2.564 & 390.9 & 2.395 & 347.3 & 2.348 & 347.3 & 2.342 & 347.7 \\
\hline 2.854 & 418.6 & .925 & 442.9 & 2.537 & 364 & 2.537 & 396.2 & 2.674 & 426.1 & 2.813 & 426.6 & 2.472 & 371.9 & 2.419 & 372.8 & 2.431 & 372.4 \\
\hline 3.147 & 453.5 & 2.993 & 467.6 & 2.647 & 392.6 & 2.677 & 420.4 & 2.765 & 448.6 & 2.824 & 455.7 & 2.537 & 401 & 2.443 & 397.9 & 2.57 & 392.2 \\
\hline 3.168 & 483.9 & 3.079 & 493.1 & 2.768 & 418.6 & 2.807 & 443.8 & 2.964 & 485.2 & 2.827 & 480.8 & 2.608 & 422.6 & 2.597 & 422.2 & 2.526 & 424.4 \\
\hline 3.274 & 502.4 & 3.177 & 516.5 & 3.035 & 456.1 & 2.869 & 472 & 3.079 & 506.3 & 2.901 & 504.1 & 2.712 & 442 & 2.656 & 453.5 & 2.611 & 448.6 \\
\hline 3.393 & 526.2 & 3.224 & 544.2 & 3.076 & 480.3 & 2.961 & 497.1 & 3.085 & 537.6 & 2.975 & 528.4 & 2.869 & 478.1 & 2.827 & 476.4 & 2.674 & 470.6 \\
\hline 3.479 & 555.3 & 3.319 & 573.8 & 3.115 & 512.1 & 2.964 & 525.7 & 3.126 & 561 & 3.064 & 557.9 & 2.827 & 510.3 & 2.91 & 500.6 & 2.727 & 495.8 \\
\hline 3.609 & 580.8 & 3.473 & 598 & 3.26 & 531.9 & 3.126 & 555.3 & 3.212 & 583 & 3.168 & 581.3 & 2.949 & 531 & 2.964 & 530.1 & 2.842 & 520.4 \\
\hline 3.748 & 605.5 & 3.576 & 621.8 & 3.328 & 559.7 & 3.188 & 580.4 & 3.31 & 608.6 & 3.396 & 618.7 & 2.993 & 554.8 & 2.961 & 558.8 & 2.86 & 550 \\
\hline 3.999 & 642.5 & 3.721 & 665.9 & 3.407 & 581.3 & 3.449 & 617.4 & 3.372 & 639.4 & 3.44 & 646 & 3.061 & 579.5 & 3.076 & 581.7 & 2.996 & 579.9 \\
\hline & 676 & & 690.5 & & & & 644.3 & 3.461 & & & 671.2 & & 617.8 & & 606.8 & 3.15 & 604.6 \\
\hline 4.136 & 701.6 & 3.869 & 713.9 & 3.706 & 646.5 & 3.626 & 669.8 & 3.57 & 686.6 & 3.57 & 697.6 & 3.171 & 629.3 & 3.41 & 648.2 & 3.191 & 628.9 \\
\hline 4.278 & 727.1 & 3.919 & 739.5 & 3.706 & 676.4 & 3.7 & 694.1 & 3.816 & 727.1 & 3.683 & 720.1 & 3.304 & 661.9 & 3.47 & 670.3 & 3.203 & 657.5 \\
\hline 4.352 & 749.2 & 3.996 & 766.3 & 3.801 & 695.4 & 3.739 & 719.6 & 3.845 & 751.4 & 3.65 & 746.5 & 3.464 & 689.2 & 3.532 & 698.9 & 3.342 & 683.5 \\
\hline 4.485 & 771.6 & 4.056 & 790.1 & 3.813 & 720.1 & 3.807 & 747 & 3.89 & 781.3 & 3.813 & 768.6 & 3.517 & 716.6 & 3.665 & 724 & 3.419 & 712.6 \\
\hline 4.568 & 800.3 & & 817.5 & & 744.3 & & 771.2 & 3.925 & 804.2 & 76 & 806.9 & & 739.5 & 3.727 & 754 & 3.659 & 750.9 \\
\hline 4.6 & 826.3 & 4.218 & 841.7 & 4.029 & 769 & 3.845 & 794.1 & 4.07 & 841.3 & 4.103 & 833.8 & 3.769 & 783.5 & 3.724 & 782.7 & 3.674 & 781.8 \\
\hline 4.908 & 863.3 & & 866.8 & & 810.4 & & 820.1 & 4.112 & 869.9 & 36 & 864.2 & & 806.4 & 3.801 & 803.8 & 3.683 & 805.6 \\
\hline 5.047 & 886.2 & 4.423 & 893.7 & 4.239 & 839.5 & 4.221 & 860.2 & 4.056 & 878.3 & & 885.8 & & 836.4 & 3.822 & 830.7 & 3.695 & 830.2 \\
\hline 5.091 & 911.8 & & 936 & & 859.8 & 4.227 & 889.7 & 4.26 & 926.8 & 4.328 & 907.4 & & 859.8 & 3.922 & 851.4 & 3.801 & 857.1 \\
\hline 5.145 & 945.3 & 4.727 & 958.5 & 4.337 & 886.7 & 4.094 & 901.6 & 4.443 & 947.5 & 4.36 & 940.9 & 4.044 & 888.4 & 4.106 & 886.7 & 4.014 & 882.7 \\
\hline 5.189 & 967.7 & 4.733 & 988.5 & 4.532 & 908.2 & 4.467 & 943.1 & 4.5 & 972.6 & 4.431 & 966.9 & 4.109 & 909.1 & 4.121 & 923.7 & 4.05 & 912.7 \\
\hline 5.316 & 992 & 4.757 & 1018 & 4.627 & 950.1 & 4.556 & 968.6 & 4.535 & 1004 & 4.511 & 992.4 & & 935.1 & 4.301 & 944.8 & 4.082 & 939.5 \\
\hline 5.367 & 1016 & 4.884 & 1037 & 4.784 & 971.7 & 4.544 & 998.1 & 4.612 & 1025 & 4.633 & 1016 & 4.195 & 959.8 & 4.289 & 973 & 4.156 & 963.8 \\
\hline 5.512 & 1042 & 4.94 & 1059 & 4.727 & 1003 & 4.645 & 1020 & 4.582 & 1048 & 4.653 & 1041 & 4.443 & 999.9 & 4.319 & 997.3 & 4.263 & 988.5 \\
\hline 5.603 & 1070 & 4.932 & 1089 & 4.831 & 1022 & 4.721 & 1044 & 4.834 & 1087 & 4.733 & 1066 & 4.482 & 1021 & 4.497 & 1029 & 4.369 & 1014 \\
\hline 5.787 & 1112 & 5.266 & 1127 & 5.003 & 1061 & 4.816 & 1077 & 4.917 & 1111 & 4.902 & 1105 & 4.532 & 1054 & 4.588 & 1057 & 4.437 & 1039 \\
\hline 5.858 & 1139 & 5.325 & 1151 & 4.929 & 1070 & 4.798 & 1100 & 5.038 & 1133 & 4.887 & 1136 & 4.532 & 1076 & 4.633 & 1079 & 4.455 & 1067 \\
\hline 5.867 & 1164 & 5.458 & 1176 & 5.154 & 1111 & 4.988 & 1136 & 5.047 & 1159 & 4.905 & 1162 & 4.662 & 1097 & 4.713 & 1103 & 4.568 & 1095 \\
\hline 6.068 & 1184 & 5.479 & 1203 & 5.325 & 1138 & 5.077 & 1162 & 5.065 & 1193 & 5.1 & 1182 & 4.831 & 1135 & 4.76 & 1137 & 4.571 & 1120 \\
\hline
\end{tabular}


Table 4. Rheometer Response for Blank - Diluted - continued

\begin{tabular}{|c|c|c|c|c|c|c|c|c|c|c|c|c|c|c|c|c|c|}
\hline run1 & & run2 & & run3 & & run4 & & run5 & & run6 & & run7 & & run8 & & run9 & \\
\hline [Pa] & [1/s] & [Pa] & [1/s] & [Pa] & [1/s] & [Pa] & {$[1 / s]$} & [Pa] & [1/s] & [Pa] & [1/s] & [Pa] & [1/s] & [Pa] & [1/s] & [Pa] & {$[1 / s]$} \\
\hline 6.139 & 1219 & 5.509 & 1236 & 5.367 & 1161 & 5.257 & 1186 & 5.233 & 1218 & 5.1 & 1206 & 4.831 & 1159 & 4.683 & 1165 & 4.852 & 1162 \\
\hline 6.243 & 1241 & 651 & 1257 & 5.367 & 1189 & 5.228 & 1212 & 5.376 & 1246 & 5.293 & 1251 & 4.985 & 1180 & 4.828 & 1187 & 4.908 & 1185 \\
\hline 6.29 & 1267 & 5.855 & 1293 & 5.568 & 1215 & 5.37 & 1237 & 5.47 & 1270 & 5.426 & 1272 & 4.911 & 1215 & 4.887 & 1214 & 4.81 & 1218 \\
\hline 6.346 & 1291 & 5.899 & 1316 & 5.473 & 1248 & 5.373 & 1270 & 5.568 & 1300 & 5.355 & 1281 & 5.091 & 1235 & 4.943 & 1242 & 4.899 & 1239 \\
\hline 6.506 & 1315 & 5.911 & 1347 & 5.55 & 1271 & 5.432 & 1294 & 5.595 & 1327 & 5.586 & 1323 & 5.251 & 1273 & 5.012 & 1266 & 4.991 & 1262 \\
\hline 6.769 & 1354 & 5.991 & 1368 & 5.805 & 1295 & 5.526 & 1316 & 5.595 & 1357 & 5.544 & 1354 & 5.233 & 1296 & 5.148 & 1293 & 5.038 & 1287 \\
\hline 6.775 & 1386 & 5.982 & 1393 & 5.728 & 1327 & 5.529 & 1340 & 5.586 & 1384 & 5.692 & 1375 & 5.304 & 1325 & 5.162 & 1317 & 5.278 & 1326 \\
\hline 6.876 & 1409 & 6.024 & 1417 & 5.814 & 1349 & 5.751 & 1382 & 5.692 & 1407 & 5.846 & 1410 & 5.523 & 1357 & 5.281 & 1341 & 5.263 & 1351 \\
\hline 6.909 & 1435 & 6.311 & 1453 & 5.84 & 1375 & 5.822 & 1405 & 5.805 & 1432 & 5.899 & 1433 & 5.47 & 1389 & 5.482 & 1378 & 5.399 & 1378 \\
\hline 6.974 & 1460 & 6.364 & 1477 & 6.106 & 1412 & 5.944 & 1430 & 5.849 & 1457 & 5.879 & 1465 & 5.376 & 1393 & 5.6 & 1402 & 5.414 & 1409 \\
\hline 7.181 & 1484 & 6.373 & 1507 & 6.151 & 1434 & 5.917 & 1462 & 5.861 & 1486 & 5.929 & 1487 & 5.722 & 1433 & 4.727 & 1627 & 5.444 & 1433 \\
\hline 7.367 & 1522 & 6.462 & 1529 & 6.254 & 1459 & 6 & 1484 & 6.041 & 1513 & 5.92 & 1510 & 5.663 & 1464 & 4.772 & 1598 & 4.793 & 1635 \\
\hline 5.364 & 1546 & 5.038 & 1524 & 5.085 & 1575 & 4.869 & 1568 & 4.692 & 1525 & 4.665 & 1542 & 4.76 & 1600 & 4.713 & 1576 & 4.606 & 1595 \\
\hline 5.275 & 1520 & 4.982 & 1498 & 4.985 & 1549 & 4.911 & 1538 & 4.393 & 1484 & 4.642 & 1514 & 4.647 & 1567 & 4.591 & 1551 & 4.574 & 1572 \\
\hline 5.168 & 1496 & 4.94 & 1471 & 4.94 & 1523 & 4.819 & 1514 & 4.47 & 1452 & 4.55 & 1494 & 4.615 & 1546 & 4.378 & 1512 & 4.565 & 1544 \\
\hline 5.071 & 1470 & 4.763 & 1448 & 4.822 & 1500 & 4.798 & 1490 & 4.378 & 1433 & 4.532 & 1466 & 4.494 & 1518 & 4.337 & 1491 & 4.446 & 1512 \\
\hline 4.858 & 1433 & 4.574 & 1410 & 4.73 & 1475 & 4.671 & 1466 & 4.245 & 1400 & 4.476 & 1440 & 4.526 & 1493 & 4.283 & 1460 & 4.372 & 1490 \\
\hline 4.76 & 1404 & 4.642 & 1403 & 4.479 & 1432 & 4.556 & 1440 & 4.207 & 1376 & 4.405 & 1411 & 4.588 & 1455 & 4.272 & 1433 & 4.177 & 1456 \\
\hline 4.647 & 1386 & 4.334 & 1357 & 4.355 & 1411 & 4.491 & 1416 & 4.118 & 1355 & 4.242 & 1384 & 4.582 & 1454 & 4.136 & 1408 & 4.121 & 1429 \\
\hline 4.633 & 1355 & 4.236 & 1335 & 4.325 & 1381 & 4.177 & 1378 & 3.955 & 1316 & 4.059 & 1359 & 4.005 & 1416 & 4.133 & 1381 & 4.041 & 1405 \\
\hline 4.571 & 1328 & 4.254 & 1304 & 4.334 & 1354 & 4.106 & 1355 & 3.843 & 1295 & 3.982 & 1322 & 3.922 & 1348 & 3.964 & 1356 & 4.05 & 1375 \\
\hline 4.423 & 1303 & 4.233 & 1284 & 4.248 & 1333 & 4.177 & 1327 & 3.875 & 1264 & 3.84 & 1301 & 3.99 & 1343 & 3.893 & 1326 & 3.985 & 1351 \\
\hline 4.34 & 1279 & 4.165 & 1259 & 4.147 & 1312 & 4.076 & 1304 & 3.774 & 1242 & 3.881 & 1271 & 3.739 & 1325 & 3.777 & 1304 & 3.878 & 1326 \\
\hline 4.289 & 1253 & 4.059 & 1237 & 3.917 & 1273 & 3.831 & 1269 & 3.668 & 1222 & 3.698 & 1243 & 3.831 & 1315 & 3.523 & 1267 & 3.854 & 1307 \\
\hline 4.171 & 1228 & 4.032 & 1209 & 3.86 & 1247 & 3.81 & 1240 & 3.473 & 1185 & 3.644 & 1220 & 3.443 & 1262 & 3.526 & 1243 & 3.612 & 1266 \\
\hline 3.993 & 1191 & 3.698 & 1162 & 3.837 & 1220 & 3.632 & 1218 & 3.479 & 1155 & 3.618 & 1196 & 3.671 & 1245 & 3.499 & 1211 & 3.487 & 1244 \\
\hline 3.848 & 1165 & 3.789 & 1154 & 3.816 & 1194 & 3.68 & 1188 & 3.529 & 1117 & 3.476 & 1172 & 3.564 & 1219 & 3.413 & 1191 & 3.434 & 1219 \\
\hline 3.724 & 1138 & 3.517 & 1113 & 3.695 & 1168 & 3.615 & 1162 & 3.585 & 1115 & 3.313 & 1137 & 3.292 & 1169 & 3.348 & 1165 & 3.437 & 1188 \\
\hline 3.763 & 1107 & 3.422 & 1090 & 3.632 & 1143 & 3.496 & 1139 & 2.857 & 1091 & 3.316 & 1104 & 3.268 & 1154 & 3.357 & 1139 & 3.39 & 1165 \\
\hline 3.677 & 1085 & 3.416 & 1064 & 3.538 & 1114 & 3.458 & 1115 & 2.946 & 1077 & 3.257 & 1080 & 3.138 & 1131 & 3.227 & 1116 & 3.141 & 1127 \\
\hline 3.547 & 1063 & 3.354 & 1030 & 3.384 & 1088 & 3.209 & 1080 & 2.588 & 1024 & 3.094 & 1056 & 3.079 & 1108 & 2.978 & 1080 & 3.106 & 1108 \\
\hline
\end{tabular}


Table 4. Rheometer Response for Blank - Diluted - continued

\begin{tabular}{|c|c|c|c|c|c|c|c|c|c|c|c|c|c|c|c|c|c|}
\hline run1 & & run2 & & run3 & & run4 & & run5 & & run6 & & run7 & & run8 & & run9 & \\
\hline [Pa] & [1/s] & [Pa] & [1/s] & [Pa] & [1/s] & [Pa] & {$[1 / s]$} & [Pa] & [1/s] & [Pa] & [1/s] & [Pa] & [1/s] & [Pa] & [1/s] & [Pa] & {$[1 / s]$} \\
\hline 3.538 & 1038 & 3.2 & 1010 & 3.191 & 1052 & 3.248 & 1048 & 2.736 & 1010 & 3.097 & 1029 & 3.144 & 1075 & 3.017 & 1051 & 3.082 & 1077 \\
\hline 3.44 & 1014 & 3.032 & 981.4 & 3.212 & 1025 & 3.126 & 1030 & 2.961 & 958.5 & 2.984 & 1006 & 2.887 & 1054 & 2.946 & 1026 & 3.076 & 1051 \\
\hline 3.141 & 971.7 & 3.017 & 952.3 & 3.005 & 1003 & 2.961 & 988.5 & 2.345 & 928.1 & 2.928 & 981 & 2.881 & 1026 & 2.842 & 1002 & 3.043 & 1028 \\
\hline 3.138 & 944.8 & 2.964 & 922.3 & 2.907 & 976.6 & 2.863 & 966.4 & 2.505 & 910.5 & 2.668 & 943.9 & 2.866 & 992.9 & 2.78 & 977.9 & 2.946 & 999.9 \\
\hline 3.159 & 922.8 & 2.898 & 899.9 & 2.919 & 947.5 & 2.804 & 936.9 & 2.422 & 888.9 & 2.709 & 915.3 & 2.75 & 966.9 & 2.608 & 936.5 & 2.762 & 969.5 \\
\hline 2.89 & 888.4 & 2.816 & 879.2 & 2.898 & 926.8 & 2.762 & 914.4 & 2.197 & 830.7 & 2.582 & 892.8 & 2.753 & 942.2 & 2.469 & 916.6 & 2.668 & 946.1 \\
\hline 2.736 & 865.9 & 2.611 & 840.8 & 2.798 & 903.4 & 2.753 & 891.5 & 2.295 & 814.4 & 2.605 & 867.7 & 2.605 & 918.8 & 2.493 & 889.7 & 2.496 & 910.9 \\
\hline 2.792 & 837.3 & 2.653 & 813.5 & 2.987 & 886.7 & 2.523 & 858 & 2.354 & 808.2 & 2.313 & 823.2 & 2.644 & 887.1 & 2.443 & 865.1 & 2.478 & 884 \\
\hline 2.712 & 814.8 & 2.594 & 789.3 & 2.508 & 840.4 & 2.505 & 827.6 & 2.037 & 783.5 & 2.188 & 802.9 & 2.443 & 862.4 & 2.159 & 830.7 & 2.36 & 861.1 \\
\hline 2.662 & 787.9 & 2.496 & 762.8 & 2.697 & 821 & 2.49 & 803.4 & 1.978 & 756.2 & 2.129 & 776 & 2.425 & 839.1 & 2.156 & 808.2 & 2.283 & 835.5 \\
\hline 2.632 & 756.2 & 2.443 & 737.7 & 2.173 & 756.7 & 2.384 & 779.6 & 2.117 & 720.1 & 2.241 & 765.9 & 2.203 & 801.6 & 2.156 & 779.6 & 2.191 & 802.5 \\
\hline 2.745 & 744.3 & 2.366 & 712.1 & 2.381 & 734.2 & 2.313 & 751.8 & 2.014 & 694.5 & 2.02 & 721.4 & 2.378 & 791 & 2.135 & 751.4 & 2.182 & 777.8 \\
\hline 1.573 & 710.4 & 2.215 & 686.1 & 1.573 & 706.4 & 2.2 & 729.3 & 1.901 & 673.4 & 1.925 & 700.7 & 1.96 & 752.2 & 2.002 & 730.2 & 2.088 & 754.9 \\
\hline 1.457 & 679.1 & 2.176 & 658.4 & 1.75 & 687.5 & 2.144 & 704.7 & 1.768 & 637.2 & 1.922 & 669 & 2.002 & 720.5 & 1.948 & 704.2 & 2.037 & 729.3 \\
\hline 1.531 & 635.9 & 1.91 & 618.7 & 1.827 & 655.3 & 1.907 & 668.5 & 1.65 & 612.5 & 1.848 & 644.3 & 2.011 & 695.4 & 1.729 & 666.3 & 1.86 & 693.2 \\
\hline 2.117 & 579.9 & 1.824 & 593.6 & 2.111 & 623.1 & 1.96 & 639 & 1.676 & 585.7 & 1.809 & 618.3 & 1.872 & 673.4 & 1.7 & 640.8 & 1.756 & 670.7 \\
\hline 1.315 & 563.2 & 1.744 & 567.2 & 2.156 & 621.4 & 1.806 & 615.2 & 1.602 & 559.7 & 1.579 & 583 & 1.842 & 648.2 & 1.667 & 617.8 & 1.673 & 639.4 \\
\hline 1.576 & 535.4 & 1.593 & 543.4 & 1.185 & 598.4 & 1.741 & 590.5 & 1.49 & 535 & 1.519 & 558.3 & 1.644 & 605.5 & 1.567 & 589.6 & 1.584 & 615.2 \\
\hline 1.543 & 504.6 & 1.576 & 516.9 & 1.354 & 581.3 & 1.703 & 562.3 & 1.481 & 509 & 1.576 & 549.5 & 1.546 & 583.5 & 1.505 & 568.5 & 1.617 & 591.4 \\
\hline 1.762 & 480.3 & 1.496 & 488.7 & 1.451 & 544.7 & 1.582 & 537.6 & 1.158 & 472 & 1.25 & 505.9 & 1.445 & 560.1 & 1.519 & 539.8 & 1.513 & 568 \\
\hline 1.12 & 457 & 1.395 & 462.3 & 1.46 & 511.2 & 1.522 & 514.7 & 1.102 & 450.8 & 1.268 & 479 & 1.499 & 526.2 & 1.354 & 513.4 & 1.342 & 529.3 \\
\hline 1.241 & 442.9 & 1.336 & 437.6 & 1.392 & 481.2 & 1.475 & 490.5 & 1.135 & 418.2 & 1.126 & 453.9 & 1.321 & 505.9 & 1.262 & 488.7 & 1.226 & 505.9 \\
\hline 1.135 & 420.4 & 1.312 & 404.5 & 1.274 & 459.6 & 1.241 & 447.3 & 1.049 & 390 & 1.105 & 427.9 & 1.173 & 482.1 & 1.238 & 461.8 & 1.232 & 473.7 \\
\hline 1.235 & 411.2 & 1.167 & 383 & 1.309 & 427.9 & 1.132 & 422.6 & 0.975 & 363.1 & 0.969 & 401.9 & 1.161 & 450.4 & 1.07 & 435.8 & 1.161 & 448.2 \\
\hline 1.241 & 381.6 & 1.176 & 357 & 1.209 & 402.3 & 1.155 & 392.2 & 0.898 & 339.3 & 0.913 & 373.7 & 1.075 & 426.1 & 0.851 & 394.9 & 1.105 & 422.2 \\
\hline 1.158 & 351.7 & 0.93 & 318.6 & 1.158 & 378.1 & 1.075 & 369.7 & 0.759 & 317.7 & 0.821 & 348.6 & 0.851 & 391.3 & 0.809 & 369.3 & 1.022 & 400.1 \\
\hline 0.954 & 314.6 & 0.854 & 291.3 & 1.075 & 357 & 1.028 & 344.2 & 0.703 & 277.2 & 0.842 & 313.3 & 0.874 & 364.4 & 0.791 & 339.3 & 0.981 & 375.5 \\
\hline 0.833 & 290.4 & 0.691 & 267.1 & 1.055 & 328.7 & 0.93 & 318.6 & 0.596 & 258.7 & 0.738 & 288.6 & 0.868 & 337.6 & & & 0.729 & 338.9 \\
\hline 0.768 & 267.1 & 0.803 & 256.5 & 0.782 & 290.9 & 0.782 & 294.8 & 0.531 & 239.3 & 0.667 & 264 & 0.782 & 315.5 & & & & \\
\hline & & & & & & & & & & & & & & & & & \\
\hline & & & & & & & & & & & & & & & & & \\
\hline & & & & & & & & & & & & & & & & & \\
\hline
\end{tabular}


Table 5. $25{ }^{\circ} \mathrm{C}$ Diluted

\begin{tabular}{|c|c|c|c|c|c|c|c|c|c|c|c|c|c|c|c|c|c|}
\hline run1 & & run2 & & run3 & & run4 & & run5 & & run6 & & run7 & & run8 & & run9 & \\
\hline [Pa] & {$[1 / \mathrm{s}]$} & {$[\mathrm{Pa}]$} & {$[1 / s]$} & {$[\mathrm{Pa}]$} & {$[1 / s]$} & [Pa] & {$[1 / \mathrm{s}]$} & {$[\mathrm{Pa}]$} & {$[1 / \mathrm{s}]$} & {$[\mathrm{Pa}]$} & {$[1 / \mathrm{s}]$} & [Pa] & {$[1 / \mathrm{s}]$} & {$[\mathrm{Pa}]$} & {$[1 / \mathrm{s}]$} & {$[\mathrm{Pa}]$} & {$[1 / \mathrm{s}]$} \\
\hline 3.737 & 341.1 & 3.908 & 345.5 & 3.393 & 329.2 & 3.183 & 312.9 & 3.879 & 342.4 & 4.183 & 370.2 & 3.663 & 309.4 & 2.325 & 337.1 & 2.867 & 312.4 \\
\hline 3.802 & 362.2 & 4.068 & 368.9 & 3.742 & 332.7 & 3.677 & 326.1 & 4.115 & 373.7 & 4.352 & 402.8 & 4.145 & 346.8 & 3.396 & 304.1 & 3.497 & 323.9 \\
\hline 4.095 & 386.9 & 4.151 & 390 & 4.101 & 352.1 & 3.648 & 334 & 4.107 & 397.1 & 4.645 & 425.7 & 4.195 & 372.4 & 4.035 & 325.2 & 3.964 & 345.9 \\
\hline 4.459 & 411.2 & 4.456 & 414.7 & 4.53 & 390 & 4.089 & 368 & 4.305 & 425.3 & 4.837 & 454.8 & 4.367 & 398.8 & 4.035 & 349 & 4.044 & 368.4 \\
\hline 4.764 & 449.5 & 4.675 & 453.5 & 4.695 & 417.3 & 4.293 & 390 & 4.565 & 450.4 & 4.985 & 480.8 & 4.352 & 406.8 & 4.26 & 372.8 & 4.133 & 393.5 \\
\hline 5.033 & 473.7 & 4.926 & 477.7 & 4.923 & 444.6 & 4.382 & 425.7 & 4.835 & 473.3 & 5.033 & 504.1 & 4.873 & 453 & 4.571 & 401.9 & 4.367 & 423.5 \\
\hline 5.098 & 505 & 5.157 & 500.2 & 5.054 & 468.9 & 4.414 & 449.9 & 5.071 & 511.6 & 5.169 & 531.5 & 5.116 & 474.6 & 4.716 & 431.4 & 4.571 & 447.7 \\
\hline 5.278 & 530.1 & 5.207 & 529.7 & 5.142 & 497.1 & 4.687 & 474.2 & 5.216 & 540.7 & 5.447 & 557.5 & 5.222 & 505 & 4.663 & 451.3 & 4.731 & 475.9 \\
\hline 5.441 & 551.7 & 5.358 & 554.4 & 5.332 & 523.1 & 4.817 & 501.1 & 5.344 & 563.2 & 5.625 & 582.1 & 5.305 & 530.6 & 4.793 & 475.1 & 4.968 & 500.6 \\
\hline 5.557 & 585.2 & 5.574 & 578.6 & 5.503 & 546.4 & 5.172 & 535.4 & 5.524 & 587 & 5.861 & 621.8 & 5.527 & 557 & 4.906 & 501.1 & 5.16 & 527.1 \\
\hline 5.713 & 609 & 5.595 & 609 & 5.728 & 577.7 & 5.311 & 560.5 & 5.684 & 617.4 & 6.143 & 646.9 & 5.666 & 580.8 & 5.142 & 525.3 & 5.385 & 551.3 \\
\hline 5.882 & 635.5 & 5.882 & 632.8 & 5.906 & 602 & 5.536 & 590.1 & 5.779 & 644.7 & 6.273 & 674.7 & 5.787 & 606.8 & 5.284 & 550.9 & 5.388 & 578.2 \\
\hline 6.163 & 658.8 & 6.107 & 657.5 & 6.036 & 625.3 & 5.687 & 616.5 & 6.012 & 668.5 & 6.344 & 698.9 & 5.906 & 631.1 & 5.696 & 595.4 & 5.622 & 600.2 \\
\hline 6.376 & 685.3 & 6.418 & 695.8 & 6.184 & 648.2 & 5.693 & 644.7 & 6.083 & 691.4 & 6.527 & 728.4 & 6.264 & 668.1 & 5.894 & 617.4 & 5.894 & 641.6 \\
\hline 6.436 & 712.1 & 6.616 & 719.6 & 6.474 & 690.5 & 5.962 & 670.3 & 6.468 & 730.7 & 6.655 & 752.2 & 6.326 & 693.6 & 5.921 & 650 & 5.932 & 668.1 \\
\hline 6.69 & 735.9 & 6.652 & 751.8 & 6.551 & 715.2 & 6.184 & 695.4 & 6.646 & 752.7 & 6.699 & 776 & 6.444 & 721.8 & 6.143 & 671.6 & 6.086 & 689.7 \\
\hline 7.131 & 776.5 & 6.879 & 774.7 & 6.791 & 743.9 & 6.37 & 719.2 & 6.853 & 778.2 & 6.98 & 804.2 & 6.705 & 745.2 & 6.249 & 698 & 6.261 & 723.2 \\
\hline 7.205 & 802 & 6.794 & 800.3 & 6.788 & 767.7 & 6.388 & 742.6 & 7.057 & 812.2 & 6.98 & 828 & 6.634 & 770.8 & 6.341 & 721.4 & 6.282 & 748.3 \\
\hline 7.522 & 827.6 & 7.116 & 824.5 & 7.042 & 790.6 & 6.687 & 780.4 & 7.101 & 837.7 & 7.323 & 851.8 & 6.874 & 795.4 & 6.749 & 757.5 & 6.486 & 771.6 \\
\hline 7.64 & 858 & 7.128 & 850.1 & 7.181 & 816.1 & 6.897 & 801.2 & 7.371 & 861.5 & 7.427 & 881.8 & 7.217 & 835.1 & 6.933 & 786.6 & 6.64 & 797.2 \\
\hline 7.631 & 883.6 & 7.548 & 872.1 & 7.457 & 858.9 & 6.948 & 832 & 7.347 & 884.9 & 7.528 & 904.7 & 7.217 & 858 & 6.909 & 811.7 & 6.734 & 817.5 \\
\hline 7.954 & 99.6 & 7.829 & 915.7 & 7.477 & 865.5 & 7.3 & 858 & 7.563 & 906.5 & 7.942 & 946.6 & 7.394 & 883.6 & 7.235 & 838.6 & 6.921 & 844.3 \\
\hline 8.01 & 1.2 & 7.927 & 941.3 & 7.738 & 892.4 & 7.35 & 891.5 & 7.631 & 930.7 & 8.063 & 969.5 & 7.643 & 913.1 & 7.294 & 861.5 & 7.264 & 891.1 \\
\hline 8.214 & 957.2 & 8.267 & 966.4 & 8.001 & 933.8 & 7.554 & 914.9 & 7.741 & 959.4 & 8.253 & 1001 & 7.69 & 937.3 & 7.383 & 891.9 & 7.448 & 915.3 \\
\hline 8.703 & 994.2 & 8.433 & 992 & 8.111 & 959.4 & 7.687 & 938.2 & 8.185 & 999.5 & 8.288 & 1025 & 7.776 & 959.4 & 7.519 & 913.1 & 7.534 & 943.1 \\
\hline 8.726 & 1025 & 8.522 & 1025 & 8.294 & 985.8 & 7.812 & 962.9 & 8.353 & 1024 & 8.356 & 1049 & 7.927 & 986.2 & 7.584 & 939.1 & 7.809 & 964.7 \\
\hline 8.871 & 1052 & 8.643 & 1051 & 8.528 & 1010 & 7.945 & 991.5 & 8.534 & 1052 & 8.486 & 1071 & 8.17 & 1010 & 8.12 & 976.6 & 7.72 & 988.5 \\
\hline 9.058 & 1076 & 8.886 & 1074 & 8.566 & 1041 & 8.146 & 1014 & 8.62 & 1080 & 8.836 & 1111 & 8.353 & 1040 & 8.081 & 1006 & 7.948 & 1017 \\
\hline 9.306 & 1099 & 8.904 & 1100 & 8.809 & 1063 & 8.327 & 1052 & 8.794 & 1107 & 8.951 & 1136 & 8.436 & 1067 & 8.244 & 1027 & 8.09 & 1050 \\
\hline 9.457 & 1123 & 9.055 & 1126 & 8.954 & 1088 & 8.475 & 1079 & 8.942 & 1138 & 9.099 & 1161 & 8.726 & 1108 & 8.38 & 1055 & 8.199 & 1076 \\
\hline 9.732 & 1161 & 9.084 & 1153 & 9.277 & 1126 & 8.489 & 1103 & 9.043 & 1165 & 9.244 & 1192 & 8.839 & 1133 & 8.525 & 1081 & 8.427 & 1097 \\
\hline
\end{tabular}


Table 5. $25^{\circ} \mathrm{C}$ Diluted - continued

\begin{tabular}{|c|c|c|c|c|c|c|c|c|c|c|c|c|c|c|c|c|c|}
\hline run1 & & run2 & & run3 & & run4 & & run5 & & run6 & & run7 & & run8 & & run9 & \\
\hline$[\mathrm{Pa}]$ & {$[1 / \mathrm{s}]$} & {$[\mathrm{Pa}]$} & {$[1 / \mathrm{s}]$} & [Pa] & {$[1 / \mathrm{s}]$} & {$[\mathrm{Pa}]$} & {$[1 / \mathrm{s}]$} & {$[\mathrm{Pa}]$} & {$[1 / \mathrm{s}]$} & {$[\mathrm{Pa}]$} & {$[1 / \mathrm{s}]$} & {$[\mathrm{Pa}]$} & {$[1 / \mathrm{s}]$} & {$[\mathrm{Pa}]$} & {$[1 / \mathbf{s}]$} & {$[\mathrm{Pa}]$} & {$[1 / \mathrm{s}]$} \\
\hline 9.735 & 1190 & 9.419 & 1179 & 9.46 & 1154 & 8.984 & 1137 & 9.126 & 1189 & 9.351 & 1216 & 9.025 & 1157 & 8.587 & 1103 & 8.478 & 1127 \\
\hline 9.913 & 1213 & 9.614 & 1204 & 9.567 & 1179 & 9.108 & 1162 & 9.413 & 1215 & 9.62 & 1237 & 9.143 & 1181 & 8.966 & 1142 & 8.549 & 1154 \\
\hline 10.11 & 1233 & 9.848 & 1230 & 9.786 & 1206 & 9.185 & 1186 & 9.587 & 1241 & 9.682 & 1265 & 9.401 & 1212 & 9.081 & 1167 & 8.812 & 1182 \\
\hline 10.42 & 1271 & 9.99 & 1254 & 9.996 & 1230 & 9.339 & 1215 & 9.765 & 1265 & 9.806 & 1289 & 9.507 & 1237 & 9.129 & 1196 & 8.99 & 1204 \\
\hline 10.56 & 1300 & 10.33 & 1298 & 10.11 & 1262 & 9.428 & 1237 & 9.792 & 1289 & 9.857 & 1317 & 9.564 & 1271 & 9.265 & 1220 & 9.152 & 1229 \\
\hline 10.77 & 1325 & 10.51 & 1321 & 10.29 & 1285 & 9.528 & 1265 & 10.2 & 1332 & 10.19 & 1343 & 9.78 & 1296 & 9.419 & 1244 & 9.41 & 1265 \\
\hline 10.95 & 1347 & 10.69 & 1348 & 10.24 & 1310 & 9.833 & 1289 & 10.41 & 1357 & 10.48 & 1384 & 9.966 & 1322 & 9.567 & 1269 & 9.478 & 1289 \\
\hline 11.11 & 1376 & 10.81 & 1380 & 10.45 & 1333 & 9.975 & 1315 & 10.46 & 1383 & 10.57 & 1407 & 10.01 & 1347 & 9.792 & 1293 & 9.587 & 1320 \\
\hline 11.31 & 1397 & 10.91 & 1402 & 10.7 & 1358 & 10.15 & 1343 & 10.67 & 1409 & 10.71 & 1434 & 10.14 & 1371 & 10.08 & 1333 & 9.75 & 1344 \\
\hline 11.66 & 1436 & 11.13 & 1430 & 10.79 & 1383 & 10.28 & 1368 & 10.81 & 1436 & 10.81 & 1464 & 10.28 & 1396 & 10.29 & 1360 & 9.895 & 1367 \\
\hline 11.79 & 1463 & 11.32 & 1450 & 10.93 & 1411 & 10.52 & 1393 & 11.02 & 1460 & 10.93 & 1490 & 10.64 & 1434 & 10.38 & 1386 & 10.23 & 1405 \\
\hline 11.97 & 1489 & 11.64 & 1490 & 11.31 & 1456 & 10.61 & 1420 & 11.23 & 1489 & 11.15 & 1514 & 10.81 & 1459 & 10.54 & 1415 & 10.37 & 1430 \\
\hline 12.11 & 1513 & 11.83 & 1515 & 11.51 & 1481 & 11.09 & 1463 & 11.32 & 1521 & 11.3 & 1538 & 11.03 & 1484 & 10.64 & 1438 & 10.51 & 1458 \\
\hline 10.57 & 1529 & 10.32 & 1556 & 10.24 & 1533 & 10.26 & 1567 & 9.948 & 1515 & 10.2 & 1526 & 10.52 & 1568 & 10.61 & 1592 & 10.52 & 1588 \\
\hline 10.46 & 1496 & 10.18 & 1532 & 10.4 & 1517 & 10.12 & 1544 & 9.999 & 1492 & 9.975 & 1500 & 10.36 & 1542 & 10.39 & 1569 & 10.39 & 1563 \\
\hline 10.64 & 1473 & 9.848 & 1491 & 10.08 & 1491 & 10.03 & 1520 & 9.67 & 1462 & 9.685 & 1461 & 10.04 & 1515 & 10.3 & 1538 & 10.11 & 1535 \\
\hline 9.765 & 1450 & 9.614 & 1465 & 10.16 & 1485 & 9.797 & 1497 & 9.635 & 1432 & 9.626 & 1439 & 9.943 & 1490 & 10.07 & 1514 & 9.925 & 1508 \\
\hline 9.88 & 1436 & 9.502 & 1441 & 10.01 & 1468 & 9.765 & 1471 & 9.46 & 1409 & 9.392 & 1408 & 9.481 & 1451 & 9.966 & 1488 & 9.721 & 1480 \\
\hline 9.715 & 1409 & 9.407 & 1415 & 9.866 & 1435 & 9.336 & 1430 & 9.351 & 1382 & 9.223 & 1386 & 9.38 & 1428 & 9.744 & 1464 & 9.475 & 1459 \\
\hline 9.599 & 1378 & 9.176 & 1389 & 9.809 & 1411 & 9.12 & 1405 & 9.214 & 1356 & 9.016 & 1361 & 9.309 & 1402 & 9.67 & 1438 & 9.413 & 1426 \\
\hline 9.472 & 1354 & 9.025 & 1354 & 9.614 & 1388 & 9.253 & 1392 & 9.049 & 1331 & 8.865 & 1329 & 9.058 & 1378 & 9.543 & 1412 & 9.283 & 1404 \\
\hline 9.33 & 1319 & 8.791 & 1329 & 9.505 & 1365 & 8.845 & 1351 & 8.824 & 1307 & 8.75 & 1300 & 8.966 & 1354 & 9.333 & 1387 & 9.061 & 1378 \\
\hline 9.058 & 1298 & 8.643 & 1306 & 9.2 & 1328 & 8.676 & 1329 & 8.62 & 1283 & 8.525 & 1276 & 8.8 & 1318 & 9.203 & 1360 & 8.922 & 1356 \\
\hline 8.96 & 1272 & 8.59 & 1284 & 8.942 & 1303 & 8.519 & 1298 & 8.383 & 1239 & 8.338 & 1252 & 8.525 & 1298 & 8.969 & 1336 & 8.904 & 1331 \\
\hline 8.694 & 1236 & 8.463 & 1259 & 8.845 & 1272 & 8.555 & 1273 & 8.143 & 1214 & 8.164 & 1229 & 8.478 & 1266 & 8.472 & 1293 & 8.386 & 1288 \\
\hline 8.513 & 1212 & 8.111 & 1217 & 8.649 & 1247 & 8.383 & 1251 & 8.09 & 1186 & 8.134 & 1202 & 8.259 & 1241 & 8.445 & 1266 & 8.297 & 1265 \\
\hline 8.389 & 1180 & 7.815 & 1192 & 8.51 & 1226 & 8.167 & 1224 & 7.824 & 1162 & 7.818 & 1174 & 8.125 & 1218 & 8.235 & 1242 & 8.276 & 1234 \\
\hline 8.158 & 1162 & 7.75 & 1168 & 8.347 & 1201 & 7.989 & 1197 & 7.542 & 1140 & 7.468 & 1133 & 7.995 & 1193 & 8.173 & 1216 & 8.096 & 1211 \\
\hline 7.942 & 1136 & 7.634 & 1140 & 8.205 & 1176 & 7.794 & 1172 & 7.59 & 1107 & 7.442 & 1111 & 7.767 & 1169 & 7.918 & 1183 & 7.936 & 1186 \\
\hline 7.841 & 1107 & 7.492 & 1117 & 7.788 & 1137 & 7.708 & 1147 & 7.445 & 1084 & 7.214 & 1081 & 7.566 & 1143 & 7.853 & 1157 & 7.69 & 1163 \\
\hline 7.676 & 1081 & 7.421 & 1090 & 7.806 & 1107 & 7.409 & 1105 & 7.181 & 1061 & 7.093 & 1054 & 7.519 & 1117 & 7.693 & 1132 & 7.575 & 1135 \\
\hline
\end{tabular}


Table 5. $25^{\circ} \mathrm{C}$ Diluted - continued

\begin{tabular}{|c|c|c|c|c|c|c|c|c|c|c|c|c|c|c|c|c|c|}
\hline run1 & & run2 & & run3 & & run4 & & run5 & & run6 & & run7 & & run8 & & run9 & \\
\hline$[\mathrm{Pa}]$ & {$[1 / s]$} & {$[\mathrm{Pa}]$} & {$[1 / \mathrm{s}]$} & {$[\mathrm{Pa}]$} & {$[1 / \mathrm{s}]$} & {$[\mathrm{Pa}]$} & {$[1 / s]$} & {$[\mathrm{Pa}]$} & {$[1 / \mathrm{s}]$} & {$[\mathrm{Pa}]$} & {$[1 / s]$} & {$[\mathrm{Pa}]$} & {$[1 / s]$} & {$[\mathrm{Pa}]$} & {$[1 / \mathbf{s}]$} & {$[\mathrm{Pa}]$} & {$[1 / s]$} \\
\hline 7.495 & 1057 & 7.199 & 1065 & 7.693 & 1081 & 7.193 & 1078 & 6.788 & 1022 & 6.989 & 1031 & 7.09 & 1074 & 7.572 & 1108 & 7.199 & 1099 \\
\hline 7.087 & 1017 & 6.965 & 1040 & 7.445 & 1058 & 7.119 & 1050 & 6.788 & 999.5 & 6.874 & 1007 & 7.104 & 1046 & 7.285 & 1085 & 7.078 & 1071 \\
\hline 7.007 & 994.6 & 6.693 & 995.9 & 7.27 & 1033 & 6.806 & 1028 & 6.61 & 968.2 & 6.595 & 984 & 6.82 & 1023 & 7.161 & 1060 & 7.022 & 1045 \\
\hline 6.945 & 964.2 & 6.782 & 988 & 7.007 & 1007 & 6.82 & 1000 & 6.607 & 943.1 & 6.35 & 945.7 & 6.847 & 999.9 & 6.856 & 1022 & 6.797 & 1025 \\
\hline 6.663 & 938.7 & 6.613 & 961.1 & 6.844 & 970.4 & 6.56 & 976.6 & 6.279 & 920.6 & 6.317 & 915.7 & 6.548 & 977.4 & 6.732 & 996.4 & 6.708 & 999.5 \\
\hline 6.64 & 915.7 & 6.157 & 919.7 & 6.601 & 947.9 & 6.521 & 948.8 & 6.296 & 893.3 & 6.119 & 888.4 & 6.379 & 950.6 & 6.598 & 965.5 & 6.607 & 974.3 \\
\hline 6.291 & 891.5 & 6.134 & 893.3 & 6.459 & 918.8 & 6.347 & 923.7 & 6.048 & 866.4 & 5.894 & 863.7 & 6.083 & 907.4 & 6.504 & 937.3 & 6.391 & 948.8 \\
\hline 6.282 & 869 & 5.974 & 867.7 & 6.418 & 895 & 6.075 & 895.5 & 5.764 & 843 & 5.79 & 839.1 & 5.956 & 885.8 & 6.187 & 914.9 & 5.974 & 904.7 \\
\hline 5.847 & 824.5 & 5.639 & 841.7 & 6.122 & 868.6 & 5.986 & 866.8 & 5.693 & 817.5 & 5.548 & 813.9 & 5.841 & 858.5 & 6.16 & 889.3 & 6.107 & 895 \\
\hline 5.826 & 803.8 & 5.625 & 810.4 & 6.072 & 845.2 & 5.637 & 831.6 & 5.551 & 792.8 & 5.382 & 790.1 & 5.737 & 827.6 & 5.873 & 864.2 & 5.657 & 855.4 \\
\hline 5.702 & 778.7 & 5.347 & 784.4 & 5.69 & 805.1 & 5.462 & 809.1 & 5.213 & 752.2 & 5.344 & 765 & 5.471 & 806 & 5.749 & 836.4 & 5.548 & 826.7 \\
\hline 5.453 & 758 & 5.326 & 760.6 & 5.551 & 778.7 & 5.338 & 778.2 & 5.059 & 721.8 & 5.059 & 738.6 & 5.503 & 780.4 & 5.548 & 810.9 & 5.373 & 800.3 \\
\hline 5.009 & 717 & 5.184 & 737.7 & 5.441 & 750.9 & 5.255 & 750.9 & 4.926 & 698 & 4.716 & 695.4 & 5.148 & 740.8 & 5.494 & 787.5 & 5.178 & 769 \\
\hline 4.852 & 692.8 & 4.885 & 700.7 & 5.169 & 731.1 & 5.16 & 728.4 & 4.764 & 676.4 & 4.55 & 671.6 & 5.056 & 718.8 & 5.255 & 761.9 & 5.225 & 743 \\
\hline 4.811 & 668.1 & 4.654 & 670.7 & 4.92 & 705.5 & 4.994 & 704.7 & 4.539 & 643 & 4.382 & 650.4 & 4.778 & 695 & 4.832 & 720.1 & 5.027 & 718.3 \\
\hline 4.71 & 641.2 & 4.556 & 645.6 & 4.897 & 672.9 & 4.74 & 678.2 & 4.34 & 619.6 & 4.485 & 617.8 & 4.66 & 663.7 & 4.778 & 693.6 & 4.722 & 690.5 \\
\hline 4.385 & 609.9 & 4.399 & 628 & 4.684 & 646.9 & 4.414 & 638.1 & 4.266 & 594.9 & 4.24 & 592.3 & 4.503 & 643.8 & 4.66 & 669.8 & 4.553 & 669.8 \\
\hline 4.249 & 585.7 & 4.204 & 597.1 & 4.388 & 622.7 & 4.157 & 612.5 & 4.127 & 570.2 & 4.133 & 569.4 & 4.281 & 606.8 & 4.382 & 638.1 & 4.556 & 636.8 \\
\hline 4.151 & 563.2 & 3.994 & 574.2 & 4.349 & 600.7 & 3.976 & 585.7 & 3.935 & 544.2 & 3.976 & 544.7 & 4.331 & 594.9 & 4.237 & 610.8 & 4.411 & 613.4 \\
\hline 3.784 & 524.4 & 3.932 & 547.8 & 4.074 & 563.2 & 3.846 & 558.3 & 3.695 & 519.1 & 3.855 & 520.4 & 4.142 & 567.6 & 4.095 & 587.4 & 3.908 & 579.1 \\
\hline 3.565 & 501.1 & 3.811 & 524 & 3.864 & 539.4 & 3.76 & 530.6 & 3.553 & 492.2 & 3.642 & 494.4 & 3.816 & 545.6 & 3.929 & 563.6 & 3.855 & 552.2 \\
\hline 3.464 & 470.2 & 3.63 & 496.2 & 3.722 & 514.3 & 3.58 & 513.4 & 3.381 & 464 & 3.207 & 453 & 3.74 & 516.9 & 3.799 & 526.2 & 3.595 & 523.5 \\
\hline 3.293 & 446 & 3.663 & 468.4 & 3.695 & 479 & 3.411 & 479.5 & 3.009 & 423.1 & 3.133 & 427.9 & 3.346 & 471.5 & 3.55 & 503.3 & 3.384 & 501.5 \\
\hline 3.228 & 420.4 & 3.136 & 425.7 & 3.381 & 455.7 & 3.278 & 453 & 2.881 & 398.4 & 2.864 & 403.2 & 3.376 & 449.1 & 3.37 & 475.1 & 3.461 & 473.7 \\
\hline 3.012 & 398.4 & 2.84 & 399.7 & 3.222 & 430.1 & 3.245 & 426.6 & 2.849 & 371.1 & 2.674 & 370.2 & 3.192 & 414.7 & 3.08 & 451.7 & 3.263 & 448.2 \\
\hline 2.784 & 376.3 & 2.754 & 371.5 & 3.124 & 405.4 & 3.05 & 401.5 & 2.751 & 346.4 & 2.653 & 347.3 & 2.807 & 394 & 2.988 & 419.1 & 3.201 & 416.4 \\
\hline 2.369 & 334.9 & 2.745 & 346.8 & 3.032 & 380.8 & 2.594 & 364.9 & 2.452 & 322.6 & 2.431 & 323.9 & 2.656 & 370.2 & 3.006 & 394 & 2.914 & 391.3 \\
\hline 2.452 & 306.7 & 2.39 & 325.7 & 2.719 & 355.6 & 2.5 & 340.2 & 2.47 & 293.1 & 2.384 & 301 & 2.772 & 336.2 & 2.852 & 369.3 & 2.739 & 367.1 \\
\hline 1.982 & 284.7 & 2.405 & 302.7 & 2.695 & 328.7 & 2.378 & 315.1 & 2.032 & 264.4 & 2.1 & 277.6 & 2.343 & 317.3 & 2.55 & 341.1 & 2.745 & 337.6 \\
\hline 2.038 & 249.9 & 2.245 & 275.9 & 2.402 & 302.7 & 2.177 & 289.1 & 2.067 & 239.7 & 2.103 & 248.5 & 2.328 & 292.2 & 2.576 & 317.3 & 2.34 & 311.6 \\
\hline & & & & & & & & & & & & & & & & & \\
\hline
\end{tabular}


Table 6. $50{ }^{\circ} \mathrm{C}$ Diluted

\begin{tabular}{|c|c|c|c|c|c|c|c|c|c|c|c|c|c|c|c|c|c|}
\hline run1 & & run2 & & run3 & & run4 & & run5 & & run6 & & run7 & & run8 & & run9 & \\
\hline [Pa] & [1/s] & [Pa] & {$[1 / s]$} & [Pa] & {$[1 / s]$} & {$[\mathrm{Pa}]$} & [1/s] & [Pa] & [1/s] & [Pa] & [1/s] & [Pa] & [1/s] & [Pa] & {$[1 / s]$} & [Pa] & {$[1 / s]$} \\
\hline 2.462 & 318.2 & 3.548 & 348.1 & 3.219 & 334.5 & 3.261 & 338.9 & 3.264 & 344.2 & 3.338 & 347.7 & 2.87 & 308.9 & 2.734 & 317.3 & 2.527 & 323 \\
\hline 2.909 & 22.6 & 583 & 368.9 & 3.53 & 361.8 & .388 & 358.7 & 3.361 & 374.1 & 314 & 375.5 & 3.136 & 321.7 & 3.024 & 324.8 & 2.873 & 322.6 \\
\hline 3.403 & 52.1 & 785 & 398.4 & 3.58 & 386.9 & .568 & 389.6 & 3.645 & 397.5 & 3.358 & 398.8 & 3.261 & 343.3 & 3.237 & 344.6 & 3.148 & 338 \\
\hline 3.497 & 75.9 & 3.93 & 420 & 3.672 & 408.5 & .847 & 411.6 & 3.773 & 420.4 & 3.628 & 421.3 & 3.506 & 372.8 & 3.607 & 375.9 & 3.385 & 362.2 \\
\hline 3.492 & 383.4 & 4.045 & 444.2 & 4.057 & 449.5 & 3.832 & 435.4 & 3.861 & 446.9 & 3.796 & 445.5 & 3.619 & 401.9 & 3.779 & 399.7 & 3.752 & 401.9 \\
\hline 3.811 & 28.8 & 4.16 & 468.4 & 4.063 & 458.8 & 4.208 & 471.5 & 3.956 & 474.6 & 3.965 & 473.3 & 3.859 & 430.1 & 3.808 & 424.8 & 3.941 & 424.8 \\
\hline 4.001 & 453.9 & 4.616 & 510.8 & 4.285 & 485.2 & 4.335 & 495.8 & 4.151 & 501.9 & 4.359 & 510.3 & 3.947 & 454.8 & 3.921 & 451.3 & 3.983 & 454.8 \\
\hline 4.098 & 484.8 & 4.835 & 535 & 4.524 & 529.3 & 4.492 & 525.3 & 4.406 & 531 & 4.521 & 533.7 & 4.027 & 478.6 & 4.134 & 478.1 & 4.042 & 479 \\
\hline 4.172 & 512.5 & 4.903 & 561.4 & 4.708 & 553.5 & 4.764 & 549.1 & 4.554 & 557 & 4.584 & 561 & 4.311 & 512.1 & 4.311 & 505.5 & 4.128 & 505 \\
\hline 4.483 & 534.1 & 4.974 & 587.9 & 4.773 & 576.4 & & 579.5 & 4.782 & 594.9 & 4.569 & 589.2 & 4.447 & 538.5 & 4.424 & 536.8 & 4.276 & 530.6 \\
\hline 4.56 & 562.8 & 4.98 & 615.6 & 4.927 & 608.1 & 4.841 & 604.2 & 4.874 & 617.4 & 4.581 & 613.9 & 4.625 & 563.6 & 4.415 & 559.2 & 4.394 & 554.8 \\
\hline 4.776 & 585.2 & & & & 632.8 & & & & 647.8 & & 638.1 & & 587.4 & & 584.3 & & 581.3 \\
\hline 5.172 & 620.9 & 5.427 & 663.2 & 5.3 & 654 & 5.22 & 661.5 & 5.181 & 672.5 & 4.862 & 677.8 & 4.868 & 612.5 & 4.764 & 609.9 & 4.726 & 604.2 \\
\hline 5.255 & 644.3 & 5.602 & 700.2 & 5.368 & 678.7 & 5.362 & 685.7 & 5.288 & 694.1 & 5.045 & 702 & 5.17 & 650 & 4.986 & 632.8 & 4.945 & 646.5 \\
\hline 5.255 & 676 & 5.779 & 725.8 & 5.397 & 707.3 & 5.59 & 720.5 & 5.273 & 719.6 & 5.172 & 728.4 & 5.359 & 673.4 & 5.226 & 672.9 & 5.066 & 668.1 \\
\hline 5.513 & 699.4 & 5.847 & 754.4 & 5.684 & 731.5 & 5.732 & 746.1 & 5.613 & 760.6 & 5.246 & 750 & 5.433 & 702 & 5.285 & 701.6 & 5.175 & 702.4 \\
\hline 5.658 & 723.2 & 5.992 & 774.7 & 5.767 & 759.3 & & 766.8 & 5.877 & 784 & 94 & 780 & 5.424 & 726.7 & 5.291 & 723.6 & 318 & 727.1 \\
\hline 5.717 & 747.8 & 6.259 & 811.7 & 5.98 & 786.6 & 5.901 & 798.1 & 5.969 & 806 & 5.421 & 802 & 5.608 & 749.6 & 5.409 & 748.7 & 5.516 & 750.9 \\
\hline 5.954 & 788.4 & & 820.6 & 6.247 & 828.9 & & 824.1 & & 837.3 & & 828 & & 774.3 & 5.628 & 773.4 & 5.542 & 775.2 \\
\hline 6.14 & 810.9 & & 863.3 & 6.451 & 851 & & 850.5 & & 861.5 & & 852.3 & 63 & 802.9 & & 799.4 & 5.699 & 800.3 \\
\hline 6.253 & 832.4 & & 892.8 & & 882.2 & & 874.8 & & & & 893.7 & & 841.3 & 92 & 826.3 & 374 & 822.3 \\
\hline 6.501 & 869.5 & 6.777 & 914.4 & 6.614 & 907.4 & & 897.7 & 6.424 & 912.2 & & 918.4 & 6.371 & 869 & 6.022 & 853.2 & 6.117 & 859.3 \\
\hline 6.691 & 893.3 & 7.034 & 942.2 & 6.788 & 930.3 & 6.694 & 930.7 & 6.484 & 940.4 & 5.883 & 928.5 & 6.593 & 888.9 & 6.105 & 881.4 & 6.267 & 887.5 \\
\hline 6.791 & 921 & 7.102 & 971.7 & 6.883 & 955.4 & & 960.7 & 6.723 & 967.3 & & 959.4 & 6.602 & 920.6 & 6.492 & 921 & 6.436 & 912.2 \\
\hline 6.794 & 946.1 & 7.268 & 996.8 & 7.013 & 979.2 & & 986.7 & 6.856 & 988.9 & & 1003 & 6.664 & 945.3 & 6.62 & 944.8 & 6.484 & 944.8 \\
\hline 6.99 & 969.9 & & & & 1003 & & 1011 & & 1026 & & 1029 & 6.83 & 969.5 & 6.652 & 972.6 & 6.495 & 968.2 \\
\hline 7.129 & 994.2 & 7.431 & 1044 & 7.546 & 1044 & & 1039 & & 1052 & 6.744 & 1055 & 7.138 & 1005 & 6.815 & 997.7 & 6.667 & 992 \\
\hline 7.212 & 1022 & 7.765 & & 7.573 & 1074 & 7.306 & & & 1082 & & 1077 & 7.262 & 1029 & & 1026 & 6.848 & 1015 \\
\hline 7.425 & 1048 & 7.984 & 1106 & 7.691 & 1095 & 7.478 & 1096 & 7.386 & 1107 & 7.007 & 1105 & 7.404 & 1054 & 6.984 & 1047 & 6.907 & 1040 \\
\hline 7.712 & 1074 & 8.025 & 1133 & 7.8 & 1119 & & 1119 & 7.439 & 1133 & 7.093 & 1132 & 7.404 & 1084 & 7.265 & 1082 & 7.004 & 1069 \\
\hline 7.795 & 1100 & 8.12 & 1163 & 7.904 & 1146 & 7.783 & 1144 & 7.623 & 1160 & 7.149 & 1159 & 7.487 & 1108 & 7.324 & 1110 & 7.081 & 1099 \\
\hline
\end{tabular}


Table 6. $50{ }^{\circ} \mathrm{C}$ Diluted - continued

\begin{tabular}{|c|c|c|c|c|c|c|c|c|c|c|c|c|c|c|c|c|c|}
\hline run1 & & run2 & & run3 & & run4 & & run5 & & run6 & & run7 & & run8 & & run9 & \\
\hline$[\mathrm{Pa}]$ & {$[1 / \mathrm{s}]$} & {$[\mathrm{Pa}]$} & {$[1 / \mathbf{s}]$} & {$[\mathrm{Pa}]$} & {$[1 / \mathrm{s}]$} & {$[\mathrm{Pa}]$} & {$[1 / \mathrm{s}]$} & {$[\mathrm{Pa}]$} & {$[1 / \mathrm{s}]$} & {$[\mathrm{Pa}]$} & {$[1 / \mathrm{s}]$} & {$[\mathrm{Pa}]$} & {$[1 / \mathrm{s}]$} & {$[\mathrm{Pa}]$} & {$[1 / \mathrm{s}]$} & {$[\mathrm{Pa}]$} & {$[1 / \mathrm{s}]$} \\
\hline 8.085 & 1142 & 8.28 & 1186 & 8.019 & 1172 & 7.895 & 1170 & 7.67 & 1183 & 7.256 & 1182 & 7.655 & 1132 & 7.389 & 1134 & 7.451 & 1140 \\
\hline 8.339 & 1170 & 8.564 & 1219 & 8.395 & 1210 & 8.203 & 1207 & 8.011 & 1223 & 7.587 & 1221 & 7.759 & 1159 & 7.587 & 1157 & 7.546 & 1164 \\
\hline 8.422 & 1195 & 8.688 & 1242 & 8.437 & 1231 & 8.333 & 1229 & 8.162 & 1248 & 7.451 & 1232 & 8.028 & 1198 & 7.703 & 1193 & 7.664 & 1187 \\
\hline 8.611 & 1220 & 8.745 & 1264 & 8.549 & 1262 & 8.348 & 1258 & 8.262 & 1277 & 7.65 & 1257 & 8.005 & 1207 & 7.821 & 1221 & 7.682 & 1218 \\
\hline 8.694 & 1249 & 9.014 & 1295 & 8.638 & 1285 & 8.531 & 1287 & 8.176 & 1284 & 7.993 & 1300 & 8.463 & 1247 & 7.951 & 1243 & 7.851 & 1240 \\
\hline 8.94 & 1275 & 9.153 & 1326 & 8.727 & 1307 & 8.665 & 1309 & 8.41 & 1315 & 8.144 & 1327 & 8.526 & 1272 & 8.176 & 1268 & 8.031 & 1275 \\
\hline 9.088 & 1303 & 9.224 & 1353 & 9.112 & 1345 & 8.715 & 1337 & 8.579 & 1344 & 8.253 & 1352 & 8.54 & 1306 & 8.159 & 1306 & 8.144 & 1304 \\
\hline 9.141 & 1332 & 9.268 & 1360 & 9.12 & 1368 & 8.771 & 1361 & 8.821 & 1383 & 8.369 & 1383 & 8.718 & 1330 & 8.318 & 1328 & 8.129 & 1329 \\
\hline 9.363 & 1359 & 9.567 & 1401 & 9.251 & 1393 & 8.904 & 1388 & 8.765 & 1392 & 8.694 & 1406 & 8.759 & 1360 & 8.395 & 1349 & 8.312 & 1354 \\
\hline 9.452 & 1382 & 9.541 & 1436 & 9.404 & 1421 & 9.168 & 1411 & 8.872 & 1424 & 8.887 & 1431 & 8.961 & 1378 & 8.49 & 1374 & 8.422 & 1378 \\
\hline 9.502 & 1412 & 9.757 & 1460 & 9.44 & 1447 & 9.277 & 1441 & 9.091 & 1450 & 9.212 & 1468 & 9.212 & 1422 & 8.774 & 1409 & 8.526 & 1400 \\
\hline 9.659 & 1435 & 9.804 & 1484 & 9.552 & 1471 & 9.547 & 1484 & 9.325 & 1495 & 9.286 & 1495 & 9.224 & 1435 & 9.029 & 1432 & 8.878 & 1436 \\
\hline 9.816 & 1459 & 9.958 & 1509 & 9.928 & 1508 & 9.629 & 1508 & 9.505 & 1517 & 9.366 & 1522 & 9.431 & 1465 & 9.055 & 1463 & 8.857 & 1466 \\
\hline 10.01 & 1484 & 10.27 & 1545 & 9.993 & 1537 & 9.792 & 1529 & 9.615 & 1546 & 9.511 & 1542 & 9.452 & 1490 & 9.114 & 1486 & 9.032 & 1488 \\
\hline 10.25 & 1520 & 10.44 & 1570 & 10.08 & 1560 & 9.825 & 1560 & 9.662 & 1571 & 9.547 & 1568 & 9.641 & 1520 & 9.18 & 1507 & 9.114 & 1512 \\
\hline 8.656 & 1521 & 8.167 & 1464 & 8.019 & 1462 & 7.854 & 1456 & 8.073 & 1477 & 7.803 & 1478 & 8.144 & 1522 & 7.294 & 1381 & 9.117 & 1534 \\
\hline 8.555 & 1496 & 8.073 & 1429 & 7.975 & 1438 & 7.721 & 1432 & 7.67 & 1434 & 7.721 & 1451 & 7.925 & 1485 & 7.194 & 1355 & 9.473 & 1571 \\
\hline 8.354 & 1471 & 7.993 & 1403 & 7.792 & 1415 & 7.599 & 1406 & 7.481 & 1411 & 7.626 & 1421 & 7.866 & 1456 & 7.084 & 1332 & 9.407 & 1602 \\
\hline 8.259 & 1451 & 7.762 & 1378 & 7.599 & 1376 & 7.484 & 1376 & 7.475 & 1378 & 7.398 & 1399 & 7.712 & 1435 & 7.061 & 1305 & 9.591 & 1628 \\
\hline 8.07 & 1422 & 7.667 & 1351 & 7.383 & 1353 & 7.36 & 1352 & 7.407 & 1355 & 7.303 & 1367 & 7.602 & 1412 & 6.658 & 1270 & 9.748 & 1653 \\
\hline 7.661 & 1382 & 7.436 & 1326 & 7.318 & 1321 & 7.191 & 1330 & 7.223 & 1333 & 7.223 & 1346 & 7.286 & 1378 & 6.552 & 1246 & 9.916 & 1676 \\
\hline 7.726 & 1354 & 7.226 & 1292 & 7.194 & 1296 & 7.016 & 1296 & 7.046 & 1310 & 7.052 & 1319 & 7.294 & 1348 & 6.469 & 1217 & 7.161 & 1376 \\
\hline 7.579 & 1328 & 7.283 & 1282 & 7.114 & 1269 & 6.901 & 1267 & 6.774 & 1273 & 6.966 & 1289 & 7.194 & 1327 & 6.365 & 1188 & 7.226 & 1367 \\
\hline 7.374 & 1306 & 6.936 & 1241 & 6.809 & 1246 & 6.578 & 1246 & 6.797 & 1241 & 6.815 & 1263 & 7.013 & 1301 & 6.188 & 1163 & 7.087 & 1339 \\
\hline 7.256 & 1282 & 6.697 & 1218 & 6.741 & 1211 & 6.563 & 1212 & 6.584 & 1217 & 6.697 & 1239 & 6.948 & 1273 & 6.102 & 1141 & 6.898 & 1315 \\
\hline 7.067 & 1259 & 6.637 & 1186 & 6.531 & 1187 & 6.336 & 1189 & 6.51 & 1194 & 6.578 & 1214 & 6.729 & 1249 & 5.791 & 1108 & 6.629 & 1272 \\
\hline 7.007 & 1232 & 6.56 & 1161 & 6.35 & 1166 & 6.339 & 1165 & 6.339 & 1170 & 6.149 & 1170 & 6.407 & 1212 & 5.797 & 1079 & 6.436 & 1245 \\
\hline 6.711 & 1205 & 6.43 & 1138 & 6.303 & 1136 & 6.232 & 1140 & 6.054 & 1136 & 5.954 & 1147 & 6.199 & 1189 & 5.682 & 1055 & 6.344 & 1222 \\
\hline 6.64 & 1176 & 6.282 & 1115 & 6.143 & 1114 & 6.072 & 1114 & 5.93 & 1104 & 5.806 & 1121 & 6.096 & 1163 & 5.377 & 1033 & 6.294 & 1188 \\
\hline 6.439 & 1152 & 5.995 & 1078 & 6.066 & 1088 & 5.862 & 1086 & 5.806 & 1078 & 5.753 & 1098 & 6.019 & 1134 & 5.427 & 1007 & 6.223 & 1161 \\
\hline 6.217 & 1110 & 5.889 & 1049 & 5.679 & 1048 & 5.741 & 1058 & 5.631 & 1055 & 5.696 & 1068 & 5.841 & 1110 & 5.17 & 965.1 & 5.998 & 1137 \\
\hline
\end{tabular}


Table 6. $50{ }^{\circ} \mathrm{C}$ Diluted - continued

\begin{tabular}{|c|c|c|c|c|c|c|c|c|c|c|c|c|c|c|c|c|c|}
\hline run1 & & run2 & & run3 & & run4 & & run5 & & run6 & & run7 & & run8 & & run9 & \\
\hline$[\mathrm{Pa}]$ & {$[1 / \mathrm{s}]$} & {$[\mathrm{Pa}]$} & {$[1 / \mathrm{s}]$} & {$[\mathrm{Pa}]$} & {$[1 / \mathbf{s}]$} & {$[\mathrm{Pa}]$} & {$[1 / \mathbf{s}]$} & {$[\mathrm{Pa}]$} & {$[1 / \mathrm{s}]$} & {$[\mathrm{Pa}]$} & {$[1 / \mathrm{s}]$} & {$[\mathrm{Pa}]$} & {$[1 / \mathrm{s}]$} & {$[\mathrm{Pa}]$} & {$[1 / \mathbf{s}]$} & {$[\mathrm{Pa}]$} & {$[1 / \mathbf{s}]$} \\
\hline 6.031 & 1082 & 5.717 & 1023 & 5.486 & 1026 & 5.463 & 1032 & 5.67 & 1032 & 5.587 & 1047 & 5.829 & 1088 & 4.983 & 945.7 & 5.865 & 1109 \\
\hline 5.841 & 1055 & 5.723 & 999.9 & 5.483 & 992.9 & 5.3 & 995.1 & 5.427 & 1009 & 5.531 & 1021 & 5.542 & 1044 & 4.853 & 919.3 & 5.69 & 1087 \\
\hline 5.711 & 1031 & 5.596 & 972.6 & 5.279 & 973 & 5.229 & 969.5 & 5.365 & 983.6 & 5.362 & 995.1 & 5.264 & 1022 & 4.749 & 886.7 & 5.445 & 1051 \\
\hline 5.702 & 1001 & 5.332 & 947 & 5.202 & 939.1 & 5.016 & 943.1 & 5.01 & 943.9 & 5.297 & 966.4 & 5.22 & 992.4 & 4.666 & 861.5 & 5.288 & 1025 \\
\hline 5.61 & 974.3 & 5.09 & 908.7 & 5.072 & 915.3 & 4.921 & 919.3 & 4.927 & 917.9 & 5.087 & 940 & 5.066 & 964.7 & 4.575 & 840.4 & 5.235 & 997.3 \\
\hline 5.335 & 951 & 4.894 & 882.7 & 4.948 & 890.2 & 4.844 & 887.5 & 4.69 & 889.7 & 4.968 & 916.2 & 5.137 & 953.6 & 4.199 & 803.8 & 5.14 & 973 \\
\hline 5.276 & 928.5 & 782 & 856.7 & 4.838 & 864.2 & 4.658 & 864.6 & 4.723 & 864.6 & 4.743 & 871.2 & 4.971 & 922.8 & 4.092 & 775.6 & 5.054 & 953.6 \\
\hline 5.131 & 900.8 & 4.672 & 833.8 & 4.723 & 838.6 & 4.743 & 840.4 & 4.51 & 839.1 & 4.554 & 847 & .894 & 894.1 & 3.894 & 750.5 & 4.953 & 928.1 \\
\hline 4.82 & 863.7 & 4.394 & 806.4 & 4.424 & 798.5 & 4.421 & 801.2 & 4.314 & 810.9 & 4.498 & 823.2 & 4.566 & 867.7 & 3.731 & 727.1 & 4.575 & 888.9 \\
\hline 4.631 & 839.1 & 4.35 & 779.6 & 4.385 & 790.6 & 4.308 & 775.6 & 4.151 & 786.6 & 4.32 & 796.3 & 4.498 & 837.7 & 3.592 & 699.4 & 4.557 & 859.8 \\
\hline 4.625 & 811.7 & 4.098 & 749.6 & 4.08 & 750 & 4.184 & 751.8 & 4.137 & 758.9 & 4.193 & 770.8 & 4.329 & 814.4 & 3.607 & 673.8 & 4.436 & 834.2 \\
\hline 4.418 & 787.5 & 4.116 & 718.3 & 4.036 & 724.5 & 3.918 & 727.1 & 3.918 & 732.4 & 3.992 & 742.1 & 4.11 & 770.3 & 3.412 & 648.2 & 4.341 & 812.2 \\
\hline 4.362 & 762.8 & 3.974 & 699.4 & 3.752 & 694.5 & 3.79 & 700.2 & 3.601 & 696.7 & 3.856 & 713.5 & 4.069 & 761.9 & 3.204 & 616.5 & 4.113 & 786.6 \\
\hline 3.986 & 727.6 & 3.719 & 669 & 3.678 & 667.6 & 3.58 & 665 & 3.521 & 674.2 & 3.77 & 687 & 3.98 & 735.1 & 3.098 & 592.7 & 4.166 & 755.3 \\
\hline 3.912 & 703.3 & 3.675 & 647.4 & 3.574 & 643 & 3.418 & 638.5 & 3.494 & 644.3 & 3.657 & 662.3 & 3.64 & 698 & 3.003 & 556.6 & 3.782 & 729.3 \\
\hline 3.915 & 676 & 3.557 & 620.9 & 3.361 & 611.7 & 3.207 & 613.4 & 3.444 & 622.2 & 3.326 & 624.9 & 3.663 & 666.8 & 2.811 & 534.5 & 3.69 & 706 \\
\hline 3.708 & 654 & 3.563 & 591.4 & 3.267 & 593.2 & 3.122 & 589.6 & 3.302 & 595.8 & 3.163 & 602.9 & 3.503 & 643.8 & 2.695 & 507.2 & 3.604 & 680 \\
\hline 3.462 & 615.2 & 3.092 & 556.6 & 3.089 & 559.7 & 3.157 & 557 & 3.222 & 570.2 & 3.095 & 571.1 & 3.329 & 620.9 & 2.465 & 481.2 & 3.548 & 653.5 \\
\hline 3.382 & 590.1 & 3.101 & 532.3 & 2.974 & 531.5 & 3.045 & 531.5 & 3.051 & 545.6 & 2.932 & 550.9 & 3.08 & 582.1 & 2.343 & 453 & 3.228 & 613.9 \\
\hline 3.166 & 567.6 & 3.03 & 505.9 & 2.885 & 507.7 & 2.894 & 505 & 2.906 & 518.7 & 2.885 & 519.1 & 2.84 & 560.5 & 2.311 & 430.1 & 3.08 & 587.9 \\
\hline 3.119 & 34.1 & & 480.3 & 2.6 & 480.3 & 2.687 & 481.2 & 2.586 & 480 & & 496.2 & 2.906 & 527.9 & 2.05 & 395.7 & 3.039 & 562.8 \\
\hline 3.006 & 511.2 & 2.598 & 455.7 & 2.639 & 459.6 & 2.539 & 459.6 & 2.485 & 454.8 & 2.601 & 466.2 & 2.716 & 505.9 & 2.027 & 371.1 & 2.87 & 535 \\
\hline 2.817 & 490.5 & 2.42 & 427 & 2.556 & 432.8 & 2.183 & 422.6 & 2.237 & 431.4 & 2.249 & 442.4 & 2.69 & 479 & 1.772 & 344.2 & 2.82 & 511.2 \\
\hline 2.61 & 467.6 & 2.299 & 394 & 2.305 & 407.6 & 2.189 & 396.6 & 2.266 & 394 & 2.13 & 420 & 2.438 & 457.4 & 1.837 & 323 & 2.58 & 488.3 \\
\hline 2.53 & 441.6 & 2.231 & 370.6 & 1.959 & 366.6 & 2.071 & 370.6 & 2.035 & 366.6 & 2.006 & 383.4 & 2.444 & 431 & 1.494 & 284.7 & 2.391 & 448.2 \\
\hline 2.172 & 405.4 & 2.009 & 336.2 & 1.902 & 345.1 & 1.893 & 342.4 & 1.864 & 349 & 2.059 & 359.2 & 2.107 & 396.6 & 1.305 & 261.3 & 2.142 & 425.7 \\
\hline 2.189 & 374.1 & 1.68 & 313.3 & 1.858 & 311.6 & 1.926 & 321.3 & 1.725 & 314.2 & 1.793 & 334 & 2.068 & 367.1 & 1.313 & 252.5 & 2.009 & 395.7 \\
\hline 2.05 & 352.5 & 1.763 & 286.9 & 1.639 & 286.9 & 1.701 & 294.4 & 1.657 & 290.9 & 1.766 & 307.6 & 1.787 & 346.4 & 0 & 173.5 & 2.109 & 373.3 \\
\hline 1.846 & 327.4 & 1.42 & 263.5 & 1.586 & 264 & 1.609 & 269.3 & 1.411 & 267.5 & 1.491 & 283.8 & 1.911 & 322.1 & 0 & 174.4 & 1.867 & 347.7 \\
\hline 1.624 & 286.9 & 1.506 & 255.6 & 1.47 & 241.5 & 1.426 & 242.4 & 1.441 & 241.9 & 1.275 & 244.1 & 1.441 & 284.2 & 0 & 152.6 & 1.686 & 318.6 \\
\hline 1.387 & 264.4 & 0 & 169.3 & 0 & 152.7 & 0 & 151.6 & 0 & 156.2 & 1.266 & 238.9 & 1.408 & 254.3 & 0 & 127.5 & 1.526 & 286.9 \\
\hline 1.473 & 258.7 & 0 & 173.9 & 0 & 162.4 & 0 & 159.3 & 0 & 164.5 & 0 & 153.4 & 0 & 152.4 & 0 & 103.3 & 1.275 & 260.4 \\
\hline
\end{tabular}


Table 6. $50^{\circ} \mathrm{C}$ Diluted - continued

\begin{tabular}{|c|c|c|c|c|c|c|c|c|c|c|c|c|c|c|c|c|c|}
\hline run1 & {$[1 / \mathrm{s}]$} & run2 & {$[1 / s]$} & run3 & {$[1 / \mathrm{s}]$} & run4 & {$[1 / \mathrm{s}]$} & run5 & [1/s] & run6 & {$[1 / 1$} & run7 & {$[1 / \mathrm{s}]$} & run8 & [1/s] & run9 & {$[1 / \mathrm{s}]$} \\
\hline & & & {$[110]$} & & {$\left[\begin{array}{lll}{[} \\
\end{array}\right.$} & & {$[101]$} & & {$[115]$} & & $[1 / 1)]$ & & {$[115]$} & & {$[175]$} & 1.381 & 252.1 \\
\hline & & & & & & & & & & & & & & & & 0 & 169.3 \\
\hline & & & & & & & & & & & & & & & & 0 & 169.6 \\
\hline & & & & & & & & & & & & & & & & 0 & 152.4 \\
\hline & & & & & & & & & & & & & & & & 0 & 129.1 \\
\hline & & & & & & & & & & & & & & & & 0 & 92.81 \\
\hline & & & & & & & & & & & & & & & & & \\
\hline
\end{tabular}


This page intentionally left blank. 


\begin{tabular}{|ll|}
\hline APPENDIX C & WSRC-TR-2000-00352 \\
ENVELOPE B RHEOLOGY DATA & SRT-RPP-2000-00026 \\
Page 119 of 514
\end{tabular}

\section{APPENDIX C}

\section{ENVELOPE B RHEOLOGY DATA}

Table 1. Rheometer Response for Blank - As Received .................................................. 121

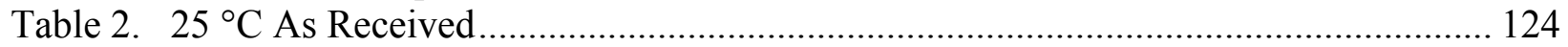

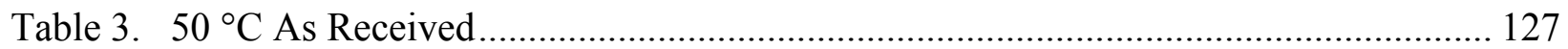

Table 4. Rheometer Response for Blank - Pretreated ....................................................... 130

Table 5. $25^{\circ} \mathrm{C}$ Pretreated ................................................................................................... 134

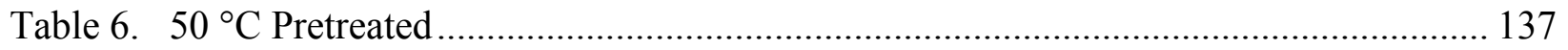

NOTE: Envelope $\mathrm{B}$ was received at $3 \mathrm{M}$ Na so there is not a diluted sample to be run as with Envelope A and Envelope C. 
This page intentionally left blank. 
Table 1. Rheometer Response for Blank - As Received

\begin{tabular}{|c|c|c|c|c|c|c|c|c|c|c|c|c|c|c|c|c|c|}
\hline run1 & & run2 & & run3 & & run4 & & run5 & & run6 & & run7 & & run8 & & run9 & \\
\hline$[\mathrm{Pa}]$ & {$[1 / \mathbf{s}]$} & {$[\mathrm{Pa}]$} & {$[1 / \mathrm{s}]$} & {$[\mathrm{Pa}]$} & {$[1 / \mathbf{s}]$} & {$[\mathrm{Pa}]$} & {$[1 / \mathrm{s}]$} & [Pa] & {$[1 / \mathrm{s}]$} & {$[\mathrm{Pa}]$} & {$[1 / \mathrm{s}]$} & {$[\mathrm{Pa}]$} & {$[1 / \mathrm{s}]$} & {$[\mathrm{Pa}]$} & {$[1 / \mathrm{s}]$} & {$[\mathrm{Pa}]$} & {$[1 / \mathrm{s}]$} \\
\hline 3.078 & 348.6 & 3.031 & 343.7 & 2.241 & 319.9 & 2.253 & 322.6 & 2.454 & 360 & 2.324 & 342 & 0.338 & 395.3 & 0.211 & 392.6 & 0.791 & 322.6 \\
\hline 3.442 & 368.4 & 179 & 364.4 & 2.723 & 321.7 & 2.522 & 344.6 & 2.714 & 379.4 & 2.43 & 372.4 & .516 & 323.9 & 1.374 & 322.6 & 1.279 & 322.6 \\
\hline 3.697 & 398.4 & 3.386 & 387.4 & 2.904 & 342.4 & 2.771 & 368.9 & 2.845 & 408.1 & 2.643 & 396.6 & 1.886 & 328.7 & 1.788 & 323.9 & 1.56 & 342.9 \\
\hline 3.913 & 420.4 & 3.552 & 410.7 & 3.167 & 362.7 & 2.883 & 394 & 3.025 & 431.9 & 2.848 & 421.7 & 2.143 & 346.4 & 2.007 & 340.6 & 1.741 & 371.5 \\
\hline 4.064 & 446.4 & 3.715 & 435.4 & 3.374 & 385.6 & 3.037 & 423.5 & 3.176 & 459.2 & 2.972 & 445.1 & 2.359 & 366.6 & 2.265 & 360.9 & 1.898 & 394.9 \\
\hline 4.514 & 485.2 & 4.191 & 475.5 & 3.774 & 424.8 & 3.185 & 446.4 & 3.3 & 484.3 & 3.135 & 470.6 & 2.484 & 390 & 2.398 & 384.7 & 2.057 & 419.5 \\
\hline 4.676 & 509.9 & 4.215 & 505.9 & 3.94 & 447.7 & 3.336 & 470.2 & 3.496 & 509 & 3.531 & 508.1 & 2.706 & 415.6 & 2.59 & 409.8 & 2.235 & 442.4 \\
\hline 4.884 & 533.7 & 4.345 & 530.1 & 4.061 & 470.6 & 3.647 & 509.9 & 3.635 & 534.1 & 3.641 & 532.8 & 3.061 & 455.7 & 2.957 & 449.9 & 2.507 & 482.1 \\
\hline 5.005 & 559.7 & 4.271 & 555.3 & 4.28 & 498 & 3.815 & 541.6 & 3.78 & 557.5 & 3.815 & 561.4 & 3.235 & 482.1 & 3.061 & 477.3 & 2.649 & 505.9 \\
\hline 5.088 & 583 & 4.416 & 577.7 & 4.381 & 531 & 3.762 & 548.2 & 4.147 & 603.3 & 3.984 & 591.4 & 3.389 & 511.2 & 3.2 & 507.2 & 2.765 & 537.2 \\
\hline 5.043 & 614.8 & 4.558 & 604.6 & 4.579 & 553.5 & 4.176 & 590.5 & 4.239 & 625.8 & 4.096 & 615.2 & 3.54 & 540.3 & 3.357 & 531.9 & 2.892 & 564.5 \\
\hline 5.147 & 639 & 4.7 & 629.7 & 4.691 & 581.3 & 4.351 & 614.3 & 4.286 & 657.1 & 4.17 & 640.8 & 3.632 & 566.7 & 3.508 & 555.3 & 3.072 & 586.5 \\
\hline 5.384 & 661.9 & 5.017 & 670.3 & 4.901 & 604.2 & 4.481 & 643.4 & 4.517 & 681.7 & 4.348 & 661.9 & 3.777 & 591.4 & 3.62 & 579.9 & 3.229 & 618.3 \\
\hline 5.703 & 699.8 & 5.177 & 694.5 & 5.058 & 628.9 & 4.6 & 666.8 & 4.617 & 707.7 & 4.375 & 686.1 & 3.928 & 615.2 & 3.972 & 617.8 & 3.271 & 643.8 \\
\hline 5.807 & 723.2 & 5.396 & 720.5 & 5.224 & 655.7 & 4.774 & 695 & 4.795 & 729.3 & 4.567 & 713.5 & 4.014 & 640.3 & 4.043 & 641.6 & 3.392 & 670.3 \\
\hline 5.949 & 753.6 & 5.473 & 744.8 & 5.558 & 697.6 & 4.934 & 720.5 & 4.789 & 757.1 & 4.762 & 741.7 & 4.173 & 665 & 4.197 & 664.1 & 3.608 & 695 \\
\hline 6.082 & 778.7 & 5.564 & 774.7 & 5.748 & 717.9 & 4.987 & 751.8 & 5.026 & 781.8 & 4.943 & 766.3 & 4.321 & 691.4 & 4.28 & 696.3 & 3.729 & 718.8 \\
\hline 6.227 & 801.6 & 5.733 & 797.6 & 5.78 & 746.1 & 5.097 & 780 & 5.322 & 821.9 & 5.265 & 810.4 & 4.431 & 717.9 & 4.401 & 721 & 3.78 & 742.6 \\
\hline 6.352 & 825.8 & 5.878 & 819.7 & 5.955 & 766.8 & 5.295 & 803.4 & 5.384 & 847 & 5.221 & 818.3 & 4.771 & 757.1 & 4.52 & 747 & 4.073 & 781.3 \\
\hline 6.801 & 863.7 & 5.976 & 844.8 & 6.301 & 805.1 & 5.603 & 837.3 & 5.526 & 875.2 & 5.689 & 863.7 & 4.895 & 784.4 & 4.611 & 772.5 & 4.188 & 802.5 \\
\hline 6.943 & 888.9 & 6.337 & 883.6 & 6.402 & 828 & 5.647 & 861.1 & 5.748 & 897.7 & 5.748 & 886.7 & 5.038 & 809.5 & 4.777 & 798.5 & 4.236 & 832.9 \\
\hline 7.154 & 911.3 & 6.402 & 914.4 & 6.55 & 860.2 & 5.538 & 871.7 & 5.967 & 922.8 & 5.843 & 918.4 & 5.085 & 836.4 & 4.931 & 822.3 & 4.354 & 856.7 \\
\hline 7.281 & 942.6 & 6.636 & 935.1 & 6.671 & 884.9 & 6.002 & 917.1 & 6.079 & 952.8 & 6.023 & 942.6 & 5.005 & 848.8 & 5.005 & 847.4 & 4.502 & 879.6 \\
\hline 7.396 & 969.1 & 6.719 & 961.6 & 6.869 & 904.3 & 6.112 & 941.3 & 6.346 & 973 & 6.218 & 969.9 & 5.467 & 891.9 & 177 & 872.1 & 4.582 & 905.6 \\
\hline 7.574 & 992.4 & & 984 & 7.08 & 930.3 & 6.1 & 971.7 & 6.736 & 999 & 6.381 & 995.1 & 5.544 & 915.3 & .487 & 912.2 & 4.777 & 932 \\
\hline 7.843 & 1016 & & 1023 & 7.299 & 959.8 & 6.295 & 995.5 & 7.041 & 1025 & 6.446 & 1021 & 5.709 & 941.3 & 641 & 937.8 & .881 & 958 \\
\hline 8.228 & 1057 & 7.352 & 1046 & 7.411 & 988.5 & 6.497 & 1019 & 7.346 & 1063 & 6.612 & 1044 & 5.792 & 969.9 & 5.644 & 970.8 & 4.996 & 984 \\
\hline 8.29 & 1084 & 7.47 & 1073 & 7.603 & 1020 & 6.585 & 1045 & 7.592 & 1089 & 6.739 & 1070 & 5.869 & 997.3 & 5.81 & 993.7 & 5.351 & 1027 \\
\hline 8.45 & 1107 & 7.577 & 1107 & 7.68 & 1047 & 6.751 & 1070 & 7.68 & 1120 & 6.792 & 1099 & 6.053 & 1025 & 5.925 & 1022 & 5.49 & 1052 \\
\hline 8.654 & 1133 & 7.722 & 1123 & 7.813 & 1070 & 6.967 & 1096 & 7.751 & 1145 & 7.003 & 1125 & 6.177 & 1054 & 6.082 & 1045 & 5.538 & 1078 \\
\hline 8.802 & 1164 & 7.961 & 1156 & 8.024 & 1095 & 7.47 & 1136 & 7.843 & 1168 & 7.08 & 1152 & 6.325 & 1077 & 6.195 & 1069 & 5.626 & 1109 \\
\hline
\end{tabular}


Table 1. Rheometer Response for Blank - As Received - continued

\begin{tabular}{|c|c|c|c|c|c|c|c|c|c|c|c|c|c|c|c|c|c|}
\hline run1 & & run2 & & run3 & & run4 & & run5 & & run6 & & run7 & & run8 & & run9 & \\
\hline [Pa] & {$[1 / s]$} & {$[\mathrm{Pa}]$} & {$[1 / s]$} & {$[\mathrm{Pa}]$} & {$[1 / s]$} & [Pa] & {$[1 / s]$} & [Pa] & {$[1 / s]$} & {$[\mathrm{Pa}]$} & {$[1 / \mathrm{s}]$} & {$[\mathrm{Pa}]$} & {$[1 / \mathrm{s}]$} & {$[\mathrm{Pa}]$} & {$[1 / \mathrm{s}]$} & {$[\mathrm{Pa}]$} & {$[1 / \mathrm{s}]$} \\
\hline 8.941 & 1189 & 8.021 & 1183 & 8.092 & 1118 & 7.417 & 1167 & 8.009 & 1192 & 7.34 & 1191 & 6.443 & 1101 & 6.298 & 1091 & 5.789 & 1133 \\
\hline 9.071 & 1212 & 8.207 & 1207 & 8.485 & 1160 & 7.544 & 1189 & 8.139 & 1215 & 7.473 & 1213 & 6.594 & 1127 & 6.618 & 1133 & 5.94 & 1155 \\
\hline 9.252 & 1236 & 8.281 & 1232 & 8.669 & 1186 & 7.648 & 1211 & 8.417 & 1257 & 7.523 & 1246 & 6.739 & 1150 & 6.742 & 1159 & 5.97 & 1182 \\
\hline 9.571 & 1272 & 8.488 & 1256 & 8.846 & 1211 & 7.731 & 1245 & 8.616 & 1282 & 7.674 & 1267 & 6.778 & 1180 & 6.828 & 1183 & 6.13 & 1207 \\
\hline 9.66 & 1302 & 8.846 & 1296 & 8.923 & 1242 & 7.852 & 1268 & 8.755 & 1312 & 7.754 & 1290 & 6.982 & 1205 & 7.011 & 1210 & 6.405 & 1245 \\
\hline 9.847 & 1322 & 9.015 & 1325 & 9.083 & 1264 & 7.976 & 1292 & 8.858 & 1337 & 8.018 & 1329 & 7.065 & 1235 & 7.213 & 1239 & 6.502 & 1267 \\
\hline 9.891 & 1344 & 9.056 & 1348 & 9.196 & 1287 & 8.151 & 1315 & 8.997 & 1362 & 8.154 & 1353 & 7.393 & 1274 & 7.304 & 1267 & 6.526 & 1294 \\
\hline 10.3 & 1382 & 9.163 & 1375 & 9.32 & 1313 & 8.379 & 1352 & 9.125 & 1388 & 8.204 & 1380 & 7.583 & 1304 & 7.473 & 1292 & 6.766 & 1331 \\
\hline 10.43 & 1407 & 9.311 & 1400 & 9.518 & 1336 & 8.583 & 1377 & 9.237 & 1412 & 8.391 & 1401 & 7.734 & 1327 & 7.562 & 1325 & 6.938 & 1352 \\
\hline 10.56 & 1431 & 9.471 & 1423 & 9.77 & 1375 & 8.695 & 1405 & 9.459 & 1437 & 8.349 & 1437 & 7.831 & 1354 & 7.63 & 1349 & 7.083 & 1377 \\
\hline 10.85 & 1464 & 9.557 & 1449 & 9.864 & 1400 & 8.888 & 1432 & 9.574 & 1464 & 8.657 & 1459 & 7.973 & 1379 & 7.781 & 1374 & 7.071 & 1409 \\
\hline 10.93 & 1490 & 9.986 & 1488 & 9.891 & 1429 & 8.962 & 1463 & 9.275 & 1504 & 8.74 & 1482 & 8.006 & 1412 & 7.885 & 1397 & 6.935 & 1646 \\
\hline 11.1 & 1513 & 10.11 & 1513 & 10.02 & 1452 & 9.122 & 1488 & 9.388 & 1526 & 8.908 & 1512 & 8.204 & 1436 & 8.971 & 1629 & 6.866 & 1622 \\
\hline 9.897 & 1523 & 8.583 & 1542 & 8.571 & 1585 & 8.459 & 1580 & 7.624 & 1519 & 7.518 & 1543 & 8.755 & 1598 & 8.891 & 1602 & 6.801 & 1590 \\
\hline 9.716 & 1497 & 8.479 & 1513 & 8.243 & 1543 & 8.26 & 1554 & 7.485 & 1494 & 7.405 & 1516 & 8.616 & 1574 & 8.642 & 1579 & 6.642 & 1572 \\
\hline 9.527 & 1470 & 8.317 & 1488 & 8.287 & 1534 & 8.121 & 1528 & 7.322 & 1461 & 7.148 & 1490 & 8.533 & 1543 & 8.462 & 1556 & 6.476 & 1544 \\
\hline 9.133 & 1428 & 8.18 & 1461 & 7.941 & 1488 & 7.751 & 1485 & 7.18 & 1436 & 7.091 & 1464 & 8.441 & 1519 & 8.175 & 1519 & 6.174 & 1517 \\
\hline 9.003 & 1406 & 8.101 & 1437 & 7.813 & 1463 & 7.58 & 1464 & 7.056 & 1413 & 6.926 & 1436 & 8.257 & 1493 & 8.077 & 1494 & 6.121 & 1487 \\
\hline 8.849 & 1375 & 7.908 & 1411 & 7.645 & 1438 & 7.494 & 1439 & 6.92 & 1391 & 6.73 & 1408 & 8.083 & 1471 & 7.944 & 1463 & 6.005 & 1459 \\
\hline 8.74 & 1352 & 7.654 & 1386 & 7.467 & 1409 & 7.402 & 1416 & 6.571 & 1351 & 6.585 & 1383 & 7.766 & 1434 & 7.825 & 1441 & 5.988 & 1432 \\
\hline 8.559 & 1324 & 7.364 & 1347 & 7.464 & 1386 & 7.091 & 1378 & 6.517 & 1325 & 6.452 & 1360 & 7.654 & 1404 & 7.71 & 1419 & 5.745 & 1411 \\
\hline 8.45 & 1299 & 7.192 & 1326 & 7.242 & 1363 & 7.597 & 1349 & 6.286 & 1299 & 6.352 & 1334 & 7.568 & 1380 & 7.396 & 1376 & 5.659 & 1387 \\
\hline 8.204 & 1274 & 7.13 & 1295 & 7.165 & 1336 & 7.449 & 1325 & 6.133 & 1276 & 6.198 & 1308 & 7.405 & 1357 & 7.479 & 1371 & 5.336 & 1346 \\
\hline 8.035 & 1250 & 6.952 & 1272 & 6.92 & 1310 & 7.299 & 1300 & 6.094 & 1250 & 6.035 & 1280 & 7.201 & 1334 & 7.035 & 1326 & 5.212 & 1317 \\
\hline 7.686 & 1211 & 6.887 & 1248 & 6.745 & 1284 & 6.967 & 1276 & 5.869 & 1219 & 5.908 & 1252 & 7.121 & 1310 & 6.864 & 1304 & 5.301 & 1308 \\
\hline 7.523 & 1189 & 6.668 & 1223 & 6.639 & 1259 & 6.689 & 1250 & 5.766 & 1191 & 5.757 & 1227 & 6.911 & 1283 & 6.748 & 1279 & 5.049 & 1283 \\
\hline 7.405 & 1161 & 6.363 & 1183 & 6.34 & 1217 & 6.423 & 1227 & 5.609 & 1167 & 5.384 & 1189 & 6.576 & 1244 & 6.585 & 1249 & 4.961 & 1252 \\
\hline 7.269 & 1130 & 6.242 & 1159 & 6.201 & 1191 & 5.949 & 1187 & 5.467 & 1144 & 5.236 & 1163 & 6.497 & 1218 & 6.497 & 1219 & 4.824 & 1222 \\
\hline 7.091 & 1105 & 6.088 & 1131 & 6.062 & 1169 & 5.848 & 1160 & 5.162 & 1106 & 5.183 & 1133 & 6.257 & 1189 & 6.278 & 1192 & 4.605 & 1196 \\
\hline 6.914 & 1081 & 5.928 & 1103 & 5.896 & 1140 & 5.949 & 1153 & 5.046 & 1081 & 5.091 & 1112 & 6.106 & 1166 & 6.165 & 1166 & 4.428 & 1166 \\
\hline 6.721 & 1052 & 5.816 & 1079 & 5.718 & 1116 & 5.499 & 1111 & 4.872 & 1059 & 4.984 & 1089 & 5.985 & 1137 & 5.964 & 1140 & 4.416 & 1143 \\
\hline
\end{tabular}


Table 1. Rheometer Response for Blank - As Received - continued

\begin{tabular}{|c|c|c|c|c|c|c|c|c|c|c|c|c|c|c|c|c|c|}
\hline run1 & & run2 & & run3 & & run4 & & run5 & & run6 & & run7 & & run8 & & run9 & \\
\hline [Pa] & {$[1 / \mathbf{s}]$} & {$[\mathrm{Pa}]$} & {$[1 / \mathrm{s}]$} & {$[\mathrm{Pa}]$} & {$[1 / \mathbf{s}]$} & {$[\mathrm{Pa}]$} & {$[1 / \mathrm{s}]$} & {$[\mathrm{Pa}]$} & {$[1 / \mathrm{s}]$} & {$[\mathrm{Pa}]$} & {$[1 / \mathrm{s}]$} & {$[\mathrm{Pa}]$} & {$[1 / \mathrm{s}]$} & {$[\mathrm{Pa}]$} & {$[1 / \mathrm{s}]$} & {$[\mathrm{Pa}]$} & {$[1 / \mathrm{s}]$} \\
\hline 6.573 & 1028 & 5.689 & 1054 & 5.591 & 1085 & 5.41 & 1080 & 4.748 & 1026 & 4.827 & 1062 & 5.887 & 1107 & 5.825 & 1109 & 4.052 & 1103 \\
\hline 6.384 & 1004 & 5.535 & 1031 & 5.47 & 1060 & 5.159 & 1054 & 4.682 & 1003 & 4.588 & 1024 & 5.697 & 1082 & 5.635 & 1090 & 3.957 & 1074 \\
\hline 6.207 & 978.8 & 5.366 & 1005 & 5.304 & 1037 & 5.002 & 1029 & 4.564 & 979.6 & 4.419 & 1002 & 5.629 & 1055 & 5.538 & 1057 & 3.848 & 1052 \\
\hline 6.02 & 952.8 & 5.212 & 980.5 & 5.038 & 996.8 & 4.774 & 1004 & 4.17 & 941.3 & 4.321 & 974.8 & 5.41 & 1029 & 5.36 & 1031 & 3.753 & 1025 \\
\hline 5.668 & 911.3 & 4.798 & 936.9 & 4.94 & 971.3 & 4.685 & 970.8 & 4.301 & 932 & 4.141 & 946.1 & 5.194 & 1000 & 5.236 & 1003 & 3.647 & 997.3 \\
\hline 5.544 & 884 & 4.718 & 913.1 & 4.893 & 950.1 & 4.605 & 945.7 & 3.963 & 889.7 & 4.093 & 923.7 & 5.07 & 974.3 & 5.023 & 981.4 & 3.481 & 970.8 \\
\hline 5.416 & 862.9 & 4.576 & 888.9 & 4.934 & 921 & 4.499 & 922.8 & 3.842 & 866.4 & 3.993 & 899 & 4.913 & 947 & 4.922 & 958 & 3.336 & 947.5 \\
\hline 5.333 & 831.6 & 4.457 & 862 & 4.573 & 891.5 & 4.357 & 898.1 & 3.706 & 834.2 & 3.688 & 859.3 & 4.819 & 921.9 & 4.558 & 916.2 & 3.238 & 922.8 \\
\hline 5.052 & 807.3 & 4.33 & 832.4 & 4.389 & 866.8 & 4.274 & 871.7 & 3.655 & 810.4 & 3.51 & 830.2 & 4.594 & 897.2 & 4.44 & 888 & 2.954 & 883.1 \\
\hline 4.981 & 781.3 & 4.256 & 810 & 4.224 & 845.2 & 4.407 & 842.6 & 3.546 & 786.6 & 3.587 & 824.5 & 4.268 & 858.9 & 4.286 & 865.1 & 2.83 & 860.7 \\
\hline 4.777 & 753.6 & 4.091 & 786.6 & 3.919 & 806 & 4.046 & 815.3 & 3.368 & 760.2 & 3.259 & 776.5 & 4.114 & 831.6 & 4.111 & 838.6 & 2.806 & 830.2 \\
\hline 4.54 & 728.9 & 3.735 & 746.1 & 3.795 & 778.2 & 3.777 & 791 & 3.271 & 729.8 & 3.084 & 754.9 & 3.996 & 807.8 & 4.049 & 812.6 & 2.676 & 804.7 \\
\hline 4.431 & 705.1 & 3.647 & 722.3 & 3.564 & 758 & 3.706 & 749.6 & 3.022 & 703.3 & 2.975 & 724 & 3.957 & 782.7 & 3.901 & 782.2 & 2.51 & 781.3 \\
\hline 4.037 & 665.4 & 3.593 & 692.8 & 3.475 & 731.1 & 3.395 & 726.2 & 2.913 & 679.5 & 2.945 & 698.9 & 3.65 & 758.9 & 3.759 & 758 & 2.466 & 757.5 \\
\hline 3.877 & 640.3 & 3.41 & 669 & 3.434 & 698 & 3.123 & 699.8 & 2.791 & 651.8 & 2.735 & 678.7 & 3.587 & 726.2 & 3.644 & 733.7 & 2.131 & 718.3 \\
\hline 3.75 & 612.5 & 3.277 & 643.8 & 3.244 & 676 & 2.993 & 673.4 & 2.661 & 627.1 & 2.7 & 655.7 & 3.457 & 702.4 & 3.472 & 709.9 & 2.161 & 709.5 \\
\hline 3.567 & 589.2 & 3.117 & 620 & 3.117 & 651.8 & 3.2 & 651.3 & 2.489 & 601.5 & 2.356 & 615.2 & 3.36 & 676.9 & 3.333 & 684.8 & 2.004 & 680.9 \\
\hline 3.49 & 555.7 & 2.948 & 596.2 & 3.001 & 625.8 & 3.271 & 615.2 & 2.194 & 559.2 & 2.356 & 581.7 & 2.978 & 639.9 & 3.01 & 644.7 & 1.924 & 653.1 \\
\hline 3.318 & 532.3 & 2.64 & 558.3 & 2.848 & 602.9 & 3.173 & 588.8 & 2.043 & 532.3 & 2.377 & 576.9 & 2.901 & 616.1 & 2.922 & 616.5 & 1.575 & 609.5 \\
\hline 3.223 & 507.2 & 2.605 & 529.7 & 2.489 & 561.9 & 3.022 & 565.4 & 1.904 & 509.4 & 1.96 & 534.1 & 2.774 & 591.4 & 2.853 & 592.3 & 1.433 & 580.4 \\
\hline 3.04 & 484.8 & 2.454 & 506.3 & 2.407 & 536.8 & 2.919 & 542 & 1.812 & 479.5 & 1.794 & 511.6 & 2.555 & 565 & 2.676 & 568 & 1.261 & 558.8 \\
\hline 2.88 & 460.5 & 2.306 & 483.9 & 2.315 & 508.1 & 2.771 & 517.8 & 1.75 & 455.7 & 1.761 & 476.4 & 2.451 & 537.6 & 2.555 & 541.2 & 1.181 & 528.8 \\
\hline 2.519 & 420.9 & 2.17 & 458.8 & 2.208 & 483.9 & 2.611 & 490.9 & 1.605 & 429.7 & 1.616 & 453.5 & 2.312 & 505.5 & 2.41 & 515.6 & 1.057 & 500.6 \\
\hline 2.362 & 395.7 & 1.995 & 433.6 & 1.977 & 460.1 & 2.469 & 464 & 1.421 & 401 & 1.525 & 430.5 & 2.196 & 479 & 2.259 & 488.7 & 0.906 & 475.9 \\
\hline 2.27 & 366.6 & 1.667 & 393.1 & 1.841 & 435 & 2.259 & 442 & 1.279 & 375.9 & 1.386 & 407.6 & 2.01 & 453.9 & 2.063 & 464 & 0.746 & 448.6 \\
\hline 2.149 & 341.1 & 1.528 & 367.5 & 1.738 & 408.1 & 1.983 & 398.4 & 1.134 & 350.3 & 1.226 & 382.1 & 1.862 & 426.6 & 1.989 & 438.9 & 0.587 & 422.6 \\
\hline 1.986 & 313.8 & 1.492 & 338.9 & 1.631 & 383.8 & 1.75 & 375.5 & 1.045 & 324.8 & 1.167 & 357.4 & 1.756 & 399.3 & 1.806 & 414.2 & 0.569 & 391.8 \\
\hline 1.812 & 292.2 & 1.309 & 315.1 & 1.229 & 343.3 & 1.466 & 347.3 & 0.909 & 299.2 & 0.764 & 316.4 & 1.631 & 371.5 & 1.466 & 371.5 & 0.501 & 366.6 \\
\hline 1.676 & 267.5 & 1.214 & 291.3 & 1.155 & 314.2 & 1.255 & 318.6 & 0.735 & 271.5 & 0.684 & 286.9 & 1.297 & 349.9 & 1.344 & 344.6 & & \\
\hline 1.468 & 245.9 & 1.063 & 265.7 & 0.971 & 290 & 0.998 & 292.6 & 0.581 & 245 & 0.495 & 260.4 & 1.158 & 324.3 & & & & \\
\hline & & & & & & & & & & & & & & & & & \\
\hline & & & & & & & & & & & & & & & & & \\
\hline
\end{tabular}


Table 2. $25^{\circ} \mathrm{C}$ As Received

\begin{tabular}{|c|c|c|c|c|c|c|c|c|c|c|c|c|c|c|c|c|c|}
\hline run1 & & run2 & & run3 & & run4 & & run5 & & run6 & & run7 & & run8 & & run9 & \\
\hline [Pa] & [1/s] & [Pa] & [1/s] & [Pa] & [1/s] & [Pa] & [1/s] & [Pa] & [1/s] & [Pa] & [1/s] & [Pa] & [1/s] & [Pa] & {$[1 / s]$} & [Pa] & [1/s] \\
\hline 4.293 & 305.8 & 3.639 & 314.6 & 3.769 & 302.7 & 3.748 & 327.9 & 2.932 & 359.2 & 3.704 & 327.9 & 3.589 & 323.5 & 3.775 & 323.5 & 3.106 & 353.9 \\
\hline 4.802 & 19.9 & .379 & 314.6 & 4.769 & 319.9 & 4.586 & 325.2 & 4.047 & 326.1 & 4.601 & 325.7 & 4.311 & 320.4 & 4.033 & 327.9 & 3.929 & 324.3 \\
\hline 4.997 & 341.5 & 4.98 & 339.8 & 4.743 & 340.2 & 5.122 & 347.7 & 4.74 & 340.6 & 5.142 & 342.4 & 4.521 & 333.6 & 4.618 & 344.6 & 4.713 & 334.5 \\
\hline 5.361 & 371.9 & 5.068 & 360.9 & 5.296 & 363.6 & 5.178 & 369.3 & 4.994 & 364.4 & 5.394 & 371.9 & 5.284 & 370.2 & 5.163 & 370.2 & 4.897 & 356.1 \\
\hline 5.811 & 397.5 & 361 & 388.2 & 5.622 & 391.8 & 5.583 & 393.1 & 5.486 & 388.2 & 5.637 & 394.4 & 5.388 & 391.3 & 5.252 & 394 & 5.077 & 379.4 \\
\hline 5.897 & 420 & 817 & 412 & 5.903 & 420.4 & 5.965 & 421.7 & 5.876 & 412.9 & 5.808 & 427 & 5.69 & 422.6 & 5.823 & 433.2 & 5.545 & 403.2 \\
\hline 6.098 & 444.2 & 6.14 & 442.4 & 5.983 & 445.1 & 6.424 & 446.4 & 5.938 & 439.4 & 6.119 & 450.8 & 6.122 & 445.5 & 5.971 & 457.9 & 5.817 & 446 \\
\hline 6.803 & 483 & 6.279 & 464.9 & 6.211 & 467.6 & 6.453 & 470.6 & 6.08 & 462.3 & 6.436 & 474.6 & 6.442 & 480.8 & 6.036 & 480.8 & 6.234 & 471.5 \\
\hline 6.906 & 505.9 & 6.397 & 487.8 & 6.936 & 508.1 & 6.959 & 508.5 & 6.82 & 500.6 & 6.717 & 498.9 & 6.699 & 505 & 6.273 & 510.8 & 6.613 & 495.3 \\
\hline 7.332 & 537.2 & & 527.9 & 7.181 & 531.5 & 7.294 & 529.7 & 7.019 & 525.3 & & 536.3 & 7.051 & 527.9 & 6.489 & 534.1 & 6.838 & 522.2 \\
\hline 7.649 & 561.4 & 7.341 & 550 & 7.554 & 560.1 & 7.394 & 561.4 & 7.175 & 550.9 & 7.412 & 557.5 & 7.128 & 561.4 & 6.646 & 559.2 & 6.877 & 554.8 \\
\hline 7.936 & 586.5 & & 576.4 & & 583.9 & 7.602 & 585.2 & & 581.7 & & 589.6 & & & 6.93 & 582.6 & 7.11 & 579.1 \\
\hline 8.155 & 610.8 & 7.664 & 607.3 & 8.075 & 609 & 7.856 & 608.1 & 7.625 & 606.8 & 7.634 & 613 & 7.613 & 606.8 & 7.406 & 623.6 & 7.454 & 605.9 \\
\hline 8.436 & 635 & 7.835 & 629.7 & 7.995 & 639.4 & 8.016 & 633.3 & 7.741 & 628.9 & 7.859 & 637.2 & 7.773 & 632.8 & 7.655 & 647.8 & 7.67 & 628.4 \\
\hline 8.492 & 661 & 8.291 & 659.3 & 8.353 & 665.4 & 8.501 & 673.4 & 7.945 & 655.7 & 8.25 & 662.3 & 8.176 & 658.8 & 7.974 & 674.2 & 7.983 & 653.5 \\
\hline 9.129 & 698.5 & 8.557 & 682.6 & 8.833 & 690.5 & 8.72 & 695 & 8.188 & 678.2 & 8.708 & 698.9 & 8.33 & 684.8 & 8.318 & 698 & 8.155 & 677.8 \\
\hline 9.247 & 721.8 & 557 & 710.4 & 8.85 & 717.4 & 8.945 & 725.8 & 578 & 706 & & 729.3 & 8.833 & 725.4 & 8.38 & 731.5 & 324 & 704.2 \\
\hline 9.505 & 754.9 & 9.01 & 734.6 & 9.167 & 740.3 & 8.987 & 750.5 & .646 & 732 & 8.788 & 735.9 & 8.948 & 748.7 & 8.581 & 758.4 & 8.735 & 733.3 \\
\hline 9.871 & 778.7 & 9.223 & 759.3 & 9.7 & 781.3 & & 772.5 & .028 & 761.5 & & 764.6 & 9.294 & 775.6 & & 783.5 & 8.93 & 756.2 \\
\hline 10.11 & 805.1 & .688 & 802.5 & 9.883 & 806.4 & 9.667 & 810.9 & .244 & 787.5 & 9.357 & 788.8 & 9.36 & 802 & 96 & 807.3 & 9.41 & 801.2 \\
\hline 10.35 & 827.6 & 10.11 & 825.8 & 10.09 & 829.8 & 9.502 & 819.2 & 9.738 & 832.9 & 9.88 & 830.2 & 9.5 & 832.9 & 17 & 830.7 & 9.605 & 823.6 \\
\hline 10.7 & 850.1 & 10.11 & 850.5 & 10.21 & 854.5 & 10.16 & 861.5 & 9.67 & 840.4 & 9.987 & 856.2 & 9.901 & 858.9 & 9.315 & 861.1 & 9.883 & 851 \\
\hline 11.04 & 889.7 & 10.4 & 879.6 & 10.54 & 884.5 & 10.38 & 885.3 & 9.931 & 864.6 & 10.23 & 888.9 & 10.16 & 881.4 & 9.661 & 889.7 & 10.1 & 879.2 \\
\hline 11.21 & 919.7 & 10.82 & 901.6 & 10.75 & 907.4 & 10.52 & 915.7 & 10.51 & 909.1 & & 912.2 & 10.38 & 913.1 & 10 & 914.4 & 10.25 & 904.3 \\
\hline 11.52 & 937.8 & 10.98 & 932.9 & 11.01 & 930.3 & 10.88 & 940 & 10.76 & 934.7 & & 937.3 & 10.7 & 932.9 & 10.03 & 939.5 & 10.37 & 936.5 \\
\hline 11.9 & 968.6 & & 956.3 & & 955 & 11.14 & 964.2 & 0.78 & 958.5 & & 963.8 & 10.74 & 957.2 & 10.37 & 965.1 & 10.69 & 961.1 \\
\hline 12.04 & 996.4 & 11.22 & 981.8 & 11.56 & 983.6 & 11.24 & 989.8 & 11.05 & 989.8 & 11.09 & 987.1 & 11.14 & 997.7 & 10.7 & 1006 & 10.76 & 981.8 \\
\hline 12.28 & 1022 & 11.56 & 1006 & 11.76 & 1008 & 11.59 & 1029 & 11.26 & 1014 & 11.61 & 1030 & 11.38 & 1022 & 11.08 & 1031 & 11.12 & 1019 \\
\hline 12.52 & 1044 & 11.71 & 1033 & 12.27 & 1050 & 11.82 & 1052 & 11.58 & 1036 & 11.51 & 1036 & 11.56 & 1051 & 11.34 & 1056 & 11.28 & 1048 \\
\hline 12.64 & 1071 & 12.09 & 1059 & 12.39 & 1079 & 12 & 1083 & 11.62 & 1065 & 12.02 & 1080 & 11.8 & 1076 & 11.23 & 1066 & 11.45 & 1073 \\
\hline 13.28 & 1111 & 12.49 & 1100 & 12.55 & 1102 & 12.19 & 1106 & 11.85 & 1092 & 12.16 & 1107 & 11.97 & 1102 & 11.72 & 1111 & 11.62 & 1097 \\
\hline
\end{tabular}


Table 2. $25^{\circ} \mathrm{C}$ As Received - continued

\begin{tabular}{|c|c|c|c|c|c|c|c|c|c|c|c|c|c|c|c|c|c|}
\hline run1 & & run2 & & run3 & & run4 & & run5 & & run6 & & run7 & & run8 & & run9 & \\
\hline [Pa] & {$[1 / s]$} & [Pa] & {$[1 / s]$} & [Pa] & {$[1 / s]$} & [Pa] & {$[1 / s]$} & [Pa] & {$[1 / s]$} & [Pa] & {$[1 / s]$} & [Pa] & {$[1 / s]$} & [Pa] & {$[1 / s]$} & [Pa] & {$[1 / s]$} \\
\hline 13.51 & 1136 & 12.69 & 1122 & 12.77 & 1125 & 12.53 & 1128 & 12.14 & 1117 & 12.38 & 1133 & 12.13 & 1126 & 11.93 & 1136 & 11.87 & 1122 \\
\hline 13.76 & 1166 & 12.83 & 1153 & 13.07 & 1149 & 12.91 & 1163 & 12.23 & 1141 & 12.67 & 1161 & 12.48 & 1152 & 12.17 & 1168 & 11.94 & 1149 \\
\hline 14.04 & 1189 & 12.95 & 1175 & 13.57 & 1187 & 13.05 & 1192 & 12.95 & 1182 & 12.88 & 1184 & 12.69 & 1184 & 12.44 & 1192 & 12.19 & 1174 \\
\hline 14.23 & 1212 & 13.11 & 1199 & 13.54 & 1216 & 13.24 & 1213 & 13.1 & 1205 & 13.04 & 1211 & 12.91 & 1211 & 12.57 & 1216 & 12.39 & 1199 \\
\hline 14.34 & 1240 & 13.48 & 1224 & 13.82 & 1239 & 13.42 & 1236 & 13.33 & 1237 & 13.32 & 1234 & 13.18 & 1237 & 12.51 & 1241 & 12.69 & 1222 \\
\hline 14.6 & 1262 & 3.91 & 1260 & 14.04 & 1264 & 13.85 & 1273 & 13.46 & 1262 & 13.55 & 1261 & 13.31 & 1263 & 12.82 & 1265 & 12.98 & 1264 \\
\hline 15.09 & 1302 & 14.01 & 1290 & 14.32 & 1287 & 14.05 & 1299 & 13.68 & 1290 & 13.77 & 1285 & 13.53 & 1287 & 13.14 & 1291 & 13.18 & 1289 \\
\hline 15.25 & 1328 & 14.19 & 1314 & 14.74 & 1326 & 14.3 & 1324 & 13.91 & 1314 & 14.25 & 1327 & 13.78 & 1312 & 13.26 & 1315 & 13.34 & 1312 \\
\hline 15.5 & 1352 & 14.4 & 1339 & 14.89 & 1347 & 14.54 & 1348 & 14.09 & 1337 & 14.18 & 1335 & 14.04 & 1339 & 13.55 & 1358 & 13.48 & 1344 \\
\hline 15.67 & 1375 & 14.61 & 1362 & 15.09 & 1379 & 14.67 & 1381 & 14.38 & 1363 & 14.41 & 1382 & 14.49 & 1379 & 13.97 & 1384 & 13.63 & 1367 \\
\hline 15.95 & 1400 & & 402 & & 1405 & 14.98 & 1408 & & 1389 & & & 14.68 & 1404 & 14.09 & 1409 & 14.01 & 1397 \\
\hline 16.15 & 1422 & 15.27 & 1423 & 15.67 & 1429 & 15.15 & 1432 & 14.97 & 1417 & 14.95 & 1416 & 14.78 & 1433 & 14.27 & 1439 & 14.12 & 1422 \\
\hline 16.63 & 1462 & 5.46 & 1454 & 15.79 & 1450 & 15.33 & 1460 & 15.16 & 1449 & 15.4 & 1460 & 15.05 & 1459 & 14.49 & 1468 & 14.3 & 1443 \\
\hline 16.75 & 1493 & 15.72 & 1477 & 16.24 & 1491 & 15.65 & 1483 & 15.39 & 1474 & 15.62 & 1487 & 15.18 & 1485 & 14.71 & 1493 & 14.53 & 1467 \\
\hline 17.06 & 1516 & 15.87 & 1504 & 16.47 & 1515 & 15.75 & 1507 & 15.75 & 1501 & 15.75 & 1519 & 15.38 & 1507 & 14.86 & 1516 & 14.85 & 1494 \\
\hline 14.88 & 1526 & 12.9 & 1313 & 14.85 & 1516 & 13.67 & 1379 & 14.32 & 1533 & 14.14 & 1497 & 13.96 & 1515 & 13.67 & 1512 & 12.93 & 1521 \\
\hline 14.5 & 1489 & 12.63 & 1291 & 14.67 & 1488 & 13.43 & 1358 & 13.93 & 1493 & 13.93 & 1471 & 13.73 & 1490 & 13.49 & 1489 & 12.81 & 1496 \\
\hline 14.26 & 1466 & 2.46 & 1261 & 14.35 & 1467 & 13.19 & 1331 & 13.67 & 1461 & 13.7 & 1441 & 13.34 & 1449 & 13.34 & 1466 & 12.51 & 1468 \\
\hline 14.08 & 1441 & 12.25 & 1233 & 14.02 & 1433 & 12.9 & 1304 & 13.58 & 1435 & 13.31 & 1414 & 13.05 & 1428 & 13.08 & 1439 & 12.48 & 1439 \\
\hline 13.93 & 1407 & 11.94 & 1202 & 13.73 & 1405 & 12.75 & 1279 & 13.31 & 1409 & 13.05 & 1378 & 12.81 & 1404 & 13.08 & 1408 & 12.31 & 1412 \\
\hline 13.7 & 1381 & 11.7 & 1178 & 13.52 & 1382 & 12.49 & 1247 & 13.02 & 1383 & 12.84 & 1346 & 12.48 & 1379 & 12.87 & 1381 & 11.96 & 1383 \\
\hline 13.46 & 1358 & 11.6 & 1148 & 13.31 & 1356 & 12.27 & 1221 & 12.9 & 1362 & 12.51 & 1322 & 12.4 & 1351 & 12.66 & 1355 & 11.82 & 1357 \\
\hline 13.17 & 1332 & 11.39 & 1123 & 13.08 & 1332 & 12 & 1196 & 12.71 & 1338 & 12.12 & 1299 & 12.3 & 1320 & 12.48 & 1327 & 11.53 & 1331 \\
\hline 12.96 & 1304 & 11.08 & 1100 & 12.96 & 1308 & 11.72 & 1171 & 12.3 & 1299 & 12.1 & 1272 & 12.01 & 1293 & 12.09 & 1303 & 11.31 & 1308 \\
\hline 12.57 & 1277 & 10.8 & 1076 & 12.51 & 1269 & 11.56 & 1148 & 12.15 & 1277 & 11.74 & 1251 & 11.82 & 1270 & 11.88 & 1280 & 11.13 & 1285 \\
\hline 12.52 & 1249 & 10.71 & 1053 & 12.32 & 1241 & 11.03 & 1111 & 11.93 & 1245 & 11.59 & 1223 & 11.71 & 1247 & 11.49 & 1239 & 10.74 & 1244 \\
\hline 12.22 & 1225 & 10.21 & 1014 & 12.11 & 1212 & 10.82 & 1084 & 11.6 & 1230 & & 1181 & 11.43 & 1223 & 11.42 & 1210 & 10.65 & 1222 \\
\hline 11.72 & 1187 & 10.01 & 987.6 & 11.94 & 1190 & 10.64 & 1059 & 11.27 & 1191 & 10.78 & 1158 & 11.32 & 1195 & 11.07 & 1188 & 10.48 & 1192 \\
\hline 11.61 & 1163 & 9.842 & 957.6 & 11.76 & 1166 & 10.67 & 1049 & 11.1 & 1165 & 10.69 & 1127 & 11.01 & 1170 & 10.94 & 1155 & 10.18 & 1168 \\
\hline 11.28 & 1137 & 9.439 & 935.6 & 11.17 & 1128 & 10.11 & 1003 & 10.87 & 1140 & 10.58 & 1102 & 10.47 & 1126 & 10.81 & 1135 & 9.913 & 1141 \\
\hline 11.15 & 1114 & 9.404 & 910 & 11.03 & 1104 & 9.854 & 977.9 & 10.67 & 1114 & 10.32 & 1078 & 10.19 & 1101 & 10.41 & 1104 & 9.744 & 1112 \\
\hline
\end{tabular}


Table 2. $25^{\circ} \mathrm{C}$ As Received - continued

\begin{tabular}{|c|c|c|c|c|c|c|c|c|c|c|c|c|c|c|c|c|c|}
\hline run1 & & run2 & & run3 & & run4 & & run5 & & run6 & & run7 & & run8 & & run9 & \\
\hline [Pa] & {$[1 / \mathrm{s}]$} & {$[\mathrm{Pa}]$} & {$[1 / s]$} & {$[\mathrm{Pa}]$} & {$[1 / \mathrm{s}]$} & [Pa] & {$[1 / s]$} & {$[\mathrm{Pa}]$} & {$[1 / \mathrm{s}]$} & {$[\mathrm{Pa}]$} & {$[1 / \mathrm{s}]$} & {$[\mathrm{Pa}]$} & {$[1 / \mathrm{s}]$} & {$[\mathrm{Pa}]$} & {$[1 / \mathrm{s}]$} & [Pa] & {$[1 / \mathrm{s}]$} \\
\hline 10.81 & 1084 & 9.049 & 886.7 & 10.9 & 1076 & 9.505 & 948.3 & 10.52 & 1088 & 10.14 & 1052 & 9.984 & 1077 & 10.25 & 1083 & 9.392 & 1088 \\
\hline 10.59 & 1062 & 8.987 & 863.3 & 10.61 & 1057 & 9.271 & 922.8 & 10.15 & 1061 & 9.866 & 1026 & 9.795 & 1048 & 9.934 & 1053 & 9.191 & 1055 \\
\hline 10.26 & 1028 & 8.3 & 823.2 & 10.45 & 1032 & 9.099 & 891.5 & 9.848 & 1037 & 9.57 & 1001 & 9.62 & 1020 & 9.608 & 1021 & 9.111 & 1028 \\
\hline 10.08 & 999.5 & 8.217 & 798.1 & 9.886 & 991.1 & 8.883 & 866.4 & 9.425 & 996.8 & 9.259 & 963.3 & 9.463 & 995.5 & 9.451 & 995.9 & 8.913 & 1003 \\
\hline 9.863 & 973.9 & 8.022 & 769.9 & 9.664 & 966 & 8.676 & 839.9 & 9.342 & 970.8 & 8.987 & 940.4 & 9.182 & 972.1 & 9.155 & 973.9 & 8.779 & 978.8 \\
\hline 9.729 & 948.3 & 7.714 & 745.6 & 9.327 & 942.2 & 8.436 & 813.1 & 9.155 & 943.1 & 8.726 & 913.1 & 9.004 & 945.7 & 8.951 & 947 & 8.537 & 957.2 \\
\hline 9.419 & 927.2 & 7.622 & 721 & 9.167 & 911.3 & 8.146 & 787.1 & 8.85 & 920.6 & 8.703 & 886.2 & 8.972 & 919.7 & 8.924 & 923.7 & 8.167 & 914.9 \\
\hline 9.058 & 887.1 & 7.389 & 696.7 & 9.123 & 887.5 & 7.948 & 763.3 & 8.67 & 899.4 & 8.442 & 857.1 & 8.794 & 895 & 8.714 & 896.8 & 7.948 & 888 \\
\hline 8.685 & 862.9 & 6.98 & 657.5 & 8.883 & 863.7 & 7.566 & 738.6 & 8.608 & 877 & 8.102 & 828.5 & 8.501 & 867.7 & 8.185 & 857.6 & 7.699 & 865.5 \\
\hline 8.593 & 837.3 & 6.675 & 632.4 & 8.587 & 840.8 & 7.347 & 713.9 & 8.037 & 836 & 7.957 & 804.2 & 8.294 & 841.3 & 8.031 & 828 & 7.46 & 838.6 \\
\hline 8.223 & 808.2 & 6.456 & 609.5 & 8.294 & 799.8 & 7.184 & 690.1 & 7.921 & 811.3 & 7.788 & 782.2 & 7.892 & 813.5 & 7.776 & 808.7 & 7.246 & 813.9 \\
\hline 8.193 & 780.4 & 6.077 & 577.7 & 8.013 & 776.5 & 6.598 & 649.1 & 7.507 & 785.7 & 7.43 & 759.3 & 7.554 & 771.6 & 7.445 & 779.1 & 7.214 & 782.2 \\
\hline 7.699 & 755.3 & 5.903 & 557 & 7.847 & 746.5 & 6.418 & 625.3 & 7.519 & 758.4 & 6.906 & 717 & 7.279 & 747.4 & 7.244 & 754.9 & 6.794 & 756.7 \\
\hline 7.572 & 731.5 & 5.746 & 531.5 & 7.557 & 720.5 & 6.424 & 592.7 & 7.152 & 728.9 & 6.649 & 695.4 & 7.045 & 721.8 & 6.894 & 722.3 & 6.672 & 730.2 \\
\hline 7.397 & 707.7 & 5.492 & 507.7 & 7.113 & 694.5 & 6.166 & 568.9 & 6.962 & 704.7 & 6.619 & 668.1 & 7.036 & 691.4 & 6.823 & 695.4 & 6.518 & 702 \\
\hline 7.075 & 679.5 & 5.281 & 482.1 & 6.989 & 669 & 5.977 & 544.7 & 6.814 & 680.9 & 6.199 & 636.8 & 6.699 & 666.3 & 6.498 & 670.3 & 6.166 & 678.7 \\
\hline 6.693 & 656.2 & 4.63 & 438.9 & 6.897 & 643.4 & 5.634 & 520 & 6.311 & 644.3 & 5.956 & 612.1 & 6.237 & 639 & 6.252 & 647.4 & 5.912 & 655.3 \\
\hline 6.409 & 615.6 & 4.438 & 413.8 & 6.557 & 617.8 & 5.267 & 496.2 & 6.072 & 619.6 & 5.767 & 583.5 & 5.989 & 613.9 & 5.897 & 609 & 5.642 & 628.4 \\
\hline 6.196 & 593.6 & 4.189 & 383 & 6.279 & 591.8 & 4.935 & 471.1 & 5.879 & 592.7 & 5.361 & 561.4 & 5.782 & 589.6 & 5.639 & 587.9 & 5.571 & 599.8 \\
\hline 5.992 & 566.7 & 3.722 & 360.9 & 5.98 & 567.2 & 4.642 & 446.4 & 5.622 & 569.4 & 5.273 & 527.5 & 5.512 & 568 & 5.533 & 555.3 & 5.273 & 576 \\
\hline 5.625 & 536.8 & 3.586 & 334.5 & 5.287 & 528.8 & 4.115 & 405.4 & 5.394 & 544.2 & 4.914 & 513 & 5.349 & 524.4 & 5.323 & 535.9 & 4.799 & 536.3 \\
\hline 5.483 & 512.5 & 3.133 & 308.5 & 5.107 & 502.4 & 3.92 & 372.8 & 5.243 & 509.9 & 4.758 & 482.5 & 5.048 & 498.4 & 5.086 & 508.5 & 4.731 & 513 \\
\hline 5.385 & 487.8 & 988 & 277.2 & 4.879 & 479.9 & 3.846 & 356.1 & 5.11 & 479 & 4.364 & 454.8 & 4.69 & 466.2 & 4.755 & 483 & 4.574 & 483.4 \\
\hline 5.077 & 460.5 & 2.624 & 254.7 & 4.728 & 447.7 & 3.42 & 319.9 & 4.636 & 455.2 & 4.237 & 421.7 & 4.583 & 459.6 & 4.429 & 460.1 & 4.408 & 457.4 \\
\hline 4.648 & 436.3 & & & 4.592 & 424.8 & 3.157 & 293.1 & 4.334 & 429.7 & 4.062 & 395.7 & 4.515 & 437.2 & 4.361 & 435.8 & 4.024 & 432.3 \\
\hline 4.346 & 410.7 & & & 4.355 & 397.1 & 2.855 & 267.9 & 4.077 & 405 & 3.695 & 368.4 & 3.757 & 391.3 & 4.121 & 405 & 3.6 & 402.8 \\
\hline 3.816 & 369.3 & & & 3.95 & 376.3 & 2.636 & 241.5 & 4.009 & 375.9 & 3.47 & 346.4 & 3.695 & 366.6 & 3.55 & 364.9 & 3.405 & 379.9 \\
\hline 3.737 & 348.1 & & & 3.426 & 334 & & & 3.524 & 350.3 & 3.284 & 320.8 & 3.29 & 338.4 & 3.278 & 338.4 & 3.127 & 342.4 \\
\hline 3.441 & 317.3 & & & 3.429 & 324.8 & & & 3.254 & 324.3 & 2.935 & 296.1 & 3.331 & 316.9 & 2.914 & 314.6 & 2.707 & 316.9 \\
\hline 3.257 & 294.8 & & & 2.911 & 285.1 & & & 2.976 & 287.3 & 2.579 & 254.3 & 2.872 & 291.3 & 2.935 & 285.6 & 2.935 & 308.9 \\
\hline 2.846 & 271.9 & & & 2.79 & 260.9 & & & 2.426 & 263.5 & 2.497 & 247.2 & 2.668 & 264.4 & 2.411 & 261.8 & 2.207 & 267.5 \\
\hline 2.716 & 244.1 & & & 2.881 & 252.5 & & & 2.505 & 255.2 & & & & & 2.278 & 237.5 & 2.215 & 238.4 \\
\hline
\end{tabular}


Table 3. $50{ }^{\circ} \mathrm{C}$ As Received

\begin{tabular}{|c|c|c|c|c|c|c|c|c|c|c|c|c|c|c|c|c|c|}
\hline run1 & & run2 & & run3 & & run4 & & run5 & & run6 & & run7 & & run8 & & run9 & \\
\hline [Pa] & {$[1 / \mathrm{s}]$} & {$[\mathrm{Pa}]$} & {$[1 / \mathrm{s}]$} & {$[\mathrm{Pa}]$} & {$[1 / s]$} & [Pa] & {$[1 / \mathrm{s}]$} & {$[\mathrm{Pa}]$} & {$[1 / \mathrm{s}]$} & {$[\mathrm{Pa}]$} & {$[1 / \mathrm{s}]$} & [Pa] & {$[1 / \mathrm{s}]$} & {$[\mathrm{Pa}]$} & {$[1 / \mathrm{s}]$} & {$[\mathrm{Pa}]$} & {$[1 / \mathrm{s}]$} \\
\hline 3.696 & 306.3 & 4.173 & 338 & 4.226 & 341.1 & 3.936 & 328.7 & 4.587 & 374.1 & 3.939 & 327.9 & 3.495 & 327.9 & 4.007 & 303.6 & 3.427 & 305.4 \\
\hline 4.084 & 314.6 & 4.703 & 373.7 & 4.611 & 361.4 & 4.256 & 349 & 4.824 & 393.5 & 4.489 & 370.2 & 4.427 & 354.7 & 3.977 & 314.2 & 3.936 & 323.9 \\
\hline 4.498 & 342.4 & 4.898 & 396.6 & 4.72 & 384.7 & 4.578 & 371.9 & 5.321 & 431.4 & 4.788 & 394 & 4.38 & 362.7 & 4.244 & 336.7 & 4.096 & 342 \\
\hline 4.694 & 367.5 & 5.203 & 426.1 & 4.904 & 407.6 & 4.88 & 394.4 & 5.333 & 453.5 & 5.123 & 417.3 & 5.043 & 403.7 & 4.303 & 344.6 & 4.359 & 372.4 \\
\hline 4.788 & 392.2 & 5.522 & 451.7 & 5.428 & 449.1 & 4.951 & 416 & 5.561 & 481.7 & 5.365 & 442.9 & 5.099 & 428.8 & 4.788 & 386 & 4.614 & 397.5 \\
\hline 5.188 & 416.4 & 5.762 & 477.3 & 5.635 & 473.7 & 5.146 & 441.1 & 5.732 & 506.8 & 5.277 & 451.3 & 5.303 & 460.1 & 4.99 & 401 & 4.933 & 423.9 \\
\hline 5.475 & 442.4 & 5.919 & 502.4 & 5.803 & 499.3 & 5.454 & 466.2 & 6.079 & 536.3 & 5.404 & 492.2 & 5.49 & 486.1 & 5.052 & 433.6 & 5.176 & 446.4 \\
\hline 5.7 & 63.6 & 6.096 & 527.9 & 6.031 & 521.8 & 5.611 & 497.1 & 6.179 & 559.2 & 5.383 & 494 & 5.727 & 508.5 & 5.623 & 483.4 & 5.146 & 469.8 \\
\hline 6.085 & 504.1 & 6.383 & 553.9 & 6.315 & 556.1 & 5.916 & 522.2 & 6.437 & 583.5 & 6.265 & 542 & 5.851 & 536.3 & 5.685 & 496.2 & 5.759 & 509.9 \\
\hline 6.339 & 529.3 & 6.466 & 581.3 & 6.543 & 579.9 & 6.431 & 566.3 & 6.765 & 622.2 & 6.789 & 594.9 & 6.144 & 563.6 & 5.839 & 536.3 & 6.005 & 534.5 \\
\hline 6.375 & 557.5 & 6.975 & 623.6 & 6.762 & 605.1 & 6.25 & 575.1 & 6.919 & 645.6 & 6.813 & 606.8 & 6.422 & 587.4 & 5.789 & 551.3 & 6.034 & 566.7 \\
\hline 6.588 & 580.4 & 7.144 & 647.4 & 6.961 & 630.2 & 6.857 & 613.4 & 7.2 & 673.8 & 6.884 & 643.8 & 6.62 & 613 & 6.413 & 572 & 6.144 & 589.6 \\
\hline 6.833 & 601.1 & 7.046 & 657.9 & 7.014 & 657.5 & 6.878 & 643 & 7.342 & 702 & 7.493 & 677.3 & 6.75 & 634.1 & 6.558 & 587.9 & 6.342 & 615.6 \\
\hline 7.236 & 643.4 & 7.517 & 698.5 & 7.227 & 681.7 & 6.916 & 662.3 & 7.487 & 723.2 & 7.538 & 693.6 & 7.277 & 677.3 & 6.665 & 628.4 & 6.759 & 643.8 \\
\hline 7.289 & 667.2 & 7.422 & 723.2 & 7.552 & 707.3 & 7.754 & 697.2 & 7.792 & 750 & 7.632 & 716.1 & 7.419 & 704.7 & 6.801 & 656.6 & 6.901 & 672.5 \\
\hline 7.57 & 690.1 & 8.346 & 747.8 & 7.733 & 732.9 & 7.544 & 713 & 7.982 & 779.6 & 7.499 & 744.8 & 7.585 & 730.7 & 6.928 & 680.9 & 7.061 & 697.2 \\
\hline 7.777 & 722.7 & 8.233 & 768.6 & 7.896 & 758.4 & 7.766 & 765 & 8.209 & 807.3 & 8.28 & 761.5 & 7.976 & 756.2 & 7.203 & 708.6 & 7.286 & 721.4 \\
\hline 7.99 & 748.7 & 8.322 & 826.7 & 8.434 & 800.3 & 7.748 & 766.3 & 8.488 & 833.3 & 8.343 & 776.5 & 7.979 & 779.6 & 7.677 & 751.4 & 7.585 & 743 \\
\hline 8.275 & 770.3 & 8.227 & 828.5 & 8.497 & 823.6 & 8.476 & 790.6 & 8.541 & 861.1 & 8.565 & 826.7 & 8.192 & 810.4 & 7.751 & 774.7 & 7.609 & 769 \\
\hline 8.372 & 793.2 & 9.094 & 849.6 & 8.739 & 852.3 & 8.585 & 803.8 & 8.884 & 886.2 & 8.683 & 856.2 & 8.266 & 833.8 & 7.902 & 803.8 & 8.047 & 810.9 \\
\hline 8.582 & 819.2 & 9.059 & 864.2 & 8.943 & 878.3 & 9.032 & 851.4 & 9.159 & 915.3 & 8.822 & 887.1 & 8.502 & 861.5 & 8.109 & 825.4 & 8.257 & 836 \\
\hline 9.035 & 856.7 & 9.677 & 920.1 & 9.21 & 909.1 & 9.041 & 873 & 9.254 & 943.9 & 9.062 & 910.5 & 8.677 & 884.5 & 8.443 & 854 & 8.573 & 861.5 \\
\hline 9.204 & 881.8 & 9.586 & 937.3 & 9.296 & 931.6 & 9.13 & 904.3 & 9.39 & 965.1 & 9.165 & 936 & 9.106 & 924.6 & 8.618 & 880.5 & 8.677 & 892.4 \\
\hline 9.393 & 905.6 & 9.805 & 959.4 & 9.586 & 955.4 & 9.642 & 956.7 & 9.606 & 988.9 & 9.426 & 958.5 & 9.287 & 950.6 & 8.893 & 909.6 & 8.751 & 917.5 \\
\hline 9.509 & 940.9 & 9.571 & 978.8 & 9.594 & 980.5 & 9.571 & 963.8 & 10.05 & 1027 & 9.589 & 981.4 & 9.547 & 977.4 & 9.065 & 940.4 & 8.979 & 941.3 \\
\hline 9.811 & 956.7 & 10.29 & 1003 & 10.06 & 1020 & 9.834 & 991.1 & 10.2 & 1059 & 9.982 & 1022 & 9.674 & 1000 & 9.168 & 963.3 & 9.053 & 965.1 \\
\hline 9.982 & 992 & 10.51 & 1043 & 10.25 & 1048 & 9.967 & 1021 & 10.34 & 1082 & 10.21 & 1051 & 9.87 & 1027 & 9.423 & 995.1 & 9.284 & 992.9 \\
\hline 10.2 & 1019 & 10.67 & 1075 & 10.46 & 1074 & 10.24 & 1046 & 10.63 & 1105 & 10.27 & 1074 & 10.01 & 1060 & 9.648 & 1026 & 9.449 & 1018 \\
\hline 10.45 & 1043 & 10.81 & 1103 & 10.62 & 1096 & 10.38 & 1073 & 10.76 & 1130 & 10.53 & 1105 & 10.17 & 1080 & 9.84 & 1052 & 9.63 & 1043 \\
\hline 10.64 & 1068 & 10.96 & 1137 & 10.88 & 1123 & 10.57 & 1097 & 10.92 & 1155 & 10.62 & 1126 & 10.28 & 1103 & 10.01 & 1076 & 10 & 1083 \\
\hline 10.77 & 1096 & 11.11 & 1159 & 11.01 & 1154 & 10.76 & 1123 & 11.23 & 1196 & 10.85 & 1150 & 10.45 & 1133 & 10.17 & 1101 & 10.24 & 1109 \\
\hline
\end{tabular}


Table 3. $50{ }^{\circ} \mathrm{C}$ As Received - continued

\begin{tabular}{|c|c|c|c|c|c|c|c|c|c|c|c|c|c|c|c|c|c|}
\hline run1 & & run2 & & run3 & & run4 & & run5 & & run6 & & run7 & & run8 & & run9 & \\
\hline [Pa] & {$[1 / \mathrm{s}]$} & {$[\mathrm{Pa}]$} & {$[1 / \mathrm{s}]$} & {$[\mathrm{Pa}]$} & {$[1 / s]$} & [Pa] & {$[1 / \mathrm{s}]$} & {$[\mathrm{Pa}]$} & {$[1 / \mathrm{s}]$} & {$[\mathrm{Pa}]$} & {$[1 / \mathrm{s}]$} & [Pa] & {$[1 / \mathrm{s}]$} & {$[\mathrm{Pa}]$} & {$[1 / \mathrm{s}]$} & {$[\mathrm{Pa}]$} & {$[1 / \mathrm{s}]$} \\
\hline 11.08 & 1118 & 11.25 & 1180 & 11.23 & 1177 & 10.92 & 1151 & 11.32 & 1222 & 11.21 & 1185 & 10.78 & 1159 & 10.34 & 1126 & 10.37 & 1133 \\
\hline 11.43 & 1159 & 11.62 & 1218 & 11.38 & 1199 & 11.38 & 1191 & 11.37 & 1231 & 11.28 & 1214 & 10.94 & 1185 & 10.46 & 1150 & 10.6 & 1159 \\
\hline 11.69 & 1185 & 11.77 & 1242 & 11.7 & 1240 & 11.49 & 1218 & 11.78 & 1272 & 11.44 & 1239 & 11.13 & 1211 & 10.6 & 1174 & 10.79 & 1188 \\
\hline 11.91 & 1215 & 11.95 & 1270 & 11.97 & 1263 & 11.77 & 1241 & 12.02 & 1300 & 11.66 & 1261 & 11.57 & 1252 & 10.86 & 1202 & 10.98 & 1219 \\
\hline 12.11 & 1236 & 12.13 & 1292 & 12.01 & 1293 & 11.87 & 1273 & 12.15 & 1327 & 11.86 & 1284 & 11.68 & 1273 & 11.06 & 1228 & 11.14 & 1242 \\
\hline 12.39 & 1272 & 12.33 & 1314 & 12.31 & 1317 & 11.99 & 1299 & 12.42 & 1352 & 12.26 & 1329 & 11.78 & 1302 & 11.41 & 1270 & 11.23 & 1267 \\
\hline 12.51 & 1296 & 12.74 & 1353 & 12.46 & 1346 & 12.25 & 1324 & 12.44 & 1378 & 12.5 & 1352 & 11.89 & 1329 & 11.58 & 1293 & 11.41 & 1293 \\
\hline 12.63 & 1325 & 12.99 & 1380 & 12.7 & 1368 & 12.36 & 1349 & 12.76 & 1401 & 12.61 & 1378 & 12.13 & 1352 & 11.72 & 1322 & 11.58 & 1320 \\
\hline 12.88 & 1349 & 13.08 & 1403 & 12.81 & 1392 & 12.58 & 1370 & 12.9 & 1427 & 12.8 & 1400 & 12.32 & 1374 & 11.89 & 1349 & 11.76 & 1351 \\
\hline 13.13 & 1374 & 13.23 & 1432 & 12.95 & 1418 & 12.65 & 1397 & 13.07 & 1453 & 12.99 & 1433 & 12.66 & 1413 & 12.06 & 1372 & 12 & 1375 \\
\hline 13.34 & 1400 & 13.36 & 1457 & 13.49 & 1457 & 13.05 & 1439 & 13.21 & 1479 & 13.08 & 1457 & 12.86 & 1436 & 12.24 & 1398 & 12.22 & 1404 \\
\hline 13.6 & 1423 & 13.51 & 1483 & 13.6 & 1481 & 13.17 & 1466 & 13.68 & 1521 & 13.34 & 1480 & 13.06 & 1468 & 12.49 & 1423 & 12.39 & 1433 \\
\hline 13.71 & 1447 & 13.84 & 1507 & 13.64 & 1510 & 13.42 & 1490 & 13.89 & 1552 & 13.59 & 1503 & 13.17 & 1490 & 12.6 & 1447 & 12.55 & 1459 \\
\hline 13.9 & 1474 & 14.14 & 1547 & 13.9 & 1530 & 13.52 & 1516 & 14.01 & 1575 & 13.94 & 1547 & 13.32 & 1517 & 13.07 & 1487 & 12.78 & 1486 \\
\hline 14.34 & 1514 & 14.31 & 1570 & 14.05 & 1554 & 13.73 & 1539 & 14.31 & 1603 & 13.81 & 1555 & 13.68 & 1552 & 13.22 & 1512 & 13.03 & 1512 \\
\hline 12.87 & 1513 & 11.99 & 1458 & 12.6 & 1502 & 12.09 & 1489 & 12.03 & 1473 & 11.92 & 1488 & 11.91 & 1493 & 12.31 & 1551 & 12.23 & 1557 \\
\hline 12.68 & 1488 & 11.78 & 1430 & 12.02 & 1461 & 11.89 & 1463 & 11.78 & 1449 & 11.82 & 1466 & 11.73 & 1468 & 12 & 1522 & 11.82 & 1518 \\
\hline 12.55 & 1464 & 11.71 & 1404 & 11.91 & 1435 & 11.6 & 1440 & 11.63 & 1428 & 11.53 & 1443 & 11.5 & 1442 & 11.77 & 1493 & 11.65 & 1489 \\
\hline 12.26 & 1440 & 11.48 & 1382 & 11.68 & 1409 & 11.42 & 1408 & 11.16 & 1389 & 11.38 & 1420 & 11.3 & 1420 & 11.52 & 1467 & 11.6 & 1465 \\
\hline 12.11 & 1412 & 11.29 & 1358 & 11.42 & 1386 & 11.29 & 1382 & 11.04 & 1363 & 11.23 & 1392 & 11.15 & 1396 & 11.42 & 1442 & 11.35 & 1441 \\
\hline 11.84 & 1388 & 11.07 & 1337 & 11.17 & 1355 & 11.17 & 1354 & 10.9 & 1335 & 11.01 & 1361 & 10.92 & 1370 & 11.02 & 1404 & 11.18 & 1414 \\
\hline 11.35 & 1348 & 10.94 & 1311 & 11.03 & 1327 & 10.88 & 1332 & 10.72 & 1311 & 10.76 & 1337 & 10.43 & 1327 & 10.79 & 1379 & 10.89 & 1389 \\
\hline 11.17 & 1319 & 10.43 & 1267 & .75 & 1300 & 10.68 & 1306 & 10.51 & 1285 & 10.49 & 1310 & 10.38 & 1304 & 10.67 & 1349 & 10.74 & 1362 \\
\hline 11.09 & 1293 & 10.24 & 1244 & 10.51 & 1273 & 10.39 & 1279 & 10.3 & 1260 & 10.32 & 1284 & 10.13 & 1272 & 10.54 & 1323 & 10.46 & 1339 \\
\hline 10.81 & 1270 & 10.11 & 1215 & 10.26 & 1248 & 10.34 & 1251 & 10.06 & 1238 & 9.92 & 1243 & 9.935 & 1250 & 10.31 & 1299 & 10.15 & 1299 \\
\hline 10.63 & 1247 & 9.932 & 1194 & 10.09 & 1225 & 10.01 & 1226 & 9.745 & 1199 & 9.719 & 1219 & 9.737 & 1225 & 10.09 & 1276 & 9.938 & 1272 \\
\hline 10.49 & 1223 & 9.751 & 1169 & 9.76 & 1190 & 9.734 & 1199 & 9.568 & 1166 & 9.452 & 1196 & 9.627 & 1201 & 10.01 & 1252 & 9.763 & 1249 \\
\hline 10.3 & 1197 & 9.577 & 1144 & 9.568 & 1167 & 9.624 & 1175 & 9.355 & 1148 & 9.452 & 1164 & 9.479 & 1174 & 9.816 & 1230 & 9.586 & 1217 \\
\hline 9.825 & 1160 & 9.139 & 1104 & 9.328 & 1137 & 9.319 & 1149 & 9.189 & 1115 & 9.23 & 1142 & 9.062 & 1134 & 9.506 & 1198 & 9.358 & 1191 \\
\hline 9.571 & 1127 & 8.994 & 1082 & 9.192 & 1114 & 8.935 & 1112 & 8.929 & 1091 & 9.056 & 1118 & 9.118 & 1118 & 9.251 & 1174 & 9.275 & 1165 \\
\hline 9.367 & 1106 & 8.724 & 1050 & 9.091 & 1091 & 8.929 & 1081 & 8.751 & 1066 & 8.526 & 1082 & 8.772 & 1091 & 8.917 & 1130 & 8.985 & 1144 \\
\hline
\end{tabular}


Table 3. $50{ }^{\circ} \mathrm{C}$ As Received - continued

\begin{tabular}{|c|c|c|c|c|c|c|c|c|c|c|c|c|c|c|c|c|c|}
\hline run1 & & run2 & & run3 & & run4 & & run5 & & run6 & & run7 & & run8 & & run9 & \\
\hline [Pa] & {$[1 / s]$} & {$[\mathrm{Pa}]$} & {$[1 / s]$} & {$[\mathrm{Pa}]$} & {$[1 / s]$} & [Pa] & {$[1 / s]$} & [Pa] & {$[1 / s]$} & [Pa] & {$[1 / \mathrm{s}]$} & [Pa] & {$[1 / s]$} & {$[\mathrm{Pa}]$} & {$[1 / \mathrm{s}]$} & {$[\mathrm{Pa}]$} & {$[1 / s]$} \\
\hline 9.272 & 1081 & 8.55 & 1027 & 8.621 & 1050 & 8.674 & 1054 & 8.621 & 1042 & 8.334 & 1056 & 8.434 & 1066 & 8.686 & 1105 & 8.866 & 1122 \\
\hline 9.014 & 1060 & 8.384 & 1002 & 8.455 & 1028 & 8.381 & 1032 & 8.135 & 1000 & 8.233 & 1026 & 8.097 & 1025 & 8.713 & 1095 & 8.63 & 1095 \\
\hline 8.579 & 1021 & .109 & 977.4 & 8.239 & 1001 & 8.328 & 1013 & 8.044 & 973.5 & 8.138 & 1003 & 8.204 & 1015 & 8.508 & 1067 & 8.236 & 1055 \\
\hline 8.399 & 995.1 & 7.979 & 952.3 & 8.106 & 970.4 & 7.928 & 978.3 & 7.709 & 949.2 & 7.985 & 979.6 & 7.467 & 969.9 & 8.31 & 1040 & 8.106 & 1031 \\
\hline 8.278 & 968.6 & 7.547 & 914 & 7.828 & 950.1 & 7.869 & 951.9 & 7.547 & 924.1 & 7.452 & 940.9 & 7.461 & 943.5 & 7.724 & 995.9 & 7.848 & 997.3 \\
\hline 8.035 & 937.3 & 7.348 & 888.4 & 7.647 & 921.5 & 7.629 & 921.5 & 7.375 & 899.9 & 7.665 & 933.4 & 7.221 & 919.7 & 7.662 & 966 & 7.745 & 976.1 \\
\hline 7.922 & 913.1 & 7.079 & 865.1 & 7.511 & 895 & 7.384 & 895.9 & 7.129 & 874.3 & 7.126 & 885.8 & 7.094 & 889.7 & 7.443 & 949.7 & 7.57 & 954.1 \\
\hline 7.68 & 887.1 & .875 & 832.9 & 7.336 & 873 & 7.153 & 869.9 & 6.91 & 839.1 & 7.15 & 877 & 6.884 & 863.7 & 7.2 & 916.6 & 7.301 & 925.4 \\
\hline 7.399 & 862.9 & 6.887 & 808.7 & 7.106 & 845.7 & 6.984 & 847.4 & 6.697 & 817 & 6.768 & 846.6 & 6.617 & 838.6 & 7.058 & 895 & 7.112 & 897.2 \\
\hline 7.233 & 838.2 & 6.594 & 785.7 & 6.742 & 819.7 & 6.611 & 806.4 & 6.419 & 787.5 & 6.398 & 806.9 & 6.531 & 809.5 & 6.89 & 859.3 & 6.86 & 872.6 \\
\hline 7.082 & 813.5 & 6.392 & 759.7 & 6.301 & 780.4 & 6.357 & 784 & 6.318 & 764.1 & 6.342 & 776.9 & 6.286 & 788.8 & 6.588 & 837.3 & 6.739 & 848.3 \\
\hline 6.597 & 772.1 & 6.292 & 732.4 & 6.227 & 755.3 & 6.241 & 754.9 & 6.185 & 741.7 & 6.117 & 751.4 & 5.987 & 748.3 & 6.531 & 808.2 & 6.567 & 821 \\
\hline 6.289 & 747 & 5.833 & 694.1 & 5.951 & 724.5 & 6.088 & 729.3 & 5.975 & 714.3 & 5.96 & 724.9 & 6.108 & 740.8 & 6.241 & 787.1 & 6.307 & 793.7 \\
\hline 6.221 & 721.8 & 5.602 & 669.8 & 5.86 & 699.4 & 5.78 & 706 & 5.638 & 675.1 & 5.729 & 701.1 & 5.712 & 712.1 & 6.04 & 763.7 & 5.872 & 752.7 \\
\hline 5.963 & 692.3 & 5.277 & 644.3 & 5.798 & 674.2 & 5.623 & 684.4 & 5.416 & 654 & 5.454 & 677.3 & 5.289 & 671.6 & 5.978 & 735.5 & 5.685 & 727.1 \\
\hline 5.744 & 671.2 & 5.179 & 613 & 5.502 & 651.3 & 5.226 & 644.3 & 5.129 & 624 & 5.259 & 652.7 & 5.087 & 643.4 & 5.552 & 694.1 & 5.59 & 696.7 \\
\hline 5.549 & 650 & 4.865 & 591.8 & 5.354 & 626.7 & 5.043 & 620 & 5.001 & 599.3 & 5.102 & 628.4 & 4.93 & 620 & 5.286 & 665 & 5.321 & 672.9 \\
\hline 5.33 & 624 & 4.652 & 564.5 & 4.83 & 585.7 & 4.812 & 591.4 & 4.771 & 576 & 4.919 & 602.9 & 4.821 & 593.2 & 4.993 & 642.1 & 5.099 & 646.5 \\
\hline 5.096 & 586.5 & 4.466 & 537.2 & 4.605 & 563.6 & 4.667 & 568 & 4.596 & 551.3 & 4.501 & 564.1 & 4.605 & 566.7 & 4.765 & 618.3 & 4.88 & 623.6 \\
\hline 4.83 & 555.3 & 4.318 & 501.5 & 4.353 & 537.6 & 4.572 & 545.6 & 4.353 & 524.9 & 4.321 & 533.7 & 4.353 & 542 & 4.694 & 590.1 & 4.856 & 596.7 \\
\hline 4.685 & 531.9 & 3.945 & 483.4 & 4.199 & 509.9 & 4.078 & 508.5 & 4.069 & 484.3 & 4.191 & 507.2 & 4.241 & 512.1 & 4.54 & 565 & 4.626 & 567.2 \\
\hline 4.51 & 509 & 3.741 & 455.2 & 3.901 & 484.8 & 3.933 & 479.5 & 4.066 & 473.3 & 4.025 & 483 & 3.989 & 487.4 & 4.235 & 535.9 & 4.383 & 544.7 \\
\hline 4.377 & 82.5 & 3.445 & 423.9 & 3.738 & 454.3 & 3.664 & 454.8 & 3.468 & 431.9 & 3.906 & 456.1 & 3.711 & 462.7 & 4.12 & 509 & 4.143 & 520 \\
\hline 3.986 & 457.4 & 3.326 & 401 & 3.687 & 434.5 & 3.427 & 425.7 & 3.362 & 405.9 & 3.705 & 430.5 & 3.537 & 436.7 & 3.977 & 479 & 3.85 & 482.5 \\
\hline 3.723 & 430.1 & 3.104 & 370.2 & 3.528 & 409.8 & 3.306 & 400.1 & 3.261 & 383 & 3.332 & 405 & 3.474 & 412 & 3.684 & 457.9 & 3.658 & 458.3 \\
\hline 3.294 & 391.8 & 2.776 & 345.1 & 3.122 & 370.6 & 3.11 & 375 & 2.954 & 351.2 & 3.128 & 380.3 & 3.394 & 383 & 3.525 & 435.4 & 3.51 & 426.1 \\
\hline 3.125 & 368 & 2.69 & 315.5 & 2.8 & 345.1 & 2.8 & 350.3 & 2.85 & 328.3 & 3.022 & 355.2 & 2.809 & 342.4 & 3.406 & 407.6 & 3.14 & 401.9 \\
\hline 2.983 & 345.1 & 2.498 & 291.3 & 2.69 & 319.1 & 2.758 & 318.6 & 2.693 & 302.7 & 2.705 & 312 & 2.871 & 334 & 2.918 & 367.5 & 3.06 & 375 \\
\hline 2.666 & 311.6 & 2.394 & 268.8 & 2.379 & 296.6 & 2.403 & 294.8 & 2.394 & 278.1 & 2.297 & 292.2 & 2.613 & 305.8 & 2.711 & 344.2 & 2.951 & 350.8 \\
\hline 2.625 & 286 & 2.092 & 243.7 & 2.267 & 265.7 & 2.433 & 272.3 & 2.199 & 254.3 & 2.205 & 264.9 & 2.299 & 275.9 & 2.501 & 314.6 & 2.61 & 326.5 \\
\hline & & & & & & & & & & & & & & & & & \\
\hline & & & & & & & & & & & & & & & & & \\
\hline
\end{tabular}


Table 4. Rheometer Response for Blank - Pretreated

\begin{tabular}{|c|c|c|c|c|c|c|c|c|c|c|c|c|c|c|c|c|c|}
\hline run1 & & run2 & & run3 & & run4 & & run5 & & run6 & & run7 & & run8 & & run9 & \\
\hline [Pa] & {$[1 / s]$} & [Pa] & {$[1 / \mathrm{s}]$} & [Pa] & {$[1 / s]$} & [Pa] & {$[1 / s]$} & [Pa] & {$[1 / s]$} & [Pa] & {$[1 / s]$} & [Pa] & {$[1 / s]$} & [Pa] & {$[1 / s]$} & [Pa] & [1/s] \\
\hline 2.739 & 306.7 & 1.771 & 322.6 & 2.144 & 329.2 & 1.602 & 318.6 & 1.25 & 304.1 & & & & & & & & \\
\hline 2.863 & 323.9 & 2.025 & 323.9 & 2.401 & 345.9 & 1.934 & 325.2 & 1.522 & 318.2 & & & & & & & & \\
\hline 3.023 & 343.3 & 2.404 & 341.1 & 2.523 & 365.8 & 2.218 & 344.2 & 1.762 & 340.6 & & & & & & & & \\
\hline 3.082 & 374.1 & 2.626 & 366.2 & 2.91 & 404.1 & 2.375 & 369.3 & 1.895 & 366.6 & & & & & & & & \\
\hline 3.203 & 399.3 & 2.804 & 396.2 & 3.02 & 426.6 & 2.543 & 394.9 & 2.067 & 397.1 & & & & & & & & \\
\hline 3.307 & 421.7 & 2.901 & 423.1 & 3.191 & 454.3 & 2.671 & 418.2 & 2.233 & 420.4 & & & & & & & & \\
\hline 3.484 & 445.5 & 3.023 & 448.2 & 3.366 & 478.6 & 2.884 & 443.3 & 2.33 & 446.9 & & & & & & & & \\
\hline 3.792 & 484.3 & 3.262 & 472 & 3.514 & 504.1 & 3.212 & 483 & 2.552 & 475.5 & & & & & & & & \\
\hline 3.89 & 508.1 & 3.348 & 498.4 & 3.65 & 536.3 & 3.399 & 505.5 & 2.765 & 501.5 & & & & & & & & \\
\hline 4.017 & 535.4 & 3.588 & 524 & 3.718 & 563.6 & 3.555 & 534.1 & 2.946 & 527.9 & & & & & & & & \\
\hline 4.15 & 561.9 & 3.686 & 548.2 & 3.985 & 584.8 & 3.686 & 563.2 & 3.135 & 555.3 & & & & & & & & \\
\hline 4.183 & 591 & 4.082 & 583.9 & 4.186 & 614.8 & 3.964 & 587 & 3.26 & 579.1 & & & & & & & & \\
\hline 4.378 & 617 & 4.192 & 614.8 & 4.263 & 639.9 & 3.993 & 619.2 & 3.452 & 604.6 & & & & & & & & \\
\hline 4.491 & 640.8 & 4.272 & 639.4 & 4.461 & 661.9 & 4.183 & 641.2 & 3.618 & 629.3 & & & & & & & & \\
\hline 4.618 & 665 & 4.458 & 661.9 & 4.576 & 691.9 & 4.34 & 666.3 & 4.05 & 671.6 & & & & & & & & \\
\hline 4.911 & 702.9 & 4.748 & 698.9 & 4.787 & 721.8 & 4.514 & 690.5 & 4.215 & 698.9 & & & & & & & & \\
\hline 5.05 & 726.2 & 4.875 & 723.6 & 5.012 & 745.6 & 4.763 & 713.5 & 4.402 & 722.3 & & & & & & & & \\
\hline 5.219 & 754.4 & 5.056 & 748.3 & 5.411 & 787.1 & 4.881 & 740.3 & 4.612 & 752.2 & & & & & & & & \\
\hline 5.37 & 779.1 & 5.145 & 779.6 & 5.535 & 809.1 & 5.272 & 781.8 & 4.84 & 775.2 & & & & & & & & \\
\hline 5.488 & 811.7 & 5.373 & 805.1 & 5.734 & 833.3 & 5.432 & 808.7 & 4.896 & 807.3 & & & & & & & & \\
\hline 5.615 & 837.7 & 5.532 & 828 & 5.793 & 866.8 & 5.651 & 833.8 & 5.071 & 830.2 & & & & & & & & \\
\hline 5.722 & 862 & 5.657 & 850.1 & 5.92 & 893.3 & 5.864 & 859.3 & 5.231 & 853.6 & & & & & & & & \\
\hline 5.89 & 885.3 & 5.887 & 890.6 & 6.157 & 917.1 & 6.035 & 886.7 & 5.337 & 877.8 & & & & & & & & \\
\hline 6.047 & 910 & 6.009 & 913.5 & 6.305 & 945.3 & 6.207 & 916.6 & 5.716 & 917.5 & & & & & & & & \\
\hline 6.172 & 936 & 6.112 & 937.3 & 6.471 & 971.7 & 6.361 & 944.4 & 5.657 & 925.9 & & & & & & & & \\
\hline 6.604 & 976.1 & 6.364 & 961.1 & 6.666 & 993.3 & 6.568 & 975.2 & 5.87 & 955 & & & & & & & & \\
\hline 6.799 & 1000 & 6.485 & 986.2 & 6.823 & 1018 & 6.684 & 996.8 & 6.225 & 996.8 & & & & & & & & \\
\hline 6.882 & 1029 & 6.775 & 1023 & 7.193 & 1058 & 6.849 & 1025 & 6.459 & 1020 & & & & & & & & \\
\hline 6.959 & 1052 & 6.932 & 1051 & 7.308 & 1087 & 7.03 & 1047 & 6.53 & 1053 & & & & & & & & \\
\hline 7.086 & 1078 & 7.116 & 1074 & 7.45 & 1109 & 7.184 & 1072 & 6.681 & 1080 & & & & & & & & \\
\hline 7.335 & 1104 & 7.264 & 1096 & 7.589 & 1133 & 7.595 & 1109 & 6.944 & 1103 & & & & & & & & \\
\hline
\end{tabular}


Table 4. Rheometer Response for Blank - Pretreated - continued

\begin{tabular}{|c|c|c|c|c|c|c|c|c|c|c|c|c|c|c|c|c|c|}
\hline run1 & & run2 & & run3 & & run4 & & run5 & & run6 & & run7 & & run8 & & run9 & \\
\hline [Pa] & [1/s] & [Pa] & {$[1 / \mathrm{s}]$} & [Pa] & {$[1 / s]$} & [Pa] & {$[1 / s]$} & [Pa] & {$[1 / s]$} & [Pa] & {$[1 / s]$} & [Pa] & {$[1 / s]$} & [Pa] & {$[1 / s]$} & [Pa] & [1/s] \\
\hline 7.746 & 1141 & 7.586 & 1137 & 7.716 & 1158 & 7.684 & 1136 & 7.148 & 1129 & & & & & & & & \\
\hline 7.921 & 1165 & 7.743 & 1160 & 7.927 & 1182 & 7.814 & 1162 & 7.204 & 1152 & & & & & & & & \\
\hline 7.998 & 1188 & 7.921 & 1184 & 8.14 & 1206 & 8.027 & 1184 & 7.56 & 1192 & & & & & & & & \\
\hline 8.163 & 1220 & 8.018 & 1211 & 8.234 & 1235 & 8.187 & 1207 & 7.554 & 1199 & & & & & & & & \\
\hline 8.335 & 1243 & 8.166 & 1234 & 8.663 & 1277 & 8.385 & 1232 & 8.006 & 1241 & & & & & & & & \\
\hline 8.462 & 1268 & 8.489 & 1274 & 8.803 & 1300 & 8.492 & 1258 & 8.11 & 1265 & & & & & & & & \\
\hline 8.507 & 1297 & 8.708 & 1299 & 8.912 & 1328 & 8.956 & 1298 & 8.095 & 1296 & & & & & & & & \\
\hline 8.847 & 1322 & 8.862 & 1324 & 9.101 & 1352 & 9.078 & 1320 & 8.273 & 1320 & & & & & & & & \\
\hline 8.921 & 1348 & 9.016 & 1353 & 9.229 & 1373 & 9.288 & 1352 & 8.444 & 1341 & & & & & & & & \\
\hline 9.137 & 1372 & 9.205 & 1378 & 9.628 & 1411 & 9.377 & 1375 & 8.616 & 1366 & & & & & & & & \\
\hline 9.374 & 1417 & 9.383 & 1406 & 9.788 & 1434 & 9.531 & 1400 & 8.977 & 1409 & & & & & & & & \\
\hline 9.584 & 1441 & 9.545 & 1434 & 9.827 & 1463 & 9.673 & 1425 & 9.187 & 1430 & & & & & & & & \\
\hline 9.924 & 1465 & 9.77 & 1456 & 10.01 & 1488 & 10.15 & 1467 & 9.294 & 1462 & & & & & & & & \\
\hline 10.04 & 1495 & 9.895 & 1481 & 10.23 & 1516 & 10.31 & 1489 & 9.386 & 1487 & & & & & & & & \\
\hline 10.28 & 1522 & 10.21 & 1518 & 10.29 & 1545 & 10.42 & 1519 & 9.386 & 1511 & & & & & & & & \\
\hline 10.42 & 1545 & 10.39 & 1540 & 10.64 & 1569 & 10.6 & 1545 & 9.699 & 1537 & & & & & & & & \\
\hline 9.714 & 1542 & 9.909 & 1548 & 8.948 & 1492 & 9.409 & 1539 & 8.563 & 1528 & & & & & & & & \\
\hline 9.46 & 1517 & 8.382 & 1519 & 8.684 & 1463 & 8.586 & 1508 & 8.391 & 1505 & & & & & & & & \\
\hline 9.317 & 1493 & 8.261 & 1482 & 8.35 & 1436 & 8.613 & 1460 & 8.279 & 1480 & & & & & & & & \\
\hline 9.164 & 1470 & 8.279 & 1432 & 8.083 & 1406 & 8.412 & 1432 & 7.719 & 1438 & & & & & & & & \\
\hline 9.004 & 1448 & 8.4 & 1409 & 7.938 & 1379 & 8.151 & 1405 & 7.61 & 1412 & & & & & & & & \\
\hline 8.658 & 1408 & 8.249 & 1378 & 7.891 & 1357 & 8.057 & 1379 & 7.545 & 1387 & & & & & & & & \\
\hline 8.498 & 1385 & 8.291 & 1367 & 7.864 & 1334 & 7.885 & 1353 & 7.237 & 1356 & & & & & & & & \\
\hline 8.276 & 1357 & 8.184 & 1349 & 7.814 & 1309 & 7.69 & 1328 & 7.145 & 1334 & & & & & & & & \\
\hline 8.107 & 1335 & 8.252 & 1340 & 7.533 & 1271 & 7.663 & 1300 & 6.888 & 1311 & & & & & & & & \\
\hline 7.953 & 1303 & 8.083 & 1315 & 7.305 & 1245 & 7.385 & 1274 & 6.814 & 1276 & & & & & & & & \\
\hline 7.856 & 1278 & 7.681 & 1277 & 7.234 & 1219 & 7.246 & 1246 & 6.607 & 1253 & & & & & & & & \\
\hline 7.755 & 1256 & 7.524 & 1252 & 7.104 & 1188 & 7.048 & 1219 & 6.488 & 1228 & & & & & & & & \\
\hline 7.388 & 1217 & 7.361 & 1223 & 6.909 & 1163 & 6.787 & 1194 & 6.284 & 1204 & & & & & & & & \\
\hline 7.225 & 1189 & 7.267 & 1192 & 6.784 & 1140 & 6.69 & 1171 & 6.053 & 1179 & & & & & & & & \\
\hline 7.113 & 1166 & 7.086 & 1170 & 6.618 & 1116 & 6.314 & 1129 & 5.595 & 1137 & & & & & & & & \\
\hline
\end{tabular}


Table 4. Rheometer Response for Blank - Pretreated - continued

\begin{tabular}{|c|c|c|c|c|c|c|c|c|c|c|c|c|c|c|c|c|c|}
\hline run1 & & run2 & & run3 & & run4 & & run5 & & run6 & & run7 & & run8 & & run9 & \\
\hline [Pa] & [1/s] & [Pa] & {$[1 / \mathrm{s}]$} & [Pa] & {$[1 / s]$} & [Pa] & {$[1 / s]$} & [Pa] & {$[1 / s]$} & [Pa] & {$[1 / s]$} & [Pa] & {$[1 / s]$} & [Pa] & {$[1 / s]$} & [Pa] & [1/s] \\
\hline 6.95 & 1141 & 6.95 & 1145 & 6.317 & 1077 & 6.166 & 1106 & 5.5 & 1113 & & & & & & & & \\
\hline 6.787 & 1116 & 6.577 & 1109 & 6.05 & 1056 & 6.006 & 1074 & 5.21 & 1087 & & & & & & & & \\
\hline 6.586 & 1088 & 6.485 & 1086 & 6.038 & 1025 & 5.763 & 1050 & 4.958 & 1064 & & & & & & & & \\
\hline 6.385 & 1064 & 6.34 & 1055 & 5.911 & 1001 & 5.763 & 1023 & 4.929 & 1030 & & & & & & & & \\
\hline 6.231 & 1036 & 6.186 & 1030 & 5.76 & 977.4 & 5.592 & 997.3 & 4.627 & 1008 & & & & & & & & \\
\hline 5.799 & 995.1 & 6.053 & 1009 & 5.592 & 955 & 5.461 & 972.1 & 4.571 & 984.5 & & & & & & & & \\
\hline 5.689 & 972.1 & 5.935 & 985.8 & 5.458 & 926.8 & 5.313 & 946.1 & 4.431 & 958.9 & & & & & & & & \\
\hline 5.491 & 949.2 & 5.547 & 946.1 & 5.014 & 888.4 & 5.085 & 922.3 & 4.056 & 920.6 & & & & & & & & \\
\hline 5.417 & 920.1 & 5.325 & 921.5 & 4.828 & 863.7 & 4.952 & 891.5 & 3.982 & 893.7 & & & & & & & & \\
\hline 5.313 & 897.7 & 5.307 & 890.6 & 4.716 & 832.9 & 4.733 & 864.6 & 3.807 & 871.7 & & & & & & & & \\
\hline 5.121 & 873.9 & 5.085 & 866.4 & 4.541 & 806 & 4.491 & 839.9 & 3.73 & 839.9 & & & & & & & & \\
\hline 4.946 & 849.2 & 4.997 & 840.4 & 4.301 & 781.8 & 4.334 & 814.8 & 3.564 & 815.7 & & & & & & & & \\
\hline 4.653 & 807.8 & 4.704 & 812.6 & 4.245 & 751.8 & 4.198 & 789.3 & 3.416 & 788.8 & & & & & & & & \\
\hline 4.428 & 782.7 & 4.547 & 788.4 & 4.112 & 726.2 & 3.964 & 761.5 & 3.203 & 764.6 & & & & & & & & \\
\hline 4.212 & 759.7 & 4.23 & 753.6 & 3.934 & 702.9 & 3.597 & 720.1 & 3.123 & 738.1 & & & & & & & & \\
\hline 4.109 & 728.4 & 4.062 & 729.3 & 3.677 & 678.7 & 3.369 & 697.2 & 2.854 & 712.1 & & & & & & & & \\
\hline 3.908 & 705.5 & 4.005 & 698.5 & 3.564 & 655.3 & 3.18 & 670.3 & 2.514 & 683.5 & & & & & & & & \\
\hline 3.703 & 680.9 & 3.843 & 674.7 & 3.18 & 616.5 & 2.981 & 640.8 & 2.33 & 661.9 & & & & & & & & \\
\hline 3.677 & 657.1 & 3.644 & 650.4 & 3.02 & 594.5 & 2.78 & 615.6 & 2.037 & 622.7 & & & & & & & & \\
\hline 3.313 & 614.8 & 3.508 & 626.7 & 2.901 & 563.2 & 2.656 & 589.6 & 2.022 & 594.9 & & & & & & & & \\
\hline 3.15 & 591.8 & 3.183 & 587.4 & 2.697 & 539.4 & 2.529 & 558.3 & 1.943 & 570.2 & & & & & & & & \\
\hline 3.046 & 561.9 & 2.996 & 565.8 & 2.582 & 511.6 & 2.395 & 531 & 1.762 & 546 & & & & & & & & \\
\hline 2.878 & 532.8 & 2.824 & 538.5 & 2.455 & 480.8 & 2.15 & 507.7 & 1.706 & 522.2 & & & & & & & & \\
\hline 2.718 & 509 & 2.739 & 508.5 & 2.265 & 453.9 & 2.04 & 481.2 & 1.419 & 482.1 & & & & & & & & \\
\hline 2.537 & 486.5 & 2.62 & 483 & 2.102 & 431 & 1.842 & 455.7 & 1.229 & 459.2 & & & & & & & & \\
\hline 2.434 & 462.7 & 2.472 & 461.8 & 1.934 & 407.6 & 1.694 & 431.4 & 1.064 & 435.4 & & & & & & & & \\
\hline 2.25 & 435.8 & 2.241 & 430.5 & 1.682 & 365.3 & 1.573 & 406.8 & 0.874 & 401.9 & & & & & & & & \\
\hline 2.014 & 410.7 & 2.082 & 406.3 & 1.552 & 342.4 & 1.363 & 379.9 & 0.64 & 383 & & & & & & & & \\
\hline 1.658 & 370.6 & 1.783 & 368 & 1.419 & 318.6 & 0.948 & 339.8 & 0.602 & 351.2 & & & & & & & & \\
\hline 1.605 & 341.1 & 1.617 & 345.1 & 1.203 & 296.1 & 0.827 & 312.4 & 0.466 & 325.7 & & & & & & & & \\
\hline 1.428 & 315.5 & 1.484 & 321.7 & 1.117 & 270.1 & 0.652 & 282.9 & 0.27 & 299.7 & & & & & & & & \\
\hline
\end{tabular}


Table 4. Rheometer Response for Blank - Pretreated - continued

\begin{tabular}{|c|c|c|c|c|c|c|c|c|c|c|c|c|c|c|c|c|c|}
\hline run1 & & run2 & & run3 & & run4 & & run5 & & run6 & & run7 & & run8 & & run9 & \\
\hline [Pa] & {$[1 / s]$} & [Pa] & {$[1 / \mathrm{s}]$} & [Pa] & {$[1 / s]$} & {$[\mathrm{Pa}]$} & {$[1 / s]$} & {$[\mathrm{Pa}]$} & {$[1 / s]$} & {$[\mathrm{Pa}]$} & {$[1 / \mathrm{s}]$} & {$[\mathrm{Pa}]$} & {$[1 / \mathrm{s}]$} & {$[\mathrm{Pa}]$} & {$[1 / \mathrm{s}]$} & {$[\mathrm{Pa}]$} & {$[1 / \mathrm{s}]$} \\
\hline 1.271 & 288.2 & 1.363 & 290.9 & 0.88 & 244.6 & 0.487 & 256.9 & 0.06333 & 275 & & & & & & & & \\
\hline 1.182 & 264.9 & 1.22 & 267.1 & & & & & & & & & & & & & & \\
\hline 1.025 & 245 & 1.064 & 243.7 & & & & & & & & & & & & & & \\
\hline & & & & & & & & & & & & & & & & & \\
\hline & & & & & & & & & & & & & & & & & \\
\hline
\end{tabular}


Table 5. $25^{\circ} \mathrm{C}$ Pretreated

\begin{tabular}{|c|c|c|c|c|c|c|c|c|c|c|c|c|c|c|c|c|c|}
\hline run1 & & run2 & & run3 & & run4 & & run5 & & run6 & & run7 & & run8 & & run9 & \\
\hline [Pa] & {$[1 / \mathrm{s}]$} & {$[\mathrm{Pa}]$} & {$[1 / \mathrm{s}]$} & {$[\mathrm{Pa}]$} & {$[1 / s]$} & [Pa] & {$[1 / \mathrm{s}]$} & {$[\mathrm{Pa}]$} & {$[1 / \mathrm{s}]$} & {$[\mathrm{Pa}]$} & {$[1 / \mathrm{s}]$} & [Pa] & {$[1 / \mathrm{s}]$} & {$[\mathrm{Pa}]$} & {$[1 / \mathrm{s}]$} & {$[\mathrm{Pa}]$} & {$[1 / \mathrm{s}]$} \\
\hline 2.62 & 331 & 3.428 & 342 & 3.383 & 345.5 & 3.351 & 346.4 & 3.016 & 313.8 & 3.185 & 324.8 & 3.963 & 423.1 & 3.454 & 375.9 & 3.365 & 372.8 \\
\hline 3.286 & 326.5 & 3.567 & 364.4 & 3.475 & 366.2 & 3.632 & 368.4 & 3.138 & 338.9 & 3.244 & 342.9 & 4.2 & 450.4 & 3.499 & 402.3 & 3.564 & 394.9 \\
\hline 3.389 & 340.6 & 3.818 & 392.2 & 3.792 & 386.9 & 3.798 & 390.9 & 3.425 & 364.4 & 3.587 & 364.4 & 4.386 & 474.6 & 3.839 & 426.6 & 3.67 & 419.5 \\
\hline 3.638 & 368.9 & 4.123 & 417.3 & 3.981 & 412 & 4.262 & 437.2 & 3.721 & 389.6 & 3.833 & 394.4 & 4.617 & 506.3 & 4.085 & 452.6 & 3.928 & 443.8 \\
\hline 3.948 & 390 & 4.321 & 441.6 & 4.425 & 451.7 & 4.558 & 459.6 & 3.786 & 412.9 & 3.972 & 422.2 & 4.842 & 531.5 & 4.434 & 476.4 & 4.176 & 470.2 \\
\hline 4.07 & 414.2 & 4.561 & 471.5 & 4.605 & 474.6 & 4.345 & 470.6 & 4.052 & 436.3 & 4.173 & 448.2 & 4.913 & 554.4 & 4.537 & 500.6 & 4.363 & 498 \\
\hline 4.259 & 438.5 & 4.768 & 495.8 & 4.863 & 500.6 & 4.662 & 497.1 & 4.236 & 463.6 & 4.378 & 474.2 & 5.123 & 576 & 4.842 & 542 & 4.623 & 522.6 \\
\hline 4.422 & 55.8 & 4.925 & 520.9 & 5.023 & 533.2 & 4.854 & 527.9 & 4.703 & 501.1 & 4.635 & 501.1 & 5.461 & 615.2 & 5.049 & 565 & 4.86 & 547.3 \\
\hline 4.981 & 503.7 & 5.336 & 564.5 & 5.188 & 554.8 & 5.422 & 566.7 & 4.993 & 531 & 5.023 & 528.4 & 5.76 & 641.2 & 5.342 & 597.1 & 5.055 & 574.2 \\
\hline 5.236 & 529.7 & 5.547 & 587 & 5.339 & 580.4 & 5.582 & 591 & 5.221 & 553.9 & 5.357 & 553.5 & 5.848 & 671.2 & 5.55 & 623.6 & 5.49 & 619.6 \\
\hline 5.36 & 561 & 5.724 & 617.8 & 5.541 & 606.8 & 5.774 & 617 & 5.413 & 581.7 & 5.476 & 577.7 & 5.982 & 694.1 & 5.816 & 646.5 & 5.698 & 640.8 \\
\hline 5.552 & 582.6 & 6.029 & 641.6 & 5.698 & 631.9 & 5.86 & 639.9 & 5.624 & 605.5 & 6.002 & 618.3 & 6.242 & 717.4 & 5.943 & 670.7 & 5.922 & 666.3 \\
\hline 5.698 & 605.5 & 6.171 & 665.4 & 5.928 & 657.1 & 6.307 & 679.5 & 5.689 & 636.8 & 6.165 & 641.2 & 6.526 & 741.2 & 6.079 & 693.2 & 6.112 & 698 \\
\hline 6.236 & 644.3 & 6.278 & 692.8 & 6.325 & 694.1 & 6.209 & 687.9 & 5.899 & 660.6 & 6.553 & 670.3 & 6.624 & 764.1 & 6.434 & 736.4 & 6.23 & 722.3 \\
\hline 6.36 & 667.6 & 6.633 & 718.3 & 6.606 & 724.5 & 6.76 & 731.1 & 6.106 & 684.4 & 6.668 & 696.3 & 6.822 & 789.3 & 6.775 & 758.4 & 6.571 & 746.1 \\
\hline 6.505 & 691 & 6.754 & 743.9 & 6.683 & 748.3 & 6.893 & 757.1 & 6.337 & 705.5 & 6.938 & 722.7 & 7.325 & 830.7 & 6.92 & 784.4 & 6.683 & 770.3 \\
\hline 6.742 & 722.7 & 7.017 & 770.3 & 6.952 & 774.3 & 7.183 & 780 & 6.807 & 746.5 & 7.171 & 748.3 & 7.414 & 853.6 & 7.088 & 814.8 & 6.973 & 799 \\
\hline 6.878 & 745.2 & 7.254 & 795.9 & 7.177 & 799.8 & 7.337 & 808.7 & 6.866 & 770.3 & 7.29 & 778.2 & 7.677 & 880 & 7.299 & 837.7 & 7.115 & 825.8 \\
\hline 7.337 & 780.9 & 7.435 & 819.7 & 7.429 & 825 & 7.627 & 834.2 & 7.219 & 795.9 & 7.556 & 802.5 & 7.737 & 903.4 & 7.529 & 862.9 & 7.414 & 850.1 \\
\hline 7.476 & 805.1 & 7.967 & 860.2 & 7.843 & 863.7 & 7.74 & 868.1 & 7.319 & 825.4 & 7.769 & 828.9 & 7.929 & 927.6 & 7.668 & 885.3 & 7.701 & 880.5 \\
\hline 7.562 & 834.7 & 8.074 & 888.4 & 7.941 & 885.8 & 8.018 & 886.2 & 7.55 & 849.6 & 7.964 & 854 & 8.175 & 957.2 & 7.976 & 908.7 & 7.902 & 903 \\
\hline 7.926 & 860.2 & 8.234 & 914 & 8.077 & 916.2 & 8.287 & 921.9 & 7.713 & 870.3 & 8.293 & 878.7 & 8.515 & 983.2 & 8.423 & 952.3 & 8.035 & 930.7 \\
\hline 8.231 & 888 & 8.417 & 938.7 & 8.382 & 939.1 & 8.539 & 945.7 & 7.994 & 89 & 8.397 & 902.5 & 8.944 & 1027 & 8.559 & 976.1 & 8.539 & 967.7 \\
\hline 8.349 & 908.7 & 8.698 & 960.2 & 8.547 & 962.5 & 8.775 & 970.4 & 8.186 & 924.1 & 8.908 & 944.4 & 9.261 & 1050 & 8.681 & 1000 & 8.648 & 998.1 \\
\hline 8.627 & 936.9 & 8.787 & 985.8 & 8.965 & 997.7 & 8.837 & 993.7 & 8.479 & 951.9 & 9.255 & 970.4 & 9.364 & 1079 & 9.065 & 1028 & 8.82 & 1020 \\
\hline 8.796 & 962.5 & 9.021 & 1011 & 9.104 & 1022 & 9.074 & 1018 & 8.645 & 977.9 & 9.494 & 993.3 & 9.625 & 1101 & 9.204 & 1056 & 9.051 & 1043 \\
\hline 9.258 & 997.7 & 9.545 & 1054 & 9.249 & 1052 & 9.503 & 1058 & 9.045 & 1016 & 9.66 & 1024 & 9.77 & 1125 & 9.497 & 1087 & 9.199 & 1071 \\
\hline 9.409 & 1020 & 9.761 & 1079 & 9.444 & 1077 & 9.699 & 1084 & 9.243 & 1044 & 9.675 & 1049 & 10 & 1150 & 9.731 & 1111 & 9.489 & 1094 \\
\hline 9.58 & 1049 & 10.01 & 1105 & 9.693 & 1101 & 9.873 & 1111 & 9.568 & 1074 & 9.773 & 1076 & 10.38 & 1189 & 9.847 & 1137 & 9.876 & 1134 \\
\hline 9.752 & 1072 & 10.2 & 1136 & 9.853 & 1129 & 10.13 & 1137 & 9.708 & 1100 & 10.07 & 1098 & 10.58 & 1213 & 10.12 & 1159 & 10.18 & 1155 \\
\hline 9.977 & 1095 & 10.41 & 1160 & 10.15 & 1154 & 10.42 & 1162 & 10.15 & 1120 & 10.16 & 1122 & 10.77 & 1242 & 10.47 & 1196 & 10.38 & 1188 \\
\hline
\end{tabular}


Table 5. $25{ }^{\circ} \mathrm{C}$ Pretreated - continued

\begin{tabular}{|c|c|c|c|c|c|c|c|c|c|c|c|c|c|c|c|c|c|}
\hline run1 & & run2 & & run3 & & run4 & & run5 & & run6 & & run7 & & run8 & & run9 & \\
\hline [Pa] & {$[1 / s]$} & [Pa] & [1/s] & [Pa] & {$[1 / s]$} & [Pa] & {$[1 / s]$} & [Pa] & [1/s] & [Pa] & [1/s] & [Pa] & [1/s] & [Pa] & [1/s] & [Pa] & {$[1 / s]$} \\
\hline 10.47 & 1133 & 10.58 & 1184 & 10.36 & 1179 & 10.61 & 1192 & 10.21 & 1155 & 10.39 & 1162 & 10.89 & 1268 & 10.71 & 1226 & 10.63 & 1211 \\
\hline 10.66 & 155 & 10.85 & 1210 & 10.63 & 1204 & 10.78 & 1216 & 10.42 & 1181 & 10.59 & 1187 & 11.24 & 1295 & 10.97 & 1250 & 10.78 & 1234 \\
\hline 10.8 & 1184 & 11.08 & 1239 & 10.85 & 1228 & 10.97 & 1237 & 10.77 & 1207 & 10.68 & 1218 & 11.43 & 1319 & 11.11 & 1274 & 10.97 & 1259 \\
\hline 10.95 & 1209 & 11.27 & 1264 & 11.1 & 1253 & 11.48 & 1275 & 10.9 & 1231 & 10.89 & 1244 & 11.61 & 1344 & 11.39 & 1305 & 11.21 & 1285 \\
\hline 11.2 & 1234 & 11.56 & 1290 & 11.5 & 1302 & 11.6 & 1301 & 11.01 & 1257 & 11.17 & 1267 & 11.9 & 1367 & 11.59 & 1326 & 11.38 & 1312 \\
\hline 11.46 & 1261 & 11.75 & 1315 & 11.78 & 1324 & 11.84 & 1326 & 11.25 & 1279 & 11.43 & 1294 & 12.3 & 1407 & 11.73 & 1350 & 12.05 & 1355 \\
\hline 11.64 & 1285 & 12.15 & 1353 & 11.84 & 1353 & 11.99 & 1357 & 11.81 & 1319 & 11.85 & 1319 & 12.47 & 1432 & 11.91 & 1373 & 12.21 & 1380 \\
\hline 12.01 & 1311 & 12.45 & 1382 & 12.16 & 1376 & 12.25 & 1378 & 12 & 1346 & 12.21 & 1348 & 12.71 & 1460 & 12.47 & 1416 & 12.44 & 1405 \\
\hline 12.2 & 1335 & 12.7 & 1411 & 12.24 & 1399 & 12.4 & 1403 & 12.27 & 1367 & 12.45 & 1372 & 12.91 & 1485 & 12.68 & 1441 & 12.67 & 1428 \\
\hline 12.41 & 1362 & 12.79 & 1431 & 12.4 & 1423 & 12.96 & 1443 & 12.46 & 1400 & 12.87 & 1397 & 13.27 & 1511 & 12.87 & 1466 & 12.78 & 1460 \\
\hline 12.89 & 1404 & 12.9 & 1458 & 12.67 & 1449 & 13.27 & 1467 & 12.65 & 1426 & 13.1 & 1423 & 13.46 & 1541 & 13.05 & 1496 & 12.95 & 1485 \\
\hline 13.12 & 1434 & 13.21 & 1480 & 12.97 & 1475 & 13.4 & 1492 & 12.74 & 1452 & 13.32 & 1449 & 13.63 & 1564 & 13.2 & 1516 & 13.35 & 1508 \\
\hline 13.2 & 1459 & 13.68 & 1524 & 13.39 & 1518 & 13.66 & 1523 & 13.05 & 1477 & 13.37 & 1490 & 13.76 & 1588 & 13.42 & 1540 & 13.46 & 1535 \\
\hline 13.5 & 1482 & 13.97 & 1545 & 13.59 & 1545 & 13.82 & 1541 & 13.35 & 1502 & 13.57 & 1517 & 13.98 & 1612 & 13.92 & 1579 & 13.98 & 1571 \\
\hline 13.63 & 1504 & 14.04 & 1575 & 13.8 & 1570 & 14.05 & 1573 & 13.53 & 1528 & 13.71 & 1540 & 14.29 & 1641 & 14.01 & 1602 & 14.15 & 1597 \\
\hline 12.68 & 1519 & 12.04 & 1463 & 12.22 & 1486 & 12.61 & 1514 & 12.51 & 1519 & 11.26 & 1398 & 11.21 & 1408 & 11.3 & 1437 & 11.97 & 1475 \\
\hline 12.5 & 1496 & 11.82 & 1431 & 11.93 & 1464 & 12.22 & 1490 & 12.33 & 1490 & 10.94 & 1371 & 11.01 & 1379 & 11.2 & 1413 & 11.72 & 1449 \\
\hline 12.12 & 1457 & 11.6 & 1408 & 11.73 & 1430 & 12.08 & 1465 & 12.12 & 1462 & 10.49 & 1329 & 10.66 & 1354 & 10.92 & 1387 & 11.45 & 1424 \\
\hline 11.88 & 1433 & 11.35 & 1383 & 11.59 & 1408 & 11.77 & 1441 & 12 & 1436 & 10.12 & 1303 & 10.45 & 1329 & 10.74 & 1364 & 10.95 & 1382 \\
\hline 11.72 & 1402 & 11.2 & 1362 & 11.32 & 1386 & 11.41 & 1401 & 11.67 & 1410 & 9.983 & 1275 & 10.28 & 1305 & 10.22 & 1322 & 10.69 & 1357 \\
\hline 11.52 & 1378 & 10.66 & 1321 & 10.96 & 1350 & 11.38 & 1391 & 11.49 & 1384 & 9.728 & 1245 & 9.779 & 1266 & 10.03 & 1299 & 10.51 & 1327 \\
\hline 11.23 & 1352 & 10.49 & 1296 & 10.73 & 1323 & 11.18 & 1365 & 11.28 & 1360 & 9.595 & 1218 & 9.684 & 1239 & 9.861 & 1269 & 10.36 & 1301 \\
\hline 11.04 & 1327 & 10.24 & 1271 & 10.47 & 1301 & 10.72 & 1320 & 11.06 & 1334 & 9.364 & 1194 & 9.373 & 1216 & 9.702 & 1246 & 10.08 & 1278 \\
\hline 10.76 & 1301 & 10.04 & 1242 & 10.25 & 1267 & 10.44 & 1296 & 10.71 & 1309 & 9.122 & 1170 & 9.119 & 1192 & 9.503 & 1221 & 9.864 & 1255 \\
\hline 10.55 & 1275 & 9.829 & 1213 & 10.06 & 1241 & 10.26 & 1266 & 10.54 & 1284 & 8.793 & 1144 & 8.968 & 1164 & 9.237 & 1195 & 9.708 & 1232 \\
\hline 10.3 & 1250 & 9.634 & 1189 & 9.853 & 1219 & 10.1 & 1243 & 10.08 & 1245 & 8.669 & 1119 & 8.743 & 1133 & 9.059 & 1165 & 9.512 & 1204 \\
\hline 10.12 & 1224 & 9.418 & 1166 & 9.651 & 1195 & 9.761 & 1217 & 9.856 & 1214 & 8.402 & 1092 & 8.577 & 1110 & 8.704 & 1135 & 9.16 & 1178 \\
\hline 9.568 & 1184 & 9.222 & 1143 & 9.21 & 1157 & 9.598 & 1194 & 9.657 & 1189 & 8.198 & 1068 & 8.379 & 1085 & 8.429 & 1109 & 8.746 & 1137 \\
\hline 9.349 & 1156 & 8.761 & 1105 & 8.944 & 1130 & 9.125 & 1157 & 9.403 & 1165 & 7.66 & 1028 & 7.961 & 1046 & 8.207 & 1082 & 8.465 & 1114 \\
\hline 9.13 & 1133 & 8.539 & 1074 & 8.624 & 1104 & 9 & 1132 & 9.204 & 1138 & 7.429 & 1003 & 7.719 & 1022 & 7.997 & 1058 & 8.328 & 1083 \\
\hline 8.906 & 1107 & 8.325 & 1055 & 8.539 & 1075 & 8.775 & 1103 & 8.977 & 1113 & 7.367 & 977 & 7.553 & 994.6 & 7.837 & 1033 & 8.195 & 1060 \\
\hline
\end{tabular}


Table 5. $25{ }^{\circ} \mathrm{C}$ Pretreated - continued

\begin{tabular}{|c|c|c|c|c|c|c|c|c|c|c|c|c|c|c|c|c|c|}
\hline run1 & & run2 & & run3 & & run4 & & run5 & & run6 & & run7 & & run8 & & run9 & \\
\hline [Pa] & {$[1 / \mathrm{s}]$} & {$[\mathrm{Pa}]$} & {$[1 / \mathrm{s}]$} & [Pa] & {$[1 / \mathrm{s}]$} & {$[\mathrm{Pa}]$} & {$[1 / \mathrm{s}]$} & [Pa] & {$[1 / \mathrm{s}]$} & {$[\mathrm{Pa}]$} & {$[1 / \mathrm{s}]$} & {$[\mathrm{Pa}]$} & {$[1 / \mathrm{s}]$} & {$[\mathrm{Pa}]$} & {$[1 / \mathrm{s}]$} & {$[\mathrm{Pa}]$} & {$[1 / \mathrm{s}]$} \\
\hline 8.769 & 1078 & 8.127 & 1024 & 8.275 & 1052 & 8.524 & 1079 & 8.82 & 1088 & 7.145 & 951.4 & 7.328 & 966.9 & 7.245 & 992.9 & 7.95 & 1035 \\
\hline 8.494 & 1055 & 7.979 & 1001 & 8.109 & 1021 & 8.18 & 1055 & 8.536 & 1064 & 6.967 & 918.4 & 7.177 & 945.3 & 7.115 & 966.9 & 7.719 & 1007 \\
\hline 8.349 & 1032 & 7.722 & 977.4 & 7.876 & 994.6 & 8.154 & 1022 & 8.269 & 1040 & 6.76 & 892.4 & 6.982 & 921.5 & 6.914 & 942.6 & 7.316 & 969.9 \\
\hline 8.124 & 1007 & 7.615 & 952.3 & 7.624 & 966.9 & 7.834 & 992.4 & 7.79 & 999 & 6.511 & 869 & 6.683 & 894.6 & 6.745 & 917.5 & 7.115 & 945.7 \\
\hline 8.044 & 980.5 & 7.094 & 911.8 & 7.302 & 947.5 & 7.523 & 972.6 & 7.731 & 973 & 6.387 & 842.1 & 6.556 & 871.2 & 6.44 & 889.3 & 6.914 & 916.2 \\
\hline 7.438 & 938.7 & 6.923 & 886.7 & 7.151 & 915.7 & 7.378 & 940.9 & 7.5 & 944.8 & 6.026 & 817.9 & 6.097 & 829.4 & 6.346 & 859.3 & 6.71 & 894.1 \\
\hline 7.201 & 913.5 & 6.713 & 856.7 & 6.92 & 889.3 & 7.287 & 917.1 & 7.233 & 921.5 & 5.834 & 793.7 & 5.94 & 806 & 6.008 & 836.9 & 6.491 & 868.6 \\
\hline 7.091 & 889.7 & 6.464 & 831.1 & 6.763 & 864.2 & 6.949 & 891.5 & 7.165 & 898.1 & 5.44 & 752.7 & 5.807 & 780.9 & 5.928 & 812.6 & 6.236 & 844.3 \\
\hline 6.733 & 861.1 & 6.346 & 808.2 & 6.479 & 836.9 & 6.79 & 864.2 & 7.032 & 870.3 & 5.277 & 727.6 & 5.428 & 754.9 & 5.552 & 773.4 & 5.848 & 807.3 \\
\hline 6.621 & 828.9 & 6.165 & 785.7 & 6.372 & 813.9 & 6.535 & 837.3 & 6.606 & 845.7 & 5.046 & 704.2 & 5.378 & 731.1 & 5.251 & 748.7 & 5.668 & 783.1 \\
\hline 6.417 & 806.9 & 5.718 & 747 & 5.837 & 772.5 & 6.319 & 812.6 & 6.467 & 818.8 & 4.771 & 677.3 & 4.972 & 686.6 & 5.112 & 724.9 & 5.591 & 758 \\
\hline 6.242 & 780 & 5.49 & 720.1 & 5.6 & 747.8 & 6.035 & 789.3 & 6.18 & 791.5 & 4.685 & 647.4 & 4.934 & 678.2 & 5.035 & 694.1 & 5.316 & 725.8 \\
\hline 5.967 & 754 & 5.319 & 696.7 & 5.443 & 724 & 5.967 & 764.6 & 5.739 & 750.9 & 4.401 & 617.4 & 4.626 & 653.5 & 4.706 & 669.4 & 5.067 & 707.7 \\
\hline 5.727 & 724.9 & 5.117 & 664.1 & 5.307 & 696.3 & 5.733 & 733.3 & 5.535 & 724.9 & 4.2 & 593.6 & 4.141 & 612.1 & 4.484 & 645.6 & 4.792 & 679.5 \\
\hline 5.431 & 700.2 & 4.83 & 647.4 & 5.02 & 667.6 & 5.132 & 692.3 & 5.31 & 702.9 & 4.085 & 570.2 & 3.931 & 586.5 & 4.321 & 620 & 4.582 & 645.6 \\
\hline 5.295 & 675.6 & 4.591 & 613 & 4.863 & 643 & 4.919 & 670.3 & 5.008 & 669.4 & 3.848 & 543.8 & 3.756 & 564.1 & 4.085 & 596.2 & 4.392 & 621.4 \\
\hline 5.109 & 652.2 & 4.386 & 590.1 & 4.697 & 618.3 & 4.795 & 645.6 & 4.866 & 644.3 & 3.658 & 520 & 3.661 & 534.1 & 3.94 & 569.8 & 4.274 & 594.5 \\
\hline 4.682 & 613.4 & 4.321 & 569.8 & 4.431 & 594.5 & 4.573 & 614.3 & 4.656 & 620.5 & 3.516 & 496.2 & 3.439 & 509 & 3.647 & 545.1 & 4.008 & 569.8 \\
\hline 4.611 & 584.8 & 3.857 & 530.1 & 4.33 & 568.5 & 4.33 & 591 & 4.62 & 592.3 & 3.067 & 457 & 3.294 & 481.2 & 3.481 & 518.7 & 3.753 & 546.9 \\
\hline 4.312 & 561 & 3.653 & 506.3 & 3.874 & 528.8 & 4.108 & 563.2 & 4.312 & 568.9 & 2.951 & 430.5 & 2.945 & 449.9 & 3.093 & 468.4 & 3.451 & 506.3 \\
\hline 4.165 & 535 & 3.46 & 478.6 & 3.718 & 504.1 & 3.869 & 536.8 & 4.102 & 542.5 & 2.788 & 399.3 & 2.809 & 421.7 & 3.09 & 461.8 & 3.235 & 481.7 \\
\hline 3.916 & 510.3 & 3.383 & 454.8 & 3.7 & 480.8 & 3.697 & 505.9 & 3.866 & 516.9 & 2.413 & 373.7 & 2.691 & 402.3 & 2.732 & 434.5 & 3.087 & 451.7 \\
\hline 3.795 & 487.8 & & 430.1 & 3.351 & 453.9 & 3.599 & 482.5 & 3.608 & 493.1 & 2.1 & 346.8 & 2.433 & 378.5 & 2.336 & 396.6 & 2.815 & 424.4 \\
\hline 3.327 & 448.6 & 3.072 & 406.8 & 3.164 & 421.3 & 3.173 & 444.2 & 3.28 & 447.3 & 1.992 & 322.1 & 2.06 & 340.2 & 2.33 & 368.9 & 2.522 & 399.3 \\
\hline 3.291 & 425.7 & 2.714 & 367.5 & 2.91 & 397.5 & 2.972 & 419.5 & 3.007 & 424.8 & 1.699 & 296.1 & 1.918 & 313.8 & 2.075 & 345.9 & 2.424 & 373.7 \\
\hline 3.149 & 395.7 & 2.386 & 343.7 & 2.803 & 372.8 & 2.768 & 397.1 & 2.708 & 397.1 & 1.676 & 267.5 & 1.581 & 290.9 & 1.901 & 322.1 & 2.155 & 350.3 \\
\hline 2.839 & 371.9 & 2.347 & 311.6 & 2.513 & 349.5 & 2.463 & 368.9 & 2.691 & 373.7 & 1.436 & 244.6 & 1.522 & 257.4 & 1.56 & 281.2 & 1.972 & 324.3 \\
\hline 2.729 & 348.6 & 2.06 & 285.1 & 2.333 & 323.9 & 2.398 & 343.3 & 2.436 & 351.7 & & & 1.27 & 236.2 & 1.178 & 260.4 & 1.898 & 300.1 \\
\hline 2.563 & 322.1 & 1.93 & 260.9 & 2.143 & 299.2 & 2.262 & 312 & 2.034 & 310.2 & & & & & 1.223 & 253 & 1.575 & 272.3 \\
\hline 2.04 & 287.8 & & & 1.874 & 271.5 & 1.924 & 290.9 & 1.865 & 288.2 & & & & & & & 1.4 & 248.1 \\
\hline 2.051 & 258.2 & & & 1.696 & 246.8 & 1.8 & 267.1 & 1.599 & 262.2 & & & & & & & & \\
\hline & & & & & & 1.696 & 244.1 & 1.685 & 255.2 & & & & & & & & \\
\hline
\end{tabular}


Table 6. $50{ }^{\circ} \mathrm{C}$ Pretreated

\begin{tabular}{|c|c|c|c|c|c|c|c|c|c|c|c|c|c|c|c|c|c|}
\hline run1 & & run2 & & run3 & & run4 & & run5 & & run6 & & run7 & & run8 & & run9 & \\
\hline [Pa] & [1/s] & [Pa] & [1/s] & [Pa] & [1/s] & [Pa] & [1/s] & [Pa] & [1/s] & [Pa] & [1/s] & [Pa] & [1/s] & [Pa] & {$[1 / s]$} & [Pa] & {$[1 / s]$} \\
\hline 2.906 & 323.9 & 3.063 & 340.2 & 2.986 & 317.7 & 3.01 & 325.2 & 2.995 & 326.1 & 3.35 & 335.8 & 2.885 & 312 & 2.51 & 326.5 & 2.865 & 314.6 \\
\hline 3.365 & 327 & 557 & 333.2 & 3.705 & 334 & 3.542 & 326.1 & 3.537 & 327.9 & 3.569 & 350.8 & 3.471 & 328.7 & 3.483 & 319.1 & 3.03 & 310.2 \\
\hline 3.572 & 343.3 & 862 & 350.8 & 4.022 & 353.9 & 3.717 & 350.3 & 3.649 & 345.1 & 3.83 & 380.3 & 3.356 & 337.1 & 3.537 & 342.4 & 3.48 & 333.6 \\
\hline 3.735 & 63.6 & 013 & 371.1 & 4.17 & 376.3 & 3.821 & 369.7 & 3.806 & 367.1 & 3.773 & 391.3 & 3.945 & 366.2 & 4.084 & 383 & 3.83 & 384.3 \\
\hline 3.918 & 389.6 & 4.164 & 394.9 & 4.217 & 404.1 & 4.043 & 394.4 & 4.072 & 393.5 & 4.223 & 418.2 & 4.229 & 409.8 & 3.966 & 392.6 & 3.729 & 399.7 \\
\hline 4.22 & 413.4 & 4.436 & 417.8 & 4.362 & 435.4 & 4.279 & 416.4 & 4.421 & 430.1 & 4.054 & 436.7 & 4.282 & 434.1 & 4.217 & 414.2 & 4.214 & 433.6 \\
\hline 4.608 & 454.3 & 4.7 & 457.9 & 4.629 & 457.4 & 4.371 & 446.4 & 4.629 & 457.4 & 4.555 & 463.6 & 4.339 & 459.2 & 4.25 & 431 & 4.04 & 450.4 \\
\hline 4.608 & 487.4 & 4.927 & 481.7 & 4.806 & 479.9 & 4.572 & 471.5 & 4.759 & 483.4 & 4.38 & 484.3 & 4.531 & 490.9 & 4.161 & 457.9 & 4.59 & 484.8 \\
\hline 4.927 & 510.3 & 5.173 & 504.1 & 5.152 & 521.3 & 4.676 & 496.6 & 4.945 & 505 & 4.839 & 511.6 & 4.667 & 513.8 & 4.596 & 511.6 & 4.51 & 502.4 \\
\hline 5.07 & 537.6 & 5.209 & 539 & 5.342 & 544.2 & 4.865 & 521.8 & 5.067 & 537.6 & & 560.5 & & 537.2 & 4.566 & 526.2 & 4.933 & 531 \\
\hline 5.327 & 561 & 5.357 & 562.8 & 5.51 & 569.4 & 5.342 & 565.4 & 5.197 & 564.1 & 5.123 & 579.1 & 5.336 & 575.1 & 5.416 & 578.2 & 4.892 & 551.7 \\
\hline 5.451 & 584.3 & & 585.7 & & 602.9 & & 590.5 & & 589.2 & & 602.9 & & & & 614.3 & & 578.6 \\
\hline 5.638 & 617 & 5.738 & 609.5 & 5.966 & 627.1 & 5.682 & 617 & 5.484 & 614.3 & 5.445 & 626.2 & 5.653 & 623.6 & 5.907 & 653.5 & 5.537 & 628 \\
\hline 5.922 & 643.8 & 6.176 & 647.8 & 6.132 & 650.4 & 5.866 & 646 & 5.629 & 639.4 & 5.747 & 652.7 & 5.821 & 648.7 & 5.771 & 680.9 & 5.422 & 645.6 \\
\hline 6.182 & 670.3 & 6.351 & 670.3 & 6.138 & 674.7 & 6.085 & 669 & 5.88 & 666.8 & 6.105 & 698.5 & 5.877 & 680.9 & 6.162 & 707.3 & 5.916 & 673.8 \\
\hline 6.33 & 693.2 & 6.499 & 701.6 & 6.428 & 700.7 & 6.236 & 693.2 & 6.067 & 693.6 & 5.984 & 718.8 & 6.12 & 704.2 & 5.957 & 727.6 & 5.732 & 695 \\
\hline 6.511 & 718.8 & 727 & 724 & & 737.3 & & 717 & 6.2 & 717.9 & & 748.3 & 6.224 & 726.7 & 05 & 754.4 & 209 & 724.9 \\
\hline 6.718 & 743.9 & 6.801 & 748.3 & 7.002 & 768.1 & 6.608 & 757.1 & .475 & 744.8 & 6.188 & 766.3 & 6.65 & 763.7 & 6.301 & 774.7 & 6.073 & 743.4 \\
\hline 7.141 & 784.9 & & 772.1 & & 775.6 & & 776.9 & & 783.5 & & 792.8 & 6.641 & 789.7 & 6.783 & 805.1 & 6.588 & 769.4 \\
\hline 7.363 & 810.4 & 7.378 & 808.2 & 7.476 & 818.3 & 6.907 & 810.4 & 6.901 & 810 & 6.674 & 814.8 & 6.922 & 813.1 & & 823.2 & 6.916 & 817 \\
\hline 7.484 & 833.8 & 7.547 & 834.2 & 7.576 & 841.3 & 7.183 & 834.7 & 7.171 & 835.1 & 7.052 & 842.6 & 6.99 & 844.8 & 6.981 & 847.4 & 6.819 & 832.9 \\
\hline 7.718 & 865.1 & 7.718 & 858.5 & 7.783 & 870.8 & 7.313 & 863.3 & 7.354 & 858.5 & 7.348 & 891.5 & 7.221 & 862.4 & 7.076 & 873 & 7.239 & 861.5 \\
\hline 7.863 & 890.6 & 7.919 & 884 & 8.002 & 899 & 7.496 & 888.4 & 7.481 & 888 & 7.295 & 909.6 & 7.336 & 899.9 & 7.523 & 905.6 & 7.316 & 887.1 \\
\hline 8.044 & 914.9 & 8.094 & 913.1 & 8.23 & 922.8 & 7.736 & 916.6 & 7.632 & 917.5 & & 938.2 & 7.532 & 924.1 & 7.265 & 921.5 & 7.493 & 913.1 \\
\hline 8.26 & 941.3 & 8.248 & 935.6 & 8.375 & 955 & 7.973 & 940.9 & 7.774 & 940 & 7.612 & 956.3 & 7.555 & 947 & 7.887 & 943.5 & 7.84 & 954.1 \\
\hline 8.594 & 978.3 & 8.591 & 973.5 & & 980.5 & 7.988 & 964.7 & 7.94 & 965.5 & & 988.9 & 7.81 & 971.7 & 7.724 & 968.6 & 8.076 & 973.9 \\
\hline 8.807 & 1003 & 8.834 & 999 & 8.775 & 1002 & 8.384 & 1001 & 8.153 & 989.3 & 7.928 & 1004 & 8.286 & 1013 & 8.183 & 996.8 & 8.153 & 1003 \\
\hline 9.05 & 1027 & 9 & 1026 & 9.094 & 1040 & 8.585 & 1026 & 8.34 & 1014 & 8.396 & 1032 & 8.467 & 1037 & 8.038 & 1014 & 8.414 & 1039 \\
\hline 9.077 & 1056 & 9.186 & 1050 & 9.284 & 1065 & 8.713 & 1057 & 8.511 & 1040 & 8.63 & 1086 & 8.689 & 1064 & 8.417 & 1040 & 8.55 & 1057 \\
\hline 9.301 & 1081 & 9.287 & 1079 & 9.526 & 1089 & 8.932 & 1081 & 8.92 & 1079 & 8.573 & 1100 & 8.751 & 1087 & 8.411 & 1059 & 8.659 & 1080 \\
\hline 9.589 & 1104 & 9.503 & 1102 & 9.757 & 1117 & 9.05 & 1106 & 8.988 & 1111 & 8.92 & 1123 & 8.914 & 1118 & 8.798 & 1085 & 9.014 & 1121 \\
\hline
\end{tabular}


Table 6. $50{ }^{\circ} \mathrm{C}$ Pretreated - continued

\begin{tabular}{|c|c|c|c|c|c|c|c|c|c|c|c|c|c|c|c|c|c|}
\hline run1 & & run2 & & run3 & & run4 & & run5 & & run6 & & run7 & & run8 & & run9 & \\
\hline [Pa] & {$[1 / \mathrm{s}]$} & [Pa] & {$[1 / s]$} & {$[\mathrm{Pa}]$} & {$[1 / \mathrm{s}]$} & [Pa] & {$[1 / \mathrm{s}]$} & {$[\mathrm{Pa}]$} & {$[1 / \mathrm{s}]$} & {$[\mathrm{Pa}]$} & {$[1 / \mathrm{s}]$} & {$[\mathrm{Pa}]$} & {$[1 / \mathrm{s}]$} & {$[\mathrm{Pa}]$} & {$[1 / \mathrm{s}]$} & [Pa] & {$[1 / \mathrm{s}]$} \\
\hline 9.757 & 1128 & 9.89 & 1139 & 9.917 & 1140 & 9.281 & 1128 & 9.154 & 1137 & 9.02 & 1148 & 9.05 & 1142 & 9.011 & 1131 & 9.183 & 1147 \\
\hline 10.14 & 1169 & 10.09 & 1162 & 10.03 & 1171 & 9.476 & 1152 & 9.423 & 1157 & 9.319 & 1169 & 9.213 & 1166 & 9 & 1148 & 9.485 & 1174 \\
\hline 10.36 & 1192 & 10.15 & 1192 & 10.26 & 1195 & 9.861 & 1192 & 9.609 & 1189 & 9.772 & 1217 & 9.441 & 1192 & 9.408 & 1177 & 9.609 & 1199 \\
\hline 10.59 & 1224 & 10.39 & 1218 & 10.38 & 1219 & 9.97 & 1218 & 9.834 & 1213 & 9.609 & 1234 & 9.606 & 1218 & 9.378 & 1196 & 9.787 & 1225 \\
\hline 10.82 & 1241 & 10.57 & 1240 & 10.61 & 1243 & 10.1 & 1248 & 9.893 & 1240 & 10.07 & 1261 & 9.87 & 1244 & 9.778 & 1224 & 9.953 & 1251 \\
\hline 10.92 & 1274 & 10.78 & 1265 & 11.05 & 1280 & 10.29 & 1272 & 10.16 & 1264 & 9.938 & 1280 & 10.24 & 1286 & 9.778 & 1244 & 10.1 & 1282 \\
\hline 11.12 & 1297 & 11.15 & 1304 & 11.17 & 1312 & 10.4 & 1296 & 10.3 & 1290 & 10.53 & 1332 & 10.47 & 1311 & 10.14 & 1278 & 10.29 & 1306 \\
\hline 11.36 & 1320 & 11.31 & 1332 & 11.37 & 1334 & 10.66 & 1321 & 10.64 & 1330 & 10.39 & 1346 & 10.53 & 1339 & 10.1 & 1294 & 10.5 & 1331 \\
\hline 11.66 & 1357 & 11.5 & 1356 & 11.91 & 1359 & 10.76 & 1345 & 10.91 & 1352 & 10.56 & 1400 & 10.74 & 1361 & 10.54 & 1326 & 10.66 & 1355 \\
\hline 11.91 & 1382 & 11.74 & 1381 & 12.35 & 1386 & 11 & 1370 & 11.01 & 1381 & 12.57 & 1563 & 10.89 & 1388 & 10.94 & 1375 & 10.83 & 1379 \\
\hline 12.1 & 1408 & 11.86 & 1404 & 12.56 & 1412 & 11.14 & 1395 & 11.24 & 1407 & 12.35 & 1577 & 11.09 & 1412 & 10.83 & 1394 & 11.01 & 1407 \\
\hline 12.28 & 1430 & 11.99 & 1432 & 12.74 & 1437 & 11.56 & 1437 & 11.46 & 1430 & 12.67 & 1629 & 11.28 & 1435 & 11.32 & 1423 & 11.39 & 1447 \\
\hline 12.49 & 1467 & 12.25 & 1454 & 13.01 & 1464 & 11.78 & 1463 & 11.56 & 1454 & 12.53 & 1652 & 11.58 & 1475 & 11.14 & 1442 & 11.65 & 1472 \\
\hline 12.77 & 1493 & 12.41 & 1479 & 13.34 & 1502 & 11.88 & 1493 & 11.74 & 1477 & 13.44 & 1689 & 11.81 & 1501 & 11.62 & 1473 & 11.88 & 1500 \\
\hline 12.86 & 1518 & 12.86 & 1519 & 13.57 & 1525 & 12.01 & 1518 & 11.94 & 1504 & 13.31 & 1724 & 12.01 & 1525 & 11.48 & 1491 & 12.04 & 1525 \\
\hline 13.05 & 1542 & 13.05 & 1544 & 13.7 & 1558 & 12.21 & 1545 & 12.41 & 1549 & 13.55 & 1751 & 12.18 & 1555 & 12.01 & 1516 & 12.18 & 1551 \\
\hline 12.52 & 1573 & 12.41 & 1604 & 11.84 & 1598 & 11.23 & 1578 & 11.01 & 1566 & 11.03 & 1560 & 11.12 & 1574 & 11.66 & 1629 & 11.46 & 1590 \\
\hline 12.42 & 1548 & 12.27 & 1581 & 11.73 & 1572 & 11.07 & 1547 & 10.92 & 1537 & 10.94 & 1536 & 10.96 & 1546 & 11.17 & 1603 & 11.06 & 1550 \\
\hline 12.15 & 1523 & 11.92 & 1542 & 11.44 & 1549 & 10.72 & 1522 & 10.82 & 1515 & 10.59 & 1511 & 10.78 & 1523 & 11.35 & 1582 & 10.84 & 1526 \\
\hline 11.94 & 1497 & 11.64 & 1514 & 11.23 & 1522 & 10.54 & 1493 & 10.49 & 1491 & 10.34 & 1467 & 10.42 & 1483 & 9.112 & 1430 & 10.74 & 1498 \\
\hline 11.48 & 1456 & 11.39 & 1494 & 11.01 & 1498 & 10.28 & 1471 & 10.31 & 1468 & 10.26 & 1443 & 10.16 & 1458 & 8.313 & 1259 & 10.56 & 1474 \\
\hline 11.19 & 1434 & 11.17 & 1464 & 10.64 & 1457 & 10.14 & 1444 & 9.988 & 1429 & 9.751 & 1415 & 9.961 & 1431 & 7.964 & 1195 & 10.35 & 1447 \\
\hline 11.06 & 1400 & .06 & 1436 & 10.55 & 1434 & 9.914 & 1421 & 9.739 & 1402 & 9.917 & 1395 & 9.757 & 1406 & 8.076 & 1166 & 10.13 & 1419 \\
\hline 10.85 & 1380 & 10.79 & 1414 & 10.27 & 1409 & 9.592 & 1381 & 9.512 & 1377 & 9.535 & 1371 & 9.571 & 1381 & 7.579 & 1123 & 9.896 & 1395 \\
\hline 10.7 & 1355 & 10.66 & 1389 & 10.04 & 1385 & 9.367 & 1356 & 9.361 & 1347 & 9.467 & 1347 & 9.384 & 1347 & 7.712 & 1106 & 9.666 & 1371 \\
\hline 10.51 & 1330 & 10.1 & 1350 & 9.885 & 1353 & 9.21 & 1332 & 9.257 & 1323 & 9.053 & 1321 & 9.165 & 1325 & 6.919 & 1062 & 9.544 & 1341 \\
\hline 10.27 & 1305 & 9.92 & 1324 & 9.666 & 1327 & 9.047 & 1301 & 9.011 & 1297 & 8.778 & 1277 & 8.914 & 1297 & 7.073 & 1036 & 9.26 & 1318 \\
\hline 9.799 & 1264 & 9.811 & 1296 & 9.396 & 1303 & 8.875 & 1275 & 8.716 & 1271 & 8.784 & 1256 & 8.757 & 1274 & 6.682 & 981 & 8.84 & 1278 \\
\hline 9.612 & 1241 & 9.811 & 1285 & 9.189 & 1279 & 8.606 & 1252 & 8.588 & 1243 & 8.28 & 1231 & 8.612 & 1249 & 6.108 & 943.9 & 8.73 & 1253 \\
\hline 9.405 & 1208 & 9.355 & 1248 & 8.852 & 1239 & 8.417 & 1226 & 8.446 & 1219 & 8.455 & 1209 & 8.437 & 1223 & 6.321 & 926.3 & 8.502 & 1226 \\
\hline 9.109 & 1185 & 9.151 & 1218 & 8.914 & 1232 & 8.34 & 1200 & 8.269 & 1194 & 8.038 & 1185 & 7.919 & 1181 & 5.812 & 897.2 & 8.337 & 1200 \\
\hline
\end{tabular}


Table 6. $50{ }^{\circ} \mathrm{C}$ Pretreated - continued

\begin{tabular}{|c|c|c|c|c|c|c|c|c|c|c|c|c|c|c|c|c|c|}
\hline run1 & & run2 & & run3 & & run4 & & run5 & & run6 & & run7 & & run8 & & run9 & \\
\hline [Pa] & {$[1 / \mathrm{s}]$} & {$[\mathrm{Pa}]$} & {$[1 / s]$} & {$[\mathrm{Pa}]$} & {$[1 / \mathrm{s}]$} & [Pa] & {$[1 / s]$} & {$[\mathrm{Pa}]$} & {$[1 / \mathrm{s}]$} & {$[\mathrm{Pa}]$} & {$[1 / \mathrm{s}]$} & {$[\mathrm{Pa}]$} & {$[1 / \mathrm{s}]$} & {$[\mathrm{Pa}]$} & {$[1 / \mathrm{s}]$} & {$[\mathrm{Pa}]$} & {$[1 / \mathrm{s}]$} \\
\hline 9.003 & 1162 & 8.997 & 1192 & 8.473 & 1188 & 8.112 & 1173 & 7.89 & 1155 & 7.78 & 1136 & 7.851 & 1155 & 5.948 & 875.2 & 8.05 & 1170 \\
\hline 8.778 & 1138 & 8.84 & 1170 & 8.278 & 1163 & 7.884 & 1147 & 7.763 & 1125 & 7.774 & 1114 & 7.541 & 1133 & 5.602 & 852.3 & 8.014 & 1139 \\
\hline 8.446 & 1102 & 8.366 & 1131 & 8.13 & 1132 & 7.733 & 1123 & 7.523 & 1101 & 7.333 & 1067 & 7.422 & 1106 & 5.552 & 829.4 & 7.641 & 1118 \\
\hline 8.248 & 1076 & 8.239 & 1107 & 7.878 & 1107 & 7.523 & 1099 & 7.455 & 1078 & 7.088 & 1045 & 7.517 & 1092 & 5.17 & 797.6 & 7.541 & 1091 \\
\hline 8.061 & 1046 & 8.023 & 1083 & 7.751 & 1085 & 7.079 & 1054 & 7.239 & 1051 & 6.999 & 1022 & 7.239 & 1060 & 5.194 & 781.8 & 7.381 & 1068 \\
\hline 7.848 & 1020 & 7.757 & 1055 & 7.618 & 1056 & 7.008 & 1030 & 7.049 & 1025 & 6.591 & 997.3 & 6.925 & 1036 & 4.741 & 753.1 & 7.171 & 1045 \\
\hline 7.686 & 992.9 & 7.603 & 1024 & 7.319 & 1033 & 6.798 & 1002 & 6.756 & 1001 & 6.659 & 976.6 & 6.579 & 994.6 & 4.927 & 732 & 6.961 & 1017 \\
\hline 7.363 & 972.1 & 7.407 & 995.5 & 7.162 & 1005 & 6.543 & 978.8 & 6.65 & 971.7 & 6.227 & 950.1 & 6.389 & 969.9 & 4.43 & 704.7 & 6.629 & 975.7 \\
\hline 7.206 & 943.9 & 7.186 & 974.3 & 7.008 & 977 & 6.366 & 949.2 & 6.383 & 946.1 & 6.31 & 926.8 & 6.283 & 943.5 & 4.537 & 685.3 & 6.431 & 953.2 \\
\hline 7.02 & 918.8 & 6.943 & 948.8 & 6.736 & 951 & 6.194 & 923.2 & 6.28 & 920.1 & 5.931 & 902.1 & 6.079 & 912.7 & 4.229 & 652.7 & 6.206 & 924.1 \\
\hline 6.765 & 893.3 & 6.804 & 921 & 6.437 & 912.2 & 6.017 & 896.3 & 6.105 & 892.4 & 5.898 & 879.2 & 5.916 & 884.9 & 4.265 & 636.3 & 6.046 & 895.9 \\
\hline 6.54 & 867.3 & 6.529 & 889.3 & 6.141 & 888.9 & 5.8 & 867.3 & 5.824 & 867.3 & 5.434 & 852.3 & 5.614 & 861.1 & 3.729 & 607.7 & 5.954 & 871.7 \\
\hline 6.301 & 839.9 & 6.348 & 863.7 & 5.984 & 864.6 & 5.581 & 842.6 & 5.747 & 839.5 & 5.602 & 831.1 & 5.558 & 833.8 & 3.924 & 586.1 & 5.777 & 849.6 \\
\hline 6.105 & 811.7 & 6.241 & 839.5 & 5.783 & 838.6 & 5.466 & 809.1 & 5.283 & 800.7 & 5.217 & 804.7 & 5.291 & 810.9 & 3.43 & 561 & 5.398 & 811.7 \\
\hline 5.907 & 788.4 & 6.049 & 812.2 & 5.661 & 807.8 & 5.247 & 787.5 & 5.084 & 778.7 & 4.927 & 758.4 & 4.933 & 772.5 & 3.531 & 539.8 & 5.25 & 787.9 \\
\hline 5.543 & 746.5 & 5.768 & 787.1 & 5.519 & 784 & 5.203 & 761.9 & 5.052 & 747.4 & 4.895 & 734.6 & 4.735 & 750 & 3.093 & 512.5 & 5.022 & 758 \\
\hline 5.407 & 721.8 & 5.508 & 756.2 & 5.333 & 761.5 & 4.987 & 736.8 & 4.803 & 724 & 4.575 & 710.8 & 4.697 & 723.6 & 3.255 & 492.2 & 4.954 & 733.7 \\
\hline 5.081 & 697.2 & 5.496 & 728.9 & 4.99 & 722.7 & 4.72 & 712.6 & 4.614 & 700.2 & 4.56 & 687.5 & 4.501 & 692.3 & 2.841 & 467.1 & 4.815 & 709.1 \\
\hline 5.022 & 666.8 & 5.191 & 705.5 & 4.924 & 698.9 & 4.537 & 689.2 & 4.327 & 662.3 & 4.235 & 663.2 & 4.377 & 669.4 & 2.971 & 446 & 4.658 & 683.9 \\
\hline 4.806 & 639 & 4.785 & 669 & 4.634 & 669.8 & 4.211 & 642.5 & 4.054 & 639 & 4.022 & 621.8 & 4.146 & 640.3 & 2.498 & 416.9 & 4.418 & 661 \\
\hline 4.537 & 610.8 & 4.667 & 646 & 4.424 & 647.4 & 4.282 & 635.5 & 3.918 & 615.6 & 3.992 & 597.6 & 4.007 & 615.2 & 2.737 & 397.9 & 4.134 & 616.1 \\
\hline 4.253 & 584.8 & 4.478 & 617 & 4.253 & 623.6 & 4.051 & 605.1 & 3.809 & 583.5 & 3.575 & 572 & 3.877 & 590.5 & 2.246 & 365.3 & 3.898 & 595.8 \\
\hline 4.037 & 559.2 & 4.312 & 594 & 4.1 & 600.2 & 3.841 & 580.4 & 3.661 & 559.7 & 3.687 & 548.7 & 3.679 & 565.8 & 2.338 & 349 & 3.782 & 572 \\
\hline 3.918 & 535.9 & 4.143 & 572.4 & 4.054 & 572.9 & 3.468 & 536.8 & 3.474 & 533.7 & 3.244 & 521.8 & 3.471 & 536.3 & 1.844 & 319.9 & 3.548 & 543.8 \\
\hline 3.791 & 511.2 & 3.726 & 531.5 & 3.56 & 531 & 3.318 & 512.5 & 3.297 & 510.3 & 3.326 & 500.2 & 3.184 & 509.9 & 1.98 & 298.3 & 3.427 & 513.8 \\
\hline 3.596 & 482.5 & 3.608 & 507.7 & 3.483 & 499.7 & 3.246 & 479.9 & 3.045 & 471.5 & 2.88 & 473.7 & 3.069 & 486.5 & 1.657 & 265.3 & 3.332 & 489.6 \\
\hline 3.409 & 458.3 & 3.371 & 475.9 & 3.48 & 494.9 & 3.066 & 458.3 & 2.959 & 441.6 & 2.856 & 428.3 & 2.992 & 456.1 & 1.684 & 249.4 & 3.155 & 463.2 \\
\hline 3.291 & 433.2 & 3.276 & 452.6 & 3.143 & 451.3 & 2.865 & 429.2 & 2.619 & 417.8 & 2.699 & 403.2 & 2.8 & 429.2 & & & 2.954 & 440.2 \\
\hline 3.004 & 407.2 & 3.119 & 430.1 & 2.915 & 427.5 & 2.672 & 405.9 & 2.542 & 397.5 & 2.412 & 381.2 & 2.498 & 404.1 & & & 2.607 & 400.6 \\
\hline 2.592 & 363.6 & 2.927 & 405.9 & 2.61 & 400.6 & 2.581 & 381.6 & 2.513 & 368.4 & 2.338 & 358.3 & 2.412 & 379.4 & & & 2.368 & 376.3 \\
\hline 2.397 & 337.1 & 2.516 & 371.5 & 2.551 & 373.7 & 2.264 & 343.7 & 2.104 & 334.5 & 2.113 & 332.3 & 1.992 & 337.6 & & & 2.347 & 353.4 \\
\hline & & & & & & & & & & & & & & & & & \\
\hline
\end{tabular}


Table 6. $50{ }^{\circ} \mathrm{C}$ Pretreated - continued

\begin{tabular}{|c|c|c|c|c|c|c|c|c|c|c|c|c|c|c|c|c|c|}
\hline run1 & & run2 & & run3 & & run4 & & run5 & & run6 & & run7 & & run8 & & run9 & \\
\hline [Pa] & {$[1 / \mathrm{s}]$} & {$[\mathrm{Pa}]$} & {$[1 / \mathbf{s}]$} & [Pa] & {$[1 / s]$} & [Pa] & {$[1 / \mathrm{s}]$} & {$[\mathrm{Pa}]$} & {$[1 / \mathrm{s}]$} & [Pa] & {$[1 / \mathrm{s}]$} & [Pa] & {$[1 / \mathrm{s}]$} & {$[\mathrm{Pa}]$} & {$[1 / \mathrm{s}]$} & [Pa] & {$[1 / \mathrm{s}]$} \\
\hline 2.237 & 313.8 & 2.453 & 342 & 2.35 & 349.5 & 1.998 & 317.7 & 2.042 & 313.8 & 2.039 & 308.9 & 1.938 & 314.6 & & & 2.119 & 322.1 \\
\hline 2.018 & 281.6 & 2.341 & 318.6 & 2.175 & 317.3 & 1.935 & 289.1 & 1.9 & 282.5 & 1.589 & 280.3 & 1.755 & 288.2 & & & 1.986 & 299.7 \\
\hline 1.933 & 256 & 2.08 & 295.3 & 2.137 & 290.4 & 1.776 & 264.9 & 1.699 & 257.4 & 1.737 & 259.1 & 1.598 & 256.5 & & & 1.802 & 275.4 \\
\hline & & 1.752 & 258.2 & 1.79 & 266.2 & 1.669 & 241.9 & 1.885 & 240.6 & & & 1.666 & 250.3 & & & 1.719 & 254.3 \\
\hline & & 1.782 & 250.3 & 1.764 & 240.6 & & & & & & & & & & & & \\
\hline
\end{tabular}




\begin{tabular}{|ll|}
\hline APPENDIX D & WSRC-TR-2000-00352 \\
ENVELOPE C RHEOLOGY DATA & SRT-RPP-2000-00026 \\
Page 141 of 514
\end{tabular}

\section{APPENDIX D}

\section{ENVELOPE C RHEOLOGY DATA}

Table 1. Rheometer Response for Blank - As Received ................................................. 143

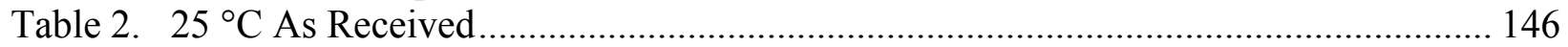

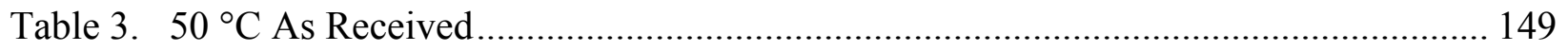

Table 4. Rheometer Response for Blank - Diluted .......................................................... 152

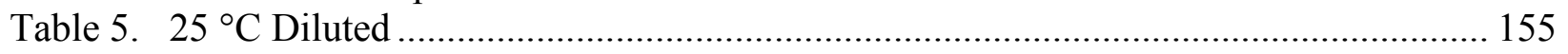

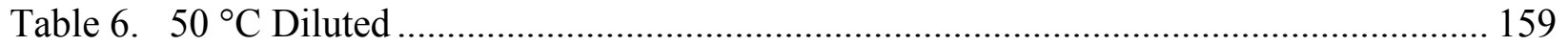

Table 7. Rheometer Response for Blank - Pretreated ..................................................... 162

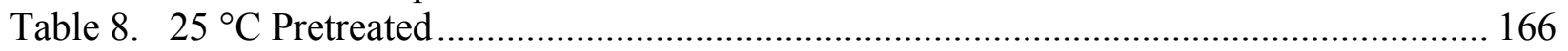

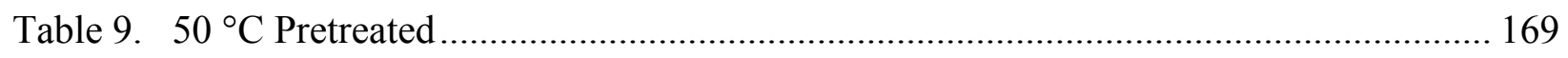


This page intentionally left blank. 
Table 1. Rheometer Response for Blank - As Received

\begin{tabular}{|c|c|c|c|c|c|c|c|c|c|c|c|c|c|c|c|c|c|}
\hline run1 & & run2 & & run3 & & run4 & & run5 & & run6 & & run7 & & run8 & & run9 & \\
\hline [Pa] & {$[1 / s]$} & [Pa] & {$[1 / s]$} & [Pa] & {$[1 / s]$} & [Pa] & {$[1 / s]$} & [Pa] & [1/s] & [Pa] & {$[1 / s]$} & [Pa] & [1/s] & [Pa] & {$[1 / \mathrm{s}]$} & [Pa] & [1/s] \\
\hline 3.13 & 341.5 & 3.87 & 385.2 & 3.956 & 401.9 & 3.252 & 360.5 & 3.716 & 371.5 & 3.737 & 374.1 & 3.16 & 327 & 1.828 & 331.4 & 2.473 & 320.4 \\
\hline 3.225 & 366.2 & 4.199 & 422.2 & 4.134 & 429.2 & 3.45 & 386 & 3.903 & 396.6 & 3.672 & 383.8 & 3.432 & 344.6 & 2.663 & 305 & 3.216 & 325.2 \\
\hline 3.373 & 394.9 & 4.388 & 446 & 4.302 & 452.1 & 3.669 & 408.5 & 4.024 & 424.4 & 4.217 & 424.8 & 3.634 & 367.5 & 3.098 & 315.5 & 3.421 & 346.4 \\
\hline 3.586 & 423.5 & 4.447 & 476.4 & 4.471 & 473.3 & 4.075 & 449.5 & 4.211 & 448.6 & 4.373 & 447.7 & 3.832 & 390.9 & 3.37 & 332.7 & 3.53 & 367.5 \\
\hline 3.734 & 445.5 & 4.619 & 499.7 & 4.817 & 511.6 & 4.175 & 482.1 & 4.388 & 474.2 & 4.48 & 480.8 & 3.983 & 413.8 & 3.776 & 372.8 & 3.696 & 388.2 \\
\hline 3.82 & 473.3 & 4.808 & 524 & 4.924 & 540.3 & 4.273 & 505.5 & 4.554 & 498.4 & 4.652 & 502.4 & 4.368 & 452.6 & 3.927 & 397.9 & 4.057 & 424.8 \\
\hline 4.107 & 497.1 & 4.977 & 550 & 5.087 & 564.1 & 4.524 & 532.8 & 4.806 & 524.4 & 4.752 & 523.1 & 4.498 & 480.8 & 3.998 & 428.8 & 4.217 & 450.4 \\
\hline 4.456 & 535.9 & 5.178 & 576.4 & 5.261 & 587.4 & 4.684 & 557.9 & 4.891 & 550 & 5.039 & 563.2 & 4.495 & 487.8 & 4.208 & 452.6 & 4.264 & 481.7 \\
\hline 4.551 & 558.3 & 5.288 & 600.2 & 5.433 & 612.1 & 4.811 & 580.4 & 5.246 & 589.6 & 5.202 & 588.3 & 4.874 & 532.8 & 4.368 & 476.8 & 4.492 & 505 \\
\hline 4.566 & 588.8 & 5.48 & 626.7 & 5.596 & 635.9 & 5.107 & 618.3 & 5.389 & 615.6 & 5.312 & 613 & 4.986 & 557.5 & 4.439 & 501.5 & 4.56 & 530.1 \\
\hline 4.776 & 611.7 & 5.785 & 661.9 & 5.72 & 661.9 & 5.279 & 639 & 5.542 & 643 & 5.412 & 643.4 & 5.113 & 587.9 & 4.675 & 523.5 & 4.779 & 552.6 \\
\hline 4.903 & 633.7 & 5.832 & 693.2 & 6.081 & 702.4 & 5.362 & 671.2 & 5.605 & 671.6 & 5.572 & 667.2 & 5.249 & 612.5 & 4.924 & 562.8 & 5.066 & 589.6 \\
\hline 5.027 & 657.5 & 6.025 & 714.8 & 6.149 & 731.1 & 5.501 & 695.8 & 5.729 & 694.5 & 5.747 & 693.2 & 5.465 & 639 & 5.116 & 588.3 & 5.199 & 614.8 \\
\hline 5.389 & 698.9 & 6.105 & 741.7 & & 758.4 & & 721.4 & 5.832 & 719.6 & & 716.1 & 5.59 & 661.9 & 5.359 & 617.4 & 5.297 & 644.7 \\
\hline 5.486 & 720.1 & 6.445 & 775.6 & 6.463 & 779.6 & 5.756 & 748.3 & 6.025 & 745.2 & 5.966 & 739 & 5.705 & 686.6 & 5.474 & 642.1 & 5.389 & 669.8 \\
\hline 5.61 & 751.8 & 6.528 & 801.2 & 6.558 & 804.2 & 6.001 & 771.6 & 6.176 & 767.2 & 6.105 & 765 & 6.048 & 726.7 & 5.584 & 669.4 & 5.587 & 695.8 \\
\hline 5.72 & 777.4 & 6.679 & 824.1 & 6.729 & 827.2 & 6.114 & 798.5 & 6.321 & 795.9 & 6.418 & 805.1 & 6.191 & 749.2 & 5.702 & 701.1 & 5.738 & 724 \\
\hline 5.936 & 801.2 & 6.815 & 856.7 & 7.013 & 865.5 & 6.309 & 825 & 6.442 & 823.6 & 6.608 & 831.1 & 6.291 & 778.2 & 5.838 & 717.9 & 5.85 & 748.7 \\
\hline 6.019 & 824.5 & 6.966 & 879.2 & 7.105 & 889.7 & 6.43 & 850.1 & 6.623 & 849.6 & 6.694 & 863.7 & 6.534 & 803.4 & 5.93 & 751.4 & 5.977 & 772.5 \\
\hline 6.35 & 863.7 & 7.004 & 905.2 & 7.309 & 915.7 & 6.478 & 879.2 & 6.753 & 875.2 & 6.815 & 886.7 & 6.705 & 826.7 & 6.137 & 780.4 & 6.256 & 811.7 \\
\hline 6.445 & 884.5 & 7.217 & 929.4 & 7.487 & 943.9 & 6.655 & 909.6 & 7.075 & 917.9 & 6.924 & 912.2 & 6.697 & 859.3 & 6.288 & 806 & 6.377 & 839.9 \\
\hline 6.472 & 915.3 & 7.389 & 954.5 & 7.694 & 972.1 & 6.877 & 936.9 & 7.274 & 943.5 & 7.108 & 936.9 & 6.797 & 882.2 & 6.418 & 830.7 & 6.519 & 860.7 \\
\hline 6.682 & 937.8 & 7.519 & 979.2 & 7.792 & 996.4 & 6.972 & 960.7 & 7.407 & 971.3 & 7.229 & 964.7 & 6.984 & 906 & 6.575 & 854 & 6.658 & 885.8 \\
\hline 6.821 & 960.2 & 7.65 & 1004 & 7.916 & 1028 & 7.203 & 998.1 & 7.573 & 995.5 & 7.38 & 989.3 & 7.096 & 930.7 & 6.646 & 879.2 & 6.759 & 917.5 \\
\hline 7.117 & 998.6 & 7.993 & 1047 & 7.978 & 1049 & 7.291 & 1022 & 7.661 & 1026 & 7.422 & 1014 & 7.247 & 955.8 & 7.025 & 917.5 & 6.88 & 940.9 \\
\hline 7.3 & 1023 & 8.132 & 1075 & 8.117 & 1075 & 7.368 & 1046 & 7.777 & 1050 & 7.653 & 1040 & 7.38 & 983.2 & 7.067 & 946.6 & 6.993 & 968.2 \\
\hline 7.374 & 1052 & 8.259 & 1098 & 8.268 & 1101 & 7.398 & 1079 & 7.901 & 1075 & 7.978 & 1080 & 7.593 & 1008 & 7.215 & 969.9 & 7.17 & 993.3 \\
\hline 7.49 & 1077 & 8.327 & 1120 & 8.49 & 1126 & 7.525 & 1103 & 8.011 & 1099 & 8.07 & 1104 & 7.925 & 1048 & 7.339 & 996.4 & 7.241 & 1015 \\
\hline 7.614 & 1101 & 8.463 & 1144 & 8.869 & 1166 & 7.638 & 1129 & 8.17 & 1125 & 8.289 & 1136 & 7.895 & 1058 & 7.475 & 1024 & 7.354 & 1043 \\
\hline 7.735 & 1126 & 8.848 & 1185 & 8.999 & 1190 & 7.756 & 1155 & 8.33 & 1152 & 8.398 & 1157 & 8.25 & 1106 & 7.641 & 1049 & 7.65 & 1087 \\
\hline 7.88 & 1151 & 8.999 & 1209 & 8.972 & 1222 & 7.869 & 1183 & 8.614 & 1190 & 8.484 & 1181 & 8.336 & 1133 & 7.818 & 1072 & 7.88 & 1110 \\
\hline
\end{tabular}


Table 1. Rheometer Response for Blank - As Received - continued

\begin{tabular}{|c|c|c|c|c|c|c|c|c|c|c|c|c|c|c|c|c|c|}
\hline run1 & & run2 & & run3 & & run4 & & run5 & & run6 & & run7 & & run8 & & run9 & \\
\hline [Pa] & {$[1 / \mathrm{s}]$} & {$[\mathrm{Pa}]$} & {$[1 / s]$} & {$[\mathrm{Pa}]$} & {$[1 / s]$} & [Pa] & {$[1 / \mathrm{s}]$} & {$[\mathrm{Pa}]$} & {$[1 / \mathrm{s}]$} & {$[\mathrm{Pa}]$} & {$[1 / \mathrm{s}]$} & [Pa] & {$[1 / \mathrm{s}]$} & {$[\mathrm{Pa}]$} & {$[1 / \mathrm{s}]$} & {$[\mathrm{Pa}]$} & {$[1 / \mathrm{s}]$} \\
\hline 8.014 & 1178 & 9.064 & 1236 & 9.194 & 1248 & 8.064 & 1211 & 8.75 & 1213 & 8.715 & 1207 & 8.475 & 1156 & 7.94 & 1097 & 7.963 & 1135 \\
\hline 8.185 & 1207 & 9.265 & 1263 & 9.265 & 1274 & 8.176 & 1236 & 8.866 & 1237 & 8.827 & 1234 & 8.487 & 1189 & 8.244 & 1138 & 8.073 & 1161 \\
\hline 8.398 & 1232 & 9.369 & 1289 & 9.505 & 1298 & 8.312 & 1258 & 8.925 & 1268 & 8.937 & 1271 & 8.674 & 1210 & 8.369 & 1163 & 8.224 & 1186 \\
\hline 8.745 & 1273 & 9.496 & 1309 & 9.635 & 1325 & 8.449 & 1285 & 9.126 & 1296 & 9.097 & 1295 & 8.724 & 1232 & 8.46 & 1186 & 8.324 & 1217 \\
\hline 8.895 & 1297 & 9.555 & 1334 & 9.774 & 1352 & 8.736 & 1328 & 9.171 & 1324 & 9.144 & 1318 & 8.895 & 1258 & 8.635 & 1210 & 8.452 & 1242 \\
\hline 8.981 & 1326 & 10.05 & 1376 & 9.958 & 1374 & 8.964 & 1351 & 9.39 & 1351 & 9.301 & 1345 & 9.251 & 1296 & 8.718 & 1244 & 8.662 & 1265 \\
\hline 9.188 & 1349 & 10.03 & 1404 & 10.06 & 1399 & 8.99 & 1376 & 9.567 & 1376 & 9.443 & 1369 & 9.307 & 1322 & 8.869 & 1266 & 8.854 & 1290 \\
\hline 9.248 & 1372 & 10.19 & 1428 & 10.37 & 1443 & 9.138 & 1409 & 9.653 & 1400 & 9.623 & 1398 & 9.458 & 1353 & 8.996 & 1290 & 8.916 & 1315 \\
\hline 9.393 & 1403 & 10.3 & 1449 & 10.55 & 1464 & 9.274 & 1434 & 9.789 & 1426 & 9.789 & 1425 & 9.57 & 1377 & 9.254 & 1330 & 9.103 & 1341 \\
\hline 9.547 & 1426 & 10.44 & 1474 & 10.69 & 1489 & 9.387 & 1461 & 10.14 & 1464 & 9.946 & 1450 & 9.656 & 1401 & 9.245 & 1336 & 9.283 & 1367 \\
\hline 9.919 & 1463 & 10.54 & 1496 & 10.74 & 1521 & 9.467 & 1486 & 10.29 & 1487 & 10.26 & 1487 & 9.831 & 1424 & 9.162 & 1371 & 9.692 & 1409 \\
\hline 9.748 & 1474 & 11.03 & 1536 & 11 & 1542 & 9.6 & 1511 & 10.35 & 1516 & 10.36 & 1519 & 10.12 & 1460 & 9.206 & 1372 & 9.813 & 1433 \\
\hline 10.17 & 1516 & 11.11 & 1563 & 11.09 & 1569 & 9.73 & 1536 & 10.34 & 1537 & 10.48 & 1543 & 10.18 & 1489 & 9.86 & 1448 & 9.884 & 1460 \\
\hline 9.561 & 1516 & 8.996 & 1471 & 8.407 & 1500 & 9.15 & 1522 & 8.999 & 1513 & 9.035 & 1523 & 9.236 & 1568 & 9.236 & 1590 & 8.91 & 1593 \\
\hline 9.422 & 1493 & 8.676 & 1432 & 8.25 & 1475 & 9.067 & 1497 & 8.857 & 1489 & 8.697 & 1483 & 9.162 & 1538 & 9.07 & 1566 & 8.975 & 1584 \\
\hline 9.301 & 1466 & 8.57 & 1408 & 7.987 & 1439 & 8.878 & 1471 & 8.721 & 1459 & 8.543 & 1461 & 8.978 & 1514 & 8.94 & 1536 & 8.662 & 1541 \\
\hline 9.058 & 1444 & 8.463 & 1377 & 8.227 & 1407 & 8.727 & 1447 & 8.54 & 1428 & 8.407 & 1432 & 8.792 & 1489 & 8.813 & 1512 & 8.561 & 1519 \\
\hline 8.94 & 1416 & 8.348 & 1357 & 8.398 & 1380 & 8.558 & 1421 & 8.342 & 1405 & 8.271 & 1410 & 8.608 & 1461 & 8.694 & 1485 & 8.389 & 1486 \\
\hline 8.727 & 1392 & 8.008 & 1326 & 8.167 & 1359 & 8.289 & 1380 & 8.315 & 1379 & 8.099 & 1385 & 8.552 & 1432 & 8.431 & 1460 & 8.247 & 1463 \\
\hline 8.383 & 1349 & 7.948 & 1303 & 8.008 & 1334 & 8.043 & 1355 & 8.147 & 1353 & 7.981 & 1353 & 8.44 & 1407 & 8.345 & 1434 & 8.203 & 1434 \\
\hline 8.173 & 1325 & 7.774 & 1277 & 7.851 & 1302 & 7.892 & 1326 & 7.984 & 1330 & 7.863 & 1330 & 8.265 & 1383 & 8.141 & 1410 & 8.005 & 1409 \\
\hline 8.008 & 1301 & 7.614 & 1253 & 7.792 & 1276 & 7.741 & 1302 & 7.874 & 1305 & 7.712 & 1303 & 8.123 & 1357 & 8.046 & 1384 & 7.815 & 1384 \\
\hline 7.948 & 269 & 7.463 & 1227 & 7.632 & 1254 & 7.555 & 1278 & 7.747 & 1282 & 7.573 & 1280 & 8.005 & 1334 & 7.697 & 1349 & 7.715 & 1360 \\
\hline 7.783 & 1245 & 7.096 & 1189 & 7.507 & 1229 & 7.425 & 1252 & 7.564 & 1259 & 7.46 & 1253 & 7.655 & 1294 & 7.614 & 1319 & 7.564 & 1337 \\
\hline 7.611 & 1222 & 7.022 & 1164 & 7.354 & 1204 & 7.297 & 1222 & 7.141 & 1215 & 7.238 & 1226 & 7.555 & 1270 & 7.496 & 1293 & 7.457 & 1312 \\
\hline 7.291 & 1186 & 6.91 & 1136 & 7.025 & 1158 & 7.17 & 1198 & 7.016 & 1189 & 6.966 & 1185 & 7.395 & 1241 & 7.315 & 1266 & 7.031 & 1270 \\
\hline 7.194 & 1156 & 6.797 & 1116 & 6.83 & 1136 & 7.072 & 1173 & 6.848 & 1163 & 6.845 & 1163 & 7.327 & 1215 & 7.143 & 1247 & 6.927 & 1241 \\
\hline 7.064 & 1137 & 6.442 & 1077 & 6.685 & 1109 & 6.874 & 1148 & 6.774 & 1136 & 6.64 & 1133 & 7.167 & 1190 & 6.93 & 1217 & 6.714 & 1216 \\
\hline 6.88 & 1103 & 6.386 & 1049 & 6.51 & 1086 & 6.774 & 1121 & 6.581 & 1113 & 6.549 & 1104 & 7.031 & 1166 & 6.708 & 1189 & 6.578 & 1191 \\
\hline 6.744 & 1077 & 6.146 & 1029 & 6.418 & 1057 & 6.602 & 1090 & 6.448 & 1077 & 6.291 & 1082 & 6.907 & 1141 & 6.54 & 1161 & 6.578 & 1160 \\
\hline 6.555 & 1053 & 5.972 & 1003 & 6.318 & 1033 & 6.353 & 1067 & 6.344 & 1051 & 6.247 & 1053 & 6.543 & 1100 & 6.383 & 1134 & 6.469 & 1137 \\
\hline
\end{tabular}


Table 1. Rheometer Response for Blank - As Received - continued

\begin{tabular}{|c|c|c|c|c|c|c|c|c|c|c|c|c|c|c|c|c|c|}
\hline run1 & & run2 & & run3 & & run4 & & run5 & & run6 & & run7 & & run8 & & run9 & \\
\hline [Pa] & {$[1 / s]$} & {$[\mathrm{Pa}]$} & {$[1 / s]$} & [Pa] & {$[1 / s]$} & [Pa] & {$[1 / s]$} & [Pa] & {$[1 / s]$} & [Pa] & {$[1 / \mathrm{s}]$} & [Pa] & {$[1 / \mathbf{s}]$} & [Pa] & {$[1 / s]$} & {$[\mathrm{Pa}]$} & {$[1 / s]$} \\
\hline 6.46 & 1030 & 5.844 & 967.7 & 6.294 & 1003 & 6.244 & 1040 & 6.12 & 1028 & 6.102 & 1032 & 6.424 & 1074 & 6.279 & 1103 & 6.33 & 1112 \\
\hline 6.051 & 991.5 & .705 & 943.1 & 6.022 & 977 & 6.161 & 1012 & 6.051 & 1004 & 6.051 & 1007 & 6.17 & 1050 & 6.22 & 1080 & 6.125 & 1088 \\
\hline 5.963 & 970.4 & 5.551 & 919.7 & 5.832 & 948.3 & 5.865 & 988.9 & 5.844 & 977 & 5.785 & 979.6 & 6.001 & 1025 & 6.111 & 1056 & 5.96 & 1061 \\
\hline 5.868 & 937.3 & 5.457 & 899 & 5.673 & 923.2 & 5.554 & 947 & 5.72 & 945.7 & 5.658 & 954.5 & 6.001 & 994.6 & 5.764 & 1017 & 5.646 & 1022 \\
\hline 5.67 & 919.3 & 5.146 & 859.8 & 5.513 & 895.9 & 5.483 & 923.2 & 5.513 & 923.7 & 5.323 & 911.3 & 5.847 & 970.8 & 5.815 & 1009 & 5.519 & 996.4 \\
\hline 5.406 & 893.7 & 5.007 & 833.3 & 5.297 & 869.9 & 5.353 & 895 & 5.282 & 885.8 & 5.167 & 884.9 & 5.711 & 944.8 & 5.667 & 979.6 & 5.338 & 973 \\
\hline 5.359 & 861.5 & 4.764 & 806.9 & 5.178 & 845.2 & 5.273 & 867.7 & 5.134 & 863.3 & 5.084 & 858.5 & 5.498 & 921 & 5.264 & 938.7 & 5.294 & 943.9 \\
\hline 5.039 & 836.9 & 4.752 & 775.6 & 5.004 & 818.8 & 5.137 & 843.9 & 5.048 & 836.9 & 4.9 & 834.2 & 5.249 & 881.8 & 5.214 & 910.5 & 5.19 & 921 \\
\hline 4.939 & 811.7 & 4.566 & 758.9 & 4.699 & 779.1 & 4.968 & 821.9 & 4.888 & 807.3 & 4.672 & 810 & 5.039 & 860.2 & 5.063 & 884.5 & 5.119 & 895.5 \\
\hline 4.874 & 777.4 & 4.468 & 725.4 & 4.557 & 756.2 & 4.563 & 782.7 & 4.764 & 784.9 & 4.581 & 783.5 & 4.971 & 827.6 & 4.912 & 863.3 & 4.921 & 869 \\
\hline 4.666 & 756.2 & 4.323 & 700.7 & 4.45 & 724.5 & 4.524 & 753.1 & 4.646 & 760.6 & 4.551 & 749.2 & 4.882 & 801.6 & 4.791 & 836.9 & 4.811 & 843 \\
\hline 4.587 & 732.9 & 4.258 & 677.3 & 4.359 & 699.4 & 4.471 & 730.7 & 4.436 & 734.6 & 4.403 & 721.4 & 4.72 & 776.9 & 4.521 & 795 & 4.616 & 817.9 \\
\hline 4.166 & 695.8 & 4.018 & 653.5 & 4.214 & 673.8 & 4.184 & 710.4 & 4.063 & 695 & 4.193 & 694.5 & 4.527 & 753.1 & 4.598 & 785.7 & 4.344 & 777.8 \\
\hline 4.054 & 671.2 & 3.64 & 613.9 & 4.06 & 650.9 & 4.16 & 674.2 & 3.95 & 666.8 & 3.965 & 676.9 & 4.394 & 728.9 & 4.35 & 758.9 & 4.261 & 747.4 \\
\hline 3.983 & 640.3 & 3.512 & 583.9 & 3.879 & 624.4 & 4.012 & 651.3 & 3.85 & 638.5 & 3.947 & 644.7 & 4.335 & 701.1 & 4.122 & 723.2 & 4.128 & 723.6 \\
\hline 3.814 & 617.8 & 3.403 & 562.3 & 3.693 & 601.5 & 3.876 & 622.7 & 3.678 & 612.1 & 3.764 & 622.2 & 4.122 & 676.9 & 4.075 & 696.3 & 3.998 & 700.7 \\
\hline 3.666 & 593.6 & 3.338 & 531.5 & 3.592 & 575.5 & 3.657 & 601.1 & 3.622 & 584.8 & 3.625 & 598.4 & 3.938 & 650 & 3.761 & 673.4 & 3.882 & 676 \\
\hline 3.577 & 567.2 & 3.169 & 509.4 & 3.243 & 535.9 & 3.468 & 573.8 & 3.388 & 560.1 & 3.341 & 559.2 & 3.61 & 608.6 & 3.749 & 641.2 & 3.767 & 654 \\
\hline 3.225 & 531.5 & 3.036 & 485.2 & 3.128 & 505.9 & 3.412 & 547.3 & 3.332 & 534.5 & 3.261 & 530.6 & 3.471 & 583.9 & 3.622 & 617 & 3.45 & 613 \\
\hline 3.148 & 505.9 & 2.713 & 449.9 & 2.917 & 483 & 3.267 & 522.6 & 3.125 & 509.4 & 3.145 & 502.4 & 3.311 & 561.9 & 3.512 & 592.7 & 3.302 & 584.3 \\
\hline 2.959 & 482.5 & 2.515 & 424.8 & 2.811 & 456.5 & 3.142 & 497.5 & 3.071 & 479.9 & 2.917 & 476.8 & 3.24 & 527.1 & 3.317 & 567.6 & 3.151 & 560.1 \\
\hline 2.823 & 449.1 & 2.539 & 395.7 & 2.722 & 427.9 & 2.764 & 457 & 2.769 & 456.5 & 2.764 & 453.5 & 3.068 & 501.1 & 3.006 & 527.5 & 3.225 & 547.8 \\
\hline 2.645 & 426.6 & 2.4 & 375.9 & 2.565 & 401 & 2.6 & 430.1 & 2.728 & 432.8 & 2.71 & 430.1 & 2.959 & 477.7 & 2.855 & 499.7 & 2.953 & 521.3 \\
\hline 2.547 & 401.5 & 2.331 & 350.8 & 2.488 & 377.2 & 2.509 & 401.5 & 2.468 & 390 & 2.346 & 393.1 & 2.846 & 449.9 & 2.746 & 476.4 & 2.926 & 488.3 \\
\hline 2.397 & 377.7 & 2.104 & 327.4 & 2.254 & 352.1 & 2.456 & 378.1 & 2.539 & 382.1 & 2.231 & 368.9 & 2.71 & 421.3 & 2.63 & 442.9 & 2.743 & 459.2 \\
\hline 2.237 & 353.9 & 1.802 & 286.4 & 2.139 & 325.2 & 2.331 & 349.9 & 2.299 & 355.6 & 2.074 & 345.5 & 2.435 & 394.9 & 2.678 & 436.3 & 2.568 & 435 \\
\hline 1.828 & 312 & 1.589 & 261.3 & 2 & 298.8 & 2.092 & 326.5 & 1.926 & 311.1 & 1.929 & 318.6 & 2.234 & 369.7 & 2.296 & 391.8 & 2.216 & 394.9 \\
\hline 1.772 & 289.1 & 1.66 & 253.4 & 1.636 & 260.9 & 2 & 300.1 & 2.003 & 301.4 & 1.858 & 285.6 & 2.118 & 337.6 & 2.13 & 368.9 & 2.133 & 370.2 \\
\hline 1.535 & 264.9 & 0 & 176.5 & 1.689 & 252.5 & 1.799 & 273.7 & 1.808 & 275 & 1.677 & 258.7 & 1.905 & 315.5 & 2.074 & 335.8 & 1.97 & 344.2 \\
\hline 1.633 & 255.6 & 0 & 174 & 0 & 159.8 & 1.642 & 247.2 & 1.633 & 248.5 & 0 & 159.7 & 1.819 & 283.8 & 1.947 & 311.1 & 1.855 & 319.9 \\
\hline & & & & & & & & & & & & & & & & & \\
\hline & & & & & & & & & & & & & & & & & \\
\hline
\end{tabular}


Table 2. $25^{\circ} \mathrm{C}$ As Received

\begin{tabular}{|c|c|c|c|c|c|c|c|c|c|c|c|c|c|c|c|c|c|}
\hline run1 & & run2 & & run3 & & run4 & & run5 & & run6 & & run7 & & run8 & & run9 & \\
\hline [Pa] & {$[1 / \mathrm{s}]$} & {$[\mathrm{Pa}]$} & {$[1 / \mathrm{s}]$} & {$[\mathrm{Pa}]$} & {$[1 / \mathbf{s}]$} & {$[\mathrm{Pa}]$} & {$[1 / \mathbf{s}]$} & {$[\mathrm{Pa}]$} & {$[1 / \mathrm{s}]$} & {$[\mathrm{Pa}]$} & {$[1 / \mathrm{s}]$} & {$[\mathrm{Pa}]$} & {$[1 / \mathrm{s}]$} & {$[\mathrm{Pa}]$} & {$[1 / \mathrm{s}]$} & {$[\mathrm{Pa}]$} & {$[1 / s]$} \\
\hline 4.849 & 317.3 & 5.042 & 324.8 & 2.867 & 372.4 & 1.603 & 490.9 & 4.864 & 325.2 & 5.095 & 325.2 & 5.275 & 326.5 & 4.213 & 314.6 & 4.755 & 319.5 \\
\hline 4.82 & 323 & 974 & 341.5 & 4.115 & 323.5 & 4.621 & 328.7 & 5.518 & 345.5 & 5.148 & 343.3 & 5.287 & 346.8 & 5.107 & 326.5 & .778 & 326.1 \\
\hline 5.524 & 340.2 & 506 & 376.3 & 4.781 & 323.5 & 4.784 & 326.5 & 5.444 & 368.4 & 5.317 & 372.4 & 5.423 & 379.4 & 5.503 & 349 & 5.278 & 346.8 \\
\hline 6.083 & 374.1 & 5.953 & 398.8 & 5.456 & 341.1 & 5.296 & 348.6 & 5.684 & 395.3 & 5.693 & 394.9 & 5.853 & 401.5 & 5.557 & 373.7 & 5.793 & 372.4 \\
\hline 6.234 & 400.6 & 6.146 & 430.5 & 5.805 & 366.6 & 5.817 & 371.5 & 5.888 & 427.5 & 6.255 & 418.6 & 6.113 & 427.5 & 5.817 & 400.6 & 5.785 & 396.2 \\
\hline 6.246 & 425.3 & 6.625 & 453.5 & 5.885 & 395.3 & 5.699 & 394 & 6.365 & 450.8 & 6.43 & 445.5 & 6.548 & 455.7 & 6.166 & 428.3 & 5.861 & 420 \\
\hline 6.542 & 449.1 & 6.865 & 478.6 & 5.962 & 419.5 & 5.977 & 417.3 & 6.77 & 472.9 & 6.734 & 473.7 & 6.874 & 482.1 & 6.536 & 453 & 6.329 & 445.1 \\
\hline 6.764 & 481.7 & 6.865 & 501.9 & 6.4 & 442.4 & 6.507 & 457.4 & 6.871 & 497.5 & 6.782 & 497.1 & 7.036 & 508.1 & 6.708 & 481.2 & 6.723 & 485.2 \\
\hline 6.998 & 505.9 & 7.377 & 531.5 & 6.649 & 479.9 & 6.699 & 479.5 & 7.093 & 522.2 & 7.093 & 524.9 & 7.119 & 531 & 7.107 & 508.1 & 6.977 & 493.1 \\
\hline 7.365 & 529.7 & 652 & 559.7 & 6.956 & 503.3 & 7.045 & 503.3 & 7.332 & 546.9 & 7.501 & 563.2 & 7.315 & 555.7 & 7.344 & 534.1 & 7.193 & 535.9 \\
\hline 7.605 & 553.9 & 7.986 & 583.9 & 7.11 & 533.7 & 7.027 & 533.2 & 7.69 & 589.6 & 7.551 & 591.8 & 7.732 & 595.4 & 7.542 & 558.8 & 7.525 & 564.1 \\
\hline 8.463 & 591.4 & 8.191 & 607.7 & 7.362 & 555.7 & 7.383 & 555.3 & 8.016 & 615.6 & 7.963 & 610.3 & 7.927 & 620 & 7.764 & 583 & 8.013 & 595.8 \\
\hline 8.714 & 617 & 8.744 & 650 & 7.596 & 579.1 & 7.72 & 577.3 & 8.478 & 642.1 & 8.309 & 642.1 & 8.341 & 646.9 & 8.019 & 605.5 & 8.057 & 621.4 \\
\hline 8.661 & 644.7 & 8.951 & 672.9 & 7.815 & 603.7 & 8.386 & 617.4 & 8.365 & 671.2 & 8.623 & 668.5 & 8.691 & 675.6 & 8.427 & 645.6 & 8.404 & 640.8 \\
\hline 8.859 & 669.8 & 9.055 & 695.8 & 8.445 & 644.3 & 8.531 & 643 & 8.552 & 695 & 8.91 & 689.7 & 8.963 & 706 & 8.563 & 672 & 8.395 & 672.5 \\
\hline 9.256 & 692.8 & 9.454 & 728 & 8.62 & 653.5 & 8.59 & 669 & 9.087 & 720.1 & 9.395 & 725.8 & 9.348 & 732.4 & 8.842 & 702 & 8.833 & 695.8 \\
\hline 9.67 & 726.2 & & 753.1 & 9.306 & 695.4 & 9.232 & 699.4 & 9.265 & 743 & 9.413 & 752.2 & 9.401 & 758.4 & 9.324 & 724.5 & 9.17 & 720.5 \\
\hline 9.96 & 748.7 & 9.889 & 778.7 & 9.333 & 724 & 9.123 & 724.5 & 9.67 & 772.1 & 9.928 & 778.7 & 9.883 & 787.1 & 9.277 & 751.4 & 9.17 & 744.3 \\
\hline 10.24 & 781.3 & 10.06 & 804.7 & 9.863 & 751.8 & 9.469 & 748.3 & 9.741 & 801.2 & 10.18 & 799.8 & 9.818 & 811.7 & 9.658 & 784.9 & 9.652 & 784 \\
\hline 10.79 & 804.7 & 10.51 & 840.8 & 10.12 & 773.4 & 9.836 & 776.5 & 10.24 & 824.1 & 10.39 & 833.3 & 10.34 & 836.9 & 9.774 & 807.8 & 10.12 & 807.3 \\
\hline 10.85 & 827.6 & 10.82 & 862.4 & 10.27 & 799.8 & 10.14 & 802.9 & 10.64 & 864.6 & 10.56 & 859.3 & 10.5 & 862.9 & 10.4 & 832 & 10.37 & 832 \\
\hline 11.27 & 851.4 & 11.28 & 883.1 & 10.56 & 833.8 & 10.36 & 828 & 11.09 & 891.9 & 10.85 & 886.2 & 10.61 & 889.7 & 10.34 & 856.7 & 10.47 & 865.1 \\
\hline 11.36 & 877.4 & 11.65 & 923.7 & 11.02 & 856.2 & 10.67 & 852.3 & 11.49 & 920.6 & 11.23 & 913.1 & 11.22 & 912.7 & 10.9 & 879.6 & 10.93 & 886.7 \\
\hline 11.91 & 903.8 & & 944.4 & 11.14 & 881.4 & 10.85 & 878.7 & 11.65 & 940.4 & 11.43 & 936.5 & 11.4 & 939.5 & 11.13 & 917.5 & 11.12 & 909.1 \\
\hline 12.65 & 944.8 & & 966.9 & 11.56 & 905.6 & 11.52 & 905.2 & 12.03 & 966.4 & 11.66 & 962.5 & 11.87 & 978.8 & 11.49 & 948.8 & 11.71 & 950.6 \\
\hline 12.8 & 969.9 & & 989.8 & 12.09 & 944.8 & & 945.3 & & 998.1 & 12.22 & 984.5 & 12.26 & 1005 & 12.03 & 970.8 & 11.85 & 978.3 \\
\hline 13.19 & 996.8 & & 1031 & 12.4 & 968.2 & 12.33 & 974.8 & 12.69 & 1020 & 12.68 & 1024 & 12.69 & 1028 & 12.3 & 999 & 12.1 & 989.8 \\
\hline 13.55 & 1024 & 3.51 & 1056 & 12.73 & 994.2 & 12.42 & 999.5 & 13.11 & 1057 & 13.02 & 1048 & 12.8 & 1059 & 12.48 & 1028 & 12.54 & 1014 \\
\hline 13.95 & 1048 & 13.89 & 1082 & 13.12 & 1020 & 12.74 & 1024 & 13.39 & 1079 & 13.44 & 1078 & 13.18 & 1087 & 12.88 & 1055 & 13.13 & 1059 \\
\hline 14.48 & 1080 & 14.31 & 1110 & 13.41 & 1051 & 13.15 & 1048 & 13.82 & 1109 & 13.7 & 1103 & 13.53 & 1112 & 13.19 & 1081 & 13.52 & 1085 \\
\hline 14.88 & 1103 & 14.81 & 1139 & 13.75 & 1076 & 13.58 & 1073 & 13.95 & 1130 & 13.93 & 1130 & 13.86 & 1137 & 13.6 & 1104 & 13.76 & 1111 \\
\hline
\end{tabular}


Table 2. $25^{\circ} \mathrm{C}$ As Received - continued

\begin{tabular}{|c|c|c|c|c|c|c|c|c|c|c|c|c|c|c|c|c|c|}
\hline run1 & & run2 & & run3 & & run4 & & run5 & & run6 & & run7 & & run8 & & run9 & \\
\hline [Pa] & [1/s] & [Pa] & [1/s] & [Pa] & [1/s] & [Pa] & [1/s] & [Pa] & [1/s] & [Pa] & [1/s] & [Pa] & [1/s] & [Pa] & {$[1 / s]$} & [Pa] & {$[1 / s]$} \\
\hline 15.21 & 1135 & 15.07 & 1163 & 14.04 & 1100 & 13.91 & 1099 & 14.34 & 1154 & 14.39 & 1155 & 14.11 & 1160 & 13.77 & 1132 & 14.16 & 1136 \\
\hline 15.45 & 1160 & 5.39 & 1186 & 14.39 & 1125 & 14.24 & 1126 & 14.66 & 1179 & 14.68 & 1178 & 14.55 & 1185 & 14.14 & 1155 & 14.31 & 1162 \\
\hline 15.82 & 1184 & 15.82 & 1210 & 14.75 & 1148 & 14.59 & 1150 & 15.48 & 1217 & 14.92 & 1203 & 15.01 & 1211 & 14.62 & 1184 & 14.8 & 1193 \\
\hline 16.28 & 1209 & 16.39 & 1250 & 15.57 & 1189 & 15.2 & 1178 & 15.85 & 1241 & 15.29 & 1229 & 15.24 & 1237 & 15.15 & 1208 & 15.17 & 1218 \\
\hline 16.63 & 1233 & 16.86 & 1277 & 15.55 & 1202 & 15.59 & 1203 & 16.19 & 1267 & 16.02 & 1273 & 16.18 & 1281 & 15.59 & 1248 & 15.33 & 1241 \\
\hline 17.07 & 1258 & 17.23 & 1302 & 16.08 & 1228 & 16.17 & 1244 & 16.52 & 1299 & 16.08 & 1284 & 16.44 & 1304 & 15.96 & 1270 & 15.61 & 1265 \\
\hline 17.39 & 1284 & 17.51 & 1326 & 16.63 & 1259 & 16.55 & 1269 & 16.81 & 1321 & 16.85 & 1325 & 16.65 & 1334 & 16.17 & 1303 & 16.07 & 1292 \\
\hline 18.1 & 1325 & 17.78 & 1352 & 16.92 & 1283 & 16.93 & 1300 & 17.48 & 1358 & 17.12 & 1345 & 17.01 & 1359 & 16.7 & 1327 & 16.76 & 1331 \\
\hline 18.58 & 1354 & 18.28 & 1381 & 17.25 & 1310 & 17.28 & 1323 & 17.75 & 1382 & 17.31 & 1373 & 17.34 & 1380 & 17.13 & 1351 & 17.15 & 1359 \\
\hline 18.81 & 1376 & 18.58 & 1404 & 18.02 & 1352 & 17.66 & 1346 & 18.1 & 1406 & 17.75 & 1404 & 17.69 & 1406 & 17.22 & 1373 & 17.48 & 1385 \\
\hline 19.14 & 1400 & 18.99 & 1427 & 18.4 & 1373 & 18.25 & 1384 & 18.49 & 1432 & 18.1 & 1427 & 18.05 & 1430 & 18.02 & 1412 & 17.75 & 1410 \\
\hline 19.53 & 1425 & & 1467 & 18.64 & 1398 & 18.58 & 1407 & 18.81 & 1458 & 18.46 & 1451 & 18.79 & 1469 & 17.93 & 1421 & 18.16 & 1441 \\
\hline 19.85 & 1449 & 19.94 & 1492 & 18.9 & 1429 & 18.96 & 1431 & 19.35 & 1485 & 18.93 & 1478 & 19.2 & 1499 & 18.87 & 1467 & 18.58 & 1466 \\
\hline 20.74 & 1490 & 20.41 & 1518 & 19.35 & 1451 & 19.35 & 1462 & 19.55 & 1516 & 19.61 & 1518 & 19.32 & 1519 & 19.14 & 1495 & 18.96 & 1490 \\
\hline 20.98 & 1516 & 20.65 & 1544 & 19.73 & 1481 & 19.7 & 1483 & 20 & 1540 & 19.55 & 1527 & 19.82 & 1551 & 19.14 & 1506 & 19.23 & 1516 \\
\hline 19.61 & 1527 & 19.05 & 1493 & 20.03 & 1555 & 19.44 & 1552 & 18.31 & 1493 & 18.37 & 1512 & 18.19 & 1486 & 18.73 & 1522 & 18.99 & 1545 \\
\hline 19.32 & 1495 & 18.81 & 1469 & 19.73 & 1526 & 18.81 & 1510 & 17.81 & 1469 & 02 & 1487 & 17.81 & 1462 & 18.34 & 1497 & 18.52 & 1518 \\
\hline 18.93 & 1473 & 18.4 & 1442 & 19.29 & 1499 & 18.31 & 1487 & 17.25 & 1430 & 17.72 & 1465 & 17.45 & 1433 & 18.1 & 1471 & 18.28 & 1491 \\
\hline 18.55 & 1449 & 17.9 & 1417 & 18.93 & 1476 & 17.93 & 1461 & 16.95 & 1405 & 17.48 & 1438 & 17.07 & 1406 & 17.28 & 1428 & 17.9 & 1467 \\
\hline 18.31 & 1426 & 17.66 & 1389 & 18.43 & 1449 & 17.54 & 1436 & 16.57 & 1376 & 17.07 & 1414 & 16.57 & 1378 & 16.89 & 1405 & 17.6 & 1436 \\
\hline 17.54 & 1388 & 16.86 & 1350 & 18.16 & 1424 & 17.31 & 1406 & 16.36 & 1353 & 16.51 & 1371 & 16.33 & 1353 & 16.51 & 1381 & 17.31 & 1412 \\
\hline 17.25 & 1364 & 16.54 & 1320 & 17.34 & 1383 & 16.86 & 1379 & 16 & 1326 & 16.06 & 1347 & 16.06 & 1331 & 16.3 & 1356 & 16.57 & 1376 \\
\hline 16.95 & 1334 & 16.12 & 1296 & 17.07 & 1359 & 16.6 & 1354 & 15.59 & 1303 & 15.74 & 1327 & 15.74 & 1306 & 15.97 & 1324 & 16.33 & 1351 \\
\hline 16.51 & 1309 & 15.8 & 1270 & 16.74 & 1334 & 16.15 & 1329 & 15.35 & 1278 & 15.44 & 1292 & 15.38 & 1278 & 15.65 & 1304 & 15.91 & 1322 \\
\hline 16.3 & 1287 & 15.41 & 1243 & 16.33 & 1308 & 15.71 & 1305 & 14.79 & 1239 & 14.91 & 1275 & 15.12 & 1253 & 15.41 & 1278 & 15.68 & 1299 \\
\hline 15.59 & 1248 & 15.12 & 1217 & 15.86 & 1279 & 15.47 & 1282 & 14.29 & 1212 & 14.82 & 1241 & 14.46 & 1225 & 14.88 & 1251 & 15.17 & 1267 \\
\hline 15.23 & 1228 & 14.7 & 1190 & 15.59 & 1250 & 15.23 & 1255 & 14.05 & 1185 & 14.38 & 1219 & 13.99 & 1188 & 14.35 & 1213 & 14.97 & 1243 \\
\hline 15 & 1196 & 14.32 & 1165 & 15.17 & 1223 & 14.49 & 1215 & 13.64 & 1159 & 14.17 & 1195 & 13.67 & 1162 & 14.02 & 1187 & 14.64 & 1219 \\
\hline 14.79 & 1173 & 13.93 & 1133 & 14.73 & 1195 & 14.17 & 1189 & 13.4 & 1136 & 13.81 & 1172 & 13.4 & 1136 & 13.7 & 1162 & 14.14 & 1188 \\
\hline 14.46 & 1149 & 13.55 & 1110 & 14.49 & 1172 & 13.93 & 1165 & 13.07 & 1107 & 13.43 & 1144 & 13.04 & 1106 & 13.46 & 1137 & 13.61 & 1168 \\
\hline 13.84 & 1112 & 13.37 & 1083 & 14.38 & 1151 & 13.55 & 1132 & 12.63 & 1078 & 13.22 & 1119 & 12.84 & 1081 & 13.16 & 1111 & 13.49 & 1133 \\
\hline
\end{tabular}


Table 2. $25^{\circ} \mathrm{C}$ As Received - continued

\begin{tabular}{|c|c|c|c|c|c|c|c|c|c|c|c|c|c|c|c|c|c|}
\hline run1 & & run2 & & run3 & & run4 & & run5 & & run6 & & run7 & & run8 & & run9 & \\
\hline [Pa] & {$[1 / s]$} & {$[\mathrm{Pa}]$} & {$[1 / s]$} & [Pa] & {$[1 / s]$} & [Pa] & {$[1 / s]$} & {$[\mathrm{Pa}]$} & {$[1 / s]$} & {$[\mathrm{Pa}]$} & {$[1 / \mathrm{s}]$} & [Pa] & {$[1 / s]$} & {$[\mathrm{Pa}]$} & {$[1 / \mathrm{s}]$} & {$[\mathrm{Pa}]$} & {$[1 / \mathrm{s}]$} \\
\hline 13.43 & 1085 & 12.93 & 1057 & 13.99 & 1127 & 13.16 & 1110 & 12.3 & 1051 & 12.42 & 1076 & 12.6 & 1056 & 12.57 & 1081 & 13.28 & 1107 \\
\hline 13.07 & 063 & 12.42 & 1020 & 13.46 & 1088 & 12.6 & 1084 & 11.95 & 1023 & 12.27 & 1049 & 12.16 & 1032 & 12.31 & 1054 & 12.9 & 1085 \\
\hline 12.66 & 036 & 12.15 & 994.6 & 12.84 & 1055 & 12.27 & 1051 & 11.74 & 999.9 & 11.81 & 1021 & 11.77 & 1008 & 12.17 & 1031 & 12.48 & 1061 \\
\hline 12.53 & 1003 & 11.77 & 968.6 & 12.69 & 1032 & 12.3 & 1029 & 11.45 & 975.7 & 11.67 & 996.4 & 11.42 & 979.2 & 11.8 & 1008 & 11.95 & 1021 \\
\hline 11.98 & 980.1 & 11.35 & 941.3 & 12.45 & 1007 & 11.97 & 1007 & 10.74 & 937.3 & 11.37 & 971.7 & 10.78 & 936.5 & 11.65 & 978.3 & 11.62 & 996.4 \\
\hline 11.94 & 957.6 & 11.35 & 919.3 & 12.09 & 979.6 & 11.72 & 978.8 & 10.63 & 912.7 & 10.87 & 947.5 & 10.56 & 913.5 & 1.18 & 949.7 & 11.49 & 970.8 \\
\hline 1.4 & 55.6 & .76 & 895.9 & & 53.6 & 1.24 & 951.9 & 10.27 & 889.7 & 0.82 & 922.8 & .28 & 882.2 & .66 & 925 & .15 & 40.9 \\
\hline 10.82 & 92.8 & 10.36 & 856.7 & .25 & 28.1 & 0.76 & 914 & 10.13 & 862.4 & 943 & 885.8 & 10.02 & 857.6 & 0.62 & 900.8 & 0.77 & 14.9 \\
\hline 10.49 & 869 & 9.848 & 831.6 & 11.09 & 904.3 & 10.29 & 888 & 9.525 & 838.2 & 9.919 & 861.5 & 9.715 & 832.9 & .679 & 859.8 & 10.38 & 387.5 \\
\hline 10.04 & 842.6 & 9.605 & 808.7 & 10.55 & 879.6 & 10.1 & 863.3 & .058 & 800.3 & 9.395 & 832.9 & 9.505 & 809.5 & 9.706 & 836.4 & 10.18 & 863.7 \\
\hline 9.827 & 817.5 & 9.167 & 777.4 & 10.03 & 839.9 & 9.88 & 834.7 & 9.016 & 773 & 9.339 & 809.5 & 8.984 & 785.7 & 9.28 & 810.9 & 9.886 & 832.9 \\
\hline 9.368 & 785.7 & 8.96 & 754 & 9.877 & 816.1 & 9.454 & 810.4 & 8.498 & 747.4 & 8.842 & 784.9 & 8.466 & 748.3 & 9.058 & 779.1 & 9.436 & 809.5 \\
\hline 9.167 & 761.9 & 8.723 & 733.7 & 9.59 & 784.4 & 8.998 & 787.9 & 8.12 & 728.4 & 8.56 & 760.2 & 8.276 & 721.8 & 8.552 & 751.4 & 9.123 & 782.7 \\
\hline 9.031 & 740.3 & & 692.8 & & 758.9 & 8.998 & 761.5 & 8.016 & 697.2 & 8.451 & 736.8 & 8.019 & 700.7 & & 728.9 & 8.927 & 758.9 \\
\hline 8.6 & 14.8 & & 669.8 & & 735.1 & 8.398 & 722.3 & 7.439 & 671.2 & 84 & 709.9 & 306 & 663.7 & & 700.7 & 566 & 734.2 \\
\hline 8.128 & 79.1 & 7.714 & 645.6 & 8.626 & 706.9 & 7.933 & 699.8 & 7.113 & 643.8 & 7.433 & 666.8 & 7.019 & 640.3 & 7.513 & 676 & 8.001 & 693.6 \\
\hline 7.906 & 650.4 & 7.199 & 613.4 & 046 & 680 & 7.676 & 675.1 & 6.992 & 615.6 & 6.927 & 639.4 & 6.853 & 617.4 & 7.258 & 652.2 & 7.433 & 668.5 \\
\hline 7.474 & 626.7 & 6.737 & 587.4 & 7.661 & 654 & 7.211 & 646.5 & 6.773 & 591 & 6.841 & 611.2 & 6.8 & 586.1 & 7.051 & 613.9 & 7.208 & 643 \\
\hline 7.146 & 604.2 & 6.619 & 566.3 & 7.315 & 628 & 6.942 & 624 & 6.521 & 565.8 & 6.465 & 588.8 & 6.536 & 559.7 & 6.806 & 591.8 & 6.951 & 618.3 \\
\hline 6.613 & 566.3 & 6.4 & 541.6 & 7.001 & 603.7 & 6.761 & 587 & 6.122 & 542 & 6.22 & 564.5 & 6.095 & 538.1 & 6.273 & 561 & 6.643 & 593.2 \\
\hline 6.403 & 539 & 5.666 & 502.8 & & 564.5 & 6.415 & 562.8 & 5.69 & 505.5 & 5.95 & 538.1 & 5.897 & 510.3 & 5.941 & 535.4 & 6.471 & 559.7 \\
\hline 5.974 & 15.6 & & 480.8 & & 38.1 & & 33.7 & 5.37 & 474.2 & 5.56 & 513 & 358 & 487 & & 512.1 & 6.22 & 536.8 \\
\hline 5.483 & 0.5 & & 449.1 & & 528.8 & 823 & 508.1 & .148 & 450.4 & 163 & 485.6 & 92 & 48.2 & & 488.3 & 5.787 & 515.2 \\
\hline 5.187 & 458.3 & 107 & 423.5 & 5.841 & 500.2 & 5.577 & 481.2 & 4.604 & 428.3 & 4.843 & 446 & 4.583 & 423.9 & 5.201 & 464.5 & 5.382 & 490.5 \\
\hline 5.006 & 434.1 & 4.849 & 399.7 & 5.459 & 470.6 & 5.187 & 460.1 & 4.598 & 405 & 4.453 & 420.4 & 4.293 & 397.9 & 4.923 & 437.6 & 5.199 & 461.4 \\
\hline 4.994 & 409 & 4.314 & 377.2 & 5.139 & 438.9 & 4.817 & 421.3 & 3.964 & 364.9 & 4.701 & 411.6 & 4.352 & 367.1 & 4.524 & 395.3 & 4.923 & 440.2 \\
\hline 4.657 & 82.1 & 3.87 & 341.1 & 4.66 & 412.9 & 4.299 & 398.8 & 3.926 & 342 & 4.246 & 368 & 3.967 & 345.5 & 4.053 & 373.3 & 4.293 & 392.6 \\
\hline 4.059 & 357.8 & 3.923 & 313.8 & 4.183 & 375.5 & 4.056 & 369.3 & 3.337 & 307.6 & 3.612 & 340.6 & 3.814 & 321.3 & 3.997 & 341.1 & 4.58 & 384.7 \\
\hline 4.216 & 333.6 & 3.236 & 287.3 & 4.284 & 353 & 4.166 & 342 & 3.378 & 286 & 3.595 & 311.1 & 3.521 & 282.9 & 3.725 & 316 & 3.985 & 345.5 \\
\hline 3.473 & 305.8 & 3.343 & 261.3 & 3.68 & 324.3 & 3.524 & 317.3 & 2.795 & 253.8 & 3.278 & 290.4 & 2.858 & 259.1 & 3.621 & 295.3 & 3.482 & 312.4 \\
\hline 3.556 & 277.6 & 3.349 & 254.7 & 3.834 & 303.2 & 3.651 & 293.9 & 0 & 159.7 & 3.216 & 264 & 2.929 & 251.2 & 3.189 & 271 & 3.695 & 290 \\
\hline 2.911 & 253.8 & 0 & 173.8 & 3.171 & 264.9 & 2.896 & 256.9 & 0 & 176.4 & 2.659 & 243.3 & & 169.9 & 3.127 & 244.6 & 3.023 & 262.2 \\
\hline
\end{tabular}


Table 3. $50{ }^{\circ} \mathrm{C}$ As Received

\begin{tabular}{|c|c|c|c|c|c|c|c|c|c|c|c|c|c|c|c|c|c|}
\hline run1 & & run2 & & run3 & & run4 & & run5 & & run6 & & run7 & & run8 & & run9 & \\
\hline [Pa] & {$[1 / s]$} & [Pa] & [1/s] & [Pa] & {$[1 / s]$} & [Pa] & {$[1 / s]$} & [Pa] & [1/s] & [Pa] & [1/s] & [Pa] & [1/s] & [Pa] & {$[1 / s]$} & [Pa] & {$[1 / s]$} \\
\hline 3.233 & 314.6 & 3.42 & 353.9 & 2.582 & 354.3 & 3.411 & 308.9 & 3.1 & 334 & 3.352 & 327 & 3.352 & 304.1 & 4 & 319.9 & 3.535 & 310.7 \\
\hline 3.479 & 20.8 & 3.444 & 346.8 & 3.834 & 323.9 & 3.497 & 311.6 & 3.769 & 327.9 & 3.899 & 328.7 & 3.811 & 315.1 & 4.166 & 344.2 & 4.027 & 322.6 \\
\hline 3.964 & 342.4 & .059 & 350.3 & .849 & 342 & 4 & 334.5 & 4.272 & 349.9 & 4.432 & 355.2 & 3.917 & 335.4 & 4.195 & 375 & 4.074 & 343.7 \\
\hline 4.127 & 364.9 & 4.219 & 368.9 & 4.172 & 360.9 & 3.994 & 351.7 & 4.32 & 379.4 & 4.577 & 378.1 & 4.186 & 363.6 & 4.542 & 398.8 & 4.142 & 372.4 \\
\hline 4.589 & 403.2 & 4.755 & 408.5 & 4.284 & 384.7 & 4.42 & 380.8 & 4.397 & 401.9 & 4.589 & 404.1 & 4.592 & 400.6 & 4.684 & 423.5 & 4.447 & 397.5 \\
\hline 4.657 & 427.9 & 4.867 & 432.8 & 4.565 & 416.4 & 4.237 & 406.3 & 4.586 & 426.6 & 4.672 & 433.6 & 4.752 & 428.3 & 4.758 & 450.4 & 4.672 & 425.7 \\
\hline 4.761 & 459.6 & 5.009 & 458.3 & 4.666 & 445.5 & 4.832 & 432.3 & 4.837 & 450.8 & 5.107 & 450.4 & 4.864 & 449.1 & 4.829 & 475.5 & 4.843 & 450.8 \\
\hline 4.864 & 482.5 & 5.204 & 482.1 & 4.755 & 473.3 & 5.11 & 483.9 & 5.024 & 472.9 & 5.092 & 483.9 & 5.03 & 475.5 & 5.335 & 513.8 & 4.959 & 476.4 \\
\hline 4.991 & 506.3 & 5.308 & 511.6 & .045 & 495.8 & 5.104 & 506.3 & 5.349 & 509 & 5.305 & 507.2 & 5.181 & 503.3 & 5.515 & 536.8 & 5.101 & 500.2 \\
\hline 5.287 & 531 & 5.45 & 537.6 & 5.302 & 515.2 & 5.471 & 555.7 & 5.299 & 536.8 & 5.545 & 543.8 & 5.19 & 527.5 & 5.613 & 559.7 & 5.326 & 529.3 \\
\hline 5.477 & 555.7 & 5.687 & 561 & 5.468 & 552.6 & 5.823 & 580.8 & 5.521 & 559.2 & 5.645 & 566.7 & 5.42 & 553.1 & 5.645 & 593.2 & 5.494 & 556.6 \\
\hline & 579.9 & & 597.1 & & 560.5 & & & 5.66 & 581.7 & 5.82 & 597.1 & & 590.5 & & 618.3 & 5.666 & 580.8 \\
\hline 5.764 & 603.7 & 6.252 & 620.9 & 5.713 & 595.8 & 6.098 & 632.4 & 5.82 & 605.1 & 6.057 & 621.4 & 5.856 & 621.8 & 5.876 & 641.2 & 5.841 & 620.9 \\
\hline 6.119 & 648.2 & 6.368 & 646.9 & 6.009 & 626.2 & 5.95 & 654.9 & 5.897 & 632.8 & 6.116 & 647.8 & 5.986 & 643.8 & 6.036 & 666.3 & 6.08 & 644.7 \\
\hline 6.373 & 677.8 & 6.527 & 677.8 & 6.001 & 657.5 & 6.48 & 680.4 & 6.128 & 659.3 & 6.279 & 671.2 & 6.187 & 667.2 & 6.288 & 693.6 & 6.252 & 672.5 \\
\hline 6.501 & 701.6 & 6.711 & 701.6 & 6.382 & 689.2 & 6.406 & 703.8 & 6.385 & 686.1 & 6.412 & 694.1 & 6.187 & 696.7 & 6.634 & 730.7 & 6.193 & 697.6 \\
\hline 6.521 & 726.7 & 6.749 & 726.7 & .501 & 715.7 & & 753.6 & 6.592 & 731.1 & 504 & 717.9 & 536 & 724.5 & 6.61 & 753.6 & .471 & 717.9 \\
\hline 6.779 & 749.2 & 6.956 & 750.5 & 6.637 & 740.3 & 7.267 & 778.7 & 6.862 & 756.7 & 6.622 & 744.3 & 6.572 & 753.1 & 6.82 & 784 & 6.734 & 744.3 \\
\hline & 773.4 & 7.235 & 775.2 & & 764.1 & & 803.8 & 7.025 & 784.9 & & 785.7 & 6.8 & 776.9 & 6.965 & 806.4 & 6.909 & 781.3 \\
\hline 6.989 & 799 & 7.365 & 819.7 & 6.977 & 790.1 & 7.412 & 830.7 & 7.075 & 813.1 & 7.332 & 811.3 & 6.939 & 804.2 & & 829.4 & 6.942 & 803.8 \\
\hline 7.51 & 836.9 & 7.684 & 839.9 & 7.14 & 817.5 & & 850.1 & 7.134 & 837.7 & 89 & 840.4 & & 828.5 & 7.448 & 865.9 & 7.312 & 829.8 \\
\hline 7.522 & 876.1 & 7.782 & 863.3 & 7.181 & 845.7 & 7.652 & 875.6 & 7.394 & 862.9 & 7.519 & 865.5 & 7.335 & 872.1 & 7.572 & 889.3 & 7.341 & 865.9 \\
\hline 7.522 & 889.7 & 7.877 & 896.3 & 7.409 & 873.9 & 7.566 & 897.2 & 7.463 & 888.4 & 7.821 & 892.8 & 7.309 & 877.8 & 7.619 & 921.5 & 7.445 & 891.9 \\
\hline 7.829 & 913.1 & 8.155 & 921 & 7.714 & 901.2 & 7.738 & 947.5 & 7.72 & 910.5 & 7.85 & 917.5 & 7.649 & 908.7 & 7.841 & 945.3 & 7.616 & 917.5 \\
\hline 8.202 & 945.3 & 8.214 & 944.4 & 7.838 & 928.5 & 8.498 & 969.5 & 7.924 & 937.3 & 8.051 & 949.7 & 7.983 & 945.3 & 7.877 & 966.9 & 7.779 & 943.1 \\
\hline 8.279 & 975.2 & 8.344 & 968.6 & 7.936 & 950.1 & 8.217 & 995.5 & 7.966 & 960.7 & 8.016 & 973 & 8.031 & 976.6 & 8.247 & 1006 & 7.942 & 967.7 \\
\hline 8.412 & 995.1 & 8.856 & 1006 & 8.35 & 990.2 & 8.478 & 1021 & 8.294 & 1003 & 8.262 & 997.3 & 8.179 & 1000 & 8.415 & 1029 & 7.983 & 992.4 \\
\hline 8.525 & 1022 & 8.978 & 1032 & 8.478 & 1014 & 8.463 & 1040 & 8.445 & 1029 & 8.442 & 1021 & 8.303 & 1022 & 8.611 & 1051 & 8.111 & 1016 \\
\hline 8.629 & 1050 & 9.138 & 1059 & 8.629 & 1044 & 8.679 & 1061 & 8.64 & 1053 & 8.771 & 1056 & 8.51 & 1051 & 8.723 & 1076 & 8.294 & 1045 \\
\hline 8.848 & 1074 & 9.214 & 1083 & 8.892 & 1070 & 8.679 & 1085 & 8.705 & 1078 & 8.865 & 1082 & 8.602 & 1074 & 8.806 & 1108 & 8.466 & 1071 \\
\hline 8.984 & 1100 & 9.404 & 1107 & 8.833 & 1079 & 9.102 & 1111 & 8.91 & 1108 & 8.995 & 1107 & 8.998 & 1111 & 8.948 & 1130 & 8.631 & 1097 \\
\hline
\end{tabular}


Table 3. $50{ }^{\circ} \mathrm{C}$ As Received - continued

\begin{tabular}{|c|c|c|c|c|c|c|c|c|c|c|c|c|c|c|c|c|c|}
\hline run1 & & run2 & & run3 & & run4 & & run5 & & run6 & & run7 & & run8 & & run9 & \\
\hline [Pa] & {$[1 / \mathrm{s}]$} & {$[\mathrm{Pa}]$} & {$[1 / \mathrm{s}]$} & {$[\mathrm{Pa}]$} & {$[1 / \mathrm{s}]$} & [Pa] & {$[1 / \mathrm{s}]$} & [Pa] & {$[1 / \mathrm{s}]$} & {$[\mathrm{Pa}]$} & {$[1 / \mathrm{s}]$} & {$[\mathrm{Pa}]$} & {$[1 / \mathrm{s}]$} & {$[\mathrm{Pa}]$} & {$[1 / \mathrm{s}]$} & {$[\mathrm{Pa}]$} & {$[1 / \mathrm{s}]$} \\
\hline 9.463 & 1138 & 9.442 & 1130 & 8.981 & 1114 & 8.998 & 1132 & 9.084 & 1138 & 9.075 & 1139 & 9.173 & 1138 & 9.161 & 1155 & 8.951 & 1138 \\
\hline 9.602 & 1164 & 9.904 & 1169 & 9.108 & 1141 & 9.294 & 1180 & 9.217 & 1163 & 9.306 & 1165 & 9.336 & 1164 & 9.309 & 1181 & 9.194 & 1162 \\
\hline 9.747 & 1188 & 10.12 & 1194 & 9.259 & 1171 & 9.981 & 1202 & 9.321 & 1188 & 9.425 & 1187 & 9.348 & 1190 & 9.348 & 1207 & 9.416 & 1189 \\
\hline 9.812 & 1220 & 10.32 & 1218 & 9.614 & 1197 & 9.724 & 1228 & 9.549 & 1215 & 9.644 & 1216 & 9.644 & 1214 & 9.765 & 1244 & 9.428 & 1220 \\
\hline 10.05 & 1243 & 10.41 & 1250 & 9.756 & 1223 & 10.11 & 1251 & 9.759 & 1239 & 9.747 & 1241 & 9.694 & 1246 & 9.824 & 1278 & 9.573 & 1243 \\
\hline 10.24 & 1267 & 10.58 & 1274 & 10.11 & 1266 & 10.01 & 1272 & 9.886 & 1268 & 10.08 & 1280 & 9.812 & 1269 & 10.06 & 1302 & 9.712 & 1265 \\
\hline 10.66 & 1304 & 10.7 & 1303 & 10.21 & 1290 & 10.35 & 1294 & 10.23 & 1296 & 10.17 & 1306 & 9.886 & 1293 & 10.13 & 1327 & 9.966 & 1304 \\
\hline 10.77 & 1327 & 10.84 & 1329 & 10.32 & 1315 & 10.27 & 1316 & 10.34 & 1320 & 10.43 & 1331 & 10.02 & 1318 & 10.39 & 1352 & 10.2 & 1328 \\
\hline 10.93 & 1354 & 11.11 & 1353 & 10.48 & 1343 & 10.7 & 1337 & 10.74 & 1357 & 10.53 & 1356 & 10.22 & 1344 & 10.46 & 1377 & 10.37 & 1352 \\
\hline 11.16 & 1385 & 11.26 & 1375 & 10.58 & 1369 & 10.98 & 1382 & 10.81 & 1382 & 10.74 & 1387 & 10.34 & 1370 & 10.68 & 1400 & 10.57 & 1379 \\
\hline 11.2 & 1412 & 11.64 & 1412 & 10.53 & 1377 & 10.9 & 1403 & 11.04 & 1410 & 10.85 & 1412 & 10.9 & 1412 & 10.9 & 1425 & 10.68 & 1405 \\
\hline 11.4 & 1431 & 11.81 & 1439 & 11.07 & 1409 & 11.29 & 1426 & 10.96 & 1416 & 11.08 & 1436 & 10.87 & 1437 & 10.95 & 1453 & 10.75 & 1440 \\
\hline 11.66 & 1464 & 11.98 & 1464 & 10.95 & 1441 & 11.22 & 1447 & 11.42 & 1467 & 11.25 & 1460 & 11.05 & 1466 & 11.18 & 1478 & 10.84 & 1463 \\
\hline 11.88 & 1492 & 11.99 & 1492 & 11.39 & 1465 & 11.59 & 1469 & 11.45 & 1493 & 11.4 & 1486 & 11.33 & 1493 & 11.54 & 1519 & 11.06 & 1487 \\
\hline 11.97 & 1512 & 12.24 & 1518 & 11.26 & 1488 & 11.93 & 1516 & 11.69 & 1517 & 11.62 & 1508 & 11.61 & 1517 & 11.72 & 1544 & 11.24 & 1509 \\
\hline 11.11 & 1522 & 10.87 & 1516 & 10.47 & 1504 & 10.77 & 1524 & 10.24 & 1517 & 10.38 & 1516 & 10.57 & 1549 & 10.32 & 1528 & 10.29 & 1543 \\
\hline 10.95 & 1490 & 10.7 & 1491 & 10.24 & 1479 & 10.73 & 1501 & 10.1 & 1486 & 10.26 & 1493 & 10.4 & 1526 & 9.957 & 1504 & 10.16 & 1513 \\
\hline 10.74 & 1467 & 10.47 & 1469 & 9.605 & 1424 & 10.07 & 1458 & 9.999 & 1466 & 9.984 & 1461 & 9.987 & 1486 & 9.916 & 1479 & 9.945 & 1489 \\
\hline 10.5 & 1444 & 10.03 & 1428 & 9.661 & 1405 & 10.02 & 1435 & 9.797 & 1444 & 9.857 & 1435 & 9.916 & 1463 & 9.386 & 1434 & 9.774 & 1463 \\
\hline 10.22 & 1419 & 9.818 & 1407 & 9.226 & 1379 & 9.827 & 1403 & 9.419 & 1403 & 9.747 & 1409 & 9.786 & 1432 & 9.365 & 1411 & 9.537 & 1438 \\
\hline 9.943 & 1380 & 9.602 & 1383 & 9.33 & 1355 & 9.638 & 1377 & 9.306 & 1379 & 9.416 & 1385 & 9.635 & 1411 & 9.217 & 1379 & 9.324 & 1412 \\
\hline 9.812 & 1356 & 9.54 & 1354 & 8.995 & 1332 & 9.365 & 1352 & 9.084 & 1352 & 9.309 & 1358 & 9.451 & 1386 & 9.028 & 1356 & 9.173 & 1389 \\
\hline 9.587 & 1326 & 9.348 & 1332 & 8.927 & 1308 & 9.271 & 1329 & 8.945 & 1324 & 9.176 & 1335 & 9.069 & 1351 & 8.913 & 1332 & 8.984 & 1360 \\
\hline 9.525 & 1304 & 9.22 & 1309 & 8.484 & 1280 & 9.052 & 1305 & 8.83 & 1301 & 8.735 & 1297 & 8.818 & 1328 & 8.824 & 1308 & 8.916 & 1335 \\
\hline 9.3 & 1278 & 8.871 & 1271 & 8.59 & 1257 & 8.886 & 1279 & 8.572 & 1276 & 8.611 & 1273 & 8.697 & 1302 & 8.501 & 1271 & 8.581 & 1307 \\
\hline 9.058 & 1251 & 8.694 & 1245 & 8.048 & 1226 & 8.685 & 1254 & 8.371 & 1252 & 8.507 & 1239 & 8.557 & 1269 & 8.377 & 1248 & 8.38 & 1283 \\
\hline 8.963 & 1228 & 8.56 & 1216 & 8.288 & 1203 & 8.546 & 1228 & 8.3 & 1223 & 8.339 & 1216 & 8.43 & 1244 & 8.285 & 1218 & 8.241 & 1254 \\
\hline 8.72 & 1204 & 8.321 & 1190 & 7.788 & 1177 & 8.069 & 1185 & 7.779 & 1184 & 8.081 & 1195 & 8.282 & 1219 & 8.025 & 1195 & 8.125 & 1225 \\
\hline 8.371 & 1160 & 8.27 & 1167 & 7.832 & 1152 & 7.948 & 1161 & 7.619 & 1159 & 8.057 & 1171 & 8.001 & 1192 & 7.835 & 1171 & 7.344 & 1200 \\
\hline 8.134 & 1135 & 7.779 & 1132 & 7.315 & 1126 & 7.732 & 1132 & 7.48 & 1134 & 7.835 & 1147 & 7.862 & 1161 & 7.531 & 1133 & 7.498 & 1178 \\
\hline 7.972 & 1110 & 7.643 & 1105 & 6.965 & 1077 & 7.463 & 1103 & 7.3 & 1108 & 7.522 & 1103 & 7.684 & 1136 & 7.32 & 1110 & 7.137 & 1115 \\
\hline
\end{tabular}


Table 3. $50{ }^{\circ} \mathrm{C}$ As Received - continued

\begin{tabular}{|c|c|c|c|c|c|c|c|c|c|c|c|c|c|c|c|c|c|}
\hline run1 & & run2 & & run3 & & run4 & & run5 & & run6 & & run7 & & run8 & & run9 & \\
\hline [Pa] & {$[1 / \mathrm{s}]$} & {$[\mathrm{Pa}]$} & {$[1 / \mathrm{s}]$} & [Pa] & {$[1 / \mathrm{s}]$} & [Pa] & {$[1 / \mathrm{s}]$} & [Pa] & {$[1 / \mathrm{s}]$} & [Pa] & {$[1 / s]$} & [Pa] & {$[1 / \mathrm{s}]$} & {$[\mathrm{Pa}]$} & {$[1 / \mathrm{s}]$} & {$[\mathrm{Pa}]$} & {$[1 / \mathrm{s}]$} \\
\hline 7.77 & 1083 & 7.471 & 1081 & 7.081 & 1054 & 7.365 & 1079 & 7.016 & 1078 & 7.255 & 1080 & 7.516 & 1111 & 7.178 & 1083 & 7.045 & 1095 \\
\hline 7.56 & 1058 & 7.391 & 1053 & 6.829 & 1030 & 7.164 & 1052 & 6.862 & 1054 & 7.152 & 1052 & 7.309 & 1085 & 7.039 & 1056 & 6.847 & 1061 \\
\hline 7.486 & 1034 & 7.11 & 1029 & 6.879 & 1006 & 7.004 & 1026 & 6.909 & 1021 & 6.791 & 1032 & 7.048 & 1060 & 6.877 & 1027 & 6.45 & 992 \\
\hline 7.229 & 1002 & 6.853 & 1001 & 6.625 & 984.9 & 6.835 & 994.6 & 6.631 & 998.1 & 6.808 & 999.5 & 6.808 & 1036 & 6.708 & 999.9 & 6.48 & 978.8 \\
\hline 7.107 & 976.6 & 6.734 & 974.8 & 6.625 & 960.2 & 6.708 & 971.3 & 6.566 & 972.1 & 6.687 & 973.5 & 6.56 & 996.8 & 6.489 & 973.9 & 6.619 & 963.8 \\
\hline 6.838 & 950.6 & 6.625 & 941.7 & 6.104 & 934.7 & 6.397 & 947.5 & 6.365 & 947.5 & 6.578 & 949.7 & 6.637 & 974.3 & 6.45 & 950.6 & 6.521 & 962.5 \\
\hline 6.729 & 928.1 & 6.356 & 916.2 & 6.249 & 910.9 & 6.409 & 923.7 & 6.193 & 921.9 & 6.193 & 924.6 & 6.797 & 973.9 & 6.261 & 918.8 & 5.169 & 923.2 \\
\hline 6.344 & 886.2 & 6.228 & 895.5 & 5.782 & 885.3 & 6.11 & 896.8 & 5.956 & 895.5 & 6.175 & 900.3 & 5.779 & 943.9 & 6.045 & 894.1 & 5.278 & 895.9 \\
\hline 6.163 & 867.3 & 6.113 & 871.2 & 5.42 & 832.9 & 5.941 & 869.5 & 5.796 & 865.5 & 5.708 & 857.6 & 5.725 & 904.7 & 5.861 & 867.3 & 5.21 & 858.5 \\
\hline 6.036 & 836.9 & 5.773 & 834.7 & 5.574 & 810.9 & 5.577 & 829.4 & 5.16 & 832 & 5.642 & 837.3 & 5.879 & 868.6 & 5.811 & 841.7 & 5.373 & 843.5 \\
\hline 5.93 & 814.8 & 5.598 & 812.2 & 5.065 & 783.5 & 5.598 & 819.7 & 5.373 & 814.4 & 5.338 & 811.7 & 5.264 & 845.2 & 5.53 & 819.2 & 5.24 & 817.5 \\
\hline 5.657 & 792.3 & 5.403 & 780 & 5.358 & 761.9 & 5.492 & 795 & 5.104 & 794.1 & 5.219 & 776.9 & 5.299 & 788.8 & 5.373 & 794.1 & 5.178 & 808.7 \\
\hline 5.311 & 754 & 5.278 & 758.4 & 4.837 & 735.9 & 5.086 & 748.7 & 5.32 & 760.6 & 5.166 & 751.4 & 5.486 & 778.7 & 5.302 & 768.1 & 5.086 & 783.1 \\
\hline 5.133 & 731.1 & 5.196 & 734.6 & 4.891 & 713 & 4.799 & 727.1 & 5.246 & 759.3 & 4.994 & 728 & 4.512 & 760.6 & 4.98 & 743 & 4.974 & 760.2 \\
\hline 5.042 & 704.7 & 5.039 & 706.4 & 4.636 & 687.9 & 4.692 & 700.7 & 4.414 & 722.3 & 4.823 & 703.3 & 4.725 & 748.3 & 4.556 & 701.6 & 4.701 & 718.3 \\
\hline 4.894 & 80.9 & 4.719 & 668.5 & 4.734 & 665 & 4.687 & 672.9 & 3.932 & 658.8 & 4.654 & 680.4 & 4.725 & 711.3 & 4.476 & 676.9 & 4.559 & 693.2 \\
\hline 4.74 & 646.5 & 4.488 & 644.3 & 4.192 & 637.7 & 4.37 & 646.5 & 4.13 & 645.2 & 4.429 & 653.1 & 4.9 & 691 & 4.491 & 645.2 & 4.349 & 669.4 \\
\hline 4.53 & 624.4 & 4.37 & 619.2 & 3.932 & 592.3 & 4.16 & 615.6 & 3.902 & 621.8 & 4.388 & 627.1 & 3.905 & 660.1 & 4.299 & 619.2 & 4.18 & 639 \\
\hline 4.388 & 601.5 & 4.104 & 587.9 & 4.056 & 568.5 & 4.009 & 590.1 & 4.266 & 588.8 & 3.964 & 585.2 & 3.864 & 625.3 & 4.136 & 596.2 & 4.006 & 616.5 \\
\hline 4.272 & 575.5 & .935 & 563.6 & 3.636 & 543.4 & 3.849 & 563.6 & 3.39 & 561 & 3.763 & 559.7 & 3.763 & 591.4 & 3.982 & 571.1 & 3.896 & 584.3 \\
\hline 3.973 & 538.5 & 3.793 & 536.8 & 3.609 & 521.3 & 3.822 & 531.9 & 3.58 & 546 & 3.633 & 536.8 & 3.657 & 565.4 & 3.846 & 546.4 & 3.757 & 557 \\
\hline 3.769 & 515.6 & 3.597 & 510.3 & 3.358 & 472.4 & 3.553 & 510.3 & 3.417 & 514.3 & 3.458 & 509.4 & 3.6 & 532.8 & 3.384 & 506.8 & 3.597 & 530.6 \\
\hline 3.731 & 484.3 & 3.411 & 485.6 & 2.976 & 446.9 & 3.37 & 477.7 & 3.216 & 487.4 & 3.254 & 475.5 & 3.482 & 512.1 & 3.651 & 496.2 & 3.491 & 503.7 \\
\hline 3.559 & 461 & 3.056 & 445.5 & 3.186 & 416 & 3.121 & 452.1 & 3.133 & 460.5 & 3.077 & 453.5 & 3.091 & 475.9 & 3.396 & 469.8 & 3.322 & 477.7 \\
\hline 3.21 & 424.4 & 3.012 & 424.4 & 2.745 & 389.1 & 2.961 & 424.4 & 3.077 & 432.3 & 3.032 & 422.2 & 3.012 & 453 & 2.911 & 423.5 & 3.038 & 452.1 \\
\hline 2.878 & 401.5 & 3 & 394.9 & 2.772 & 371.1 & 3.174 & 411.2 & 2.893 & 405 & 2.914 & 398.8 & 3.02 & 424.4 & 3.009 & 415.1 & 2.775 & 428.3 \\
\hline 2.727 & 376.3 & 2.721 & 375.9 & 1.268 & 316.4 & 2.707 & 372.8 & 2.615 & 376.3 & 2.576 & 374.6 & 2.846 & 401 & 2.574 & 371.5 & 2.843 & 402.8 \\
\hline 2.807 & 345.1 & 2.387 & 339.3 & 1.408 & 292.6 & 2.464 & 343.7 & 2.426 & 350.8 & 2.653 & 349 & 2.547 & 375 & 2.437 & 348.1 & 2.769 & 375.5 \\
\hline 2.331 & 327.9 & 2.502 & 312 & & & 2.168 & 313.3 & 2.103 & 312 & 2.458 & 320.4 & 2.541 & 350.8 & 2.452 & 317.7 & 2.381 & 349.9 \\
\hline 2.313 & 292.2 & 2.156 & 292.6 & & & 2.215 & 286 & 2.011 & 284.7 & 2.198 & 297.9 & 2.381 & 323.5 & 2.144 & 293.5 & 2.434 & 324.8 \\
\hline 2.05 & 270.6 & 2.041 & 262.2 & & & 2.091 & 266.6 & 1.834 & 255.6 & 1.982 & 257.4 & 2.124 & 298.8 & 2.159 & 271.9 & 2.079 & 284.2 \\
\hline \multirow[t]{2}{*}{2.115} & 249 & 2.053 & 255.2 & & & 1.813 & 243.3 & & & 2.005 & 250.3 & 1.917 & 253 & 1.905 & 249.4 & 1.766 & 260.4 \\
\hline & & & & & & & & & & & & 1.846 & 245.9 & & & 1.786 & 252.1 \\
\hline
\end{tabular}


Table 4. Rheometer Response for Blank - Diluted

\begin{tabular}{|c|c|c|c|c|c|c|c|c|c|c|c|c|c|c|c|c|c|}
\hline run1 & & run2 & & run3 & & run4 & & run5 & & run6 & & run7 & & run8 & & run9 & \\
\hline [Pa] & {$[1 / s]$} & [Pa] & [1/s] & [Pa] & {$[1 / s]$} & [Pa] & {$[1 / s]$} & [Pa] & [1/s] & [Pa] & [1/s] & [Pa] & {$[1 / s]$} & [Pa] & {$[1 / s]$} & [Pa] & {$[1 / s]$} \\
\hline 2.984 & 351.7 & 2.948 & 385.2 & 2.723 & 375.5 & 2.563 & 369.3 & 2.611 & 365.3 & 2.484 & 367.1 & 1.812 & 325.7 & 1.226 & 312 & 1.264 & 309.4 \\
\hline 3.144 & 376.8 & 291 & 419.1 & .969 & 397.9 & 2.747 & 394 & 2.762 & 391.3 & 2.658 & 393.1 & 2.143 & 338.4 & 1.883 & 320.4 & 1.841 & 322.1 \\
\hline 3.294 & 399.3 & 3.434 & 447.3 & 3.078 & 422.6 & 2.877 & 417.8 & 2.951 & 416.9 & 2.892 & 418.2 & 2.339 & 362.2 & 2.022 & 338 & 1.782 & 330.1 \\
\hline 3.442 & 428.8 & 3.579 & 468 & 3.259 & 449.1 & 3.075 & 438.9 & 3.078 & 445.5 & 3.078 & 446.9 & 2.537 & 386.9 & 2.262 & 361.8 & 2.223 & 370.6 \\
\hline 3.611 & 453 & 3.7 & 498.4 & 3.398 & 474.6 & 3.398 & 481.7 & 3.223 & 477.3 & 3.155 & 478.6 & 2.706 & 411.6 & 2.451 & 388.2 & 2.401 & 396.6 \\
\hline 3.75 & 476.8 & 3.812 & 522.6 & 3.727 & 509.9 & 3.552 & 506.8 & 3.484 & 501.1 & 3.306 & 502.8 & 2.762 & 440.7 & 2.634 & 420 & 2.513 & 421.7 \\
\hline 3.94 & 501.1 & 3.99 & 547.3 & 3.792 & 539 & 3.724 & 531 & 3.658 & 525.7 & 3.481 & 524.4 & 2.904 & 466.7 & 2.729 & 444.2 & 2.791 & 448.6 \\
\hline 4.04 & 527.9 & 4.277 & 582.6 & 3.934 & 563.2 & 3.895 & 557 & 3.709 & 557 & 3.614 & 550.4 & 3.161 & 488.7 & 2.922 & 468 & 2.856 & 480.8 \\
\hline 4.466 & 565.4 & 4.422 & 606.8 & 4.064 & 585.2 & 4.034 & 581.7 & 3.898 & 579.9 & 3.706 & 579.1 & 3.185 & 516 & 3.052 & 494 & 3.052 & 506.3 \\
\hline 4.579 & 590.1 & 4.543 & 633.7 & 4.17 & 608.6 & 4.17 & 614.3 & 4.064 & 605.1 & 3.978 & 602 & 3.374 & 539.4 & 3.238 & 518.2 & 3.164 & 527.5 \\
\hline 4.783 & 619.6 & 4.727 & 660.6 & 4.378 & 645.2 & 4.238 & 641.2 & 4.253 & 631.1 & 4.286 & 643.4 & 3.51 & 568.5 & 3.567 & 558.3 & 3.303 & 552.2 \\
\hline 4.839 & 644.7 & & 683.5 & & 661 & & 665.9 & & 657.1 & & 668.5 & & 596.2 & & 584.3 & 3.395 & 576 \\
\hline 5.002 & 666.3 & 4.898 & 710.8 & 4.839 & 703.3 & 4.552 & 689.2 & 4.674 & 692.8 & 4.591 & 695 & 3.928 & 624.4 & 3.848 & 606.8 & 3.801 & 616.1 \\
\hline 5.141 & 689.7 & 5.132 & 734.6 & 4.907 & 732 & 4.721 & 713.9 & 4.75 & 721.4 & 632 & 725.4 & 4.212 & 663.7 & 3.943 & 632.8 & 4.005 & 643.4 \\
\hline 5.186 & 714.8 & 5.274 & 759.3 & 5.123 & 754.4 & 4.81 & 740.3 & 4.857 & 746.5 & 4.739 & 747.4 & 4.372 & 687 & 4.093 & 665.4 & 4.093 & 670.3 \\
\hline 5.647 & 754.4 & 5.351 & 785.7 & 5.18 & 777.4 & 5.206 & 778.7 & 5.035 & 772.1 & 4.907 & 771.6 & 4.434 & 717 & 4.182 & 692.3 & 4.203 & 693.6 \\
\hline 5.848 & 780.4 & 5.739 & 825.8 & 5.286 & 802.9 & 5.283 & 806.4 & 5.123 & 797.2 & 43 & 799.4 & & 736.4 & 54 & 716.1 & 4.369 & 726.7 \\
\hline 5.99 & 805.6 & 5.819 & 849.6 & 5.455 & 827.6 & 5.555 & 831.6 & 5.298 & 819.7 & 5.177 & 822.3 & 4.739 & 765 & 4.579 & 740.3 & 4.443 & 750.9 \\
\hline 6.041 & 835.5 & 5.999 & 876.1 & 5.647 & 854.9 & & 857.1 & 5.588 & 854.5 & 5.28 & 849.6 & & 792.3 & 4.824 & 766.3 & 4.617 & 775.6 \\
\hline 6.251 & 858.9 & 6.097 & 909.1 & 5.81 & 878.3 & 5.739 & 888.4 & 5.76 & 880.5 & 5.407 & 877.4 & 961 & 824.5 & 5.029 & 805.6 & 4.685 & 804.2 \\
\hline 6.343 & 882.7 & 6.254 & 931.2 & 6.091 & 920.6 & 5.854 & 913.5 & 5.854 & 906.5 & .532 & 902.1 & 5.156 & 847.9 & 5.1 & 832 & 4.898 & 832 \\
\hline 6.674 & 924.6 & 6.411 & 952.8 & 6.242 & 950.6 & 5.996 & 935.6 & 5.866 & 932 & 5.881 & 943.5 & 186 & 870.8 & 5.316 & 853.6 & 5.052 & 859.8 \\
\hline 6.878 & 946.1 & 6.716 & 990.7 & 6.349 & 975.7 & 6.165 & 959.4 & 6.035 & 953.6 & 5.996 & 965.1 & 5.328 & 897.2 & 5.369 & 886.7 & 5.221 & 883.6 \\
\hline 7 & 972.1 & 6.831 & 1014 & 6.514 & 999.9 & 6.266 & 985.8 & 6.346 & 994.2 & 6.118 & 999.5 & & 933.4 & 5.484 & 913.1 & 5.499 & 909.1 \\
\hline 7.248 & 994.6 & 6.961 & 1041 & 6.71 & 1020 & 6.464 & 1010 & 6.517 & 1016 & 6.171 & 1024 & 5.786 & 962.9 & 5.624 & 938.2 & 5.736 & 934.2 \\
\hline 7.254 & 1027 & 7.077 & 1072 & 6.84 & 1053 & 6.73 & 1052 & 6.582 & 1048 & 6.298 & 1048 & 5.958 & 988 & 5.801 & 965.1 & 5.931 & 960.2 \\
\hline 7.367 & 1052 & 7.257 & 1089 & 6.905 & 1079 & 6.932 & 1075 & 6.739 & 1070 & 6.431 & 1074 & 6.085 & 1012 & 5.955 & 986.2 & 6.097 & 984.9 \\
\hline 7.571 & 1076 & 7.411 & 1122 & 7.118 & 1103 & 7.009 & 1107 & 6.846 & 1098 & 6.556 & 1100 & 6.307 & 1038 & 6.047 & 1011 & 6.482 & 1026 \\
\hline 7.71 & 1097 & 7.506 & 1149 & 7.387 & 1143 & 7.142 & 1130 & 7.009 & 1122 & 6.775 & 1124 & 6.378 & 1067 & 6.331 & 1050 & 6.52 & 1055 \\
\hline 8.032 & 1137 & 7.698 & 1172 & 7.565 & 1166 & 7.239 & 1152 & 7.248 & 1159 & 6.819 & 1148 & 6.559 & 1098 & 6.47 & 1078 & 6.642 & 1080 \\
\hline 8.124 & 1161 & 7.997 & 1211 & 7.689 & 1197 & 7.553 & 1189 & 7.384 & 1181 & 7.213 & 1189 & 6.591 & 1126 & 6.568 & 1103 & 6.837 & 1104 \\
\hline
\end{tabular}


Table 4. Rheometer Response for Blank - Diluted - continued

\begin{tabular}{|c|c|c|c|c|c|c|c|c|c|c|c|c|c|c|c|c|c|}
\hline run1 & & run2 & & run3 & & run4 & & run5 & & run6 & & run7 & & run8 & & run9 & \\
\hline [Pa] & {$[1 / \mathrm{s}]$} & {$[\mathrm{Pa}]$} & {$[1 / s]$} & {$[\mathrm{Pa}]$} & {$[1 / s]$} & [Pa] & {$[1 / s]$} & {$[\mathrm{Pa}]$} & {$[1 / \mathrm{s}]$} & {$[\mathrm{Pa}]$} & {$[1 / \mathrm{s}]$} & {$[\mathrm{Pa}]$} & {$[1 / \mathrm{s}]$} & {$[\mathrm{Pa}]$} & {$[1 / \mathrm{s}]$} & [Pa] & {$[1 / \mathrm{s}]$} \\
\hline 8.263 & 1193 & 8.104 & 1233 & 7.861 & 1218 & 7.701 & 1215 & 7.479 & 1208 & 7.349 & 1216 & 6.822 & 1148 & 6.665 & 1126 & 6.89 & 1133 \\
\hline 8.509 & 1216 & 8.198 & 1263 & 7.97 & 1244 & 7.834 & 1240 & 7.677 & 1237 & 7.452 & 1240 & 6.899 & 1175 & 6.79 & 1149 & 6.751 & 1155 \\
\hline 8.553 & 1240 & 8.391 & 1282 & 8.104 & 1275 & 7.911 & 1270 & 7.796 & 1263 & 7.639 & 1266 & 7.065 & 1201 & 6.861 & 1177 & 6.748 & 1180 \\
\hline 8.944 & 1277 & 8.465 & 1312 & 8.169 & 1297 & 8.148 & 1293 & 8 & 1286 & 7.825 & 1294 & 7.18 & 1228 & 7.121 & 1201 & 6.917 & 1207 \\
\hline 9.053 & 1299 & 8.642 & 1338 & 8.334 & 1321 & 8.331 & 1318 & 8.104 & 1310 & 7.917 & 1319 & 7.284 & 1256 & 7.219 & 1228 & 7.204 & 1242 \\
\hline 9.08 & 1329 & 8.758 & 1360 & 8.473 & 1345 & 8.355 & 1348 & 8.186 & 1337 & 7.973 & 1342 & 7.447 & 1279 & 7.55 & 1270 & 7.337 & 1268 \\
\hline 9.181 & 1356 & 9.056 & 1400 & 8.562 & 1373 & 8.438 & 1374 & 8.385 & 1363 & 8.127 & 1366 & 7.55 & 1305 & 7.757 & 1294 & 7.31 & 1299 \\
\hline 9.435 & 1381 & 9.083 & 1408 & 8.725 & 1400 & 8.66 & 1398 & 8.462 & 1387 & 8.302 & 1393 & 7.876 & 1346 & 7.879 & 1319 & 7.414 & 1324 \\
\hline 9.545 & 1407 & 9.175 & 1434 & 8.882 & 1427 & 8.74 & 1425 & 8.616 & 1419 & 8.405 & 1421 & 8.047 & 1371 & 7.893 & 1349 & 7.621 & 1349 \\
\hline 9.66 & 1431 & 9.574 & 1478 & 9.252 & 1468 & 8.95 & 1450 & 8.778 & 1445 & 8.802 & 1463 & 8.186 & 1395 & 7.902 & 1374 & 7.757 & 1374 \\
\hline 9.873 & 1455 & 9.713 & 1510 & 9.335 & 1492 & 9.127 & 1482 & 9.169 & 1487 & 8.932 & 1487 & 8.275 & 1423 & 8.041 & 1401 & 7.959 & 1399 \\
\hline 10.26 & 1493 & 9.867 & 1525 & 9.432 & 1523 & 9.278 & 1509 & 9.252 & 1513 & 9.056 & 1512 & 8.34 & 1455 & 8.213 & 1425 & 7.973 & 1426 \\
\hline 10.39 & 1519 & 10.04 & 1558 & 9.583 & 1543 & 9.441 & 1534 & 9.4 & 1538 & 9.157 & 1540 & 8.533 & 1475 & 8.34 & 1450 & 7.864 & 1633 \\
\hline 8.512 & 1512 & 8.302 & 1482 & 8.37 & 1519 & 8.118 & 1533 & 7.571 & 1487 & 7.586 & 1510 & 7.423 & 1510 & 7.654 & 1592 & 7.701 & 1563 \\
\hline 8.482 & 1486 & 8.308 & 1473 & 8.169 & 1495 & 7.825 & 1493 & 7.627 & 1480 & 7.411 & 1483 & 7.497 & 1495 & 7.544 & 1567 & 7.639 & 1549 \\
\hline 8.281 & 1462 & 7.944 & 1434 & 7.908 & 1456 & 7.612 & 1467 & 7.094 & 1436 & 7.233 & 1458 & 7.606 & 1481 & 7.373 & 1543 & 7.666 & 1544 \\
\hline 8.065 & 1437 & 7.811 & 1404 & 7.725 & 1433 & 7.494 & 1443 & 7.118 & 1421 & 7.103 & 1434 & 6.582 & 1440 & 7.307 & 1511 & 7.103 & 1522 \\
\hline 8.012 & 1404 & 7.698 & 1381 & 7.689 & 1403 & 7.287 & 1419 & 7.204 & 1384 & 7.023 & 1401 & 6.701 & 1425 & 7.213 & 1490 & 7.171 & 1510 \\
\hline 7.808 & 1382 & 7.571 & 1358 & 7.666 & 1363 & 7.251 & 1387 & 6.387 & 1354 & 6.84 & 1376 & 6.671 & 1387 & 7.115 & 1467 & 6.991 & 1462 \\
\hline 7.781 & 1356 & 7.435 & 1334 & 6.736 & 1332 & 6.961 & 1364 & 6.565 & 1340 & 6.745 & 1352 & 6.887 & 1361 & 7.011 & 1444 & 6.852 & 1436 \\
\hline 7.553 & 1331 & 7.331 & 1308 & 6.926 & 1316 & 6.795 & 1336 & 6.411 & 1308 & 6.594 & 1329 & 6.156 & 1334 & 6.872 & 1413 & 6.751 & 1414 \\
\hline 7.482 & 1289 & 7.142 & 1283 & 6.991 & 1257 & 6.819 & 1302 & 6.112 & 1263 & 6.304 & 1291 & 6.301 & 1317 & 6.647 & 1388 & 6.547 & 1386 \\
\hline 6.674 & 1264 & 6.887 & 1256 & 7.059 & 1255 & 6.597 & 1277 & 6.035 & 1249 & 6.091 & 1264 & 6.124 & 1295 & 6.434 & 1359 & 6.488 & 1355 \\
\hline 6.79 & 1250 & 6.612 & 1214 & 6.233 & 1233 & 6.354 & 1259 & 5.925 & 1227 & 5.985 & 1238 & 6.207 & 1285 & 6.31 & 1336 & 6.322 & 1330 \\
\hline 6.787 & 1215 & 6.417 & 1189 & 6.062 & 1151 & 6.147 & 1229 & 5.979 & 1216 & 5.807 & 1214 & 5.899 & 1244 & 6.076 & 1297 & 6.138 & 1304 \\
\hline 6.905 & 1192 & 6.292 & 1157 & 6.094 & 1135 & 5.996 & 1199 & 6.053 & 1175 & 5.76 & 1181 & 5.84 & 1218 & 5.925 & 1266 & 6.109 & 1279 \\
\hline 6.186 & 1164 & 6.204 & 1133 & 6.133 & 1128 & 5.881 & 1173 & 6.047 & 1174 & 5.6 & 1155 & 5.683 & 1192 & 5.825 & 1243 & 5.961 & 1254 \\
\hline 6.31 & 1147 & 6.094 & 1108 & 5.857 & 1089 & 5.706 & 1144 & 5.292 & 1118 & 5.479 & 1131 & 5.606 & 1161 & 5.745 & 1213 & 5.606 & 1215 \\
\hline 5.745 & 1106 & 5.931 & 1085 & 5.931 & 1073 & 5.662 & 1111 & 5.177 & 1086 & 5.351 & 1108 & 5.443 & 1135 & 5.484 & 1186 & 5.413 & 1190 \\
\hline 5.686 & 1092 & 5.777 & 1060 & 5.144 & 1056 & 5.428 & 1087 & 5.135 & 1054 & 5.156 & 1083 & 5.328 & 1112 & 5.313 & 1160 & 5.336 & 1162 \\
\hline 5.644 & 1047 & 5.467 & 1022 & 4.816 & 1030 & 5.316 & 1063 & 5.046 & 1030 & 5.073 & 1057 & 5.174 & 1087 & 5.242 & 1138 & 5.162 & 1137 \\
\hline
\end{tabular}


Table 4. Rheometer Response for Blank - Diluted - continued

\begin{tabular}{|c|c|c|c|c|c|c|c|c|c|c|c|c|c|c|c|c|c|}
\hline run1 & & run2 & & run3 & & run4 & & run5 & & run6 & & run7 & & run8 & & run9 & \\
\hline$[\mathrm{Pa}]$ & {$[1 / \mathrm{s}]$} & {$[\mathrm{Pa}]$} & {$[1 / s]$} & {$[\mathrm{Pa}]$} & {$[1 / \mathrm{s}]$} & {$[\mathrm{Pa}]$} & {$[1 / \mathrm{s}]$} & {$[\mathrm{Pa}]$} & {$[1 / \mathrm{s}]$} & {$[\mathrm{Pa}]$} & {$[1 / \mathrm{s}]$} & {$[\mathrm{Pa}]$} & {$[1 / \mathrm{s}]$} & {$[\mathrm{Pa}]$} & {$[1 / \mathrm{s}]$} & {$[\mathrm{Pa}]$} & {$[1 / \mathrm{s}]$} \\
\hline 5.896 & 1019 & 5.298 & 998.6 & 4.863 & 987.6 & 5.289 & 1040 & 4.94 & 1004 & 4.682 & 1015 & 5.02 & 1064 & 5.114 & 1114 & 5.058 & 1107 \\
\hline 5.896 & 1018 & 5.194 & 967.7 & 5.014 & 967.3 & 5.094 & 1016 & 4.789 & 981 & 4.605 & 992.9 & 4.869 & 1039 & 4.946 & 1091 & 4.964 & 1086 \\
\hline 4.904 & 978.8 & 5.094 & 942.6 & 4.786 & 944.8 & 4.768 & 973.5 & 4.57 & 956.7 & 4.419 & 965.1 & 4.591 & 997.3 & 4.644 & 1048 & 4.75 & 1051 \\
\hline 5.005 & 960.7 & 4.978 & 919.7 & 4.872 & 932.9 & 4.585 & 951 & 4.366 & 918.4 & 4.351 & 942.2 & 4.644 & 990.7 & 4.52 & 1025 & 4.466 & 1023 \\
\hline 4.86 & 931.2 & 4.863 & 894.1 & 4.724 & 912.2 & 4.463 & 924.6 & 4.244 & 894.1 & 4.129 & 914.4 & 4.318 & 942.6 & 4.487 & 992.4 & 4.558 & 1012 \\
\hline 4.745 & 894.6 & 4.721 & 869 & 4.585 & 887.5 & 4.324 & 899.4 & 4.162 & 864.6 & 4.102 & 880.9 & 4.247 & 917.5 & 4.345 & 966.9 & 4.431 & 980.5 \\
\hline 4.668 & 858.9 & 4.46 & 845.2 & 4.437 & 863.3 & 4.262 & 867.7 & 4.04 & 841.3 & 3.969 & 858.5 & 4.028 & 901.6 & 4.173 & 944.4 & 4.283 & 956.3 \\
\hline 4.57 & 837.7 & 167 & 803.8 & 4.384 & 831.1 & 4.108 & 843.9 & 3.744 & 809.1 & 3.824 & 834.7 & 3.889 & 862.4 & 4.093 & 918.4 & 3.99 & 916.2 \\
\hline 4.428 & 812.2 & 4.055 & 776 & 4.247 & 806 & 4.025 & 820.6 & 3.691 & 777.8 & 3.667 & 812.6 & 3.756 & 835.1 & 3.963 & 891.5 & 3.86 & 888.4 \\
\hline 4.295 & 791 & 3.904 & 751.8 & 4.014 & 779.6 & 3.845 & 795.9 & 3.596 & 756.7 & 3.363 & 772.1 & 3.682 & 807.8 & 3.762 & 867.7 & 3.685 & 865.1 \\
\hline 3.969 & 749.2 & 3.815 & 721 & 3.836 & 753.1 & 3.759 & 770.3 & 3.36 & 732.4 & 3.259 & 744.3 & 3.368 & 786.2 & 3.721 & 842.6 & 3.629 & 833.3 \\
\hline 3.88 & 721.8 & 3.611 & 697.6 & 3.771 & 724.9 & 3.608 & 744.8 & 3.265 & 708.2 & 3.037 & 718.3 & 3.306 & 754.4 & 3.265 & 801.2 & 3.49 & 810 \\
\hline 3.806 & 698 & 3.537 & 673.4 & 3.555 & 703.8 & 3.398 & 717.4 & 3.152 & 679.5 & 2.951 & 690.1 & 3.217 & 727.1 & 3.191 & 775.6 & 3.38 & 784 \\
\hline 3.62 & 671.2 & 3.395 & 650 & 3.466 & 677.3 & 3.141 & 679.1 & 2.981 & 654.9 & 2.788 & 668.1 & 3.019 & 700.7 & 3.067 & 748.3 & 3.286 & 760.2 \\
\hline 3.478 & 644.7 & 3.185 & 624 & 3.294 & 655.7 & 3.064 & 649.1 & 2.75 & 616.5 & 2.652 & 641.6 & 2.851 & 682.2 & 2.969 & 719.2 & 3.114 & 735.1 \\
\hline 3.315 & 622.2 & 3.064 & 598.9 & 3.191 & 625.3 & 2.913 & 624.9 & 2.561 & 591.8 & 2.596 & 610.3 & 2.694 & 650.9 & 2.815 & 698 & 3.001 & 704.7 \\
\hline 3.191 & 598 & 2.785 & 560.1 & 2.812 & 590.5 & 2.738 & 601.5 & 2.543 & 561.4 & 2.427 & 586.1 & 2.623 & 627.5 & 2.729 & 670.3 & 2.874 & 680.9 \\
\hline 2.919 & 559.2 & 2.608 & 534.5 & 2.708 & 561.4 & 2.697 & 575.1 & 2.377 & 539 & 2.333 & 560.1 & 2.525 & 602.9 & 2.563 & 647.4 & 2.569 & 640.3 \\
\hline 2.75 & 535.4 & 2.43 & 509 & 2.572 & 531 & 2.478 & 551.7 & 2.294 & 517.4 & 2.161 & 536.8 & 2.167 & 559.2 & 2.46 & 621.8 & 2.43 & 615.6 \\
\hline 2.691 & 506.3 & 2.389 & 476.4 & 2.463 & 509.9 & 2.185 & 513.4 & 1.972 & 477.7 & 2.117 & 509 & 2.034 & 537.2 & 2.306 & 598.9 & 2.528 & 605.9 \\
\hline 2.555 & 483.9 & 2.262 & 451.3 & 2.448 & 484.8 & 2.04 & 488.7 & 1.776 & 451.3 & 1.895 & 484.3 & 1.912 & 511.6 & 2.001 & 557.5 & 2.087 & 563.6 \\
\hline 2.407 & 460.5 & 2.072 & 428.8 & 2.253 & 457.9 & 1.939 & 463.2 & 1.685 & 426.6 & 1.803 & 459.6 & 1.841 & 481.2 & 1.886 & 533.2 & 2.01 & 535.4 \\
\hline 2.241 & 432.3 & 1.989 & 406.8 & 1.918 & 419.1 & .838 & 431 & 1.83 & 416.4 & 1.646 & 434.1 & 1.705 & 457.9 & 1.815 & 510.8 & 1.812 & 509.9 \\
\hline 2.128 & 402.8 & 1.895 & 383 & 2.025 & 408.5 & 1.693 & 410.7 & 1.32 & 371.5 & 1.273 & 390 & 1.619 & 428.3 & 1.764 & 478.6 & 1.726 & 482.5 \\
\hline 1.948 & 379.9 & 1.454 & 340.6 & 1.575 & 369.7 & 1.572 & 378.1 & 1.264 & 344.6 & 1.134 & 365.8 & 1.403 & 402.8 & 1.581 & 453.9 & 1.613 & 456.1 \\
\hline 1.619 & 342.9 & 1.335 & 317.7 & 1.522 & 338.4 & 1.389 & 355.2 & 1.063 & 319.1 & 1.042 & 341.1 & 1.285 & 376.8 & 1.427 & 427.5 & 1.43 & 432.8 \\
\hline 1.504 & 311.1 & 1.211 & 286 & 1.241 & 319.1 & 1.276 & 327 & 0.891 & 291.7 & 0.992 & 306.7 & 0.942 & 340.6 & 1.326 & 403.7 & 1.297 & 406.8 \\
\hline 1.377 & 288.2 & 1.119 & 268.8 & 1.143 & 290 & 1.143 & 302.3 & 0.767 & 267.1 & 0.72 & 289.5 & 0.829 & 314.6 & 1.211 & 378.5 & 1.264 & 382.5 \\
\hline 1.264 & 267.1 & 1.175 & 258.2 & 1.042 & 259.6 & 1.028 & 274.5 & 0.835 & 258.2 & 0.646 & 259.1 & 0.666 & 293.1 & 1.036 & 351.7 & 0.862 & 338.9 \\
\hline 1.173 & 245.5 & 0 & 177.9 & 0.897 & 235.8 & 0.773 & 247.7 & 0 & 187.4 & 0.518 & 236.2 & 0.542 & 267.1 & 0.69 & 312.9 & & \\
\hline & & & & & & & & & & & & & & & & & \\
\hline & & & & & & & & & & & & & & & & & \\
\hline
\end{tabular}


Table 5. $25^{\circ} \mathrm{C}$ Diluted

\begin{tabular}{|c|c|c|c|c|c|c|c|c|c|c|c|c|c|c|c|c|c|}
\hline run1 & & run2 & & run3 & & run4 & & run5 & & run6 & & run7 & & run8 & & run9 & \\
\hline [Pa] & {$[1 / s]$} & {$[\mathrm{Pa}]$} & {$[1 / \mathrm{s}]$} & {$[\mathrm{Pa}]$} & {$[1 / \mathrm{s}]$} & {$[\mathrm{Pa}]$} & {$[1 / \mathrm{s}]$} & [Pa] & {$[1 / \mathrm{s}]$} & {$[\mathrm{Pa}]$} & {$[1 / \mathrm{s}]$} & [Pa] & {$[1 / \mathrm{s}]$} & {$[\mathrm{Pa}]$} & {$[1 / \mathrm{s}]$} & [Pa] & {$[1 / \mathrm{s}]$} \\
\hline 4.883 & 302.7 & 5.925 & 367.5 & 5.078 & 309.4 & 4.303 & 304.5 & 4.303 & 330.5 & 4.339 & 308 & 4.682 & 328.3 & 5.084 & 305.4 & 4.211 & 305 \\
\hline 5.315 & 313.3 & 463 & 395.3 & 5.309 & 321.3 & 5.247 & 312 & 5.454 & 323.5 & 5.51 & 318.2 & 5.653 & 327.9 & 5.54 & 322.6 & 5.247 & 320.8 \\
\hline 6.088 & 348.1 & 6.833 & 428.8 & 5.99 & 340.2 & 5.434 & 334 & 5.676 & 339.8 & 5.661 & 337.1 & 5.59 & 341.5 & 5.478 & 344.6 & 5.457 & 344.6 \\
\hline 6.582 & 371.1 & 6.972 & 448.2 & 6.15 & 361.8 & 5.638 & 361.4 & 5.892 & 361.4 & 5.883 & 366.2 & 6.135 & 367.5 & 6.073 & 371.5 & 5.567 & 368.4 \\
\hline 6.694 & 396.2 & 7.405 & 481.2 & 6.238 & 386.5 & 6.2 & 397.9 & 6.561 & 387.4 & 6.318 & 391.3 & 6.594 & 397.5 & 6.555 & 395.3 & 6.014 & 397.1 \\
\hline 6.934 & 423.5 & 7.511 & 507.2 & 6.65 & 412.5 & 6.549 & 424.4 & 6.819 & 410.7 & 6.821 & 413.4 & 6.916 & 420.4 & 6.653 & 422.6 & 6.378 & 421.7 \\
\hline 7.191 & 449.9 & 7.7 & 530.6 & 7.15 & 438.9 & 7.109 & 447.3 & 6.91 & 435.8 & 6.777 & 438.5 & 7.017 & 447.3 & 6.712 & 446 & 6.703 & 450.8 \\
\hline 7.49 & 482.1 & 7.955 & 555.7 & 7.378 & 479 & 7.345 & 473.3 & 7.049 & 461.4 & 7.514 & 481.2 & 7.126 & 472.9 & 7.446 & 485.2 & 6.978 & 477.3 \\
\hline 7.745 & 506.3 & 8.106 & 578.6 & 7.763 & 501.9 & 7.476 & 504.6 & 8.05 & 503.3 & 7.754 & 499.7 & 7.464 & 494.4 & 7.751 & 511.6 & 7.342 & 504.6 \\
\hline 8.079 & 530.1 & 8.535 & 617.8 & 8.008 & 529.3 & 7.834 & 529.3 & 8.061 & 524.9 & 7.866 & 525.3 & 7.733 & 520.4 & 8.008 & 536.3 & 7.538 & 531.5 \\
\hline 8.248 & 555.3 & 8.683 & 644.7 & 8.159 & 554.8 & 8.047 & 553.5 & 8.408 & 557 & 8.059 & 549.1 & 8.479 & 561.9 & 8.28 & 564.5 & 7.842 & 559.2 \\
\hline 8.402 & 580.4 & 9.248 & 672.9 & 8.476 & 585.7 & 8.201 & 579.1 & 8.615 & 580.4 & 8.254 & 586.1 & 8.65 & 585.7 & 8.529 & 591.8 & 8.05 & 584.8 \\
\hline 8.677 & 606.8 & 9.183 & 699.4 & 8.81 & 609.9 & 8.307 & 604.6 & 8.766 & 603.7 & 8.692 & 605.1 & 8.819 & 611.7 & 8.757 & 615.2 & 8.215 & 608.6 \\
\hline 9.296 & 644.7 & 9.506 & 725.8 & 9.097 & 633.3 & 8.426 & 630.6 & 8.852 & 628.9 & 8.884 & 639.4 & 8.908 & 639 & 8.866 & 639 & 8.159 & 635.9 \\
\hline 9.355 & 668.5 & 9.828 & 750.5 & 9.408 & 657.5 & 8.653 & 652.7 & 9.112 & 651.3 & 9.233 & 663.2 & 9.278 & 664.5 & 9.011 & 661 & 8.801 & 676.4 \\
\hline 9.858 & 700.7 & 9.769 & 773.4 & 9.497 & 681.3 & 9.299 & 698 & 9.183 & 677.3 & 9.269 & 690.5 & 9.5 & 696.3 & 9.574 & 698 & 8.884 & 686.1 \\
\hline 9.781 & 724.5 & 10.33 & 816.6 & 9.805 & 724 & 9.66 & 721 & 9.748 & 705.5 & 9.452 & 718.3 & 9.562 & 717.9 & 9.52 & 723.2 & 9.301 & 711.3 \\
\hline 10.05 & 749.6 & 10.07 & 825 & 10.14 & 749.2 & 9.568 & 750.9 & 10.13 & 745.6 & 9.923 & 741.7 & 9.722 & 742.1 & 10.01 & 750.9 & 9.745 & 753.1 \\
\hline 10.43 & 771.2 & 10.63 & 848.8 & 10.32 & 775.6 & 9.911 & 774.3 & 10.19 & 775.6 & 10.04 & 764.6 & 10.25 & 765.9 & 9.967 & 782.2 & 10.2 & 781.3 \\
\hline 10.32 & 798.5 & 10.83 & 891.5 & 10.67 & 804.7 & 10.02 & 799.8 & 10.48 & 798.1 & 9.982 & 789.3 & 10.48 & 807.3 & 10.21 & 809.1 & 10.25 & 806 \\
\hline 10.91 & 823.6 & 11.14 & 918.4 & 10.6 & 828 & 10.35 & 829.8 & 10.33 & 821 & 10.69 & 830.7 & 10.72 & 829.8 & 10.53 & 828.9 & 10.63 & 831.6 \\
\hline 11 & 853.2 & 11.55 & 943.5 & 10.88 & 851.4 & 10.6 & 855.4 & 10.82 & 847.4 & 10.66 & 855.4 & 10.79 & 859.3 & 10.73 & 855.8 & 10.65 & 862.9 \\
\hline 11.18 & 881.4 & & 67.7 & 10.98 & 876.1 & 10.82 & 888.4 & 11. & 884 & 10.76 & 885.8 & 11.18 & 883.1 & 10.76 & 891.1 & 10.78 & 885.8 \\
\hline 11.38 & 906.9 & & 994.6 & 11.49 & 914.9 & 10.7 & 911.8 & 11.41 & 908.2 & 11.17 & 908.7 & 11.37 & 910.5 & 21 & 916.2 & 11.13 & 912.7 \\
\hline 11.96 & 949.2 & 12.17 & 1025 & 11.54 & 939.1 & 11.15 & 936.5 & 11.44 & 936.5 & 11.15 & 929.8 & 11.46 & 935.6 & 11.17 & 939.1 & 11.13 & 936.5 \\
\hline 12 & 972.6 & 12.31 & 1055 & 11.91 & 962 & 11.3 & 962.9 & 11.47 & 959.8 & 11.53 & 974.3 & 11.74 & 958.9 & 11.53 & 966.9 & 11.34 & 963.8 \\
\hline 12.19 & 998.1 & 12.32 & 1081 & 11.96 & 992.9 & 11.33 & 985.4 & 11.78 & 985.8 & 11.89 & 996.8 & 11.75 & 991.1 & 11.61 & 994.6 & 11.58 & 993.3 \\
\hline 12.45 & 1028 & 12.59 & 1107 & 12.2 & 1019 & 11.75 & 1025 & 12.2 & 1010 & 11.92 & 1018 & 12.16 & 1012 & 11.76 & 1022 & 12.04 & 1022 \\
\hline 12.44 & 1051 & 12.84 & 1130 & 12.41 & 1044 & 11.67 & 1035 & 12.04 & 1032 & 12.24 & 1051 & 12.51 & 1053 & 11.97 & 1046 & 11.96 & 1047 \\
\hline 12.71 & 1076 & 12.95 & 1154 & 12.51 & 1066 & 12.13 & 1075 & 12.68 & 1076 & 12.44 & 1076 & 12.36 & 1074 & 12.15 & 1072 & 12.13 & 1070 \\
\hline 12.97 & 1099 & 12.81 & 1182 & 12.8 & 1093 & 12.21 & 1101 & 12.81 & 1103 & 12.5 & 1097 & 12.51 & 1097 & 12.61 & 1111 & 12.3 & 1096 \\
\hline
\end{tabular}


Table 5. $25^{\circ} \mathrm{C}$ Diluted - continued

\begin{tabular}{|c|c|c|c|c|c|c|c|c|c|c|c|c|c|c|c|c|c|}
\hline run1 & & run2 & & run3 & & run4 & & run5 & & run6 & & run7 & & run8 & & run9 & \\
\hline [Pa] & {$[1 / \mathrm{s}]$} & {$[\mathrm{Pa}]$} & {$[1 / \mathrm{s}]$} & {$[\mathrm{Pa}]$} & {$[1 / s]$} & [Pa] & {$[1 / \mathrm{s}]$} & {$[\mathrm{Pa}]$} & {$[1 / \mathrm{s}]$} & {$[\mathrm{Pa}]$} & {$[1 / \mathrm{s}]$} & {$[\mathrm{Pa}]$} & {$[1 / \mathrm{s}]$} & {$[\mathrm{Pa}]$} & {$[1 / \mathrm{s}]$} & {$[\mathrm{Pa}]$} & {$[1 / \mathrm{s}]$} \\
\hline 13.3 & 1137 & 13.05 & 1205 & 12.89 & 1120 & 12.5 & 1133 & 13 & 1126 & 12.77 & 1123 & 12.83 & 1122 & 12.84 & 1136 & 12.72 & 1134 \\
\hline 13.35 & 1165 & 13.62 & 1247 & 13.22 & 1159 & 12.74 & 1159 & 13.23 & 1152 & 13.08 & 1162 & 13.07 & 1148 & 13.02 & 1167 & 12.97 & 1166 \\
\hline 13.62 & 1188 & 14.12 & 1272 & 13.37 & 1182 & 12.7 & 1185 & 13.19 & 1180 & 13.26 & 1185 & 13.46 & 1187 & 13.29 & 1188 & 13.13 & 1185 \\
\hline 13.89 & 1213 & 14.45 & 1298 & 13.54 & 1211 & 12.89 & 1209 & 13.45 & 1205 & 13.46 & 1211 & 13.57 & 1215 & 13.37 & 1212 & 13.23 & 1217 \\
\hline 14.02 & 1245 & 14.52 & 1329 & 13.57 & 1240 & 13.07 & 1234 & 13.56 & 1229 & 13.57 & 1236 & 13.77 & 1241 & 13.49 & 1244 & 13.46 & 1245 \\
\hline 14.23 & 1267 & 14.76 & 1351 & 13.89 & 1265 & 13.44 & 1259 & 13.79 & 1253 & 13.7 & 1267 & 13.86 & 1270 & 13.72 & 1267 & 13.78 & 1263 \\
\hline 14.45 & 1291 & 14.92 & 1375 & 14.13 & 1293 & 13.6 & 1300 & 14.1 & 1294 & 13.86 & 1295 & 14.17 & 1296 & 13.82 & 1294 & 13.97 & 1300 \\
\hline 14.8 & 1330 & 15.21 & 1398 & 14.12 & 1318 & 13.96 & 1325 & 14.24 & 1317 & 14.06 & 1321 & 14.24 & 1325 & 13.98 & 1318 & 13.9 & 1325 \\
\hline 15 & 1352 & 15.48 & 1436 & 14.4 & 1341 & 13.96 & 1352 & 14.41 & 1347 & 14.21 & 1344 & 14.38 & 1352 & 14.2 & 1343 & 14.23 & 1351 \\
\hline 15.12 & 1383 & 15.53 & 1447 & 14.56 & 1366 & 14.15 & 1376 & 14.53 & 1370 & 14.47 & 1368 & 14.56 & 1374 & 14.54 & 1382 & 14.34 & 1377 \\
\hline 15.14 & 1408 & 15.89 & 1490 & 14.79 & 1392 & 14.38 & 1401 & 14.72 & 1394 & 14.59 & 1394 & 14.74 & 1400 & 14.69 & 1407 & 14.69 & 1402 \\
\hline 15.51 & 1433 & 16.15 & 1519 & 15.2 & 1431 & 14.74 & 1430 & 14.81 & 1422 & 14.92 & 1417 & 14.97 & 1426 & 14.89 & 1439 & 14.63 & 1425 \\
\hline 15.54 & 1458 & 16.29 & 1544 & 15.28 & 1456 & 14.83 & 1453 & 15.4 & 1457 & 14.93 & 1445 & 15.3 & 1464 & 14.96 & 1464 & 14.84 & 1450 \\
\hline 15.79 & 1483 & 16.27 & 1567 & 15.56 & 1486 & 14.8 & 1487 & 15.38 & 1479 & 15.34 & 1481 & 15.43 & 1489 & 15.18 & 1491 & 15.31 & 1491 \\
\hline 15.98 & 1508 & 16.75 & 1603 & 15.78 & 1509 & 15.08 & 1511 & 15.67 & 1512 & 15.47 & 1515 & 15.72 & 1513 & 15.41 & 1515 & 15.64 & 1519 \\
\hline 14.94 & 1501 & 14.47 & 1466 & 15.15 & 1574 & 15.27 & 1577 & 15.12 & 1551 & 14.88 & 1543 & 15.06 & 1577 & 14.82 & 1547 & 14.56 & 1548 \\
\hline 14.62 & 1456 & 14.32 & 1440 & 14.88 & 1550 & 15.15 & 1549 & 14.59 & 1511 & 14.74 & 1518 & 14.71 & 1540 & 14.56 & 1523 & 14.38 & 1525 \\
\hline 14.71 & 1448 & 14.05 & 1405 & 14.82 & 1518 & 14.94 & 1527 & 14.29 & 1484 & 14.38 & 1496 & 14.5 & 1516 & 14.32 & 1495 & 14.32 & 1504 \\
\hline 14.02 & 1408 & 13.76 & 1386 & 14.65 & 1493 & 14.71 & 1503 & 14.11 & 1460 & 14.2 & 1473 & 14.38 & 1493 & 14.17 & 1473 & 13.91 & 1464 \\
\hline 13.85 & 1385 & 13.55 & 1359 & 14.35 & 1467 & 14.38 & 1477 & 14.02 & 1434 & 13.7 & 1431 & 14.11 & 1460 & 13.61 & 1430 & 13.76 & 1433 \\
\hline 13.76 & 1354 & 13.26 & 1328 & 14.2 & 1441 & 14.38 & 1453 & 13.82 & 1404 & 13.64 & 1408 & 13.97 & 1438 & 13.88 & 1422 & 13.43 & 1411 \\
\hline 13.61 & 1331 & 13.26 & 1306 & 14.08 & 1419 & 13.91 & 1412 & 13.7 & 1377 & 13.49 & 1381 & 13.7 & 1411 & 13.31 & 1378 & 13.26 & 1388 \\
\hline 13.2 & 1296 & 12.96 & 1280 & 13.64 & 1380 & 13.7 & 1387 & 13.52 & 1352 & 3.23 & 1356 & 13.55 & 1387 & 13.05 & 1354 & 12.93 & 1351 \\
\hline 13.02 & 1273 & 12.57 & 1241 & 13.37 & 1356 & 13.43 & 1359 & 13.17 & 1329 & 12.99 & 1329 & 13.34 & 1364 & 12.87 & 1323 & 12.66 & 1328 \\
\hline 12.72 & 1247 & 12.47 & 1218 & 13.2 & 1332 & 13.28 & 1336 & 12.9 & 1304 & 12.84 & 1300 & 12.99 & 1325 & 12.75 & 1299 & 12.43 & 1304 \\
\hline 12.52 & 1216 & 12.29 & 1188 & 13.02 & 1299 & 13.02 & 1306 & 12.87 & 1276 & 12.66 & 1274 & 12.75 & 1300 & 12.59 & 1275 & 12.36 & 1278 \\
\hline 12.2 & 1192 & 12.04 & 1163 & 12.87 & 1274 & 12.72 & 1282 & 12.63 & 1251 & 12.57 & 1248 & 12.67 & 1268 & 12.48 & 1250 & 12.11 & 1251 \\
\hline 12.04 & 1165 & 11.82 & 1139 & 12.64 & 1252 & 12.66 & 1256 & 12.43 & 1226 & 12.28 & 1222 & 12.24 & 1247 & 12.28 & 1226 & 12.03 & 1225 \\
\hline 11.88 & 1141 & 11.62 & 1116 & 12.23 & 1214 & 12.48 & 1223 & 12.03 & 1184 & 12.04 & 1196 & 12.11 & 1221 & 11.82 & 1184 & 11.64 & 1192 \\
\hline 11.85 & 1110 & 11.46 & 1092 & 12.03 & 1189 & 12.31 & 1200 & 11.71 & 1162 & 11.84 & 1171 & 12.07 & 1190 & 11.58 & 1161 & 11.5 & 1169 \\
\hline 11.63 & 1084 & 11.4 & 1063 & 11.7 & 1164 & 12.12 & 1174 & 11.6 & 1130 & 11.73 & 1144 & 11.76 & 1169 & 11.65 & 1132 & 11.41 & 1145 \\
\hline
\end{tabular}


Table 5. $25^{\circ} \mathrm{C}$ Diluted - continued

\begin{tabular}{|c|c|c|c|c|c|c|c|c|c|c|c|c|c|c|c|c|c|}
\hline run1 & & run2 & & run3 & & run4 & & run5 & & run6 & & run7 & & run8 & & run9 & \\
\hline$[\mathrm{Pa}]$ & {$[1 / \mathrm{s}]$} & {$[\mathrm{Pa}]$} & {$[1 / \mathrm{s}]$} & {$[\mathrm{Pa}]$} & {$[1 / \mathrm{s}]$} & {$[\mathrm{Pa}]$} & {$[1 / \mathrm{s}]$} & {$[\mathrm{Pa}]$} & {$[1 / \mathrm{s}]$} & {$[\mathrm{Pa}]$} & {$[1 / \mathrm{s}]$} & {$[\mathrm{Pa}]$} & {$[1 / s]$} & {$[\mathrm{Pa}]$} & {$[1 / \mathrm{s}]$} & {$[\mathrm{Pa}]$} & {$[1 / \mathrm{s}]$} \\
\hline 11.35 & 1059 & 11.11 & 1033 & 11.58 & 1141 & 11.84 & 1143 & 11.3 & 1103 & 11.57 & 1116 & 11.71 & 1140 & 11.38 & 1106 & 11.19 & 1115 \\
\hline 11.04 & 1036 & 10.92 & 1003 & 11.47 & 1110 & 11.44 & 1118 & 11.1 & 1079 & 11.04 & 1077 & 11.56 & 1114 & 11.18 & 1080 & 10.85 & 1092 \\
\hline 10.97 & 1010 & 10.66 & 976.1 & 11.22 & 1086 & 11.34 & 1089 & 10.99 & 1052 & 10.93 & 1050 & 11.36 & 1089 & 10.9 & 1054 & 10.56 & 1053 \\
\hline 10.73 & 982.7 & 10.38 & 946.6 & 11.06 & 1062 & 11.09 & 1065 & 10.91 & 1029 & 10.95 & 1040 & 11.12 & 1063 & 10.8 & 1026 & 10.48 & 1028 \\
\hline 10.63 & 958.5 & 9.964 & 922.8 & 10.76 & 1021 & 10.94 & 1041 & 10.69 & 1003 & 10.48 & 1008 & 10.83 & 1038 & 10.55 & 1001 & 10.3 & 997.7 \\
\hline 10.12 & 912.7 & 10 & 896.3 & 10.64 & 998.6 & 10.81 & 1015 & 10.37 & 977 & 10.38 & 978.8 & 10.3 & 997.3 & 10.15 & 977 & 10.22 & 972.6 \\
\hline 9.66 & 891.5 & 9.426 & 858.9 & 10.37 & 971.3 & 10.33 & 977 & 10.12 & 938.7 & 9.97 & 939.1 & 9.97 & 967.7 & 9.976 & 948.3 & 9.87 & 947.9 \\
\hline 9.583 & 865.5 & 9.491 & 832 & 9.958 & 945.7 & 10.05 & 951 & 9.852 & 914 & 9.58 & 916.2 & 9.885 & 945.3 & 9.855 & 921.5 & 9.668 & 924.1 \\
\hline 9.408 & 842.6 & 8.958 & 807.8 & 9.837 & 915.3 & 10.01 & 926.3 & 9.541 & 891.5 & 9.615 & 891.1 & 9.745 & 913.1 & 9.704 & 894.1 & 9.692 & 897.7 \\
\hline 9.37 & 810.9 & 8.858 & 783.1 & 9.63 & 888.4 & 9.92 & 895.9 & 9.287 & 857.1 & 9.287 & 864.6 & 9.411 & 890.6 & 9.393 & 873 & 9.124 & 860.7 \\
\hline 9.103 & 789.3 & 8.559 & 750 & 9.331 & 866.8 & 9.506 & 873.4 & 9.023 & 834.2 & 9.08 & 835.1 & 9.299 & 865.5 & 8.822 & 831.1 & 8.763 & 836.4 \\
\hline 8.855 & 761.9 & 8.42 & 727.1 & 9.183 & 840.8 & 9.393 & 843.9 & 8.816 & 802 & 8.949 & 809.5 & 9.26 & 836.9 & 8.677 & 804.2 & 8.488 & 806.4 \\
\hline 8.671 & 739.5 & 8.322 & 698.9 & 9.133 & 810.9 & 9.014 & 820.1 & 8.881 & 776.9 & 8.665 & 782.7 & 8.769 & 813.5 & 8.375 & 780.4 & 8.384 & 781.8 \\
\hline 8.221 & 698 & 7.837 & 670.7 & 8.979 & 788.4 & 9.103 & 793.7 & 8.624 & 752.7 & 8.399 & 757.1 & 8.884 & 784 & 8.346 & 756.2 & 8.328 & 756.7 \\
\hline 8.053 & 672.9 & 7.502 & 646.5 & 8.485 & 750 & 8.636 & 769.9 & 8.192 & 727.1 & 8.414 & 732.4 & 8.331 & 759.3 & 7.952 & 724.5 & 7.902 & 732.4 \\
\hline 7.668 & 649.6 & 7.227 & 623.1 & 8.076 & 723.6 & 8.159 & 730.2 & 7.94 & 704.2 & 7.967 & 701.1 & 8.121 & 732 & 7.931 & 697.6 & 7.97 & 699.4 \\
\hline 7.316 & 618.3 & 7.011 & 602 & 7.976 & 699.4 & 8.156 & 700.7 & 7.484 & 664.1 & 7.866 & 673.4 & 8.135 & 707.7 & 7.615 & 668.5 & 7.561 & 674.7 \\
\hline 7.15 & 593.6 & 6.706 & 557 & 7.878 & 670.7 & 8.109 & 675.6 & 7.283 & 641.6 & 7.552 & 645.2 & 7.727 & 682.6 & 7.171 & 642.5 & 7.135 & 649.1 \\
\hline 7.085 & 565 & 6.611 & 537.2 & 7.517 & 646.5 & 7.635 & 651.3 & 7.085 & 616.5 & 7.186 & 617.8 & 7.339 & 642.1 & 6.964 & 618.7 & 6.904 & 622.7 \\
\hline 6.733 & 542 & 6.301 & 505 & 7.301 & 621.8 & 7.443 & 628.4 & 7.188 & 583.9 & 6.816 & 589.2 & 7.236 & 617.4 & 6.742 & 595.4 & 6.795 & 596.2 \\
\hline 6.771 & 511.2 & 6.224 & 482.5 & 7.07 & 592.7 & 7.091 & 602.4 & 6.925 & 559.7 & 6.487 & 564.1 & 6.671 & 586.1 & 6.549 & 567.6 & 6.531 & 572.9 \\
\hline 6.277 & 482.1 & 6.022 & 457 & 6.576 & 571.1 & 6.816 & 577.7 & 6.688 & 534.5 & 6.244 & 539.4 & 6.543 & 561.9 & 6.23 & 543.8 & 6.292 & 534.1 \\
\hline 5.786 & 456.1 & 5.434 & 431.9 & 6.52 & 548.2 & 6.715 & 554.4 & 6.321 & 510.3 & 6.052 & 513.8 & 6.209 & 535.4 & 6.022 & 505 & .111 & 510.8 \\
\hline 5.451 & 433.6 & 5.102 & 405.9 & 6.283 & 507.7 & 6.321 & 509.4 & 6.028 & 482.1 & 5.62 & 476.8 & 6.011 & 509.4 & 5.783 & 477.3 & 5.806 & 485.6 \\
\hline 5.519 & 408.1 & 5.229 & 383.8 & 5.676 & 486.1 & 5.874 & 485.6 & 5.502 & 457.9 & 5.33 & 451.3 & 5.478 & 473.7 & 5.434 & 456.1 & 5.508 & 456.5 \\
\hline 5.289 & 381.2 & 4.717 & 355.6 & 5.537 & 455.2 & 5.46 & 461.4 & 5.111 & 421.7 & 5.209 & 426.1 & 5.389 & 452.1 & 5.143 & 424.4 & 5.031 & 431.9 \\
\hline 4.62 & 357 & 4.306 & 329.6 & 5.294 & 430.5 & 5.342 & 432.8 & 5.061 & 397.5 & 5.182 & 398.4 & 5.232 & 443.3 & 5.164 & 399.7 & 5.075 & 408.5 \\
\hline 4.051 & 318.6 & 4.022 & 289.1 & 5.493 & 409.8 & 5.407 & 406.8 & 4.907 & 371.5 & 4.484 & 376.3 & 4.889 & 410.3 & 4.732 & 376.3 & 4.418 & 366.2 \\
\hline 4.214 & 287.3 & 3.403 & 264.9 & 4.913 & 385.2 & 5.167 & 379.4 & 4.531 & 338 & 4.235 & 342.9 & 4.501 & 365.3 & 4.324 & 349.5 & 4.359 & 357.8 \\
\hline 3.534 & 266.6 & 3.696 & 255.6 & 4.599 & 359.2 & 4.492 & 357.4 & 3.948 & 312 & 4.392 & 316.9 & 4.528 & 347.3 & 4.398 & 322.6 & 3.664 & 316.9 \\
\hline 3.605 & 243.7 & & & 4.001 & 317.7 & 4.67 & 332.3 & 4.054 & 289.5 & 3.702 & 292.2 & 4.229 & 317.3 & 3.788 & 286.4 & 3.895 & 292.2 \\
\hline
\end{tabular}




APPENDIX D
ENVELOPE C RHEOLOGY DATA

Table 5. $25^{\circ} \mathrm{C}$ Diluted - continued

\begin{tabular}{|c|c|c|c|c|c|c|c|c|c|c|c|c|c|c|c|c|c|}
\hline run1 & & run2 & & run3 & & run4 & & run5 & & run6 & & run7 & & run8 & & run9 & \\
\hline$[\mathrm{Pa}]$ & {$[1 / \mathrm{s}]$} & {$[\mathrm{Pa}]$} & {$[1 / s]$} & [Pa] & {$[1 / \mathbf{s}]$} & [Pa] & {$[1 / \mathbf{s}]$} & [Pa] & {$[1 / s]$} & [Pa] & {$[1 / \mathbf{s}]$} & [Pa] & {$[1 / s]$} & [Pa] & {$[1 / s]$} & [Pa] & {$[1 / s]$} \\
\hline & & & & 4.072 & 287.8 & 3.975 & 306.7 & 3.516 & 264.9 & 3.776 & 266.6 & 3.687 & 293.1 & 3.223 & 257.4 & 3.17 & 266.6 \\
\hline & & & & 3.495 & 267.9 & 3.986 & 278.5 & 3.285 & 241.9 & 2.995 & 240.2 & 3.64 & 268.4 & 3.104 & 249.9 & 3.38 & 238 \\
\hline & & & & 3.445 & 259.6 & 3.285 & 251.6 & & & & & 3.359 & 245.9 & & & & \\
\hline & & & & & & & & & & & & & & & & & \\
\hline
\end{tabular}


Table 6. $50{ }^{\circ} \mathrm{C}$ Diluted

\begin{tabular}{|c|c|c|c|c|c|c|c|c|c|c|c|c|c|c|c|c|c|}
\hline run1 & & run2 & & run3 & & run4 & & run5 & & run6 & & run7 & & run8 & & run9 & \\
\hline [Pa] & {$[1 / \mathrm{s}]$} & [Pa] & {$[1 / \mathrm{s}]$} & [Pa] & {$[1 / s]$} & [Pa] & [1/s] & [Pa] & {$[1 / s]$} & [Pa] & {$[1 / \mathrm{s}]$} & [Pa] & [1/s] & [Pa] & {$[1 / \mathrm{s}]$} & [Pa] & [1/s] \\
\hline 3.977 & 305.4 & 3.673 & 342.9 & 2.776 & 374.6 & 3.788 & 307.6 & 4.605 & 325.2 & 4.705 & 343.3 & 4.294 & 327.9 & 4.383 & 328.7 & 3.661 & 312.4 \\
\hline 4.146 & 318.6 & 4.078 & 327.9 & 3.803 & 331 & 4.214 & 318.6 & 4.67 & 347.7 & 5.138 & 367.1 & 4.694 & 346.4 & 4.815 & 347.7 & 4.578 & 330.5 \\
\hline 4.646 & 340.2 & 4.513 & 348.6 & 4.282 & 324.8 & 4.765 & 336.7 & 5.084 & 368.4 & 5.36 & 396.6 & 4.815 & 367.5 & 4.951 & 365.8 & 4.664 & 349.9 \\
\hline 4.75 & 361.4 & 5.01 & 370.2 & 4.88 & 353 & 4.856 & 364.9 & 5.309 & 390.9 & 5.445 & 421.3 & 5.164 & 393.1 & 5.324 & 388.7 & 4.998 & 372.8 \\
\hline 5.454 & 401 & 5.031 & 392.2 & 5.327 & 371.5 & 5.138 & 389.6 & 5.842 & 430.1 & 5.653 & 446 & 5.584 & 418.6 & 5.508 & 413.8 & 5.36 & 399.7 \\
\hline 5.694 & 423.5 & 5.185 & 416.9 & 5.407 & 396.6 & 5.596 & 414.2 & 5.869 & 451.7 & 5.969 & 469.3 & 5.771 & 445.5 & 5.629 & 438.9 & 5.36 & 420.4 \\
\hline 5.937 & 455.2 & 5.552 & 442.4 & 5.617 & 427.5 & 5.806 & 440.7 & 6.238 & 485.2 & 6.212 & 496.6 & 6.102 & 475.1 & 5.886 & 468.4 & 5.564 & 448.2 \\
\hline 6.315 & 475.5 & 5.928 & 481.7 & 5.866 & 451.7 & 6.247 & 479 & 6.395 & 509 & 6.499 & 521.8 & 6.333 & 500.2 & 6.141 & 494 & 5.602 & 473.7 \\
\hline 6.57 & 499.3 & 6.197 & 506.8 & 6.218 & 474.2 & 6.469 & 506.3 & 6.517 & 532.8 & 6.688 & 548.2 & 6.543 & 526.6 & 6.739 & 532.3 & 5.824 & 500.6 \\
\hline 6.848 & 538.5 & 6.434 & 534.1 & 6.478 & 511.6 & 6.676 & 531.5 & 6.762 & 558.3 & 7.236 & 593.2 & 6.742 & 551.7 & 7.02 & 558.3 & 6.529 & 539 \\
\hline 7.129 & 568.9 & 6.632 & 558.3 & 6.623 & 539 & 6.916 & 557.5 & 6.996 & 582.6 & 7.511 & 615.6 & 7.064 & 591 & 6.955 & 591.4 & 6.816 & 564.5 \\
\hline 7.484 & 591 & 6.866 & 584.3 & 6.952 & 566.3 & 7.049 & 586.1 & 7.313 & 624 & 7.629 & 637.7 & 7.251 & 617 & 7.257 & 623.1 & 7.079 & 591 \\
\hline 7.81 & 620.5 & 7.2 & 617.4 & 7.18 & 591.4 & 7.366 & 615.6 & 7.544 & 644.3 & 7.623 & 668.5 & 7.437 & 641.2 & 6.934 & 634.1 & 7.203 & 620.9 \\
\hline 8.026 & 643 & 7.319 & 645.2 & 7.402 & 613.4 & 7.632 & 641.6 & 7.828 & 671.2 & 7.851 & 691.4 & 7.733 & 671.2 & 8.695 & 688.8 & 7.393 & 647.8 \\
\hline 8.097 & 674.7 & 7.517 & 670.3 & 7.766 & 639.9 & 7.751 & 669.4 & 7.979 & 699.8 & 8.15 & 714.8 & 7.754 & 694.5 & 8.485 & 719.2 & 7.591 & 672 \\
\hline 8.375 & 698 & 7.795 & 694.1 & 7.896 & 663.2 & 7.881 & 691 & 8.073 & 725.4 & 8.153 & 743.4 & 8.097 & 715.7 & 8.659 & 762.8 & 7.538 & 687 \\
\hline 8.653 & 722.7 & 8.091 & 717.4 & 8.067 & 686.6 & 8.067 & 713 & 8.479 & 750 & 8.609 & 781.8 & 8.337 & 747 & 8.39 & 802.5 & 8.085 & 729.3 \\
\hline 8.665 & 748.3 & 8.053 & 746.5 & 8.461 & 729.3 & 8.541 & 754.4 & 8.464 & 774.7 & 8.929 & 806 & 8.562 & 776 & 9.233 & 829.8 & 7.955 & 742.1 \\
\hline 8.935 & 769.4 & 8.428 & 773.4 & 8.798 & 755.3 & 8.701 & 782.2 & 8.683 & 799 & 8.902 & 830.2 & 8.671 & 808.7 & 9.216 & 844.8 & 8.307 & 768.1 \\
\hline 9.529 & 809.5 & 8.565 & 796.3 & 8.896 & 779.1 & 8.905 & 806.9 & 9.13 & 840.8 & 9.275 & 857.6 & 8.985 & 828.9 & 9.414 & 868.6 & 8.716 & 812.2 \\
\hline 9.47 & 837.7 & 8.976 & 835.1 & 9.177 & 810.4 & 9.103 & 838.2 & 9.301 & 865.5 & 8.887 & 879.2 & 9.133 & 861.5 & 9.094 & 896.8 & 8.94 & 836.9 \\
\hline 9.799 & 863.7 & 9.041 & 863.7 & 9.299 & 831.1 & 9.343 & 864.2 & 9.414 & 889.7 & 9.858 & 901.6 & 9.23 & 885.8 & 9.95 & 929 & 9.032 & 863.7 \\
\hline 9.944 & 886.2 & 9.281 & 886.2 & 9.328 & 853.2 & 9.458 & 888 & 9.704 & 916.2 & 9.739 & 919.7 & 9.509 & 910 & 9.825 & 943.5 & 9.31 & 888 \\
\hline 10.1 & 910.9 & 9.58 & 914.9 & 9.695 & 895.9 & 9.686 & 911.3 & 9.849 & 947.5 & 9.899 & 958.9 & 9.559 & 937.8 & 10.01 & 964.7 & 9.423 & 920.6 \\
\hline 10.43 & 934.7 & 9.677 & 937.3 & 10.09 & 919.3 & 9.908 & 935.6 & 10.04 & 973 & 10.54 & 998.6 & 9.713 & 968.6 & 10.11 & 974.8 & 9.452 & 943.9 \\
\hline 10.71 & 975.7 & 9.873 & 961.1 & 10.17 & 949.2 & 10.01 & 962 & 10.12 & 998.1 & 10.3 & 1014 & 10.06 & 990.7 & 10.25 & 995.9 & 9.642 & 966 \\
\hline 10.86 & 999 & 10 & 989.3 & 10.23 & 974.3 & 10.38 & 1002 & 10.33 & 1022 & 10.65 & 1040 & 10.08 & 1018 & 10.25 & 1019 & 10.01 & 1004 \\
\hline 10.97 & 1030 & 10.34 & 1014 & 10.42 & 997.7 & 10.4 & 1010 & 10.65 & 1063 & 10.92 & 1080 & 10.34 & 1040 & 10.42 & 1046 & 10.15 & 1031 \\
\hline 11.28 & 1046 & 10.68 & 1056 & 10.62 & 1023 & 10.4 & 1050 & 10.84 & 1092 & 11 & 1094 & 10.44 & 1072 & 10.79 & 1074 & 10.18 & 1053 \\
\hline 11.44 & 1076 & 10.77 & 1079 & 10.87 & 1049 & 10.45 & 1051 & 10.77 & 1097 & 11.21 & 1119 & 10.69 & 1096 & 10.85 & 1101 & 10.31 & 1076 \\
\hline 11.73 & 1105 & 10.81 & 1120 & 11.15 & 1073 & 11.05 & 1092 & 11.24 & 1136 & 10.97 & 1149 & 11.06 & 1140 & 10.88 & 1134 & 10.67 & 1113 \\
\hline
\end{tabular}


Table 6. $50{ }^{\circ} \mathrm{C}$ Diluted - continued

\begin{tabular}{|c|c|c|c|c|c|c|c|c|c|c|c|c|c|c|c|c|c|}
\hline run1 & & run2 & & run3 & & run4 & & run5 & & run6 & & run7 & & run8 & & run9 & \\
\hline [Pa] & {$[1 / \mathrm{s}]$} & {$[\mathrm{Pa}]$} & {$[1 / \mathrm{s}]$} & {$[\mathrm{Pa}]$} & {$[1 / s]$} & [Pa] & {$[1 / \mathrm{s}]$} & {$[\mathrm{Pa}]$} & {$[1 / \mathrm{s}]$} & {$[\mathrm{Pa}]$} & {$[1 / \mathrm{s}]$} & [Pa] & {$[1 / \mathrm{s}]$} & {$[\mathrm{Pa}]$} & {$[1 / \mathrm{s}]$} & {$[\mathrm{Pa}]$} & {$[1 / \mathrm{s}]$} \\
\hline 12.08 & 1134 & 11.86 & 1147 & 11.41 & 1114 & 11.05 & 1140 & 11.42 & 1168 & 11.84 & 1174 & 11.02 & 1149 & 11.03 & 1160 & 10.8 & 1140 \\
\hline 12.23 & 1159 & 11.75 & 1174 & 11.6 & 1139 & 10.96 & 1142 & 11.54 & 1192 & 11.8 & 1189 & 11.3 & 1191 & 11.3 & 1186 & 11.03 & 1167 \\
\hline 12.33 & 1189 & 12.42 & 1234 & 11.83 & 1162 & 11.67 & 1173 & 11.64 & 1218 & 11.83 & 1232 & 11.45 & 1215 & 11.44 & 1211 & 11.2 & 1193 \\
\hline 12.49 & 1212 & 12.36 & 1249 & 11.99 & 1185 & 11.67 & 1204 & 11.85 & 1247 & 11.96 & 1259 & 11.64 & 1244 & 11.59 & 1239 & 11.35 & 1219 \\
\hline 12.66 & 1241 & 12.68 & 1276 & 12.03 & 1215 & 11.7 & 1228 & 12.06 & 1270 & 12.18 & 1288 & 11.74 & 1267 & 11.78 & 1262 & 11.44 & 1248 \\
\hline 12.93 & 1267 & 12.55 & 1304 & 12.18 & 1238 & 12.27 & 1253 & 12.09 & 1292 & 12.42 & 1327 & 11.89 & 1293 & 11.96 & 1286 & 11.59 & 1274 \\
\hline 13.18 & 1292 & 13.34 & 1337 & 12.38 & 1263 & 12.43 & 1283 & 12.21 & 1318 & 12.61 & 1352 & 12.11 & 1317 & 12.03 & 1314 & 11.58 & 1301 \\
\hline 13.28 & 1320 & 13.18 & 1357 & 12.73 & 1302 & 12.2 & 1315 & 12.52 & 1359 & 12.76 & 1378 & 12.31 & 1355 & 12.3 & 1339 & 11.85 & 1326 \\
\hline 13.39 & 1342 & 13.9 & 1398 & 12.82 & 1326 & 12.12 & 1315 & 12.73 & 1382 & 12.91 & 1402 & 12.49 & 1377 & 12.43 & 1367 & 11.93 & 1352 \\
\hline 13.89 & 1385 & 13.86 & 1419 & 12.9 & 1351 & 12.92 & 1355 & 12.88 & 1412 & 12.94 & 1434 & 12.67 & 1404 & 12.73 & 1412 & 12.46 & 1386 \\
\hline 14.05 & 1409 & 13.97 & 1452 & 13.12 & 1382 & 13.02 & 1394 & 13.14 & 1434 & 13 & 1460 & 12.67 & 1434 & 12.94 & 1430 & 12.49 & 1413 \\
\hline 14.06 & 1434 & 13.89 & 1463 & 13.3 & 1405 & 13.14 & 1423 & 13.3 & 1470 & 13.24 & 1486 & 12.9 & 1462 & 13.1 & 1457 & 12.62 & 1440 \\
\hline 14.37 & 1465 & 14.08 & 1485 & 13.6 & 1430 & 13.47 & 1465 & 13.45 & 1498 & 13.42 & 1510 & 13.04 & 1488 & 13.22 & 1487 & 12.85 & 1459 \\
\hline 14.47 & 1491 & 14.05 & 1494 & 13.65 & 1459 & 13.59 & 1488 & 13.28 & 1506 & 13.68 & 1537 & 13.16 & 1513 & 12.36 & 1571 & 12.15 & 1570 \\
\hline 14.74 & 1515 & 14.21 & 1510 & 13.91 & 1485 & 13.8 & 1512 & 13.73 & 1535 & 13.81 & 1561 & 13.3 & 1536 & 12.17 & 1546 & 11.99 & 1543 \\
\hline 13.1 & 1542 & 12.81 & 1537 & 12.73 & 1547 & 12.47 & 1524 & 12.04 & 1482 & 11.6 & 1456 & 11.96 & 1518 & 12.06 & 1524 & 11.73 & 1518 \\
\hline 12.98 & 1516 & 12.67 & 1507 & 12.61 & 1521 & 12.31 & 1498 & 11.84 & 1455 & 11.62 & 1431 & 11.86 & 1494 & 11.85 & 1503 & 11.63 & 1490 \\
\hline 12.8 & 1491 & 12.49 & 1486 & 12.45 & 1497 & 12.15 & 1469 & 11.62 & 1430 & 11.44 & 1406 & 11.7 & 1468 & 11.51 & 1462 & 11.47 & 1461 \\
\hline 12.58 & 1464 & 12.17 & 1461 & 12.28 & 1476 & 11.81 & 1443 & 11.44 & 1408 & 11.33 & 1382 & 11.55 & 1443 & 11.37 & 1440 & 11.12 & 1442 \\
\hline 12.45 & 1437 & 12.02 & 1429 & 11.85 & 1436 & 11.78 & 1417 & 11.33 & 1373 & 11.14 & 1355 & 11.34 & 1416 & 11.3 & 1409 & 11.03 & 1406 \\
\hline 12.27 & 1408 & 11.94 & 1407 & 11.66 & 1411 & 11.53 & 1392 & 11.13 & 1349 & 10.96 & 1330 & 11.08 & 1387 & 11.02 & 1384 & 10.96 & 1382 \\
\hline 12.12 & 1382 & 11.81 & 1382 & .53 & 1380 & 11.22 & 1353 & 11.01 & 1323 & 10.75 & 1307 & 11.03 & 1355 & 10.64 & 1350 & 10.74 & 1353 \\
\hline 11.78 & 1359 & 11.41 & 1344 & 43 & 1357 & 10.95 & 1327 & 10.7 & 1300 & 10.25 & 1267 & 10.8 & 1334 & 10.65 & 1324 & 10.49 & 1329 \\
\hline 11.74 & 1334 & 11.23 & 1315 & 11.36 & 1332 & 10.85 & 1300 & 10.63 & 1276 & 10.22 & 1243 & 10.69 & 1305 & 10.41 & 1298 & 10.39 & 1306 \\
\hline 11.52 & 1310 & 11.24 & 1289 & 11.11 & 1308 & 10.49 & 1274 & 10.22 & 1239 & 10.06 & 1211 & 10.48 & 1282 & 10.43 & 1288 & 10.2 & 1280 \\
\hline 11.34 & 1284 & 11.01 & 1265 & 10.74 & 1269 & 10.57 & 1244 & 10.13 & 1212 & 9.87 & 1187 & 10.31 & 1256 & 10.26 & 1261 & 10.12 & 1256 \\
\hline 10.93 & 1245 & 10.79 & 1243 & 10.53 & 1243 & 10.2 & 1225 & 9.964 & 1183 & 9.606 & 1162 & 10.16 & 1230 & 9.769 & 1217 & 9.671 & 1215 \\
\hline 10.77 & 1223 & 10.46 & 1217 & 10.39 & 1223 & 10.2 & 1190 & 9.802 & 1159 & 9.447 & 1137 & 9.953 & 1204 & 9.559 & 1194 & 9.55 & 1189 \\
\hline 10.58 & 1192 & 10.31 & 1192 & 10.22 & 1189 & 9.982 & 1166 & 9.636 & 1134 & 9.411 & 1109 & 9.71 & 1176 & 9.532 & 1161 & 9.334 & 1166 \\
\hline 10.46 & 1170 & 10.22 & 1166 & 10.12 & 1164 & 9.674 & 1142 & 9.319 & 1108 & 9.103 & 1084 & 9.319 & 1137 & 9.438 & 1137 & 9.148 & 1138 \\
\hline 10.27 & 1144 & 9.793 & 1125 & 9.825 & 1140 & 9.538 & 1118 & 9.278 & 1084 & 8.855 & 1059 & 9.248 & 1112 & 9.165 & 1111 & 9.074 & 1106 \\
\hline
\end{tabular}


Table 6. $50{ }^{\circ} \mathrm{C}$ Diluted - continued

\begin{tabular}{|c|c|c|c|c|c|c|c|c|c|c|c|c|c|c|c|c|c|}
\hline run1 & & run2 & & run3 & & run4 & & run5 & & run6 & & run7 & & run8 & & run9 & \\
\hline [Pa] & {$[1 / \mathrm{s}]$} & {$[\mathrm{Pa}]$} & {$[1 / \mathbf{s}]$} & [Pa] & {$[1 / \mathrm{s}]$} & [Pa] & {$[1 / s]$} & [Pa] & {$[1 / s]$} & {$[\mathrm{Pa}]$} & {$[1 / \mathrm{s}]$} & {$[\mathrm{Pa}]$} & {$[1 / \mathrm{s}]$} & {$[\mathrm{Pa}]$} & {$[1 / \mathrm{s}]$} & {$[\mathrm{Pa}]$} & {$[1 / \mathrm{s}]$} \\
\hline 10 & 1120 & 9.636 & 1097 & 9.609 & 1116 & 9.408 & 1094 & 8.917 & 1045 & 8.787 & 1029 & 9.05 & 1081 & 9.08 & 1085 & 8.872 & 1081 \\
\hline 9.683 & 1083 & 9.358 & 1074 & 9.39 & 1092 & 9.18 & 1067 & 8.713 & 1022 & 8.535 & 1003 & 8.858 & 1056 & 8.899 & 1060 & 8.73 & 1057 \\
\hline 9.452 & 1054 & .263 & 1051 & 9.148 & 1052 & 8.792 & 1026 & 8.544 & 995.1 & 8.352 & 977.4 & 8.742 & 1034 & 8.689 & 1034 & 8.526 & 1034 \\
\hline 9.174 & 1028 & 9.156 & 1019 & 9.026 & 1029 & 8.576 & 999.5 & 8.387 & 966 & 8.076 & 933.4 & 8.689 & 1007 & 8.494 & 1007 & 8.301 & 1007 \\
\hline 9.177 & 1004 & 8.887 & 994.2 & 8.837 & 1003 & 8.464 & 975.7 & 8.218 & 943.1 & 7.754 & 910.9 & 8.396 & 984 & 8.301 & 980.1 & 8.31 & 981.4 \\
\hline 9.009 & 974.3 & 8.633 & 967.7 & 8.686 & 970.8 & 8.375 & 943.1 & 8.183 & 916.6 & 7.887 & 901.6 & 8.204 & 960.7 & 8.026 & 955 & 7.774 & 941.7 \\
\hline 8.76 & 945.7 & 541 & 943.9 & 8.473 & 944.4 & 8.106 & 917.5 & 7.754 & 892.8 & 7.47 & 856.2 & 8.014 & 933.8 & 7.715 & 10.5 & 7.632 & 915.3 \\
\hline 8.497 & 920.1 & 201 & 919.7 & 8.218 & 923.7 & 7.955 & 893.7 & 7.712 & 867.3 & 7.126 & 833.8 & 7.659 & 890.6 & 7.449 & 889.7 & 7.526 & 891.9 \\
\hline 8.272 & 895.9 & 7.955 & 878.7 & 8.138 & 896.8 & 7.866 & 865.1 & 7.464 & 840.4 & 7.129 & 800.7 & 7.381 & 862.4 & 7.425 & 865.1 & 7.354 & 862.4 \\
\hline 7.993 & 870.8 & 7.635 & 857.6 & 7.863 & 873 & 7.499 & 843 & 7.268 & 814.8 & 6.836 & 777.4 & 7.268 & 836 & 7.076 & 839.9 & 7.171 & 836 \\
\hline 7.925 & 847.4 & 7.603 & 831.6 & 7.588 & 828.9 & 7.313 & 818.3 & 6.756 & 774.7 & 6.679 & 756.7 & 6.946 & 809.1 & 6.958 & 809.1 & 6.928 & 810.4 \\
\hline 7.609 & 821.4 & 7.277 & 800.3 & 7.262 & 808.2 & 7.079 & 778.2 & 6.638 & 748.3 & 6.31 & 717 & 6.765 & 781.3 & 6.827 & 786.2 & 6.842 & 787.5 \\
\hline 7.236 & 780 & 7.286 & 774.7 & 7.061 & 783.1 & 6.884 & 756.2 & 6.455 & 718.3 & 6.227 & 692.3 & 6.472 & 754.9 & 6.768 & 762.8 & 6.626 & 762.4 \\
\hline 6.955 & 754.9 & 7.029 & 752.7 & 6.999 & 756.7 & 6.682 & 726.2 & 6.274 & 696.3 & 6.025 & 665.4 & 6.416 & 732 & 6.449 & 734.6 & 6.238 & 722.7 \\
\hline 6.863 & 724.5 & 6.564 & 713 & 6.7 & 731.1 & 6.52 & 702 & 6.144 & 665.9 & 5.67 & 641.2 & 6.298 & 705.1 & 6.301 & 707.7 & 5.898 & 699.4 \\
\hline 6.878 & 699.4 & 6.301 & 689.2 & 6.529 & 701.1 & 6.404 & 677.8 & 6.025 & 640.8 & 5.46 & 611.2 & 5.934 & 675.6 & 6.058 & 686.1 & 5.901 & 672.5 \\
\hline 6.514 & 674.2 & 6.147 & 664.5 & 6.487 & 676 & 6.019 & 649.6 & 5.806 & 616.5 & 5.389 & 585.7 & 5.789 & 650.9 & 5.694 & 642.5 & 5.795 & 646.5 \\
\hline 6.197 & 651.8 & 6.055 & 631.5 & 6.212 & 652.7 & 5.732 & 624.9 & 5.682 & 593.2 & 5.126 & 561 & 5.62 & 630.6 & 5.466 & 618.7 & 5.543 & 620.5 \\
\hline 5.987 & 627.1 & 5.721 & 609 & 5.969 & 626.7 & 5.54 & 597.6 & 5.374 & 567.6 & 4.998 & 532.3 & 5.291 & 591.4 & 5.336 & 595.8 & 5.141 & 587.4 \\
\hline 5.866 & 602.4 & 5.605 & 582.1 & 5.676 & 602.4 & 5.362 & 560.5 & 4.697 & 527.5 & 4.691 & 508.5 & 5.173 & 566.7 & 5.108 & 566.7 & 4.972 & 563.6 \\
\hline 5.549 & 561.9 & 5.274 & 555.3 & 5.362 & 559.7 & 5.105 & 539.4 & 4.632 & 503.7 & 4.463 & 481.7 & 4.922 & 537.2 & 4.871 & 539.8 & 4.865 & 542 \\
\hline 5.28 & 537.2 & 5.141 & 531.5 & 5.164 & 535 & 4.874 & 511.6 & 4.38 & 472 & 4.344 & 456.5 & 4.948 & 516.5 & 4.833 & 508.1 & 4.922 & 518.7 \\
\hline 5.12 & 6.8 & 39 & 499.7 & & 07.7 & 4.629 & 486.5 & 4.38 & 447.3 & 3.85 & 418.2 & 4.643 & 492.2 & 4.611 & 485.2 & 4.608 & 490 \\
\hline 4.916 & 483.9 & 4.771 & 472 & 4.655 & 479.9 & 4.309 & 458.3 & 4.226 & 423.9 & 3.756 & 394 & 4.238 & 453.9 & 4.241 & 459.6 & 4.418 & 465.4 \\
\hline 4.534 & 459.2 & 4.537 & 447.7 & 4.336 & 462.7 & 4.099 & 426.1 & 3.88 & 395.3 & 3.504 & 368.9 & 4.155 & 426.6 & 4.028 & 435.4 & 3.821 & 420.9 \\
\hline 4.262 & 433.6 & 4.262 & 420 & 4.134 & 428.3 & 3.85 & 402.3 & 3.445 & 371.5 & 3.196 & 336.2 & 3.788 & 401.9 & 3.531 & 397.5 & 3.794 & 396.2 \\
\hline 4.182 & 409 & 3.862 & 395.7 & 4.161 & 404.5 & 3.714 & 375.9 & 3.439 & 341.1 & 3.149 & 311.6 & 3.593 & 376.8 & 3.48 & 373.3 & 3.359 & 374.1 \\
\hline 3.658 & 368.9 & 3.824 & 370.6 & 3.747 & 381.6 & 3.427 & 349.5 & 3.104 & 313.8 & 2.773 & 285.6 & 3.557 & 349 & 3.246 & 349 & 3.193 & 342 \\
\hline 3.498 & 346.4 & 3.492 & 346.4 & 3.451 & 356.1 & 3.232 & 327.4 & 2.912 & 290.9 & 2.675 & 259.6 & 3.09 & 325.7 & 2.9 & 317.3 & 2.989 & 327 \\
\hline 3.122 & 318.6 & 2.9 & 308 & 3.048 & 316.4 & 2.897 & 287.3 & 2.607 & 265.7 & 2.243 & 234.9 & 3.06 & 302.3 & 2.954 & 290 & 2.637 & 289.1 \\
\hline 3.081 & 292.2 & 3.004 & 286.4 & 3.019 & 287.3 & 2.45 & 267.1 & 2.516 & 241.5 & 0 & 153 & 2.619 & 273.7 & & & & \\
\hline 2.604 & 262.2 & 2.545 & 255.2 & 2.619 & 263.1 & 2.542 & 234.9 & & 151.9 & 0 & 155.6 & 2.539 & 249 & & & & \\
\hline
\end{tabular}


Table 7. Rheometer Response for Blank - Pretreated

\begin{tabular}{|c|c|c|c|c|c|c|c|c|c|c|c|c|c|c|c|c|c|}
\hline run1 & & run2 & & run3 & & run4 & & run5 & & run6 & & run7 & & run8 & & run9 & \\
\hline [Pa] & {$[1 / s]$} & [Pa] & [1/s] & [Pa] & {$[1 / s]$} & [Pa] & {$[1 / s]$} & [Pa] & [1/s] & [Pa] & [1/s] & [Pa] & [1/s] & [Pa] & {$[1 / s]$} & [Pa] & {$[1 / s]$} \\
\hline 3.753 & 323.9 & 3.463 & 321.3 & 3.46 & 319.9 & 3.283 & 317.7 & 3.493 & 323 & 3.451 & 328.7 & 3.641 & 347.7 & 3.351 & 324.3 & 3.324 & 325.2 \\
\hline 4.025 & 343.3 & 768 & 341.1 & 3.759 & 335.8 & 3.546 & 338.4 & 3.741 & 345.5 & 3.798 & 343.3 & 3.848 & 371.1 & 3.617 & 344.6 & 3.644 & 340.6 \\
\hline 4.194 & 364.9 & 037 & 363.6 & .996 & 360.5 & 3.801 & 358.7 & 3.972 & 370.2 & 3.922 & 373.7 & 4.058 & 394.4 & 3.765 & 372.4 & 3.827 & 368.4 \\
\hline 4.455 & 387.4 & 4.212 & 388.7 & 4.215 & 385.2 & 4.218 & 402.3 & 4.114 & 396.2 & 4.135 & 396.2 & 4.141 & 416.4 & 3.972 & 396.2 & 3.993 & 389.6 \\
\hline 4.851 & 426.6 & 4.434 & 411.2 & 4.392 & 411.2 & 4.404 & 424.8 & 4.265 & 427.9 & 4.446 & 435 & 4.342 & 442.9 & 4.135 & 417.3 & 4.218 & 413.8 \\
\hline 5.079 & 451.3 & 4.54 & 434.5 & 4.564 & 444.2 & 4.534 & 457.4 & 4.425 & 450.4 & 4.638 & 458.3 & 4.57 & 467.6 & 4.28 & 440.2 & 4.588 & 453 \\
\hline 5.224 & 478.6 & 4.967 & 474.2 & 4.753 & 470.6 & 4.715 & 480.3 & 4.508 & 476.8 & 4.845 & 482.1 & 4.819 & 496.6 & 4.694 & 481.2 & 4.756 & 478.6 \\
\hline 5.464 & 501.9 & 5.153 & 499.7 & 4.922 & 498.4 & 4.842 & 501.1 & 4.822 & 499.7 & 4.943 & 505 & 5.141 & 539.4 & 4.848 & 504.1 & 4.922 & 501.5 \\
\hline 5.644 & 525.7 & 5.333 & 525.7 & 5.088 & 524.4 & 5.162 & 538.5 & 4.981 & 525.7 & 5.117 & 539.8 & 5.339 & 562.3 & 4.928 & 536.8 & 5.088 & 525.7 \\
\hline 6.044 & 565 & 5.467 & 553.1 & 5.319 & 551.7 & 5.399 & 560.5 & 5.103 & 549.5 & 5.316 & 564.1 & 5.467 & 592.3 & 5.106 & 560.1 & 5.251 & 552.6 \\
\hline 6.281 & 589.6 & 5.624 & 576 & 5.47 & 576 & 5.39 & 593.2 & 5.523 & 591 & 5.44 & 589.2 & 5.621 & 616.1 & 5.28 & 583.9 & 5.405 & 585.7 \\
\hline & 613.9 & & 598.9 & 5.618 & 602 & 5.576 & 617 & & 617.4 & & 612.5 & & 638.1 & & 606.8 & 5.505 & 611.7 \\
\hline 6.609 & 644.7 & 6.109 & 637.2 & 5.745 & 626.2 & 5.78 & 641.2 & 5.831 & 644.7 & 6.05 & 651.8 & 5.928 & 662.3 & 5.624 & 630.6 & 5.76 & 635 \\
\hline 6.849 & 666.3 & 6.316 & 663.7 & 5.911 & 650.9 & 5.946 & 665 & 5.999 & 670.3 & 5.952 & 663.7 & 6.106 & 687.5 & & 675.1 & 5.872 & 659.3 \\
\hline 7.044 & 691.4 & 6.473 & 688.8 & 6.257 & 690.1 & 6.141 & 689.7 & 6.112 & 701.1 & 6.431 & 704.2 & 6.278 & 719.2 & 5.928 & 683.1 & 6.053 & 683.9 \\
\hline 7.304 & 716.1 & 6.757 & 716.6 & 6.458 & 712.1 & 6.31 & 712.1 & 6.307 & 725.8 & 6.541 & 729.3 & 6.71 & 757.5 & 6.411 & 728.4 & 6.204 & 711.7 \\
\hline & 757.1 & & 739.5 & 6.535 & 744.8 & 6.668 & 754.9 & & 748.7 & 36 & 758.4 & & 780.4 & & 752.7 & 6.417 & 736.8 \\
\hline 7.846 & 780.9 & 7.026 & 771.2 & 6.733 & 770.8 & 6.872 & 780 & 6.645 & 773.4 & 6.872 & 784.9 & 6.961 & 811.3 & 6.787 & 776.5 & 6.52 & 767.7 \\
\hline & 805.6 & & 795.9 & 6.875 & 791.9 & 6.94 & 809.1 & & 797.2 & & 808.7 & & 833.8 & & 806.4 & 6.822 & 791.9 \\
\hline 8.257 & 832 & 7.287 & 818.3 & 7.207 & 828.5 & 7.18 & 839.1 & 6.985 & 822.8 & & 833.8 & & 856.7 & & 832.4 & 6.911 & 821 \\
\hline 8.355 & 862.9 & 7.618 & 855.8 & 7.367 & 854.9 & 7.275 & 865.1 & 51 & 849.6 & 449 & 860.7 & & 892.8 & 7.192 & 856.7 & 7.219 & 850.5 \\
\hline 8.589 & 887.5 & 7.799 & 878.7 & 7.432 & 879.2 & 7.535 & 888.4 & 7.515 & 890.6 & 7.574 & 885.8 & 7.793 & 920.6 & 7.521 & 893.7 & 7.387 & 878.7 \\
\hline 8.755 & 909.1 & 7.976 & 905.2 & 7.689 & 903.8 & 7.621 & 912.7 & 7.71 & 912.7 & 7.964 & 923.7 & 7.825 & 945.3 & 7.734 & 918.8 & 7.509 & 904.3 \\
\hline 8.879 & 934.2 & 8.098 & 933.4 & 7.772 & 928.1 & 7.748 & 941.3 & 7.816 & 939.5 & 8.13 & 948.8 & & 973.5 & 7.852 & 947 & 7.734 & 927.6 \\
\hline 9.272 & 973.9 & 8.228 & 955 & 7.938 & 952.8 & 7.961 & 965.1 & 7.982 & 969.9 & 8.246 & 977 & 8.272 & 999.9 & 8.145 & 971.7 & 7.867 & 954.5 \\
\hline 9.453 & 996.8 & & 991.5 & 8.095 & 979.6 & 8.251 & 992.4 & 8.115 & 998.1 & 8.423 & 999.5 & 8.435 & 1023 & 8.251 & 996.8 & 8.246 & 995.5 \\
\hline 9.642 & 1030 & 8.725 & 1014 & 8.426 & 1023 & 8.343 & 1019 & 8.296 & 1020 & 8.595 & 1031 & 8.592 & 1048 & 88 & 1022 & 8.429 & 1022 \\
\hline 9.912 & 1055 & 8.935 & 1045 & 8.669 & 1044 & 8.515 & 1044 & 8.544 & 1050 & 8.787 & 1053 & 8.737 & 1070 & 8.598 & 1050 & 8.565 & 1049 \\
\hline 10.12 & 1081 & 9.19 & 1069 & 8.823 & 1069 & 8.846 & 1085 & 8.698 & 1074 & 9.006 & 1078 & 9.136 & 1112 & 8.719 & 1080 & 8.74 & 1076 \\
\hline 10.29 & 1103 & 9.284 & 1093 & 8.858 & 1099 & 9.03 & 1110 & 8.947 & 1098 & 9.172 & 1101 & 9.329 & 1136 & & 1110 & 8.95 & 1099 \\
\hline 10.62 & 1139 & 9.477 & 1118 & 9.122 & 1119 & 9.187 & 1140 & 9.255 & 1135 & 9.589 & 1141 & 9.432 & 1166 & 9.009 & 1137 & 8.971 & 1130 \\
\hline
\end{tabular}


Table 7. Rheometer Response for Blank - Pretreated - continued

\begin{tabular}{|c|c|c|c|c|c|c|c|c|c|c|c|c|c|c|c|c|c|}
\hline run1 & & run2 & & run3 & & run4 & & run5 & & run6 & & run7 & & run8 & & run9 & \\
\hline [Pa] & {$[1 / \mathrm{s}]$} & [Pa] & {$[1 / \mathrm{s}]$} & {$[\mathrm{Pa}]$} & {$[1 / \mathrm{s}]$} & [Pa] & {$[1 / s]$} & [Pa] & {$[1 / \mathrm{s}]$} & {$[\mathrm{Pa}]$} & {$[1 / \mathrm{s}]$} & [Pa] & {$[1 / \mathrm{s}]$} & {$[\mathrm{Pa}]$} & {$[1 / \mathrm{s}]$} & {$[\mathrm{Pa}]$} & {$[1 / \mathrm{s}]$} \\
\hline 10.83 & 1161 & 9.563 & 1142 & 9.252 & 1144 & 9.299 & 1165 & 9.326 & 1160 & 9.773 & 1162 & 9.518 & 1188 & 9.252 & 1162 & 9.107 & 1154 \\
\hline 10.9 & 1190 & 9.882 & 1182 & 9.394 & 1171 & 9.554 & 1185 & 9.503 & 1189 & 9.879 & 1196 & 9.713 & 1211 & 9.477 & 1188 & 9.329 & 1178 \\
\hline 11.11 & 1216 & 10.04 & 1204 & 9.554 & 1197 & 9.645 & 1223 & 9.675 & 1217 & 10.04 & 1220 & 10.05 & 1250 & 9.56 & 1215 & 9.497 & 1205 \\
\hline 11.33 & 1238 & 10.18 & 1232 & 9.752 & 1223 & 9.879 & 1246 & 9.776 & 1241 & 10.17 & 1243 & 10.27 & 1274 & 9.764 & 1241 & 9.69 & 1230 \\
\hline 11.46 & 1265 & 10.35 & 1261 & 10.12 & 1266 & 9.968 & 1270 & 9.953 & 1264 & 10.36 & 1266 & 10.41 & 1303 & 9.947 & 1264 & 9.838 & 1253 \\
\hline 11.72 & 1290 & 10.5 & 1286 & 10.34 & 1293 & 10.14 & 1293 & 10.12 & 1289 & 10.54 & 1293 & 10.55 & 1326 & 10.24 & 1303 & 10.18 & 1295 \\
\hline 12.1 & 1333 & 10.65 & 1310 & 10.44 & 1319 & 10.6 & 1330 & 10.55 & 1329 & 10.8 & 1317 & 10.68 & 1348 & 10.42 & 1331 & 10.32 & 1322 \\
\hline 12.27 & 1354 & 10.82 & 1331 & 10.6 & 1341 & 10.73 & 1355 & 10.69 & 1352 & 11.17 & 1361 & 10.81 & 1375 & 10.57 & 1353 & 10.47 & 1351 \\
\hline 12.51 & 1382 & 11.1 & 1370 & 10.73 & 1366 & 10.9 & 1379 & 10.79 & 1383 & 11.34 & 1391 & 11.26 & 1413 & 10.62 & 1378 & 10.53 & 1373 \\
\hline 12.7 & 1410 & 11.27 & 1401 & 10.94 & 1389 & 10.97 & 1413 & 11.04 & 1408 & 11.59 & 1410 & 11.42 & 1436 & 10.87 & 1402 & 10.76 & 1396 \\
\hline 12.88 & 1434 & 11.47 & 1423 & 11.13 & 1413 & 11.29 & 1431 & 11.16 & 1430 & 11.75 & 1435 & 11.54 & 1464 & 11.04 & 1434 & 10.92 & 1421 \\
\hline 13.02 & 1457 & 11.68 & 1443 & 11.23 & 1442 & 11.42 & 1459 & 11.33 & 1455 & 11.89 & 1466 & 11.62 & 1489 & 11.26 & 1456 & 11.3 & 1461 \\
\hline 13.18 & 1480 & 11.9 & 1479 & 11.74 & 1482 & 11.55 & 1492 & 11.46 & 1479 & 12.05 & 1493 & 11.85 & 1510 & 11.43 & 1483 & 11.27 & 1470 \\
\hline 13.46 & 1504 & 12.07 & 1504 & 11.84 & 1512 & 11.73 & 1516 & 11.65 & 1502 & 12.21 & 1516 & 12.21 & 1551 & 11.6 & 1507 & 11.69 & 1512 \\
\hline 13.78 & 1542 & 12.21 & 1527 & 11.93 & 1534 & 11.89 & 1539 & 11.97 & 1542 & 12.37 & 1541 & 12.21 & 1560 & 11.69 & 1534 & 11.85 & 1540 \\
\hline 11.33 & 1536 & 10.64 & 1513 & 10.74 & 1547 & 10.02 & 1536 & 10.88 & 1541 & 10.86 & 1547 & 10.56 & 1549 & 10.41 & 1545 & 10.43 & 1538 \\
\hline 11.06 & 1512 & 10.5 & 1488 & 10.56 & 1519 & 9.737 & 1510 & 10.44 & 1515 & 10.58 & 1523 & 10.39 & 1515 & 10.22 & 1522 & 10.31 & 1514 \\
\hline 10.9 & 1489 & 10.04 & 1447 & 10.44 & 1497 & 9.725 & 1483 & 10.27 & 1493 & 10.44 & 1492 & 10.19 & 1491 & 10.12 & 1490 & 10.12 & 1489 \\
\hline 10.73 & 1463 & 9.802 & 1420 & 9.924 & 1461 & 9.518 & 1459 & 10.08 & 1460 & 10.33 & 1467 & 10.05 & 1467 & 10 & 1467 & 9.882 & 1463 \\
\hline 10.56 & 1441 & 9.648 & 1394 & 9.708 & 1438 & 9.361 & 1425 & 9.938 & 1436 & 10.14 & 1443 & 9.876 & 1445 & 9.811 & 1441 & 9.767 & 1434 \\
\hline 10.15 & 1402 & 9.512 & 1369 & 9.687 & 1407 & 9.13 & 1400 & 9.814 & 1413 & 9.918 & 1419 & 9.761 & 1419 & 9.568 & 1416 & 9.474 & 1412 \\
\hline 9.986 & 1379 & 9.335 & 1345 & 9.554 & 1381 & 8.935 & 1375 & 9.607 & 1391 & 9.767 & 1396 & 9.287 & 1380 & 9.477 & 1391 & 9.344 & 1390 \\
\hline 9.743 & 1348 & 9.213 & 1314 & 9.338 & 1358 & 8.802 & 1348 & 9.465 & 1353 & 9.539 & 1352 & 9.166 & 1356 & 9.287 & 1366 & 8.953 & 1349 \\
\hline 9.592 & 1322 & 8.944 & 1289 & 9.163 & 1337 & 8.385 & 1324 & 9.021 & 1328 & 9.166 & 1329 & 8.935 & 1332 & 9.101 & 1337 & 8.734 & 1326 \\
\hline 9.361 & 1300 & 8.752 & 1264 & 8.817 & 1297 & 8.414 & 1300 & 8.891 & 1304 & 8.991 & 1299 & 8.855 & 1300 & 8.737 & 1309 & 8.645 & 1293 \\
\hline 9.207 & 1274 & 8.61 & 1238 & 8.613 & 1270 & 7.956 & 1255 & 8.725 & 1276 & 8.808 & 1274 & 8.672 & 1275 & 8.636 & 1281 & 8.482 & 1269 \\
\hline 9.08 & 1250 & 8.423 & 1214 & 8.648 & 1259 & 7.808 & 1248 & 8.607 & 1251 & 8.692 & 1249 & 8.533 & 1251 & 8.429 & 1253 & 8.269 & 1246 \\
\hline 8.666 & 1210 & 8.29 & 1183 & 8.479 & 1232 & 7.879 & 1208 & 8.367 & 1215 & 8.527 & 1226 & 8.379 & 1227 & 8.213 & 1232 & 8.092 & 1222 \\
\hline 8.441 & 1186 & 8.003 & 1158 & 8.086 & 1192 & 7.399 & 1179 & 8.186 & 1190 & 8.284 & 1200 & 8.192 & 1204 & 7.87 & 1192 & 7.988 & 1196 \\
\hline 8.308 & 1157 & 7.802 & 1134 & 7.873 & 1166 & 7.248 & 1171 & 8.018 & 1168 & 8.198 & 1167 & 7.745 & 1162 & 7.645 & 1165 & 7.811 & 1170 \\
\hline 8.216 & 1135 & 7.423 & 1092 & 7.781 & 1134 & 7.34 & 1129 & 7.837 & 1140 & 8.021 & 1143 & 7.577 & 1139 & 7.556 & 1142 & 7.556 & 1144 \\
\hline
\end{tabular}


Table 7. Rheometer Response for Blank - Pretreated - continued

\begin{tabular}{|c|c|c|c|c|c|c|c|c|c|c|c|c|c|c|c|c|c|}
\hline run1 & & run2 & & run3 & & run4 & & run5 & & run6 & & run7 & & run8 & & run9 & \\
\hline [Pa] & {$[1 / s]$} & {$[\mathrm{Pa}]$} & {$[1 / s]$} & {$[\mathrm{Pa}]$} & {$[1 / s]$} & {$[\mathrm{Pa}]$} & {$[1 / s]$} & {$[\mathrm{Pa}]$} & {$[1 / \mathrm{s}]$} & {$[\mathrm{Pa}]$} & {$[1 / \mathrm{s}]$} & [Pa] & {$[1 / \mathrm{s}]$} & {$[\mathrm{Pa}]$} & {$[1 / \mathbf{s}]$} & {$[\mathrm{Pa}]$} & {$[1 / s]$} \\
\hline 8.047 & 1112 & 7.263 & 1066 & 7.529 & 1111 & 6.807 & 1103 & 7.816 & 1114 & 7.822 & 1120 & 7.452 & 1108 & 7.399 & 1109 & 7.21 & 1102 \\
\hline 7.651 & 1072 & 7.088 & 1043 & 7.432 & 1085 & 6.65 & 1091 & 7.538 & 1091 & 7.645 & 1091 & 7.228 & 1089 & 7.186 & 1085 & 7.047 & 1079 \\
\hline 7.432 & 1049 & 6.982 & 1014 & 7.228 & 1062 & 6.497 & 1046 & 7.316 & 1054 & 7.532 & 1066 & 7.032 & 1056 & 7.091 & 1057 & 6.905 & 1049 \\
\hline 7.307 & 1018 & 6.875 & 988 & 6.914 & 1019 & 6.354 & 1021 & 7.009 & 1026 & 7.299 & 1022 & 6.896 & 1032 & 6.861 & 1030 & 6.795 & 1026 \\
\hline 7.148 & 995.1 & 6.647 & 965.5 & 7.017 & 1009 & 6.159 & 995.1 & 6.866 & 1002 & 6.834 & 999.9 & 6.813 & 1008 & 6.662 & 1002 & 6.568 & 999.9 \\
\hline 6.955 & 969.5 & 6.479 & 939.5 & 6.63 & 985.4 & 5.993 & 965.1 & 6.686 & 977.9 & 6.621 & 970.8 & 6.591 & 981.4 & 6.437 & 974.3 & 6.372 & 973.9 \\
\hline 6.778 & 944.8 & 6.195 & 914.4 & 6.156 & 943.9 & 5.837 & 937.8 & 6.476 & 954.1 & 6.505 & 951.4 & 6.381 & 956.7 & 6.289 & 950.6 & 6.221 & 950.6 \\
\hline 6.606 & 921.9 & 5.982 & 890.2 & 6.121 & 921 & 5.635 & 910 & 6.337 & 911.3 & 6.272 & 923.7 & 6.011 & 918.8 & 6.156 & 924.6 & 5.845 & 910.5 \\
\hline 6.209 & 882.2 & 5.689 & 848.8 & 6.02 & 887.1 & 5.446 & 883.1 & 5.958 & 891.1 & 6.156 & 889.3 & 5.843 & 893.7 & 5.967 & 899 & 5.783 & 883.1 \\
\hline 6.011 & 857.6 & 5.561 & 818.8 & 5.834 & 864.2 & 5.307 & 855.8 & 5.804 & 859.3 & 5.958 & 862.9 & 5.659 & 869 & 5.837 & 875.2 & 5.612 & 858.9 \\
\hline 5.845 & 832 & 5.348 & 793.7 & 5.709 & 841.3 & 5.153 & 832 & 5.65 & 838.6 & 5.798 & 836.9 & 5.529 & 841.7 & 5.322 & 832.9 & 5.455 & 834.2 \\
\hline 5.552 & 806.9 & 5.117 & 768.6 & 5.49 & 815.3 & 4.727 & 806.4 & 5.544 & 810.9 & 5.606 & 811.7 & 5.31 & 811.7 & 5.248 & 806 & 5.304 & 809.1 \\
\hline 5.508 & 775.2 & 5.064 & 747 & 5.366 & 792.3 & 4.81 & 780.4 & 5.348 & 789.3 & 5.449 & 787.1 & 5.197 & 788.4 & 4.999 & 782.7 & 5.112 & 785.3 \\
\hline 5.322 & 750.9 & 4.857 & 722.3 & 4.931 & 753.6 & 4.395 & 739.9 & 5.132 & 750.9 & 5.188 & 764.6 & 5.005 & 765.5 & 5.15 & 769.4 & 4.739 & 746.1 \\
\hline 5.153 & 730.7 & 4.555 & 682.6 & 4.718 & 721.8 & 4.469 & 732 & 4.795 & 723.6 & 5.103 & 723.6 & 4.608 & 724.5 & 4.614 & 725.4 & 4.54 & 723.6 \\
\hline 5.029 & 700.7 & 4.357 & 661.5 & 4.511 & 699.8 & 4.049 & 690.5 & 4.688 & 699.4 & 4.745 & 699.8 & 4.505 & 699.4 & 4.404 & 702.4 & 4.339 & 698.5 \\
\hline 4.774 & 674.2 & 4.191 & 635 & 4.478 & 672.5 & 3.863 & 680 & 4.457 & 668.1 & 4.6 & 676.4 & 4.339 & 672.5 & 4.336 & 672.9 & 4.197 & 672 \\
\hline 4.549 & 648.7 & 4.049 & 603.3 & 4.241 & 651.3 & 3.715 & 636.3 & 4.339 & 644.7 & 4.36 & 643 & 4.17 & 647.8 & 4.236 & 648.2 & 4.12 & 643 \\
\hline 4.197 & 612.1 & 3.883 & 578.2 & 4.07 & 616.1 & 3.416 & 611.7 & 4.126 & 619.2 & 4.218 & 616.1 & 3.946 & 622.2 & 4.028 & 624.4 & 3.99 & 614.8 \\
\hline 4.046 & 584.8 & 3.676 & 552.6 & 3.954 & 592.3 & 3.312 & 583 & 3.851 & 594 & 4.046 & 592.7 & 3.883 & 589.6 & 3.86 & 600.7 & 3.741 & 591 \\
\hline 3.904 & 557 & 3.552 & 528.8 & 3.747 & 568 & 3.185 & 559.2 & 3.667 & 565.4 & 3.922 & 565 & 3.712 & 565 & 3.706 & 574.7 & 3.582 & 563.6 \\
\hline 3.694 & 527.9 & 3.407 & 505.9 & 3.611 & 545.6 & 3.081 & 533.2 & 3.641 & 542.9 & 3.765 & 539 & 3.552 & 538.1 & 3.522 & 549.5 & 3.363 & 539.8 \\
\hline 3.502 & 501.1 & 3.173 & 480.8 & 3.454 & 519.1 & 2.933 & 500.6 & 3.448 & 518.7 & 3.596 & 515.2 & 3.315 & 510.8 & 3.146 & 507.2 & 3.265 & 512.5 \\
\hline 3.286 & 475.1 & 2.833 & 441.1 & 3.081 & 480.3 & 2.714 & 477.3 & 3.315 & 491.8 & 3.386 & 492.7 & 3.114 & 485.6 & 2.978 & 482.1 & 3.084 & 489.2 \\
\hline 3.182 & 449.9 & 2.753 & 411.2 & 2.898 & 457 & 2.371 & 453.5 & 3.194 & 466.7 & 3.191 & 454.8 & 2.966 & 462.7 & 2.788 & 453 & 2.714 & 447.7 \\
\hline 3.001 & 424.8 & 2.626 & 386.5 & 2.785 & 423.9 & 2.41 & 428.8 & 2.922 & 441.1 & 2.845 & 425.3 & 2.714 & 437.2 & 2.587 & 429.7 & 2.584 & 422.2 \\
\hline 2.821 & 402.8 & 2.371 & 361.4 & 2.634 & 400.6 & 1.983 & 388.7 & 2.785 & 395.7 & 2.7 & 401.9 & 2.537 & 400.1 & 2.504 & 396.2 & 2.356 & 397.5 \\
\hline 2.711 & 377.2 & 2.146 & 335.8 & 2.442 & 375.9 & 1.821 & 379.4 & 2.401 & 373.3 & 2.466 & 370.6 & 2.35 & 377.7 & 2.294 & 370.2 & 2.232 & 365.3 \\
\hline 2.552 & 349.9 & 2.051 & 312.9 & 2.297 & 349.9 & 1.599 & 333.2 & 2.253 & 343.3 & 2.374 & 342.4 & 2.238 & 354.3 & 2.108 & 343.7 & 2.093 & 341.1 \\
\hline 2.279 & 325.7 & 1.649 & 273.7 & 2.14 & 323.9 & 1.448 & 306.3 & 2.114 & 317.3 & 2.235 & 319.5 & 2.143 & 327.9 & 1.972 & 320.4 & 1.957 & 319.5 \\
\hline 1.927 & 283.8 & 1.575 & 246.3 & 1.927 & 300.5 & 1.477 & 282 & 2.007 & 293.9 & 2.01 & 293.1 & 1.886 & 299.7 & 1.803 & 300.1 & 1.803 & 290.9 \\
\hline & & & & & & & & & & & & & & & & & \\
\hline
\end{tabular}


Table 7. Rheometer Response for Blank - Pretreated - continued

\begin{tabular}{|c|c|c|c|c|c|c|c|c|c|c|c|c|c|c|c|c|c|}
\hline run1 & & run2 & & run3 & & run4 & & run5 & & run6 & & run7 & & run8 & & run9 & \\
\hline$[\mathrm{Pa}]$ & {$[1 / \mathrm{s}]$} & {$[\mathrm{Pa}]$} & {$[1 / s]$} & [Pa] & {$[1 / \mathbf{s}]$} & {$[\mathrm{Pa}]$} & {$[1 / \mathrm{s}]$} & [Pa] & {$[1 / s]$} & [Pa] & {$[1 / \mathbf{s}]$} & [Pa] & {$[1 / s]$} & [Pa] & {$[1 / s]$} & [Pa] & {$[1 / s]$} \\
\hline 1.696 & 258.7 & & & 1.779 & 276.3 & & & 1.815 & 268.4 & 1.88 & 267.5 & 1.687 & 273.7 & 1.445 & 259.6 & 1.599 & 268.8 \\
\hline 1.779 & 250.3 & & & 1.613 & 250.3 & & & 1.622 & 243.7 & 1.643 & 238.4 & 1.528 & 248.5 & 1.507 & 251.6 & 1.43 & 242.4 \\
\hline & & & & & & & & 1.466 & 154.1 & 1.477 & 148.9 & & & & & & \\
\hline & & & & & & & & & & & & & & & & & \\
\hline
\end{tabular}


Table 8. $25^{\circ} \mathrm{C}$ Pretreated

\begin{tabular}{|c|c|c|c|c|c|c|c|c|c|c|c|c|c|c|c|c|c|}
\hline run1 & & run2 & & run3 & & run4 & & run5 & & run6 & & run7 & & run8 & & run9 & \\
\hline [Pa] & {$[1 / \mathrm{s}]$} & {$[\mathrm{Pa}]$} & {$[1 / \mathrm{s}]$} & {$[\mathrm{Pa}]$} & {$[1 / \mathbf{s}]$} & {$[\mathrm{Pa}]$} & {$[1 / \mathrm{s}]$} & {$[\mathrm{Pa}]$} & {$[1 / \mathrm{s}]$} & {$[\mathrm{Pa}]$} & {$[1 / \mathrm{s}]$} & {$[\mathrm{Pa}]$} & {$[1 / \mathrm{s}]$} & {$[\mathrm{Pa}]$} & {$[1 / \mathrm{s}]$} & {$[\mathrm{Pa}]$} & {$[1 / s]$} \\
\hline 3.331 & 318.6 & 4.283 & 346.8 & 3.908 & 312 & 4.174 & 328.7 & 3.742 & 325.2 & 3.443 & 303.2 & 3.828 & 325.2 & 3.245 & 331.4 & 3.239 & 312.4 \\
\hline 4.212 & 23.9 & 766 & 372.8 & 4.621 & 343.3 & 4.295 & 345.9 & 4.112 & 328.3 & 3.754 & 310.2 & 4.13 & 340.2 & 4.062 & 327.9 & 4.008 & 315.5 \\
\hline 4.136 & 339.8 & 115 & 392.6 & 5.009 & 365.8 & 4.905 & 368.9 & 4.393 & 347.7 & 4.301 & 346.4 & 4.766 & 363.1 & 4.295 & 342 & 3.985 & 334 \\
\hline 4.65 & 361.4 & 5.074 & 416.9 & 5.062 & 389.6 & 5.124 & 393.1 & 4.952 & 372.4 & 4.887 & 369.7 & 4.923 & 386.9 & 4.47 & 366.2 & 4.562 & 371.1 \\
\hline 5.044 & 402.8 & 5.772 & 454.3 & 5.316 & 420.9 & 5.775 & 429.7 & 5.106 & 395.3 & 4.967 & 390.9 & 5.55 & 427.5 & 4.804 & 398.8 & 4.911 & 394.4 \\
\hline 5.606 & 425.7 & 6.056 & 483 & 5.725 & 445.5 & 5.902 & 455.7 & 5.467 & 426.1 & 5.189 & 423.9 & 5.879 & 454.8 & 5.266 & 423.5 & 5.068 & 425.7 \\
\hline 6.027 & 449.9 & 6.151 & 505.5 & 6.089 & 471.1 & 6.16 & 485.6 & 5.535 & 450.4 & 5.331 & 449.1 & 5.911 & 478.6 & 5.609 & 449.5 & 5.529 & 451.3 \\
\hline 6.29 & 475.9 & 6.417 & 526.6 & 6.482 & 494 & 6.263 & 506.3 & 5.787 & 481.7 & 5.651 & 473.7 & 6.29 & 508.5 & 5.606 & 471.1 & 5.704 & 473.3 \\
\hline 6.497 & 501.9 & 7.045 & 560.5 & 6.731 & 521.3 & 6.71 & 531 & 6.018 & 504.6 & 6.006 & 496.6 & 6.334 & 532.3 & 6.189 & 509 & 5.796 & 496.6 \\
\hline 6.817 & 534.1 & 326 & 592.3 & 7.048 & 555.7 & 7.213 & 564.1 & 6.281 & 531.9 & 6.343 & 520.9 & 6.592 & 555.7 & 6.45 & 536.8 & 5.89 & 520.4 \\
\hline 7.062 & 557.5 & 7.574 & 614.8 & 7.187 & 584.8 & 7.409 & 591.4 & 6.672 & 556.1 & 6.775 & 561.4 & 7.062 & 583 & 6.755 & 557.5 & 6.237 & 546 \\
\hline 7.346 & 581.7 & 7.746 & 638.1 & 7.595 & 610.8 & 7.548 & 616.1 & 6.858 & 579.5 & 6.95 & 583.9 & 7.281 & 608.6 & 6.692 & 587.9 & 6.663 & 589.6 \\
\hline 7.657 & 606.4 & 8.483 & 674.2 & 8.021 & 634.1 & 7.841 & 638.5 & 7.551 & 618.3 & 7.385 & 615.2 & 7.471 & 632.8 & 7.015 & 608.6 & 6.911 & 614.8 \\
\hline 8.258 & 646 & 8.687 & 694.1 & 8.335 & 658.4 & 7.977 & 676 & 7.77 & 642.5 & 7.311 & 642.1 & 7.932 & 672.9 & 7.054 & 634.1 & 7.418 & 641.2 \\
\hline 8.545 & 669 & 8.995 & 728.9 & 8.551 & 680.9 & 8.228 & 678.2 & 8.178 & 671.2 & 7.637 & 668.1 & 7.761 & 681.7 & 7.326 & 657.1 & 7.412 & 671.2 \\
\hline 8.527 & 693.6 & 9.024 & 756.2 & 8.578 & 705.5 & 10 & 708.6 & 8.504 & 695.8 & 8.187 & 694.1 & 8.542 & 726.7 & 7.823 & 697.2 & 7.583 & 693.6 \\
\hline 8.924 & 720.1 & & 780.9 & 9.35 & 750.5 & 10.15 & 737.7 & 8.69 & 728.9 & 8.172 & 721.4 & 8.557 & 754.9 & 8.4 & 724.5 & 8.06 & 721 \\
\hline 9.113 & 750.9 & 9.776 & 806.9 & 9.59 & 775.2 & 10.15 & 775.2 & 9.119 & 752.2 & 8.557 & 746.5 & 8.915 & 776.5 & 8.436 & 749.6 & 8.285 & 744.8 \\
\hline 9.412 & 771.6 & 10.11 & 831.6 & 9.948 & 802.5 & 10.08 & 796.8 & 9.11 & 779.6 & 8.98 & 770.8 & 9.066 & 808.7 & 8.853 & 781.3 & 8.385 & 769.9 \\
\hline 10.12 & 810.9 & 10.75 & 868.6 & 10.21 & 830.2 & 10.22 & 827.6 & 9.687 & 802.9 & 8.995 & 795.4 & 9.581 & 832 & 8.862 & 805.1 & 8.942 & 795 \\
\hline 10.39 & 834.2 & 10.92 & 897.2 & 10.67 & 857.1 & 10.48 & 863.7 & 9.797 & 830.7 & 9.758 & 834.7 & 9.531 & 856.7 & 9.383 & 830.2 & 8.888 & 820.1 \\
\hline 10.7 & 858 & 11.22 & 921 & 10.89 & 881.8 & 10.97 & 890.6 & 10.15 & 856.7 & 9.785 & 863.7 & 9.906 & 883.1 & 9.421 & 854.5 & 9.315 & 846.1 \\
\hline 10.88 & 887.1 & 11.65 & 944.8 & 11.16 & 913.5 & 11.4 & 914.9 & 10.44 & 881.8 & 10.1 & 889.3 & 10.45 & 909.1 & 9.853 & 879.2 & 9.889 & 888 \\
\hline 11.2 & 914 & 11.86 & 975.7 & 11.56 & 934.7 & 11.5 & 944.8 & 10.69 & 907.8 & 10.52 & 913.1 & 10.36 & 933.8 & 10.23 & 918.8 & 10.08 & 910.9 \\
\hline 11.46 & 943.9 & 12.27 & 998.6 & 11.83 & 960.2 & 11.84 & 969.9 & 11.29 & 947.9 & 10.62 & 935.1 & 10.75 & 956.7 & .24 & 926.8 & 10.25 & 944.8 \\
\hline 11.82 & 971.3 & 12.25 & 1022 & 12.03 & 983.6 & & 997.7 & & 970.4 & 11.08 & 958.9 & 11.4 & 999 & 54 & 955.4 & 10.51 & 968.6 \\
\hline 12.25 & 993.7 & 12.6 & 1047 & 12.29 & 1008 & 12.57 & 1020 & 11.92 & 1001 & 11.29 & 984.9 & 11.47 & 1029 & 10.79 & 984 & 10.83 & 995.1 \\
\hline 12.31 & 1016 & 12.96 & 1069 & 13.05 & 1053 & 13.18 & 1058 & 12.11 & 1025 & 11.58 & 1012 & 11.7 & 1054 & 11.37 & 1027 & 11.16 & 1021 \\
\hline 12.58 & 1039 & 3.66 & 1113 & 12.93 & 1061 & 13.39 & 1084 & 12.44 & 1054 & 11.76 & 1038 & 12.11 & 1075 & 11.42 & 1036 & 11.5 & 1047 \\
\hline 12.89 & 1068 & 13.86 & 1138 & 13.56 & 1101 & 13.66 & 1112 & 12.71 & 1077 & 12.2 & 1075 & 12.38 & 1110 & 11.67 & 1065 & 11.71 & 1071 \\
\hline 13.74 & 1109 & 14.24 & 1161 & 13.98 & 1133 & 14.03 & 1136 & 12.86 & 1099 & 12.53 & 1107 & 12.48 & 1118 & 12.26 & 1105 & 11.99 & 1098 \\
\hline
\end{tabular}


Table 8. $25{ }^{\circ} \mathrm{C}$ Pretreated - continued

\begin{tabular}{|c|c|c|c|c|c|c|c|c|c|c|c|c|c|c|c|c|c|}
\hline run1 & & run2 & & run3 & & run4 & & run5 & & run6 & & run7 & & run8 & & run9 & \\
\hline [Pa] & {$[1 / \mathrm{s}]$} & [Pa] & {$[1 / \mathrm{s}]$} & {$[\mathrm{Pa}]$} & {$[1 / \mathrm{s}]$} & [Pa] & {$[1 / \mathrm{s}]$} & [Pa] & {$[1 / \mathrm{s}]$} & {$[\mathrm{Pa}]$} & {$[1 / \mathrm{s}]$} & {$[\mathrm{Pa}]$} & {$[1 / \mathrm{s}]$} & {$[\mathrm{Pa}]$} & {$[1 / \mathrm{s}]$} & {$[\mathrm{Pa}]$} & {$[1 / \mathrm{s}]$} \\
\hline 14.03 & 1134 & 14.41 & 1191 & 14.24 & 1156 & 14.08 & 1164 & 13.43 & 1138 & 12.86 & 1136 & 13.16 & 1162 & 12.53 & 1130 & 12.29 & 1122 \\
\hline 14.3 & 1159 & 14.79 & 1213 & 14.54 & 1181 & 14.43 & 1188 & 13.73 & 1160 & 13.16 & 1156 & 13.4 & 1187 & 12.82 & 1158 & 12.52 & 1148 \\
\hline 14.53 & 1185 & 15.06 & 1237 & 14.8 & 1214 & 14.82 & 1214 & 14.06 & 1189 & 13.34 & 1181 & 13.66 & 1210 & 13.07 & 1184 & 12.96 & 1188 \\
\hline 14.9 & 1209 & 15.65 & 1276 & 15.26 & 1239 & 15.11 & 1242 & 14.2 & 1211 & 13.65 & 1204 & 13.93 & 1241 & 13.37 & 1207 & 13.16 & 1213 \\
\hline 15.2 & 1243 & 16 & 1300 & 15.51 & 1262 & 15.45 & 1267 & 14.7 & 1249 & 13.81 & 1228 & 14.09 & 1264 & 13.76 & 1244 & 13.55 & 1237 \\
\hline 15.56 & 1270 & 16.24 & 1322 & 15.8 & 1287 & 15.83 & 1293 & 14.97 & 1272 & 14.24 & 1255 & 14.51 & 1301 & 14.07 & 1271 & 13.77 & 1269 \\
\hline 15.93 & 1293 & 16.47 & 1355 & 16.15 & 1311 & 16.06 & 1319 & 15.35 & 1300 & 14.78 & 1298 & 14.84 & 1326 & 14.3 & 1304 & 14.01 & 1291 \\
\hline 16.1 & 1319 & 16.9 & 1379 & 16.43 & 1335 & 16.46 & 1342 & 15.63 & 1323 & 14.98 & 1325 & 15.2 & 1351 & 14.6 & 1325 & 14.24 & 1317 \\
\hline 16.41 & 1342 & 16.99 & 1405 & 17.04 & 1375 & 17.03 & 1384 & 15.96 & 1353 & 15.34 & 1352 & 15.34 & 1376 & 14.81 & 1352 & 14.53 & 1341 \\
\hline 17 & 1380 & 17.36 & 1427 & 17.37 & 1401 & 17.3 & 1408 & 16.16 & 1376 & 15.5 & 1376 & 15.58 & 1402 & 15.05 & 1374 & 15.01 & 1380 \\
\hline 17.37 & 1409 & 17.54 & 1452 & 17.54 & 1434 & 17.62 & 1435 & 16.4 & 1397 & 15.82 & 1402 & 15.88 & 1435 & 15.25 & 1407 & 15.32 & 1409 \\
\hline 17.45 & 1433 & 17.92 & 1479 & 17.92 & 1454 & 17.8 & 1466 & 17.01 & 1438 & 16.09 & 1424 & 16.09 & 1460 & 15.59 & 1428 & 15.62 & 1435 \\
\hline 17.89 & 1460 & 18.39 & 1520 & 18.22 & 1482 & 18.31 & 1492 & 17.32 & 1461 & 16.38 & 1449 & 16.46 & 1486 & 15.95 & 1457 & 15.69 & 1458 \\
\hline 18.22 & 1478 & 18.9 & 1542 & 18.48 & 1508 & 18.51 & 1515 & 17.33 & 1493 & 16.87 & 1490 & 16.29 & 1582 & 15.91 & 1570 & 15.98 & 1480 \\
\hline 18.57 & 1516 & 19.05 & 1573 & 18.78 & 1531 & 19.1 & 1560 & 16.88 & 1548 & 17.28 & 1517 & 15.94 & 1556 & 15.58 & 1545 & 14.67 & 1478 \\
\hline 17.74 & 1523 & 17.24 & 1467 & 17.57 & 1494 & 18.84 & 1528 & 16.23 & 1507 & 16.09 & 1540 & 15.55 & 1530 & 15.32 & 1513 & 14.4 & 1451 \\
\hline 17.39 & 1501 & 16.56 & 1427 & 17.21 & 1471 & 18.36 & 1501 & 15.97 & 1483 & 15.76 & 1515 & 15.23 & 1486 & 14.99 & 1489 & 14.34 & 1441 \\
\hline 16.83 & 1462 & 16.32 & 1404 & 16.86 & 1448 & 17.98 & 1478 & 15.61 & 1461 & 15.32 & 1475 & 14.84 & 1463 & 14.84 & 1465 & 13.98 & 1412 \\
\hline 16.62 & 1431 & 15.85 & 1375 & 16.23 & 1408 & 17.86 & 1451 & 15.38 & 1429 & 15.32 & 1466 & 14.64 & 1435 & 14.43 & 1440 & 13.78 & 1387 \\
\hline 16.35 & 1408 & 15.82 & 1352 & 15.91 & 1382 & 17.48 & 1428 & 15.02 & 1407 & 14.75 & 1422 & 14.22 & 1411 & 14.31 & 1415 & 13.19 & 1344 \\
\hline 16.06 & 1384 & 15.41 & 1325 & 15.76 & 1356 & 16.83 & 1385 & 14.96 & 1383 & 14.31 & 1399 & 13.98 & 1380 & 13.98 & 1390 & 13.1 & 1321 \\
\hline 15.64 & 1361 & 15.2 & 1298 & 15.41 & 1333 & 16.62 & 1362 & 14.46 & 1358 & 14.1 & 1367 & 13.72 & 1356 & 13.63 & 1364 & 12.65 & 1296 \\
\hline 15.2 & 1321 & 14.78 & 1277 & 14.96 & 1305 & 16.06 & 1333 & 14.1 & 1333 & 13.93 & 1341 & 13.6 & 1332 & 13.39 & 1337 & 12.36 & 1260 \\
\hline 14.81 & 1297 & 14.58 & 1251 & 14.52 & 1273 & 15.88 & 1308 & 13.78 & 1304 & 13.45 & 1316 & 13.19 & 1307 & 12.92 & 1297 & 12.23 & 1235 \\
\hline 14.55 & 1274 & 13.81 & 1211 & 14.37 & 1246 & 15.58 & 1283 & 13.6 & 1279 & 13.22 & 1292 & 12.83 & 1282 & 12.61 & 1274 & 11.93 & 1208 \\
\hline 14.19 & 1243 & 13.51 & 1187 & 14.13 & 1220 & 15.2 & 1252 & 13.3 & 1254 & 12.98 & 1269 & 12.51 & 1244 & 12.57 & 1243 & 11.53 & 1182 \\
\hline 14.01 & 1220 & 13.33 & 1160 & 13.81 & 1197 & 14.9 & 1227 & 12.98 & 1225 & 12.9 & 1242 & 12.21 & 1217 & 12.2 & 1218 & 11.3 & 1157 \\
\hline 13.63 & 1196 & 13.07 & 1134 & 13.42 & 1173 & 14.64 & 1204 & 12.64 & 1201 & 12.48 & 1216 & 11.87 & 1193 & 11.91 & 1196 & 11.07 & 1133 \\
\hline 13.01 & 1158 & 12.6 & 1101 & 13.19 & 1149 & 14.31 & 1179 & 12.19 & 1159 & 12.22 & 1190 & 11.71 & 1161 & 11.66 & 1170 & 10.87 & 1109 \\
\hline 12.86 & 1135 & 12.31 & 1076 & 12.99 & 1121 & 13.6 & 1139 & 11.88 & 1129 & 11.81 & 1166 & 11.29 & 1140 & 11.23 & 1144 & 10.57 & 1083 \\
\hline 12.69 & 1110 & 12.24 & 1053 & 12.27 & 1079 & 13.36 & 1115 & 11.69 & 1106 & 11.61 & 1140 & 10.84 & 1106 & 10.74 & 1104 & 9.998 & 1043 \\
\hline
\end{tabular}


Table 8. $25{ }^{\circ} \mathrm{C}$ Pretreated - continued

\begin{tabular}{|c|c|c|c|c|c|c|c|c|c|c|c|c|c|c|c|c|c|}
\hline run1 & & run2 & & run3 & & run4 & & run5 & & run6 & & run7 & & run8 & & run9 & \\
\hline [Pa] & {$[1 / \mathrm{s}]$} & {$[\mathrm{Pa}]$} & {$[1 / \mathrm{s}]$} & [Pa] & {$[1 / \mathrm{s}]$} & {$[\mathrm{Pa}]$} & {$[1 / \mathrm{s}]$} & [Pa] & {$[1 / \mathrm{s}]$} & {$[\mathrm{Pa}]$} & {$[1 / \mathrm{s}]$} & {$[\mathrm{Pa}]$} & {$[1 / \mathrm{s}]$} & {$[\mathrm{Pa}]$} & {$[1 / \mathrm{s}]$} & {$[\mathrm{Pa}]$} & {$[1 / \mathrm{s}]$} \\
\hline 12.33 & 1082 & 11.82 & 1030 & 12.05 & 1056 & 13.1 & 1091 & 11.47 & 1080 & 11.24 & 1095 & 10.89 & 1082 & 10.61 & 1080 & 9.755 & 1013 \\
\hline 11.84 & 1051 & 11.28 & 991.5 & 11.72 & 1027 & 12.7 & 1059 & 11.22 & 1054 & 10.91 & 1067 & 10.55 & 1060 & 10.25 & 1054 & 9.906 & 1003 \\
\hline 11.68 & 1029 & 10.89 & 964.2 & 11.39 & 997.3 & 12.5 & 1036 & 10.98 & 1029 & 10.61 & 1040 & 10.16 & 1027 & 10.05 & 1031 & 9.454 & 978.3 \\
\hline 11.48 & 1005 & 10.73 & 941.3 & 10.95 & 978.8 & 12.24 & 1011 & 10.62 & 1003 & 10.17 & 1022 & 10.11 & 1002 & 9.868 & 999.5 & 9.066 & 951.9 \\
\hline 11.04 & 982.7 & 10.44 & 910.9 & 10.81 & 943.9 & 11.86 & 987.6 & 10.2 & 977.4 & 9.972 & 989.3 & 9.779 & 976.1 & 9.655 & 973 & 8.542 & 906.9 \\
\hline 10.96 & 958.5 & 10.04 & 886.7 & 10.48 & 922.8 & 11.5 & 960.7 & 9.809 & 939.1 & 9.619 & 964.7 & 9.516 & 949.2 & 9.323 & 946.1 & 8.444 & 877.4 \\
\hline 10.34 & 916.6 & 871 & 859.3 & 10.16 & 899 & 11.33 & 935.1 & 9.605 & 911.3 & 9.468 & 943.9 & 8.986 & 921.5 & 8.888 & 921.5 & 8.098 & 854.9 \\
\hline 9.883 & 886.2 & 9.386 & 834.2 & 9.986 & 875.6 & 11.05 & 907.4 & 9.288 & 890.2 & 8.915 & 903.4 & 8.85 & 900.8 & 8.817 & 896.8 & 8.134 & 830.7 \\
\hline 9.844 & 863.3 & 9.326 & 809.1 & 9.714 & 850.1 & 10.35 & 865.5 & 9.016 & 865.9 & 8.794 & 878.3 & 8.415 & 859.3 & 8.427 & 872.1 & 7.607 & 806.4 \\
\hline 9.285 & 841.7 & 8.749 & 785.3 & 9.217 & 824.1 & 10.23 & 843 & 8.456 & 824.5 & 8.409 & 855.8 & 8.243 & 834.2 & 8.196 & 844.8 & 7.639 & 782.2 \\
\hline 9.152 & 818.3 & 8.489 & 761.5 & 8.708 & 779.1 & 9.705 & 817.5 & 8.036 & 803.8 & 8.143 & 822.8 & 7.962 & 811.7 & 7.983 & 818.3 & 7.077 & 741.7 \\
\hline 8.696 & 777.8 & 7.85 & 722.7 & 8.4 & 752.7 & 9.451 & 791 & 7.983 & 778.2 & 7.918 & 799 & 7.764 & 780.4 & 7.58 & 779.6 & 6.589 & 715.7 \\
\hline 8.314 & 755.3 & 7.858 & 692.3 & 7.992 & 728 & 9.107 & 760.2 & 7.58 & 748.3 & 7.912 & 774.7 & 7.489 & 755.3 & 7.045 & 748.7 & 6.45 & 687 \\
\hline 8.172 & 724 & & 665 & 7.989 & 697.2 & 8.977 & 736.4 & 7.29 & 725.8 & 7.429 & 750.5 & 7.074 & 732.9 & 6.911 & 725.8 & 6.201 & 666.8 \\
\hline 7.826 & 698 & 7.142 & 647.4 & 7.761 & 676.9 & 8.779 & 714.3 & 7.045 & 692.8 & 6.959 & 710.4 & 6.84 & 706.9 & 6.938 & 702.4 & 6.056 & 634.6 \\
\hline 7.468 & 674.2 & 6.716 & 614.8 & 7.468 & 653.1 & 8.181 & 674.2 & 6.769 & 669.4 & 6.482 & 687.9 & 6.885 & 680.9 & 6.589 & 676.4 & 5.991 & 613.9 \\
\hline 7.181 & 651.8 & 6.459 & 589.6 & 7.042 & 628 & 7.861 & 650.4 & 6.382 & 642.1 & 6.497 & 659.7 & 6.53 & 656.6 & 6.083 & 651.3 & 5.751 & 591 \\
\hline 6.775 & 624 & 6.151 & 567.2 & 6.459 & 591 & 7.367 & 624.4 & 6.213 & 618.3 & 6.231 & 633.7 & 6.133 & 630.6 & 5.935 & 614.3 & 5.127 & 546.4 \\
\hline 6.417 & 599.3 & 5.917 & 529.3 & 6.219 & 565 & 6.941 & 598.4 & 5.959 & 593.2 & 6.035 & 611.7 & 5.76 & 605.5 & 5.704 & 587 & 5.251 & 539.4 \\
\hline 6.207 & 572.9 & 5.592 & 503.7 & 6.035 & 537.6 & 6.669 & 568.9 & 5.624 & 556.6 & 5.556 & 577.3 & 5.408 & 559.7 & 5.331 & 558.3 & 4.861 & 512.1 \\
\hline 5.905 & 546.9 & 5.266 & 477.3 & 5.621 & 514.3 & 6.494 & 541.6 & 5.157 & 529.3 & 5.37 & 553.9 & 5.186 & 550.9 & 5.18 & 535 & 4.153 & 468.9 \\
\hline 5.609 & 506.8 & 4.864 & 452.1 & 5.287 & 487.4 & 6.083 & 517.4 & 5.1 & 507.2 & 5.257 & 530.1 & 4.946 & 509.9 & 5.003 & 510.8 & 4.094 & 444.6 \\
\hline 5.293 & 483.4 & & 427 & 4.932 & 464 & 5.825 & 491.8 & 4.858 & 480.8 & 5.127 & 506.8 & 4.582 & 478.1 & 4.781 & 487 & 4.041 & 420.9 \\
\hline 4.769 & 459.2 & 4.455 & 403.2 & 4.476 & 423.5 & 5.701 & 468.4 & 4.745 & 449.1 & 4.846 & 481.2 & 4.44 & 455.7 & 4.195 & 443.8 & 3.884 & 388.2 \\
\hline 4.553 & 426.1 & 4.13 & 377.7 & 4.224 & 403.7 & 5.574 & 442.4 & 4.26 & 423.9 & 4.526 & 454.8 & 3.961 & 430.1 & 4.053 & 423.5 & 3.369 & 363.6 \\
\hline 4.248 & 402.8 & 3.594 & 334.5 & 4.153 & 371.1 & 5.231 & 415.1 & 3.914 & 400.6 & 4.064 & 428.3 & 3.745 & 397.9 & 3.576 & 400.6 & 3.286 & 338.4 \\
\hline 4.263 & 379.4 & 3.055 & 309.8 & 3.712 & 347.3 & 4.695 & 375.5 & 4.011 & 378.1 & 3.671 & 386.5 & 3.538 & 370.6 & 3.39 & 368.9 & 2.949 & 313.3 \\
\hline 3.745 & 352.1 & 3.07 & 282.9 & 3.351 & 321.3 & 4.15 & 350.3 & 3.49 & 338 & 3.301 & 362.2 & 3.366 & 350.8 & 3.476 & 343.7 & 2.83 & 291.3 \\
\hline 3.082 & 312.4 & 2.57 & 260.9 & 3.304 & 295.3 & 3.976 & 320.8 & 2.901 & 315.1 & 3.325 & 331.4 & 2.78 & 314.6 & 2.913 & 318.6 & 2.52 & 264 \\
\hline 3.177 & 290 & 2.534 & 254.3 & 2.7 & 268.8 & 3.848 & 293.5 & 2.898 & 289.1 & 2.863 & 307.2 & 2.789 & 287.3 & 2.893 & 291.3 & 2.401 & 238.4 \\
\hline 2.549 & 262.6 & & & 2.611 & 244.6 & 3.399 & 271.5 & 2.345 & 261.8 & 2.81 & 282 & & & & & & \\
\hline 2.617 & 256 & & & & & & & & & 2.413 & 260 & & & & & & \\
\hline
\end{tabular}


Table 9. $50^{\circ} \mathrm{C}$ Pretreated

\begin{tabular}{|c|c|c|c|c|c|c|c|c|c|c|c|c|c|c|c|c|c|}
\hline run1 & & run2 & & run3 & & run4 & & run5 & & run6 & & run7 & & run8 & & run9 & \\
\hline$[\mathrm{Pa}]$ & {$[1 / \mathrm{s}]$} & {$[\mathrm{Pa}]$} & {$[1 / s]$} & [Pa] & {$[1 / \mathrm{s}]$} & [Pa] & {$[1 / \mathrm{s}]$} & {$[\mathrm{Pa}]$} & {$[1 / \mathrm{s}]$} & {$[\mathrm{Pa}]$} & {$[1 / \mathrm{s}]$} & {$[\mathrm{Pa}]$} & {$[1 / \mathrm{s}]$} & {$[\mathrm{Pa}]$} & {$[1 / \mathrm{s}]$} & {$[\mathrm{Pa}]$} & {$[1 / \mathrm{s}]$} \\
\hline 3.286 & 303.2 & 3.368 & 317.3 & 3.152 & 315.1 & 2.963 & 322.6 & 3.729 & 349 & 3.658 & 343.7 & 3.037 & 315.5 & 2.966 & 304.1 & 2.655 & 314.6 \\
\hline 3.877 & 318.2 & 3.886 & 349.5 & 3.653 & 331.4 & 3.371 & 323.5 & 3.839 & 356.5 & 3.777 & 367.1 & 3.7 & 346.8 & 3.383 & 323.5 & 3.09 & 322.6 \\
\hline 4.085 & 337.6 & 4.253 & 370.2 & 3.857 & 353 & 3.783 & 345.1 & 4.437 & 397.1 & 4.2 & 391.3 & 4.093 & 369.7 & 3.724 & 345.9 & 3.522 & 343.7 \\
\hline 4.289 & 364.9 & 4.709 & 401.9 & 4.413 & 390 & 4.111 & 368 & 4.582 & 422.2 & 4.478 & 415.6 & 4.224 & 398.4 & 3.786 & 369.7 & 3.676 & 365.8 \\
\hline 4.756 & 389.6 & 4.81 & 426.1 & 4.496 & 412.5 & 4.227 & 389.1 & 4.851 & 453.9 & 4.688 & 441.6 & 4.416 & 421.7 & 4.064 & 394 & 3.874 & 390.4 \\
\hline 5.094 & 417.8 & 4.878 & 449.1 & 4.682 & 444.2 & 4.46 & 415.1 & 5.002 & 480.3 & 5.106 & 483 & 4.603 & 449.5 & 4.384 & 418.6 & 4.17 & 416.9 \\
\hline 5.517 & 448.2 & 5.419 & 482.5 & 4.969 & 466.7 & 4.816 & 441.6 & 5.265 & 503.7 & 5.39 & 506.3 & 4.904 & 470.6 & 4.579 & 445.5 & 4.428 & 444.2 \\
\hline 5.745 & 473.7 & 677 & 510.8 & 5.283 & 489.2 & 5.117 & 467.6 & 5.425 & 529.3 & 5.55 & 531.9 & 4.999 & 502.8 & 4.78 & 472 & 4.715 & 468.9 \\
\hline 6.011 & 502.8 & 5.73 & 531 & 5.558 & 511.6 & 5.467 & 507.7 & 5.771 & 555.7 & 5.745 & 556.1 & 5.363 & 524.4 & 5.052 & 496.6 & 4.943 & 496.2 \\
\hline 6.295 & 533.2 & 6.257 & 562.8 & 5.908 & 551.7 & 5.695 & 529.7 & 5.949 & 578.2 & 5.934 & 584.3 & 5.745 & 561.4 & 5.224 & 523.1 & 5.251 & 538.1 \\
\hline 6.568 & 560.5 & 6.449 & 590.5 & 6.183 & 577.3 & 5.925 & 561.9 & 6.428 & 617 & 6.115 & 613.9 & 6.035 & 591.4 & 5.511 & 548.2 & 5.47 & 565 \\
\hline 6.935 & 583 & 6.63 & 609 & 6.473 & 601.5 & 6.115 & 584.3 & 6.621 & 641.2 & 6.334 & 639 & 6.26 & 614.3 & 5.695 & 572.9 & 5.81 & 592.3 \\
\hline 7.148 & 609.5 & 7.085 & 647.4 & 6.787 & 630.6 & 6.408 & 609.5 & 6.952 & 665.9 & 6.704 & 665.4 & 6.337 & 642.1 & 5.976 & 602 & 6.047 & 618.3 \\
\hline 7.37 & 639.9 & 7.325 & 670.3 & 6.988 & 657.1 & 6.674 & 635.9 & 7.127 & 698 & 6.872 & 689.7 & 6.582 & 667.2 & 6.488 & 646.9 & 6.221 & 642.1 \\
\hline 7.76 & 664.5 & 7.405 & 701.6 & 7.242 & 684.4 & 6.89 & 660.6 & 7.452 & 722.3 & 7.068 & 714.8 & 6.923 & 696.7 & 6.612 & 675.6 & 6.328 & 671.6 \\
\hline 7.964 & 692.3 & 7.734 & 721.8 & 7.606 & 708.6 & 7.097 & 684.4 & 7.775 & 749.2 & 7.375 & 738.1 & 7.08 & 721.8 & 6.713 & 682.6 & 6.565 & 695.8 \\
\hline 8.207 & 714.8 & 7.976 & 744.8 & 7.71 & 738.1 & 7.574 & 726.2 & 7.926 & 774.7 & 7.485 & 767.7 & 7.325 & 746.1 & 7.201 & 726.7 & 6.692 & 727.1 \\
\hline 8.707 & 753.1 & 8.509 & 784.4 & 8 & 769 & 7.929 & 747.8 & 8.018 & 807.3 & 8.062 & 807.3 & 7.663 & 772.5 & 7.364 & 753.1 & 6.878 & 750.5 \\
\hline 9.154 & 776.9 & 8.663 & 807.8 & 8.186 & 792.3 & 8.121 & 774.3 & 8.485 & 834.2 & 8.237 & 835.1 & 7.71 & 801.2 & 7.651 & 780 & 7.207 & 773.4 \\
\hline 9.272 & 802.9 & 8.962 & 836.4 & 8.42 & 821.4 & 8.515 & 804.2 & 8.589 & 858.9 & 8.604 & 862 & 7.967 & 826.3 & 7.737 & 802.9 & 7.287 & 803.8 \\
\hline 9.512 & 824.1 & 9.071 & 859.8 & 8.891 & 846.1 & 8.61 & 832.4 & 8.864 & 882.7 & 8.793 & 888 & 8.243 & 850.1 & 7.941 & 835.1 & 7.668 & 825.8 \\
\hline 10.12 & 866.4 & 9.441 & 880.5 & 8.985 & 871.2 & 8.938 & 855.8 & 9.027 & 906.5 & 8.917 & 912.7 & 8.722 & 887.1 & 8.311 & 860.7 & 7.935 & 865.1 \\
\hline 10.33 & 887.5 & $9.7 !$ & 917.9 & 379 & 894.6 & 9.074 & 877 & 9.5 & 946.6 & 9.095 & 942.2 & 8.917 & 917.1 & 8.402 & 885.3 & 8.124 & 892.4 \\
\hline 10.65 & 910 & & 944.4 & 9.489 & 920.1 & 9.261 & 905.6 & 9.885 & 969.9 & 9.483 & 966 & 8.938 & 940 & 8.698 & 910 & 8.343 & 917.1 \\
\hline 10.88 & 942.2 & 10.25 & 971.3 & 10.05 & 959.8 & 9.634 & 929 & 10.19 & 998.6 & 9.663 & 990.2 & 9.252 & 966 & 9.009 & 936 & 8.417 & 942.6 \\
\hline 11.21 & 966.9 & 10.43 & 991.5 & 10.14 & 988.5 & 9.915 & 954.5 & 10.41 & 1026 & 9.909 & 1018 & 9.489 & 989.8 & 9.107 & 960.2 & 8.669 & 965.5 \\
\hline 11.52 & 992.9 & 10.9 & 1028 & 10.51 & 1010 & 10.04 & 980.5 & 10.61 & 1049 & 10.22 & 1044 & 9.755 & 1015 & 9.551 & 999 & 8.903 & 992 \\
\hline 11.87 & 1019 & 11.03 & 1056 & 10.86 & 1049 & 10.6 & 1023 & 10.71 & 1072 & 10.29 & 1067 & 10.17 & 1052 & 9.743 & 1029 & 9.252 & 1017 \\
\hline 12.03 & 1041 & 11.42 & 1077 & 11.13 & 1071 & 10.9 & 1047 & 11.21 & 1112 & 10.47 & 1107 & 10.31 & 1075 & 10.1 & 1049 & 9.415 & 1045 \\
\hline 12.59 & 1078 & 11.52 & 1104 & 11.37 & 1094 & 11.13 & 1079 & 11.41 & 1135 & 10.36 & 1108 & 10.58 & 1105 & 10.38 & 1074 & 9.642 & 1072 \\
\hline 12.86 & 1106 & 12.16 & 1139 & 11.72 & 1125 & 11.4 & 1104 & 11.76 & 1162 & 12.06 & 1150 & 10.89 & 1129 & 10.6 & 1108 & 9.861 & 1098 \\
\hline
\end{tabular}


Table 9. $50{ }^{\circ} \mathrm{C}$ Pretreated - continued

\begin{tabular}{|c|c|c|c|c|c|c|c|c|c|c|c|c|c|c|c|c|c|}
\hline run1 & & run2 & & run3 & & run4 & & run5 & & run6 & & run7 & & run8 & & run9 & \\
\hline [Pa] & {$[1 / \mathrm{s}]$} & {$[\mathrm{Pa}]$} & {$[1 / \mathrm{s}]$} & {$[\mathrm{Pa}]$} & {$[1 / s]$} & {$[\mathrm{Pa}]$} & {$[1 / \mathrm{s}]$} & {$[\mathrm{Pa}]$} & {$[1 / \mathrm{s}]$} & {$[\mathrm{Pa}]$} & {$[1 / \mathrm{s}]$} & [Pa] & {$[1 / \mathrm{s}]$} & {$[\mathrm{Pa}]$} & {$[1 / \mathrm{s}]$} & {$[\mathrm{Pa}]$} & {$[1 / \mathrm{s}]$} \\
\hline 13.17 & 1130 & 12.35 & 1161 & 11.87 & 1147 & 11.6 & 1131 & 11.96 & 1187 & 12.07 & 1185 & 11.07 & 1158 & 10.91 & 1134 & 10.3 & 1137 \\
\hline 13.41 & 1157 & 12.67 & 1194 & 12.11 & 1175 & 11.86 & 1157 & 12.15 & 1213 & 12.32 & 1223 & 11.42 & 1182 & 11.11 & 1159 & 10.55 & 1159 \\
\hline 13.86 & 1180 & 12.84 & 1216 & 12.45 & 1200 & 12.24 & 1186 & 12.49 & 1238 & 12.3 & 1238 & 11.74 & 1215 & 11.31 & 1182 & 10.7 & 1189 \\
\hline 14.11 & 1205 & 13.02 & 1237 & 12.7 & 1227 & 12.52 & 1210 & 12.79 & 1266 & 12.51 & 1262 & 11.93 & 1237 & 11.48 & 1207 & 10.92 & 1220 \\
\hline 14.4 & 1229 & 13.43 & 1270 & 13.08 & 1251 & 12.78 & 1233 & 12.98 & 1289 & 12.63 & 1293 & 12.2 & 1259 & 11.72 & 1230 & 11.14 & 1243 \\
\hline 14.96 & 1269 & 13.83 & 1297 & 13.28 & 1276 & 13.05 & 1257 & 13.24 & 1315 & 12.92 & 1322 & 12.6 & 1296 & 11.94 & 1256 & 11.34 & 1267 \\
\hline 15.24 & 1293 & 14.12 & 1329 & 13.54 & 1301 & 13.21 & 1282 & 13.52 & 1340 & 13.25 & 1345 & 12.85 & 1322 & 12.4 & 1298 & 11.6 & 1292 \\
\hline 15.47 & 1323 & 14.27 & 1348 & 13.89 & 1331 & 13.76 & 1323 & 14.16 & 1384 & 13.39 & 1381 & 13.06 & 1348 & 12.76 & 1322 & 11.97 & 1329 \\
\hline 15.77 & 1346 & 14.46 & 1371 & 14.37 & 1356 & 14 & 1354 & 14.34 & 1409 & 13.7 & 1401 & 13.29 & 1370 & 12.91 & 1352 & 12.25 & 1358 \\
\hline 16.06 & 1370 & 15.11 & 1408 & 14.53 & 1386 & 14.31 & 1377 & 14.62 & 1434 & 13.98 & 1433 & 13.55 & 1401 & 13.09 & 1374 & 12.38 & 1380 \\
\hline 16.29 & 1391 & 15.31 & 1436 & 15.01 & 1416 & 14.5 & 1404 & 14.91 & 1463 & 14.19 & 1458 & 13.69 & 1426 & 13.39 & 1398 & 12.62 & 1404 \\
\hline 16.85 & 1434 & 15.47 & 1460 & 15.2 & 1443 & 14.87 & 1429 & 15.18 & 1487 & 14.47 & 1485 & 13.96 & 1452 & 13.86 & 1434 & 12.82 & 1432 \\
\hline 17.17 & 1457 & 15.86 & 1485 & 15.58 & 1471 & 15.06 & 1457 & 15.32 & 1517 & 14.74 & 1514 & 14.31 & 1478 & 13.98 & 1465 & 13.1 & 1460 \\
\hline 17.26 & 1489 & 16.31 & 1523 & 15.9 & 1495 & 15.28 & 1482 & 15.56 & 1538 & 14.94 & 1541 & 14.5 & 1501 & 14.16 & 1486 & 13.26 & 1485 \\
\hline 17.58 & 1514 & 16.51 & 1548 & 16.19 & 1521 & 15.58 & 1504 & 15.83 & 1563 & 15.28 & 1565 & 14.65 & 1528 & 14.39 & 1509 & 13.54 & 1513 \\
\hline 15.54 & 1515 & 14.65 & 1482 & 14.59 & 1514 & 14.18 & 1508 & 13.94 & 1495 & 13.2 & 1496 & 13.76 & 1511 & 12.6 & 1506 & 12.64 & 1542 \\
\hline 15.27 & 1486 & 14.33 & 1457 & 14.48 & 1489 & 13.79 & 1485 & 13.68 & 1473 & 12.52 & 1433 & 13.44 & 1489 & 12.67 & 1475 & 12.39 & 1516 \\
\hline 14.95 & 1465 & 13.97 & 1433 & 14.15 & 1458 & 13.62 & 1456 & 13.03 & 1434 & 12.67 & 1423 & 13.03 & 1455 & 12.69 & 1474 & 12.15 & 1491 \\
\hline 14.65 & 1436 & 13.74 & 1407 & 13.91 & 1431 & 13.41 & 1418 & 12.79 & 1410 & 12.47 & 1391 & 12.73 & 1430 & 11.82 & 1441 & 11.9 & 1462 \\
\hline 14.48 & 1412 & 13.53 & 1382 & 13.56 & 1404 & 12.58 & 1393 & 12.71 & 1381 & 11.65 & 1349 & 12.57 & 1404 & 11.92 & 1428 & 11.69 & 1435 \\
\hline 14.24 & 1391 & 13.17 & 1363 & 13.35 & 1378 & 12.67 & 1375 & 12.34 & 1357 & 11.63 & 1334 & 12.37 & 1377 & 11.5 & 1404 & 11.52 & 1411 \\
\hline 13.94 & 1363 & 12.7 & 1332 & 13 & 1355 & 12.42 & 1354 & 12.04 & 1330 & 11.44 & 1304 & 12.13 & 1352 & 11.57 & 1393 & 11.24 & 1384 \\
\hline 13.32 & 1326 & 12.56 & 1314 & 12.92 & 13 & 12.51 & 1332 & 11.86 & 1304 & 10.84 & 1255 & .88 & 1330 & 11.4 & 1364 & 10.93 & 1360 \\
\hline 13.11 & 1295 & 12.35 & 1282 & 12.36 & 1292 & 11.73 & 1300 & 11.59 & 1271 & 10.86 & 1235 & 11.48 & 1293 & 11.17 & 1338 & 10.76 & 1335 \\
\hline 12.84 & 1270 & 12.15 & 1241 & 12.08 & 1269 & 11.71 & 1285 & 11.34 & 1246 & 10.84 & 1222 & 11.26 & 1263 & 10.85 & 1309 & 10.6 & 1310 \\
\hline 12.6 & 1245 & 12.32 & 1240 & 11.89 & 1242 & 11.43 & 1250 & 11.13 & 1225 & 10.32 & 1206 & 10.87 & 1239 & 10.5 & 1284 & 10.04 & 1266 \\
\hline 12.33 & 1222 & 11.47 & 1207 & 11.59 & 1211 & 11.52 & 1227 & 10.92 & 1201 & 10.07 & 1162 & 10.72 & 1213 & 10.33 & 1258 & 9.823 & 1243 \\
\hline 12.05 & 1200 & 11.41 & 1166 & 11.33 & 1185 & 10.72 & 1196 & 10.38 & 1162 & 9.95 & 1138 & 10.49 & 1190 & 9.87 & 1221 & 9.675 & 1218 \\
\hline 11.41 & 1160 & 11.32 & 1155 & 11.12 & 1158 & 10.78 & 1180 & 10.18 & 1137 & 9.657 & 1111 & 10.2 & 1169 & 9.693 & 1186 & 9.483 & 1187 \\
\hline 11.31 & 1131 & 10.31 & 1138 & 10.78 & 1134 & 10.02 & 1145 & 9.906 & 1106 & 9.435 & 1081 & 10.02 & 1141 & 9.391 & 1163 & 9.24 & 1163 \\
\hline 10.92 & 1111 & 10.39 & 1118 & 10.49 & 1111 & 9.897 & 1128 & 9.675 & 1085 & 9.19 & 1052 & 9.527 & 1100 & 9.181 & 1137 & 8.994 & 1141 \\
\hline
\end{tabular}


Table 9. $50{ }^{\circ} \mathrm{C}$ Pretreated - continued

\begin{tabular}{|c|c|c|c|c|c|c|c|c|c|c|c|c|c|c|c|c|c|}
\hline run1 & & run2 & & run3 & & run4 & & run5 & & run6 & & run7 & & run8 & & run9 & \\
\hline [Pa] & {$[1 / \mathrm{s}]$} & {$[\mathrm{Pa}]$} & {$[1 / s]$} & [Pa] & {$[1 / s]$} & {$[\mathrm{Pa}]$} & {$[1 / \mathrm{s}]$} & [Pa] & {$[1 / \mathrm{s}]$} & {$[\mathrm{Pa}]$} & {$[1 / \mathrm{s}]$} & {$[\mathrm{Pa}]$} & {$[1 / \mathrm{s}]$} & {$[\mathrm{Pa}]$} & {$[1 / \mathrm{s}]$} & {$[\mathrm{Pa}]$} & {$[1 / \mathrm{s}]$} \\
\hline 10.74 & 1082 & 9.66 & 1062 & 10.23 & 1083 & 9.811 & 1090 & 9.509 & 1062 & 8.835 & 1027 & 9.213 & 1075 & 9.077 & 1113 & 8.811 & 1116 \\
\hline 10.41 & 1051 & 9.79 & 1048 & .953 & 1057 & 9.533 & 1062 & 8.959 & 1025 & 8.63 & 1001 & 8.98 & 1051 & 8.796 & 1086 & 8.539 & 1088 \\
\hline 10.23 & 1026 & 9.438 & 1013 & 9.571 & 1031 & 9.053 & 1019 & 8.775 & 1003 & 8.462 & 977 & 8.71 & 1022 & 8.544 & 1060 & 8.296 & 1060 \\
\hline 9.755 & 1004 & .181 & 981.8 & 9.441 & 1005 & 8.787 & 992.9 & 8.598 & 976.6 & 8.098 & 953.2 & 8.37 & 997.3 & 8.121 & 1023 & 8.089 & 1034 \\
\hline 9.373 & 966.9 & 8.805 & 941.3 & 9.009 & 964.2 & 8.586 & 969.5 & 8.314 & 944.8 & 7.63 & 913.1 & 8.284 & 971.3 & 7.864 & 995.1 & 7.594 & 996.4 \\
\hline 9.053 & 943.9 & 8.429 & 914.9 & 8.704 & 940 & 8.334 & 942.2 & 8.041 & 920.1 & 7.565 & 886.2 & 8.041 & 948.8 & 7.627 & 974.8 & 7.449 & 971.3 \\
\hline 8.802 & 914.9 & 8.183 & 886.7 & 8.379 & 915.3 & 8.053 & 917.1 & 7.876 & 897.7 & 7.219 & 865.1 & .805 & 920.1 & 7.529 & 945.3 & 171 & 946.6 \\
\hline 8.826 & 891.1 & 044 & 863.3 & 8.216 & 886.7 & 7.778 & 886.2 & 7.346 & 860.7 & 7.091 & 833.3 & .435 & 893.7 & 7.281 & 919.7 & 7.148 & 913.5 \\
\hline 8.385 & 869.5 & 7.642 & 828.9 & 8.092 & 859.3 & 7.568 & 862.4 & 7.091 & 839.5 & 6.736 & 808.2 & 7.325 & 867.3 & 7.148 & 888.9 & 6.816 & 891.1 \\
\hline 8.32 & 842.6 & 7.216 & 803.4 & 7.577 & 834.7 & 7.411 & 838.6 & 6.926 & 809.1 & 6.568 & 777.8 & 6.784 & 828.9 & 6.677 & 865.9 & 6.659 & 864.6 \\
\hline 7.843 & 818.3 & 7.159 & 775.2 & 7.452 & 809.5 & 6.878 & 800.3 & 6.668 & 785.7 & 6.18 & 759.7 & 6.609 & 799.8 & 6.446 & 832.9 & 6.399 & 839.5 \\
\hline 7.378 & 776.5 & 6.748 & 746.1 & 7.239 & 783.1 & 6.698 & 771.2 & 6.508 & 764.1 & 6.002 & 724.9 & 6.384 & 773.8 & 6.118 & 808.7 & 6.227 & 814.8 \\
\hline 7.154 & 752.2 & 6.565 & 722.7 & 6.84 & 760.6 & 6.44 & 745.6 & 6.097 & 725.8 & 5.697 & 698.9 & 6.064 & 750.9 & 6.091 & 781.3 & 5.973 & 790.6 \\
\hline 6.819 & 721 & 6.408 & 698 & 6.417 & 714.3 & 6.168 & 723.6 & 5.869 & 700.2 & 5.44 & 674.7 & 5.925 & 728 & 5.774 & 756.7 & 5.538 & 751.4 \\
\hline 6.609 & 697.6 & 6.097 & 670.7 & 6.251 & 689.2 & 5.925 & 699.4 & 5.541 & 671.2 & 5.354 & 646.9 & 5.789 & 702.9 & 5.535 & 733.3 & 203 & 728.4 \\
\hline 6.417 & 674.2 & 5.828 & 648.2 & 6.088 & 681.3 & 5.766 & 675.6 & 5.523 & 643 & 5.159 & 623.1 & 5.387 & 674.2 & 5.47 & 706.4 & 5.233 & 696.3 \\
\hline 6.215 & 648.2 & 5.443 & 609.9 & 5.837 & 654.4 & 5.585 & 650.4 & 5.191 & 617 & 4.866 & 595.4 & 5.129 & 649.1 & 5.188 & 680 & 5.008 & 673.4 \\
\hline 5.757 & 622.7 & 5.224 & 584.3 & 5.26 & 610.3 & 5.035 & 608.6 & 4.955 & 592.3 & 4.594 & 574.2 & 4.901 & 623.1 & 4.884 & 655.3 & 4.759 & 650.4 \\
\hline 5.564 & 599.8 & 4.916 & 555.3 & 5.041 & 584.3 & 4.774 & 585.7 & 4.762 & 568.9 & 3.999 & 530.1 & 4.771 & 595.4 & 4.653 & 628 & 4.395 & 612.5 \\
\hline 5.1 & 557.9 & 4.629 & 537.6 & 4.771 & 560.5 & 4.561 & 560.5 & 4.469 & 545.6 & 4.224 & 521.3 & 4.493 & 571.1 & 4.543 & 602 & 4.132 & 591 \\
\hline 4.822 & 532.8 & 4.487 & 505 & 4.605 & 530.1 & 4.31 & 532.3 & 4.194 & 521.8 & 3.848 & 495.8 & 3.969 & 524.9 & 4.002 & 560.1 & 4.046 & 561.9 \\
\hline 4.647 & 503.7 & 4.17 & 481.2 & 04 & 506.8 & 4.025 & 506.8 & 3.685 & 478.6 & 3.306 & 449.5 & 3.688 & 499.7 & 3.718 & 535.4 & 3.795 & 539.8 \\
\hline 4.274 & 481.2 & 3.818 & 457.9 & & 480.3 & & 471.5 & 3.555 & 453.5 & 79 & 420.9 & 3.448 & 472 & 3.543 & 504.6 & & 515.2 \\
\hline 4.046 & 457.9 & 3.463 & 415.6 & 3.809 & 454.3 & 3.472 & 450.4 & 3.33 & 427.9 & 2.877 & 397.1 & 3.28 & 448.6 & 3.365 & 479.5 & 3.297 & 476.4 \\
\hline 3.522 & 420.4 & 3.114 & 394 & 3.584 & 425.7 & 3.182 & 419.1 & 3.07 & 395.7 & 2.492 & 368.9 & 3.096 & 423.5 & 3.297 & 456.5 & 3.055 & 447.3 \\
\hline 3.445 & 397.5 & 3.055 & 367.1 & 3.389 & 398.8 & 2.966 & 396.6 & 2.756 & 368.9 & 2.466 & 342.4 & 2.794 & 394.9 & 3.164 & 429.7 & 3.046 & 440.2 \\
\hline 3.179 & 373.3 & 2.652 & 344.2 & 3.001 & 373.7 & 2.806 & 371.1 & 2.643 & 348.1 & 2.241 & 314.6 & 2.525 & 365.8 & 2.812 & 404.1 & 2.445 & 394.9 \\
\hline 2.803 & 343.3 & 2.439 & 313.3 & 2.815 & 349.9 & 2.572 & 337.1 & 2.294 & 316 & 1.936 & 290 & 2.525 & 342.4 & 2.575 & 378.5 & 2.466 & 371.1 \\
\hline 2.756 & 317.3 & 2.365 & 289.5 & 2.315 & 308.9 & 2.268 & 313.3 & 2.099 & 291.3 & 1.847 & 259.6 & 2.173 & 318.6 & 2.442 & 352.5 & 2.143 & 345.5 \\
\hline 2.374 & 294.4 & 2.01 & 266.2 & 2.022 & 286.4 & 2.146 & 284.2 & 1.862 & 265.7 & 0 & 161.6 & 2.046 & 294.4 & 2.031 & 312 & 1.912 & 314.6 \\
\hline 2.265 & 272.3 & 1.809 & 237.1 & 1.927 & 260.4 & 1.838 & 262.6 & 1.711 & 242.8 & 0 & 176.8 & 1.605 & 257.4 & 1.649 & 285.6 & 1.915 & 289.1 \\
\hline 1.847 & 247.7 & 0 & 149.8 & 1.824 & 251.6 & 1.634 & 237.1 & & 158.6 & 0 & 177.3 & 1.687 & 249.4 & 1.797 & 275 & 1.525 & 264.9 \\
\hline
\end{tabular}


This page intentionally left blank. 


\section{APPENDIX E \\ ENVELOPE C SR/TRU PRECIPITATE SLURRY RHEOLOGY DATA}

Table 1. Rheometer Response for Blank - 2 wt \% ......................................................... 175

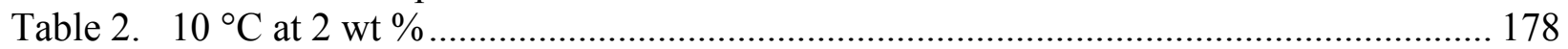

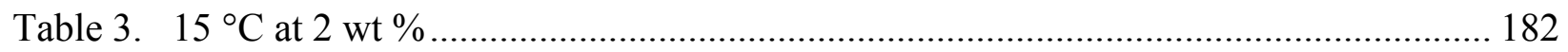

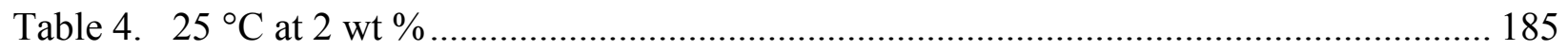

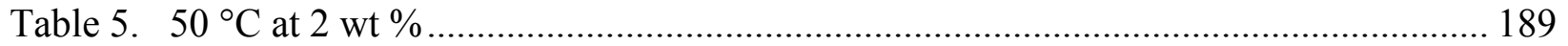

Table 6. Rheometer Response for Blank - 10 wt \% .......................................................... 192

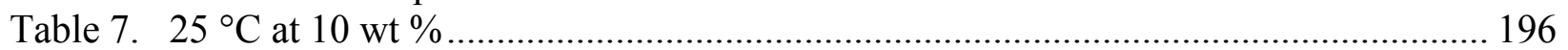

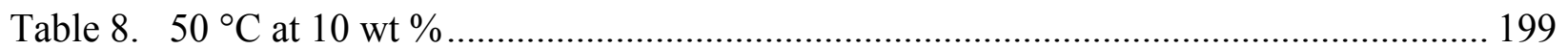

Table 9. Rheometer Response for Blank - 13 wt \% ........................................................ 202

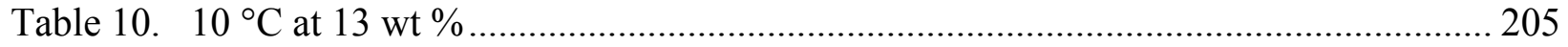

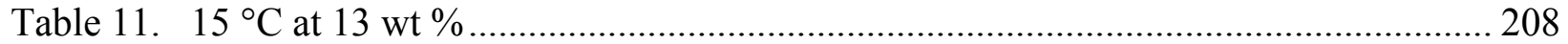

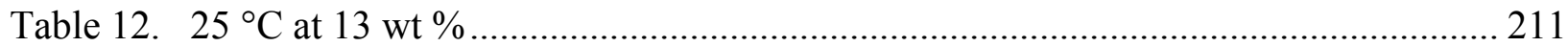

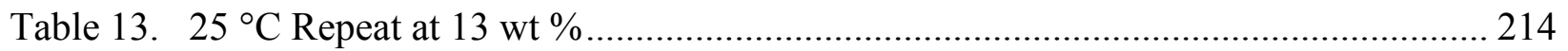

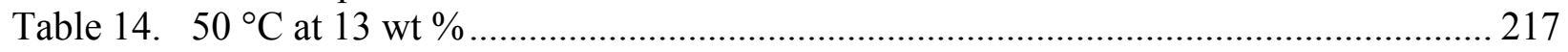

Table 15. Rheometer Response for Blank - 16 wt \% ..................................................... 220

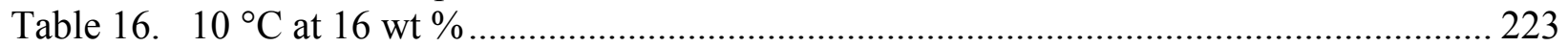

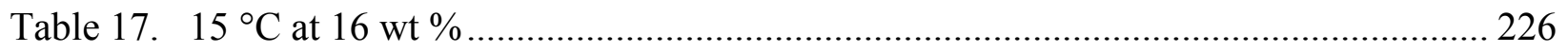

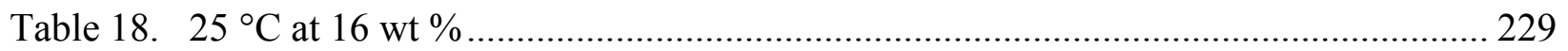

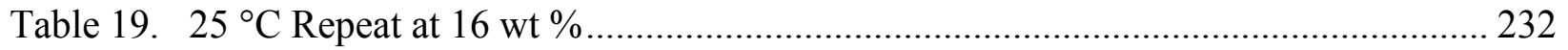

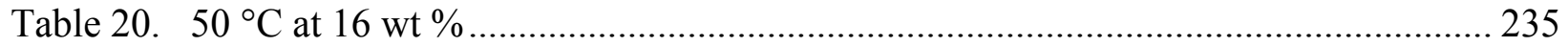




\begin{tabular}{|ll|}
\hline APPENDIX E & WRC-TR-2000-00352 \\
ENVELOPE C & SRT-RPP-2000-00026 \\
SR/TRU PRECIPITATE SLURRY RHEOLOGY DATA & Page 174 of 514 \\
\hline
\end{tabular}

This page intentionally left blank. 
Table 1. Rheometer Response for Blank - 2 wt \%

\begin{tabular}{|c|c|c|c|c|c|c|c|c|c|c|c|c|c|c|c|c|c|}
\hline run1 & & run2 & & run3 & & run4 & & run5 & & run6 & & run7 & & run8 & & run9 & \\
\hline [Pa] & {$[1 / \mathrm{s}]$} & [Pa] & {$[1 / \mathrm{s}]$} & [Pa] & [1/s] & [Pa] & {$[1 / s]$} & [Pa] & {$[1 / \mathrm{s}]$} & [Pa] & {$[1 / \mathrm{s}]$} & [Pa] & {$[1 / s]$} & [Pa] & {$[1 / \mathrm{s}]$} & [Pa] & {$[1 / s]$} \\
\hline 2.379 & 272.3 & 2.417 & 279 & 2.5 & 275.4 & 2.58 & 279 & 2.61 & 272.3 & 2.568 & 274.5 & 2.633 & 275 & 2.497 & 279.8 & 2.497 & 275 \\
\hline 2.784 & 294.8 & 2.663 & 297 & 2.698 & 290.4 & 2.746 & 292.6 & 2.811 & 296.1 & 2.725 & 294.4 & 2.849 & 297 & 2.778 & 293.1 & 2.722 & 291.3 \\
\hline 2.971 & 321.3 & 2.826 & 321.7 & 2.95 & 323.5 & 3.045 & 323.9 & 2.926 & 319.9 & 2.968 & 325.7 & 3.039 & 321.7 & 3.021 & 325.2 & 2.867 & 314.6 \\
\hline 3.098 & 349 & 3.039 & 349.5 & 3.196 & 350.3 & 3.216 & 352.5 & 3.092 & 345.1 & 3.16 & 349.5 & 3.193 & 351.7 & 3.169 & 349 & 3.157 & 346.8 \\
\hline 3.305 & 374.1 & 3.29 & 382.1 & 3.287 & 377.2 & 3.305 & 375.5 & 3.216 & 371.5 & 3.284 & 377.2 & 3.299 & 375.5 & 3.296 & 379.4 & 3.317 & 375.9 \\
\hline 3.557 & 398.4 & 3.385 & 408.1 & 3.429 & 401 & 3.613 & 407.6 & 3.483 & 404.5 & 3.465 & 402.3 & 3.418 & 399.3 & 3.468 & 404.5 & 3.527 & 401.9 \\
\hline 3.841 & 432.3 & 3.563 & 431.4 & 3.657 & 433.6 & 3.737 & 432.3 & 3.687 & 432.3 & 3.613 & 428.3 & 3.702 & 431.4 & 3.672 & 429.2 & 3.619 & 430.5 \\
\hline 4.004 & 457.4 & 3.82 & 464 & 3.861 & 458.8 & 3.903 & 458.8 & 3.764 & 456.5 & 3.708 & 455.2 & 3.805 & 460.1 & 3.859 & 460.1 & 3.734 & 455.7 \\
\hline 4.107 & 485.6 & 3.992 & 488.3 & 3.977 & 486.5 & 4.012 & 488.7 & 3.912 & 481.7 & 3.998 & 487.8 & 3.977 & 484.8 & 3.995 & 484.8 & 3.918 & 487 \\
\hline 4.297 & 507.7 & & 516.9 & & 509.4 & & 512.5 & & 515.2 & & 513.8 & & 509.9 & & 514.7 & & 505.9 \\
\hline 4.548 & 540.7 & 4.237 & 541.6 & 4.329 & 542 & 4.341 & 537.2 & 4.332 & 543.4 & 4.208 & 536.8 & 4.368 & 542.5 & 4.314 & 542.5 & 4.214 & 534.1 \\
\hline 4.705 & 565 & 4.433 & 569.4 & 4.462 & 570.7 & 4.607 & 571.6 & 4.518 & 568.5 & 4.468 & 568 & 4.554 & 566.7 & 394 & 571.1 & 4.436 & 565.4 \\
\hline 4.877 & 595.4 & 4.572 & 592.3 & 4.563 & 593.6 & 4.752 & 597.6 & 4.566 & 594 & 4.598 & 595.4 & 4.637 & 597.6 & 4.572 & 595.4 & 4.595 & 594.5 \\
\hline 5.06 & 620.5 & 4.85 & 626.7 & 4.77 & 618.7 & 4.826 & 625.3 & 4.743 & 619.6 & 4.803 & 625.3 & 4.832 & 623.6 & 735 & 618.7 & 4.77 & 620.9 \\
\hline 5.205 & 646.5 & 4.974 & 649.1 & 4.977 & 650.4 & 4.951 & 648.2 & 4.894 & 646 & 4.945 & 651.3 & 4.945 & 648.7 & 4.962 & 655.3 & 4.88 & 644.7 \\
\hline 5.347 & 671.2 & 5.175 & 678.2 & 5.184 & 676.4 & 5.122 & 674.2 & 5.087 & 671.6 & 5.042 & 678.2 & 5.175 & 672.9 & 5.158 & 680.4 & 5.128 & 677.8 \\
\hline 5.649 & 704.2 & & 705.5 & & 706 & & 708.6 & & 703.8 & & 701.1 & 5.294 & 699.4 & & 706.9 & & 703.3 \\
\hline 5.859 & 729.8 & 5.445 & 729.3 & 5.463 & 732.9 & 5.465 & 726.7 & 5.38 & 732 & 5.477 & 732.4 & 5.516 & 733.7 & 5.394 & 733.3 & 5.448 & 732.4 \\
\hline 5.939 & 757.5 & 5.56 & 752.2 & 5.667 & 755.3 & 5.75 & 759.7 & 5.631 & 758.4 & 5.643 & 758.9 & 5.75 & 758 & 5.539 & 757.5 & 31 & 755.3 \\
\hline 6.105 & 780 & 5.862 & 780 & 5.892 & 786.2 & 5.832 & 787.1 & 5.761 & 782.7 & 5.779 & 786.6 & 5.892 & 787.1 & 5.72 & 782.7 & 5.655 & 780 \\
\hline 6.309 & 805.6 & 5.741 & 806 & 5.998 & 811.7 & 5.933 & 810 & 5.939 & 807.8 & 5.942 & 813.1 & 6.046 & 813.1 & 5.871 & 814.8 & 5.898 & 810.4 \\
\hline 6.448 & 840.4 & 6.182 & 826.3 & 6.259 & 843 & 6.146 & 844.8 & 6.069 & 835.5 & 6.155 & 841.3 & 6.173 & 836.9 & 6.081 & 840.4 & 6.048 & 836.4 \\
\hline 6.634 & 865.5 & 6.149 & 855.4 & 6.386 & 868.1 & 6.306 & 868.1 & 6.318 & 869 & 6.309 & 867.3 & 6.371 & 862 & 6.22 & 869.9 & 6.17 & 868.1 \\
\hline 6.815 & 888.4 & 6.525 & 889.7 & 6.543 & 895.9 & 6.528 & 893.7 & 6.448 & 897.2 & 6.478 & 891.1 & 6.682 & 895.5 & 6.359 & 893.7 & 6.418 & 891.1 \\
\hline 6.948 & 914.9 & 6.744 & 913.5 & 6.726 & 920.6 & 6.768 & 921.9 & 6.605 & 921 & 6.726 & 922.8 & 6.812 & 922.3 & 492 & 918.4 & 6.51 & 917.1 \\
\hline 7.206 & 948.3 & 6.942 & 949.7 & 6.981 & 952.3 & 6.948 & 951.9 & 6.877 & 951.9 & 6.975 & 949.2 & 6.904 & 948.3 & & 951.9 & 6.774 & 947.9 \\
\hline 7.315 & 973.9 & 7.093 & 978.8 & 7.067 & 979.2 & 7.09 & 975.7 & 6.987 & 979.6 & 7.025 & 972.6 & 7.173 & 971.7 & 957 & 981 & 6.919 & 975.2 \\
\hline 7.546 & 1005 & 7.188 & 1003 & 7.206 & 1003 & 7.253 & 1002 & 7.182 & 1003 & 7.274 & 1005 & 7.448 & 1005 & 7.167 & 1003 & 7.046 & 1002 \\
\hline 7.765 & 1032 & 7.312 & 1028 & 7.36 & 1028 & 7.457 & 1029 & 7.371 & 1032 & 7.478 & 1031 & 7.561 & 1034 & 7.253 & 1033 & 7.271 & 1028 \\
\hline 7.901 & 1059 & 7.617 & 1062 & 7.558 & 1053 & 7.706 & 1062 & 7.507 & 1056 & 7.638 & 1057 & 7.812 & 1058 & 7.448 & 1057 & 7.525 & 1057 \\
\hline 8.019 & 1081 & 7.815 & 1087 & 7.839 & 1089 & 7.8 & 1089 & 7.753 & 1088 & 7.783 & 1086 & 8.07 & 1088 & 7.605 & 1084 & 7.614 & 1086 \\
\hline 8.265 & 1111 & 7.99 & 1116 & 8.093 & 1114 & 7.981 & 1113 & 7.984 & 1114 & 7.987 & 1112 & 8.221 & 1113 & 7.771 & 1109 & 7.759 & 1110 \\
\hline
\end{tabular}


Table 1. Rheometer Response for Blank - 2 wt \% - continued

\begin{tabular}{|c|c|c|c|c|c|c|c|c|c|c|c|c|c|c|c|c|c|}
\hline run1 & & run2 & & run3 & & run4 & & run5 & & run6 & & run7 & & run8 & & run9 & \\
\hline [Pa] & {$[1 / \mathrm{s}]$} & [Pa] & {$[1 / \mathrm{s}]$} & [Pa] & [1/s] & [Pa] & {$[1 / s]$} & [Pa] & {$[1 / \mathrm{s}]$} & [Pa] & {$[1 / \mathrm{s}]$} & [Pa] & {$[1 / s]$} & [Pa] & {$[1 / \mathrm{s}]$} & [Pa] & {$[1 / \mathrm{s}]$} \\
\hline 8.407 & 1139 & 8.144 & 1140 & 8.114 & 1140 & 8.164 & 1139 & 8.138 & 1141 & 8.138 & 1137 & 8.383 & 1142 & 8.04 & 1142 & 7.978 & 1140 \\
\hline 8.647 & 1170 & .366 & 1173 & 8.298 & 1163 & 8.455 & 1172 & 8.256 & 1166 & 8.33 & 1167 & 8.594 & 1166 & 8.194 & 1166 & 8.162 & 1164 \\
\hline 8.86 & 1194 & 8.585 & 1196 & 8.549 & 1196 & 8.647 & 1197 & 8.452 & 1192 & 8.52 & 1191 & 8.706 & 1193 & 8.333 & 1197 & 8.309 & 1192 \\
\hline 9.017 & 1221 & 8.733 & 1221 & 8.73 & 1224 & 8.827 & 1225 & 8.747 & 1226 & 8.795 & 1223 & 8.922 & 1220 & 8.478 & 1222 & 8.564 & 1222 \\
\hline 9.15 & 1245 & 8.952 & 1254 & 8.881 & 1248 & 9.046 & 1249 & 8.981 & 1248 & 8.961 & 1252 & 9.097 & 1244 & 8.682 & 1247 & 8.632 & 1249 \\
\hline 9.422 & 1276 & 9.117 & 1281 & 9.12 & 1277 & 9.103 & 1274 & 9.153 & 1276 & 9.079 & 1277 & 9.366 & 1278 & 8.89 & 1281 & 8.848 & 1275 \\
\hline 9.6 & 1304 & 9.274 & 1303 & 9.274 & 1303 & 9.393 & 1308 & 9.348 & 1301 & 9.268 & 1301 & 9.532 & 1304 & 9.1 & 1304 & 9.026 & 1305 \\
\hline 9.689 & 1327 & 9.588 & 1335 & 9.47 & 1327 & 9.555 & 1332 & 9.544 & 1334 & 9.473 & 1330 & 9.937 & 1335 & 9.268 & 1329 & 9.141 & 1329 \\
\hline 10.04 & 1358 & 9.745 & 1361 & 9.724 & 1359 & 9.721 & 1360 & 9.683 & 1359 & 9.609 & 1355 & 10.19 & 1360 & 9.369 & 1354 & 9.354 & 1354 \\
\hline 10.12 & 1385 & 9.887 & 1386 & 9.934 & 1385 & 9.857 & 1383 & 9.828 & 1378 & 9.84 & 1386 & 10.33 & 1388 & 9.632 & 1388 & 9.659 & 1386 \\
\hline 10.35 & 1410 & 10.14 & 1418 & 10 & 1412 & 10.19 & 1409 & 10.15 & 1416 & 10.02 & 1410 & 10.51 & 1410 & 9.81 & 1413 & 9.854 & 1414 \\
\hline 10.57 & 1440 & 10.31 & 1444 & 10.28 & 1438 & 10.27 & 1436 & 10.3 & 1441 & 10.29 & 1442 & 10.84 & 1441 & 9.985 & 1442 & 9.99 & 1438 \\
\hline 10.7 & 1467 & 10.53 & 1469 & 10.48 & 1470 & 10.45 & 1470 & 10.48 & 1468 & 10.43 & 1469 & 10.97 & 1470 & 10.22 & 1466 & 10.15 & 1465 \\
\hline 10.86 & 1492 & 10.7 & 1497 & 10.6 & 1497 & 10.69 & 1497 & 10.65 & 1495 & 10.6 & 1496 & 11.16 & 1494 & 10.3 & 1492 & 10.26 & 1490 \\
\hline 10.55 & 1510 & 10.53 & 1516 & 10.62 & 1512 & 10.55 & 1507 & 10.23 & 1477 & 10.34 & 1480 & 10.08 & 1508 & 10.29 & 1508 & 10.23 & 1510 \\
\hline 10.32 & 1481 & 10.18 & 1481 & 10.4 & 1488 & 10.29 & 1481 & 10.04 & 1452 & 10.17 & 1456 & 9.842 & 1456 & 10.14 & 1485 & 10.08 & 1481 \\
\hline 10.09 & 1452 & 10.02 & 1456 & 10.06 & 1452 & & 1454 & 9.837 & 1423 & 9.967 & 1430 & 9.689 & 1430 & 9.845 & 1453 & 9.887 & 1457 \\
\hline 9.931 & 1428 & 9.866 & 1429 & 9.837 & 1427 & 9.905 & 1428 & 9.697 & 1399 & 9.804 & 1406 & 9.481 & 1401 & 9.683 & 1428 & 9.57 & 1425 \\
\hline 9.73 & 1403 & 9.718 & 1399 & 9.647 & 1401 & & 1404 & 9.493 & 1374 & 9.461 & 1372 & 9.316 & 1376 & 9.514 & 1400 & 9.473 & 1399 \\
\hline 9.422 & 1372 & 9.505 & 1375 & 9.514 & 1374 & 9.576 & 1378 & 9.313 & 1348 & 9.381 & 1345 & 9.188 & 1352 & 9.378 & 1375 & 9.274 & 1375 \\
\hline 9.336 & 1345 & 9.224 & 1345 & 9.233 & 1350 & 9.248 & 1345 & 9.159 & 1321 & 9.183 & 1318 & 8.981 & 1324 & 9.085 & 1345 & 9.049 & 1349 \\
\hline 9.046 & 1320 & 9.12 & 1317 & 9.07 & 1323 & 9.073 & 1317 & 8.884 & 1288 & 8.984 & 1296 & 8.602 & 1292 & 8.987 & 1318 & 8.795 & 1314 \\
\hline 8.946 & 1290 & 8.863 & 1292 & 8.759 & 1291 & 8.931 & 1292 & 8.638 & 1261 & 8.614 & 1264 & 8.517 & 1267 & 8.736 & 1292 & 8.511 & 1292 \\
\hline 8.768 & 1268 & 8.682 & 1268 & 8.641 & 1265 & 8.676 & 1263 & 8.463 & 1231 & 8.416 & 1237 & 8.345 & 1240 & 8.561 & 1268 & 8.336 & 1265 \\
\hline 8.493 & 1243 & 8.431 & 1237 & 8.496 & 1237 & 8.567 & 1239 & 8.298 & 1206 & 8.312 & 1209 & 8.185 & 1215 & 8.253 & 1237 & 8.138 & 1240 \\
\hline 8.227 & 1207 & 8.259 & 1208 & 8.339 & 1211 & 8.28 & 1207 & 8.111 & 1179 & 8.096 & 1182 & 7.901 & 1183 & 8.141 & 1207 & 8.055 & 1214 \\
\hline 8.088 & 1181 & 8.12 & 1185 & 8.061 & 1187 & 8.105 & 1182 & 7.928 & 1155 & 7.922 & 1157 & 7.872 & 1162 & 7.963 & 1181 & 7.877 & 1186 \\
\hline 7.957 & 1155 & 7.845 & 1153 & 7.913 & 1161 & 7.874 & 1155 & 7.741 & 1126 & 7.599 & 1126 & 7.484 & 1127 & 7.732 & 1155 & 7.525 & 1155 \\
\hline 7.729 & 1129 & 7.673 & 1126 & 7.664 & 1126 & 7.753 & 1128 & 7.567 & 1102 & 7.558 & 1103 & 7.407 & 1098 & 7.448 & 1126 & 7.315 & 1131 \\
\hline 7.579 & 1102 & 7.516 & 1102 & 7.496 & 1100 & 7.57 & 1103 & 7.256 & 1070 & 7.268 & 1074 & 7.212 & 1075 & 7.3 & 1100 & 7.167 & 1100 \\
\hline 7.318 & 1076 & 7.259 & 1070 & 7.33 & 1074 & 7.188 & 1072 & 7.081 & 1044 & 7.117 & 1047 & 7.013 & 1051 & 7.132 & 1075 & 6.96 & 1072 \\
\hline 7.084 & 1044 & 7.034 & 1044 & 7.146 & 1047 & 7.043 & 1046 & 6.942 & 1019 & 6.984 & 1022 & 6.824 & 1018 & 6.963 & 1047 & 6.842 & 1046 \\
\hline
\end{tabular}


Table 1. Rheometer Response for Blank - 2 wt \% - continued

\begin{tabular}{|c|c|c|c|c|c|c|c|c|c|c|c|c|c|c|c|c|c|}
\hline run1 & & run2 & & run3 & & run4 & & run5 & & run6 & & run7 & & run8 & & run9 & \\
\hline [Pa] & {$[1 / s]$} & [Pa] & {$[1 / \mathrm{s}]$} & [Pa] & {$[1 / s]$} & [Pa] & {$[1 / s]$} & [Pa] & {$[1 / \mathrm{s}]$} & [Pa] & {$[1 / \mathrm{s}]$} & [Pa] & {$[1 / \mathrm{s}]$} & [Pa] & [1/s] & [Pa] & {$[1 / s]$} \\
\hline 6.927 & 1018 & 6.874 & 1017 & 6.927 & 1022 & 6.924 & 1017 & 6.67 & 985.4 & 6.679 & 991.5 & 6.596 & 992.9 & 6.711 & 1016 & 6.617 & 1019 \\
\hline 6.809 & 995.1 & .714 & 991.5 & 6.708 & 995.5 & 6.711 & 995.1 & 6.563 & 962.5 & 6.543 & 964.2 & 6.38 & 963.3 & 6.513 & 992 & 6.448 & 990.2 \\
\hline 6.531 & 960.2 & 6.546 & 966 & 6.507 & 969.1 & 6.549 & 963.3 & 6.356 & 937.3 & 6.347 & 939.5 & 6.276 & 939.1 & 6.392 & 964.7 & 6.25 & 963.8 \\
\hline 6.374 & 937.3 & 6.374 & 941.3 & 6.279 & 934.7 & 6.389 & 941.3 & 6.128 & 906 & 6.161 & 913.5 & 6.051 & 911.8 & 6.324 & 953.6 & 6.09 & 936.9 \\
\hline 6.241 & 911.8 & 6.102 & 906.5 & 6.069 & 910 & 6.241 & 914.9 & 5.859 & 879.2 & 5.951 & 880.9 & 5.892 & 885.3 & 5.995 & 910.9 & 5.972 & 913.1 \\
\hline 5.951 & 879.6 & 5.939 & 881.4 & 5.951 & 880.5 & 6.063 & 889.3 & 5.667 & 853.2 & 5.732 & 852.3 & 5.649 & 860.7 & 6.037 & 900.8 & 5.732 & 888.4 \\
\hline 5.75 & 854 & 5.785 & 855.8 & 5.764 & 856.7 & 5.758 & 854 & 5.498 & 828.5 & 5.569 & 826.3 & 5.56 & 834.7 & 5.741 & 881.8 & 5.628 & 859.8 \\
\hline 5.548 & 826.3 & 5.661 & 829.8 & 5.56 & 832 & 5.569 & 826.7 & 5.353 & 801.6 & 5.433 & 801.6 & 5.312 & 799.4 & 5.394 & 835.1 & 5.391 & 833.3 \\
\hline 5.457 & 802 & 5.288 & 797.6 & 5.433 & 805.6 & 5.445 & 802.9 & 5.273 & 776.9 & 5.09 & 769.4 & 5.104 & 774.7 & 5.463 & 807.8 & 5.107 & 801.2 \\
\hline 5.232 & 776.9 & 5.161 & 770.3 & 5.099 & 771.2 & 5.276 & 776.5 & 4.927 & 743 & 4.983 & 743 & 4.956 & 750.5 & 5.09 & 787.1 & 5.054 & 773.8 \\
\hline 4.977 & 745.6 & 5.057 & 748.3 & 4.956 & 745.6 & 5.007 & 741.2 & 4.785 & 715.2 & 4.785 & 721.8 & 4.735 & 719.2 & 4.877 & 744.3 & 4.9 & 750.9 \\
\hline 4.829 & 717.4 & 4.776 & 716.6 & 4.767 & 720.5 & 4.758 & 717.9 & 4.663 & 690.5 & 4.625 & 691.9 & 4.569 & 694.1 & 4.782 & 719.6 & 4.616 & 717.9 \\
\hline 4.711 & 695.4 & 4.628 & 691.4 & 4.666 & 690.1 & 4.563 & 691 & 4.498 & 665.9 & 4.353 & 661 & 4.323 & 664.5 & 4.569 & 693.6 & 4.474 & 690.1 \\
\hline 4.403 & 662.3 & 4.373 & 662.3 & 4.492 & 667.2 & 4.483 & 662.8 & 4.184 & 631.9 & 4.249 & 635.5 & 4.184 & 641.2 & 4.368 & 661.9 & 4.326 & 666.3 \\
\hline 4.311 & 635.9 & 4.243 & 638.1 & 4.353 & 642.5 & 4.326 & 635.5 & 4.048 & 605.5 & 4.06 & 610.8 & 4.089 & 613.4 & 4.214 & 637.7 & 4.134 & 639.9 \\
\hline 4.08 & 610.8 & 4.163 & 609.9 & 4.015 & 609 & 4.113 & 607.7 & 3.915 & 580.8 & 3.782 & 579.5 & 3.85 & 583 & 4.001 & 615.2 & 3.918 & 607.7 \\
\hline 3.965 & 581.7 & 3.864 & 577.3 & 4.021 & 591 & 3.935 & 582.6 & 3.74 & 555.3 & 3.687 & 556.6 & 3.687 & 557 & 3.856 & 583 & 3.731 & 580.4 \\
\hline 3.755 & 556.6 & 3.713 & 556.6 & 3.705 & 552.6 & 3.785 & 559.2 & 3.453 & 523.5 & 3.506 & 528.4 & 3.58 & 530.6 & 3.699 & 559.2 & 3.634 & 553.1 \\
\hline 3.497 & 524.9 & 3.61 & 528.4 & 3.687 & 535.4 & 3.518 & 526.6 & 3.352 & 498 & 3.281 & 497.5 & 3.352 & 501.9 & 3.435 & 524.9 & 3.494 & 528.4 \\
\hline 3.412 & 497.5 & 3.403 & 501.5 & 3.358 & 499.7 & 3.361 & 498.4 & 3.172 & 470.6 & 3.157 & 471.5 & 3.19 & 476.8 & 3.299 & 501.9 & 3.196 & 498 \\
\hline 3.246 & 472.4 & 3.139 & 472.4 & 3.178 & 473.7 & 3.234 & 473.7 & 3.068 & 446.9 & 3.024 & 445.1 & 2.935 & 448.2 & 3.169 & 472.9 & 3.065 & 473.3 \\
\hline 3.071 & 450.8 & 3.024 & 445.5 & 3.003 & 445.5 & 3.092 & 449.5 & 2.808 & 415.1 & 2.826 & 421.3 & 2.796 & 421.3 & 2.974 & 447.7 & 2.873 & 448.6 \\
\hline 2.814 & 418.2 & 2.882 & 421.7 & 2.814 & 419.5 & 2.838 & 414.2 & 2.66 & 386.9 & 2.583 & 387.4 & 2.633 & 394 & 2.835 & 422.2 & 2.764 & 417.3 \\
\hline 2.71 & 390.4 & 2.633 & 388.2 & 2.704 & 390.9 & 2.687 & 389.1 & 2.533 & 362.7 & 2.408 & 363.1 & 2.429 & 363.6 & 2.61 & 388.7 & 2.672 & 391.8 \\
\hline 2.521 & 365.3 & 2.503 & 363.6 & 2.491 & 366.2 & 2.518 & 363.1 & 2.272 & 330.1 & 2.299 & 334 & 2.32 & 337.1 & 2.488 & 362.7 & 2.468 & 364.4 \\
\hline 2.397 & 339.8 & 2.32 & 337.6 & 2.376 & 343.3 & 2.414 & 340.2 & 2.059 & 305.8 & 2.109 & 312 & 2.133 & 310.2 & 2.32 & 336.7 & 2.305 & 339.8 \\
\hline 2.151 & 310.7 & 2.186 & 309.8 & 2.139 & 309.4 & 2.249 & 313.8 & 1.976 & 277.6 & 1.997 & 282 & 1.967 & 285.6 & 2.163 & 308.5 & 2.062 & 306.3 \\
\hline 2.009 & 282 & 2.035 & 286 & 1.962 & 279 & 1.988 & 281.6 & 1.828 & 251.6 & 1.837 & 256 & 1.834 & 260.9 & 2.003 & 284.7 & 1.893 & 281.6 \\
\hline 1.834 & 258.2 & 1.805 & 251.6 & 1.867 & 253 & 1.914 & 253.8 & 1.651 & 227.4 & 1.648 & 231.8 & 1.663 & 235.3 & 1.828 & 259.6 & 1.757 & 253 \\
\hline 1.713 & 233.1 & 1.713 & 237.5 & 1.701 & 228.7 & 1.719 & 229.6 & 0 & 179.1 & 0 & 184 & 0 & 186.8 & 1.725 & 236.6 & 1.84 & 245.9 \\
\hline & & & & & & & & & & & & & & & & & \\
\hline & & & & & & & & & & & & & & & & & \\
\hline
\end{tabular}


Table 2. $10{ }^{\circ} \mathrm{C}$ at 2 wt $\%$

\begin{tabular}{|c|c|c|c|c|c|c|c|c|c|c|c|c|c|c|c|c|c|}
\hline run1 & & run2 & & run3 & & run4 & & run5 & & run6 & & run7 & & run8 & & run9 & \\
\hline [Pa] & {$[1 / s]$} & [Pa] & {$[1 / s]$} & [Pa] & {$[1 / s]$} & [Pa] & {$[1 / s]$} & [Pa] & {$[1 / s]$} & [Pa] & {$[1 / \mathrm{s}]$} & [Pa] & {$[1 / s]$} & [Pa] & {$[1 / s]$} & [Pa] & [1/s] \\
\hline 2.893 & 275.9 & 2.975 & 273.7 & 2.626 & 274.1 & 2.688 & 271 & 2.851 & 277.2 & 2.922 & 275.9 & 2.753 & 271 & & & & \\
\hline 3.221 & 290 & 3.206 & 293.1 & 3.106 & 288.6 & 3.1 & 294.8 & 3.082 & 295.3 & 3.109 & 296.6 & 3.046 & 288.6 & & & & \\
\hline 3.624 & 323 & 3.348 & 320.8 & 3.372 & 316.9 & 3.387 & 321.7 & 3.322 & 321.3 & 3.257 & 321.7 & 3.18 & 315.5 & & & & \\
\hline 3.902 & 349.9 & 3.7 & 351.7 & 3.567 & 338.4 & 3.588 & 350.8 & 3.502 & 348.6 & 3.751 & 354.3 & 3.452 & 341.5 & & & & \\
\hline 4.07 & 374.1 & 3.917 & 379.4 & 3.893 & 371.5 & 3.786 & 373.3 & 3.695 & 375 & 3.789 & 381.6 & 3.73 & 376.8 & & & & \\
\hline 4.461 & 404.1 & 4.059 & 403.2 & 4.085 & 398.8 & 4.041 & 400.6 & 4.029 & 401 & 4.032 & 404.5 & 4.026 & 401.5 & & & & \\
\hline 4.574 & 431 & 4.396 & 429.7 & 4.209 & 421.7 & 4.224 & 426.6 & 4.313 & 431.9 & 4.159 & 430.5 & 4.136 & 425.7 & & & & \\
\hline 4.914 & 456.1 & 4.719 & 464 & 4.532 & 454.8 & 4.414 & 455.2 & 4.485 & 461 & 4.508 & 464 & 4.464 & 452.1 & & & & \\
\hline 5.269 & 486.5 & 4.923 & 490 & 4.784 & 478.6 & 4.674 & 483.4 & 4.645 & 485.6 & 4.801 & 487.8 & 4.775 & 484.8 & & & & \\
\hline 5.491 & 511.2 & 5.213 & 515.2 & 4.982 & 508.5 & 4.872 & 508.1 & 4.911 & 511.6 & 5 & 514.7 & 4.911 & 511.6 & & & & \\
\hline 5.748 & 540.7 & 5.358 & 539.8 & 5.245 & 534.5 & 5.159 & 533.2 & 5.109 & 537.2 & 5.257 & 542.5 & 5.136 & 532.8 & & & & \\
\hline 6.035 & 566.7 & 5.606 & 564.5 & 5.408 & 559.2 & 5.529 & 566.7 & 5.535 & 570.2 & 5.408 & 567.6 & 5.331 & 559.2 & & & & \\
\hline 6.24 & 593.6 & 5.89 & 590.5 & 5.63 & 583.9 & 5.722 & 593.6 & 5.79 & 595.4 & 5.674 & 593.6 & 5.787 & 594.9 & & & & \\
\hline 6.494 & 618.3 & 6.136 & 621.4 & 5.947 & 618.3 & 5.911 & 620 & 5.959 & 623.1 & 5.994 & 626.7 & 6.012 & 620.9 & & & & \\
\hline 6.909 & 649.6 & 6.429 & 649.6 & 6.172 & 641.6 & 6.139 & 646 & 6.136 & 646 & 6.254 & 652.2 & 6.154 & 644.3 & & & & \\
\hline 7.21 & 677.3 & 6.574 & 674.7 & 6.533 & 673.8 & 6.391 & 671.6 & 6.521 & 677.8 & 6.453 & 679.1 & 6.352 & 670.3 & & & & \\
\hline 7.592 & 705.5 & 6.897 & 702 & 6.852 & 698.9 & 6.719 & 703.3 & 6.823 & 702.9 & 6.74 & 703.3 & 6.542 & 697.6 & & & & \\
\hline 7.844 & 736.4 & 7.213 & 728 & 6.974 & 723.2 & 6.9 & 729.3 & 7.119 & 732.4 & 7.018 & 737.3 & 6.909 & 723.2 & & & & \\
\hline 7.767 & 740.8 & 7.53 & 759.3 & 7.267 & 748.3 & 7.116 & 753.1 & 7.302 & 759.7 & 7.364 & 762.4 & 7.163 & 750.5 & & & & \\
\hline 8.279 & 761.9 & 7.722 & 788.8 & 7.625 & 781.3 & 7.563 & 786.6 & 7.601 & 786.6 & 7.628 & 790.6 & 7.506 & 783.1 & & & & \\
\hline 8.734 & 808.7 & 8.006 & 810.4 & 7.888 & 806.4 & 7.861 & 811.7 & 7.743 & 809.1 & 7.79 & 815.3 & 7.669 & 809.5 & & & & \\
\hline 8.808 & 813.9 & 8.391 & 843.5 & 8.104 & 834.2 & 8.057 & 840.4 & 8.116 & 841.7 & 8.107 & 839.9 & 8.08 & 840.8 & & & & \\
\hline 9.285 & 858 & 8.652 & 867.7 & 8.362 & 859.3 & 8.329 & 864.2 & 8.483 & 868.6 & 8.368 & 872.6 & 8.394 & 864.2 & & & & \\
\hline 9.344 & 879.2 & 8.915 & 893.3 & 8.77 & 889.3 & 8.684 & 893.7 & 8.779 & 895.5 & 8.66 & 895.5 & 8.607 & 893.3 & & & & \\
\hline 10.02 & 917.5 & 9.276 & 922.8 & 9.004 & 916.6 & 8.894 & 919.7 & 8.983 & 922.8 & 9.11 & 926.8 & 8.921 & 921 & & & & \\
\hline 10.4 & 950.1 & 9.566 & 951.4 & 9.3 & 940.4 & 9.252 & 946.6 & 9.312 & 947.5 & 9.317 & 952.8 & 9.152 & 944.8 & & & & \\
\hline 10.67 & 977 & 9.841 & 978.8 & 9.738 & 970.8 & 9.513 & 969.9 & 9.575 & 972.1 & 9.643 & 982.3 & 9.427 & 970.4 & & & & \\
\hline 10.96 & 1002 & 10.11 & 1002 & 10.03 & 998.1 & 9.995 & 1003 & 9.951 & 1002 & 9.874 & 1004 & 9.871 & 1002 & & & & \\
\hline 11.26 & 1027 & 10.6 & 1033 & 10.43 & 1029 & 10.24 & 1029 & 10.22 & 1028 & 10.4 & 1036 & 10.23 & 1029 & & & & \\
\hline 11.73 & 1056 & 10.9 & 1058 & 10.77 & 1053 & 10.51 & 1056 & 10.69 & 1060 & 10.64 & 1064 & 10.5 & 1056 & & & & \\
\hline 11.97 & 1084 & 11.21 & 1087 & 11.08 & 1081 & 10.83 & 1081 & 11.02 & 1087 & 10.94 & 1087 & 10.81 & 1083 & & & & \\
\hline
\end{tabular}


Table 2. $10{ }^{\circ} \mathrm{C}$ at $2 \mathrm{wt} \%$ - continued

\begin{tabular}{|c|c|c|c|c|c|c|c|c|c|c|c|c|c|c|c|c|c|}
\hline run1 & & run2 & & run3 & & run4 & & run5 & & run6 & & run7 & & run8 & & run9 & \\
\hline [Pa] & {$[1 / s]$} & [Pa] & [1/s] & [Pa] & [1/s] & [Pa] & {$[1 / \mathrm{s}]$} & [Pa] & [1/s] & [Pa] & [1/s] & [Pa] & {$[1 / s]$} & [Pa] & {$[1 / s]$} & [Pa] & [1/s] \\
\hline 12.28 & 1110 & 11.5 & 1112 & 11.32 & 1105 & 11.18 & 1107 & 11.35 & 1117 & 11.3 & 1119 & 11.18 & 1107 & & & & \\
\hline 12.8 & 1140 & 11.87 & 1143 & 11.75 & 1136 & 11.63 & 1138 & 11.69 & 1141 & 11.55 & 1143 & 11.57 & 1139 & & & & \\
\hline 13.11 & 1168 & 12.21 & 1171 & 12.12 & 1162 & 11.88 & 1165 & 11.99 & 1168 & 11.92 & 1172 & 11.86 & 1166 & & & & \\
\hline 13.38 & 1192 & 12.53 & 1196 & 12.35 & 1188 & 12.22 & 1191 & 12.25 & 1197 & 12.15 & 1195 & 12.25 & 1194 & & & & \\
\hline 14.08 & 1224 & 13.02 & 1229 & 12.57 & 1212 & 12.6 & 1216 & 12.6 & 1223 & 12.56 & 1222 & 12.5 & 1219 & & & & \\
\hline 14.42 & 1250 & 13.3 & 1252 & 13.09 & 1244 & 13.01 & 1246 & 12.96 & 1249 & 12.86 & 1249 & 12.9 & 1245 & & & & \\
\hline 14.73 & 1278 & 13.67 & 1281 & 13.4 & 1273 & 13.27 & 1274 & 13.3 & 1273 & 13.21 & 1278 & 13.32 & 1277 & & & & \\
\hline 15.01 & 1302 & 13.88 & 1305 & 13.72 & 1297 & 13.58 & 1302 & 13.59 & 1299 & 13.6 & 1302 & 13.63 & 1302 & & & & \\
\hline 15.33 & 1326 & 14.24 & 1330 & 14.05 & 1323 & 13.98 & 1327 & 14.06 & 1331 & 13.98 & 1335 & 13.94 & 1330 & & & & \\
\hline 15.71 & 1357 & 14.73 & 1361 & 14.32 & 1349 & 14.27 & 1352 & 14.45 & 1360 & 14.4 & 1360 & 14.25 & 1356 & & & & \\
\hline 16.02 & 1385 & 15.06 & 1386 & 14.78 & 1375 & 14.79 & 1384 & 14.69 & 1386 & 14.68 & 1389 & 14.66 & 1382 & & & & \\
\hline 16.28 & 1408 & 15.25 & 1411 & 15.2 & 1409 & 15.04 & 1411 & 15.04 & 1411 & 15.05 & 1415 & 14.96 & 1407 & & & & \\
\hline 16.88 & 1440 & 15.73 & 1438 & 15.51 & 1434 & 15.41 & 1439 & 15.38 & 1437 & 15.41 & 1442 & 15.5 & 1439 & & & & \\
\hline 17.02 & 1466 & 16.11 & 1472 & 15.83 & 1461 & 15.66 & 1460 & 15.87 & 1468 & 15.79 & 1472 & 15.74 & 1467 & & & & \\
\hline 17.4 & 1491 & 16.33 & 1487 & 16.2 & 1485 & 16.19 & 1493 & 16.16 & 1497 & 16.23 & 1498 & 16.06 & 1493 & & & & \\
\hline 17.84 & 1523 & 16.47 & 1507 & 16.67 & 1517 & 16.53 & 1522 & 16.56 & 1522 & 16.54 & 1526 & 16.41 & 1520 & & & & \\
\hline 16.42 & 1510 & 15.86 & 1478 & 16.21 & 1508 & 16.18 & 1508 & 16.15 & 1511 & 15.83 & 1513 & 16.36 & 1508 & & & & \\
\hline 16.06 & 1484 & 15.5 & 1453 & 15.83 & 1486 & 15.92 & 1482 & 15.77 & 1487 & 15.71 & 1494 & 15.98 & 1482 & & & & \\
\hline 15.77 & 1457 & 15.09 & 1424 & 15.44 & 1455 & 15.38 & 1457 & 15.41 & 1461 & 15 & 1431 & 15.8 & 1458 & & & & \\
\hline 15.44 & 1431 & 14.79 & 1400 & 15 & 1430 & 14.97 & 1422 & 15.12 & 1436 & 14.82 & 1401 & 15.24 & 1427 & & & & \\
\hline 14.91 & 1398 & 14.47 & 1376 & 14.64 & 1405 & 14.7 & 1399 & 14.64 & 1405 & 14.38 & 1374 & 14.91 & 1402 & & & & \\
\hline 14.53 & 1374 & 13.99 & 1346 & 14.35 & 1374 & 14.32 & 1371 & 14.29 & 1376 & 13.99 & 1349 & 14.56 & 1371 & & & & \\
\hline 14.2 & 1345 & 13.7 & 1316 & 14.08 & 1350 & 13.87 & 1341 & 13.93 & 1351 & 13.64 & 1321 & 14.2 & 1345 & & & & \\
\hline 13.87 & 1319 & 13.37 & 1292 & 13.76 & 1326 & 13.58 & 1315 & 13.61 & 1326 & 13.4 & 1294 & 13.79 & 1321 & & & & \\
\hline 13.52 & 1293 & 12.9 & 1260 & 13.34 & 1298 & 13.25 & 1287 & 13.43 & 1302 & 13.05 & 1271 & 13.46 & 1296 & & & & \\
\hline 13.16 & 1268 & 12.61 & 1235 & 12.9 & 1267 & 12.9 & 1261 & 12.87 & 1268 & 12.7 & 1239 & 13.02 & 1262 & & & & \\
\hline 12.73 & 1237 & 12.32 & 1207 & 12.6 & 1239 & 12.62 & 1235 & 12.59 & 1241 & 12.29 & 1213 & 12.72 & 1237 & & & & \\
\hline 12.44 & 1211 & 11.9 & 1177 & 12.23 & 1210 & 12.21 & 1208 & 12.21 & 1218 & 12.06 & 1184 & 12.32 & 1208 & & & & \\
\hline 12.18 & 1189 & 11.49 & 1154 & 11.98 & 1184 & 11.93 & 1184 & 11.81 & 1187 & 11.7 & 1161 & 11.92 & 1182 & & & & \\
\hline 11.71 & 1153 & 11.21 & 1126 & 11.6 & 1159 & 11.44 & 1150 & 11.5 & 1160 & 11.46 & 1137 & 11.7 & 1160 & & & & \\
\hline 11.33 & 1126 & 10.91 & 1099 & 11.23 & 1127 & 11.16 & 1123 & 11.21 & 1136 & 11.05 & 1108 & 11.24 & 1127 & & & & \\
\hline
\end{tabular}


Table 2. $10{ }^{\circ} \mathrm{C}$ at 2 wt $\%$ - continued

\begin{tabular}{|c|c|c|c|c|c|c|c|c|c|c|c|c|c|c|c|c|c|}
\hline run1 & & run2 & & run3 & & run4 & & run5 & & run6 & & run7 & & run8 & & run9 & \\
\hline [Pa] & {$[1 / s]$} & [Pa] & {$[1 / s]$} & [Pa] & {$[1 / s]$} & [Pa] & {$[1 / s]$} & [Pa] & [1/s] & [Pa] & [1/s] & [Pa] & {$[1 / \mathrm{s}]$} & [Pa] & [1/s] & [Pa] & {$[1 / \mathrm{s}]$} \\
\hline 11.06 & 1099 & 10.45 & 1070 & 10.85 & 1100 & 10.76 & 1097 & 10.86 & 1105 & 10.62 & 1074 & 11 & 1101 & & & & \\
\hline 10.7 & 1074 & 10.2 & 1044 & 10.51 & 1075 & 10.46 & 1072 & 10.55 & 1075 & 10.31 & 1050 & 10.63 & 1075 & & & & \\
\hline 10.39 & 1050 & 9.892 & 1019 & 10.29 & 1051 & 10.15 & 1042 & 10.21 & 1050 & 10 & 1023 & 10.18 & 1044 & & & & \\
\hline 10.1 & 1024 & 9.536 & 986.7 & 9.847 & 1019 & 9.785 & 1018 & 9.983 & 1026 & 9.761 & 999.5 & 9.9 & 1021 & & & & \\
\hline 9.557 & 990.2 & 9.229 & 962.9 & 9.489 & 992.9 & 9.474 & 988.9 & 9.474 & 993.3 & 9.184 & 967.7 & 9.584 & 991.5 & & & & \\
\hline 9.338 & 963.3 & 8.897 & 937.8 & 9.249 & 968.2 & 9.214 & 963.8 & 9.267 & 966 & 8.998 & 940.4 & 9.3 & 965.1 & & & & \\
\hline 9.004 & 935.1 & 8.69 & 911.8 & 8.945 & 936.9 & 8.912 & 932 & 8.903 & 941.7 & 8.634 & 913.5 & 8.986 & 941.3 & & & & \\
\hline 8.64 & 908.2 & 8.317 & 885.8 & 8.681 & 911.8 & 8.521 & 906.5 & 8.495 & 910.9 & 8.368 & 889.7 & 8.743 & 913.5 & & & & \\
\hline 8.329 & 883.1 & 7.956 & 852.7 & 8.314 & 886.2 & 8.291 & 878.3 & 8.217 & 885.8 & 8.042 & 855.8 & 8.323 & 888 & & & & \\
\hline 8.092 & 859.3 & 7.631 & 825.8 & 7.9 & 855.4 & 7.998 & 851.8 & 7.95 & 860.7 & 7.642 & 830.7 & 8.199 & 862 & & & & \\
\hline 7.716 & 827.6 & 7.486 & 802.9 & 7.725 & 827.6 & 7.657 & 827.6 & 7.696 & 831.6 & 7.4 & 804.7 & 7.734 & 843 & & & & \\
\hline 7.423 & 800.3 & 6.926 & 769.9 & 7.37 & 804.7 & 7.429 & 802.5 & 7.474 & 806 & 7.095 & 778.7 & 7.622 & 813.9 & & & & \\
\hline 7.222 & 777.4 & 6.752 & 745.2 & 7.201 & 777.8 & 7.009 & 767.2 & 7.139 & 781.3 & 6.941 & 752.2 & 7.323 & 778.7 & & & & \\
\hline 6.775 & 745.2 & 6.55 & 718.3 & 6.793 & 753.1 & 6.835 & 750.9 & 6.778 & 747.4 & 6.503 & 720.1 & 6.82 & 756.2 & & & & \\
\hline 6.598 & 717.9 & 6.183 & 693.2 & 6.53 & 721.4 & 6.471 & 716.1 & 6.559 & 722.3 & 6.337 & 700.7 & 6.607 & 721 & & & & \\
\hline 6.299 & 695.8 & 5.879 & 662.3 & 6.183 & 693.2 & 6.24 & 688.8 & 6.252 & 698 & 6.053 & 671.6 & 6.237 & 694.5 & & & & \\
\hline 5.902 & 662.3 & 5.642 & 636.3 & 5.95 & 666.3 & 5.979 & 664.1 & 6.009 & 672.9 & 5.796 & 645.2 & 6.062 & 668.5 & & & & \\
\hline 5.645 & 636.3 & 5.464 & 612.1 & 5.686 & 639.9 & 5.633 & 633.3 & 5.654 & 641.2 & 5.544 & 618.7 & 5.66 & 635.5 & & & & \\
\hline 5.355 & 608.6 & 5.233 & 588.3 & 5.467 & 615.6 & 5.39 & 607.3 & 5.387 & 611.2 & 5.159 & 583 & 5.438 & 611.2 & & & & \\
\hline 5.112 & 583 & 4.748 & 552.6 & 5.041 & 581.7 & 5.127 & 578.6 & 5.207 & 588.3 & 4.935 & 560.1 & 5.127 & 582.6 & & & & \\
\hline 4.843 & 554.8 & 4.609 & 523.5 & 4.973 & 562.3 & 4.887 & 555.7 & 4.985 & 564.1 & 4.763 & 530.6 & 4.92 & 559.2 & & & & \\
\hline 4.612 & 530.1 & 4.272 & 500.2 & 4.801 & 536.3 & 4.659 & 528.4 & 4.778 & 538.5 & 4.485 & 504.6 & 4.5 & 528.4 & & & & \\
\hline 4.334 & 498.4 & 3.982 & 469.8 & 4.387 & 503.3 & 4.245 & 497.1 & 4.278 & 503.7 & 4.221 & 477.7 & 4.316 & 502.4 & & & & \\
\hline 3.967 & 473.3 & 3.822 & 443.8 & 4.153 & 475.1 & 4.091 & 468.9 & 4.15 & 477.7 & 3.905 & 451.7 & 4.056 & 473.3 & & & & \\
\hline 3.928 & 447.7 & 3.553 & 414.2 & 3.973 & 451.7 & 3.925 & 443.3 & 3.851 & 447.3 & 3.754 & 427 & 3.896 & 446 & & & & \\
\hline 3.618 & 416 & 3.487 & 395.7 & 3.624 & 419.1 & 3.629 & 416.4 & 3.709 & 421.7 & 3.325 & 395.3 & 3.695 & 420.9 & & & & \\
\hline 3.283 & 389.6 & 3.138 & 364.9 & 3.407 & 393.1 & 3.416 & 391.3 & 3.292 & 392.6 & 3.177 & 366.6 & 3.295 & 390 & & & & \\
\hline 3.094 & 362.2 & 2.934 & 339.8 & 3.132 & 365.3 & 3.132 & 367.1 & 3.26 & 365.8 & 2.967 & 344.2 & 3.168 & 361.4 & & & & \\
\hline 2.967 & 339.3 & 2.567 & 305.8 & 2.955 & 343.3 & 2.913 & 334 & 3.041 & 342 & 2.688 & 309.8 & 2.94 & 335.4 & & & & \\
\hline 2.502 & 308 & 2.366 & 280.3 & 2.715 & 309.4 & 2.756 & 308 & 2.789 & 317.7 & 2.455 & 292.6 & 2.721 & 312.4 & & & & \\
\hline 2.342 & 281.6 & 2.117 & 253.8 & 2.428 & 282.9 & 2.537 & 283.4 & 2.469 & 283.4 & 2.289 & 257.8 & 2.36 & 280.7 & & & & \\
\hline 2.179 & 257.8 & 1.913 & 228.3 & 2.247 & 254.7 & 2.342 & 257.8 & 2.25 & 260.9 & 2.031 & 229.2 & 2.173 & 256 & & & & \\
\hline
\end{tabular}


Table 2. $10{ }^{\circ} \mathrm{C}$ at $2 \mathrm{wt} \%$ - continued

\begin{tabular}{|c|c|c|c|c|c|c|c|c|c|c|c|c|c|c|c|c|c|}
\hline run1 & & run2 & & run3 & & run4 & & run5 & & run6 & & run7 & & run8 & & run9 & \\
\hline$[\mathrm{Pa}]$ & {$[1 / \mathrm{s}]$} & {$[\mathrm{Pa}]$} & {$[1 / s]$} & {$[\mathrm{Pa}]$} & {$[1 / \mathrm{s}]$} & {$[\mathrm{Pa}]$} & {$[1 / \mathrm{s}]$} & {$[\mathrm{Pa}]$} & {$[1 / s]$} & {$[\mathrm{Pa}]$} & {$[1 / \mathbf{s}]$} & {$[\mathrm{Pa}]$} & {$[1 / \mathrm{s}]$} & {$[\mathrm{Pa}]$} & {$[1 / \mathrm{s}]$} & {$[\mathrm{Pa}]$} & {$[1 / s]$} \\
\hline & & & & & & & & & & & & 0 & 11.26 & & & & \\
\hline & & & & & & & & & & & & 0 & 49.31 & & & & \\
\hline & & & & & & & & & & & & 0 & 72.71 & & & & \\
\hline & & & & & & & & & & & & 0 & 102 & & & & \\
\hline & & & & & & & & & & & & 0 & 124.8 & & & & \\
\hline & & & & & & & & & & & & 34.41 & 2592 & & & & \\
\hline & & & & & & & & & & & & 0 & 27.09 & & & & \\
\hline & & & & & & & & & & & & 33.35 & 2604 & & & & \\
\hline & & & & & & & & & & & & & & & & & \\
\hline
\end{tabular}


Table 3. $15^{\circ} \mathrm{C}$ at 2 wt \%

\begin{tabular}{|c|c|c|c|c|c|c|c|c|c|c|c|c|c|c|c|c|c|}
\hline run1 & & run2 & & run3 & & run4 & & run5 & & run6 & & run7 & & run8 & & run9 & \\
\hline [Pa] & {$[1 / s]$} & [Pa] & {$[1 / s]$} & [Pa] & {$[1 / s]$} & [Pa] & {$[1 / s]$} & [Pa] & {$[1 / s]$} & [Pa] & [1/s] & [Pa] & {$[1 / \mathrm{s}]$} & [Pa] & {$[1 / s]$} & [Pa] & {$[1 / \mathrm{s}]$} \\
\hline 3.023 & 272.8 & 2.99 & 277.6 & 2.83 & 276.7 & 2.765 & 273.2 & 2.958 & 276.3 & 2.878 & 275.9 & 2.78 & 277.2 & 2.89 & 271.9 & 2.789 & 281.2 \\
\hline 3.41 & 292.6 & 3.227 & 293.5 & 3.197 & 293.9 & 2.795 & 269.3 & 3.212 & 296.6 & 3.076 & 295.3 & 3.123 & 291.7 & 3.103 & 295.3 & 3.073 & 290.4 \\
\hline 3.6 & 319.5 & .541 & 317.7 & 3.573 & 324.8 & 3.215 & 307.6 & 3.464 & 321.7 & 3.328 & 319.1 & 3.461 & 324.3 & 3.39 & 319.9 & 3.319 & 316.4 \\
\hline 3.917 & 345.5 & 3.872 & 348.6 & 3.674 & 350.8 & 3.668 & 346.4 & 3.692 & 345.1 & 3.677 & 353 & 3.65 & 349.9 & 3.635 & 345.9 & 3.514 & 342.9 \\
\hline 4.198 & 370.2 & 4.079 & 373.3 & 3.952 & 377.7 & 4.124 & 364.4 & 4.02 & 380.8 & 3.893 & 378.5 & 3.86 & 376.3 & 3.813 & 371.1 & 3.878 & 372.8 \\
\hline 4.579 & 402.3 & 4.357 & 401 & 4.23 & 405.9 & 4.109 & 395.3 & 4.192 & 403.2 & 4.141 & 404.1 & 4.094 & 401 & 4.1 & 397.9 & 3.988 & 398.4 \\
\hline 4.754 & 430.5 & 4.497 & 424.4 & 4.514 & 431.9 & 4.491 & 413.4 & 4.458 & 430.5 & 4.325 & 430.5 & 4.393 & 437.6 & 4.431 & 431.9 & 4.221 & 421.3 \\
\hline 5.032 & 456.1 & 4.861 & 456.1 & 4.73 & 460.1 & 4.665 & 452.1 & 4.585 & 457 & 4.704 & 461.8 & 4.656 & 460.1 & 4.553 & 459.6 & 4.541 & 454.3 \\
\hline 5.358 & 482.1 & 5.13 & 481.7 & 4.852 & 485.2 & 4.958 & 479.5 & 4.958 & 489.6 & 4.849 & 489.2 & 4.801 & 489.6 & 4.855 & 484.3 & 4.766 & 481.2 \\
\hline 5.725 & 508.5 & 5.299 & 509 & 5.112 & 509.9 & 5.13 & 502.4 & 5.088 & 513 & 5.068 & 513 & 4.973 & 514.7 & 5.035 & 507.7 & 4.911 & 505.9 \\
\hline 6.237 & 539.4 & & 540.7 & & 536.8 & & & & 540.7 & & 538.5 & 5.162 & 539 & 5.263 & 539.8 & & 533.7 \\
\hline 6.512 & 565.8 & 5.885 & 564.5 & 5.701 & 567.2 & 5.521 & 558.3 & 5.515 & 566.3 & 5.535 & 562.8 & 5.39 & 566.7 & 5.414 & 568 & 5.278 & 559.7 \\
\hline 6.906 & 589.2 & 074 & 590.1 & 5.923 & 594.9 & 5.802 & 585.7 & 5.725 & 590.5 & 5.734 & 588.8 & 5.707 & 592.3 & 5.71 & 595.8 & & 584.3 \\
\hline 7.483 & 620 & 6.399 & 620.9 & 6.136 & 617.4 & 6.059 & 613.4 & 6.098 & 623.1 & 5.976 & 617.8 & 6.024 & 625.3 & 5.932 & 618.7 & 5.893 & 616.1 \\
\hline 7.746 & 648.7 & 6.687 & 649.1 & 6.355 & 648.2 & 6.364 & 646.5 & 6.399 & 650.4 & 6.361 & 650 & 6.287 & 648.7 & 6.234 & 650.4 & 6.074 & 642.5 \\
\hline 7.924 & 672.5 & 6.941 & 673.4 & 6.621 & 672 & 6.577 & 672.5 & 6.512 & 673.8 & 6.536 & 676 & 6.468 & 676.9 & 6.287 & 669.8 & 6.246 & 669.8 \\
\hline 8.149 & 702 & 7.104 & 697.6 & 6.941 & 705.5 & 6.855 & 701.1 & 6.879 & 703.3 & 6.965 & 707.7 & 6.737 & 702.4 & 6.568 & 695.4 & 6.503 & 693.6 \\
\hline 8.288 & 728 & 474 & 728.4 & 7.169 & 729.3 & & 727.1 & 7.11 & 730.2 & 7.204 & 731.5 & 7.03 & 734.6 & 6.944 & 31.1 & 6.728 & 719.6 \\
\hline 8.601 & 754 & 693 & 754 & 7.438 & 754.9 & 7.302 & 752.7 & 7.474 & 761.5 & 7.344 & 758.9 & 7.296 & 761.5 & 7.175 & 758.9 & 6.941 & 746.5 \\
\hline 8.868 & 782.7 & 8.089 & 784 & 7.779 & 785.3 & 7.693 & 784 & 7.699 & 786.6 & 7.515 & 783.5 & 7.489 & 784.9 & 7.45 & 781.3 & 415 & 781.8 \\
\hline 9.093 & 809.5 & 8.296 & 811.7 & 7.962 & 814.8 & 7.927 & 810.9 & 7.953 & 816.6 & 7.752 & 807.3 & 7.782 & 815.7 & 7.675 & 811.7 & 7.619 & 805.6 \\
\hline 9.356 & 833.3 & 8.578 & 837.7 & 8.279 & 840.8 & 8.163 & 836.4 & 8.184 & 839.5 & 8.151 & 839.9 & 8.122 & 842.6 & 7.95 & 835.5 & 7.773 & 832 \\
\hline 9.673 & 865.5 & 8.797 & 862 & 8.483 & 864.6 & 8.444 & 861.1 & 8.394 & 863.3 & 8.397 & 868.1 & 8.291 & 866.8 & 8.282 & 865.9 & 83 & 857.6 \\
\hline 10.01 & 893.7 & 9.241 & 893.7 & 8.773 & 890.6 & 8.607 & 888.4 & 8.69 & 889.3 & 8.829 & 892.8 & 8.515 & 891.1 & 8.441 & 894.1 & 8.347 & 882.2 \\
\hline 10.32 & 918.8 & 9.406 & 921 & 9.178 & 925 & 9.042 & 920.1 & 9.081 & 923.2 & 9.187 & 925 & 8.974 & 925 & 8.717 & 919.7 & 8.702 & 917.1 \\
\hline 10.65 & 944.4 & 9.681 & 943.9 & 9.359 & 951.4 & 9.317 & 945.3 & 9.288 & 950.6 & 9.386 & 951.4 & 9.19 & 953.2 & 8.951 & 943.9 & 8.977 & 943.1 \\
\hline 10.89 & 973.5 & & 977.9 & 9.625 & 975.2 & & 975.2 & 9.56 & 974.3 & 9.673 & 978.3 & 9.51 & 977 & 9.347 & 976.6 & 9.172 & 971.3 \\
\hline 11.07 & 999 & 10.33 & 1002 & 9.898 & 1001 & 9.827 & 1001 & 9.832 & 994.2 & 9.93 & 1007 & 9.755 & 1005 & 9.613 & 1001 & 9.436 & 997.7 \\
\hline 11.6 & 1030 & 10.59 & 1030 & 10.27 & 1031 & 10.19 & 1027 & 10.29 & 1026 & 10.14 & 1031 & 10 & 1030 & 9.892 & 1031 & 9.69 & 1025 \\
\hline 11.92 & 1058 & 10.88 & 1055 & 10.62 & 1058 & 10.41 & 1052 & 10.27 & 1043 & 10.34 & 1056 & 10.39 & 1062 & 10.15 & 1053 & 10.03 & 1049 \\
\hline 12.18 & 1086 & 11.29 & 1087 & 10.78 & 1081 & 10.79 & 1078 & 10.77 & 1072 & 10.65 & 1082 & 10.68 & 1081 & 10.52 & 1087 & 10.32 & 1076 \\
\hline
\end{tabular}


Table 3. $15^{\circ} \mathrm{C}$ at $2 \mathrm{wt} \%$ - continued

\begin{tabular}{|c|c|c|c|c|c|c|c|c|c|c|c|c|c|c|c|c|c|}
\hline run1 & & run2 & & run3 & & run4 & & run5 & & run6 & & run7 & & run8 & & run9 & \\
\hline [Pa] & {$[1 / s]$} & [Pa] & {$[1 / s]$} & [Pa] & {$[1 / s]$} & [Pa] & {$[1 / s]$} & [Pa] & {$[1 / s]$} & [Pa] & {$[1 / s]$} & [Pa] & {$[1 / s]$} & [Pa] & {$[1 / s]$} & [Pa] & {$[1 / s]$} \\
\hline 12.49 & 1113 & 11.62 & 1111 & 11.09 & 1108 & 11 & 1104 & 11.19 & 1111 & 11.1 & 1113 & 10.99 & 1114 & 10.8 & 1110 & 10.64 & 1108 \\
\hline 12.77 & 1134 & 11.8 & 1138 & 11.5 & 1137 & 11.5 & 1140 & 11.33 & 1131 & 11.37 & 1142 & 11.2 & 1140 & 11.08 & 1140 & 10.95 & 1135 \\
\hline 13.03 & 1166 & 12.13 & 1163 & 11.94 & 1168 & 11.74 & 1165 & 11.68 & 1158 & 11.67 & 1169 & 11.46 & 1164 & 11.41 & 1166 & 11.26 & 1161 \\
\hline 13.4 & 1192 & 12.66 & 195 & 12.15 & 1194 & 11.94 & 1192 & 11.97 & 1189 & 12.04 & 1195 & 11.98 & 1197 & 11.66 & 1192 & 11.53 & 1185 \\
\hline 13.81 & 1220 & 12.87 & 1219 & 12.42 & 1217 & 12.29 & 1217 & 12.45 & 1225 & 12.35 & 1225 & 12.28 & 1222 & 12.08 & 1219 & 11.94 & 1216 \\
\hline 14.02 & 1243 & 13 & 1247 & 12.79 & 1244 & 12.61 & 1242 & 12.78 & 1251 & 12.61 & 1249 & 12.6 & 1249 & 12.49 & 1250 & 12.19 & 1243 \\
\hline 14.38 & 1268 & 13.29 & 1271 & 13.24 & 1276 & 13.13 & 1274 & 13.08 & 1278 & 12.99 & 1274 & 12.9 & 1275 & 12.81 & 1276 & 12.52 & 1268 \\
\hline 14.81 & 1298 & 3.67 & 1305 & 13.49 & 1306 & 13.36 & 1302 & 13.35 & 1302 & 13.41 & 1307 & 13.19 & 1302 & 13.05 & 1301 & 12.85 & 1293 \\
\hline 15.15 & 1324 & 14.01 & 1330 & 13.85 & 1328 & 13.65 & 1326 & 13.74 & 1327 & 13.72 & 1333 & 13.68 & 1336 & 13.32 & 1326 & 13.33 & 1325 \\
\hline 15.59 & 1356 & 14.3 & 1356 & 14.29 & 1359 & 14.06 & 1352 & 14.17 & 1360 & 14.06 & 1362 & 13.9 & 1360 & 13.68 & 1350 & 13.59 & 1354 \\
\hline 15.98 & 1383 & 14.62 & 380 & & 1386 & 14.45 & 1382 & & 1385 & & & 14.23 & 1388 & 14.1 & 1386 & 13.96 & 1379 \\
\hline 16.16 & 1408 & 15.02 & 1412 & 14.91 & 1411 & 14.86 & 1409 & 14.72 & 1413 & 14.68 & 1409 & 14.56 & 1413 & 14.44 & 1409 & 14.36 & 1405 \\
\hline 16.62 & 1438 & .51 & 1440 & 5.54 & 1442 & 15.12 & 1437 & 15.06 & 1439 & 15.14 & 1444 & 14.86 & 1438 & 14.74 & 1434 & 14.59 & 1433 \\
\hline 16.91 & 1465 & 15.76 & 1464 & 15.88 & 1469 & 15.42 & 1462 & 15.45 & 1465 & 15.51 & 1467 & 15.3 & 1469 & 15.25 & 1469 & 14.9 & 1461 \\
\hline 17.29 & 1488 & 0.03 & 1489 & 16.25 & 1493 & 15.7 & 1487 & 15.88 & 1499 & 15.83 & 1497 & 15.63 & 1498 & 15.59 & 1496 & 15.34 & 1492 \\
\hline 17.65 & 1521 & 6.44 & 1520 & 16.5 & 1518 & 16.18 & 1519 & 16.14 & 1525 & 16.09 & 1520 & 15.96 & 1523 & 15.83 & 1520 & 15.51 & 1506 \\
\hline 16.17 & 1506 & 5.93 & 1509 & 5.78 & 1507 & 15.54 & 1508 & 15.4 & 1489 & 15.28 & 1488 & 15.66 & 1511 & 15.57 & 1514 & 15.54 & 1515 \\
\hline 15.75 & 1481 & .51 & 1484 & & 1479 & 15.28 & 1481 & 14.89 & 1457 & 14.77 & 1456 & 15.34 & 1483 & 15.28 & 1486 & 15.1 & 1482 \\
\hline 15.43 & 1454 & 5.25 & 1461 & 5.07 & 1456 & 15.04 & 1457 & 14.51 & 1429 & 14.42 & 1427 & 15.04 & 1461 & 14.75 & 1453 & 14.77 & 1454 \\
\hline 15.13 & 1430 & 14.89 & 1429 & 14.69 & 1430 & 14.51 & 1425 & 14.3 & 1408 & 14.18 & 1402 & 14.63 & 1428 & 14.42 & 1426 & 14.54 & 1429 \\
\hline 14.57 & 1397 & 4.48 & 1400 & 14.39 & 1403 & 14.09 & 1399 & 13.98 & 1382 & 13.77 & 1377 & 14.24 & 1403 & 14.03 & 1402 & 14.18 & 1406 \\
\hline 14.27 & 1372 & 14.09 & 1376 & 14.01 & 1370 & 13.86 & 1370 & 13.53 & 1348 & 13.47 & 1346 & 13.98 & 1374 & 13.68 & 1376 & 13.77 & 1373 \\
\hline 13.92 & 1343 & 13.77 & 1346 & 13.65 & 1346 & 13.5 & 1347 & 13.21 & 1322 & 13.09 & 1321 & 13.62 & 1348 & 13.41 & 1352 & 13.41 & 1346 \\
\hline 13.68 & 1321 & 13.38 & 1318 & 13.29 & 1318 & 13.09 & 1316 & 12.95 & 1297 & 12.77 & 1291 & 13.29 & 1323 & 13.09 & 1326 & 13.18 & 1322 \\
\hline 13.18 & 1291 & 13.09 & 1293 & 13.03 & 1291 & 12.75 & 1289 & 12.51 & 1264 & 12.49 & 1268 & 12.95 & 1296 & 12.64 & 1290 & 12.82 & 1294 \\
\hline 12.98 & 1263 & 12.84 & 1268 & 12.62 & 1266 & 12.54 & 1265 & 12.19 & 1238 & 12.25 & 1244 & 12.58 & 1268 & 12.36 & 1263 & 12.44 & 1265 \\
\hline 12.56 & 1237 & 12.45 & 1237 & 12.25 & 1232 & 12.09 & 1233 & 11.89 & 1213 & 11.74 & 1210 & 12.21 & 1237 & 12.01 & 1237 & 12.13 & 1242 \\
\hline 12.32 & 1207 & 12.01 & 1208 & 12.04 & 1211 & 11.83 & 1209 & 11.59 & 1184 & 11.52 & 1182 & 11.9 & 1211 & 11.84 & 1212 & 11.81 & 1209 \\
\hline 11.95 & 1182 & 11.82 & 1184 & 11.59 & 1178 & 11.56 & 1182 & 11.25 & 1158 & 11.11 & 1156 & 11.54 & 1185 & 11.51 & 1186 & 11.52 & 1184 \\
\hline 11.48 & 1160 & 11.39 & 1154 & 11.21 & 1152 & 11.32 & 1159 & 10.85 & 1132 & 10.87 & 1130 & 11.21 & 1155 & 11.07 & 1155 & 11.19 & 1159 \\
\hline 11.35 & 1140 & 11.1 & 1129 & 10.92 & 1122 & 11.04 & 1132 & 10.67 & 1106 & 10.61 & 1106 & 10.93 & 1129 & 10.72 & 1125 & 10.85 & 1134 \\
\hline
\end{tabular}


Table 3. $15^{\circ} \mathrm{C}$ at $2 \mathrm{wt} \%$ - continued

\begin{tabular}{|c|c|c|c|c|c|c|c|c|c|c|c|c|c|c|c|c|c|}
\hline run1 & & run2 & & run3 & & run4 & & run5 & & run6 & & run7 & & run8 & & run9 & \\
\hline$[\mathrm{Pa}]$ & {$[1 / s]$} & {$[\mathrm{Pa}]$} & {$[1 / \mathbf{s}]$} & [Pa] & {$[1 / s]$} & [Pa] & {$[1 / s]$} & [Pa] & {$[1 / s]$} & [Pa] & {$[1 / \mathrm{s}]$} & [Pa] & {$[1 / s]$} & [Pa] & {$[1 / s]$} & [Pa] & {$[1 / s]$} \\
\hline 11.02 & 1108 & 10.75 & 1098 & 10.71 & 1098 & 10.49 & 1099 & 10.2 & 1077 & 10.1 & 1075 & 10.68 & 1102 & 10.33 & 1101 & 10.62 & 1107 \\
\hline 10.83 & 1082 & 10.43 & 1074 & 10.24 & 1068 & 10.28 & 1071 & 9.989 & 1051 & 9.859 & 1047 & 10.32 & 1076 & 10.05 & 1072 & 10.18 & 1075 \\
\hline 10.23 & 1049 & 10.13 & 1047 & 10.06 & 1043 & 10.06 & 1050 & 9.693 & 1025 & 9.631 & 1022 & 9.963 & 1051 & 9.877 & 1048 & 9.874 & 1048 \\
\hline 10.18 & 1033 & 868 & 1023 & & 1021 & 9.536 & 1018 & 9.309 & 991.5 & 155 & 989.3 & 9.738 & 1026 & .545 & 1024 & .622 & 1024 \\
\hline 9.631 & 993.7 & 9.43 & 990.2 & 338 & 986.7 & 9.264 & 990.2 & .078 & 968.6 & 983 & 963.8 & 9.261 & 991.1 & .184 & 991.1 & .347 & 997.3 \\
\hline 9.279 & 2.5 & 104 & 63.3 & 9.081 & 959.8 & 9.084 & 962.9 & 8.791 & 943.1 & 3.666 & 936.5 & 9.042 & 965.1 & 838 & 965.1 & .903 & 965.1 \\
\hline 9.11 & 936.9 & 871 & 938.2 & 8.74 & 932 & 8.797 & 940.4 & 8.415 & 914.4 & 8.447 & 911.3 & 8.761 & 938.2 & 658 & 941.3 & 8.604 & 938.2 \\
\hline 8.655 & 905.2 & 619 & 910 & 8.477 & 908.7 & 8.379 & 906.9 & 8.193 & 888 & 8.075 & 887.1 & 8.53 & 913.1 & 8.193 & 909.6 & 8.365 & 910.5 \\
\hline 8.376 & 878.3 & 8.359 & 885.8 & 8.225 & 883.1 & 8.143 & 880 & 7.808 & 858 & 7.787 & 853.2 & 8.249 & 888 & 8.06 & 881.4 & 8.137 & 885.8 \\
\hline 8.211 & 855.8 & 8.042 & 859.3 & 7.853 & 850.1 & 7.906 & 855.4 & 7.554 & 828.5 & 7.503 & 829.4 & 7.858 & 854 & 7.779 & 857.1 & 7.752 & 860.7 \\
\hline 7.799 & 826.3 & 7.639 & 827.6 & 7.604 & 824.1 & 7.465 & 824.1 & 7.258 & 803.8 & 7.231 & 803.8 & 7.568 & 826.3 & 7.551 & 832.4 & 7.48 & 829.8 \\
\hline 7.518 & 799 & & 801.6 & & 795.4 & 7.2 & 799.4 & 7.107 & 780 & 6.92 & 778.7 & 7.341 & 799.8 & 16 & 800.3 & 7.199 & 802.5 \\
\hline 7.32 & 774.7 & 559 & 771.6 & 7.071 & 769.9 & 6.982 & 772.1 & 6.758 & 753.6 & 6.645 & 745.6 & 7.012 & 776.5 & 79 & 771.6 & 8.897 & 771.6 \\
\hline 6.92 & 741.7 & 846 & 744.8 & 6.793 & 745.2 & .761 & 745.6 & 6.429 & 722.3 & 6.364 & 717.9 & 6.707 & 744.8 & 6.642 & 745.2 & .595 & 746.1 \\
\hline 6.695 & 718.3 & 607 & 717.9 & 6.613 & 720.5 & 6.506 & 717.9 & 6.234 & 696.7 & 6.124 & 694.1 & 6.42 & 719.6 & 6.349 & 720.1 & 0.432 & 723.6 \\
\hline 6.42 & 688.8 & 254 & 695 & 6.299 & 692.8 & 6.186 & 692.8 & .012 & 671.2 & 5.917 & 669.4 & 6.195 & 692.8 & 6.16 & 696.7 & 6.077 & 690.5 \\
\hline 6.192 & 665 & 6.035 & 664.1 & 5.917 & 659.7 & 5.879 & 661.5 & 5.589 & 637.2 & 5.562 & 638.5 & 5.917 & 665.4 & 5.811 & 662.3 & 5.748 & 665.9 \\
\hline 5.834 & 638.1 & 5.816 & 640.3 & 5.68 & 635.5 & 5.595 & 634.1 & 5.331 & 613.4 & 5.408 & 609.5 & 5.648 & 642.5 & 5.624 & 639.9 & 5.615 & 638.5 \\
\hline 5.731 & 612.5 & 5.55 & 616.5 & 5.381 & 608.1 & 5.447 & 609.9 & 5.077 & 585.7 & 5.124 & 585.7 & 5.322 & 610.8 & 5.307 & 614.8 & 5.402 & 614.8 \\
\hline 5.198 & 9.9 & & 583 & & 576.4 & 5.0 & 580.8 & 4.843 & 561.4 & 4.716 & 554.8 & 5.065 & 586.1 & 5.006 & 587.9 & 5.006 & 584.3 \\
\hline 5.05 & 550.9 & & 554.8 & 908 & 552.2 & 4.8 & 556.6 & 4.609 & 528.8 & 4.482 & 527.5 & 4.923 & 556.6 & 63 & 555.3 & 4.763 & 555.3 \\
\hline 4.766 & 527.9 & 784 & 531.9 & 4.633 & 525.7 & 4.576 & 526.2 & 4.357 & 501.1 & 4.357 & 502.8 & 4.701 & 532.3 & 4.538 & 528.8 & 4.568 & 530.1 \\
\hline 4.547 & 505 & 381 & 499.3 & 4.396 & 497.1 & 4.36 & 501.9 & 4.109 & 476.4 & 4.13 & 476.8 & 4.426 & 506.8 & 4.289 & 503.7 & 4.319 & 506.3 \\
\hline 4.337 & 476.8 & 4.198 & 472 & 4.18 & 468.9 & 4.044 & 470.6 & 3.955 & 453.9 & 3.804 & 451.3 & 4.085 & 473.3 & 4.008 & 478.1 & 4.038 & 471.1 \\
\hline 3.97 & 444.6 & 3.996 & 447.3 & 3.828 & 442 & 3.884 & 443.3 & 3.6 & 419.5 & 3.487 & 416.9 & 3.783 & 445.5 & 3.709 & 444.2 & 3.721 & 448.2 \\
\hline 3.724 & 418.6 & 3.78 & 423.9 & 3.727 & 416.4 & 3.635 & 420.4 & 3.319 & 394.4 & 3.319 & 392.2 & 3.635 & 418.6 & 3.443 & 416.4 & 3.632 & 417.8 \\
\hline 3.49 & 391.3 & 3.674 & 404.5 & 3.384 & 390 & 3.325 & 388.2 & 3.18 & 368.9 & 3.055 & 366.2 & 3.407 & 395.3 & 3.31 & 391.8 & 3.319 & 394.4 \\
\hline 3.227 & 361.8 & 289 & 385.2 & 3.197 & 364 & 2.978 & 363.1 & 2.937 & 342 & 2.878 & 338 & 3.271 & 369.3 & 3.138 & 366.2 & 3.076 & 363.1 \\
\hline 3.076 & 334.5 & & 342 & 3.052 & 339.8 & & 334.9 & 2.529 & 312 & 2.7 & 313.3 & 2.851 & 336.2 & 2.819 & 339.8 & .845 & 338 \\
\hline 2.842 & 309.8 & .002 & 331.8 & 2.603 & 306.7 & 2.709 & 310.7 & 2.443 & 285.6 & 2.404 & 282.9 & 2.671 & 310.7 & 311 & 308.5 & 2.611 & 312.4 \\
\hline 2.537 & 283.4 & & 293.5 & 2.431 & 277.2 & 2.434 & 282 & 2.247 & 259.6 & 2.215 & 255.2 & 2.505 & 284.2 & 2.395 & 283.4 & 2.375 & 283.4 \\
\hline 2.236 & 252.5 & 2.514 & 271 & 2.135 & 252.1 & 2.271 & 257.8 & 2.025 & 237.1 & & 3.8 & 2.218 & 259.1 & 2.159 & 255.2 & 2.129 & 257.4 \\
\hline
\end{tabular}


Table 4. $25{ }^{\circ} \mathrm{C}$ at 2 wt \%

\begin{tabular}{|c|c|c|c|c|c|c|c|c|c|c|c|c|c|c|c|c|c|}
\hline run1 & & run2 & & run3 & & run4 & & run5 & & run6 & & run7 & & run8 & & run9 & \\
\hline [Pa] & {$[1 / s]$} & [Pa] & {$[1 / s]$} & [Pa] & {$[1 / s]$} & [Pa] & {$[1 / s]$} & [Pa] & {$[1 / s]$} & [Pa] & [1/s] & [Pa] & {$[1 / \mathrm{s}]$} & [Pa] & {$[1 / \mathrm{s}]$} & [Pa] & {$[1 / \mathrm{s}]$} \\
\hline 2.652 & 275 & 2.62 & 279.4 & 2.605 & 275 & 2.605 & 277.6 & 2.525 & 273.2 & 2.484 & 275.4 & 2.247 & 272.3 & 2.208 & 274.5 & 2.108 & 278.1 \\
\hline 3.102 & 296.1 & 3.034 & 293.5 & 2.865 & 296.1 & 2.836 & 297.9 & 2.774 & 297.5 & 2.717 & 297 & 2.575 & 294.8 & 2.463 & 295.3 & 2.531 & 294.8 \\
\hline 3.312 & 321.7 & 265 & 318.6 & 3.129 & 318.2 & .141 & 321.7 & 3.043 & 318.6 & 2.998 & 323.9 & 2.874 & 323.9 & 2.809 & 327.4 & 2.717 & 323 \\
\hline 3.552 & 346.4 & .558 & 346.4 & 3.454 & 349.9 & .454 & 351.2 & 3.256 & 347.3 & 3.209 & 349 & 3.158 & 350.3 & 3.034 & 357 & 2.922 & 347.3 \\
\hline 3.842 & 369.7 & 3.774 & 370.6 & 3.709 & 379.4 & 3.667 & 378.1 & 3.641 & 379 & 3.413 & 373.7 & 3.392 & 375.5 & 3.212 & 382.1 & 3.274 & 378.1 \\
\hline 4.209 & 402.3 & 4.185 & 403.2 & 3.922 & 404.1 & 3.916 & 400.6 & 3.851 & 404.1 & 3.768 & 405.9 & 3.579 & 403.7 & 3.496 & 407.6 & 3.425 & 403.7 \\
\hline 4.561 & 427.9 & 4.345 & 428.8 & 4.292 & 434.5 & 4.093 & 426.1 & 4.064 & 431.4 & 4.008 & 431.9 & 3.771 & 430.5 & 3.694 & 433.6 & 3.688 & 431 \\
\hline 4.904 & 460.1 & 4.608 & 453.5 & 4.268 & 438.5 & 4.484 & 461 & 4.333 & 456.5 & 4.173 & 460.1 & 4.102 & 454.8 & 4.043 & 468.9 & 3.886 & 458.8 \\
\hline 5.186 & 485.2 & 4.907 & 486.5 & 4.759 & 483.9 & 4.706 & 489.2 & 4.558 & 482.1 & 4.401 & 485.6 & 4.425 & 484.8 & 4.244 & 492.7 & 4.064 & 485.2 \\
\hline 5.443 & 513 & 5.224 & 511.6 & 5.135 & 505 & 4.961 & 513.8 & 4.786 & 508.5 & 4.676 & 517.4 & 4.6 & 515.2 & 4.457 & 521.3 & 4.265 & 509 \\
\hline 5.656 & 535 & & & & 528.8 & & 539 & & 534.1 & & 542 & 4.854 & & 4.629 & 546 & & 532.8 \\
\hline 6.171 & 568 & 5.698 & 562.3 & 5.511 & 560.1 & 5.479 & 565 & 5.41 & 568 & 5.171 & 570.7 & 4.987 & 564.1 & 5.014 & 577.3 & 4.893 & 569.8 \\
\hline 6.363 & 594 & 5.97 & 586.1 & 5.668 & 575.1 & 5.795 & 595.8 & 5.606 & 591 & 5.39 & 593.6 & 5.262 & & 5.058 & 597.1 & 5.005 & 597.6 \\
\hline 6.615 & 618.7 & 6.352 & 618.7 & 6.133 & 623.1 & 6.005 & 625.3 & 5.825 & 621.4 & 5.715 & 624 & 5.558 & 621.8 & 5.366 & 623.6 & 5.227 & 622.7 \\
\hline 6.935 & 643.8 & 6.508 & 646 & 6.461 & 652.2 & 6.289 & 649.1 & 6.076 & 645.2 & 5.928 & 651.8 & 5.718 & 649.1 & 5.505 & 650 & 5.493 & 647.8 \\
\hline 7.296 & 676.9 & 6.745 & 672 & 6.642 & 676.9 & 6.47 & 678.2 & & 677.8 & 6.171 & 676.9 & 6.109 & 675.6 & 5.813 & 683.1 & 5.718 & 672.5 \\
\hline 7.541 & 703.8 & 7.177 & 702.4 & 7.009 & 700.7 & 6.79 & 706 & 6.733 & 703.8 & 6.431 & 708.6 & 6.233 & 703.3 & 6.02 & 710.8 & 5.99 & 703.3 \\
\hline 7.858 & 726.7 & & 730.7 & 7.293 & 733.7 & & 732.4 & & 723.2 & 6.674 & 733.3 & 6.461 & 728 & 6.405 & 741.7 & 6.275 & 735.1 \\
\hline 8.287 & 760.2 & 7.648 & 754.9 & 7.621 & 758.9 & 7.361 & 759.7 & 7.21 & 761.5 & 6.976 & 760.2 & 6.76 & 754.9 & 6.719 & 763.7 & 6.467 & 761.5 \\
\hline 8.556 & 787.1 & 7.985 & 785.3 & 7.825 & 786.6 & 7.671 & 786.6 & 7.29 & 777.8 & 7.21 & 789.3 & 7.006 & 781.3 & 6.852 & 793.7 & 6.736 & 786.2 \\
\hline 8.894 & 810.4 & 8.151 & 804.2 & 8.068 & 812.6 & 7.831 & 814.4 & 7.722 & 814.4 & 7.387 & 814.8 & 7.358 & 815.3 & 7.009 & 818.8 & 6.884 & 812.2 \\
\hline 9.113 & 838.2 & 8.515 & 839.1 & 8.323 & 836.9 & 8.071 & 839.1 & 7.929 & 838.6 & 7.657 & 839.5 & 7.556 & 841.7 & 7.307 & 846.1 & 7.115 & 840.4 \\
\hline 9.536 & 869 & 8.808 & 862 & 8.604 & 862.9 & 8.349 & 867.7 & 8.195 & 864.6 & 7.95 & 864.6 & 7.763 & 865.5 & 7.464 & 870.8 & 7.449 & 868.6 \\
\hline 9.826 & 893.7 & 9.107 & 891.1 & 8.982 & 896.3 & 8.666 & 894.6 & 8.438 & 889.7 & 8.178 & 891.1 & 8.071 & 897.2 & 7.802 & 897.2 & 7.704 & 895 \\
\hline 10.11 & 922.3 & 9.426 & 922.3 & 9.142 & 923.2 & 8.855 & 918.8 & 8.728 & 921.9 & 8.586 & 925.4 & 8.37 & 922.8 & 8.08 & 924.1 & 7.855 & 920.1 \\
\hline 10.37 & 945.7 & 9.696 & 946.6 & 9.403 & 951.4 & 9.116 & 945.3 & 9.039 & 947.9 & 8.808 & 954.1 & 8.527 & 950.1 & 8.394 & 957.2 & 8.083 & 945.7 \\
\hline 10.76 & 977 & & 971.3 & 9.705 & 975.2 & 9.551 & 978.3 & 9.222 & 975.7 & & 979.6 & 8.766 & 974.3 & 8.598 & 984.5 & 8.453 & 979.6 \\
\hline 11.05 & 1005 & 10.29 & 999.5 & 9.941 & 1002 & 9.71 & 1007 & 9.512 & 1001 & 9.332 & 1004 & 8.977 & 999.9 & 8.897 & 1009 & 8.687 & 1007 \\
\hline 11.32 & 1028 & 10.52 & 1025 & 10.23 & 1030 & 9.995 & 1031 & 9.743 & 1027 & 9.604 & 1029 & 9.465 & 1033 & 9.127 & 1034 & 8.95 & 1031 \\
\hline 11.58 & 1053 & 10.78 & 1050 & 10.62 & 1062 & 10.24 & 1055 & 10.13 & 1059 & 9.87 & 1056 & 9.663 & 1059 & 9.497 & 1066 & 9.172 & 1056 \\
\hline 12.01 & 1087 & 11.15 & 1084 & 10.89 & 1086 & 10.48 & 1081 & 10.44 & 1088 & 10.23 & 1090 & 9.95 & 1084 & 9.687 & 1092 & 9.491 & 1090 \\
\hline
\end{tabular}


Table 4. $25{ }^{\circ} \mathrm{C}$ at 2 wt $\%$ - continued

\begin{tabular}{|c|c|c|c|c|c|c|c|c|c|c|c|c|c|c|c|c|c|}
\hline run1 & & run2 & & run3 & & run4 & & run5 & & run6 & & run7 & & run8 & & run9 & \\
\hline [Pa] & [1/s] & [Pa] & [1/s] & [Pa] & [1/s] & [Pa] & [1/s] & [Pa] & [1/s] & [Pa] & [1/s] & [Pa] & {$[1 / s]$} & [Pa] & {$[1 / s]$} & [Pa] & [1/s] \\
\hline 12.31 & 1110 & 11.42 & 1113 & 11.1 & 1113 & 10.88 & 1117 & 10.64 & 1111 & 10.5 & 1117 & 10.13 & 1109 & 9.98 & 1122 & 9.814 & 1113 \\
\hline 12.62 & 1137 & 11.66 & 1134 & 11.36 & 1140 & 11.19 & 1140 & 11.02 & 1142 & 10.66 & 1142 & 10.51 & 1141 & 10.23 & 1144 & 10.04 & 1141 \\
\hline 13.08 & 1167 & 11.97 & 1159 & 11.62 & 1164 & 11.53 & 1172 & 11.25 & 1169 & 10.92 & 1169 & 10.67 & 1170 & 10.53 & 1175 & 10.2 & 1166 \\
\hline 13.38 & 1194 & 12.38 & 1192 & 12.05 & 1196 & 11.82 & 1198 & 11.49 & 1193 & 11.2 & 1194 & 11 & 1192 & 10.8 & 1203 & 10.62 & 1199 \\
\hline 13.58 & 1217 & 12.61 & 1221 & 12.31 & 1221 & 11.89 & 1218 & 11.79 & 1218 & 11.65 & 1227 & 11.2 & 1218 & 11.06 & 1228 & 10.85 & 1223 \\
\hline 14.08 & 1249 & 12.95 & 1247 & 12.57 & 1250 & 12.29 & 1237 & 12 & 1244 & 11.88 & 1252 & 11.64 & 1252 & 11.43 & 1258 & 11.2 & 1251 \\
\hline 14.37 & 1277 & 13.27 & 1275 & 12.86 & 1274 & 12.39 & 1250 & 12.41 & 1272 & 12.12 & 1280 & 11.9 & 1278 & 11.61 & 1285 & 11.43 & 1277 \\
\hline 14.52 & 1299 & 13.62 & 1304 & 13.25 & 1308 & 12.89 & 1308 & 12.76 & 1304 & 12.38 & 1304 & 12.18 & 1304 & 11.9 & 1310 & 11.67 & 1301 \\
\hline 14.93 & 1328 & 13.84 & 1330 & 13.55 & 1334 & 12.95 & 1315 & 13.12 & 1332 & 12.76 & 1334 & 12.4 & 1329 & 12.14 & 1338 & 12 & 1334 \\
\hline 15.19 & 1353 & 14.2 & 1354 & 13.76 & 1356 & 13.38 & 1336 & 13.32 & 1358 & 13.08 & 1359 & 12.85 & 1360 & 12.41 & 1362 & 12.34 & 1359 \\
\hline 15.65 & 1384 & 14.56 & 1384 & 14.19 & 1389 & 13.9 & 1385 & 13.61 & 1383 & 13.32 & 1387 & 13.15 & 1386 & 12.82 & 1396 & 12.62 & 1384 \\
\hline 15.95 & 1412 & 14.8 & 1412 & 14.55 & 1416 & 14.19 & 1412 & 13.89 & 1407 & & 1414 & 13.41 & 1411 & 13.09 & 1419 & 12.74 & 1411 \\
\hline 16.25 & 1438 & 15.12 & 1436 & 14.86 & 1442 & 14.67 & 1444 & 14.28 & 1441 & 13.99 & 1446 & 13.74 & 1438 & 13.43 & 1443 & 13.16 & 1436 \\
\hline 16.53 & 1462 & 15.4 & 1461 & 15.09 & 1466 & 14.84 & 1468 & 14.67 & 1467 & 14.31 & 1471 & 13.91 & 1464 & 13.82 & 1475 & 13.54 & 1470 \\
\hline 16.95 & 1489 & 15.83 & 1493 & 15.37 & 1491 & 15.17 & 1495 & 14.92 & 1492 & 14.64 & 1498 & 14.37 & 1497 & 14.09 & 1500 & 13.81 & 1494 \\
\hline 17.39 & 1523 & 16.09 & 1520 & 15.72 & 1517 & 15.49 & 1524 & 15.26 & 1516 & 14.85 & 1523 & 14.71 & 1523 & 14.59 & 1531 & 14.15 & 1523 \\
\hline 16.26 & 1535 & 5.7 & 1534 & 15.4 & 1541 & 14.93 & 1513 & 14.34 & 1511 & 14.72 & 1539 & 12.93 & 1419 & 13.87 & 1535 & 13.63 & 1504 \\
\hline 16 & 1509 & 15.35 & 1510 & 14.93 & 1509 & 14.58 & 1486 & 14.04 & 1485 & 14.22 & 1506 & 12.61 & 1394 & 13.57 & 1507 & 13.48 & 1478 \\
\hline 15.58 & 1478 & 5.05 & 1487 & 14.69 & 1486 & 14.13 & 1453 & 13.87 & 1456 & 14.01 & 1480 & 12.21 & 1360 & 13.42 & 1482 & 13.1 & 1453 \\
\hline 15.14 & 1452 & 4.63 & 1453 & 14.43 & 1458 & 13.9 & 1429 & 13.45 & 1434 & 13.72 & 1455 & 12.13 & 1344 & 13.18 & 1458 & 12.82 & 1426 \\
\hline 14.9 & 1425 & 14.37 & 1430 & 14.25 & 1431 & 13.57 & 1399 & 13.16 & 1408 & & 1431 & 11.65 & 1308 & 12.74 & 1425 & 12.52 & 1399 \\
\hline 14.61 & 1402 & 14.1 & 1407 & 13.9 & 1405 & 13.24 & 1374 & 12.86 & 1376 & 13.04 & 1402 & 11.39 & 1280 & 12.55 & 1400 & 12.29 & 1374 \\
\hline 14.16 & 1370 & 13.63 & 1371 & 13.36 & 1372 & 12.89 & 1347 & 12.54 & 1348 & 12.74 & 1370 & 11.1 & 1256 & 12.3 & 1374 & 11.81 & 1342 \\
\hline 13.87 & 1343 & 3.24 & 1346 & 13.04 & 1348 & 12.7 & 1318 & 12.37 & 1322 & 12.4 & 1343 & 10.84 & 1230 & 11.86 & 1342 & 11.88 & 1338 \\
\hline 13.57 & 1320 & 13.02 & 1317 & 12.87 & 1320 & 12.41 & 1292 & 11.81 & 1291 & 12.21 & 1320 & 10.53 & 1204 & 11.59 & 1318 & 11.19 & 1307 \\
\hline 13.3 & 1294 & 12.72 & 1296 & 12.64 & 1295 & 12.03 & 1267 & 11.59 & 1266 & 11.86 & 1293 & 10.13 & 1173 & 11.2 & 1290 & 11.2 & 1293 \\
\hline 12.82 & 1261 & 12.44 & 1269 & 12.27 & 1270 & 11.76 & 1243 & 11.4 & 1237 & 11.4 & 1262 & 9.882 & 1145 & 11.02 & 1264 & 10.4 & 1219 \\
\hline 12.5 & 1237 & 12.07 & 1237 & 11.78 & 1237 & 11.38 & 1208 & 11.11 & 1212 & 11.13 & 1236 & 9.577 & 1114 & 10.75 & 1233 & 10.37 & 1212 \\
\hline 12.25 & 1207 & 11.84 & 1209 & 11.48 & 1214 & 11.06 & 1183 & 10.77 & 1186 & 10.9 & 1208 & 9.346 & 1097 & 10.48 & 1209 & 10.44 & 1207 \\
\hline 11.89 & 1181 & 11.38 & 1180 & 11.23 & 1184 & 10.81 & 1157 & 10.49 & 1155 & 10.58 & 1181 & 9 & 1063 & 10.05 & 1178 & 9.805 & 1157 \\
\hline 11.63 & 1156 & 11.07 & 1154 & 10.96 & 1158 & 10.48 & 1130 & 10.19 & 1128 & 10.33 & 1155 & 8.695 & 1034 & 9.98 & 1160 & 9.444 & 1123 \\
\hline
\end{tabular}


Table 4. $25{ }^{\circ} \mathrm{C}$ at $2 \mathrm{wt} \%$ - continued

\begin{tabular}{|c|c|c|c|c|c|c|c|c|c|c|c|c|c|c|c|c|c|}
\hline run1 & & run2 & & run3 & & run4 & & run5 & & run6 & & run7 & & run8 & & run9 & \\
\hline [Pa] & {$[1 / \mathrm{s}]$} & {$[\mathrm{Pa}]$} & {$[1 / s]$} & {$[\mathrm{Pa}]$} & {$[1 / \mathrm{s}]$} & [Pa] & {$[1 / s]$} & {$[\mathrm{Pa}]$} & {$[1 / \mathrm{s}]$} & {$[\mathrm{Pa}]$} & {$[1 / \mathrm{s}]$} & {$[\mathrm{Pa}]$} & {$[1 / \mathrm{s}]$} & {$[\mathrm{Pa}]$} & {$[1 / \mathrm{s}]$} & [Pa] & {$[1 / \mathrm{s}]$} \\
\hline 11.18 & 1128 & 10.8 & 1129 & 10.73 & 1133 & 10.16 & 1104 & 10.01 & 1106 & 10.08 & 1130 & 8.533 & 1011 & 9.456 & 1127 & 9.249 & 1096 \\
\hline 10.78 & 1097 & 10.52 & 1105 & 10.2 & 1100 & 9.932 & 1078 & 9.779 & 1078 & 9.651 & 1097 & 8.133 & 979.6 & 9.249 & 1097 & 8.929 & 1071 \\
\hline 10.57 & 1072 & 10.15 & 1073 & 10.03 & 1073 & 9.48 & 1046 & 9.5 & 1051 & 9.302 & 1071 & 7.961 & 952.3 & 8.982 & 1071 & 8.701 & 1045 \\
\hline 10.26 & 1046 & 9.873 & 1046 & 9.69 & 1050 & 9.267 & 1021 & 9.19 & 1019 & 9.074 & 1044 & 7.71 & 929 & 8.695 & 1046 & 8.426 & 1019 \\
\hline 9.929 & 1019 & .601 & 1022 & 9.394 & 1021 & 8.938 & 991.1 & 8.959 & 990.7 & 8.929 & 1018 & 7.319 & 899.4 & 8.426 & 1017 & 8.104 & 984.9 \\
\hline 9.705 & 995.1 & 9.222 & 991.5 & 9.116 & 994.2 & 8.716 & 964.2 & 8.681 & 966.9 & 8.598 & 988.5 & 7.08 & 872.6 & 8.201 & 988 & 7.822 & 960.2 \\
\hline 9.204 & 60.7 & 8.994 & 963.8 & 8.855 & 969.5 & 8.423 & 937.8 & 8.405 & 943.1 & 8.346 & 962.9 & 6.855 & 845.2 & .961 & 962.5 & 7.583 & 932.9 \\
\hline 8.956 & 33.4 & 8.737 & 937.8 & 8.465 & 936.9 & 8.175 & 913.1 & 7.97 & 909.6 & 8.032 & 936.5 & 6.568 & 816.1 & 7.737 & 936 & 7.426 & 909.6 \\
\hline 8.684 & 905.6 & 8.358 & 905.6 & 8.154 & 911.8 & 7.745 & 883.1 & 7.734 & 884.5 & 7.76 & 912.7 & 6.292 & 789.3 & 7.455 & 911.3 & 7.159 & 884.5 \\
\hline 8.435 & 881.4 & 8.024 & 881.8 & 7.923 & 882.7 & 7.503 & 855.4 & 7.452 & 856.7 & 7.523 & 884.9 & 6.109 & 762.4 & 7.106 & 880.5 & 6.766 & 849.6 \\
\hline 8.148 & 854.9 & 7.861 & 852.3 & 7.683 & 859.3 & 7.296 & 832.4 & 7.156 & 832.9 & 7.154 & 850.5 & 5.792 & 735.9 & 6.801 & 854 & 6.485 & 825 \\
\hline 7.808 & 827.2 & 7.615 & 828.9 & 7.316 & 827.2 & 6.899 & 801.2 & 6.792 & 802.5 & 6.875 & 825 & 5.6 & 711.7 & 6.615 & 827.2 & 6.281 & 797.2 \\
\hline 7.518 & 801.6 & 7.302 & 806 & 7.091 & 802.9 & 6.621 & 772.5 & 6.517 & 775.2 & 6.603 & 802 & 5.319 & 681.7 & 6.405 & 803.8 & 5.982 & 767.2 \\
\hline 7.145 & 769.9 & 6.908 & 770.8 & 6.763 & 774.3 & 6.431 & 748.7 & 6.322 & 749.6 & 6.396 & 772.5 & 5.005 & 654 & 6.156 & 777.8 & 5.689 & 742.1 \\
\hline 6.884 & 743 & 6.689 & 746.1 & 6.568 & 748.7 & 6.147 & 723.2 & 6.07 & 724 & 6.112 & 747.4 & 4.824 & 626.7 & 5.727 & 743 & 5.49 & 714.3 \\
\hline 6.653 & 721 & 6.476 & 717.4 & 6.331 & 724.5 & 5.774 & 690.5 & 5.668 & 690.5 & 5.819 & 716.6 & 4.511 & 601.5 & 5.547 & 720.1 & 5.268 & 687 \\
\hline 6.257 & 687.5 & 6.189 & 692.8 & 5.925 & 690.1 & 5.579 & 665 & 5.41 & 666.3 & 5.561 & 687.9 & 4.375 & 576 & 5.348 & 689.7 & 4.996 & 659.7 \\
\hline 6.038 & 660.6 & 5.881 & 669 & 5.692 & 666.3 & 5.333 & 639.4 & 5.171 & 638.5 & 5.283 & 661.5 & 4.167 & 550.9 & 5.112 & 665.9 & 4.774 & 635.5 \\
\hline 5.786 & 635.5 & 5.564 & 635.9 & 5.552 & 640.8 & 5.138 & 613 & 4.987 & 613.9 & 5.058 & 637.7 & 3.836 & 522.2 & 4.863 & 641.6 & 4.502 & 610.8 \\
\hline 5.499 & 608.1 & 5.372 & 613.4 & 5.191 & 613.9 & 4.866 & 587 & 4.718 & 587.9 & 4.771 & 607.7 & 3.567 & 489.6 & 4.543 & 608.1 & 4.286 & 579.1 \\
\hline 5.159 & 579.1 & 5.103 & 587.9 & 4.981 & 589.6 & 4.413 & 555.3 & 4.407 & 554.4 & 4.543 & 583 & 3.336 & 464.5 & 4.271 & 580.8 & 4.043 & 550.9 \\
\hline 4.866 & 553.9 & 4.753 & 555.3 & 4.617 & 558.3 & 4.209 & 527.5 & 4.117 & 528.4 & 4.363 & 556.6 & 3.194 & 440.7 & 4.005 & 553.9 & 3.798 & 524.9 \\
\hline 4.644 & 529.3 & 4.52 & 528.4 & 4.3 & 528.8 & 3.969 & 500.2 & 3.872 & 501.5 & 3.975 & 523.5 & 2.868 & 412.9 & 3.792 & 529.7 & 3.599 & 499.7 \\
\hline 4.304 & 495.8 & 4.318 & 503.7 & 4.111 & 503.3 & 3.756 & 476.8 & 3.605 & 473.7 & 3.753 & 497.1 & 2.717 & 384.3 & 3.516 & 499.7 & 3.336 & 474.2 \\
\hline 4.046 & 469.3 & 3.943 & 472 & 3.907 & 472.9 & 3.54 & 450.8 & 3.404 & 447.7 & 3.57 & 468.9 & 2.294 & 353.4 & 3.363 & 473.3 & 3.093 & 447.7 \\
\hline 3.809 & 442.9 & 3.718 & 443.3 & 3.623 & 449.5 & 3.212 & 416.4 & 3.176 & 424.4 & 3.303 & 445.1 & 2.185 & 326.5 & 3.084 & 445.1 & 2.91 & 421.3 \\
\hline 3.534 & 417.8 & 3.481 & 418.2 & 3.472 & 425.3 & 2.886 & 392.6 & 2.794 & 391.8 & 3.155 & 420.9 & 1.951 & 299.7 & 2.851 & 419.5 & 2.593 & 386 \\
\hline 3.354 & 393.5 & 3.294 & 394.9 & 3.135 & 397.9 & 2.711 & 363.6 & 2.667 & 364.4 & 2.75 & 388.7 & 1.747 & 274.5 & 2.543 & 390 & 2.389 & 361.8 \\
\hline 3.001 & 360.9 & 3.016 & 370.6 & 2.797 & 365.8 & 2.492 & 337.1 & 2.421 & 338 & 2.492 & 361.4 & 1.486 & 247.7 & 2.368 & 361.8 & 2.078 & 329.6 \\
\hline 2.759 & 333.6 & 2.632 & 336.2 & 2.569 & 338.9 & 2.259 & 312.4 & 2.155 & 313.8 & 2.273 & 334.5 & & & 2.202 & 333.6 & 1.841 & 303.6 \\
\hline 2.537 & 307.2 & 2.457 & 306.3 & 2.324 & 312 & 2.034 & 287.3 & 1.96 & 289.1 & 2.078 & 308 & & & 1.945 & 310.2 & 1.69 & 278.1 \\
\hline & & & & & & & & & & & & & & & & & \\
\hline
\end{tabular}


APPENDIX E

ENVELOPE C

SR/TRU PRECIPITATE SLURRY RHEOLOGY DATA
WRC-TR-2000-00352

SRT-RPP-2000-00026

Page 188 of 514

Table 4. $25^{\circ} \mathrm{C}$ at 2 wt $\%$ - continued

\begin{tabular}{|c|c|c|c|c|c|c|c|c|c|c|c|c|c|c|c|c|c|}
\hline run1 & & run2 & & run3 & & run4 & & run5 & & run6 & & run7 & & run8 & & run9 & \\
\hline [Pa] & {$[1 / s]$} & [Pa] & {$[1 / \mathrm{s}]$} & [Pa] & [1/s] & [Pa] & {$[1 / s]$} & [Pa] & [1/s] & [Pa] & {$[1 / s]$} & [Pa] & [1/s] & [Pa] & [1/s] & [Pa] & {$[1 / s]$} \\
\hline 2.268 & 281.6 & 2.208 & 282.9 & 2.108 & 287.3 & 1.693 & 253.8 & 1.548 & 256.9 & 1.824 & 282.5 & & & 1.587 & 277.6 & 1.483 & 250.3 \\
\hline 2.131 & 269.7 & 1.995 & 258.7 & 1.835 & 257.4 & 1.477 & 229.2 & 1.394 & 227.8 & 1.646 & 257.4 & & & 1.415 & 253 & 1.32 & 238 \\
\hline 1.809 & 239.3 & 1.726 & 232.2 & 1.557 & 228.7 & 0 & 177.9 & 0 & 169.4 & 1.457 & 232.7 & & & 1.241 & 226.1 & 0 & 191.8 \\
\hline
\end{tabular}


Table 5. $50{ }^{\circ} \mathrm{C}$ at 2 wt $\%$

\begin{tabular}{|c|c|c|c|c|c|c|c|c|c|c|c|c|c|c|c|c|c|}
\hline run1 & & run2 & & run3 & & run4 & & run5 & & run6 & & run7 & & run8 & & run9 & \\
\hline [Pa] & {$[1 / s]$} & [Pa] & {$[1 / s]$} & [Pa] & {$[1 / s]$} & [Pa] & {$[1 / s]$} & [Pa] & {$[1 / s]$} & [Pa] & [1/s] & [Pa] & {$[1 / s]$} & [Pa] & {$[1 / \mathrm{s}]$} & [Pa] & {$[1 / \mathrm{s}]$} \\
\hline 3.587 & 274.5 & 3.232 & 282 & 3.229 & 278.5 & 2.989 & 272.3 & 3.087 & 275.4 & 2.912 & 276.7 & 2.743 & 278.5 & 2.696 & 271.9 & 2.776 & 277.6 \\
\hline 3.927 & 292.6 & .643 & 290.9 & 3.687 & 297.5 & 3.309 & 291.7 & 3.406 & 295.7 & 3.187 & 293.5 & 3.223 & 298.8 & 2.983 & 291.7 & 3.137 & 300.5 \\
\hline 4.288 & 319.1 & .936 & 318.6 & 3.895 & 321.7 & .628 & 316 & 3.495 & 314.2 & 3.534 & 325.2 & 3.495 & 322.6 & 3.335 & 324.3 & 3.377 & 324.8 \\
\hline 4.691 & 347.3 & .244 & 344.6 & 4.137 & 348.1 & .057 & 348.6 & 3.927 & 349 & 3.732 & 350.3 & 3.643 & 349 & 3.593 & 349.9 & 3.684 & 355.2 \\
\hline 4.939 & 375.5 & 4.448 & 367.5 & 4.398 & 372.8 & 4.259 & 376.3 & 4.12 & 376.8 & 3.989 & 376.3 & 3.909 & 373.3 & 3.782 & 378.5 & 3.853 & 383.4 \\
\hline 5.188 & 397.5 & 4.922 & 399.7 & 4.608 & 399.3 & 4.569 & 401.5 & 4.401 & 401 & 4.297 & 403.2 & 4.161 & 400.6 & 4.049 & 404.5 & 4.105 & 409 \\
\hline 5.632 & 429.7 & 5.078 & 423.5 & 5.099 & 432.3 & 4.791 & 428.3 & 4.744 & 432.8 & 4.478 & 430.1 & 4.454 & 433.6 & 4.297 & 430.5 & 4.291 & 419.1 \\
\hline 5.83 & 457 & 5.401 & 453 & 5.339 & 459.6 & 5.087 & 453.9 & 4.975 & 458.3 & 4.7 & 453.5 & 4.697 & 459.2 & 4.652 & 461.8 & 4.708 & 467.6 \\
\hline 6.123 & 479.9 & 5.664 & 476.8 & 5.709 & 482.5 & 5.436 & 486.5 & 5.191 & 483.4 & 5.138 & 486.1 & 4.871 & 483.4 & 4.803 & 486.1 & 4.673 & 479.9 \\
\hline 6.514 & 511.2 & 6.07 & 509.9 & 5.922 & 509.4 & 5.738 & 514.7 & 5.472 & 509.4 & 5.306 & 514.7 & 5.087 & 507.7 & 5.061 & 516 & 5.01 & 507.2 \\
\hline 6.727 & 539 & & 536.3 & & & & & & 534.1 & & & 5.46 & 542 & & 539.4 & 5.442 & 533.2 \\
\hline 6.984 & 563.2 & 6.576 & 561.4 & 6.576 & 568.5 & 6.138 & 567.6 & 5.972 & 560.5 & 5.857 & 569.8 & 5.732 & 567.6 & 5.484 & 564.1 & 5.505 & 557.5 \\
\hline 7.292 & 588.3 & 6.958 & 585.7 & 6.789 & 594.9 & 6.457 & 591.8 & 6.404 & 597.6 & 6.206 & 596.2 & 5.907 & 594.9 & 5.833 & 597.1 & 5.943 & 585.2 \\
\hline 7.706 & 620 & 7.286 & 618.7 & 7.085 & 620 & 6.807 & 622.7 & 6.638 & 622.7 & 6.327 & 623.6 & 6.108 & 621.8 & 6.04 & 621.4 & 6.221 & 628.9 \\
\hline 8.02 & 645.2 & 7.612 & 643.4 & 7.381 & 645.2 & 7.126 & 649.6 & 6.848 & 646.5 & 6.626 & 647.8 & 6.354 & 646.5 & 6.262 & 647.8 & 6.381 & 658.4 \\
\hline 8.42 & 670.7 & & 670.7 & 7.721 & 680 & 7.325 & 675.6 & & 672.5 & 7.026 & 679.5 & 6.65 & 671.6 & 6.493 & 676.4 & 6.579 & 681.7 \\
\hline 8.671 & 698 & 8.118 & 694.5 & 7.931 & 703.8 & 7.635 & 702 & 7.526 & 704.2 & 7.227 & 704.7 & 6.961 & 707.3 & 6.736 & 701.6 & 6.792 & 708.2 \\
\hline 9.106 & 728.4 & & 728.9 & & 727.1 & & 728.9 & & 733.3 & 7.464 & & 7.171 & 731.1 & 7.126 & 734.2 & 7.097 & 732.9 \\
\hline 9.346 & 757.1 & 8.837 & 754 & 8.594 & 758.9 & 8.112 & 758.9 & 8.023 & 758.9 & 7.78 & 757.1 & 7.449 & 757.1 & 7.325 & 760.2 & 7.354 & 766.8 \\
\hline 9.639 & 781.3 & 9.097 & 779.1 & 8.872 & 786.2 & 8.381 & 780.9 & 8.251 & 784.4 & 8.044 & 789.3 & 7.677 & 784 & 7.511 & 785.7 & 7.6 & 793.2 \\
\hline 10.09 & 813.9 & 9.556 & 811.3 & 9.124 & 815.7 & 8.772 & 811.7 & 8.428 & 808.7 & 8.28 & 814.4 & 7.993 & 814.4 & 7.78 & 810.4 & 7.816 & 817 \\
\hline 10.23 & 831.1 & 9.837 & 835.1 & 9.411 & 839.9 & 9 & 838.2 & 8.917 & 842.1 & 8.455 & 842.1 & 8.227 & 842.1 & 8.109 & 841.7 & 8.263 & 848.8 \\
\hline 10.63 & 864.6 & 10.09 & 863.3 & 9.802 & 869.9 & 9.225 & 862.4 & 94 & 869.9 & 8.683 & 866.4 & 8.479 & 867.7 & 369 & 867.3 & 8.514 & 873.9 \\
\hline 10.99 & 889.3 & 10.36 & 885.8 & 9.923 & 894.6 & 9.603 & 896.3 & 9.358 & 893.7 & 9.085 & 897.7 & 8.671 & 891.5 & 8.568 & 898.6 & 8.716 & 898.6 \\
\hline 11.34 & 918.4 & 10.73 & 917.1 & 10.3 & 924.6 & 9.885 & 919.7 & 9.618 & 917.5 & 9.328 & 923.2 & 9.032 & 924.1 & 8.852 & 923.2 & 8.967 & 924.1 \\
\hline 11.75 & 943.1 & 11.05 & 945.3 & 10.64 & 952.8 & 10.13 & 947.5 & 9.938 & 948.8 & 9.494 & 950.6 & 9.236 & 949.2 & 9.127 & 950.6 & 9.352 & 955.8 \\
\hline 12.1 & 974.3 & & 968.2 & 10.86 & 976.6 & & 972.6 & & 977.4 & 9.793 & 974.8 & 9.586 & 978.3 & 9.322 & 974.3 & 9.654 & 983.6 \\
\hline 12.4 & 999.5 & 11.72 & 998.1 & 11.22 & 1005 & 10.77 & 1003 & 10.38 & 1002 & 10.04 & 1001 & 9.748 & 1003 & 9.603 & 1002 & 9.834 & 1008 \\
\hline 12.7 & 1022 & 11.9 & 1027 & 11.43 & 1033 & 11.07 & 1029 & 10.85 & 1034 & 10.42 & 1033 & 10.03 & 1028 & 9.84 & 1027 & 10.22 & 1039 \\
\hline 13.13 & 1057 & 12.18 & 1053 & 11.77 & 1059 & 11.31 & 1058 & 11.11 & 1059 & 10.62 & 1060 & 10.29 & 1053 & 10.17 & 1060 & 10.55 & 1064 \\
\hline 13.38 & 1080 & 12.46 & 1077 & 12.04 & 1085 & 11.48 & 1081 & 11.31 & 1084 & 10.89 & 1086 & 10.57 & 1079 & 10.47 & 1089 & 10.78 & 1093 \\
\hline
\end{tabular}


Table 5. $50{ }^{\circ} \mathrm{C}$ at $2 \mathrm{wt} \%$ - continued

\begin{tabular}{|c|c|c|c|c|c|c|c|c|c|c|c|c|c|c|c|c|c|}
\hline run1 & & run2 & & run3 & & run4 & & run5 & & run6 & & run7 & & run8 & & run9 & \\
\hline [Pa] & {$[1 / s]$} & [Pa] & {$[1 / s]$} & [Pa] & {$[1 / s]$} & [Pa] & {$[1 / s]$} & [Pa] & {$[1 / s]$} & [Pa] & {$[1 / s]$} & [Pa] & {$[1 / s]$} & [Pa] & {$[1 / s]$} & [Pa] & {$[1 / s]$} \\
\hline 13.57 & 1106 & 12.94 & 1108 & 12.48 & 1117 & 12.02 & 1114 & 11.49 & 1112 & 11.22 & 1115 & 10.96 & 1116 & 10.59 & 1107 & 11.01 & 1119 \\
\hline 14.07 & 137 & 13.09 & 1137 & 12.71 & 1141 & 12.22 & 1138 & 11.77 & 1135 & 11.52 & 1143 & 11.23 & 1141 & 11.04 & 1141 & 11.33 & 1147 \\
\hline 14.42 & 163 & 3.47 & 1166 & 13.03 & 1171 & 12.55 & 1164 & 12.11 & 1168 & 11.73 & 1167 & 11.43 & 1168 & 11.24 & 1169 & 11.65 & 1172 \\
\hline 14.82 & 1191 & 13.78 & 1192 & 13.29 & 1195 & 12.8 & 1193 & 12.39 & 1196 & 12.06 & 1196 & 11.72 & 1192 & 11.56 & 1196 & 12.06 & 1201 \\
\hline 15.09 & 1218 & 14.08 & 1218 & 13.59 & 1219 & 13.13 & 1217 & 12.7 & 1222 & 12.32 & 1221 & 12.05 & 1222 & 11.84 & 1223 & 12.24 & 1229 \\
\hline 15.21 & 1243 & 14.34 & 1243 & 13.8 & 1246 & 13.48 & 1251 & 12.99 & 1247 & 12.66 & 1254 & 12.29 & 1250 & 12.12 & 1249 & 12.51 & 1254 \\
\hline 15.54 & 1268 & 14.6 & 1268 & 14.2 & 1279 & 13.75 & 1277 & 13.26 & 1272 & 12.98 & 1281 & 12.6 & 1275 & 12.39 & 1276 & 12.78 & 1279 \\
\hline 15.95 & 1301 & 15.06 & 1299 & 14.51 & 1305 & 14.1 & 1304 & 13.61 & 1308 & 13.2 & 1304 & 12.92 & 1306 & 12.67 & 1302 & 13.27 & 1313 \\
\hline 16.24 & 1329 & 15.33 & 1327 & 14.74 & 1330 & 14.34 & 1328 & 13.9 & 1331 & 13.49 & 1329 & 13.2 & 1330 & 13.06 & 1333 & 13.64 & 1338 \\
\hline 16.48 & 1351 & 15.57 & 1352 & 15.12 & 1360 & 14.63 & 1353 & 14.21 & 1359 & 13.71 & 1354 & 13.53 & 1361 & 13.3 & 1359 & 13.93 & 1366 \\
\hline 16.92 & 1382 & & 376 & 15.4 & 1389 & 15.04 & 1386 & 14.42 & 1384 & & & 13.68 & 1383 & 13.57 & 1383 & 14.18 & 1393 \\
\hline 17.12 & 1410 & 16.27 & 1408 & 15.69 & 1414 & 15.39 & 1411 & 14.77 & 1412 & 14.52 & 1416 & 14.12 & 1415 & 13.99 & 1416 & 14.44 & 1419 \\
\hline 17.52 & 1439 & 0.62 & 1434 & 5.94 & 1440 & 15.61 & 1436 & 15.02 & 1435 & 14.8 & 1441 & 14.36 & 1442 & 14.29 & 1441 & 14.8 & 1449 \\
\hline 17.67 & 1463 & 17.04 & 1465 & 16.31 & 1471 & 16.08 & 1468 & 15.45 & 1468 & 15.07 & 1470 & 14.61 & 1465 & 14.56 & 1467 & 14.93 & 1474 \\
\hline 18.17 & 1478 & 17.24 & 1492 & 16.57 & 1497 & 16.35 & 1495 & 15.76 & 1496 & 15.32 & 1495 & 14.89 & 1491 & 14.79 & 1497 & 15.25 & 1503 \\
\hline 18.32 & 1501 & 17.43 & 1516 & 16.85 & 1525 & 16.72 & 1521 & 16.03 & 1520 & 15.64 & 1521 & 15.3 & 1523 & 15.08 & 1521 & 15.47 & 1528 \\
\hline 17.46 & 1510 & 16.22 & 1487 & 16.55 & 1508 & 16.01 & 1510 & 15.21 & 1488 & 15.3 & 1509 & 15.07 & 1508 & 14.89 & 1510 & 14.86 & 1487 \\
\hline 16.99 & 1480 & .81 & 1456 & 16.28 & 1482 & 15.66 & 1485 & 14.98 & 1452 & 14.98 & 82 & 14.77 & 1482 & 14.59 & 1482 & 14.56 & 1463 \\
\hline 16.72 & 1463 & 5.54 & 1430 & 15.9 & 1458 & 15.24 & 1452 & 14.62 & 1426 & 14.68 & 1459 & 14.47 & 1456 & 14.36 & 1454 & 14.15 & 1429 \\
\hline 16.25 & 1428 & 15.18 & 1398 & 15.66 & 1432 & 14.86 & 1424 & 14.33 & 1400 & 14.39 & 1434 & 14.21 & 1432 & 14.06 & 1429 & 13.85 & 1401 \\
\hline 15.92 & 1400 & 4.83 & 1374 & 15.27 & 1400 & 14.68 & 1400 & 13.97 & 1371 & 14 & 1400 & 13.82 & 1397 & 13.76 & 1406 & 13.59 & 1378 \\
\hline 15.54 & 1376 & 14.56 & 1346 & 14.89 & 1371 & 14.39 & 1373 & 13.65 & 1343 & 13.76 & 1371 & 13.5 & 1371 & 13.32 & 1372 & 13.2 & 1353 \\
\hline 15.16 & 1344 & 14.3 & 1324 & 14.68 & 1348 & 14.03 & 1346 & 13.29 & 1314 & 13.47 & 1348 & 13.2 & 1348 & 13.05 & 1348 & 12.82 & 1320 \\
\hline 14.8 & 1318 & 13.85 & 1291 & 14.24 & 1316 & 13.79 & 1319 & 13.08 & 1294 & 13.2 & 1324 & 12.97 & 1325 & 12.84 & 1321 & 12.6 & 1293 \\
\hline 14.56 & 1294 & 13.56 & 1264 & 13.97 & 1293 & 13.26 & 1286 & 12.73 & 1258 & 12.82 & 1291 & 12.49 & 1288 & 12.55 & 1294 & 12.3 & 1268 \\
\hline 14.24 & 1270 & 13.23 & 1239 & 13.59 & 1263 & 13.02 & 1259 & 12.42 & 1235 & 12.58 & 1263 & 12.33 & 1264 & 12.23 & 1268 & 11.98 & 1241 \\
\hline 13.85 & 1236 & 12.94 & 1208 & 13.35 & 1237 & 12.78 & 1234 & 12.16 & 1206 & 12.28 & & 12.03 & 1236 & 11.9 & 1243 & 11.63 & 1215 \\
\hline 13.53 & 1213 & 12.64 & 1181 & 13.08 & 1214 & 12.52 & 1210 & 11.89 & 1182 & 11.93 & 1208 & 11.73 & 1212 & 11.55 & 1207 & 11.27 & 1183 \\
\hline 13.17 & 1182 & 12.41 & 1157 & 12.66 & 1181 & 12.1 & 1177 & 11.44 & 1154 & 11.64 & 1183 & 11.27 & 1180 & 11.3 & 1183 & 10.97 & 1159 \\
\hline 12.84 & 1155 & 11.98 & 1126 & 12.42 & 1157 & 11.91 & 1158 & 11.29 & 1125 & 11.38 & 1160 & 11.07 & 1153 & 11.02 & 1155 & 10.77 & 1130 \\
\hline 12.64 & 1131 & 11.83 & 1107 & 12.34 & 1128 & 11.34 & 1124 & 10.99 & 1101 & 11.08 & 1132 & 10.84 & 1128 & 10.67 & 1127 & 10.38 & 1106 \\
\hline
\end{tabular}


Table 5. $50{ }^{\circ} \mathrm{C}$ at $2 \mathrm{wt} \%$ - continued

\begin{tabular}{|c|c|c|c|c|c|c|c|c|c|c|c|c|c|c|c|c|c|}
\hline run1 & & run2 & & run3 & & run4 & & run5 & & run6 & & run7 & & run8 & & run9 & \\
\hline [Pa] & {$[1 / s]$} & [Pa] & {$[1 / s]$} & [Pa] & {$[1 / s]$} & [Pa] & {$[1 / s]$} & [Pa] & [1/s] & [Pa] & [1/s] & [Pa] & [1/s] & [Pa] & {$[1 / s]$} & [Pa] & [1/s] \\
\hline 12.27 & 1106 & 11.3 & 1074 & 12.02 & 1104 & 11.06 & 1099 & 10.66 & 1076 & 10.76 & 1107 & 10.52 & 1104 & 10.49 & 1103 & 10.21 & 1077 \\
\hline 11.96 & 079 & 11.09 & 1046 & 1.47 & 1073 & 10.8 & 1074 & 10.37 & 1049 & 10.55 & 1081 & 10.3 & 1077 & 10.12 & 1077 & 9.87 & 1050 \\
\hline 11.51 & 047 & .69 & 1016 & 11.18 & 1049 & 10.49 & 1042 & 9.917 & 1017 & 10.18 & 1053 & 9.908 & 1051 & 9.748 & 1044 & 9.574 & 1025 \\
\hline 11.24 & 1018 & .38 & 990.7 & 10.84 & 1025 & 10.15 & 1014 & 9.725 & 987.6 & 9.802 & 1018 & 9.583 & 1016 & 9.544 & 1019 & 9.278 & 999.5 \\
\hline 10.94 & 995.9 & 0.09 & 962.9 & 10.42 & 989.8 & 9.873 & 988.9 & 9.432 & 961.1 & 9.544 & 992.9 & 9.373 & 990.2 & 9.343 & 995.5 & 8.979 & 964.2 \\
\hline 10.66 & 65.1 & .828 & 938.2 & 10.1 & 963.8 & 9.606 & 961.6 & 9.195 & 938.2 & 9.272 & 965.1 & 8.961 & 966 & 8.92 & 963.8 & 8.692 & 939.1 \\
\hline 10.29 & 938.2 & 9.574 & 914.9 & 9.799 & 939.5 & 9.275 & 931.2 & 8.781 & 903 & 9.029 & 941.3 & 8.787 & 935.1 & 8.65 & 936 & 8.405 & 915.3 \\
\hline 9.973 & 15.3 & 9.183 & 882.2 & 9.518 & 910 & 8.985 & 904.3 & 8.565 & 884.9 & 8.727 & 920.1 & 8.52 & 913.5 & 8.434 & 911.8 & 7.973 & 884 \\
\hline 9.737 & 887.5 & 8.955 & 855.8 & 9.278 & 884.5 & 8.701 & 880.5 & 8.201 & 851.8 & & 895.5 & 8.115 & 881.8 & 8.109 & 880.5 & 7.769 & 857.1 \\
\hline 9.287 & 853.2 & 8.692 & 831.6 & 8.905 & 857.6 & 8.346 & 850.1 & 7.931 & 822.8 & 8.242 & 885.8 & 7.934 & 857.1 & 7.795 & 852.3 & 7.428 & 827.6 \\
\hline 9.059 & 830.7 & 245 & 796.3 & 8.647 & 831.6 & 8.07 & 824.1 & 7.706 & 798.1 & 7.771 & 836 & 7.653 & 828.5 & 7.561 & 828.5 & 7.174 & 804.7 \\
\hline 8.724 & 806.4 & 7.973 & 772.1 & 8.212 & 799.8 & 7.801 & 799 & 7.413 & 774.3 & 7.511 & 804.2 & 7.259 & 797.2 & 7.28 & 802.9 & 6.969 & 776.9 \\
\hline 8.405 & 777.8 & 7.703 & 744.3 & 7.996 & 772.1 & 7.55 & 769 & 7.046 & 741.7 & 7.328 & 790.6 & 7.005 & 773.4 & 6.937 & 776.5 & 653 & 749.2 \\
\hline 8.026 & 743.9 & 7.487 & 721.4 & 7.724 & 748.3 & 7.283 & 743.9 & 6.789 & 715.2 & 6.913 & 750 & 6.789 & 745.2 & 6.632 & 747.8 & 6.366 & 721 \\
\hline 7.789 & 725.8 & 7.052 & 689.7 & 7.393 & 722.3 & 7.014 & 719.6 & 6.561 & 691.4 & 6.694 & 721 & 6.534 & 721 & 6.434 & 720.1 & 6.167 & 696.7 \\
\hline 7.384 & & & 662.8 & & & & & & & & & & 688.8 & 6.203 & & & 669.4 \\
\hline 7.052 & 666.3 & 6.546 & 638.5 & 6.765 & 662.8 & 6.324 & 660.6 & 5.895 & 633.3 & 6.126 & 665.4 & 5.889 & 662.3 & 5.94 & 669.8 & 5.555 & 642.1 \\
\hline 6.83 & 639.9 & 221 & 608.1 & 6.487 & 640.3 & 6.126 & & & 606.8 & & 642.1 & & 635.9 & 5.543 & 636.3 & 5.33 & 616.5 \\
\hline 6.552 & 612.1 & .972 & 581.7 & 6.123 & 609 & 5.827 & 609.9 & 5.401 & 581.3 & 5.519 & 607.7 & 5.425 & 608.6 & 5.327 & 612.1 & 4.963 & 583 \\
\hline 6.233 & 589.6 & 5.667 & 557.5 & 5.854 & 584.3 & 5.576 & 585.2 & 5.09 & 555.7 & 5.22 & 583 & 5.117 & 583.9 & 5.105 & 581.7 & 4.729 & 556.6 \\
\hline 5.981 & 561.4 & 5.321 & 526.2 & 5.57 & 553.9 & 5.217 & 549.1 & 4.779 & 524.4 & 5.013 & 553.5 & 4.824 & 550.4 & 4.916 & 557.5 & 4.519 & 534.1 \\
\hline 5.59 & 527.9 & 5.087 & 497.5 & 5.318 & 528.4 & 5.01 & 525.3 & 4.608 & 498.9 & 4.75 & 527.5 & 4.62 & 525.7 & 4.623 & 532.8 & 4.155 & 500.2 \\
\hline 5.309 & 501.1 & 4.948 & 473.7 & 5.052 & 502.8 & 4.777 & 500.2 & 4.217 & 469.8 & 4.546 & 500.6 & 4.356 & 499.3 & 4.347 & 507.2 & 915 & 476.4 \\
\hline 5.037 & 477.3 & 4.572 & 443.8 & 4.777 & 479 & 4.504 & 475.9 & 3.963 & 441.6 & 4.2 & 475.9 & 4.066 & 472 & 4.028 & 472 & 3.753 & 446.9 \\
\hline 4.679 & 446 & 4.336 & 416 & 4.469 & 447.3 & 4.093 & 442.4 & 3.812 & 419.5 & 4.007 & 447.7 & 3.871 & 447.7 & 3.711 & 444.2 & 3.409 & 423.1 \\
\hline 4.457 & 420.4 & 4.134 & 394 & 4.306 & 420.4 & 3.809 & 414.7 & 3.415 & 386.5 & 3.75 & 424.8 & 3.504 & 415.6 & 3.501 & 419.1 & 3.255 & 395.3 \\
\hline 4.164 & 394.4 & 3.753 & 362.7 & 4.279 & 390 & 3.619 & 386 & 3.214 & 362.7 & 3.451 & 390.4 & 3.276 & 388.7 & 3.232 & 392.2 & 2.877 & 363.6 \\
\hline 3.812 & 364.9 & 3.412 & 335.8 & 4.069 & 361.8 & 3.38 & 363.6 & 2.995 & 335.4 & 3.175 & 365.3 & 3.063 & 363.6 & 3.048 & 368 & 2.714 & 338.9 \\
\hline 3.528 & 335.8 & 3.196 & 307.6 & 3.69 & 334.5 & 3.104 & 335.4 & 2.749 & 312 & 2.921 & 338.9 & 2.859 & 338.9 & 2.681 & 335.8 & 2.516 & 313.8 \\
\hline 3.267 & 312.4 & 2.924 & 285.6 & 3.323 & 310.7 & 2.779 & 305.4 & 2.391 & 276.3 & 2.649 & 307.6 & 2.477 & 307.2 & 2.427 & 310.7 & 2.19 & 282.9 \\
\hline 2.998 & 281.2 & 2.699 & 259.6 & 3.019 & 282.9 & 2.563 & 277.6 & 2.125 & 251.2 & 2.371 & 282.9 & 2.252 & 279 & 2.184 & 281.6 & 1.965 & 256.5 \\
\hline 2.702 & 253.4 & 2.368 & 236.2 & 2.761 & 257.4 & 2.297 & 253.8 & 2.051 & 236.2 & 2.205 & 253.8 & 1.995 & 253.8 & 2.015 & 256.9 & 1.799 & 232.7 \\
\hline
\end{tabular}


Table 6. Rheometer Response for Blank - $10 \mathrm{wt} \%$

\begin{tabular}{|c|c|c|c|c|c|c|c|c|c|c|c|c|c|c|c|c|c|}
\hline run1 & & run2 & & run3 & & run4 & & run5 & & run6 & & run7 & & run8 & & run9 & \\
\hline [Pa] & {$[1 / s]$} & [Pa] & {$[1 / s]$} & [Pa] & [1/s] & [Pa] & [1/s] & [Pa] & [1/s] & [Pa] & [1/s] & [Pa] & [1/s] & {$[\mathrm{Pa}]$} & [1/s] & [Pa] & [1/s] \\
\hline 2.743 & 319.1 & 2.885 & 305.8 & 3.021 & 305.4 & 3.048 & 303.6 & 2.592 & 324.3 & 2.411 & 314.6 & 2.867 & 304.1 & 2.864 & 304.5 & 2.613 & 331 \\
\hline 3.193 & 317.7 & 3.323 & 314.6 & .314 & 325.2 & 3.462 & 322.1 & 3.065 & 327.9 & 2.959 & 317.7 & 3.139 & 325.2 & 3.095 & 324.3 & 3.163 & 326.1 \\
\hline 3.483 & 334.5 & 3.542 & 331.8 & 3.568 & 346.8 & 3.681 & 346.8 & 3.261 & 345.9 & 3.258 & 333.6 & 3.403 & 341.5 & 3.329 & 345.5 & 3.367 & 348.6 \\
\hline 3.713 & 357 & 3.743 & 355.6 & 3.764 & 371.1 & 3.9 & 373.3 & 3.459 & 369.7 & 3.409 & 361.8 & 3.533 & 372.4 & 3.494 & 370.2 & 3.536 & 372.4 \\
\hline 3.986 & 384.3 & 4.246 & 395.3 & 3.947 & 396.6 & 4.151 & 400.6 & 3.705 & 399.3 & 3.648 & 386.9 & 3.734 & 397.1 & 3.687 & 392.6 & 3.802 & 396.2 \\
\hline 4.193 & 409.8 & 4.305 & 426.6 & 4.175 & 417.8 & 4.338 & 430.1 & 3.9 & 422.6 & 3.838 & 413.4 & 3.918 & 419.5 & 3.856 & 417.3 & 3.956 & 420 \\
\hline 4.338 & 442.9 & 4.459 & 449.1 & 4.581 & 456.5 & 4.575 & 454.3 & 4.107 & 446.9 & 3.992 & 436.3 & 4.08 & 445.1 & 4.217 & 455.7 & 4.196 & 446 \\
\hline 4.516 & 470.2 & 4.675 & 472.4 & 4.675 & 479.9 & 4.69 & 485.2 & 4.391 & 483.4 & 4.116 & 459.6 & 4.498 & 486.5 & 4.382 & 476.8 & 4.347 & 472.9 \\
\hline 4.749 & 498.4 & 4.835 & 499.3 & 4.794 & 507.7 & 4.912 & 511.2 & 4.584 & 506.8 & 4.536 & 504.1 & 4.69 & 511.6 & 4.554 & 506.3 & 4.498 & 496.2 \\
\hline 5.051 & 522.2 & 5.048 & 524.9 & 5.125 & 535.4 & 5.054 & 531.9 & 4.729 & 538.1 & 4.755 & 530.1 & 4.865 & 535 & 4.764 & 532.3 & 4.699 & 523.5 \\
\hline 5.161 & 547.8 & 5.208 & 548.7 & 5.329 & 558.8 & 5.223 & 561 & 4.948 & 562.3 & 4.953 & 559.7 & 5.063 & 562.8 & 5.007 & 560.1 & 4.88 & 551.3 \\
\hline 5.403 & 572 & & 590.5 & 5.519 & 594.5 & 5.457 & 583.5 & & 586.1 & & 568.5 & & 587 & & 584.3 & 5.264 & 591 \\
\hline 5.56 & 596.7 & 5.918 & 613.9 & 5.75 & 620 & 5.634 & 610.8 & 5.255 & 611.7 & 5.35 & 608.6 & 5.448 & 617.8 & 5.309 & 618.7 & 5.427 & 612.5 \\
\hline 6.01 & 635.5 & 6.152 & 636.8 & 5.924 & 645.2 & 5.844 & 633.7 & 5.483 & 635.9 & 5.477 & 635 & 5.687 & 639.9 & 5.474 & 643 & 5.513 & 644.7 \\
\hline 6.173 & 658.8 & 6.3 & 667.2 & 6.143 & 666.3 & 6.285 & 674.2 & 5.705 & 661.5 & 5.711 & 661.5 & 5.806 & 671.2 & 5.584 & 667.2 & 5.676 & 666.3 \\
\hline 6.41 & 683.1 & 6.507 & 691 & 6.22 & 691.4 & 6.38 & 702 & 5.847 & 686.6 & 5.883 & 685.3 & 6.102 & 695.8 & 5.812 & 690.5 & 5.915 & 689.7 \\
\hline 6.608 & 718.3 & & 712.6 & 6.451 & 715.2 & 6.691 & 724.5 & 6.028 & 713 & & 718.8 & 47 & 729.8 & 5.98 & 717.9 & 6.244 & 726.2 \\
\hline 6.771 & 744.8 & 7.022 & 754 & 6.637 & 740.8 & 6.871 & 752.2 & 6.469 & 754 & 6.164 & 743.9 & 6.395 & 755.8 & 6.173 & 744.8 & 6.38 & 749.2 \\
\hline 7.013 & 765 & 7.404 & 777.4 & 7.105 & 783.1 & 7.016 & 780.4 & 6.605 & 778.2 & 6.436 & 765.5 & 6.623 & 778.2 & 6.54 & 784.4 & 6.472 & 780.9 \\
\hline 7.247 & 796.8 & 7.611 & 804.7 & 7.286 & 806.9 & 7.321 & 806.9 & 6.738 & 811.3 & 6.46 & 790.6 & 6.726 & 805.1 & 6.463 & 795 & 6.676 & 803.8 \\
\hline 7.425 & 824.5 & 7.741 & 833.8 & 7.505 & 839.1 & 7.481 & 832.9 & 6.862 & 839.5 & 6.649 & 818.3 & 6.927 & 830.7 & 6.833 & 839.5 & 6.871 & 827.6 \\
\hline 7.629 & 847.4 & 7.874 & 859.3 & 7.644 & 865.1 & 7.703 & 859.3 & 7.173 & 854.9 & 6.868 & 848.3 & 7.111 & 858 & 7.052 & 863.3 & 6.972 & 852.7 \\
\hline 7.792 & 873.4 & 8.102 & 882.2 & 7.83 & 891.5 & 7.806 & 891.9 & 7.365 & 886.2 & 7.126 & 870.3 & 7.294 & 880.5 & 7.265 & 885.3 & 7.149 & 877.4 \\
\hline 7.934 & 897.2 & 8.256 & 904.3 & 8.049 & 915.3 & 8.04 & 915.7 & 7.534 & 918.8 & 7.407 & 907.4 & 7.451 & 906 & 7.309 & 916.2 & 7.318 & 905.2 \\
\hline 8.138 & 925.4 & 8.502 & 931.6 & 8.224 & 942.2 & 8.162 & 940 & 7.641 & 942.2 & 7.584 & 932 & 7.869 & 950.1 & 7.466 & 941.3 & 7.457 & 930.7 \\
\hline 8.656 & 963.3 & 8.668 & 958.5 & 8.419 & 964.7 & 8.375 & 966.4 & 7.824 & 968.2 & 7.706 & 965.5 & 8.046 & 973.5 & 7.721 & 963.3 & 7.91 & 971.7 \\
\hline 8.845 & 988 & 9.114 & 995.5 & 8.742 & 1000 & 8.623 & 990.2 & 8.061 & 991.1 & 7.91 & 988 & 8.126 & 1004 & 7.966 & 991.5 & 8.046 & 998.1 \\
\hline 8.958 & 1017 & 9.316 & 1025 & 8.913 & 1027 & 9.082 & 1033 & 8.33 & 1029 & 8.031 & 1012 & 8.339 & 1026 & 8.052 & 1020 & 8.197 & 1018 \\
\hline 9.218 & 1039 & 9.514 & 1048 & 9.144 & 1057 & 9.33 & 1052 & 8.372 & 1051 & 8.244 & 1042 & 8.446 & 1051 & 8.307 & 1042 & 8.386 & 1054 \\
\hline 9.345 & 1069 & 9.757 & 1073 & 9.322 & 1082 & 9.422 & 1086 & 8.594 & 1081 & 8.502 & 1066 & 8.662 & 1083 & 8.395 & 1066 & 8.552 & 1078 \\
\hline 9.538 & 1092 & 9.976 & 1099 & 9.493 & 1109 & 9.547 & 1111 & 8.759 & 1105 & 8.555 & 1093 & 8.842 & 1107 & 8.839 & 1107 & 8.709 & 1105 \\
\hline
\end{tabular}


Table 6. Rheometer Response for Blank - $10 \mathrm{wt} \%$ - continued

\begin{tabular}{|c|c|c|c|c|c|c|c|c|c|c|c|c|c|c|c|c|c|}
\hline run1 & & run2 & & run3 & & run4 & & run5 & & run6 & & run7 & & run8 & & run9 & \\
\hline [Pa] & [1/s] & [Pa] & {$[1 / s]$} & [Pa] & {$[1 / s]$} & [Pa] & {$[1 / s]$} & [Pa] & [1/s] & [Pa] & {$[1 / s]$} & [Pa] & [1/s] & [Pa] & [1/s] & [Pa] & {$[1 / s]$} \\
\hline 9.872 & 1127 & 10.26 & 1126 & 9.742 & 1131 & 9.816 & 1129 & 8.961 & 1129 & 8.801 & 1118 & 9.002 & 1132 & 8.961 & 1132 & 8.919 & 1131 \\
\hline 10.09 & 1151 & 10.53 & 1150 & 9.914 & 1156 & 9.955 & 1163 & 9.114 & 1152 & 8.993 & 1144 & 9.153 & 1157 & 9.091 & 1165 & 9.079 & 1155 \\
\hline 10.27 & 1180 & 11.01 & 1188 & 10.27 & 1196 & 10.13 & 1186 & 9.476 & 1191 & 9.15 & 1169 & 9.316 & 1184 & 9.227 & 1187 & 9.301 & 1176 \\
\hline 10.39 & 1204 & 11.21 & 1214 & 10.38 & 1220 & 10.39 & 1213 & 9.674 & 1214 & 9.526 & 1211 & 9.564 & 1209 & 9.393 & 1210 & 9.366 & 1204 \\
\hline 10.62 & 1228 & 11.38 & 1237 & 10.63 & 1245 & 10.48 & 1238 & 9.763 & 1245 & 9.712 & 1236 & 9.697 & 1236 & 9.567 & 1234 & 9.579 & 1230 \\
\hline 10.77 & 1253 & 11.54 & 1268 & 10.78 & 1276 & 10.94 & 1277 & 9.979 & 1269 & 9.84 & 1265 & 10.11 & 1275 & 9.721 & 1261 & 9.985 & 1270 \\
\hline 11.04 & 1278 & 11.75 & 1289 & 10.98 & 1302 & 11.07 & 1302 & 10.16 & 1294 & 9.985 & 1290 & 10.23 & 1300 & 9.902 & 1290 & 10.05 & 1301 \\
\hline 11.46 & 1317 & 11.92 & 1313 & 11.24 & 1326 & 11.2 & 1326 & 10.32 & 1319 & 10.16 & 1315 & 10.42 & 1327 & 10.18 & 1316 & 10.27 & 1325 \\
\hline 11.6 & 1343 & 12.3 & 1353 & 11.38 & 1350 & 11.45 & 1356 & 10.48 & 1347 & 10.31 & 1342 & 10.59 & 1356 & 10.36 & 1342 & 10.44 & 1348 \\
\hline 11.85 & 1367 & 12.47 & 1378 & 11.55 & 1376 & 11.59 & 1376 & 10.68 & 1368 & 10.53 & 1365 & 10.75 & 1380 & 10.45 & 1367 & 10.58 & 1368 \\
\hline 12.12 & 1394 & 12.67 & 1400 & 11.83 & 1399 & 11.8 & 1402 & 11.06 & 1411 & 10.65 & 1392 & 10.98 & 1403 & 10.92 & 1412 & 10.71 & 1399 \\
\hline 12.27 & 1424 & 12.83 & 1433 & 11.96 & 1425 & 11.95 & 1427 & 11.19 & 1436 & 10.77 & 1415 & 11.17 & 1430 & 11.16 & 1436 & 10.98 & 1423 \\
\hline 12.44 & 1447 & 13.1 & 1458 & 12.35 & 1462 & 12.12 & 1451 & 11.42 & 1460 & 11.24 & 1453 & 11.33 & 1455 & 11.33 & 1460 & 11.14 & 1453 \\
\hline 12.66 & 1469 & 13.22 & 1484 & 12.61 & 1490 & 12.47 & 1477 & 11.62 & 1494 & 11.42 & 1482 & 11.45 & 1482 & 11.49 & 1490 & 11.5 & 1492 \\
\hline 13.06 & 1510 & 13.46 & 1507 & 12.91 & 1517 & 12.8 & 1517 & 11.83 & 1519 & 11.55 & 1508 & 11.77 & 1522 & 11.75 & 1519 & 11.71 & 1516 \\
\hline 11.29 & 1526 & 12.09 & 1596 & 11.3 & 1568 & 11.17 & 1597 & 10.71 & 1565 & 11.12 & 1596 & 10.8 & 1564 & 11.06 & 1575 & 10.97 & 1595 \\
\hline 10.95 & 1487 & 11.95 & 1573 & 11.12 & 1545 & 10.99 & 1572 & 10.49 & 1542 & 10.86 & 1569 & 10.71 & 1534 & 10.95 & 1549 & 10.78 & 1573 \\
\hline 10.69 & 1465 & 11.75 & 1549 & 10.96 & 1520 & 10.9 & 1542 & 10.42 & 1511 & 10.71 & 1545 & 10.54 & 1510 & 10.71 & 1522 & 10.6 & 1549 \\
\hline 10.46 & 1441 & 11.31 & 1510 & 10.7 & 1493 & 10.68 & 1518 & 10.17 & 1485 & 10.54 & 1519 & 10.35 & 1486 & 10.25 & 1485 & 10.39 & 1524 \\
\hline 10.39 & 1406 & 11.09 & 1488 & 10.3 & 1456 & 10.46 & 1493 & 9.993 & 1461 & 10.33 & 1490 & 10.14 & 1458 & 10.29 & 1476 & 10.03 & 1484 \\
\hline 10.12 & 1382 & 10.93 & 1458 & 10.11 & 1430 & 10.24 & 1469 & 9.801 & 1438 & 10.07 & 1466 & 9.967 & 1434 & 9.816 & 1434 & 10.13 & 1475 \\
\hline 9.99 & 1359 & 10.79 & 1432 & 10.03 & 1400 & 10.08 & 1442 & 9.529 & 1402 & 9.878 & 1440 & 9.766 & 1408 & 9.641 & 1401 & 9.893 & 1448 \\
\hline 9.786 & 1337 & 10.6 & 1408 & 9.792 & 1377 & 9.837 & 1418 & 9.304 & 1376 & 9.748 & 1411 & 9.597 & 1382 & 9.499 & 1376 & 9.476 & 1404 \\
\hline 9.369 & 1300 & 10.39 & 1387 & 9.603 & 1347 & 9.449 & 1378 & 9.1 & 1352 & 9.455 & 1385 & 9.413 & 1353 & 9.378 & 1352 & 9.307 & 1381 \\
\hline 9.156 & 1274 & 9.967 & 1349 & 9.369 & 1322 & 9.304 & 1356 & 8.937 & 1326 & 9.342 & 1359 & 9.165 & 1329 & 9.233 & 1326 & 9.168 & 1351 \\
\hline 8.961 & 1244 & 9.774 & 1323 & 9.197 & 1296 & 9.144 & 1327 & 8.789 & 1296 & 9.18 & 1335 & 8.996 & 1303 & 9.046 & 1302 & 8.913 & 1325 \\
\hline 8.866 & 1218 & 9.576 & 1298 & 8.952 & 1270 & 9.002 & 1302 & 8.597 & 1270 & 9.026 & 1306 & 8.549 & 1261 & 8.83 & 1274 & 8.733 & 1297 \\
\hline 8.561 & 1194 & 9.295 & 1273 & 8.872 & 1245 & 8.75 & 1276 & 8.348 & 1244 & 8.813 & 1281 & 8.309 & 1237 & 8.428 & 1237 & 8.526 & 1273 \\
\hline 8.357 & 1168 & 9.212 & 1244 & 8.576 & 1223 & 8.555 & 1248 & 8.221 & 1218 & 8.363 & 1239 & 8.274 & 1212 & 8.259 & 1213 & 8.33 & 1246 \\
\hline 8.156 & 1144 & 9.082 & 1219 & 8.221 & 1182 & 8.363 & 1226 & 8.073 & 1191 & 8.153 & 1209 & 7.937 & 1187 & 8.058 & 1181 & 8.162 & 1223 \\
\hline 8.028 & 1121 & 8.86 & 1196 & 8.04 & 1157 & 8.028 & 1186 & 7.765 & 1166 & 7.945 & 1185 & 7.898 & 1153 & 7.812 & 1160 & 8.04 & 1198 \\
\hline
\end{tabular}


Table 6. Rheometer Response for Blank - $10 \mathrm{wt} \%$ - continued

\begin{tabular}{|c|c|c|c|c|c|c|c|c|c|c|c|c|c|c|c|c|c|}
\hline run1 & & run2 & & run3 & & run4 & & run5 & & run6 & & run7 & & run8 & & run9 & \\
\hline [Pa] & {$[1 / s]$} & [Pa] & {$[1 / s]$} & [Pa] & {$[1 / s]$} & [Pa] & [1/s] & [Pa] & [1/s] & [Pa] & [1/s] & [Pa] & {$[1 / s]$} & [Pa] & [1/s] & [Pa] & {$[1 / s]$} \\
\hline 7.827 & 1094 & 8.455 & 1158 & 7.874 & 1134 & 7.809 & 1163 & 7.472 & 1129 & 7.803 & 1157 & 7.712 & 1129 & 7.691 & 1133 & 7.877 & 1173 \\
\hline 7.33 & 1052 & .304 & 1133 & 7.673 & 1100 & 7.644 & 1137 & 7.274 & 1106 & 7.632 & 1133 & 7.581 & 1103 & 7.522 & 1102 & 7.685 & 1148 \\
\hline 7.188 & 1029 & 8.082 & 1103 & 7.487 & 1075 & 7.537 & 1106 & 7.123 & 1076 & 7.309 & 1106 & 7.351 & 1077 & 7.38 & 1077 & 7.217 & 1104 \\
\hline 7.072 & 998.1 & 7.954 & 1078 & 7.294 & 1053 & 7.286 & 1079 & 6.966 & 1051 & 7.149 & 1081 & 7.182 & 1051 & 7.152 & 1051 & 7.312 & 1095 \\
\hline 7.093 & 988.5 & 7.798 & 1054 & 7.072 & 1025 & 6.966 & 1059 & 6.853 & 1026 & 6.951 & 1054 & 6.942 & 1023 & 6.981 & 1027 & 6.859 & 1055 \\
\hline 6.498 & 943.9 & 7.57 & 1031 & 6.85 & 995.1 & 6.874 & 1024 & 6.67 & 999.5 & 6.848 & 1027 & 6.779 & 993.3 & 6.623 & 992.4 & 6.735 & 1026 \\
\hline 6.341 & 918.4 & 7.371 & 1001 & 6.705 & 969.5 & 6.723 & 997.7 & 6.41 & 975.2 & 6.694 & 995.5 & 6.525 & 969.5 & 6.427 & 967.3 & 6.608 & 994.6 \\
\hline 6.17 & 891.1 & 7.064 & 975.2 & 6.469 & 941.3 & 6.54 & 970.8 & 6.3 & 951.9 & 6.478 & 970.8 & 6.333 & 945.3 & 6.247 & 943.5 & 6.436 & 974.8 \\
\hline 5.835 & 868.1 & 6.88 & 945.7 & 6.276 & 917.9 & 6.321 & 942.6 & 6.137 & 926.3 & 6.383 & 947.5 & 5.989 & 905.6 & 6.087 & 917.1 & 6.146 & 938.7 \\
\hline 5.77 & 836 & 6.643 & 921 & 6.084 & 888.4 & 6.117 & 925 & 5.756 & 887.5 & 6.122 & 919.3 & 5.847 & 880.9 & 5.874 & 886.7 & 5.966 & 915.7 \\
\hline 5.539 & 810.9 & 6.534 & 897.7 & 5.877 & 862 & 5.93 & 894.1 & 5.608 & 858.5 & 5.779 & 880 & 5.684 & 857.6 & 5.693 & 858.5 & 5.8 & 885.3 \\
\hline 5.344 & 788.8 & & 873 & & 835.5 & 5.782 & 869.9 & 5.406 & 831.6 & 5.605 & 858 & 5.51 & 828.9 & 5.442 & 832.9 & 5.643 & 861.1 \\
\hline 5.167 & 765.5 & 5.862 & 831.6 & 5.477 & 811.7 & 5.664 & 844.8 & 5.282 & 806.9 & 5.468 & 834.2 & 5.285 & 802 & 5.261 & 806.4 & 5.531 & 838.2 \\
\hline 4.844 & 725.8 & 5.761 & 801.6 & 5.261 & 786.2 & 5.421 & 821.9 & 5.155 & 781.3 & 5.359 & 802 & 5.122 & 770.8 & 5.199 & 773.8 & 5.356 & 815.7 \\
\hline 4.643 & 702 & 5.631 & 777.4 & 5.134 & 757.1 & 5.013 & 778.7 & 4.974 & 755.3 & 5.17 & 779.1 & 4.962 & 747.4 & 4.927 & 748.3 & 5.244 & 790.1 \\
\hline 4.442 & 677.8 & 5.436 & 753.6 & 4.675 & 718.3 & 4.936 & 754.4 & 4.74 & 729.3 & 4.971 & 751.4 & 4.803 & 725.4 & 4.746 & 724.5 & 4.977 & 765.9 \\
\hline 4.297 & 644.3 & 5.282 & 731.5 & 4.746 & 704.2 & 4.687 & 729.8 & 4.578 & 703.3 & 4.856 & 728 & 4.622 & 701.1 & 4.601 & 699.4 & 4.797 & 737.7 \\
\hline 4.083 & 620.5 & 5.054 & 706.4 & 4.51 & 679.5 & 4.56 & 698.5 & 4.397 & 677.8 & 4.415 & 690.5 & 4.187 & 662.3 & 4.453 & 675.1 & 4.696 & 711.3 \\
\hline 3.956 & 596.7 & 4.616 & 665.4 & 4.297 & 654.9 & 4.35 & 678.2 & 4.001 & 638.1 & 4.249 & 667.2 & 4.063 & 635 & 4.039 & 638.5 & 65 & 684.8 \\
\hline 3.755 & 571.1 & 4.465 & 642.5 & 3.894 & 609.9 & 4.166 & 645.6 & 3.87 & 616.1 & 4.083 & 640.3 & 3.903 & 609.9 & 3.873 & 613.4 & 33 & 640.3 \\
\hline 3.566 & 547.8 & 4.214 & 618.3 & 3.696 & 585.2 & 3.941 & 619.2 & 3.77 & 584.3 & 3.888 & 614.3 & 3.749 & 584.8 & 3.746 & 589.6 & 3.891 & 616.1 \\
\hline 3.184 & 506.3 & 4.116 & 585.2 & 3.533 & 556.1 & 3.829 & 595.8 & 3.586 & 560.1 & 3.681 & 583.5 & 3.601 & 551.7 & 3.586 & 561 & 3.793 & 586.1 \\
\hline 2.965 & 482.1 & 3.897 & 560.5 & 3.302 & 531.9 & 3.702 & 569.8 & 3.441 & 535.4 & 3.574 & 558.8 & 3.335 & 534.5 & 3.492 & 536.3 & 3.592 & 561.9 \\
\hline 2.811 & 457.4 & 3.74 & 539 & 3.19 & 501.1 & 3.326 & 534.1 & 3.32 & 507.7 & 3.453 & 531.5 & 3.228 & 501.9 & 3.299 & 516.5 & 3.429 & 538.1 \\
\hline 2.695 & 427.5 & 3.37 & 499.7 & 3.033 & 477.7 & 3.157 & 508.1 & 3.092 & 480.3 & 3.225 & 508.1 & 3.083 & 478.1 & 2.941 & 470.6 & 3.273 & 516.9 \\
\hline 2.503 & 403.2 & 3.21 & 475.1 & 2.873 & 456.5 & 3.065 & 483.9 & 2.835 & 457.4 & 3.089 & 483 & 2.891 & 451.7 & 3.039 & 464 & 2.926 & 476.4 \\
\hline 2.352 & 378.5 & 3.003 & 449.1 & 2.752 & 430.1 & 2.923 & 451.3 & 2.695 & 431 & 2.935 & 457.9 & 2.565 & 413.8 & 2.799 & 439.4 & 2.731 & 452.6 \\
\hline 2.145 & 350.8 & 2.823 & 418.6 & 2.337 & 389.1 & 2.639 & 430.5 & 2.553 & 404.5 & 2.545 & 416.4 & 2.402 & 390 & 2.382 & 395.3 & 2.681 & 422.2 \\
\hline 1.935 & 327 & 2.672 & 394.9 & 2.109 & 364.4 & 2.426 & 403.7 & 2.37 & 379.9 & 2.367 & 395.3 & 2.237 & 364.9 & 2.278 & 368.4 & 2.479 & 397.9 \\
\hline 1.757 & 301.9 & 2.476 & 371.1 & 2.169 & 353.9 & 2.32 & 368.4 & 2.166 & 354.3 & 2.266 & 363.1 & 2.104 & 331.8 & 2 & 342 & 2.337 & 374.1 \\
\hline 1.331 & 265.7 & 2.323 & 346.4 & 1.89 & 327.4 & 2.127 & 342.4 & 1.772 & 312.4 & 2.109 & 338.4 & 1.834 & 306.7 & 1.879 & 311.1 & 2.145 & 350.8 \\
\hline
\end{tabular}


APPENDIX E

ENVELOPE C

SR/TRU PRECIPITATE SLURRY RHEOLOGY DATA
WRC-TR-2000-00352

SRT-RPP-2000-00026

Page 195 of 514

Table 6. Rheometer Response for Blank - $10 \mathrm{wt} \%$ - continued

\begin{tabular}{|c|c|c|c|c|c|c|c|c|c|c|c|c|c|c|c|c|c|}
\hline run1 & & run2 & & run3 & & run4 & & run5 & & run6 & & run7 & & run8 & & run9 & \\
\hline [Pa] & {$[1 / s]$} & [Pa] & {$[1 / \mathrm{s}]$} & [Pa] & {$[1 / s]$} & [Pa] & {$[1 / s]$} & [Pa] & {$[1 / \mathrm{s}]$} & [Pa] & {$[1 / \mathrm{s}]$} & [Pa] & {$[1 / \mathrm{s}]$} & [Pa] & {$[1 / \mathrm{s}]$} & [Pa] & {$[1 / \mathrm{s}]$} \\
\hline 1.254 & 235.8 & 2.118 & 319.9 & 1.592 & 285.1 & 1.926 & 315.5 & 1.663 & 285.1 & 1.92 & 315.1 & 1.674 & 284.2 & 1.713 & 289.5 & 2.035 & 327 \\
\hline & & 1.728 & 284.2 & 1.337 & 261.8 & 1.805 & 292.2 & 1.405 & 263.1 & 1.763 & 289.1 & 1.521 & 251.6 & 1.538 & 264.9 & 1.645 & 290.4 \\
\hline & & 1.58 & 259.6 & 1.458 & 245.9 & 1.639 & 264.9 & 1.494 & 255.2 & 1.521 & 259.6 & & & 1.37 & 239.7 & 1.518 & 258.7 \\
\hline & & 1.666 & 248.5 & & & 1.399 & 240.6 & & & & & & & & & & \\
\hline & & & & & & & & & & & & & & & & & \\
\hline & & & & & & & & & & & & & & & & & \\
\hline & & & & & & & & & & & & & & & & & \\
\hline & & & & & & & & & & & & & & & & & \\
\hline & & & & & & & & & & & & & & & & & \\
\hline
\end{tabular}


Table 7. $25^{\circ} \mathrm{C}$ at $10 \mathrm{wt} \%$

\begin{tabular}{|c|c|c|c|c|c|c|c|c|c|c|c|c|c|c|c|c|c|}
\hline run1 & & run2 & & run3 & & run4 & & run5 & & run6 & & run7 & & run8 & & run9 & \\
\hline [Pa] & {$[1 / s]$} & [Pa] & {$[1 / s]$} & [Pa] & {$[1 / s]$} & [Pa] & {$[1 / s]$} & [Pa] & {$[1 / s]$} & [Pa] & [1/s] & [Pa] & {$[1 / \mathrm{s}]$} & [Pa] & {$[1 / s]$} & [Pa] & {$[1 / \mathrm{s}]$} \\
\hline 6.701 & 325.2 & 6.402 & 308 & 5.606 & 320.8 & 5.45 & 332.7 & 6.325 & 323.9 & 6.716 & 306.3 & 5.55 & 310.7 & 5.846 & 334.9 & 6.681 & 306.7 \\
\hline 7.749 & 342.4 & .172 & 320.8 & 6.891 & 324.3 & 6.488 & 328.7 & 6.382 & 324.8 & 6.814 & 321.7 & 6.864 & 313.3 & 7.184 & 329.2 & 6.962 & 319.9 \\
\hline 8.166 & 365.8 & 7.166 & 342.9 & 6.811 & 342.4 & 7.27 & 346.8 & 7.394 & 344.2 & 7.358 & 342.4 & 7.737 & 346.8 & 6.849 & 343.7 & 7.429 & 339.8 \\
\hline 8.119 & 394 & 7.601 & 367.1 & 7.199 & 369.7 & 7.172 & 370.2 & 7.974 & 373.7 & 7.992 & 365.3 & 7.503 & 368.4 & 7.986 & 361.4 & 8.184 & 375.9 \\
\hline 8.592 & 420 & 7.554 & 388.7 & 8.187 & 392.6 & 7.959 & 393.5 & 7.844 & 394.4 & 7.989 & 389.6 & 8.243 & 395.7 & 8.122 & 385.2 & 8.894 & 401.9 \\
\hline 9.365 & 444.2 & 8.598 & 430.1 & 8.267 & 415.1 & 8.578 & 414.7 & 8.231 & 424.4 & 8.368 & 419.1 & 9.069 & 423.5 & 9.243 & 426.6 & 9.448 & 429.2 \\
\hline 9.966 & 468.4 & 8.877 & 453 & 9.362 & 453.5 & 8.696 & 439.8 & 9.024 & 447.3 & 9.226 & 441.6 & 9.566 & 446.4 & 9.202 & 447.7 & 9.525 & 452.1 \\
\hline 10.03 & 490.9 & 9.531 & 479.5 & 9.445 & 475.9 & 9.915 & 483 & 9.619 & 475.1 & 9.628 & 470.6 & 10.11 & 479 & 10.15 & 481.7 & 9.948 & 478.1 \\
\hline 10.75 & 520.9 & 10.1 & 506.3 & 9.986 & 504.1 & 10.44 & 505.9 & 10.19 & 499.7 & 10.4 & 495.8 & 10.32 & 501.9 & 10.4 & 506.3 & 10.71 & 511.2 \\
\hline 11.27 & 558.8 & 10.46 & 535.9 & 10.16 & 526.2 & 10.77 & 531 & 10.78 & 523.5 & 10.67 & 535 & 10.61 & 524.9 & 10.79 & 530.6 & 10.98 & 535.4 \\
\hline 11.81 & 589.2 & & 559.2 & & 564.1 & & 557.5 & & & & 565.8 & & 550.4 & & & 11.52 & 560.1 \\
\hline 12.33 & 612.1 & 11.54 & 582.6 & 11.86 & 587.9 & 11.79 & 582.6 & 11.79 & 590.1 & 12.14 & 591.4 & 11.84 & 589.6 & 12.04 & 584.8 & 12.12 & 585.7 \\
\hline 13.08 & 639.4 & 11.96 & 607.7 & 12.27 & 613 & 12.06 & 609 & 12.27 & 612.1 & 12.71 & 615.6 & 12.21 & 614.3 & 12.54 & 607.7 & 12.64 & 609.5 \\
\hline 13.53 & 669.8 & 13.16 & 646.5 & 12.47 & 640.8 & 12.33 & 635 & 13.06 & 640.8 & 13.11 & 638.1 & 13.17 & 646 & 13.14 & 647.8 & 13.06 & 644.7 \\
\hline 13.91 & 694.1 & 13.51 & 670.3 & 12.93 & 662.8 & 13.16 & 664.1 & 13.12 & 671.2 & 13.13 & 666.8 & 13.42 & 672.5 & 13.7 & 671.2 & 13.93 & 674.7 \\
\hline 14.53 & 717.9 & 14 & 699.8 & 13.55 & 685.7 & 13.73 & 691.4 & 13.84 & 696.3 & 13.93 & 691.4 & 13.74 & 696.7 & 14.04 & 698.5 & 14.19 & 699.8 \\
\hline 15.25 & 741.2 & 14.6 & 723.2 & 14.72 & 729.8 & 14.05 & 714.8 & 14.43 & 720.5 & 14.6 & 729.3 & 14.65 & 724 & 14.36 & 722.3 & 14.51 & 724 \\
\hline 16.06 & 781.3 & 15.04 & 752.2 & & 750.9 & & 739 & 14.66 & 744.8 & 15.42 & 755.3 & 14.85 & 751.8 & .14 & 747.4 & 15.25 & 748.3 \\
\hline 16.32 & 804.2 & 15.71 & 774.3 & 15.55 & 780.4 & 5.45 & 778.2 & 15.2 & 769 & 15.61 & 781.3 & 15.41 & 775.6 & 15.46 & 777.4 & 15.6 & 774.7 \\
\hline 17.06 & 835.1 & 16.6 & 812.6 & 16.23 & 802.5 & 15.98 & 803.4 & 15.87 & 795.4 & 16.48 & 808.7 & 16 & 800.7 & 16.19 & 802 & 16.29 & 800.7 \\
\hline 17.53 & 859.3 & 16.95 & 835.1 & 16.38 & 825.4 & 16.67 & 832.4 & 16.51 & 835.5 & 16.66 & 832.4 & 16.45 & 825.8 & 16.38 & 828.5 & 16.58 & 825 \\
\hline 18 & 882.7 & 17.3 & 857.1 & 17.13 & 851 & 16.83 & 856.2 & 17.23 & 858.5 & 17.09 & 863.7 & 17.1 & 848.8 & 17.17 & 851.8 & 17.56 & 871.2 \\
\hline 18.33 & 903.8 & 17.94 & 889.7 & 17.38 & 876.1 & 17.5 & 880.5 & 17.68 & 890.2 & 17.68 & 892.4 & 17.65 & 889.3 & 17.5 & 891.1 & 17.44 & 877.4 \\
\hline 18.92 & 931.6 & 18.39 & 913.5 & 17.91 & 902.5 & 18.15 & 918.4 & 17.89 & 913.1 & 18.36 & 917.5 & 18.27 & 914.4 & 18.27 & 915.3 & 18.33 & 919.3 \\
\hline 19.63 & 957.2 & 18.86 & 936 & 18.54 & 932 & 18.68 & 940.4 & 18.65 & 935.6 & 18.57 & 940.9 & 18.83 & 946.1 & 18.65 & 947.5 & 18.98 & 945.3 \\
\hline 20.34 & 983.2 & 19.63 & 975.2 & 19.6 & 973 & 19.25 & 972.6 & 18.77 & 956.7 & 19.07 & 967.7 & 18.8 & 954.1 & 19.19 & 973.5 & 19.54 & 971.7 \\
\hline 20.87 & 1009 & 20.37 & 997.3 & 20.02 & 997.7 & & 998.1 & & 998.6 & 19.81 & 991.1 & 19.84 & 995.5 & 19.78 & 999.9 & 19.78 & 1005 \\
\hline 21.7 & 1049 & 20.9 & 1025 & 20.58 & 1026 & 20.16 & 1023 & 20.19 & 1025 & 20.28 & 1018 & 20.37 & 1025 & 20.02 & 1024 & 20.28 & 1024 \\
\hline 22.35 & 1080 & 21.38 & 1054 & 20.93 & 1050 & 20.76 & 1053 & 20.79 & 1056 & 21.14 & 1058 & 20.84 & 1053 & 20.4 & 1048 & 20.81 & 1058 \\
\hline 22.89 & 1103 & 21.94 & 1077 & 21.29 & 1075 & 21.29 & 1076 & 21.2 & 1077 & 21.5 & 1081 & 21.35 & 1075 & 21.02 & 1070 & 21.29 & 1084 \\
\hline 23.36 & 1126 & 22.27 & 1106 & 21.97 & 1099 & 21.53 & 1099 & 21.67 & 1102 & 22 & 1110 & 21.61 & 1100 & 21.76 & 1111 & 21.82 & 1111 \\
\hline
\end{tabular}


Table 7. $25^{\circ} \mathrm{C}$ at 10 wt $\%$ - continued

\begin{tabular}{|c|c|c|c|c|c|c|c|c|c|c|c|c|c|c|c|c|c|}
\hline run1 & & run2 & & run3 & & run4 & & run5 & & run6 & & run7 & & run8 & & run9 & \\
\hline [Pa] & [1/s] & [Pa] & [1/s] & [Pa] & [1/s] & [Pa] & [1/s] & [Pa] & [1/s] & [Pa] & [1/s] & [Pa] & [1/s] & [Pa] & {$[1 / s]$} & [Pa] & [1/s] \\
\hline 23.95 & 1153 & 22.89 & 1132 & 22.38 & 1123 & 22.06 & 1123 & 22.27 & 1129 & 22.56 & 1136 & 21.97 & 1123 & 22.18 & 1134 & 22.38 & 1137 \\
\hline 24.43 & 1175 & 3.45 & 1159 & 23.42 & 1164 & 23.09 & 1164 & 22.92 & 1159 & 22.92 & 1159 & 22.65 & 1151 & 22.71 & 1160 & 22.83 & 1164 \\
\hline 24.84 & 1203 & 23.95 & 1183 & 23.77 & 1187 & 23.54 & 1187 & 23.33 & 1184 & 23.36 & 1182 & 23.39 & 1189 & 23.27 & 1189 & 23.3 & 1189 \\
\hline 25.96 & 1245 & 24.43 & 1208 & 24.45 & 1216 & 23.92 & 1216 & 23.92 & 1210 & 23.8 & 1209 & 23.89 & 1218 & 23.66 & 1212 & 23.83 & 1212 \\
\hline 26.35 & 1266 & 25.4 & 1246 & 24.9 & 1240 & 24.43 & 1239 & 24.4 & 1244 & 24.37 & 1235 & 24.45 & 1241 & 23.92 & 1237 & 24.1 & 1237 \\
\hline 26.82 & 1295 & 25.91 & 1269 & 25.19 & 1263 & 24.93 & 1266 & 24.87 & 1267 & 25.17 & 1275 & 24.93 & 1267 & 24.43 & 1262 & 24.51 & 1263 \\
\hline 27.27 & 1316 & 26.35 & 1298 & 26.17 & 1301 & 25.67 & 1300 & 25.25 & 1289 & 25.52 & 1301 & 25.37 & 1300 & 25.34 & 1303 & 25.43 & 1303 \\
\hline 27.86 & 1342 & 26.85 & 1321 & 26.62 & 1324 & 26.02 & 1324 & 25.82 & 1315 & 26.02 & 1328 & 25.7 & 1322 & 25.7 & 1329 & 25.82 & 1326 \\
\hline 28.81 & 1384 & 27.24 & 1348 & 27.06 & 1347 & 26.5 & 1348 & 26.62 & 1354 & 26.44 & 1352 & 26.14 & 1348 & 26.29 & 1354 & 26.29 & 1352 \\
\hline 28.95 & 1395 & 28.15 & 1388 & 27.5 & 1378 & 26.91 & 1370 & 26.59 & 1362 & 26.97 & 1385 & 26.59 & 1371 & 26.73 & 1385 & 26.85 & 1382 \\
\hline 29.43 & 1418 & 28.72 & 1409 & 28.01 & 1401 & 27.74 & 1409 & 27.65 & 1407 & 27.53 & 1407 & 27.03 & 1397 & 27.15 & 1404 & 27.15 & 1408 \\
\hline 30.4 & 1462 & & 1432 & 28.6 & 1425 & 28.27 & 1432 & 28.07 & 1436 & & 1430 & 27.59 & 1421 & 27.62 & 1436 & 27.59 & 1434 \\
\hline 31.05 & 1489 & 29.72 & 1464 & 28.98 & 1450 & 28.83 & 1459 & 28.6 & 1456 & 28.24 & 1458 & 28.24 & 1462 & 28.07 & 1464 & 28.18 & 1458 \\
\hline 31.5 & 1514 & 30.26 & 1489 & 29.96 & 1486 & 29.37 & 1486 & 29.07 & 1486 & 28.89 & 1487 & 28.81 & 1488 & 28.72 & 1486 & 28.54 & 1487 \\
\hline 32.09 & 1538 & 30.61 & 1514 & 30.37 & 1511 & 29.9 & 1518 & 29.55 & 1516 & 29.43 & 1511 & 29.34 & 1513 & 29.1 & 1508 & 29.07 & 1509 \\
\hline 29.66 & 1516 & 29.57 & 1524 & 29.16 & 1543 & 28.81 & 1549 & 28.24 & 1546 & 28.1 & 1538 & 28.39 & 1570 & 28.3 & 1572 & 27.62 & 1545 \\
\hline 29.16 & 1492 & & 1489 & 28.51 & 1517 & 28.21 & 1525 & 27.77 & 1520 & 27.59 & 1516 & 27.89 & 1545 & 27.92 & 1546 & 27.27 & 1515 \\
\hline 28.66 & 1460 & 28.21 & 1466 & 28.1 & 1490 & 27.8 & 1500 & 26.97 & 1477 & 27.09 & 1488 & 27.41 & 1517 & 27.53 & 1520 & 26.82 & 1490 \\
\hline 28.12 & 1436 & 27.53 & 1434 & 27.65 & 1467 & 26.88 & 1458 & 26.94 & 1468 & 26.64 & 1462 & 26.85 & 1487 & 27 & 1496 & 26.44 & 1467 \\
\hline 27.68 & 1415 & 27.09 & 1408 & 27.03 & 1434 & 26.38 & 1434 & 25.96 & 1427 & 26.11 & 1430 & 26.41 & 1460 & 26.53 & 1472 & 25.91 & 1443 \\
\hline 26.88 & 1376 & 26.64 & 1387 & 26.5 & 1407 & 25.88 & 1407 & 25.52 & 1403 & 25.61 & 1404 & 25.91 & 1433 & 26.08 & 1447 & 25.46 & 1416 \\
\hline 26.26 & 1351 & 26.08 & 1365 & 25.96 & 1383 & 25.37 & 1383 & 24.99 & 1370 & 25.11 & 1380 & 25.46 & 1407 & 25.61 & 1417 & 24.84 & 1378 \\
\hline 25.76 & 1326 & 25.28 & 1323 & 25.58 & 1358 & 24.9 & 1353 & 24.51 & 1343 & 24.69 & 1352 & 24.99 & 1382 & 24.72 & 1371 & 24.37 & 1352 \\
\hline 25.17 & 1296 & 24.84 & 1301 & 25.17 & 1335 & 24.43 & 1331 & 24.07 & 1319 & 24.13 & 1329 & 24.6 & 1361 & 24.28 & 1349 & 23.92 & 1324 \\
\hline 24.72 & 1272 & 24.25 & 1272 & 24.54 & 1309 & 24.19 & 1306 & 23.6 & 1293 & 23.83 & 1304 & 23.77 & 1323 & 23.86 & 1326 & 23.45 & 1305 \\
\hline 24.19 & 1246 & 23.69 & 1244 & 24.13 & 1283 & 23.72 & 1283 & 23.12 & 1269 & 22.83 & 1263 & 23.42 & 1300 & 23.39 & 1294 & 22.89 & 1276 \\
\hline 23.63 & 1226 & 23.15 & 1218 & 23.15 & 1243 & 22.71 & 1243 & 22.56 & 1245 & 22.5 & 1234 & 22.8 & 1270 & 22.89 & 1269 & 22.5 & 1252 \\
\hline 23 & 1191 & 22.8 & 1191 & 22.65 & 1215 & 22.21 & 1220 & 22.12 & 1220 & 21.94 & 1210 & 22.38 & 1248 & 22.44 & 1244 & 21.88 & 1215 \\
\hline 22.38 & 1167 & 22.15 & 1165 & 22.03 & 1185 & 21.82 & 1192 & 21.79 & 1194 & 21.35 & 1179 & 21.79 & 1215 & 22.03 & 1219 & 21.44 & 1191 \\
\hline 21.94 & 1144 & 21.7 & 1141 & 21.53 & 1162 & 21.38 & 1167 & 21.32 & 1169 & 20.9 & 1158 & 21.41 & 1190 & 21.47 & 1195 & 20.99 & 1166 \\
\hline 21.02 & 1104 & 21.2 & 1116 & 21.11 & 1138 & 20.64 & 1136 & 20.13 & 1126 & 20.52 & 1126 & 20.87 & 1166 & 21.08 & 1170 & 20.64 & 1141 \\
\hline
\end{tabular}


Table 7. $25^{\circ} \mathrm{C}$ at 10 wt $\%$ - continued

\begin{tabular}{|c|c|c|c|c|c|c|c|c|c|c|c|c|c|c|c|c|c|}
\hline run1 & & run2 & & run3 & & run4 & & run5 & & run6 & & run7 & & run8 & & run9 & \\
\hline [Pa] & {$[1 / s]$} & [Pa] & {$[1 / \mathrm{s}]$} & [Pa] & {$[1 / s]$} & [Pa] & {$[1 / s]$} & [Pa] & {$[1 / \mathrm{s}]$} & [Pa] & {$[1 / \mathrm{s}]$} & [Pa] & {$[1 / s]$} & [Pa] & {$[1 / \mathrm{s}]$} & [Pa] & {$[1 / \mathrm{s}]$} \\
\hline 20.52 & 1077 & 20.73 & 1090 & 20.46 & 1107 & 20.34 & 1113 & 19.75 & 1097 & 20.1 & 1101 & 20.55 & 1140 & 20.52 & 1141 & 19.9 & 1110 \\
\hline 20.13 & 1055 & 20.1 & 1066 & 20.28 & 1087 & 9.84 & 1089 & 19.51 & 1075 & 19.57 & 1079 & 20.02 & 1116 & 19.84 & 1103 & 19.6 & 1086 \\
\hline 19.78 & 1030 & 19.6 & 1038 & 19.78 & 1064 & 9.13 & 1056 & 18.71 & 1038 & 19.28 & 1056 & 19.48 & 1091 & 19.42 & 1079 & 19.31 & 1063 \\
\hline 19.07 & 999 & 18.45 & 998.6 & 18.89 & 1025 & 18.8 & 1033 & 18.18 & 1014 & 18.36 & 1016 & 19.13 & 1066 & 19.01 & 1051 & 18.86 & 1038 \\
\hline 18.62 & 972.6 & 18.27 & 975.7 & 18.33 & 994.2 & 18.42 & 1010 & 17.68 & 989.3 & 17.97 & 992.9 & 18.21 & 1023 & 18.48 & 1028 & 17.91 & 999.9 \\
\hline 17.94 & 949.7 & 17.8 & 945.7 & 17.77 & 972.1 & 17.89 & 981.8 & 17.47 & 967.3 & 17.44 & 961.1 & 17.77 & 994.6 & 18 & 996.4 & 17.53 & 968.6 \\
\hline 17.62 & 925.4 & 17.12 & 921 & 17.35 & 942.2 & 17.2 & 956.7 & 17.03 & 934.2 & 16.79 & 936.9 & 17.59 & 971.3 & 17.53 & 969.5 & 17.12 & 946.1 \\
\hline 16.94 & 902.1 & 16.43 & 892.4 & 16.97 & 915.7 & 16.49 & 915.7 & 16.46 & 909.1 & 16.49 & 911.8 & 17.17 & 943.1 & 16.94 & 947 & 16.79 & 921 \\
\hline 16.55 & 874.3 & 16.17 & 869 & 16.29 & 891.9 & 6.11 & 887.5 & 15.81 & 882.2 & 16.08 & 880.9 & 16.41 & 918.8 & 16.67 & 921.9 & 16.05 & 895.9 \\
\hline 16.08 & 848.8 & 15.46 & 844.8 & & 867.7 & & 862.9 & & 856.7 & & 856.7 & & 895 & & 896.3 & 15.34 & 859.8 \\
\hline 15.34 & 819.2 & 14.54 & 807.8 & 15.34 & 844.3 & 14.98 & 832.4 & 14.78 & 832.4 & 15.16 & 831.6 & 15.67 & 866.4 & 15.58 & 867.7 & 15.22 & 837.7 \\
\hline 14.75 & 791.5 & 14.36 & 782.2 & 15.1 & 818.3 & 14.48 & 808.7 & 14.63 & 802.9 & 14.48 & 806.4 & 15.34 & 847.9 & 15.28 & 840.8 & & 809.5 \\
\hline 13.98 & 751.8 & 13.62 & 755.8 & 14.45 & 793.2 & 14.36 & 784.4 & 13.86 & 780 & 14.19 & 782.7 & 14.45 & 806.4 & 14.54 & 816.1 & 14.22 & 780.4 \\
\hline 13.36 & 727.6 & 13.33 & 724.9 & 13.42 & 751.8 & 13.77 & 761.1 & 13.53 & 754 & 13.68 & 756.7 & 13.83 & 781.3 & 13.74 & 776 & 13.74 & 751.4 \\
\hline 12.97 & 695.8 & 12.68 & 707.3 & 13.12 & 727.1 & 13.09 & 735.9 & 13.24 & 728 & 12.97 & 716.1 & 13.68 & 754.9 & 13.53 & 750 & 13.39 & 726.7 \\
\hline 12.54 & 670.3 & 11.91 & 672 & 12.79 & 704.7 & 12.85 & 709.5 & 12.35 & 686.1 & 12.47 & 694.1 & 12.97 & 728.4 & 12.68 & 722.3 & 12.91 & 710.4 \\
\hline 11.84 & 645.6 & & 646 & & & & 684.4 & & 661 & & 669 & 12.47 & 705.5 & 12.47 & 691.9 & 12.39 & 671.2 \\
\hline 11.34 & 621.4 & 11.23 & 620 & 11.66 & 643.8 & 11.61 & 641.2 & 11.26 & 637.7 & 11.87 & 637.7 & 12.12 & 681.7 & 12.2 & 683.9 & 11.96 & 645.2 \\
\hline 11.13 & 601.5 & 10.63 & 598 & 10.68 & 605.5 & 11.16 & 615.2 & 10.67 & 613 & 11.45 & 613 & 11.25 & 639.9 & 11.34 & 641.6 & 11.5 & 618.7 \\
\hline 9.806 & 562.8 & 9.972 & 559.2 & 11.01 & 596.7 & 10.75 & 591.8 & 10.49 & 579.5 & 10.85 & 586.1 & 11.01 & 612.5 & 10.85 & 617.4 & 11.18 & 597.1 \\
\hline 9.4 & 534.1 & 9.483 & 533.7 & 10.23 & 566.7 & 9.889 & 561.4 & 10.01 & 554.8 & 10.54 & 565.4 & 10.48 & 591.4 & 10.37 & 588.3 & 10.72 & 571.1 \\
\hline 8.808 & 509.4 & 9.087 & 505.5 & 9.693 & 535.9 & 9.341 & 535 & 9.572 & 526.6 & 9.386 & 527.5 & 10.08 & 565.4 & 10.13 & 561.4 & 9.516 & 531.5 \\
\hline 8.296 & 485.2 & 8.646 & 478.1 & 9.454 & 513 & 8.918 & 509.4 & 8.939 & 502.4 & 9.11 & 502.4 & 9.903 & 532.8 & 9.536 & 538.1 & 9.119 & 504.1 \\
\hline 8.187 & 459.6 & 8.214 & 453.9 & 8.871 & 486.5 & 8.794 & 484.3 & 8.131 & 479 & 8.971 & 479.9 & 9.187 & 512.1 & 9.01 & 513 & 8.515 & 480.8 \\
\hline 7.358 & 425.3 & 7.246 & 429.7 & 8.027 & 463.6 & 8.477 & 463.6 & 8.042 & 453.5 & 8.515 & 450.8 & 8.545 & 488.7 & 8.737 & 489.2 & 8.169 & 450.4 \\
\hline 7.095 & 400.6 & 7.045 & 405.9 & 7.622 & 423.5 & 7.675 & 423.1 & 7.87 & 426.1 & 7.986 & 421.3 & 8.43 & 465.4 & 7.962 & 448.6 & 8.205 & 426.6 \\
\hline 6.26 & 368.9 & 6.959 & 380.3 & 6.692 & 397.9 & 7.054 & 399.7 & 7.385 & 401.9 & 7.133 & 397.9 & 7.743 & 434.5 & 7.432 & 420 & 7.841 & 397.5 \\
\hline 6.003 & 350.3 & 5.867 & 356.5 & 6.257 & 369.7 & 6.299 & 374.1 & 6.704 & 361.4 & 7.012 & 371.5 & 7.802 & 410.7 & 7.317 & 393.5 & 6.784 & 374.6 \\
\hline 5.621 & 318.2 & 5.171 & 317.7 & 6.331 & 345.1 & 6.145 & 342.4 & 5.751 & 334.5 & 6.843 & 345.1 & 6.923 & 366.2 & 6.911 & 375.5 & 6.87 & 349.5 \\
\hline 4.647 & 294.8 & 5.31 & 293.9 & 5.334 & 314.6 & 5.683 & 319.1 & 5.876 & 311.1 & 5.796 & 320.4 & 6.293 & 338.9 & 6.109 & 343.3 & 6.305 & 325.7 \\
\hline 4.686 & 272.3 & 4.186 & 263.1 & 5.367 & 288.2 & 5.266 & 294.8 & 4.869 & 282.5 & 5.589 & 282.9 & 5.716 & 316.9 & 5.973 & 312.4 & 6.006 & 301.4 \\
\hline 4.242 & 250.3 & 4.467 & 236.2 & 4.337 & 264.9 & 4.878 & 268.4 & 4.733 & 251.6 & 4.76 & 259.6 & 5.509 & 290.9 & 5.556 & 286.9 & 5.615 & 261.3 \\
\hline
\end{tabular}


Table 8. $50{ }^{\circ} \mathrm{C}$ at $10 \mathrm{wt} \%$

\begin{tabular}{|c|c|c|c|c|c|c|c|c|c|c|c|c|c|c|c|c|c|}
\hline run1 & & run2 & & run3 & & run4 & & run5 & & run6 & & run7 & & run8 & & run9 & \\
\hline [Pa] & {$[1 / s]$} & [Pa] & {$[1 / s]$} & [Pa] & {$[1 / s]$} & [Pa] & {$[1 / s]$} & [Pa] & {$[1 / s]$} & [Pa] & [1/s] & [Pa] & {$[1 / s]$} & [Pa] & {$[1 / \mathrm{s}]$} & [Pa] & {$[1 / s]$} \\
\hline 6.562 & 313.3 & 6.861 & 325.7 & 5.893 & 334.9 & 5.31 & 371.1 & 6.758 & 317.3 & 6.574 & 312.9 & 6.468 & 316.4 & 6.397 & 303.2 & 6.926 & 350.3 \\
\hline 6.763 & 312 & .432 & 349 & 7.065 & 324.8 & 5.822 & 325.7 & 6.977 & 332.7 & 7.11 & 339.8 & 6.769 & 322.1 & 6.746 & 319.1 & 7.441 & 374.1 \\
\hline 7.225 & 331.4 & 7.873 & 368.9 & 7.299 & 342 & 6.624 & 320.4 & 7.465 & 366.2 & 7.332 & 368.9 & 7.349 & 345.5 & 7.438 & 344.6 & 7.867 & 392.6 \\
\hline 7.882 & 371.1 & 7.79 & 392.2 & 7.358 & 363.6 & 7.524 & 339.8 & 7.696 & 396.6 & 7.965 & 392.6 & 7.601 & 373.7 & 7.506 & 371.9 & 8.009 & 420.9 \\
\hline 8.495 & 397.9 & 8.199 & 418.2 & 8.08 & 386.9 & 7.48 & 367.1 & 8.282 & 418.2 & 8.341 & 416.4 & 7.675 & 396.2 & 7.61 & 393.1 & 8.672 & 457 \\
\hline 8.24 & 407.2 & 8.708 & 453.5 & 8.299 & 412.9 & 7.799 & 391.3 & 8.726 & 443.8 & 8.273 & 438 & 8.261 & 418.6 & 8.042 & 430.1 & 8.924 & 485.2 \\
\hline 8.628 & 436.7 & 9.042 & 485.6 & 8.444 & 437.6 & 8.391 & 415.6 & 8.927 & 468.9 & 9.267 & 477.3 & 8.51 & 450.4 & 8.453 & 458.3 & 9.036 & 507.2 \\
\hline 8.948 & 468 & 9.332 & 510.8 & 8.681 & 464.9 & 8.767 & 453.5 & 9.528 & 502.8 & 9.418 & 501.9 & 9.036 & 472 & 8.817 & 483 & 9.175 & 530.6 \\
\hline 9.406 & 494 & 9.829 & 539 & 8.974 & 490 & 9.273 & 476.4 & 9.729 & 533.7 & 9.61 & 528.8 & 9.229 & 511.2 & 9.338 & 505.9 & 9.46 & 552.6 \\
\hline 10.2 & 536.3 & 10.14 & 561.9 & 9.347 & 516 & 9.462 & 500.6 & 10.1 & 554.4 & 10.02 & 557.5 & 9.699 & 532.3 & 9.427 & 535.4 & 9.72 & 579.1 \\
\hline 10.52 & 559.2 & & 589.6 & & 557.5 & & & 10.33 & & & & 9.874 & & 9.554 & & 10.31 & 605.9 \\
\hline 10.72 & 584.8 & 10.92 & 612.1 & 10.55 & 582.1 & 10.1 & 556.6 & 10.65 & 616.1 & 10.45 & 608.1 & 10.19 & 580.8 & 9.862 & 586.1 & 10.66 & 643.4 \\
\hline 11.17 & 613.9 & 11.29 & 638.5 & .66 & 613.9 & 10.42 & 579.1 & 11.05 & 639.4 & 10.82 & 630.2 & 10.74 & 621.8 & 10.16 & 610.8 & 11.25 & 674.7 \\
\hline 11.33 & 639.9 & 11.52 & 663.2 & 10.97 & 633.7 & 10.67 & 599.3 & 11.34 & 669.4 & 11.47 & 669 & 10.94 & 649.6 & 11 & 647.8 & 11.4 & 698.9 \\
\hline 11.62 & 661.5 & 12.22 & 699.4 & 11.13 & 657.1 & 10.71 & 631.5 & 11.57 & 695.4 & 11.49 & 698.5 & 10.95 & 658.4 & 11 & 677.8 & 11.53 & 721.8 \\
\hline 11.84 & 685.3 & 12.38 & 724 & & 682.2 & 11.2 & 654.4 & 12.09 & 720.1 & 11.95 & 721.8 & 11.79 & 702 & 11.58 & 702.9 & 11.9 & 745.6 \\
\hline 12.58 & 726.7 & 12.83 & 750.5 & 12.06 & 719.2 & 11.75 & 693.6 & 12.38 & 742.1 & 12.28 & 743.9 & 11.96 & 728.9 & 11.77 & 728 & 12.68 & 788.4 \\
\hline 13.09 & 750 & & 781.3 & & 750 & 12.21 & 725.8 & 12.9 & 779.1 & 12.45 & 769.4 & 12.27 & 753.6 & 11.9 & 750.5 & 12.85 & 808.7 \\
\hline 13.19 & 780.4 & 13.47 & 806.4 & 12.58 & 771.6 & 12.34 & 751.4 & 13.06 & 807.3 & 12.95 & 793.7 & 12.62 & 777.8 & 12.41 & 775.2 & 13.29 & 834.2 \\
\hline 13.59 & 801.6 & 13.69 & 834.2 & 3.14 & 795.9 & 12.8 & 774.7 & 13.49 & 828 & 13.19 & 817 & 12.91 & 809.5 & 12.54 & 801.2 & 13.46 & 865.5 \\
\hline 13.88 & 825 & 14.01 & 859.3 & 13.37 & 821 & 13.11 & 797.6 & 13.65 & 849.2 & 13.71 & 855.8 & 13.24 & 831.6 & 13.14 & 839.5 & 13.76 & 888.9 \\
\hline 14.35 & 859.3 & 14.41 & 884 & 13.67 & 847 & 13.48 & 825.4 & 14.25 & 888.4 & 13.93 & 886.7 & 13.39 & 856.7 & 13.39 & 868.1 & 14.12 & 913.1 \\
\hline 14.85 & 885.8 & 14.88 & 922.3 & 14.38 & 881.4 & 13.7 & 850.5 & 14.46 & 912.7 & 14.34 & 904.7 & 14.14 & 891.1 & 13.88 & 891.1 & 14.28 & 936 \\
\hline 15.15 & 915.7 & 15.33 & 945.3 & 14.53 & 914 & 14.23 & 877 & 14.92 & 939.1 & 14.87 & 936.5 & 14.32 & 920.6 & 14.08 & 911.8 & 14.83 & 978.3 \\
\hline 15.35 & 937.3 & 15.59 & 972.6 & 15 & 940 & 14.67 & 915.3 & 15.25 & 965.5 & 15.1 & 962 & 14.67 & 942.2 & 14.51 & 938.7 & 15.31 & 1003 \\
\hline 15.71 & 960.2 & 15.88 & 997.7 & 15.28 & 957.2 & 15.11 & 942.2 & 15.41 & 988 & 15.36 & 984.5 & 14.73 & 972.6 & 14.89 & 965.1 & 15.61 & 1028 \\
\hline 16.36 & 999.9 & 16.21 & 1029 & 15.64 & 987.6 & 15.4 & 971.3 & 16.05 & 1024 & 15.96 & & 15.27 & 992.9 & 15.21 & 992.9 & 15.8 & 1049 \\
\hline 16.48 & 1022 & 16.53 & 1054 & 16 & 1016 & 15.72 & 996.4 & 16.4 & 1048 & 16.35 & 1048 & 15.48 & 1017 & 15.48 & 1018 & 16.34 & 1085 \\
\hline 16.91 & 1052 & 16.84 & 1080 & 16.33 & 1048 & 16.08 & 1019 & 16.72 & 1075 & 16.49 & 1071 & 15.75 & 1044 & 15.66 & 1042 & 16.58 & 1108 \\
\hline 17.02 & 1075 & 17.11 & 1101 & 16.66 & 1069 & 16.46 & 1047 & 16.94 & 1101 & 16.75 & 1092 & 16.3 & 1072 & 16.13 & 1072 & 16.92 & 1132 \\
\hline 17.37 & 1097 & 17.34 & 1125 & 16.96 & 1093 & 16.61 & 1067 & 17.38 & 1125 & 17.07 & 1119 & 16.7 & 1108 & 16.64 & 1097 & 17.19 & 1166 \\
\hline
\end{tabular}


Table 8. $50{ }^{\circ} \mathrm{C}$ at 10 wt $\%$ - continued

\begin{tabular}{|c|c|c|c|c|c|c|c|c|c|c|c|c|c|c|c|c|c|}
\hline run1 & & run2 & & run3 & & run4 & & run5 & & run6 & & run7 & & run8 & & run9 & \\
\hline [Pa] & {$[1 / s]$} & [Pa] & {$[1 / s]$} & [Pa] & {$[1 / s]$} & [Pa] & {$[1 / s]$} & [Pa] & {$[1 / s]$} & [Pa] & {$[1 / \mathrm{s}]$} & [Pa] & {$[1 / s]$} & [Pa] & {$[1 / \mathrm{s}]$} & [Pa] & {$[1 / s]$} \\
\hline 18.02 & 1131 & 17.96 & 1163 & 17.61 & 1130 & 17.13 & 1098 & 17.49 & 1149 & 17.79 & 1158 & 17.15 & 1140 & 17.24 & 1137 & 17.52 & 1189 \\
\hline 18.26 & 1159 & 18.32 & 1189 & 17.79 & 1159 & 17.34 & 1121 & 18.05 & 1188 & 17.94 & 1184 & 17.25 & 1164 & 17.39 & 1161 & 17.67 & 1214 \\
\hline 18.5 & 1185 & 18.65 & 1221 & 18.05 & 1180 & 18.05 & 1163 & 18.44 & 1211 & 18.41 & 1205 & 17.64 & 1189 & 17.64 & 1192 & 18.08 & 1241 \\
\hline 18.94 & 1210 & 19 & 1244 & 18.38 & 1207 & 18.35 & 1188 & 18.68 & 1242 & 18.53 & 1237 & 17.94 & 1212 & 17.88 & 1214 & 18.44 & 1267 \\
\hline 19.27 & 1234 & 19.27 & 1269 & 18.73 & 1233 & 18.56 & 1212 & 19.03 & 1267 & 18.85 & 1260 & 18.17 & 1234 & 18.44 & 1249 & 18.73 & 1295 \\
\hline 19.62 & 1259 & 19.62 & 1290 & 19.09 & 1257 & 18.97 & 1243 & 19.36 & 1288 & 19.18 & 1284 & 18.82 & 1271 & 18.65 & 1279 & 19.09 & 1317 \\
\hline 20.21 & 1294 & 20.15 & 1329 & 19.47 & 1287 & 19.33 & 1266 & 19.98 & 1326 & 19.68 & 1320 & 19.12 & 1297 & 19.06 & 1304 & 19.39 & 1348 \\
\hline 20.63 & 1325 & 20.57 & 1357 & 19.77 & 1309 & 19.53 & 1290 & 20.21 & 1349 & 20.15 & 1348 & 19.33 & 1323 & 19.44 & 1326 & 19.8 & 1371 \\
\hline 20.89 & 1347 & 20.81 & 1379 & 20.36 & 1349 & 20.01 & 1316 & 20.57 & 1379 & 20.39 & 1369 & 19.74 & 1351 & 19.56 & 1352 & 19.95 & 1397 \\
\hline 21.4 & 1379 & 21.13 & 1403 & 20.75 & 1379 & 20.27 & 1344 & 20.81 & 1400 & 20.72 & 1402 & 19.98 & 1374 & 19.98 & 1374 & 20.42 & 1421 \\
\hline 21.66 & 1404 & 21.4 & 1426 & 20.75 & 1389 & & 1371 & 21.28 & 1434 & 21.07 & 1426 & 20.33 & 1396 & 20.21 & 1400 & 20.63 & 1449 \\
\hline 22.08 & 1432 & 22.05 & 1464 & 21.43 & 1428 & 21.37 & 1407 & 21.61 & 1458 & 21.25 & 1450 & 20.66 & 1425 & 20.87 & 1439 & 21.4 & 1494 \\
\hline 22.52 & 1465 & 22.34 & 1491 & 21.81 & 1460 & 21.61 & 1430 & 21.99 & 1487 & 21.69 & 1477 & 21.01 & 1453 & 21.16 & 1461 & 21.72 & 1521 \\
\hline 22.85 & 1485 & 22.64 & 1512 & 22.11 & 1486 & 21.9 & 1459 & 22.29 & 1511 & 21.99 & 1505 & 21.28 & 1480 & 21.37 & 1492 & 21.99 & 1544 \\
\hline 23.23 & 1515 & 23.08 & 1553 & 22.4 & 1510 & 22.11 & 1481 & 22.52 & 1534 & 22.26 & 1528 & 21.96 & 1523 & 21.66 & 1515 & 22.34 & 1571 \\
\hline 21.22 & 1519 & 20.72 & 1516 & 20.87 & 1541 & 21.13 & 1536 & 20.1 & 1484 & 20.27 & 1522 & 20.3 & 1526 & 19.59 & 1497 & 19.24 & 1463 \\
\hline 20.95 & 1497 & 20.33 & 1491 & 20.69 & 1516 & 20.87 & 1515 & 19.8 & 1460 & 19.98 & 1495 & 19.77 & 1491 & 19.3 & 1473 & 18.85 & 1430 \\
\hline 20.3 & 1461 & 20.18 & 1465 & 20.1 & 1481 & 20.51 & 1492 & 19.53 & 1441 & 19.62 & 1472 & 19.44 & 1460 & 18.97 & 1450 & 18.56 & 1408 \\
\hline 19.95 & 1437 & 19.89 & 1434 & 19.86 & 1458 & 19.92 & 1454 & 18.88 & 1405 & 19.36 & 1445 & 19.15 & 1440 & 18.47 & 1407 & 18.17 & 1383 \\
\hline 19.62 & 1412 & 19.47 & 1412 & 19.56 & 1427 & 19.65 & 1431 & 18.68 & 1379 & 19 & 1419 & 18.97 & 1415 & 18.08 & 1385 & 18.05 & 1359 \\
\hline 19.27 & 1384 & 19.21 & 1388 & 19.15 & 1402 & 19.27 & 1404 & 18.32 & 1354 & 18.65 & 1394 & 18.53 & 1392 & 17.76 & 1363 & 17.64 & 1336 \\
\hline 18.97 & 1360 & 18.88 & 1367 & 18.79 & 1382 & 18.91 & 1378 & 18.05 & 1325 & 18.02 & 1358 & 18.23 & 1361 & 17.52 & 1330 & 16.96 & 1297 \\
\hline 18.56 & 1326 & 18.29 & 1330 & 18.38 & 1346 & 18.65 & 1355 & 17.76 & 1304 & 17.76 & 1334 & 17.58 & 1324 & 17.25 & 1302 & 16.84 & 1269 \\
\hline 18.14 & 1299 & 18.05 & 1296 & 18.05 & 1325 & 18.17 & 1325 & 17.49 & 1277 & 17.46 & 1299 & 17.37 & 1300 & 16.84 & 1277 & 16.46 & 1243 \\
\hline 17.79 & 1274 & 17.67 & 1274 & 17.67 & 1296 & 17.85 & 1293 & 17.2 & 1254 & 17.17 & 1281 & 17.25 & 1289 & 16.37 & 1253 & 16.13 & 1222 \\
\hline 17.61 & 1246 & 17.37 & 1248 & 17.28 & 1266 & 17.67 & 1270 & 16.81 & 1224 & 16.93 & 1257 & 16.81 & 1257 & 16.22 & 1231 & 15.8 & 1198 \\
\hline 17.28 & 1221 & 17.02 & 1226 & 17.02 & 1237 & 17.23 & 1241 & 16.46 & 1201 & 16.43 & 1223 & 16.25 & 1216 & 15.92 & 1206 & 15.21 & 1157 \\
\hline 16.9 & 1194 & 16.9 & 1206 & 16.57 & 1207 & 16.81 & 1219 & 15.86 & 1160 & 16.25 & 1197 & 16.19 & 1196 & 15.77 & 1178 & 15.39 & 1144 \\
\hline 16.49 & 1170 & 16.28 & 1164 & 16.28 & 1181 & 16.57 & 1194 & 15.57 & 1133 & 16.01 & 1173 & 15.98 & 1178 & 14.95 & 1144 & 14.62 & 1103 \\
\hline 15.86 & 1133 & 15.83 & 1142 & 16.13 & 1163 & 15.98 & 1156 & 15.21 & 1110 & 15.51 & 1145 & 15.21 & 1133 & 14.8 & 1116 & 14.41 & 1074 \\
\hline 15.69 & 1110 & 15.69 & 1111 & 15.69 & 1141 & 15.63 & 1130 & 14.83 & 1085 & 15.18 & 1120 & 14.92 & 1110 & 14.35 & 1083 & 14.12 & 1056 \\
\hline
\end{tabular}


Table 8. $50{ }^{\circ} \mathrm{C}$ at 10 wt $\%$ - continued

\begin{tabular}{|c|c|c|c|c|c|c|c|c|c|c|c|c|c|c|c|c|c|}
\hline run1 & & run2 & & run3 & & run4 & & run5 & & run6 & & run7 & & run8 & & run9 & \\
\hline [Pa] & {$[1 / s]$} & [Pa] & {$[1 / s]$} & [Pa] & {$[1 / s]$} & [Pa] & {$[1 / s]$} & [Pa] & {$[1 / s]$} & [Pa] & {$[1 / s]$} & [Pa] & {$[1 / s]$} & [Pa] & {$[1 / s]$} & [Pa] & {$[1 / s]$} \\
\hline 15.3 & 1085 & 15.24 & 1090 & 15.18 & 1106 & 15.33 & 1101 & 14.59 & 1052 & 15.01 & 1097 & 14.62 & 1085 & 14.24 & 1061 & 13.88 & 1022 \\
\hline 15.04 & 050 & 5.04 & 1062 & 4.98 & 1078 & 15.18 & 1075 & 14.3 & 1027 & 14.68 & 1070 & 14.35 & 1056 & 13.76 & 1038 & 13.38 & 1001 \\
\hline 14.62 & 1025 & 4.56 & 037 & 4.68 & 1048 & 14.74 & 1053 & 13.91 & 1004 & 14.06 & 1031 & 13.94 & 1031 & 13.53 & 1014 & 13.26 & 975.7 \\
\hline 14.24 & 998.6 & 14.3 & 013 & 4.15 & 1016 & 14.27 & 1015 & 13.53 & 977.9 & 13.82 & 999.9 & 13.67 & 1006 & 13.26 & 986.7 & 12.99 & 951.4 \\
\hline 14.09 & 977.4 & 13.88 & 983.2 & 13.79 & 993.7 & 13.91 & 995.5 & 13.35 & 954.5 & 13.29 & 978.8 & 13.44 & 984 & 12.79 & 948.3 & 12.24 & 911.8 \\
\hline 13.64 & 955 & 3.38 & 943.1 & 3.47 & 967.3 & 13.61 & 971.3 & 12.7 & 912.7 & 13.29 & 954.5 & 12.93 & 942.6 & 12.32 & 924.1 & 12.14 & 888.4 \\
\hline 13.11 & 915.3 & 12.93 & 920.1 & 13.08 & 932.9 & 13.29 & 946.1 & 12.45 & 888.4 & 12.85 & 929.4 & 12.58 & 915.7 & 12.09 & 896.3 & 11.7 & 861.1 \\
\hline 12.82 & 889.7 & 2.64 & 888.4 & 2.99 & 912.7 & 12.87 & 921.5 & 12.17 & 866.4 & 12.47 & 903.4 & 12.42 & 892.4 & 11.94 & 866.8 & 11.46 & 832.9 \\
\hline 12.57 & 861.1 & 12.45 & 865.1 & 12.72 & 895 & 12.69 & 886.2 & 11.76 & 832 & 12.15 & 875.2 & 12.01 & 872.1 & 11.47 & 845.7 & 11.2 & 806.4 \\
\hline 12.35 & 835.1 & 2.07 & 833.8 & 12.34 & 864.6 & 12.4 & 863.7 & 11.65 & 810.4 & 12.04 & 847.9 & 11.88 & 844.8 & 11.25 & 820.6 & 10.47 & 773.4 \\
\hline 11.91 & 809.1 & & & & 829.8 & 11.91 & 840.8 & & & & 821 & 11.3 & 808.7 & & & 10.47 & 748.3 \\
\hline 11.66 & 785.7 & 1.37 & 782.2 & 11.55 & 798.1 & 11.56 & 806.9 & 10.73 & 747.8 & 11.38 & 794.1 & 10.77 & 785.3 & 10.25 & 756.2 & 10.05 & 720.1 \\
\hline 11.32 & 758.9 & .19 & 759.3 & 1.14 & 780.9 & 11.42 & 779.6 & 0.26 & 723.2 & 10.7 & 755.3 & 10.76 & 755.8 & 10.08 & & 732 & 698.9 \\
\hline 10.87 & 733.7 & 10.51 & 725.8 & 10.81 & 747.4 & 10.89 & 756.2 & 10.29 & 695.4 & 10.15 & 730.7 & 10.16 & 724.5 & 9.898 & 700.2 & 9.539 & 672.5 \\
\hline 10.76 & 706.4 & .25 & 703.8 & 10.62 & 725.8 & 10.6 & 721 & 10.04 & 671.6 & 10.05 & 703.3 & 9.989 & 701.1 & 9.421 & 676.9 & 9.38 & 648.2 \\
\hline 10.19 & 677.3 & 945 & 670.7 & 9.972 & 694.5 & 10.22 & 697.6 & 9.631 & 650 & 9.98 & 677.8 & 9.705 & 674.2 & 9.078 & 652.2 & 8.622 & 612.5 \\
\hline 9.791 & 654 & 613 & 652.2 & 9.652 & 666.8 & 9.815 & 669.4 & 9.054 & 621.4 & 9.693 & 654 & 9.072 & 645.2 & 8.811 & 629.3 & 222 & 586.5 \\
\hline 9.569 & 616.5 & 229 & 621.8 & & 642.1 & & 642.5 & 8.77 & 583.9 & & & .826 & 621.8 & 66 & 594 & 84 & 554.4 \\
\hline 9.235 & 592.7 & 8.965 & 598.9 & 8.885 & 608.6 & 9.356 & 618.7 & 8.542 & 573.8 & 8.714 & 591.8 & 8.598 & 597.1 & 8.264 & 565 & 7.817 & 529.3 \\
\hline 8.681 & 59.7 & & 575.1 & & & & 584.3 & & & & & & 561.4 & 072 & 541.6 & 41 & 502.8 \\
\hline 8.382 & 534.5 & 8.356 & 534.5 & 8.589 & 559.7 & 8.527 & 561 & 7.722 & 507.2 & 8.3 & 539 & 8.086 & 537.2 & 7.867 & 514.3 & 92 & 479 \\
\hline 8.255 & 510.8 & 8.057 & 525.7 & 7.856 & 527.9 & 8.009 & 528.4 & 7.536 & 478.6 & 7.947 & 516 & 7.817 & 512.1 & 7.492 & 491.8 & 06 & 457.4 \\
\hline 8.006 & 486.1 & 7.737 & 479 & 7.512 & 498.4 & 7.808 & 503.7 & 6.968 & 454.8 & 7. & 490.5 & 7.631 & 482.5 & 213 & 458.3 & 254 & 415.6 \\
\hline 7.74 & 460.5 & 7.249 & 457 & 7.625 & 479 & 7.731 & 481.7 & 6.864 & 432.3 & 7.068 & 463.2 & 7.042 & 460.5 & 6.829 & 431 & 6.302 & 395.7 \\
\hline 7.175 & 423.1 & 6.861 & 428.8 & 7.338 & 450.8 & 7.311 & 461 & 6.281 & 395.7 & 6.817 & 438.9 & 6.761 & 437.6 & 6.328 & 402.8 & 5.953 & 366.2 \\
\hline 6.763 & 396.2 & 6.494 & 402.3 & 6.914 & 429.7 & 6.879 & 417.3 & 6.169 & 385.6 & & 412.9 & 6.669 & 410.7 & 5.935 & 379.4 & 5.376 & 341.5 \\
\hline 6.394 & 374.6 & 6.503 & 379 & 6.37 & 391.3 & 6.435 & 393.5 & 5.577 & 342.9 & & 376.8 & 6.323 & 387.4 & 5.97 & 355.6 & 5.559 & 318.2 \\
\hline 6.399 & 352.5 & 6.077 & 348.1 & 5.843 & 367.1 & 5.843 & 365.8 & 5.37 & 313.8 & 5.793 & 348.6 & 5.814 & 341.1 & 5.328 & 316.4 & 4.828 & 289.5 \\
\hline 5.666 & 316.9 & 5.645 & 324.8 & 5.882 & 339.8 & 6.006 & 344.2 & 5.316 & 284.7 & 6.047 & 337.1 & 5.077 & 319.1 & 4.804 & 292.6 & 4.935 & 265.3 \\
\hline 5.438 & 292.6 & 5.526 & 299.2 & 5.364 & 312.4 & 5.473 & 312.4 & 4.721 & 261.3 & 5.328 & 293.1 & 5.31 & 292.2 & 4.733 & 267.5 & 4.212 & 242.8 \\
\hline 5.195 & 263.5 & 4.985 & 272.3 & 5.367 & 289.1 & 5.432 & 291.3 & 4.624 & 255.6 & & 269.7 & 4.511 & 266.6 & 4.852 & 258.2 & 0 & 154.6 \\
\hline 5.065 & 243.3 & 4.884 & 246.3 & 4.858 & 252.5 & 4.772 & 265.3 & 0 & 179.3 & 4.686 & 259.1 & 4.624 & 258.7 & 0 & 176.4 & 0 & 161.7 \\
\hline
\end{tabular}


Table 9. Rheometer Response for Blank - 13 wt \%

\begin{tabular}{|c|c|c|c|c|c|c|c|c|c|c|c|c|c|c|c|c|c|}
\hline run1 & & run2 & & run3 & & run4 & & run5 & & run6 & & run7 & & run8 & & run9 & \\
\hline [Pa] & {$[1 / s]$} & [Pa] & {$[1 / \mathrm{s}]$} & [Pa] & [1/s] & [Pa] & [1/s] & [Pa] & [1/s] & [Pa] & [1/s] & [Pa] & {$[1 / s]$} & [Pa] & [1/s] & [Pa] & {$[1 / s]$} \\
\hline 2.355 & 273.2 & 2.435 & 276.3 & 2.426 & 278.1 & 2.607 & 276.3 & 2.556 & 274.5 & 2.414 & 272.3 & 2.417 & 271 & 2.26 & 275.9 & 1.831 & 314.6 \\
\hline 2.71 & 293.5 & 2.666 & 292.2 & 2.835 & 294.8 & 2.829 & 293.5 & 2.758 & 293.5 & 2.642 & 291.7 & 2.613 & 294.4 & 2.382 & 297 & 2.414 & 302.7 \\
\hline 2.882 & 320.4 & 2.92 & 322.6 & 2.98 & 317.7 & 3.021 & 319.9 & 2.917 & 317.7 & 2.82 & 316.9 & 2.784 & 317.3 & 2.601 & 319.9 & 2.583 & 323.5 \\
\hline 3.024 & 343.7 & 3.033 & 347.7 & 3.113 & 344.2 & 3.261 & 343.7 & 3.122 & 347.7 & 3.071 & 349 & 3.08 & 349.9 & 2.758 & 345.1 & 2.781 & 346.4 \\
\hline 3.273 & 376.8 & 3.196 & 372.4 & 3.204 & 368.9 & 3.509 & 375.5 & 3.261 & 373.3 & 3.193 & 376.8 & 3.222 & 377.2 & 2.997 & 378.5 & 3.003 & 377.2 \\
\hline 3.48 & 401.5 & 3.45 & 398.8 & 3.489 & 399.3 & 3.657 & 401.9 & 3.474 & 400.6 & 3.293 & 399.3 & 3.403 & 401.5 & 3.107 & 404.5 & 3.133 & 401.5 \\
\hline 3.509 & 427 & 3.959 & 431.4 & 3.651 & 426.1 & 3.787 & 426.1 & 3.678 & 427 & 3.554 & 433.6 & 3.61 & 427 & 3.255 & 431 & 3.308 & 423.9 \\
\hline 3.743 & 453.9 & 4.001 & 454.8 & 3.79 & 456.5 & 3.998 & 452.6 & 3.921 & 458.3 & 3.722 & 458.3 & 3.728 & 453 & 3.391 & 456.1 & 3.509 & 458.3 \\
\hline 3.903 & 480.3 & 4.16 & 484.3 & 3.977 & 482.1 & 4.199 & 480.3 & 4.086 & 483.4 & 3.903 & 486.1 & 3.95 & 479.9 & 3.663 & 488.7 & 3.634 & 484.3 \\
\hline 4.098 & 505.9 & 4.341 & 508.5 & 4.14 & 507.2 & 4.486 & 513.8 & 4.214 & 512.1 & 4.012 & 511.6 & 4.113 & 507.2 & 3.799 & 513 & 3.746 & 512.1 \\
\hline 4.299 & 538.1 & 4.658 & 536.3 & 4.412 & 538.1 & 4.69 & 539 & 4.439 & 538.1 & 4.146 & 535.4 & 4.347 & 540.3 & 3.927 & 540.3 & 4.027 & 539 \\
\hline 4.456 & 564.1 & 4.897 & 561.9 & 4.557 & 563.2 & & 563.2 & 4.613 & 562.8 & 4.35 & 569.4 & 4.45 & 564.1 & 4.116 & 563.6 & & 564.1 \\
\hline 4.545 & 588.8 & 5.27 & 593.6 & 5.093 & 593.2 & 5.125 & 592.3 & 4.803 & 594.9 & 4.527 & 591 & 4.69 & 589.2 & 4.24 & 588.3 & 4.308 & 591.8 \\
\hline 4.859 & 620 & 5.492 & 619.6 & 5.545 & 620 & 5.291 & 617.4 & 4.945 & 623.1 & 4.752 & 624 & 4.82 & 616.1 & 4.424 & 617.4 & 4.516 & 621.4 \\
\hline 5.022 & 646.9 & 5.761 & 643.4 & 5.634 & 649.1 & 5.548 & 650.9 & 5.175 & 647.4 & 4.909 & 649.1 & 4.986 & 646.5 & 4.652 & 648.7 & 4.669 & 649.1 \\
\hline 5.22 & 677.8 & 5.699 & 676.9 & 5.856 & 674.7 & 5.67 & 677.3 & 5.297 & 672.9 & 5.045 & 674.2 & 5.211 & 672.5 & 4.755 & 675.6 & 4.897 & 677.3 \\
\hline 5.46 & 701.1 & & 702.9 & 6.182 & 697.2 & & 705.1 & 5.593 & 704.7 & 5.187 & 703.3 & 5.43 & 704.7 & 356 & 698.9 & 5.01 & 704.2 \\
\hline 5.531 & 729.8 & 5.969 & 726.7 & 6.531 & 729.8 & 6.075 & 729.8 & 5.732 & 731.1 & 5.312 & 727.6 & 5.537 & 729.3 & 5.131 & 729.8 & 5.143 & 728 \\
\hline 5.773 & 754 & 6.179 & 757.1 & 6.623 & 748.3 & 6.22 & 752.7 & 5.88 & 759.7 & 5.545 & 760.2 & 5.679 & 754.9 & 5.279 & 759.7 & 353 & 759.3 \\
\hline 5.924 & 779.1 & 6.306 & 784 & 7.096 & 782.7 & 6.549 & 786.2 & 5.957 & 782.7 & 5.732 & 784 & 5.844 & 780 & 5.445 & 785.7 & 5.539 & 784 \\
\hline 6.137 & 811.7 & 6.501 & 808.7 & 7.436 & 809.1 & 6.741 & 812.6 & 6.247 & 808.7 & 5.809 & 813.1 & 6.099 & 813.9 & 5.551 & 810.9 & 5.69 & 813.5 \\
\hline 6.309 & 838.6 & 6.883 & 839.1 & 7.587 & 835.1 & 6.904 & 839.5 & 6.457 & 839.5 & 6.007 & 837.3 & 6.235 & 840.4 & 5.821 & 843 & 5.862 & 839.1 \\
\hline 6.498 & 863.3 & 6.945 & 866.4 & 8.002 & 864.2 & 7.129 & 862 & 6.629 & 866.8 & 6.134 & 863.3 & 6.401 & 868.6 & 5.957 & 867.7 & 5.975 & 865.1 \\
\hline 6.688 & 888.4 & 7.081 & 893.7 & 8.238 & 892.4 & 7.383 & 892.8 & 6.75 & 891.5 & 6.454 & 895 & 6.634 & 893.3 & 6.173 & 893.7 & 6.093 & 890.2 \\
\hline 6.993 & 920.1 & 7.034 & 919.3 & 8.431 & 915.7 & 7.596 & 919.7 & 6.936 & 916.2 & 6.611 & 921.5 & 6.708 & 922.3 & 6.265 & 924.1 & 6.247 & 914.9 \\
\hline 7.149 & 947.5 & 7.164 & 943.9 & 8.821 & 948.8 & 7.774 & 947.9 & 7.265 & 950.6 & 6.738 & 947 & 6.916 & 945.7 & 6.401 & 947.9 & 6.427 & 942.6 \\
\hline 7.354 & 972.6 & 7.389 & 973 & 9.1 & 973.9 & 7.978 & 977 & 7.419 & 975.2 & 6.916 & 972.6 & 7.105 & 972.6 & 6.566 & 974.3 & 6.581 & 969.1 \\
\hline 7.478 & 996.8 & 7.472 & 1001 & 9.39 & 1002 & 8.153 & 1003 & 7.531 & 1002 & 7.049 & 998.6 & 7.188 & 998.1 & 6.812 & 1006 & 6.836 & 1002 \\
\hline 7.815 & 1027 & 7.682 & 1024 & 9.766 & 1027 & 8.312 & 1027 & 7.706 & 1028 & 7.315 & 1031 & 7.49 & 1033 & 6.978 & 1030 & 6.969 & 1030 \\
\hline 8.025 & 1056 & 7.877 & 1059 & 10.22 & 1058 & 8.478 & 1052 & 7.895 & 1055 & 7.395 & 1059 & 7.658 & 1060 & 7.093 & 1057 & 7.078 & 1058 \\
\hline 8.218 & 1081 & 8.04 & 1082 & 10.43 & 1083 & 8.706 & 1078 & 8.114 & 1081 & 7.62 & 1082 & 7.812 & 1085 & 7.33 & 1089 & 7.336 & 1083 \\
\hline
\end{tabular}


Table 9. Rheometer Response for Blank - 13 wt \% - continued

\begin{tabular}{|c|c|c|c|c|c|c|c|c|c|c|c|c|c|c|c|c|c|}
\hline run1 & & run2 & & run3 & & run4 & & run5 & & run6 & & run7 & & run8 & & run9 & \\
\hline [Pa] & [1/s] & [Pa] & {$[1 / \mathrm{s}]$} & [Pa] & {$[1 / s]$} & [Pa] & {$[1 / s]$} & [Pa] & [1/s] & [Pa] & {$[1 / s]$} & [Pa] & [1/s] & [Pa] & [1/s] & [Pa] & {$[1 / s]$} \\
\hline 8.588 & 1111 & 8.105 & 1108 & 10.74 & 1107 & 9.038 & 1113 & 8.312 & 1107 & 7.83 & 1114 & 8.019 & 1112 & 7.428 & 1115 & 7.602 & 1114 \\
\hline 8.789 & 1140 & 8.354 & 1138 & 11.08 & 1140 & 9.248 & 1139 & 8.558 & 1142 & 7.996 & 1142 & 8.185 & 1139 & 7.573 & 1141 & 7.718 & 1138 \\
\hline 9.088 & 1165 & 8.425 & 1167 & 11.33 & 1166 & 9.478 & 1165 & 8.73 & 1169 & 8.111 & 1165 & 8.301 & 1165 & 7.75 & 1167 & 7.854 & 1167 \\
\hline 9.268 & 1189 & 8.644 & 1190 & 11.62 & 1191 & 9.573 & 1191 & 8.89 & 1194 & 8.372 & 1194 & 8.508 & 1190 & 7.94 & 1193 & 7.969 & 1195 \\
\hline 9.464 & 1212 & 8.824 & 1215 & 11.83 & 1216 & 9.831 & 1218 & 9.046 & 1219 & 8.496 & 1223 & 8.759 & 1223 & 8.108 & 1218 & 8.23 & 1222 \\
\hline 9.837 & 1240 & 9.091 & 1247 & 12.15 & 1241 & 10.21 & 1250 & 9.227 & 1243 & 8.697 & 1246 & 8.884 & 1248 & 8.369 & 1252 & 8.413 & 1247 \\
\hline 10.32 & 1274 & 9.245 & 1275 & 12.48 & 1276 & 10.23 & 1269 & 9.552 & 1275 & 8.839 & 1279 & 9.097 & 1274 & 8.511 & 1278 & 8.537 & 1273 \\
\hline 10.42 & 1295 & 9.547 & 1304 & 12.88 & 1300 & 10.57 & 1304 & 9.686 & 1304 & 8.984 & 1304 & 9.156 & 1299 & 8.736 & 1303 & 8.736 & 1304 \\
\hline 10.59 & 1317 & 9.638 & 1328 & 13.18 & 1329 & 10.8 & 1328 & 9.893 & 1330 & 9.23 & 1334 & 9.369 & 1326 & 8.821 & 1333 & 8.869 & 1332 \\
\hline 10.96 & 1352 & 9.792 & 1356 & 13.41 & 1353 & 10.99 & 1356 & 10.07 & 1355 & 9.428 & 1360 & 9.615 & 1360 & 8.975 & 1359 & 9.04 & 1356 \\
\hline 11.5 & 1368 & 10.07 & 1386 & 13.8 & 1384 & 11.21 & 1382 & 10.33 & 1387 & 9.597 & 1388 & 9.768 & 1387 & 9.156 & 1385 & 9.203 & 1379 \\
\hline 11.72 & 1407 & 10.31 & 1410 & 14.08 & 1409 & 11.43 & 1409 & 10.53 & 1414 & 9.754 & 1410 & 9.911 & 1412 & 9.345 & 1408 & 9.514 & 1413 \\
\hline 12.12 & 1441 & 10.45 & 1438 & 14.5 & 1438 & 11.69 & 1436 & 10.72 & 1441 & 10.01 & 1443 & 10.13 & 1437 & 9.558 & 1434 & 9.668 & 1437 \\
\hline 12.56 & 1465 & 10.61 & 1462 & 14.62 & 1462 & 11.98 & 1468 & 10.96 & 1467 & 10.19 & 1466 & 10.23 & 1464 & 9.804 & 1468 & 9.771 & 1464 \\
\hline 12.93 & 1494 & 10.85 & 1487 & 14.89 & 1487 & 12.12 & 1492 & 11.19 & 1497 & 10.3 & 1493 & 10.48 & 1496 & 9.911 & 1497 & 9.863 & 1489 \\
\hline 13.25 & 1520 & 11.13 & 1519 & 15.31 & 1520 & 12.31 & 1516 & 11.34 & 1523 & 10.49 & 1517 & 10.67 & 1522 & 10.07 & 1521 & 10.16 & 1523 \\
\hline 10.4 & 1498 & 10.63 & 1516 & 12.2 & 1531 & 11.55 & 1538 & 12.25 & 1534 & 11.17 & 1506 & 10.2 & 1540 & 9.928 & 1537 & 9.899 & 1512 \\
\hline 10.36 & 1471 & 10.27 & 1481 & 11.91 & 1506 & 11.37 & 1514 & 12.08 & 1508 & 11.01 & 1482 & 10.02 & 1516 & 9.979 & 1511 & 9.715 & 1486 \\
\hline 10.21 & 1447 & 10.21 & 1455 & 11.72 & 1480 & 11.13 & 1487 & 11.82 & 1484 & 10.72 & 1454 & 9.783 & 1481 & 9.869 & 1483 & 9.381 & 1452 \\
\hline 9.914 & 1420 & 9.922 & 1431 & 11.46 & 1449 & 10.84 & 1455 & 11.5 & 1456 & 10.56 & 1428 & 9.576 & 1457 & 9.671 & 1455 & 9.23 & 1424 \\
\hline 9.635 & 1388 & 9.763 & 1407 & 11.58 & 1422 & 10.69 & 1425 & 11.17 & 1424 & 10.36 & 1403 & 9.328 & 1427 & 9.535 & 1429 & 9.005 & 1399 \\
\hline 9.52 & 1362 & 9.502 & 1374 & 11.38 & 1397 & 10.44 & 1402 & 10.93 & 1397 & 10.09 & 1376 & 9.138 & 1400 & 9.387 & 1407 & 8.848 & 1371 \\
\hline 9.322 & 1335 & 9.333 & 1348 & 11.13 & 1372 & 10.26 & 1378 & 10.73 & 1376 & 9.928 & 1350 & 8.952 & 1377 & 9.129 & 1373 & 8.623 & 1347 \\
\hline 9.135 & 1308 & 8.969 & 1317 & 10.97 & 1348 & 9.993 & 1345 & 10.5 & 1351 & 9.606 & 1322 & 8.727 & 1345 & 8.928 & 1347 & 8.41 & 1319 \\
\hline 8.916 & 1286 & 8.836 & 1289 & 10.6 & 1313 & 9.783 & 1317 & 10.3 & 1318 & 9.36 & 1286 & 8.49 & 1321 & 8.697 & 1322 & 8.141 & 1289 \\
\hline 8.65 & 1254 & 8.7 & 1266 & 10.41 & 1288 & 9.52 & 1290 & 10.03 & 1292 & 9.277 & 1260 & 8.345 & 1294 & 8.555 & 1293 & 8.049 & 1261 \\
\hline 8.537 & 1230 & 8.496 & 1240 & 10.22 & 1262 & 9.354 & 1265 & 9.771 & 1267 & 9.005 & 1237 & 8.04 & 1261 & 8.354 & 1270 & 7.88 & 1239 \\
\hline 8.36 & 1204 & 8.203 & 1208 & 9.982 & 1235 & 9.162 & 1241 & 9.428 & 1236 & 8.765 & 1216 & 7.877 & 1238 & 8.011 & 1237 & 7.617 & 1209 \\
\hline 8.17 & 1177 & 8.073 & 1181 & 9.795 & 1210 & 8.904 & 1208 & 9.162 & 1210 & 8.378 & 1190 & 7.777 & 1210 & 7.898 & 1210 & 7.395 & 1181 \\
\hline 7.869 & 1142 & 7.969 & 1158 & 9.473 & 1178 & 8.647 & 1182 & 9.02 & 1181 & 8.392 & 1168 & 7.617 & 1187 & 7.638 & 1179 & 7.229 & 1157 \\
\hline 7.62 & 1117 & 7.75 & 1133 & 9.218 & 1150 & 8.502 & 1158 & 8.813 & 1157 & 8.105 & 1139 & 7.386 & 1154 & 7.442 & 1155 & 7.016 & 1133 \\
\hline
\end{tabular}


Table 9. Rheometer Response for Blank - 13 wt \% - continued

\begin{tabular}{|c|c|c|c|c|c|c|c|c|c|c|c|c|c|c|c|c|c|}
\hline run1 & & run2 & & run3 & & run4 & & run5 & & run6 & & run7 & & run8 & & run9 & \\
\hline [Pa] & {$[1 / s]$} & [Pa] & {$[1 / \mathrm{s}]$} & [Pa] & [1/s] & [Pa] & {$[1 / s]$} & [Pa] & {$[1 / \mathrm{s}]$} & [Pa] & {$[1 / \mathrm{s}]$} & [Pa] & {$[1 / s]$} & [Pa] & {$[1 / \mathrm{s}]$} & [Pa] & {$[1 / s]$} \\
\hline 7.496 & 1090 & 7.49 & 1101 & 8.987 & 1124 & 8.289 & 1132 & 8.57 & 1124 & 7.812 & 1100 & 7.141 & 1128 & 7.265 & 1130 & 6.747 & 1100 \\
\hline 7.36 & 1066 & 7.241 & 1076 & 8.824 & 1102 & 7.975 & 1098 & 8.457 & 1096 & 7.895 & 1085 & 7.004 & 1099 & 7.108 & 1101 & 6.516 & 1072 \\
\hline 7.075 & 1035 & .123 & 1048 & 8.472 & 1070 & 7.815 & 1072 & 8.333 & 1071 & 7.374 & 1061 & 6.83 & 1075 & 6.809 & 1071 & 0.368 & 1046 \\
\hline 6.883 & 1009 & 6.919 & 1025 & 8.256 & 1041 & 7.581 & 1049 & 8.244 & 1046 & 7.179 & 1015 & 6.694 & 1050 & 6.708 & 1044 & 6.265 & 1017 \\
\hline 6.753 & 981.4 & .708 & 992.9 & 8.008 & 1015 & 7.416 & 1020 & 7.916 & 1020 & 6.99 & 989.3 & 6.398 & 1017 & 6.513 & 1019 & 6.087 & 993.7 \\
\hline 6.498 & 951.4 & 6.537 & 967.7 & 7.824 & 992.9 & 7.259 & 995.1 & 7.718 & 995.1 & 6.762 & 960.2 & 6.185 & 991.5 & 6.362 & 992.4 & 5.909 & 965.1 \\
\hline 6.282 & 926.8 & 6.306 & 934.7 & 7.65 & 966.4 & 6.916 & 963.8 & 7.321 & 965.1 & 6.552 & 934.7 & 5.983 & 962.9 & 6.173 & 969.1 & 5.72 & 936 \\
\hline 6.182 & 899 & 6.075 & 908.2 & 7.265 & 933.4 & 6.777 & 936 & 7.019 & 936.5 & 6.356 & 907.4 & 5.782 & 937.8 & 6.001 & 942.2 & 5.483 & 907.8 \\
\hline 5.986 & 874.3 & 5.921 & 882.2 & 7.084 & 904.3 & 6.59 & 909.6 & 6.791 & 914.4 & 6.194 & 882.2 & 5.693 & 909.1 & 5.711 & 909.1 & 5.312 & 883.1 \\
\hline 5.827 & 850.1 & & 860.2 & 6.91 & 880 & & 884 & .522 & 880.9 & & & & 881.8 & & 883.1 & & 858 \\
\hline 5.596 & 817.5 & 5.528 & 827.6 & 6.691 & 854.5 & 6.167 & 858.9 & 6.256 & 854.5 & 5.696 & 826.3 & 5.362 & 856.7 & 5.344 & 854 & 4.965 & 831.6 \\
\hline 5.424 & 793.2 & 5.4 & 799.8 & 6.486 & 829.4 & 5.856 & 825 & 6.004 & 825 & 5.516 & 797.2 & 5.193 & 832 & & 830.2 & & 797.2 \\
\hline 5.205 & 767.7 & 5.238 & 777.4 & 6.191 & 794.1 & 5.661 & 799.8 & 5.877 & 801.2 & 5.383 & 772.5 & 4.918 & 798.5 & 5.001 & 802.5 & 4.521 & 772.1 \\
\hline 5.099 & 739.5 & 4.951 & 744.3 & 6.057 & 771.6 & 5.507 & 772.5 & 5.679 & 775.2 & 5.164 & 744.8 & 4.788 & 771.2 & 4.794 & 777.4 & 341 & 743.4 \\
\hline 4.803 & 708.6 & 4.82 & 717.9 & 5.785 & 745.2 & 5.329 & 748.3 & 5.362 & 741.7 & 4.965 & 721.4 & 4.646 & 746.5 & 4.607 & 750.5 & 157 & 718.8 \\
\hline 4.696 & 681.3 & 4.661 & 693.6 & 5.468 & 713.9 & 5.119 & 722.3 & 5.178 & 717.9 & 4.672 & 687.5 & 4.53 & 721.4 & 4.347 & 719.2 & 998 & 691 \\
\hline 4.465 & 656.2 & & 665.4 & 5.273 & 686.1 & & 695.4 & & 688.3 & 4.542 & & 4.223 & 693.6 & & 692.8 & & 665.4 \\
\hline 4.359 & 633.3 & 4.308 & 639 & 5.09 & 663.2 & 4.655 & 662.3 & 4.776 & 664.1 & 4.37 & 636.8 & 4.149 & 665.9 & 4.042 & 668.1 & 3.678 & 638.1 \\
\hline 4.146 & 598.9 & 4.122 & 610.8 & 4.808 & 631.9 & 4.587 & 642.5 & 4.587 & 636.3 & 4.223 & & 918 & 642.5 & 376 & 643.4 & 97 & 613.4 \\
\hline 3.965 & 574.7 & 3.956 & 585.7 & 4.566 & 603.3 & 4.397 & 616.1 & 4.32 & 608.6 & 3.95 & 583.5 & 3.672 & 609 & 616 & 609.9 & 3.329 & 586.1 \\
\hline 3.796 & 548.2 & 3.696 & 556.6 & 4.412 & 580.8 & 4.009 & 579.1 & 4.228 & 582.6 & 3.663 & 552.6 & 3.497 & 581.3 & 3.518 & 582.6 & 3.068 & 552.6 \\
\hline 3.64 & 524 & 3.521 & 527.5 & 4.255 & 555.7 & 4.033 & 558.3 & 4.03 & 557.5 & 3.53 & 526.6 & 3.376 & 553.5 & 3.367 & 558.8 & 2.935 & 524.9 \\
\hline 3.355 & 489.2 & 3.403 & 504.6 & 3.921 & 523.5 & 3.687 & 524.9 & 3.817 & 530.1 & 3.332 & 499.3 & 3.267 & 530.1 & 3.086 & 527.9 & 2.775 & 500.6 \\
\hline 3.196 & 461.4 & 3.281 & 478.6 & 3.82 & 496.6 & 3.53 & 498.9 & 3.56 & 505 & 3.237 & 475.9 & 3.092 & 505 & 2.965 & 500.6 & 2.627 & 476.4 \\
\hline 3.03 & 439.4 & 3.006 & 445.1 & 3.53 & 466.7 & 3.37 & 476.8 & 3.258 & 469.8 & 2.956 & 443.8 & 3.003 & 492.7 & 2.761 & 472.9 & 2.376 & 443.3 \\
\hline 2.873 & 407.6 & 2.888 & 418.2 & 3.352 & 442.4 & 3.278 & 450.4 & 3.119 & 445.1 & 2.802 & 418.2 & 2.728 & 449.5 & 2.66 & 446.9 & 2.269 & 415.1 \\
\hline 2.734 & 382.5 & 2.663 & 391.3 & 3.181 & 417.3 & 3.006 & 424.4 & 2.929 & 416.4 & 2.616 & 391.3 & 2.394 & 432.3 & 2.488 & 421.7 & 2.074 & 390.4 \\
\hline 2.536 & 358.3 & 2.542 & 368 & 2.956 & 392.2 & 2.746 & 389.1 & 2.734 & 390.4 & 2.444 & 365.8 & 2.414 & 406.3 & 2.228 & 390 & 1.935 & 365.3 \\
\hline 2.246 & 326.5 & 2.275 & 335.8 & 2.69 & 359.6 & 2.53 & 360.9 & 2.542 & 365.3 & 2.257 & 339.8 & 2.107 & 366.2 & 2.044 & 365.8 & 1.731 & 334.5 \\
\hline 2.118 & 299.2 & 2.16 & 312 & 2.5 & 334.5 & 2.411 & 334.5 & 2.373 & 340.2 & 2.024 & 306.7 & 2.183 & 361.4 & 2.003 & 337.6 & 1.532 & 308 \\
\hline 1.938 & 273.7 & 2 & 282.5 & 2.326 & 307.6 & 2.24 & 311.6 & 2.166 & 312 & 1.849 & 279.8 & 1.772 & 312 & 1.834 & 313.3 & 1.435 & 281.6 \\
\hline 1.834 & 247.2 & 1.731 & 252.5 & 2.133 & 281.2 & 2.018 & 279 & 1.917 & 278.5 & 1.743 & 256 & 1.689 & 280.7 & 1.592 & 279.8 & 1.201 & 249.4 \\
\hline
\end{tabular}


Table 10. $10{ }^{\circ} \mathrm{C}$ at 13 wt $\%$

\begin{tabular}{|c|c|c|c|c|c|c|c|c|c|c|c|c|c|c|c|c|c|}
\hline run1 & & run2 & & run3 & & run4 & & run5 & & run6 & & run7 & & run8 & & run9 & \\
\hline [Pa] & {$[1 / s]$} & [Pa] & {$[1 / s]$} & [Pa] & {$[1 / s]$} & [Pa] & {$[1 / s]$} & [Pa] & {$[1 / s]$} & [Pa] & [1/s] & [Pa] & {$[1 / \mathrm{s}]$} & [Pa] & {$[1 / \mathrm{s}]$} & [Pa] & {$[1 / \mathrm{s}]$} \\
\hline 16.53 & 272.3 & 15.95 & 272.8 & 16.63 & 276.3 & 16.64 & 271.9 & 16.67 & 276.3 & 16.83 & 276.7 & 16.32 & 273.2 & 17.12 & 279.8 & 17.29 & 271.9 \\
\hline 17.7 & 295.3 & 17.39 & 295.7 & 17.51 & 296.1 & 17.47 & 292.6 & 17.42 & 298.8 & 17.73 & 291.7 & 16.8 & 270.6 & 18.01 & 294.8 & 18.01 & 296.1 \\
\hline 18.4 & 320.4 & 18.1 & 319.9 & 18.25 & 319.5 & 18.43 & 325.2 & 18.37 & 322.6 & 18.43 & 319.9 & 17.92 & 291.7 & 18.9 & 318.6 & 18.75 & 321.3 \\
\hline 19.2 & 344.6 & 18.66 & 346.8 & 19.31 & 352.1 & 19.58 & 352.5 & 19.2 & 345.1 & 19.14 & 345.9 & 19.64 & 344.6 & 19.97 & 351.7 & 19.46 & 344.6 \\
\hline 20.62 & 375 & 20.11 & 377.2 & 20.26 & 375.9 & 20.11 & 377.7 & 20.26 & 378.5 & 20.56 & 375.9 & 20.71 & 373.7 & 20.91 & 379 & 20.85 & 379 \\
\hline 21.27 & 401 & 20.73 & 405 & 20.94 & 405 & 21.3 & 404.5 & 21.47 & 405.9 & 21.12 & 403.2 & 20.97 & 389.1 & 21.5 & 403.2 & 21.47 & 401.9 \\
\hline 22.42 & 425.3 & 21.86 & 428.8 & 21.95 & 430.1 & 21.98 & 427.9 & 22.21 & 433.2 & 22.21 & 427.9 & 21.74 & 411.2 & 22.51 & 433.2 & 22.54 & 429.2 \\
\hline 23.66 & 457.4 & 22.36 & 453.5 & 22.81 & 455.7 & 22.95 & 453.5 & 23.1 & 460.1 & 23.34 & 460.1 & 23.28 & 450.4 & 23.4 & 455.7 & 23.46 & 456.1 \\
\hline 24.43 & 483.9 & 23.69 & 485.2 & 23.46 & 481.2 & 23.72 & 480.3 & 24.14 & 489.2 & 24.02 & 482.5 & 24.4 & 487.8 & 24.43 & 486.1 & 24.23 & 482.5 \\
\hline 25.2 & 508.5 & 24.61 & 513.8 & 24.79 & 513.4 & 24.97 & 513.8 & 25.03 & 514.7 & 24.85 & 510.8 & 25.2 & 513.4 & 25.23 & 512.5 & 25.5 & 514.3 \\
\hline 26.57 & 539.4 & & 541.2 & & 541.6 & 25.8 & & 25.8 & & & & 26.06 & & 26 & 536.8 & 26.36 & 542.5 \\
\hline 27.51 & 567.2 & 26.48 & 567.6 & 26.42 & 567.2 & 26.57 & 564.1 & 26.71 & 568.5 & 26.86 & 567.6 & 27.19 & 569.8 & 27.19 & 568.5 & 27.22 & 568.5 \\
\hline 28.28 & 592.3 & 27.19 & 591 & 27.16 & 590.5 & 27.36 & 591.4 & 27.39 & 592.7 & 27.54 & 593.6 & 28.07 & 597.6 & 27.99 & 593.6 & 27.99 & 594.9 \\
\hline 29.29 & 622.7 & 28.1 & 622.2 & 28.07 & 621.8 & 28.55 & 624.9 & 28.28 & 620 & 28.28 & 618.7 & 28.7 & 622.2 & 28.67 & 621.4 & 28.78 & 619.6 \\
\hline 30.23 & 649.6 & 28.99 & 648.7 & 29.08 & 649.6 & 29.17 & 648.2 & 29.08 & 643.8 & 29.26 & 646 & 29.47 & 646 & 29.73 & 652.7 & 29.64 & 645.2 \\
\hline 30.97 & 675.6 & 29.79 & 675.1 & 29.67 & 673.8 & & 676 & & 679.1 & & 670.7 & 30.56 & 678.7 & 30.59 & 679.1 & 30.71 & 679.5 \\
\hline 31.86 & 700.7 & 30.74 & 702 & 30.71 & 705.5 & 31.03 & 706.4 & 30.83 & 704.2 & 30.89 & 702 & 31.39 & 704.7 & 31.3 & 704.7 & 31.42 & 706.4 \\
\hline 32.72 & 728.4 & & 733.7 & & 732.9 & & & .57 & 729.3 & 74 & 730.2 & 32.28 & 732.4 & 32.1 & 729.8 & 32.19 & 729.3 \\
\hline 33.52 & 756.2 & 32.51 & 761.1 & 32.37 & 758 & 32.48 & 759.3 & 32.37 & 756.2 & 32.54 & 755.8 & 32.78 & 755.3 & 32.9 & 757.1 & 32.9 & 755.3 \\
\hline 34.35 & 783.1 & 33.13 & 783.1 & 32.99 & 782.7 & 33.19 & 783.5 & 33.34 & 787.9 & 33.43 & 787.1 & 33.87 & 788.8 & 33.79 & 787.9 & 33.85 & 787.1 \\
\hline 35.12 & 807.8 & 34.11 & 814.4 & 33.76 & 809.1 & 33.96 & 807.8 & 34.17 & 815.3 & 34.2 & 810.4 & 34.64 & 816.6 & 34.5 & 812.6 & 34.59 & 813.5 \\
\hline 36.01 & 838.2 & 34.88 & 838.2 & 34.5 & 835.5 & 35 & 841.7 & 34.76 & 838.6 & 34.91 & 837.3 & 35.38 & 841.3 & 35.21 & 839.9 & 35.38 & 840.8 \\
\hline 36.75 & 864.6 & 35.71 & 865.5 & 35.65 & 869.5 & 35.77 & 869.5 & 35.5 & 862.9 & 35.47 & 858.5 & 36.15 & 866.4 & 36.24 & 869.9 & 36.24 & 868.6 \\
\hline 37.43 & 888 & 36.54 & 896.8 & 36.36 & 896.8 & 36.48 & 896.3 & 36.48 & 895.5 & 36.66 & 894.1 & 36.8 & 892.4 & 36.89 & 894.1 & 36.98 & 892.4 \\
\hline 38.46 & 921.5 & 37.25 & 917.9 & 36.98 & 920.1 & 37.19 & 922.3 & 37.25 & 923.2 & 37.37 & 920.1 & 37.93 & 925.9 & 37.69 & 922.3 & 37.69 & 917.5 \\
\hline 39.23 & 946.6 & 38.02 & 946.6 & 37.99 & 952.3 & 37.9 & 945.3 & 37.93 & 947 & 38.11 & 946.1 & 38.61 & 951.9 & 38.55 & 952.8 & 38.61 & 949.7 \\
\hline 39.85 & 970.8 & 38.73 & 973 & 38.79 & 979.2 & & 978.3 & 38.73 & 973.5 & & & & 979.6 & 39.23 & 974.3 & 39.26 & 972.6 \\
\hline 40.89 & 1002 & 39.59 & 999.5 & 39.56 & 1005 & 39.53 & 1003 & 39.65 & 1006 & 39.56 & 999.5 & 40.06 & 1004 & 39.2 & 985.8 & 39.97 & 998.1 \\
\hline 41.57 & 1029 & 40.53 & 1032 & 40.33 & 1033 & 40.39 & 1033 & 40.42 & 1035 & 40.5 & 1031 & 40.95 & 1034 & 40.03 & 1002 & 40.8 & 1027 \\
\hline 42.28 & 1055 & 41.36 & 1060 & 41.04 & 1058 & 41.07 & 1059 & 41.18 & 1061 & 41.16 & 1057 & 41.66 & 1059 & 40.47 & 1029 & 41.81 & 1060 \\
\hline 42.96 & 1079 & 42.07 & 1087 & 41.78 & 1086 & 41.84 & 1085 & 41.84 & 1086 & 41.84 & 1082 & 42.4 & 1085 & 41.27 & 1046 & 42.4 & 1085 \\
\hline
\end{tabular}


Table $10 . \quad 10{ }^{\circ} \mathrm{C}$ at 13 wt $\%$ - continued

\begin{tabular}{|c|c|c|c|c|c|c|c|c|c|c|c|c|c|c|c|c|c|}
\hline run1 & & run2 & & run3 & & run4 & & run5 & & run6 & & run7 & & run8 & & run9 & \\
\hline [Pa] & [1/s] & [Pa] & {$[1 / s]$} & [Pa] & {$[1 / s]$} & [Pa] & {$[1 / \mathrm{s}]$} & [Pa] & {$[1 / s]$} & [Pa] & {$[1 / s]$} & [Pa] & {$[1 / s]$} & [Pa] & {$[1 / s]$} & [Pa] & {$[1 / s]$} \\
\hline 43.67 & 1104 & 42.75 & 1112 & 42.46 & 1111 & 42.61 & 1110 & 42.49 & 1111 & 42.46 & 1106 & 42.99 & 1110 & 42.99 & 1117 & 43.23 & 1114 \\
\hline 44.59 & 1138 & 43.46 & 1138 & 43.4 & 1144 & 43.55 & 1142 & 43.23 & 1137 & 43.55 & 1141 & 43.61 & 1136 & 43.43 & 1126 & 43.97 & 1142 \\
\hline 45.33 & 1167 & 44.14 & 1164 & 44 & 1170 & 44.32 & 1170 & 44.2 & 1170 & 44.2 & 1166 & 44.29 & 1164 & 44.47 & 1165 & 44.8 & 1168 \\
\hline 45.98 & 1191 & 44.85 & 1189 & 44.62 & 1192 & 44.97 & 1196 & 44.82 & 1197 & 44.8 & 1191 & 45.09 & 1191 & 45.27 & 1196 & 45.48 & 1193 \\
\hline 46.96 & 1223 & 45.89 & 1222 & 45.24 & 1216 & 45.65 & 1222 & 45.42 & 1221 & 45.56 & 1220 & 46.04 & 1226 & 45.92 & 1220 & 46.25 & 1223 \\
\hline 47.61 & 1247 & 46.48 & 1247 & 46.3 & 1251 & 46.33 & 1249 & 46.45 & 1252 & 46.3 & 1248 & 46.57 & 1249 & 46.57 & 1244 & 46.87 & 1249 \\
\hline 48.26 & 1272 & 47.37 & 1278 & 46.99 & 1280 & 46.99 & 1273 & 47.1 & 1279 & 47.07 & 1274 & 47.22 & 1274 & 47.52 & 1278 & 47.61 & 1274 \\
\hline 48.97 & 1299 & 48.08 & 1307 & 47.61 & 1304 & 47.81 & 1300 & 47.73 & 1305 & 47.7 & 1298 & 48.2 & 1306 & 48.2 & 1304 & 48.2 & 1298 \\
\hline 49.83 & 1330 & 48.76 & 1329 & 48.32 & 1331 & 48.49 & 1327 & 48.44 & 1330 & 48.58 & 1330 & 48.85 & 1333 & 48.88 & 1330 & 49.09 & 1330 \\
\hline 50.51 & 1354 & 49.65 & 1359 & 49.2 & 1361 & 49.41 & 1359 & 49.38 & 1363 & 49.32 & 1358 & 49.62 & 1360 & 49.53 & 1357 & 49.8 & 1358 \\
\hline 51.22 & 1384 & 50.27 & 1386 & 49.71 & 1380 & 49.97 & 1385 & 49.77 & 1380 & 49.91 & 1382 & 50.24 & 1385 & 50.27 & 1382 & 50.39 & 1382 \\
\hline 51.96 & 1409 & 51.04 & 1415 & 50.39 & 1407 & 50.74 & 1412 & 50.74 & 1415 & 50.74 & 1412 & 50.86 & 1410 & 50.89 & 1407 & 51.16 & 1413 \\
\hline 52.82 & 1441 & 51.66 & 1436 & 51.1 & 1435 & 51.37 & 1438 & 51.45 & 1441 & 51.16 & 1430 & 51.57 & 1435 & 51.72 & 1440 & 51.84 & 1441 \\
\hline 53.44 & 1465 & 52.58 & 1470 & 52.02 & 1469 & 52.37 & 1471 & 52.13 & 1469 & 52.13 & 1465 & 52.49 & 1471 & 52.4 & 1467 & 52.52 & 1465 \\
\hline 54.21 & 1494 & 53.26 & 1494 & 52.7 & 1495 & 52.96 & 1497 & 52.67 & 1493 & 52.87 & 1494 & 53.17 & 1496 & 52.96 & 1490 & 53.32 & 1492 \\
\hline 54.89 & 1520 & 53.94 & 1519 & 53.47 & 1523 & 53.58 & 1521 & 53.35 & 1519 & 53.53 & 1519 & 53.82 & 1524 & 53.91 & 1524 & 54 & 1519 \\
\hline 51.37 & 1539 & 51.31 & 1542 & 50.57 & 1516 & 50.51 & 1510 & 51.25 & 1536 & 51.45 & 1538 & 51.39 & 1533 & 51.66 & 1542 & 50.92 & 1509 \\
\hline 50.63 & 1514 & 50.42 & 1511 & 49.71 & 1482 & 49.89 & 1487 & 50.6 & 1511 & 50.74 & 1508 & 50.86 & 1510 & 50.65 & 1510 & 50.3 & 1486 \\
\hline 49.71 & 1483 & 49.77 & 1482 & 49.03 & 1455 & 49.03 & 1455 & 50 & 1484 & 50.15 & 1486 & 50.15 & 1481 & 50 & 1481 & 49.44 & 1453 \\
\hline 49.09 & 1458 & 49.09 & 1457 & 48.49 & 1434 & 48.35 & 1430 & 49.35 & 1461 & 49.35 & 1456 & 49.47 & 1456 & 49.35 & 1455 & 48.76 & 1426 \\
\hline 48.38 & 1432 & 48.38 & 1431 & 47.55 & 1404 & 47.7 & 1400 & 48.32 & 1425 & 48.64 & 1427 & 48.76 & 1430 & 48.67 & 1430 & 48.02 & 1400 \\
\hline 47.49 & 1400 & 47.37 & 1399 & 46.87 & 1378 & 46.93 & 1374 & 48.05 & 1410 & 48.02 & 1405 & 47.9 & 1398 & 48.02 & 1404 & 47.28 & 1371 \\
\hline 46.84 & 1375 & 46.78 & 1375 & 46.04 & 1346 & 46.3 & 1348 & 46.96 & 1372 & 47.19 & 1375 & 47.19 & 1373 & 47.34 & 1379 & 46.72 & 1348 \\
\hline 46.04 & 1347 & 46.13 & 1348 & 45.39 & 1319 & 45.56 & 1322 & 46.33 & 1348 & 46.54 & 1349 & 46.54 & 1344 & 46.39 & 1345 & 45.83 & 1316 \\
\hline 45.51 & 1325 & 45.39 & 1322 & 44.77 & 1297 & 44.88 & 1298 & 45.62 & 1319 & 45.86 & 1323 & 45.92 & 1319 & 45.77 & 1319 & 45.15 & 1292 \\
\hline 44.59 & 1292 & 44.47 & 1290 & 43.91 & 1266 & 44.23 & 1272 & 44.88 & 1293 & 44.97 & 1291 & 45.12 & 1294 & 45 & 1291 & 44.35 & 1261 \\
\hline 43.85 & 1266 & 43.91 & 1268 & 43.23 & 1238 & 43.26 & 1236 & 44.32 & 1270 & 44.23 & 1265 & 44.44 & 1267 & 44.35 & 1265 & 43.7 & 1237 \\
\hline 43.11 & 1238 & 43.14 & 1241 & 42.55 & 1215 & 42.58 & 1210 & 43.61 & 1243 & 43.52 & 1237 & 43.55 & 1236 & 43.61 & 1240 & 43.05 & 1211 \\
\hline 42.4 & 1212 & 42.4 & 1211 & 41.72 & 1183 & 41.87 & 1184 & 42.72 & 1210 & 42.9 & 1212 & 42.84 & 1210 & 43.05 & 1215 & 42.25 & 1179 \\
\hline 41.66 & 1186 & 41.78 & 1187 & 40.95 & 1154 & 41.1 & 1155 & 41.98 & 1185 & 42.19 & 1186 & 42.19 & 1182 & 42.04 & 1182 & 41.57 & 1156 \\
\hline 40.95 & 1161 & 41.01 & 1161 & 40.3 & 1131 & 40.47 & 1131 & 41.24 & 1155 & 41.3 & 1155 & 41.48 & 1158 & 41.39 & 1155 & 40.86 & 1129 \\
\hline
\end{tabular}


Table $10 . \quad 10{ }^{\circ} \mathrm{C}$ at 13 wt $\%$ - continued

\begin{tabular}{|c|c|c|c|c|c|c|c|c|c|c|c|c|c|c|c|c|c|}
\hline run1 & & run2 & & run3 & & run4 & & run5 & & run6 & & run7 & & run8 & & run9 & \\
\hline [Pa] & {$[1 / s]$} & [Pa] & {$[1 / \mathrm{s}]$} & [Pa] & [1/s] & [Pa] & {$[1 / s]$} & [Pa] & {$[1 / \mathrm{s}]$} & [Pa] & {$[1 / \mathrm{s}]$} & [Pa] & {$[1 / s]$} & [Pa] & {$[1 / \mathrm{s}]$} & [Pa] & {$[1 / s]$} \\
\hline 40 & 1129 & 40.15 & 1130 & 39.44 & 1099 & 39.76 & 1106 & 40.56 & 1131 & 40.53 & 1129 & 40.62 & 1128 & 40.8 & 1132 & 40 & 1100 \\
\hline 39.41 & 1104 & 9.41 & 1103 & 38.64 & 1073 & 39.08 & 1081 & 39.7 & 1100 & 39.88 & 1101 & 39.91 & 1100 & 40.15 & 1107 & 39.26 & 1074 \\
\hline 38.76 & 1080 & 38.85 & 1081 & 37.9 & 1046 & 8.28 & 1055 & 39.08 & 1077 & 39.29 & 1079 & 39.17 & 1072 & 39.11 & 1074 & 38.64 & 1047 \\
\hline 37.75 & 1047 & 37.84 & 1046 & 37.31 & 1020 & 38.11 & 1047 & 38.34 & 1050 & 38.4 & 1046 & 38.49 & 1047 & 38.43 & 1048 & 38.02 & 1023 \\
\hline 37.01 & 1019 & 37.16 & 1020 & 36.57 & 993.7 & 36.75 & 999 & 37.49 & 1019 & 37.69 & 1019 & 37.69 & 1018 & 37.72 & 1022 & 37.07 & 990.2 \\
\hline 36.48 & 999.5 & 36.45 & 996.4 & 35.65 & 962.5 & 36.04 & 974.8 & 36.83 & 993.7 & 36.95 & 995.5 & 36.98 & 993.7 & 36.86 & 989.3 & 36.27 & 962 \\
\hline 35.47 & 964.7 & 35.56 & 966 & 34.97 & 938.7 & 35.5 & 954.1 & 36.06 & 966 & 36.01 & 963.3 & 36.24 & 967.3 & 36.18 & 965.1 & 35.65 & 938.7 \\
\hline 34.76 & 936.9 & 34.91 & 939.5 & 34.23 & 909.6 & 34.44 & 918.8 & 35.35 & 941.7 & 35.41 & 940 & 35.38 & 933.8 & 35.41 & 935.6 & 34.76 & 905.6 \\
\hline 34.05 & 912.7 & 34.11 & 912.2 & 33.43 & 883.1 & 33.46 & 884.5 & 34.38 & 908.2 & 34.56 & 911.3 & 34.7 & 910 & 34.79 & 913.1 & 34.05 & 880.5 \\
\hline 33.08 & 881.4 & & 885.3 & & 857.6 & & 856.7 & 33.73 & 880.5 & 33.9 & 883.6 & 33.79 & 881.8 & & 884 & & 854 \\
\hline 32.4 & 854.9 & 32.75 & 859.8 & 31.8 & 825.8 & 31.8 & 821.9 & 33.05 & 857.1 & 33.28 & 859.3 & 33.19 & 852.7 & 33.13 & 854.5 & 32.51 & 828 \\
\hline 31.63 & 827.6 & 31.83 & 828.9 & 31.15 & 801.2 & 31.36 & 807.3 & 32.31 & 832.4 & 32.45 & 834.2 & 32.48 & 830.2 & 32.42 & 827.6 & 31.66 & 795.9 \\
\hline 30.89 & 801.6 & 31 & 803.8 & 30.41 & 773.8 & 30.35 & 772.5 & 31.6 & 807.3 & 31.48 & 800.7 & 31.71 & 802.9 & 31.71 & 801.6 & 30.92 & 771.6 \\
\hline 30.15 & 774.7 & 30.23 & 773.4 & 29.58 & 749.2 & 29.7 & 747 & 30.53 & 773.8 & 30.8 & 776 & 30.77 & 772.1 & 31.06 & 776.5 & 30.15 & 744.3 \\
\hline 29.32 & 749.6 & 29.44 & 749.6 & 28.76 & 718.3 & 28.99 & 721 & 29.85 & 748.3 & 29.97 & 748.3 & 29.97 & 743 & 30.18 & 749.6 & 29.52 & 720.5 \\
\hline 28.37 & 718.3 & 28.64 & 721.8 & 27.84 & 692.3 & 28.02 & 689.2 & 29.11 & 721.8 & 29.11 & 719.6 & 29.29 & 720.1 & 29.49 & 724.5 & 28.4 & 687.9 \\
\hline 27.54 & 691 & & 696.3 & & 666.8 & & 663.2 & 28.25 & 694.1 & & 695.4 & & 690.1 & & 691 & 27.72 & 663.2 \\
\hline 26.89 & 669.4 & 26.98 & 664.5 & 26.3 & 637.7 & 26.45 & 637.7 & 27.3 & 662.8 & 27.78 & 670.3 & 27.69 & 663.2 & 27.72 & 663.7 & 26.89 & 634.6 \\
\hline 25.85 & 637.2 & 26.27 & 638.1 & 25.68 & 611.2 & 25.62 & 609 & 26.71 & 639.9 & 26.8 & 639.4 & 26.71 & 634.6 & 26.89 & 639 & .09 & 609.5 \\
\hline 25.23 & 609 & 25.53 & 615.2 & 24.91 & 584.8 & 24.73 & 581.3 & 25.83 & 609.5 & 25.91 & 611.2 & 26.06 & 608.1 & 26.09 & 613.4 & .47 & 581.7 \\
\hline 24.58 & 584.3 & 24.61 & 583 & 23.78 & 553.1 & 23.93 & 556.1 & 25.03 & 585.2 & 25.2 & 587.4 & 25.38 & 584.8 & 25.41 & 588.8 & 24.64 & 556.1 \\
\hline 23.75 & 561.4 & 23.87 & 556.6 & 23.43 & 535.9 & 23.19 & 530.1 & 24.23 & 559.2 & 24.35 & 555.7 & 24.26 & 553.5 & 24.76 & 561.9 & 23.72 & 526.6 \\
\hline 22.92 & 534.5 & 23.22 & 533.7 & 22.04 & 500.2 & 22.27 & 498.4 & 23.4 & 527.1 & 23.58 & 527.9 & 23.43 & 525.7 & 23.87 & 534.1 & 22.98 & 499.7 \\
\hline 21.95 & 502.4 & 22.39 & 506.8 & 21.59 & 475.5 & 21.33 & 471.1 & 22.54 & 502.4 & 22.87 & 505.5 & 22.92 & 502.4 & 22.72 & 501.5 & 22.04 & 476.8 \\
\hline 21.15 & 473.7 & 21.36 & 473.3 & 20.59 & 445.5 & 20.76 & 446.9 & 21.65 & 473.7 & 21.8 & 472.9 & 21.8 & 470.2 & 21.77 & 471.5 & 21 & 444.2 \\
\hline 20.2 & 449.9 & 20.53 & 446.9 & 19.7 & 418.2 & 19.91 & 418.2 & 21 & 450.8 & 20.97 & 445.1 & 21.12 & 444.2 & 21.15 & 449.1 & 20.35 & 417.8 \\
\hline 19.11 & 419.1 & 19.46 & 418.6 & 18.93 & 392.2 & 19.2 & 394.4 & 20.08 & 419.1 & 20.29 & 421.7 & 20.05 & 416.4 & 20.2 & 417.8 & 19.46 & 393.1 \\
\hline 18.6 & 393.5 & 18.99 & 395.3 & 18.04 & 369.3 & 18.19 & 361.8 & 19.14 & 391.3 & 19.31 & 391.3 & 19.52 & 392.6 & 19.37 & 390.9 & 18.93 & 368 \\
\hline 17.48 & 364.9 & 18.04 & 368.9 & 17.24 & 335.8 & 17.48 & 337.6 & 18.49 & 365.8 & 18.37 & 364.4 & 18.52 & 366.6 & 18.4 & 365.8 & 17.51 & 334 \\
\hline 16.74 & 339.8 & 17.09 & 337.1 & 16.5 & 312 & 16.59 & 311.1 & 17.86 & 354.7 & 17.51 & 341.5 & 17.69 & 340.6 & 17.69 & 342.9 & 16.83 & 308 \\
\hline 16 & 308.5 & 16.41 & 312 & 15.53 & 284.2 & 15.67 & 285.1 & 17.69 & 344.6 & 16.77 & 308 & 16.83 & 307.6 & 16.89 & 309.8 & 16.18 & 282.5 \\
\hline 15.29 & 284.7 & 15.53 & 282.9 & 14.82 & 259.6 & 14.99 & 260 & 17.12 & 326.1 & 16.03 & 284.2 & 16 & 279 & 16.06 & 282.5 & 15.29 & 255.6 \\
\hline
\end{tabular}


Table 11. $15{ }^{\circ} \mathrm{C}$ at 13 wt $\%$

\begin{tabular}{|c|c|c|c|c|c|c|c|c|c|c|c|c|c|c|c|c|c|}
\hline run1 & & run2 & & run3 & & run4 & & run5 & & run6 & & run7 & & run8 & & run9 & \\
\hline [Pa] & {$[1 / s]$} & [Pa] & {$[1 / s]$} & [Pa] & {$[1 / s]$} & [Pa] & {$[1 / s]$} & [Pa] & {$[1 / s]$} & [Pa] & [1/s] & [Pa] & {$[1 / s]$} & [Pa] & {$[1 / \mathrm{s}]$} & [Pa] & {$[1 / \mathrm{s}]$} \\
\hline 12.92 & 275 & 12.44 & 301.4 & 12.95 & 276.7 & 14.66 & 301.9 & 14.4 & 280.7 & 14.5 & 274.5 & 14.11 & 276.7 & 14.75 & 278.5 & 14.9 & 273.2 \\
\hline 13.82 & 291.7 & 13.79 & 300.5 & 13.9 & 290.4 & 5.35 & 327.9 & 15.06 & 300.1 & 15.17 & 293.5 & 14.93 & 287.3 & 15.51 & 293.1 & 15.87 & 295.7 \\
\hline 14.65 & 322.6 & 14.56 & 320.4 & 14.78 & 315.1 & 16.72 & 362.7 & 15.61 & 322.6 & 15.85 & 319.9 & 16.13 & 316.9 & 16.11 & 318.6 & 16.56 & 319.1 \\
\hline 15.71 & 348.6 & 15.33 & 345.1 & 15.55 & 347.3 & 7.28 & 387.4 & 16.41 & 349.5 & 16.79 & 344.6 & 16.98 & 344.6 & 16.83 & 342 & 17.17 & 345.1 \\
\hline 16.56 & 371.1 & 15.72 & 371.5 & 16.37 & 372.8 & 18.22 & 415.1 & 17.31 & 373.7 & 17.7 & 370.6 & 17.48 & 370.2 & 18.1 & 374.1 & 17.7 & 370.2 \\
\hline 17.68 & 405 & 17.01 & 405.4 & 17.16 & 397.9 & 18.87 & 442 & 18.26 & 407.6 & 18.66 & 403.7 & 18.52 & 397.9 & 18.69 & 401.5 & 18.75 & 404.1 \\
\hline 18.38 & 429.7 & 17.88 & 433.6 & 18.21 & 430.1 & 19.37 & 468 & 18.75 & 435.8 & 19.25 & 428.3 & 18.96 & 423.5 & 19.46 & 427.5 & 19.7 & 428.8 \\
\hline 19.37 & 455.2 & 18.28 & 457 & 18.57 & 453.5 & 20.47 & 501.1 & 19.7 & 458.8 & 20.2 & 455.2 & 19.85 & 454.3 & 20.26 & 452.1 & 20.41 & 457.9 \\
\hline 20.14 & 480.8 & 19.23 & 479.9 & 19.34 & 481.7 & 21.03 & 524.9 & 20.62 & 490.9 & 20.68 & 479 & 20.73 & 479.9 & 21.15 & 485.2 & 21.03 & 481.2 \\
\hline 20.85 & 506.3 & 19.99 & 514.3 & 20.17 & 506.3 & 21.77 & 550 & 21.21 & 513.8 & 21.89 & 512.5 & 21.36 & 504.1 & 21.89 & 510.8 & 21.83 & 512.5 \\
\hline 22.27 & 540.3 & & 540.7 & & 532.8 & & 578.2 & & & & & & & & 536.8 & & 538.5 \\
\hline 22.92 & 561.9 & 21.44 & 566.3 & 21.62 & 559.7 & 23.37 & 604.2 & 22.72 & 569.4 & 23.37 & 563.2 & 22.98 & 563.2 & 23.22 & 561.9 & 23.46 & 566.7 \\
\hline 23.78 & 587.9 & 22.13 & 591.4 & 22.63 & 591 & 24.23 & 635.5 & 23.4 & 594.5 & 24.26 & 594 & 23.75 & 590.5 & 23.99 & 588.3 & 24.17 & 591 \\
\hline 24.82 & 620 & 23.07 & 623.6 & 23.31 & 618.3 & 24.97 & 661 & 24.02 & 619.2 & 25 & 621.4 & 24.52 & 616.1 & 24.61 & 614.8 & 24.91 & 615.6 \\
\hline 25.65 & 647.8 & 23.84 & 650 & 23.96 & 641.6 & 25.65 & 689.2 & 24.88 & 646.5 & 25.35 & 643.8 & 25.17 & 645.2 & 25.23 & 639.4 & 25.85 & 648.2 \\
\hline 26.48 & 673.8 & 24.61 & 679.1 & 24.46 & 666.3 & 26.27 & 713 & 25.47 & 673.8 & 25.82 & 669.4 & 25.8 & 668.1 & 26.42 & 676 & 26.51 & 678.2 \\
\hline 27.25 & 697.6 & 25.38 & 705.5 & 25.59 & 698.9 & 26.83 & 736.4 & 26.51 & 708.6 & 26.51 & 695.4 & 26.71 & 699.4 & 26.92 & 700.7 & 27.25 & 702.4 \\
\hline 28.22 & 731.5 & 26.06 & 729.8 & & 725.8 & & 772.1 & & 732.9 & 27.19 & 72 & 27.48 & 728.9 & 27.75 & 726.2 & 27.96 & 728.9 \\
\hline 29.08 & 758.9 & 26.62 & 754.4 & 26.89 & 750.5 & 28.52 & 798.1 & 27.87 & 761.9 & 28.22 & 756.7 & 28.1 & 754 & 28.49 & 754.4 & 28.49 & 753.6 \\
\hline 29.88 & 782.7 & 27.36 & 780.4 & 27.78 & 783.5 & 29.29 & 826.7 & 28.58 & 786.2 & 28.9 & 783.5 & 28.84 & 779.6 & 28.99 & 776.9 & 29.46 & 785.3 \\
\hline 30.74 & 813.5 & 28.01 & 808.2 & 28.46 & 807.8 & 29.94 & 849.2 & 29.32 & 816.1 & 29.52 & 807.3 & 29.55 & 806 & 30 & 810.4 & 30.17 & 811.3 \\
\hline 31.24 & 829.4 & 29.02 & 839.5 & 29.29 & 838.2 & 30.53 & 874.3 & 30 & 842.6 & 30.29 & 834.7 & 30.2 & 831.6 & 30.62 & 836.9 & 30.8 & 837.3 \\
\hline 32.04 & 858.9 & 29.67 & 869 & 29.85 & 861.1 & 31.48 & 909.1 & 30.8 & 869.9 & 30.97 & 860.7 & 30.97 & 862.9 & 31.48 & 866.8 & 31.48 & 867.7 \\
\hline 33.08 & 894.1 & 30.35 & 893.7 & 30.2 & 873.9 & 32.1 & 936 & 31.42 & 897.7 & 31.71 & 894.1 & 31.63 & 891.9 & 32.07 & 892.4 & 32.22 & 892.8 \\
\hline 33.84 & 920.1 & 31 & 918.4 & 31.18 & 910.9 & 32.78 & 961.1 & 32.01 & 922.3 & 32.45 & 920.1 & 32.31 & 917.1 & 32.69 & 918.4 & 32.84 & 916.6 \\
\hline 34.55 & 945.7 & 31.83 & 950.1 & 31.92 & 940.4 & 33.4 & 985.4 & 32.78 & 947.5 & 33.16 & 946.6 & 33.05 & 942.6 & 33.46 & 949.2 & 33.67 & 948.8 \\
\hline 35.21 & 971.3 & 32.48 & 975.7 & 32.31 & 956.3 & 34.08 & 1011 & 33.55 & 979.6 & 33.82 & 973.9 & 33.58 & 967.3 & 34.11 & 973 & 34.23 & 975.7 \\
\hline 36.01 & 996.8 & 33.16 & 1005 & 33.43 & 994.6 & 34.7 & 1038 & 34.14 & 1003 & 34.58 & 998.6 & 34.61 & 1001 & 34.73 & 1000 & 34.82 & 1000 \\
\hline 36.66 & 1022 & 33.84 & 1029 & 33.46 & 1001 & 35.68 & 1072 & 34.85 & 1031 & 35.41 & 1024 & 35.09 & 1025 & 35.47 & 1030 & 35.62 & 1031 \\
\hline 37.69 & 1059 & 34.7 & 1061 & 34.88 & 1055 & 36.24 & 1098 & 35.41 & 1055 & 36.21 & 1056 & 35.71 & 1052 & 36.01 & 1054 & 36.27 & 1058 \\
\hline 38.43 & 1086 & 35.38 & 1088 & 35.53 & 1081 & 36.86 & 1124 & 36.12 & 1083 & 37.04 & 1084 & 36.33 & 1079 & 36.6 & 1078 & 36.86 & 1083 \\
\hline
\end{tabular}


Table 11. $15^{\circ} \mathrm{C}$ at 13 wt $\%$ - continued

\begin{tabular}{|c|c|c|c|c|c|c|c|c|c|c|c|c|c|c|c|c|c|}
\hline run1 & & run2 & & run3 & & run4 & & run5 & & run6 & & run7 & & run8 & & run9 & \\
\hline [Pa] & {$[1 / s]$} & {$[\mathrm{Pa}]$} & {$[1 / s]$} & [Pa] & {$[1 / s]$} & [Pa] & {$[1 / s]$} & [Pa] & {$[1 / s]$} & [Pa] & {$[1 / \mathrm{s}]$} & [Pa] & {$[1 / s]$} & [Pa] & [1/s] & [Pa] & {$[1 / s]$} \\
\hline 39.02 & 1110 & 35.92 & 1111 & 36.09 & 1108 & 37.57 & 1151 & 36.92 & 1116 & 38.22 & 1109 & 36.95 & 1104 & 37.51 & 1112 & 37.43 & 1107 \\
\hline 39.67 & 1134 & 36.6 & 1140 & 36.77 & 1132 & 38.34 & 1182 & 37.6 & 1144 & 39.67 & 1141 & 37.69 & 1131 & 38.17 & 1137 & 38.37 & 1140 \\
\hline 40.44 & 1169 & 37.46 & 1170 & 37.66 & 1166 & 38.93 & 1207 & 38.17 & 1166 & 40.47 & 1166 & 38.55 & 1165 & 38.82 & 1166 & 38.99 & 1166 \\
\hline 41.18 & 1196 & 38.05 & 1196 & 38.25 & 1191 & 39.53 & 1234 & 38.93 & 1200 & 40.98 & 1193 & 39.17 & 1191 & 39.44 & 1192 & 39.56 & 1192 \\
\hline 41.81 & 1222 & 38.67 & 1220 & 38.85 & 1218 & 40.09 & 1258 & 39.53 & 1224 & 41.98 & 1218 & 39.73 & 1214 & 39.97 & 1216 & 40.27 & 1221 \\
\hline 42.46 & 1246 & 39.44 & 1251 & 39.41 & 1241 & 40.77 & 1283 & 40.12 & 1248 & 42.52 & 1242 & 40.41 & 1245 & 40.59 & 1241 & 40.83 & 1247 \\
\hline 43.2 & 1275 & 40.15 & 1278 & 40.18 & 1275 & 41.6 & 1318 & 41.01 & 1281 & 43.67 & 1276 & 41.15 & 1272 & 41.27 & 1268 & 41.48 & 1271 \\
\hline 43.91 & 1302 & 40.74 & 1304 & 40.8 & 1299 & 42.19 & 1345 & 41.63 & 1307 & 44.71 & 1300 & 41.66 & 1296 & 42.13 & 1304 & 42.28 & 1304 \\
\hline 44.44 & 1326 & 41.33 & 1328 & 41.48 & 1326 & 42.78 & 1369 & 42.22 & 1334 & 48.05 & 1327 & 42.34 & 1322 & 42.49 & 1322 & 42.87 & 1332 \\
\hline 45.12 & 1351 & 42.16 & 1359 & 42.16 & 1353 & 43.67 & 1399 & 42.78 & 1361 & 47.69 & 1351 & 42.96 & 1348 & 43.14 & 1351 & 43.52 & 1357 \\
\hline 46.01 & 1388 & 42.81 & 1386 & 42.87 & 1383 & & 1424 & 43.49 & 1386 & 44.29 & 1383 & 43.58 & 1374 & 44.08 & 1382 & 44.14 & 1383 \\
\hline 46.6 & 1413 & 43.4 & 1410 & 43.52 & 1409 & 44.76 & 1452 & 44.29 & 1417 & 44.5 & 1408 & 44.44 & 1408 & 44.59 & 1408 & 44.74 & 1412 \\
\hline 47.19 & 1438 & 44.23 & 1442 & 44.03 & 1434 & 45.45 & 1476 & 44.85 & 1441 & 45.12 & 1438 & 45.06 & 1437 & 45.24 & 1434 & 45.42 & 1437 \\
\hline 47.84 & 1464 & 44.88 & 1469 & 44.68 & 1461 & 46.07 & 1502 & 45.53 & 1469 & 45.83 & 1467 & 45.71 & 1462 & 45.92 & 1467 & 46.13 & 1469 \\
\hline 48.46 & 1488 & 45.5 & 1491 & 45.3 & 1486 & 46.81 & 1534 & 46.1 & 1495 & 46.39 & 1493 & 46.33 & 1490 & 46.57 & 1492 & 46.51 & 1488 \\
\hline 49.32 & 1521 & 46.39 & 1523 & 46.1 & 1519 & 47.4 & 1559 & 46.87 & 1526 & 47.04 & 1520 & 46.95 & 1516 & 47.07 & 1516 & 47.37 & 1520 \\
\hline 44.38 & 1535 & 44.23 & 1537 & 43.85 & 1510 & 43.88 & 1490 & 45.03 & 1534 & 45.15 & 1535 & 45.33 & 1534 & 46.19 & 1558 & 45.59 & 1535 \\
\hline 43.55 & 1504 & 43.64 & 1510 & 43.26 & 1486 & & 1464 & 44.35 & 1508 & 44.47 & 1508 & 44.74 & 1508 & 44.97 & 1514 & 44.85 & 1506 \\
\hline 42.96 & 1481 & 42.99 & 1483 & 42.49 & 1453 & 42.34 & 1430 & 43.76 & 1482 & 44 & 1484 & 44.14 & 1481 & 44.91 & 1510 & 44.26 & 1478 \\
\hline 42.28 & 1454 & 42.22 & 1456 & 41.81 & 1425 & 41.66 & 1404 & 42.9 & 1450 & 43.2 & 1454 & 43.43 & 1455 & 43.97 & 1471 & 43.64 & 1453 \\
\hline 41.72 & 1430 & 41.51 & 1426 & 41.18 & 1399 & 41.04 & 1378 & 42.37 & 1423 & 42.57 & 1428 & 42.78 & 1426 & 43.4 & 1442 & 43.11 & 1430 \\
\hline 40.92 & 1397 & 40.92 & 1404 & 40.5 & 1372 & 40.24 & 1347 & 41.75 & 1399 & 41.98 & 1403 & 42.13 & 1397 & 42.43 & 1404 & 42.37 & 1399 \\
\hline 40.24 & 1368 & 40.33 & 1374 & 39.76 & 1342 & 39.62 & 1321 & 41.15 & 1374 & 41.18 & 1371 & 41.51 & 1373 & 41.78 & 1378 & 41.78 & 1374 \\
\hline 39.5 & 1345 & 39.56 & 1346 & 39.17 & 1316 & 39.02 & 1296 & 40.3 & 1343 & 40.62 & 1345 & 40.95 & 1347 & 41.18 & 1352 & 41.01 & 1342 \\
\hline 38.79 & 1317 & 38.88 & 1320 & 38.52 & 1292 & 38.46 & 1272 & 39.76 & 1318 & 40.06 & 1322 & 40.33 & 1321 & 40.5 & 1324 & 40.36 & 1318 \\
\hline 38.28 & 1295 & 38.25 & 1295 & 37.93 & 1264 & 37.72 & 1242 & 39.23 & 1292 & 39.14 & 1289 & 39.65 & 1294 & 39.88 & 1299 & 39.73 & 1291 \\
\hline 37.37 & 1259 & 37.57 & 1266 & 37.4 & 1241 & 36.98 & 1213 & 38.58 & 1268 & 38.61 & 1263 & 38.85 & 1262 & 39.2 & 1270 & 39.11 & 1262 \\
\hline 36.8 & 1234 & 36.86 & 1234 & 36.48 & 1207 & 36.3 & 1188 & 37.72 & 1233 & 37.99 & 1235 & 38.14 & 1235 & 38.31 & 1237 & 38.49 & 1236 \\
\hline 36.21 & 1211 & 36.18 & 1208 & 35.86 & 1182 & 35.8 & 1159 & 37.13 & 1207 & 37.37 & 1210 & 37.57 & 1209 & 37.78 & 1211 & 37.9 & 1210 \\
\hline 35.32 & 1178 & 35.62 & 1185 & 35.24 & 1153 & 35.15 & 1135 & 36.54 & 1182 & 36.8 & 1186 & 36.98 & 1185 & 37.22 & 1189 & 37.25 & 1185 \\
\hline 34.7 & 1153 & 35.09 & 1161 & 34.55 & 1127 & 34.32 & 1103 & 35.71 & 1153 & 35.92 & 1154 & 36.15 & 1153 & 36.39 & 1155 & 36.63 & 1159 \\
\hline
\end{tabular}


Table 11. $15^{\circ} \mathrm{C}$ at 13 wt $\%$ - continued

\begin{tabular}{|c|c|c|c|c|c|c|c|c|c|c|c|c|c|c|c|c|c|}
\hline run1 & & run2 & & run3 & & run4 & & run5 & & run6 & & run7 & & run8 & & run9 & \\
\hline [Pa] & {$[1 / \mathrm{s}]$} & [Pa] & {$[1 / \mathrm{s}]$} & [Pa] & {$[1 / \mathrm{s}]$} & [Pa] & {$[1 / s]$} & [Pa] & {$[1 / \mathrm{s}]$} & [Pa] & {$[1 / \mathrm{s}]$} & [Pa] & {$[1 / s]$} & [Pa] & {$[1 / \mathrm{s}]$} & [Pa] & {$[1 / s]$} \\
\hline 33.9 & 1122 & 34.5 & 1135 & 33.9 & 1099 & 33.67 & 1074 & 35.09 & 1124 & 35.38 & 1126 & 35.56 & 1126 & 35.8 & 1128 & 36.01 & 1133 \\
\hline 33.31 & 1100 & 33.49 & 1101 & 33.25 & 1074 & 33.05 & 1049 & 4.44 & 1096 & 34.7 & 1099 & 34.97 & 1102 & 35.15 & 1103 & 35.09 & 1098 \\
\hline 32.6 & 071 & 32.9 & 075 & 32.66 & 1048 & 32.31 & 1020 & 3.87 & 1072 & 34.05 & 1077 & 34.41 & 1077 & 34.47 & 1076 & 34.44 & 1071 \\
\hline 31.83 & 1041 & 32.34 & 1049 & 31.98 & 1020 & 31.63 & 994.6 & 33.28 & 1048 & 33.49 & 1050 & 33.55 & 1045 & 33.82 & 1050 & 33.73 & 1044 \\
\hline 31.21 & 1014 & 31.6 & 1023 & 31.27 & 993.7 & 30.91 & 968.2 & 32.31 & 1013 & 32.63 & 1015 & 32.9 & 1017 & 32.99 & 1018 & 33.22 & 1018 \\
\hline 30.47 & 988.9 & 30.71 & 989.3 & 30.59 & 966.4 & 30.26 & 941.3 & 31.71 & 988.5 & 31.95 & 989.8 & 32.28 & 990.2 & 32.63 & 999.9 & 32.63 & 993.7 \\
\hline 29.94 & 965.1 & 0.12 & 964.2 & 29.97 & 940 & 29.58 & 915.3 & 31.12 & 963.3 & 31.3 & 962 & 31.68 & 966 & 31.71 & 963.8 & 32.01 & 968.2 \\
\hline 29.14 & 932 & 29.44 & 937.3 & 29.17 & 909.6 & 28.99 & 886.7 & 30.41 & 934.7 & 30.62 & 936 & 30.8 & 933.4 & 31.15 & 939.5 & 31.06 & 936.5 \\
\hline 28.46 & 907.4 & 28.75 & 911.8 & 28.46 & 880.5 & 28.31 & 861.5 & 29.73 & 904.7 & 30.06 & 911.3 & 30.12 & 905.6 & 30.35 & 909.1 & 30.44 & 910 \\
\hline 27.63 & 876.5 & 28.13 & 885.3 & 27.9 & 856.2 & 27.69 & 836.9 & 29.08 & 880.9 & & 884.9 & & & & 885.8 & 29.7 & 874.8 \\
\hline 27.01 & 852.7 & 27.51 & 860.7 & 26.92 & 825.4 & 26.8 & 802.5 & 28.4 & 851.4 & 28.52 & 852.7 & 28.96 & 857.1 & 29.2 & 861.1 & 28.93 & 854.5 \\
\hline 26.39 & 825.8 & 26.62 & 828.9 & 26.27 & 798.5 & 26.12 & 774.7 & 27.63 & 826.7 & 27.99 & 826.3 & 28.01 & 824.5 & 28.22 & 828 & 28.81 & 836.9 \\
\hline 25.71 & 800.3 & 26 & 800.7 & 25.56 & 772.1 & 25.44 & 751.4 & 27.07 & 801.2 & 27.33 & 801.2 & 27.39 & 800.3 & 27.6 & 800.7 & 27.9 & 811.7 \\
\hline 25.06 & 773.8 & 25.08 & 770.8 & 25.11 & 747.8 & 24.52 & 718.8 & 26.24 & 770.8 & 26.59 & 775.6 & 26.62 & 772.5 & 26.89 & 774.7 & 26.92 & 772.5 \\
\hline 24.23 & 743 & 24.52 & 744.3 & 24.14 & 714.8 & 24.08 & 695.4 & 25.62 & 745.6 & 25.85 & 748.7 & 26.21 & 749.6 & 26.27 & 749.6 & 26.39 & 741.7 \\
\hline 23.46 & 719.2 & 23.75 & 719.2 & 23.49 & 691 & 23.22 & 668.5 & 24.82 & 714.8 & 25.06 & 716.6 & 25.47 & 724 & 25.41 & 719.2 & 25.65 & 728.9 \\
\hline 22.98 & 692.8 & & 695.8 & & 664.1 & & 641.2 & & 688.8 & & 691.9 & & 690.5 & & 691 & & 691 \\
\hline 21.95 & 658.8 & 22.48 & 664.5 & 22.15 & 636.8 & 21.83 & 612.1 & 23.43 & 662.3 & 23.78 & 662.8 & 23.84 & 662.3 & 24.11 & 667.6 & 24.05 & 662.3 \\
\hline 21.27 & 635 & 21.62 & 636.8 & 21.53 & 609.9 & 21.18 & 587 & 22.89 & 639 & 23.1 & 638.1 & 23.22 & 639.9 & 23.61 & 642.1 & 23.46 & 634.1 \\
\hline 20.65 & 610.8 & 21.15 & 614.8 & 20.91 & 584.8 & 20.56 & 565.4 & 21.95 & 604.2 & 22.24 & 605.9 & 22.27 & 605.5 & 22.57 & 612.1 & 22.78 & 609 \\
\hline 19.7 & 576.4 & 20.29 & 581.7 & 20.2 & 557.9 & 19.88 & 539.8 & 21.3 & 581.3 & 21.59 & 579.9 & 21.68 & 578.6 & 22.01 & 583.5 & 22.24 & 584.3 \\
\hline 18.99 & 549.5 & 19.52 & 553.5 & 19.23 & 525.3 & 19.11 & 503.7 & 20.62 & 555.3 & 20.79 & 551.3 & 21 & 553.5 & 21.24 & 554.4 & 21.09 & 552.2 \\
\hline 18.46 & 526.2 & 18.9 & 528.8 & 18.43 & 498 & 18.37 & 477.3 & 19.94 & 528.8 & 20.14 & 525.3 & 20.29 & 530.6 & 20.59 & 527.9 & 20.44 & 525.3 \\
\hline 17.51 & 495.3 & 18.28 & 503.3 & 18.01 & 475.5 & 17.69 & 453.9 & 19.2 & 502.4 & 46 & 500.2 & 19.49 & 497.1 & 19.91 & 502.4 & 19.79 & 499.7 \\
\hline 16.95 & 469.8 & 17.57 & 476.4 & 17.18 & 445.5 & 16.71 & 421.3 & 18.34 & 469.8 & & 471.1 & 18.75 & 472.9 & 19.08 & 479 & 19.34 & 475.1 \\
\hline 16.3 & 444.2 & 16.71 & 443.3 & 16.35 & 420.4 & 16.15 & 393.5 & 17.69 & 445.1 & 17.89 & 445.5 & 17.98 & 442.9 & 18.19 & 447.7 & 18.6 & 450.4 \\
\hline 15.41 & 416.9 & & 416 & 15.64 & 390 & 15.53 & 368.9 & 17.12 & 415.6 & 17.24 & 417.3 & 17.45 & 419.5 & 17.66 & 420.4 & 17.51 & 416.4 \\
\hline 14.93 & 391.8 & 15.35 & 392.2 & 14.82 & 362.2 & 14.55 & 338.4 & 16.18 & 390.4 & 16.53 & 391.8 & 16.62 & 391.8 & 16.8 & 396.6 & 16.98 & 390.9 \\
\hline 13.81 & 360 & 14.7 & 368.9 & 14.4 & 339.3 & 14.05 & 312 & 15.56 & 366.2 & 15.67 & 360.5 & 15.94 & 365.3 & 15.97 & 363.6 & 16.09 & 360.9 \\
\hline 13.36 & 332.3 & 13.69 & 334.9 & 13.42 & 306.7 & 13.42 & 287.3 & 14.58 & 330.5 & 14.85 & 337.1 & 15.05 & 332.7 & 15.14 & 339.3 & 15.56 & 337.6 \\
\hline 12.79 & 309.4 & 13.17 & 310.7 & 12.89 & 281.6 & 12.8 & 262.2 & 13.81 & 305.4 & & 305.8 & 14.34 & 308 & 14.7 & 309.8 & 14.93 & 311.1 \\
\hline 11.92 & 277.6 & 12.58 & 284.7 & 12.31 & 256.5 & 11.92 & 238 & 13.07 & 278.5 & 13.76 & 281.6 & 13.51 & 282.9 & 14.05 & 285.6 & 13.96 & 281.2 \\
\hline
\end{tabular}


Table 12. $25^{\circ} \mathrm{C}$ at 13 wt $\%$

\begin{tabular}{|c|c|c|c|c|c|c|c|c|c|c|c|c|c|c|c|c|c|}
\hline run1 & & run2 & & run3 & & run4 & & run5 & & run6 & & run7 & & run8 & & run9 & \\
\hline [Pa] & {$[1 / s]$} & [Pa] & {$[1 / s]$} & [Pa] & {$[1 / s]$} & [Pa] & {$[1 / s]$} & [Pa] & {$[1 / s]$} & [Pa] & [1/s] & [Pa] & {$[1 / \mathrm{s}]$} & [Pa] & {$[1 / s]$} & [Pa] & {$[1 / \mathrm{s}]$} \\
\hline 13.77 & 280.3 & 14.93 & 278.5 & 15.04 & 276.3 & 14.7 & 272.8 & 14.93 & 272.3 & 15.06 & 276.7 & 14.93 & 273.7 & 14.82 & 271.5 & 14.86 & 275 \\
\hline 14.89 & 298.3 & 15.55 & 295.7 & 15.57 & 292.6 & 15.6 & 298.8 & 15.59 & 296.6 & 15.59 & 295.3 & 15.65 & 293.1 & 15.23 & 293.5 & 15.67 & 296.6 \\
\hline 15.31 & 319.9 & 15.8 & 322.6 & 15.9 & 324.3 & 16.21 & 323.5 & 15.93 & 321.3 & 15.97 & 321.7 & 15.99 & 320.4 & 16.13 & 323.9 & 16.1 & 321.7 \\
\hline 15.82 & 349.5 & 16.63 & 351.2 & 16.45 & 349.9 & 6.65 & 347.7 & 16.51 & 349.5 & 16.72 & 353.9 & 16.35 & 345.1 & 16.64 & 347.7 & 16.42 & 350.3 \\
\hline 16.3 & 377.2 & 17.2 & 375.9 & 17.09 & 372.8 & 16.85 & 373.3 & 17.15 & 372.4 & 17.13 & 378.5 & 16.97 & 371.1 & 16.85 & 372.4 & 16.89 & 374.1 \\
\hline 16.9 & 402.3 & 18.18 & 404.5 & 17.32 & 400.1 & 17.68 & 405.9 & 17.65 & 405 & 17.47 & 404.1 & 17.38 & 396.6 & 17.68 & 405.4 & 17.47 & 407.2 \\
\hline 17.63 & 433.2 & 18.06 & 435.8 & 18.09 & 433.6 & 18.21 & 434.5 & 17.88 & 433.2 & 18.06 & 435.4 & 17.88 & 422.6 & 17.88 & 429.2 & 18.24 & 435 \\
\hline 17.77 & 456.5 & 18.59 & 462.3 & 18.71 & 457.4 & 18.54 & 458.3 & 18.65 & 456.5 & 18.62 & 460.5 & 18.54 & 454.3 & 18.54 & 457.4 & 18.65 & 459.2 \\
\hline 18.42 & 487.8 & 19.22 & 485.2 & 19.19 & 486.5 & 19.04 & 484.8 & 18.89 & 478.6 & 19.07 & 483.4 & 18.95 & 482.5 & 19.19 & 481.7 & 19.07 & 482.5 \\
\hline 19.01 & 509.4 & 19.75 & 516.9 & 19.72 & 512.5 & 19.63 & 512.5 & 19.63 & 509.9 & 19.51 & 510.3 & 19.48 & 505.9 & 19.6 & 505.9 & 19.66 & 509 \\
\hline 19.57 & & & & & & & & & 530.6 & & & & 531.5 & & & & 542 \\
\hline 20.34 & 568.9 & 20.81 & 568.5 & 20.9 & 568.9 & 20.76 & 561.9 & 20.78 & 564.1 & 20.87 & 566.7 & 20.78 & 565 & 20.81 & 568.5 & 20.96 & 569.4 \\
\hline 20.81 & 592.3 & 21.2 & 595.4 & 21.2 & 597.1 & 21.49 & 596.2 & 21.44 & 594.5 & 21.32 & 597.1 & 21.26 & 591 & 21.41 & 594.5 & 21.32 & 591.4 \\
\hline 21.52 & 624.9 & 22.03 & 624.9 & 21.7 & 621.4 & 21.82 & 625.3 & 21.47 & 611.7 & 21.94 & 623.1 & 21.61 & 612.5 & 22 & 620.5 & 21.94 & 622.7 \\
\hline 22.23 & 652.2 & 22.38 & 652.7 & 22.26 & 643.8 & 22.47 & 650.9 & 22.21 & 631.1 & 22.41 & 652.7 & 22.23 & 643.8 & 22.44 & 647.8 & 22.59 & 650.4 \\
\hline 22.62 & 676.9 & 23 & 676.9 & 22.97 & 677.3 & 23.03 & 677.3 & 23.03 & 674.7 & 23.09 & 677.8 & 22.8 & 667.6 & 23.12 & 675.1 & 23.03 & 676 \\
\hline 23.24 & 702.4 & 23.66 & 707.7 & 23.42 & 704.7 & 23.45 & 700.7 & 23.54 & 706 & 23.48 & 700.2 & 23.57 & 700.7 & 23.77 & 704.7 & 23.57 & 699.4 \\
\hline 24.07 & 731.5 & 24.01 & 734.2 & 24.1 & 729.3 & 24.22 & 735.9 & & 728.9 & 24.07 & & 23.98 & 726.2 & 24.25 & 732.4 & 24.31 & 732.4 \\
\hline 24.81 & 758.9 & 24.63 & 761.1 & 24.54 & 754 & 24.66 & 757.5 & 24.66 & 758.4 & 24.63 & 759.3 & 24.42 & 752.7 & 24.6 & 756.7 & 24.84 & 761.1 \\
\hline 25.02 & 782.7 & 25.11 & 787.9 & 25.22 & 784.4 & 25.31 & 787.9 & 25.08 & 784 & 25.11 & 787.9 & 25.02 & 778.7 & 25.19 & 781.8 & 25.34 & 786.6 \\
\hline 25.55 & 814.4 & 25.67 & 814.4 & 25.76 & 813.1 & 25.73 & 813.1 & 25.67 & 810 & 25.67 & 813.1 & 25.67 & 808.2 & 25.73 & 807.8 & 25.87 & 813.1 \\
\hline 25.85 & 842.1 & 26.11 & 839.1 & 26.23 & 839.9 & 26.26 & 838.2 & 26.23 & 839.1 & 26.17 & 837.7 & 26.23 & 835.5 & 26.26 & 836.4 & 26.35 & 839.5 \\
\hline 26.41 & 870.8 & 26.79 & 870.3 & 26.76 & 863.3 & 26.79 & 863.7 & 26.67 & 864.6 & 26.88 & 868.6 & 26.7 & 860.2 & 26.73 & 860.7 & 26.88 & 863.3 \\
\hline 27.06 & 897.7 & 27.21 & 894.6 & 27.41 & 896.3 & 27.5 & 899.4 & 27.35 & 895 & 27.3 & 896.8 & 27.35 & 891.9 & 27.33 & 887.1 & 27.35 & 889.7 \\
\hline 27.62 & 924.1 & 27.95 & 924.1 & 28.01 & 923.2 & 28.04 & 925 & 27.89 & 921.5 & 27.74 & 918.8 & 27.83 & 918.4 & 27.95 & 916.2 & 28.09 & 922.8 \\
\hline 28.12 & 951.4 & 28.45 & 951 & 28.42 & 947 & 28.54 & 951.4 & 28.51 & 951.9 & 28.72 & 953.2 & 28.39 & 943.5 & 28.36 & 943.5 & 28.6 & 950.1 \\
\hline 28.8 & 981.8 & 28.92 & 978.3 & 28.83 & 971.7 & & 975.2 & & 978.3 & 29.04 & 979.6 & 28.92 & 969.9 & 28.98 & 971.3 & 29.13 & 974.3 \\
\hline 29.16 & 1005 & 29.49 & 1001 & 29.43 & 998.6 & 29.46 & 1002 & 29.52 & 1003 & 29.46 & 1005 & 29.52 & 1001 & 29.6 & 1003 & 29.78 & 1006 \\
\hline 29.81 & 1034 & 30.14 & 1033 & 30.23 & 1032 & 29.9 & 1025 & 30.05 & 1033 & 30.02 & 1030 & 30.08 & 1029 & 30.08 & 1031 & 30.28 & 1033 \\
\hline 30.31 & 1060 & 30.64 & 1059 & 30.82 & 1060 & 30.7 & 1059 & 30.55 & 1059 & 30.4 & 1055 & 30.52 & 1055 & 30.64 & 1055 & 30.82 & 1060 \\
\hline 30.79 & 1087 & 31.08 & 1087 & 31.29 & 1083 & 31.17 & 1087 & 31.08 & 1084 & 31.23 & 1090 & 31.08 & 1080 & 31.29 & 1088 & 31.35 & 1085 \\
\hline
\end{tabular}


Table 12. $25^{\circ} \mathrm{C}$ at 13 wt $\%$ - continued

\begin{tabular}{|c|c|c|c|c|c|c|c|c|c|c|c|c|c|c|c|c|c|}
\hline run1 & & run2 & & run3 & & run4 & & run5 & & run6 & & run7 & & run8 & & run9 & \\
\hline [Pa] & {$[1 / \mathrm{s}]$} & [Pa] & [1/s] & [Pa] & {$[1 / \mathrm{s}]$} & [Pa] & {$[1 / s]$} & [Pa] & {$[1 / \mathrm{s}]$} & [Pa] & {$[1 / \mathrm{s}]$} & [Pa] & [1/s] & [Pa] & {$[1 / \mathrm{s}]$} & [Pa] & {$[1 / s]$} \\
\hline 31.5 & 1113 & 31.56 & 1110 & 32.06 & 1116 & 31.73 & 1111 & 31.56 & 1110 & 31.76 & 1116 & 31.59 & 1107 & 31.79 & 1114 & 31.79 & 1113 \\
\hline 32.06 & 1144 & 32.27 & 1142 & 32.53 & 1140 & 32.44 & 1144 & 32.09 & 1135 & 32.21 & 1141 & 32.18 & 1139 & 32.3 & 1140 & 32.33 & 1138 \\
\hline 32.53 & 1166 & 32.77 & 1166 & 32.89 & 1163 & 32.8 & 1171 & 32.77 & 1169 & 32.8 & 1171 & 32.71 & 1163 & 32.86 & 1169 & 32.89 & 1163 \\
\hline 32.92 & 1194 & 33.3 & 1197 & 33.75 & 1196 & 33.3 & 1194 & 33.21 & 1196 & 33.18 & 1193 & 33.21 & 1189 & 33.24 & 1194 & 33.51 & 1196 \\
\hline 33.69 & 1223 & 33.95 & 1225 & 34.19 & 1221 & 33.81 & 1221 & 33.75 & 1220 & 33.48 & 1209 & 33.66 & 1217 & 33.75 & 1219 & 34.01 & 1223 \\
\hline 34.22 & 1252 & 34.37 & 1248 & 34.72 & 1250 & 34.4 & 1253 & 34.22 & 1245 & 33.36 & 1214 & 34.19 & 1243 & 34.46 & 1250 & 34.52 & 1247 \\
\hline 34.72 & 1278 & 34.9 & 1279 & 35.26 & 1276 & 34.99 & 1279 & 34.96 & 1279 & 34.63 & 1250 & 34.63 & 1267 & 35.05 & 1277 & 35.14 & 1280 \\
\hline 35.14 & 1302 & 35.4 & 1304 & 35.88 & 1305 & 35.4 & 1307 & 35.32 & 1305 & 35.61 & 1313 & 35.11 & 1293 & 35.49 & 1303 & 35.61 & 1304 \\
\hline 35.79 & 1330 & 36 & 1335 & 36.41 & 1330 & 35.91 & 1333 & 35.82 & 1328 & 35.76 & 1324 & 35.85 & 1329 & 36 & 1331 & 36.17 & 1331 \\
\hline 36.29 & 1356 & 36.41 & 1357 & 36.82 & 1359 & 36.41 & 1356 & 36.35 & 1354 & 36.35 & 1347 & 36.35 & 1354 & 36.47 & 1355 & 36.65 & 1360 \\
\hline 36.85 & 1386 & 37.15 & 1386 & 37.33 & 1384 & 37 & 1388 & 36.97 & 1386 & 36.94 & 1386 & 36.82 & 1378 & 36.97 & 1381 & 37.12 & 1384 \\
\hline 37.33 & 1413 & 37.65 & 1415 & 37.77 & 1408 & 37.51 & 1415 & 37.54 & 1415 & 37.45 & 1416 & 37.54 & 1412 & 37.65 & 1415 & 37.65 & 1411 \\
\hline 37.8 & 1435 & 38.22 & 1440 & 39.22 & 1440 & 37.86 & 1439 & 37.98 & 1441 & 37.98 & 1442 & 37.92 & 1435 & 38.13 & 1441 & 38.13 & 1437 \\
\hline 38.51 & 1471 & 38.9 & 1471 & 39.61 & 1469 & 38.42 & 1464 & 38.51 & 1464 & 38.51 & 1471 & 38.48 & 1462 & 38.63 & 1469 & 38.81 & 1470 \\
\hline 38.96 & 1497 & 39.37 & 1493 & 40.02 & 1491 & 39.01 & 1491 & 39.19 & 1498 & 39.01 & 1499 & 38.96 & 1490 & 39.07 & 1495 & 39.28 & 1495 \\
\hline 39.55 & 1521 & 40.02 & 1525 & 40.64 & 1523 & 39.78 & 1523 & 39.67 & 1523 & 39.55 & 1523 & 39.37 & 1513 & 39.64 & 1523 & 39.73 & 1521 \\
\hline 39.75 & 1510 & 41.29 & 1505 & & 1541 & & 1508 & 42.63 & 1539 & & 1509 & .51 & 1535 & 38.54 & 1508 & 36.62 & 1426 \\
\hline 39.19 & 1484 & 40.85 & 1482 & 38.25 & 1514 & 37.54 & 1483 & 41.86 & 1508 & 37.65 & 1483 & 37.95 & 1507 & 38.04 & 1482 & 36.2 & 1403 \\
\hline 38.54 & 1458 & 39.73 & 1451 & 37.71 & 1489 & 37.09 & 1460 & 40.85 & 1482 & 36.97 & 1451 & 37.62 & 1484 & 37.42 & 1452 & 35.64 & 1375 \\
\hline 38.16 & 1431 & 39.22 & 1423 & 37.03 & 1453 & 36.59 & 1432 & 40.38 & 1453 & 36.53 & 1425 & 37.12 & 1459 & 36.85 & 1426 & 35.17 & 1349 \\
\hline 37.51 & 1404 & 38.69 & 1399 & 36.8 & 1436 & 35.85 & 1399 & 40.05 & 1430 & 35.97 & 1400 & 36.62 & 1433 & 36.38 & 1400 & 34.72 & 1325 \\
\hline 36.91 & 1376 & 38.13 & 1370 & 36.08 & 1400 & 35.35 & 1372 & 39.49 & 1398 & 35.55 & 1371 & 35.94 & 1397 & 35.85 & 1375 & 34.04 & 1292 \\
\hline 36.35 & 1348 & 37.68 & 1342 & 35.49 & 1375 & 34.84 & 1347 & 38.9 & 1374 & 35.08 & 1347 & 35.43 & 1372 & 35.14 & 1343 & 33.54 & 1265 \\
\hline 35.76 & 1322 & 36.91 & 1319 & 35.02 & 1349 & 34.37 & 1317 & 37.98 & 1345 & 34.61 & 1322 & 34.99 & 1348 & 34.66 & 1319 & 33.01 & 1241 \\
\hline 35.14 & 1291 & 36.08 & 1286 & 34.55 & 1320 & 33.92 & 1292 & 37.54 & 1319 & 33.78 & 1288 & 34.58 & 1322 & 34.16 & 1292 & 32.53 & 1217 \\
\hline 34.66 & 1266 & 35.55 & 1259 & 34.07 & 1296 & 33.42 & 1267 & 36.74 & 1295 & 33.27 & 1263 & 33.98 & 1296 & 33.75 & 1268 & 31.88 & 1182 \\
\hline 34.22 & 1240 & 34.96 & 1235 & 33.33 & 1263 & 32.74 & 1235 & 34.66 & 1263 & 32.8 & 1235 & 33.57 & 1269 & 33.01 & 1236 & 31.41 & 1157 \\
\hline 33.51 & 1208 & 34.43 & 1210 & 32.89 & 1238 & 32.21 & 1207 & 33.39 & 1235 & 32.36 & 1211 & 32.86 & 1236 & 32.53 & 1207 & 30.88 & 1131 \\
\hline 32.92 & 1181 & 33.78 & 1182 & 32.3 & 1211 & 31.82 & 1182 & 33.3 & 1207 & 31.91 & 1187 & 32.36 & 1211 & 32.03 & 1183 & 30.46 & 1108 \\
\hline 32.42 & 1153 & 33.24 & 1157 & 31.82 & 1181 & 31.08 & 1152 & 32.77 & 1181 & 31.35 & 1154 & 31.85 & 1186 & 31.5 & 1158 & 29.72 & 1073 \\
\hline 31.82 & 1129 & 32.56 & 1125 & 31.29 & 1159 & 30.61 & 1126 & 31.97 & 1155 & 30.73 & 1126 & 31.47 & 1160 & 30.82 & 1125 & 29.25 & 1047 \\
\hline
\end{tabular}


Table 12. $25^{\circ} \mathrm{C}$ at 13 wt $\%$ - continued

\begin{tabular}{|c|c|c|c|c|c|c|c|c|c|c|c|c|c|c|c|c|c|}
\hline run1 & & run2 & & run3 & & run4 & & run5 & & run6 & & run7 & & run8 & & run9 & \\
\hline [Pa] & {$[1 / s]$} & [Pa] & {$[1 / \mathrm{s}]$} & [Pa] & [1/s] & [Pa] & {$[1 / s]$} & [Pa] & {$[1 / \mathrm{s}]$} & [Pa] & {$[1 / \mathrm{s}]$} & [Pa] & {$[1 / s]$} & [Pa] & {$[1 / \mathrm{s}]$} & [Pa] & {$[1 / s]$} \\
\hline 31.26 & 1100 & 32.21 & 1099 & 30.7 & 1128 & 30.14 & 1099 & 31.47 & 1130 & 30.2 & 1100 & 30.61 & 1126 & 30.31 & 1100 & 28.72 & 1019 \\
\hline 30.7 & 1075 & 31.5 & 1071 & 30.25 & 1101 & 29.66 & 1073 & 30.64 & 1097 & 29.6 & 1070 & 30.14 & 1098 & 29.81 & 1072 & 28.18 & 992.9 \\
\hline 30.28 & 1047 & 30.76 & 1047 & 29.84 & 1074 & 29.1 & 1047 & 30.2 & 1072 & 29.1 & 1044 & 29.75 & 1076 & 29.43 & 1048 & 27.71 & 969.5 \\
\hline 29.66 & 1021 & 30.23 & 1022 & 29.28 & 1048 & 28.57 & 1018 & 29.66 & 1046 & 28.57 & 1022 & 29.1 & 1043 & 29.16 & 1023 & 26.94 & 936 \\
\hline 29.13 & 996.8 & 29.49 & 988.5 & 28.63 & 1018 & 28.09 & 992 & 28.8 & 1017 & 28.09 & 991.5 & 28.54 & 1020 & 28.3 & 995.9 & 26.47 & 910.9 \\
\hline 28.42 & 963.8 & 29.01 & 961.1 & 28.09 & 990.2 & 27.65 & 968.2 & 28.06 & 994.2 & 27.53 & 966 & 28.06 & 990.2 & 27.62 & 964.7 & 25.96 & 884.5 \\
\hline 27.77 & 938.2 & 28.66 & 943.1 & 27.62 & 966.9 & 27.09 & 942.6 & 27.56 & 958.5 & 27.12 & 942.6 & 27.59 & 965.5 & 27.12 & 936 & 25.37 & 856.7 \\
\hline 27.21 & 909.6 & 27.68 & 911.3 & 27.09 & 943.9 & 26.64 & 916.2 & 27.24 & 942.2 & 26.35 & 908.7 & 27.09 & 939.5 & 26.61 & 907.8 & 24.93 & 832 \\
\hline 26.7 & 883.1 & 27.47 & 891.5 & 26.5 & 910.5 & 25.99 & 883.6 & 26.41 & 907.8 & 25.79 & 880 & 26.59 & 913.5 & 26.05 & 884.9 & 24.4 & 806 \\
\hline 26.26 & 861.1 & 26.44 & 847.9 & & 884 & 25.4 & 855.8 & 25.87 & 879.6 & 25.31 & 851.8 & 25.9 & 880.9 & 25.52 & 856.7 & 23.74 & 773.4 \\
\hline 25.49 & 827.2 & 25.9 & 833.3 & 25.28 & 856.7 & 24.93 & 832 & 25.46 & 855.4 & 24.87 & 826.7 & 25.34 & 852.3 & 25.08 & 832 & 23.18 & 748.3 \\
\hline 24.93 & 800.7 & 25.43 & 803.8 & 24.78 & 827.6 & 24.25 & 798.5 & 25.02 & 828.9 & 24.37 & 802.9 & 24.96 & 828.5 & 24.28 & 797.2 & 22.65 & 720.5 \\
\hline 24.31 & 772.1 & 24.78 & 784.4 & 24.28 & 801.6 & 23.8 & 772.5 & 24.31 & 796.8 & 23.77 & 776 & 24.45 & 803.4 & 23.83 & 770.8 & 22.21 & 693.6 \\
\hline 23.71 & 748.3 & 23.95 & 741.2 & 23.68 & 775.6 & 23.3 & 744.3 & 23.86 & 770.3 & 23.21 & 748.7 & 23.68 & 771.6 & 23.21 & 745.2 & 21.58 & 667.6 \\
\hline 23.12 & 716.6 & 23.36 & 717.9 & 23.15 & 747 & 22.71 & 721 & 23.39 & 747 & 22.53 & 714.3 & 23.27 & 747 & 22.71 & 718.3 & 21.2 & 643 \\
\hline 22.5 & 691.4 & 22.8 & 688.8 & 22.8 & 723.2 & 21.94 & 689.2 & 22.86 & 721 & 22.03 & 688.8 & 22.74 & 717.9 & 22.21 & 693.6 & 20.34 & 612.1 \\
\hline 22.15 & 664.5 & & 663.7 & & 697.2 & & 665 & 22.32 & 696.3 & 21.61 & 663.2 & & 693.6 & & 668.1 & 0.04 & 583 \\
\hline 21.55 & 640.3 & 21.67 & 636.3 & 21.61 & 669 & 21.02 & 636.8 & 21.44 & 661 & 21.05 & 636.8 & 22.21 & 669 & 20.96 & 634.1 & 19.45 & 556.6 \\
\hline 20.78 & 613.4 & 21.08 & 612.1 & 21.2 & 642.1 & 20.64 & 610.3 & 20.9 & 635 & 20.4 & 0.8 & 21.29 & 637.2 & 20.4 & 609 & .77 & 528.8 \\
\hline 20.34 & 583 & 20.7 & 587.4 & 20.7 & 616.1 & 20.1 & 586.1 & 20.34 & 604.6 & 20.07 & 584.8 & 20.55 & 608.6 & 19.99 & 581.3 & .39 & 502.4 \\
\hline 19.72 & 556.1 & 20.04 & 559.2 & 20.02 & 580.8 & 19.69 & 560.1 & 19.72 & 579.1 & 19.28 & 552.6 & 19.99 & 583.5 & 19.45 & 556.6 & 17.85 & 477.7 \\
\hline 19.28 & 531.9 & 19.22 & 525.7 & 19.51 & 557 & 18.95 & 528.4 & 19.28 & 552.6 & 18.68 & 524.4 & 19.66 & 561.4 & 19.01 & 529.3 & 17.26 & 453.5 \\
\hline 18.68 & 504.1 & 18.71 & 498.9 & 18.95 & 528.4 & 18.27 & 498.9 & 18.83 & 528.8 & 18.36 & 499.3 & 18.95 & 528.8 & 18.36 & 498.9 & 16.58 & 420.9 \\
\hline 18.18 & 479.9 & 18.21 & 475.5 & 18.54 & 502.8 & 17.88 & 471.5 & 18.45 & 503.3 & 17.91 & 472.4 & 18.39 & 498.4 & 17.74 & 473.7 & .38 & 392.6 \\
\hline 17.47 & 442.9 & 17.56 & 440.7 & 17.91 & 478.6 & 17.44 & 445.1 & 17.59 & 469.8 & 17.29 & 447.3 & 17.77 & 475.1 & 17.17 & 445.1 & 5.72 & 368.4 \\
\hline 16.73 & 419.1 & 16.85 & 416.4 & 17.29 & 451.7 & 16.73 & 417.8 & 17.29 & 445.5 & 16.82 & 421.7 & 17.11 & 442.4 & 16.85 & 422.2 & 15.19 & 345.1 \\
\hline 16.46 & 391.8 & 16.52 & 388.7 & 16.76 & 418.6 & 16.46 & 392.2 & 16.7 & 416.4 & 16.23 & 390 & 16.79 & 418.2 & 16.14 & 392.6 & 14.69 & 312.9 \\
\hline 15.69 & 361.4 & 15.75 & 362.7 & 16.38 & 391.3 & 15.81 & 366.6 & 16.35 & 392.6 & 15.55 & 361.4 & 16.08 & 391.3 & 15.9 & 367.1 & 14.24 & 286 \\
\hline 15.25 & 343.3 & 15.43 & 333.6 & 15.61 & 364.9 & 15.28 & 339.8 & 15.49 & 360.9 & 15.25 & 335.4 & 15.81 & 362.7 & 14.9 & 333.6 & 13.68 & 255.2 \\
\hline 14.84 & 308.5 & 14.87 & 305.8 & 15.31 & 336.7 & 14.81 & 308 & 15.25 & 334.5 & 14.78 & 309.4 & 15.31 & 337.6 & 14.48 & 309.4 & 13.27 & 229.2 \\
\hline 14.39 & 282.9 & 14.51 & 282 & 14.95 & 312.4 & 14.3 & 282.5 & 14.84 & 309.8 & 14.21 & 279.8 & 14.39 & 306.7 & 14.19 & 280.7 & 0 & 172.9 \\
\hline 13.98 & 260.4 & 13.74 & 249.9 & 14.45 & 286.4 & 13.74 & 253 & 14.21 & 279 & 13.74 & 253 & 13.92 & 280.3 & 13.53 & 256.5 & 0 & 171.6 \\
\hline
\end{tabular}


Table 13. $25{ }^{\circ} \mathrm{C}$ Repeat at 13 wt \%

\begin{tabular}{|c|c|c|c|c|c|c|c|c|c|c|c|c|c|c|c|c|c|}
\hline run1 & & run2 & & run3 & & run4 & & run5 & & run6 & & run7 & & run8 & & run9 & \\
\hline [Pa] & {$[1 / s]$} & [Pa] & {$[1 / s]$} & [Pa] & {$[1 / s]$} & [Pa] & {$[1 / s]$} & [Pa] & {$[1 / s]$} & [Pa] & [1/s] & [Pa] & {$[1 / s]$} & [Pa] & {$[1 / \mathrm{s}]$} & [Pa] & {$[1 / s]$} \\
\hline 13.05 & 275 & 14.21 & 305 & 13.29 & 274.1 & 13.37 & 274.5 & 13.2 & 275.4 & 13.14 & 272.8 & 13.62 & 280.3 & 13.62 & 276.3 & 13.36 & 274.1 \\
\hline 13.88 & 293.1 & 14.75 & 332.3 & 13.83 & 296.6 & 13.89 & 293.5 & 13.72 & 295.7 & 13.78 & 290.4 & 14.19 & 301 & 14.28 & 298.8 & 14.18 & 297 \\
\hline 14.19 & 321.7 & 15 & 357.4 & 14.44 & 322.6 & 4.25 & 318.2 & 14.28 & 318.6 & 14.6 & 320.4 & 14.85 & 327.9 & 14.79 & 322.1 & 14.74 & 317.7 \\
\hline 14.63 & 344.6 & 15.9 & 388.7 & 15.13 & 345.9 & 4.77 & 343.7 & 15.1 & 345.5 & 15.29 & 345.5 & 15.64 & 355.2 & 15.25 & 346.8 & 15.36 & 345.9 \\
\hline 15.52 & 371.1 & 16.12 & 414.7 & 15.8 & 377.2 & 15.68 & 375.9 & 15.64 & 378.5 & 15.7 & 369.7 & 15.87 & 384.7 & 16.14 & 379 & 16.35 & 375.5 \\
\hline 16.1 & 404.5 & 16.83 & 438.5 & 17.09 & 403.7 & 16.03 & 400.6 & 16.52 & 404.5 & 16.53 & 403.7 & 16.68 & 409 & 16.41 & 405.4 & 16.71 & 400.6 \\
\hline 16.68 & 430.1 & 17.09 & 462.7 & 17.54 & 429.7 & 16.56 & 429.2 & 16.89 & 432.8 & 17.06 & 431 & 17.24 & 440.2 & 17.24 & 432.3 & 17.39 & 430.5 \\
\hline 17.27 & 454.8 & 18.01 & 496.6 & 18.07 & 457.9 & 17.27 & 453.5 & 17.45 & 457.4 & 17.6 & 453.9 & 17.68 & 463.6 & 17.86 & 461.4 & 18.16 & 457 \\
\hline 18.07 & 486.5 & 18.57 & 521.3 & 18.34 & 484.8 & 17.77 & 480.8 & 18.07 & 488.7 & 18.28 & 479.5 & 18.34 & 490.5 & 18.31 & 487 & 18.78 & 482.1 \\
\hline 18.57 & 513 & 19.19 & 550.9 & 18.9 & 507.7 & 18.6 & 513.4 & 18.69 & 514.3 & 18.93 & 507.7 & 18.81 & 519.6 & 18.9 & 514.3 & 19.34 & 507.7 \\
\hline 19.16 & 539.4 & 19.7 & & 19.82 & & 19.1 & 537.6 & & & & & & & 19.4 & 539.4 & 20.2 & 538.1 \\
\hline 19.7 & 563.6 & 20.29 & 607.7 & 20.32 & 565.4 & 19.67 & 565 & 19.79 & 567.2 & 20.17 & 568.5 & 20.14 & 576.9 & 20.08 & 565 & 20.82 & 565.8 \\
\hline 20.23 & 588.8 & 21.09 & 633.7 & 20.64 & 591 & 20.2 & 592.3 & 20.56 & 596.2 & 20.82 & & 20.41 & 594.9 & 21.12 & 597.1 & & 593.2 \\
\hline 20.88 & 622.7 & 21.71 & 657.5 & 21.29 & 622.7 & 20.73 & 617.4 & 21.06 & 624 & 21.56 & 619.6 & 21.38 & 629.3 & 21.56 & 624.4 & 21.98 & 621.4 \\
\hline 21.29 & 645.2 & 22.06 & 687.9 & 21.56 & 639.9 & 21.32 & 642.1 & 21.59 & 650 & 22.12 & 643.8 & 22.03 & 657.5 & 22.48 & 649.6 & 22.54 & 645.2 \\
\hline 21.86 & 669 & 22.6 & 712.1 & 22.42 & 665.9 & 22.15 & 674.2 & 22.24 & 676.4 & 22.57 & 669 & 22.42 & 682.2 & 23.04 & 677.8 & 23.16 & 676.9 \\
\hline 22.51 & 702 & 23.1 & 739.9 & 23.34 & 713.9 & 22.69 & 703.3 & 22.48 & 701.1 & 23.6 & 703.3 & 23.1 & 707.3 & 23.84 & 702.9 & 23.46 & 702.4 \\
\hline 23.16 & 728.9 & 23.69 & 764.1 & 23.4 & 723.6 & & 728.9 & 22.95 & 727.6 & 24.05 & 728.9 & 23.84 & 738.6 & 24.4 & 728.4 & 24.11 & 727.6 \\
\hline 23.51 & 752.2 & 24.43 & 795.9 & 23.34 & 731.1 & 23.87 & 752.7 & 23.81 & 761.5 & 24.7 & 758 & 24.46 & 766.3 & 25.35 & 762.8 & 24.7 & 758.9 \\
\hline 24.37 & 784 & 24.88 & 821.4 & 24.58 & 773.8 & 24.52 & 781.8 & 24.34 & 779.1 & 25.29 & 781.3 & 24.82 & 789.7 & 26 & 787.5 & 25.32 & 782.2 \\
\hline 24.88 & 812.2 & 25.41 & 846.1 & 25.35 & 803.4 & 25.11 & 811.7 & 25.35 & 814.4 & 25.82 & 806.4 & 25.23 & 814.8 & 26.53 & 813.1 & 26.21 & 810 \\
\hline 25.47 & 835.1 & 26.06 & 877.4 & 25.97 & 840.4 & 25.73 & 835.1 & 25.91 & 843.5 & 26.65 & 841.7 & 25.91 & 845.7 & 27.18 & 839.1 & 26.83 & 835.1 \\
\hline 26.09 & 865.9 & 26.74 & 905.6 & 26.62 & 865.9 & 26.24 & 865.5 & 26.44 & 867.7 & 27.18 & 865.1 & 26.68 & 877.4 & 27.86 & 868.1 & 27.6 & 864.6 \\
\hline 26.62 & 894.6 & 27.15 & 930.3 & 27.01 & 890.2 & 26.86 & 893.3 & 26.95 & 894.1 & 27.66 & 890.6 & 27.01 & 903 & 28.58 & 898.1 & 28.25 & 893.7 \\
\hline 27.24 & 916.2 & 27.69 & 955.8 & 27.75 & 922.8 & 27.39 & 918.8 & 27.6 & 918.8 & 28.43 & 923.7 & 27.54 & 930.3 & 29.29 & 924.6 & 28.72 & 918.4 \\
\hline 27.78 & 943.1 & 28.52 & 988 & 28.43 & 951.9 & 28.07 & 943.1 & 28.04 & 943.1 & 29.11 & 950.6 & 27.89 & 955.8 & 30.11 & 949.2 & 29.43 & 943.9 \\
\hline 28.6 & 977 & 29.02 & 1016 & 28.93 & 976.1 & & 977.9 & 28.9 & 977 & 29.55 & 973.9 & 28.37 & 978.3 & 30.59 & 975.2 & 30.23 & 972.6 \\
\hline 29.08 & 1000 & 29.46 & 1039 & 29.46 & 1006 & 29.37 & 1003 & 29.4 & 1005 & 30.35 & 1006 & 29.26 & 1012 & 31.18 & 999.9 & 31.03 & 1004 \\
\hline 29.46 & 1028 & 30.23 & 1070 & 29.88 & 1029 & 29.91 & 1030 & 29.82 & 1029 & 30.85 & 1030 & 29.64 & 1038 & 31.95 & 1034 & 31.45 & 1027 \\
\hline 29.91 & 1053 & 30.77 & 1096 & 30.44 & 1056 & 30.47 & 1056 & 30.56 & 1061 & 31.42 & 1059 & 30.17 & 1061 & 32.6 & 1060 & 32.04 & 1053 \\
\hline 30.65 & 1085 & 31.27 & 1120 & 31.27 & 1088 & 31.03 & 1082 & 31.09 & 1088 & 32.07 & 1086 & 30.68 & 1087 & 33.28 & 1088 & 32.96 & 1085 \\
\hline
\end{tabular}


Table 13. $25^{\circ} \mathrm{C}$ Repeat at $13 \mathrm{wt} \%$ - continued

\begin{tabular}{|c|c|c|c|c|c|c|c|c|c|c|c|c|c|c|c|c|c|}
\hline run1 & & run2 & & run3 & & run4 & & run5 & & run6 & & run7 & & run8 & & run9 & \\
\hline [Pa] & {$[1 / s]$} & [Pa] & {$[1 / s]$} & [Pa] & {$[1 / s]$} & [Pa] & {$[1 / s]$} & [Pa] & {$[1 / s]$} & [Pa] & {$[1 / \mathrm{s}]$} & [Pa] & {$[1 / s]$} & [Pa] & {$[1 / \mathrm{s}]$} & [Pa] & {$[1 / s]$} \\
\hline 31.12 & 1111 & 31.98 & 1152 & 31.8 & 1114 & 31.53 & 1108 & 31.71 & 1116 & 32.57 & 1111 & 31.36 & 1114 & 33.93 & 1112 & 33.55 & 1112 \\
\hline 31.62 & 1134 & 32.54 & 1179 & 32.48 & 1137 & 32.19 & 1134 & 32.27 & 1142 & 33.22 & 1137 & 33.19 & 1150 & 34.67 & 1138 & 34.29 & 1141 \\
\hline 32.36 & 1168 & 33.04 & 1202 & 33.22 & 1169 & 32.81 & 1163 & 32.96 & 1168 & 33.75 & 1164 & 33.67 & 1175 & 35.35 & 1171 & 34.85 & 1165 \\
\hline 32.87 & 1192 & 33.64 & 1226 & 33.84 & 1193 & 33.34 & 1189 & 33.46 & 1194 & 34.32 & 1190 & 34.38 & 1200 & 35.94 & 1197 & 35.23 & 1188 \\
\hline 33.4 & 1218 & 34.52 & 1259 & 34.61 & 1219 & 34.08 & 1223 & 34.05 & 1226 & 35.12 & 1220 & 34.85 & 1226 & 36.65 & 1224 & 35.8 & 1219 \\
\hline 33.75 & 1241 & 35.2 & 1284 & 35.5 & 1250 & 34.55 & 1249 & 34.7 & 1253 & 35.74 & 1251 & 35.38 & 1255 & 37.19 & 1248 & 36.42 & 1246 \\
\hline 34.64 & 1275 & 35.71 & 1311 & 36.03 & 1275 & 35.17 & 1275 & 35.29 & 1278 & 36.27 & 1277 & 36.21 & 1285 & 37.69 & 1274 & 37.19 & 1278 \\
\hline 35.23 & 1303 & 36.27 & 1342 & 36.48 & 1306 & 35.62 & 1299 & 35.8 & 1301 & 36.68 & 1301 & 36.65 & 1311 & 38.37 & 1301 & 37.81 & 1302 \\
\hline 35.8 & 1327 & 36.74 & 1370 & 37.34 & 1333 & 36.42 & 1331 & 36.24 & 1327 & 37.42 & 1334 & 37.57 & 1337 & 39.23 & 1333 & 38.31 & 1331 \\
\hline 36.48 & 1359 & 37.36 & 1399 & 37.84 & 1354 & 36.83 & 1356 & 36.8 & 1355 & 38.05 & 1356 & 38.43 & 1367 & 39.73 & 1361 & 38.96 & 1356 \\
\hline 37.01 & 1386 & & 1425 & & 1388 & & 1386 & 37.39 & 1382 & 38.34 & 1376 & 38.96 & 1390 & 40.29 & 1386 & 39.55 & 1384 \\
\hline 37.51 & 1409 & 38.58 & 1452 & 39.35 & 1411 & 37.57 & 1396 & 38.19 & 1417 & 39.35 & 1415 & 39.41 & 1416 & 40.92 & 1411 & 40.12 & 1409 \\
\hline 38.28 & 1438 & 38.96 & 1474 & 39.76 & 1438 & 38.46 & 1444 & 38.7 & 1443 & 39.76 & 1439 & 40.21 & 1447 & 41.6 & 1443 & 40.71 & 1433 \\
\hline 38.79 & 1464 & 39.61 & 1507 & 40.38 & 1467 & 38.99 & 1453 & 39.2 & 1471 & 40.24 & 1462 & 40.5 & 1473 & 42.16 & 1471 & 41.51 & 1467 \\
\hline 39.35 & 1490 & 40.18 & 1533 & 41.06 & 1495 & 39.47 & 1485 & 39.55 & 1495 & 41.12 & 1496 & 41.21 & 1503 & 42.6 & 1496 & 41.95 & 1494 \\
\hline 37.16 & 1516 & 38.02 & 1483 & 38.61 & 1509 & 37.66 & 1510 & 37.87 & 1510 & 40.38 & 1485 & 40.12 & 1479 & 40.47 & 1516 & 40.47 & 1513 \\
\hline 36.86 & 1483 & 37.42 & 1456 & 37.93 & 1481 & 37.28 & 1482 & 37.54 & 1485 & 39.79 & 1457 & 39.5 & 1450 & 39.67 & 1482 & 39.79 & 1482 \\
\hline 36.62 & 1458 & 36.95 & 1426 & 37.28 & 1456 & & 1457 & 36.27 & 1452 & 39.26 & 1432 & 38.9 & 1423 & 39.17 & 1456 & 39.23 & 1454 \\
\hline 35.62 & 1432 & 36.39 & 1403 & 36.92 & 1432 & 36.15 & 1432 & 35.53 & 1427 & 38.49 & 1400 & 38.28 & 1397 & 38.34 & 1432 & 38.61 & 1429 \\
\hline 34.91 & 1401 & 35.77 & 1376 & 36 & 1397 & 35.88 & 1405 & 35 & 1402 & 38.13 & 1374 & 37.78 & 1374 & 37.78 & 1399 & 38.02 & 1404 \\
\hline 34.41 & 1374 & 35.2 & 1345 & 35.5 & 1372 & 35.44 & 1379 & 34.41 & 1372 & 37.51 & 1348 & 36.89 & 1344 & 37.36 & 1375 & 37.36 & 1370 \\
\hline 33.75 & 1343 & 34.64 & 1319 & 35.09 & 1343 & 34.85 & 1343 & 33.9 & 1350 & 36.89 & 1321 & 36.33 & 1317 & 36.71 & 1350 & 36.68 & 1347 \\
\hline 33.22 & 1318 & 34.14 & 1291 & 34.58 & 1316 & 34.46 & 1317 & 33.22 & 1317 & 36.21 & 1294 & 35.91 & 1291 & 35.97 & 1317 & 36.09 & 1319 \\
\hline 32.78 & 1292 & 33.67 & 1266 & 34.11 & 1293 & 34.05 & 1293 & 32.72 & 1289 & 35.65 & 1270 & 35.32 & 1266 & 35.41 & 1292 & 35.47 & 1293 \\
\hline 32.33 & 1266 & 33.16 & 1241 & 33.61 & 1267 & 33.46 & 1262 & 32.16 & 1265 & 34.79 & 1235 & 34.73 & 1241 & 35 & 1265 & 35.12 & 1268 \\
\hline 31.8 & 1242 & 32.3 & 1209 & 32.84 & 1235 & 33.04 & 1244 & 31.71 & 1237 & 34.26 & 1211 & 33.9 & 1208 & 34.46 & 1241 & 34.46 & 1236 \\
\hline 31.3 & 1217 & 31.74 & 1183 & 32.57 & 1216 & 32.07 & 1208 & 31.15 & 1213 & 33.69 & 1185 & 33.31 & 1182 & 33.81 & 1214 & 33.87 & 1210 \\
\hline 30.74 & 1182 & 30.91 & 1153 & 31.86 & 1186 & 31.48 & 1180 & 30.41 & 1179 & 33.01 & 1159 & 32.81 & 1152 & 33.25 & 1186 & 33.13 & 1180 \\
\hline 30.03 & 1155 & 30.47 & 1129 & 31.18 & 1159 & 31.09 & 1155 & 29.97 & 1151 & 32.33 & 1128 & 32.24 & 1129 & 32.6 & 1155 & 32.69 & 1156 \\
\hline 29.43 & 1126 & 30.03 & 1100 & 30.44 & 1126 & 30.53 & 1130 & 29.43 & 1128 & 31.95 & 1102 & 31.71 & 1099 & 32.1 & 1130 & 32.66 & 1150 \\
\hline 29.05 & 1103 & 29.55 & 1077 & 29.85 & 1097 & 29.97 & 1105 & 28.96 & 1102 & 31.3 & 1080 & 31 & 1070 & 31.42 & 1104 & 31.48 & 1108 \\
\hline
\end{tabular}


Table 13. $25^{\circ} \mathrm{C}$ Repeat at $13 \mathrm{wt} \%$ - continued

\begin{tabular}{|c|c|c|c|c|c|c|c|c|c|c|c|c|c|c|c|c|c|}
\hline run1 & & run2 & & run3 & & run4 & & run5 & & run6 & & run7 & & run8 & & run9 & \\
\hline [Pa] & {$[1 / s]$} & [Pa] & {$[1 / s]$} & [Pa] & {$[1 / s]$} & [Pa] & {$[1 / s]$} & [Pa] & {$[1 / s]$} & [Pa] & [1/s] & [Pa] & {$[1 / \mathrm{s}]$} & [Pa] & {$[1 / \mathrm{s}]$} & [Pa] & {$[1 / s]$} \\
\hline 28.49 & 1072 & 28.69 & 1045 & 29.43 & 1076 & 29.4 & 1072 & 28.31 & 1070 & 30.53 & 1045 & 30.41 & 1045 & 30.82 & 1076 & 30.85 & 1080 \\
\hline 27.86 & 1046 & 28.25 & 1017 & 28.9 & 1050 & 28.9 & 1047 & 27.95 & 1046 & 29.91 & 1023 & 29.82 & 1022 & 30.17 & 1048 & 30.41 & 1057 \\
\hline 27.36 & 1018 & 27.86 & 994.2 & 28.07 & 1018 & 28.34 & 1020 & 27.54 & 1019 & 29.23 & 992.9 & 29.02 & 990.2 & 29.4 & 1018 & 29.43 & 1010 \\
\hline 26.8 & 994.6 & 27.1 & 961.6 & 27.51 & 987.6 & 27.72 & 990.2 & 26.86 & 993.7 & 28.66 & 964.2 & 28.46 & 962.5 & 28.93 & 991.5 & 28.78 & 997.3 \\
\hline 26.15 & 963.8 & 26.39 & 937.8 & 27.01 & 961.1 & 27.21 & 965.1 & 26.3 & 962 & 28.19 & 940.9 & 27.81 & 941.3 & 28.49 & 965.5 & 28.31 & 970.4 \\
\hline 25.65 & 935.1 & 25.94 & 908.7 & 26.39 & 936.9 & 26.47 & 933.4 & 25.59 & 936 & 27.45 & 914.9 & 27.18 & 907.4 & 27.81 & 940.4 & 27.66 & 940.9 \\
\hline 25.08 & 912.2 & 25.23 & 883.1 & 25.88 & 913.1 & 26.03 & 906.9 & 25.02 & 908.7 & 26.68 & 883.1 & 26.5 & 878.7 & 27.3 & 915.3 & 27.01 & 914.9 \\
\hline 24.7 & 883.6 & 25.02 & 856.7 & 25.17 & 880.9 & 25.5 & 883.1 & 24.52 & 881.8 & 26.09 & 856.7 & 25.91 & 852.7 & 26.71 & 885.8 & 26.33 & 880 \\
\hline 24.2 & 858.9 & 24.64 & 832 & 24.7 & 852.7 & 25.02 & 855.8 & 23.87 & 852.7 & 25.53 & 828.5 & 25.32 & 828.9 & 26.12 & 860.7 & 25.82 & 855.4 \\
\hline 23.46 & 826.7 & 23.63 & 799 & 24.05 & 827.2 & 24.43 & 830.7 & 23.46 & 826.3 & 24.79 & 805.1 & 24.82 & 802.5 & 25.26 & 827.6 & 25.02 & 827.6 \\
\hline 22.86 & 802.5 & 23.31 & 773.4 & 23.43 & 796.8 & 23.96 & 804.7 & 22.92 & 800.3 & 24.08 & 772.1 & 24.17 & 777.8 & 24.7 & 802.5 & 24.52 & 802.9 \\
\hline 22.42 & 773.8 & 22.6 & 747 & 22.89 & 772.5 & 22.98 & 771.6 & 22.39 & 775.6 & 23.57 & 745.6 & 23.34 & 744.8 & 24.05 & 773.8 & 23.93 & 776 \\
\hline 21.95 & 750 & 22.06 & 723.2 & 22.42 & 747.4 & 22.51 & 744.8 & 21.95 & 750 & 22.8 & 719.6 & 22.69 & 717.9 & 23.48 & 750.5 & 23.1 & 742.6 \\
\hline 21.15 & 717 & 21.15 & 689.7 & 21.8 & 722.3 & 22.03 & 720.1 & 21.06 & 713.9 & 22.12 & 693.2 & 22.12 & 690.5 & 22.8 & 724.5 & 22.6 & 718.8 \\
\hline 20.73 & 690.5 & 20.85 & 667.6 & 21.29 & 695.4 & 21.38 & 695.4 & 20.64 & 689.7 & 21.71 & 664.1 & 21.41 & 666.3 & 22.27 & 696.7 & 21.98 & 691.9 \\
\hline 20.17 & 665.9 & 20.64 & 635.9 & 20.58 & 663.7 & 20.56 & 662.3 & 20.05 & 661.5 & 21.03 & 640.3 & 20.7 & 635.9 & 21.59 & 669.8 & 21.18 & 663.7 \\
\hline 19.76 & 640.3 & 19.79 & 608.6 & 19.96 & 635.9 & 20.17 & 635 & 19.58 & 636.3 & 20.23 & 607.3 & 20.11 & 609.9 & 20.7 & 636.8 & 20.76 & 634.6 \\
\hline 19.25 & 615.2 & 19.16 & 583.5 & 19.49 & 612.1 & 19.55 & 610.3 & 19.08 & 609.9 & 19.67 & 583 & 19.67 & 580.4 & 20.08 & 607.3 & 20.14 & 612.1 \\
\hline 18.57 & 581.7 & 18.75 & 558.8 & 18.75 & 581.7 & 18.87 & 582.6 & 18.31 & 579.9 & 19.02 & 556.6 & 19.05 & 555.3 & 19.55 & 583.9 & 19.31 & 580.8 \\
\hline 17.95 & 554.4 & 18.13 & 525.3 & 18.1 & 555.7 & 18.25 & 551.3 & 17.86 & 553.9 & 18.63 & 533.2 & 18.45 & 529.7 & 19.05 & 555.7 & 18.63 & 555.7 \\
\hline 17.51 & 531 & 18.63 & 501.1 & 17.48 & 525.7 & 17.71 & 524.9 & 17.42 & 527.5 & 17.54 & 500.2 & 17.8 & 504.1 & 18.22 & 530.1 & 17.95 & 528.8 \\
\hline 16.86 & 506.8 & 18.25 & 472.4 & 16.94 & 500.6 & 17 & 500.2 & 16.83 & 498.4 & 17.15 & 473.3 & 17.06 & 478.1 & 17.77 & 506.3 & 17.45 & 501.1 \\
\hline 16.35 & 473.3 & 17.98 & 449.5 & 16.65 & 477.3 & 16.56 & 474.2 & 16.29 & 469.8 & 16.41 & 448.2 & 16.62 & 443.3 & 16.8 & 473.3 & 16.86 & 476.4 \\
\hline 15.58 & 447.7 & 17.33 & 419.1 & 15.79 & 441.6 & 16.23 & 448.6 & 15.64 & 445.5 & 15.97 & 423.9 & 15.79 & 417.8 & 16.38 & 446 & 16.23 & 446.9 \\
\hline 15.32 & 420 & 17.15 & 392.2 & 15.23 & 418.2 & 15.44 & 422.2 & 15.29 & 419.1 & 15.23 & 390.4 & 15.17 & 390.9 & 15.61 & 421.3 & 15.7 & 420.4 \\
\hline 14.64 & 395.3 & 16.38 & 368 & 14.72 & 389.1 & 14.61 & 387.4 & 14.49 & 392.6 & 14.43 & 364.4 & 14.64 & 365.3 & 15.32 & 395.3 & 15.05 & 394 \\
\hline 14.49 & 371.5 & 16.2 & 343.3 & 14.07 & 365.8 & 14.34 & 363.6 & 14.07 & 362.2 & 13.96 & 339.8 & 13.75 & 332.7 & 14.4 & 367.5 & 14.43 & 363.1 \\
\hline 13.39 & 337.1 & 15.58 & 308.5 & 13.66 & 334.5 & 13.87 & 337.1 & 13.54 & 336.2 & 13.51 & 309.4 & 13.33 & 315.1 & 13.84 & 342.9 & 13.6 & 334.5 \\
\hline 13.13 & 310.2 & 14.96 & 283.4 & 13.01 & 305.8 & 13.42 & 312.9 & 13.01 & 312 & 12.92 & 284.7 & 12.8 & 280.7 & 13.36 & 310.7 & 12.92 & 311.1 \\
\hline 12.62 & 286 & 14.72 & 260 & 12.42 & 279.8 & 12.42 & 281.6 & 12.3 & 278.1 & 12.09 & 252.5 & 12.24 & 256 & 12.71 & 284.2 & 12.42 & 281.2 \\
\hline 12.18 & 259.1 & 14.34 & 235.8 & 12.06 & 255.2 & 11.8 & 253.8 & 11.62 & 254.7 & 11.8 & 237.1 & 11.49 & 231.4 & 12.15 & 256.9 & 11.77 & 255.6 \\
\hline
\end{tabular}


Table 14. $50{ }^{\circ} \mathrm{C}$ at 13 wt $\%$

\begin{tabular}{|c|c|c|c|c|c|c|c|c|c|c|c|c|c|c|c|c|c|}
\hline run1 & & run2 & & run3 & & run4 & & run5 & & run6 & & run7 & & run8 & & run9 & \\
\hline [Pa] & {$[1 / s]$} & [Pa] & {$[1 / s]$} & [Pa] & {$[1 / s]$} & [Pa] & {$[1 / s]$} & [Pa] & {$[1 / s]$} & [Pa] & [1/s] & [Pa] & {$[1 / \mathrm{s}]$} & [Pa] & {$[1 / \mathrm{s}]$} & [Pa] & {$[1 / \mathrm{s}]$} \\
\hline 12.28 & 273.2 & 11.8 & 276.3 & 11.04 & 275 & 11.07 & 275.4 & 10.64 & 277.6 & 10.76 & 275 & 10.89 & 273.7 & 10.75 & 273.7 & 10.57 & 271 \\
\hline 12.8 & 296.6 & 12.2 & 296.1 & 11.53 & 290.9 & 1.44 & 294.8 & 11.42 & 297 & 10.96 & 292.2 & 11.15 & 292.6 & 11.09 & 295.3 & 11.07 & 293.9 \\
\hline 13.49 & 326.5 & 12.32 & 315.1 & 12.12 & 323.9 & 11.81 & 318.6 & 11.7 & 321.7 & 11.74 & 322.6 & 11.6 & 319.5 & 11.33 & 320.8 & 11.27 & 322.1 \\
\hline 13.67 & 353 & 12.7 & 348.1 & 12.55 & 348.6 & 2.21 & 351.7 & 11.91 & 351.2 & 12.01 & 349 & 11.91 & 343.3 & 11.53 & 345.9 & 11.74 & 345.5 \\
\hline 13.99 & 376.3 & 13.3 & 376.8 & 12.79 & 378.5 & 12.71 & 379.4 & 12.56 & 378.1 & 12.55 & 378.5 & 12.51 & 376.8 & 12.29 & 379.9 & 12.29 & 380.8 \\
\hline 14.59 & 400.6 & 13.56 & 399.7 & 13.38 & 405.4 & 13.01 & 405 & 12.8 & 402.8 & 12.96 & 403.2 & 12.72 & 404.5 & 12.59 & 405 & 12.82 & 404.5 \\
\hline 15.16 & 432.3 & 14.19 & 433.2 & 13.63 & 431.4 & 13.47 & 429.7 & 13.35 & 427.5 & 13.26 & 428.3 & 13.3 & 429.2 & 12.98 & 433.2 & 13.01 & 431.9 \\
\hline 15.42 & 457 & 14.53 & 456.5 & 14.1 & 457 & 14.13 & 463.2 & 13.94 & 462.7 & 13.86 & 462.3 & 13.46 & 453.5 & 13.5 & 456.5 & 13.62 & 453.9 \\
\hline 15.87 & 486.1 & 14.9 & 486.1 & 14.57 & 487.8 & 14.44 & 489.6 & 14.08 & 485.2 & 14.05 & 486.5 & 13.94 & 483.4 & 14.09 & 488.3 & 14.11 & 487.8 \\
\hline 16.47 & 509.9 & 15.24 & 511.6 & 14.97 & 515.6 & 14.75 & 513.4 & 14.47 & 509.9 & 14.53 & 515.6 & 14.33 & 511.6 & 14.43 & 514.3 & 14.64 & 514.3 \\
\hline 16.9 & 542.5 & 15.6 & 537.6 & & 539.4 & & & & 542 & & & & & & 541.2 & & 542.5 \\
\hline 17.24 & 569.4 & 16.11 & 561 & 15.97 & 572 & 15.66 & 568.5 & 15.53 & 571.1 & 15.47 & 569.8 & 15.41 & 567.2 & 15.08 & 564.5 & 15.19 & 568 \\
\hline 17.74 & 594.9 & 16.78 & 593.6 & 16.26 & 587.9 & 16.03 & 591.8 & 15.93 & 594.5 & 15.89 & 597.1 & 15.78 & 592.7 & 15.61 & 591.8 & 15.74 & 595.8 \\
\hline 18.25 & 621.4 & 17.06 & 621.4 & 16.88 & 623.6 & 16.6 & 624.9 & 16.25 & 618.7 & 16.25 & 624.9 & 16.21 & 624 & 16.1 & 624 & 16.27 & 620.9 \\
\hline 18.51 & 646.5 & 17.42 & 647.4 & 17.2 & 648.7 & 17.19 & 651.8 & 16.7 & 653.1 & 16.73 & 650.4 & 16.68 & 647.8 & 16.46 & 652.2 & 16.66 & 645.6 \\
\hline 19.1 & 678.2 & 17.86 & 672 & 17.6 & 676.4 & & 675.6 & 17.24 & 680 & 17.17 & 680 & 17.04 & 675.1 & 16.91 & 676.9 & 17.24 & 677.8 \\
\hline 19.61 & 706.4 & 18.16 & 697.6 & 17.92 & 699.4 & 17.92 & 706.9 & 17.57 & 705.1 & 17.54 & 706.4 & 17.42 & 698.5 & 17.24 & 701.6 & 17.48 & 702 \\
\hline 19.9 & 730.2 & & 731.1 & & 727.6 & & 732 & 18.1 & 731.1 & 17.98 & 732.4 & 17.98 & 732.4 & 17.8 & 733.7 & 17.89 & 732.4 \\
\hline 20.5 & 759.3 & 19.25 & 757.5 & 18.99 & 761.1 & 8.39 & 744.8 & 18.37 & 755.8 & 18.28 & 755.8 & 18.45 & 758 & 18.25 & 757.1 & 18.39 & 754 \\
\hline 20.88 & 785.3 & .73 & 784.9 & 9.31 & 787.1 & 25 & 785.3 & .69 & 781.3 & 18.72 & 78 & 18.84 & 786.2 & .54 & 787.5 & 18.81 & 783.5 \\
\hline 21.18 & 810.9 & 20.11 & 811.3 & 19.82 & 814.4 & 19.19 & 789.3 & 19.22 & 807.3 & 19.31 & 815.7 & 19.1 & 809.5 & 19.02 & 812.6 & 19.19 & 808.7 \\
\hline 21.83 & 844.3 & 20.53 & 837.7 & 20.29 & 841.7 & 20.05 & 838.2 & 19.82 & 842.1 & 19.7 & 843 & 19.7 & 841.7 & 19.58 & 843.5 & 19.76 & 841.7 \\
\hline 22.3 & 868.6 & 20.94 & 864.2 & 20.67 & 867.7 & 20.53 & 858.5 & 20.23 & 868.1 & 20.08 & 868.6 & 20.08 & 868.1 & 19.84 & 860.7 & 20.08 & 869.5 \\
\hline 22.77 & 897.2 & 21.56 & 896.3 & 21.03 & 890.2 & 20.85 & 891.5 & 20.58 & 891.9 & 20.58 & 894.6 & 20.44 & 890.6 & 20.44 & 895.9 & 20.56 & 892.8 \\
\hline 23.1 & 920.1 & 21.89 & 919.7 & 21.5 & 925.4 & 21.35 & 914.4 & 21.24 & 925 & 20.97 & 919.7 & 20.85 & 915.7 & 20.76 & 921.5 & 20.97 & 921 \\
\hline 23.63 & 954.1 & 22.39 & 950.6 & 21.95 & 951.9 & 21.89 & 947.5 & 21.35 & 943.1 & 21.5 & 951 & 21.41 & 949.7 & 21.21 & 949.2 & 21.27 & 946.1 \\
\hline 24.17 & 977.9 & 22.86 & 975.7 & 22.36 & 977 & 22.3 & 971.7 & & 980.1 & 21.8 & 975.7 & 21.77 & 974.8 & 21.62 & 974.3 & 21.71 & 973 \\
\hline 24.55 & 1006 & 23.19 & 1003 & 22.83 & 1001 & 22.77 & 1006 & 22.48 & 1004 & 22.21 & 1002 & 22.27 & 1001 & 22.03 & 1000 & 22.3 & 1007 \\
\hline 24.88 & 1032 & 23.6 & 1028 & 23.28 & 1033 & 23.28 & 1033 & 22.95 & 1033 & 22.75 & 1030 & 22.69 & 1026 & 22.63 & 1033 & 22.69 & 1033 \\
\hline 25.38 & 1056 & 24.02 & 1054 & 23.63 & 1060 & 23.69 & 1057 & 23.28 & 1056 & 23.16 & 1054 & 23.28 & 1058 & 23.04 & 1062 & 23.16 & 1062 \\
\hline 25.88 & 1089 & 24.58 & 1087 & 24.14 & 1083 & 24.31 & 1089 & 23.63 & 1081 & 23.51 & 1075 & 23.72 & 1087 & 23.37 & 1084 & 23.51 & 1088 \\
\hline
\end{tabular}


Table $14.50{ }^{\circ} \mathrm{C}$ at 13 wt $\%$ - continued

\begin{tabular}{|c|c|c|c|c|c|c|c|c|c|c|c|c|c|c|c|c|c|}
\hline run1 & & run2 & & run3 & & run4 & & run5 & & run6 & & run7 & & run8 & & run9 & \\
\hline [Pa] & [1/s] & [Pa] & [1/s] & [Pa] & [1/s] & [Pa] & [1/s] & [Pa] & [1/s] & [Pa] & [1/s] & [Pa] & [1/s] & [Pa] & {$[1 / s]$} & [Pa] & {$[1 / s]$} \\
\hline 26.27 & 1115 & 25.23 & 1112 & 24.52 & 1109 & 24.7 & 1117 & 24.31 & 1117 & 24.11 & 1108 & 24.05 & 1110 & 24.02 & 1116 & 24.02 & 1115 \\
\hline 26.65 & 1138 & 25.53 & 1139 & 25.08 & 1143 & 25.11 & 1141 & 24.7 & 1141 & 24.37 & 1131 & 24.46 & 1134 & 24.34 & 1141 & 24.22 & 1137 \\
\hline 27.18 & 1170 & 25.85 & 1163 & 25.44 & 1170 & 25.47 & 1167 & 25.17 & 1169 & 25.2 & 1164 & 25.08 & 1166 & 24.67 & 1164 & 24.91 & 1169 \\
\hline 27.72 & 1196 & 26.33 & 1194 & 25.82 & 1193 & 25.91 & 1191 & 25.47 & 1192 & 25.47 & 1195 & 25.38 & 1194 & 25.26 & 1196 & 25.23 & 1195 \\
\hline 28.1 & 1225 & 26.74 & 1221 & 26.39 & 1224 & 26.53 & 1222 & 26.09 & 1224 & 25.88 & 1218 & 25.82 & 1218 & 25.67 & 1222 & 25.62 & 1222 \\
\hline 28.37 & 1247 & 27.12 & 1244 & 26.77 & 1252 & 26.95 & 1250 & 26.5 & 1247 & 26.21 & 1241 & 26.41 & 1251 & 26.15 & 1251 & 26.27 & 1248 \\
\hline 28.81 & 1274 & 27.75 & 1278 & 27.15 & 1278 & 27.36 & 1278 & 26.92 & 1275 & 26.68 & 1272 & 26.65 & 1269 & 26.44 & 1275 & 26.59 & 1279 \\
\hline 29.34 & 1305 & 28.1 & 1304 & 27.6 & 1305 & 27.86 & 1306 & 27.51 & 1305 & 27.24 & 1305 & 27.24 & 1302 & 27.04 & 1307 & 27.01 & 1305 \\
\hline 29.82 & 1334 & 28.37 & 1324 & 28.01 & 1329 & 28.31 & 1334 & 27.78 & 1334 & 27.72 & 1331 & 27.72 & 1330 & 27.42 & 1334 & 27.36 & 1334 \\
\hline 30.14 & 1357 & 28.99 & 1359 & 28.58 & 1360 & 28.72 & 1358 & 28.28 & 1360 & 27.95 & 1351 & 28.1 & 1355 & 27.75 & 1359 & 27.86 & 1359 \\
\hline 30.56 & 1383 & 29.4 & 1386 & 28.96 & 1388 & 29.08 & 1382 & 28.69 & 1386 & 28.63 & 1385 & 28.58 & 1379 & 28.19 & 1383 & 28.19 & 1382 \\
\hline 30.94 & 1409 & 29.76 & 1408 & 29.4 & 1411 & 29.73 & 1416 & & 1411 & & 1412 & 29.26 & 1413 & 28.75 & 1415 & 28.58 & 1408 \\
\hline 31.56 & 1443 & 30.35 & 1442 & 29.73 & 1436 & 30.03 & 1442 & 29.49 & 1437 & 29.34 & 1436 & 29.52 & 1438 & 29.55 & 1442 & 29.11 & 1438 \\
\hline 31.92 & 1470 & 30.71 & 1469 & 30.32 & 1467 & 30.44 & 1466 & 30.05 & 1470 & 29.88 & 1466 & 30.05 & 1466 & 29.73 & 1469 & 29.55 & 1466 \\
\hline 32.33 & 1497 & 31.18 & 1494 & 30.74 & 1496 & 30.79 & 1491 & 30.26 & 1486 & 30.32 & 1496 & 30.41 & 1490 & 30.14 & 1493 & 29.94 & 1490 \\
\hline 31.36 & 1535 & 30.88 & 1535 & 30.41 & 1537 & 30.47 & 1536 & 29.82 & 1508 & 30.94 & 1544 & 30.11 & 1544 & 29.94 & 1542 & 29.82 & 1536 \\
\hline 30.94 & 1510 & & 1510 & 29.94 & 1510 & 29.97 & 1512 & 29.43 & 1483 & 41 & 1512 & 29.49 & 1509 & 29.31 & 1508 & 29.37 & 1512 \\
\hline 30.44 & 1485 & 29.97 & 1482 & 29.58 & 1485 & 29.64 & 1487 & 28.87 & 1454 & 29.85 & 1484 & 29.08 & 1481 & 28.93 & 1482 & 28.93 & 1486 \\
\hline 29.94 & 1453 & 29.76 & 1459 & 29.08 & 1454 & 29.02 & 1453 & 28.46 & 1426 & 43 & 1461 & 28.63 & 1455 & 28.49 & 1456 & 28.37 & 1453 \\
\hline 29.49 & 1426 & 29.11 & 1426 & 28.6 & 1427 & 28.69 & 1431 & 28.04 & 1400 & 28.99 & 1432 & 28.25 & 1430 & 28.07 & 1430 & 27.95 & 1425 \\
\hline 29.17 & 1402 & 28.66 & 1400 & 28.22 & 1402 & 28.16 & 1401 & 27.51 & 1372 & 28.63 & 1405 & 27.81 & 1405 & 27.72 & 1405 & 27.6 & 1400 \\
\hline 28.72 & 1376 & 28.25 & 1376 & 27.75 & 1376 & 27.78 & 1374 & 27.12 & 1346 & 28.1 & 1379 & 27.3 & 1373 & 27.1 & 1371 & 27.12 & 1375 \\
\hline 28.25 & 1346 & 27.6 & 1344 & 27.39 & 1352 & 27.39 & 1351 & 26.74 & 1317 & 27.48 & 1347 & 26.77 & 1348 & 26.71 & 1347 & 26.71 & 1348 \\
\hline 27.78 & 1318 & 27.3 & 1320 & 26.74 & 1319 & 26.8 & 1320 & 26.3 & 1292 & 27.27 & 1320 & 26.44 & 1319 & 26.3 & 1318 & 26.21 & 1316 \\
\hline 27.36 & 1294 & 26.83 & 1293 & 26.36 & 1292 & 26.44 & 1295 & 25.76 & 1267 & 26.59 & 1288 & 26.06 & 1296 & 25.88 & 1293 & 25.76 & 1289 \\
\hline 26.86 & 1263 & 26.47 & 1268 & 26.03 & 1268 & 25.94 & 1263 & 25.44 & 1241 & 26.09 & 1270 & 25.53 & 1265 & 25.41 & 1265 & 25.38 & 1265 \\
\hline 26.39 & 1236 & 25.82 & 1236 & 25.56 & 1243 & 25.38 & 1237 & 24.79 & 1209 & 25.7 & 1241 & 25.02 & 1237 & 24.99 & 1242 & 24.94 & 1242 \\
\hline 26 & 1212 & 25.47 & 1210 & 24.99 & 1210 & 24.96 & 1207 & 24.46 & 1184 & 25.29 & 1214 & 24.67 & 1211 & 24.46 & 1210 & 24.43 & 1210 \\
\hline 25.44 & 1181 & 25.08 & 1186 & 24.58 & 1181 & 24.52 & 1183 & 23.84 & 1154 & 24.85 & 1187 & 24.28 & 1188 & 24.02 & 1181 & 24.08 & 1181 \\
\hline 24.96 & 1155 & 24.52 & 1153 & 24.22 & 1157 & 24.08 & 1154 & 23.54 & 1127 & 24.49 & 1164 & 23.72 & 1155 & 23.63 & 1158 & 23.57 & 1157 \\
\hline 24.46 & 1126 & 24.14 & 1127 & 23.66 & 1128 & 23.66 & 1130 & 23.13 & 1104 & 23.96 & 1130 & 23.37 & 1131 & 23.19 & 1133 & 23.19 & 1129 \\
\hline
\end{tabular}


Table $14.50{ }^{\circ} \mathrm{C}$ at 13 wt $\%$ - continued

\begin{tabular}{|c|c|c|c|c|c|c|c|c|c|c|c|c|c|c|c|c|c|}
\hline run1 & & run2 & & run3 & & run4 & & run5 & & run6 & & run7 & & run8 & & run9 & \\
\hline [Pa] & {$[1 / s]$} & [Pa] & {$[1 / \mathrm{s}]$} & [Pa] & [1/s] & [Pa] & {$[1 / s]$} & [Pa] & {$[1 / \mathrm{s}]$} & [Pa] & {$[1 / \mathrm{s}]$} & [Pa] & {$[1 / s]$} & [Pa] & {$[1 / \mathrm{s}]$} & [Pa] & {$[1 / s]$} \\
\hline 24.05 & 1102 & 23.66 & 1103 & 23.28 & 1104 & 23.13 & 1096 & 22.6 & 1071 & 23.46 & 1103 & 22.83 & 1101 & 22.86 & 1104 & 22.63 & 1095 \\
\hline 23.48 & 1070 & 3.07 & 1071 & 22.86 & 1074 & 22.75 & 1070 & 22.12 & 1044 & 23.1 & 1075 & 22.45 & 1075 & 22.42 & 1077 & 22.24 & 1071 \\
\hline 23.1 & 1044 & 22.72 & 1047 & 22.36 & 1046 & 22.3 & 1046 & 21.71 & 1017 & 22.6 & 1051 & 21.86 & 1044 & 21.92 & 1050 & 21.83 & 1045 \\
\hline 22.66 & 1016 & 22.3 & 1018 & 21.92 & 1018 & 21.89 & 1017 & 21.32 & 991.1 & 22.12 & 1024 & 21.44 & 1018 & 21.38 & 1016 & 21.38 & 1017 \\
\hline 22.27 & 992.4 & 21.89 & 992.4 & 21.5 & 991.5 & 21.53 & 995.1 & 20.82 & 964.7 & 21.65 & 996.4 & 21.06 & 990.7 & 20.97 & 988 & 20.94 & 991.5 \\
\hline 21.95 & 969.1 & 21.53 & 968.2 & 21.12 & 970.8 & 21.15 & 969.5 & 20.47 & 940.9 & 21.24 & 970.8 & 20.7 & 967.7 & 20.56 & 964.7 & 20.47 & 959.8 \\
\hline 21.21 & 934.7 & 20.91 & 936 & 20.64 & 938.7 & 20.44 & 933.4 & 19.96 & 906.9 & 20.67 & 939.1 & 20.29 & 941.7 & 20.14 & 936.9 & 20.14 & 940.9 \\
\hline 20.94 & 907.4 & 20.47 & 908.7 & 20.11 & 910.9 & 20.02 & 909.1 & 19.55 & 881.8 & 20.2 & 912.2 & 19.79 & 914 & 19.79 & 913.1 & 19.58 & 906.9 \\
\hline 20.53 & 884 & 20.05 & 882.2 & 19.73 & 887.1 & 19.58 & 880.9 & 19.13 & 856.7 & 19.76 & 888.4 & 19.46 & 890.2 & 19.34 & 887.1 & 19.19 & 883.1 \\
\hline 19.93 & 854 & 19.55 & 855.8 & 19.16 & 855.8 & 9.28 & 858 & 18.72 & 832 & 19.22 & 858 & 19.02 & 862.4 & & 854.5 & & 858.5 \\
\hline 19.46 & 827.2 & 19.16 & 831.1 & 18.81 & 827.2 & 18.72 & 827.6 & 18.16 & 799.4 & 18.84 & 832 & 18.42 & 828 & 18.37 & 828 & 18.25 & 825.8 \\
\hline 19.25 & 807.8 & 18.78 & 805.1 & 18.37 & 801.2 & 18.31 & 800.7 & 17.71 & 771.6 & 18.28 & 801.2 & 17.98 & 798.5 & 17.89 & 798.5 & 17.83 & 798.5 \\
\hline 18.57 & 773.4 & 18.28 & 771.2 & 17.92 & 776 & 17.92 & 774.3 & 17.33 & 747 & 17.83 & 772.5 & 17.54 & 775.6 & 17.51 & 773.8 & 17.45 & 772.5 \\
\hline 18.13 & 745.6 & 17.68 & 747 & 17.57 & 750.5 & 17.42 & 748.3 & 16.91 & 720.5 & 17.24 & 747 & 17.09 & 747 & 17.15 & 749.2 & 17.12 & 747 \\
\hline 17.83 & 721.8 & 17.21 & 717.4 & 16.89 & 717.9 & 16.97 & 719.6 & 16.35 & 689.7 & 17 & 720.5 & 16.62 & 719.2 & 16.68 & 721.4 & 16.41 & 714.8 \\
\hline 17.36 & 697.6 & 17.03 & 693.2 & 16.56 & 690.5 & 16.56 & 695 & 15.88 & 662.8 & 6.59 & 696.3 & 16.32 & 693.6 & 16.12 & 688.8 & 16.09 & 691.4 \\
\hline 16.86 & 676 & & 665 & & 666.8 & & 661.9 & & 639 & 15.97 & & 15.85 & 666.8 & .82 & 665.9 & & 664.5 \\
\hline 16.65 & 655.7 & 16.12 & 637.2 & 15.7 & 636.3 & 15.55 & 637.2 & 15.08 & 611.2 & 15.67 & 641.2 & 15.55 & 642.1 & 15.32 & 635.9 & 15.29 & 635 \\
\hline 15.91 & 613.9 & 15.58 & 612.1 & 15.29 & 611.7 & 5.08 & 609.9 & 14.61 & 585.7 & 15.02 & 9.5 & 14.93 & 608.1 & .99 & 611.2 & 14.9 & 609.9 \\
\hline 15.94 & 602 & 15.17 & 580.4 & 14.87 & 587 & 4.84 & 581.7 & 14.22 & 559.2 & 14.58 & 585.7 & 14.61 & 582.1 & 14.64 & 586.5 & 14.37 & 582.1 \\
\hline 15.11 & 561.4 & 14.72 & 555.3 & 14.46 & 553.9 & 14.34 & 557 & 13.78 & 534.1 & 14.25 & 558.3 & 14.07 & 561 & 14.22 & 561.4 & 14.1 & 559.2 \\
\hline 14.55 & 531.9 & 14.37 & 526.6 & 14.07 & 528.8 & 13.99 & 530.1 & 13.33 & 498.9 & 13.81 & 528.8 & 13.66 & 527.5 & 13.51 & 526.2 & 13.48 & 526.6 \\
\hline 14.34 & 509 & 13.87 & 504.1 & 13.72 & 500.2 & 13.42 & 498.4 & 12.92 & 471.5 & 13.45 & 501.9 & 13.27 & 500.6 & 13.04 & 499.7 & 13.04 & 498.4 \\
\hline 13.9 & 470.2 & 13.45 & 478.1 & 13.07 & 474.2 & 13.16 & 471.1 & 12.4 & 446 & 12.92 & 476.4 & 12.83 & 475.1 & 12.83 & 475.5 & 12.83 & 475.9 \\
\hline 13.3 & 446.4 & 12.8 & 445.1 & 12.68 & 447.7 & 2.57 & 446 & 12.23 & 420.4 & 12.53 & 451.7 & 12.34 & 446.4 & 12.29 & 442.9 & 12.21 & 442.9 \\
\hline 12.89 & 417.8 & 12.62 & 418.6 & 12.43 & 423.5 & 12.36 & 422.2 & 11.53 & 393.5 & 12.15 & 419.5 & 11.95 & 420.4 & 11.87 & 417.8 & 11.94 & 416 \\
\hline 12.66 & 390.4 & 12.13 & 395.3 & 11.74 & 391.3 & 11.75 & 395.3 & 11.35 & 367.5 & 11.56 & 393.1 & 11.58 & 392.6 & 11.59 & 392.2 & 11.6 & 394 \\
\hline 12.37 & 367.5 & 11.85 & 364 & 11.51 & 363.6 & 11.55 & 370.6 & 11 & 340.6 & 11.38 & 366.2 & 11.24 & 367.5 & 11.02 & 367.5 & 11.02 & 362.2 \\
\hline 11.56 & 335.4 & 11.37 & 338 & 10.95 & 336.7 & 10.76 & 334.9 & 10.25 & 306.7 & 10.87 & 340.2 & 10.7 & 342.4 & 10.75 & 335.8 & 10.77 & 334.5 \\
\hline 11.39 & 309.4 & 10.96 & 315.1 & 10.52 & 310.7 & 10.53 & 308.9 & 10.01 & 282.9 & 10.35 & 316 & 10.39 & 309.4 & 10.32 & 310.2 & 10.41 & 310.7 \\
\hline 11.08 & 283.4 & 10.68 & 280.3 & 10.28 & 282 & 10.31 & 279.4 & 9.584 & 256.5 & 9.993 & 288.6 & 10.1 & 282 & 9.857 & 280.7 & 10.06 & 286 \\
\hline 10.76 & 255.2 & 10.34 & 254.3 & 9.869 & 259.1 & 9.951 & 256 & 9.306 & 231.4 & 9.658 & 263.1 & 9.818 & 258.7 & 9.614 & 256.5 & 9.422 & 252.5 \\
\hline
\end{tabular}


Table 15. Rheometer Response for Blank - $16 \mathrm{wt} \%$

\begin{tabular}{|c|c|c|c|c|c|c|c|c|c|c|c|c|c|c|c|c|c|}
\hline run1 & & run2 & & run3 & & run4 & & run5 & & run6 & & run7 & & run8 & & run9 & \\
\hline [Pa] & {$[1 / s]$} & [Pa] & {$[1 / \mathrm{s}]$} & [Pa] & [1/s] & [Pa] & [1/s] & [Pa] & [1/s] & [Pa] & [1/s] & [Pa] & [1/s] & [Pa] & [1/s] & [Pa] & {$[1 / s]$} \\
\hline 2.399 & 274.1 & 2.562 & 271.9 & 2.473 & 275.4 & 2.455 & 278.5 & 1.955 & 302.3 & 2.174 & 297 & 2.497 & 268.8 & 2.224 & 276.3 & 2.13 & 298.8 \\
\hline 2.784 & 294.8 & .784 & 294.4 & 2.716 & 293.1 & 2.642 & 293.5 & 2.615 & 286 & 2.642 & 290.9 & 2.698 & 289.5 & 2.701 & 300.5 & 2.618 & 288.6 \\
\hline 2.858 & 311.6 & 2.896 & 323.9 & 2.964 & 320.4 & 2.94 & 323.9 & 2.763 & 313.3 & 2.943 & 324.3 & 2.813 & 312.9 & 2.834 & 322.1 & 2.757 & 312.9 \\
\hline 3.236 & 346.4 & 3.103 & 349.9 & 3.044 & 349.9 & 3.047 & 349.9 & 3.142 & 351.2 & 3.118 & 350.3 & 2.938 & 338.9 & 2.929 & 349.9 & 2.911 & 336.7 \\
\hline 3.148 & 354.3 & 3.222 & 372.8 & 3.207 & 374.6 & 3.239 & 379.9 & 3.266 & 375 & 3.272 & 371.9 & 3.091 & 359.6 & 3.103 & 371.9 & 3.05 & 360.5 \\
\hline 3.464 & 400.1 & 3.352 & 397.1 & 3.411 & 398.8 & 3.381 & 401.9 & 3.396 & 401.9 & 3.408 & 396.2 & 3.109 & 394 & 3.26 & 394.9 & 3.305 & 408.5 \\
\hline 3.396 & 412.5 & 3.544 & 431.9 & 3.615 & 432.3 & 3.482 & 429.7 & 3.497 & 424.8 & 3.58 & 421.7 & 3.417 & 430.5 & 3.396 & 421.7 & 3.423 & 435 \\
\hline 3.701 & 439.8 & 3.737 & 457.9 & 3.742 & 454.8 & 3.606 & 458.8 & 3.6 & 446.4 & 3.719 & 444.2 & 3.541 & 456.1 & 3.473 & 442.4 & 3.55 & 455.2 \\
\hline 3.973 & 479.5 & 3.858 & 483.9 & 3.953 & 480.8 & 3.757 & 482.5 & 3.592 & 479.9 & 3.71 & 478.6 & 3.692 & 479.5 & 3.497 & 477.3 & 3.704 & 481.2 \\
\hline 4.047 & 513.8 & 3.947 & 513 & 4.169 & 511.6 & 3.973 & 508.5 & 3.677 & 503.7 & 3.964 & 515.6 & 3.816 & 503.7 & 3.834 & 513.4 & 3.748 & 503.3 \\
\hline 4.118 & 537.6 & 4.151 & 542.5 & 4.323 & 536.8 & 4.092 & 533.7 & 4.035 & 542 & 4.041 & 539.4 & 3.932 & 528.4 & 3.861 & 540.3 & 3.876 & 525.7 \\
\hline 4.249 & 561.9 & & 567.2 & 4.559 & 565 & 4.219 & 567.2 & & 567.2 & & 559.7 & & 560.5 & 3.95 & 560.1 & & 552.6 \\
\hline 4.426 & 596.7 & 4.388 & 592.7 & 4.84 & 596.2 & 4.34 & 596.2 & 4.216 & 589.6 & 4.257 & 583 & 4.266 & 600.7 & 4.121 & 588.3 & 4.213 & 598.9 \\
\hline 4.542 & 620.5 & 4.55 & 617.4 & 4.956 & 620 & 4.488 & 620 & 4.326 & 614.8 & 4.373 & 611.2 & 4.352 & 623.6 & 4.249 & 610.8 & 4.305 & 625.3 \\
\hline 4.728 & 646.5 & 4.604 & 647.8 & 5.095 & 646.5 & 4.598 & 643.8 & 4.411 & 639.9 & 4.473 & 634.1 & 4.429 & 647.8 & 4.337 & 634.6 & 4.497 & 646 \\
\hline 4.911 & 678.2 & 4.728 & 674.2 & 5.19 & 672.9 & 4.716 & 678.2 & 4.473 & 660.6 & 4.462 & 669 & 4.429 & 672.5 & 4.373 & 669 & 4.583 & 670.7 \\
\hline 4.988 & 707.3 & & 698.9 & 5.296 & 706 & & 704.2 & 4.725 & 711.3 & 4.781 & 707.3 & 4.642 & 695.4 & 4.648 & 706 & 4.681 & 696.7 \\
\hline 5.083 & 732.4 & 4.974 & 732 & 5.385 & 729.8 & 4.971 & 732.4 & 4.817 & 733.3 & 4.962 & 728.4 & 4.775 & 720.5 & 4.755 & 730.2 & 4.82 & 718.8 \\
\hline 5.27 & 761.5 & 5.006 & 757.5 & 5.456 & 758 & 5.077 & 753.6 & 4.9 & 754.4 & 4.983 & 754.9 & 4.781 & 753.1 & 4.873 & 753.1 & 4.903 & 752.2 \\
\hline 5.329 & 785.7 & 5.11 & 781.3 & 5.515 & 783.1 & 5.202 & 786.2 & 5.056 & 781.8 & 5.11 & 777.4 & 5.012 & 791.9 & 4.9 & 780 & 5.24 & 786.2 \\
\hline 5.5 & 812.2 & 5.204 & 807.8 & 5.571 & 809.5 & 5.311 & 810.4 & 5.151 & 803.4 & 5.193 & 801.2 & 5.148 & 813.9 & 5.086 & 802 & 5.358 & 810.4 \\
\hline 5.616 & 843.5 & 5.373 & 840.8 & 5.764 & 836.4 & 5.388 & 835.5 & 5.302 & 826.3 & 5.341 & 826.3 & 5.258 & 838.6 & 5.122 & 835.1 & 5.497 & 834.7 \\
\hline 5.773 & 866.8 & 5.486 & 867.3 & 5.785 & 859.8 & 5.539 & 867.3 & 5.338 & 860.7 & 5.347 & 859.3 & 5.344 & 864.6 & 5.311 & 873.9 & 5.604 & 856.2 \\
\hline 5.861 & 895 & 5.589 & 891.5 & 6.027 & 895 & 5.708 & 896.3 & 5.681 & 898.1 & 5.648 & 896.8 & 5.459 & 886.2 & 5.42 & 895 & 5.696 & 881.8 \\
\hline 5.944 & 917.9 & 5.705 & 916.2 & 6.069 & 919.3 & 5.808 & 919.7 & 5.841 & 921.9 & 5.69 & 920.1 & 5.471 & 909.6 & 5.574 & 921.5 & 5.773 & 914.9 \\
\hline 6.154 & 951.4 & 5.932 & 949.2 & 6.261 & 945.3 & 5.856 & 945.7 & 5.956 & 944.8 & 5.841 & 945.3 & 5.705 & 936 & 5.663 & 945.3 & 6.166 & 951.9 \\
\hline 6.225 & 979.2 & 6.024 & 977.4 & 6.4 & 976.1 & 6.08 & 976.6 & 5.974 & 969.5 & 5.932 & 971.3 & 5.731 & 969.1 & 5.779 & 968.6 & 6.359 & 976.6 \\
\hline 6.285 & 1004 & 6.134 & 1000 & 6.566 & 1001 & 6.137 & 1003 & 6.119 & 992 & 6.066 & 992.4 & 5.947 & 1007 & 5.879 & 991.5 & 6.498 & 998.1 \\
\hline 6.468 & 1028 & 6.368 & 1032 & 6.652 & 1029 & 6.252 & 1027 & 6.116 & 1025 & 6.19 & 1018 & 6.033 & 1030 & 5.921 & 1025 & 6.595 & 1023 \\
\hline 6.56 & 1055 & 6.385 & 1059 & 6.788 & 1055 & 6.391 & 1060 & 6.397 & 1061 & 6.169 & 1052 & 6.246 & 1053 & 6.279 & 1064 & 6.658 & 1050 \\
\hline 6.823 & 1089 & 6.779 & 1108 & 6.945 & 1082 & 6.548 & 1087 & 6.507 & 1084 & 6.518 & 1088 & 6.225 & 1079 & 6.258 & 1089 & 6.865 & 1071 \\
\hline
\end{tabular}


Table 15. Rheometer Response for Blank - 16 wt \% - continued

\begin{tabular}{|c|c|c|c|c|c|c|c|c|c|c|c|c|c|c|c|c|c|}
\hline run1 & & run2 & & run3 & & run4 & & run5 & & run6 & & run7 & & run8 & & run9 & \\
\hline [Pa] & {$[1 / s]$} & [Pa] & {$[1 / s]$} & [Pa] & {$[1 / s]$} & [Pa] & {$[1 / s]$} & [Pa] & {$[1 / s]$} & [Pa] & {$[1 / \mathrm{s}]$} & [Pa] & {$[1 / s]$} & [Pa] & {$[1 / \mathrm{s}]$} & [Pa] & {$[1 / s]$} \\
\hline 6.962 & 1114 & 6.669 & 1116 & 7.107 & 1107 & 6.675 & 1113 & 6.729 & 1108 & 6.652 & 1112 & 6.427 & 1100 & 6.4 & 1112 & 7.03 & 1106 \\
\hline 7.007 & 1141 & 6.554 & 1126 & 7.199 & 1135 & 6.791 & 1139 & 6.838 & 1130 & 6.705 & 1136 & 6.43 & 1133 & 6.566 & 1136 & 7.359 & 1146 \\
\hline 7.122 & 1167 & 7.013 & 1147 & 7.389 & 1165 & 6.856 & 1166 & 6.856 & 1157 & 6.844 & 1159 & 6.663 & 1172 & 6.607 & 1157 & 7.457 & 1169 \\
\hline 7.223 & 1191 & 6.918 & 1181 & 7.536 & 1192 & 7.11 & 1195 & 6.983 & 1178 & 6.945 & 1182 & 6.782 & 1194 & 6.817 & 1183 & 7.599 & 1194 \\
\hline 7.341 & 1234 & 7.172 & 1220 & 7.652 & 1216 & 7.246 & 1221 & 7.205 & 1227 & 6.906 & 1216 & 6.977 & 1220 & 6.877 & 1208 & 7.726 & 1216 \\
\hline 7.279 & 1235 & 7.167 & 1231 & 7.788 & 1251 & 7.329 & 1250 & 7.32 & 1251 & 7.211 & 1254 & 7.096 & 1243 & 6.936 & 1240 & 7.915 & 1242 \\
\hline 8.072 & 1251 & 7.468 & 1274 & 7.93 & 1273 & 7.4 & 1276 & 7.397 & 1271 & 7.371 & 1276 & 7.164 & 1267 & 7.291 & 1280 & 8.004 & 1263 \\
\hline 7.957 & 1288 & 7.687 & 1299 & 8.048 & 1297 & 7.616 & 1306 & 7.551 & 1299 & 7.418 & 1304 & 7.282 & 1291 & 7.403 & 1303 & 8.134 & 1297 \\
\hline 7.972 & 1331 & 7.723 & 1333 & 8.196 & 1331 & 7.818 & 1333 & 7.705 & 1323 & 7.56 & 1328 & 7.353 & 1325 & 7.492 & 1326 & 8.602 & 1334 \\
\hline 8.122 & 1355 & 7.874 & 1355 & 8.324 & 1356 & 7.853 & 1360 & 7.806 & 1346 & 7.661 & 1353 & 7.474 & 1348 & 7.596 & 1352 & 8.664 & 1354 \\
\hline 8.341 & 1386 & 7.957 & 1386 & 8.362 & 1385 & 8.007 & 1385 & 7.753 & 1372 & 7.815 & 1376 & 7.794 & 1384 & 7.758 & 1374 & 8.791 & 1377 \\
\hline 8.566 & 1412 & 8.09 & 1413 & 8.543 & 1408 & 8.14 & 1408 & 7.88 & 1398 & 8.46 & 1400 & 7.909 & 1410 & 7.838 & 1398 & 9.052 & 1400 \\
\hline 8.676 & 1441 & 8.262 & 1442 & 8.72 & 1434 & 8.247 & 1441 & 8.001 & 1430 & 9.043 & 1425 & 7.954 & 1433 & 7.903 & 1433 & 9.214 & 1425 \\
\hline 8.806 & 1466 & 8.359 & 1468 & 8.969 & 1467 & 8.421 & 1467 & 8.333 & 1471 & 8.708 & 1458 & 8.256 & 1457 & 8.137 & 1469 & 9.22 & 1460 \\
\hline 8.871 & 1494 & 8.51 & 1496 & 9.031 & 1493 & 8.528 & 1492 & 8.383 & 1492 & 8.951 & 1497 & 8.241 & 1482 & 8.196 & 1497 & 9.502 & 1500 \\
\hline 9.004 & 1519 & 8.655 & 1519 & 9.135 & 1518 & 8.623 & 1516 & 8.516 & 1519 & 9.034 & 1521 & 8.365 & 1514 & 8.412 & 1517 & 9.65 & 1522 \\
\hline 8.048 & 1508 & 8.164 & 1534 & 8.137 & 1512 & 8.33 & 1581 & 6.101 & 1212 & 7.933 & 1541 & 7.673 & 1546 & 7.613 & 1532 & 8.401 & 1544 \\
\hline 7.844 & 1483 & 7.974 & 1510 & 7.933 & 1486 & 8.122 & 1553 & 5.992 & 1188 & 7.705 & 1506 & 7.699 & 1518 & 7.545 & 1507 & 8.46 & 1521 \\
\hline 7.797 & 1455 & 7.838 & 1483 & 7.673 & 1452 & 7.901 & 1528 & 5.844 & 1165 & 7.495 & 1478 & 7.56 & 1494 & 7.374 & 1482 & 7.903 & 1494 \\
\hline 7.658 & 1431 & 7.646 & 1457 & 7.519 & 1426 & 7.785 & 1478 & 5.826 & 1132 & 7.332 & 1453 & 7.486 & 1460 & 7.258 & 1459 & 7.522 & 1461 \\
\hline 7.51 & 1404 & 7.528 & 1432 & 7.457 & 1400 & 7.572 & 1457 & 5.447 & 1095 & 7.17 & 1431 & 7.181 & 1423 & 7.164 & 1434 & 7.223 & 1421 \\
\hline 7.326 & 1378 & 7.347 & 1399 & 7.306 & 1372 & 7.433 & 1430 & 5.317 & 1069 & 7.116 & 1404 & 7.069 & 1399 & 6.956 & 1411 & 7.007 & 1399 \\
\hline 7.134 & 1345 & 7.252 & 1373 & 7.125 & 1348 & 7.208 & 1408 & 5.275 & 1047 & 6.817 & 1382 & 7.042 & 1377 & 7.019 & 1378 & 6.927 & 1375 \\
\hline 7.042 & 1318 & 7.045 & 1343 & 6.992 & 1322 & 7.155 & 1382 & 5.113 & 1023 & 6.826 & 1358 & 6.803 & 1352 & 6.874 & 1354 & 6.764 & 1354 \\
\hline 6.829 & 1291 & 6.808 & 1319 & 6.714 & 1289 & 7.027 & 1358 & 5.03 & 997.3 & 6.749 & 1324 & 6.729 & 1327 & 6.492 & 1315 & 6.749 & 1327 \\
\hline 6.663 & 1266 & 6.788 & 1293 & 6.592 & 1263 & 6.9 & 1326 & 4.923 & 975.2 & 6.453 & 1286 & 6.501 & 1305 & 6.388 & 1290 & 6.592 & 1304 \\
\hline 6.471 & 1235 & 6.655 & 1266 & 6.507 & 1236 & 6.655 & 1290 & 4.796 & 948.3 & 6.359 & 1266 & 6.539 & 1270 & 6.296 & 1266 & 6.634 & 1270 \\
\hline 6.344 & 1208 & 6.43 & 1238 & 6.376 & 1210 & 6.521 & 1265 & 4.704 & 915.3 & 6.217 & 1242 & 6.223 & 1233 & 6.143 & 1242 & 6.285 & 1234 \\
\hline 6.294 & 1183 & 6.35 & 1214 & 6.205 & 1185 & 6.356 & 1242 & 4.414 & 876.5 & 6.075 & 1216 & 6.051 & 1207 & 6.051 & 1220 & 6.202 & 1212 \\
\hline 6.134 & 1159 & 6.149 & 1184 & 6.048 & 1153 & 6.314 & 1220 & 4.243 & 853.2 & 5.888 & 1194 & 5.897 & 1183 & 6.012 & 1197 & 6.077 & 1185 \\
\hline 5.998 & 1133 & 6.119 & 1154 & 5.844 & 1127 & 6.146 & 1193 & 4.142 & 828.9 & 5.87 & 1166 & 5.731 & 1161 & 5.879 & 1166 & 5.915 & 1163 \\
\hline
\end{tabular}


Table 15. Rheometer Response for Blank - 16 wt \% - continued

\begin{tabular}{|c|c|c|c|c|c|c|c|c|c|c|c|c|c|c|c|c|c|}
\hline run1 & & run2 & & run3 & & run4 & & run5 & & run6 & & run7 & & run8 & & run9 & \\
\hline [Pa] & [1/s] & [Pa] & [1/s] & [Pa] & {$[1 / s]$} & [Pa] & {$[1 / s]$} & [Pa] & [1/s] & [Pa] & {$[1 / s]$} & [Pa] & [1/s] & [Pa] & [1/s] & [Pa] & {$[1 / s]$} \\
\hline 5.861 & 1105 & 5.98 & 1133 & 5.702 & 1098 & 5.965 & 1172 & 4.003 & 803.4 & 5.77 & 1133 & 5.705 & 1136 & 5.708 & 1134 & 5.829 & 1137 \\
\hline 5.604 & 1072 & 5.743 & 1101 & 5.598 & 1074 & 5.941 & 1137 & 3.935 & 782.2 & 5.69 & 1108 & 5.557 & 1113 & 5.489 & 1119 & 5.731 & 1106 \\
\hline 5.506 & 1044 & 5.58 & 1075 & 5.494 & 1050 & 5.787 & 1114 & 3.816 & 755.8 & 5.332 & 1068 & 5.515 & 1079 & 5.24 & 1080 & 5.394 & 1066 \\
\hline 5.397 & 1019 & 5.512 & 1048 & 5.267 & 1015 & 5.492 & 1072 & 3.654 & 732 & 5.184 & 1044 & 5.237 & 1044 & 5.344 & 1063 & 5.299 & 1044 \\
\hline 5.234 & 994.2 & 5.275 & 1018 & 5.178 & 991.1 & 5.376 & 1051 & 3.473 & 684.8 & 5.13 & 1021 & 5.083 & 1022 & 5.009 & 1040 & 5.184 & 1019 \\
\hline 5.006 & 962.9 & 5.083 & 992.9 & 4.988 & 963.8 & 5.234 & 1026 & 3.328 & 659.7 & 5.012 & 999.5 & 4.941 & 998.1 & 4.722 & 996.4 & 5.054 & 995.5 \\
\hline 4.977 & 938.2 & 5.122 & 966.4 & 4.876 & 940 & 5.042 & 1003 & 3.207 & 638.5 & 4.903 & 971.7 & 4.837 & 973.5 & 4.929 & 974.8 & 4.897 & 971.7 \\
\hline 4.734 & 906.9 & 4.938 & 942.2 & 4.734 & 913.1 & 5.021 & 979.6 & 3.091 & 612.5 & 4.787 & 947.5 & 4.725 & 951.4 & 4.465 & 953.2 & 4.772 & 946.6 \\
\hline 4.645 & 882.7 & 4.698 & 910.9 & 4.542 & 878.3 & 4.873 & 954.5 & 2.97 & 587.9 & 4.71 & 913.5 & 4.636 & 916.2 & 4.468 & 918.4 & 4.707 & 921 \\
\hline 4.456 & 855.4 & 4.509 & 884.5 & 4.444 & 854 & 4.784 & 921.5 & 2.849 & 565 & 4.414 & 873.9 & 4.361 & 879.6 & 4.308 & 893.3 & 4.432 & 876.5 \\
\hline 4.346 & 828.9 & 4.491 & 855.8 & 4.293 & 825 & 4.53 & 881.8 & 2.727 & 539.8 & 4.222 & 851.4 & 4.249 & 854.9 & 4.328 & 859.3 & 4.29 & 854.5 \\
\hline 4.225 & 803.4 & 4.349 & 828.9 & 4.186 & 798.5 & 4.405 & 858 & 2.716 & 506.3 & 4.148 & 825 & 4.127 & 831.6 & 4.012 & 820.6 & 4.219 & 828.9 \\
\hline 4.068 & 773.4 & 4.222 & 802.9 & 4.077 & 774.3 & 4.216 & 834.7 & 2.426 & 470.2 & 3.953 & 802.5 & 4.047 & 809.5 & 3.87 & 799.8 & 4.062 & 806.4 \\
\hline 3.964 & 746.5 & 4.089 & 777.4 & 3.816 & 743.9 & 4.127 & 810.4 & 2.304 & 444.2 & 3.885 & 774.7 & 3.956 & 786.2 & 3.74 & 777.8 & 3.923 & 782.2 \\
\hline 3.802 & 721.8 & 3.979 & 749.2 & 3.769 & 717 & 3.991 & 786.2 & 2.195 & 420.4 & 3.757 & 752.7 & 3.873 & 750.9 & 3.618 & 753.6 & 3.852 & 758 \\
\hline 3.592 & 691 & 3.87 & 723.2 & 3.55 & 686.6 & 3.867 & 758.9 & & 397.5 & 3.621 & 730.2 & 3.589 & 714.8 & 3.615 & 730.7 & 3.808 & 724.5 \\
\hline 3.521 & 662.3 & 3.751 & 698 & 3.479 & 661.5 & 3.666 & 738.1 & 1.958 & 372.8 & 3.455 & 703.8 & 3.42 & 690.1 & 3.435 & 704.7 & 3.538 & 687.5 \\
\hline 3.376 & 634.6 & 3.512 & 665 & 3.34 & 634.1 & 3.695 & 702.9 & 1.846 & 348.6 & 3.482 & 671.6 & 3.337 & 669.8 & 3.435 & 671.6 & 3.325 & 665 \\
\hline 3.263 & 613 & 3.414 & 637.2 & 3.263 & 606.8 & 3.42 & 662.3 & 1.627 & 301 & 3.157 & 635.5 & 3.195 & 644.3 & 3.162 & 630.6 & 3.254 & 641.2 \\
\hline 3.109 & 583 & 3.278 & 610.8 & 3.083 & 582.6 & 3.21 & 639.4 & 1.467 & 279 & 3.068 & 613.9 & 3.142 & 621.4 & 3.029 & 609.5 & 3.139 & 615.6 \\
\hline 2.979 & 555.3 & 3.154 & 585.7 & 2.872 & 550.4 & 3.086 & 615.2 & 1.387 & 253.4 & 2.991 & 590.5 & 3.094 & 588.3 & 2.914 & 587 & 3.038 & 592.3 \\
\hline 2.852 & 528.8 & 2.878 & 553.5 & 2.763 & 525.3 & 3.041 & 588.3 & 1.464 & 244.1 & 2.914 & 563.2 & 2.801 & 553.5 & 2.813 & 559.7 & 2.982 & 559.7 \\
\hline 2.73 & 505 & 2.775 & 528.8 & 2.665 & 499.3 & 2.858 & 566.3 & & & 2.73 & 539.4 & 2.713 & 527.5 & 2.727 & 539 & 2.855 & 543.4 \\
\hline 2.568 & 473.7 & 2.713 & 503.3 & 2.606 & 475.5 & 2.787 & 546 & & & 2.727 & 505.5 & 2.594 & 505 & 2.553 & 510.3 & 2.5 & 515.2 \\
\hline 2.429 & 446 & 2.579 & 472.9 & 2.369 & 442.9 & 2.798 & 511.6 & & & 2.378 & 469.3 & 2.39 & 481.2 & 2.455 & 485.2 & 2.68 & 489.2 \\
\hline 2.363 & 421.3 & 2.446 & 444.6 & 2.254 & 416.9 & 2.529 & 476.8 & & & 2.31 & 445.5 & 2.357 & 456.1 & 2.39 & 452.1 & 2.349 & 467.6 \\
\hline 2.15 & 388.7 & 2.319 & 422.2 & 2.168 & 390 & 2.349 & 450.8 & & & 2.23 & 419.1 & 2.346 & 423.1 & 2.07 & 414.2 & 2.233 & 427.9 \\
\hline 2.082 & 364.9 & 2.248 & 396.6 & 2.053 & 367.1 & 2.189 & 427.5 & & & 2.106 & 399.3 & 2.023 & 386 & 1.999 & 390 & 2.307 & 403.2 \\
\hline 1.979 & 340.2 & 2.041 & 362.7 & 1.896 & 332.3 & 2.094 & 403.7 & & & 2.002 & 372.8 & 1.94 & 361.8 & 1.902 & 368 & 2.047 & 392.2 \\
\hline 1.884 & 313.8 & 1.979 & 335.8 & 1.748 & 307.2 & 2.062 & 378.5 & & & 1.878 & 349.9 & 1.819 & 336.2 & 1.772 & 341.5 & 1.763 & 353.4 \\
\hline 1.709 & 279.4 & 1.837 & 311.6 & 1.671 & 281.2 & 1.834 & 355.6 & & & 1.653 & 301.4 & 1.86 & 316 & 1.57 & 319.1 & 1.692 & 323 \\
\hline 1.579 & 254.7 & 1.609 & 281.6 & 1.529 & 257.8 & 1.644 & 308 & & & 1.449 & 278.5 & 1.825 & 289.5 & 1.597 & 286 & 1.706 & 287.3 \\
\hline
\end{tabular}


Table 16. $10{ }^{\circ} \mathrm{C}$ at $16 \mathrm{wt} \%$

\begin{tabular}{|c|c|c|c|c|c|c|c|c|c|c|c|c|c|c|c|c|c|}
\hline run1 & & run2 & & run3 & & run4 & & run5 & & run6 & & run7 & & run8 & & run9 & \\
\hline [Pa] & {$[1 / s]$} & [Pa] & {$[1 / s]$} & [Pa] & {$[1 / s]$} & [Pa] & {$[1 / s]$} & [Pa] & [1/s] & [Pa] & {$[1 / s]$} & [Pa] & {$[1 / s]$} & [Pa] & {$[1 / s]$} & [Pa] & {$[1 / s]$} \\
\hline 15.79 & 274.1 & 15.38 & 268.4 & 14.27 & 323.5 & 16.48 & 274.5 & 17.07 & 273.7 & 16.93 & 272.3 & 15.8 & 274.1 & 14.64 & 274.5 & 17.45 & 274.5 \\
\hline 17.03 & 293.9 & 5.71 & 295.3 & 6.06 & 287.8 & 16.65 & 296.1 & 16.17 & 295.3 & 17.28 & 289.5 & 16.55 & 293.5 & 15.74 & 296.1 & 17.31 & 295.7 \\
\hline 17.64 & 23.9 & .63 & 320.8 & 7.84 & 327 & 16.59 & 324.3 & 16.49 & 319.9 & 18.37 & 319.9 & 17.59 & 316.4 & 16.69 & 318.2 & 16.8 & 322.6 \\
\hline 20.31 & 349.5 & .63 & 349 & 8.71 & 349.5 & 18.75 & 349 & 19.35 & 348.6 & 17.54 & 346.4 & 18.31 & 350.3 & 19.86 & 353 & 18.58 & 347.3 \\
\hline 20.14 & 377.2 & 9.43 & 371.1 & 19.57 & 371.5 & 20.44 & 375 & 20.89 & 372.8 & 19.97 & 371.5 & 19.37 & 375 & 19.63 & 379.4 & 21.16 & 372.4 \\
\hline 22.37 & 405.9 & .99 & 395.7 & 20.41 & 396.2 & 21.83 & 406.3 & 19.62 & 397.9 & 19.86 & 401.5 & 20.93 & 402.8 & 21.96 & 405.4 & 20.11 & 396.6 \\
\hline 22.11 & 433.6 & 21.85 & 430.5 & 21.52 & 421.7 & 20.89 & 432.8 & 21.07 & 429.7 & 22.82 & 428.8 & 21.98 & 427.9 & 22.08 & 432.3 & 21.67 & 429.7 \\
\hline 24.2 & 457 & 21.93 & 456.1 & 21.66 & 453.9 & 22.24 & 463.2 & 23.58 & 458.3 & 21.71 & 451.3 & 23.1 & 459.6 & 22.26 & 459.6 & 23.91 & 456.5 \\
\hline 24.79 & 481.2 & 23.14 & 484.8 & 23.59 & 489.6 & 24.61 & 486.1 & 24.1 & 482.5 & 24.36 & 474.6 & 23.14 & 485.2 & 24.61 & 483.4 & 24.17 & 482.5 \\
\hline 25.77 & 508.5 & 25.27 & 509 & 24.79 & 510.8 & 25.12 & 516 & 24.88 & 509.9 & 23.22 & 493.6 & 24.14 & 512.5 & 25.74 & 509.9 & 25.74 & 513.8 \\
\hline 26.39 & & & 535 & & & & 539 & & & & & & & 25.5 & & & 537.2 \\
\hline 27.75 & 568 & 27.01 & 560.1 & 26.39 & 560.1 & 26.72 & 572.4 & 27.25 & 567.2 & 27.13 & 560.1 & 26.04 & 565.4 & 26.6 & 569.4 & 26.83 & 567.2 \\
\hline 28.7 & 597.1 & 27.69 & 594 & 27.07 & 583.9 & 28.25 & 598.4 & 27.28 & 594.9 & 27.37 & 577.7 & 27.31 & 590.5 & 28.08 & 594.9 & 27.46 & 591.8 \\
\hline 30.15 & 620.9 & 29.08 & 619.6 & 27.72 & 607.3 & 28.55 & 626.7 & 29.32 & 621.8 & 29.17 & 635.5 & 28.08 & 624 & 29.38 & 619.6 & 27.99 & 617.4 \\
\hline 31.21 & 646 & 29.68 & 650 & 28.49 & 639.9 & 29.08 & 650.9 & 29.35 & 650 & 29.41 & 644.7 & 30.15 & 650.9 & 30.39 & 652.7 & 29.59 & 642.5 \\
\hline 31.95 & 679.5 & & 674.7 & 30.5 & 678.2 & & 675.1 & 31.24 & 676 & & & 30.15 & 675.1 & 30.3 & 677.8 & 31.1 & 676.4 \\
\hline 33.11 & 705.1 & 31.87 & 703.3 & 31.33 & 703.8 & 31.95 & 706.9 & 31.13 & 702.4 & 31.42 & 703.3 & 31.39 & 700.2 & 32.19 & 704.2 & 31.13 & 701.6 \\
\hline 33.32 & 729.8 & & 727.1 & & 725.4 & & 734.6 & 32.9 & 727.1 & 32.4 & 727.1 & & 724.9 & & 732.4 & .93 & 727.1 \\
\hline 34.71 & 755.8 & 3.32 & 750.9 & 32.78 & 749.2 & 32.78 & 758.9 & 33.64 & 761.9 & 33.94 & 758 & 33.82 & 757.5 & 33.29 & 758 & .17 & 758.4 \\
\hline 35.42 & 788.4 & 4.23 & 785.7 & 33.49 & 771.6 & 33.97 & 783.5 & 34.32 & 785.3 & 34.74 & 784.4 & 34.71 & 786.6 & 33.97 & 782.2 & 3.85 & 785.7 \\
\hline 35.98 & 813.1 & 34.56 & 808.7 & 34 & 804.2 & 35.42 & 817 & 34.91 & 813.9 & 35.21 & 812.2 & 35.48 & 812.6 & 35.15 & 807.8 & .54 & 808.2 \\
\hline 37.25 & 838.6 & 36.22 & 839.9 & 35 & 828.9 & 36.07 & 842.6 & 35.98 & 839.5 & 36.39 & 836.4 & 36.25 & 838.2 & 36.33 & 839.9 & 35.83 & 838.6 \\
\hline 38.05 & 868.1 & & 866.4 & 36.84 & 869.9 & & 867.3 & 36.75 & 863.3 & & & 36.9 & 863.7 & 36.6 & 868.6 & 37.1 & 864.6 \\
\hline 39.2 & 891.9 & 37.34 & 891.9 & 37.43 & 891.9 & 37.87 & 896.3 & 37.81 & 895 & 37.67 & & 37.58 & 888.9 & 37.9 & 897.2 & 37.67 & 891.5 \\
\hline 40.39 & 922.3 & & 922.8 & & 914.4 & 38.85 & 921 & 38.7 & 919.7 & 38.55 & 920.1 & 38.29 & 921.5 & 38.49 & 922.8 & 38.52 & 915.3 \\
\hline 40.98 & 950.1 & 39.41 & 946.6 & 39.26 & 938.7 & 39.41 & 947.9 & 39.35 & 947.9 & 39.8 & 946.6 & 39.83 & 947.5 & 39.12 & 945.3 & 39.32 & 945.3 \\
\hline 41.66 & 975.7 & 39.92 & 973 & 39.94 & 962.9 & & 981.8 & & 975.2 & 40.74 & 974.3 & 40.12 & 971.7 & 40.27 & 969.1 & 40.65 & 972.1 \\
\hline 42.31 & 1005 & 41.04 & 996.8 & 40.8 & 995.9 & 41.39 & 1007 & 41.34 & 1003 & 41.22 & 1001 & 41.42 & 1006 & 41.28 & 1004 & 41.39 & 999.9 \\
\hline 43.26 & 1027 & 41.78 & 1031 & 41.69 & 1035 & 42.13 & 1034 & 42.28 & 1033 & 41.81 & 1026 & 42.05 & 1030 & 41.93 & 1032 & 41.72 & 1026 \\
\hline 44.62 & 1061 & 42.87 & 1057 & 42.87 & 1062 & 42.76 & 1059 & 43.17 & 1058 & 42.93 & & 42.96 & 1057 & 43.11 & 1055 & 42.87 & 1059 \\
\hline 45.27 & 1087 & 43.47 & 1083 & 43.76 & 1088 & 43.79 & 1086 & 43.88 & 1085 & 43.91 & 1083 & 43.97 & 1080 & 43.94 & 1088 & 43.94 & 1083 \\
\hline
\end{tabular}


Table 16. $10{ }^{\circ} \mathrm{C}$ at 16 wt $\%$ - continued

\begin{tabular}{|c|c|c|c|c|c|c|c|c|c|c|c|c|c|c|c|c|c|}
\hline run1 & & run2 & & run3 & & run4 & & run5 & & run6 & & run7 & & run8 & & run9 & \\
\hline [Pa] & {$[1 / s]$} & [Pa] & {$[1 / s]$} & [Pa] & {$[1 / s]$} & [Pa] & {$[1 / s]$} & [Pa] & {$[1 / s]$} & [Pa] & {$[1 / s]$} & [Pa] & {$[1 / s]$} & [Pa] & {$[1 / s]$} & [Pa] & {$[1 / s]$} \\
\hline 46.16 & 1116 & 44.3 & 1108 & 44.74 & 1111 & 44.68 & 1118 & 44.83 & 1111 & 44.86 & 1111 & 44.71 & 1115 & 44.5 & 1115 & 44.62 & 1111 \\
\hline 47.11 & 1140 & 45.39 & 1138 & 45.48 & 1136 & 45.66 & 1144 & 45.63 & 1140 & 45.57 & 1137 & 45.72 & 1141 & 45.21 & 1139 & 45.54 & 1137 \\
\hline 47.82 & 1163 & 46.16 & 166 & 45.57 & 1159 & 46.19 & 1171 & 46.25 & 1167 & 46.66 & 1164 & 46.31 & 1168 & 46.43 & 1167 & 46.07 & 1160 \\
\hline 48.7 & 1192 & 47.2 & 1191 & 46.72 & 1183 & 46.84 & 1195 & 46.99 & 1192 & 47.67 & 1197 & 46.87 & 1191 & 47.37 & 1191 & 47.25 & 1195 \\
\hline 49.8 & 1226 & 47.96 & 1218 & 47.46 & 1215 & 48.11 & 1226 & 47.79 & 1216 & 47.91 & 1215 & 47.91 & 1214 & 48.41 & 1227 & 47.88 & 1219 \\
\hline 50.39 & 1244 & 48.79 & 1242 & 49 & 1253 & 48.76 & 1253 & 48.62 & 1243 & 49.15 & 1247 & 48.56 & 1246 & 48.85 & 1252 & 48.73 & 1247 \\
\hline 51.43 & 1278 & 49.59 & 1274 & 49.59 & 1278 & 49.53 & 1280 & 49.83 & 1276 & 49.89 & 1276 & 49.33 & 1276 & 49.95 & 1277 & 49.77 & 1274 \\
\hline 52.4 & 1302 & 50.66 & 1303 & 50.04 & 1301 & 50.27 & 1306 & 50.72 & 1302 & 50.69 & 1300 & 50.04 & 1286 & 50.21 & 1301 & 50.69 & 1305 \\
\hline 53.11 & 1333 & 51.13 & 1327 & 51.1 & 1324 & 51.22 & 1330 & 51.22 & 1330 & 51.22 & 1326 & 51.07 & 1330 & 51.52 & 1330 & 51.46 & 1330 \\
\hline 53.94 & 1360 & 51.87 & 1352 & 51.84 & 1354 & 51.93 & 1357 & 52.2 & 1356 & 52.4 & 1358 & 52.05 & 1345 & 52.29 & 1360 & 52.17 & 1356 \\
\hline 54.89 & 1389 & & 1383 & & 1383 & 52.61 & 1385 & 52.94 & 1381 & & & 52.97 & 1384 & 53.14 & 1387 & 52.97 & 1386 \\
\hline 55.42 & 1412 & 53.74 & 1409 & 53.29 & 1404 & 53.71 & 1417 & 53.91 & 1413 & 53.97 & 1408 & 53.62 & 1410 & 53.88 & 1412 & 53.74 & 1412 \\
\hline 56.4 & 1442 & 4.65 & 1441 & 54.15 & 1430 & 54.68 & 1445 & 54.8 & 1441 & 54.86 & 1438 & 54.39 & 1435 & 54.59 & 1436 & 54.48 & 1437 \\
\hline 57.05 & 1467 & 55.42 & 1467 & 55.51 & 1471 & 55.42 & 1470 & 55.51 & 1464 & 55.45 & 1462 & 55.66 & 1470 & 55.66 & 1470 & 55.1 & 1461 \\
\hline 57.73 & 1493 & 56.16 & 1492 & 56.07 & 1497 & 56.07 & 1498 & 56.46 & 1495 & 56.46 & 1496 & 56.37 & 1496 & 56.52 & 1496 & 55.84 & 1486 \\
\hline 58.59 & 1518 & 56.93 & 1516 & 56.84 & 1519 & 56.72 & 1521 & 57.41 & 1523 & 57.29 & 1521 & 57.17 & 1520 & 57.35 & 1522 & 57.05 & 1522 \\
\hline 54.83 & 1535 & 54.71 & 1537 & 54.18 & 1514 & 55.78 & 1540 & 56.4 & 1536 & 53.82 & 1505 & 54.83 & 1534 & 53.77 & 1506 & 55.19 & 1544 \\
\hline 54.15 & 1510 & 3.97 & 1512 & 53.08 & 1478 & 55.16 & 1507 & 55.66 & 1514 & 53.26 & 1481 & 54.15 & 1507 & 53.14 & 1479 & 54.15 & 1509 \\
\hline 53.41 & 1481 & 53.71 & 1502 & 52.14 & 1452 & 54.42 & 1482 & 54.33 & 1483 & 52.58 & 1456 & 53.32 & 1482 & 52.4 & 1452 & 53.47 & 1486 \\
\hline 52.46 & 1454 & 52.79 & 1475 & 51.37 & 1426 & 53.56 & 1454 & 53.41 & 1455 & 51.81 & 1431 & 52.46 & 1450 & 51.6 & 1425 & 52.61 & 1457 \\
\hline 51.96 & 1430 & 52.46 & 1457 & 50.66 & 1397 & 52.97 & 1428 & 52.58 & 1429 & 50.54 & 1398 & 51.58 & 1423 & 50.98 & 1400 & 51.75 & 1430 \\
\hline 50.69 & 1397 & 52.05 & 1438 & 50.1 & 1376 & 52.11 & 1404 & 51.9 & 1403 & 49.92 & 1370 & 50.6 & 1398 & 50.07 & 1374 & 50.81 & 1404 \\
\hline 50.15 & 1375 & 51.43 & 1417 & 48.88 & 1341 & 51.04 & 1371 & 51.01 & 1378 & 49.15 & 1345 & 50.01 & 1372 & 49.06 & 1344 & 49.95 & 1372 \\
\hline 49.39 & 1351 & 49.89 & 1394 & 48.05 & 1315 & 50.3 & 1346 & 50.33 & 1347 & 48.47 & 1321 & 48.97 & 1341 & 48.2 & 1318 & 49.3 & 1348 \\
\hline 48.32 & 1315 & 48.26 & 1321 & 47.46 & 1289 & 49.53 & 1318 & 49.65 & 1319 & 47.46 & 1293 & 48.53 & 1317 & 47.43 & 1290 & 48.38 & 1319 \\
\hline 47.58 & 1291 & 46.84 & 1277 & 46.6 & 1266 & 48.79 & 1295 & 48.53 & 1293 & 46.84 & 1267 & 47.61 & 1288 & 46.99 & 1264 & 47.55 & 1293 \\
\hline 46.84 & 1268 & 47.02 & 1274 & 45.72 & 1235 & 47.76 & 1263 & 47.88 & 1267 & 45.75 & 1233 & 46.78 & 1260 & 46.07 & 1239 & 46.93 & 1267 \\
\hline 45.89 & 1242 & 46.04 & 1256 & 44.92 & 1208 & 46.72 & 1238 & 46.66 & 1234 & 44.92 & 1206 & 45.75 & 1235 & 45.33 & 1212 & 45.83 & 1237 \\
\hline 45.12 & 1208 & 44.71 & 1220 & 44.3 & 1180 & 46.28 & 1213 & 45.83 & 1209 & 44.06 & 1182 & 45.21 & 1209 & 44.12 & 1182 & 45.15 & 1208 \\
\hline 43.94 & 1182 & 43.58 & 1175 & 43.41 & 1157 & 44.92 & 1180 & 44.89 & 1183 & 43.47 & 1155 & 44.06 & 1182 & 43.53 & 1153 & 44.15 & 1182 \\
\hline 43.23 & 1158 & 43.26 & 1151 & 42.43 & 1123 & 44.24 & 1155 & 44.15 & 1158 & 42.7 & 1131 & 43.17 & 1152 & 42.52 & 1126 & 43.38 & 1158 \\
\hline
\end{tabular}


Table 16. $10{ }^{\circ} \mathrm{C}$ at 16 wt $\%$ - continued

\begin{tabular}{|c|c|c|c|c|c|c|c|c|c|c|c|c|c|c|c|c|c|}
\hline run1 & & run2 & & run3 & & run4 & & run5 & & run6 & & run7 & & run8 & & run9 & \\
\hline [Pa] & {$[1 / s]$} & [Pa] & {$[1 / \mathrm{s}]$} & [Pa] & {$[1 / s]$} & [Pa] & {$[1 / s]$} & [Pa] & {$[1 / \mathrm{s}]$} & [Pa] & {$[1 / \mathrm{s}]$} & [Pa] & {$[1 / s]$} & [Pa] & {$[1 / \mathrm{s}]$} & [Pa] & {$[1 / s]$} \\
\hline 42.46 & 1124 & 42.13 & 1128 & 41.1 & 1094 & 43.64 & 1128 & 43.67 & 1133 & 41.63 & 1097 & 42.67 & 1123 & 41.9 & 1101 & 42.58 & 1133 \\
\hline 41.6 & 1100 & 1.31 & 1104 & 40.57 & 1070 & 42.58 & 1104 & 42.34 & 1102 & 40.89 & 1071 & 41.87 & 1097 & 41.07 & 1074 & 41.6 & 1100 \\
\hline 40.77 & 1073 & 40.98 & 1081 & 39.89 & 1044 & 41.6 & 1070 & 41.57 & 1075 & 39.89 & 1044 & 40.92 & 1073 & 9.83 & 1044 & 40.74 & 1075 \\
\hline 39.65 & 1043 & 39.83 & 1058 & 39.18 & 1018 & 40.8 & 1047 & 40.6 & 1050 & 39.23 & 1018 & 40.36 & 1045 & 39.26 & 1020 & 39.97 & 1046 \\
\hline 39.09 & 1018 & 39.12 & 1023 & 38.46 & 990.7 & 40.18 & 1019 & 39.86 & 1017 & 38.08 & 991.5 & 38.76 & 1013 & 38.29 & 988.5 & 39.15 & 1021 \\
\hline 38.32 & 989.3 & 38.08 & 987.6 & 37.43 & 959.8 & 39.5 & 993.7 & 39.06 & 991.5 & 37.84 & 967.7 & 38.26 & 987.1 & 37.55 & 962.5 & 38.67 & 994.6 \\
\hline 37.73 & 966 & 36.87 & 964.7 & 36.45 & 941.3 & 38.08 & 966.4 & 37.78 & 965.5 & 36.45 & 935.1 & 37.31 & 962.5 & 36.48 & 937.3 & 37.78 & 965.1 \\
\hline 35.95 & 933.4 & 36.13 & 942.6 & 35.89 & 907.8 & 37.31 & 934.7 & 37.46 & 939.1 & 35.39 & 909.6 & 36.22 & 932.5 & 36.04 & 911.3 & 36.33 & 939.1 \\
\hline 35.15 & 908.7 & 35.06 & 911.3 & 34.5 & 880.5 & 36.39 & 908.2 & 36.63 & 914.4 & 35.12 & 881.8 & 35.83 & 906.9 & 35.06 & 879.2 & .83 & 914.9 \\
\hline 35 & 881.8 & 34.35 & 877.8 & & 854 & 35.74 & 884.5 & & 883.1 & 33.4 & 851.4 & & 883.1 & & 854 & & 888.9 \\
\hline 34.11 & 856.2 & 33.7 & 852.7 & 33.49 & 848.8 & 34.53 & 856.7 & 34.29 & 854 & 33.11 & 824.5 & 33.55 & 851 & 32.72 & 824.1 & 34.2 & 854.5 \\
\hline 33.43 & 832 & 33.02 & 833.8 & 33.17 & 827.2 & 33.55 & 832.4 & 33.23 & 829.4 & 31.95 & 802 & 32.69 & 824.5 & 32.04 & 798.5 & 33.46 & 828.9 \\
\hline 31.84 & 799.4 & 32.16 & 811.7 & 30.92 & 769.4 & 32.49 & 799 & 32.22 & 803.4 & 31.57 & 769.9 & 32.52 & 794.6 & 31.66 & 773.4 & 32.84 & 803.4 \\
\hline 31.07 & 770.3 & 30.74 & 778.7 & 31.24 & 757.5 & 31.78 & 778.7 & 31.89 & 778.7 & 30.53 & 742.1 & 31.45 & 768.6 & 30.36 & 743 & 31.45 & 773.4 \\
\hline 30.68 & 748.3 & 30.06 & 744.3 & 30.12 & 732.9 & 30.98 & 744.8 & 31.21 & 743.9 & 28.88 & 715.2 & 30.15 & 745.6 & 30.21 & 716.1 & 31.01 & 746.5 \\
\hline 28.61 & 715.2 & 29.2 & 719.6 & 28.37 & 698.9 & 30.09 & 720.5 & 29.65 & 720.5 & 28.85 & 688.8 & 29.97 & 718.3 & 28.49 & 692.8 & 29.62 & 724.9 \\
\hline 28.61 & 691.9 & & 695.8 & & 667.2 & & 689.7 & & 690.1 & & & & 693.6 & 28.2 & 668.1 & & 691 \\
\hline 27.78 & 667.2 & 27.66 & 674.2 & 26.75 & 639.9 & 28.64 & 666.3 & 28.46 & 664.1 & 27.25 & 639.4 & 27.84 & 658.4 & 26.54 & 635.9 & 28.46 & 667.6 \\
\hline 27.04 & 636.3 & 27.37 & 641.6 & 25.47 & 604.2 & 27.19 & 634.1 & 27.31 & 638.5 & 25.35 & 606.8 & 27.43 & 635.5 & 26.63 & 609 & & 636.8 \\
\hline 24.97 & 608.1 & 25.21 & 604.2 & 24.14 & 577.7 & 26.57 & 610.3 & 25.86 & 609.5 & 25.47 & 579.5 & 25.35 & 604.6 & 24.41 & 579.5 & .36 & 608.6 \\
\hline 24.14 & 583 & 24.53 & 585.2 & 23.55 & 554.8 & 26.04 & 583.5 & 25.98 & 583 & 24.38 & 555.7 & 25.27 & 578.6 & 23.49 & 554.4 & 24.73 & 583 \\
\hline 24.38 & 551.3 & 23.82 & 558.8 & 22.84 & 528.8 & 24.85 & 558.8 & 24.88 & 559.2 & 23.82 & 526.2 & 24.7 & 551.3 & 22.6 & 527.5 & .96 & 557.5 \\
\hline 22.99 & 527.5 & 23.25 & 536.8 & 21.27 & 500.2 & 24.2 & 532.3 & 24.17 & 533.2 & 23.02 & 498.4 & 23.85 & 523.5 & 22.28 & 502.4 & 22.96 & 530.6 \\
\hline 22.69 & 498.9 & 21.89 & 494 & 22.13 & 475.1 & 23.22 & 505.9 & 23.25 & 505.5 & 21.86 & 472 & 22.04 & 499.7 & 22.19 & 475.1 & 21.74 & 498.9 \\
\hline 20.74 & 472.9 & 20.8 & 472 & 21.21 & 444.6 & 21.06 & 471.5 & 21.42 & 479 & 20.18 & 442.9 & 20.62 & 473.3 & 20.5 & 451.3 & 21.06 & 472.9 \\
\hline 20.31 & 448.2 & 20.32 & 449.5 & & 417.8 & 21.42 & 443.8 & 20.83 & 445.1 & 20.65 & 419.5 & 19.32 & 441.6 & 19.02 & 423.5 & 21.48 & 447.7 \\
\hline 19.9 & 416.4 & 19.49 & 425.7 & 17.27 & 385.6 & 18.79 & 419.5 & 18.51 & 417.8 & 19.19 & 387.8 & 19.47 & 412 & 18.62 & 389.1 & 19.28 & 424.4 \\
\hline 17.19 & 391.3 & 18.43 & 394.9 & 16.6 & 368 & 19.3 & 392.6 & 19.82 & 390.9 & 18.02 & 364.4 & 17.72 & 388.7 & 17.28 & 362.2 & 19.13 & 392.2 \\
\hline 17.69 & 367.5 & 17.7 & 373.7 & 16.42 & 341.5 & 17.01 & 367.5 & 17.46 & 366.6 & 15.43 & 336.7 & 18.9 & 362.7 & 18.1 & 335.8 & 17.21 & 363.6 \\
\hline 15.14 & 334.9 & 16.88 & 349.9 & 16.17 & 307.6 & 17.79 & 335.8 & 16.4 & 337.1 & 15.54 & 308.5 & 17.79 & 337.6 & 17.38 & 311.6 & 17.41 & 339.8 \\
\hline 14.06 & 310.7 & 15.49 & 315.5 & 13.66 & 279 & 16.97 & 310.7 & 17.05 & 309.8 & 15.95 & 280.7 & 14.67 & 305.4 & 13.47 & 277.2 & 16.02 & 308 \\
\hline 13.76 & 282 & 14.65 & 277.6 & 13.7 & 254.7 & 13.85 & 280.3 & 16.11 & 282 & 14.42 & 254.7 & 14.34 & 277.6 & 13.62 & 253.8 & 14.08 & 281.2 \\
\hline
\end{tabular}


Table 17. $15{ }^{\circ} \mathrm{C}$ at 16 wt $\%$

\begin{tabular}{|c|c|c|c|c|c|c|c|c|c|c|c|c|c|c|c|c|c|}
\hline run1 & & run2 & & run3 & & run4 & & run5 & & run6 & & run7 & & run8 & & run9 & \\
\hline [Pa] & {$[1 / s]$} & [Pa] & {$[1 / s]$} & [Pa] & {$[1 / s]$} & [Pa] & {$[1 / s]$} & [Pa] & [1/s] & [Pa] & {$[1 / s]$} & [Pa] & {$[1 / s]$} & [Pa] & {$[1 / s]$} & [Pa] & {$[1 / s]$} \\
\hline 13.46 & 272.3 & 11.87 & 271.5 & 9.993 & 278.5 & 11.93 & 272.8 & 12.42 & 272.3 & 12.42 & 271.5 & 11.77 & 274.1 & 11.91 & 275 & 11.07 & 275.4 \\
\hline 14.29 & 294.4 & 13 & 293.5 & 11.1 & 291.7 & 13.7 & 296.1 & 11.31 & 294.4 & 13.46 & 291.3 & 13.33 & 294.8 & 12.92 & 293.1 & 11.86 & 298.8 \\
\hline 14.12 & 19.1 & 1.79 & 321.3 & 4.58 & 322.6 & 13.45 & 320.4 & 13.45 & 322.1 & 12.87 & 324.8 & 14.86 & 323 & 12.5 & 321.3 & 13.83 & 325.2 \\
\hline 13.43 & 345.1 & 2.46 & 342.9 & 5.03 & 346.4 & 13.03 & 347.3 & 15.37 & 346.4 & 14.01 & 351.7 & 13.71 & 351.2 & 15.62 & 348.6 & 16.15 & 353 \\
\hline 16.41 & 374.6 & 5.44 & 375.5 & 3.63 & 372.8 & 16.15 & 378.5 & 14.19 & 376.3 & 16.38 & 378.5 & 14.8 & 374.1 & 16.12 & 372.8 & 16.12 & 374.6 \\
\hline 15.52 & 401.9 & 4.94 & 402.3 & 0.16 & 397.1 & 15.03 & 403.2 & 17.06 & 401.9 & 15.27 & 401.5 & 16.64 & 402.8 & 15.37 & 398.8 & 17.16 & 409 \\
\hline 17.83 & 425.3 & 16.73 & 427.9 & 17.19 & 430.5 & 17.67 & 428.3 & 16.94 & 432.3 & 16.44 & 434.5 & 17.9 & 432.3 & 16.7 & 432.8 & 18 & 436.7 \\
\hline 19.22 & 457.4 & 7.85 & 453.9 & 0.62 & 457.4 & 17.82 & 456.1 & 17.29 & 457 & 18.84 & 459.2 & 17.72 & 459.6 & 18.79 & 456.1 & 17.41 & 461 \\
\hline 18.66 & 482.5 & 18.15 & 481.7 & 18.88 & 480.8 & 17.47 & 480.8 & 19.27 & 480.8 & 19.41 & 486.1 & 17.97 & 485.2 & 19.65 & 483.9 & 18.33 & 491.4 \\
\hline 19.63 & 510.3 & 9.76 & 513 & 9.46 & 510.8 & 19.78 & 514.7 & 19.69 & 513.4 & 18.66 & 509 & 18.78 & 512.5 & 18.85 & 506.8 & 19.69 & 515.6 \\
\hline 21.57 & & & 538.1 & & & & & & & & 542 & 20.09 & 541.6 & & & .21 & 543.4 \\
\hline 22.43 & 565.4 & 21.1 & 563.2 & 21.12 & 568.9 & 21.69 & 568.5 & 20.8 & 565.8 & 21.31 & 568.9 & 21.7 & 568.9 & 20.86 & 568.5 & 1.59 & 571.1 \\
\hline 22.96 & 594.5 & 22.03 & 588.3 & 21.19 & 589.2 & 21.57 & 594.9 & 21.75 & 594 & 21.48 & 596.7 & 22.58 & 594.9 & 21.87 & 594.9 & 22.64 & 596.7 \\
\hline 23.85 & 623.6 & 22.73 & 620.9 & 22.19 & 614.8 & 21.96 & 619.6 & 23.17 & 621.4 & 23.32 & 624 & 22.37 & 623.6 & 22.52 & 618.3 & 23.7 & 620.9 \\
\hline 25.15 & 650.4 & 23.47 & 647.8 & 23.47 & 641.2 & 22.7 & 643.8 & 23.08 & 647.8 & 24.21 & 647.4 & 23.2 & 647.8 & 23.41 & 650 & 24.53 & 654 \\
\hline 25.51 & 676.9 & 24.47 & 675.6 & 24.35 & 675.1 & 24.65 & 678.2 & 24.35 & 672.5 & 23.82 & 673.8 & 24.86 & 672 & 25.18 & 676 & 24.35 & 681.7 \\
\hline 26.99 & 700.7 & 25.18 & 698 & 24.41 & 698.9 & 25.54 & 700.7 & 24.71 & 697.6 & 25.75 & 698.9 & 25.42 & 705.5 & 25.66 & 703.3 & 25.95 & 706.9 \\
\hline 27.85 & 728.4 & & 730.7 & & 726.2 & & & & 732 & & & 26.63 & 732 & & 728.9 & .07 & 731.1 \\
\hline 28.41 & 756.2 & 26.43 & 757.5 & 26.37 & 754 & 27.17 & 757.1 & 26.72 & 755.8 & 26.49 & 761.1 & 26.4 & 756.2 & 27.05 & 752.7 & 27.76 & 762.8 \\
\hline 29.3 & 782.7 & 27.64 & 782.7 & 27.52 & 786.2 & 27.26 & 781.8 & 27.05 & 785.3 & 27.4 & 787.9 & 28.17 & 787.9 & 27.31 & 777.4 & 27.94 & 786.6 \\
\hline 30.3 & 810.4 & 27.97 & 806.4 & 28.17 & 811.7 & 28.29 & 813.9 & 27.97 & 810.4 & 28.29 & 3.9 & 28.88 & 812.6 & 28.5 & 813.1 & 29.3 & 815.7 \\
\hline 30.75 & 838.2 & 28.73 & 839.9 & 28.73 & 836.9 & 28.88 & 839.1 & 28.76 & 835.5 & 28.82 & 839.1 & 29.59 & 839.5 & 29.8 & 840.8 & 29.33 & 843.5 \\
\hline 31.52 & 866.4 & 29.36 & 864.2 & 30.16 & 864.2 & 30.24 & 869 & 29.3 & 860.7 & & 862.4 & 29.92 & 865.1 & 30.54 & 866.4 & .04 & 868.6 \\
\hline 32.32 & 895 & 30.78 & 890.2 & 30.78 & 895.5 & 30.45 & 895.9 & 30.42 & 892.4 & 30.92 & & 30.78 & 893.7 & 30.63 & 892.4 & 30.75 & 894.1 \\
\hline 32.64 & 910 & & 919.3 & & 918.8 & 31.07 & 919.7 & 31.72 & 921 & 31.4 & 920.6 & 31.55 & 920.6 & 31.22 & 917.1 & 31.9 & 925.9 \\
\hline 33.74 & 945.7 & 32.05 & 946.6 & 32.32 & 947.5 & 32.11 & 951 & 32.26 & 948.3 & 32.67 & 951.9 & 32.38 & 946.6 & 32.32 & 948.8 & 32.58 & 954.5 \\
\hline 34.06 & 957.2 & 32.7 & 977.4 & & 971.7 & & 978.3 & & 977 & & 974.3 & 32.79 & 973.5 & 33.2 & 976.1 & 33.23 & 981 \\
\hline 35.01 & 991.5 & 33.68 & 1003 & 33.47 & 1003 & 33.77 & 1002 & 33.65 & 999.5 & 33.91 & 1002 & 34.06 & 999 & 33.77 & 1005 & 33.94 & 1004 \\
\hline 36.31 & 1016 & 34.39 & 1028 & 34.68 & 1028 & 34.56 & 1031 & 34.39 & 1033 & 34.62 & & 34.33 & 1032 & 34.74 & 1031 & 34.68 & 1031 \\
\hline 36.55 & 1040 & & 1060 & 35.04 & 1052 & 34.8 & 1054 & 35.19 & 1059 & 35.25 & 1061 & 35.39 & 1058 & 35.66 & 1057 & 35.75 & 1064 \\
\hline 38.15 & 1082 & 35.96 & 1085 & 35.6 & 1077 & 36.07 & 1086 & 35.81 & 1085 & 36.19 & 1084 & 36.07 & 1082 & 36.37 & 1090 & 36.78 & 1088 \\
\hline
\end{tabular}


Table $17.15^{\circ} \mathrm{C}$ at 16 wt $\%$ - continued

\begin{tabular}{|c|c|c|c|c|c|c|c|c|c|c|c|c|c|c|c|c|c|}
\hline run1 & & run2 & & run3 & & run4 & & run5 & & run6 & & run7 & & run8 & & run9 & \\
\hline [Pa] & {$[1 / s]$} & [Pa] & {$[1 / s]$} & [Pa] & {$[1 / s]$} & [Pa] & {$[1 / s]$} & [Pa] & {$[1 / s]$} & [Pa] & {$[1 / \mathrm{s}]$} & [Pa] & {$[1 / s]$} & [Pa] & {$[1 / \mathrm{s}]$} & [Pa] & {$[1 / s]$} \\
\hline 38.62 & 1109 & 36.78 & 1110 & 36.81 & 1111 & 36.64 & 1112 & 36.64 & 1110 & 36.73 & 1107 & 36.9 & 1115 & 37.35 & 1114 & 37.29 & 1112 \\
\hline 39.74 & 1142 & 37.17 & 1136 & 37.55 & 1137 & 37.47 & 1138 & 37.32 & 1134 & 37.55 & 1134 & 37.58 & 1142 & 37.94 & 1139 & 38.12 & 1144 \\
\hline 40.57 & 1168 & 38.26 & 1167 & 38.32 & 1162 & 38.09 & 1170 & 38.21 & 1167 & 38.53 & 1171 & 38.44 & 1167 & 38.38 & 1166 & 38.74 & 1169 \\
\hline 41.13 & 1192 & 39.03 & 1195 & 38.89 & 1195 & 38.77 & 1195 & 38.97 & 1195 & 39.18 & 1195 & 39.15 & 1192 & 39.24 & 1189 & 39.71 & 1197 \\
\hline 41.79 & 1224 & 39.77 & 1218 & 39.6 & 1224 & 39.68 & 1222 & 39.57 & 1220 & 40.04 & 1225 & 39.86 & 1225 & 40.04 & 1221 & 40.1 & 1220 \\
\hline 42.32 & 1245 & 40.34 & 1244 & 40.4 & 1248 & 40.54 & 1247 & 40.42 & 1249 & 40.45 & 1250 & 40.42 & 1243 & 40.66 & 1247 & 41.05 & 1256 \\
\hline 43.5 & 1276 & 41.16 & 1276 & 41.13 & 1276 & 41.16 & 1277 & 41.08 & 1274 & 41.11 & 1273 & 41.4 & 1277 & 41.25 & 1276 & 41.55 & 1280 \\
\hline 44.18 & 1302 & 41.79 & 1303 & 41.93 & 1303 & 41.79 & 1304 & 41.85 & 1304 & 41.99 & 1304 & 41.9 & 1304 & 42.02 & 1300 & 42.5 & 1309 \\
\hline 44.86 & 1332 & 42.73 & 1327 & 42.41 & 1328 & 42.53 & 1333 & 42.53 & 1329 & 42.59 & 1331 & 42.85 & 1334 & 43.03 & 1333 & 43.06 & 1334 \\
\hline 45.75 & 1357 & 43.56 & 1359 & 43.18 & 1355 & 43.24 & 1355 & 43.41 & 1353 & 43.21 & 1357 & 43.3 & 1360 & 43.47 & 1352 & 43.74 & 1359 \\
\hline 46.2 & 1381 & 44.21 & 1383 & 44.01 & 1384 & & 1384 & & 1387 & 44.27 & 1389 & 44.27 & 1388 & 44.39 & 1385 & 44.3 & 1385 \\
\hline 46.99 & 1406 & 45.04 & 1410 & 44.75 & 1408 & 44.83 & 1414 & 44.72 & 1411 & 44.89 & 1413 & 44.8 & 1412 & 45.1 & 1409 & 44.98 & 1409 \\
\hline 47.94 & 1439 & 45.96 & 1442 & 45.6 & 1441 & 45.49 & 1441 & 45.31 & 1437 & 45.72 & 1440 & 45.4 & 1437 & 45.6 & 1434 & 45.9 & 1445 \\
\hline 48.53 & 1464 & 46.34 & 1466 & 46.17 & 1464 & 46.14 & 1467 & 46.05 & 1462 & 46.25 & 1465 & 46.25 & 1470 & 46.52 & 1467 & 46.55 & 1468 \\
\hline 49.27 & 1492 & 47.17 & 1493 & 46.88 & 1493 & 46.82 & 1491 & 46.64 & 1489 & 47.08 & 1498 & 46.99 & 1495 & 47.26 & 1496 & 47.47 & 1499 \\
\hline 49.98 & 1519 & 47.7 & 1517 & 47.68 & 1519 & 47.53 & 1523 & 47.7 & 1522 & 47.68 & 1523 & 47.68 & 1524 & 47.85 & 1519 & 48.12 & 1527 \\
\hline 46.31 & 1541 & 44.95 & 1505 & 45.16 & 1516 & 45.22 & 1510 & 46.05 & 1537 & 45.99 & 1531 & 45.37 & 1507 & 45.31 & 1507 & 46.14 & 1538 \\
\hline 45.34 & 1513 & 44.33 & 1480 & 44.39 & 1483 & 44.48 & 1482 & 45.4 & 1508 & 37 & 1517 & 44.69 & 1482 & 44.72 & 1480 & 45.37 & 1510 \\
\hline 44.78 & 1490 & 43.68 & 1452 & 43.8 & 1455 & 43.95 & 1457 & 44.66 & 1485 & 45.13 & 1507 & 44.09 & 1456 & 44.04 & 1457 & 44.51 & 1484 \\
\hline 44.04 & 1458 & 43.03 & 1428 & 42.85 & 1431 & 43.21 & 1432 & 43.74 & 1456 & 43.71 & 1449 & 43.38 & 1429 & 43.53 & 1431 & 44.06 & 1458 \\
\hline 43.18 & 1432 & 41.99 & 1398 & 42.17 & 1399 & 42.53 & 1407 & 43.24 & 1427 & 43.71 & 1440 & 42.61 & 1403 & 42.35 & 1399 & 43.35 & 1432 \\
\hline 42.64 & 1408 & 41.46 & 1370 & 41.28 & 1371 & 41.49 & 1372 & 42.56 & 1403 & 42.53 & 1409 & 41.55 & 1369 & 41.87 & 1371 & 42.73 & 1407 \\
\hline 41.7 & 1382 & 40.69 & 1346 & 40.72 & 1347 & 40.87 & 1348 & 41.49 & 1369 & 42.11 & 1390 & 41.02 & 1345 & 41.19 & 1345 & 42.14 & 1379 \\
\hline 41.31 & 1355 & 39.92 & 1321 & 39.89 & 1315 & 40.28 & 1319 & 41.05 & 1354 & 40.81 & 1341 & 40.37 & 1319 & 40.42 & 1322 & 41.19 & 1355 \\
\hline 40.31 & 1329 & 39.24 & 1294 & 39.54 & 1300 & 39.57 & 1294 & 40.22 & 1318 & 39.98 & 1315 & 39.63 & 1291 & 39.66 & 1289 & 40.34 & 1321 \\
\hline 39.57 & 1303 & 38.41 & 1261 & 38.65 & 1264 & 38.92 & 1269 & 39.54 & 1290 & 39.66 & 1291 & 38.89 & 1267 & 39.03 & 1263 & 39.74 & 1295 \\
\hline 38.62 & 1267 & 37.49 & 1236 & 37.85 & 1237 & 37.97 & 1238 & 38.8 & 1267 & 38.62 & 1258 & 38.09 & 1236 & 38.21 & 1239 & 39.12 & 1266 \\
\hline 37.82 & 1239 & 37.02 & 1205 & 37.14 & 1211 & 37.26 & 1210 & 38.03 & 1239 & 37.88 & 1232 & 37.29 & 1207 & 37.67 & 1210 & 38.06 & 1240 \\
\hline 37.26 & 1216 & 35.99 & 1182 & 36.19 & 1180 & 36.55 & 1185 & 37.23 & 1214 & 37.29 & 1206 & 36.64 & 1181 & 36.87 & 1181 & 37.55 & 1212 \\
\hline 36.52 & 1188 & 35.45 & 1154 & 35.54 & 1154 & 35.84 & 1160 & 36.55 & 1180 & 36.37 & 1176 & 35.96 & 1156 & 36.16 & 1158 & 36.78 & 1185 \\
\hline 35.84 & 1160 & 34.98 & 1126 & 35.01 & 1129 & 34.77 & 1128 & 35.87 & 1155 & 35.69 & 1154 & 35.22 & 1131 & 35.1 & 1127 & 36.37 & 1160 \\
\hline
\end{tabular}


Table $17.15^{\circ} \mathrm{C}$ at 16 wt $\%$ - continued

\begin{tabular}{|c|c|c|c|c|c|c|c|c|c|c|c|c|c|c|c|c|c|}
\hline run1 & & run2 & & run3 & & run4 & & run5 & & run6 & & run7 & & run8 & & run9 & \\
\hline [Pa] & {$[1 / s]$} & [Pa] & {$[1 / \mathrm{s}]$} & [Pa] & {$[1 / s]$} & [Pa] & {$[1 / s]$} & [Pa] & {$[1 / \mathrm{s}]$} & [Pa] & {$[1 / \mathrm{s}]$} & [Pa] & {$[1 / s]$} & [Pa] & {$[1 / \mathrm{s}]$} & [Pa] & {$[1 / s]$} \\
\hline 34.98 & 1137 & 33.8 & 1102 & 33.88 & 1100 & 34.15 & 1102 & 34.86 & 1128 & 34.77 & 1120 & 34.39 & 1099 & 34.56 & 1102 & 35.45 & 1130 \\
\hline 34.24 & 1111 & 3.14 & 1071 & 33.23 & 1075 & 33.44 & 1074 & 34.06 & 1103 & 34.06 & 1095 & 33.62 & 1071 & 33.88 & 1072 & 34.48 & 1106 \\
\hline 33.23 & 1075 & 32.64 & 1044 & 32.82 & 1051 & 32.67 & 1047 & 33.68 & 1079 & 33.68 & 1067 & 32.79 & 1046 & 33.23 & 1048 & 34.06 & 1077 \\
\hline 32.61 & 1048 & 31.66 & 1020 & 31.99 & 1022 & 31.78 & 1021 & 32.73 & 1044 & 32.97 & 1040 & 32.32 & 1019 & 32.29 & 1023 & 33.03 & 1052 \\
\hline 31.81 & 1024 & 30.75 & 987.1 & 31.34 & 994.2 & 31.58 & 997.3 & 32.32 & 1019 & 32.02 & 1017 & 31.52 & 995.5 & 31.66 & 996.8 & 32.64 & 1027 \\
\hline 31.19 & 994.6 & 30.16 & 962.9 & 30.6 & 969.9 & 30.54 & 965.1 & 31.37 & 988.9 & 31.13 & 992 & 30.81 & 966.9 & 30.75 & 967.7 & 31.58 & 995.5 \\
\hline 30.75 & 971.3 & 29.42 & 935.1 & 29.65 & 936 & 29.83 & 938.7 & 30.42 & 962.9 & 30.57 & 963.3 & 29.47 & 934.7 & 30.19 & 941.3 & 30.75 & 966.9 \\
\hline 29.74 & 940 & 28.68 & 909.6 & 28.94 & 910 & 28.68 & 909.6 & 29.5 & 934.7 & 30.01 & 937.3 & 29.15 & 906.5 & 29.3 & 914.4 & 30.04 & 940.4 \\
\hline 28.5 & 914.4 & 28.14 & 884.5 & 27.91 & 882.2 & 28.23 & 886.7 & 29.27 & 908.7 & 28.76 & 911.3 & 28.62 & 883.1 & 28.11 & 880 & 29.27 & 914.9 \\
\hline 27.52 & 883.1 & & 859.3 & 27.49 & 855.8 & 27.26 & 862 & & 879.6 & 28.02 & 877 & 27.7 & 850.5 & & 856.2 & & 888.4 \\
\hline 27.31 & 859.8 & 26.22 & 826.3 & 26.6 & 830.2 & 26.28 & 828 & 27.58 & 852.7 & 27.88 & 849.6 & 26.57 & 827.6 & 26.84 & 831.6 & 27.79 & 855.8 \\
\hline 26.19 & 831.6 & 25.66 & 799.4 & 25.86 & 800.3 & 26.43 & 807.3 & 26.81 & 828.5 & 26.49 & 826.7 & 26.25 & 808.7 & 26.22 & 797.6 & & 830.7 \\
\hline 25.69 & 802.5 & 24.89 & 773 & 24.56 & 771.6 & 24.68 & 772.1 & 26.04 & 801.2 & 25.45 & 794.6 & 26.66 & 802.5 & 25.78 & 772.1 & 26.1 & 800.3 \\
\hline 25.09 & 775.2 & 23.85 & 747 & 24.59 & 745.6 & 24.65 & 748.3 & 24.83 & 776.5 & 25.36 & 770.3 & 24.59 & 783.1 & 24.77 & 747 & 25.89 & 776.9 \\
\hline 23.64 & 751.8 & 22.67 & 717.4 & 22.96 & 721.4 & 23.14 & 725.4 & 24.89 & 751.8 & 24.83 & 742.1 & 24.24 & 741.2 & 23.44 & 717.9 & 24.53 & 754.9 \\
\hline 23.59 & 728.9 & 22.11 & 688.3 & 22.7 & 694.5 & 22.96 & 691.4 & 23.2 & 717.4 & 23.76 & 712.6 & 22.52 & 696.7 & 23.32 & 692.3 & 23.41 & 722.7 \\
\hline 22.19 & 694.1 & & 664.5 & & 663.2 & & 669 & & 692.3 & 22 & 686.1 & & 668.1 & & 667.6 & & 694.1 \\
\hline 21.34 & 669 & 21.54 & 638.5 & 20.45 & 639.4 & 21.34 & 637.2 & 22.02 & 665.4 & 22.55 & 661 & 21.31 & 639.9 & 20.8 & 633.7 & 22.11 & 670.3 \\
\hline 20.18 & 638.5 & & 613 & 20.74 & 608.1 & 21.04 & 611.2 & 20.74 & 637.7 & 21.87 & 637.2 & 20.92 & 608.6 & 21.07 & 608.1 & 21.69 & 642.5 \\
\hline 19.44 & 613.9 & 18.82 & 586.5 & 19.47 & 581.7 & 19.24 & 580.4 & 20.63 & 608.1 & 20.92 & 603.7 & 20.27 & 584.3 & 20.54 & 583 & 21.31 & 610.3 \\
\hline 19.68 & 583.9 & 19 & 551.7 & 19.09 & 557 & 18.94 & 554.8 & 19.71 & 583.9 & 19.95 & 577.3 & 18.11 & 554.4 & 19.62 & 556.1 & 20 & 583.5 \\
\hline 19.12 & 560.5 & 18.38 & 525.7 & 17.05 & 525.3 & 17.58 & 529.7 & 19.09 & 558.3 & 18.5 & 550 & 17.96 & 529.7 & 17.55 & 522.2 & 19.47 & 558.3 \\
\hline 18.5 & 534.1 & 17.6 & 501.5 & 16.9 & 500.2 & 16.53 & 503.3 & 18.05 & 526.2 & 18.17 & 524.9 & 17.13 & 496.2 & 17.1 & 498.4 & 19.09 & 533.7 \\
\hline 16.92 & 502.4 & 15.4 & 477.3 & 16.88 & 474.2 & 15.83 & 473.7 & 17.41 & 497.5 & 18.17 & 499.7 & 16.38 & 473.3 & 17.19 & 471.1 & 18.58 & 508.1 \\
\hline 16.89 & 476.4 & 15.1 & 442.4 & 16.44 & 445.1 & 15.41 & 446.4 & 16.34 & 475.9 & 16.27 & 466.2 & 16.85 & 446.9 & 15.94 & 447.3 & 17.03 & 476.4 \\
\hline 14.36 & 453.5 & 14.99 & 417.8 & 13.86 & 420.4 & 14.53 & 422.6 & & 443.3 & 15.77 & 441.6 & 15.15 & 416.4 & .62 & 420.4 & 16.88 & 448.2 \\
\hline 13.62 & 420.4 & 14.5 & 387.8 & 14.37 & 397.1 & 13.03 & 391.8 & 14.92 & 419.1 & 14.27 & 414.7 & 13.96 & 390 & 15.25 & 394 & 14.5 & 424.4 \\
\hline 14.43 & 396.6 & 12.72 & 360.9 & 12 & 364.9 & 14.26 & 364 & 15.15 & 389.6 & 15.12 & 387.4 & 13.59 & 361.8 & 12.74 & 359.6 & 14.22 & 391.3 \\
\hline 11.66 & 365.3 & 11.35 & 334 & 12.54 & 337.6 & 13.9 & 341.1 & 12.74 & 362.7 & 12.15 & 358.7 & 13.09 & 339.8 & 13.64 & 334.5 & 13.09 & 365.3 \\
\hline 11.99 & 340.2 & 10.9 & 310.2 & 12.9 & 312 & 10.65 & 310.7 & 11.51 & 338.4 & 11.39 & 335.4 & 12.98 & 312.4 & 13.33 & 308.5 & 13.98 & 338.4 \\
\hline 12.25 & 315.5 & 11.79 & 279.4 & 11.41 & 281.2 & 9.786 & 285.1 & 12.08 & 310.7 & 11.29 & 309.8 & 10.14 & 280.3 & 12.58 & 283.8 & 13 & 312.9 \\
\hline 11.82 & 291.3 & 10.64 & 254.3 & 10.36 & 253 & 8.939 & 254.7 & 11.55 & 286.4 & 12.34 & 275 & 10.5 & 256.5 & 10.08 & 250.7 & 11.21 & 285.1 \\
\hline
\end{tabular}


Table 18. $25^{\circ} \mathrm{C}$ at 16 wt $\%$

\begin{tabular}{|c|c|c|c|c|c|c|c|c|c|c|c|c|c|c|c|c|c|}
\hline run1 & & run2 & & run3 & & run4 & & run5 & & run6 & & run7 & & run8 & & run9 & \\
\hline [Pa] & {$[1 / s]$} & [Pa] & {$[1 / s]$} & [Pa] & {$[1 / s]$} & [Pa] & {$[1 / s]$} & [Pa] & {$[1 / s]$} & [Pa] & {$[1 / s]$} & [Pa] & {$[1 / s]$} & [Pa] & {$[1 / s]$} & [Pa] & {$[1 / s]$} \\
\hline 13.45 & 278.1 & 13.52 & 276.7 & 12.8 & 276.3 & 12.05 & 273.2 & 13.7 & 272.8 & 13.36 & 281.6 & 13.62 & 279 & 14.17 & 272.3 & 14.94 & 276.3 \\
\hline 13.85 & 293.1 & 2.62 & 292.2 & 13.84 & 289.1 & 12.76 & 294.4 & 15.13 & 296.1 & 14.74 & 294.4 & 14.18 & 292.6 & 13.05 & 294.4 & 15.45 & 290.9 \\
\hline 14.12 & 15.1 & 3.98 & 318.6 & 3.25 & 313.3 & 15.14 & 323 & 15.75 & 323 & 13.85 & 323.9 & 14.38 & 315.5 & 13.97 & 320.4 & 14.15 & 317.7 \\
\hline 13.08 & 340.6 & .82 & 341.5 & 5.83 & 345.1 & 16.33 & 348.1 & 14.96 & 346.8 & 16.67 & 352.1 & 16.51 & 349 & 15.82 & 344.2 & 14.2 & 342.4 \\
\hline 15.87 & 372.8 & 14.9 & 374.1 & 15.55 & 371.1 & 15.7 & 371.5 & 16.85 & 378.1 & 15.33 & 379.4 & 15.39 & 376.3 & 14.95 & 373.3 & 17.05 & 373.3 \\
\hline 14.7 & 401 & 16.92 & 399.3 & 7.17 & 400.6 & 16.95 & 405.4 & 16.17 & 402.8 & 17.43 & 405 & 16.47 & 401 & 16.73 & 399.3 & 15.68 & 397.1 \\
\hline 15.37 & 431 & 0.19 & 428.3 & 16.26 & 415.1 & 17.14 & 433.2 & 17.29 & 427 & 16.49 & 433.2 & 17.1 & 426.6 & 16.14 & 427.5 & 17.69 & 425.7 \\
\hline 17.7 & 456.1 & 34 & 453 & 7.95 & 450.8 & 17.37 & 456.5 & 16.86 & 452.6 & 18.63 & 457 & 18.47 & 453 & 17.09 & 459.2 & 18.23 & 452.1 \\
\hline 16.67 & 479.9 & 18.06 & 476.8 & 18.41 & 465.4 & 18.68 & 482.5 & 18.64 & 478.6 & 18.66 & 488.7 & 17.7 & 479 & 18.4 & 485.6 & 18.05 & 479 \\
\hline 18.58 & 512.1 & 8.37 & 503.3 & 17.76 & 486.5 & 19.23 & 508.1 & 19.02 & 513.8 & 19.91 & 513 & 19.8 & 513 & 19.15 & 513.4 & 18.3 & 505.5 \\
\hline 18.77 & & 19.9 & & & & & & & 539 & & 539 & 19.92 & 540.7 & & & & 537.6 \\
\hline 19.55 & 562.8 & 20.78 & 561.9 & 18.92 & 543.8 & 19.54 & 567.2 & 19.85 & 564.5 & 19.88 & 569.8 & 19.88 & 568.5 & 20.59 & 563.2 & 20.92 & 562.8 \\
\hline 19.57 & 594.5 & 20.81 & 587.9 & 21.13 & 578.6 & 20.16 & 592.7 & 21.67 & 593.2 & 20.51 & 594.5 & 20.46 & 593.6 & 20.48 & 590.5 & 21.16 & 592.3 \\
\hline 20.31 & 618.7 & 21.19 & 616.5 & 22.01 & 612.1 & 21.24 & 617.4 & 22.18 & 617.4 & 22.32 & 624.4 & 21.83 & 620.5 & 22.38 & 622.2 & 21.49 & 616.5 \\
\hline 21.83 & 646.5 & 22.54 & 644.7 & 21.38 & 639.9 & 22.5 & 652.2 & 22.65 & 651.3 & 22.02 & 649.6 & 22.65 & 644.3 & 22.45 & 650.4 & 22.95 & 646 \\
\hline 21.53 & 673.4 & 23 & 672 & 23.19 & 667.2 & 23.08 & 676.4 & 22.41 & 675.1 & 23.26 & 677.8 & 22.5 & 672.5 & 22.89 & 677.8 & 22.29 & 659.3 \\
\hline 22.89 & 698 & 23.8 & 694.1 & 22.59 & 693.2 & 23.57 & 703.3 & 23.77 & 700.2 & 23.92 & 705.1 & 23.54 & 704.2 & 23.83 & 702 & 23.54 & 700.7 \\
\hline 22.89 & 731.1 & & 728.4 & & & & 728.4 & 4.45 & 728.9 & & 731.5 & 24.13 & 729.3 & & 726.7 & 24.6 & 727.1 \\
\hline 23.86 & 757.5 & 5.08 & 752.2 & 24.87 & 746.5 & 24.93 & 761.1 & 24.25 & 754 & 24.45 & 759.3 & 25.05 & 756.2 & 24.99 & 758.9 & 25.55 & 754 \\
\hline 24.99 & 782.2 & 25.61 & 779.6 & 25.64 & 781.8 & 25.43 & 788.4 & 24.84 & 780.9 & 25.25 & 785.3 & 25.25 & 784.9 & 25.76 & 787.5 & 25.25 & 778.2 \\
\hline 24.66 & 805.6 & 25.58 & 810.4 & 26.2 & 809.1 & 25.31 & 811.7 & 25.82 & 815.3 & 26.53 & 816.6 & 26.53 & 808.7 & 26.5 & 813.9 & 26.35 & 810.9 \\
\hline 25.73 & 839.9 & 26.2 & 837.3 & 26.05 & 831.6 & 26.76 & 839.5 & 26.53 & 842.6 & 26.5 & 839.9 & 27.15 & 842.1 & 27.41 & 840.8 & 27 & 835.1 \\
\hline 26.05 & 863.7 & 27.06 & 862.9 & 26.88 & 863.3 & 26.85 & 862.9 & 27.24 & 868.1 & 27.8 & 865.9 & 27.74 & 867.3 & 27.77 & 864.6 & 27.68 & 864.6 \\
\hline 26.88 & 889.7 & 27.8 & 888.4 & 27.62 & 889.7 & 27.83 & 896.3 & 27.83 & 894.6 & 28.45 & 897.7 & 28.33 & 891.5 & 28.27 & 895 & 28.21 & 888.9 \\
\hline 27.41 & 918.8 & 28.42 & 912.2 & 27.89 & 917.9 & 28.57 & 921 & 28.24 & 918.8 & 28.77 & 925.4 & 28.36 & 917.9 & 29.19 & 920.1 & 29.04 & 913.1 \\
\hline 28.48 & 946.6 & 28.77 & 944.4 & 28.95 & 943.9 & 28.86 & 944.8 & 29.25 & 952.3 & 29.75 & 950.1 & 29.25 & 944.8 & 29.87 & 950.1 & 29.28 & 938.2 \\
\hline 29.01 & 972.1 & & & 29.6 & & & 978.3 & 29.78 & 977.4 & & & 29.63 & 970.4 & 30.05 & 977 & 30.05 & 971.3 \\
\hline 29.69 & 996.8 & 30.28 & 999.5 & 29.63 & 992.4 & 29.99 & 1003 & 30.7 & 1004 & 30.91 & 1003 & 30.17 & 997.7 & 30.67 & 1002 & 30.28 & 997.7 \\
\hline 29.9 & 1029 & 30.61 & 1023 & 30.55 & 1029 & 30.79 & 1030 & 31.2 & 1033 & 31.32 & 1033 & 30.79 & 1025 & 31.44 & 1028 & 31.2 & 1023 \\
\hline 30.7 & 1056 & 31.47 & 1056 & 31.02 & 1055 & 31.02 & 1059 & 31.44 & 1056 & 32 & 1062 & 31.82 & 1062 & 31.91 & 1054 & 31.73 & 1048 \\
\hline 31.56 & 1082 & 31.56 & 1074 & 31.91 & 1078 & 32.06 & 1081 & 31.91 & 1081 & 32.59 & 1086 & 32.27 & 1087 & 32.41 & 1084 & 32.3 & 1080 \\
\hline
\end{tabular}


Table 18. $25^{\circ} \mathrm{C}$ at 16 wt $\%$ - continued

\begin{tabular}{|c|c|c|c|c|c|c|c|c|c|c|c|c|c|c|c|c|c|}
\hline run1 & & run2 & & run3 & & run4 & & run5 & & run6 & & run7 & & run8 & & run9 & \\
\hline [Pa] & {$[1 / s]$} & [Pa] & {$[1 / s]$} & [Pa] & {$[1 / s]$} & [Pa] & {$[1 / s]$} & [Pa] & {$[1 / s]$} & [Pa] & {$[1 / \mathrm{s}]$} & [Pa] & {$[1 / s]$} & [Pa] & {$[1 / s]$} & [Pa] & {$[1 / s]$} \\
\hline 32.03 & 1112 & 32.5 & 1105 & 32.39 & 1112 & 32.41 & 1106 & 32.44 & 1107 & 32.95 & 1111 & 32.95 & 1112 & 33.24 & 1112 & 33.15 & 1108 \\
\hline 32.89 & 1136 & 33.07 & 1137 & 33.12 & 1135 & 32.95 & 1142 & 33.45 & 1141 & 33.78 & 1136 & 33.48 & 1138 & 33.63 & 1140 & 33.57 & 1132 \\
\hline 33.33 & 1166 & 33.75 & 1162 & 33.84 & 1165 & 33.54 & 1161 & 33.86 & 1165 & 34.87 & 1170 & 34.16 & 1166 & 34.52 & 1169 & 34.52 & 1164 \\
\hline 33.95 & 1194 & 34.16 & 1188 & 34.25 & 1190 & 34.19 & 1199 & 34.52 & 1190 & 35.4 & 1195 & 34.66 & 1191 & 34.99 & 1194 & 34.99 & 1189 \\
\hline 34.52 & 1216 & 34.66 & 1219 & 34.81 & 1212 & 34.04 & 1204 & 35.31 & 1224 & 35.82 & 1225 & 35.4 & 1218 & 35.49 & 1225 & 35.7 & 1217 \\
\hline 35.02 & 1243 & 35.26 & 1247 & 35.14 & 1238 & 35.43 & 1226 & 35.58 & 1252 & 36.82 & 1250 & 35.97 & 1251 & 36.03 & 1248 & 36.29 & 1248 \\
\hline 35.85 & 1274 & 35.76 & 1270 & 36.11 & 1273 & 36.14 & 1266 & 36.38 & 1275 & 37.5 & 1280 & 36.77 & 1275 & 36.56 & 1274 & 36.85 & 1272 \\
\hline 36.38 & 1303 & 36.47 & 1300 & 36.56 & 1302 & 36.77 & 1302 & 36.62 & 1300 & 38.04 & 1305 & 37.24 & 1304 & 37.18 & 1302 & 37.45 & 1301 \\
\hline 36.82 & 1326 & 37.06 & 1328 & 37.18 & 1327 & 37.24 & 1325 & 37.48 & 1333 & 38.81 & 1332 & 37.65 & 1327 & 37.92 & 1328 & 37.68 & 1325 \\
\hline 37.5 & 1356 & 37.71 & 1352 & 37.56 & 1352 & 38.07 & 1360 & 37.95 & 1358 & 39.49 & 1364 & 38.39 & 1355 & 38.48 & 1359 & 38.27 & 1348 \\
\hline 38.07 & 1380 & 38.1 & 1377 & 38.1 & 1377 & & 1382 & 38.54 & 1386 & 39.87 & 1380 & 38.96 & 1380 & 39.13 & 1384 & 38.78 & 1375 \\
\hline 38.72 & 1405 & 38.84 & 1411 & 38.69 & 1402 & 38.96 & 1409 & 39.19 & 1411 & 40.79 & 1416 & 39.69 & 1411 & 39.61 & 1408 & 39.55 & 1409 \\
\hline 39.4 & 1437 & 39.4 & 1436 & 39.49 & 1437 & 39.67 & 1434 & 39.75 & 1438 & 41.26 & 1442 & 40.11 & 1438 & 40.32 & 1440 & 40.17 & 1437 \\
\hline 39.93 & 1467 & 40.02 & 1463 & 40.08 & 1463 & 40.14 & 1461 & 40.29 & 1470 & 41.77 & 1468 & 40.64 & 1464 & 40.91 & 1467 & 40.91 & 1465 \\
\hline 40.55 & 1491 & 40.61 & 1487 & 40.7 & 1488 & 40.94 & 1497 & 40.82 & 1497 & 42.65 & 1492 & 41.47 & 1495 & 41.35 & 1493 & 41.29 & 1488 \\
\hline 41.03 & 1515 & 41 & 1513 & 41.14 & 1512 & 41.53 & 1524 & 41.44 & 1522 & 43.51 & 1524 & 41.88 & 1522 & 41.91 & 1522 & 41.94 & 1515 \\
\hline 40.14 & 1538 & 40.23 & 1507 & 39.81 & 1540 & 39.78 & 1533 & 40.17 & 1535 & 40.88 & 1538 & 37.42 & 1394 & 40.67 & 1538 & 40.67 & 1540 \\
\hline 39.64 & 1509 & 39.52 & 1484 & 39.34 & 1517 & & 1507 & 39.55 & 1513 & 40.29 & 1514 & 36.79 & 1371 & 40.14 & 1512 & 39.96 & 1507 \\
\hline 39.07 & 1485 & 39.01 & 1452 & 38.78 & 1486 & 38.69 & 1481 & 39.16 & 1488 & 39.9 & 1489 & 36.2 & 1341 & 39.25 & 1483 & 39.52 & 1489 \\
\hline 38.24 & 1453 & 38.22 & 1425 & 38.19 & 1458 & 38.01 & 1453 & 38.27 & 1455 & 38.93 & 1456 & 35.55 & 1313 & 38.9 & 1458 & 38.72 & 1458 \\
\hline 37.98 & 1436 & 37.71 & 1400 & 37.53 & 1434 & 37.33 & 1426 & 37.59 & 1427 & 38.57 & 1430 & 35.05 & 1288 & 38.07 & 1426 & 38.42 & 1440 \\
\hline 37.15 & 1402 & 37.06 & 1376 & 37 & 1405 & 36.94 & 1401 & 37.21 & 1403 & 37.71 & 1406 & 34.58 & 1262 & 37.65 & 1399 & 37.77 & 1410 \\
\hline 36.59 & 1375 & 36.56 & 1345 & 36.5 & 1376 & 36.08 & 1367 & 36.62 & 1374 & 37.18 & 1372 & 33.81 & 1236 & 37.09 & 1375 & 37.48 & 1384 \\
\hline 35.85 & 1351 & 36.03 & 1319 & 35.7 & 1352 & 35.49 & 1342 & 36.05 & 1348 & 36.79 & 1347 & 33.1 & 1206 & 36.44 & 1350 & 36.62 & 1362 \\
\hline 35.37 & 1326 & 35.43 & 1296 & 35.34 & 1327 & 34.87 & 1315 & 35.46 & 1322 & 36.11 & 1323 & 32.59 & 1178 & 35.85 & 1324 & 36.47 & 1336 \\
\hline 34.72 & 1290 & 34.66 & 1262 & 34.52 & 1297 & 34.28 & 1288 & 34.63 & 1291 & 35.34 & 1291 & 32.15 & 1151 & 35.14 & 1296 & 35.17 & 1289 \\
\hline 34.01 & 1264 & 33.81 & 1237 & 33.98 & 1268 & 34.07 & 1263 & 34.13 & 1263 & 34.81 & 1264 & 31.53 & 1123 & 34.43 & 1263 & 34.72 & 1261 \\
\hline 33.54 & 1241 & 33.36 & 1210 & 33.66 & 1245 & 33.36 & 1234 & 33.69 & 1235 & 34.16 & 1237 & 30.76 & 1098 & 34.1 & 1236 & 34.22 & 1238 \\
\hline 32.74 & 1207 & 32.71 & 1182 & 32.77 & 1218 & 32.68 & 1207 & 33.07 & 1211 & 33.48 & 1211 & 29.93 & 1068 & 33.63 & 1213 & 33.24 & 1205 \\
\hline 32.12 & 1181 & 32.21 & 1155 & 32.27 & 1186 & 31.94 & 1183 & 32.41 & 1185 & 32.89 & 1183 & 29.43 & 1041 & 32.62 & 1181 & 32.65 & 1181 \\
\hline 31.67 & 1154 & 31.41 & 1125 & 31.53 & 1158 & 31.62 & 1149 & 31.79 & 1154 & 32.47 & 1161 & 29.25 & 1017 & 32.3 & 1155 & 32.36 & 1155 \\
\hline
\end{tabular}


Table 18. $25^{\circ} \mathrm{C}$ at 16 wt $\%$ - continued

\begin{tabular}{|c|c|c|c|c|c|c|c|c|c|c|c|c|c|c|c|c|c|}
\hline run1 & & run2 & & run3 & & run4 & & run5 & & run6 & & run7 & & run8 & & run9 & \\
\hline [Pa] & {$[1 / \mathrm{s}]$} & [Pa] & {$[1 / \mathrm{s}]$} & [Pa] & {$[1 / \mathrm{s}]$} & [Pa] & {$[1 / s]$} & [Pa] & {$[1 / \mathrm{s}]$} & [Pa] & {$[1 / \mathrm{s}]$} & [Pa] & {$[1 / s]$} & [Pa] & {$[1 / \mathrm{s}]$} & [Pa] & {$[1 / s]$} \\
\hline 30.96 & 1127 & 30.76 & 1099 & 30.99 & 1134 & 30.73 & 1127 & 31.47 & 1126 & 31.94 & 1134 & 28.3 & 991.1 & 31.5 & 1126 & 31.79 & 1130 \\
\hline 30.73 & 104 & 9.99 & 1074 & 30.31 & 1103 & 30.17 & 1097 & 30.73 & 1101 & 31.08 & 1101 & 27.92 & 965.5 & 30.93 & 1101 & 30.85 & 1096 \\
\hline 29.93 & 072 & 29.4 & 047 & 29.75 & 1077 & 29.54 & 1069 & 0.28 & 1078 & 0.49 & 1074 & 26.7 & 932.9 & 30.43 & 1074 & 30.49 & 1070 \\
\hline 28.98 & 047 & 28.63 & 1021 & 29.04 & 1049 & 29.07 & 1044 & 29.04 & 1042 & 30.17 & 1050 & 26.55 & 905.6 & 29.93 & 1049 & 29.93 & 1044 \\
\hline 28.42 & 1021 & 28.6 & 995.9 & 28.66 & 1025 & 28.74 & 1018 & 28.6 & 1026 & 29.22 & 1020 & 25.46 & 875.2 & 28.92 & 1018 & 28.92 & 1014 \\
\hline 28.36 & 995.1 & 27.74 & 958.9 & 28.03 & 993.7 & 27.68 & 986.7 & 27.89 & 989.3 & 28.54 & 991.1 & 24.93 & 850.1 & 28.39 & 991.5 & 28.45 & 990.7 \\
\hline 27.68 & 965.1 & 26.61 & 933.4 & 27.86 & 974.8 & 27.35 & 961.1 & 27.5 & 965.1 & 27.98 & 968.6 & 24.37 & 825 & 28.09 & 963.8 & 28.27 & 966.4 \\
\hline 26.73 & 936.9 & 26.08 & 909.6 & 26.5 & 940.4 & 26.55 & 936 & 27.32 & 940.4 & 27.15 & 937.8 & 24.37 & 795 & 27.27 & 940.9 & 27.21 & 940 \\
\hline 26.11 & 910.5 & 25.96 & 882.7 & 26.32 & 911.3 & 26.14 & 909.6 & 25.99 & 906.9 & 27.12 & 912.2 & 23.54 & 770.3 & 26.94 & 912.2 & 26.64 & 906 \\
\hline 25.19 & 883.6 & & 852.3 & & 886.7 & & 884.9 & 25.4 & 882.7 & & 881.8 & & 745.6 & 26.08 & 880.5 & 25.73 & 877.8 \\
\hline 24.63 & 858.5 & 23.92 & 826.3 & 24.87 & 862 & 24.75 & 851.8 & 24.75 & 858 & 25.67 & 856.2 & 21.67 & 714.3 & 25.52 & 855.4 & 25.16 & 852.7 \\
\hline 24.31 & 832.9 & 23.77 & 798.5 & 24.28 & 836.4 & 24.6 & 824.5 & 24.01 & 825 & 25.13 & 830.7 & 21.41 & 686.6 & 25.31 & 828.9 & 24.51 & 826.3 \\
\hline 23.12 & 799 & 22.89 & 772.5 & 23.48 & 802.9 & 23.27 & 801.2 & 23.95 & 799.8 & 24.75 & 802.9 & 20.43 & 661.5 & 24.6 & 804.2 & 24.42 & 795 \\
\hline 23.45 & 776 & 21.94 & 743.4 & 23.09 & 784 & 23.03 & 767.2 & 23.21 & 773 & 23.33 & 779.1 & 20.78 & 636.8 & 23.98 & 774.3 & 23.39 & 771.2 \\
\hline 21.97 & 744.3 & 21.41 & 716.6 & 22.94 & 749.6 & 22.5 & 740.3 & 23.15 & 747.4 & 23.42 & 747.4 & 18.89 & 605.5 & 23.12 & 745.2 & 22.56 & 744.3 \\
\hline 22.2 & 717.9 & 21.73 & 690.5 & 22.47 & 721 & 21.7 & 714.8 & 21.64 & 722.3 & 22.65 & 719.6 & 19.18 & 578.2 & 21.91 & 722.3 & 22.74 & 721.8 \\
\hline 20.7 & 693.2 & & 669 & & 699.8 & & 687.9 & & 696.7 & & 689.2 & & 547.8 & & 697.2 & 21.58 & 687 \\
\hline 21.08 & 667.2 & 19.63 & 643 & 20.9 & 666.8 & 20.07 & 665 & 21.08 & 663.7 & 21.08 & 664.5 & 18.22 & 524.9 & 20.93 & 670.3 & 20.78 & 662.3 \\
\hline 19.93 & 641.6 & 9.25 & 609.9 & 19.78 & 639.9 & 20.1 & 637.7 & 19.72 & 638.5 & 01 & 638.5 & 58 & 497.1 & 20.22 & 635.9 & 20.55 & 637.2 \\
\hline 19.63 & 611.2 & .14 & 583.5 & 19.99 & 613.9 & 19.19 & 612.1 & 20.1 & 609 & 20.01 & 612.5 & 16.11 & 469.8 & 20.16 & 608.1 & 19.19 & 607.3 \\
\hline 18.83 & 584.3 & 17.52 & 558.8 & 19.48 & 587.4 & 18.44 & 576.4 & 19.33 & 583.9 & 19.42 & 583.5 & 15.76 & 445.1 & 18.65 & 580.8 & 18.48 & 582.1 \\
\hline 17.4 & 553.9 & 7.77 & 527.1 & 17.47 & 557.9 & 18.4 & 552.6 & 17.7 & 555.3 & 18.59 & 558.3 & 15.88 & 419.5 & 17.84 & 554.8 & 19.17 & 551.3 \\
\hline 16.82 & 528.8 & 17.21 & 498.9 & 16.82 & 530.6 & 16.83 & 521.8 & 16.98 & 530.6 & 18.64 & 533.7 & 14.46 & 384.7 & 17.46 & 529.7 & 18.59 & 526.2 \\
\hline 16.02 & 502.4 & & 473.3 & 16.17 & 504.1 & 17.66 & 499.7 & 16.5 & 501.5 & & 507.2 & 14.88 & 362.2 & 16.57 & 497.5 & 17.95 & 498.4 \\
\hline 15.39 & 472.4 & 15.25 & 446.9 & 16.52 & 477.7 & 17.03 & 471.5 & 15.87 & 472 & .06 & 473.7 & 12.26 & 329.2 & 16.77 & 473.3 & 16.01 & 472.9 \\
\hline 16.55 & 447.3 & 15.7 & 420 & 16.28 & 453.5 & 16.07 & 440.7 & 16.38 & 445.1 & 16.69 & 445.5 & 13.21 & 312 & 16.86 & 446.4 & 15.67 & 438.9 \\
\hline 14.26 & 423.9 & 14.06 & 394.9 & 15.37 & 420.4 & 14.25 & 414.7 & 14.76 & 418.2 & 14.47 & 420.9 & 11.41 & 277.6 & 15.53 & 416 & 16.05 & 416.4 \\
\hline 14.99 & 392.6 & 14.84 & 364.4 & 15.2 & 396.2 & 14.99 & 388.7 & 15.56 & 393.5 & 14.44 & 390.4 & 11.14 & 249 & 15.73 & 396.2 & 13.89 & 391.3 \\
\hline 12.71 & 367.1 & 13.99 & 338.9 & 13.03 & 369.7 & 13.76 & 362.2 & 13.31 & 369.3 & & 366.6 & 10.05 & 226.5 & 14.06 & 360.5 & 15.27 & 362.7 \\
\hline 13.64 & 342.4 & 11.68 & 311.6 & 14.31 & 337.6 & 12.15 & 335.4 & 13.7 & 340.6 & 14.65 & 338.9 & 0 & 178.6 & 12.81 & 344.6 & 12.84 & 336.7 \\
\hline 12.85 & 310.7 & 10.86 & 285.6 & 14.07 & 317.7 & 12.07 & 310.7 & 13.23 & 308.9 & & 312.4 & 0 & 166.8 & 14.33 & 315.5 & 12.7 & 304.1 \\
\hline 12.26 & 297 & 10.96 & 251.2 & 11.12 & 283.8 & 13.39 & 279.4 & 12.69 & 283.8 & 11.82 & 282.5 & 0 & 141.3 & 12.86 & 283.4 & 12.51 & 279.4 \\
\hline
\end{tabular}


Table 19. $25^{\circ} \mathrm{C}$ Repeat at $16 \mathrm{wt} \%$

\begin{tabular}{|c|c|c|c|c|c|c|c|c|c|c|c|c|c|c|c|c|c|}
\hline run1 & & run2 & & run3 & & run4 & & run5 & & run6 & & run7 & & run8 & & run9 & \\
\hline [Pa] & {$[1 / s]$} & [Pa] & {$[1 / s]$} & [Pa] & {$[1 / s]$} & [Pa] & {$[1 / s]$} & [Pa] & {$[1 / s]$} & [Pa] & [1/s] & [Pa] & {$[1 / s]$} & [Pa] & {$[1 / \mathrm{s}]$} & [Pa] & {$[1 / \mathrm{s}]$} \\
\hline 16.08 & 275.4 & 16.12 & 275.9 & 18.62 & 272.3 & 16.09 & 280.3 & 16.83 & 277.6 & 17.86 & 273.2 & 16.29 & 274.1 & 16.65 & 274.5 & 15.98 & 273.7 \\
\hline 17.51 & 294.4 & 16.83 & 290.9 & 17.44 & 298.3 & 16.72 & 293.1 & 17.66 & 292.2 & 18.86 & 292.2 & 17.25 & 290 & 18.45 & 297.5 & 18.11 & 294.8 \\
\hline 18.79 & 319.1 & 17.08 & 316 & 17.39 & 325.2 & 17.71 & 317.3 & 17.41 & 317.3 & 19.68 & 319.9 & 19.36 & 322.6 & 18.51 & 321.3 & 19.76 & 319.5 \\
\hline 18.84 & 347.7 & 20.26 & 347.7 & 19.24 & 349.5 & 20.09 & 343.7 & 18.6 & 342.4 & 18.91 & 344.6 & 18.22 & 348.1 & 18.97 & 338.9 & 19.39 & 348.6 \\
\hline 18.81 & 370.2 & 18.78 & 374.1 & 18.97 & 383.4 & 18.95 & 376.3 & 19.26 & 375.5 & 20.9 & 376.8 & 20.34 & 374.6 & 20 & 357.4 & 19.08 & 372.8 \\
\hline 20.81 & 402.8 & 19.94 & 397.5 & 20.99 & 405.9 & 21.33 & 402.8 & 21.04 & 402.3 & 20.21 & 400.6 & 19.81 & 403.2 & 21.2 & 388.7 & 21.29 & 402.8 \\
\hline 20.55 & 427 & 20.37 & 429.7 & 20.29 & 435 & 20.31 & 425.3 & 20.09 & 428.3 & 21.97 & 428.8 & 21.49 & 427.9 & 20.96 & 419.5 & 20.66 & 427 \\
\hline 21.46 & 452.6 & 21.18 & 456.5 & 22.27 & 458.8 & 20.91 & 457.4 & 22.37 & 453.5 & 20.97 & 453.5 & 20.77 & 453 & 21.64 & 440.7 & 22.01 & 460.5 \\
\hline 21.66 & 483.9 & 22.66 & 480.8 & 21.76 & 481.2 & 22.25 & 483.9 & 21.79 & 478.6 & 21.99 & 479.9 & 22.36 & 487 & 22.32 & 468 & 22.98 & 477.3 \\
\hline 22.34 & 511.6 & 23.49 & 508.5 & 23.56 & 515.6 & 23.64 & 507.7 & 22.29 & 504.6 & 22.49 & 511.6 & 22.51 & 513 & 22.66 & 515.6 & 22.75 & 512.1 \\
\hline 23.19 & 536.8 & & 537.2 & & & & & & 537.6 & & & & 539 & & & & 541.2 \\
\hline 24.35 & 567.2 & 24.38 & 562.8 & 23.88 & 570.2 & 23.86 & 567.2 & 24.68 & 565 & 23.88 & 563.2 & 23.74 & 562.8 & 23.98 & 566.7 & 24.5 & 568 \\
\hline 25.43 & 592.7 & 25.17 & 595.4 & 24.8 & 597.6 & 24.74 & 592.3 & 25.3 & 589.2 & 24.38 & 587 & 24.94 & 597.1 & 25.04 & 594 & 25.73 & 594.5 \\
\hline 25.63 & 620 & 26.03 & 621.4 & 26.18 & 623.6 & 26.33 & 619.6 & 25.69 & 618.7 & 26.19 & 616.1 & 25.46 & 624 & 25.7 & 626.7 & 26.41 & 618.7 \\
\hline 26.84 & 649.6 & 25.66 & 649.1 & 25.89 & 648.7 & 25.89 & 646 & 26.28 & 646.9 & 25.89 & 642.5 & 26.69 & 647.8 & 26.45 & 649.6 & 26.96 & 650.4 \\
\hline 26.25 & 675.1 & 26.07 & 669.8 & 26.39 & 673.4 & 26.87 & 670.3 & 26.42 & 671.6 & 26.75 & 676.9 & 27.4 & 671.6 & 27.22 & 676.9 & 26.75 & 678.2 \\
\hline 27.9 & 700.7 & 27.07 & 701.1 & 27.96 & 707.3 & 27.9 & 702 & 27.13 & 701.1 & 27.58 & 703.3 & 27.4 & 706 & 27.93 & 703.3 & 27.37 & 700.2 \\
\hline 27.9 & 727.6 & 27.61 & 727.6 & & 733.7 & & 727.1 & 27.84 & 730.7 & 28.41 & 727.6 & 28.67 & 731.5 & 28.88 & 735.5 & 28.7 & 723.2 \\
\hline 28.88 & 751.4 & 28.38 & 755.8 & 28.85 & 761.1 & 28.67 & 757.1 & 29.2 & 754.9 & 28.85 & 754.4 & 29.06 & 761.1 & 29.53 & 760.6 & 29.18 & 758.9 \\
\hline 29.83 & 786.6 & 29.09 & 785.3 & 29.09 & 787.1 & 29.29 & 782.2 & 29.77 & 780.9 & 29.18 & 780.4 & 29.77 & 784 & .89 & 790.1 & .35 & 784.4 \\
\hline 30.39 & 811.3 & 29.62 & 810.4 & 30.27 & 814.4 & 30.33 & 811.7 & 29.89 & 811.7 & 30.33 & 814.4 & 29.89 & 808.7 & 30.92 & 814.8 & 30.12 & 809.1 \\
\hline 30.09 & 834.7 & 30.18 & 836 & 30.3 & 838.2 & 30.98 & 839.5 & 30.74 & 839.1 & 31.01 & 839.1 & 30.77 & 840.8 & 30.95 & 838.2 & 30.98 & 832.9 \\
\hline 31.07 & 865.9 & 31.37 & 866.4 & 31.1 & 869.9 & 31.84 & 863.3 & 31.25 & 862.4 & 31.45 & 863.7 & 31.45 & 868.1 & 31.6 & 868.6 & 69 & 862 \\
\hline 31.66 & 891.5 & 31.78 & 893.7 & 32.16 & 898.1 & 32.16 & 888 & 32.13 & 892.8 & 32.34 & 895.9 & 32.43 & 892.8 & 32.82 & 897.7 & 32.67 & 896.3 \\
\hline 32.67 & 917.9 & 32.79 & 920.6 & 32.76 & 922.3 & 32.49 & 913.5 & 32.76 & 919.3 & 32.55 & 920.1 & 32.9 & 918.4 & 33.29 & 921 & 32.99 & 921 \\
\hline 32.73 & 944.4 & 33.11 & 943.9 & 33.47 & 954.1 & 33.61 & 947.9 & 33.58 & 944.8 & 33.47 & 949.2 & 33.47 & 953.2 & 34.03 & 950.6 & 33.47 & 945.3 \\
\hline 34.15 & 975.7 & & 975.7 & 34.24 & 981 & 34.3 & 977 & & 969.1 & 34.03 & & 34.32 & 976.1 & 34.59 & 980.5 & 34.74 & 978.8 \\
\hline 34.24 & 995.1 & 34.03 & 1003 & 34.35 & 1006 & 34.56 & 1003 & 34.8 & 1002 & 34.56 & 999.5 & 34.56 & 1002 & 35.15 & 1007 & 34.86 & 1004 \\
\hline 35.12 & 1025 & 34.83 & 1026 & 34.83 & 1032 & 35.36 & 1028 & 35.45 & 1029 & 35.21 & 1026 & 35.21 & 1028 & 35.39 & 1031 & 35.36 & 1022 \\
\hline 35.6 & 1057 & 35.57 & 1056 & 35.89 & 1064 & 35.83 & 1054 & 36.1 & 1054 & 35.83 & 1051 & 35.83 & 1054 & 36.4 & 1062 & 36.51 & 1058 \\
\hline 36.46 & 1085 & 36.01 & 1080 & 36.31 & 1091 & 36.31 & 1078 & 36.57 & 1081 & 36.4 & 1081 & 36.75 & 1087 & 37.08 & 1089 & 36.54 & 1072 \\
\hline
\end{tabular}


Table 19. $25^{\circ} \mathrm{C}$ Repeat at $16 \mathrm{wt} \%$ - continued

\begin{tabular}{|c|c|c|c|c|c|c|c|c|c|c|c|c|c|c|c|c|c|}
\hline run1 & & run2 & & run3 & & run4 & & run5 & & run6 & & run7 & & run8 & & run9 & \\
\hline [Pa] & [1/s] & [Pa] & {$[1 / s]$} & [Pa] & [1/s] & [Pa] & {$[1 / s]$} & [Pa] & {$[1 / s]$} & [Pa] & {$[1 / s]$} & [Pa] & {$[1 / s]$} & [Pa] & [1/s] & [Pa] & {$[1 / s]$} \\
\hline 36.43 & 1107 & 36.57 & 1111 & 36.99 & 1116 & 37.25 & 1112 & 37.05 & 1106 & 37.31 & 1111 & 37.08 & 1114 & 37.64 & 1114 & 36.96 & 1090 \\
\hline 37.55 & 1141 & 37.46 & 1138 & 37.55 & 1140 & 37.58 & 1140 & 37.73 & 1133 & 37.82 & 1135 & 38.05 & 1144 & 38.02 & 1140 & 38.05 & 1131 \\
\hline 38.26 & 1166 & 37.73 & 1166 & 37.99 & 1173 & 38.35 & 1163 & 38.35 & 1166 & 38.05 & 1163 & 38.44 & 1168 & 38.5 & 1165 & 38.38 & 1159 \\
\hline 38.7 & 1192 & 38.5 & 1189 & 38.73 & 1197 & 38.88 & 1195 & 39.06 & 1193 & 38.85 & 1189 & 39.24 & 1195 & 39.33 & 1199 & 38.91 & 1179 \\
\hline 39.09 & 1215 & 39.06 & 1216 & 39.39 & 1226 & 39.62 & 1220 & 39.59 & 1217 & 39.56 & 1222 & 39.53 & 1223 & 39.95 & 1222 & 39.92 & 1215 \\
\hline 39.95 & 1249 & 40.01 & 1251 & 40.07 & 1252 & 40.04 & 1247 & 39.98 & 1243 & 40.27 & 1250 & 40.18 & 1250 & 40.42 & 1249 & 40.63 & 1244 \\
\hline 40.39 & 1274 & 40.36 & 1274 & 40.63 & 1279 & 40.78 & 1272 & 40.92 & 1273 & 40.87 & 1275 & 41.01 & 1276 & 41.16 & 1282 & 41.19 & 1278 \\
\hline 40.89 & 1299 & 40.87 & 1303 & 41.25 & 1305 & 41.04 & 1296 & 41.46 & 1301 & 41.31 & 1300 & 41.4 & 1301 & 41.66 & 1307 & 41.72 & 1304 \\
\hline 41.75 & 1331 & 41.58 & 1326 & 41.52 & 1330 & 41.78 & 1328 & 41.75 & 1325 & 41.93 & 1324 & 41.99 & 1327 & 42.43 & 1331 & 42.37 & 1332 \\
\hline 42.17 & 1359 & 42.23 & 1359 & 42.55 & 1363 & 42.4 & 1355 & 42.34 & 1350 & 42.37 & 1352 & 42.67 & 1359 & 42.94 & 1362 & 42.94 & 1356 \\
\hline 42.76 & 1386 & 42.82 & 1383 & 43 & 1388 & 43.14 & 1382 & 43.23 & 1384 & 43.11 & 1383 & 43.32 & 1388 & 43.53 & 1387 & 43.71 & 1386 \\
\hline 43.47 & 1411 & 43.14 & 1411 & 43.5 & 1411 & 43.5 & 1406 & 43.65 & 1408 & 43.74 & 1412 & 43.85 & 1412 & 43.94 & 1412 & 44.18 & 1413 \\
\hline 44.03 & 1441 & 43.94 & 1441 & 44.12 & 1445 & 44.24 & 1438 & 44.33 & 1435 & 44.24 & 1438 & 44.53 & 1436 & 44.8 & 1447 & 44.71 & 1438 \\
\hline 44.51 & 1467 & 44.42 & 1463 & 44.86 & 1472 & 44.86 & 1465 & 44.89 & 1466 & 44.77 & 1463 & 45.1 & 1467 & 45.24 & 1470 & 45.22 & 1467 \\
\hline 45.13 & 1493 & 44.98 & 1491 & 45.3 & 1495 & 45.42 & 1494 & 45.51 & 1493 & 45.39 & 1494 & 45.66 & 1496 & 45.84 & 1495 & 45.98 & 1497 \\
\hline 45.63 & 1519 & 45.51 & 1515 & 45.98 & 1524 & 46.01 & 1521 & 45.98 & 1516 & 46.07 & 1520 & 46.19 & 1522 & 46.28 & 1522 & 46.58 & 1522 \\
\hline 43.91 & 1537 & 43.97 & 1534 & 44.18 & 1538 & 44.42 & 1543 & 44.3 & 1538 & 43.94 & 1509 & 43.85 & 1512 & 44.24 & 1516 & 44.48 & 1535 \\
\hline 43.41 & 1511 & 43.38 & 1512 & 43.77 & 1513 & 43.77 & 1511 & 43.82 & 1514 & 43.2 & 1484 & 43.38 & 1486 & 43.56 & 1489 & 43.88 & 1507 \\
\hline 42.88 & 1481 & 42.55 & 1481 & 43.17 & 1489 & 43.08 & 1484 & 43.06 & 1477 & 42.82 & 1460 & 42.46 & 1455 & 42.91 & 1457 & 43.2 & 1484 \\
\hline 42.23 & 1456 & 42.32 & 1454 & 42.49 & 1457 & 42.46 & 1458 & 42.43 & 1455 & 42.2 & 1434 & 42.17 & 1427 & 42.17 & 1430 & 42.85 & 1453 \\
\hline 41.69 & 1431 & 41.63 & 1430 & 41.93 & 1430 & 41.66 & 1423 & 42.02 & 1426 & 41.31 & 1399 & 41.58 & 1404 & 41.9 & 1407 & 42.14 & 1430 \\
\hline 40.84 & 1398 & 41.1 & 1398 & 41.43 & 1402 & 41.28 & 1399 & 41.37 & 1400 & 40.78 & 1374 & 40.98 & 1377 & 41.43 & 1382 & 41.43 & 1397 \\
\hline 40.39 & 1371 & 40.3 & 1371 & 40.72 & 1378 & 40.84 & 1370 & 40.75 & 1376 & 40.39 & 1345 & 40.15 & 1342 & 40.54 & 1347 & 41.07 & 1373 \\
\hline 39.65 & 1348 & 39.86 & 1348 & 40.1 & 1348 & 40.33 & 1348 & 40.24 & 1351 & 39.86 & 1320 & 39.83 & 1318 & 39.95 & 1319 & 40.27 & 1345 \\
\hline 39.24 & 1317 & 39.12 & 1315 & 39.33 & 1322 & 39.71 & 1323 & 39.56 & 1316 & 39.18 & 1291 & 39.3 & 1291 & 39.36 & 1292 & 39.68 & 1315 \\
\hline 38.47 & 1293 & 38.41 & 1291 & 38.88 & 1296 & 38.91 & 1289 & 39.09 & 1292 & 38.5 & 1264 & 38.62 & 1265 & 38.65 & 1265 & 39.21 & 1290 \\
\hline 37.85 & 1265 & 37.85 & 1260 & 38.5 & 1267 & 38.38 & 1261 & 38.47 & 1266 & 38.02 & 1239 & 37.94 & 1242 & 37.94 & 1238 & 38.79 & 1261 \\
\hline 37.2 & 1236 & 37.55 & 1237 & 37.73 & 1243 & 37.7 & 1236 & 37.79 & 1240 & 37.31 & 1213 & 37.4 & 1214 & 37.61 & 1210 & 38.17 & 1237 \\
\hline 36.66 & 1211 & 37.17 & 1213 & 37.28 & 1217 & 37.14 & 1207 & 37.25 & 1210 & 36.9 & 1187 & 36.72 & 1179 & 36.72 & 1184 & 37.76 & 1213 \\
\hline 35.95 & 1183 & 36.16 & 1179 & 36.4 & 1182 & 36.72 & 1180 & 36.6 & 1181 & 36.25 & 1161 & 35.86 & 1154 & 36.28 & 1159 & 37.02 & 1183 \\
\hline 35.51 & 1159 & 35.77 & 1154 & 36.19 & 1156 & 35.98 & 1153 & 36.04 & 1158 & 35.33 & 1129 & 35.66 & 1127 & 35.57 & 1129 & 36.4 & 1152 \\
\hline
\end{tabular}


Table 19. $25^{\circ} \mathrm{C}$ Repeat at $16 \mathrm{wt} \%$ - continued

\begin{tabular}{|c|c|c|c|c|c|c|c|c|c|c|c|c|c|c|c|c|c|}
\hline run1 & & run2 & & run3 & & run4 & & run5 & & run6 & & run7 & & run8 & & run9 & \\
\hline [Pa] & [1/s] & [Pa] & [1/s] & [Pa] & {$[1 / s]$} & [Pa] & {$[1 / \mathrm{s}]$} & [Pa] & {$[1 / s]$} & [Pa] & [1/s] & [Pa] & {$[1 / s]$} & [Pa] & {$[1 / s]$} & [Pa] & {$[1 / s]$} \\
\hline 34.86 & 1128 & 34.98 & 1128 & 35.27 & 1133 & 35.45 & 1123 & 35.42 & 1133 & 35.01 & 1104 & 34.89 & 1103 & 35.01 & 1103 & 35.66 & 1126 \\
\hline 34.18 & 1102 & 4.53 & 1101 & 34.8 & 1100 & 34.71 & 1096 & 34.83 & 1098 & 33.97 & 1070 & 34.41 & 1075 & 34.56 & 1075 & 35.09 & 1100 \\
\hline 33.79 & 1071 & 34.03 & 1075 & 34.27 & 1073 & 34.15 & 1072 & 34.3 & 1071 & 34.03 & 1053 & 33.79 & 1045 & 34 & 1049 & 34.32 & 1072 \\
\hline 33.23 & 1044 & 33.32 & 1042 & 33.35 & 1048 & 33.44 & 1042 & 33.73 & 1055 & 32.96 & 1020 & 32.99 & 1017 & 33.35 & 1024 & 33.91 & 1046 \\
\hline 32.82 & 1019 & 32.31 & 1016 & 33.02 & 1023 & 32.73 & 1018 & 33.29 & 1032 & 32.34 & 992.9 & 32.55 & 991.5 & 32.43 & 992.4 & 33.41 & 1021 \\
\hline 31.96 & 994.2 & 32.4 & 992.9 & 32.31 & 998.1 & 32.55 & 989.3 & 32.76 & 1014 & 32.22 & 968.2 & 32.13 & 966.9 & 32.28 & 967.7 & 32.19 & 988 \\
\hline 31.63 & 968.2 & 31.07 & 959.8 & 31.9 & 966.4 & 31.93 & 965.5 & 32.11 & 962 & 30.98 & 941.7 & 31.04 & 940.9 & 31.51 & 938.7 & 32.19 & 961.1 \\
\hline 30.6 & 941.7 & 30.77 & 930.3 & 31.31 & 937.3 & 30.68 & 931.6 & 31.39 & 956.7 & 30.24 & 909.6 & 30.89 & 906.9 & 30.71 & 912.7 & 31.34 & 935.1 \\
\hline 30.12 & 907.4 & & 906.9 & 30.66 & 912.2 & 30.48 & 906 & 31.13 & 935.6 & 30 & 880.9 & 30.12 & 882.2 & 30.06 & 888.4 & & 909.6 \\
\hline 29.41 & 883.1 & 29.35 & 880 & 29.5 & 885.3 & 29.97 & 879.2 & 29.8 & 883.6 & 29.62 & 858 & 29.86 & 858 & 29.68 & 856.7 & 30.36 & 883.6 \\
\hline 28.91 & 855.4 & 29.15 & 854.5 & 29.06 & 860.7 & 29.59 & 855.8 & 29.74 & 858.5 & 29.15 & 832.9 & 28.61 & 832.9 & 28.61 & 828.5 & 29.41 & 857.6 \\
\hline 28.73 & 831.6 & 28.73 & 827.6 & 28.11 & 827.6 & 28.73 & 828.9 & 28.91 & 825 & 28.58 & 806.9 & 28.32 & 797.6 & 28.73 & 806.4 & 28.94 & 823.6 \\
\hline 27.34 & 801.2 & 28.17 & 801.6 & 27.61 & 801.6 & 27.99 & 802.5 & 28.23 & 799 & 26.96 & 773.4 & 27.99 & 779.6 & 27.55 & 772.1 & 27.87 & 796.3 \\
\hline 26.66 & 774.3 & 26.66 & 769.4 & 26.9 & 773.8 & 27.49 & 767.7 & 27.73 & 770.8 & 26.48 & 745.2 & 26.99 & 743.4 & 27.1 & 749.2 & 27.93 & 773 \\
\hline 26.33 & 748.3 & 26.13 & 741.7 & 27.19 & 749.6 & 26.19 & 743 & 26.6 & 745.6 & 25.98 & 717.9 & 25.77 & 716.6 & 26.78 & 724.5 & 26.75 & 747.4 \\
\hline 26.04 & 722.7 & & 716.1 & & 722.7 & & 714.8 & 25.68 & 717.4 & & 691.4 & & 689.7 & 26.19 & 692.8 & & 717.4 \\
\hline 25.21 & 691.4 & 25.24 & 693.2 & 25.09 & 694.5 & 25.74 & 691 & 25.71 & 691.9 & 25.15 & 667.6 & 24.68 & 664.5 & 24.53 & 665.9 & 26.22 & 693.2 \\
\hline 23.85 & 664.1 & 24.65 & 662.3 & 24.14 & 664.5 & 24.2 & 661 & 24.56 & 668.5 & 23.85 & 642.5 & 24.59 & 640.3 & 24.59 & 640.3 & 24.62 & 668.1 \\
\hline 23.35 & 641.2 & 23.64 & 634.1 & 24.41 & 638.1 & 24.68 & 635 & 24.56 & 642.1 & 23.11 & 609.5 & 23.32 & 607.7 & 24.29 & 617 & 23.91 & 635.9 \\
\hline 22.9 & 609.5 & 22.93 & 612.1 & 24.06 & 613.4 & 23.23 & 602.9 & 24.17 & 616.1 & 22.34 & 583.5 & 23.55 & 578.6 & 23.88 & 591 & 23.76 & 608.1 \\
\hline 21.9 & 581.7 & 22.16 & 579.5 & 22.52 & 586.1 & 22.31 & 580.4 & 23.46 & 581.7 & 22.75 & 552.6 & 22.81 & 552.2 & 22.22 & 557.5 & 23.7 & 580.4 \\
\hline 21.4 & 557.5 & 21.64 & 550.4 & 22.37 & 562.3 & 22.1 & 553.5 & 22.31 & 553.9 & 22.29 & 526.6 & 22.47 & 526.6 & 22.25 & 529.7 & 22.61 & 553.9 \\
\hline 20.7 & 531.9 & 20.65 & 526.2 & 21.91 & 528.8 & 21.14 & 528.4 & 21.42 & 525.3 & 20.98 & 502.4 & 20.91 & 503.7 & 20.77 & 505 & 22.49 & 528.4 \\
\hline 20.62 & 499.7 & 20.92 & 501.9 & 20.4 & 504.6 & 21.19 & 497.1 & 21.82 & 501.9 & 19.79 & 473.7 & 20.87 & 472 & 20.37 & 474.2 & 21.91 & 502.8 \\
\hline 19.26 & 473.3 & 19.15 & 468.4 & 19.53 & 474.6 & 19.51 & 471.1 & 21.09 & 472 & 18.86 & 446 & 19.09 & 444.2 & 19.06 & 446.4 & 20.97 & 469.8 \\
\hline 20.1 & 448.6 & 20.33 & 444.2 & 18.91 & 444.2 & 18.71 & 440.2 & 19.75 & 444.2 & 20.01 & 420 & 20.05 & 419.5 & 20.19 & 421.7 & 19.53 & 444.6 \\
\hline 19.65 & 417.8 & 17.95 & 418.2 & 19.92 & 417.8 & 19.77 & 414.7 & 19.31 & 420.4 & 18.33 & 387.8 & 17.55 & 394.4 & 17.69 & 394 & 18.3 & 416.4 \\
\hline 17.2 & 392.6 & 19.11 & 394.9 & 17.83 & 394 & 17.45 & 389.1 & 18.74 & 393.1 & 18.79 & 365.3 & 18.54 & 362.2 & 18.34 & 370.6 & 18.42 & 393.5 \\
\hline 18.01 & 365.3 & 17.15 & 360.9 & 17.11 & 370.6 & 17.93 & 365.3 & 18.98 & 368.9 & 16.91 & 338.4 & 16.36 & 336.2 & 16.42 & 337.6 & 17.15 & 361.4 \\
\hline 18.26 & 341.5 & 18.06 & 334.9 & 18.37 & 342.9 & 16.91 & 333.2 & 17 & 344.2 & 16.07 & 334 & 15.59 & 312 & 15.69 & 311.6 & 17.24 & 334 \\
\hline 15.1 & 309.4 & 17.77 & 312 & 15.65 & 312 & 15.45 & 307.2 & 17.51 & 308.5 & 14.98 & 293.5 & 15.13 & 287.3 & 15.32 & 286 & 17.37 & 307.6 \\
\hline 14.59 & 282.5 & 15.42 & 280.3 & 14.81 & 282.9 & 14.85 & 282.9 & 17.3 & 280.7 & 15.34 & 266.6 & 16.14 & 253.8 & 15.26 & 256.5 & 16.81 & 282.9 \\
\hline
\end{tabular}


Table 20. $50{ }^{\circ} \mathrm{C}$ at $16 \mathrm{wt} \%$

\begin{tabular}{|c|c|c|c|c|c|c|c|c|c|c|c|c|c|c|c|c|c|}
\hline run1 & & run2 & & run3 & & run4 & & run5 & & run6 & & run7 & & run8 & & run9 & \\
\hline [Pa] & {$[1 / s]$} & [Pa] & {$[1 / s]$} & [Pa] & {$[1 / s]$} & [Pa] & {$[1 / s]$} & [Pa] & {$[1 / s]$} & [Pa] & [1/s] & [Pa] & {$[1 / \mathrm{s}]$} & [Pa] & {$[1 / \mathrm{s}]$} & [Pa] & {$[1 / s]$} \\
\hline 12.39 & 273.7 & 10.57 & 279.8 & 8.905 & 279.8 & 10.97 & 274.1 & 10.59 & 273.2 & 10.91 & 271 & 9.287 & 283.4 & 10.62 & 271 & 9.316 & 275.4 \\
\hline 12.39 & 293.9 & 10.62 & 292.2 & 9.204 & 292.2 & 11.2 & 294.4 & 11.13 & 292.2 & 11.17 & 286.9 & 9.239 & 297.5 & 11.16 & 293.5 & 9.156 & 296.1 \\
\hline 11.98 & 319.5 & 1.25 & 323 & 10.21 & 318.2 & 10.87 & 317.7 & 10.36 & 317.7 & 9.731 & 317.7 & 9.843 & 321.3 & 11.01 & 320.4 & 9.529 & 319.1 \\
\hline 11.41 & 342.9 & 10.38 & 347.3 & 11.87 & 344.2 & 10.78 & 349.5 & 11.44 & 349.9 & 10.2 & 343.7 & 11.51 & 346.8 & 9.825 & 347.7 & 11.95 & 350.8 \\
\hline 11.67 & 368 & 12.17 & 375.9 & 11.01 & 370.2 & 11.98 & 375.9 & 10.85 & 378.5 & 11.61 & 373.3 & 10.49 & 378.1 & 12.11 & 378.1 & 10.35 & 377.7 \\
\hline 12.04 & 399.7 & 11.94 & 399.7 & 12.07 & 404.1 & 11.27 & 405 & 11.88 & 405.9 & 10.62 & 398.4 & 11.57 & 400.6 & 10.78 & 404.5 & 12.28 & 403.2 \\
\hline 13.6 & 428.3 & 12.68 & 432.8 & 11.66 & 429.2 & 12.36 & 428.3 & 11.61 & 430.1 & 12.62 & 423.9 & 11.15 & 430.5 & 12.63 & 427.9 & 11.27 & 427.9 \\
\hline 12.49 & 451.7 & 13.05 & 458.8 & 12.56 & 452.1 & 12.67 & 460.1 & 12.67 & 454.8 & 12.07 & 449.9 & 12.12 & 457.4 & 12.48 & 456.1 & 11.61 & 460.1 \\
\hline 13.29 & 482.5 & 12.27 & 483.4 & 12.15 & 485.2 & 12.78 & 483.9 & 13.21 & 487.8 & 12.35 & 481.2 & 13.38 & 484.3 & 12.1 & 479.9 & 12.68 & 486.1 \\
\hline 13.66 & 511.6 & 13.82 & 515.6 & 13.18 & 510.3 & 13.34 & 513.8 & 13.1 & 511.6 & 13.45 & 511.2 & 13.11 & 508.5 & 12.89 & 511.6 & 13.59 & 512.5 \\
\hline 13.98 & 533.7 & & & & & & 538.1 & & & & & 13.39 & & 13.9 & 540.3 & & 542.9 \\
\hline 15.22 & 566.3 & 14.47 & 566.7 & 13.5 & 562.8 & 14.05 & 568 & 14.31 & 566.3 & 13.22 & 559.7 & 13.84 & 563.2 & 14.36 & 565.8 & 13.35 & 568.5 \\
\hline 15.79 & 592.3 & 15.06 & 591.8 & 13.86 & 588.3 & 13.7 & 595.4 & 14.52 & 589.2 & 14.28 & 590.5 & 13.79 & 592.3 & 13.92 & 596.7 & 14.21 & 595.8 \\
\hline 15.86 & 616.1 & 15.29 & 624.4 & 14.8 & 621.4 & 13.92 & 620 & 14.23 & 620.9 & 14.7 & 620 & 14.11 & 616.5 & 14.51 & 621.8 & 14.97 & 620.5 \\
\hline 16.73 & 648.2 & 15.9 & 649.6 & 14.75 & 646.9 & 5.23 & 647.8 & 14.81 & 649.1 & 15.15 & 645.6 & 15.17 & 647.8 & 15.45 & 650 & 15.57 & 653.5 \\
\hline 16.9 & 670.3 & 15.26 & 674.7 & 15.25 & 676.4 & & 672.5 & 14.87 & 673.8 & 15.14 & 669.4 & 16.13 & 675.6 & 15.11 & 675.6 & 15.65 & 677.3 \\
\hline 16.9 & 702 & 16.56 & 699.8 & 15.78 & 698.5 & 5.31 & 704.7 & 15.44 & 703.8 & 15.94 & 695.4 & 15.75 & 698.9 & 16.27 & 699.4 & 16.27 & 705.5 \\
\hline 17.79 & 728.4 & 16.41 & 733.3 & 5.84 & 727.1 & & 725.8 & & 731.1 & 16.15 & 72 & 16.91 & 726.2 & 16.52 & 727.1 & 15.84 & 729.3 \\
\hline 18.03 & 755.8 & 17.18 & 759.3 & 16.95 & 752.2 & 6.31 & 761.1 & 16.86 & 758 & 16.81 & 754.4 & 17.3 & 753.1 & 16.93 & 754.9 & 17.2 & 764.1 \\
\hline 17.84 & 779.1 & 17.72 & 786.2 & 16.79 & 784 & 7.16 & 786.2 & 17.41 & 784 & 17.26 & 783.1 & 17.14 & 786.2 & 17.11 & 780 & 17.64 & 788.8 \\
\hline 18.6 & 813.1 & 18.1 & 810.4 & 17.35 & 809.5 & 17.14 & 814.4 & 17.8 & 809.5 & 17.72 & 809.1 & 17.58 & 812.2 & 17.57 & 814.8 & 17.63 & 813.5 \\
\hline 19.17 & 832.9 & 18.33 & 843.5 & 18.1 & 837.7 & 17.81 & 839.1 & 17.74 & 840.4 & 18.04 & 832.4 & 17.96 & 838.6 & 17.95 & 840.8 & 18 & 843.5 \\
\hline 19.09 & 858.5 & 18.93 & 868.1 & 18.77 & 863.7 & 18.03 & 863.3 & 18.37 & 865.9 & 18.38 & 864.6 & 18.44 & 862 & 18.48 & 866.8 & 18.81 & 867.3 \\
\hline 20.3 & 892.8 & 19.41 & 892.8 & 18.95 & 889.3 & 18.77 & 896.3 & 18.96 & 893.3 & 19.04 & 891.1 & 19.07 & 895 & 19.32 & 892.8 & 19.1 & 890.6 \\
\hline 20.31 & 919.3 & 19.75 & 923.2 & 18.98 & 914 & 19.06 & 921.9 & 19.05 & 920.1 & 19.01 & 916.2 & 19.87 & 921.5 & 19.55 & 917.5 & 19.39 & 925.4 \\
\hline 21 & 945.3 & 20.09 & 947.5 & 19.85 & 948.8 & 19.83 & 946.6 & 19.63 & 951.4 & 19.71 & 943.5 & 19.72 & 947.9 & 20 & 943.9 & 20.12 & 949.7 \\
\hline 20.98 & 971.3 & 20.45 & 973.9 & 19.79 & 975.2 & 20.2 & 978.3 & & 977.4 & 19.81 & 969.1 & 20.3 & 970.4 & 19.98 & 970.8 & 20.56 & 978.3 \\
\hline 21.85 & 1004 & 20.7 & 1007 & 20.71 & 999 & 20.72 & 1005 & 20.83 & 1003 & 20.82 & 1001 & 20.65 & 995.9 & 20.89 & 1004 & 20.74 & 1003 \\
\hline 21.94 & 1030 & 21.3 & 1031 & 20.97 & 1028 & 20.87 & 1035 & 20.93 & 1034 & 21 & 1027 & 20.99 & 1024 & 21.17 & 1033 & 21.12 & 1030 \\
\hline 22.59 & 1054 & 21.67 & 1058 & 21.35 & 1052 & 21.43 & 1060 & 21.61 & 1058 & 21.24 & 1054 & 21.46 & 1051 & 21.43 & 1056 & 21.49 & 1057 \\
\hline 23.03 & 1079 & 21.99 & 1091 & 21.79 & 1085 & 21.76 & 1086 & 21.67 & 1083 & 21.82 & 1083 & 22.08 & 1087 & 21.94 & 1088 & 22.23 & 1082 \\
\hline
\end{tabular}


Table 20. $50{ }^{\circ} \mathrm{C}$ at 16 wt $\%$ - continued

\begin{tabular}{|c|c|c|c|c|c|c|c|c|c|c|c|c|c|c|c|c|c|}
\hline run1 & & run2 & & run3 & & run4 & & run5 & & run6 & & run7 & & run8 & & run9 & \\
\hline [Pa] & {$[1 / s]$} & [Pa] & [1/s] & [Pa] & {$[1 / s]$} & [Pa] & {$[1 / s]$} & [Pa] & [1/s] & [Pa] & [1/s] & [Pa] & [1/s] & [Pa] & [1/s] & [Pa] & {$[1 / s]$} \\
\hline 23.53 & 1112 & 22.53 & 1116 & 22.05 & 1111 & 22.17 & 1111 & 22.02 & 1113 & 22.29 & 1107 & 22.44 & 1113 & 22.41 & 1113 & 22.62 & 1115 \\
\hline 23.86 & 137 & 22.82 & 1140 & 22.53 & 1139 & 22.47 & 1137 & 22.5 & 1138 & 22.68 & 1133 & 23.06 & 1139 & 22.94 & 1140 & 22.91 & 1143 \\
\hline 24.1 & 1164 & 23.12 & 1168 & 22.94 & 1163 & 22.88 & 1166 & 22.79 & 1163 & 22.97 & 1166 & 23.3 & 1163 & 23.36 & 1164 & 23.18 & 1167 \\
\hline 24.57 & 1190 & 23.42 & 1193 & 23.44 & 1195 & 23.62 & 1196 & 23.53 & 1196 & 23.3 & 1191 & 23.56 & 1189 & 23.62 & 1189 & 23.83 & 1198 \\
\hline 25.22 & 1205 & 24.3 & 1224 & 23.92 & 1220 & 23.71 & 1222 & 23.95 & 1224 & 23.83 & 1215 & 24.15 & 1224 & 24.13 & 1216 & 24.33 & 1223 \\
\hline 25.16 & 1236 & 24.66 & 1252 & 24.24 & 1248 & 24.45 & 1248 & 24.24 & 1249 & 24.33 & 1247 & 24.45 & 1248 & 24.75 & 1248 & 24.57 & 1248 \\
\hline 25.04 & 1243 & 25.01 & 1277 & 24.66 & 1273 & 24.72 & 1273 & 24.6 & 1273 & 24.84 & 1272 & 24.95 & 1276 & 25.19 & 1274 & 25.13 & 1280 \\
\hline 26.05 & 1257 & 25.34 & 1301 & 25.19 & 1299 & 25.34 & 1307 & 25.1 & 1298 & 25.34 & 1300 & 25.49 & 1300 & 25.49 & 1302 & 25.61 & 1308 \\
\hline 26.55 & 1300 & 25.84 & 1335 & 25.63 & 1330 & 25.46 & 1331 & 25.63 & 1332 & 25.87 & 1328 & 25.93 & 1330 & 25.96 & 1329 & 26.14 & 1332 \\
\hline 27.41 & 1351 & 26.23 & 1359 & 25.96 & 1356 & 26.05 & 1360 & 26.17 & 1359 & 26.05 & 1348 & 26.55 & 1359 & 26.29 & 1353 & 26.52 & 1360 \\
\hline 27.82 & 1384 & 26.58 & 1384 & 26.52 & 1383 & 26.46 & 1386 & 26.61 & 1386 & 26.73 & 1384 & 26.73 & 1386 & 26.7 & 1385 & 26.85 & 1385 \\
\hline 28.09 & 1408 & 27.26 & 1415 & & 1406 & 26.88 & 1412 & 27.03 & 1410 & 27 & 1412 & 27.11 & 1411 & 27.11 & 1412 & 27.32 & 1417 \\
\hline 28.68 & 1438 & 27.62 & 1438 & 27.35 & 1438 & 27.14 & 1436 & 27.2 & 1437 & 27.32 & 1436 & 27.71 & 1434 & 27.65 & 1437 & 27.79 & 1444 \\
\hline 28.89 & 1466 & 28.06 & 1469 & 27.68 & 1466 & 27.74 & 1470 & 27.88 & 1469 & 27.85 & 1462 & 28.3 & 1467 & 28.18 & 1464 & 28.12 & 1471 \\
\hline 29.3 & 1493 & 28.45 & 1496 & 28.03 & 1492 & 27.94 & 1490 & 28.36 & 1494 & 28.15 & 1487 & 28.65 & 1495 & 28.36 & 1487 & 28.68 & 1496 \\
\hline 29.84 & 1519 & 28.8 & 1523 & 28.65 & 1523 & 28.62 & 1524 & 28.68 & 1523 & 28.51 & 1512 & 29.16 & 1519 & 29.07 & 1523 & 28.92 & 1519 \\
\hline 27.62 & 1533 & 27.2 & 1511 & 27.68 & 1543 & 27.29 & 1511 & 27.56 & 1534 & 27.2 & 1507 & 27.77 & 1534 & 27.97 & 1536 & 28.15 & 1534 \\
\hline 27.26 & 1507 & 26.55 & 1480 & 27.26 & 1514 & 26.85 & 1482 & 27.14 & 1507 & 26.97 & 1481 & 27.47 & 1508 & 27.53 & 1509 & 27.77 & 1511 \\
\hline 26.73 & 1482 & & 1452 & 26.88 & 1488 & 26.37 & 1458 & 26.7 & 1483 & 26.61 & 1457 & 27.11 & 1485 & 27.2 & 1484 & 27.23 & 1485 \\
\hline 26.46 & 1457 & 25.84 & 1430 & 26.29 & 1453 & 26.08 & 1433 & 26.23 & 1451 & 25.93 & 1423 & 26.46 & 1454 & 26.85 & 1460 & 26.73 & 1452 \\
\hline 25.61 & 1423 & 25.25 & 1404 & 25.99 & 1430 & 25.49 & 1401 & 25.84 & 1424 & 25.55 & 1398 & 26.2 & 1429 & 26.11 & 1428 & 26.32 & 1424 \\
\hline 25.63 & 1404 & 24.78 & 1370 & 25.55 & 1406 & 25.19 & 1374 & 25.55 & 1400 & 25.19 & 1374 & 25.72 & 1400 & 25.72 & 1399 & 25.9 & 1400 \\
\hline 25.1 & 1371 & 24.45 & 1346 & 24.87 & 1372 & 24.6 & 1347 & 24.92 & 1371 & 24.54 & 1342 & 25.25 & 1375 & 25.34 & 1372 & 25.49 & 1376 \\
\hline 24.48 & 1342 & 23.92 & 1316 & 24.48 & 1345 & 24.07 & 1323 & 24.78 & 1347 & 24.24 & 1315 & 24.98 & 1349 & 24.98 & 1348 & 24.81 & 1343 \\
\hline 24.42 & 1319 & 23.62 & 1292 & 24.1 & 1319 & 23.89 & 1294 & 24.24 & 1323 & 24.04 & 1290 & 24.45 & 1323 & 24.36 & 1318 & 24.45 & 1316 \\
\hline 23.56 & 1288 & 23.24 & 1265 & 23.77 & 1295 & 23.21 & 1269 & 23.74 & 1296 & 23.3 & 1265 & 23.92 & 1291 & 24.13 & 1292 & 24.01 & 1290 \\
\hline 23.24 & 1261 & 22.62 & 1235 & 23.18 & 1268 & 22.94 & 1243 & 23.21 & 1260 & 23.33 & 1256 & 23.62 & 1263 & 23.39 & 1265 & 23.47 & 1261 \\
\hline 22.85 & 1238 & 22.14 & 1207 & 22.59 & 1234 & 22.23 & 1209 & 22.7 & 1233 & 22.76 & 1216 & 23.21 & 1238 & 23.12 & 1240 & 23.27 & 1236 \\
\hline 22.56 & 1212 & 21.76 & 1183 & 22.08 & 1211 & 22.02 & 1183 & 22.5 & 1209 & 22.11 & 1185 & 22.82 & 1211 & 22.5 & 1207 & 22.88 & 1209 \\
\hline 21.96 & 1180 & 21.55 & 1158 & 21.99 & 1184 & 21.52 & 1155 & 22.11 & 1182 & 21.46 & 1141 & 22.32 & 1187 & 22.38 & 1180 & 22.53 & 1183 \\
\hline 21.64 & 1152 & 21.2 & 1124 & 21.73 & 1159 & 21.2 & 1129 & 21.46 & 1155 & 21.2 & 1132 & 21.67 & 1152 & 22.02 & 1155 & 22.14 & 1159 \\
\hline
\end{tabular}


Table 20. $50{ }^{\circ} \mathrm{C}$ at 16 wt $\%$ - continued

\begin{tabular}{|c|c|c|c|c|c|c|c|c|c|c|c|c|c|c|c|c|c|}
\hline run1 & & run2 & & run3 & & run4 & & run5 & & run6 & & run7 & & run8 & & run9 & \\
\hline [Pa] & {$[1 / s]$} & [Pa] & {$[1 / \mathrm{s}]$} & [Pa] & {$[1 / s]$} & [Pa] & {$[1 / s]$} & [Pa] & {$[1 / \mathrm{s}]$} & [Pa] & {$[1 / \mathrm{s}]$} & [Pa] & {$[1 / s]$} & [Pa] & {$[1 / \mathrm{s}]$} & [Pa] & {$[1 / s]$} \\
\hline 21.25 & 1127 & 20.49 & 1097 & 20.96 & 1126 & 20.46 & 1105 & 21.2 & 1126 & 21.43 & 1125 & 21.2 & 1135 & 21.37 & 1129 & 21.43 & 1127 \\
\hline 20.96 & 1101 & 20.28 & 1074 & 20.69 & 1103 & 20.01 & 1072 & 20.51 & 1097 & 20.54 & 1079 & 20.69 & 1100 & 20.69 & 1102 & 21.14 & 1100 \\
\hline 20.28 & 1077 & 19.6 & 1048 & 19.89 & 1074 & 9.63 & 1045 & 19.95 & 1073 & 20.04 & 1042 & 20.51 & 1074 & 20.37 & 1069 & 20.34 & 1071 \\
\hline 19.69 & 1042 & 19.18 & 1015 & 19.83 & 1051 & 19.42 & 1019 & 19.92 & 1047 & 19.33 & 1016 & 20.13 & 1051 & 19.89 & 1044 & 20.07 & 1046 \\
\hline 19.69 & 1020 & 18.98 & 990.7 & 19.15 & 1025 & 19.27 & 993.7 & 19.39 & 1023 & 19.09 & 986.2 & 19.6 & 1024 & 19.57 & 1021 & 20.04 & 1020 \\
\hline 19.21 & 993.3 & 18.77 & 967.7 & 19.21 & 996.4 & 18.77 & 964.2 & 18.65 & 988 & 18.38 & 961.1 & 19.33 & 989.8 & 19.42 & 994.6 & 19.6 & 993.3 \\
\hline 18.86 & 964.2 & 17.82 & 933.4 & 18.56 & 965.1 & 18.24 & 942.2 & 18.68 & 963.3 & 17.88 & 936.9 & 18.41 & 962.9 & 19.15 & 966.9 & 18.8 & 966.4 \\
\hline 18.32 & 937.3 & 17.35 & 909.6 & 18.27 & 940.4 & 17.58 & 907.8 & 17.97 & 936 & 17.47 & 906 & 18.53 & 937.8 & 18.27 & 941.3 & 18.32 & 935.1 \\
\hline 18.15 & 908.7 & 17.35 & 884.5 & 17.67 & 908.7 & 17.02 & 883.1 & 17.67 & 912.2 & 17.41 & 879.2 & 18.18 & 912.7 & 17.7 & 912.7 & 17.79 & 908.2 \\
\hline 17.32 & 877 & 16.46 & 852.7 & & 880.5 & 17.2 & 854 & & 887.1 & & 854.5 & & 882.2 & 17.61 & 879.2 & 17.29 & 881.4 \\
\hline 16.82 & 853.6 & 16.66 & 824.1 & 17.01 & 854.5 & 16.68 & 829.4 & 17.06 & 854 & 16.2 & 822.3 & 17.29 & 857.6 & 16.87 & 862 & 17.17 & 854.9 \\
\hline 17 & 825 & 15.67 & 801.2 & 16.32 & 830.7 & 15.69 & 806 & 16.76 & 826.7 & 16.16 & 805.1 & 16.73 & 825.8 & 16.49 & 828.9 & 16.78 & 828.9 \\
\hline 16.29 & 801.2 & 15.6 & 776.5 & 15.83 & 805.1 & 15.39 & 779.1 & 16.24 & 802.9 & 15.35 & 769.9 & 15.77 & 801.2 & 16.37 & 798.1 & 16.27 & 802.9 \\
\hline 15.8 & 768.6 & 15.17 & 742.1 & 15.4 & 777.8 & 15.56 & 744.3 & 15.57 & 777.4 & 14.95 & 741.7 & 15.79 & 770.8 & 15.55 & 774.3 & 15.5 & 770.3 \\
\hline 15.74 & 743.9 & 14.67 & 717.9 & 15.07 & 744.8 & 14.58 & 719.2 & 15.08 & 743 & 15.26 & 717 & 15.29 & 743.4 & 14.85 & 744.3 & 15.77 & 745.6 \\
\hline 14.95 & 716.6 & 14.77 & 691 & 14.26 & 720.1 & 14.88 & 696.3 & 15.2 & 715.7 & 14.23 & 693.6 & 14.35 & 717.4 & 14.87 & 717.9 & 15.19 & 718.3 \\
\hline 14.71 & 691.4 & & 665.9 & & 694.1 & & 669.8 & 3.84 & 691.4 & & 659.7 & 14.58 & 689.7 & & 688.8 & & 692.8 \\
\hline 14.15 & 658.8 & 14.05 & 634.6 & 13.72 & 668.1 & 13.01 & 635.9 & 13.86 & 667.6 & 13.23 & 641.6 & 13.55 & 665 & 13.81 & 664.1 & 14.02 & 660.1 \\
\hline 13.18 & 633.3 & 12.8 & 606.8 & 12.93 & 636.3 & 13.43 & 609.9 & 13.32 & 634.6 & 12.96 & 606.4 & .72 & 640.3 & 13.85 & 635.5 & .59 & 634.1 \\
\hline 13.89 & 605.5 & 12.63 & 581.3 & 13.53 & 608.1 & 13.26 & 585.7 & 13.49 & 609.9 & 12.26 & 578.2 & 12.65 & 609 & 12.8 & 609 & 3.47 & 609.5 \\
\hline 13.47 & 582.1 & 11.79 & 557 & 13.24 & 584.8 & 12.96 & 559.2 & 12.31 & 580.4 & 11.86 & 555.3 & 12.9 & 581.3 & 12.9 & 585.7 & 12.91 & 580.8 \\
\hline 12.79 & 559.2 & 11.41 & 530.6 & 11.64 & 552.6 & 12.59 & 531.9 & 11.95 & 555.3 & 11.72 & 524.4 & 12.41 & 553.5 & 12.98 & 551.7 & 12.54 & 555.3 \\
\hline 12.26 & 526.2 & 12.17 & 496.6 & 11.54 & 528.4 & 12.09 & 503.7 & 11.82 & 529.3 & 10.97 & 498 & 12.6 & 541.6 & 12.4 & 527.1 & 12.57 & 530.1 \\
\hline 11.94 & 498.4 & 10.69 & 472.4 & 11.16 & 502.4 & 11.52 & 479 & 12 & 505 & 10.54 & 468.9 & 12.42 & 515.6 & 11.85 & 500.6 & 10.93 & 496.6 \\
\hline 11.79 & 474.2 & 10.27 & 445.1 & 11.51 & 474.2 & 11.21 & 446.4 & 10.31 & 469.8 & 11.2 & 445.1 & 11.73 & 475.9 & 11.39 & 472 & 11.21 & 472 \\
\hline 11.45 & 442.4 & 10.6 & 421.7 & 10.74 & 448.2 & 9.734 & 418.6 & 11.35 & 442.9 & 9.698 & 421.3 & 11.27 & 470.6 & 10.11 & 445.5 & 11.54 & 444.2 \\
\hline 9.994 & 414.7 & 9.754 & 388.7 & 9.606 & 420.4 & 10.85 & 394.4 & 10.2 & 417.8 & 9.162 & 386.5 & 11.3 & 425.3 & 11.22 & 417.8 & 9.828 & 420 \\
\hline 11.2 & 389.1 & 10.6 & 362.7 & 10.71 & 394.9 & 10.16 & 369.3 & 10.96 & 393.1 & 10.03 & 361.4 & 9.885 & 396.2 & 9.337 & 390.9 & 10.98 & 391.3 \\
\hline 9.994 & 363.6 & 8.804 & 333.6 & 8.949 & 364.4 & 9.663 & 337.1 & 9.763 & 360 & 10.38 & 337.1 & 10.59 & 374.6 & 10.25 & 366.6 & 9.68 & 367.1 \\
\hline 9.843 & 331.8 & 8.585 & 311.1 & 9.802 & 336.7 & 9.42 & 315.1 & 10.38 & 333.6 & 9.722 & 312 & 8.872 & 335.8 & 10.51 & 342.4 & 9.876 & 334 \\
\hline 10.12 & 306.7 & 8.02 & 282.5 & 10.08 & 308 & 9.739 & 283.4 & 9.825 & 308.9 & 7.789 & 276.7 & 9.864 & 307.6 & 8.183 & 308 & 10.1 & 309.4 \\
\hline 10.26 & 279.8 & 7.771 & 254.3 & 9.352 & 280.7 & 8.872 & 254.3 & 9.633 & 284.2 & 7.449 & 252.5 & 9.87 & 280.7 & 7.964 & 282.9 & 9.494 & 280.3 \\
\hline
\end{tabular}


This page intentionally left blank. 


\section{APPENDIX F}

\section{ENVELOPE A RHEOGRAMS}

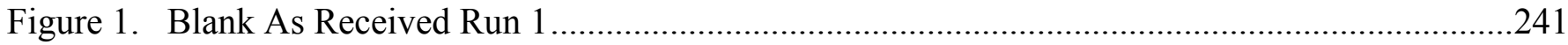

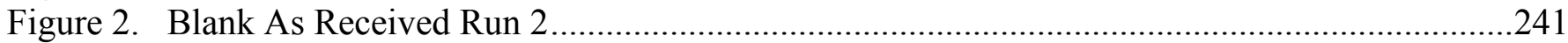

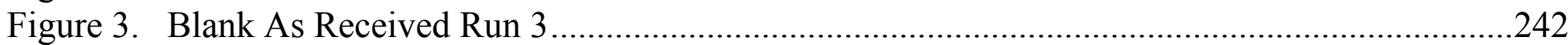

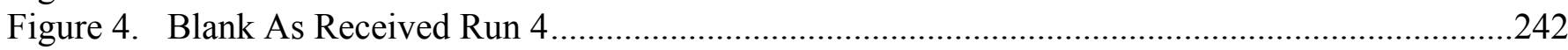

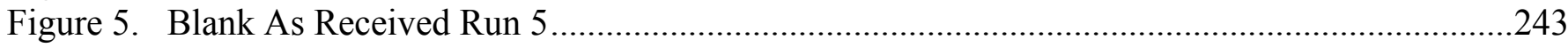

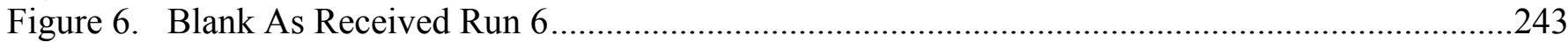

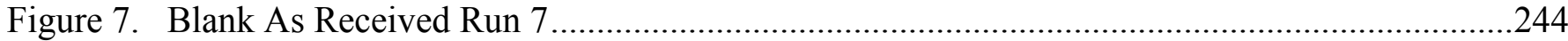

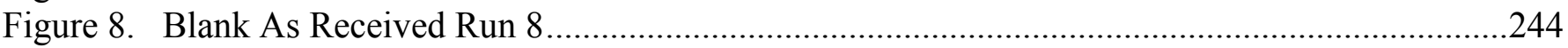

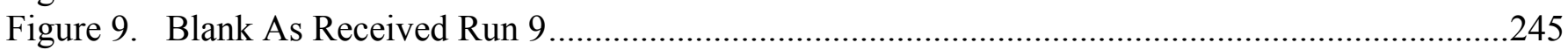

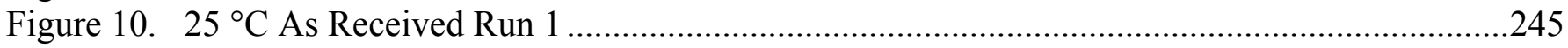

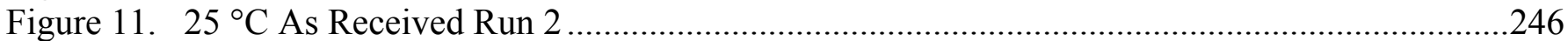

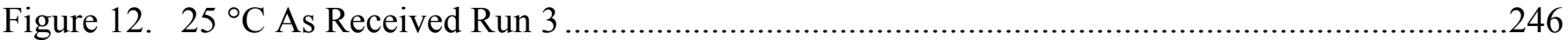

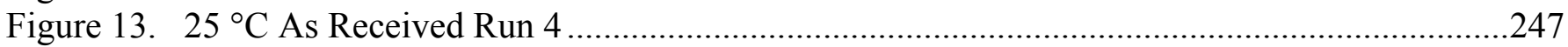

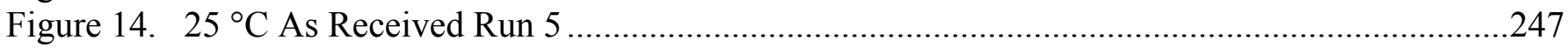

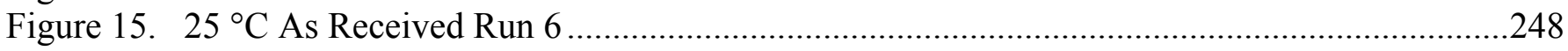

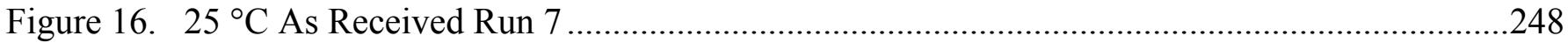

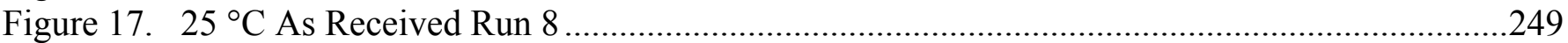

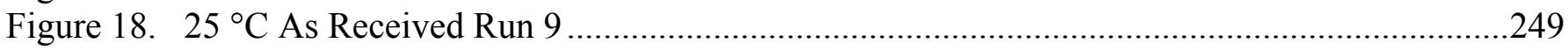

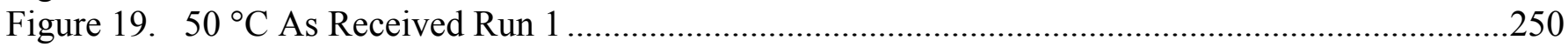

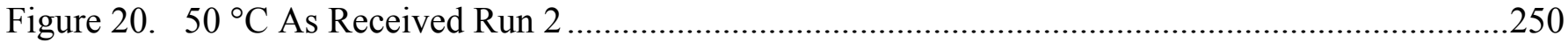

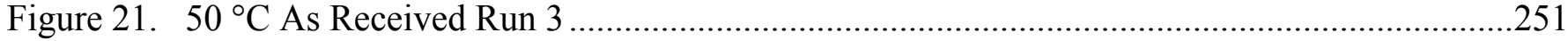

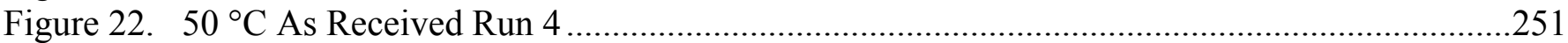

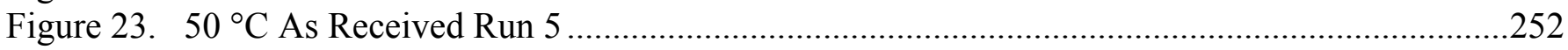

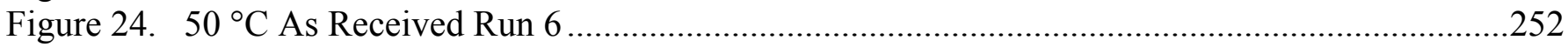

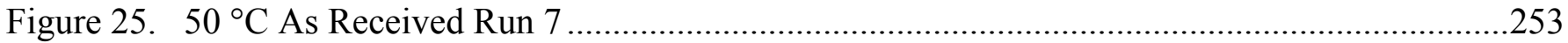

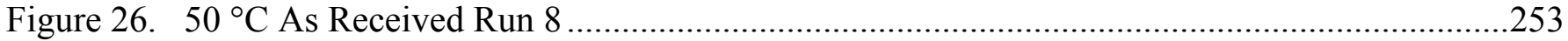

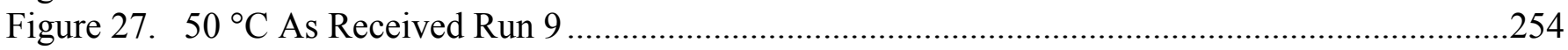

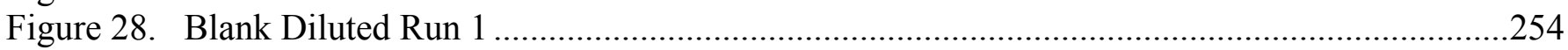

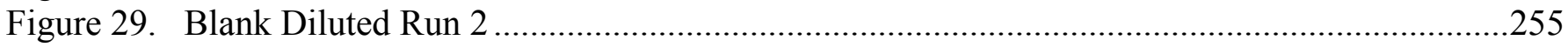

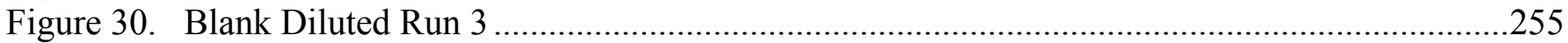

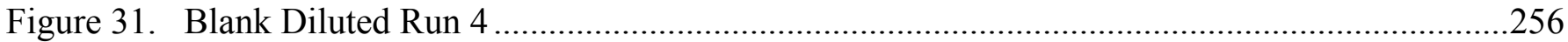

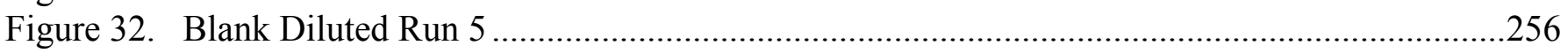

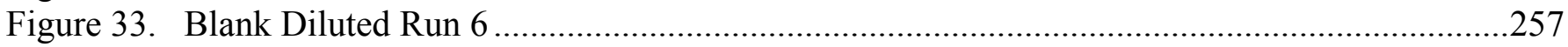

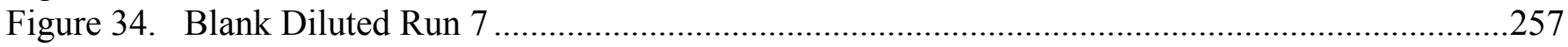

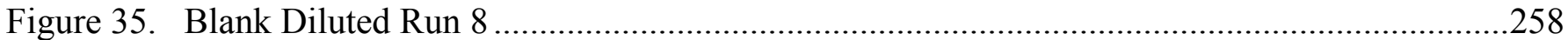

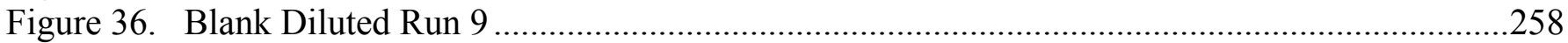

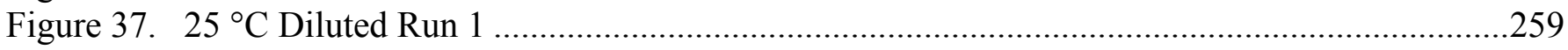

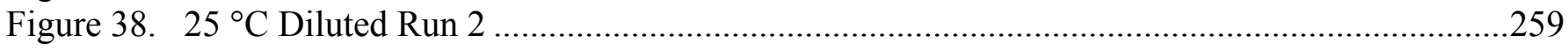

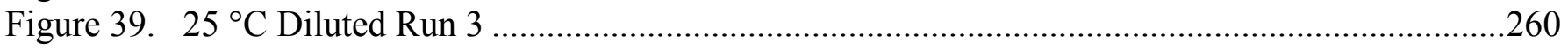


Figure 40. $25^{\circ} \mathrm{C}$ Diluted Run 4 .260

Figure 41. $25^{\circ} \mathrm{C}$ Diluted Run 5 261

Figure 42. $25^{\circ} \mathrm{C}$ Diluted Run 6 261

Figure 43. $25^{\circ} \mathrm{C}$ Diluted Run 7 262

Figure 44. $25^{\circ} \mathrm{C}$ Diluted Run 8 .262

Figure 45. $25^{\circ} \mathrm{C}$ Diluted Run 9 263

Figure 46. $50^{\circ} \mathrm{C}$ Diluted Run 1 263

Figure 47. $50{ }^{\circ} \mathrm{C}$ Diluted Run 2 264

Figure 48. $50^{\circ} \mathrm{C}$ Diluted Run 3 264

Figure 49. $50{ }^{\circ} \mathrm{C}$ Diluted Run 4 265

Figure 50. $50{ }^{\circ} \mathrm{C}$ Diluted Run 5 265

Figure 51. $50{ }^{\circ} \mathrm{C}$ Diluted Run 6 266

Figure 52. $50{ }^{\circ} \mathrm{C}$ Diluted Run 7 266

Figure 53. $50{ }^{\circ} \mathrm{C}$ Diluted Run 8 267

Figure 54. $50{ }^{\circ} \mathrm{C}$ Diluted Run 9 
$\begin{array}{ll}\text { APPENDIX F } & \text { WSRC-TR-2000-00352 }\end{array}$

ENVELOPE A RHEOGRAMS

SRT-RPP-2000-00026

Page 241 of 514

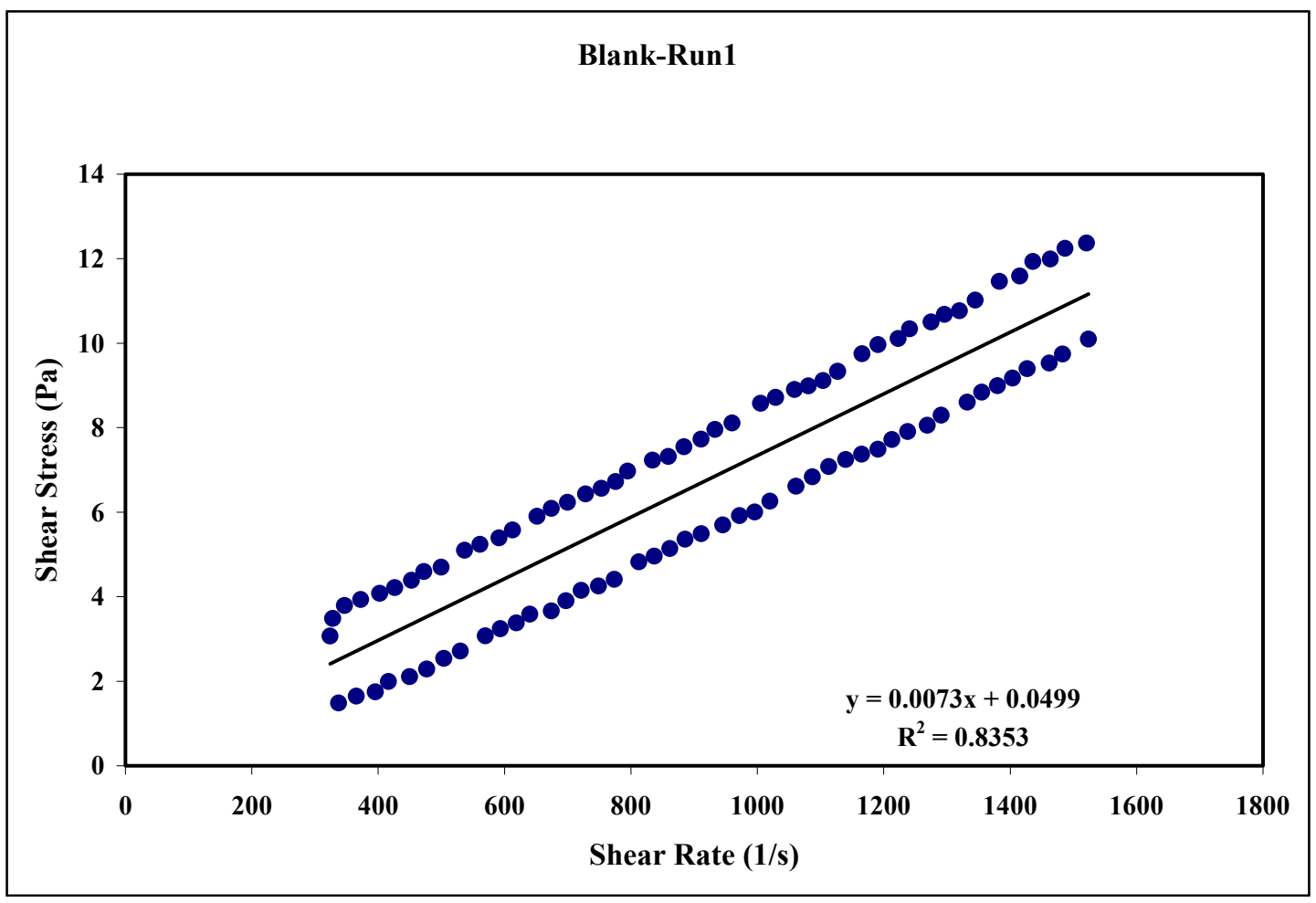

Figure 1. Blank As Received Run 1

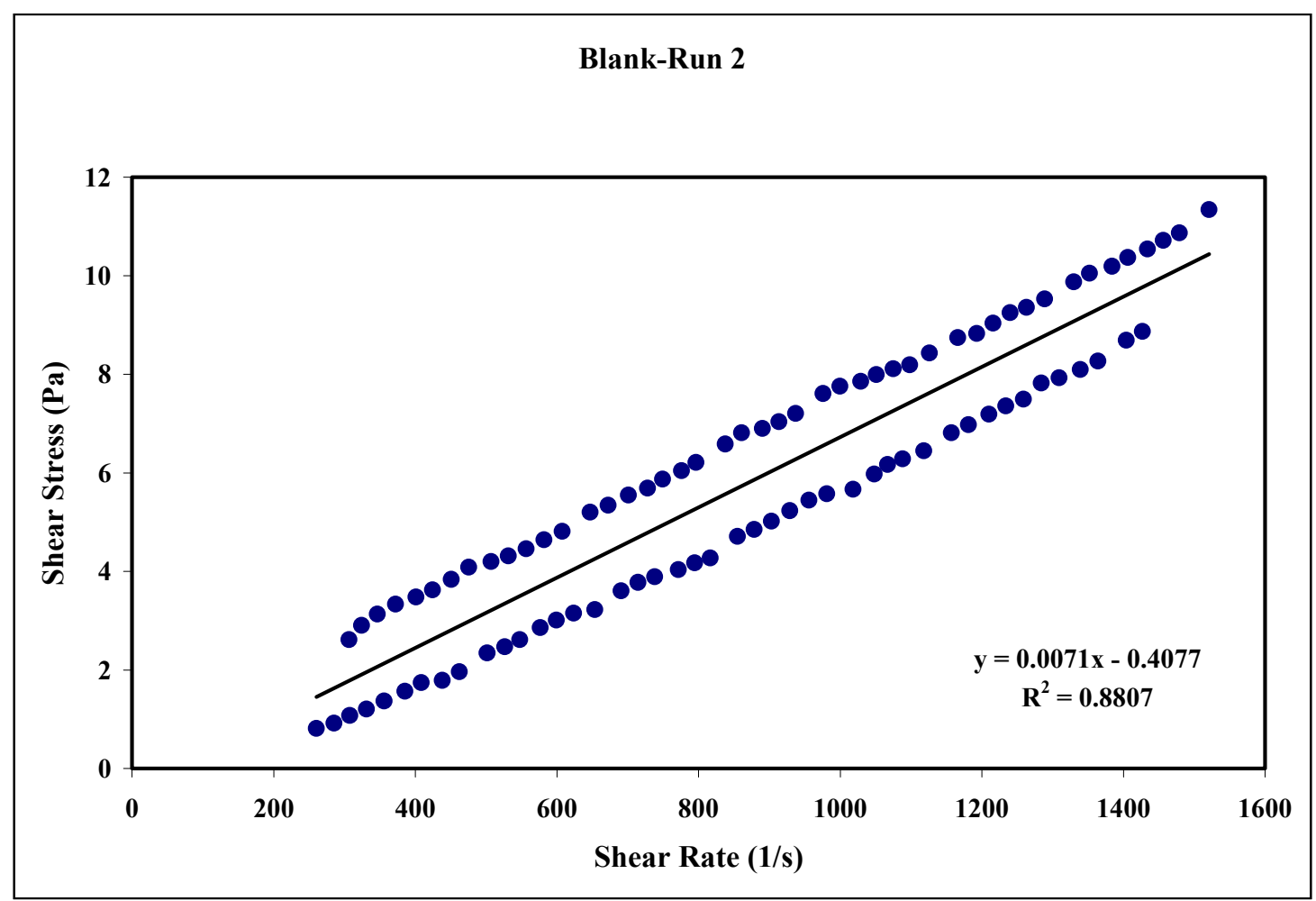

Figure 2. Blank As Received Run 2 
\begin{tabular}{|lc}
\hline APPENDIX F & WSRC-TR-2000-00352
\end{tabular}

ENVELOPE A RHEOGRAMS

SRT-RPP-2000-00026

Page 242 of 514

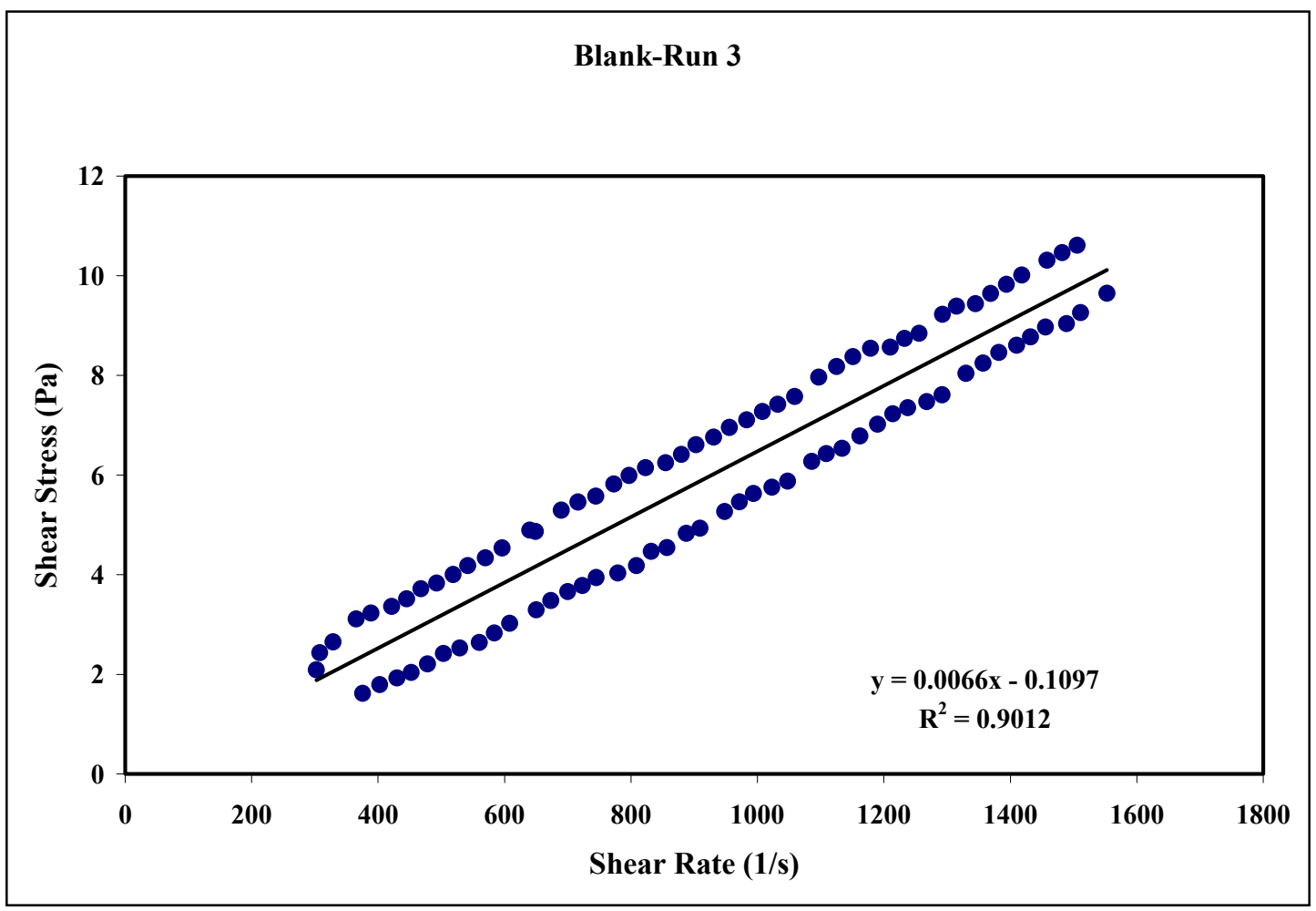

Figure 3. Blank As Received Run 3

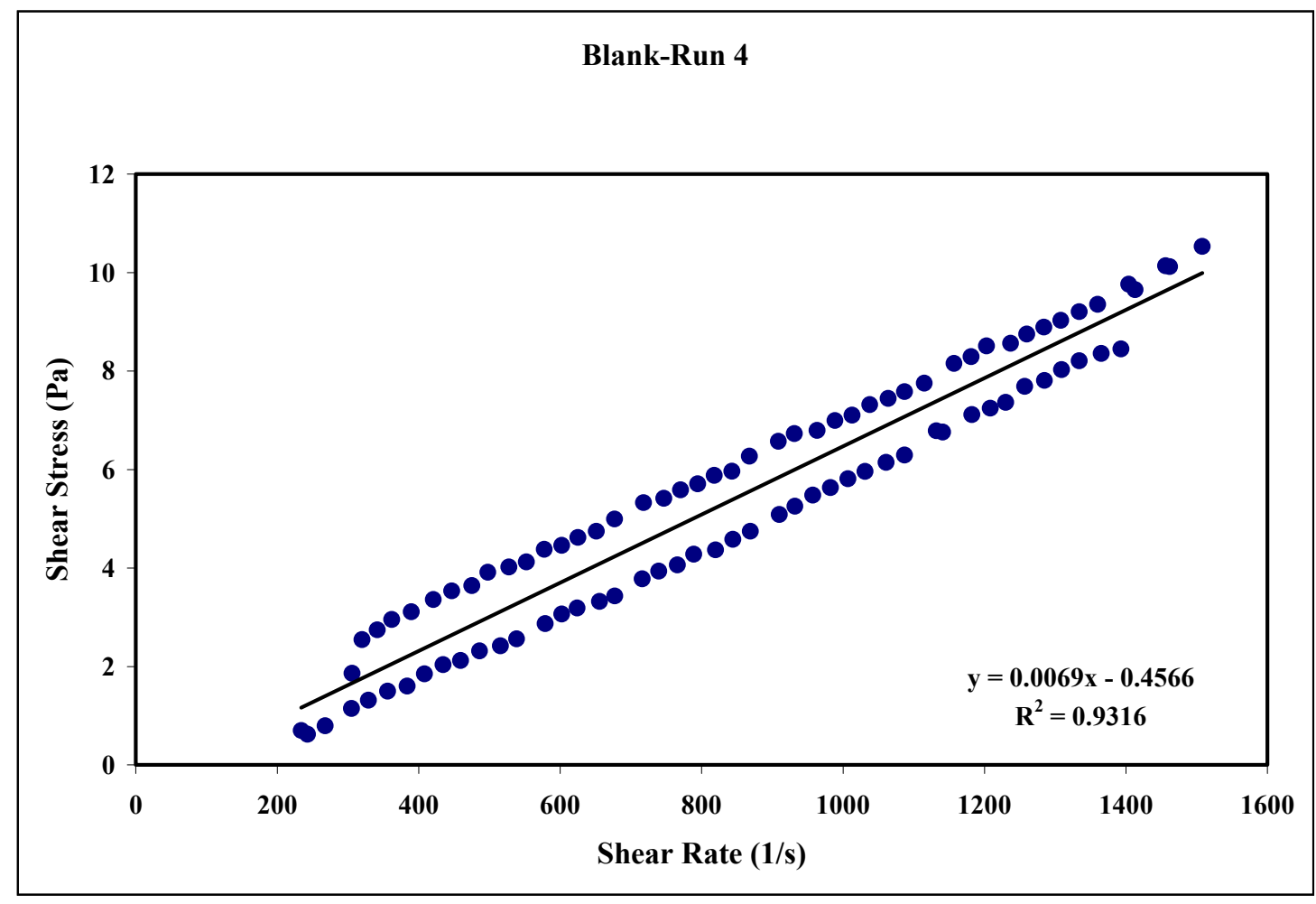

Figure 4. Blank As Received Run 4 
\begin{tabular}{|lc}
\hline APPENDIX F & WSRC-TR-2000-00352
\end{tabular}

ENVELOPE A RHEOGRAMS

SRT-RPP-2000-00026

Page 243 of 514

\section{Blank-Run 5}

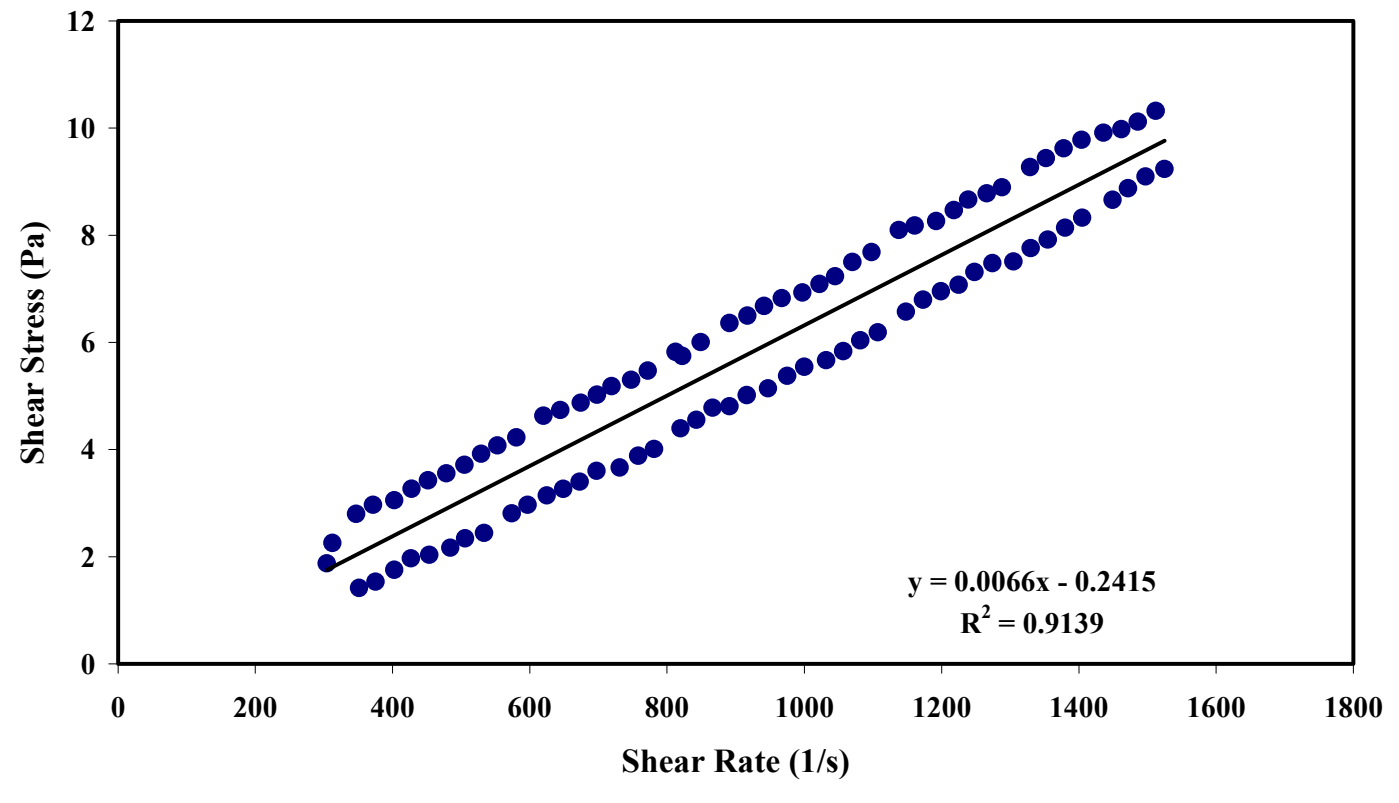

Figure 5. Blank As Received Run 5

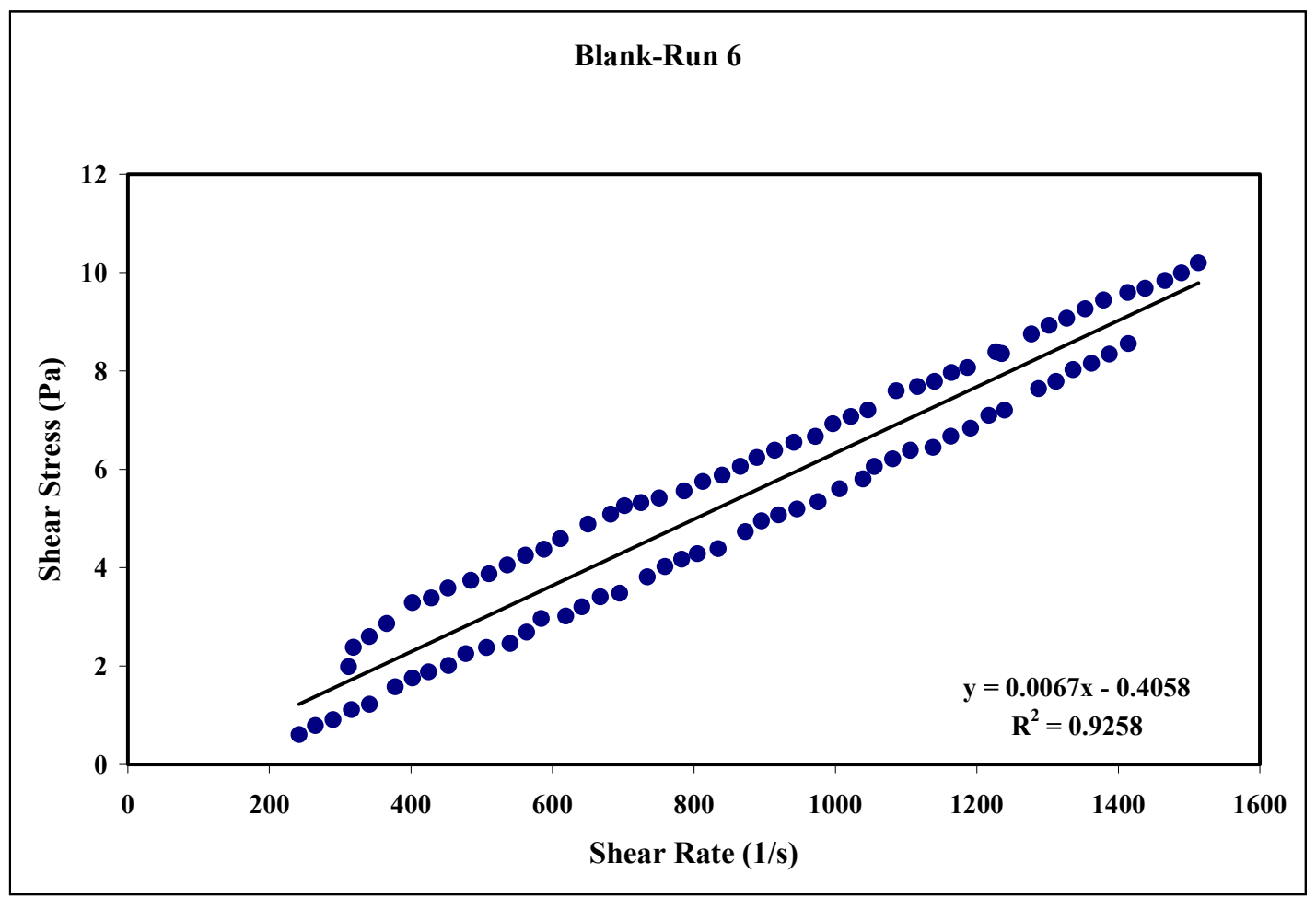

Figure 6. Blank As Received Run 6 
\begin{tabular}{|lc}
\hline APPENDIX F & WSRC-TR-2000-00352
\end{tabular}

ENVELOPE A RHEOGRAMS

SRT-RPP-2000-00026

Page 244 of 514

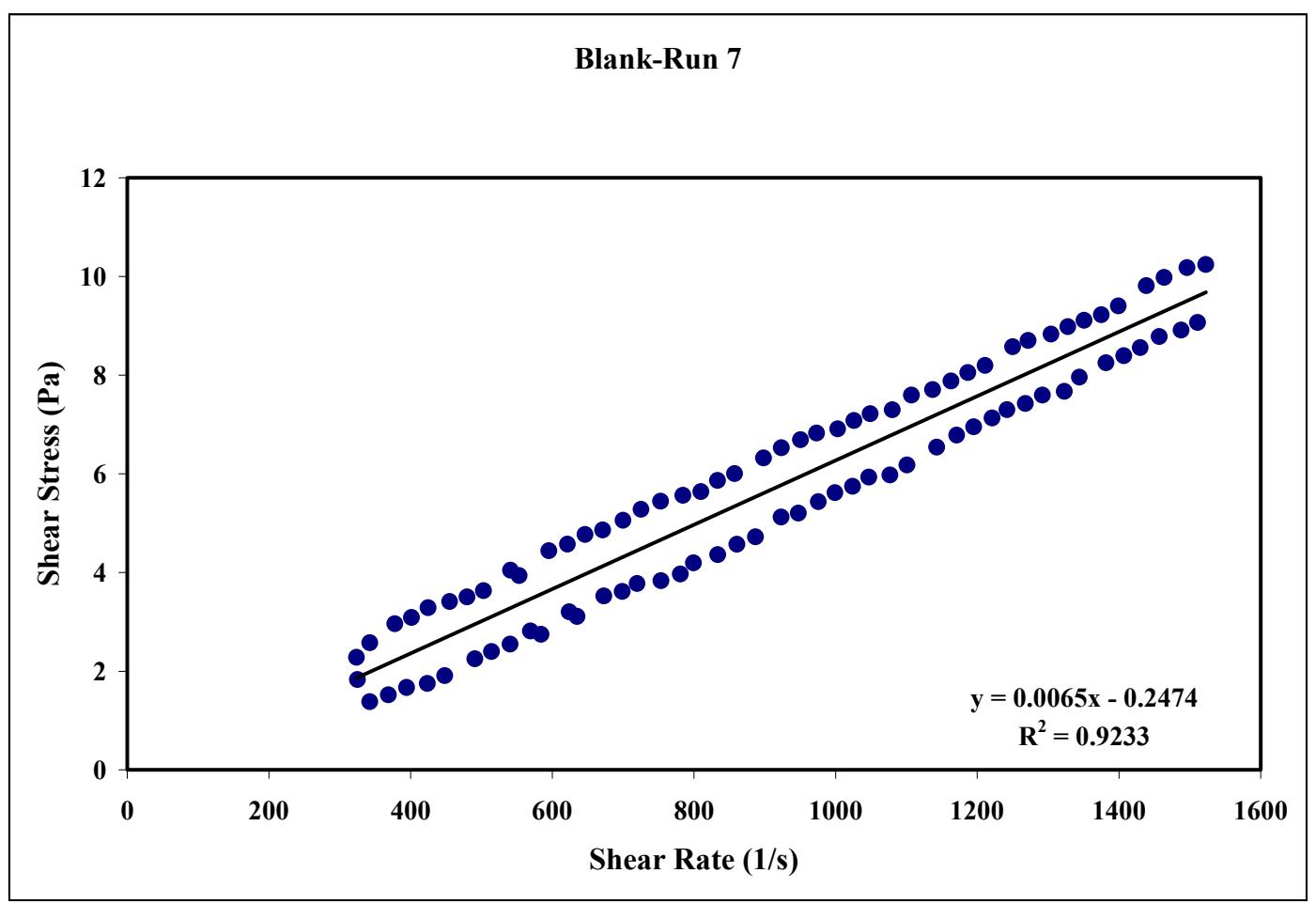

Figure 7. Blank As Received Run 7

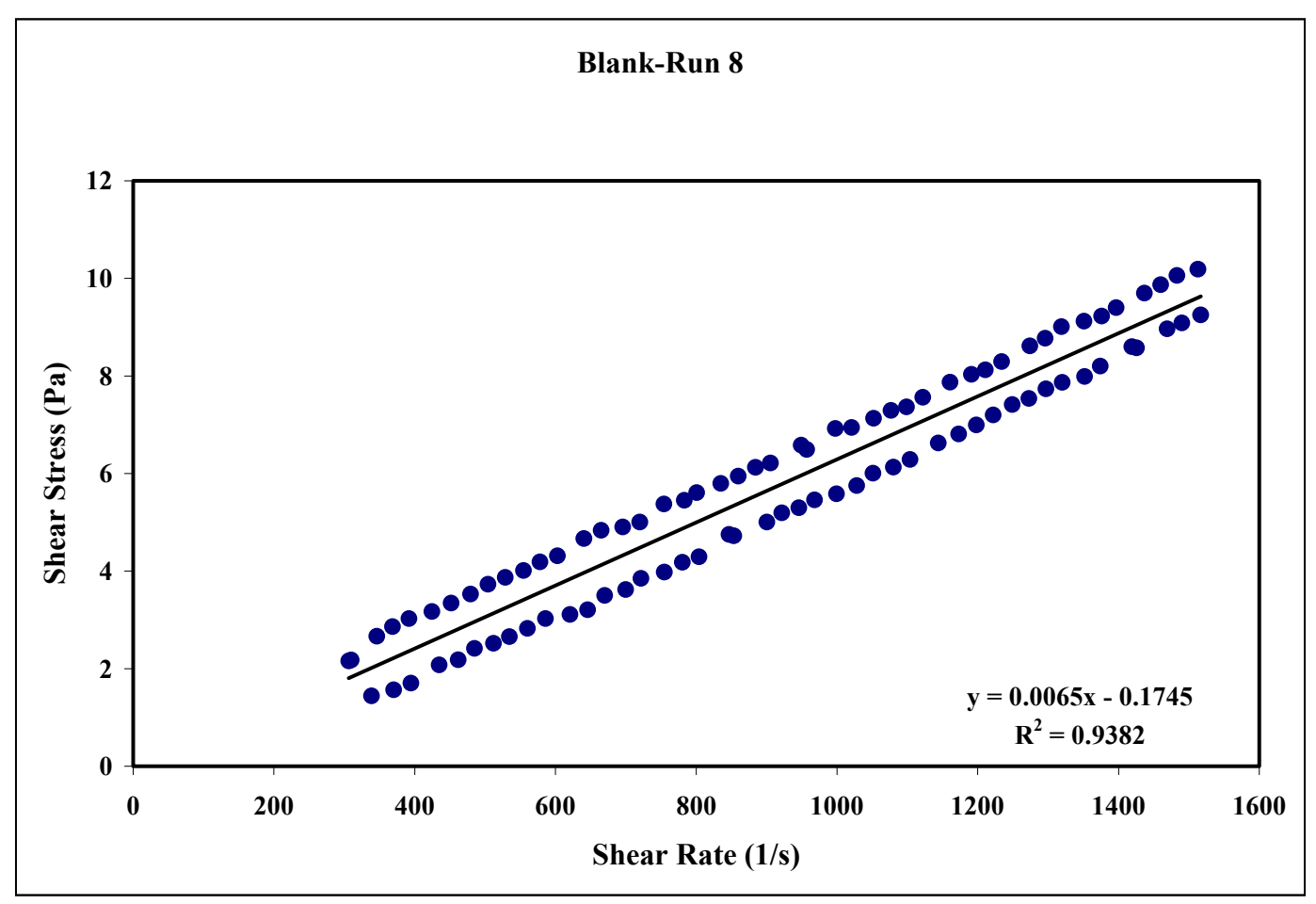

Figure 8. Blank As Received Run 8 
\begin{tabular}{|lc}
\hline APPENDIX F & WSRC-TR-2000-00352
\end{tabular}

ENVELOPE A RHEOGRAMS

SRT-RPP-2000-00026

Page 245 of 514

Blank-Run 9

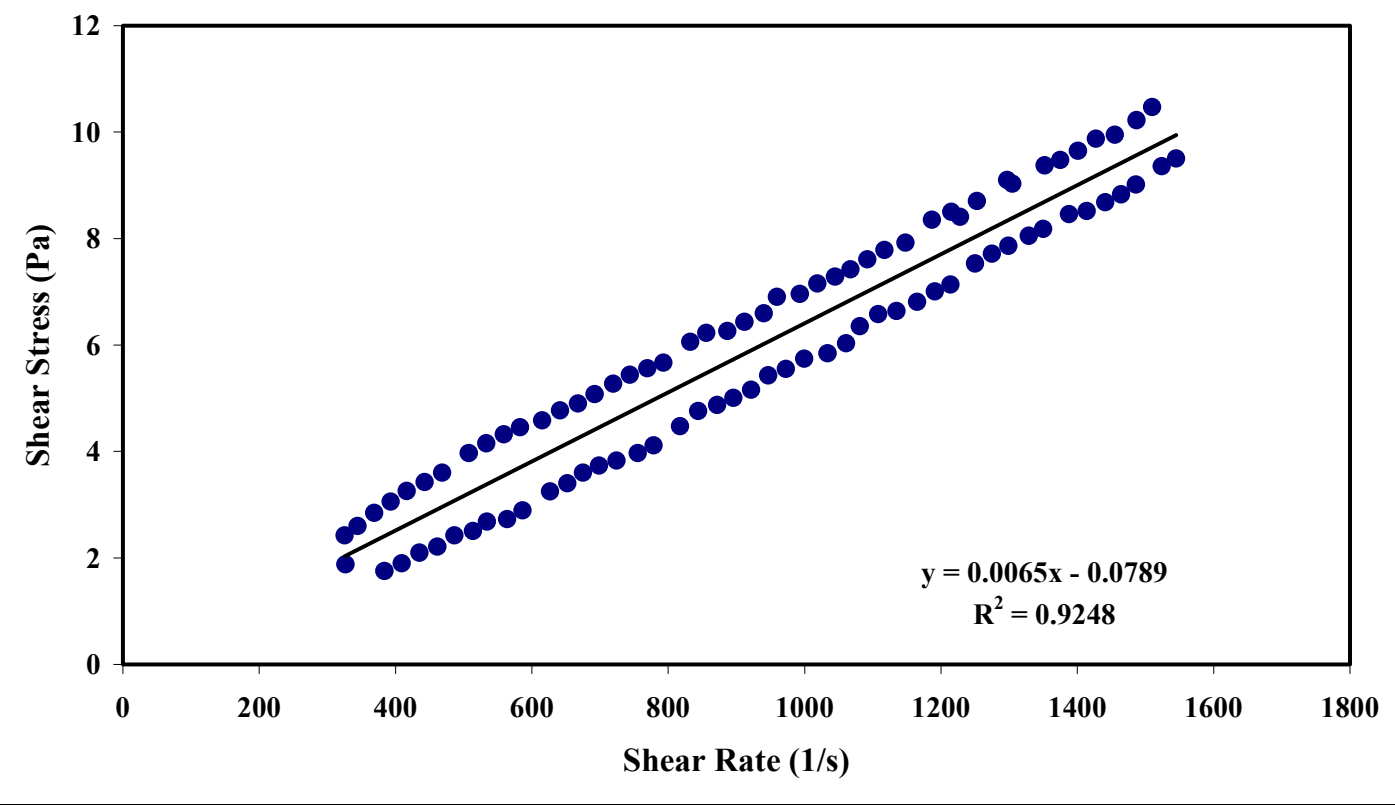

Figure 9. Blank As Received Run 9

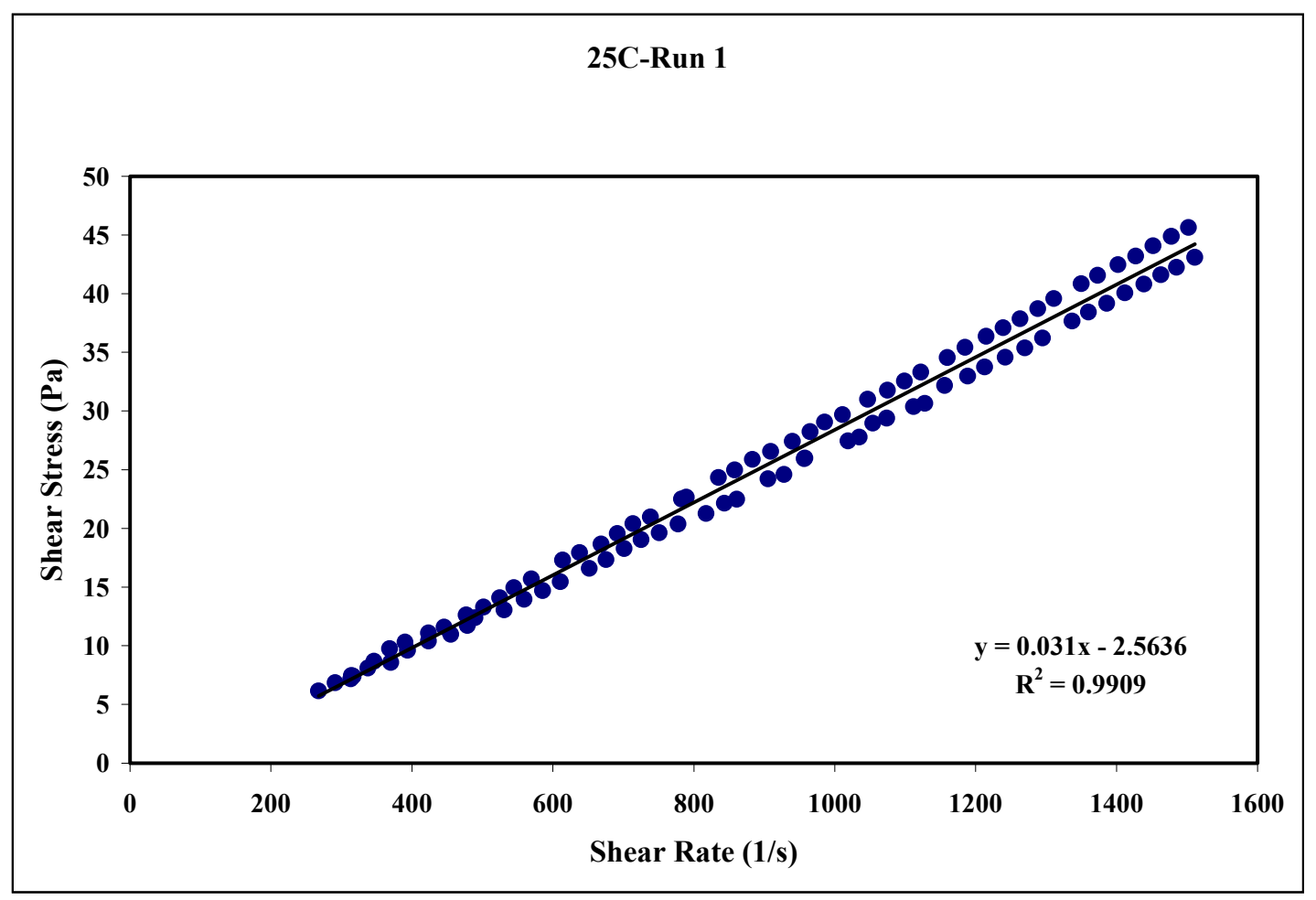

Figure 10. $25^{\circ} \mathrm{C}$ As Received Run 1 
\begin{tabular}{ll}
\hline APPENDIX F & WSRC-TR-2000-00352
\end{tabular}

ENVELOPE A RHEOGRAMS

SRT-RPP-2000-00026

Page 246 of 514

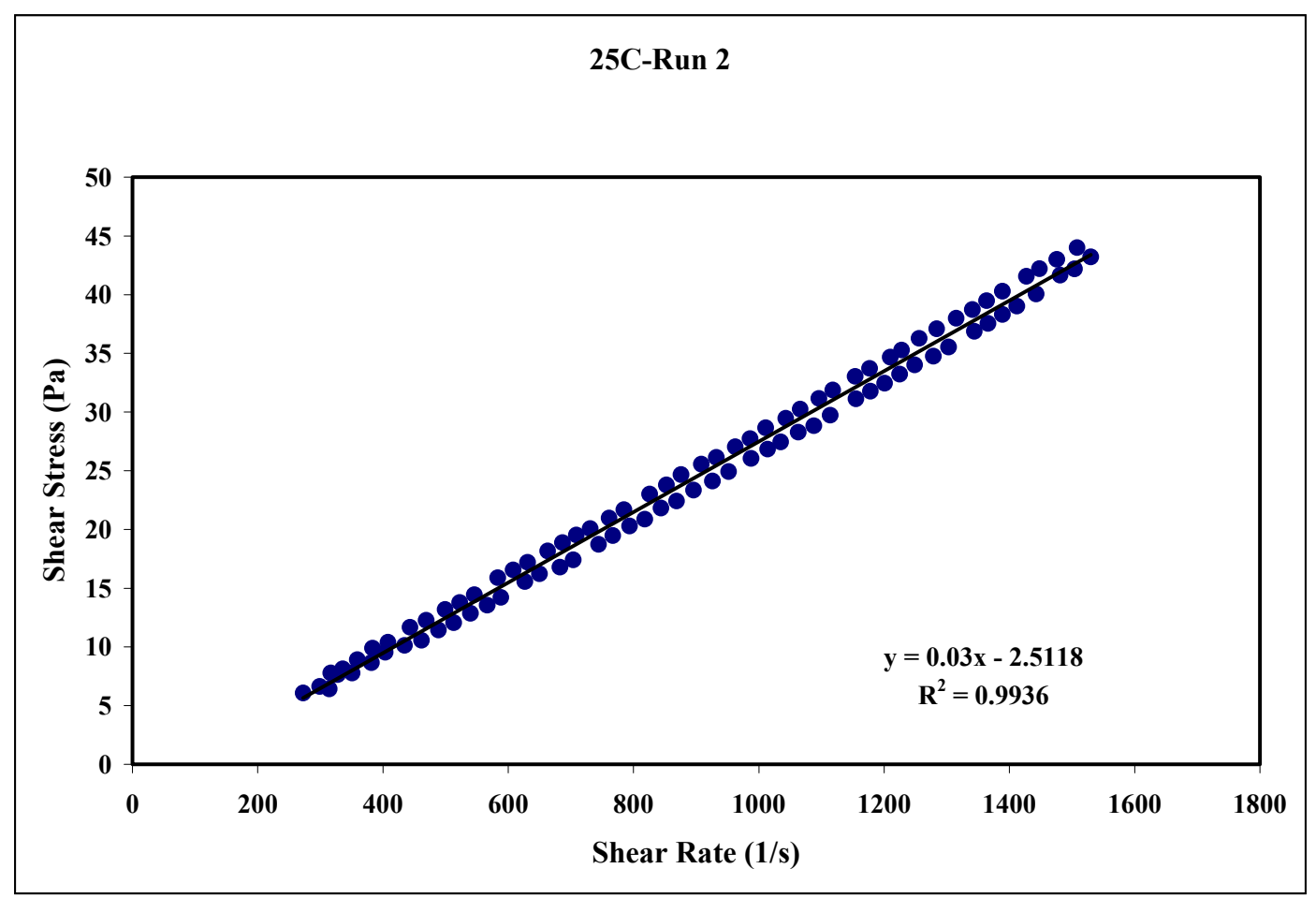

Figure 11. $25^{\circ} \mathrm{C}$ As Received Run 2

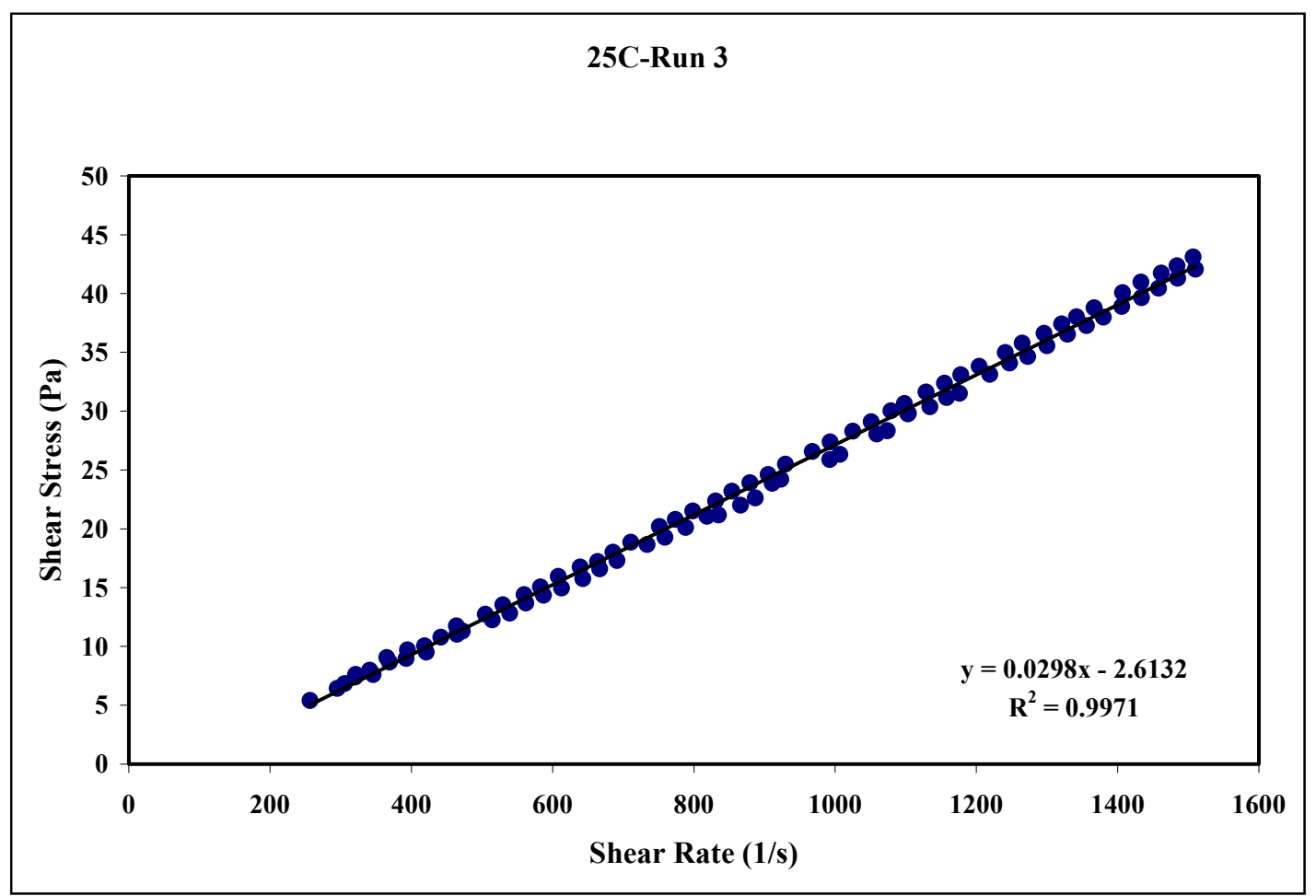

Figure 12. $25^{\circ} \mathrm{C}$ As Received Run 3 
\begin{tabular}{ll}
\hline APPENDIX F & WSRC-TR-2000-00352
\end{tabular}

ENVELOPE A RHEOGRAMS

SRT-RPP-2000-00026

Page 247 of 514

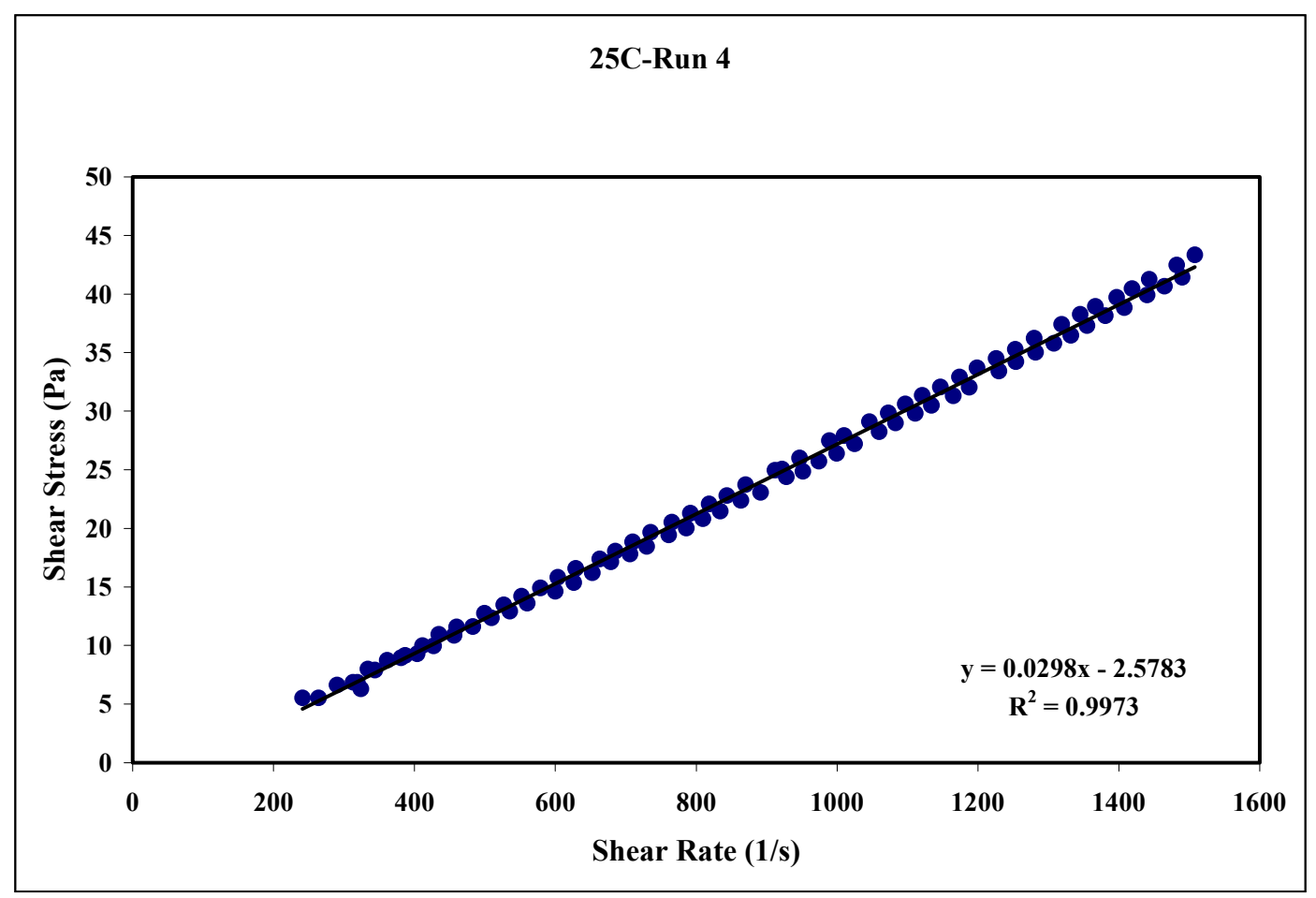

Figure 13. $25^{\circ} \mathrm{C}$ As Received Run 4

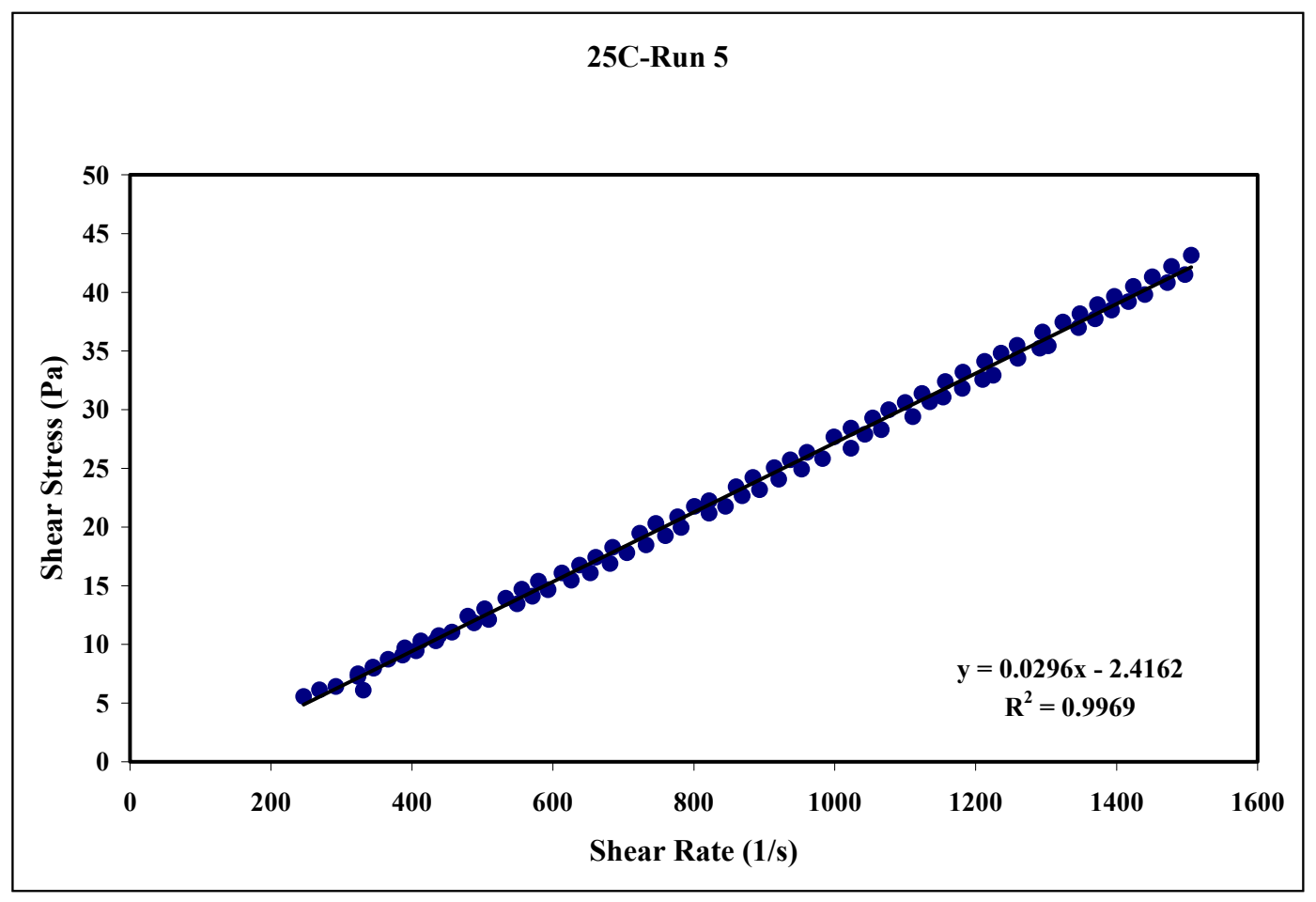

Figure 14. $25^{\circ} \mathrm{C}$ As Received Run 5 
$\begin{array}{ll}\text { APPENDIX F } & \text { WSRC-TR-2000-00352 }\end{array}$

ENVELOPE A RHEOGRAMS

SRT-RPP-2000-00026

Page 248 of 514

\section{C-Run 6}

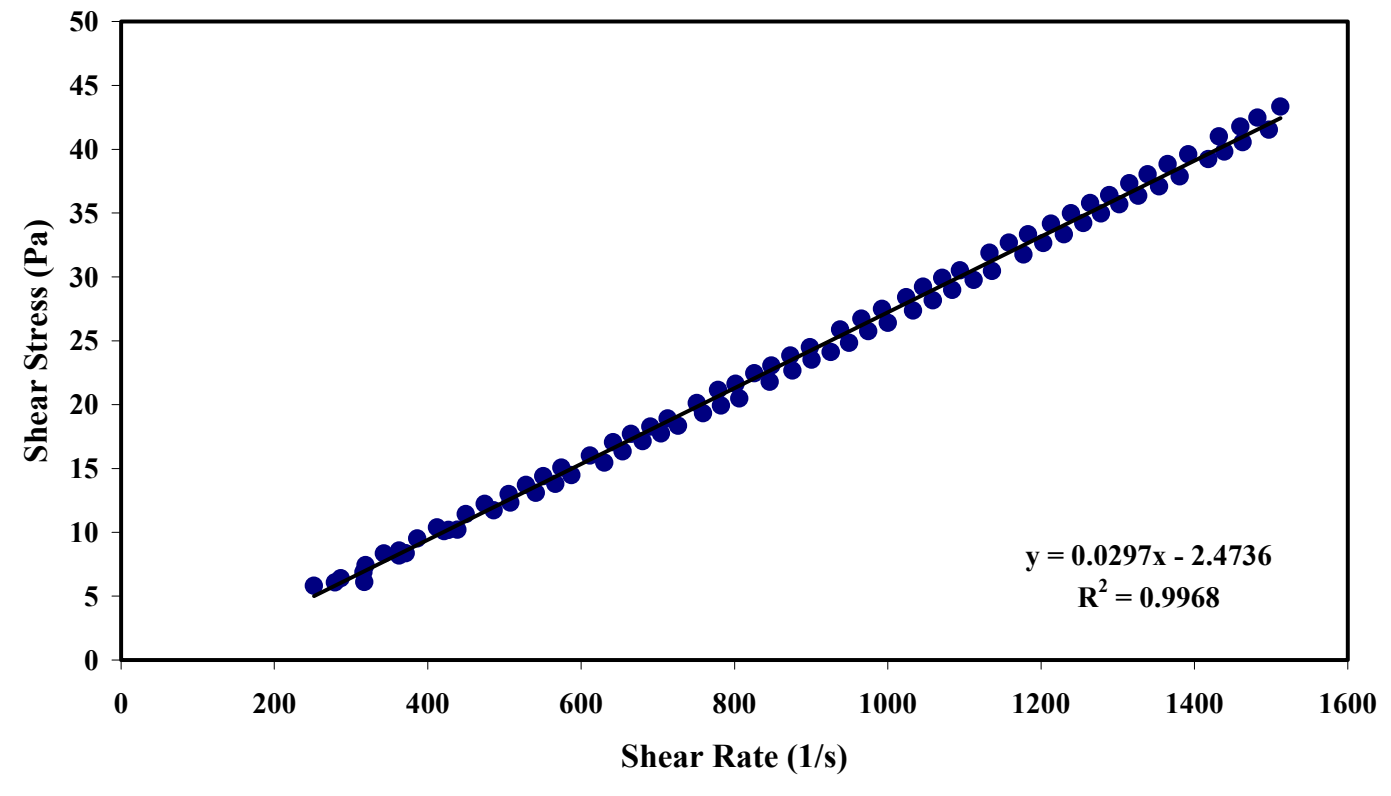

Figure 15. $25^{\circ} \mathrm{C}$ As Received Run 6

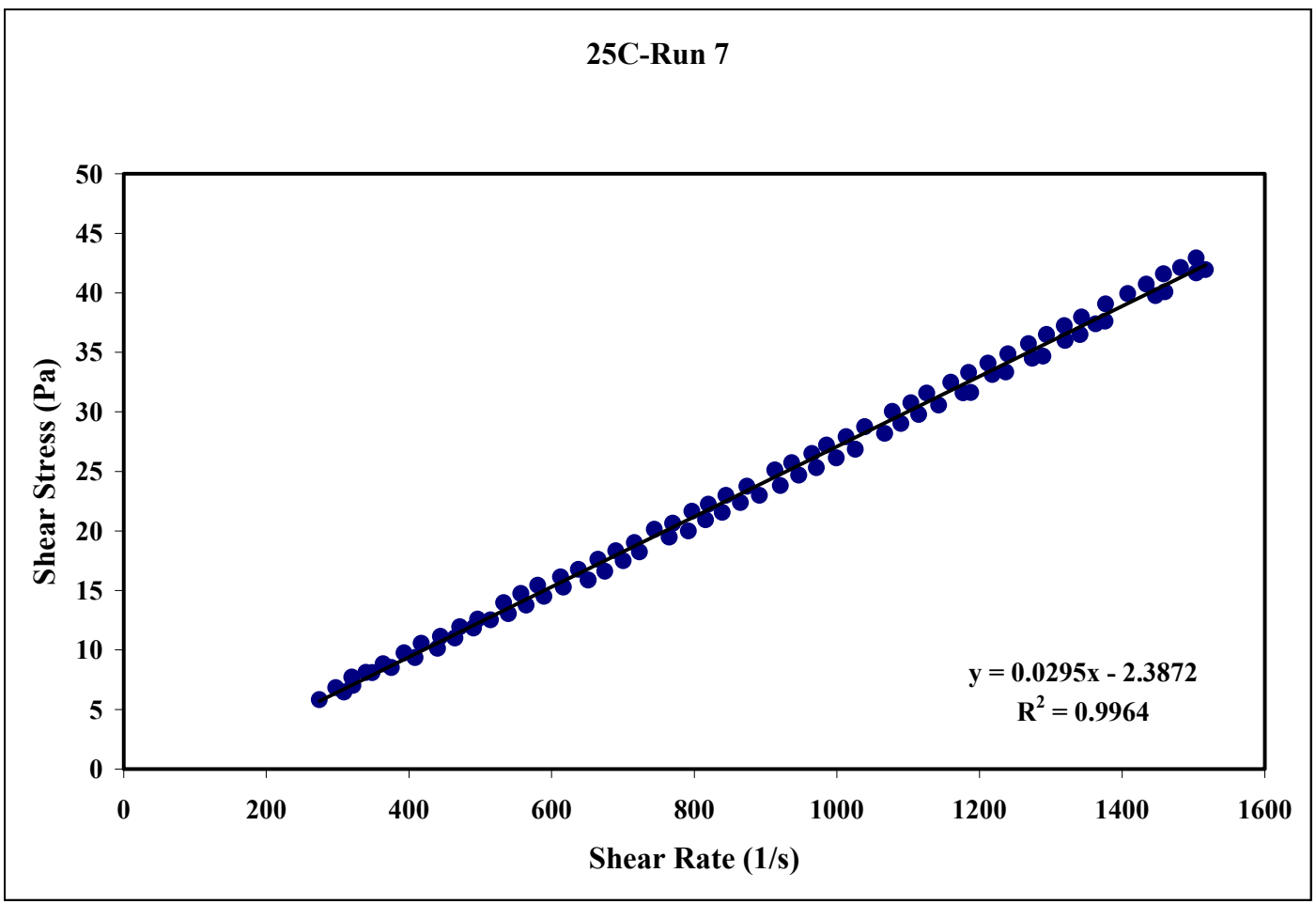

Figure 16. $25^{\circ} \mathrm{C}$ As Received Run 7 
$\begin{array}{ll}\text { APPENDIX } F & \text { WSRC-TR-2000-00352 }\end{array}$

ENVELOPE A RHEOGRAMS

SRT-RPP-2000-00026

Page 249 of 514

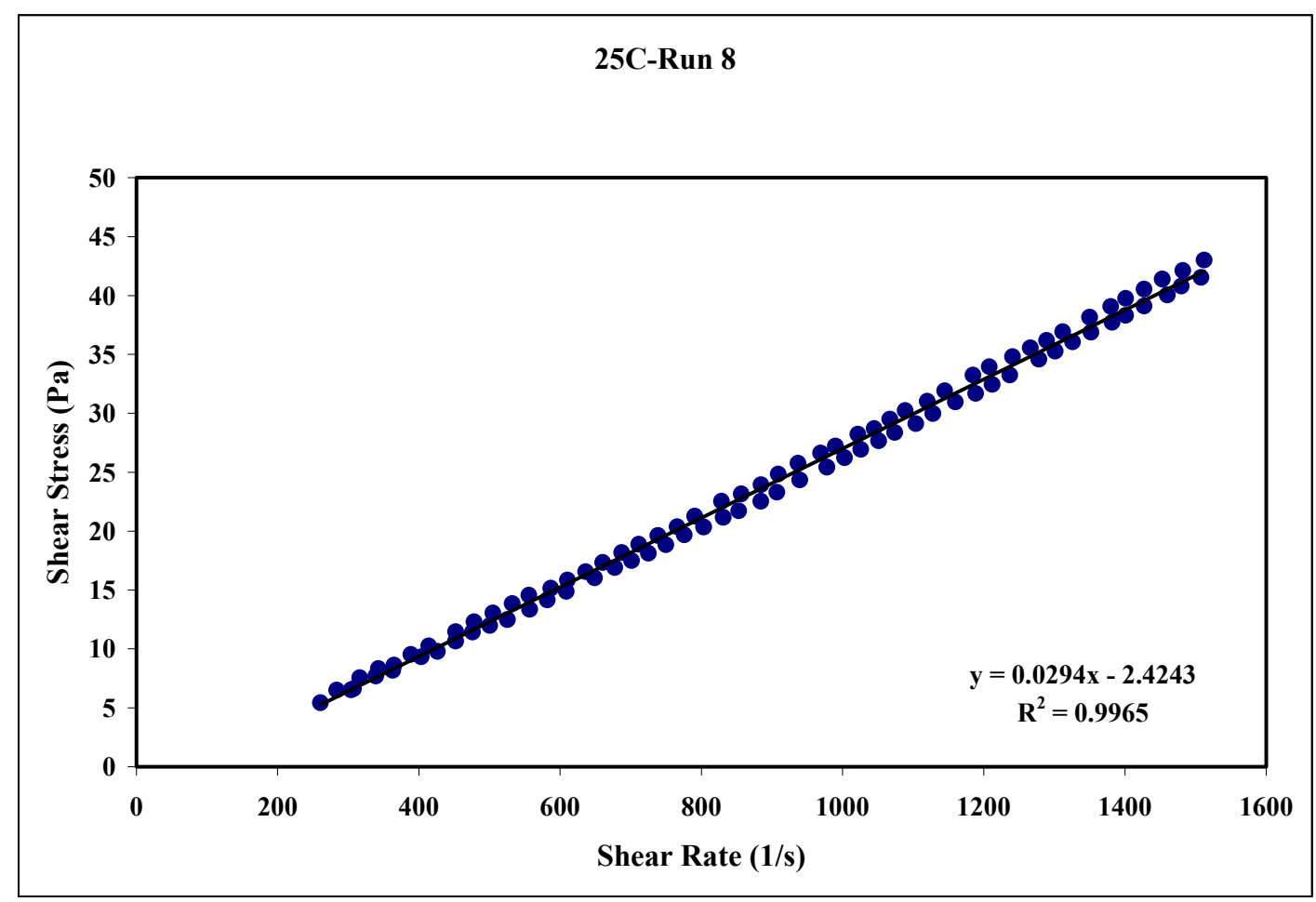

Figure 17. $25^{\circ} \mathrm{C}$ As Received Run 8

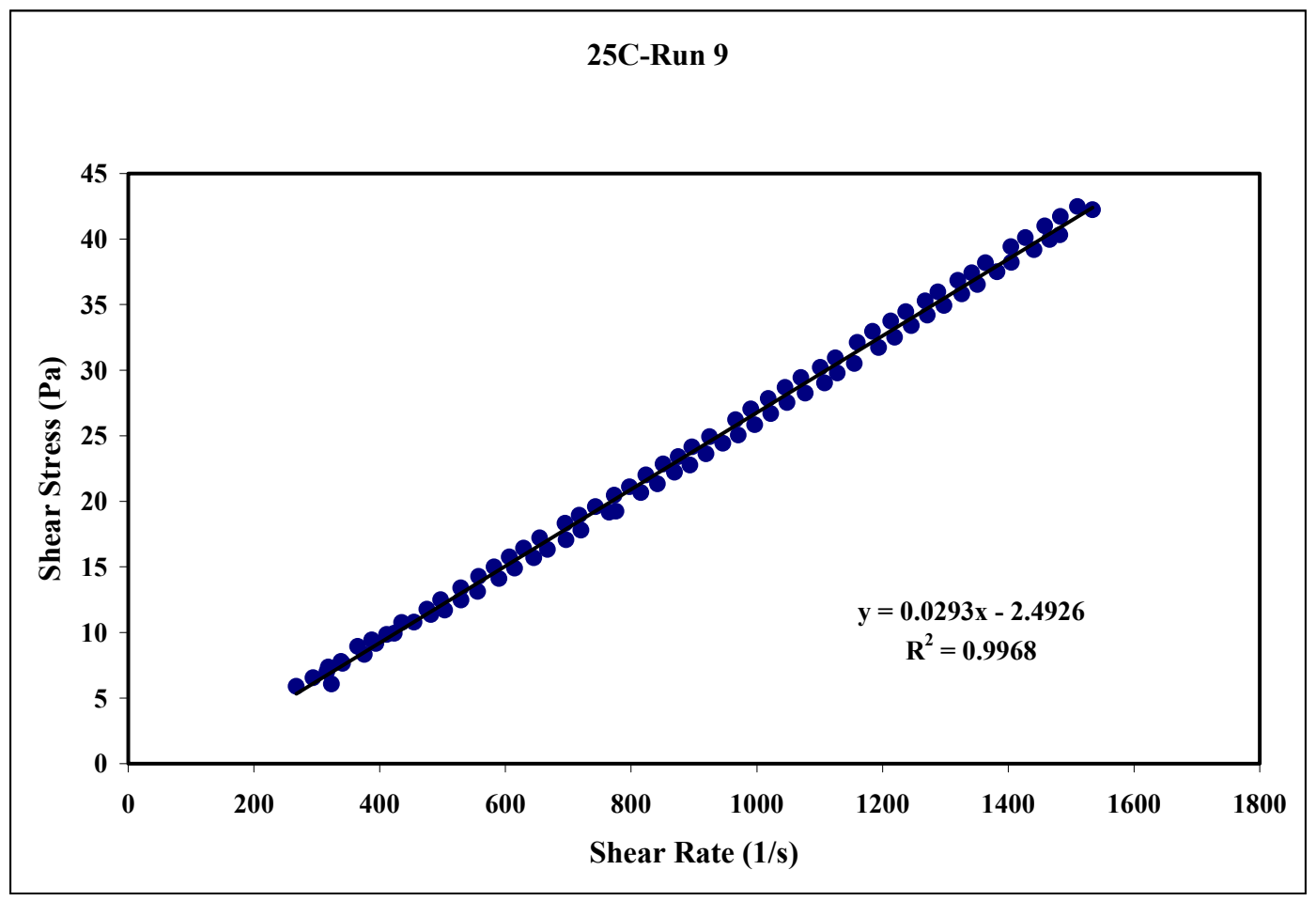

Figure 18. $25^{\circ} \mathrm{C}$ As Received Run 9 
$\begin{array}{ll}\text { APPENDIX F } & \text { WSRC-TR-2000-00352 }\end{array}$

ENVELOPE A RHEOGRAMS

SRT-RPP-2000-00026

Page 250 of 514

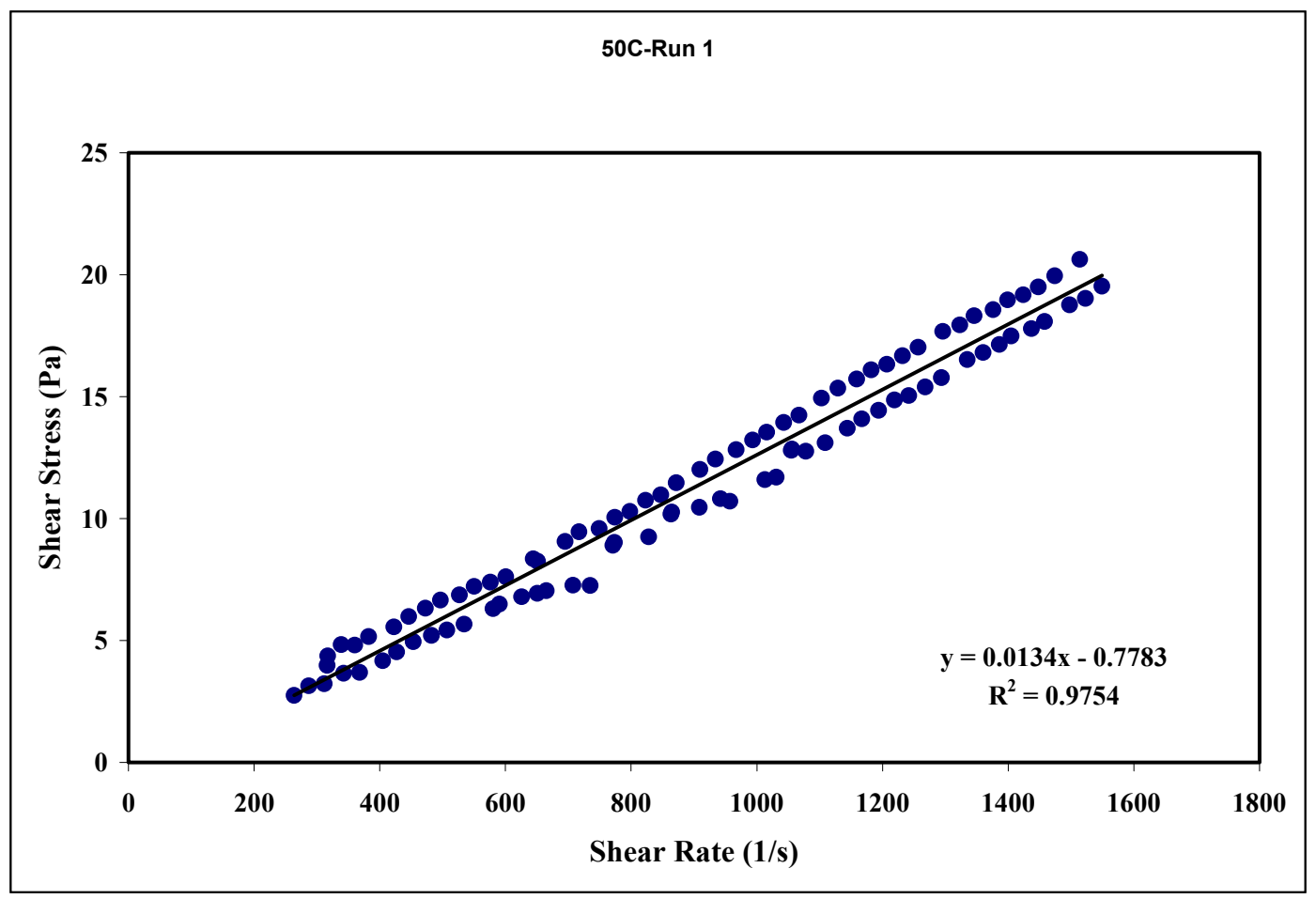

Figure 19. $50{ }^{\circ} \mathrm{C}$ As Received Run 1

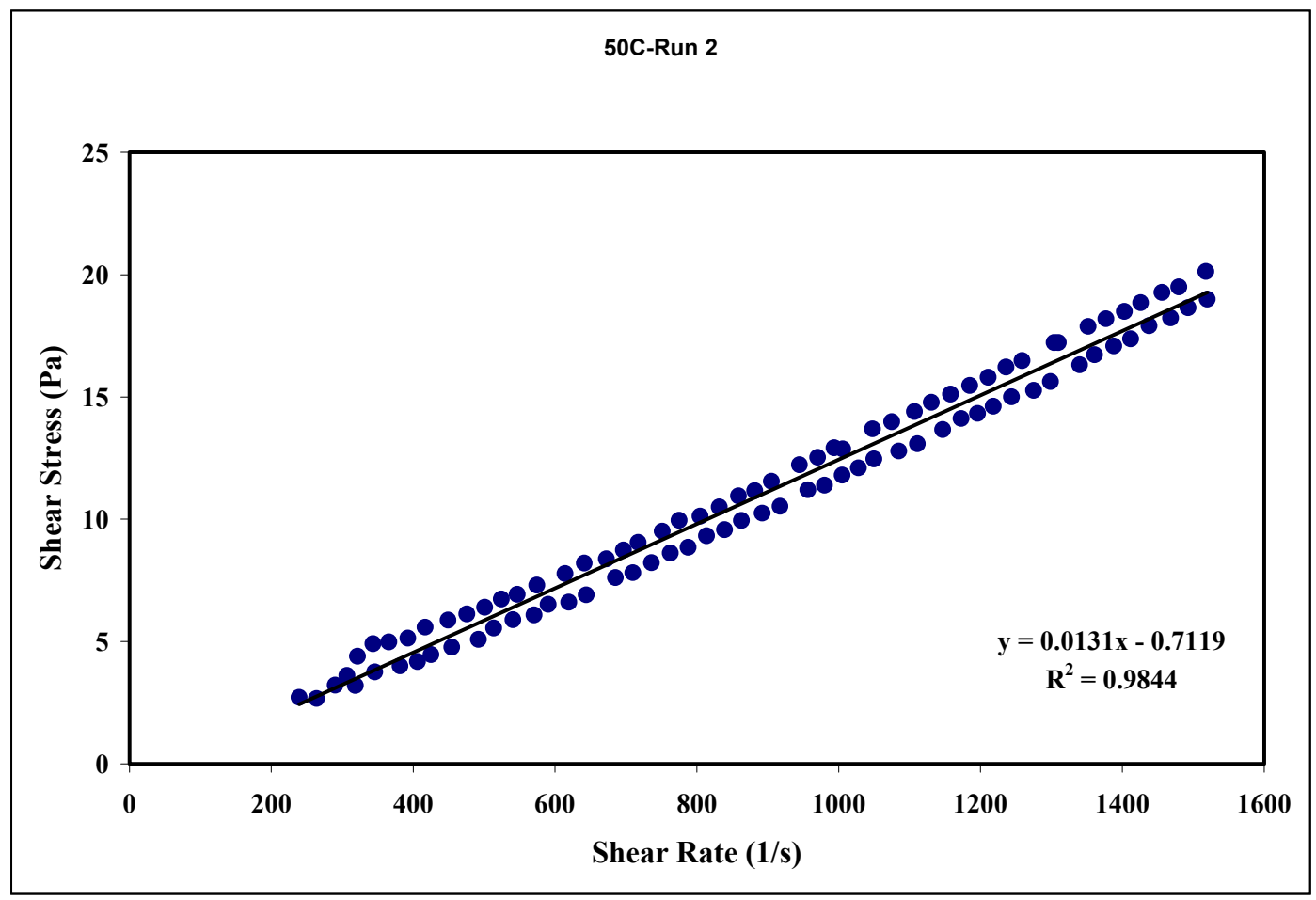

Figure 20. $50{ }^{\circ} \mathrm{C}$ As Received Run 2 
$\begin{array}{ll}\text { APPENDIX F } & \text { WSRC-TR-2000-00352 }\end{array}$

ENVELOPE A RHEOGRAMS

SRT-RPP-2000-00026

Page 251 of 514

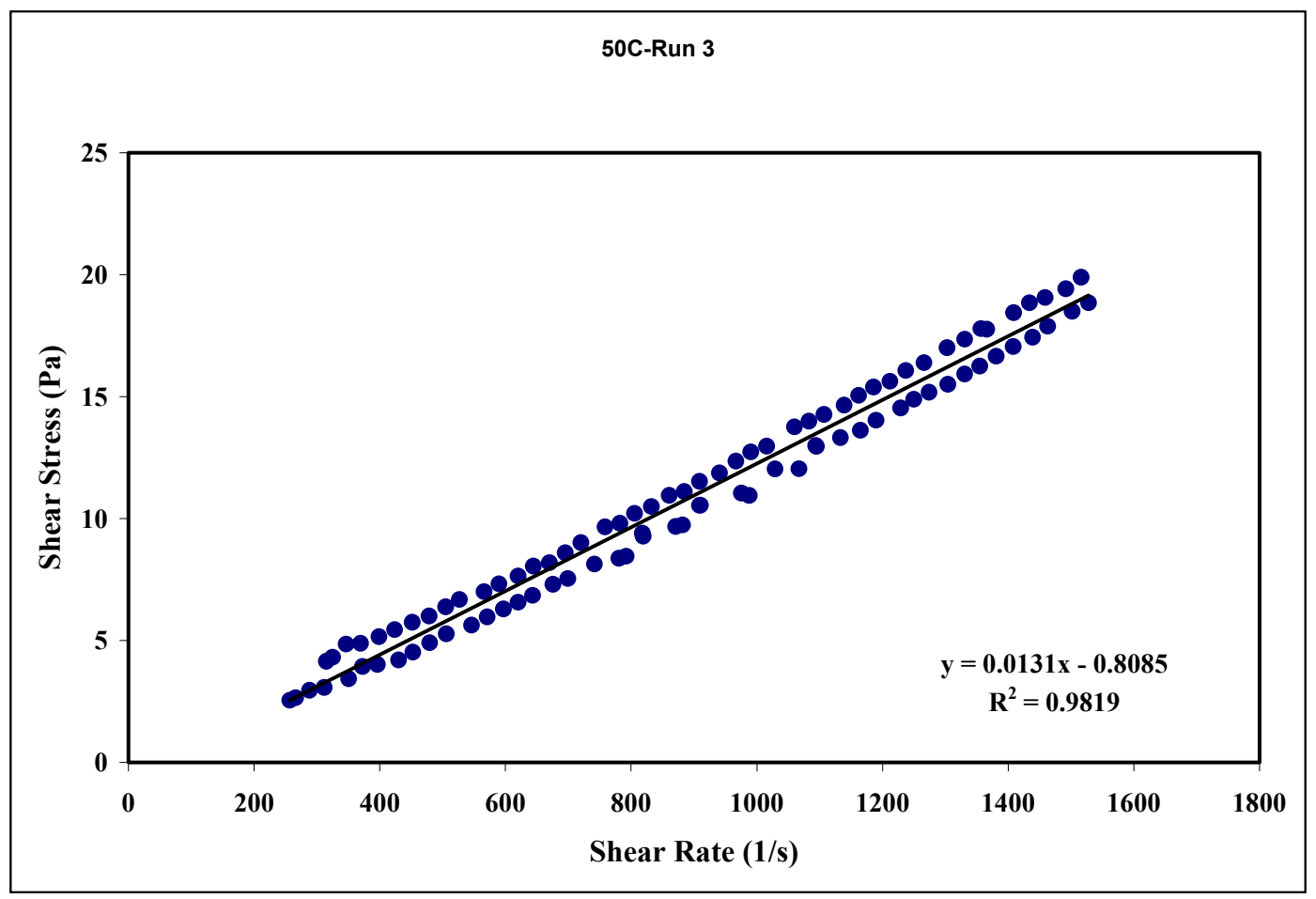

Figure 21. $50{ }^{\circ} \mathrm{C}$ As Received Run 3

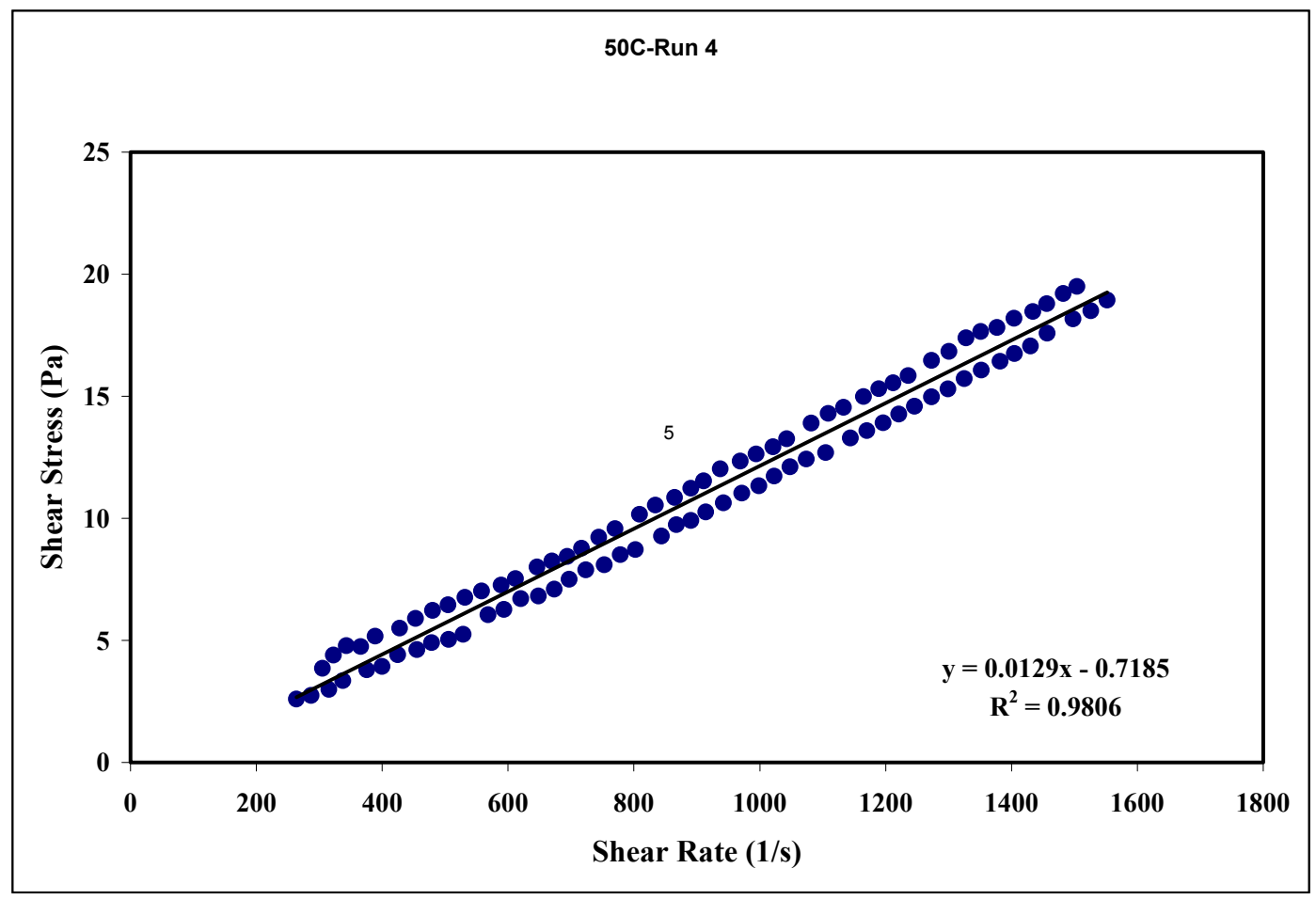

Figure 22. $50^{\circ} \mathrm{C}$ As Received Run 4 
$\begin{array}{ll}\text { APPENDIX F } & \text { WSRC-TR-2000-00352 }\end{array}$

ENVELOPE A RHEOGRAMS

SRT-RPP-2000-00026

Page 252 of 514

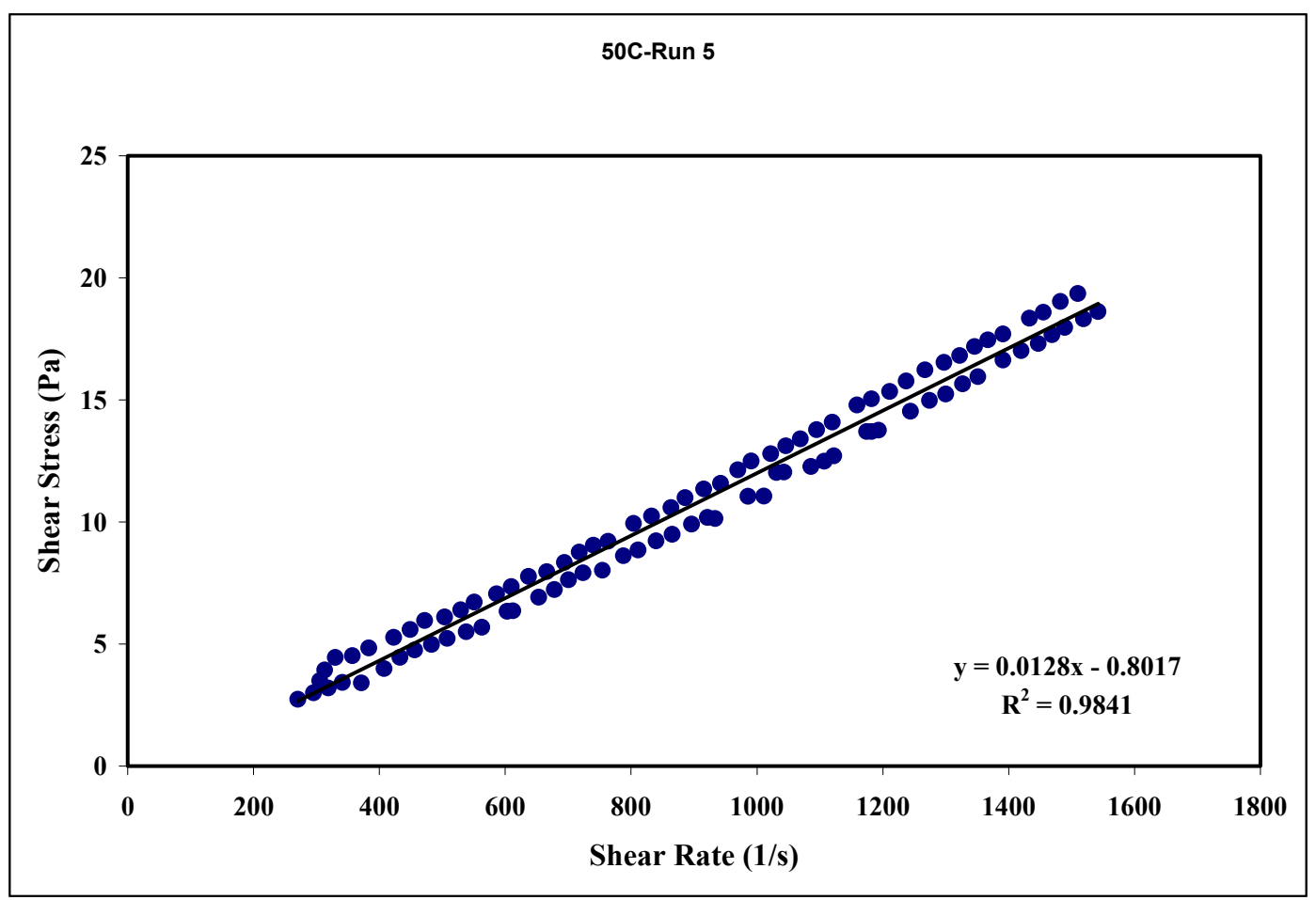

Figure 23. $50{ }^{\circ} \mathrm{C}$ As Received Run 5

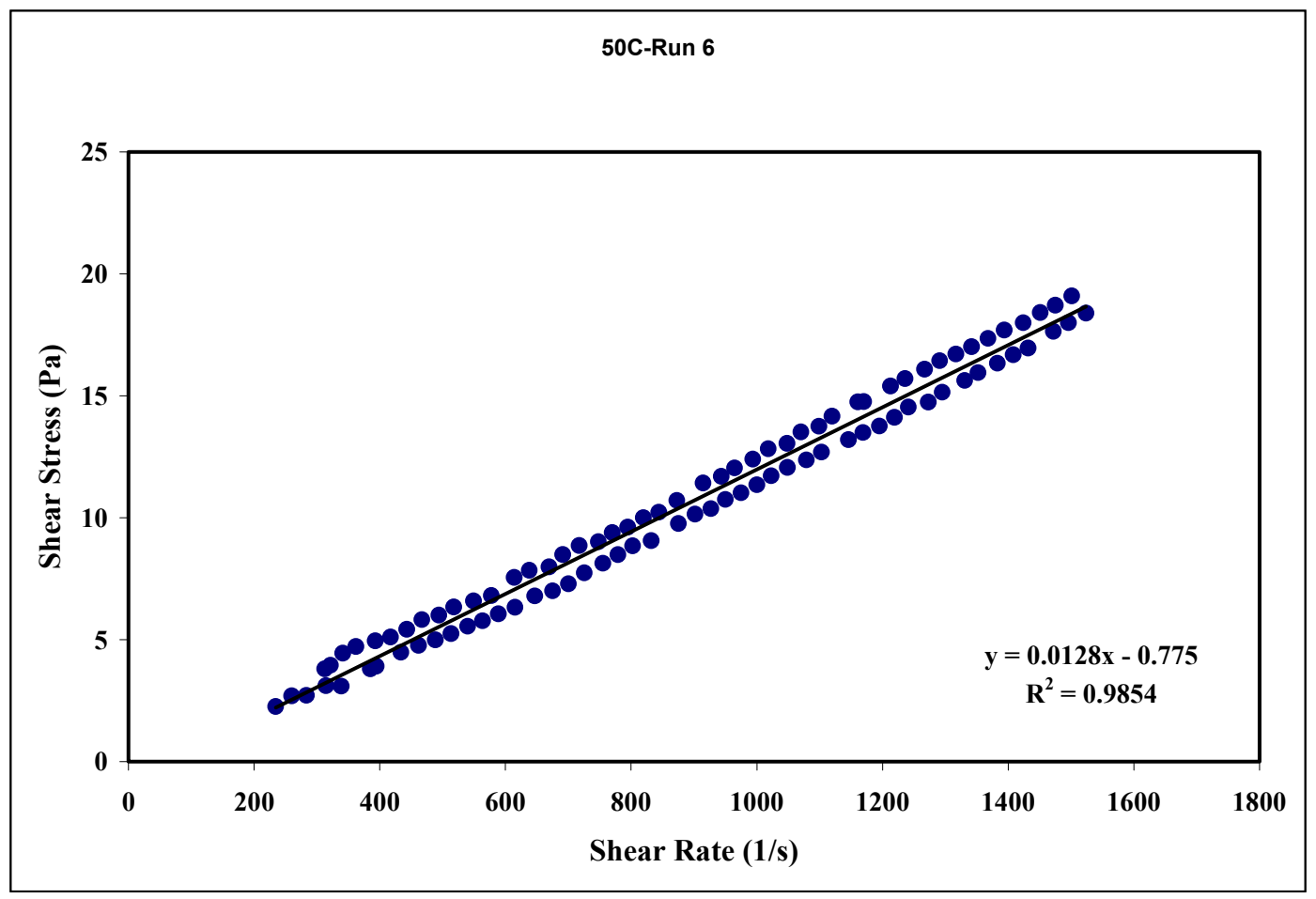

Figure 24. $50^{\circ} \mathrm{C}$ As Received Run 6 
\begin{tabular}{|lc}
\hline APPENDIX F & WSRC-TR-2000-00352
\end{tabular}

ENVELOPE A RHEOGRAMS

SRT-RPP-2000-00026

Page 253 of 514

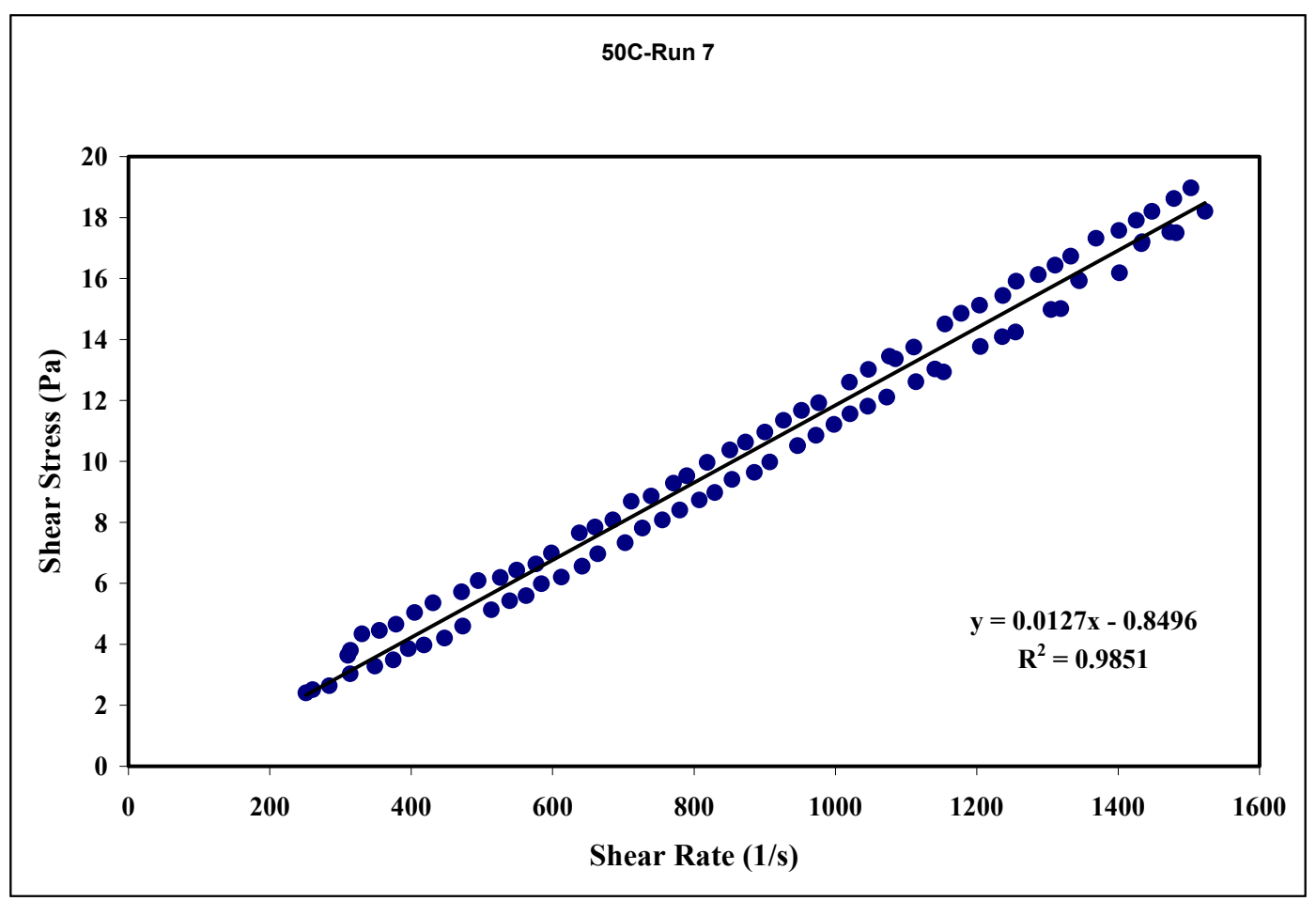

Figure 25. $50^{\circ} \mathrm{C}$ As Received Run 7

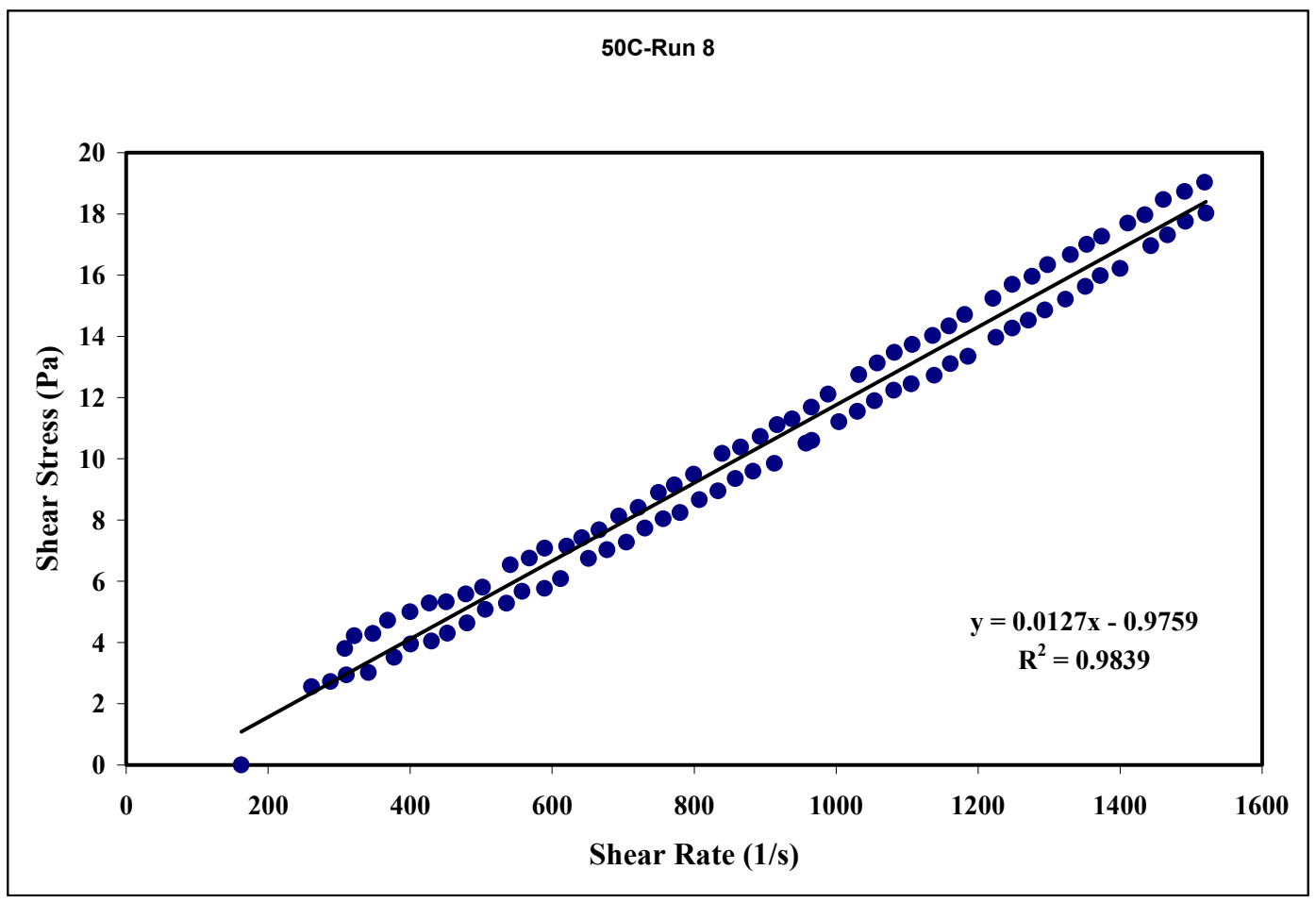

Figure 26. $50{ }^{\circ} \mathrm{C}$ As Received Run 8 
\begin{tabular}{|lc}
\hline APPENDIX F & WSRC-TR-2000-00352
\end{tabular}

ENVELOPE A RHEOGRAMS

SRT-RPP-2000-00026

Page 254 of 514

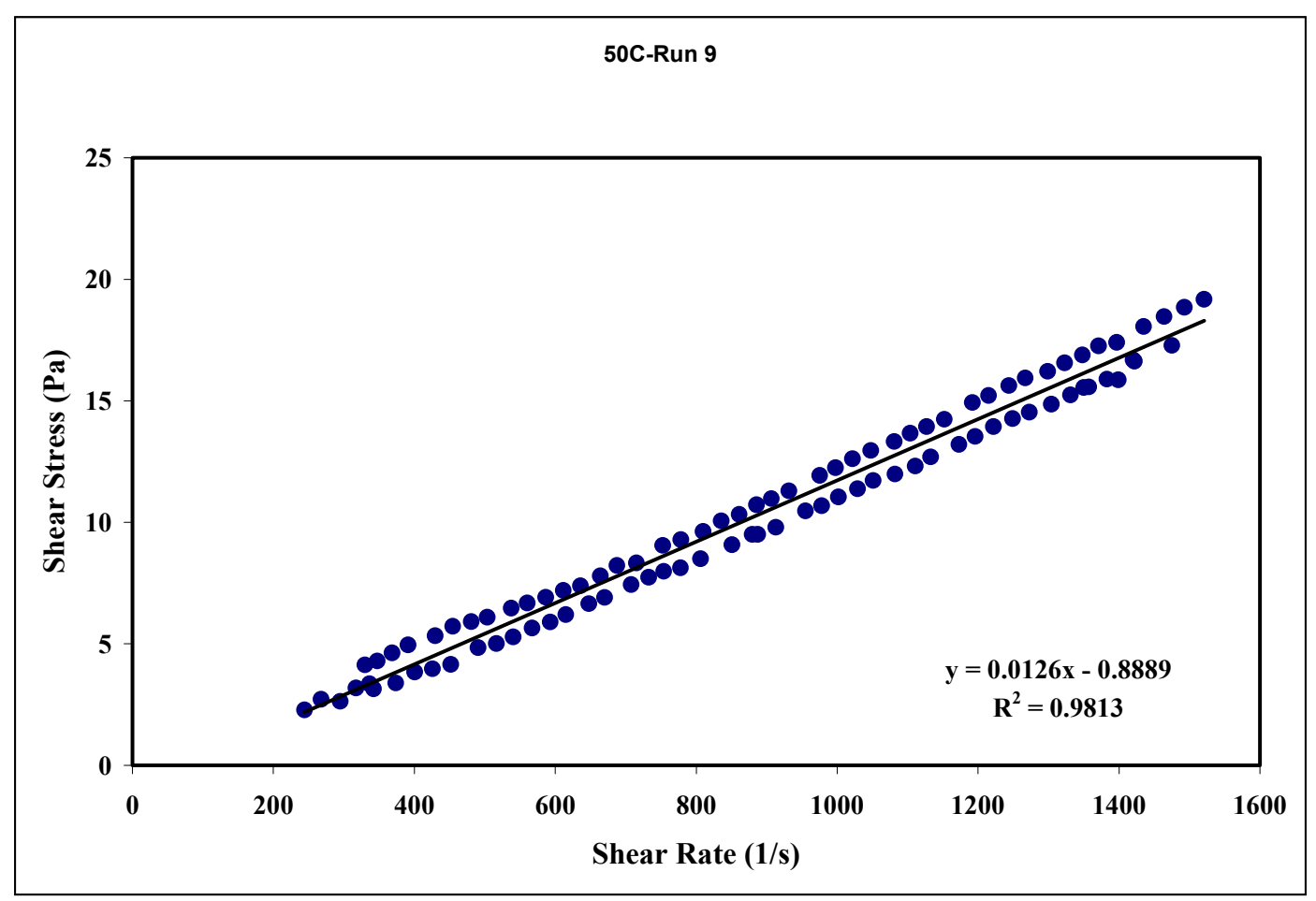

Figure 27. $50^{\circ} \mathrm{C}$ As Received Run 9

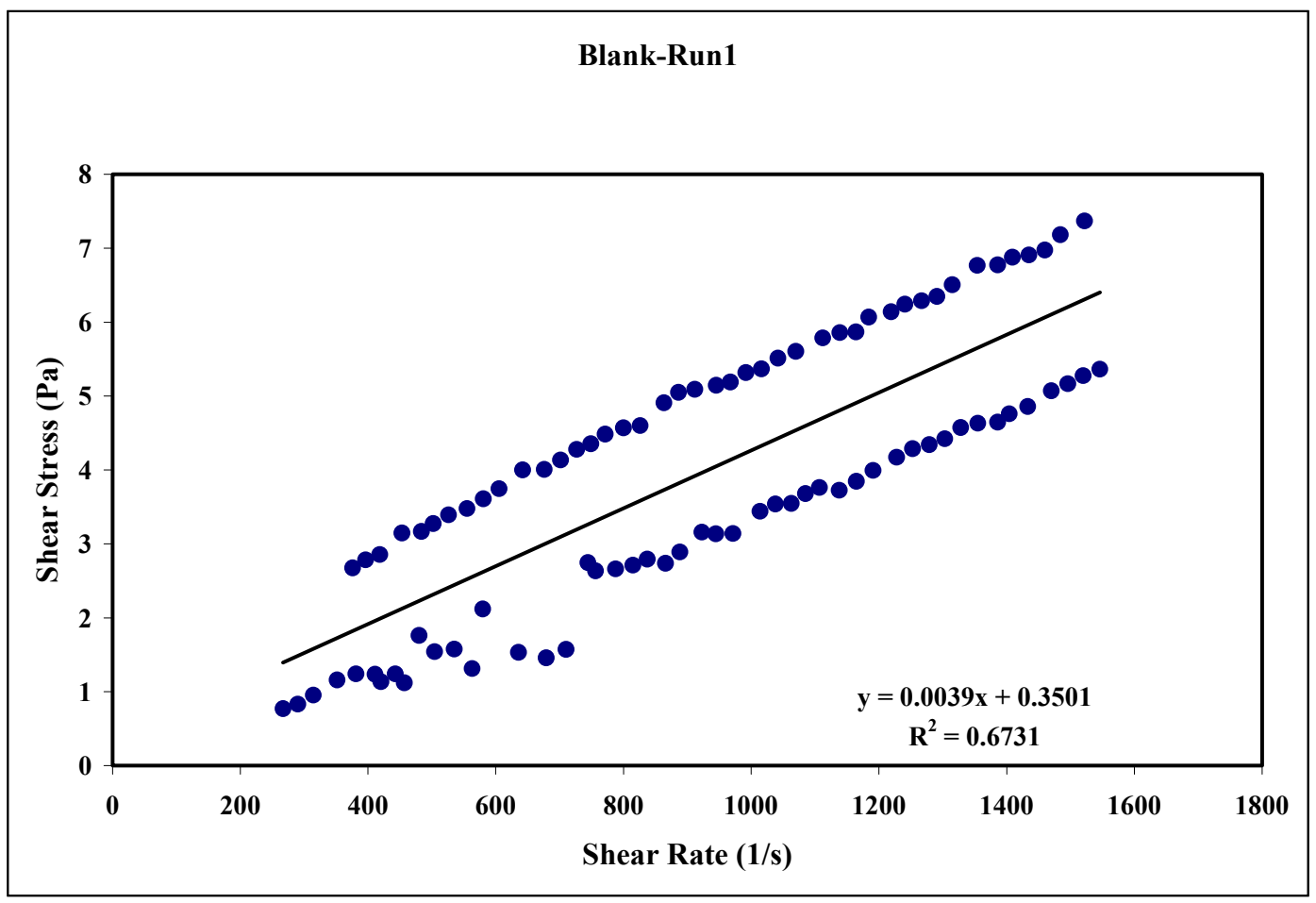

Figure 28. Blank Diluted Run 1 


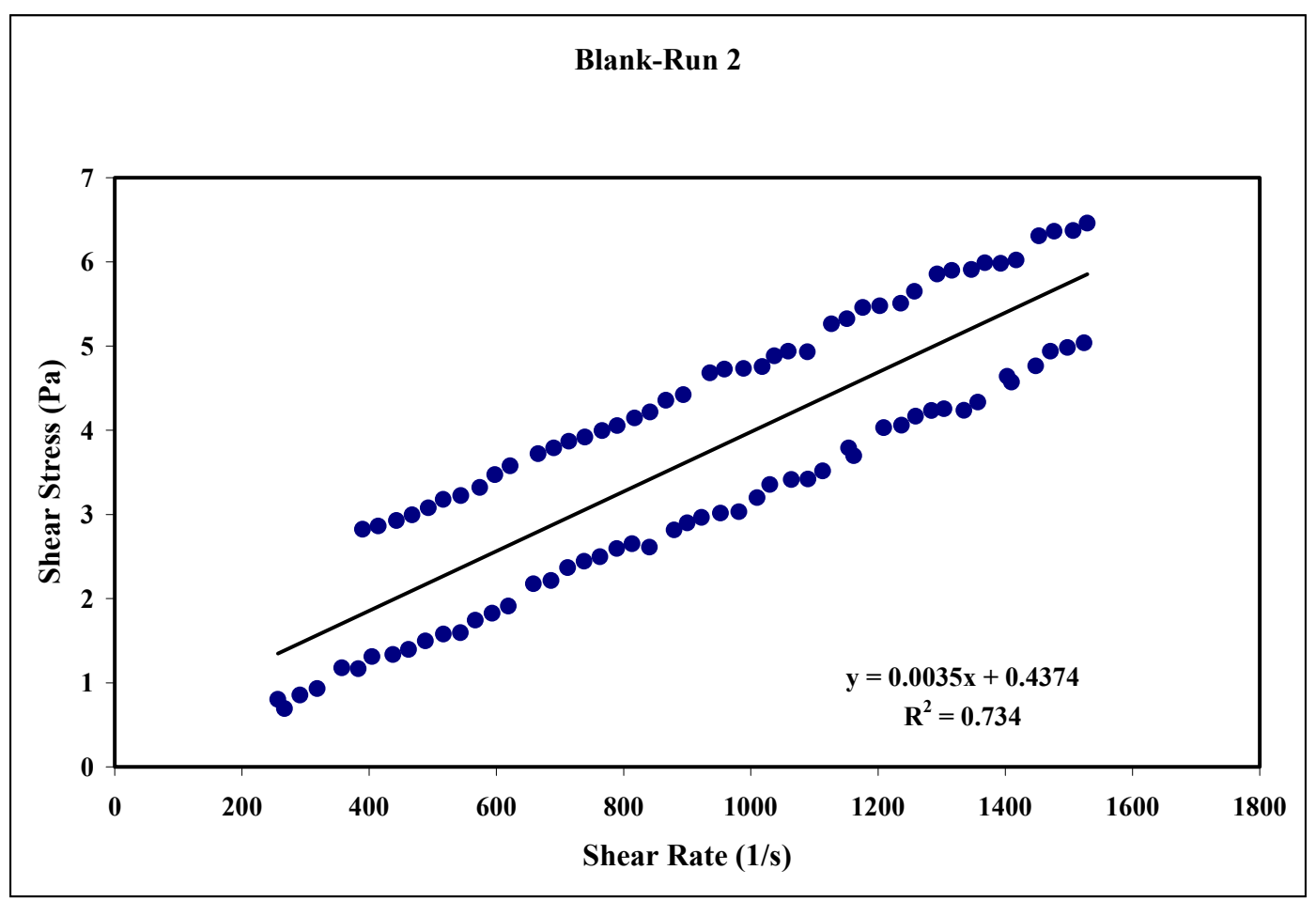

Figure 29. Blank Diluted Run 2

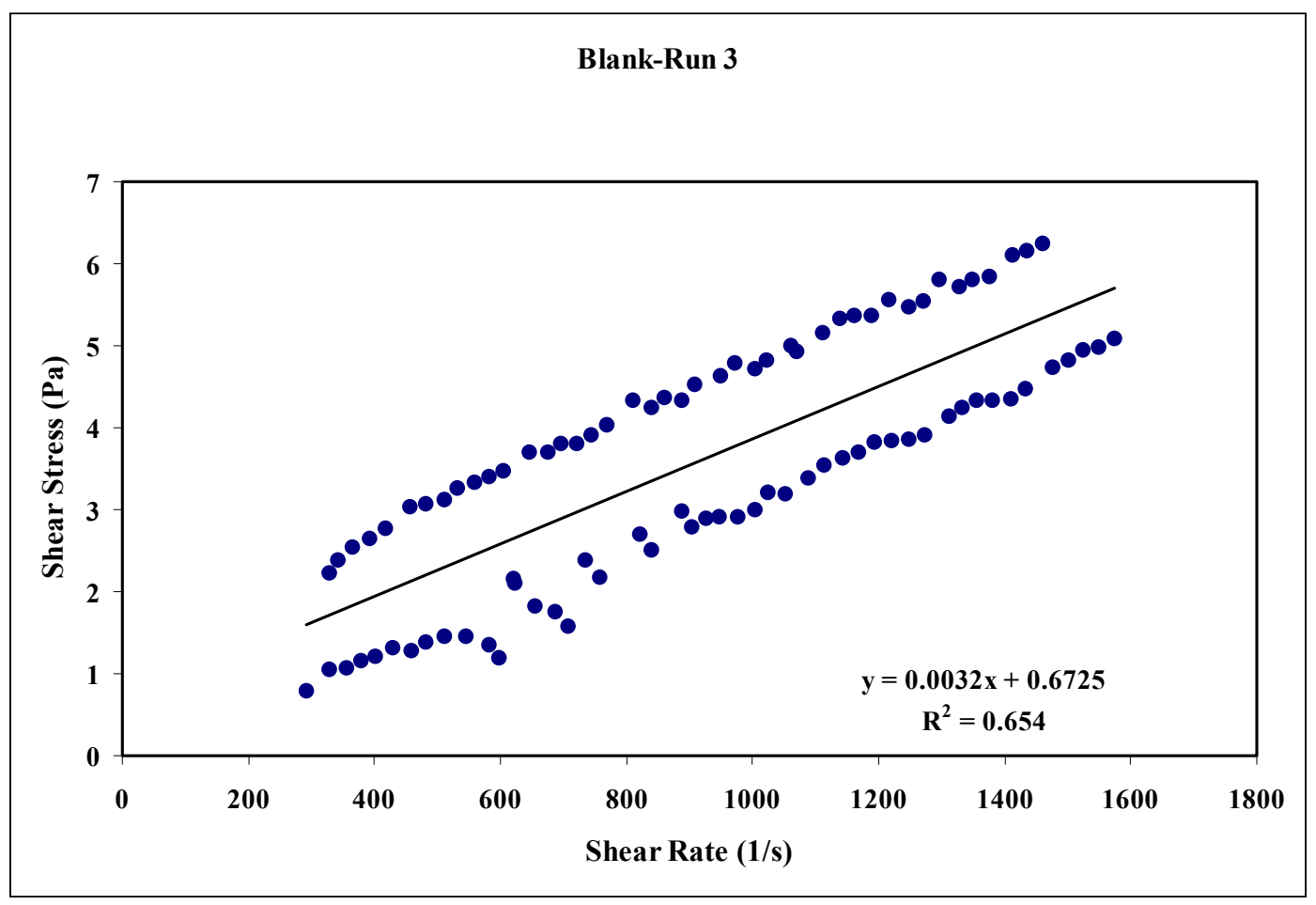

Figure 30. Blank Diluted Run 3 


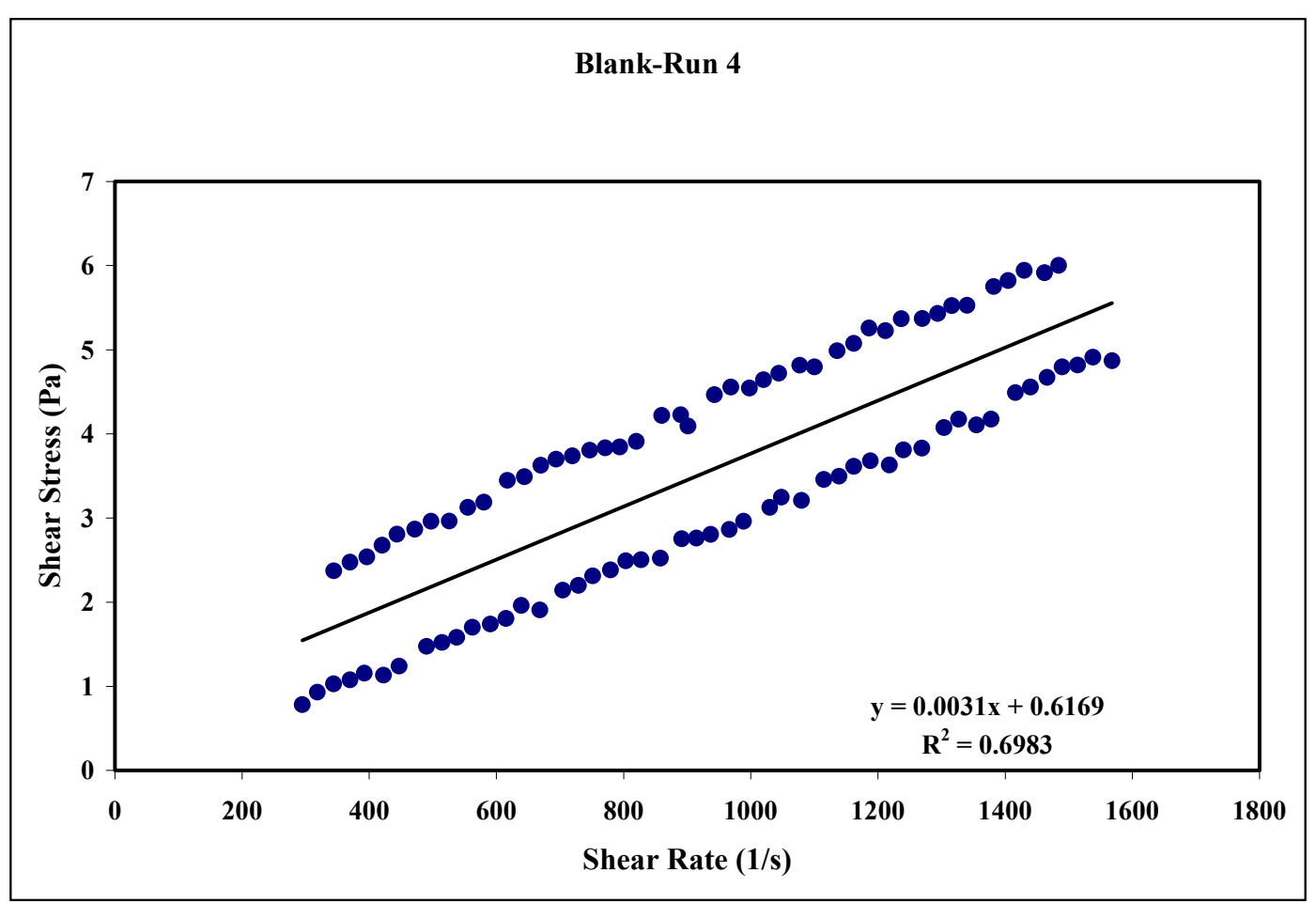

Figure 31. Blank Diluted Run 4

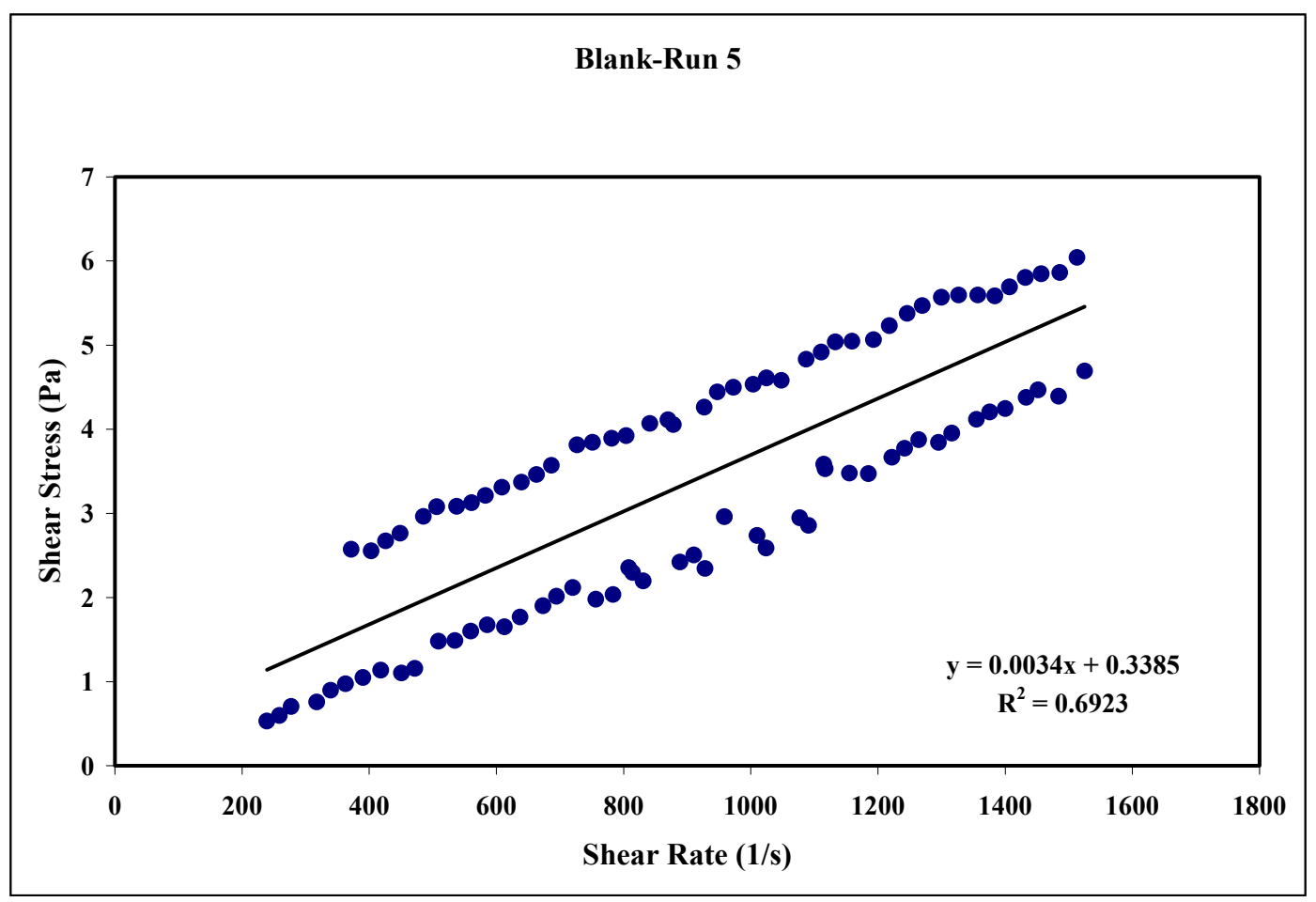

Figure 32. Blank Diluted Run 5 


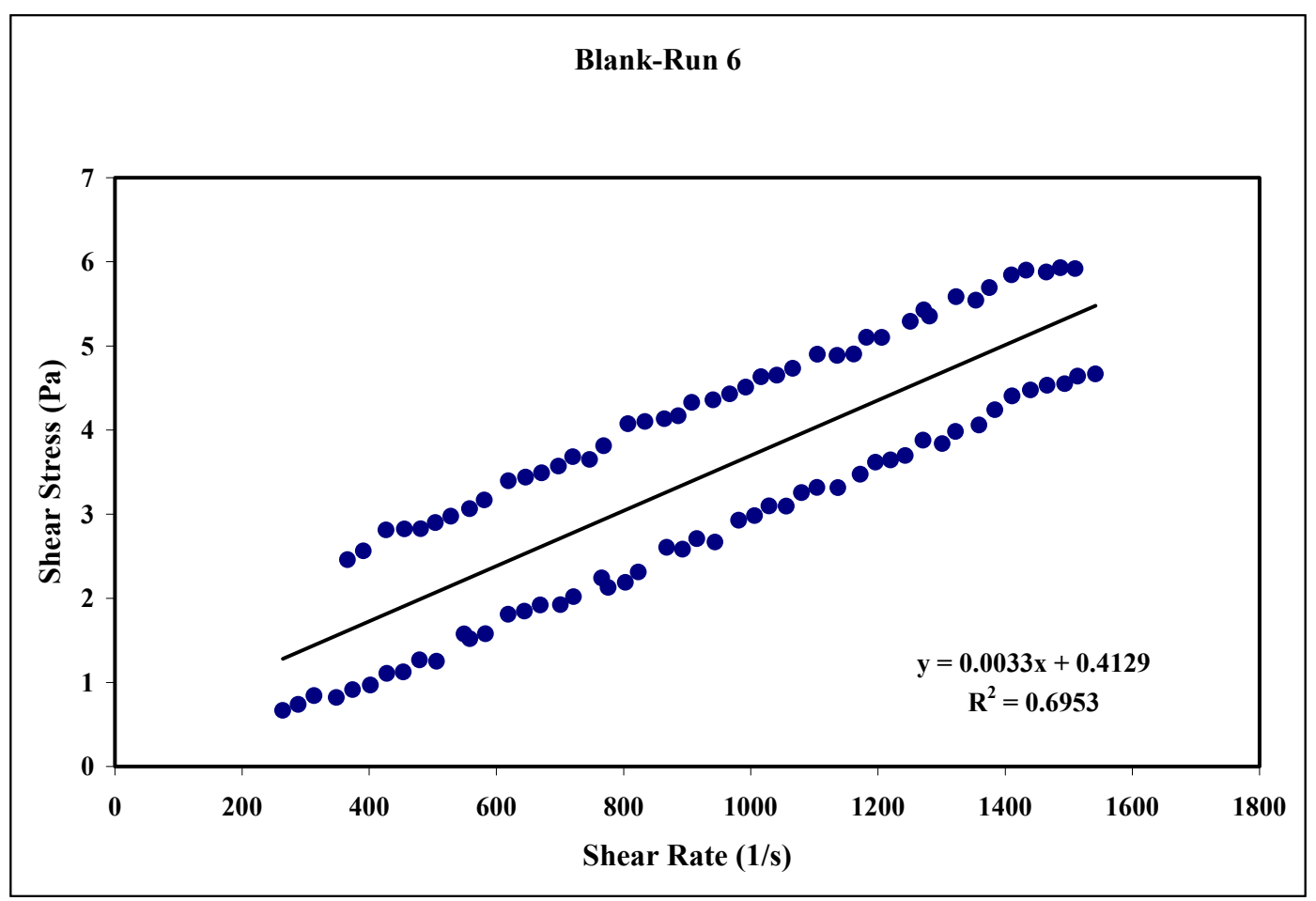

Figure 33. Blank Diluted Run 6

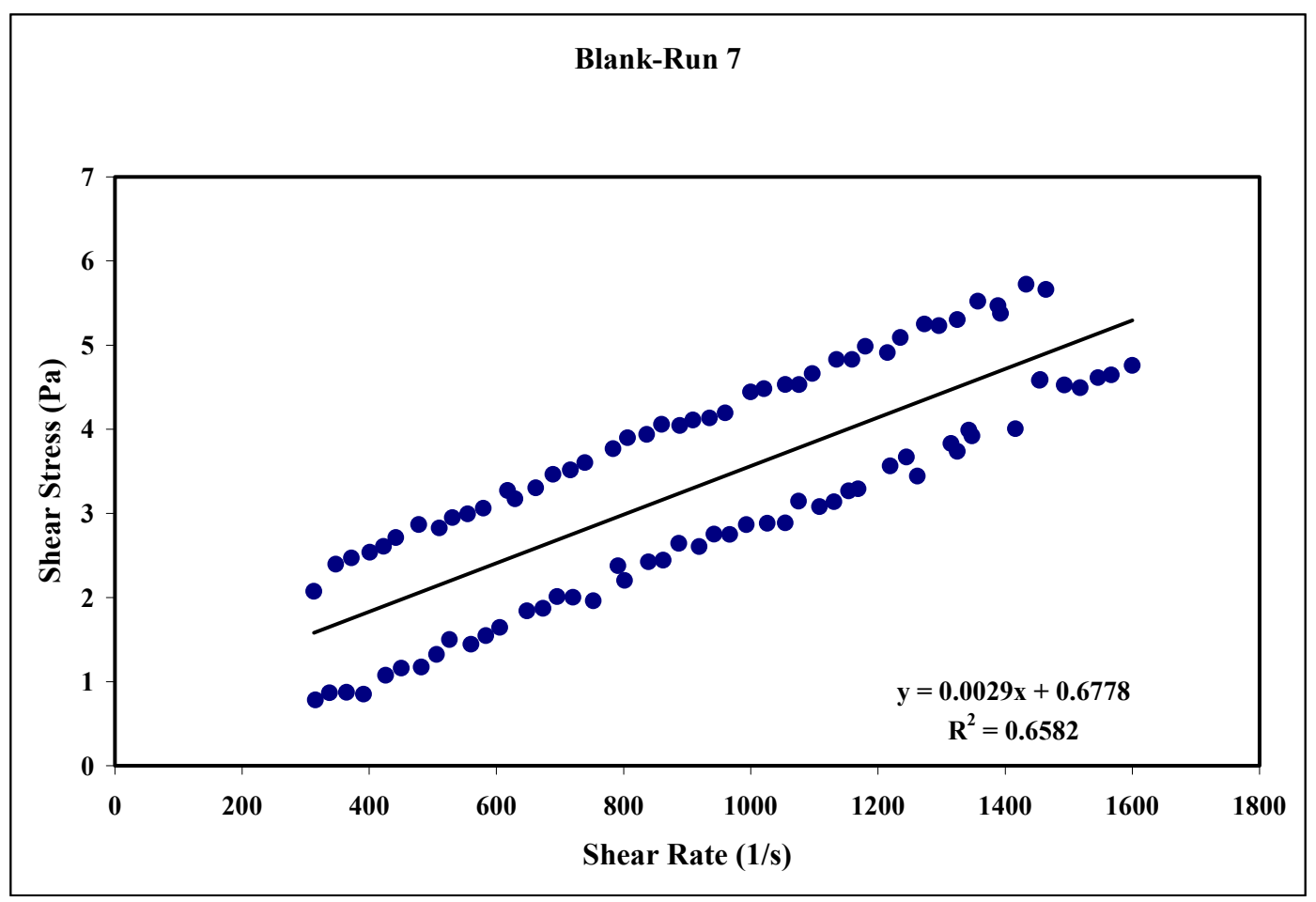

Figure 34. Blank Diluted Run 7 


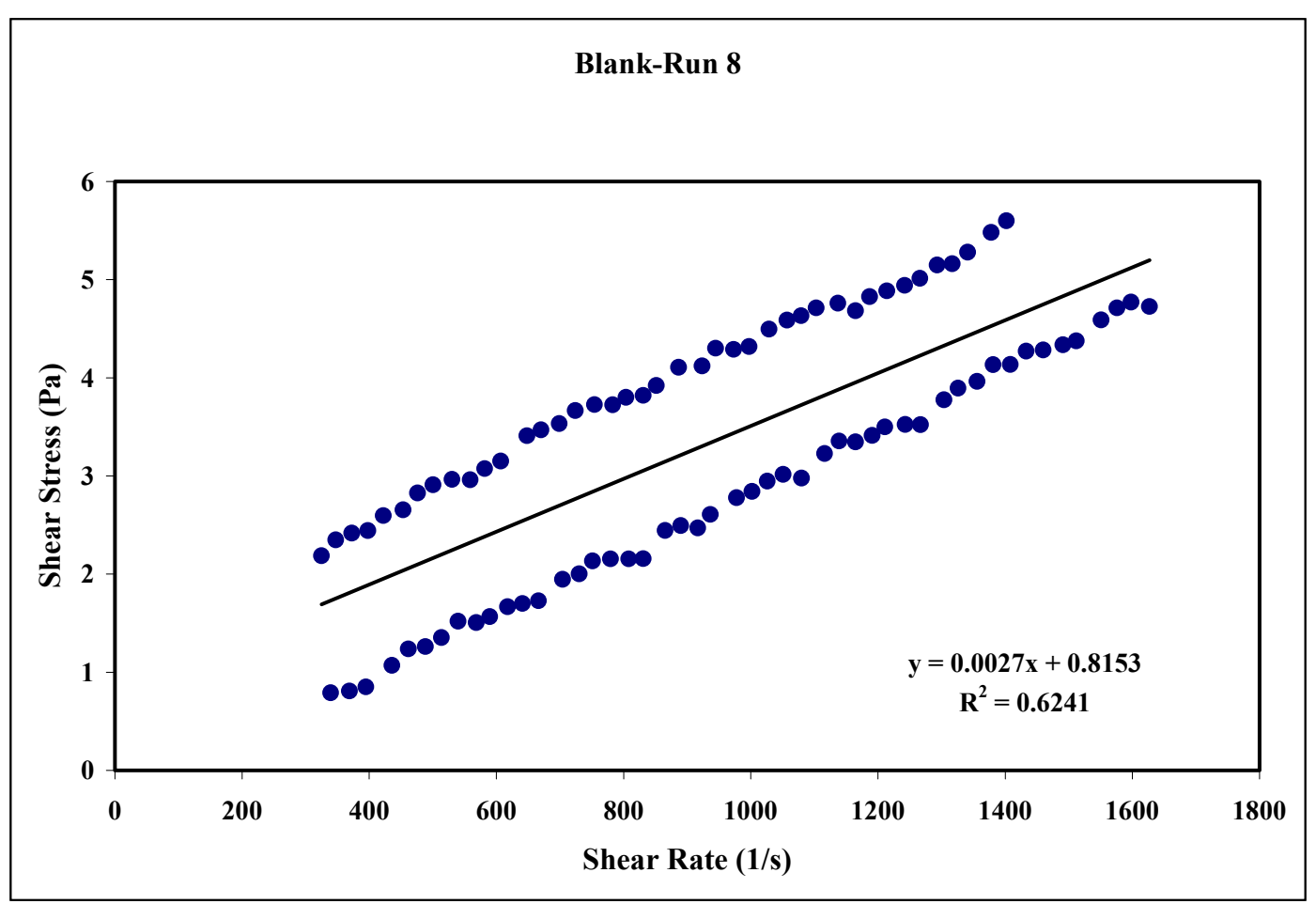

Figure 35. Blank Diluted Run 8

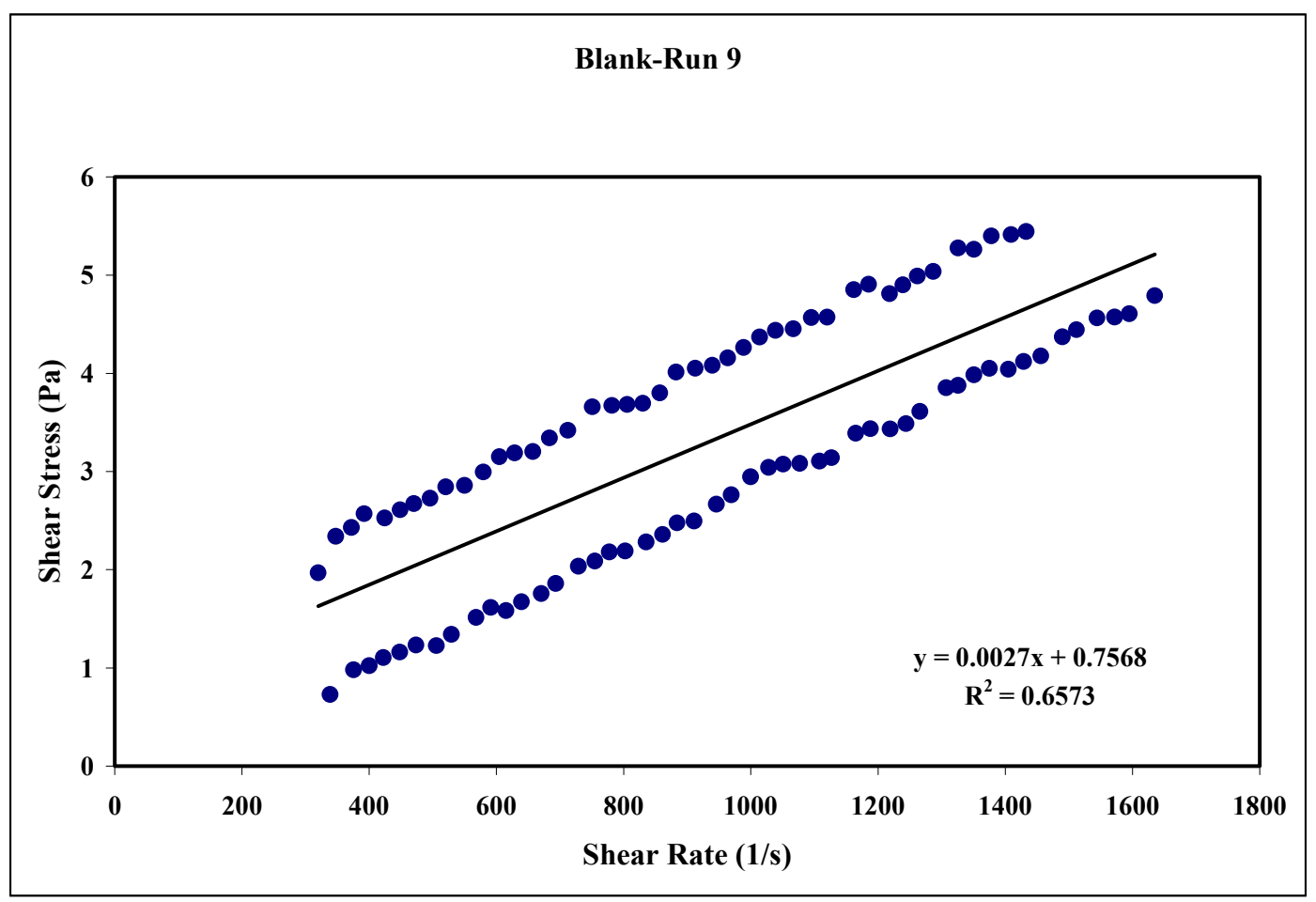

Figure 36. Blank Diluted Run 9 
$\begin{array}{ll}\text { APPENDIX F } & \text { WSRC-TR-2000-00352 }\end{array}$

ENVELOPE A RHEOGRAMS

SRT-RPP-2000-00026

Page 259 of 514

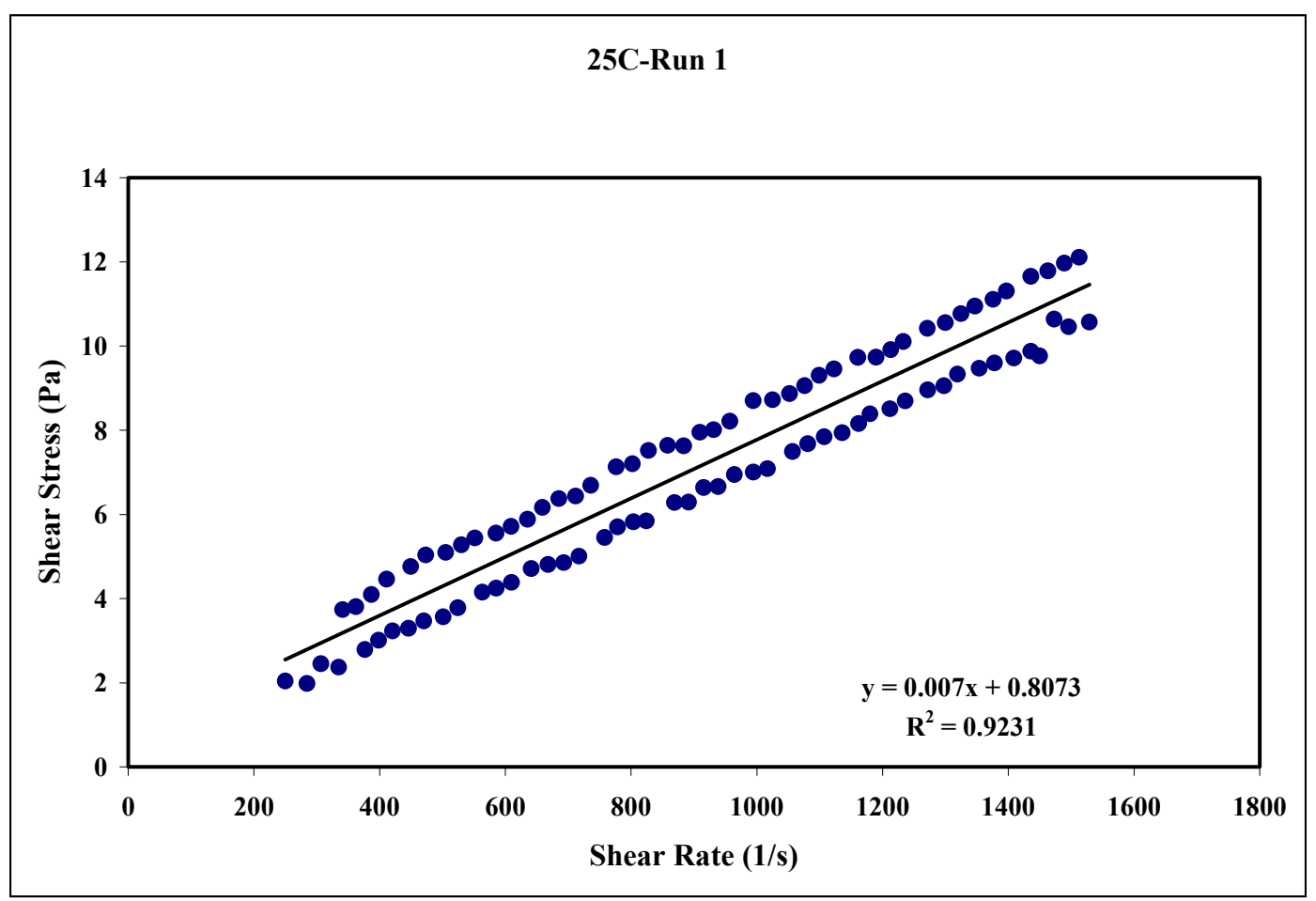

Figure 37. $25^{\circ} \mathrm{C}$ Diluted Run 1

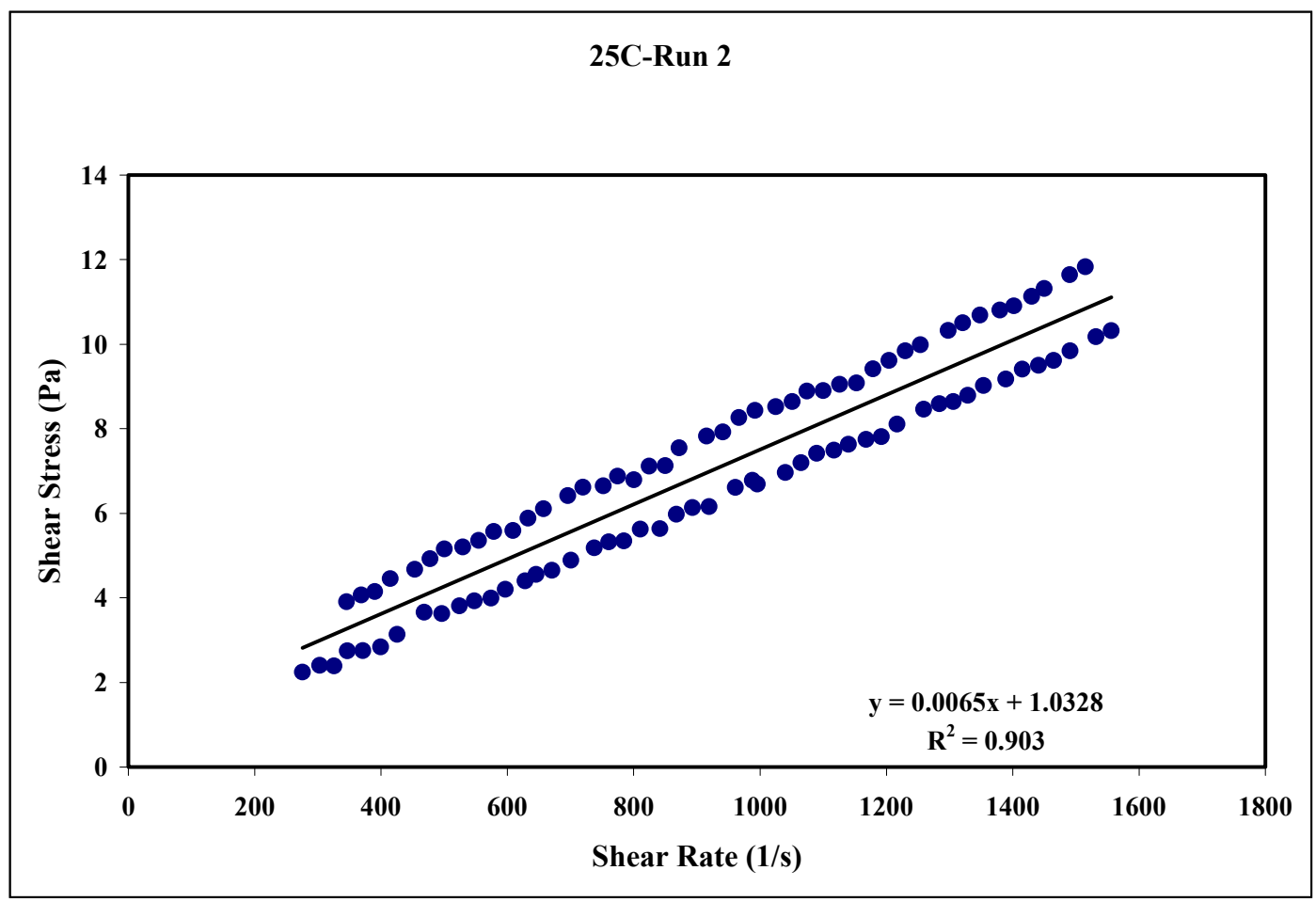

Figure 38. $25^{\circ} \mathrm{C}$ Diluted Run 2 
$\begin{array}{ll}\text { APPENDIX F } & \text { WSRC-TR-2000-00352 }\end{array}$

ENVELOPE A RHEOGRAMS

SRT-RPP-2000-00026

Page 260 of 514

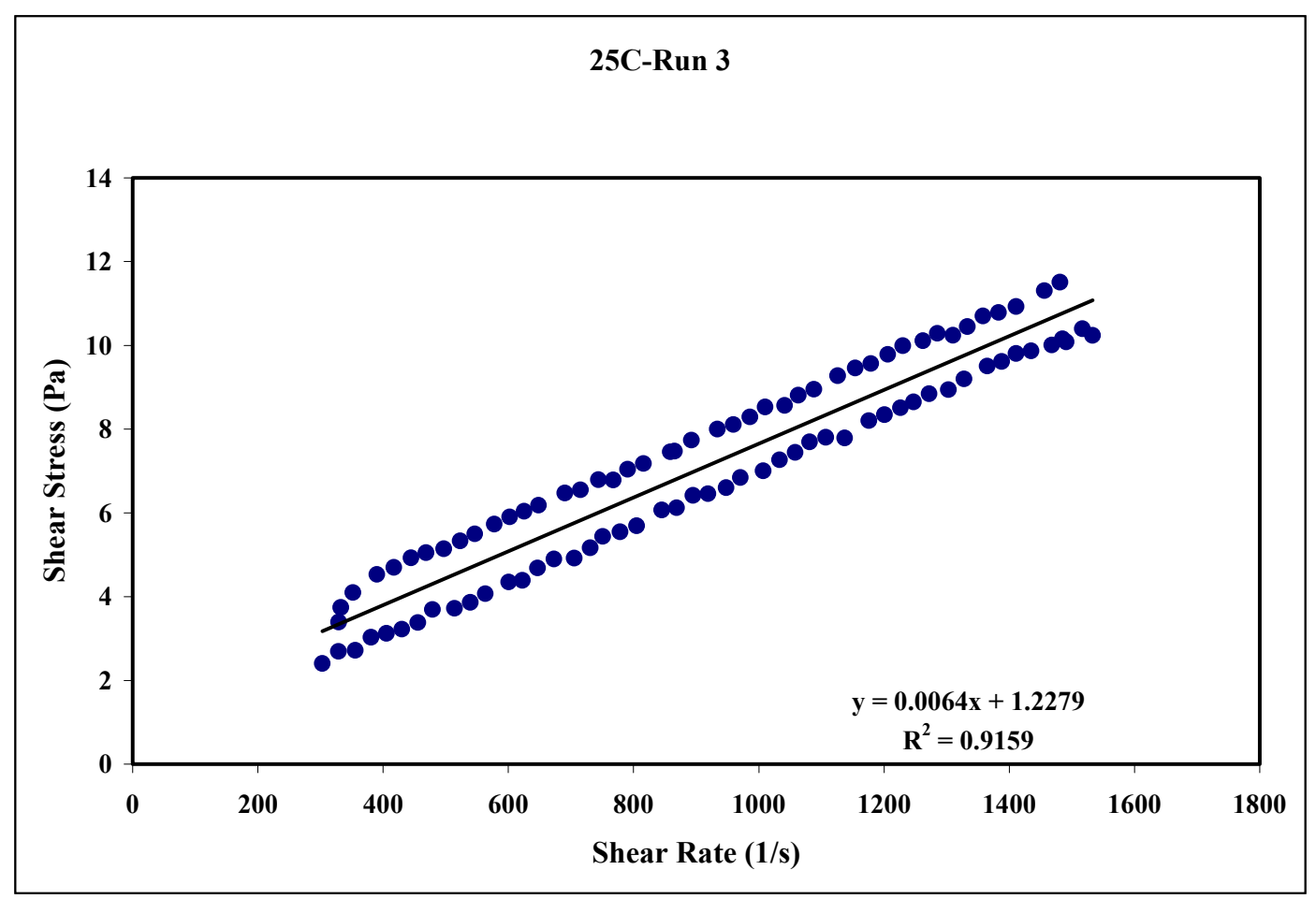

Figure 39. $25^{\circ} \mathrm{C}$ Diluted Run 3

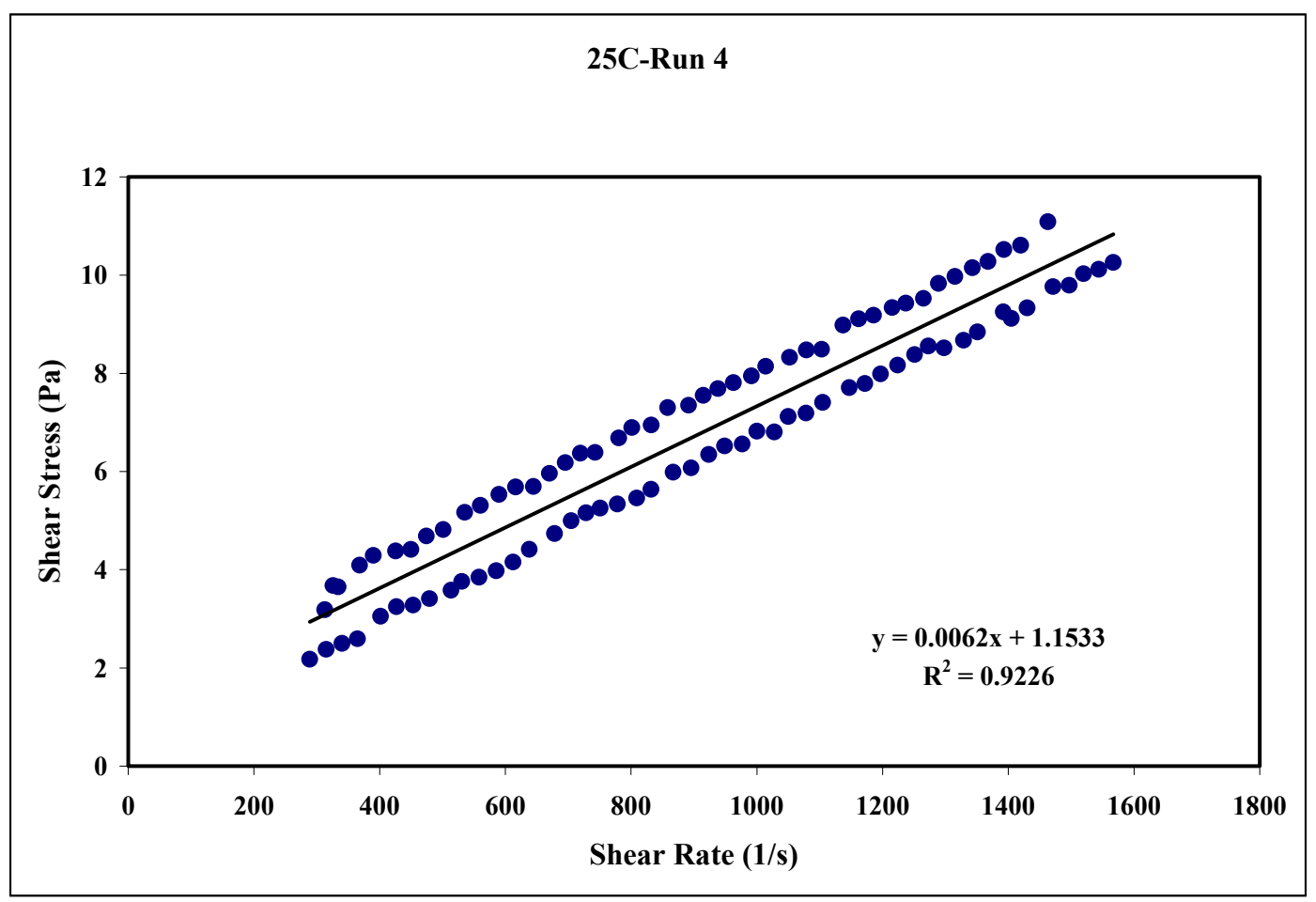

Figure 40. $25^{\circ} \mathrm{C}$ Diluted Run 4 
$\begin{array}{ll}\text { APPENDIX F } & \text { WSRC-TR-2000-00352 }\end{array}$

ENVELOPE A RHEOGRAMS

SRT-RPP-2000-00026

Page 261 of 514

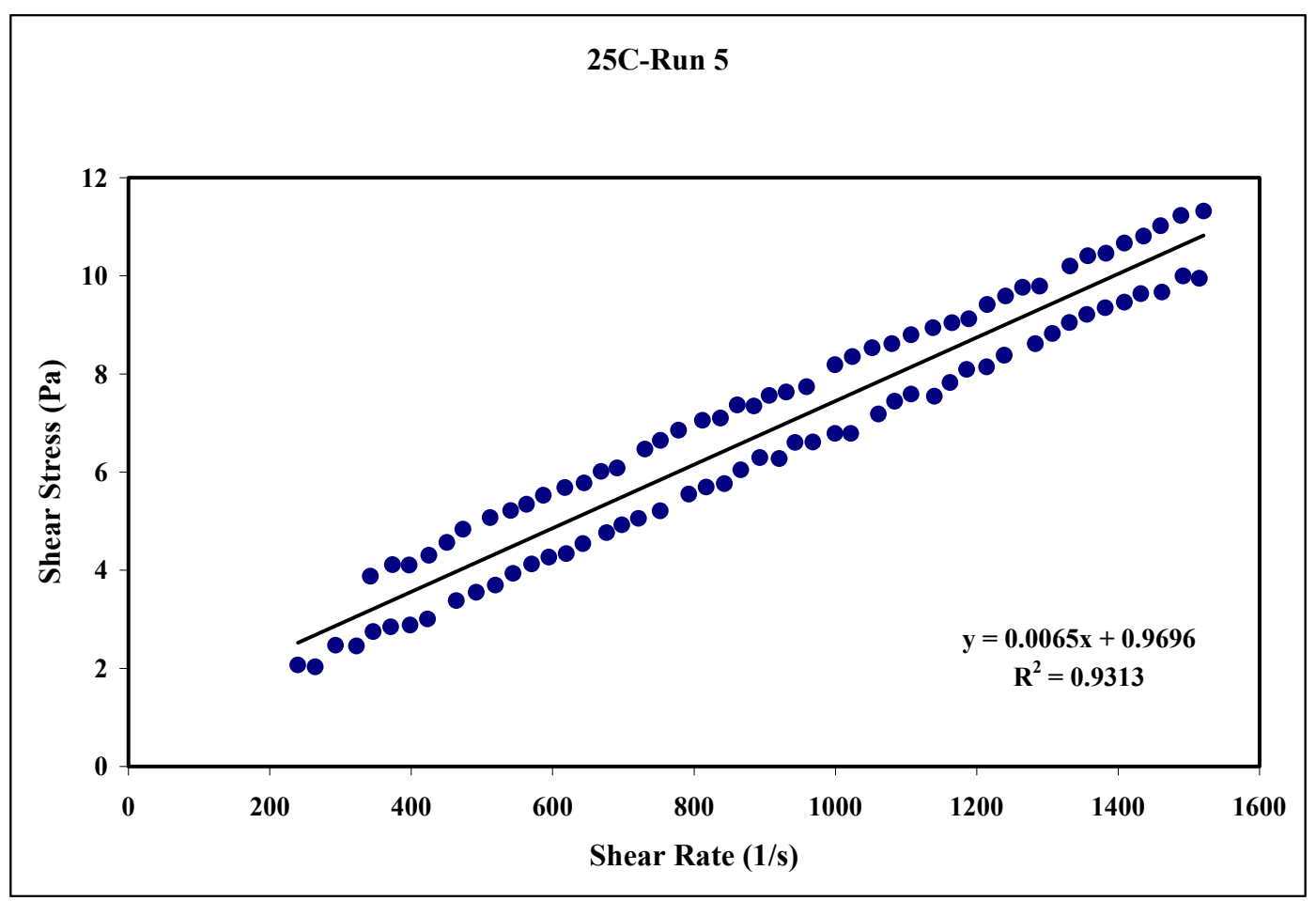

Figure 41. $25^{\circ} \mathrm{C}$ Diluted Run 5

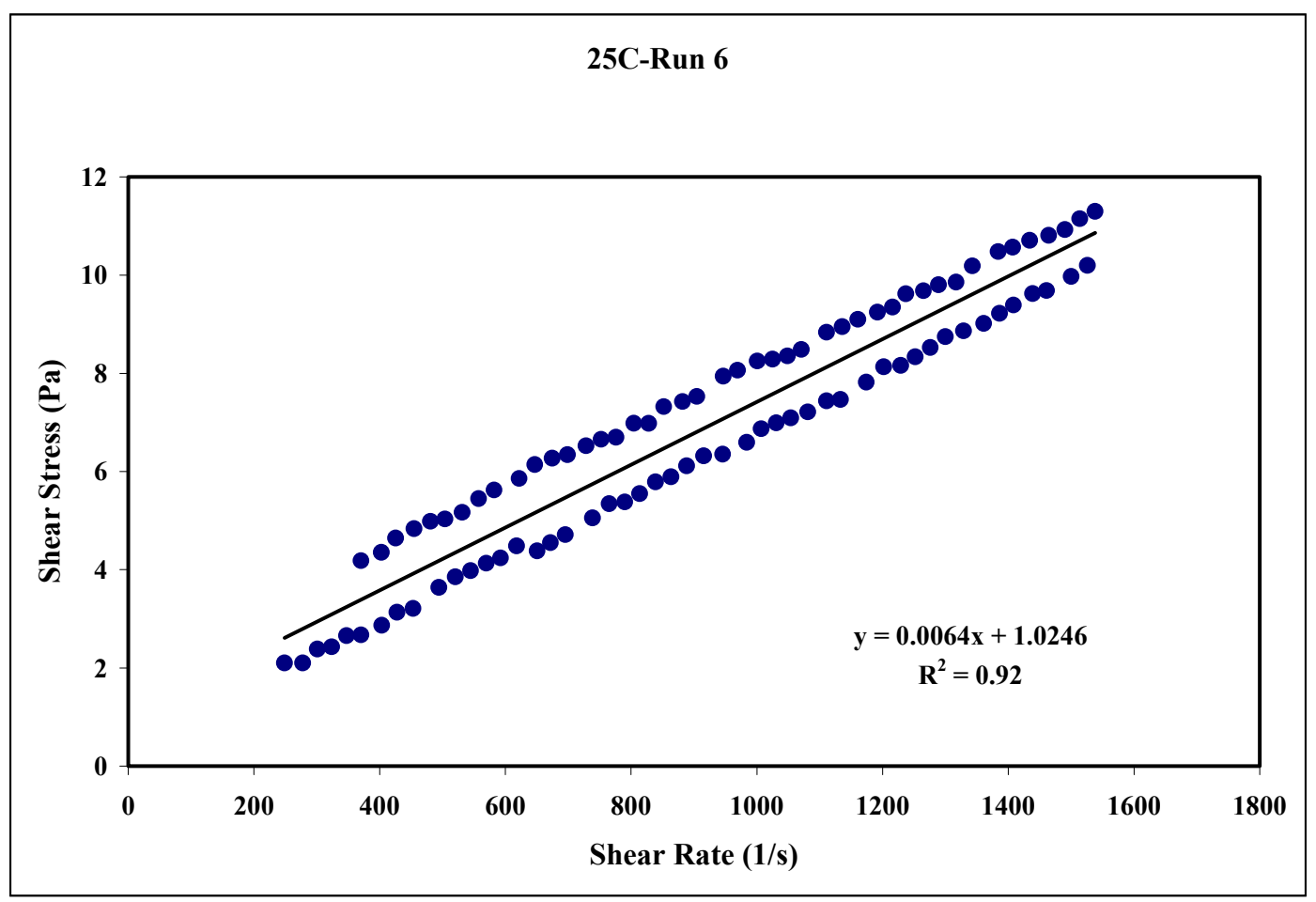

Figure 42. $25^{\circ} \mathrm{C}$ Diluted Run 6 
$\begin{array}{ll}\text { APPENDIX F } & \text { WSRC-TR-2000-00352 }\end{array}$

ENVELOPE A RHEOGRAMS

SRT-RPP-2000-00026

Page 262 of 514

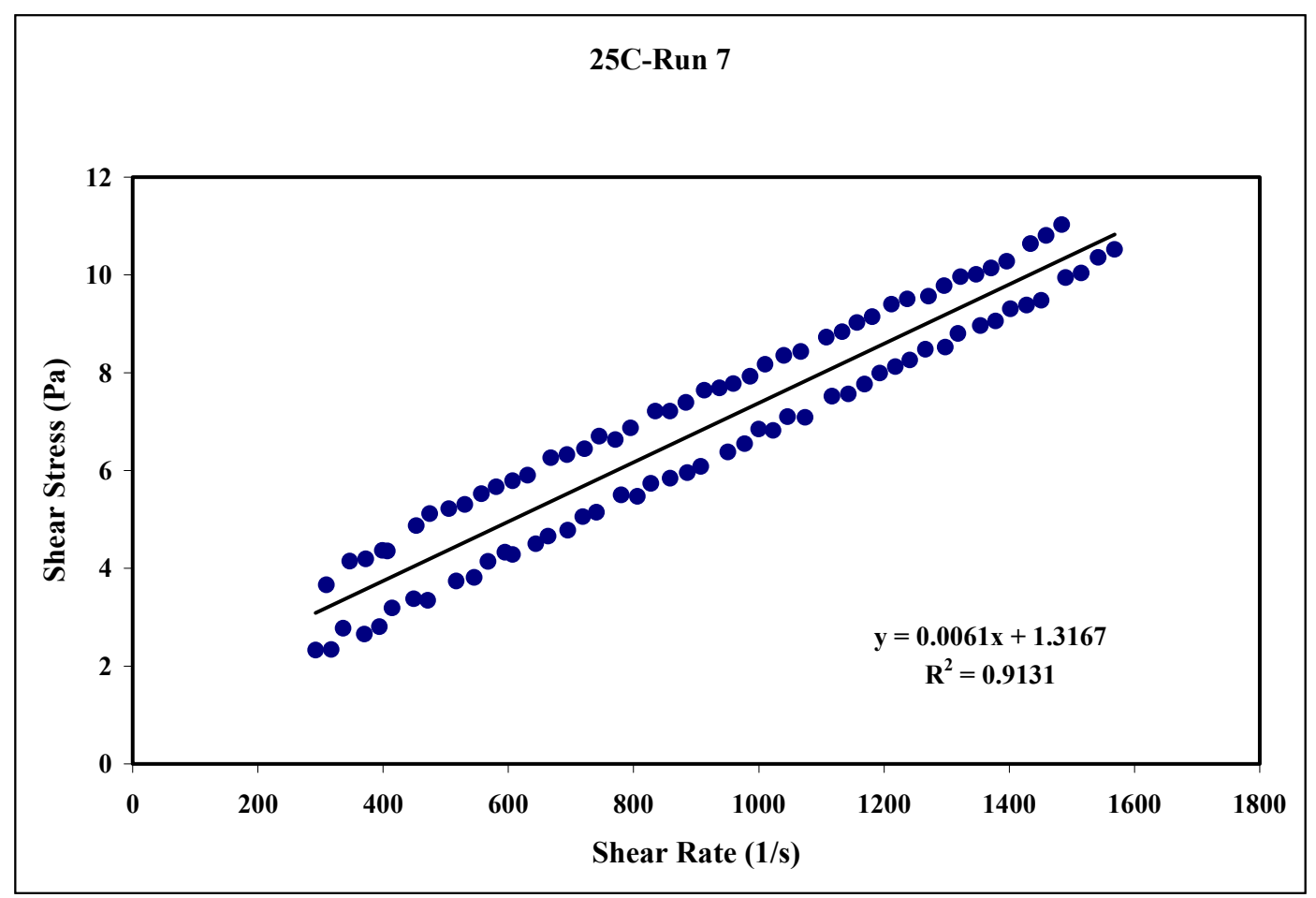

Figure 43. $25^{\circ} \mathrm{C}$ Diluted Run 7

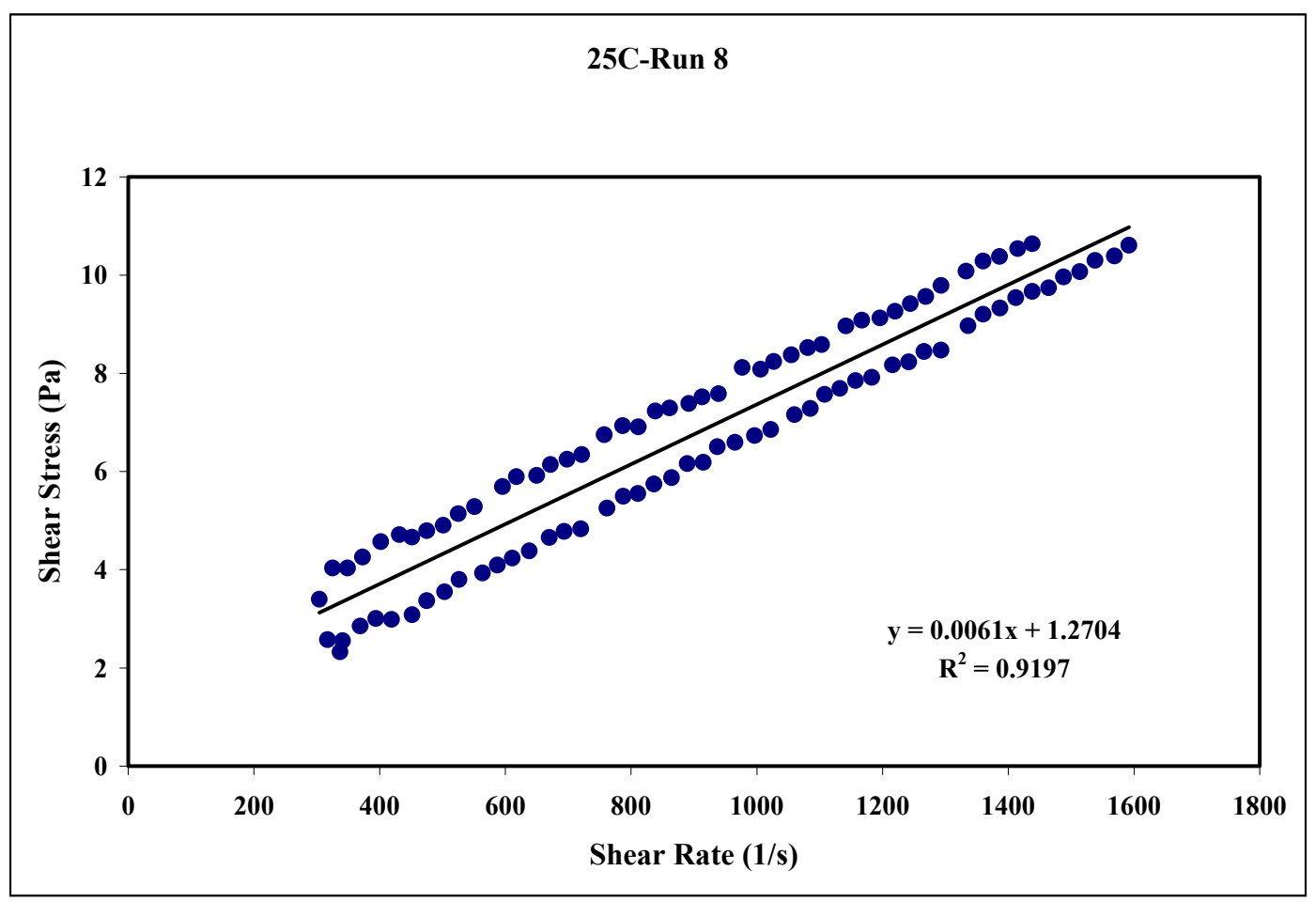

Figure 44. $25^{\circ} \mathrm{C}$ Diluted Run 8 
\begin{tabular}{|lc}
\hline APPENDIX F & WSRC-TR-2000-00352
\end{tabular}

ENVELOPE A RHEOGRAMS

SRT-RPP-2000-00026

Page 263 of 514

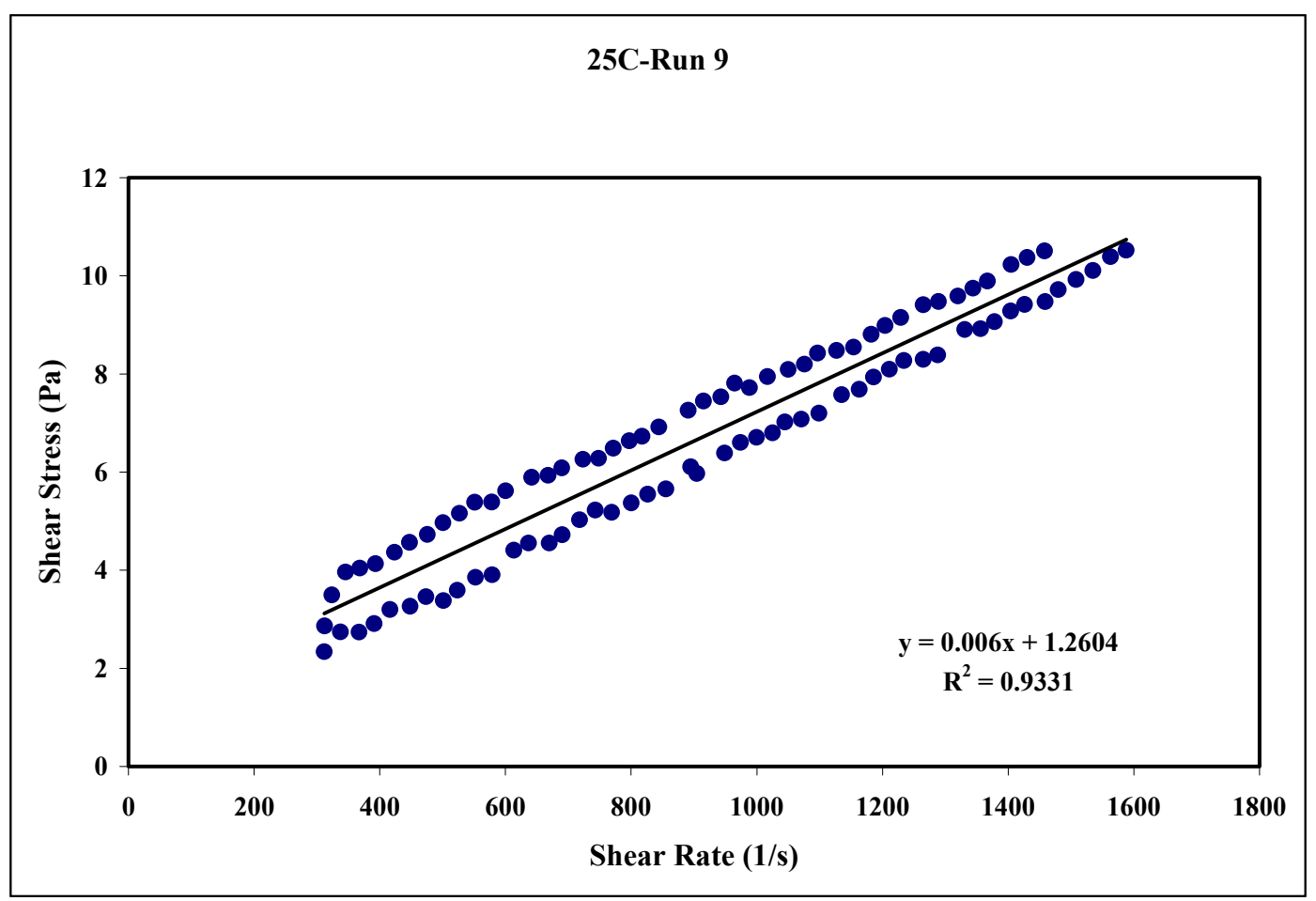

Figure 45. $25^{\circ} \mathrm{C}$ Diluted Run 9

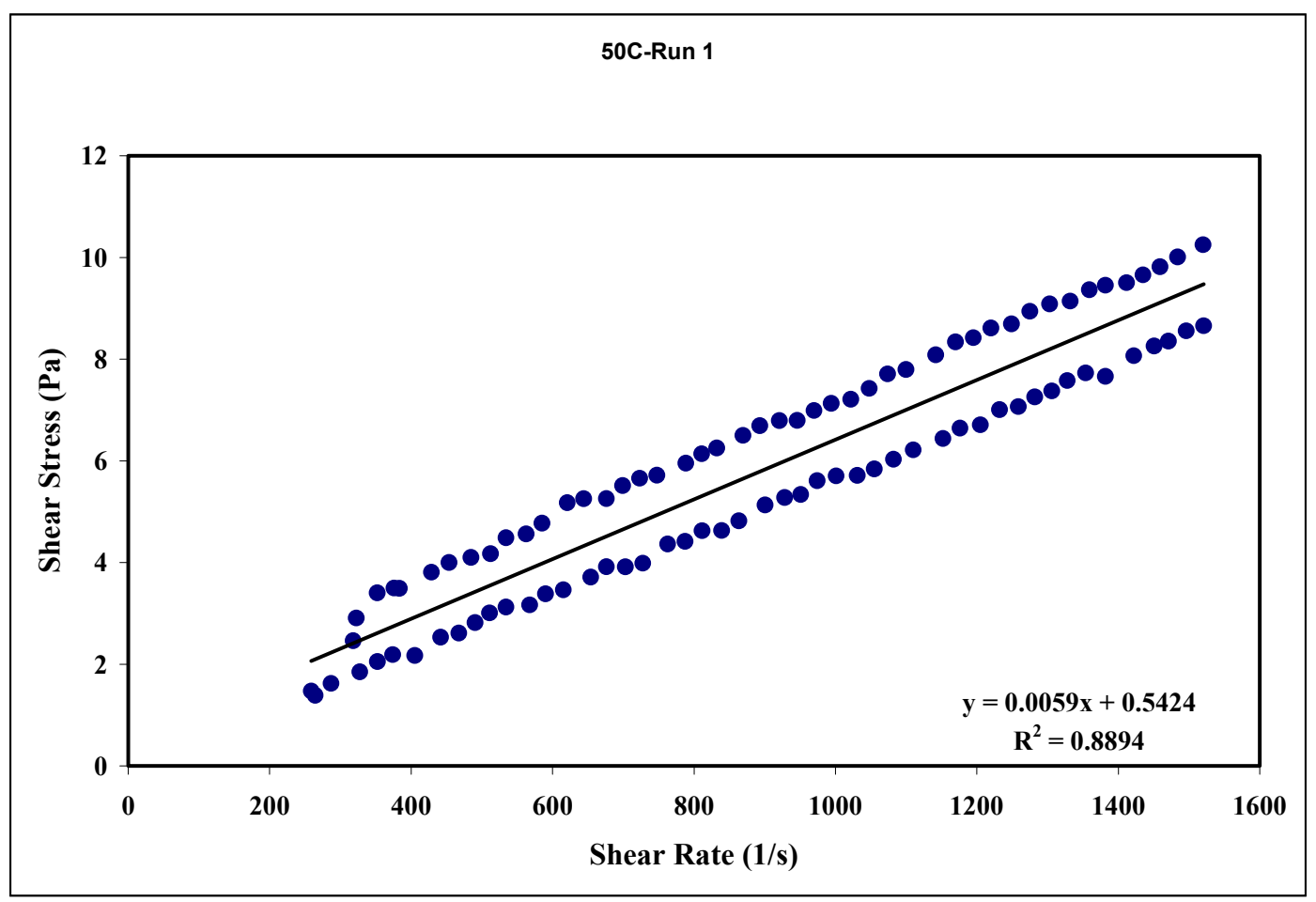

Figure 46. $50^{\circ} \mathrm{C}$ Diluted Run 1 
$\begin{array}{ll}\text { APPENDIX F } & \text { WSRC-TR-2000-00352 }\end{array}$

ENVELOPE A RHEOGRAMS

SRT-RPP-2000-00026

Page 264 of 514

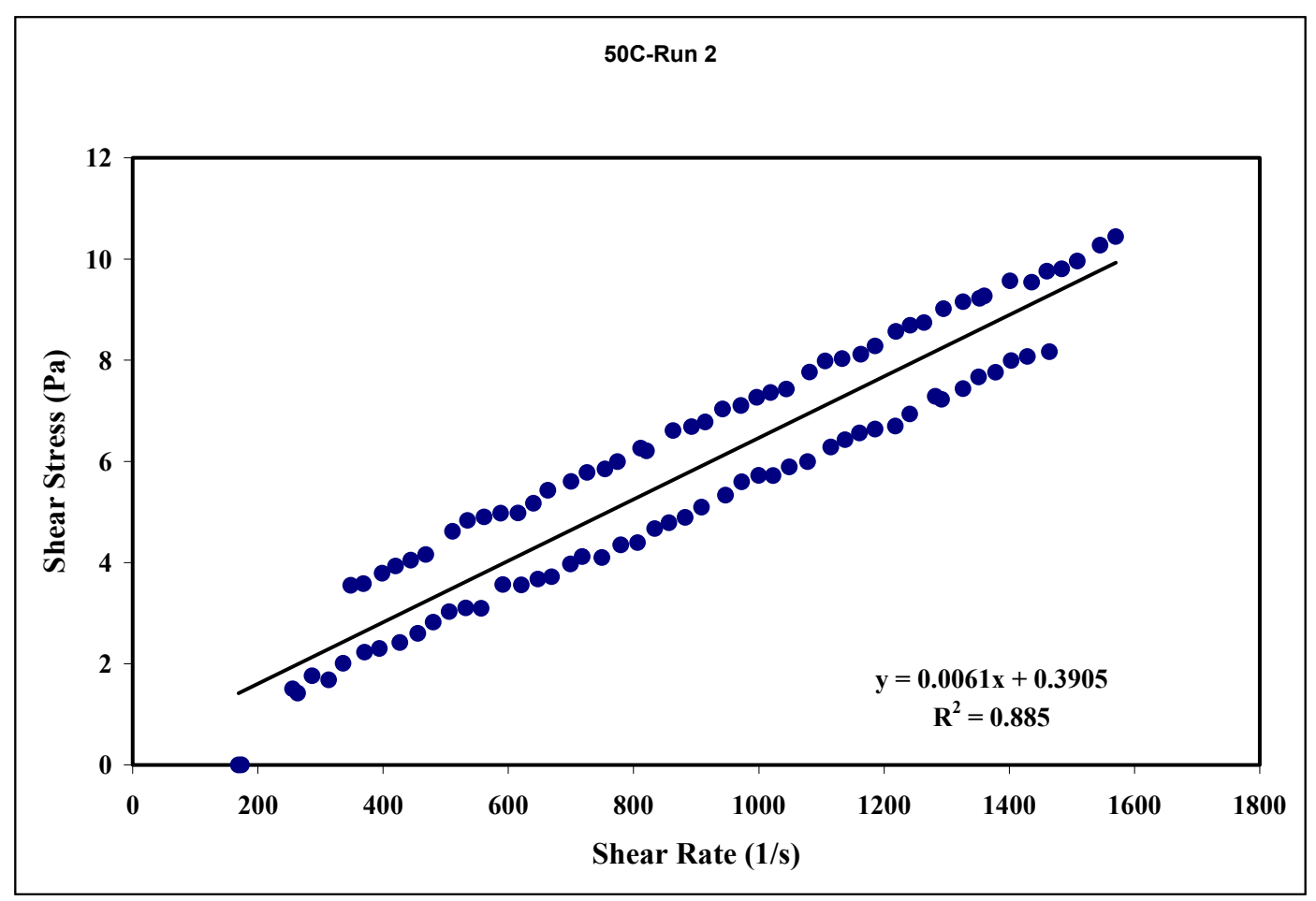

Figure 47. $50{ }^{\circ} \mathrm{C}$ Diluted Run 2

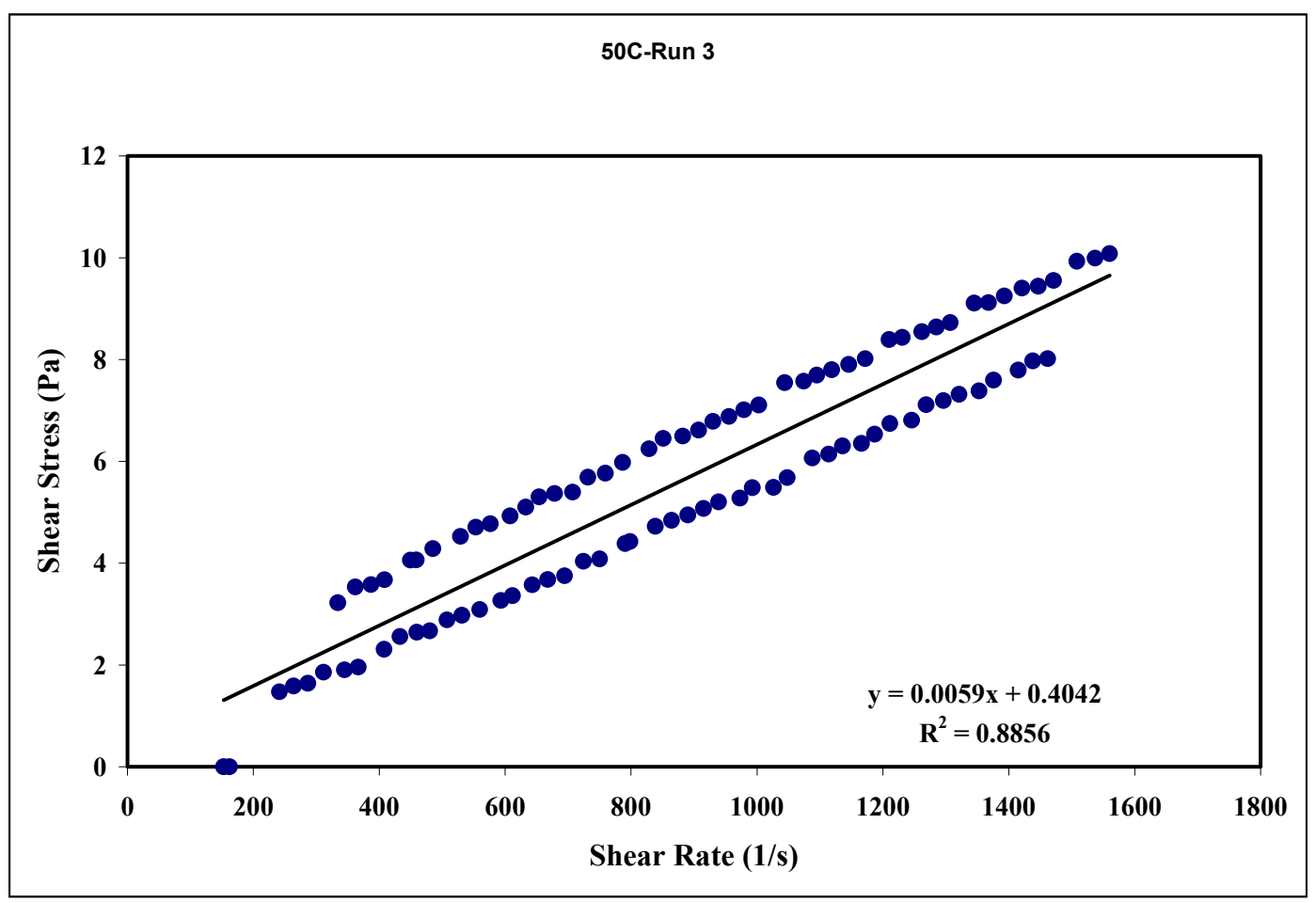

Figure 48. $50^{\circ} \mathrm{C}$ Diluted Run 3 
$\begin{array}{ll}\text { APPENDIX F } & \text { WSRC-TR-2000-00352 }\end{array}$

ENVELOPE A RHEOGRAMS

SRT-RPP-2000-00026

Page 265 of 514

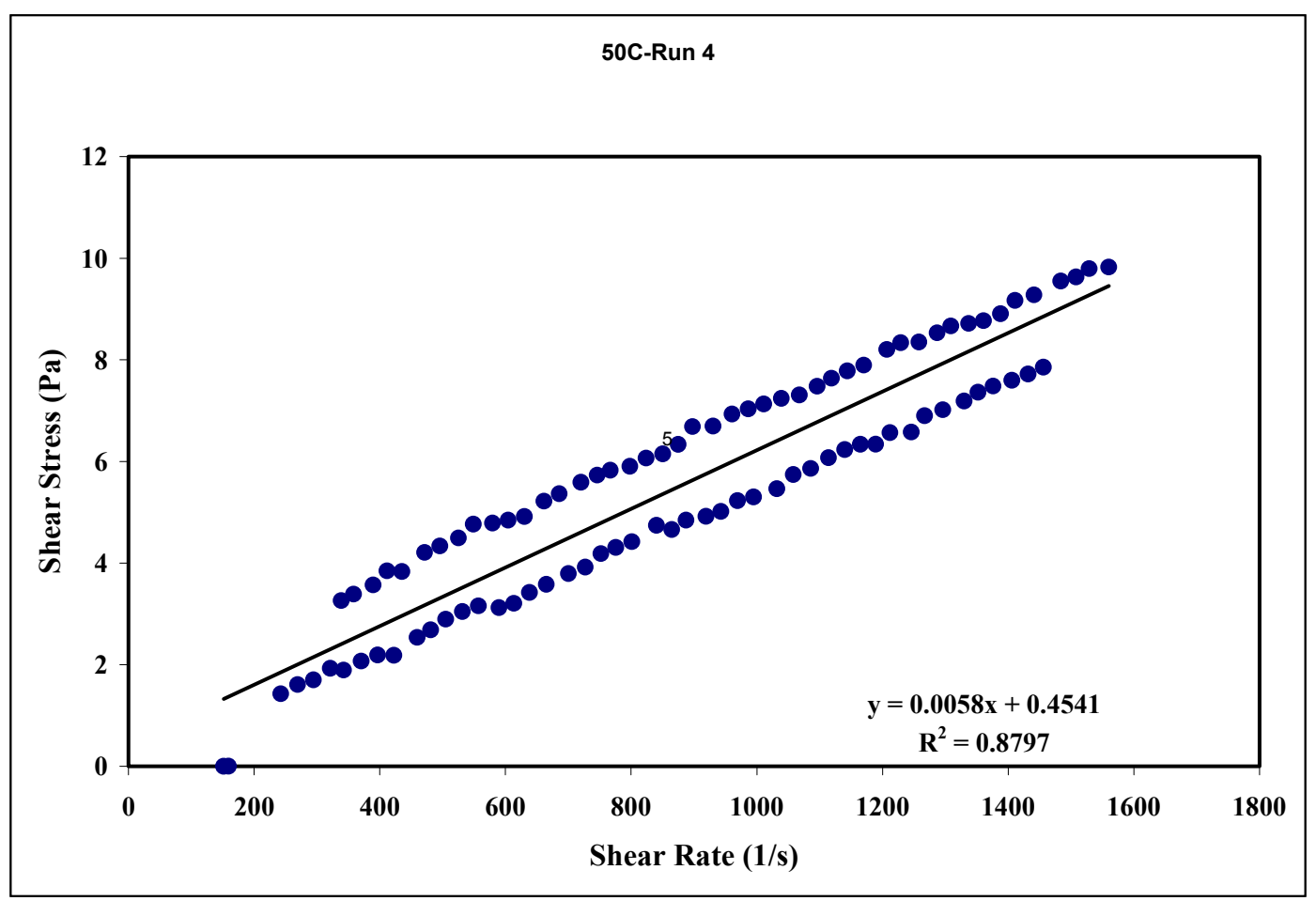

Figure 49. $50{ }^{\circ} \mathrm{C}$ Diluted Run 4

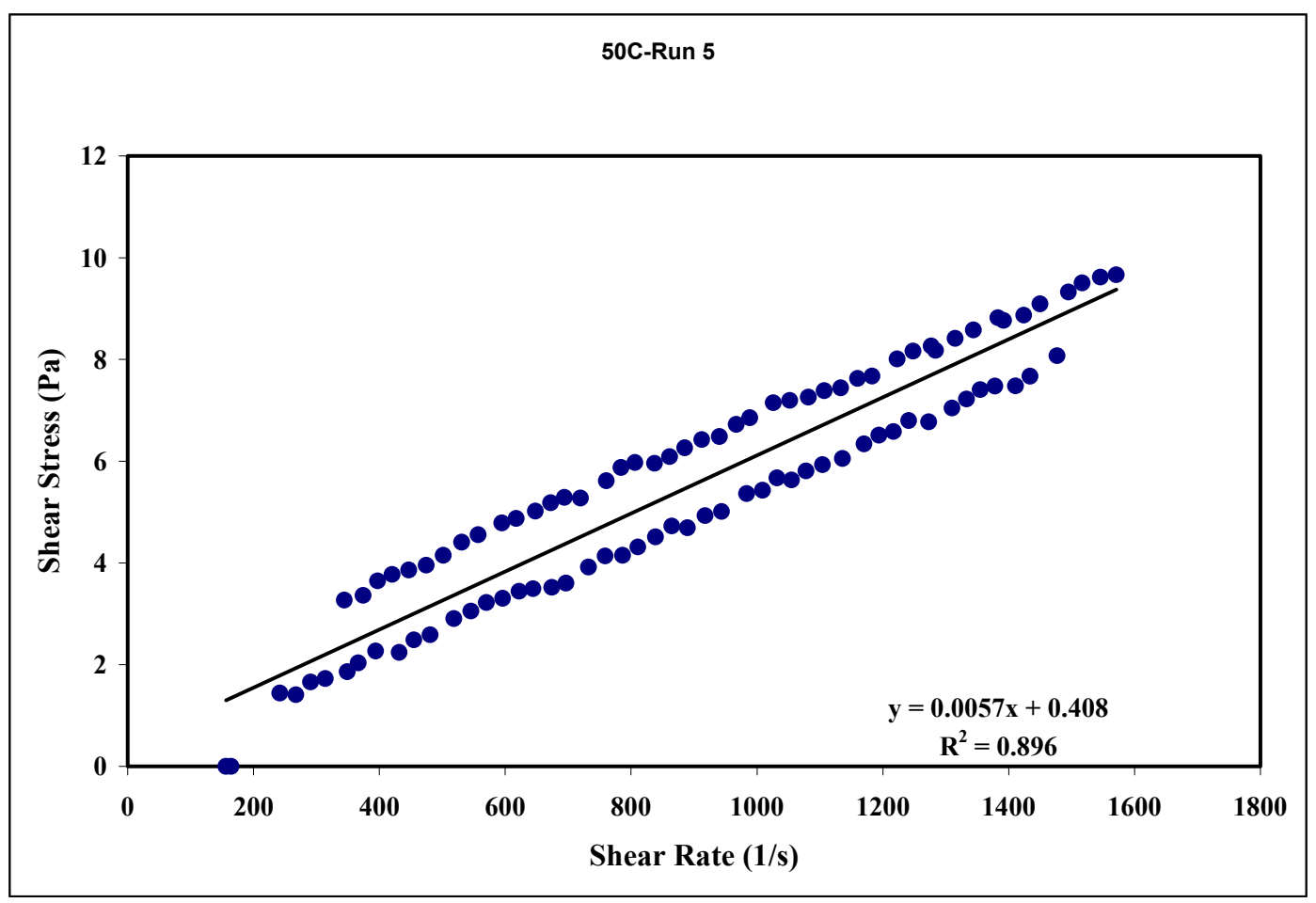

Figure 50. $50{ }^{\circ} \mathrm{C}$ Diluted Run 5 
$\begin{array}{ll}\text { APPENDIX F } & \text { WSRC-TR-2000-00352 }\end{array}$

ENVELOPE A RHEOGRAMS

SRT-RPP-2000-00026

Page 266 of 514

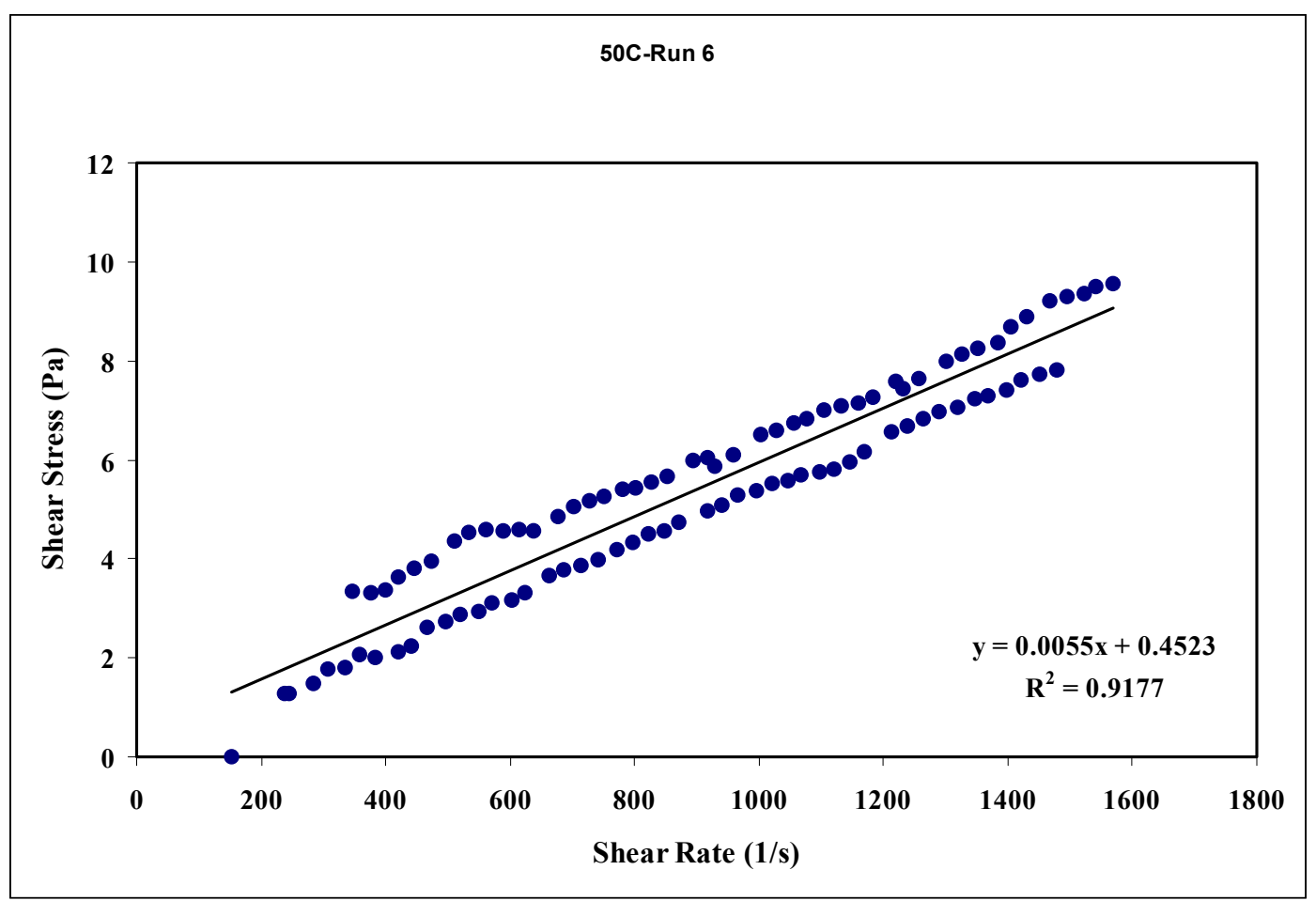

Figure 51. $50{ }^{\circ} \mathrm{C}$ Diluted Run 6

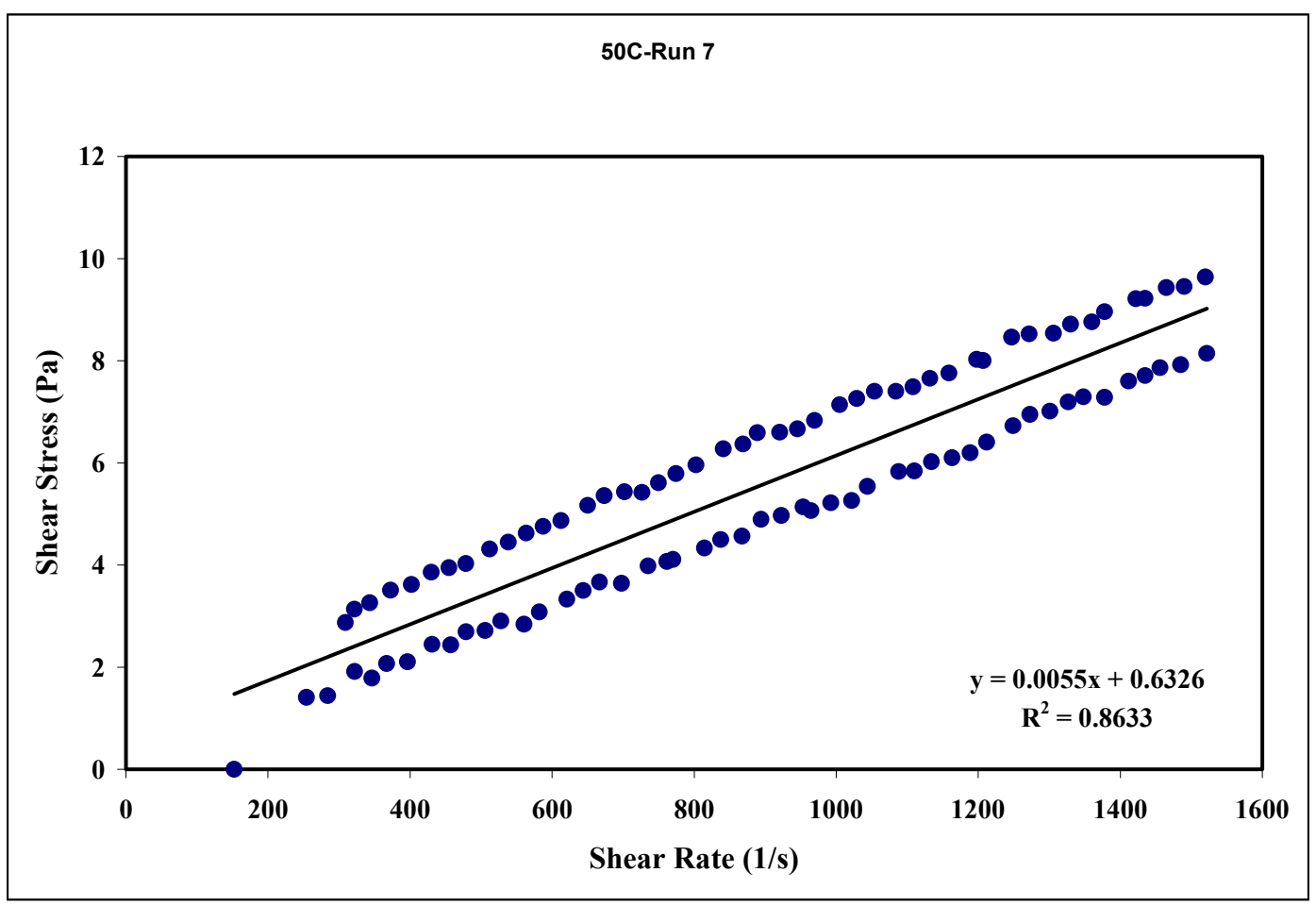

Figure 52. $50^{\circ} \mathrm{C}$ Diluted Run 7 
\begin{tabular}{|lc}
\hline APPENDIX F & WSRC-TR-2000-00352
\end{tabular}

ENVELOPE A RHEOGRAMS

SRT-RPP-2000-00026

Page 267 of 514

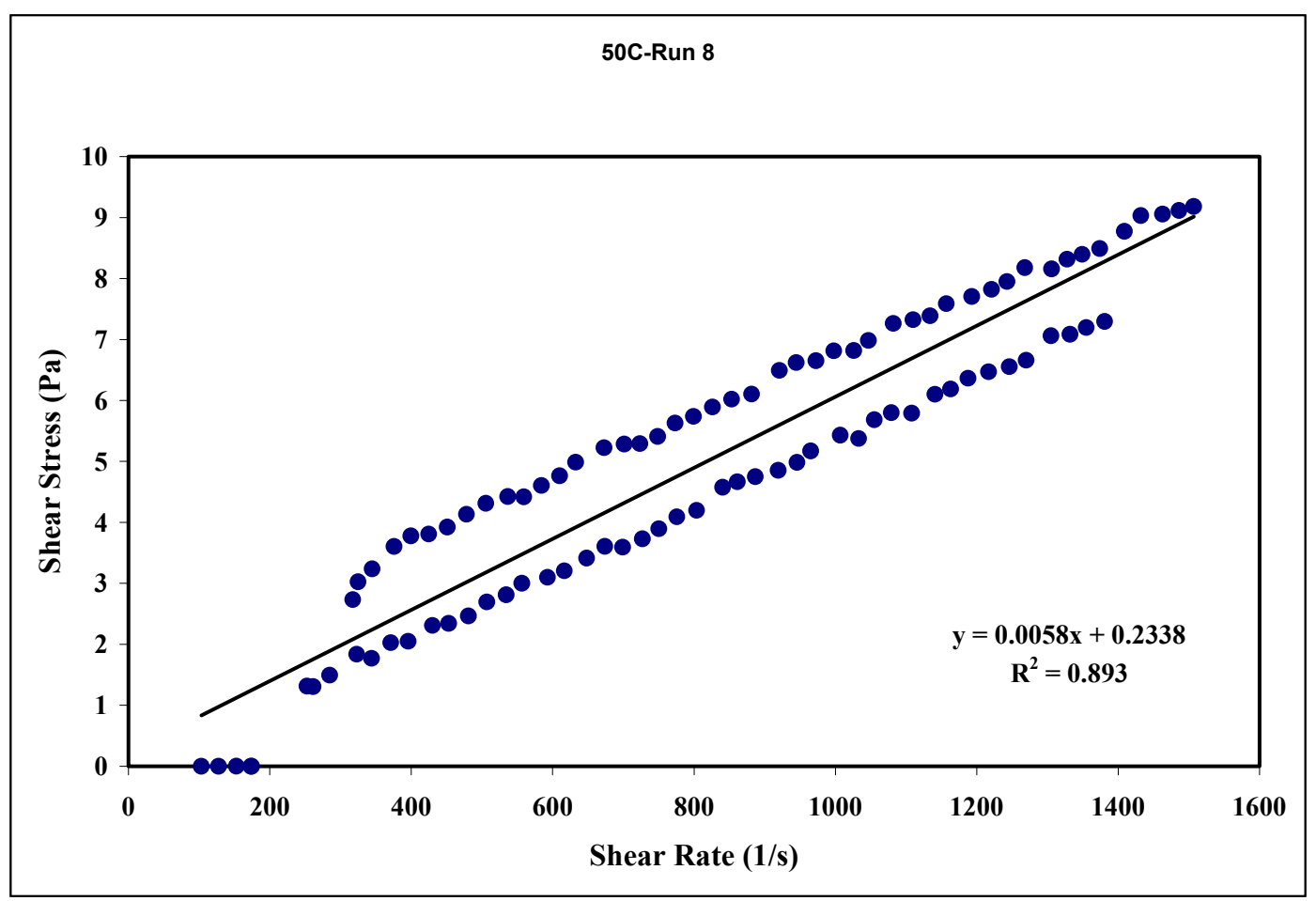

Figure 53. $50^{\circ} \mathrm{C}$ Diluted Run 8

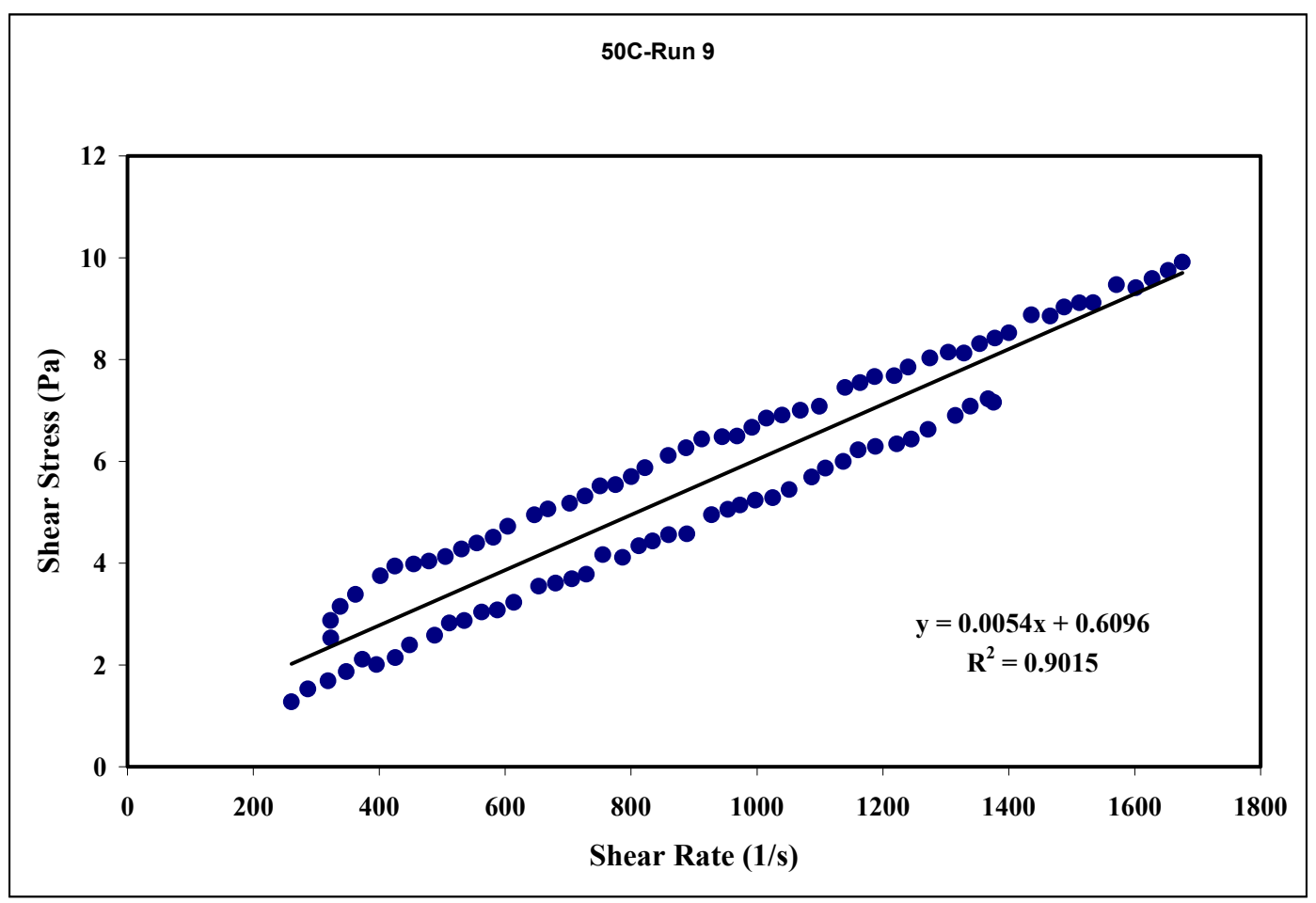

Figure 54. $50{ }^{\circ} \mathrm{C}$ Diluted Run 9 
This page intentionally left blank. 


\section{APPENDIX G}

\section{ENVELOPE B RHEOGRAMS}

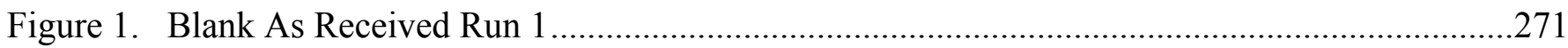

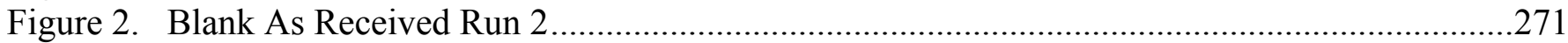

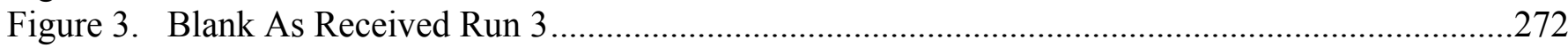

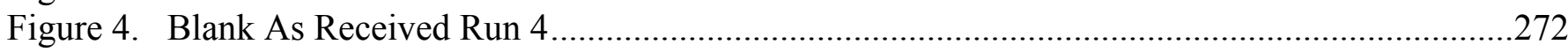

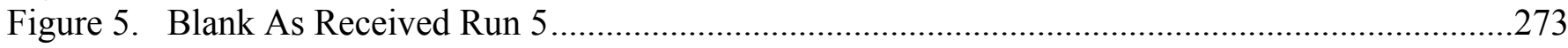

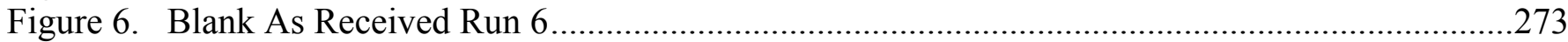

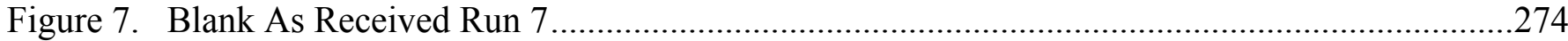

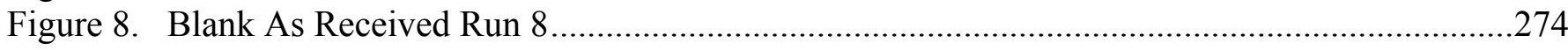

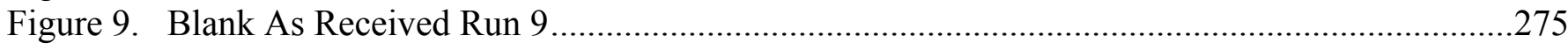

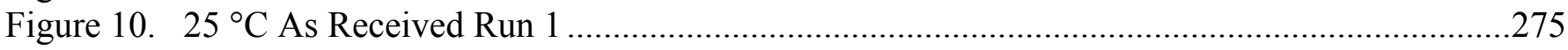

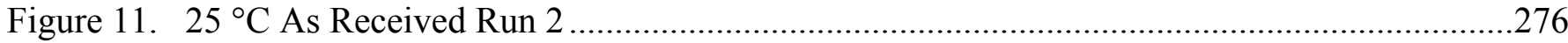

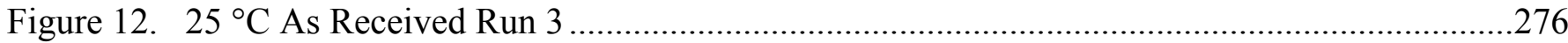

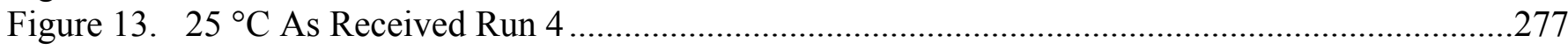

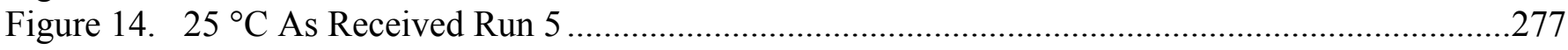

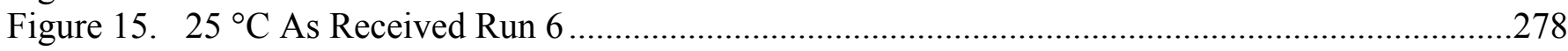

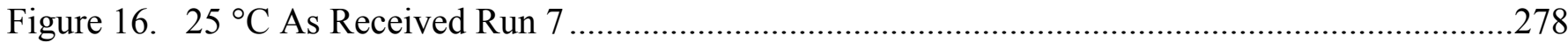

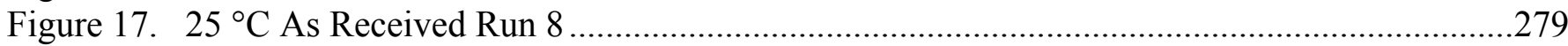

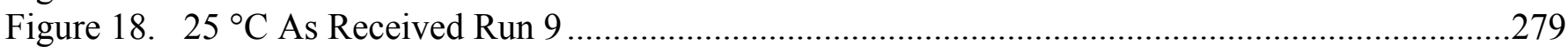

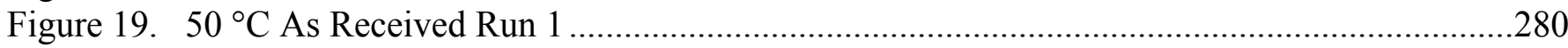

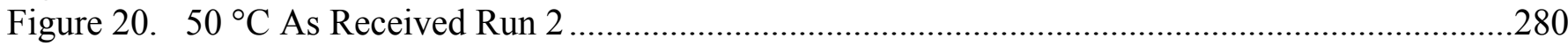

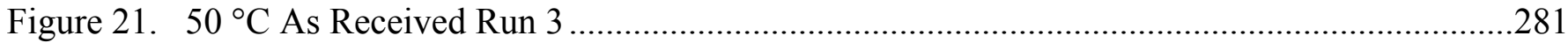

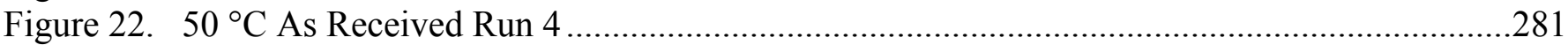

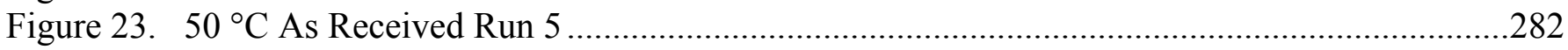

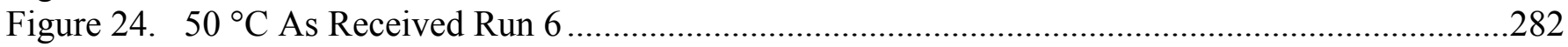

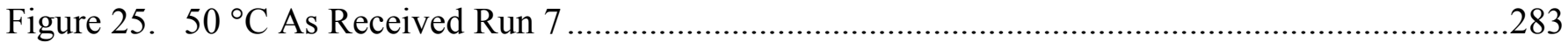

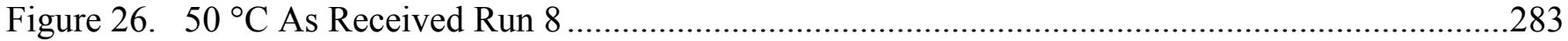

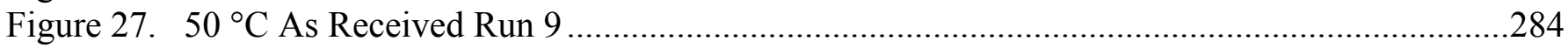

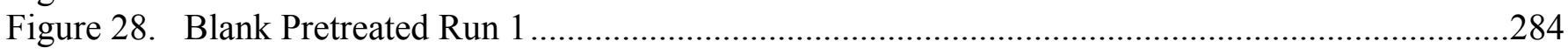

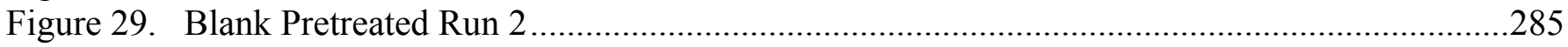

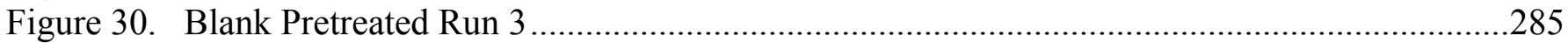

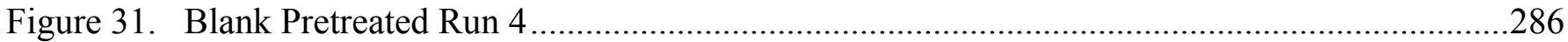

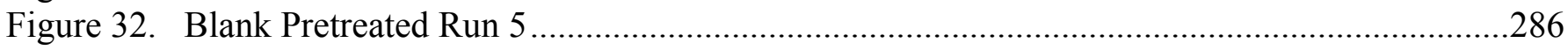

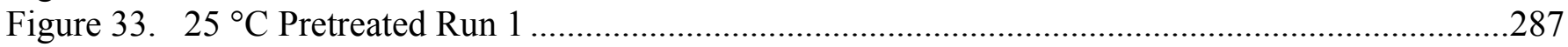

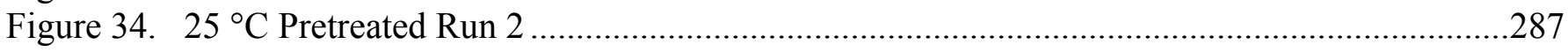

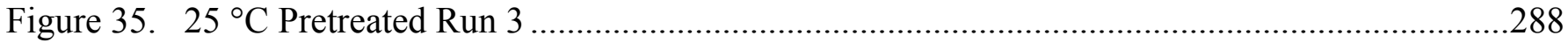

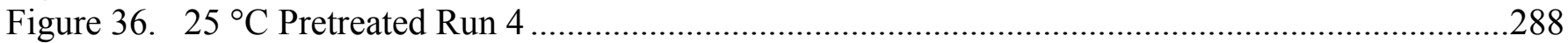

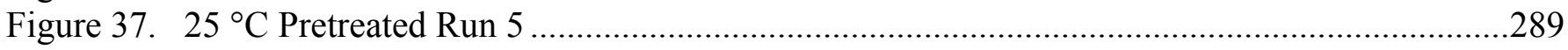

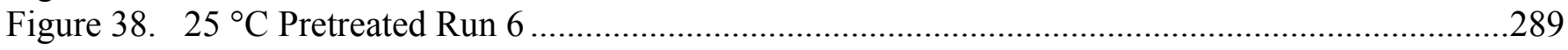

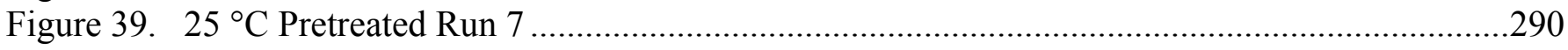




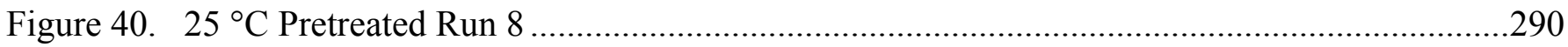

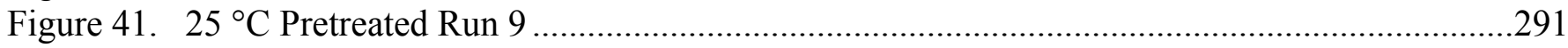

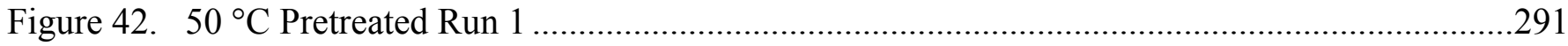

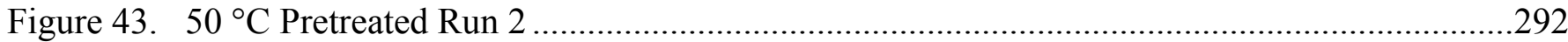

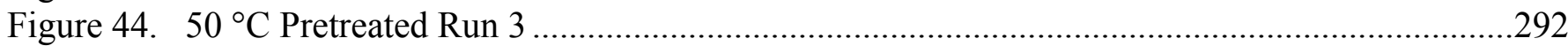

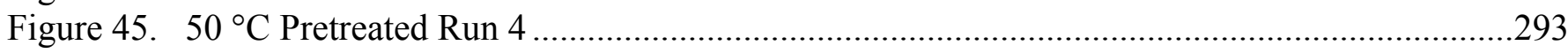

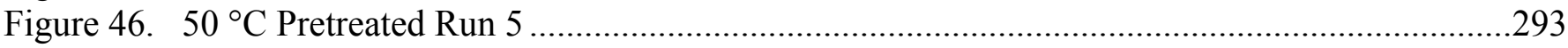

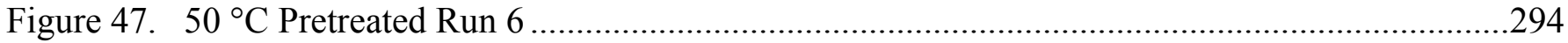

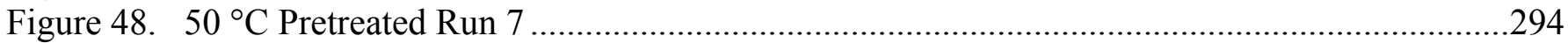

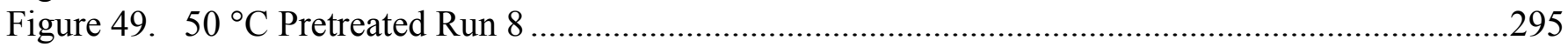

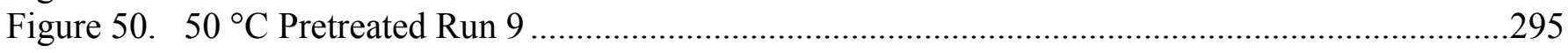


\begin{tabular}{|lc}
\hline APPENDIX G & WSRC-TR-2000-00352
\end{tabular}

ENVELOPE B RHEOGRAMS

SRT-RPP-2000-00026

Page 271 of 514

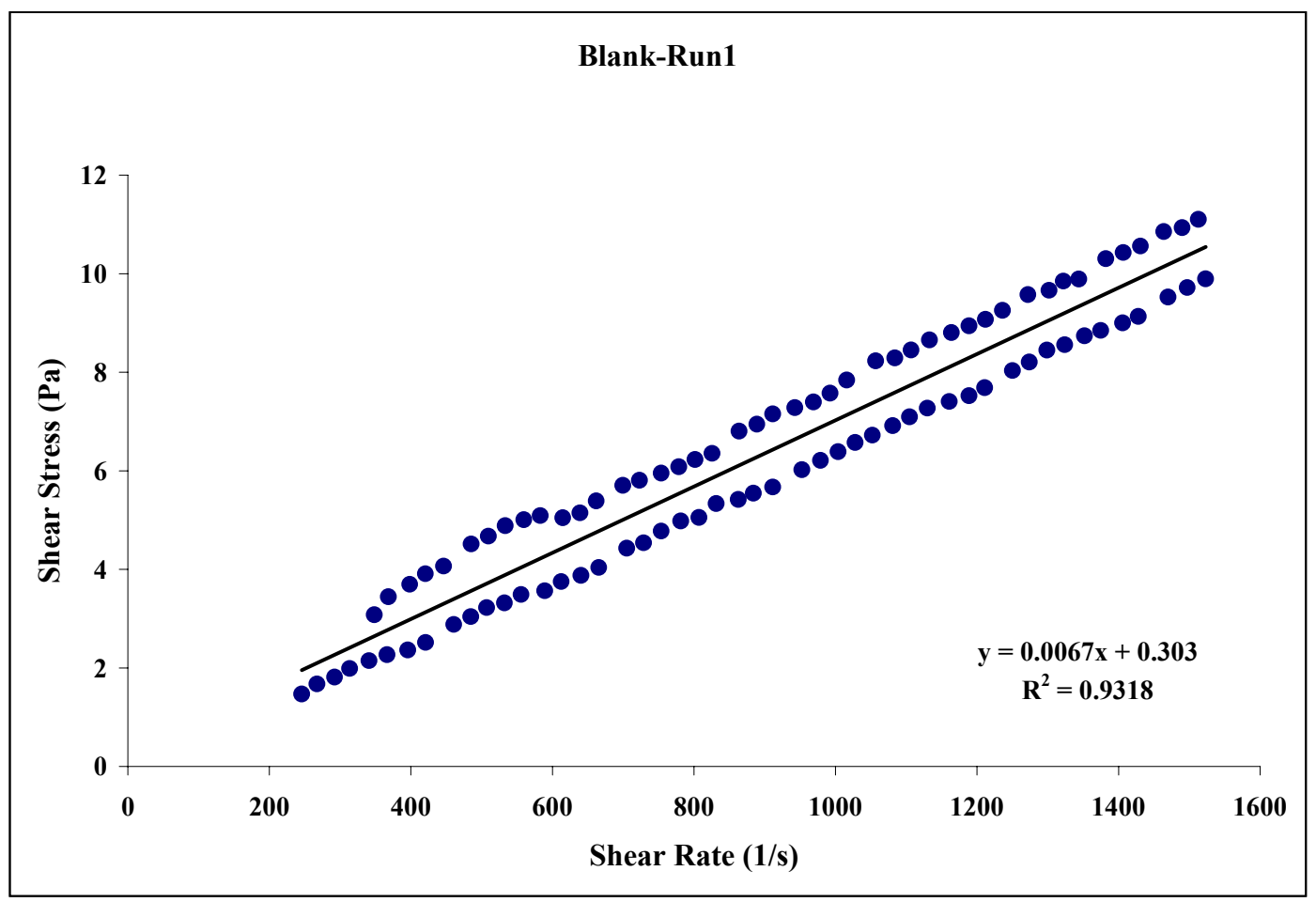

Figure 1. Blank As Received Run 1

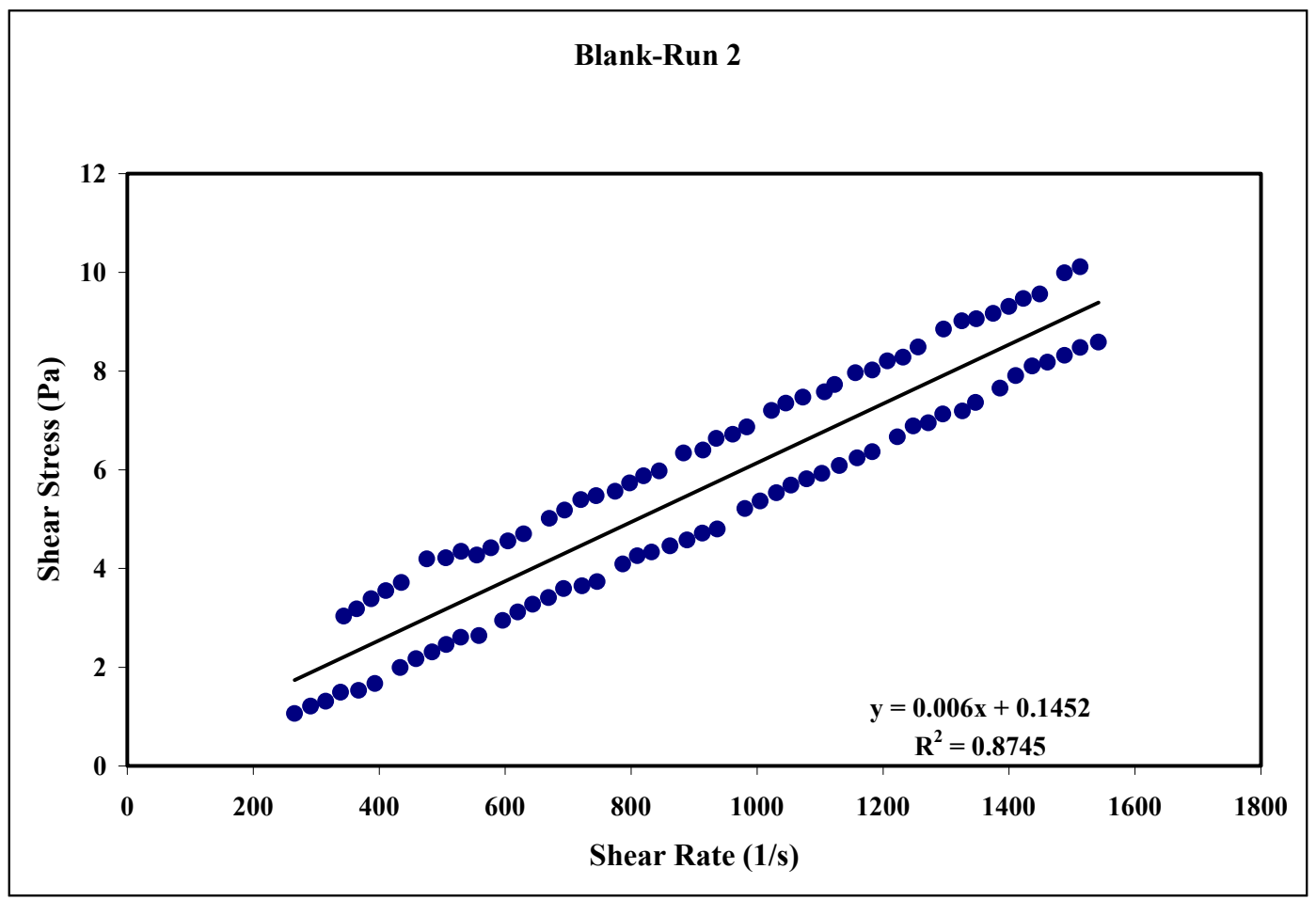

Figure 2. Blank As Received Run 2 
$\begin{array}{ll}\text { APPENDIX G } & \text { WSRC-TR-2000-00352 }\end{array}$

ENVELOPE B RHEOGRAMS

SRT-RPP-2000-00026

Page 272 of 514

\section{Blank-Run 3}

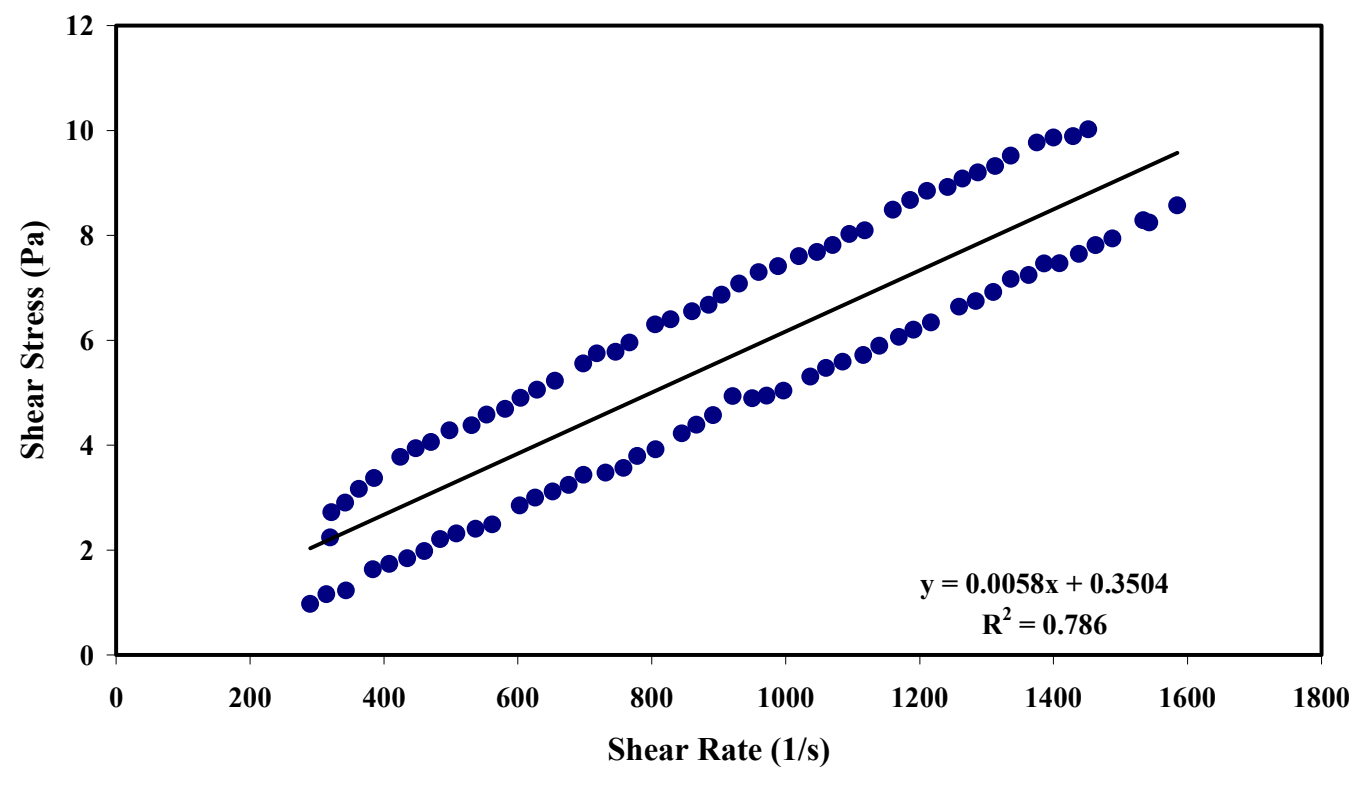

Figure 3. Blank As Received Run 3

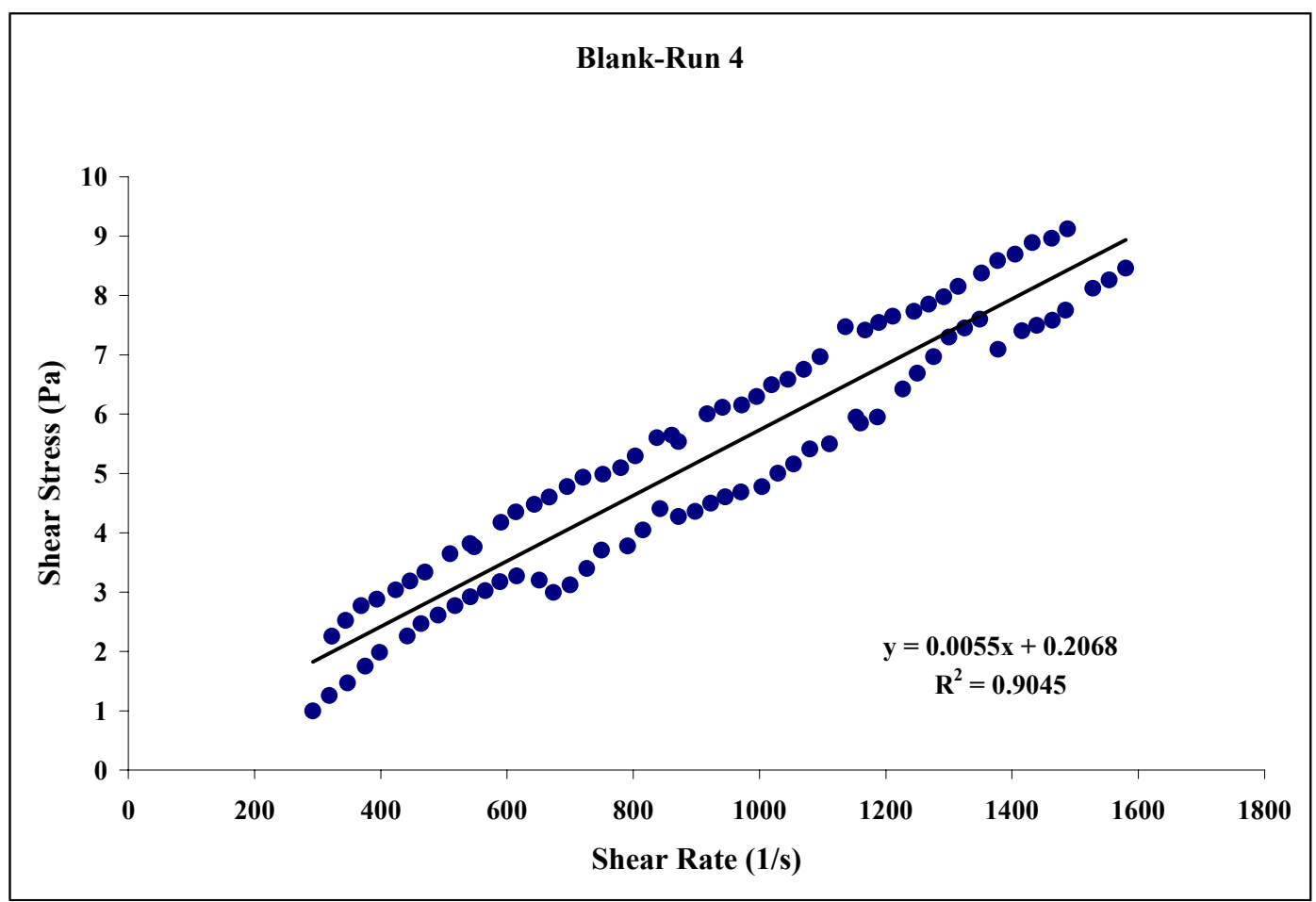

Figure 4. Blank As Received Run 4 
$\begin{array}{ll}\text { APPENDIX G } & \text { WSRC-TR-2000-00352 }\end{array}$

ENVELOPE B RHEOGRAMS

SRT-RPP-2000-00026

Page 273 of 514

\section{Blank-Run 5}

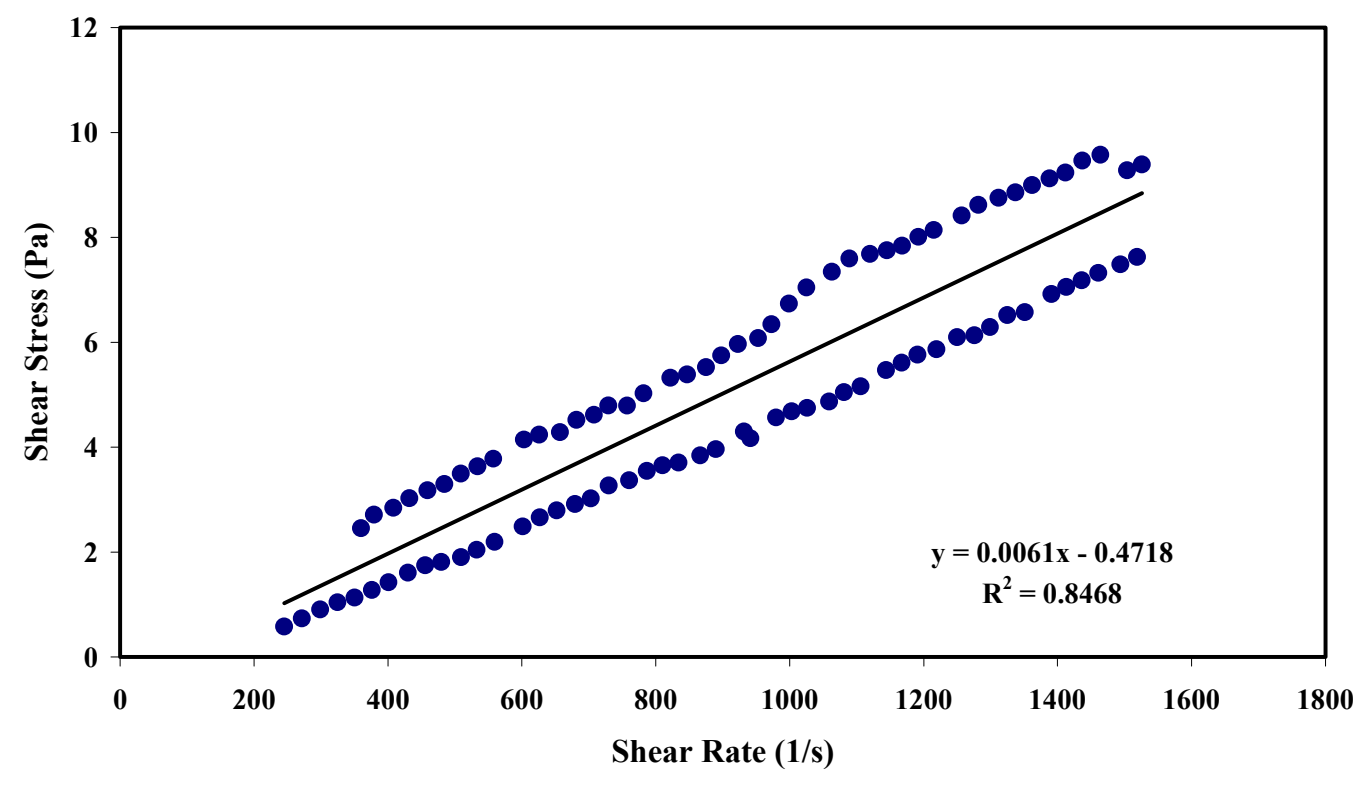

Figure 5. Blank As Received Run 5

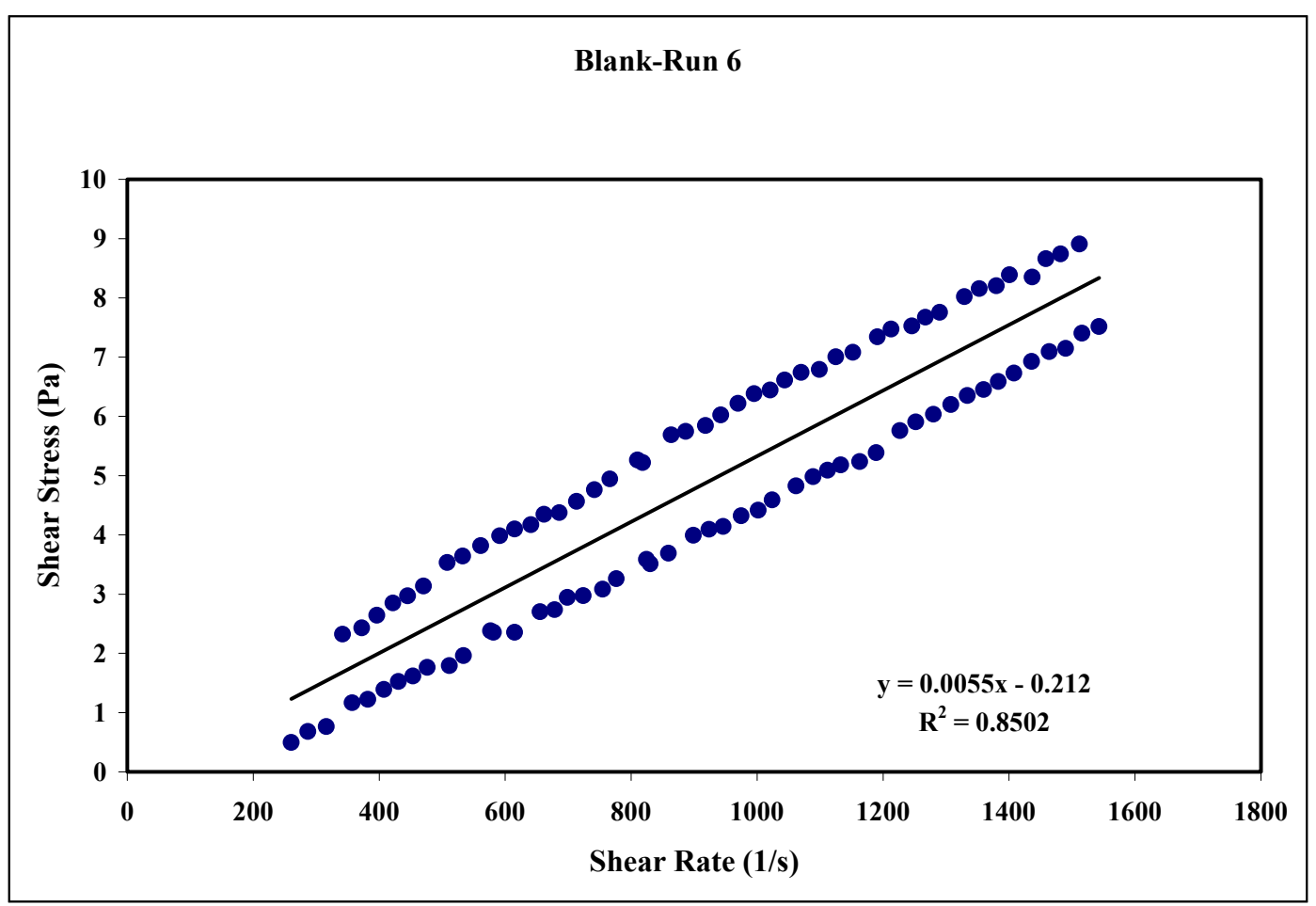

Figure 6. Blank As Received Run 6 
$\begin{array}{ll}\text { APPENDIX G } & \text { WSRC-TR-2000-00352 }\end{array}$

ENVELOPE B RHEOGRAMS

SRT-RPP-2000-00026

Page 274 of 514

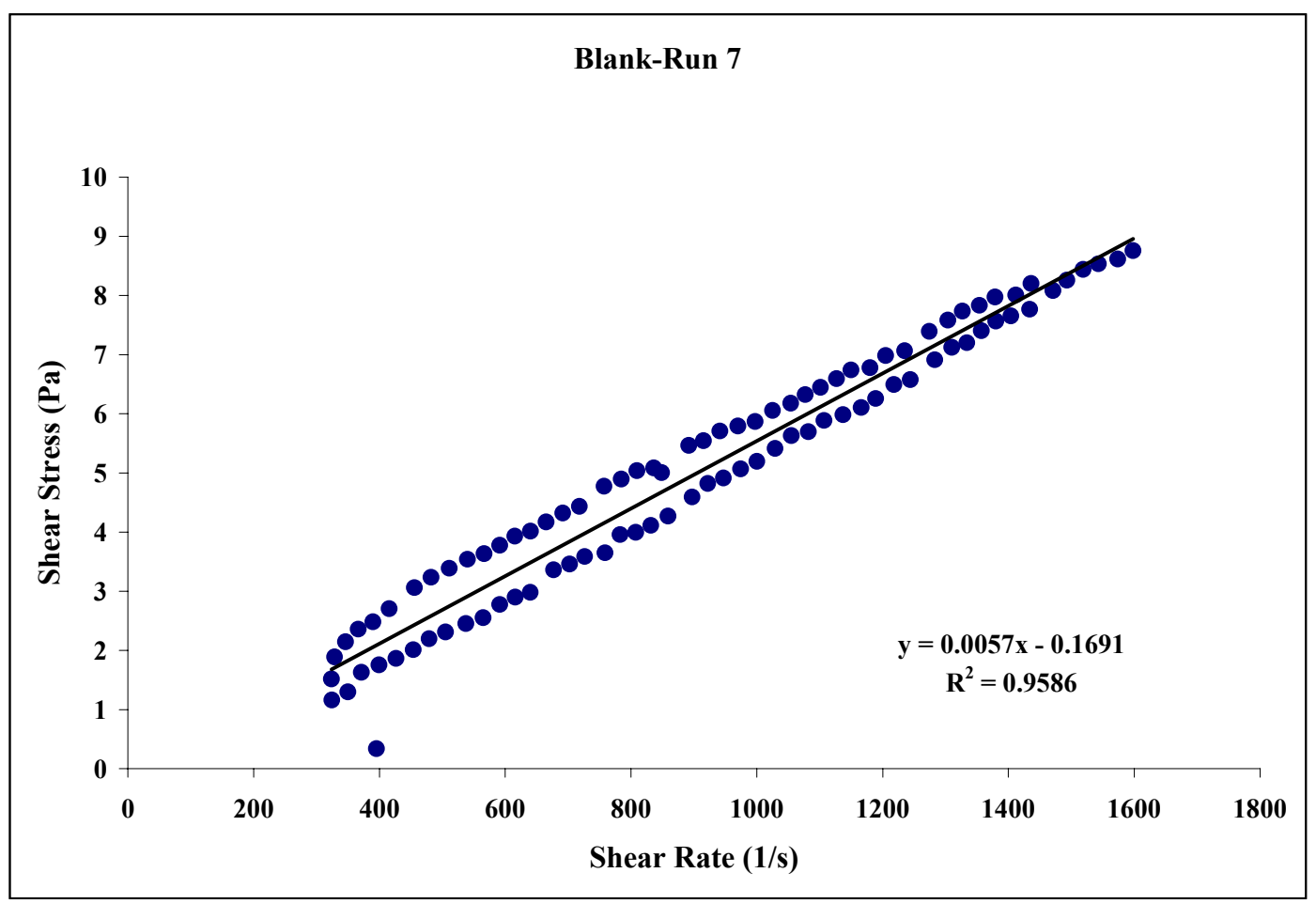

Figure 7. Blank As Received Run 7

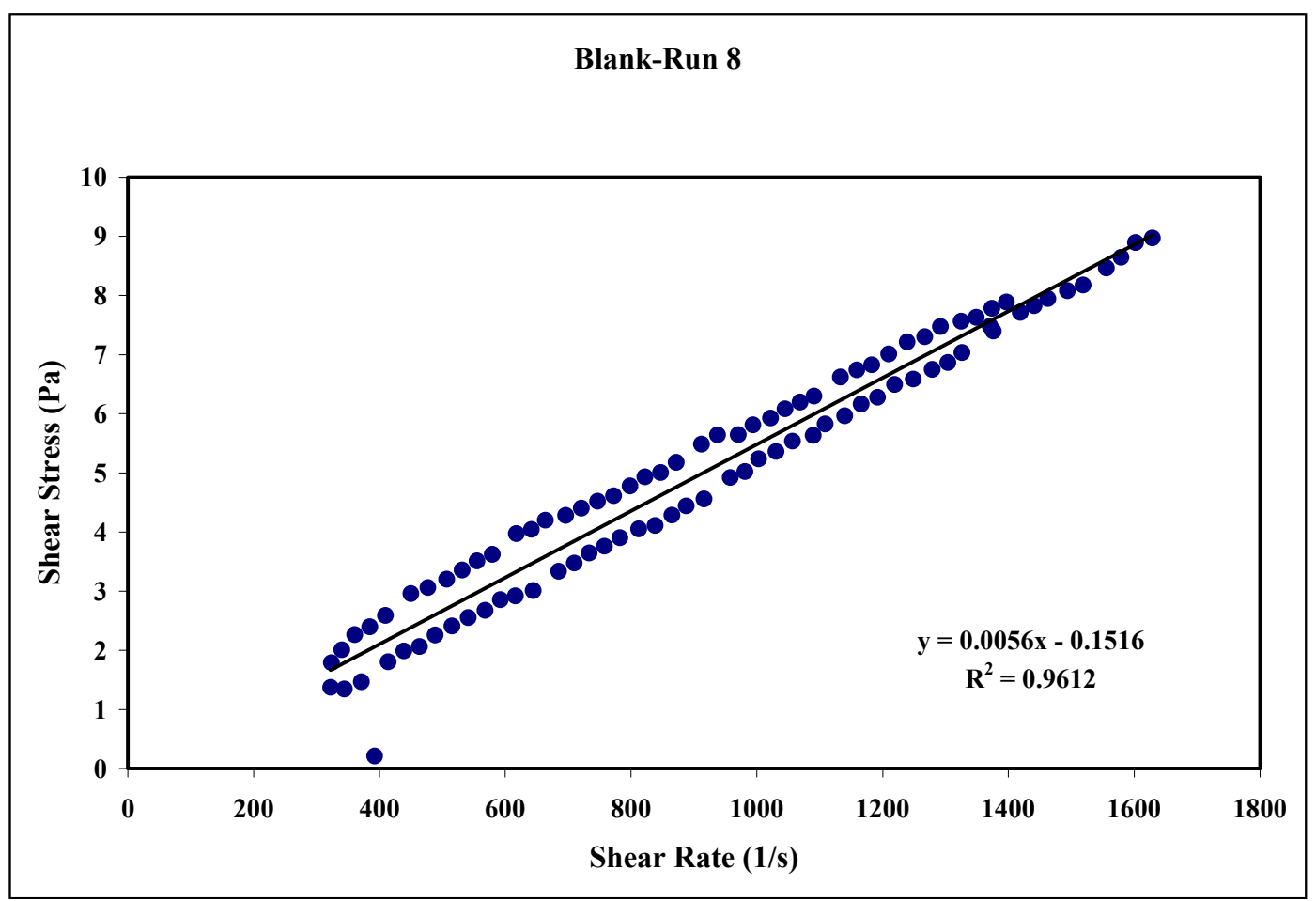

Figure 8. Blank As Received Run 8 
$\begin{array}{ll}\text { APPENDIX G } & \text { WSRC-TR-2000-00352 }\end{array}$

ENVELOPE B RHEOGRAMS

SRT-RPP-2000-00026

Page 275 of 514

\section{Blank-Run 9}

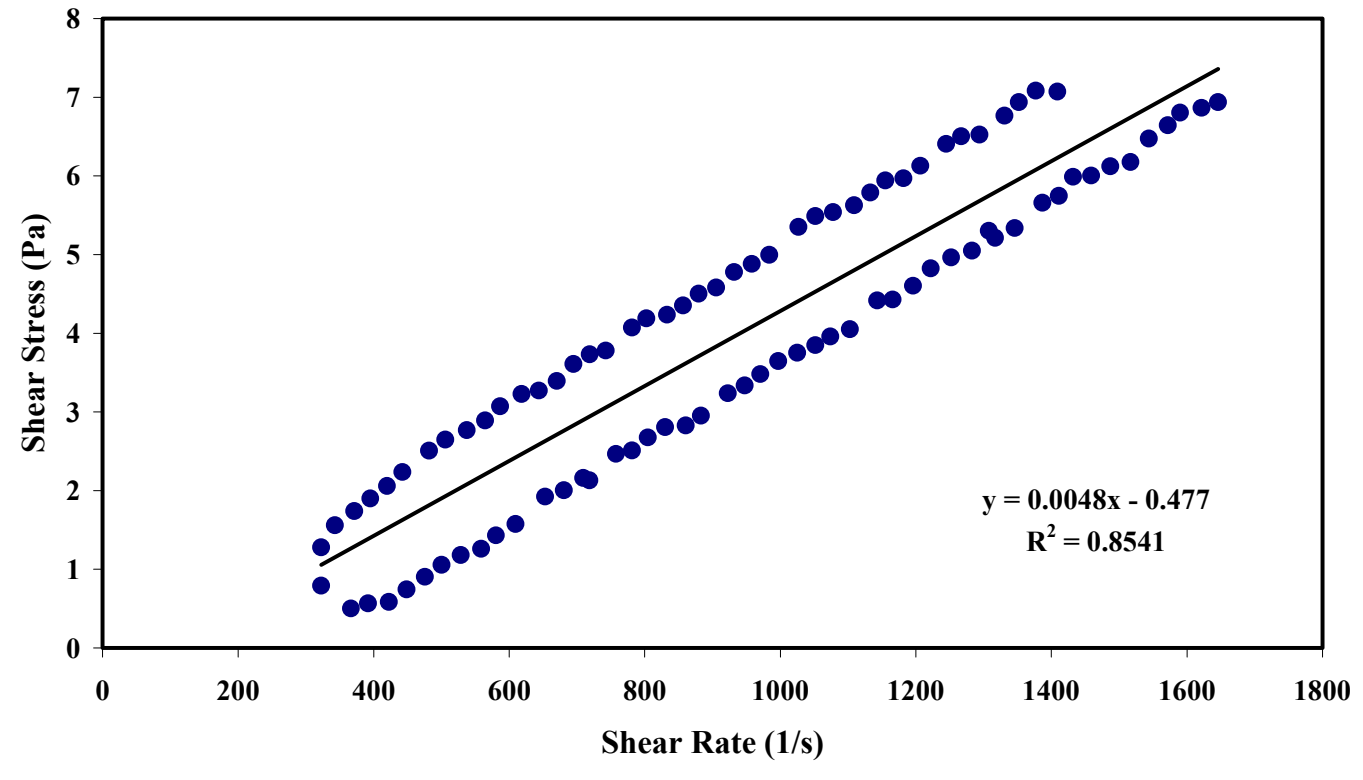

Figure 9. Blank As Received Run 9

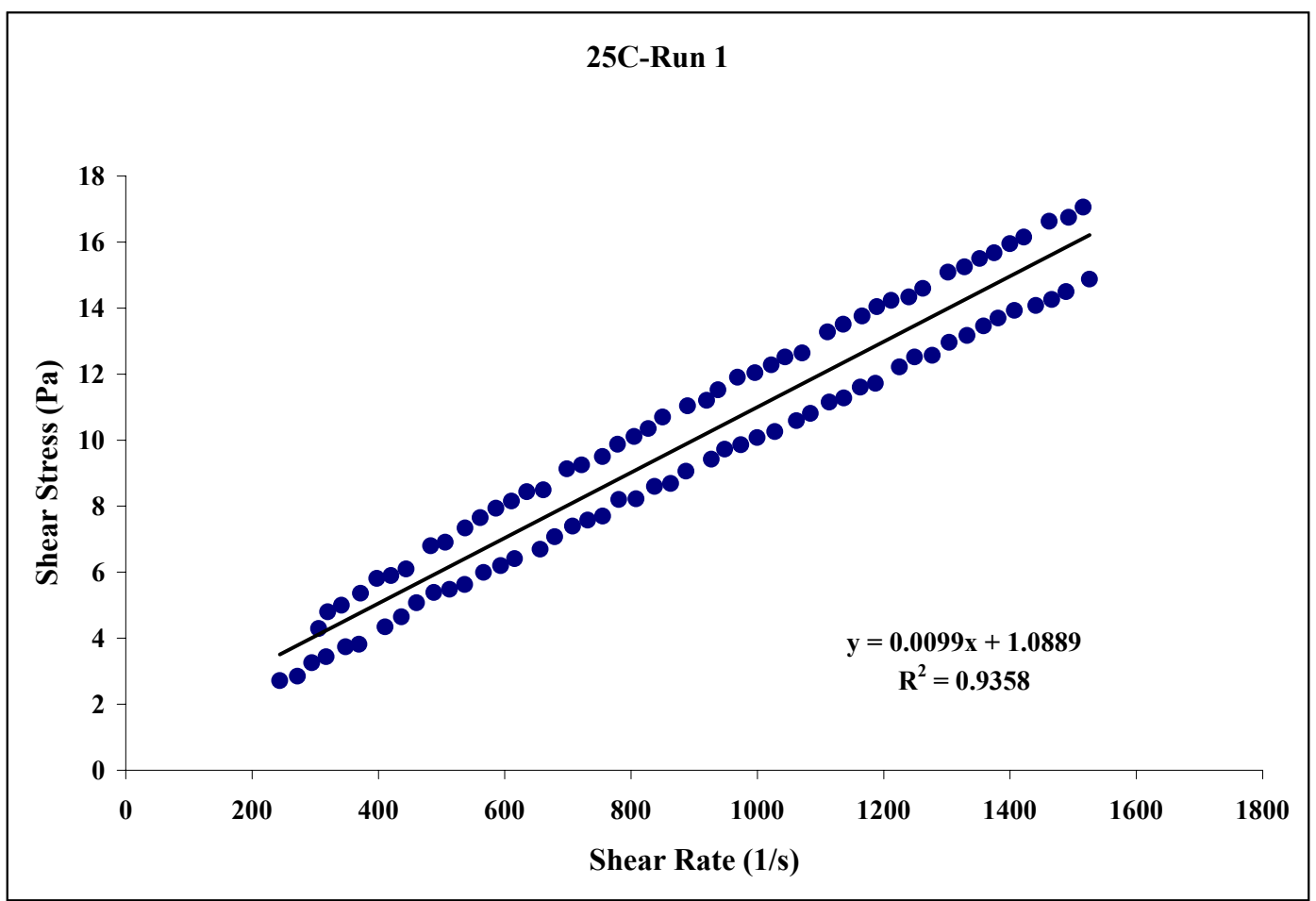

Figure 10. $25^{\circ} \mathrm{C}$ As Received Run 1 
$\begin{array}{ll}\text { APPENDIX G } & \text { WSRC-TR-2000-00352 }\end{array}$

ENVELOPE B RHEOGRAMS

SRT-RPP-2000-00026

Page 276 of 514

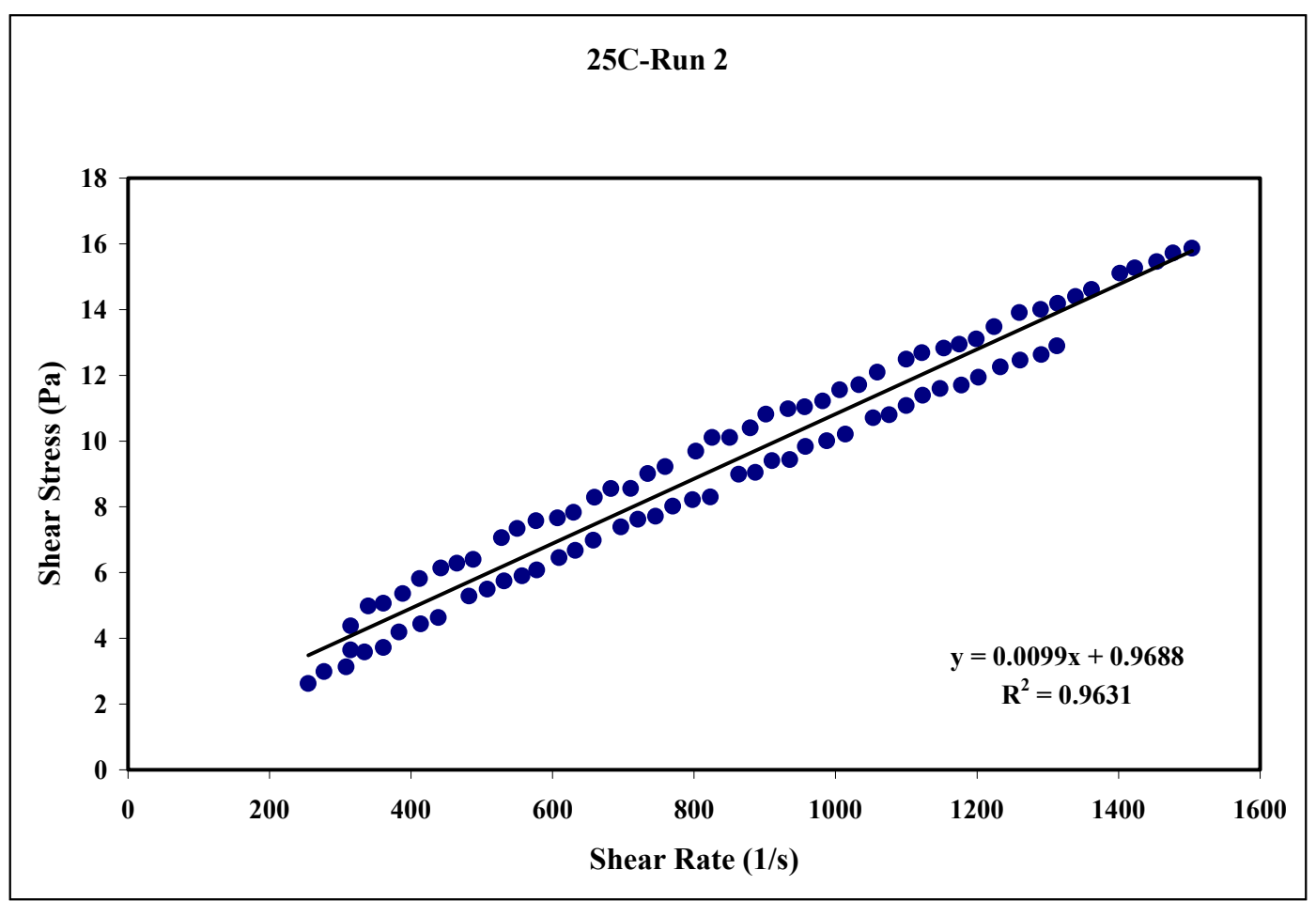

Figure 11. $25^{\circ} \mathrm{C}$ As Received Run 2

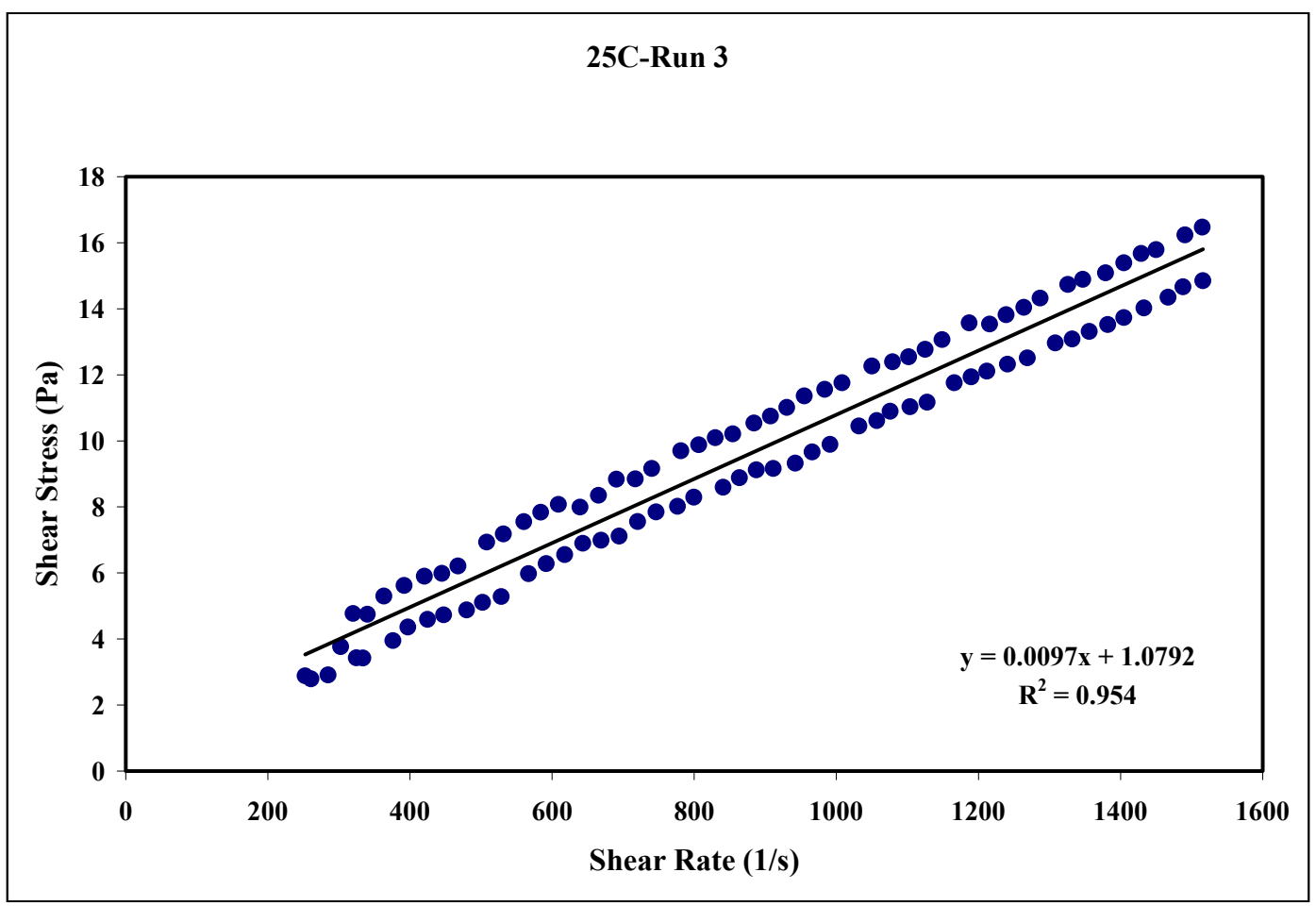

Figure 12. $25^{\circ} \mathrm{C}$ As Received Run 3 


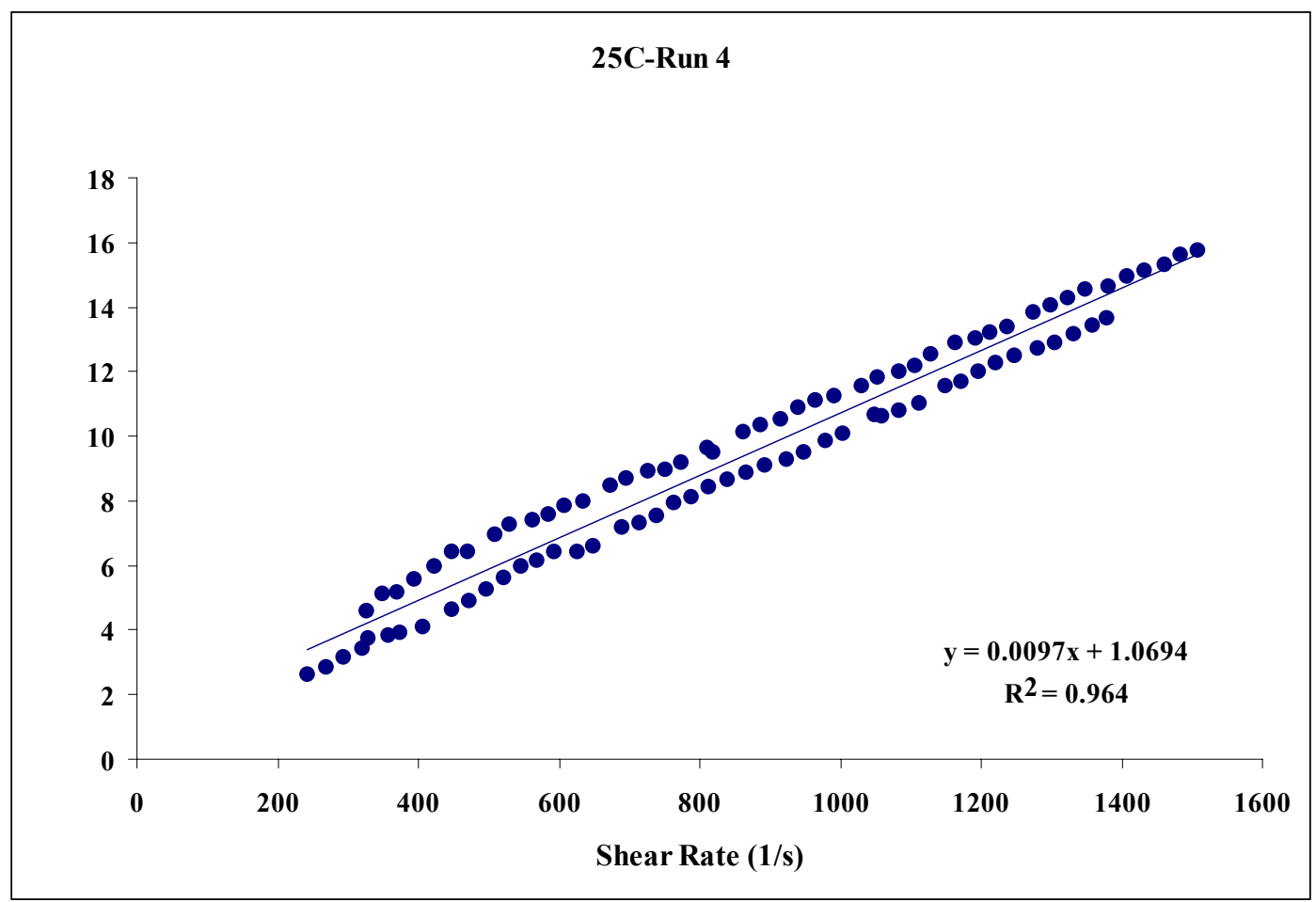

Figure 13. $25^{\circ} \mathrm{C}$ As Received Run 4

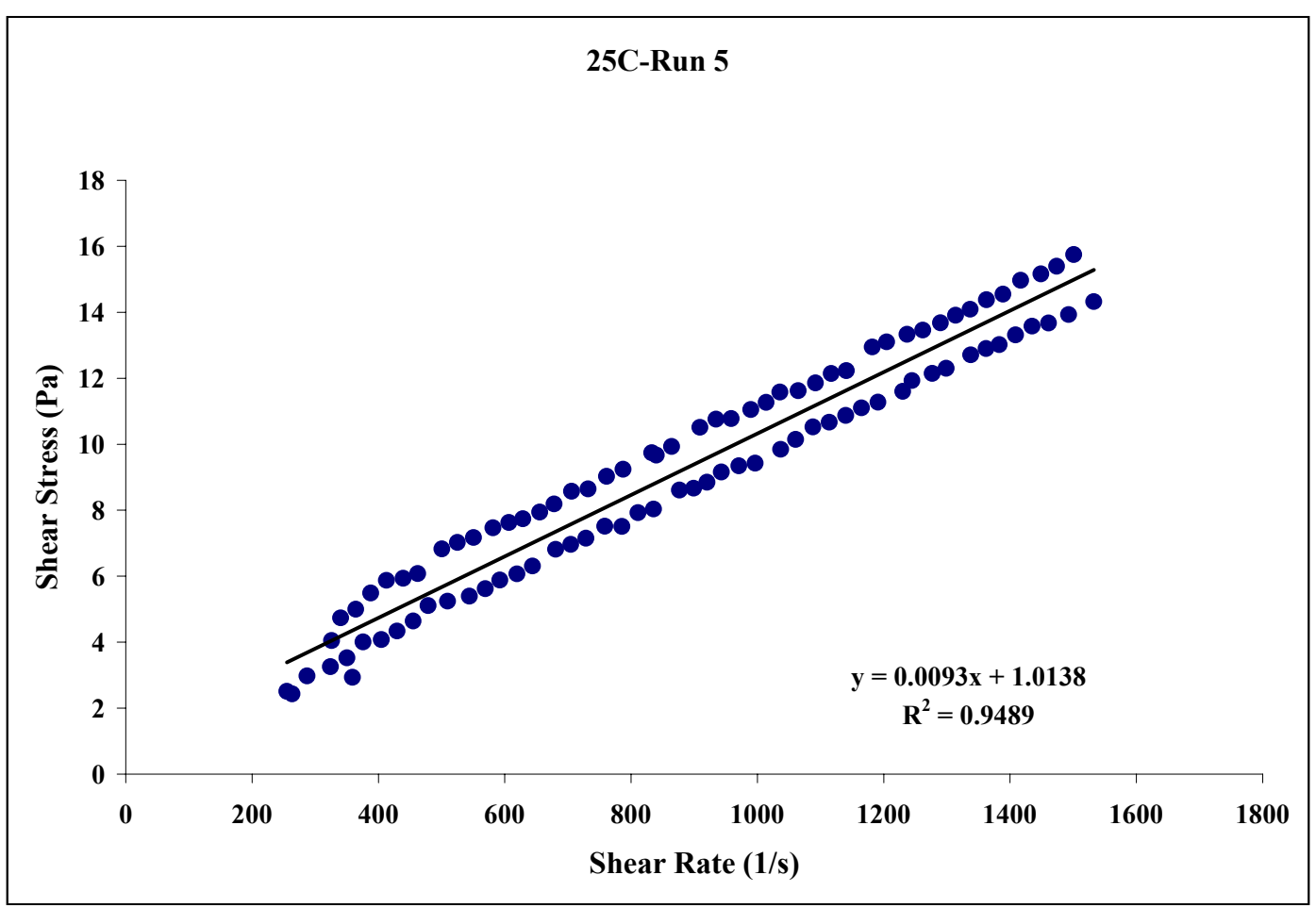

Figure 14. $25^{\circ} \mathrm{C}$ As Received Run 5 
$\begin{array}{ll}\text { APPENDIX G } & \text { WSRC-TR-2000-00352 }\end{array}$

ENVELOPE B RHEOGRAMS

SRT-RPP-2000-00026

Page 278 of 514

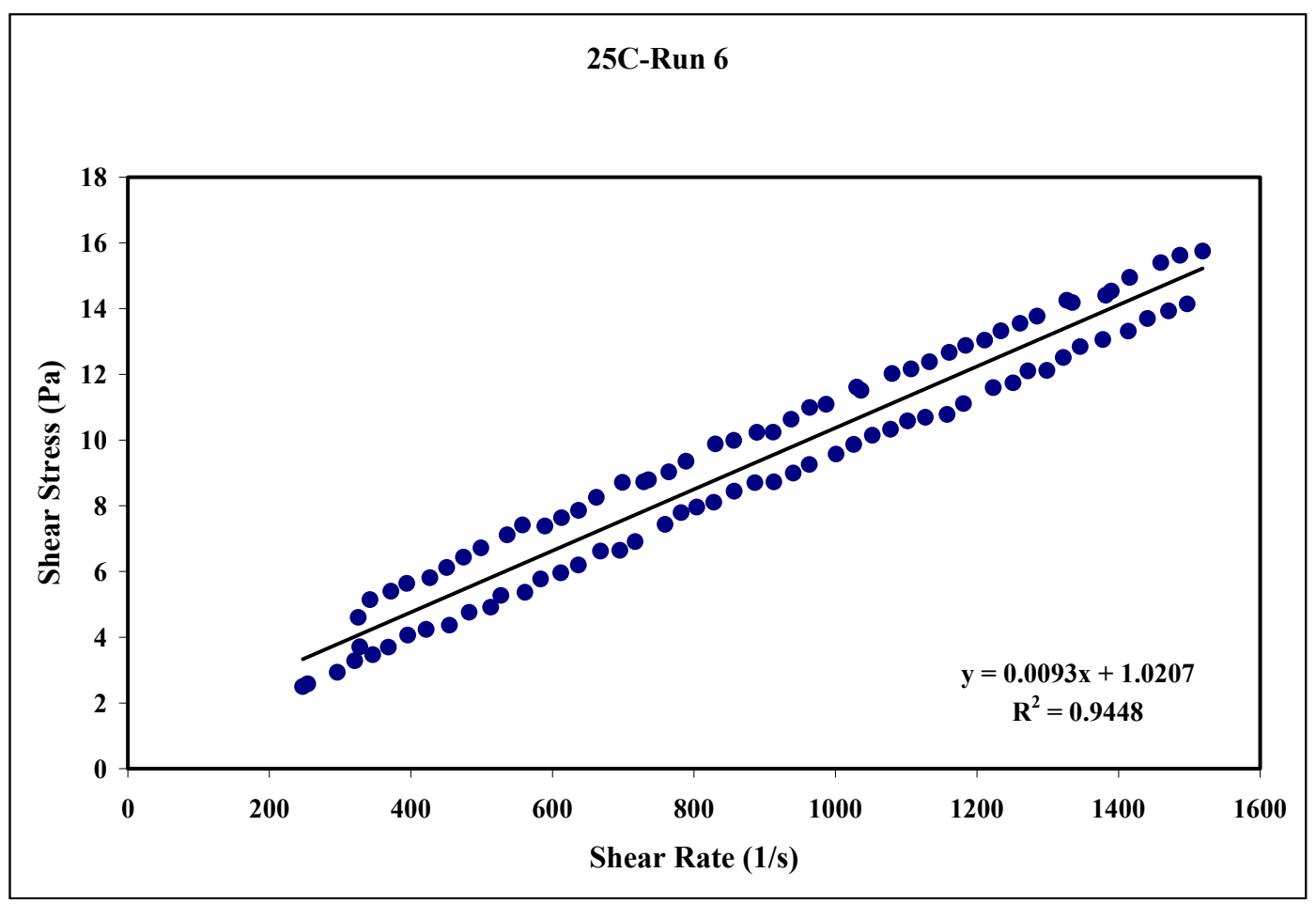

Figure 15. $25^{\circ} \mathrm{C}$ As Received Run 6

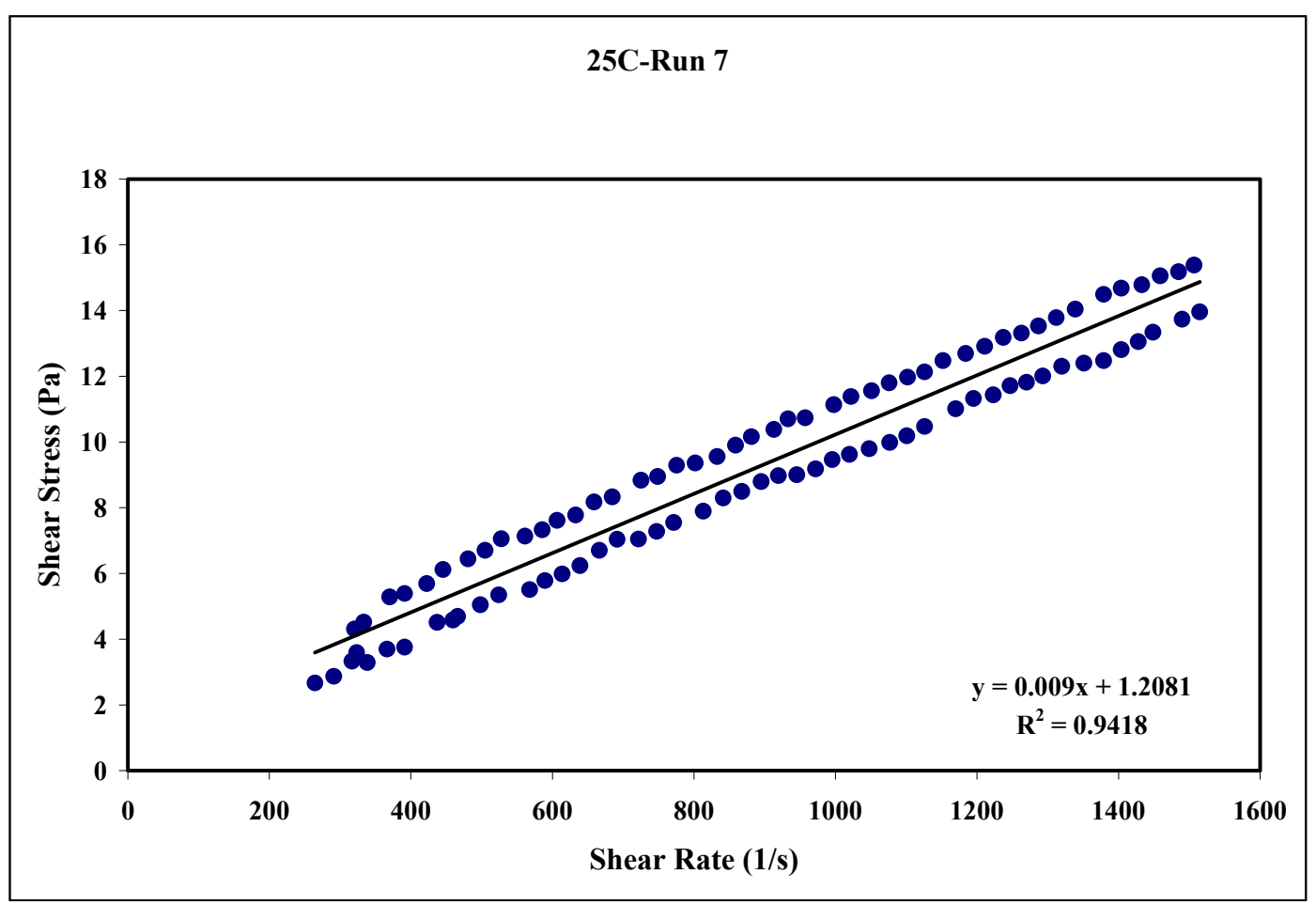

Figure 16. $25^{\circ} \mathrm{C}$ As Received Run 7 
$\begin{array}{ll}\text { APPENDIX G } & \text { WSRC-TR-2000-00352 }\end{array}$

ENVELOPE B RHEOGRAMS

SRT-RPP-2000-00026

Page 279 of 514

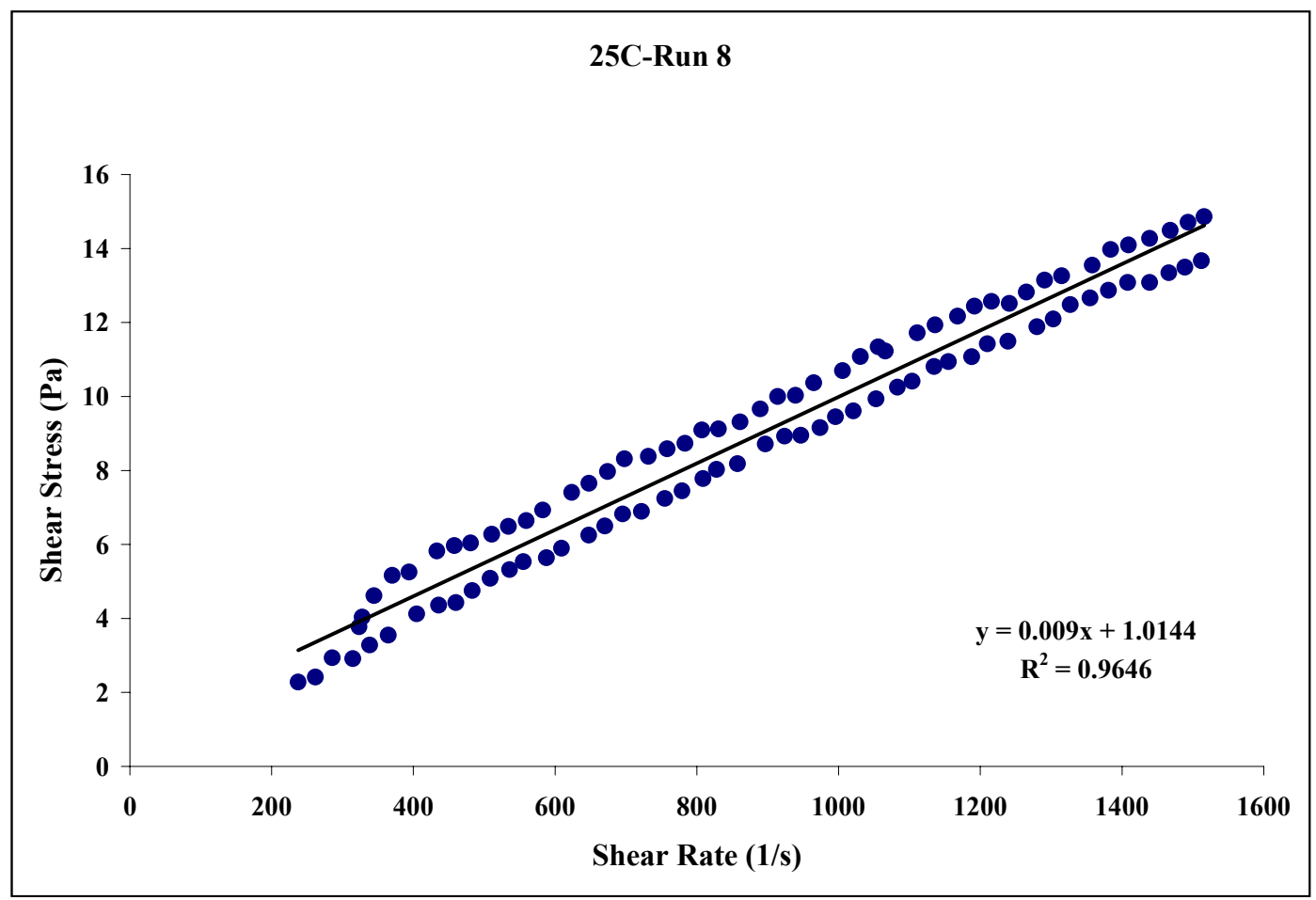

Figure 17. $25^{\circ} \mathrm{C}$ As Received Run 8

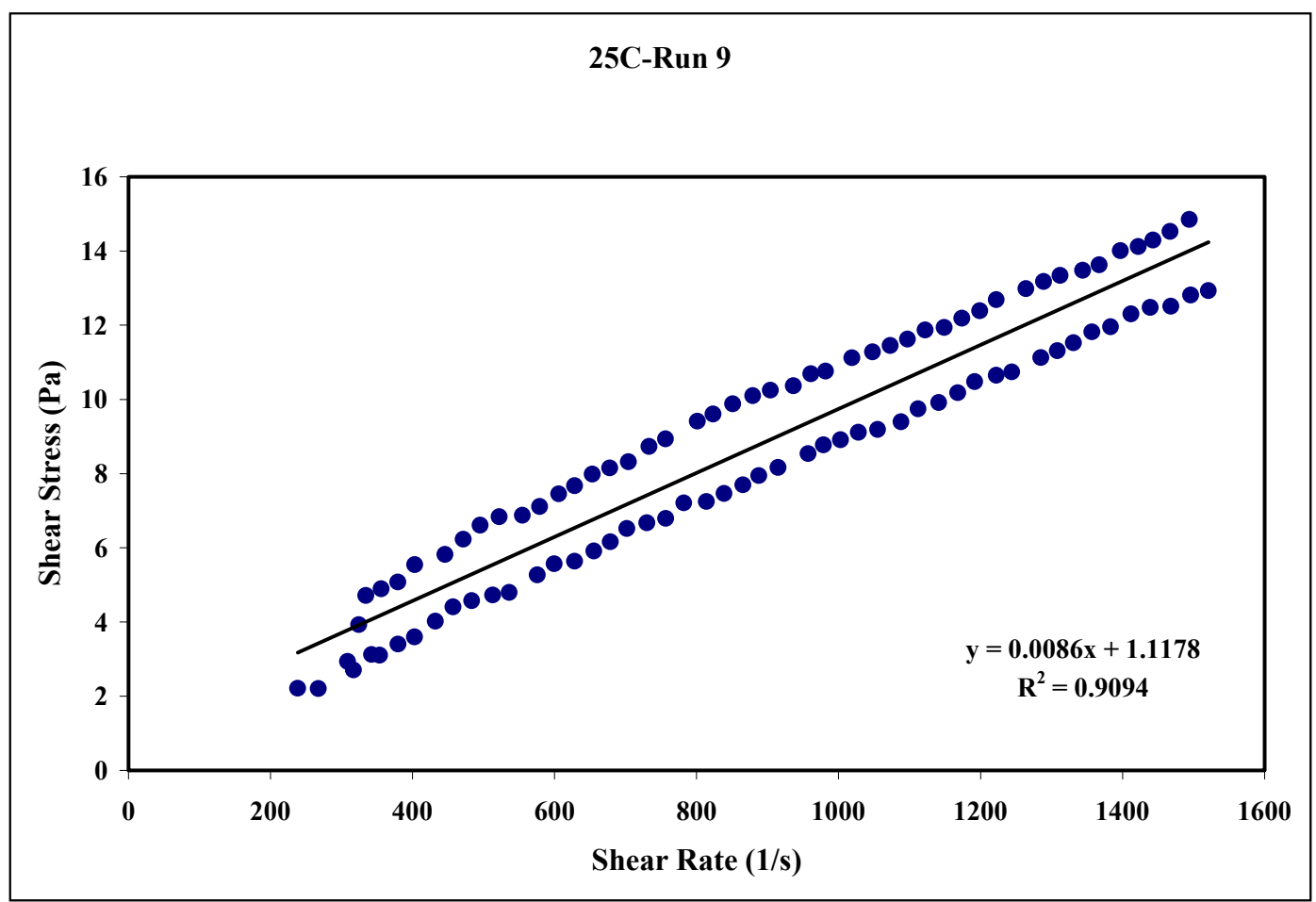

Figure 18. $25^{\circ} \mathrm{C}$ As Received Run 9 
$\begin{array}{ll}\text { APPENDIX G } & \text { WSRC-TR-2000-00352 }\end{array}$

ENVELOPE B RHEOGRAMS

SRT-RPP-2000-00026

Page 280 of 514

50C-Run 1

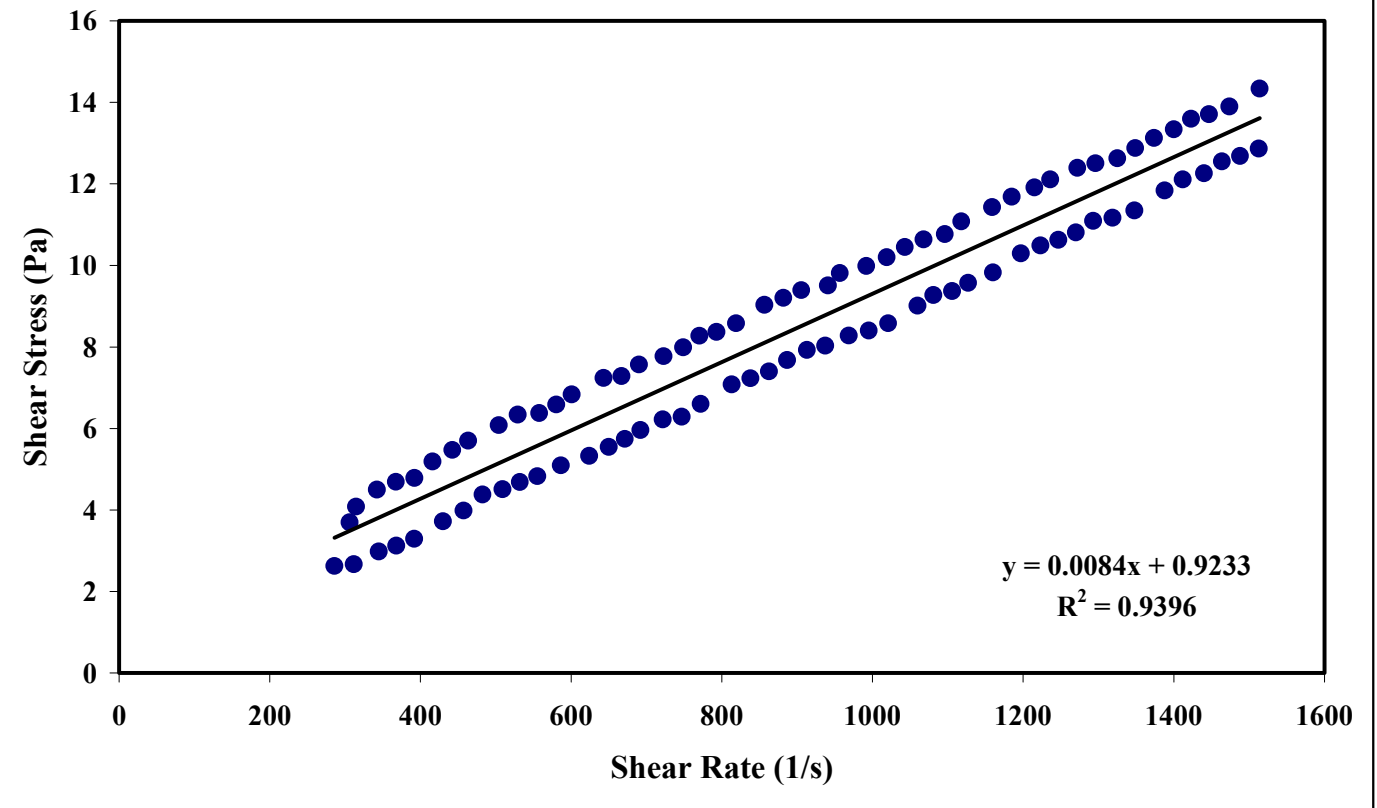

Figure 19. $50^{\circ} \mathrm{C}$ As Received Run 1

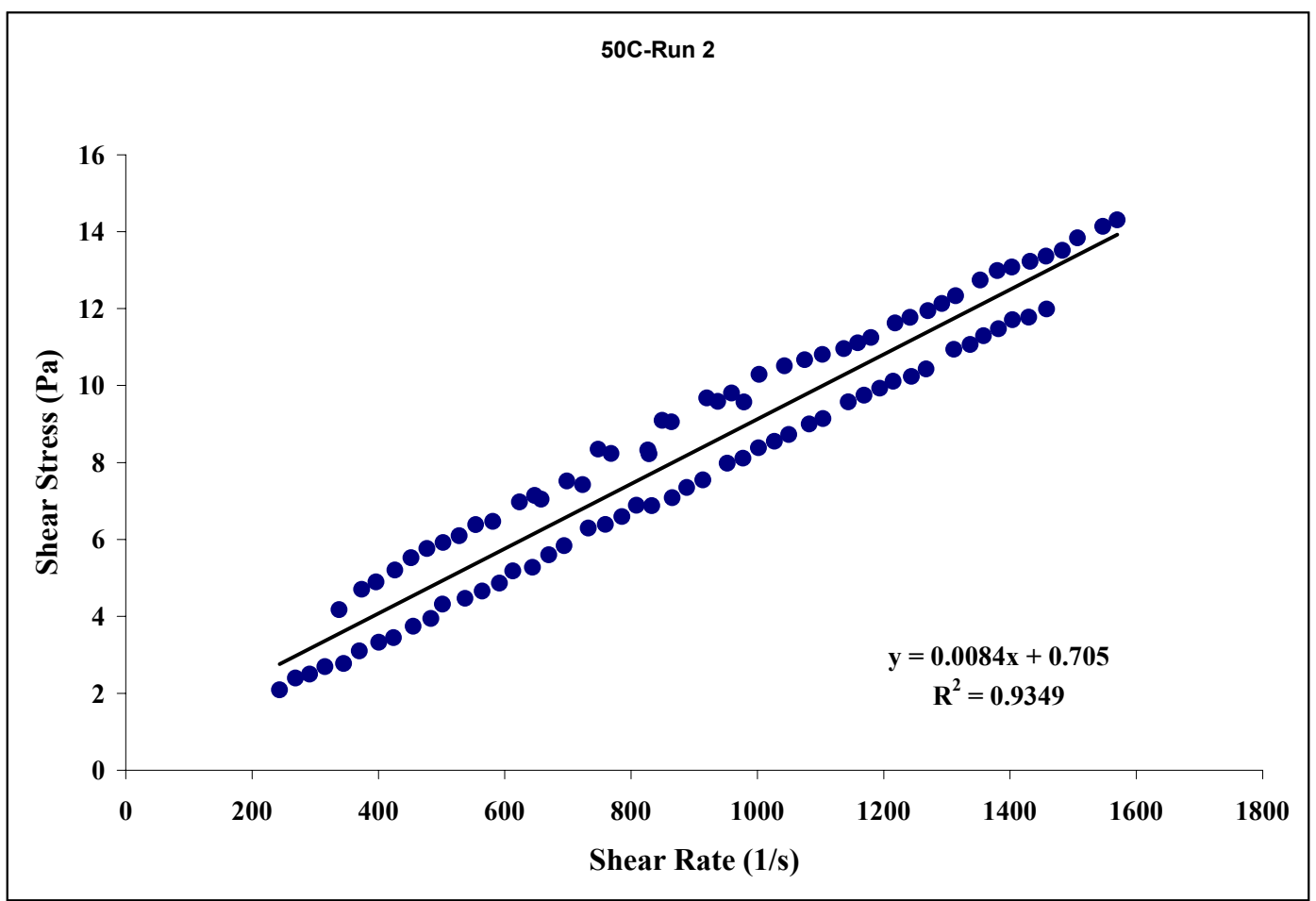

Figure 20. $50^{\circ} \mathrm{C}$ As Received Run 2 
$\begin{array}{ll}\text { APPENDIX G } & \text { WSRC-TR-2000-00352 }\end{array}$

ENVELOPE B RHEOGRAMS

SRT-RPP-2000-00026

Page 281 of 514

50C-Run 3

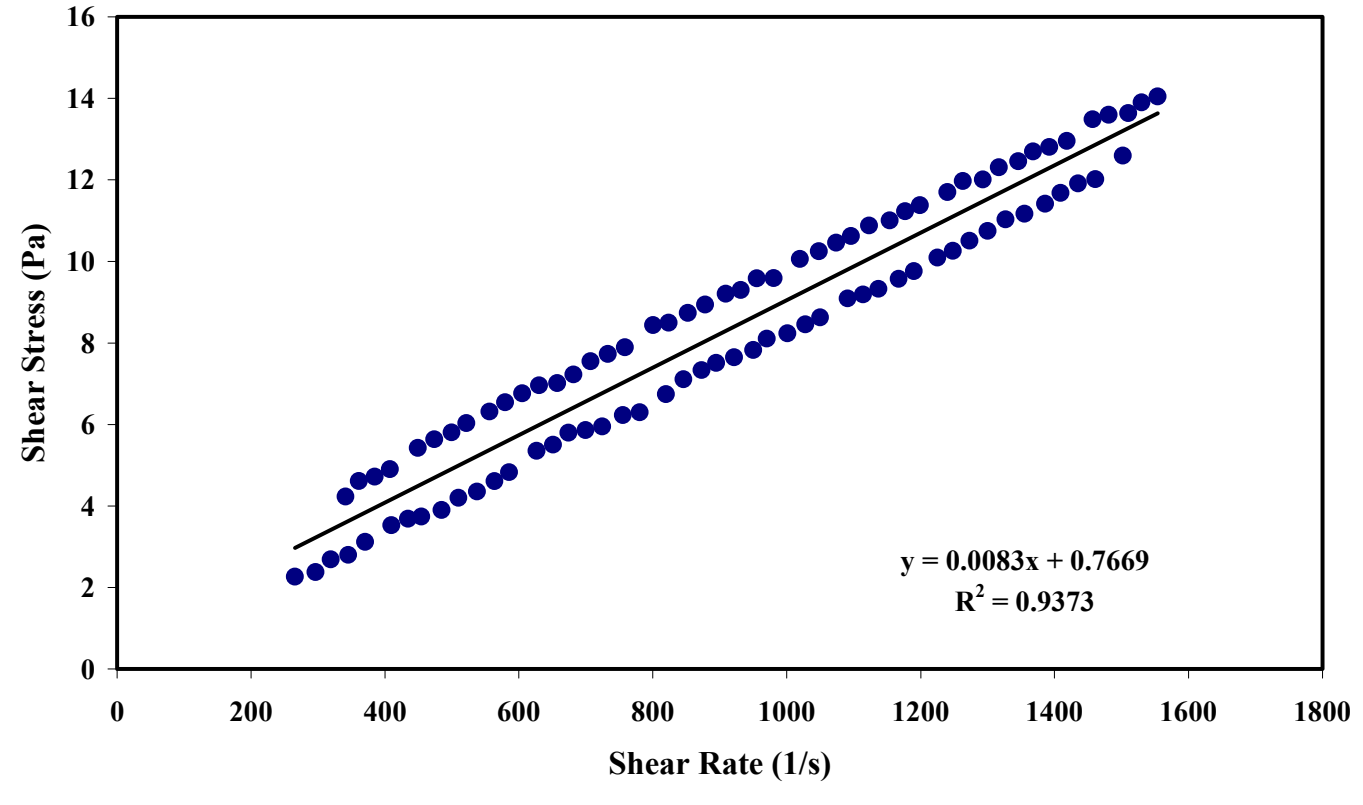

Figure 21. $50{ }^{\circ} \mathrm{C}$ As Received Run 3

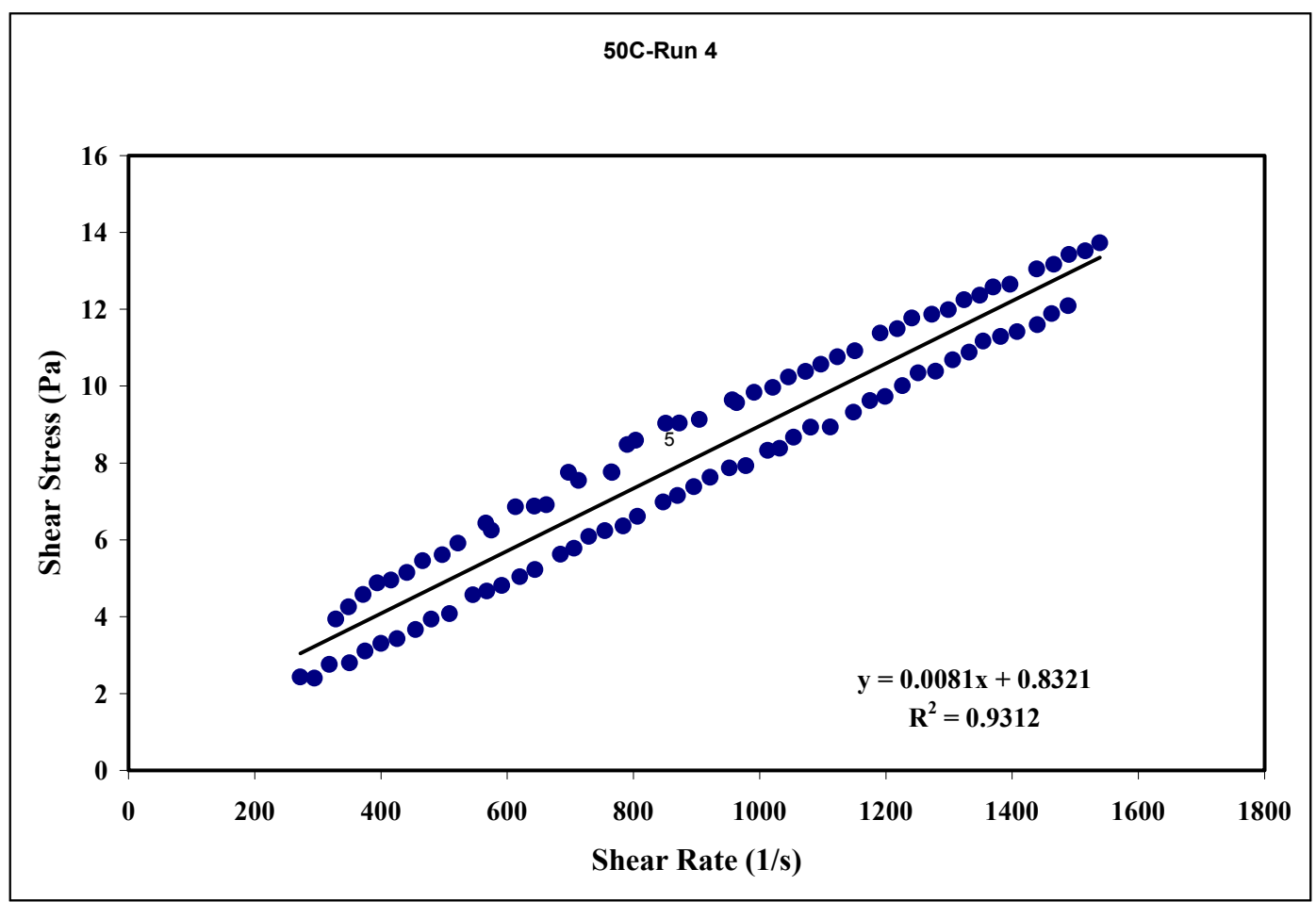

Figure 22. $50^{\circ} \mathrm{C}$ As Received Run 4 
$\begin{array}{ll}\text { APPENDIX G } & \text { WSRC-TR-2000-00352 }\end{array}$

ENVELOPE B RHEOGRAMS

SRT-RPP-2000-00026

Page 282 of 514

50C-Run 5

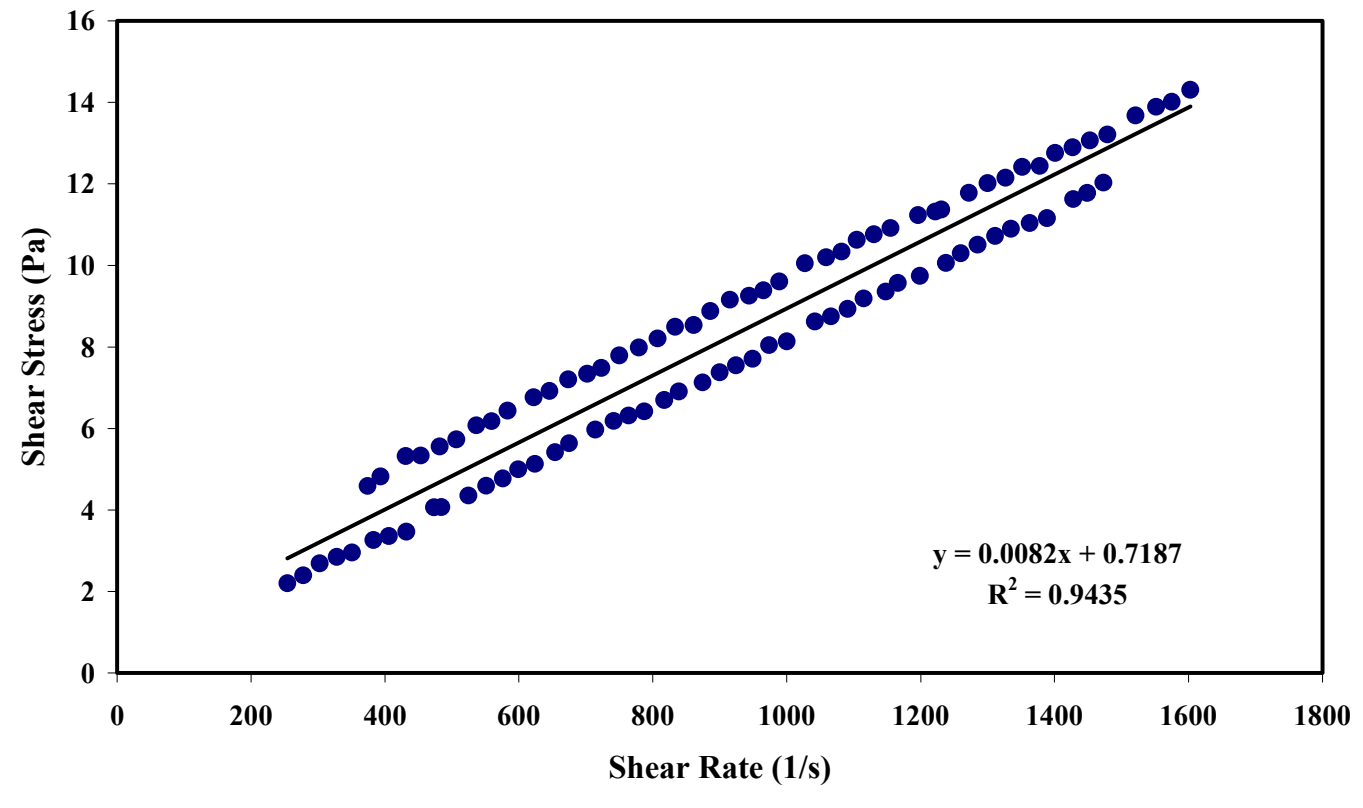

Figure 23. $50^{\circ} \mathrm{C}$ As Received Run 5

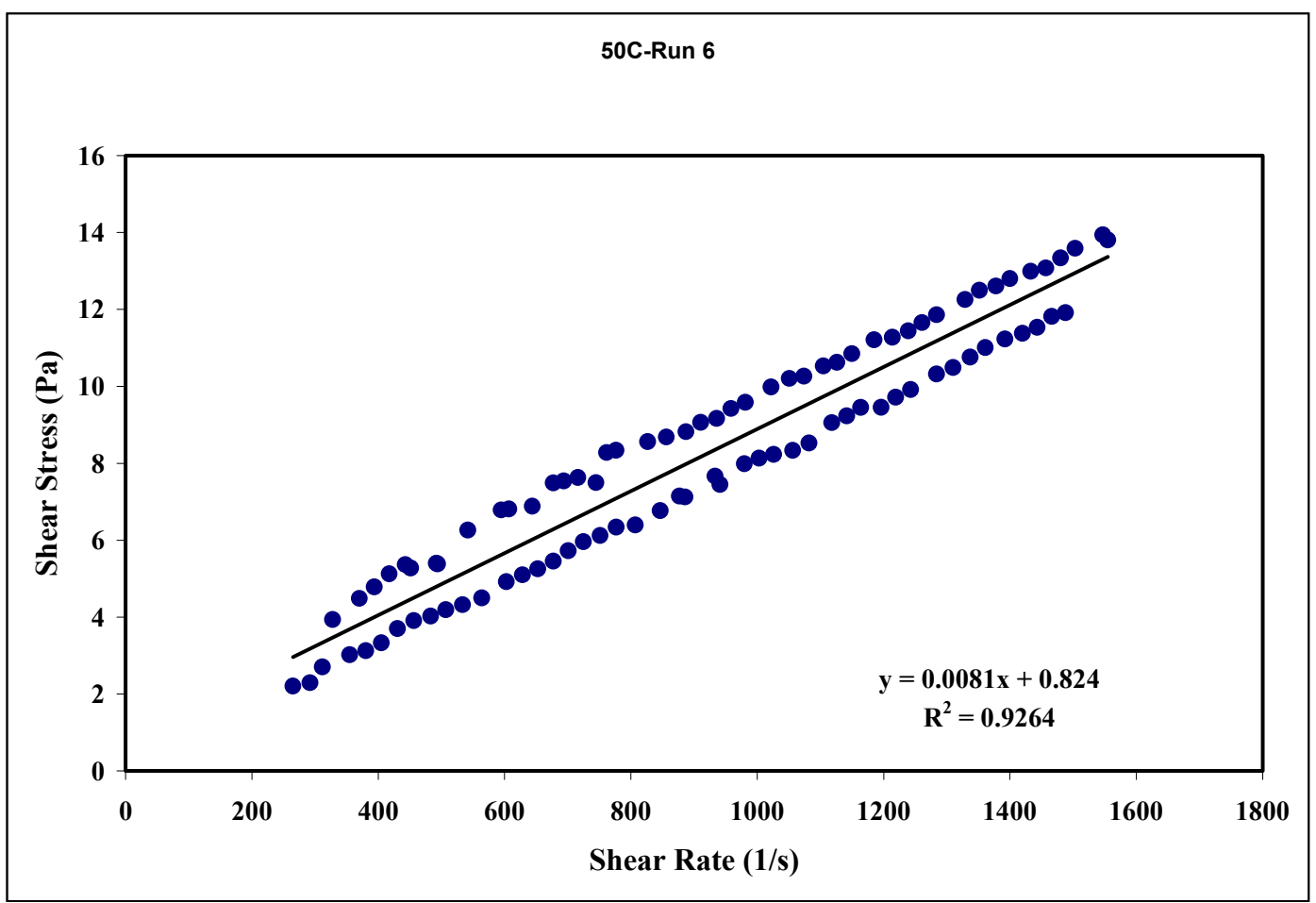

Figure 24. $50{ }^{\circ} \mathrm{C}$ As Received Run 6 
$\begin{array}{ll}\text { APPENDIX G } & \text { WSRC-TR-2000-00352 }\end{array}$

ENVELOPE B RHEOGRAMS

SRT-RPP-2000-00026

Page 283 of 514

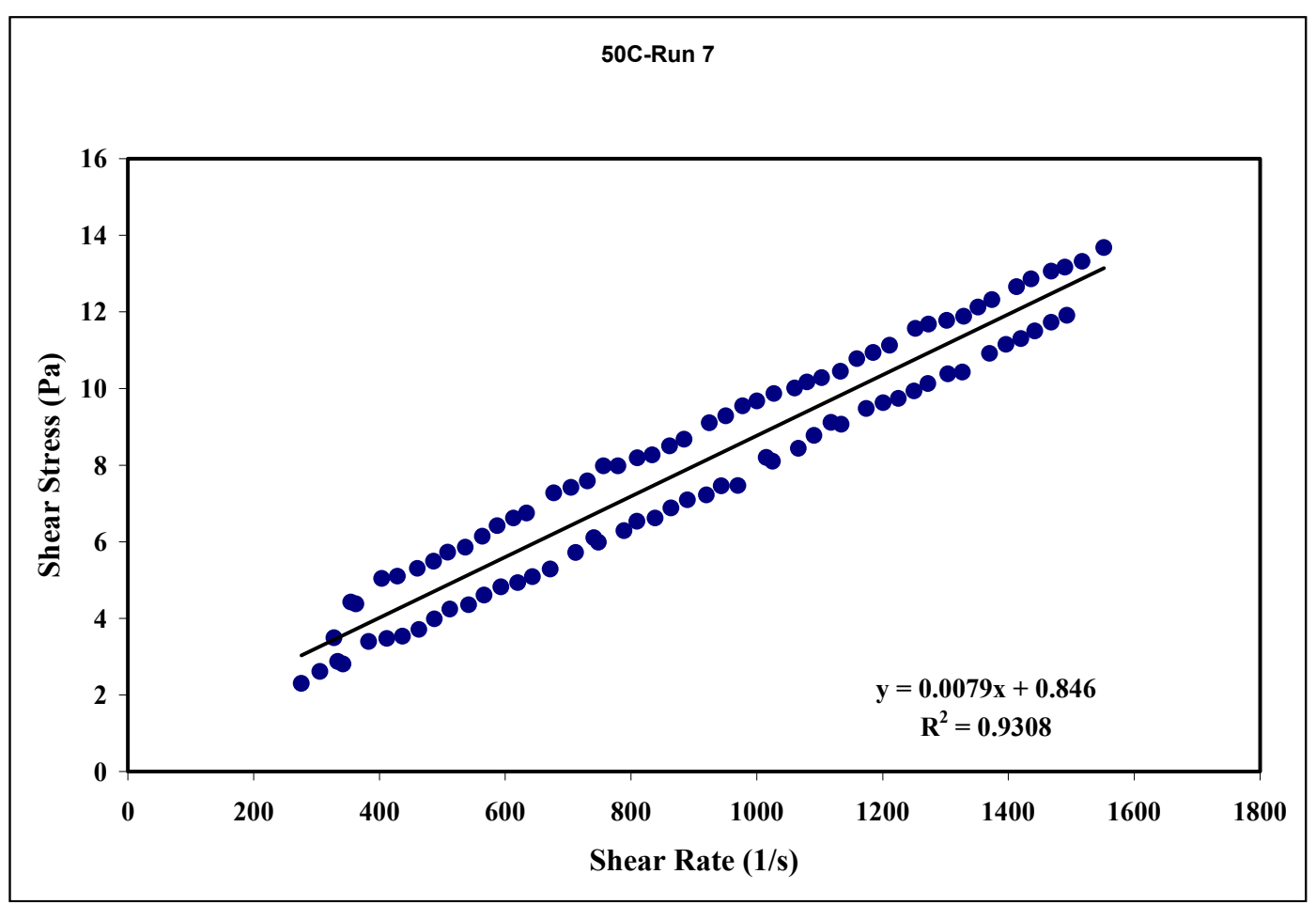

Figure 25. $50^{\circ} \mathrm{C}$ As Received Run 7

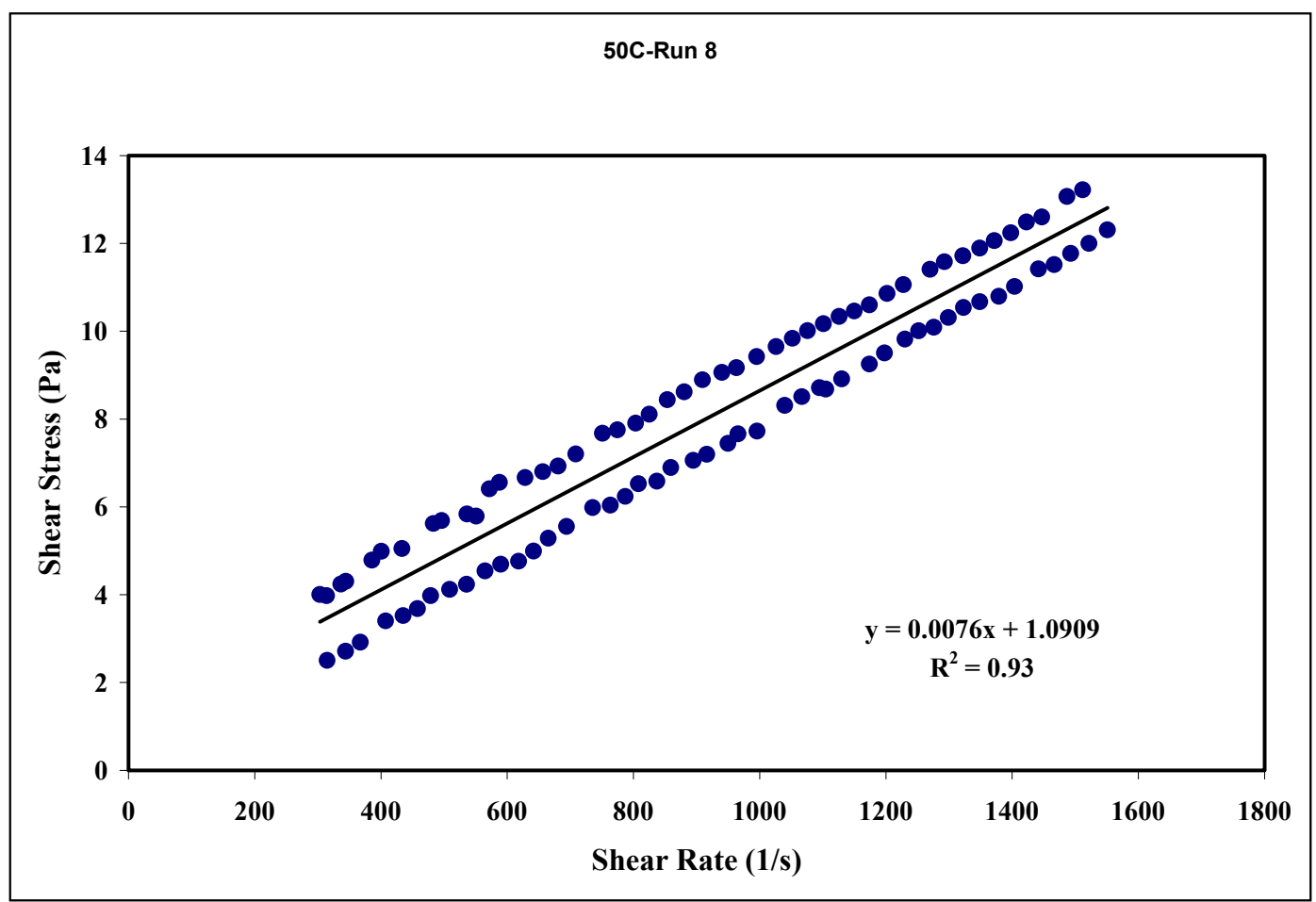

Figure 26. $50^{\circ} \mathrm{C}$ As Received Run 8 
$\begin{array}{ll}\text { APPENDIX G } & \text { WSRC-TR-2000-00352 }\end{array}$

ENVELOPE B RHEOGRAMS

SRT-RPP-2000-00026

Page 284 of 514

50C-Run 9

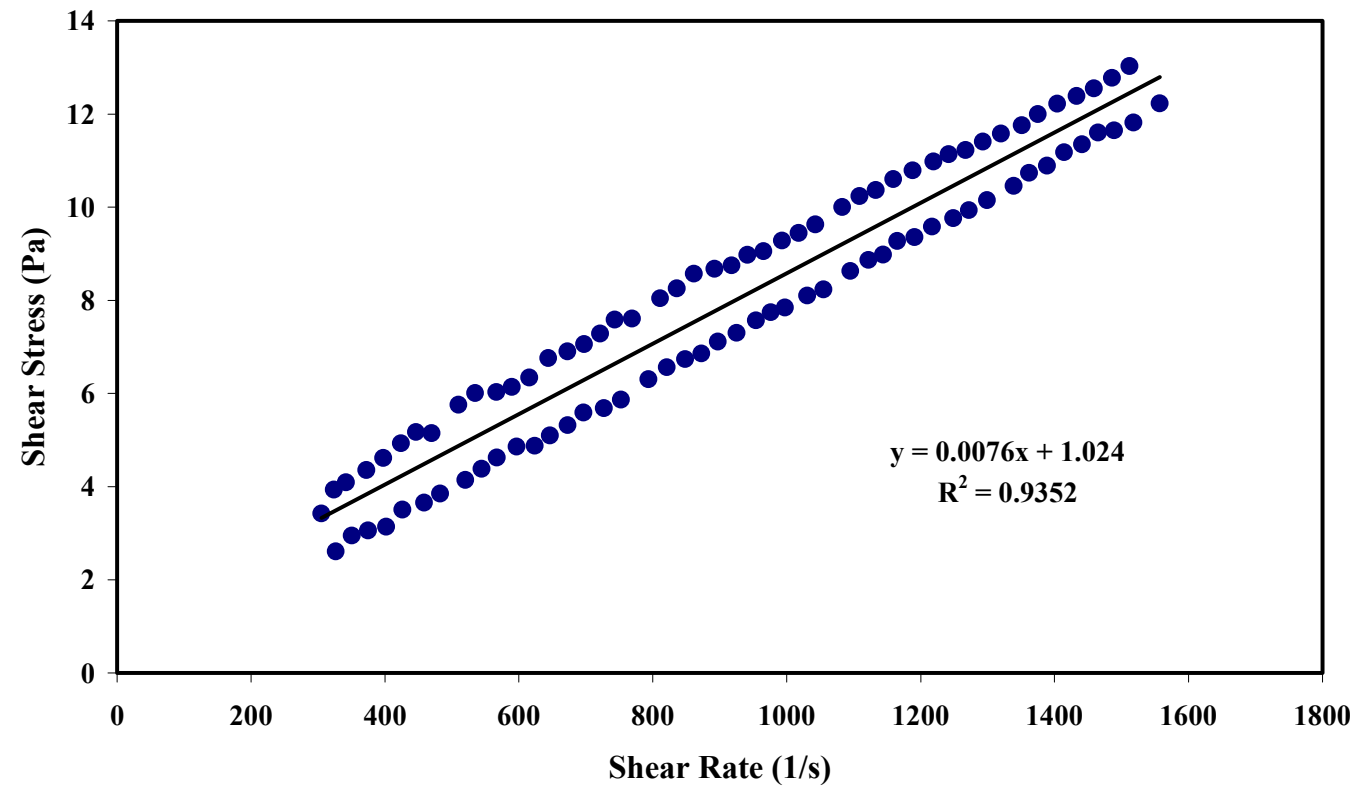

Figure 27. $50{ }^{\circ} \mathrm{C}$ As Received Run 9

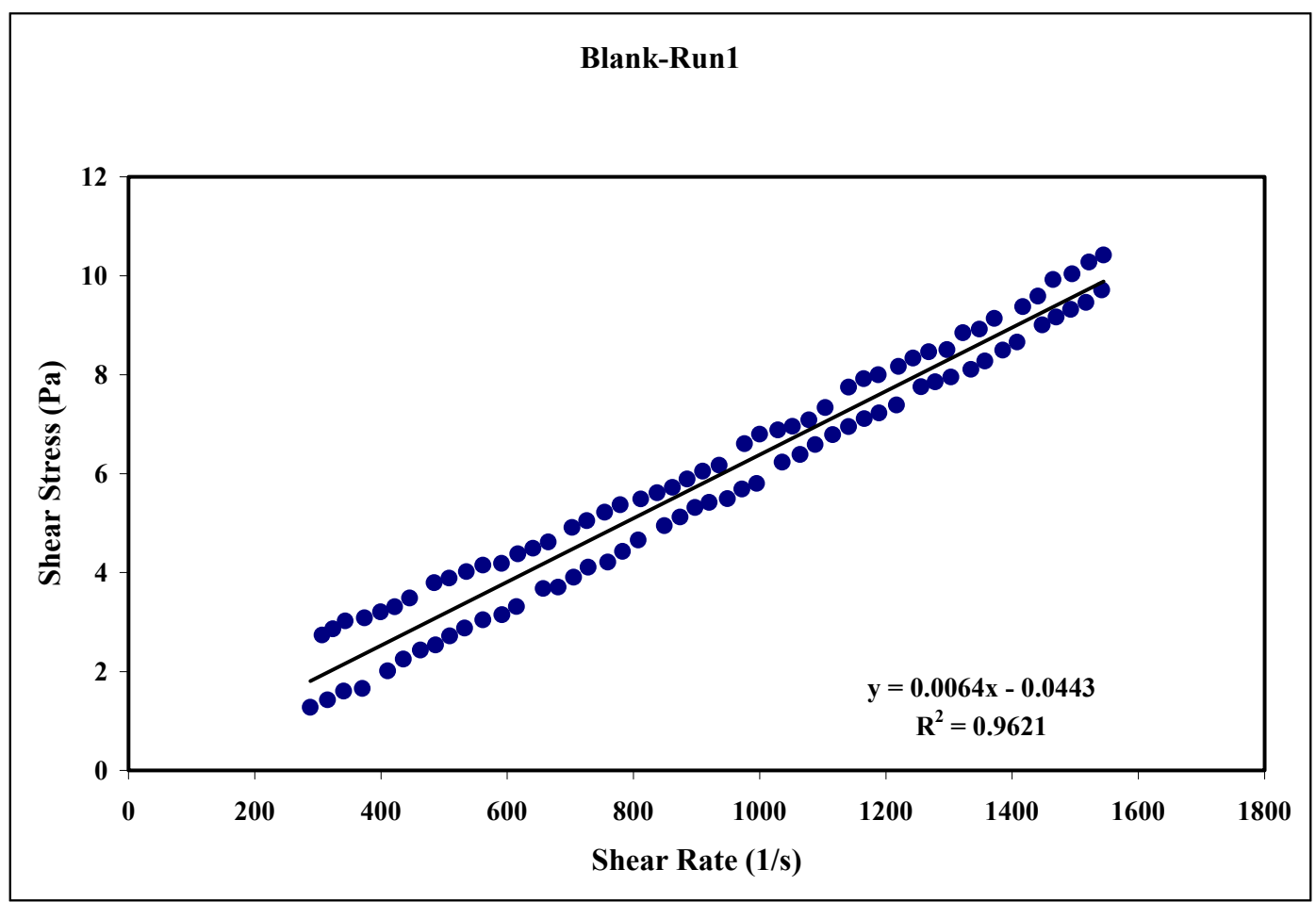

Figure 28. Blank Pretreated Run 1 
\begin{tabular}{ll}
\hline APPENDIX G & WSRC-TR-2000-00352
\end{tabular}

ENVELOPE B RHEOGRAMS

SRT-RPP-2000-00026

Page 285 of 514

\section{Blank-Run 2}

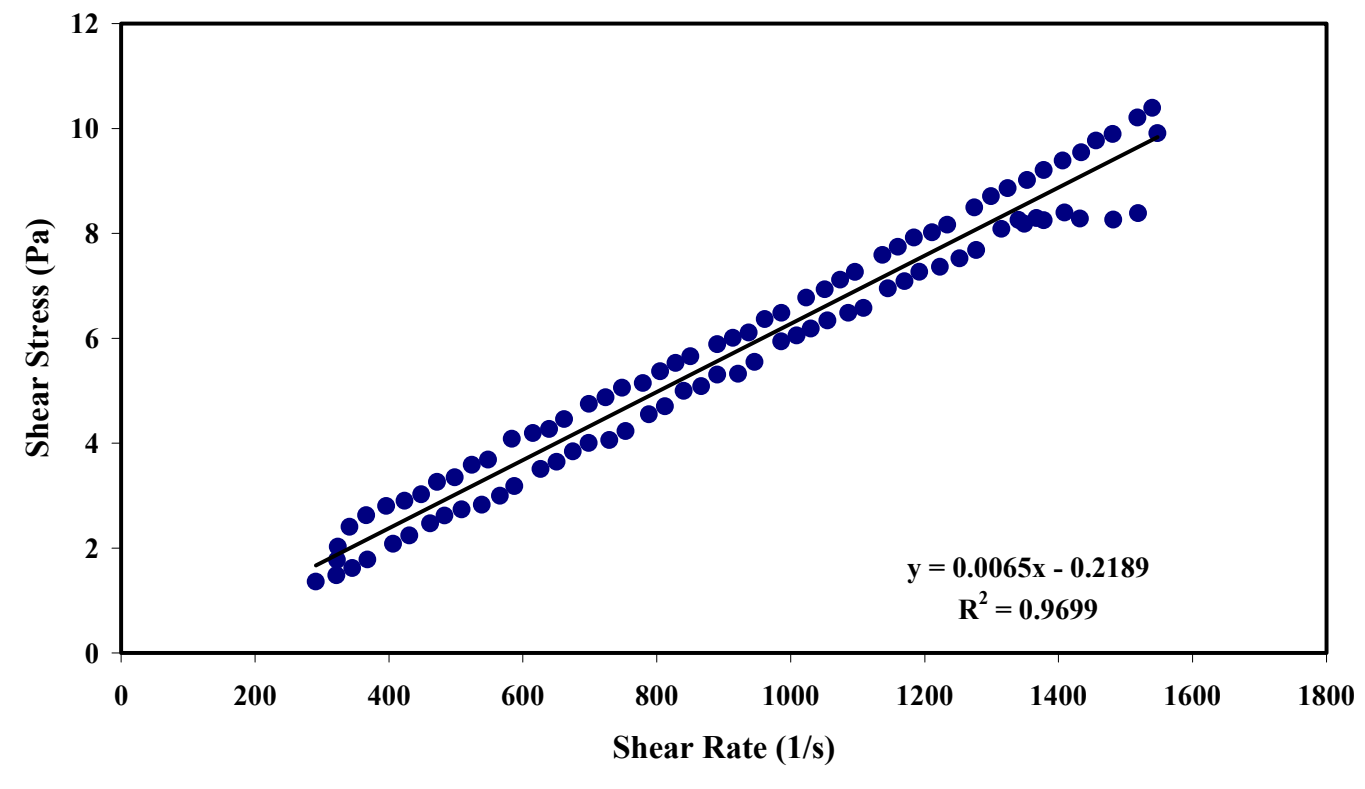

Figure 29. Blank Pretreated Run 2

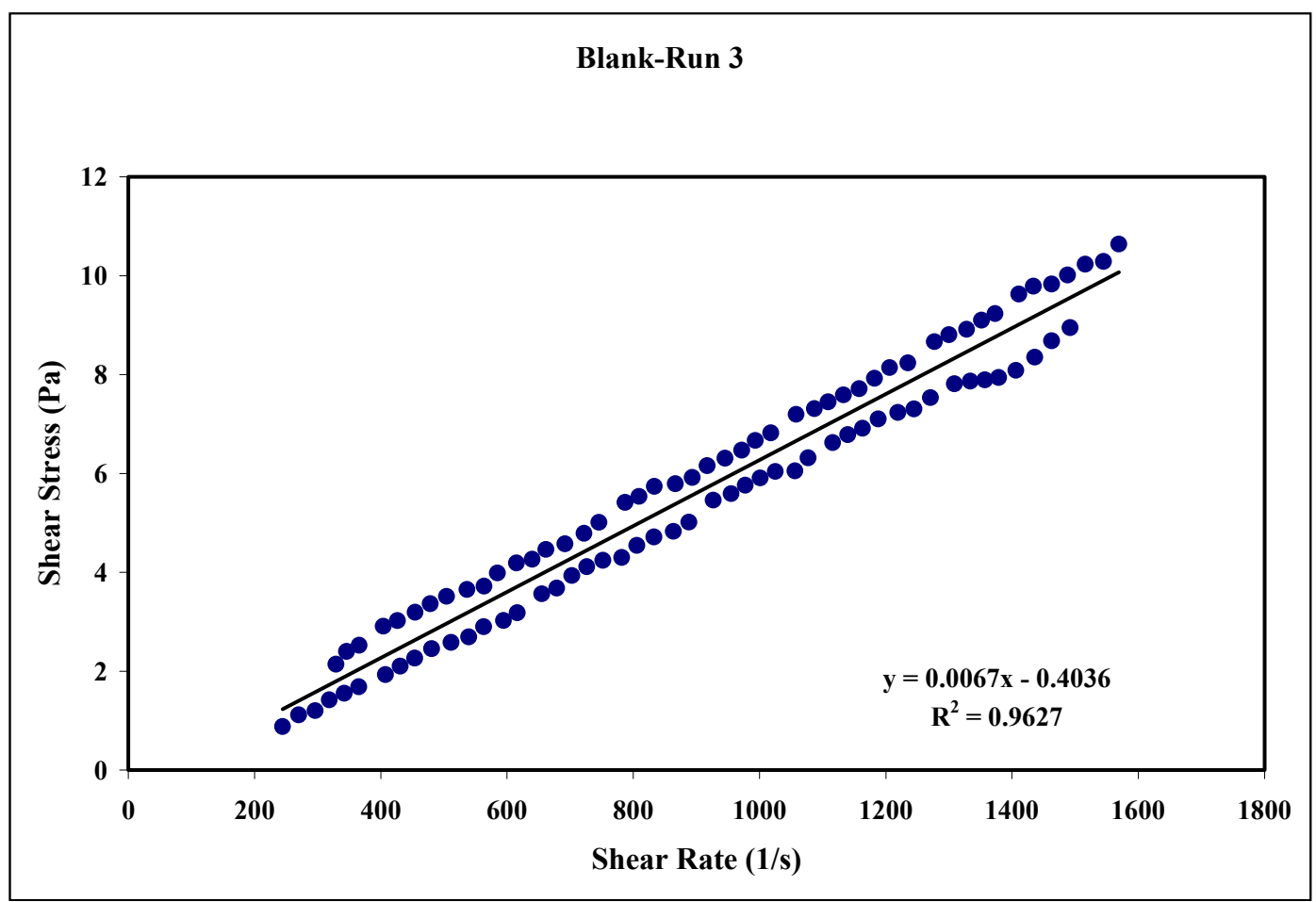

Figure 30. Blank Pretreated Run 3 
$\begin{array}{ll}\text { APPENDIX G } & \text { WSRC-TR-2000-00352 }\end{array}$

ENVELOPE B RHEOGRAMS

SRT-RPP-2000-00026

Page 286 of 514

\section{Blank-Run 4}

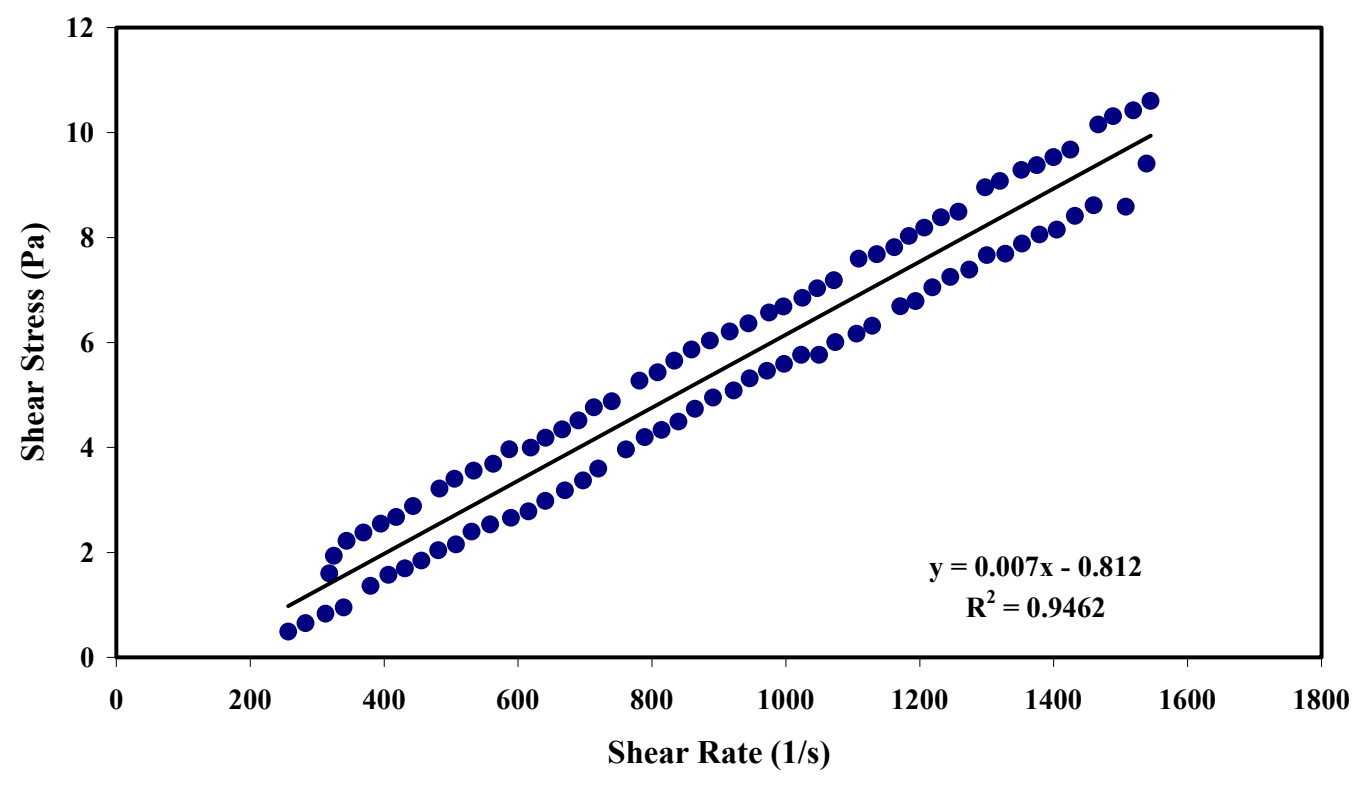

Figure 31. Blank Pretreated Run 4

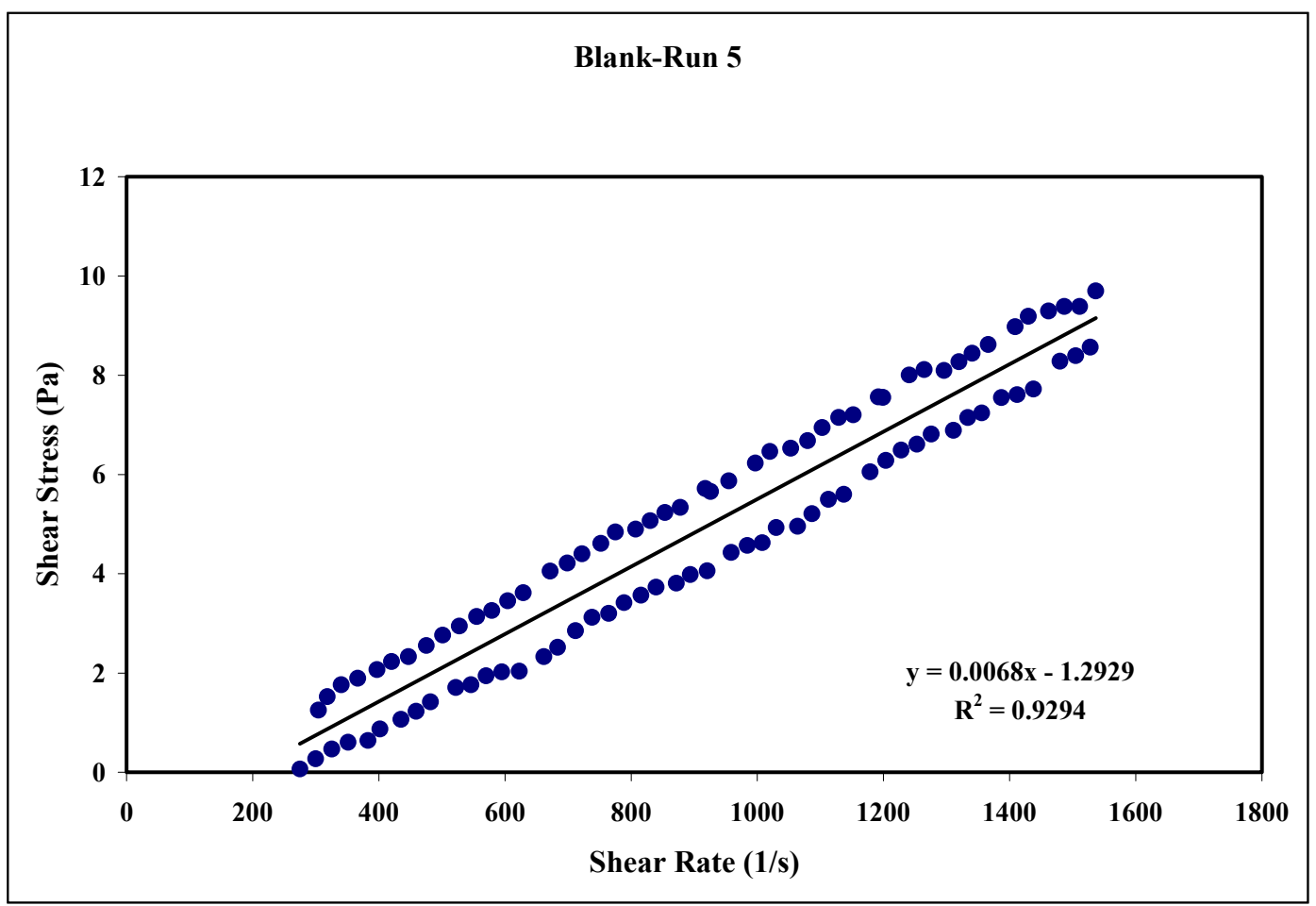

Figure 32. Blank Pretreated Run 5 
$\begin{array}{ll}\text { APPENDIX G } & \text { WSRC-TR-2000-00352 }\end{array}$

ENVELOPE B RHEOGRAMS

SRT-RPP-2000-00026

Page 287 of 514

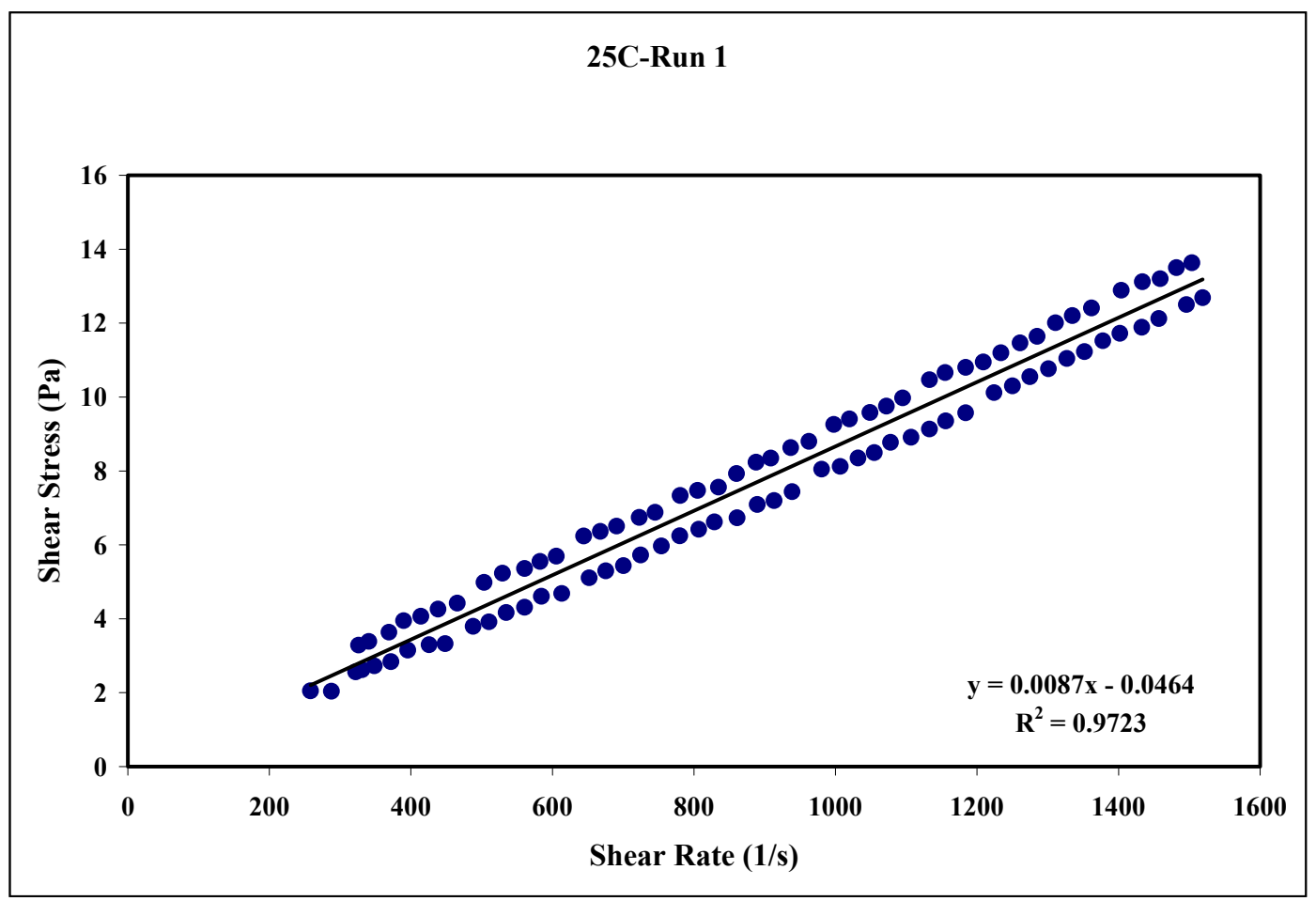

Figure 33. $25^{\circ} \mathrm{C}$ Pretreated Run 1

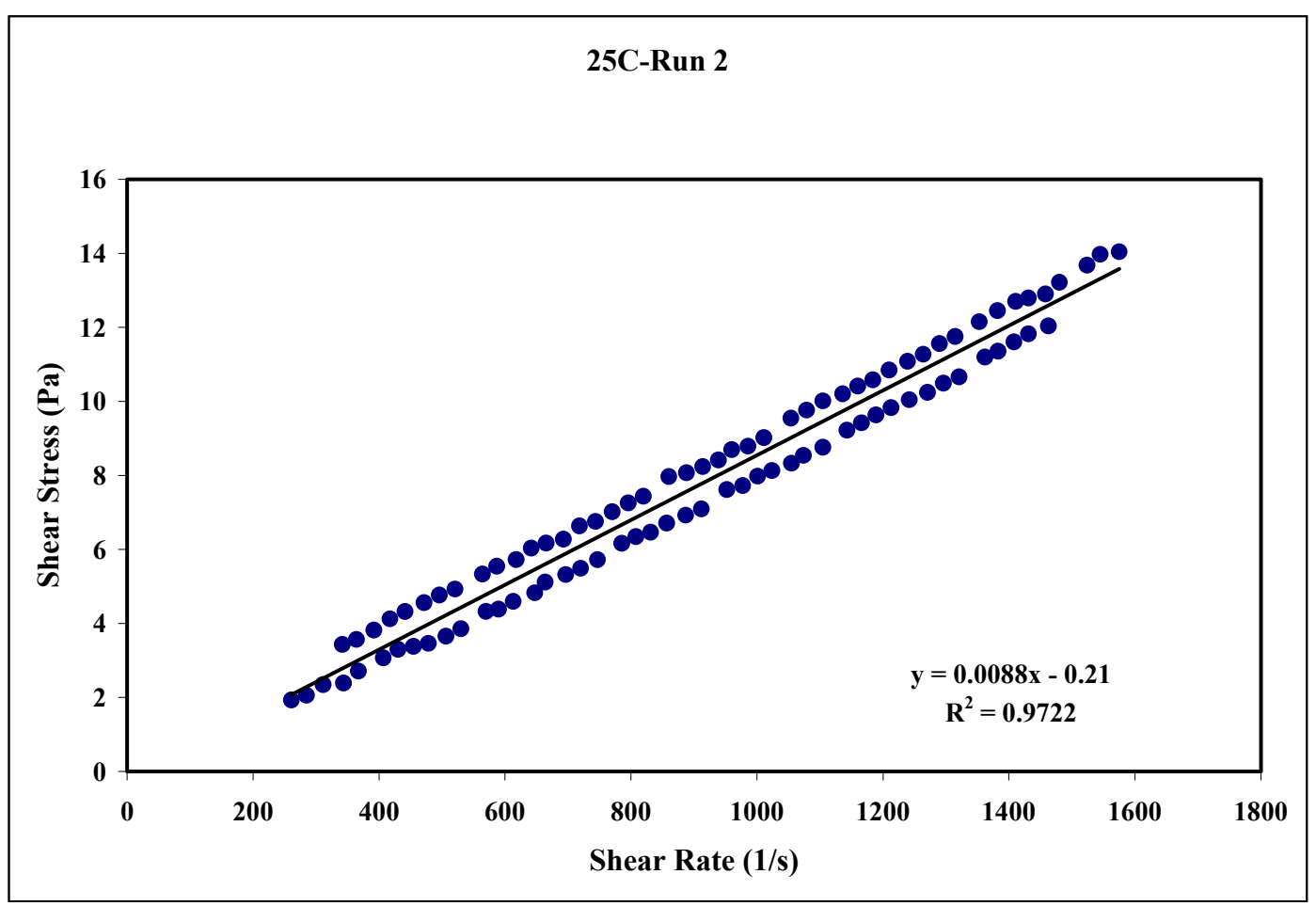

Figure 34. $25^{\circ} \mathrm{C}$ Pretreated Run 2 
$\begin{array}{ll}\text { APPENDIX G } & \text { WSRC-TR-2000-00352 }\end{array}$

ENVELOPE B RHEOGRAMS

SRT-RPP-2000-00026

Page 288 of 514

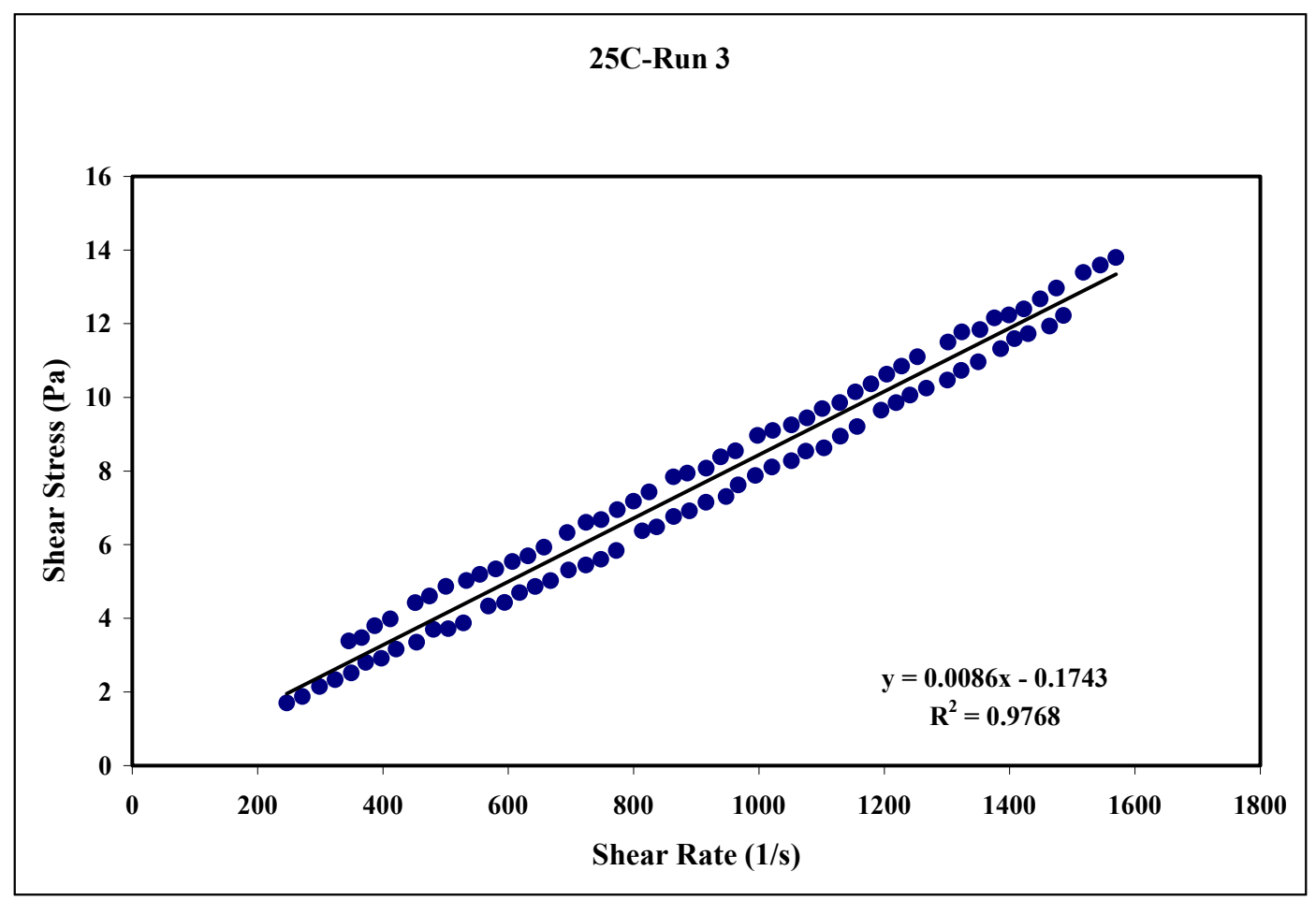

Figure 35. $25^{\circ} \mathrm{C}$ Pretreated Run 3

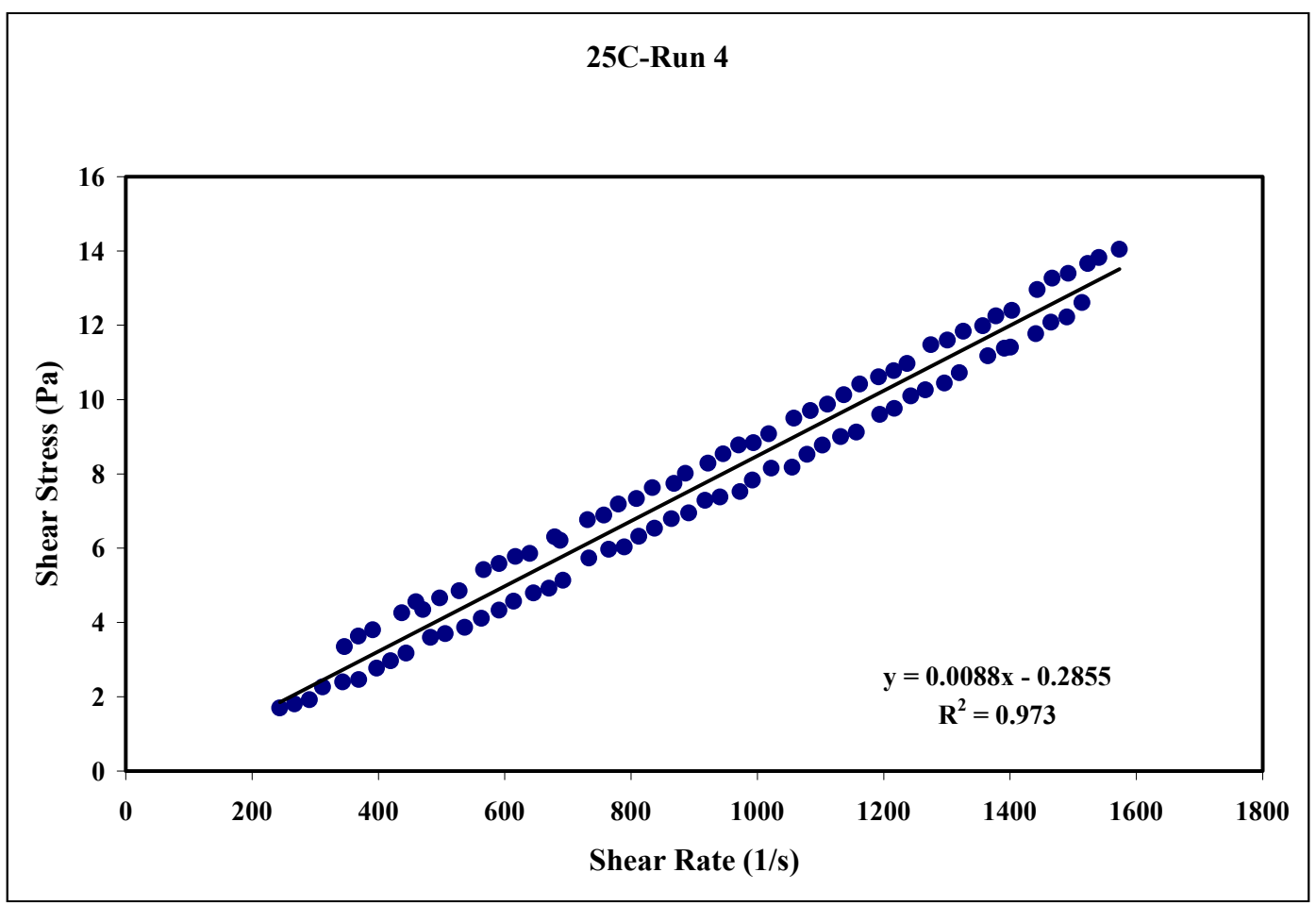

Figure 36. $25^{\circ} \mathrm{C}$ Pretreated Run 4 
$\begin{array}{ll}\text { APPENDIX G } & \text { WSRC-TR-2000-00352 }\end{array}$

ENVELOPE B RHEOGRAMS

SRT-RPP-2000-00026

Page 289 of 514

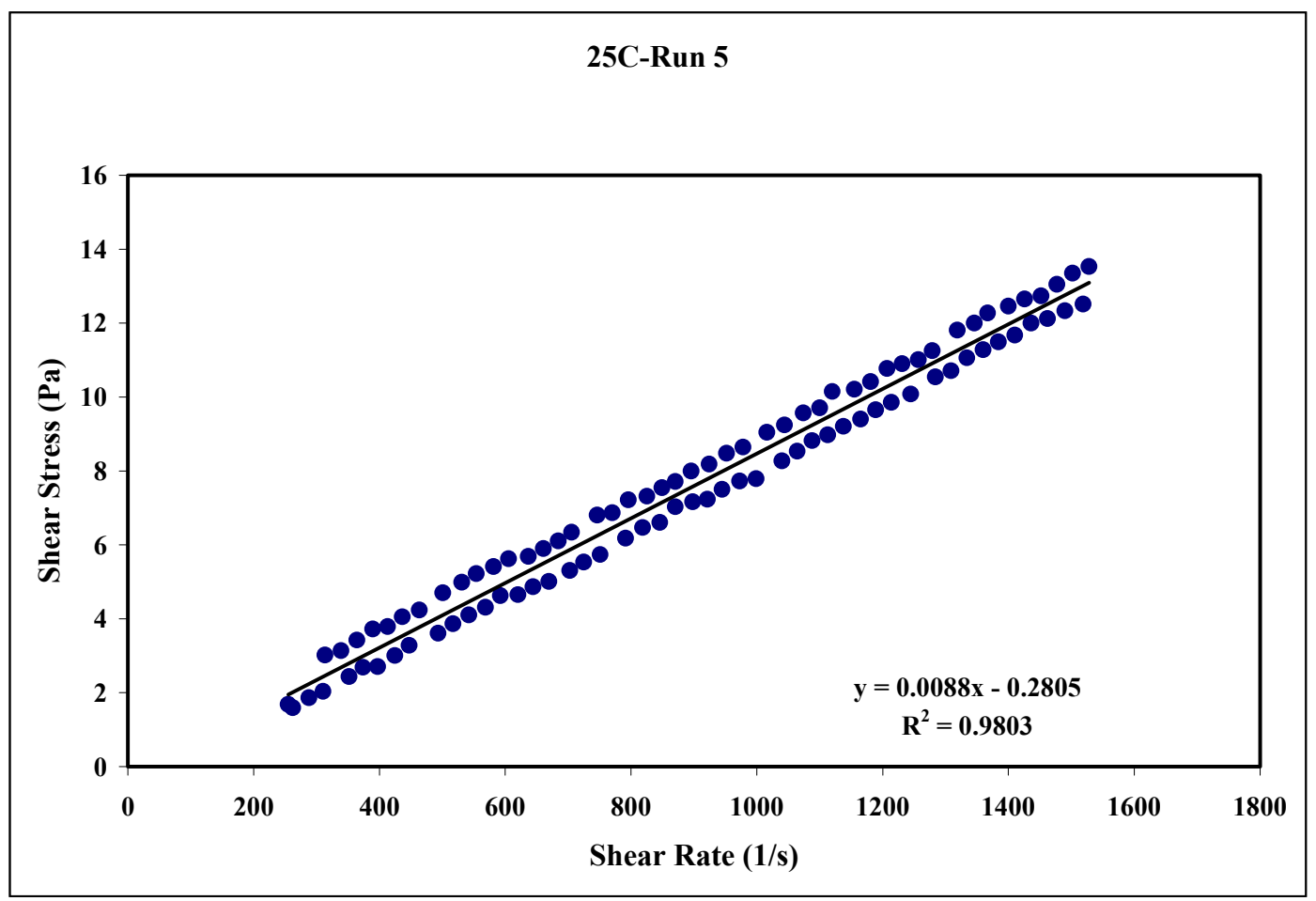

Figure 37. $25^{\circ} \mathrm{C}$ Pretreated Run 5

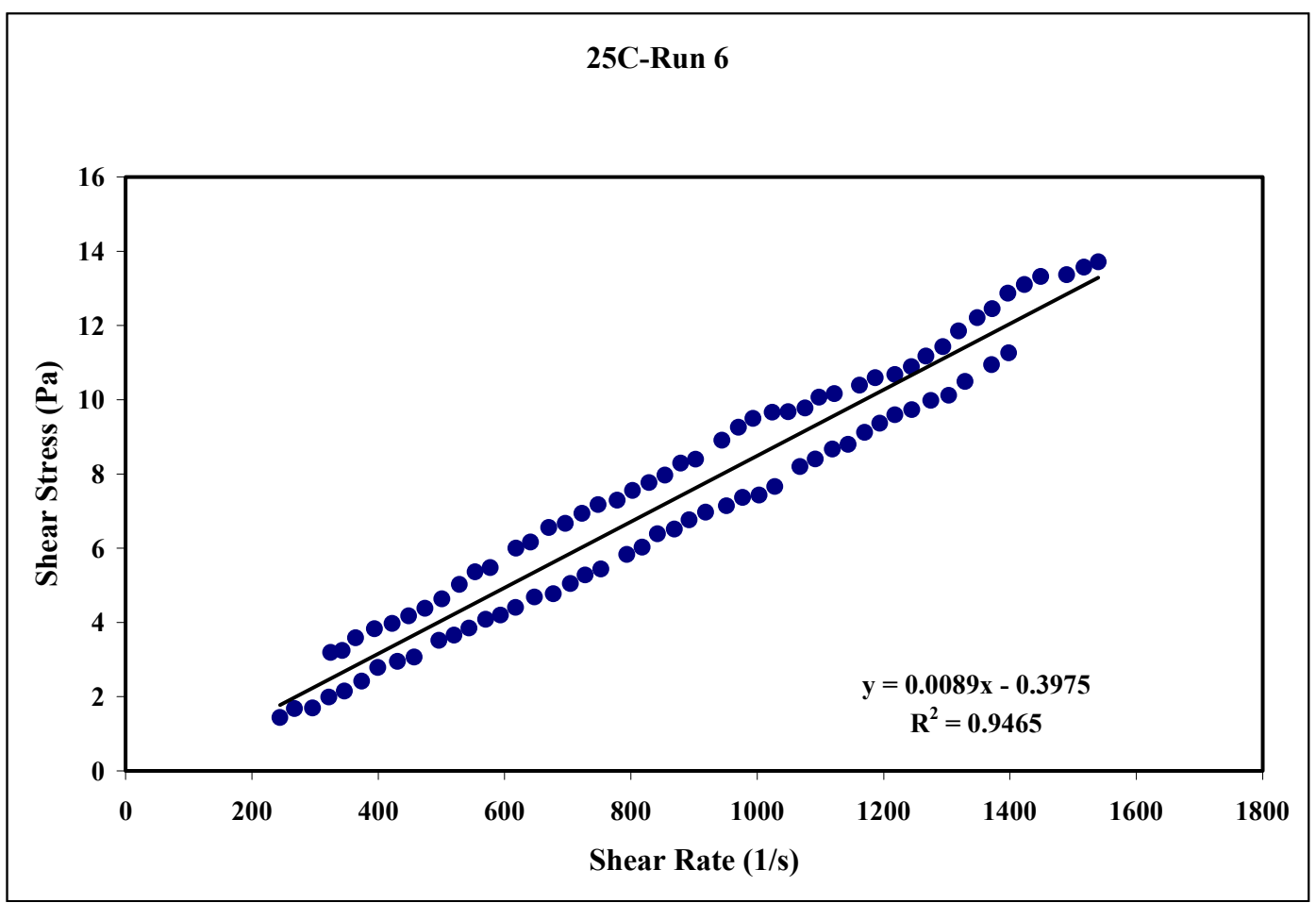

Figure 38. $25^{\circ} \mathrm{C}$ Pretreated Run 6 
$\begin{array}{ll}\text { APPENDIX G } & \text { WSRC-TR-2000-00352 }\end{array}$

ENVELOPE B RHEOGRAMS

SRT-RPP-2000-00026

Page 290 of 514

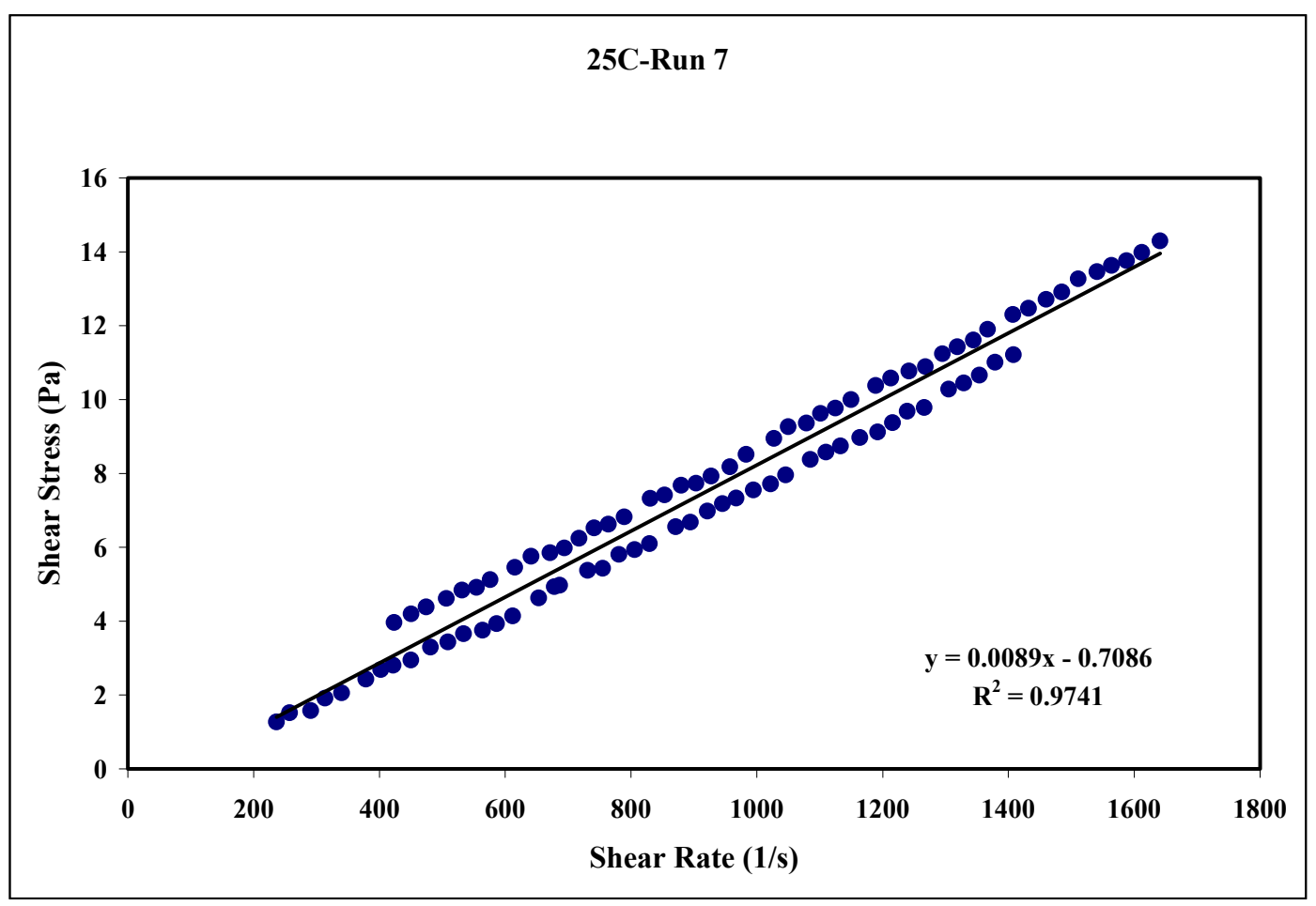

Figure 39. $25{ }^{\circ} \mathrm{C}$ Pretreated Run 7

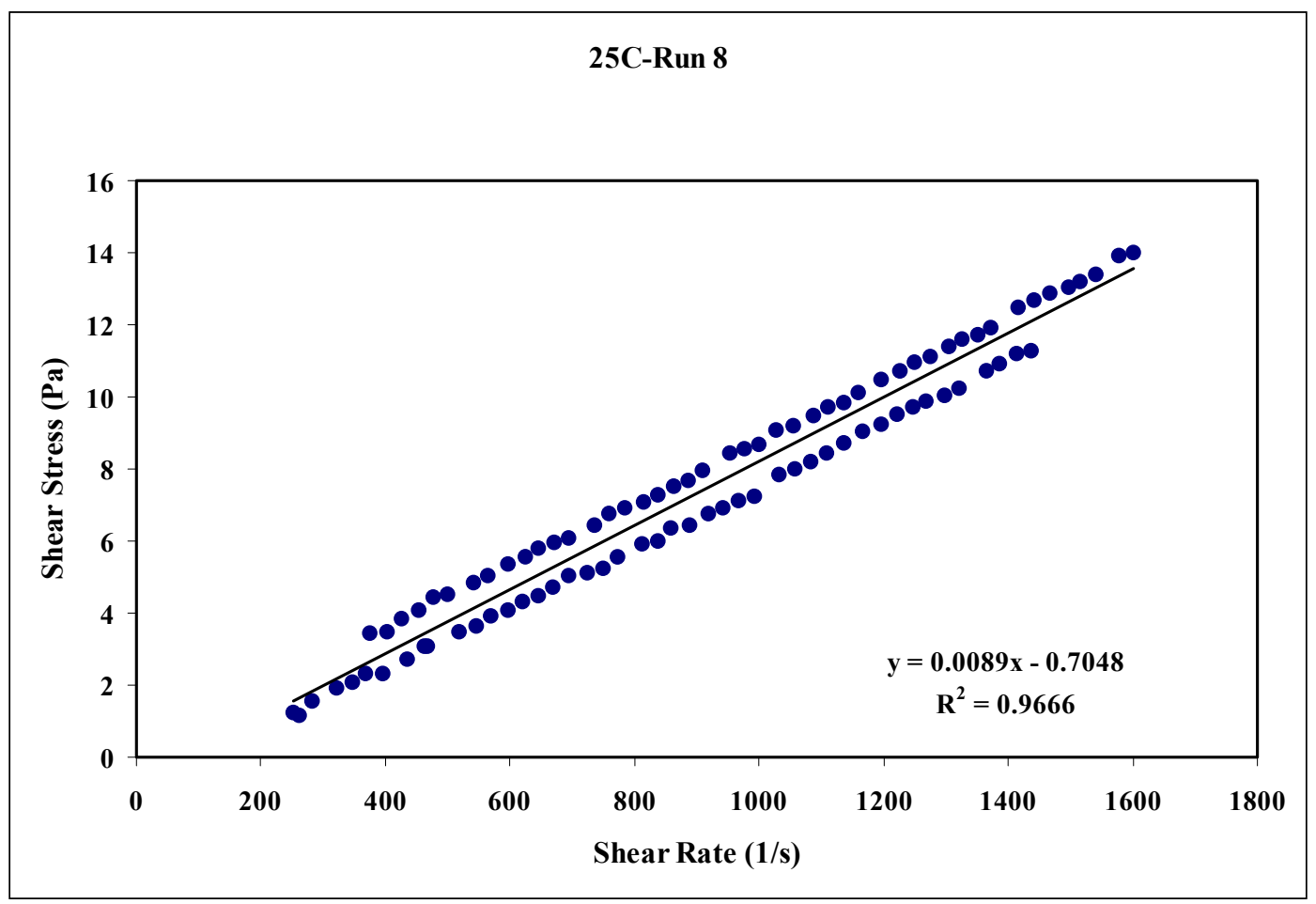

Figure 40. $25{ }^{\circ} \mathrm{C}$ Pretreated Run 8 
\begin{tabular}{|lc}
\hline APPENDIX G & WSRC-TR-2000-00352
\end{tabular}

ENVELOPE B RHEOGRAMS

SRT-RPP-2000-00026

Page 291 of 514

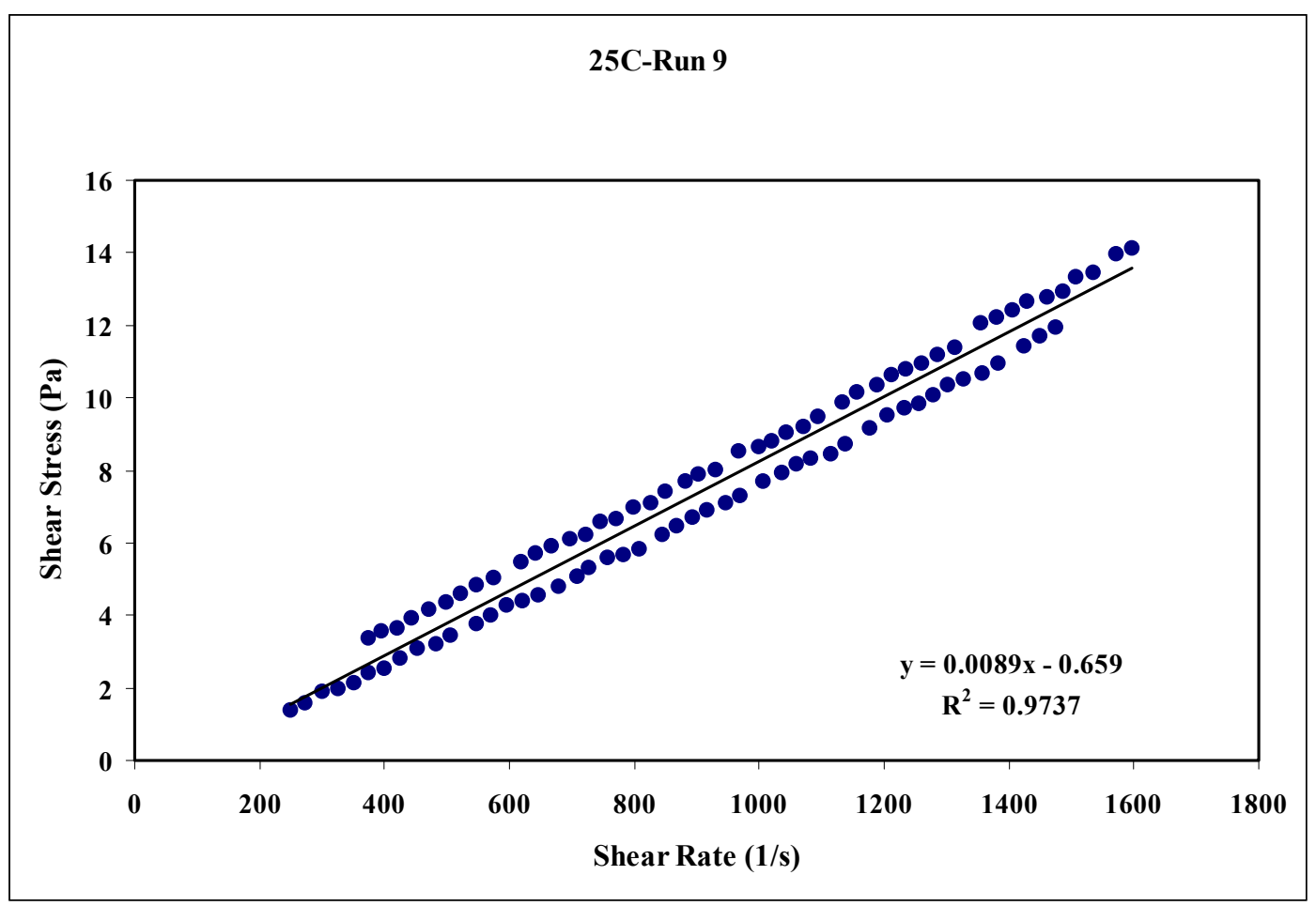

Figure 41. $25{ }^{\circ} \mathrm{C}$ Pretreated Run 9

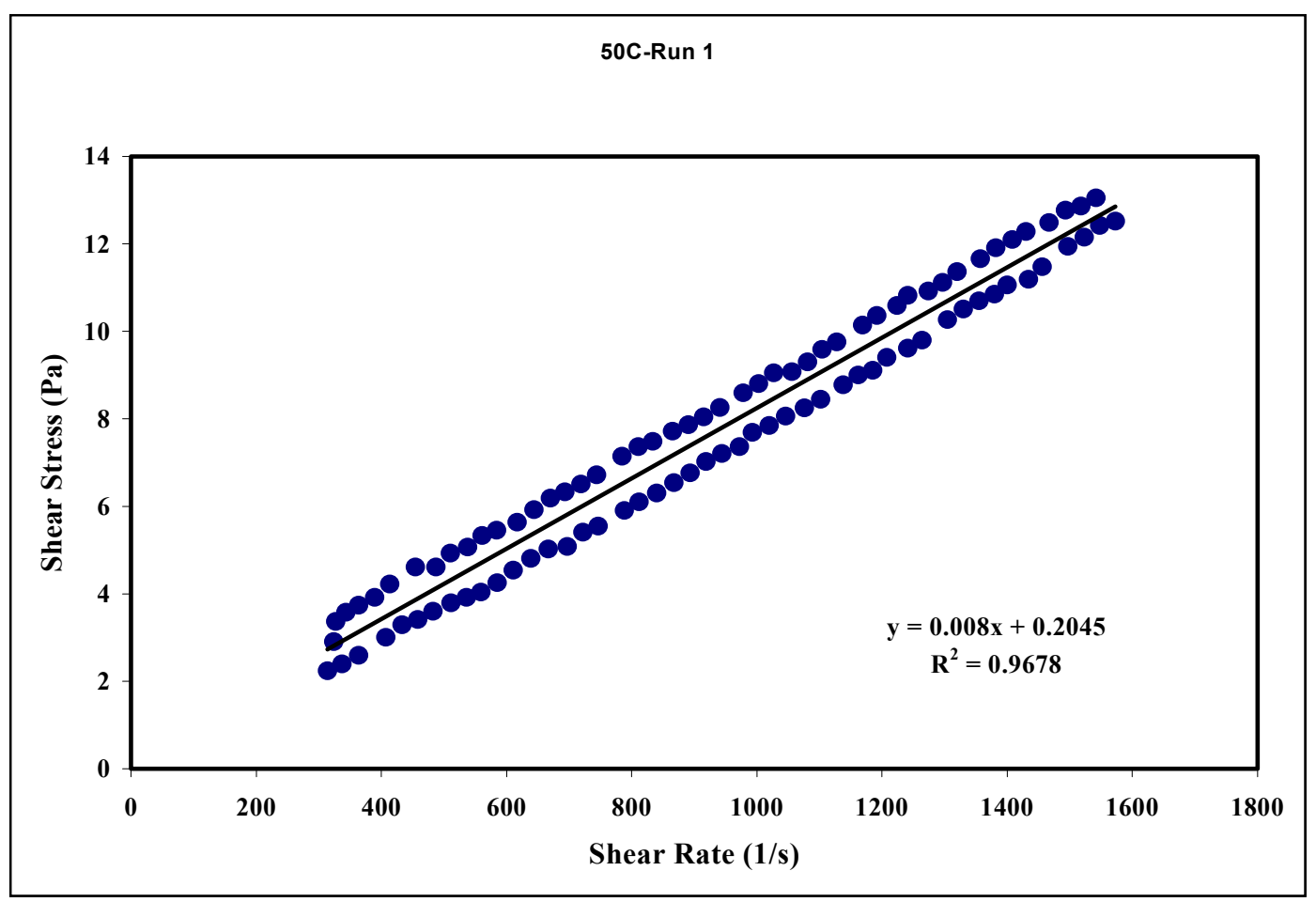

Figure 42. $50{ }^{\circ} \mathrm{C}$ Pretreated Run 1 
$\begin{array}{ll}\text { APPENDIX G } & \text { WSRC-TR-2000-00352 }\end{array}$

ENVELOPE B RHEOGRAMS

SRT-RPP-2000-00026

Page 292 of 514

50C-Run 2

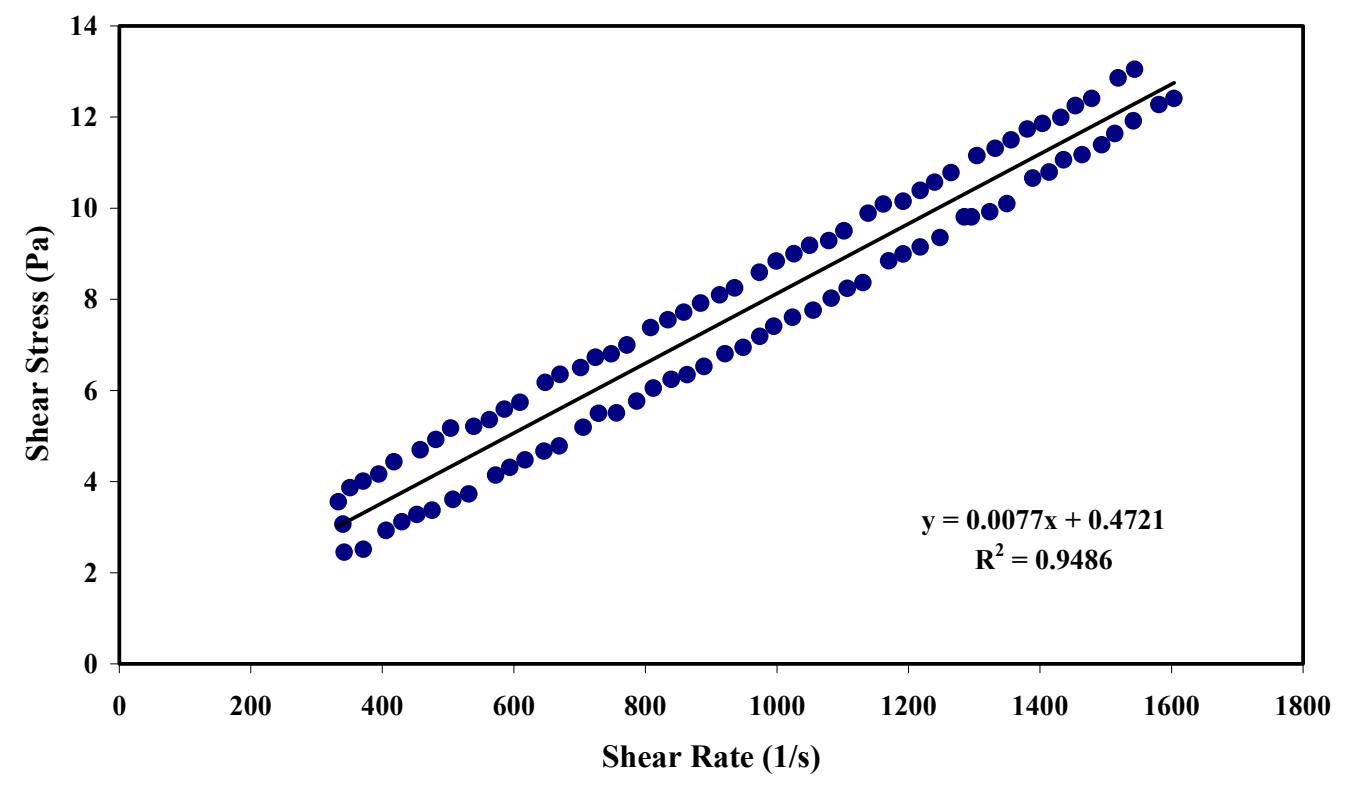

Figure 43. $50^{\circ} \mathrm{C}$ Pretreated Run 2

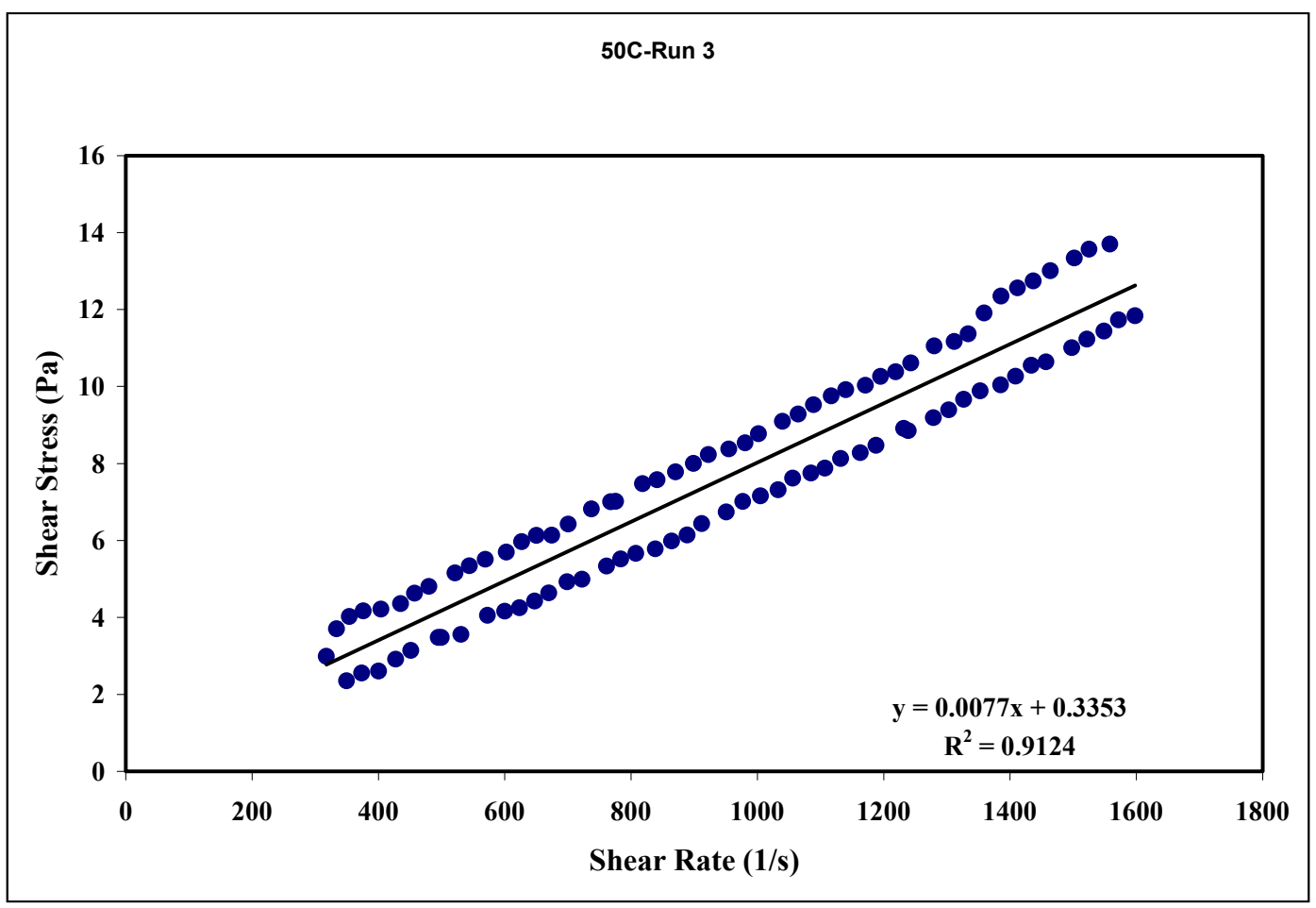

Figure 44. $50{ }^{\circ} \mathrm{C}$ Pretreated Run 3 
$\begin{array}{ll}\text { APPENDIX G } & \text { WSRC-TR-2000-00352 }\end{array}$

ENVELOPE B RHEOGRAMS

SRT-RPP-2000-00026

Page 293 of 514

50C-Run 4

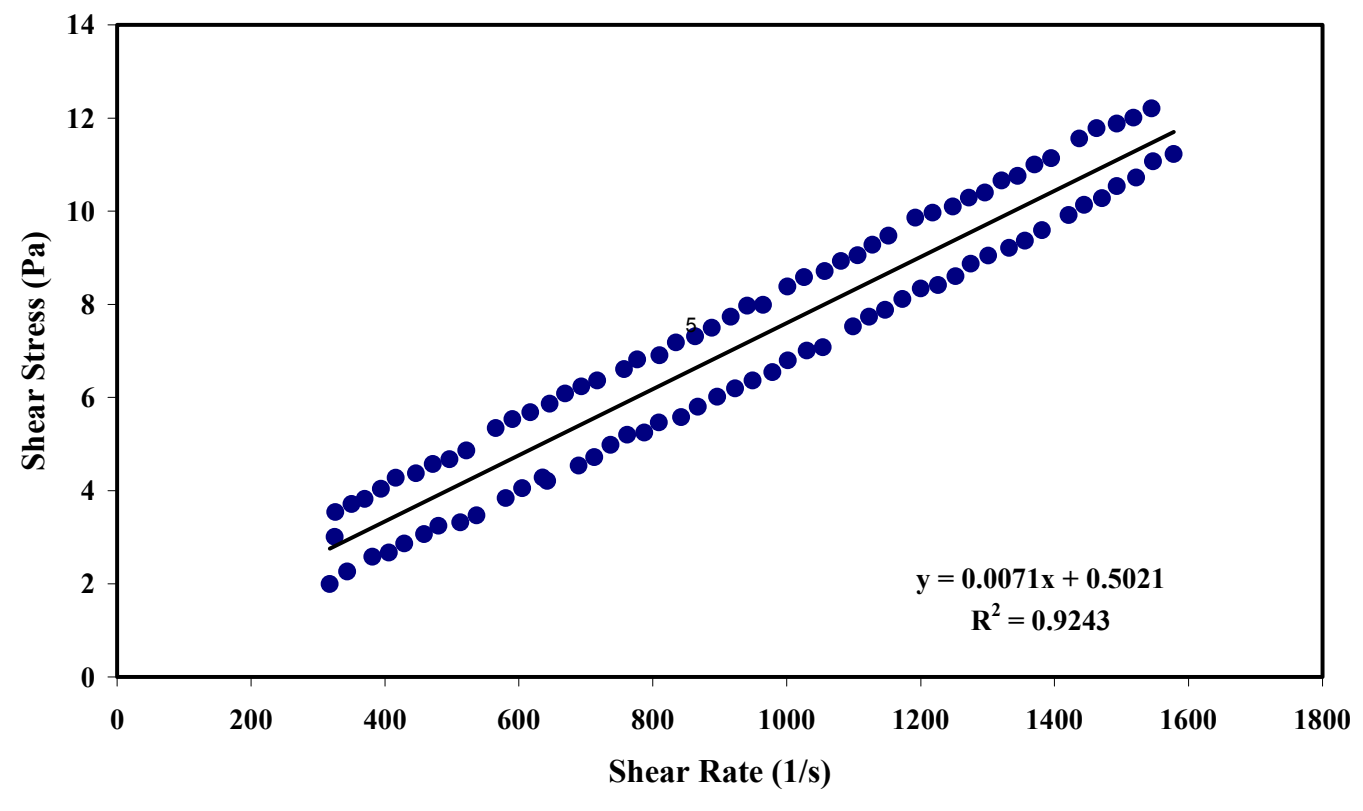

Figure 45. $50{ }^{\circ} \mathrm{C}$ Pretreated Run 4

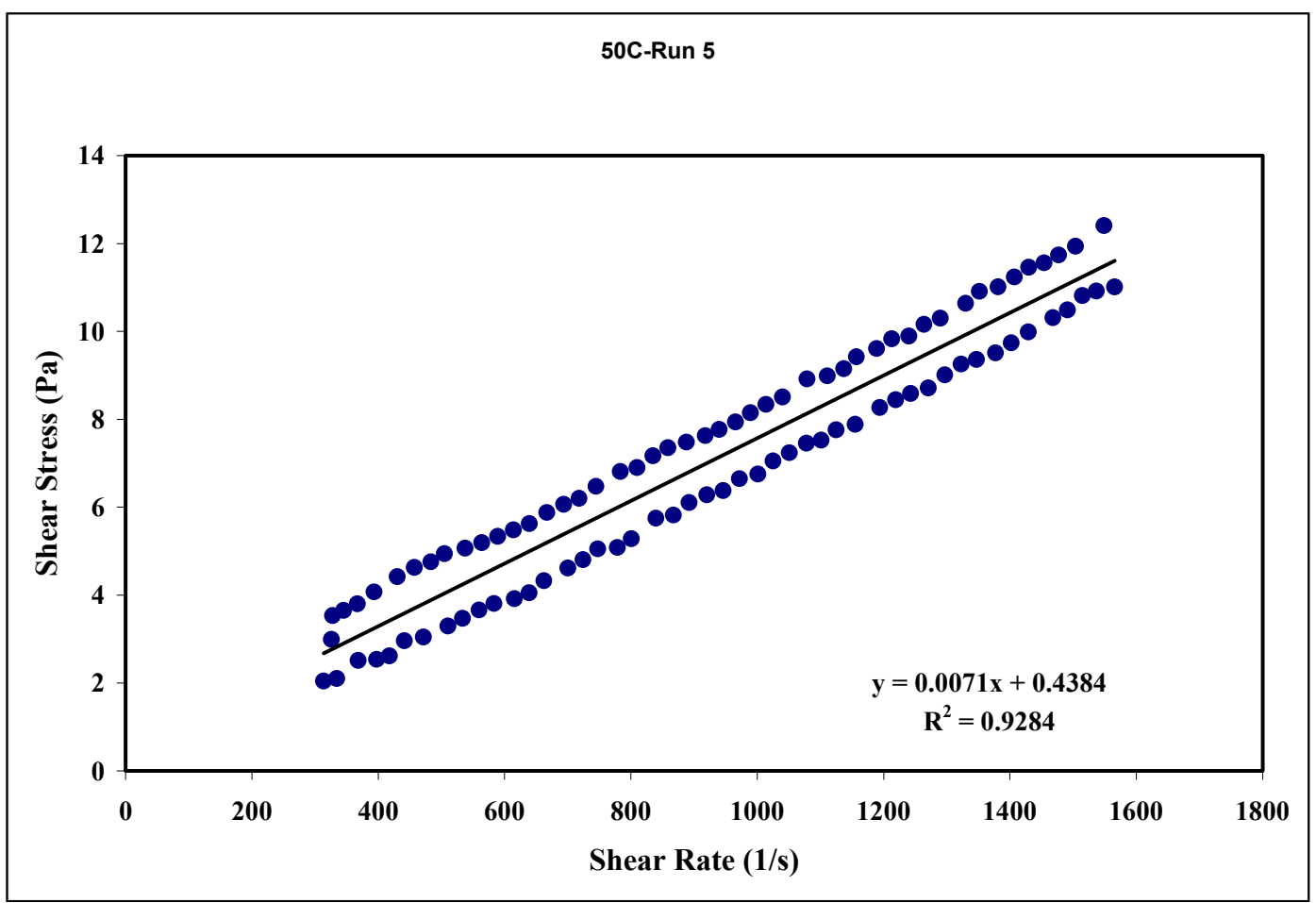

Figure 46. $50{ }^{\circ} \mathrm{C}$ Pretreated Run 5 
$\begin{array}{ll}\text { APPENDIX G } & \text { WSRC-TR-2000-00352 }\end{array}$

ENVELOPE B RHEOGRAMS

SRT-RPP-2000-00026

Page 294 of 514

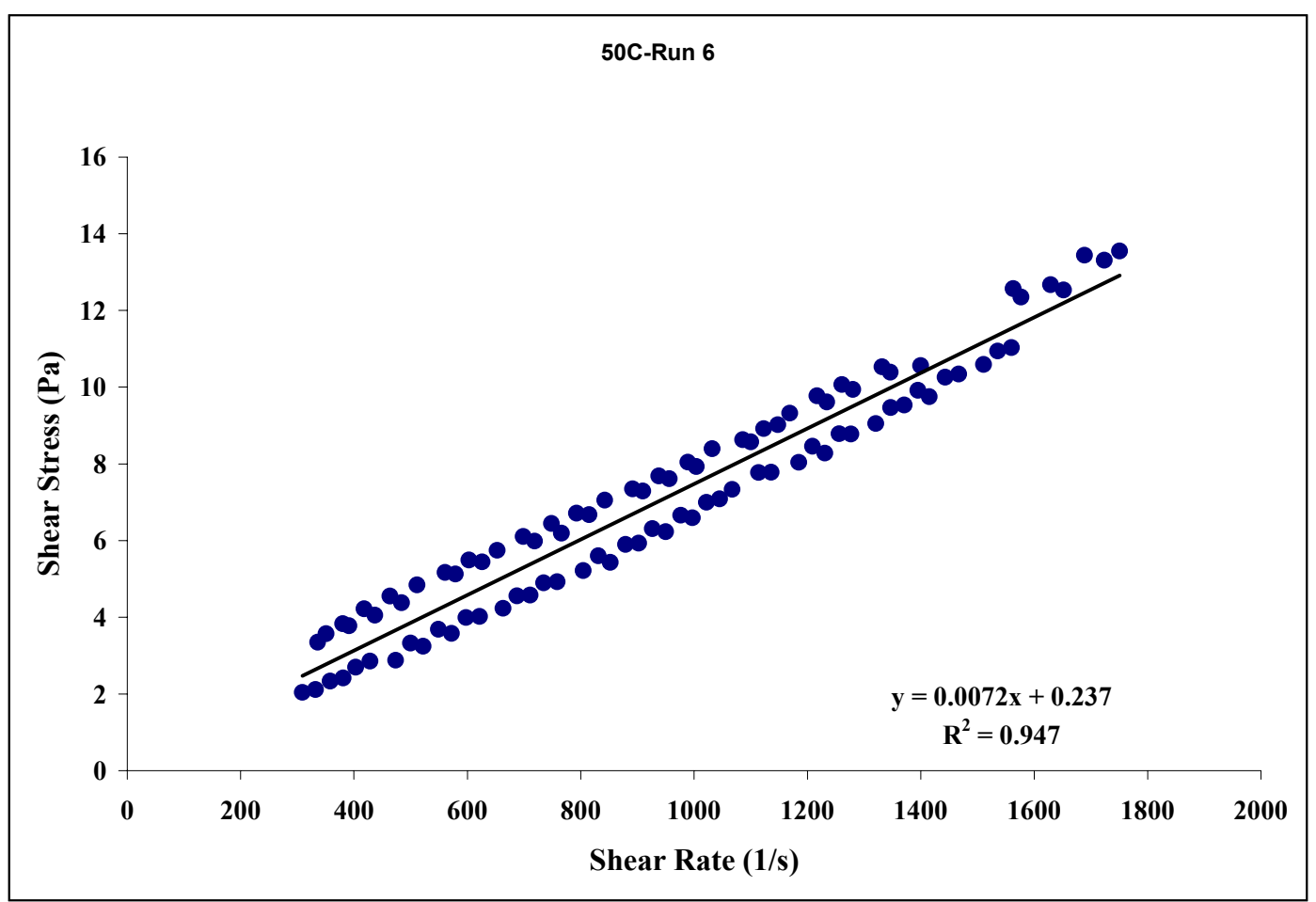

Figure 47. $50{ }^{\circ} \mathrm{C}$ Pretreated Run 6

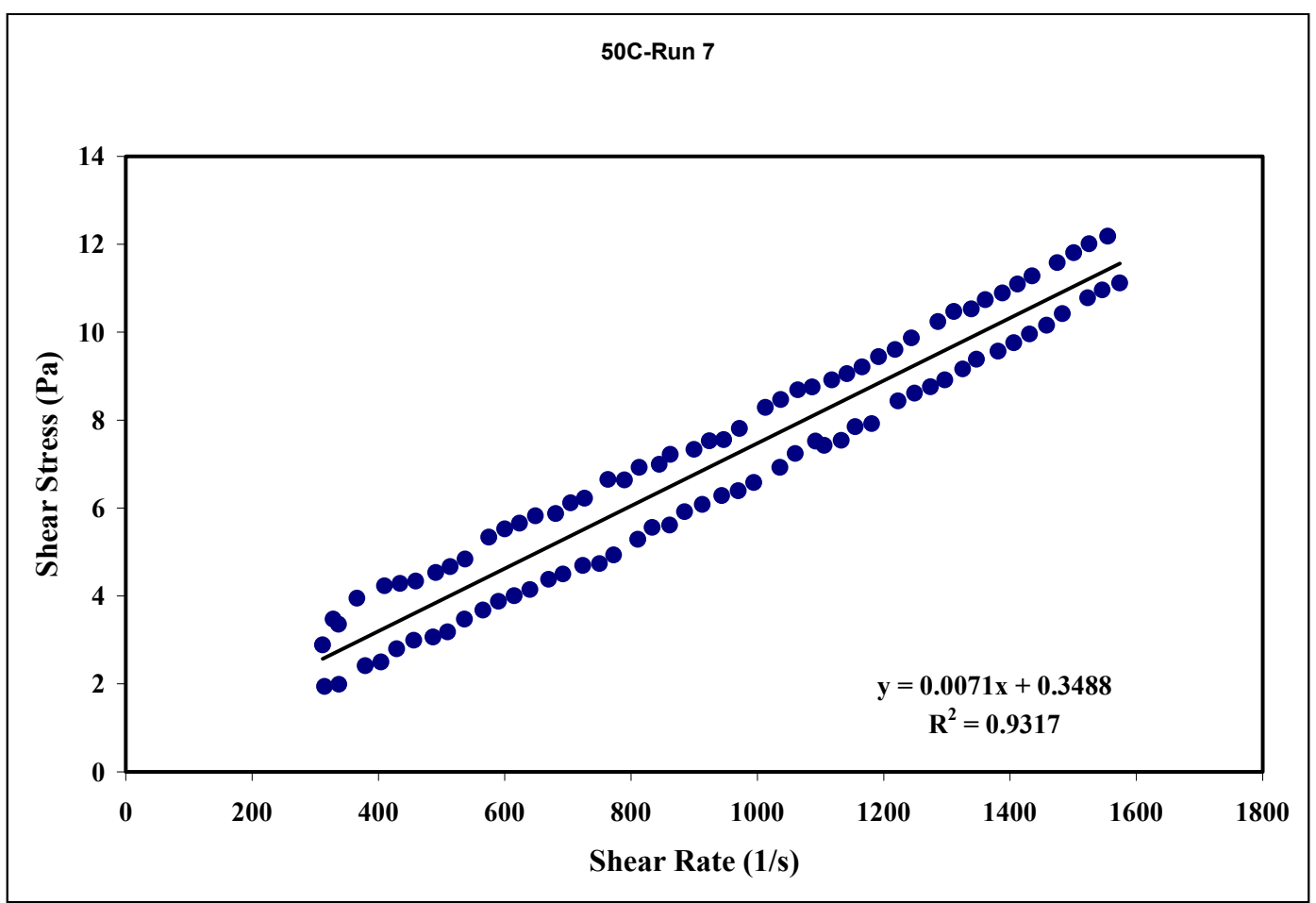

Figure 48. $50{ }^{\circ} \mathrm{C}$ Pretreated Run 7 
$\begin{array}{ll}\text { APPENDIX G } & \text { WSRC-TR-2000-00352 }\end{array}$

ENVELOPE B RHEOGRAMS

SRT-RPP-2000-00026

Page 295 of 514

50C-Run 8

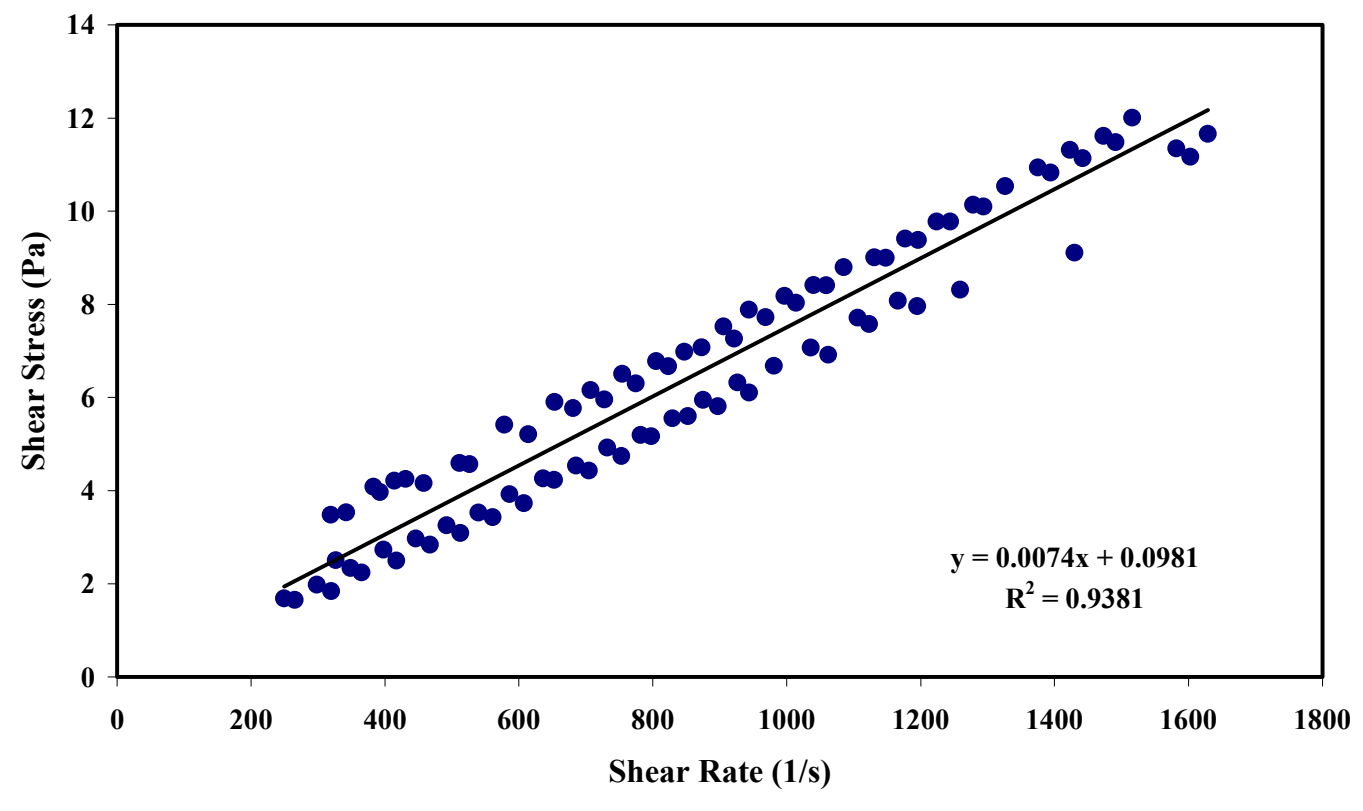

Figure 49. $50{ }^{\circ} \mathrm{C}$ Pretreated Run 8

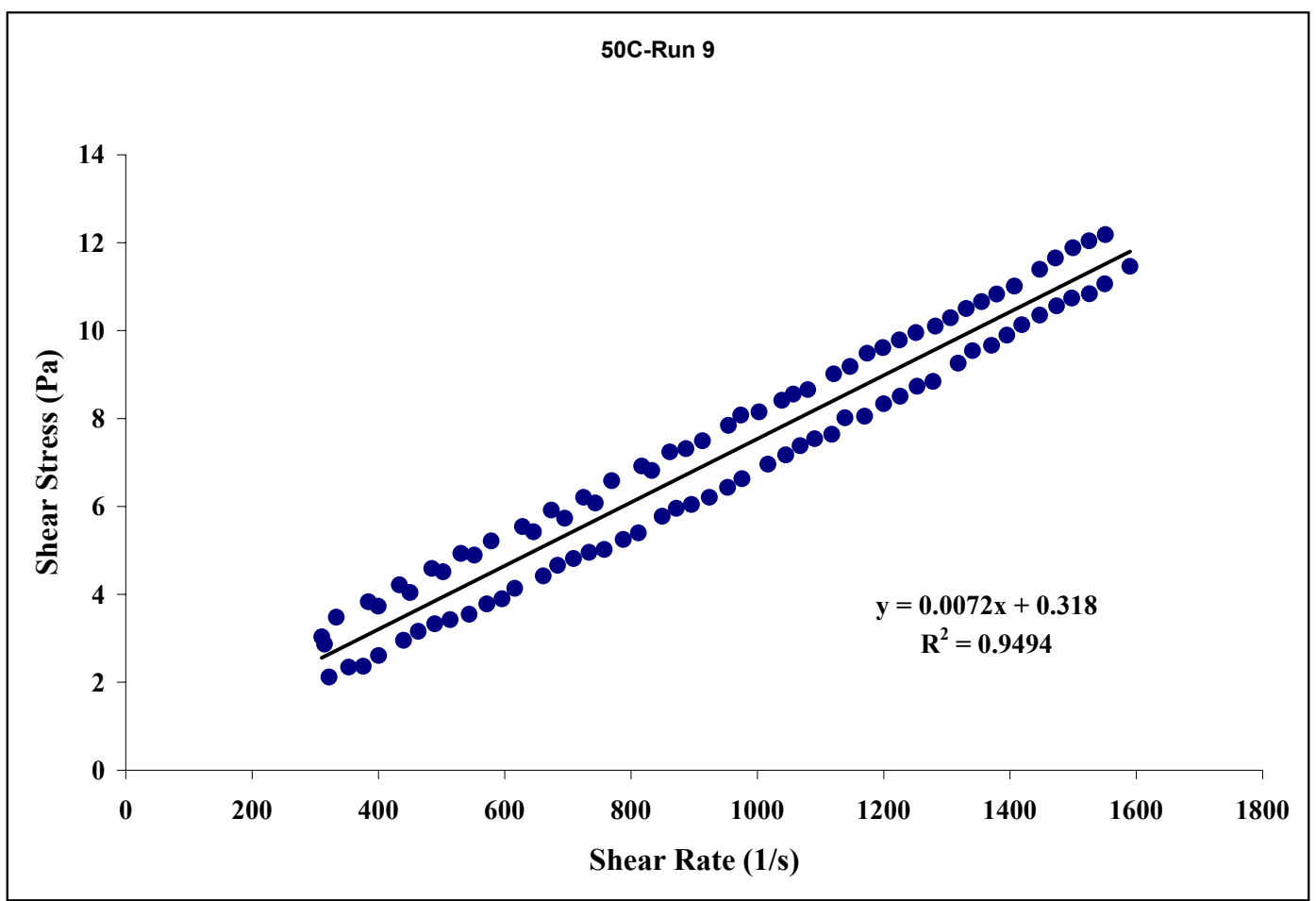

Figure 50. $50{ }^{\circ} \mathrm{C}$ Pretreated Run 9 
This page intentionally left blank. 


\section{APPENDIX H}

PART $1 \quad$ Page 299

ENVELOPE C RHEOGRAMS

As Received

Diluted

PART 2 Page 329

ENVELOPE C RHEOGRAMS

Pretreated 
This page intentionally left blank. 


\section{APPENDIX H - PART 1}

\section{ENVELOPE C RHEOGRAMS}

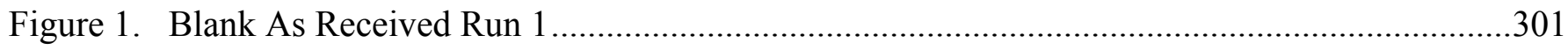

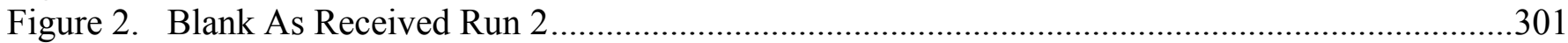

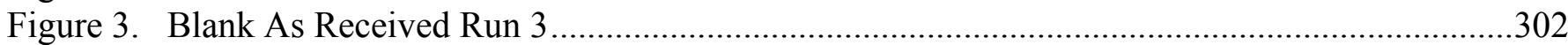

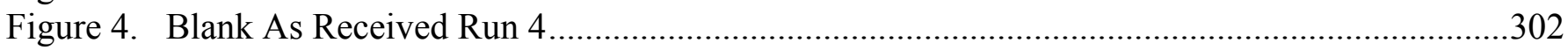

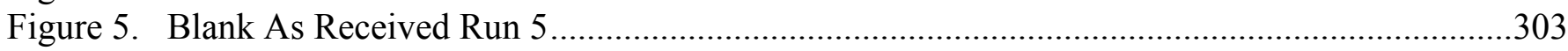

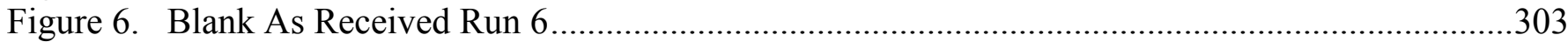

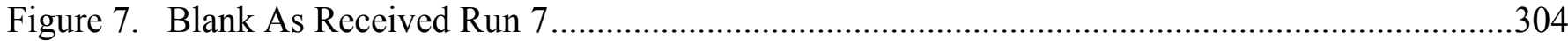

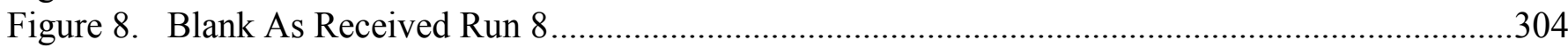

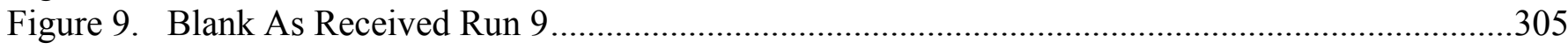

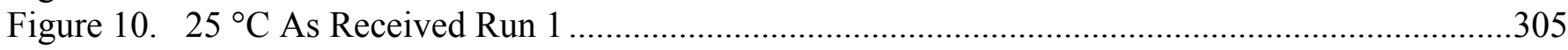

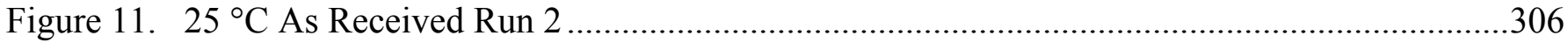

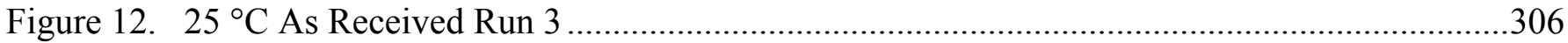

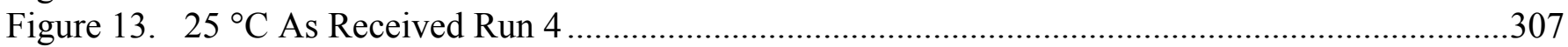

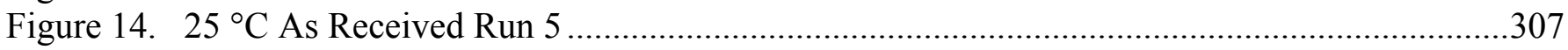

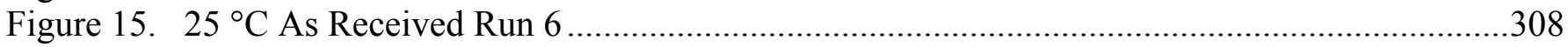

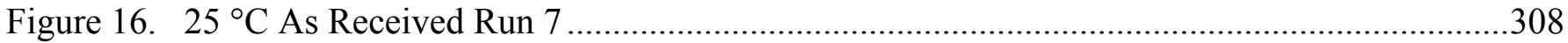

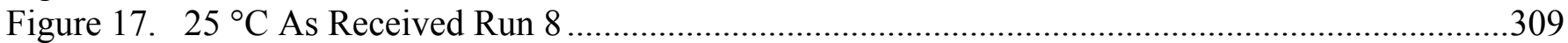

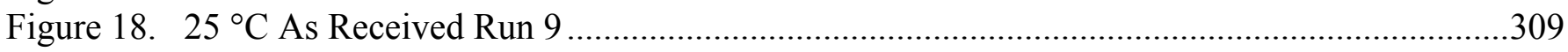

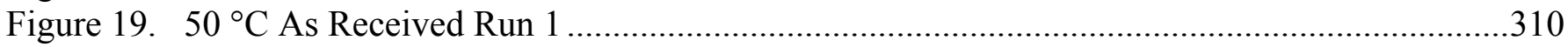

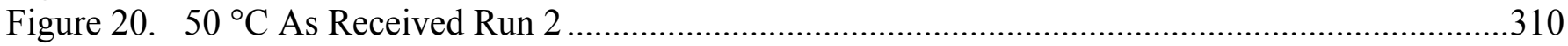

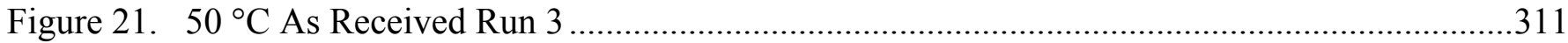

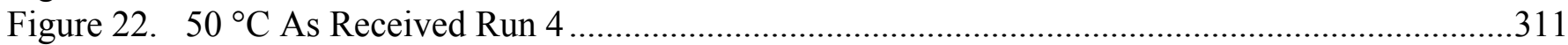

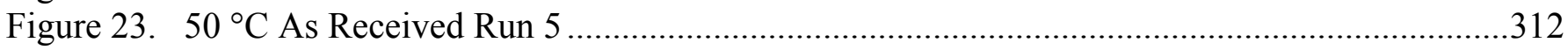

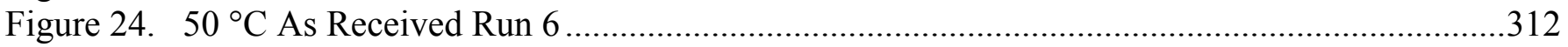

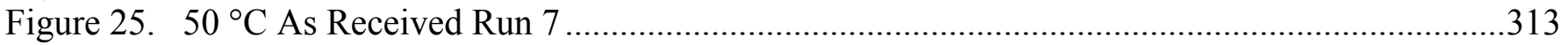

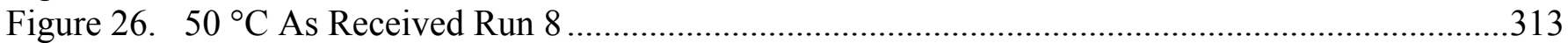

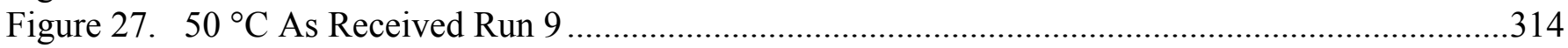

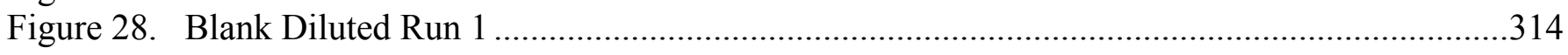

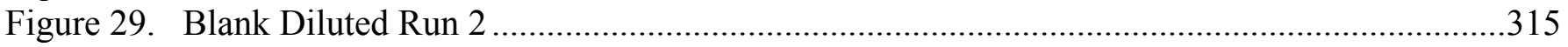

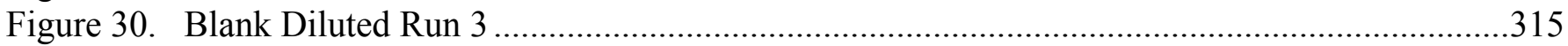

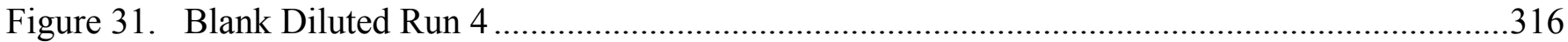

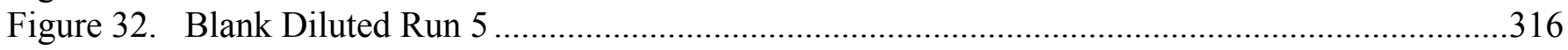

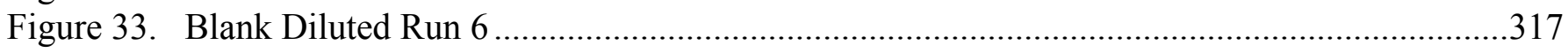

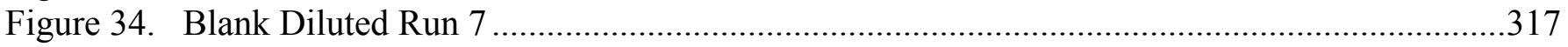

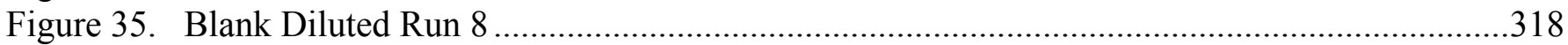

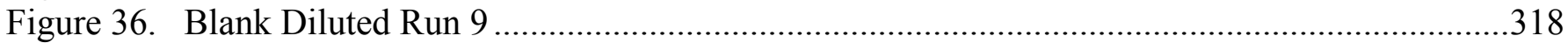

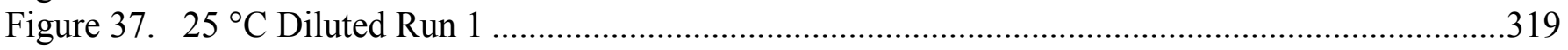

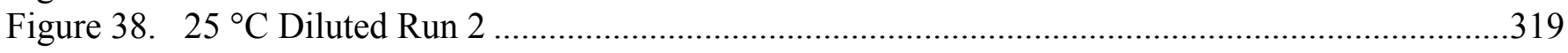

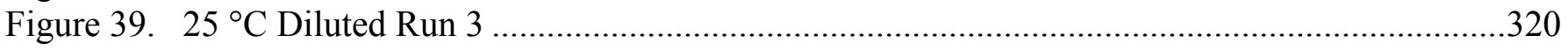


Figure 40. $25^{\circ} \mathrm{C}$ Diluted Run 4 320

Figure 41. $25^{\circ} \mathrm{C}$ Diluted Run 5

Figure 42. $25^{\circ} \mathrm{C}$ Diluted Run 6

Figure 43. $25^{\circ} \mathrm{C}$ Diluted Run 7

Figure 44. $25^{\circ} \mathrm{C}$ Diluted Run 8

Figure 45. $25^{\circ} \mathrm{C}$ Diluted Run 9

Figure 46. $50^{\circ} \mathrm{C}$ Diluted Run 1

Figure 47. $50{ }^{\circ} \mathrm{C}$ Diluted Run 2

Figure 48. $50{ }^{\circ} \mathrm{C}$ Diluted Run 3

Figure 49. $50{ }^{\circ} \mathrm{C}$ Diluted Run 4 325

Figure 50. $50{ }^{\circ} \mathrm{C}$ Diluted Run 5

Figure 51. $50{ }^{\circ} \mathrm{C}$ Diluted Run 6

Figure 52. $50{ }^{\circ} \mathrm{C}$ Diluted Run 7

Figure 53. $50{ }^{\circ} \mathrm{C}$ Diluted Run 8

Figure 54. $50{ }^{\circ} \mathrm{C}$ Diluted Run 9 
$\begin{array}{ll}\text { APPENDIX H - PART } 1 & \text { WSRC-TR-2000-00352 }\end{array}$

ENVELOPE C RHEOGRAMS

SRT-RPP-2000-00026

Page 301 of 514

Blank-Run1

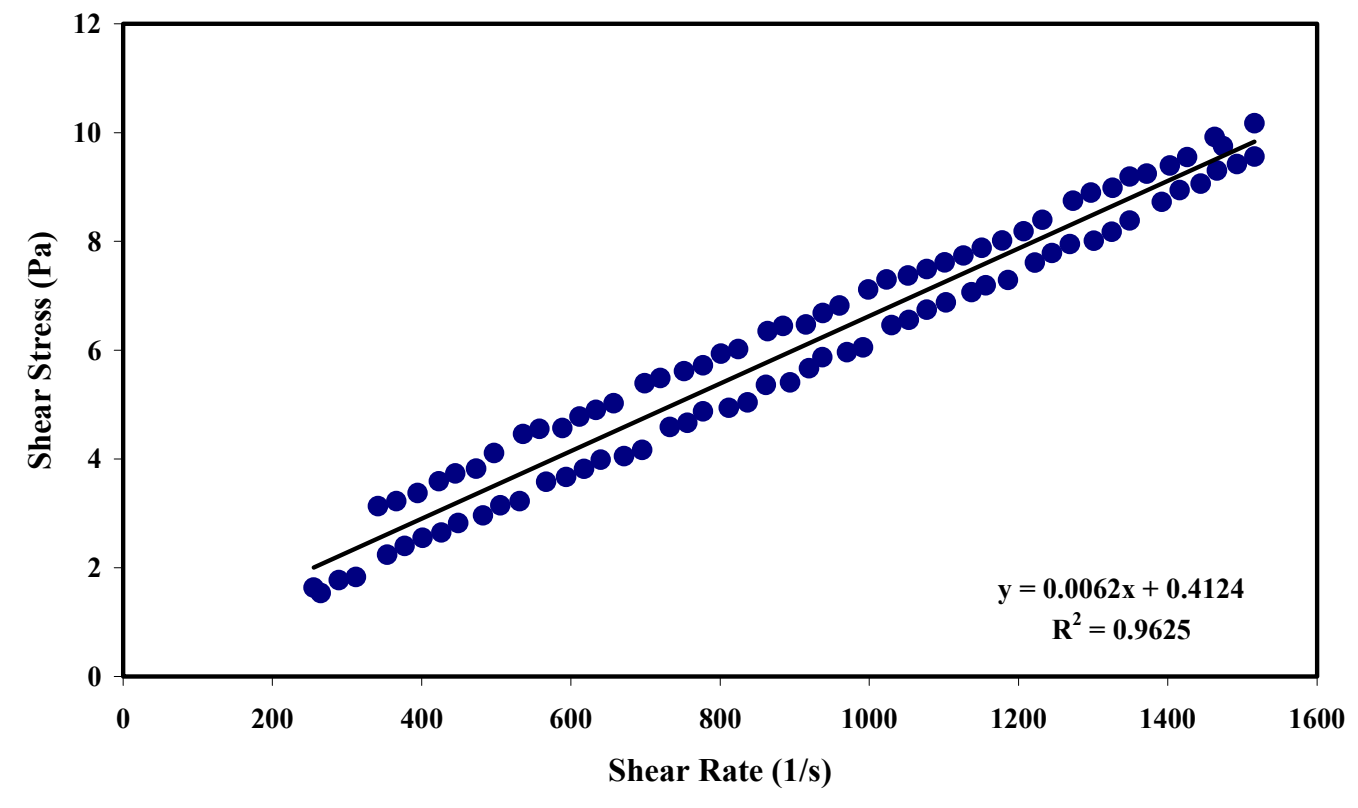

Figure 1. Blank As Received Run 1

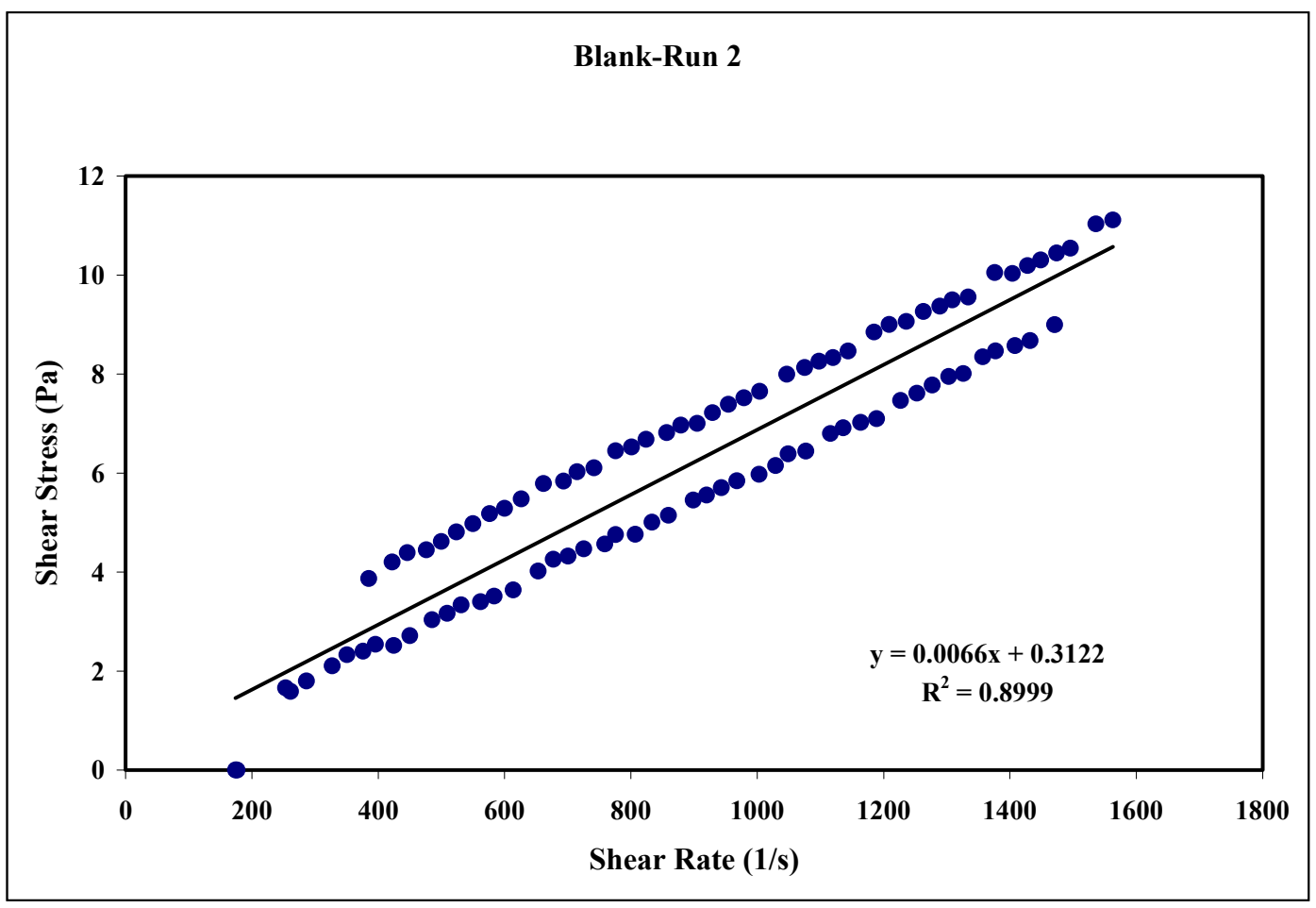

Figure 2. Blank As Received Run 2 
$\begin{array}{ll}\text { APPENDIX H - PART } 1 & \text { WSRC-TR-2000-00352 }\end{array}$

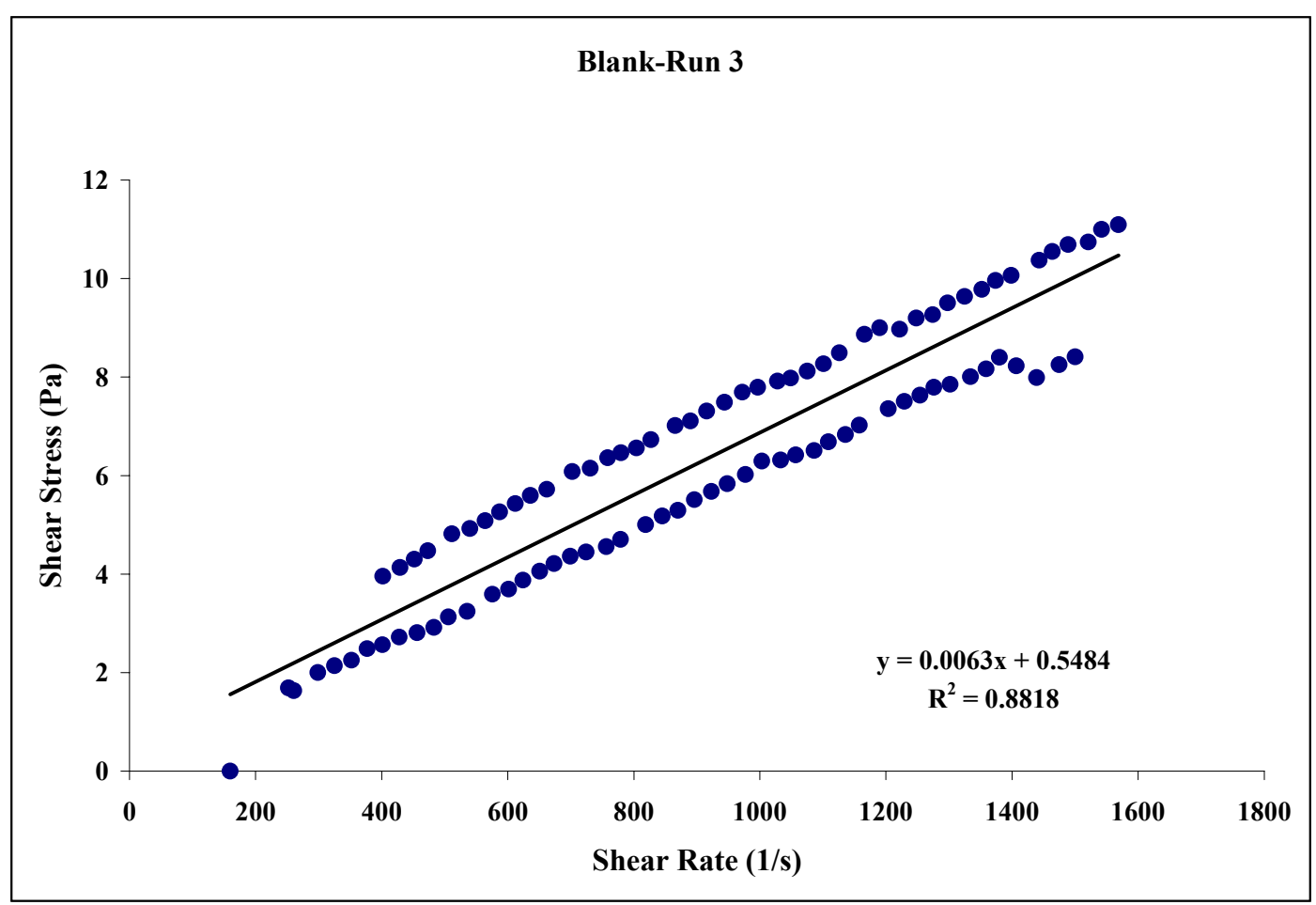

Figure 3. Blank As Received Run 3

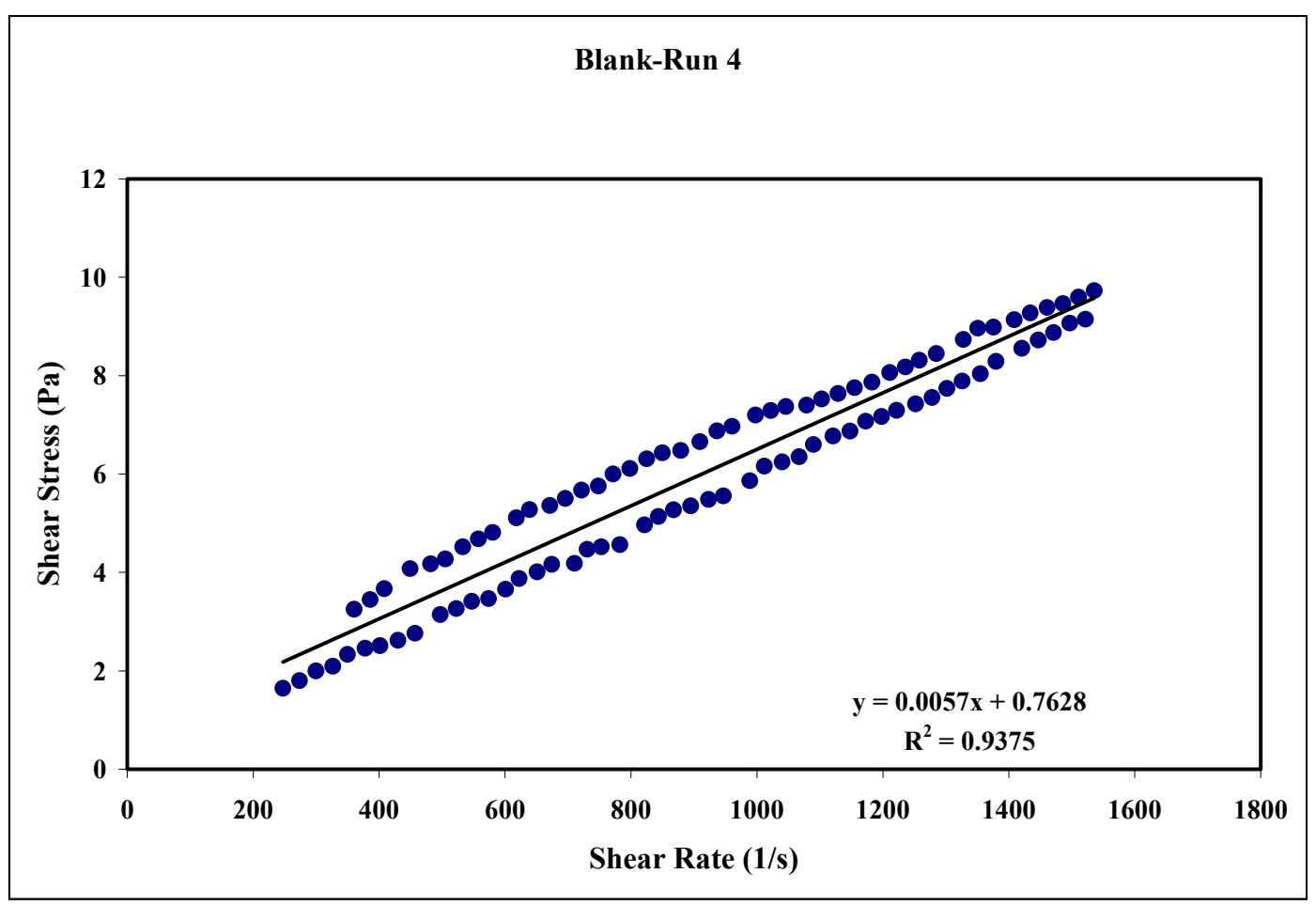

Figure 4. Blank As Received Run 4 
$\begin{array}{ll}\text { APPENDIX H - PART } 1 & \text { WSRC-TR-2000-00352 }\end{array}$

ENVELOPE C RHEOGRAMS

SRT-RPP-2000-00026

Page 303 of 514

\section{Blank-Run 5}

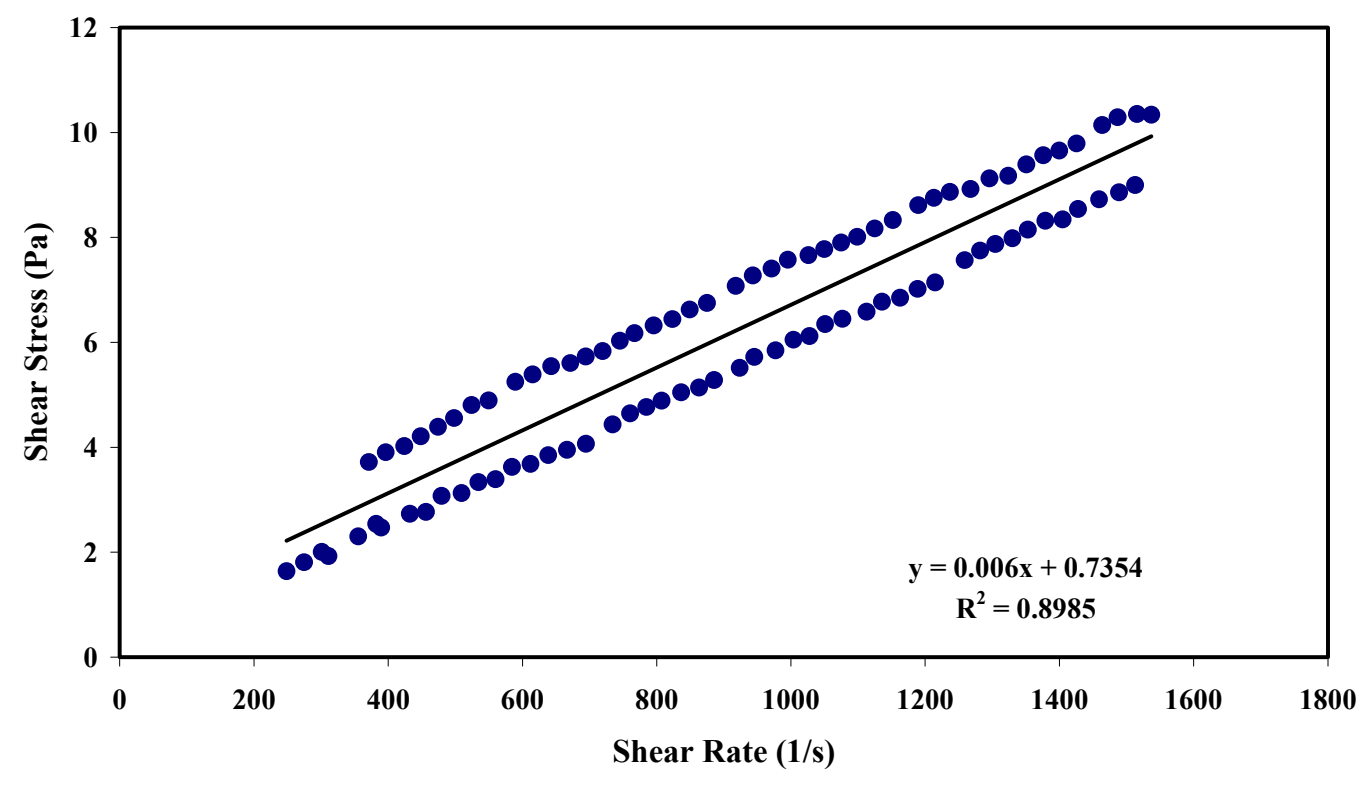

Figure 5. Blank As Received Run 5

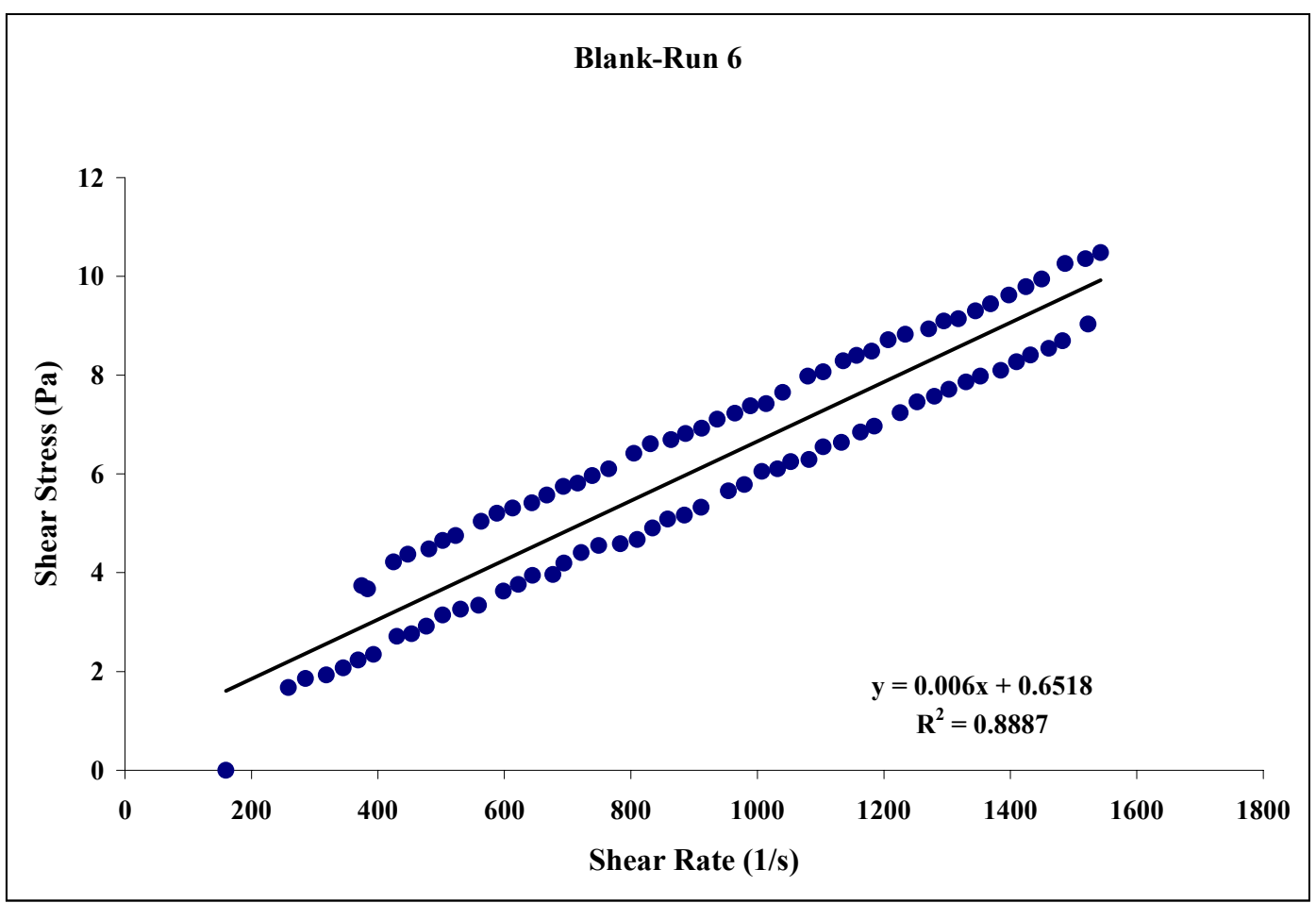

Figure 6. Blank As Received Run 6 
$\begin{array}{ll}\text { APPENDIX H - PART } 1 & \text { WSRC-TR-2000-00352 }\end{array}$

ENVELOPE C RHEOGRAMS

SRT-RPP-2000-00026

Page 304 of 514

\section{Blank-Run 7}

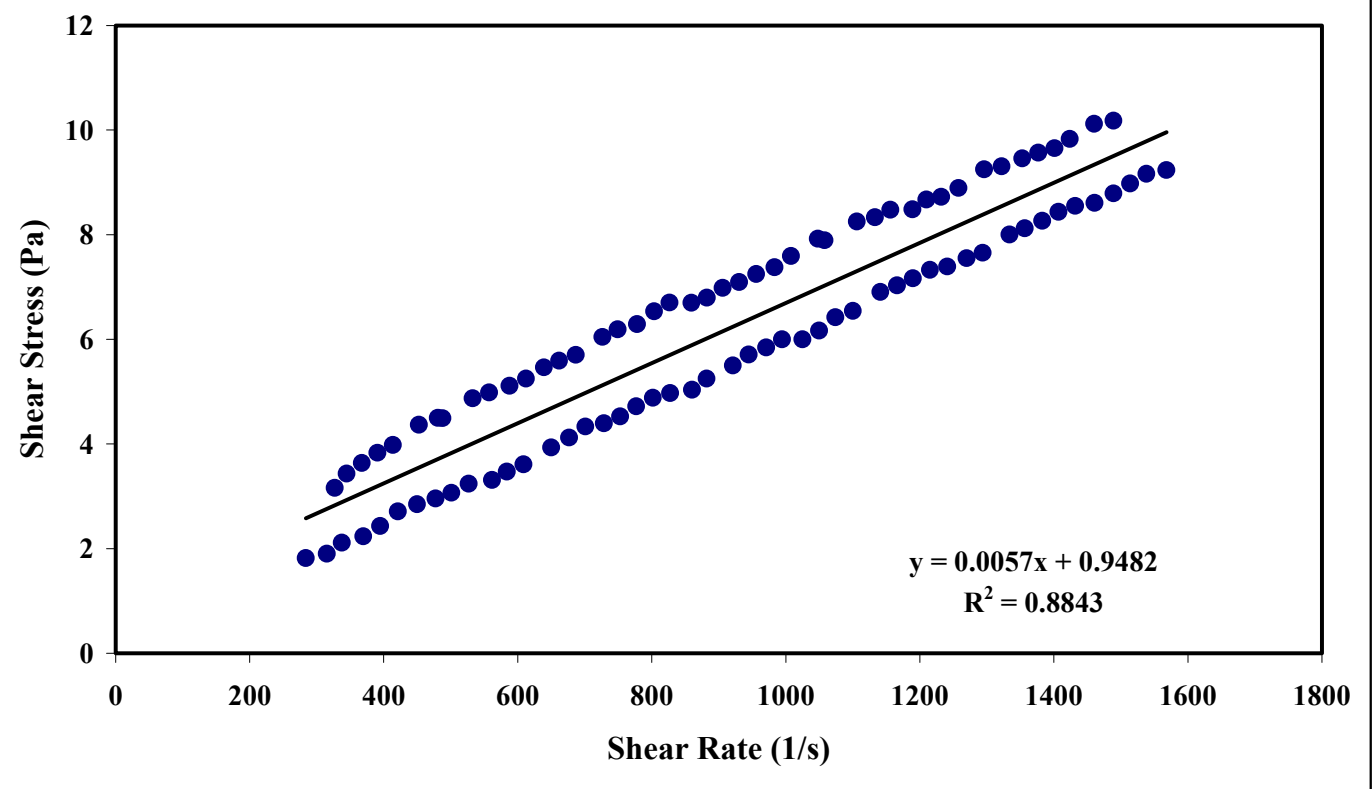

Figure 7. Blank As Received Run 7

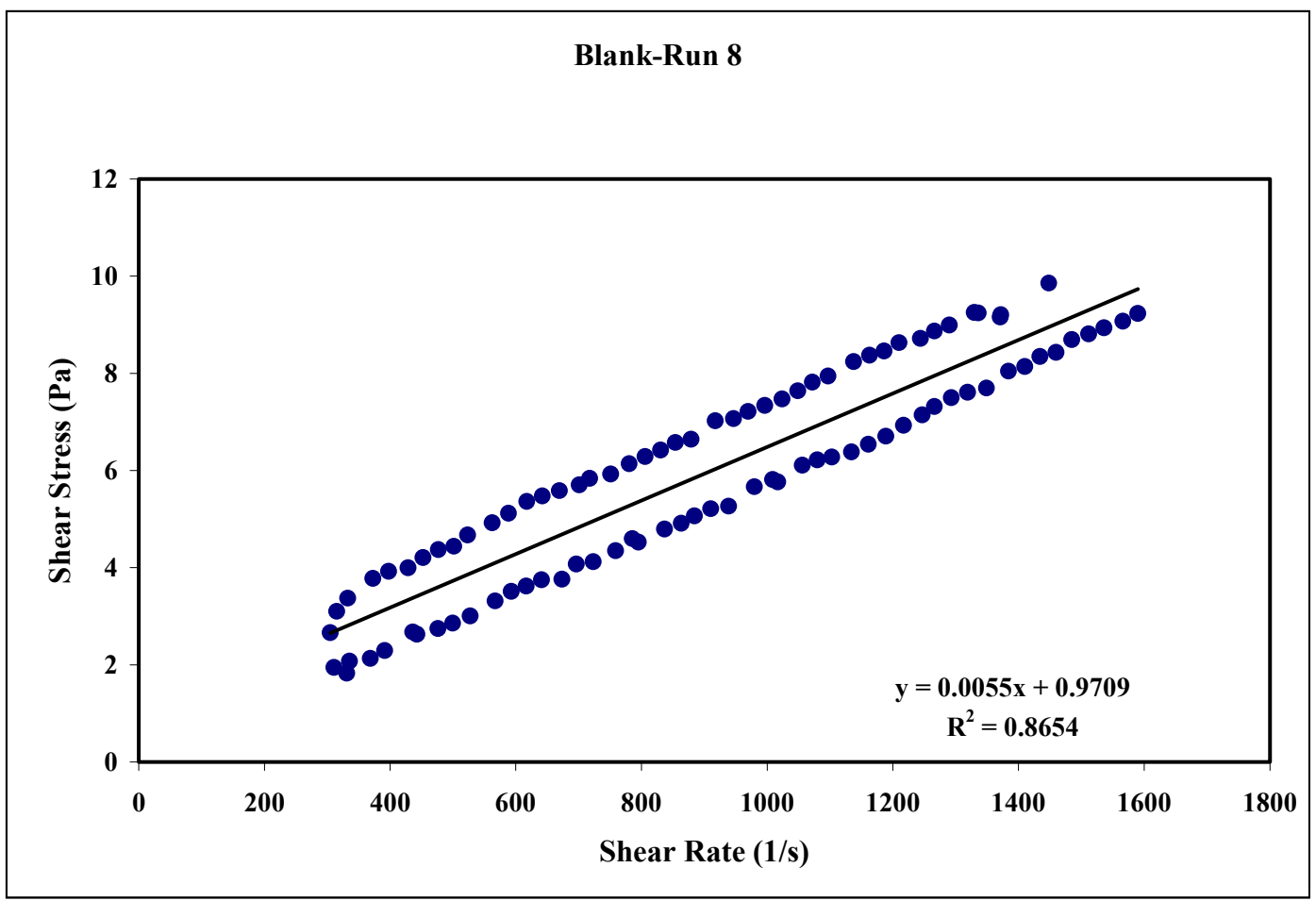

Figure 8. Blank As Received Run 8 
$\begin{array}{ll}\text { APPENDIX H - PART } 1 & \text { WSRC-TR-2000-00352 }\end{array}$

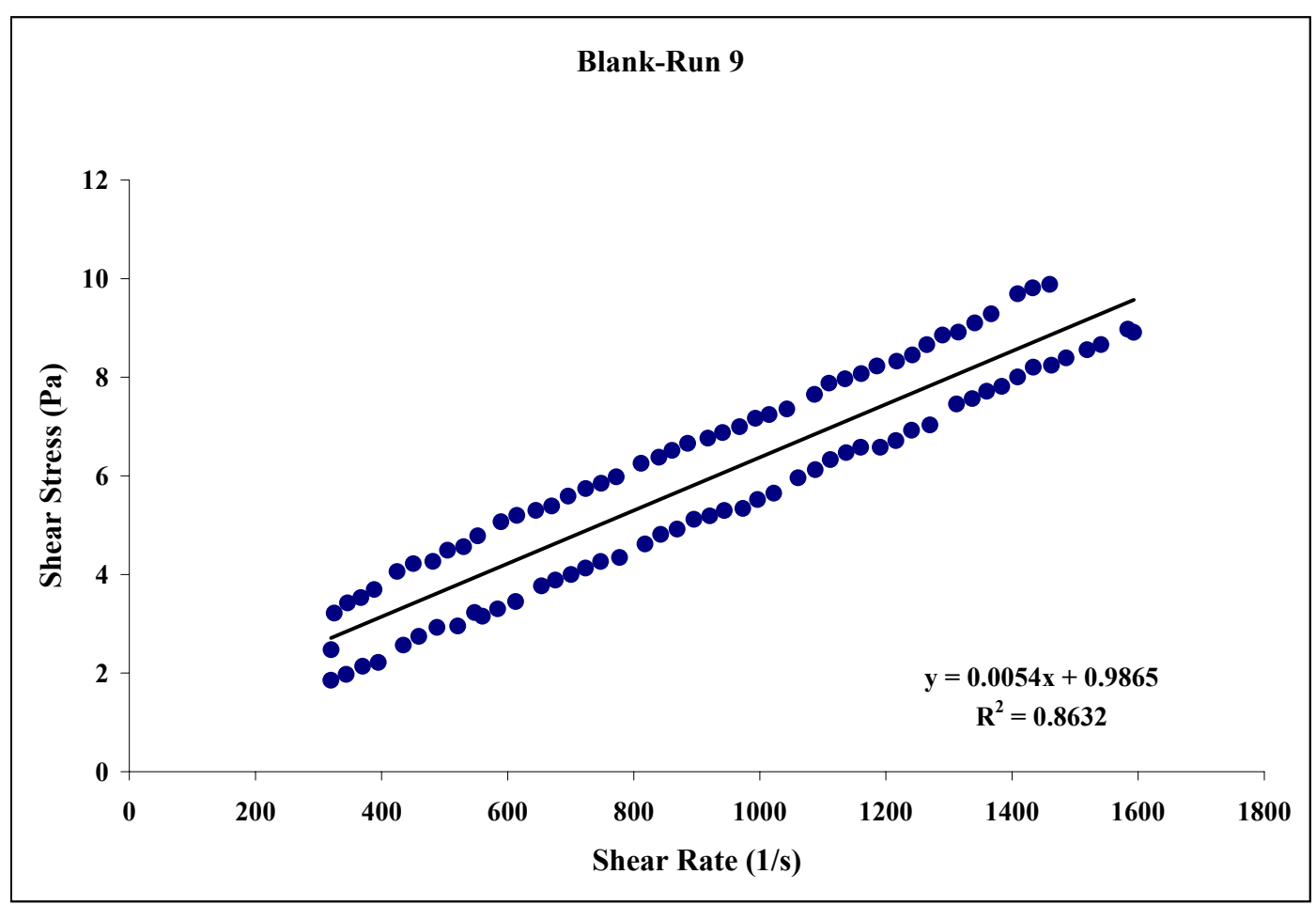

Figure 9. Blank As Received Run 9

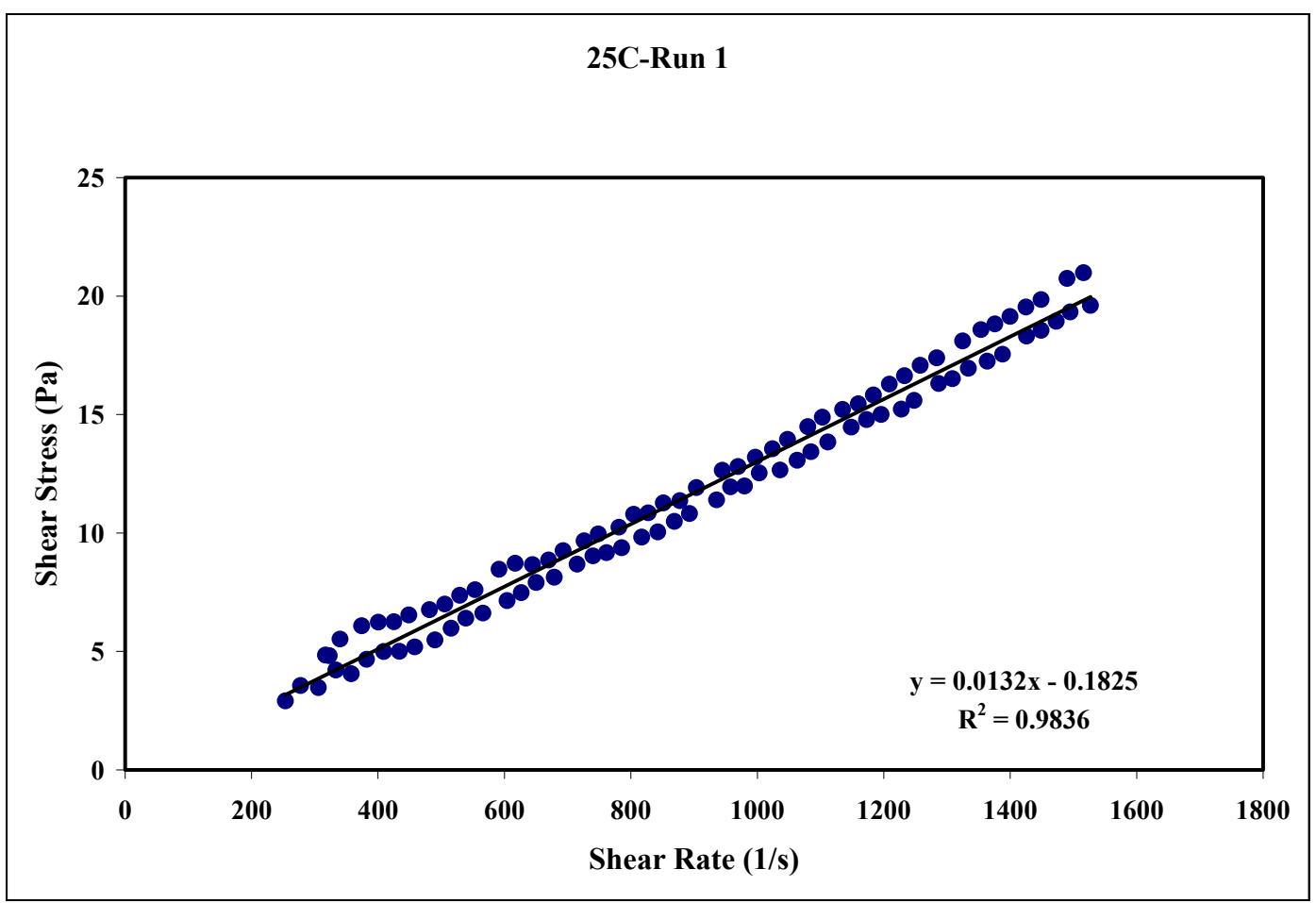

Figure 10. $25^{\circ} \mathrm{C}$ As Received Run 1 
$\begin{array}{ll}\text { APPENDIX H - PART } 1 & \text { WSRC-TR-2000-00352 }\end{array}$

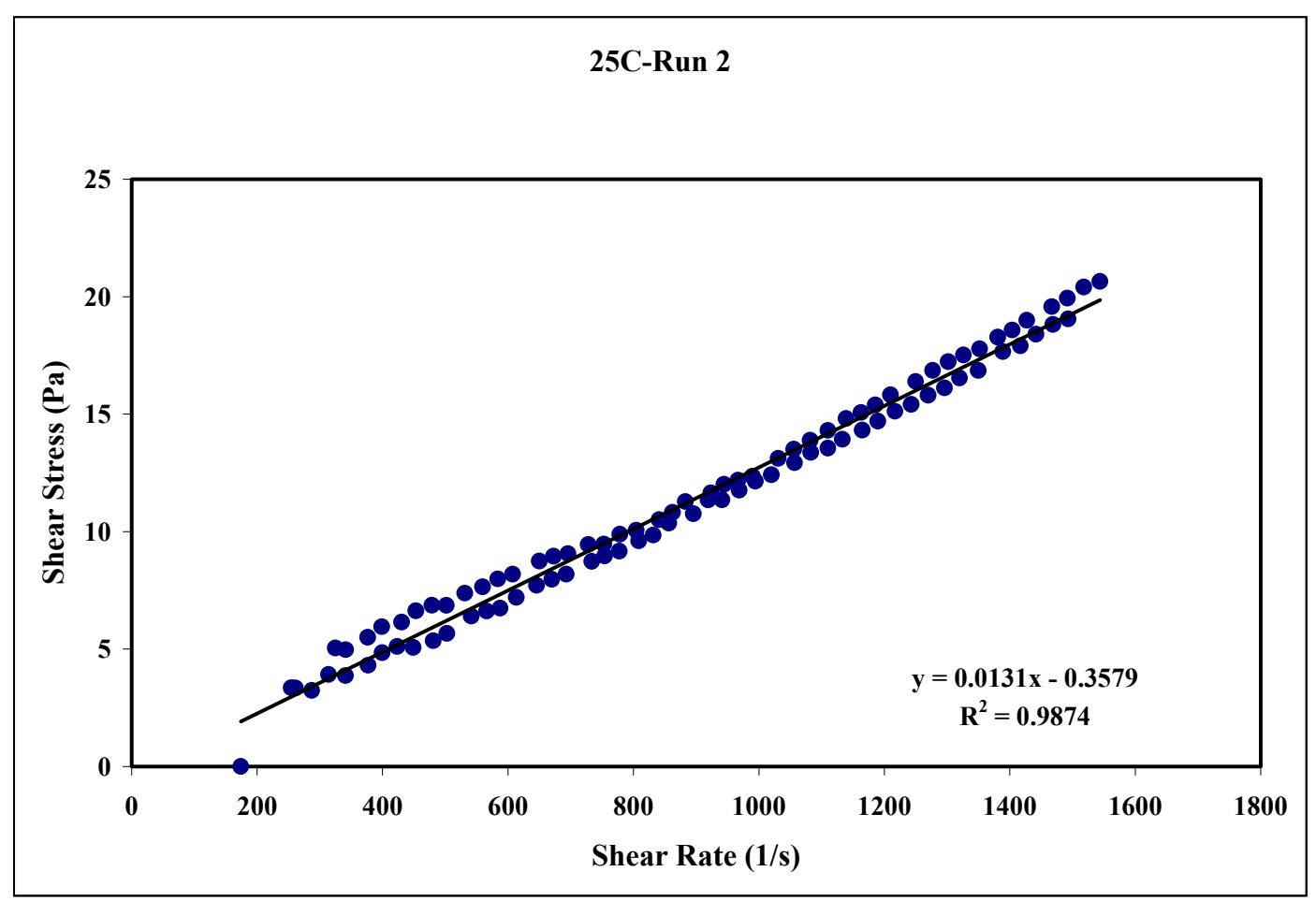

Figure 11. $25^{\circ} \mathrm{C}$ As Received Run 2

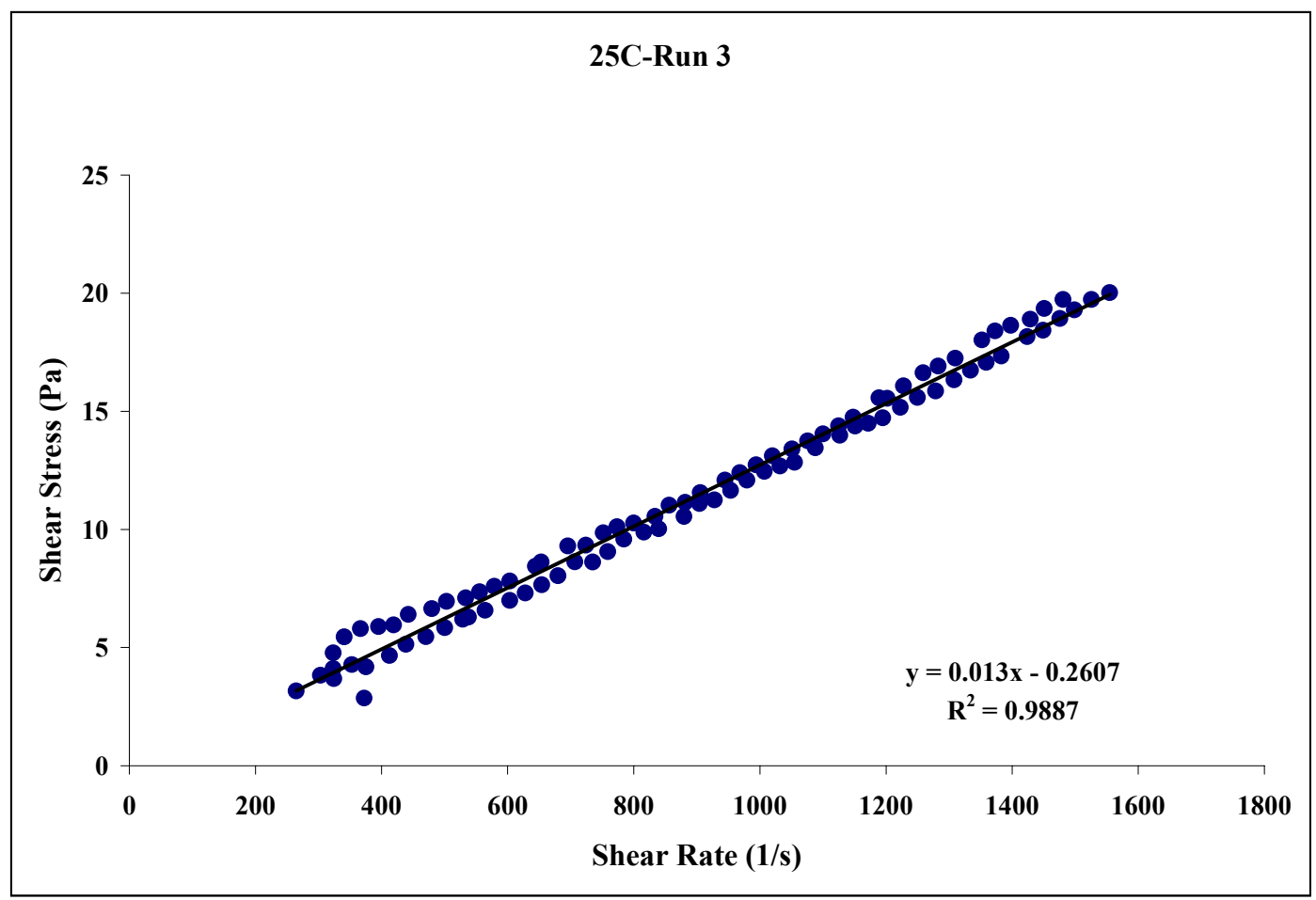

Figure 12. $25^{\circ} \mathrm{C}$ As Received Run 3 
$\begin{array}{ll}\text { APPENDIX H - PART } 1 & \text { WSRC-TR-2000-00352 }\end{array}$

ENVELOPE C RHEOGRAMS

SRT-RPP-2000-00026

Page 307 of 514

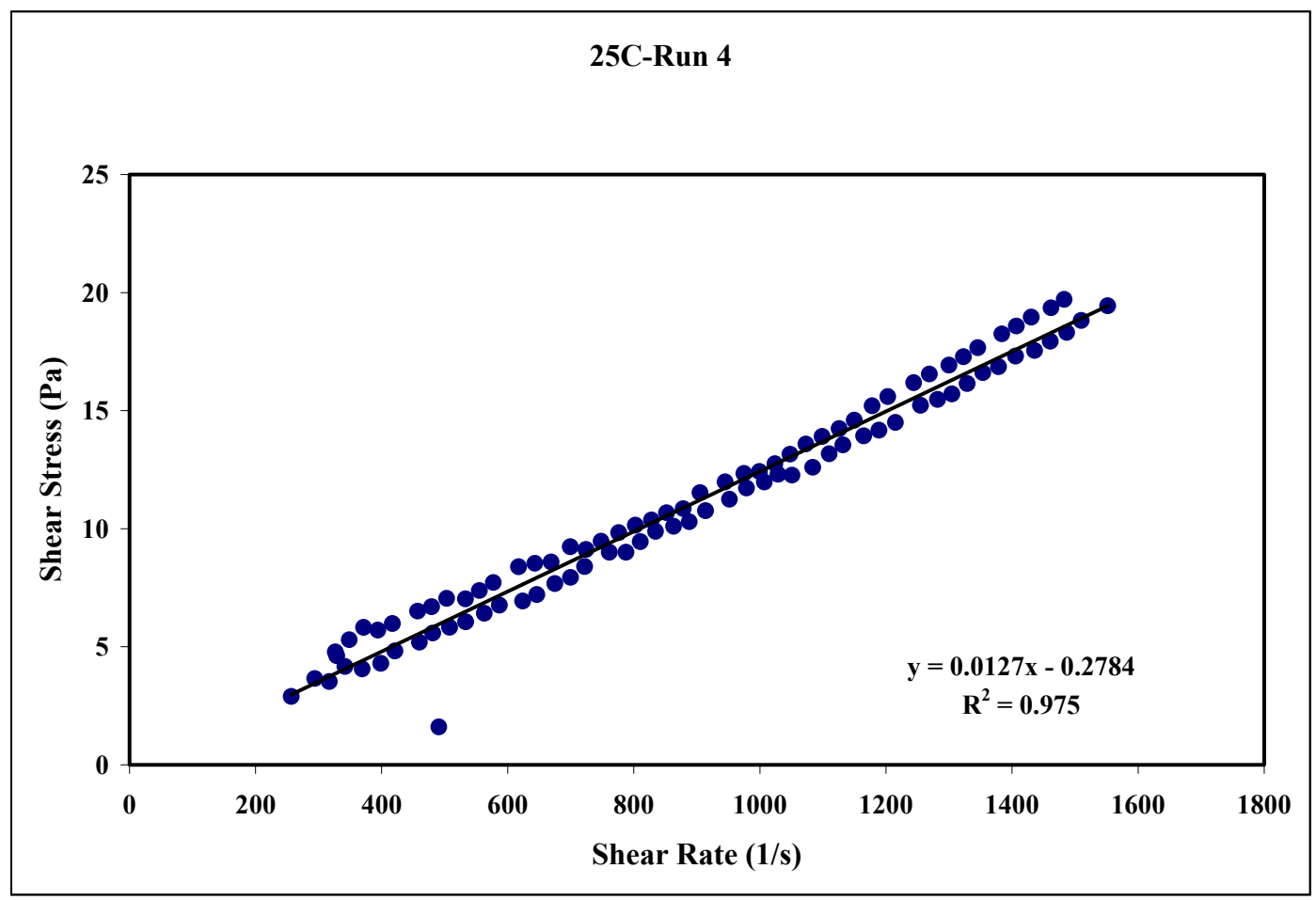

Figure 13. $25^{\circ} \mathrm{C}$ As Received Run 4

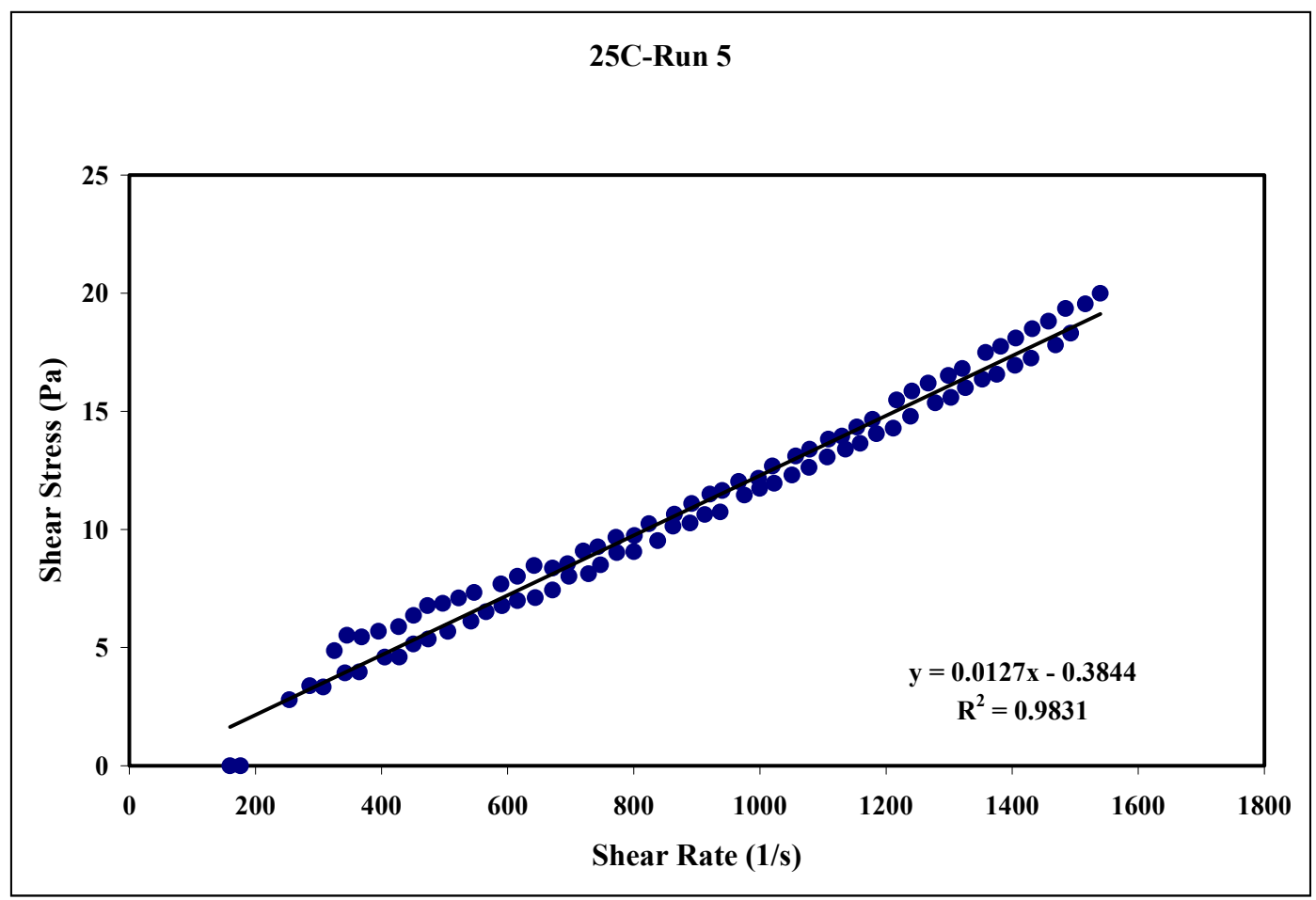

Figure 14. $25^{\circ} \mathrm{C}$ As Received Run 5 
$\begin{array}{ll}\text { APPENDIX H - PART } 1 & \text { WSRC-TR-2000-00352 }\end{array}$

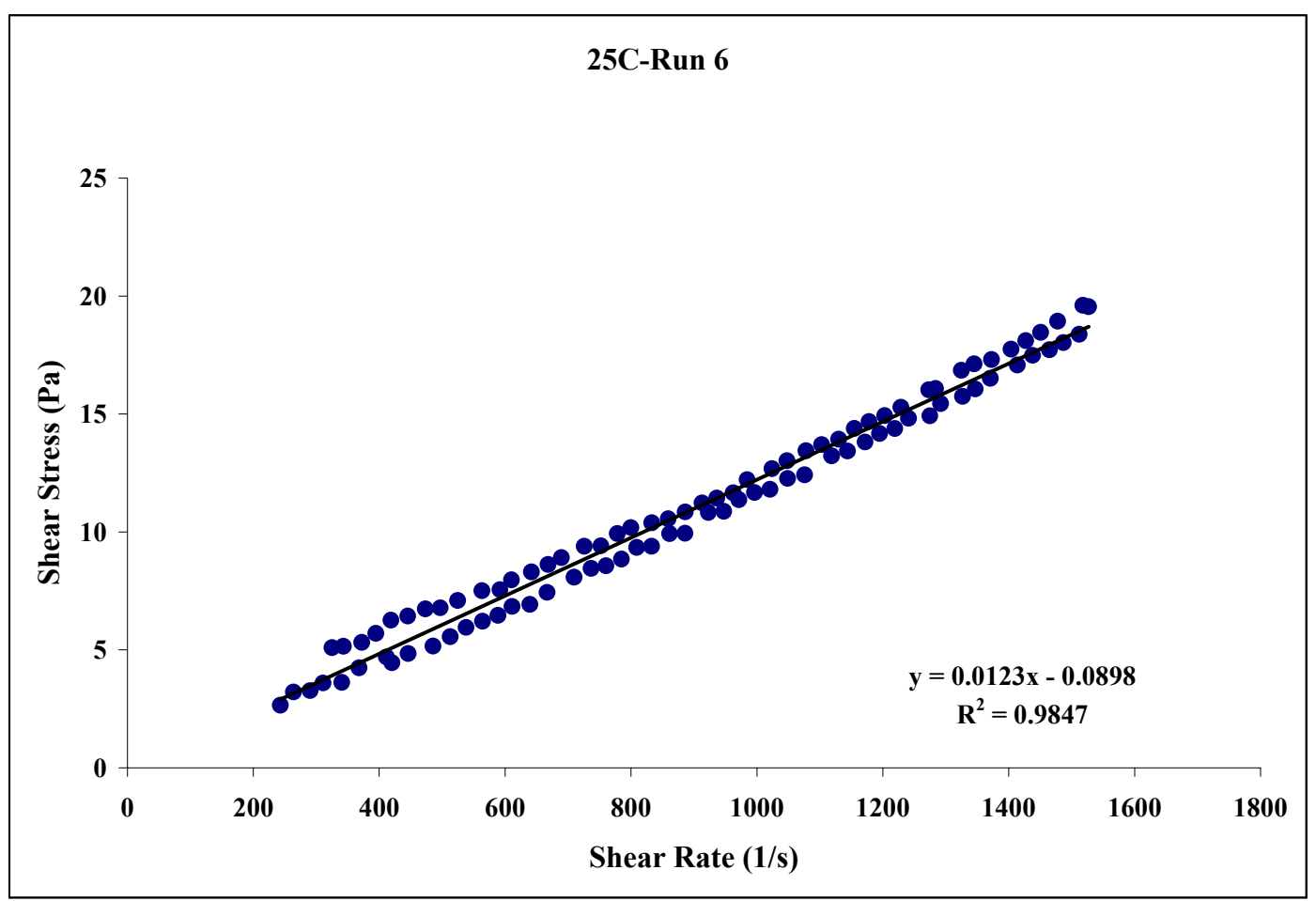

Figure 15. $25^{\circ} \mathrm{C}$ As Received Run 6

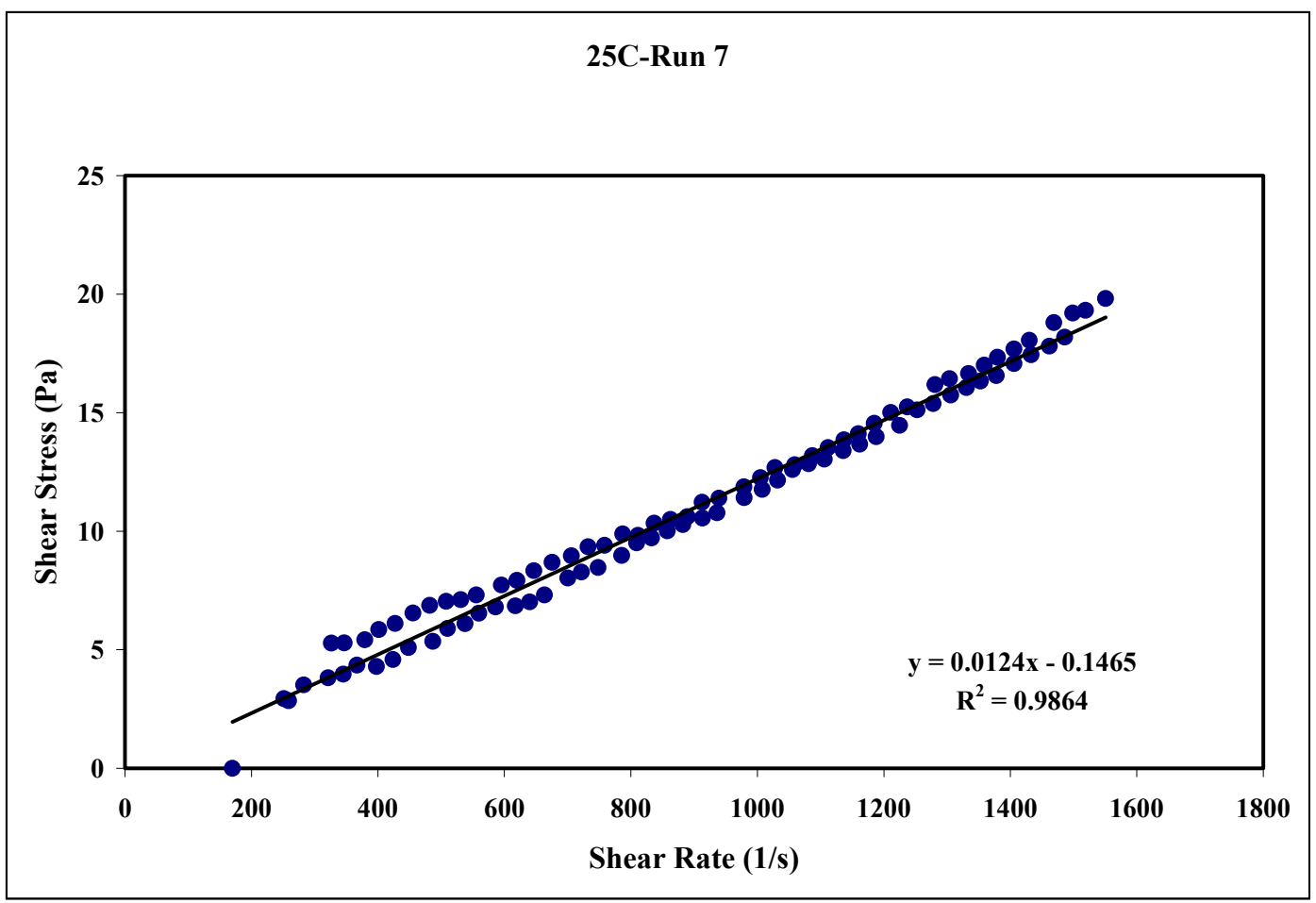

Figure 16. $25^{\circ} \mathrm{C}$ As Received Run 7 
$\begin{array}{ll}\text { APPENDIX H - PART } 1 & \text { WSRC-TR-2000-00352 }\end{array}$

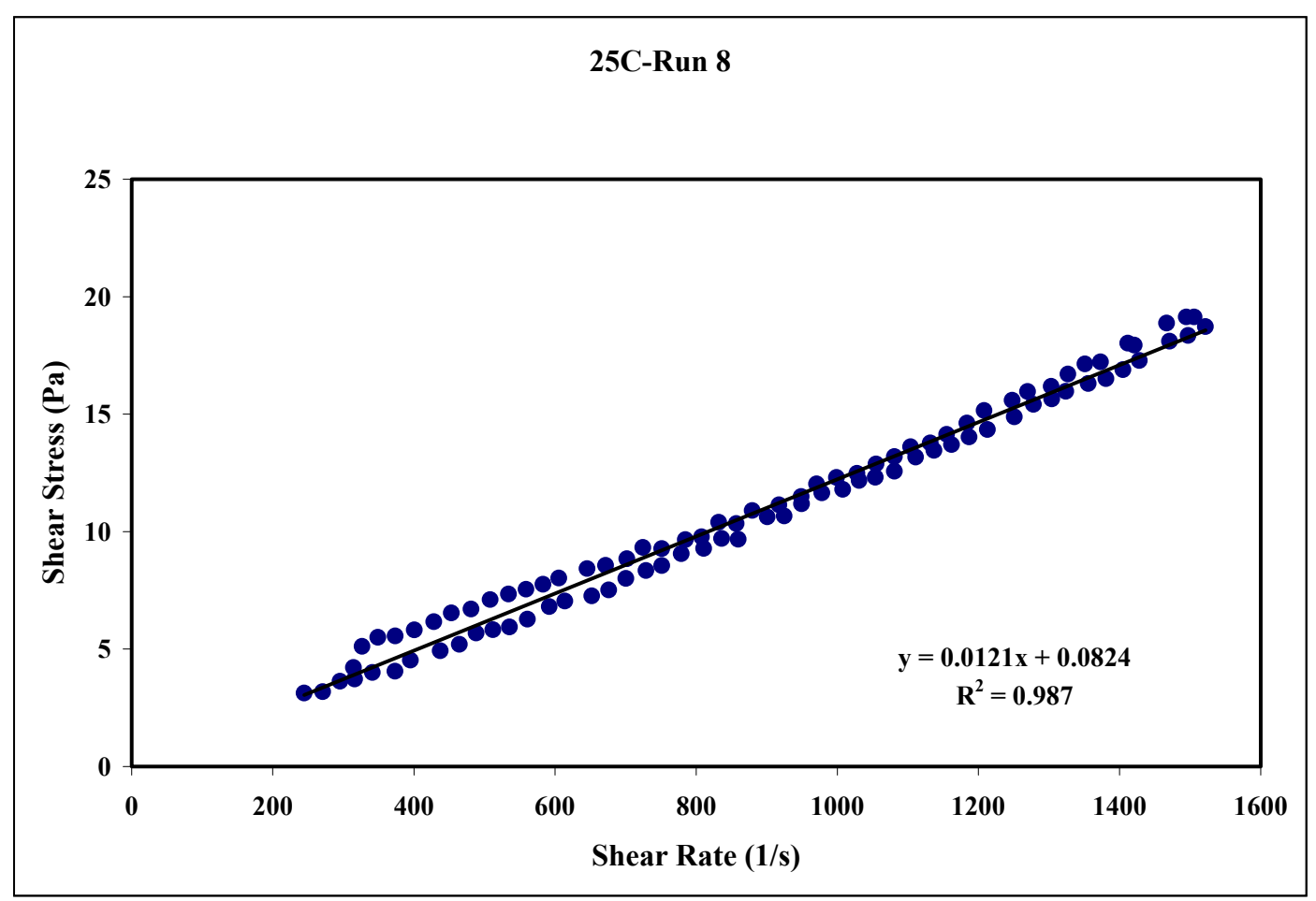

Figure 17. $25^{\circ} \mathrm{C}$ As Received Run 8

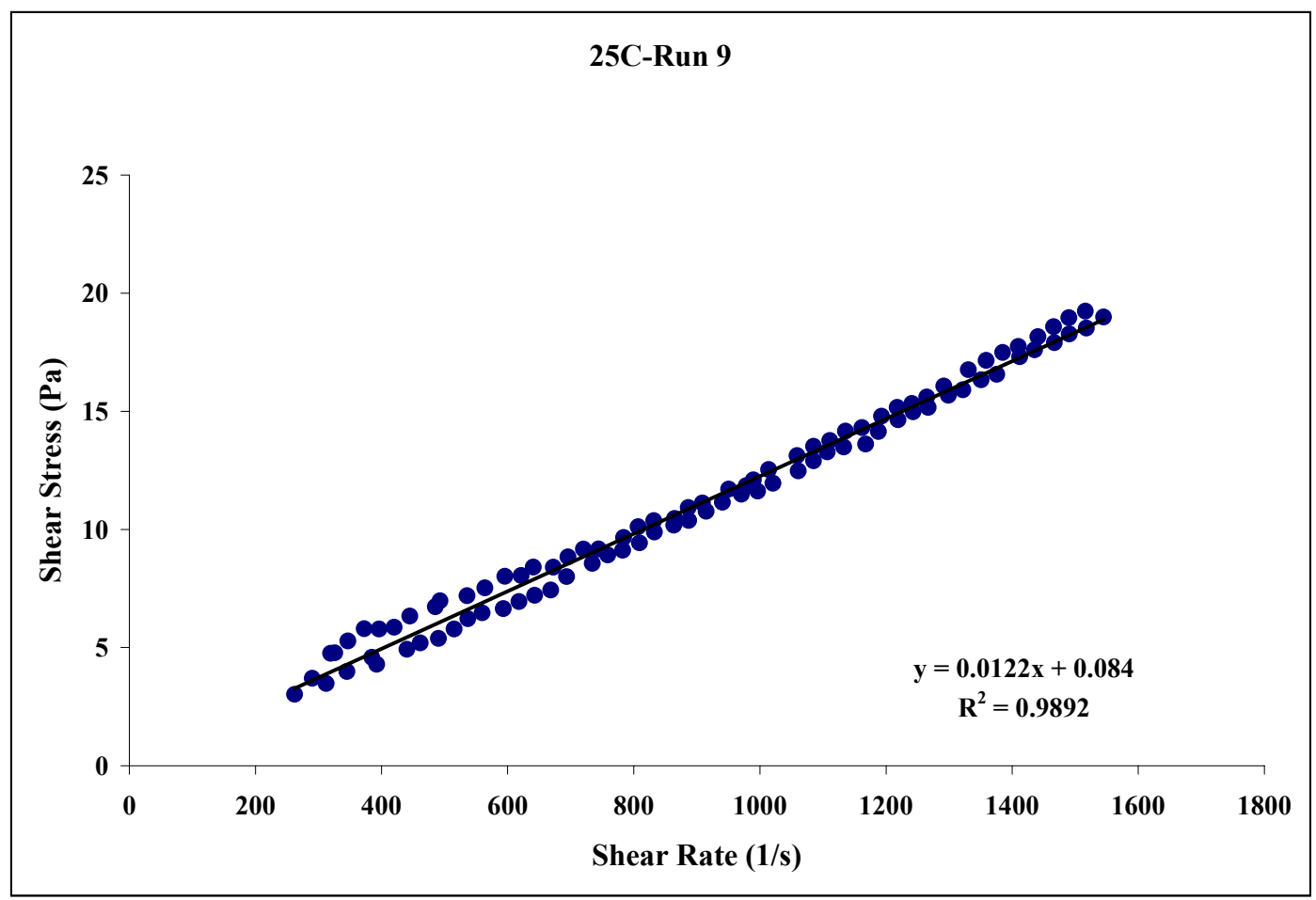

Figure 18. $25^{\circ} \mathrm{C}$ As Received Run 9 
$\begin{array}{ll}\text { APPENDIX H - PART } 1 & \text { WSRC-TR-2000-00352 }\end{array}$

ENVELOPE C RHEOGRAMS

SRT-RPP-2000-00026

Page 310 of 514

50C-Run 1

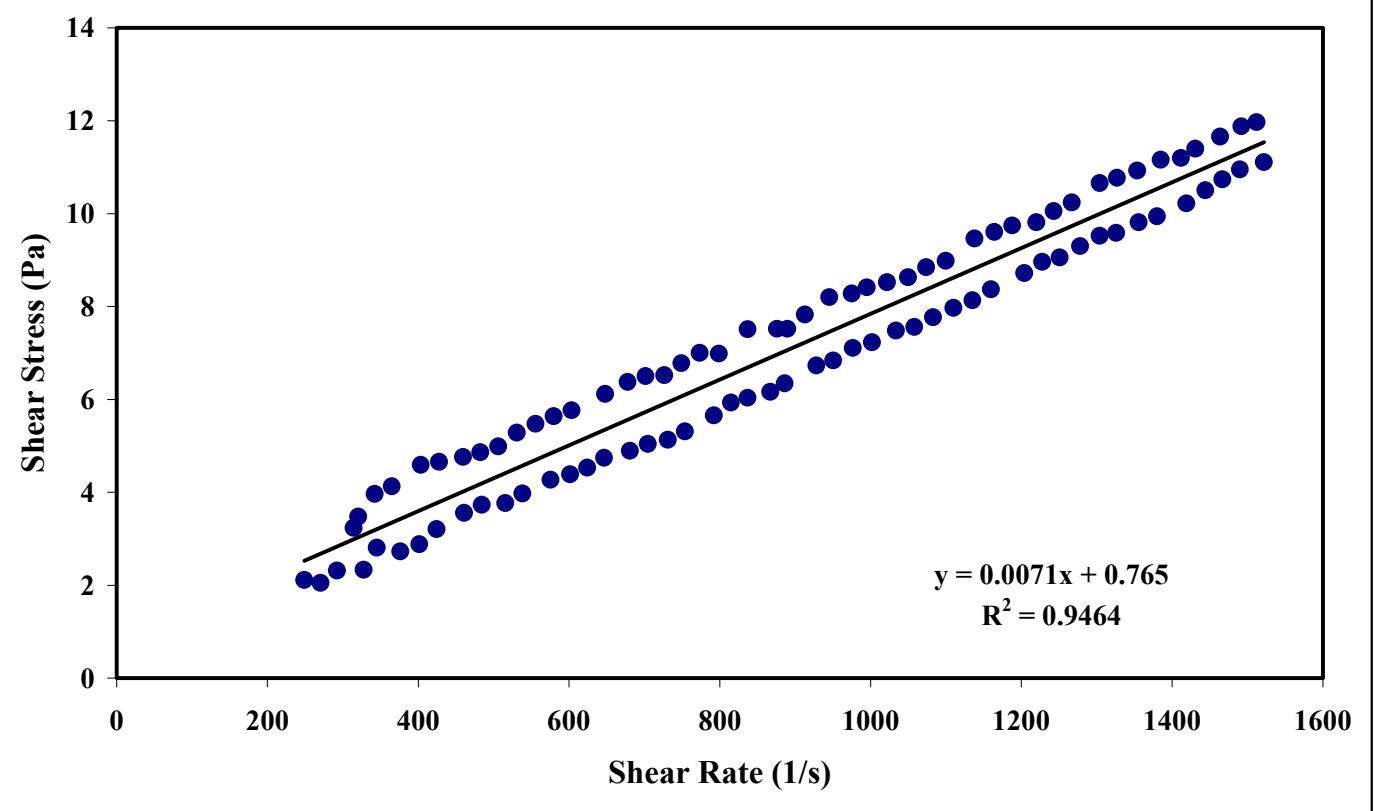

Figure 19. $50{ }^{\circ} \mathrm{C}$ As Received Run 1

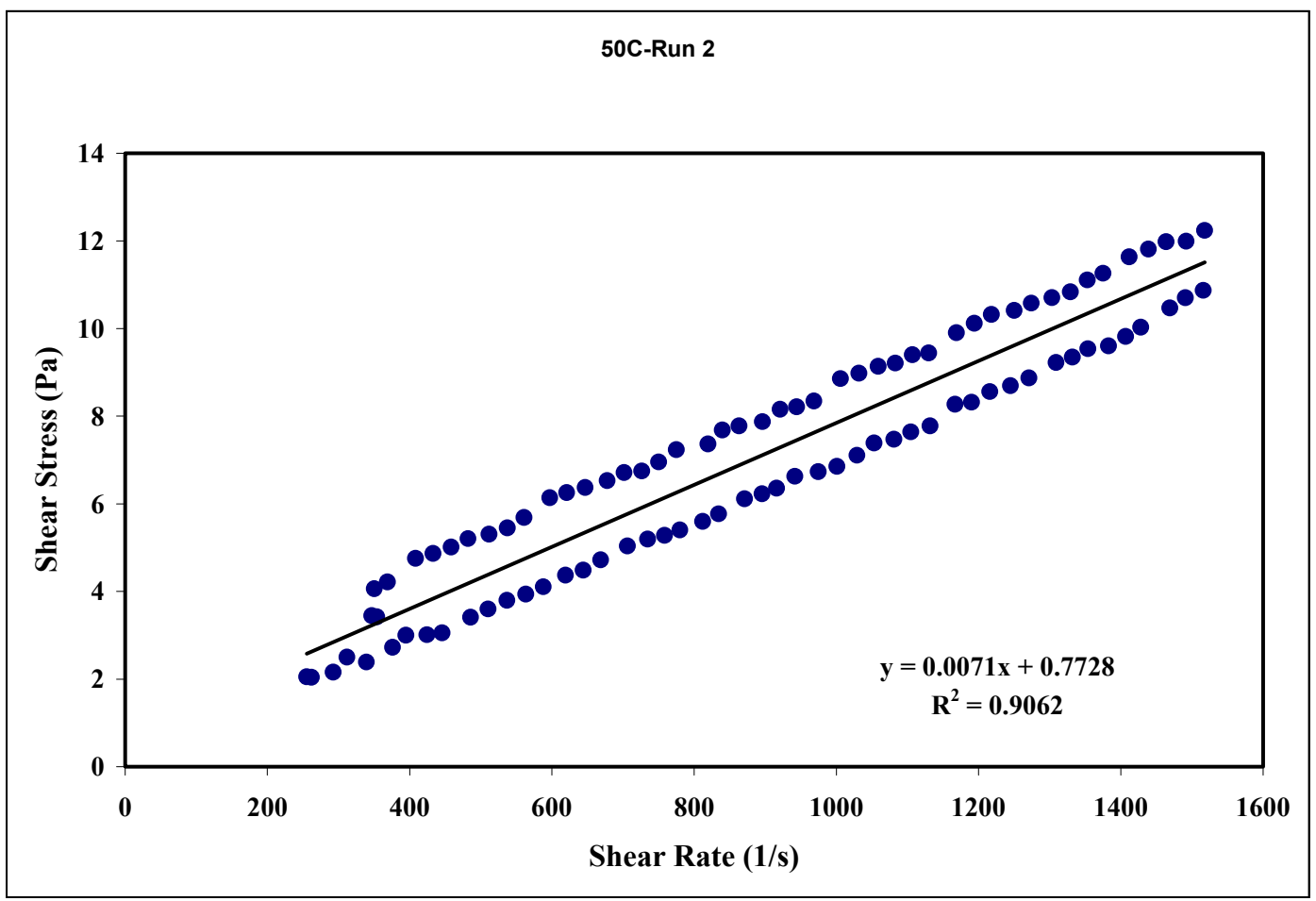

Figure 20. $50{ }^{\circ} \mathrm{C}$ As Received Run 2 
$\begin{array}{ll}\text { APPENDIX H - PART } 1 & \text { WSRC-TR-2000-00352 }\end{array}$

ENVELOPE C RHEOGRAMS

SRT-RPP-2000-00026

Page 311 of 514

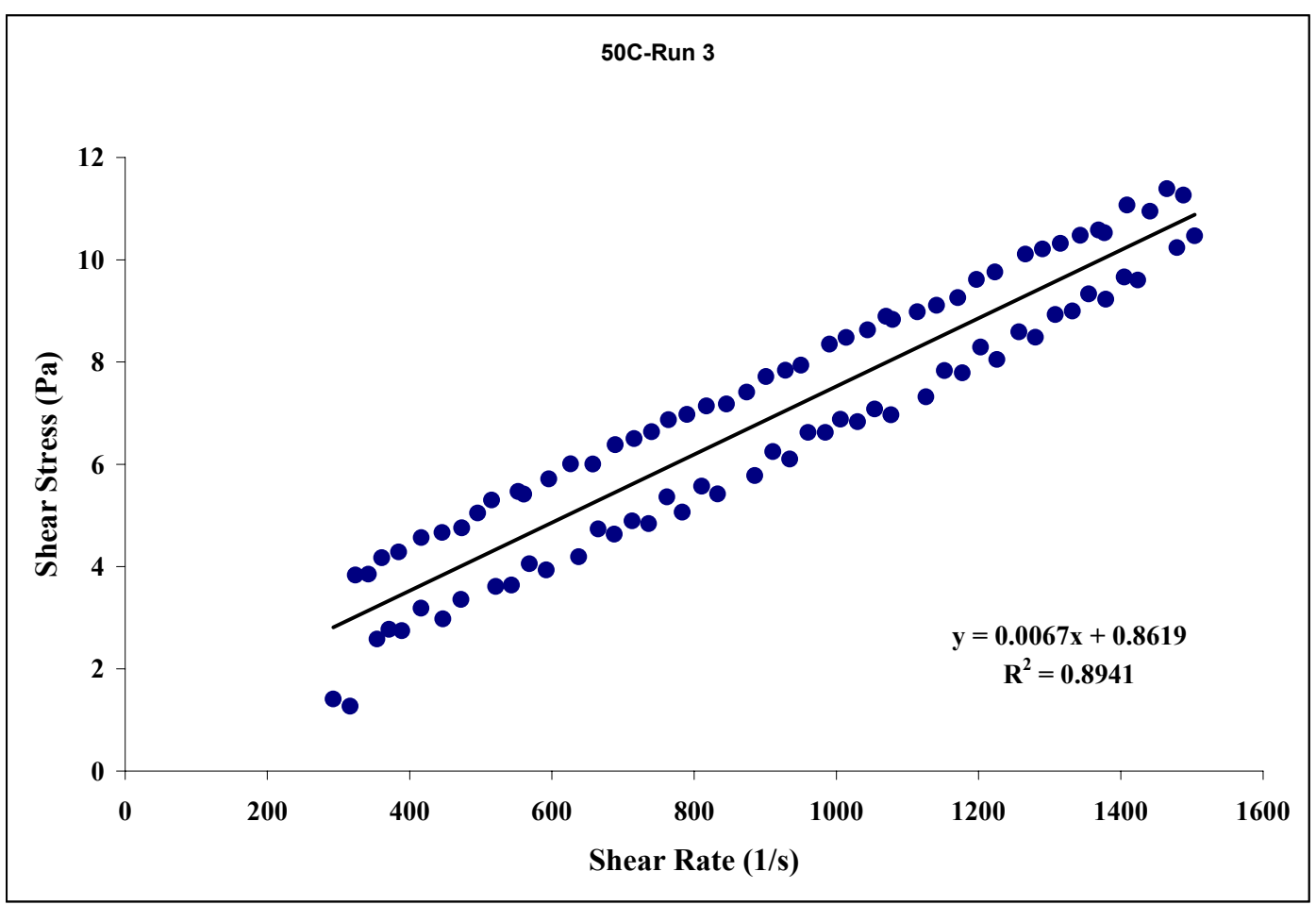

Figure 21. $50{ }^{\circ} \mathrm{C}$ As Received Run 3

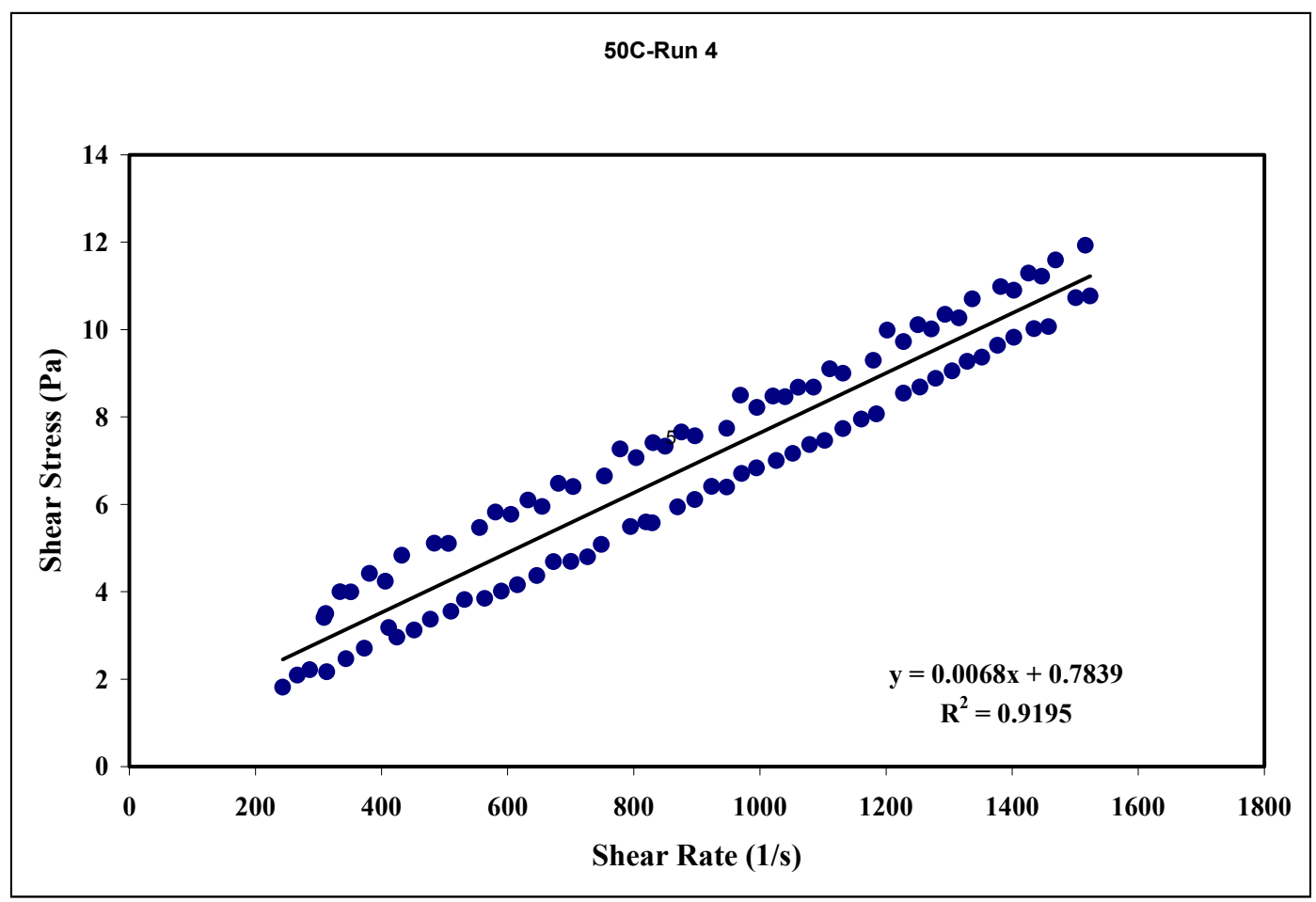

Figure 22. $50{ }^{\circ} \mathrm{C}$ As Received Run 4 
$\begin{array}{ll}\text { APPENDIX H - PART } 1 & \text { WSRC-TR-2000-00352 }\end{array}$

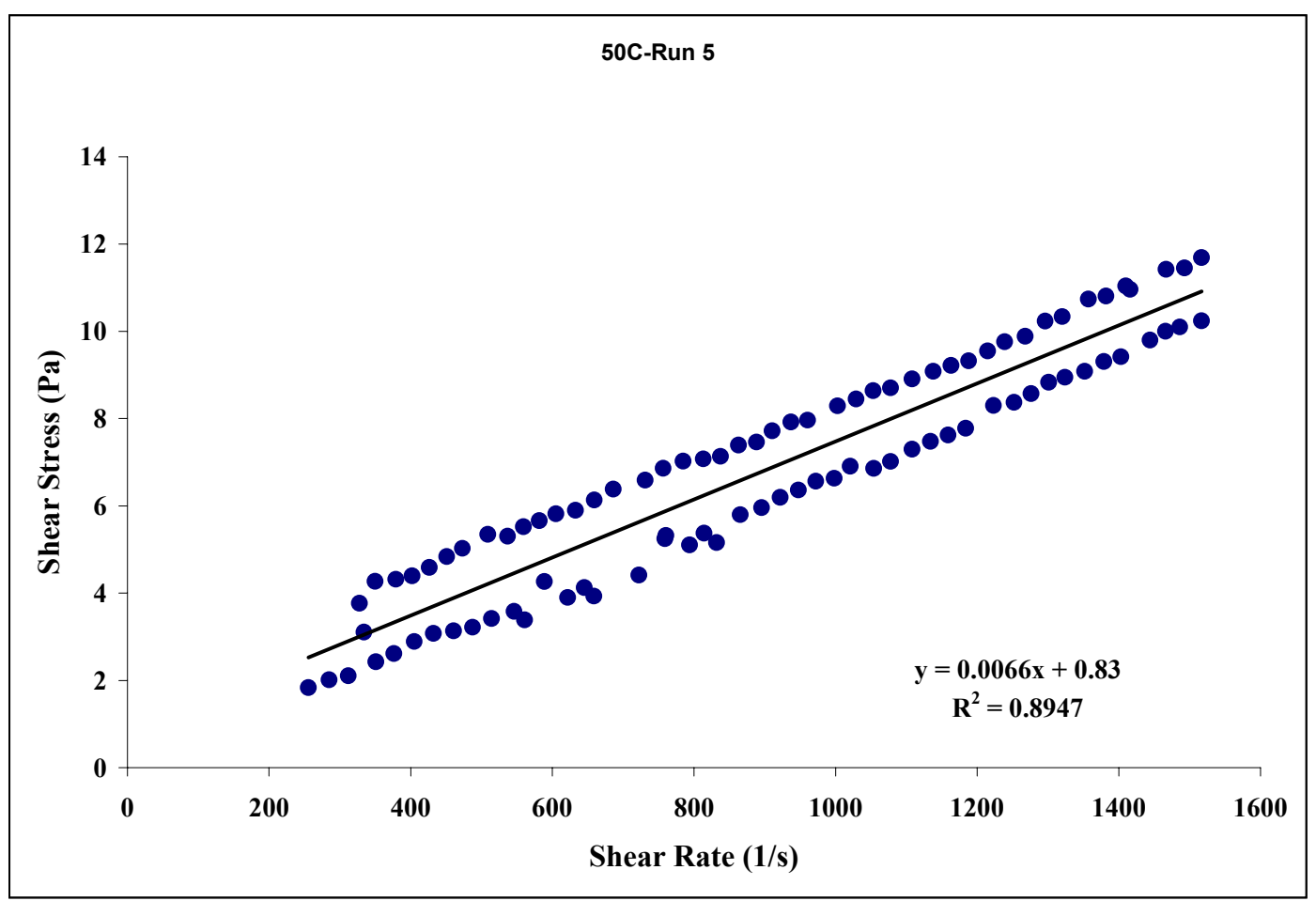

Figure 23. $50{ }^{\circ} \mathrm{C}$ As Received Run 5

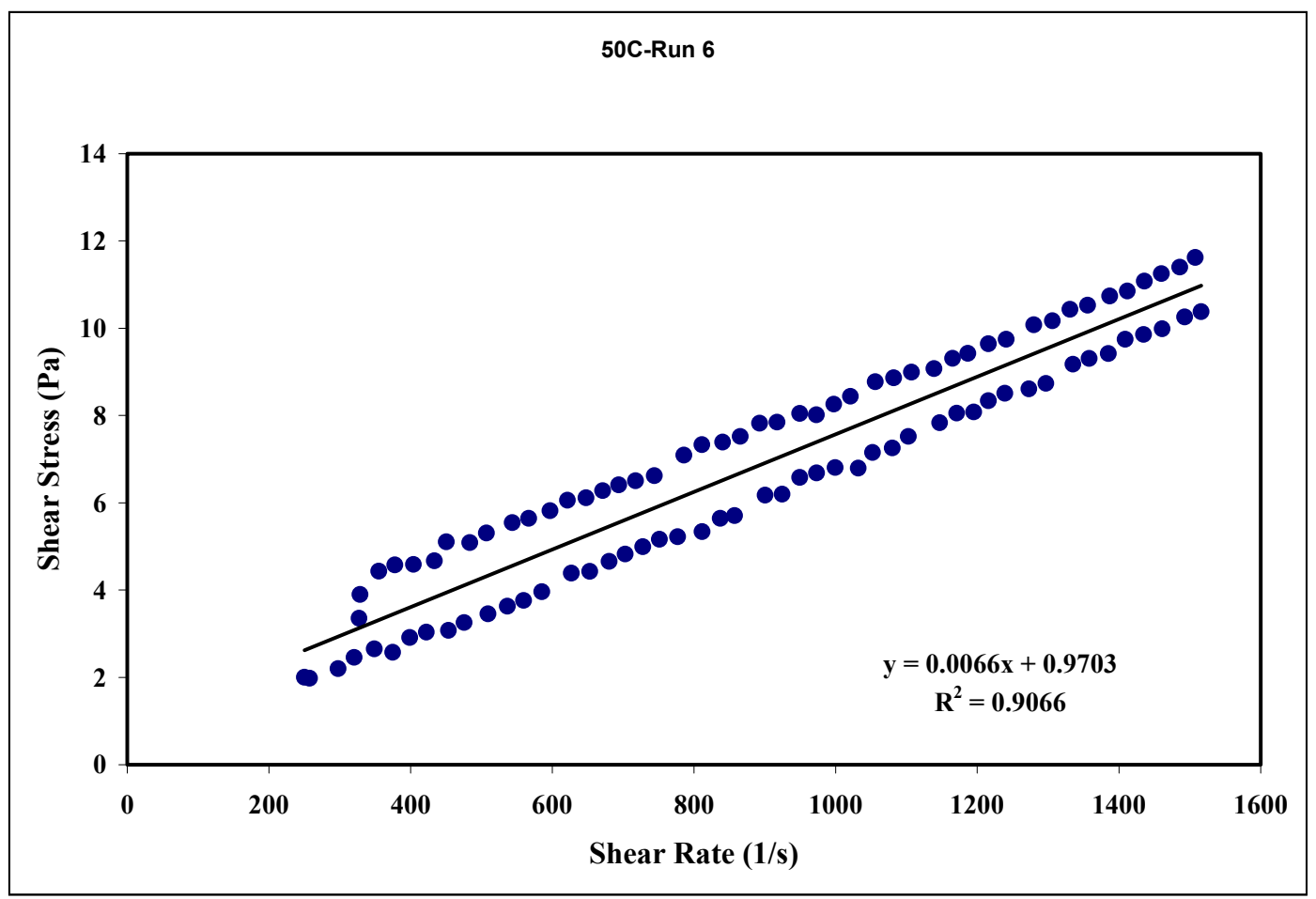

Figure 24. $50{ }^{\circ} \mathrm{C}$ As Received Run 6 
$\begin{array}{ll}\text { APPENDIX H - PART } 1 & \text { WSRC-TR-2000-00352 }\end{array}$

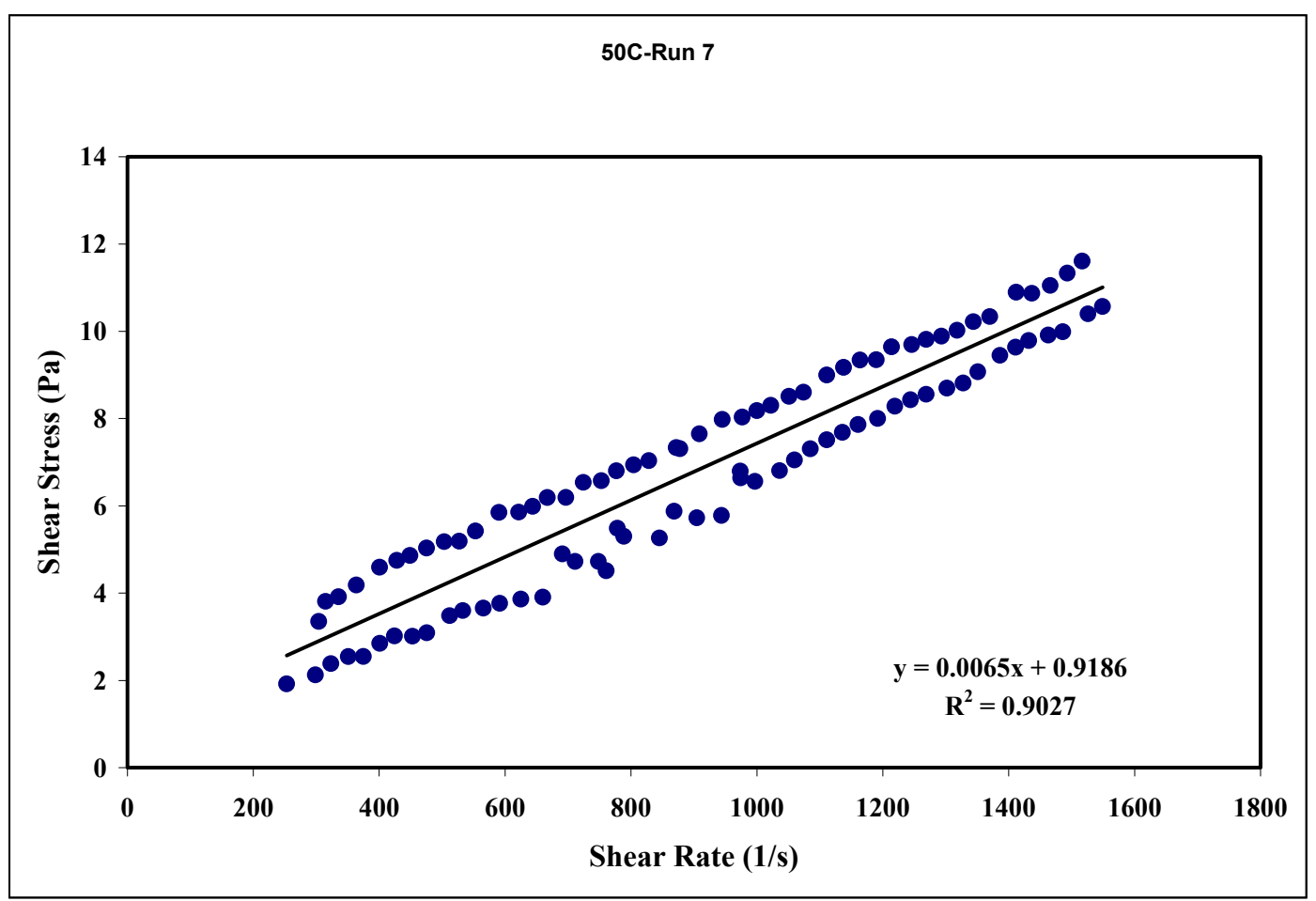

Figure 25. $5{ }^{\circ} \mathrm{C}$ As Received Run 7

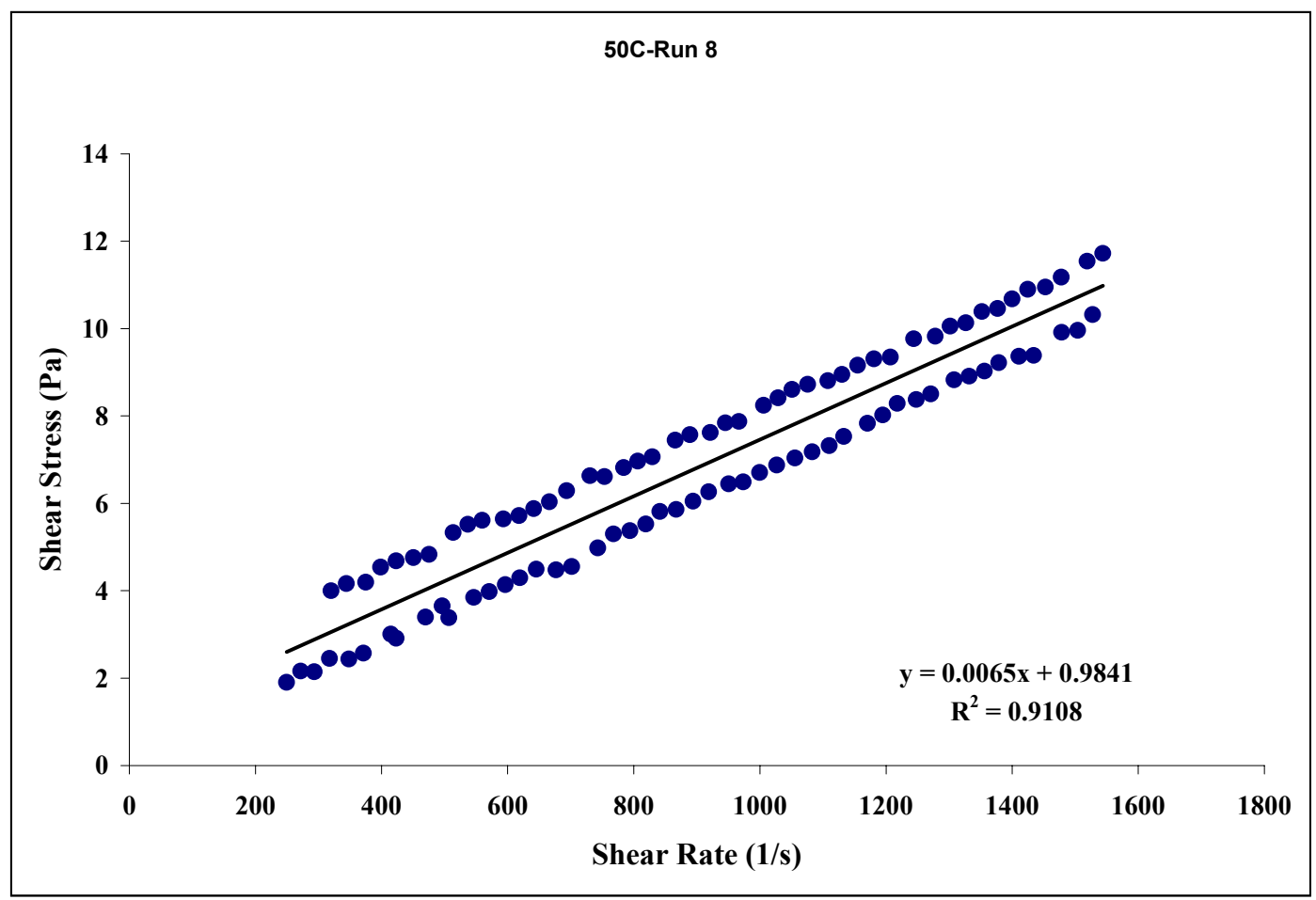

Figure 26. $5{ }^{\circ} \mathrm{C}$ As Received Run 8 
$\begin{array}{ll}\text { APPENDIX H - PART } 1 & \text { WSRC-TR-2000-00352 }\end{array}$

ENVELOPE C RHEOGRAMS

SRT-RPP-2000-00026

Page 314 of 514

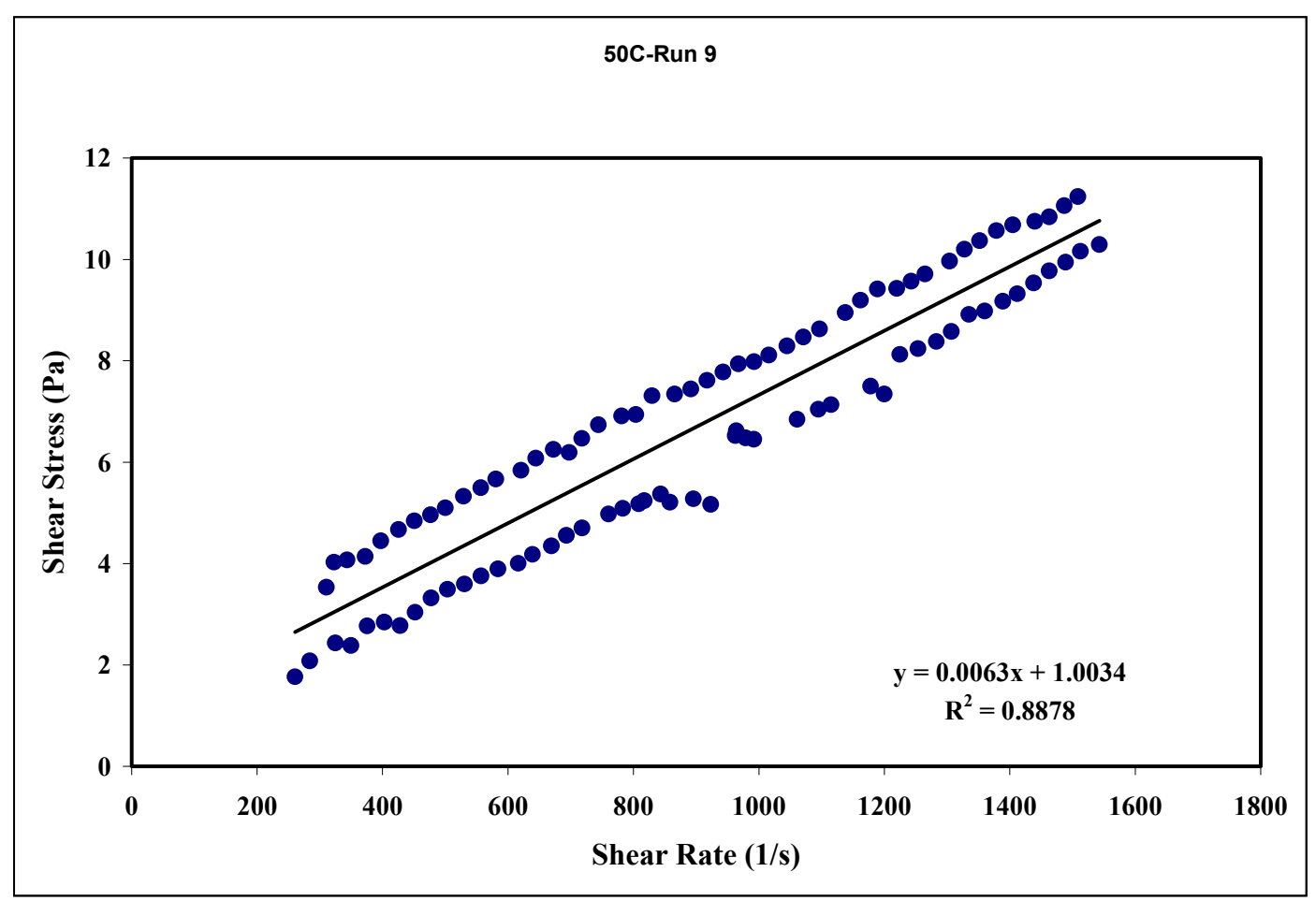

Figure 27. $50{ }^{\circ} \mathrm{C}$ As Received Run 9

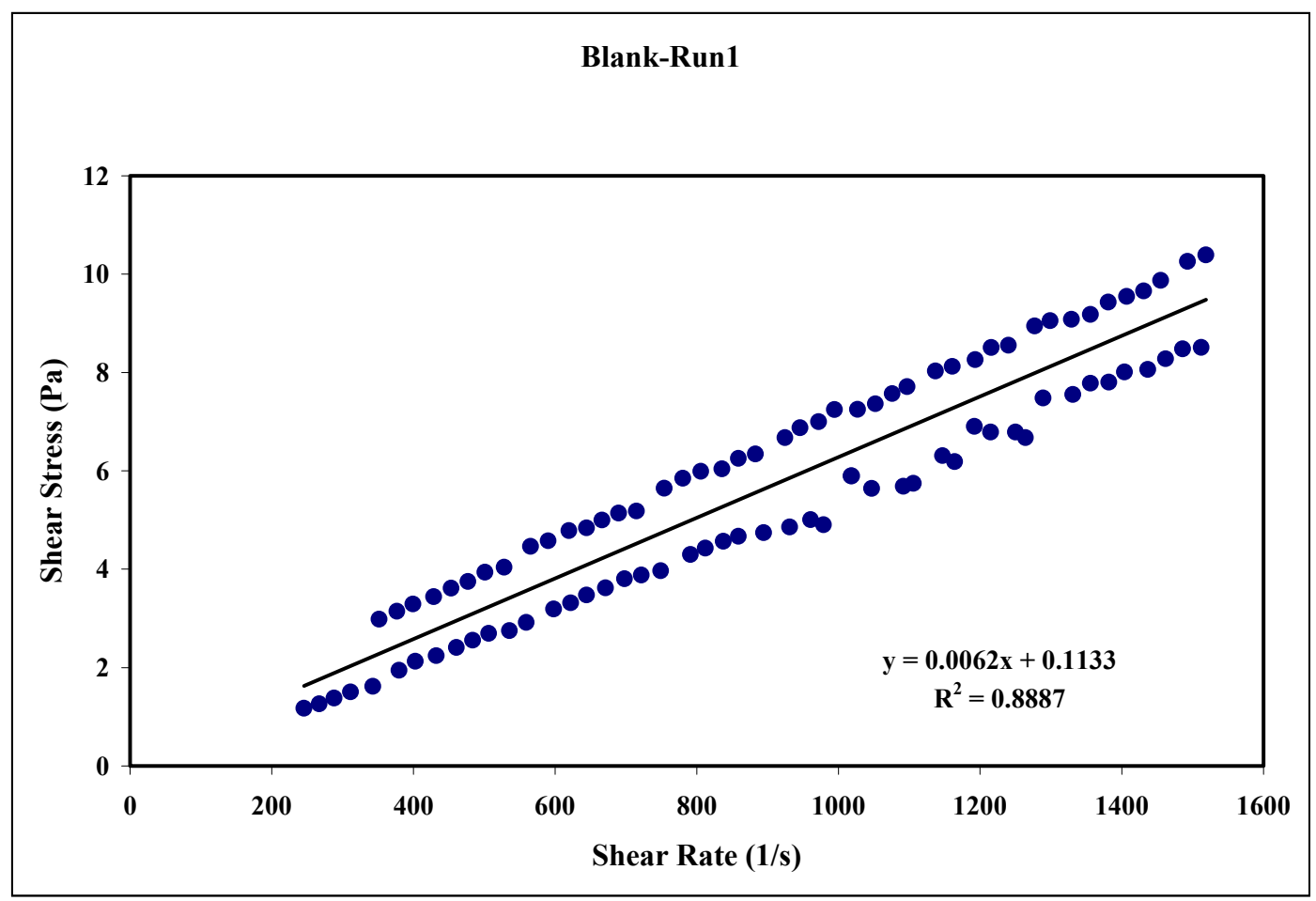

Figure 28. Blank Diluted Run 1 
$\begin{array}{ll}\text { APPENDIX H - PART } 1 & \text { WSRC-TR-2000-00352 }\end{array}$

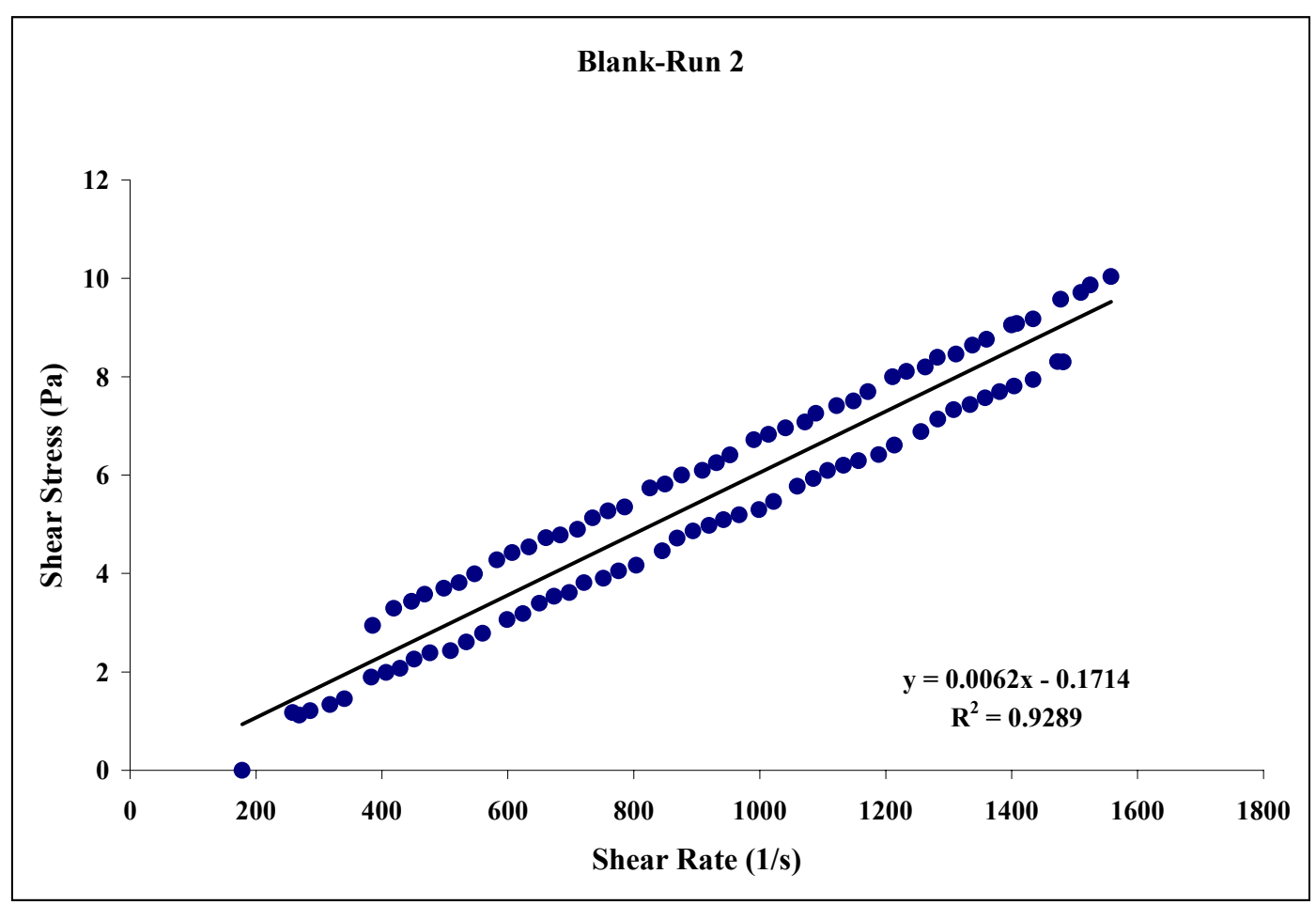

Figure 29. Blank Diluted Run 2

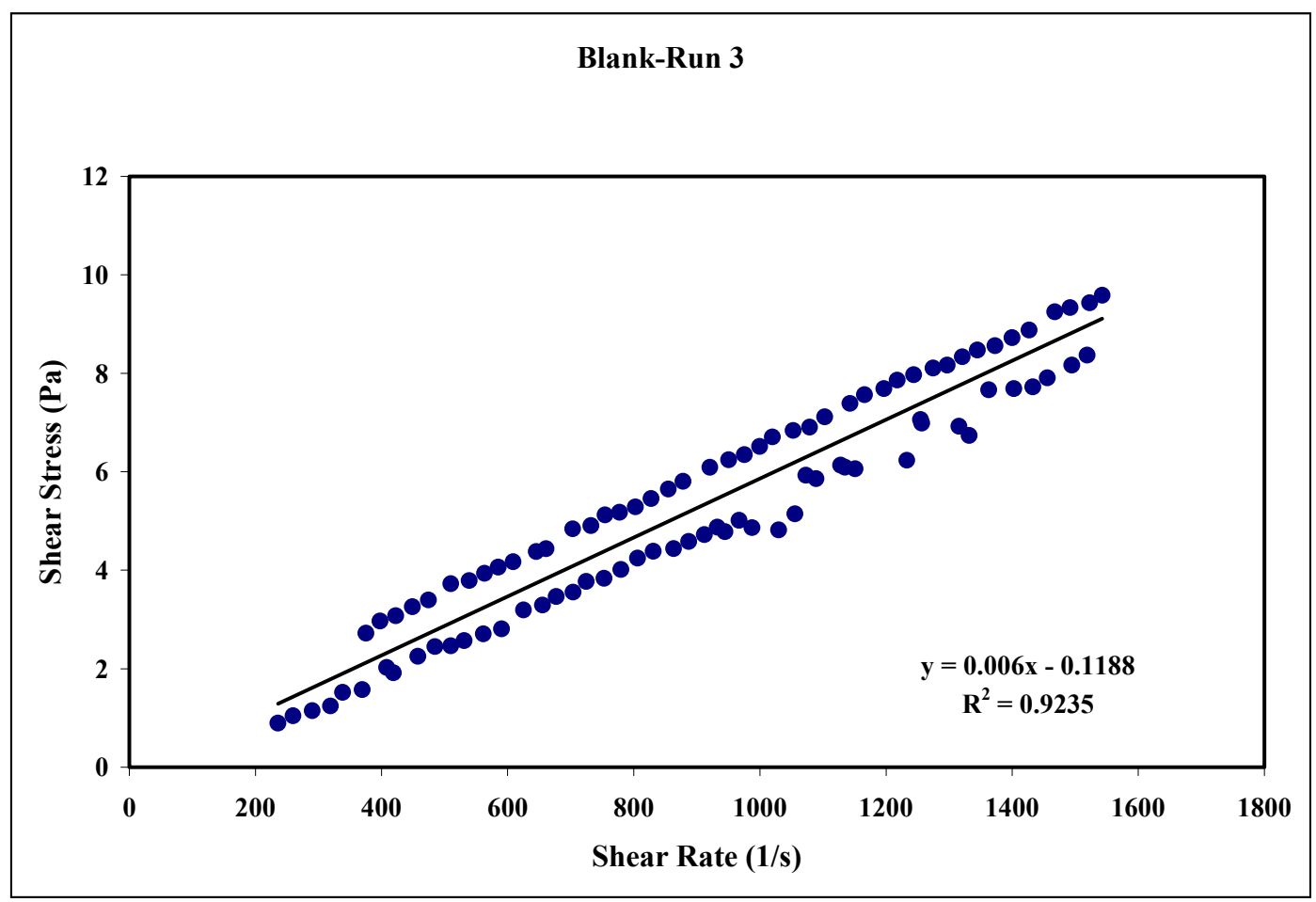

Figure 30. Blank Diluted Run 3 
$\begin{array}{ll}\text { APPENDIX H - PART } 1 & \text { WSRC-TR-2000-00352 }\end{array}$

ENVELOPE C RHEOGRAMS

SRT-RPP-2000-00026

Page 316 of 514

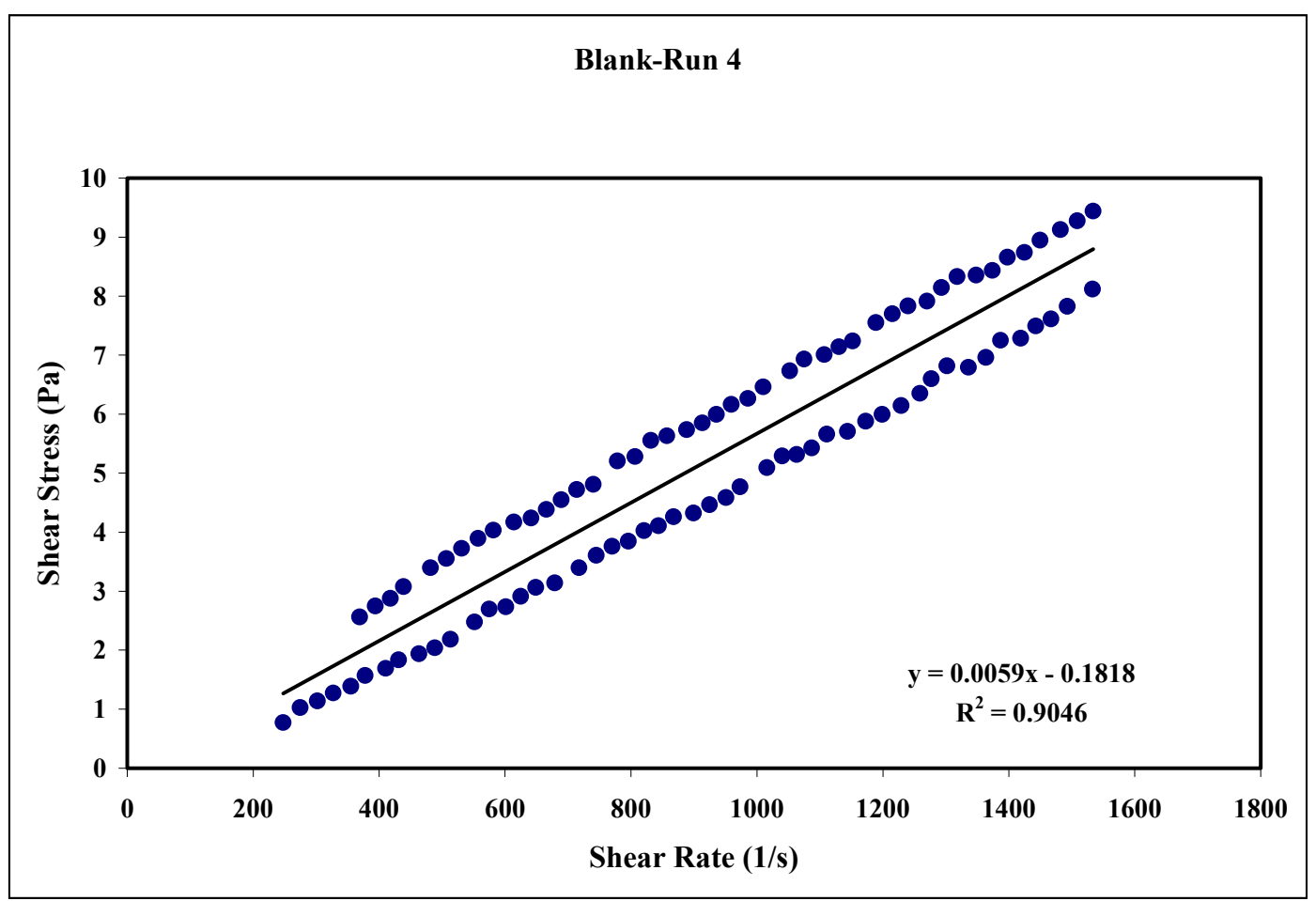

Figure 31. Blank Diluted Run 4

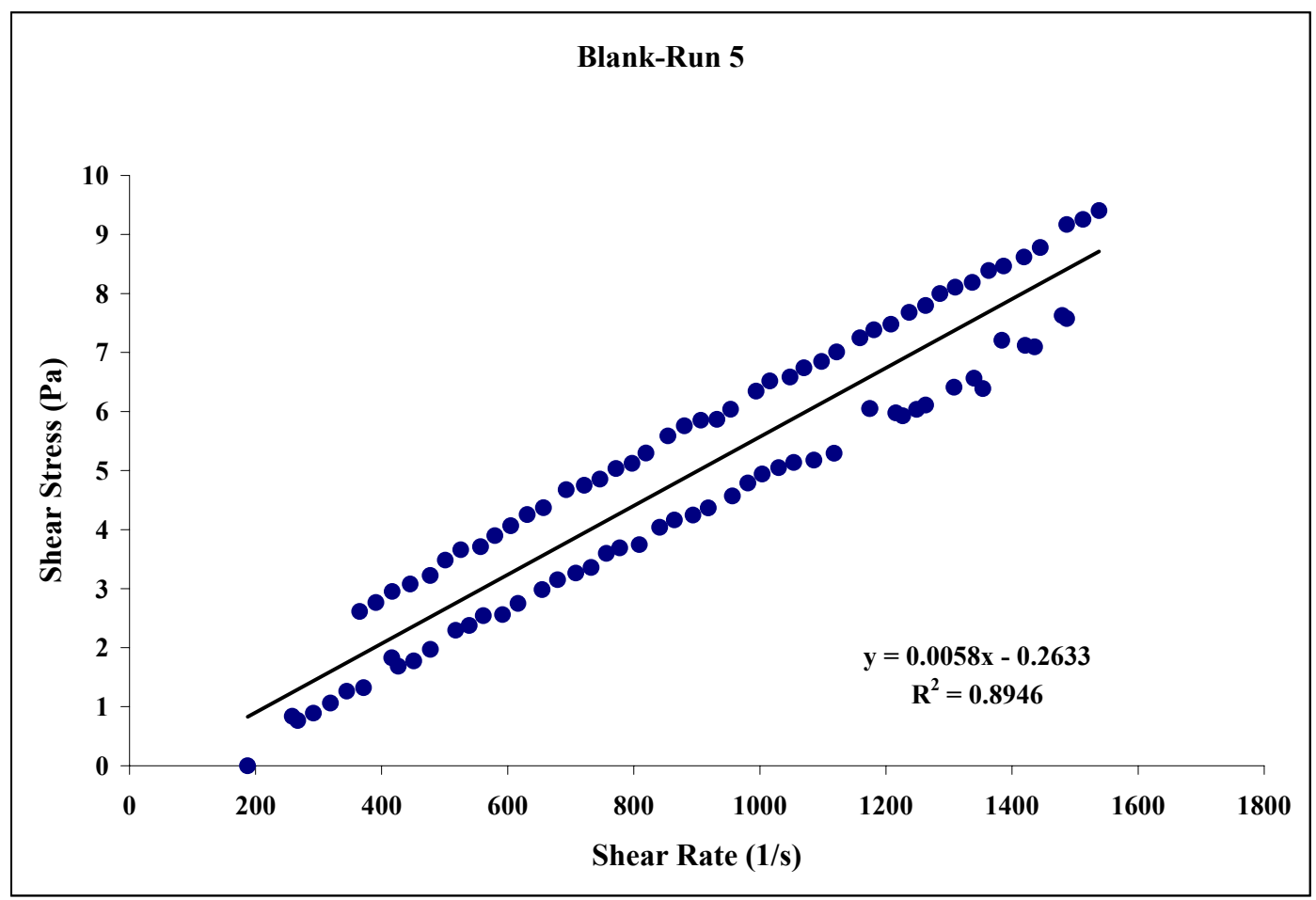

Figure 32. Blank Diluted Run 5 
$\begin{array}{ll}\text { APPENDIX H - PART } 1 & \text { WSRC-TR-2000-00352 }\end{array}$

ENVELOPE C RHEOGRAMS

SRT-RPP-2000-00026

Page 317 of 514

\section{Blank-Run 6}

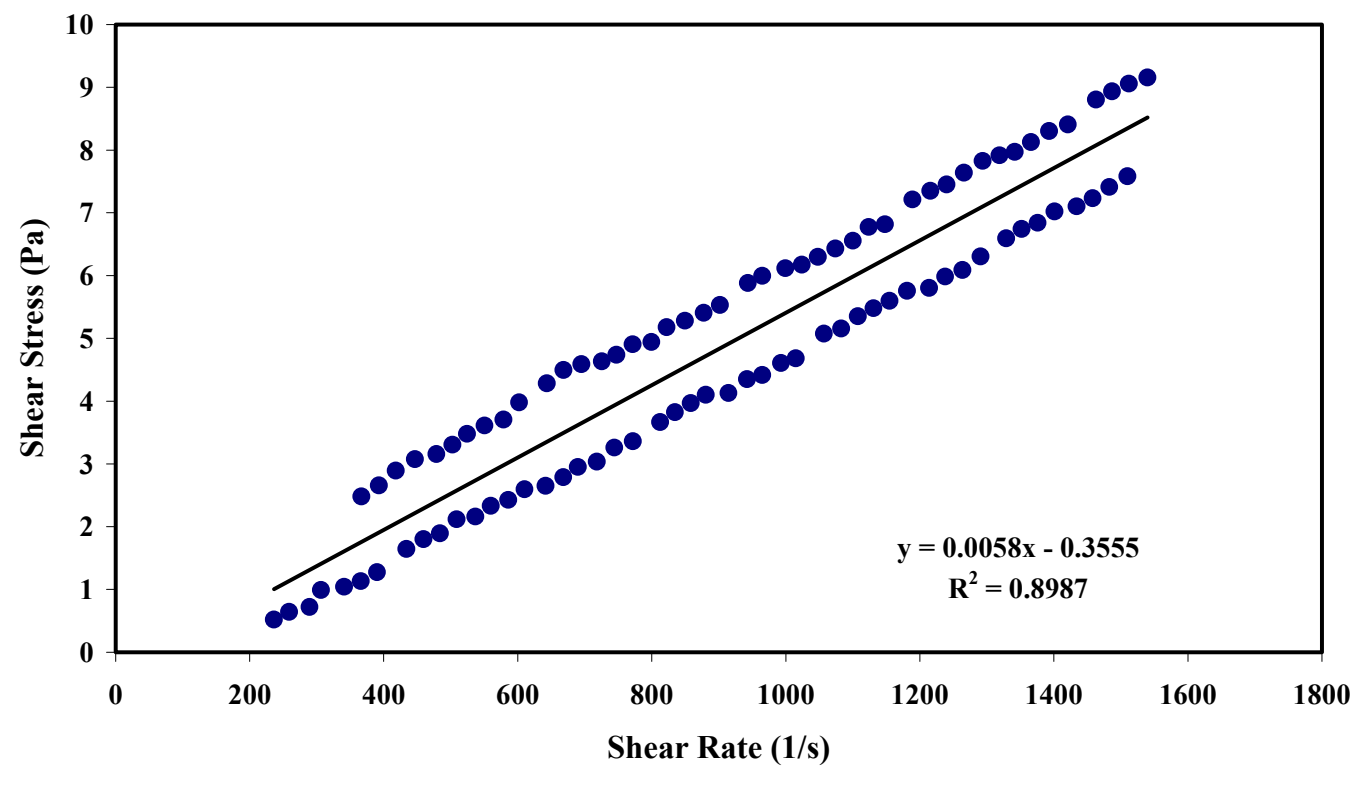

Figure 33. Blank Diluted Run 6

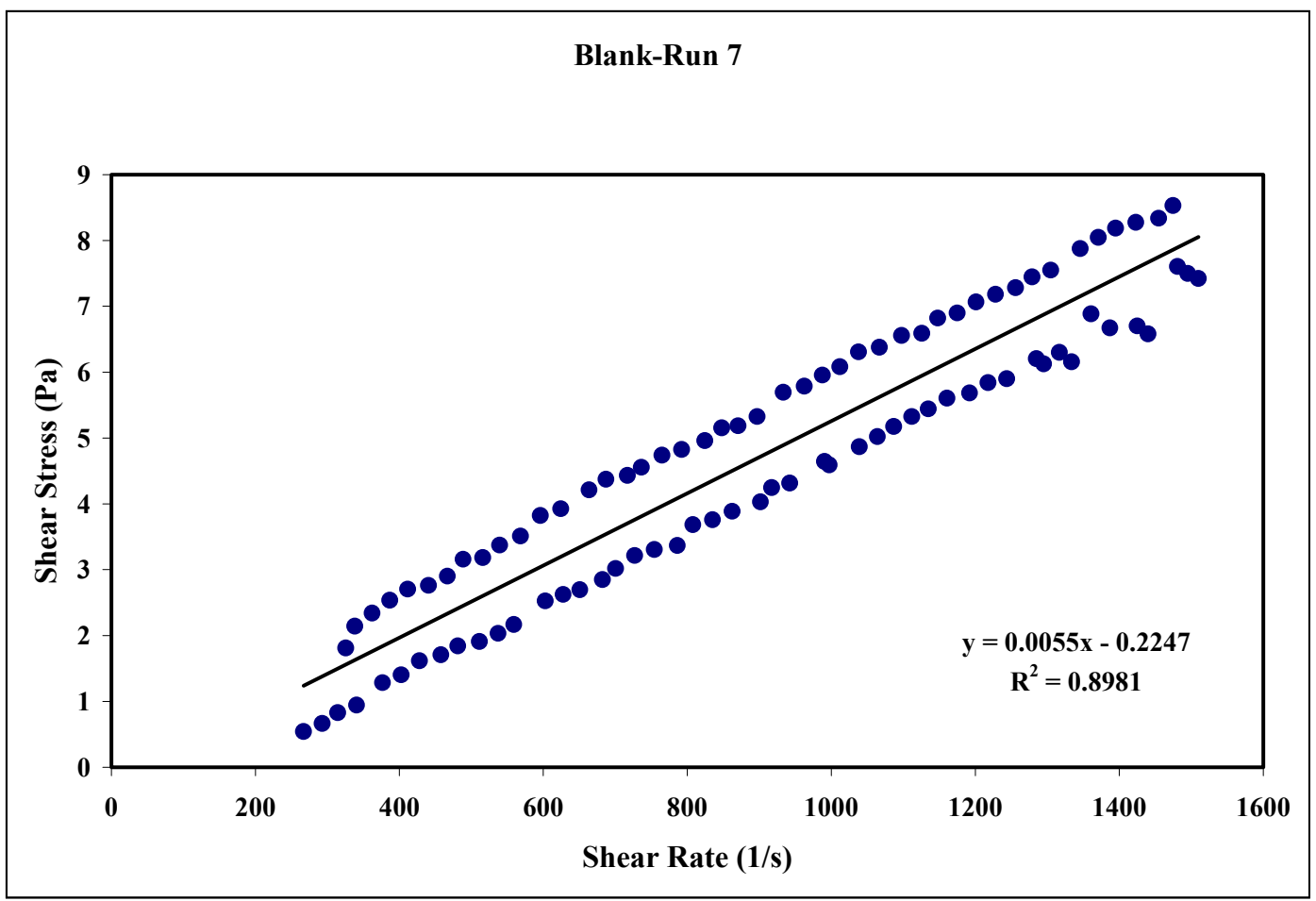

Figure 34. Blank Diluted Run 7 
\begin{tabular}{|ll}
\hline APPENDIX H - PART 1 & WSRC-TR-2000-00352
\end{tabular}

ENVELOPE C RHEOGRAMS

SRT-RPP-2000-00026

Page 318 of 514

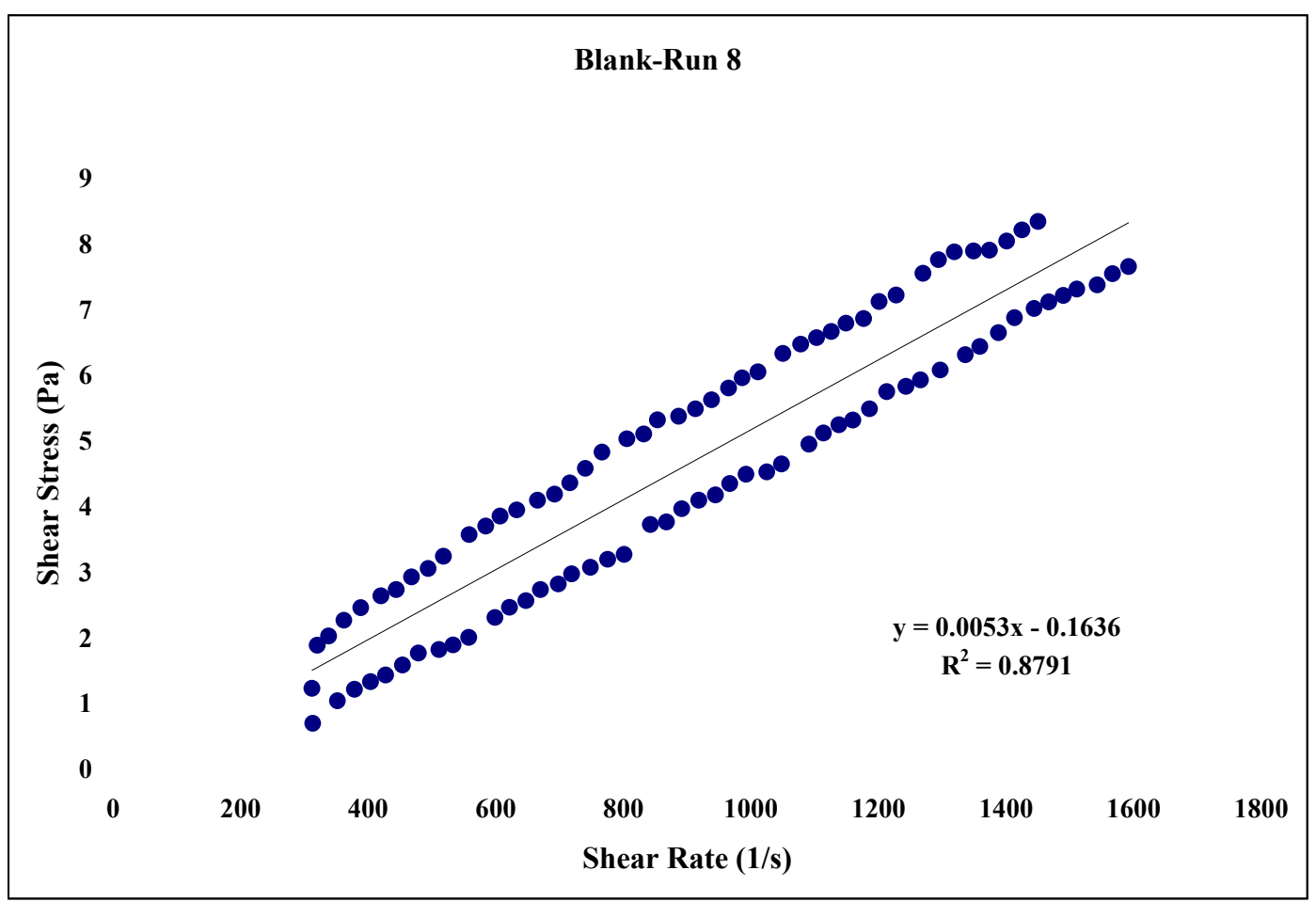

Figure 35. Blank Diluted Run 8

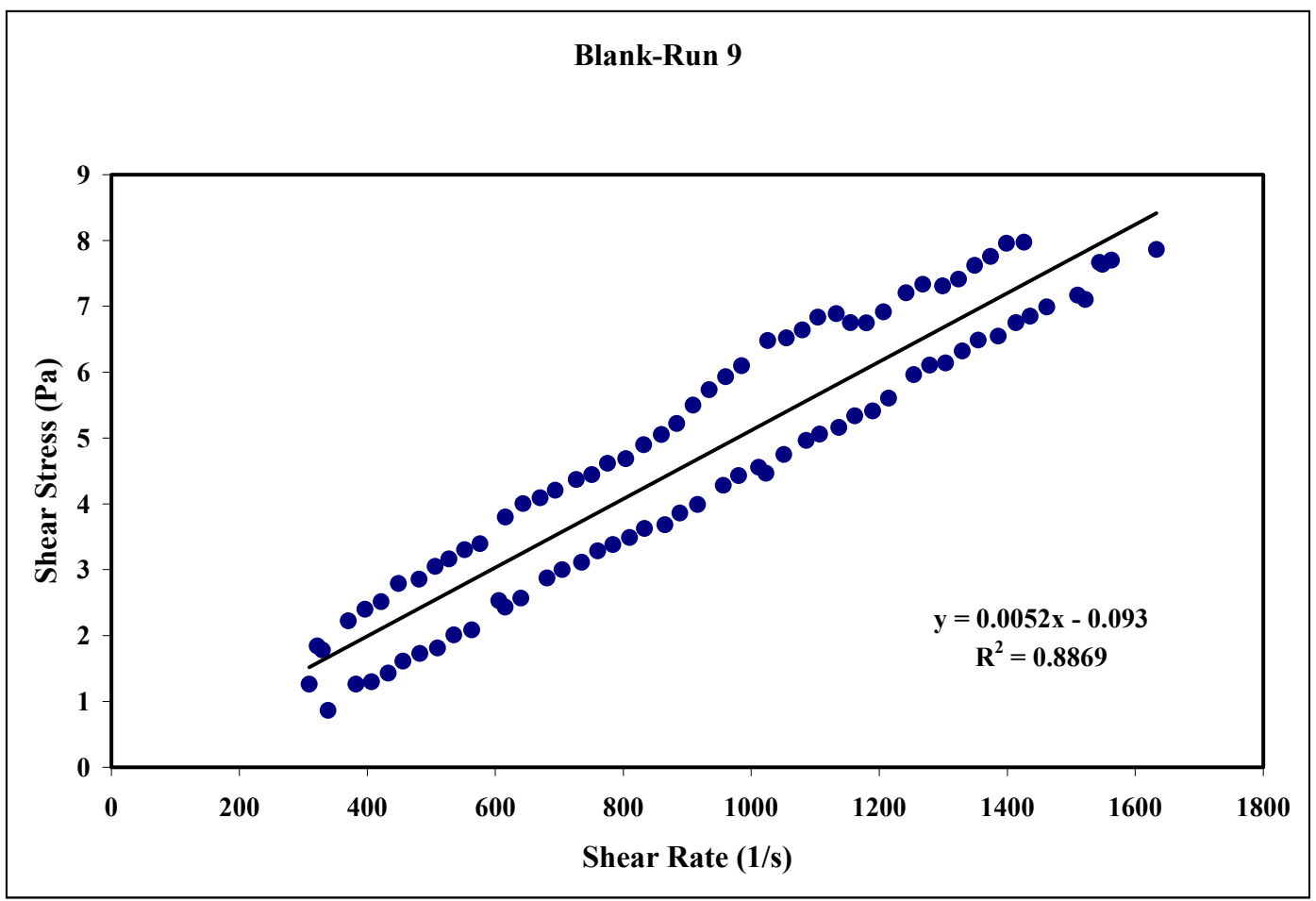

Figure 36. Blank Diluted Run 9 
\begin{tabular}{|ll}
\hline APPENDIX H - PART 1 & WSRC-TR-2000-00352
\end{tabular}

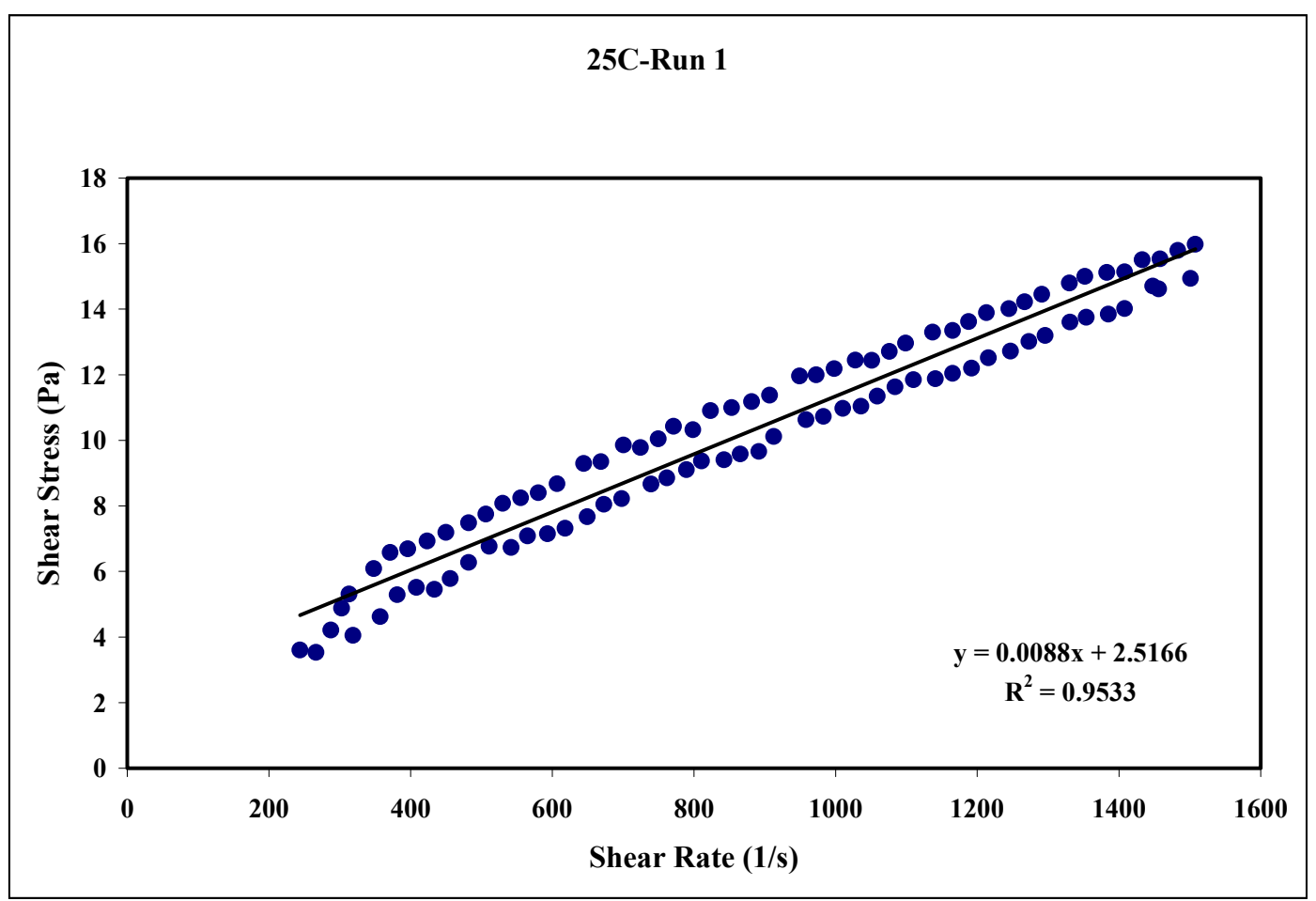

Figure 37. $25^{\circ} \mathrm{C}$ Diluted Run 1

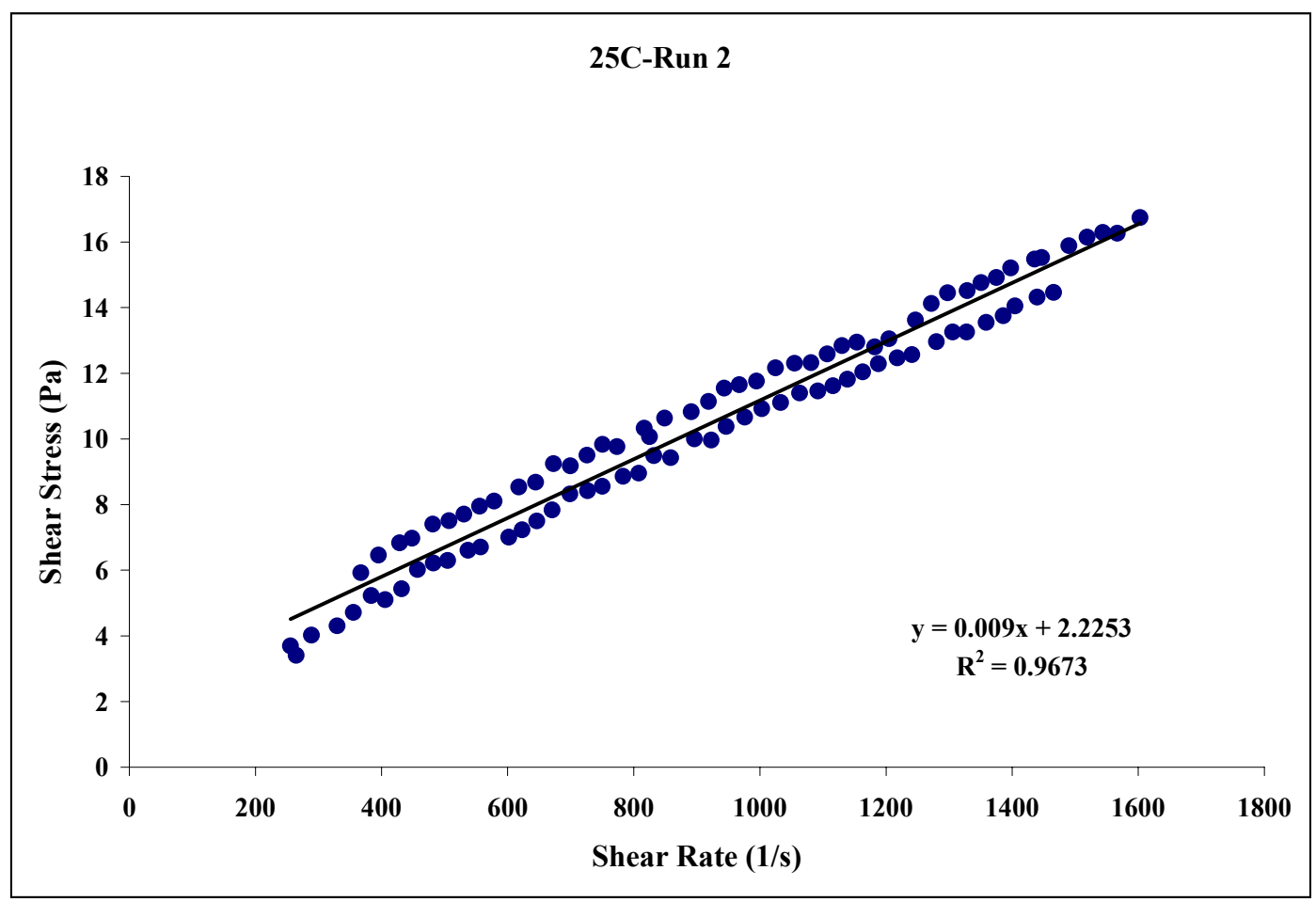

Figure 38. $25^{\circ} \mathrm{C}$ Diluted Run 2 
$\begin{array}{ll}\text { APPENDIX H - PART } 1 & \text { WSRC-TR-2000-00352 }\end{array}$

ENVELOPE C RHEOGRAMS

SRT-RPP-2000-00026

Page 320 of 514

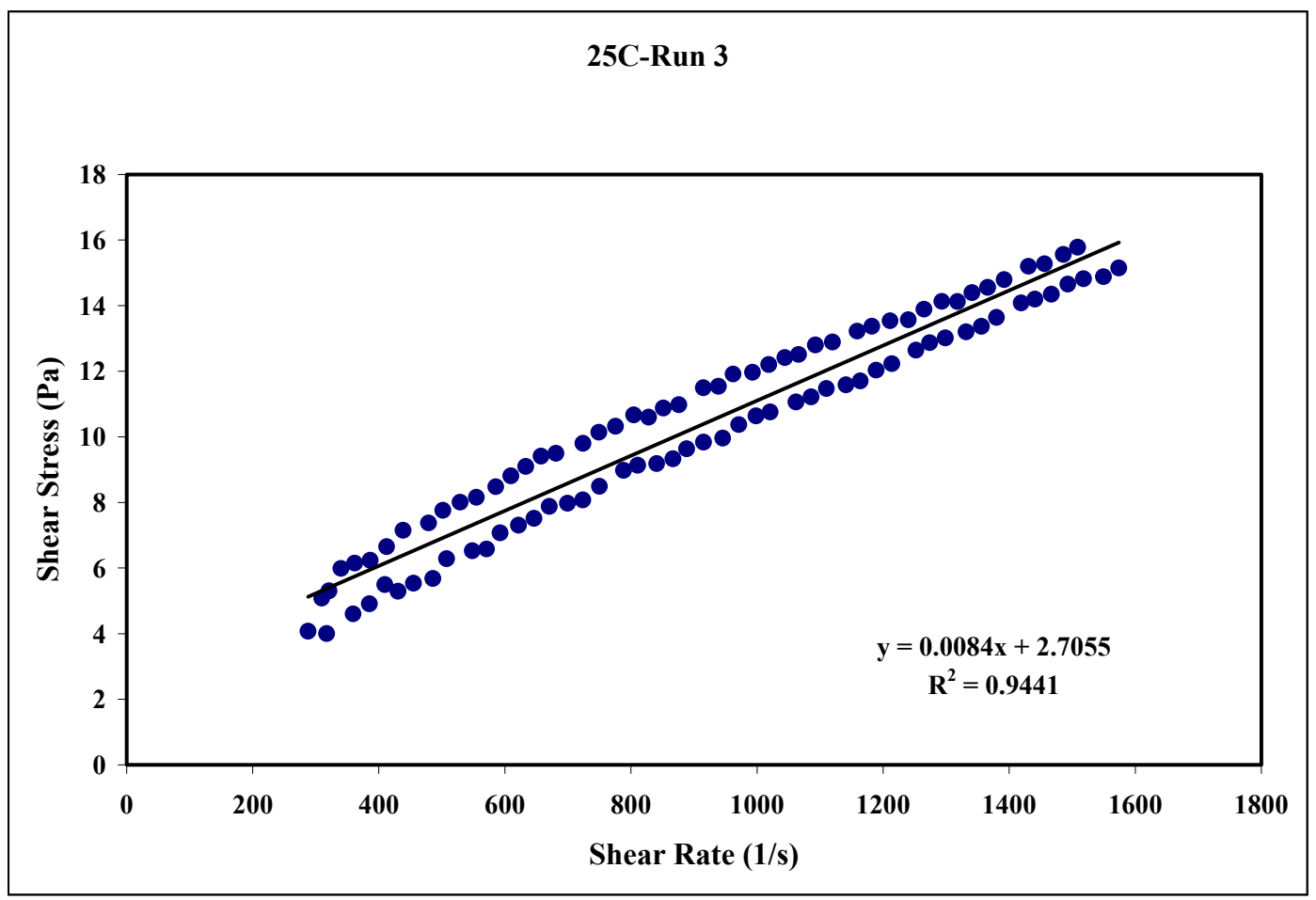

Figure 39. $25^{\circ} \mathrm{C}$ Diluted Run 3

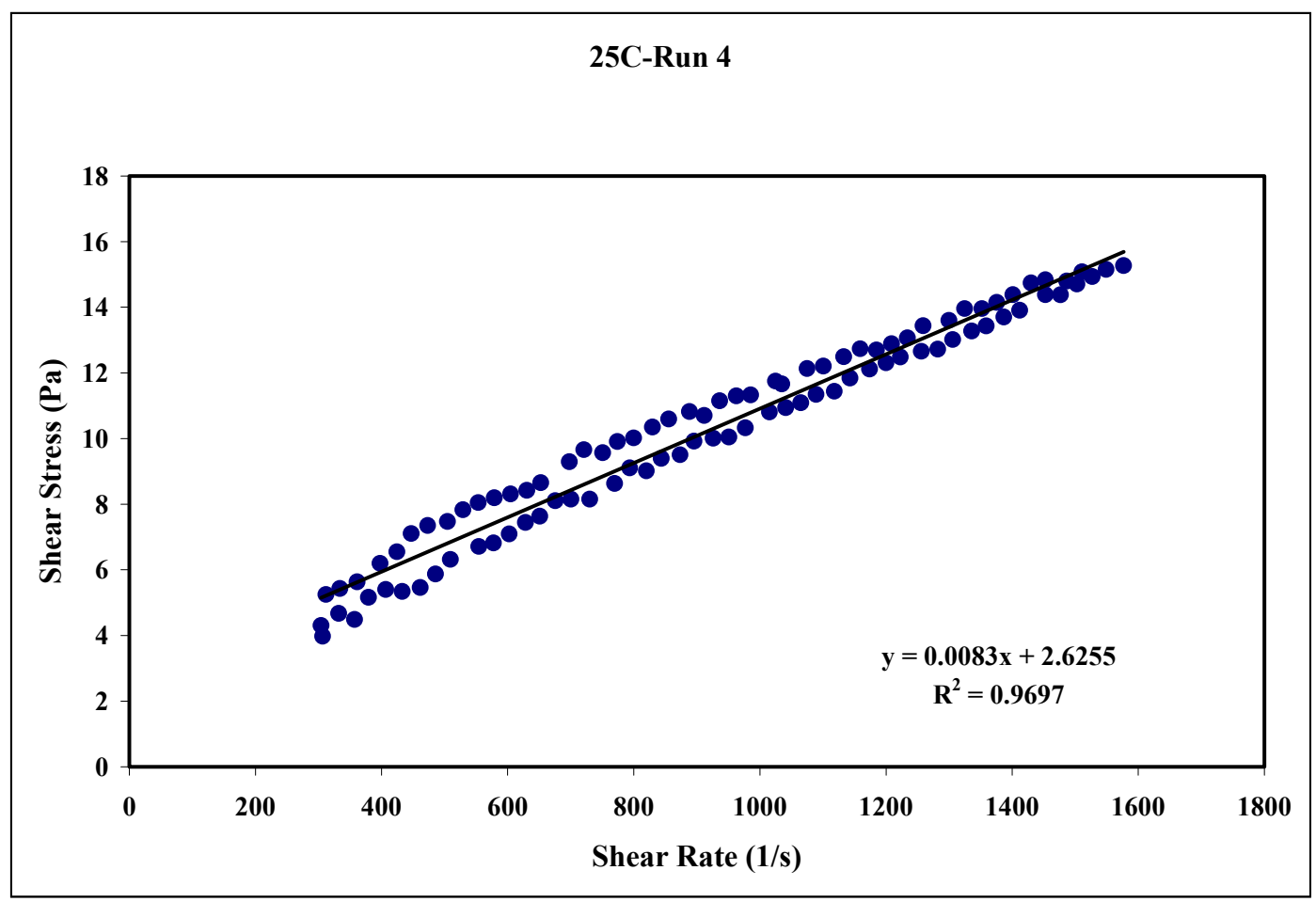

Figure 40. $25^{\circ} \mathrm{C}$ Diluted Run 4 
$\begin{array}{ll}\text { APPENDIX H - PART } 1 & \text { WSRC-TR-2000-00352 }\end{array}$

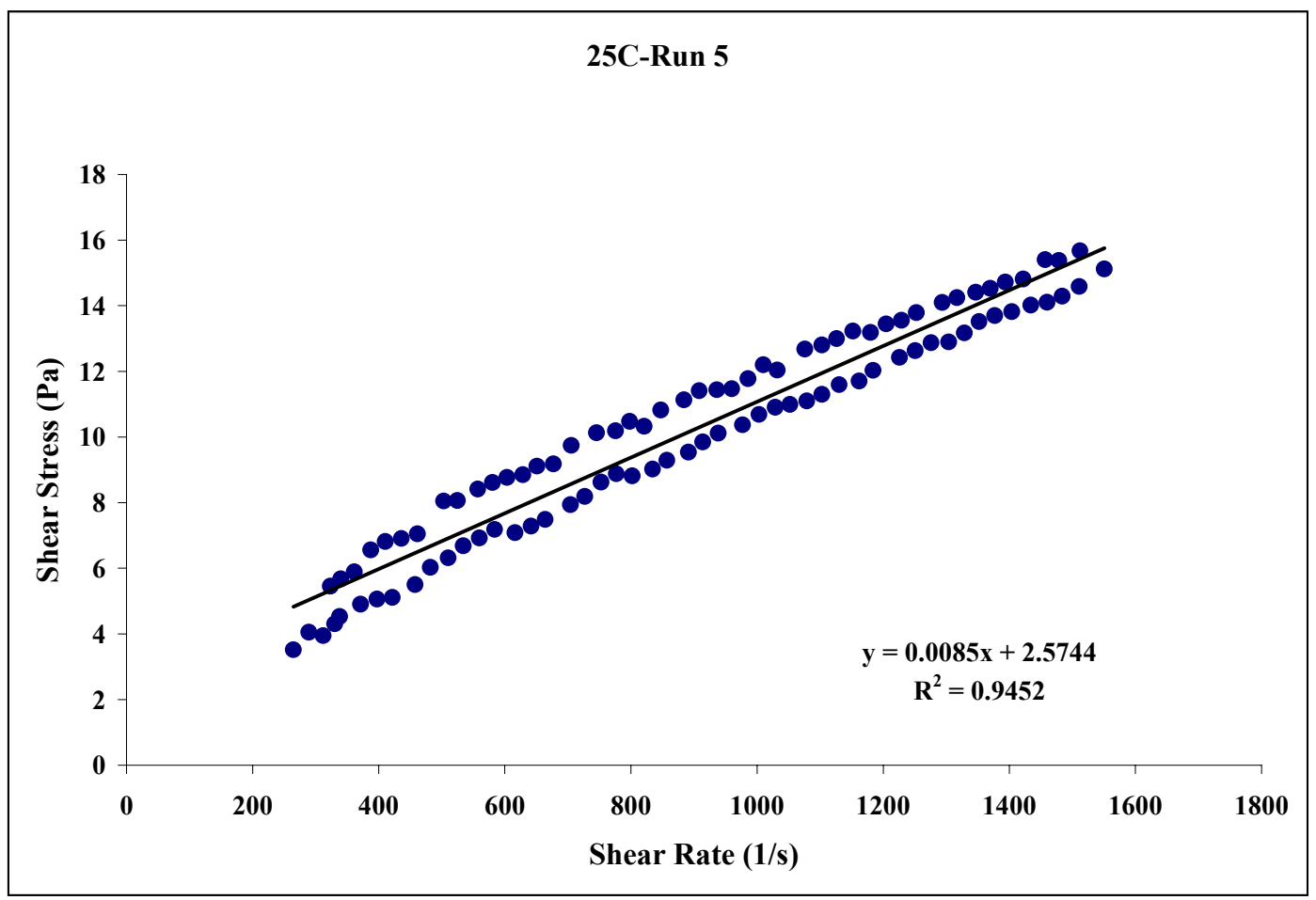

Figure 41. $25^{\circ} \mathrm{C}$ Diluted Run 5

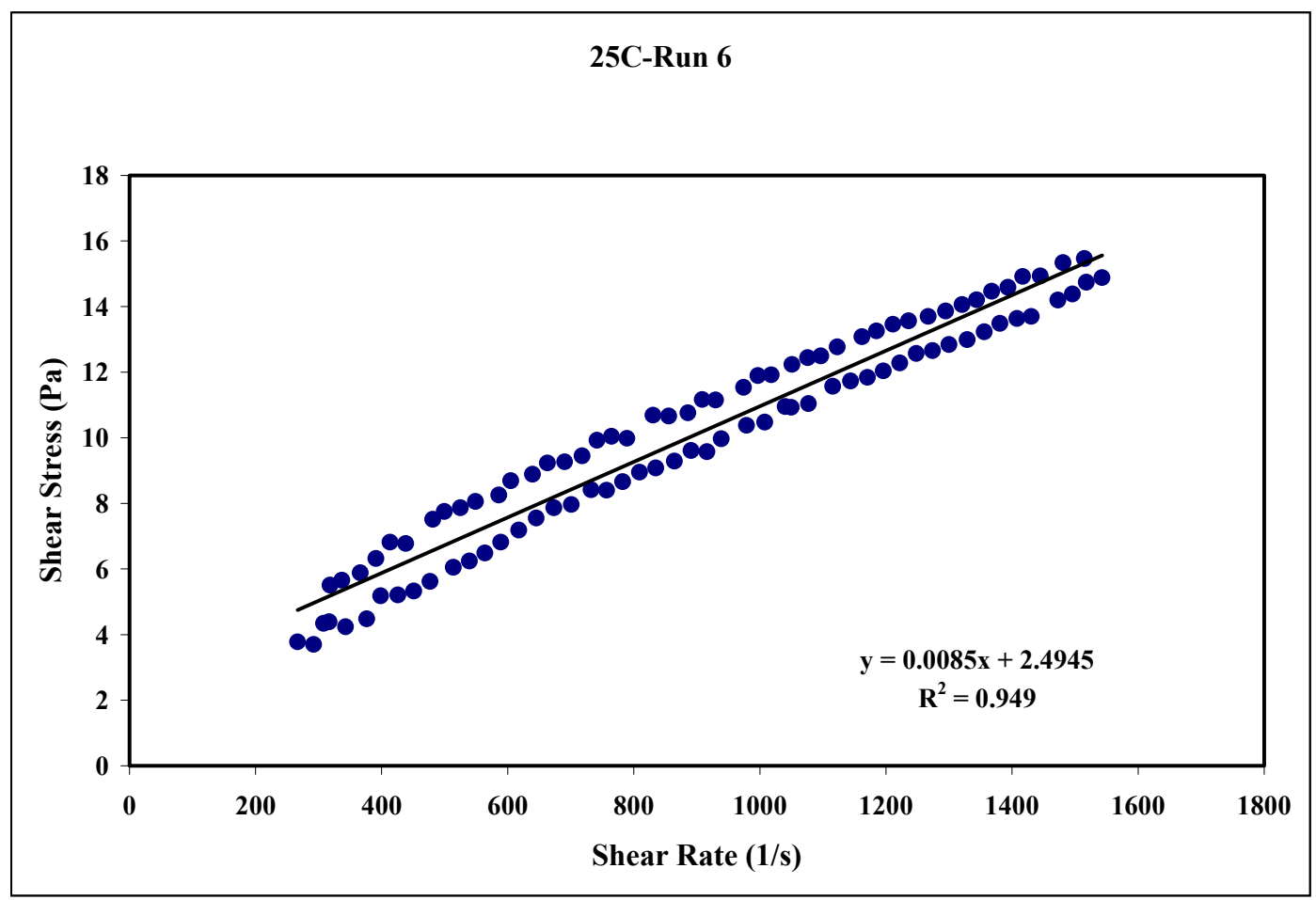

Figure 42. $25^{\circ} \mathrm{C}$ Diluted Run 6 
$\begin{array}{ll}\text { APPENDIX H - PART } 1 & \text { WSRC-TR-2000-00352 }\end{array}$

ENVELOPE C RHEOGRAMS

SRT-RPP-2000-00026

Page 322 of 514

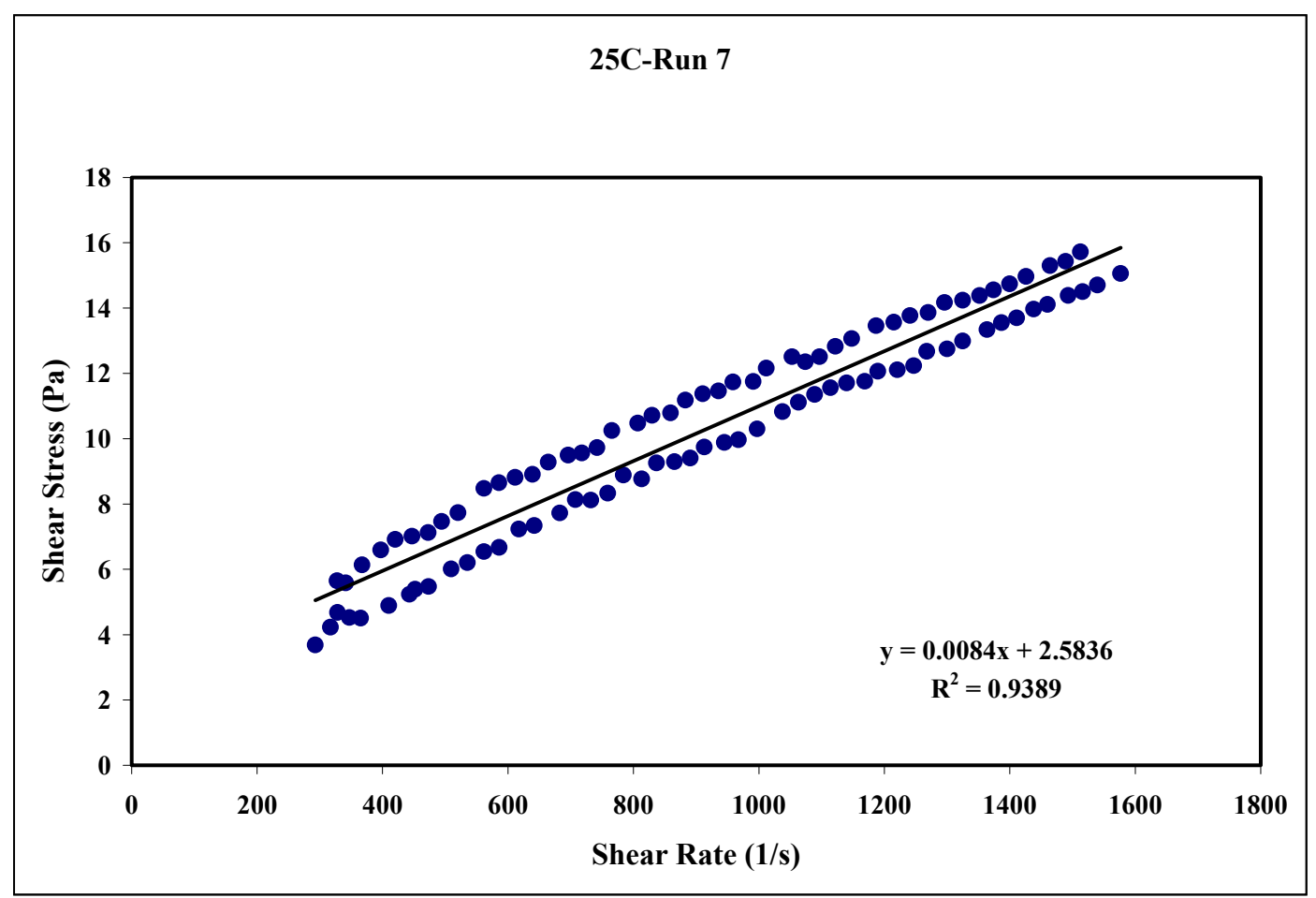

Figure 43. $25^{\circ} \mathrm{C}$ Diluted Run 7

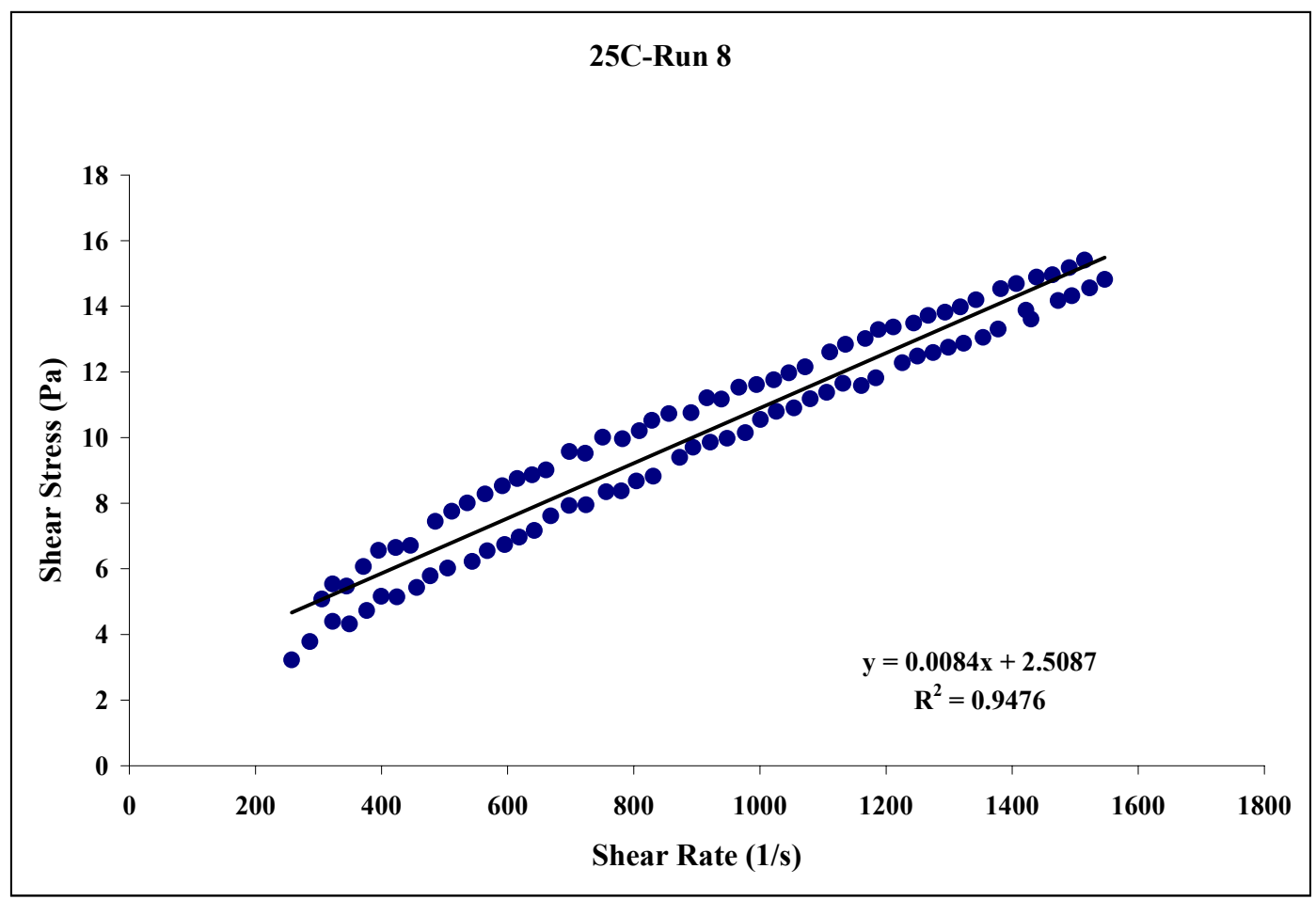

Figure 44. $25^{\circ} \mathrm{C}$ Diluted Run 8 
$\begin{array}{ll}\text { APPENDIX H - PART } 1 & \text { WSRC-TR-2000-00352 }\end{array}$

ENVELOPE C RHEOGRAMS

SRT-RPP-2000-00026

Page 323 of 514

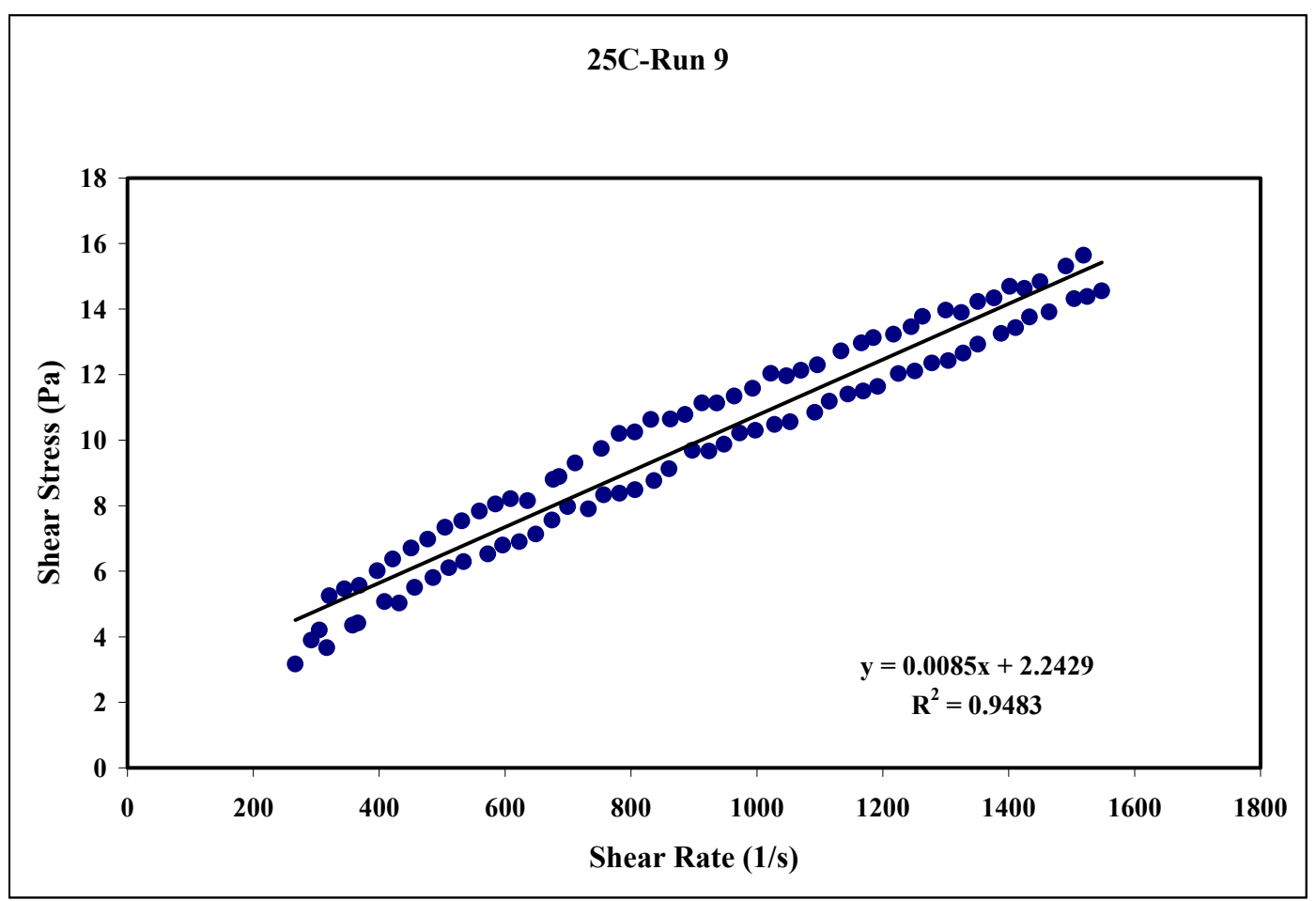

Figure 45. $25^{\circ} \mathrm{C}$ Diluted Run 9

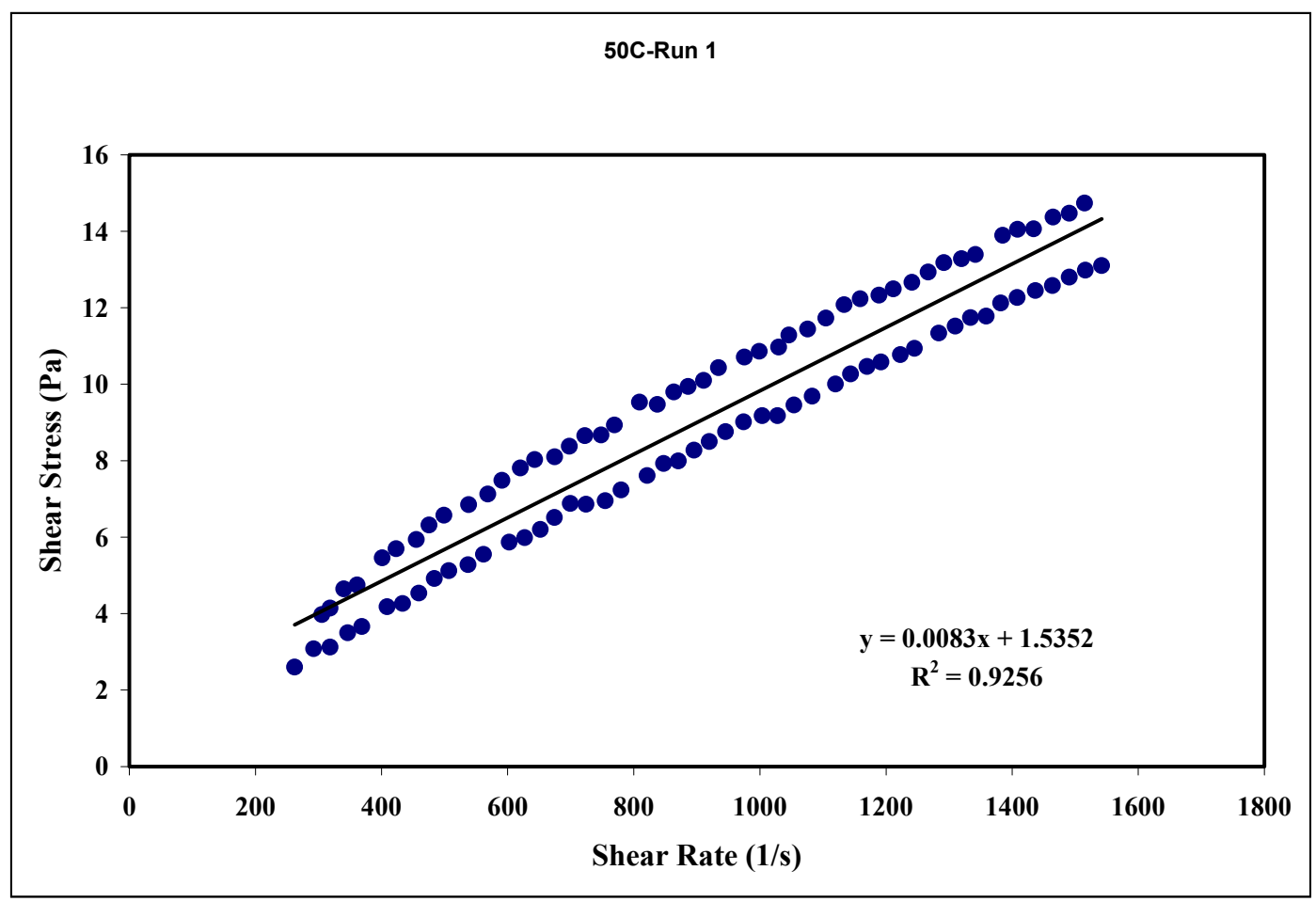

Figure 46. $50^{\circ} \mathrm{C}$ Diluted Run 1 
$\begin{array}{ll}\text { APPENDIX H - PART } 1 & \text { WSRC-TR-2000-00352 }\end{array}$

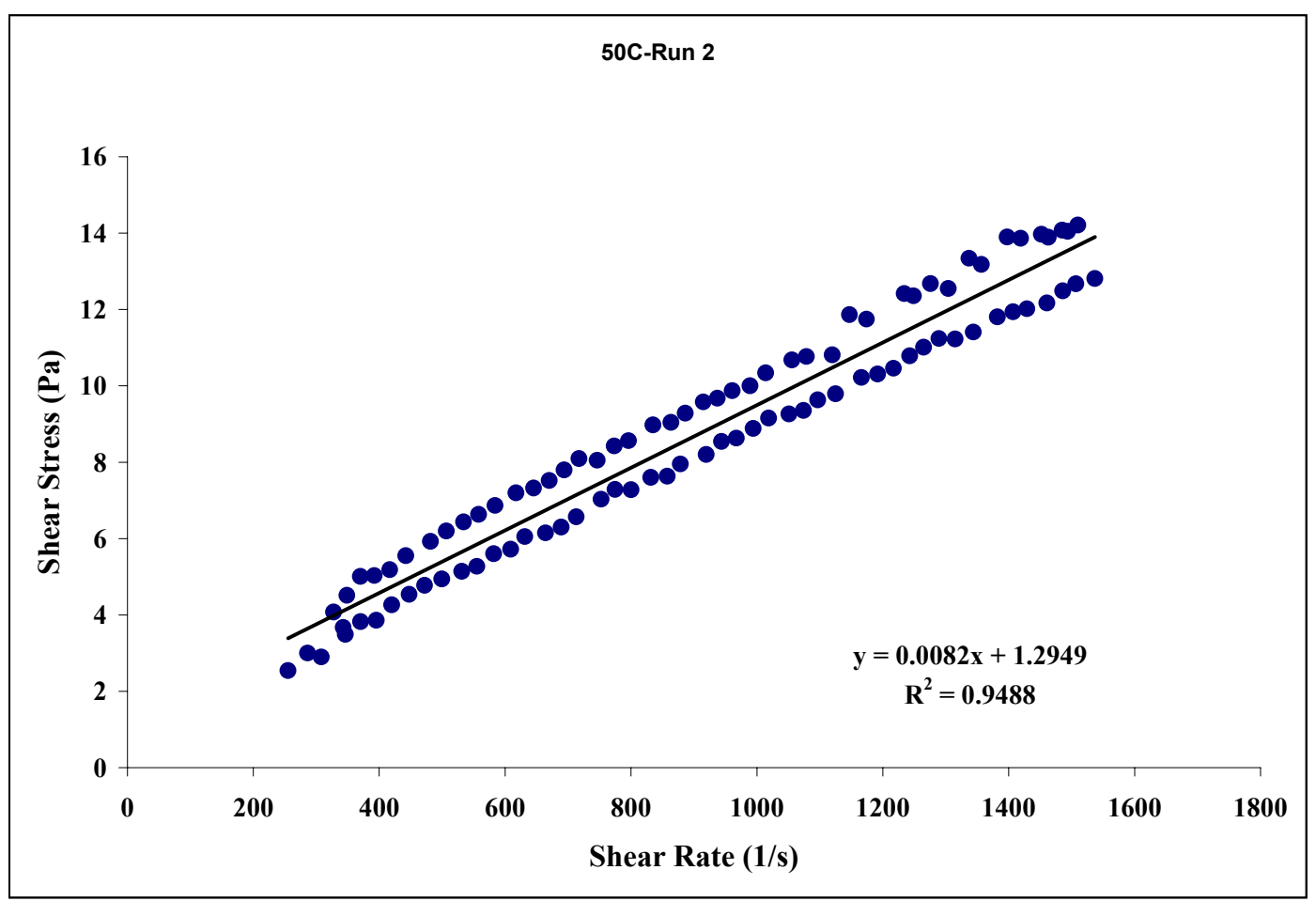

Figure 47. $50{ }^{\circ} \mathrm{C}$ Diluted Run 2

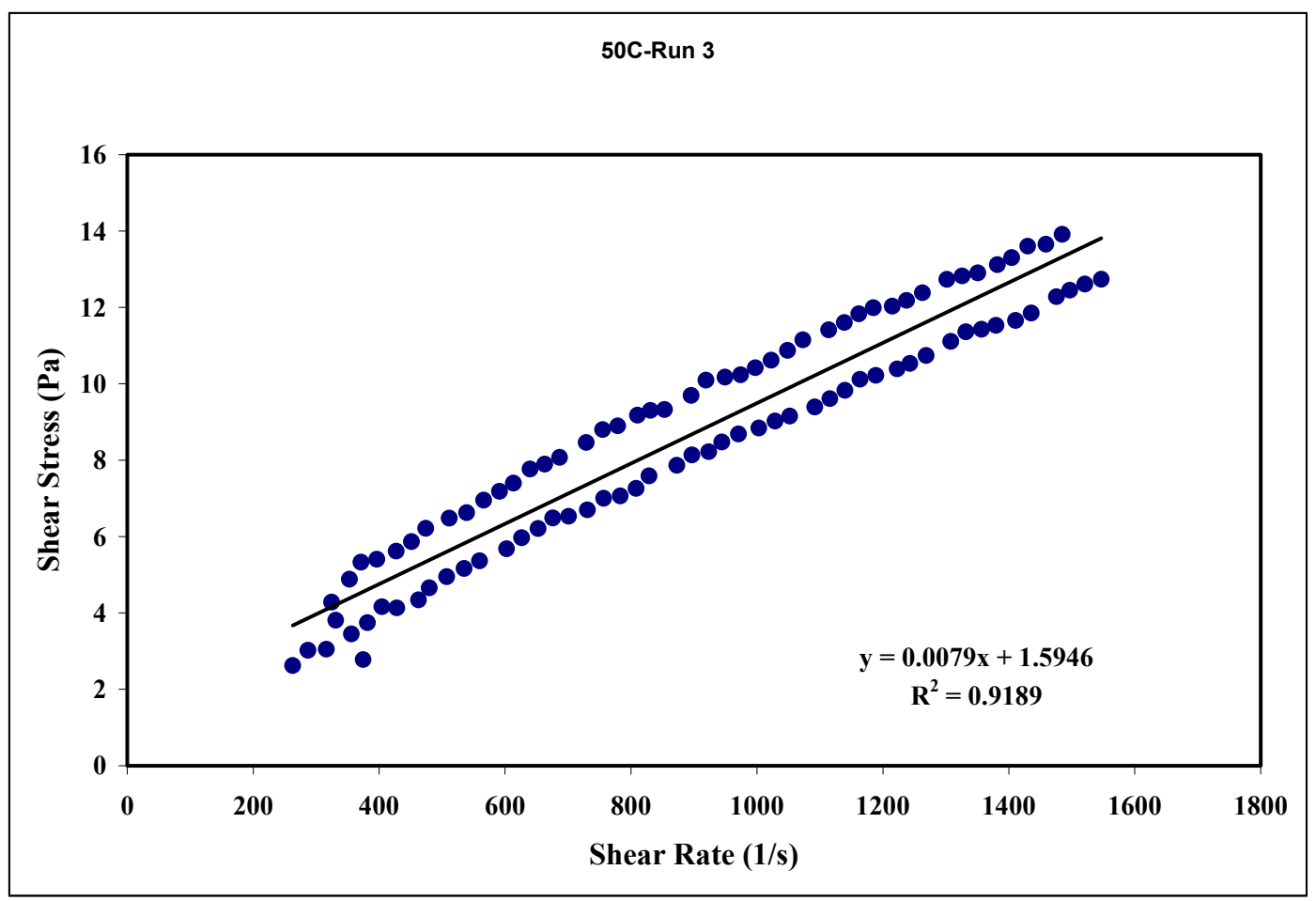

Figure 48. $50^{\circ} \mathrm{C}$ Diluted Run 3 
$\begin{array}{ll}\text { APPENDIX H - PART } 1 & \text { WSRC-TR-2000-00352 }\end{array}$

ENVELOPE C RHEOGRAMS

SRT-RPP-2000-00026

Page 325 of 514

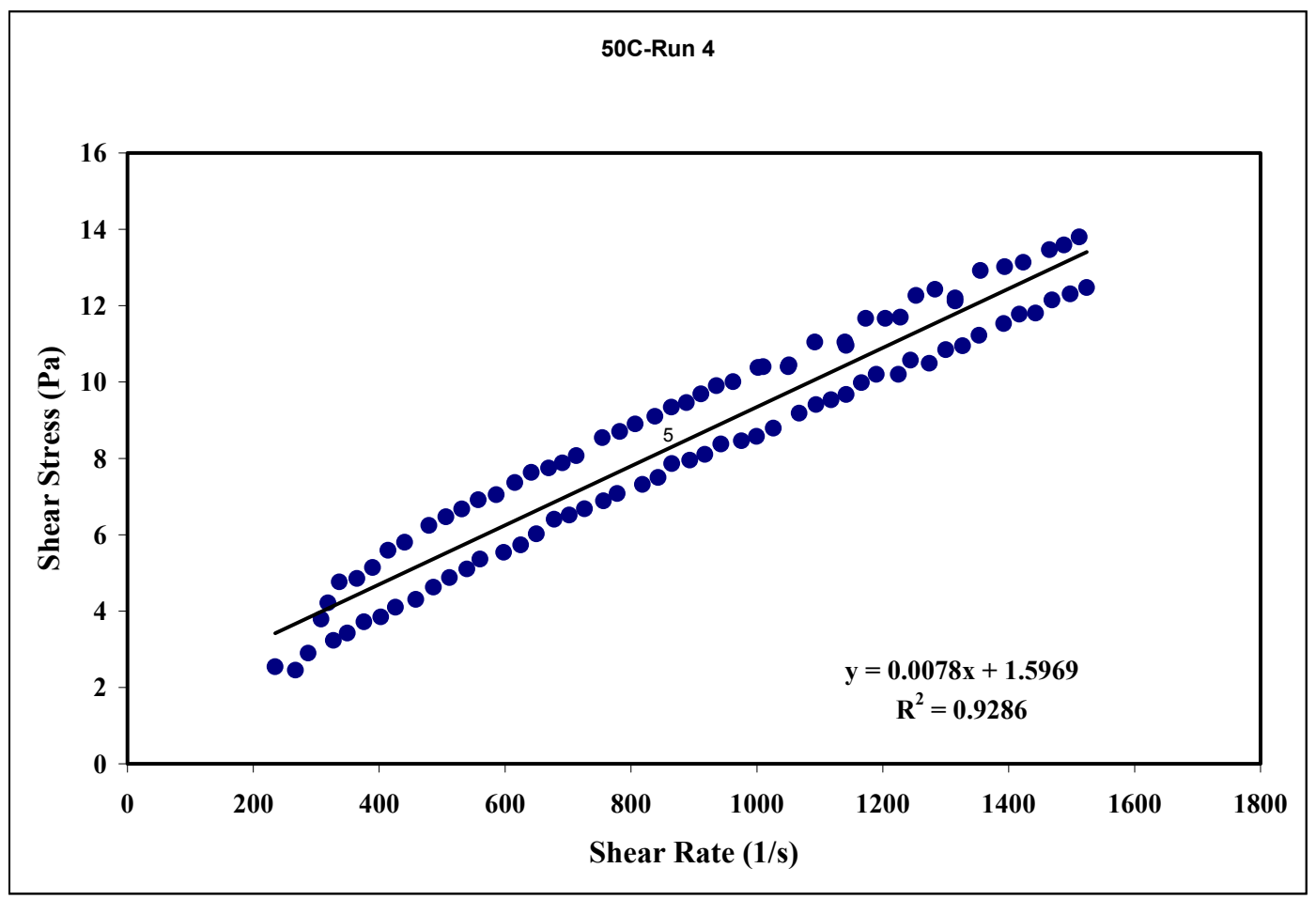

Figure 49. $50{ }^{\circ} \mathrm{C}$ Diluted Run 4

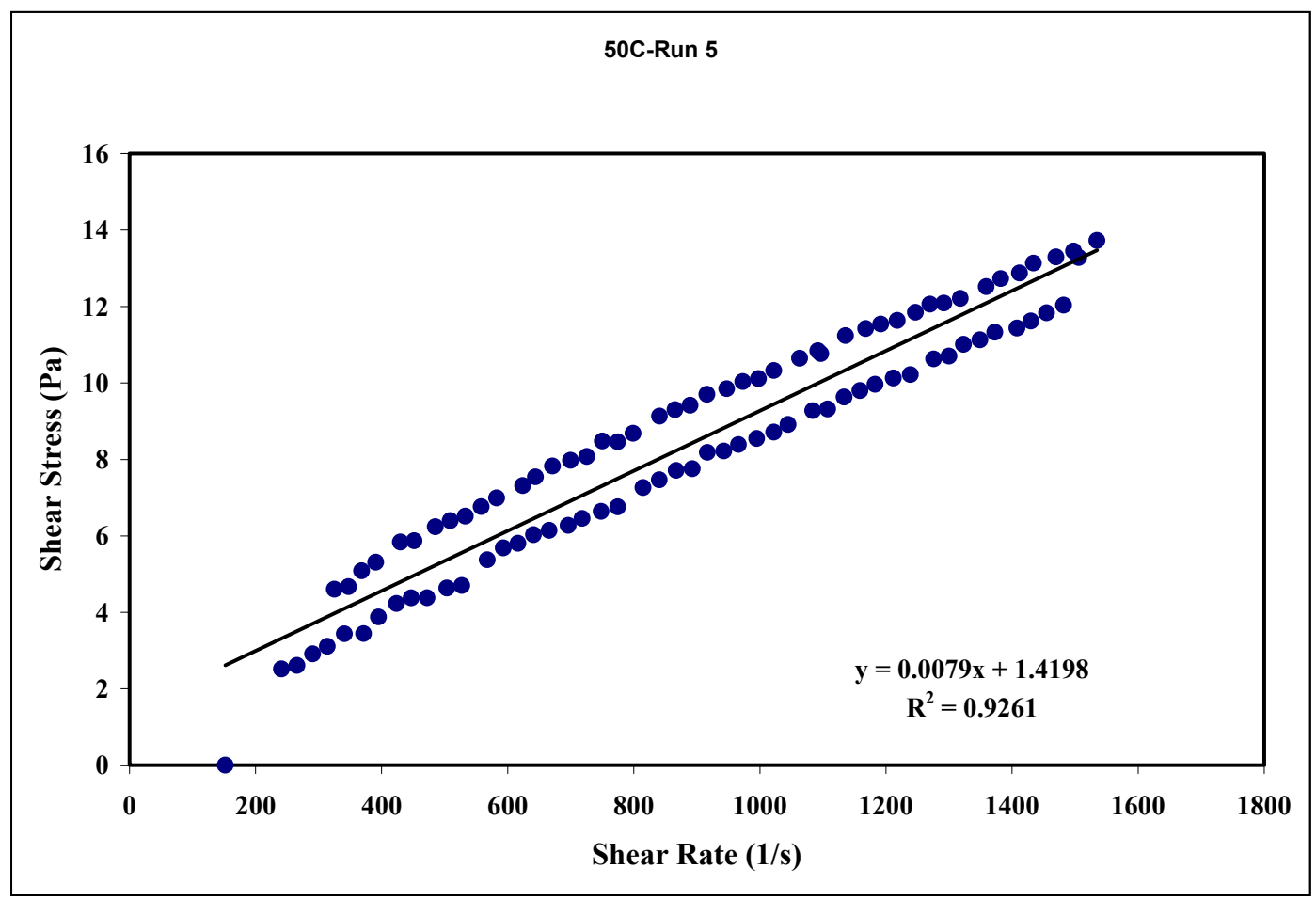

Figure 50. $50^{\circ} \mathrm{C}$ Diluted Run 5 
$\begin{array}{ll}\text { APPENDIX H - PART } 1 & \text { WSRC-TR-2000-00352 }\end{array}$

ENVELOPE C RHEOGRAMS

SRT-RPP-2000-00026

Page 326 of 514

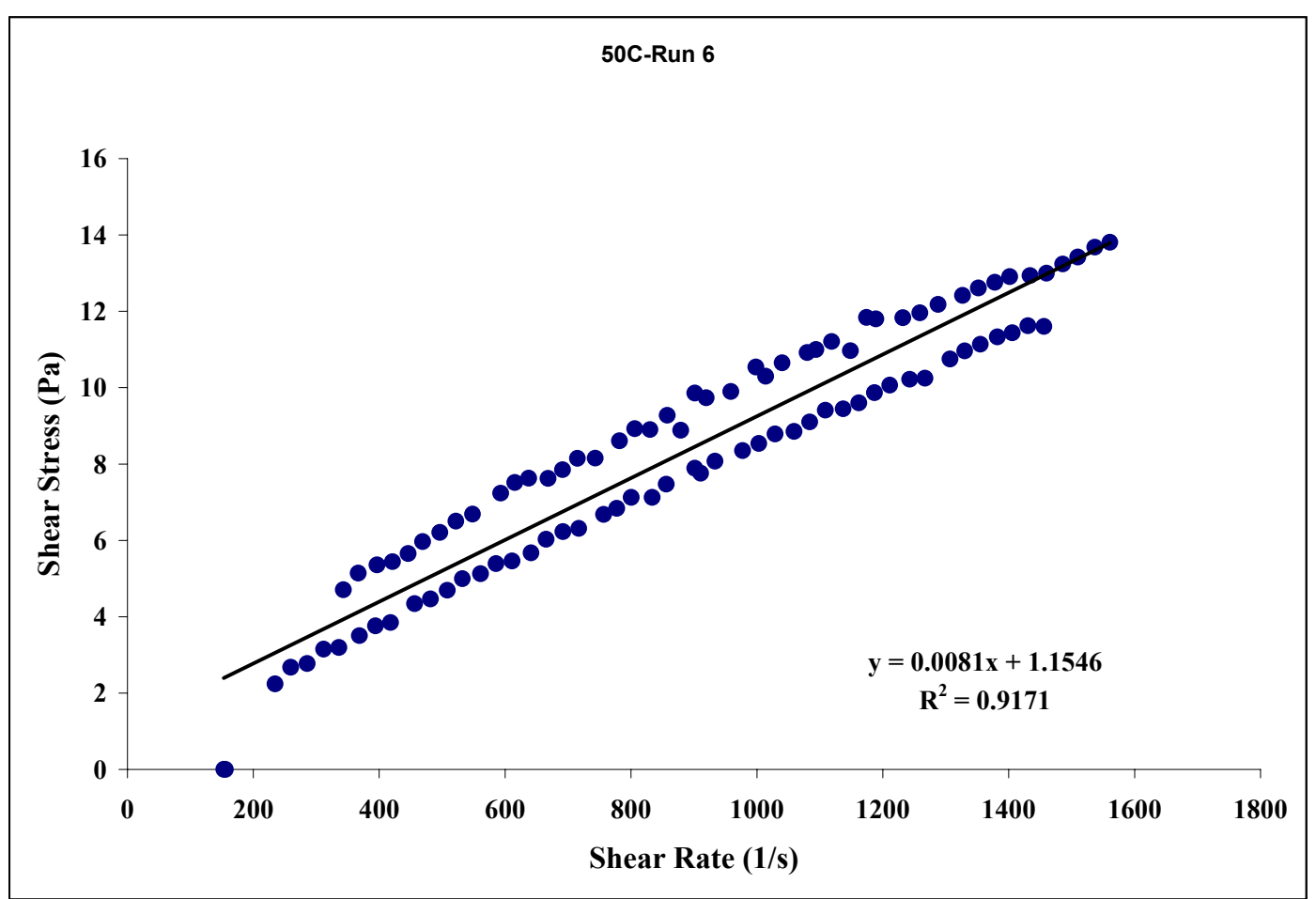

Figure 51. $50{ }^{\circ} \mathrm{C}$ Diluted Run 6

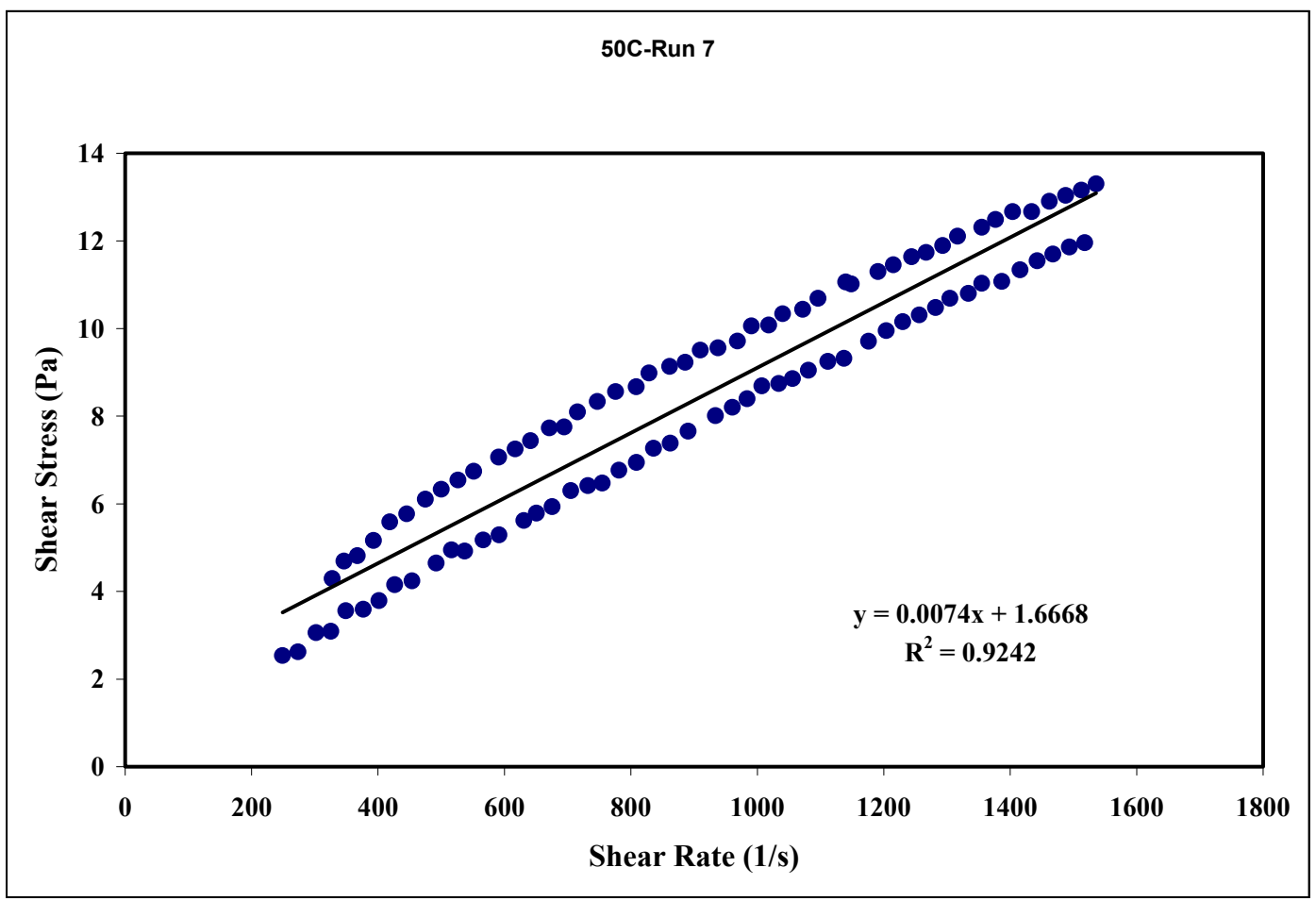

Figure 52. $50^{\circ} \mathrm{C}$ Diluted Run 7 
$\begin{array}{ll}\text { APPENDIX H - PART } 1 & \text { WSRC-TR-2000-00352 }\end{array}$

ENVELOPE C RHEOGRAMS

SRT-RPP-2000-00026

Page 327 of 514

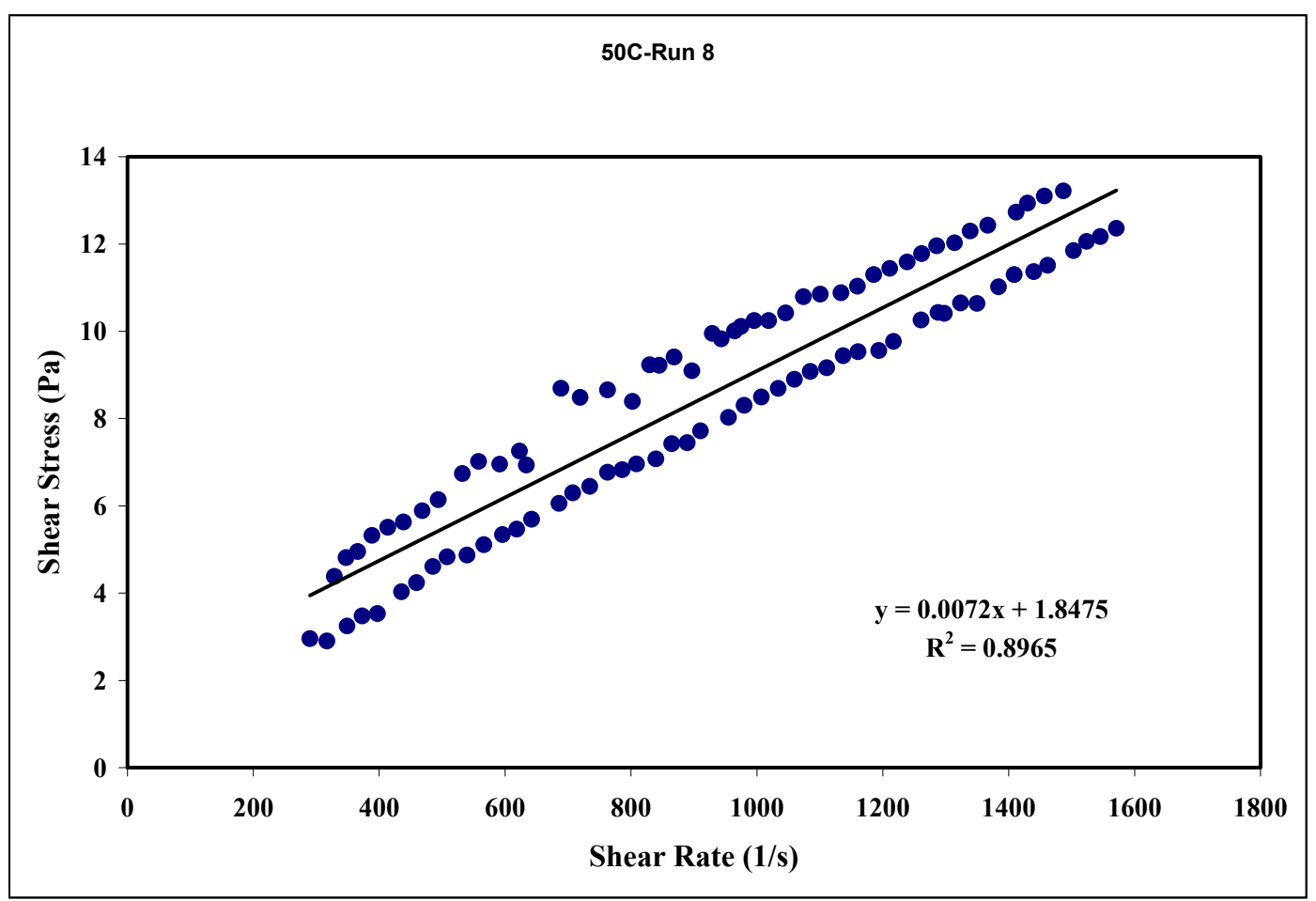

Figure 53. $50^{\circ} \mathrm{C}$ Diluted Run 8

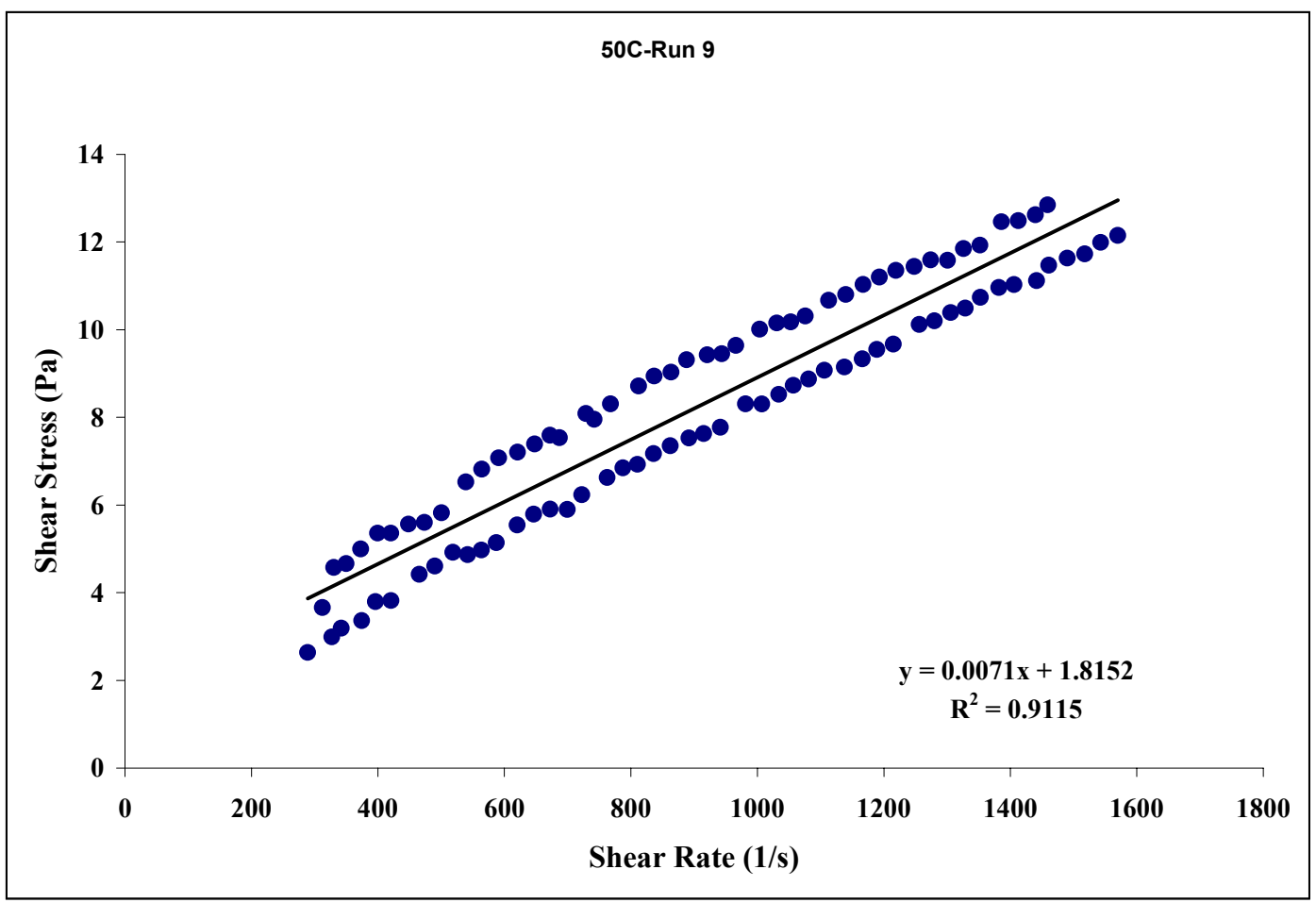

Figure 54. $50^{\circ} \mathrm{C}$ Diluted Run 9 
This page intentionally left blank. 


\begin{tabular}{|ll|}
\hline APPENDIX H - PART 2 & WSRC-TR-2000-00352 \\
ENVELOPE C RHEOGRAMS & SRT-RPP-2000-00026 \\
\hline
\end{tabular}

\section{APPENDIX H - PART 2}

\section{ENVELOPE C RHEOGRAMS}

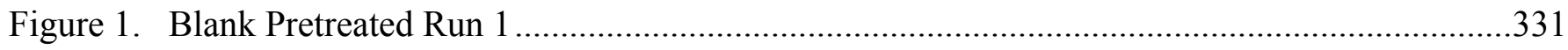

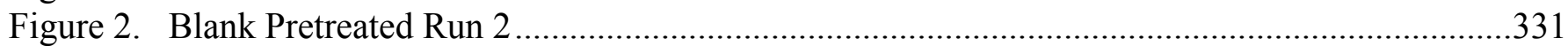

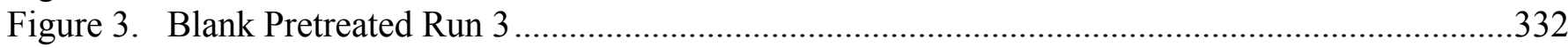

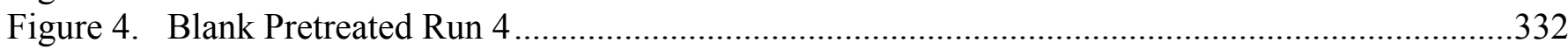

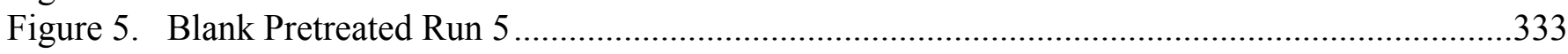

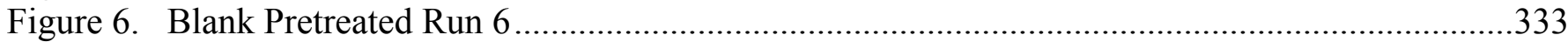

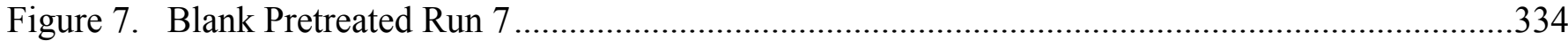

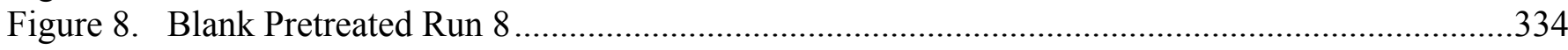

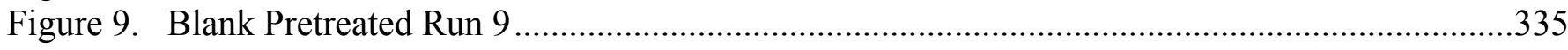

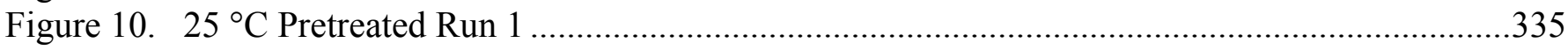

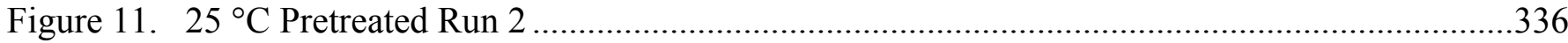

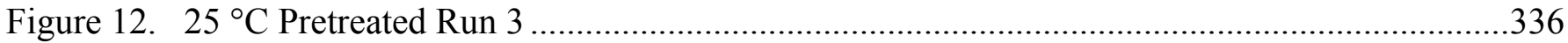

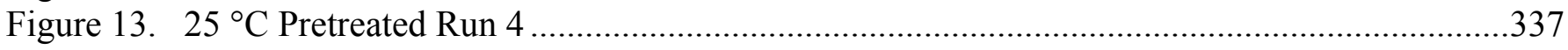

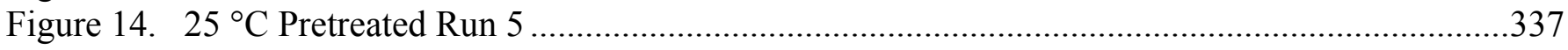

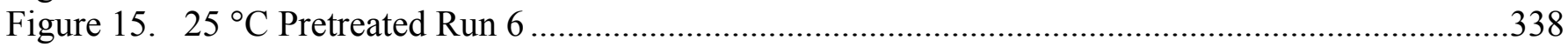

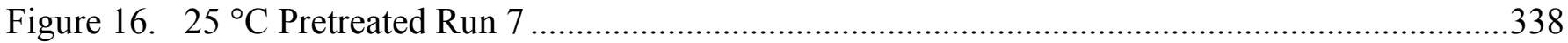

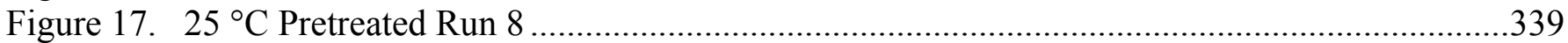

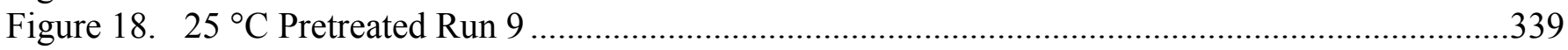

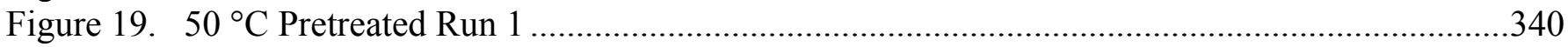

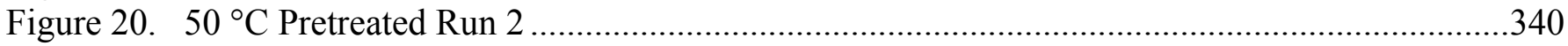

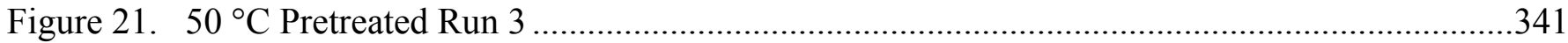

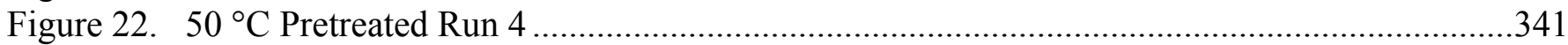

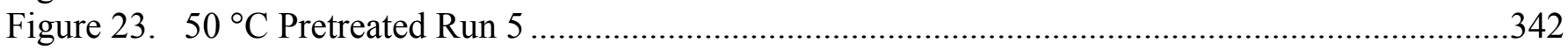

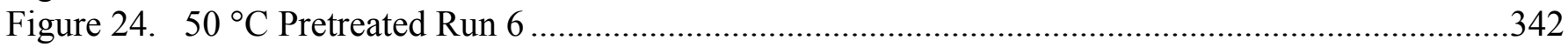

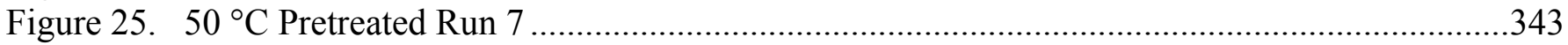

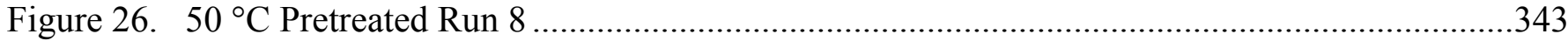

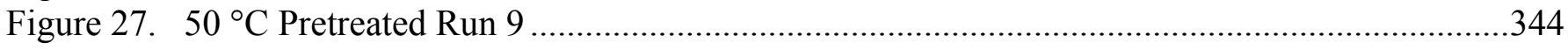


This page intentionally left blank. 
$\begin{array}{ll}\text { APPENDIX H - PART } 2 & \text { WSRC-TR-2000-00352 }\end{array}$

ENVELOPE C RHEOGRAMS

SRT-RPP-2000-00026

Page 331 of 514

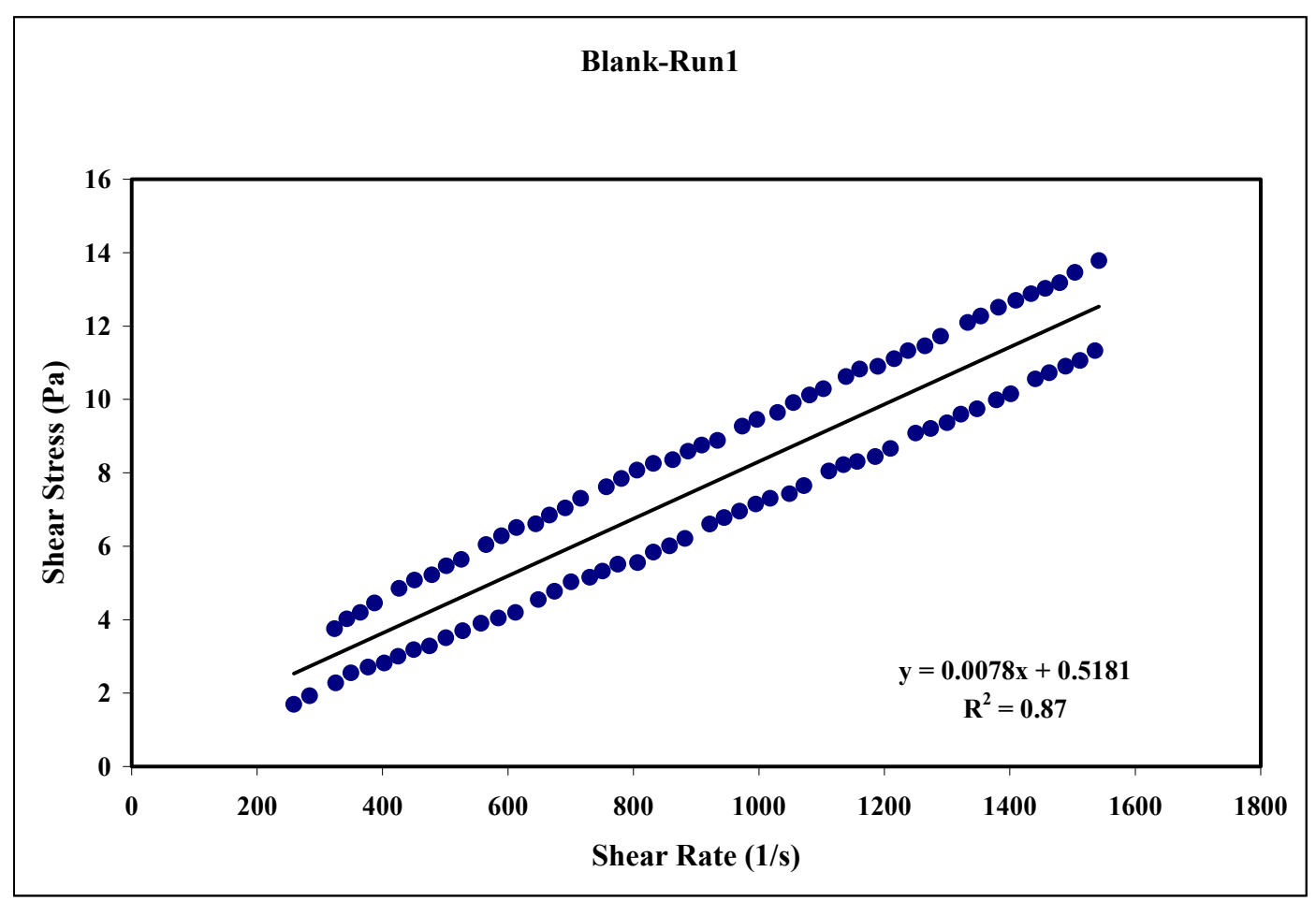

Figure 1. Blank Pretreated Run 1

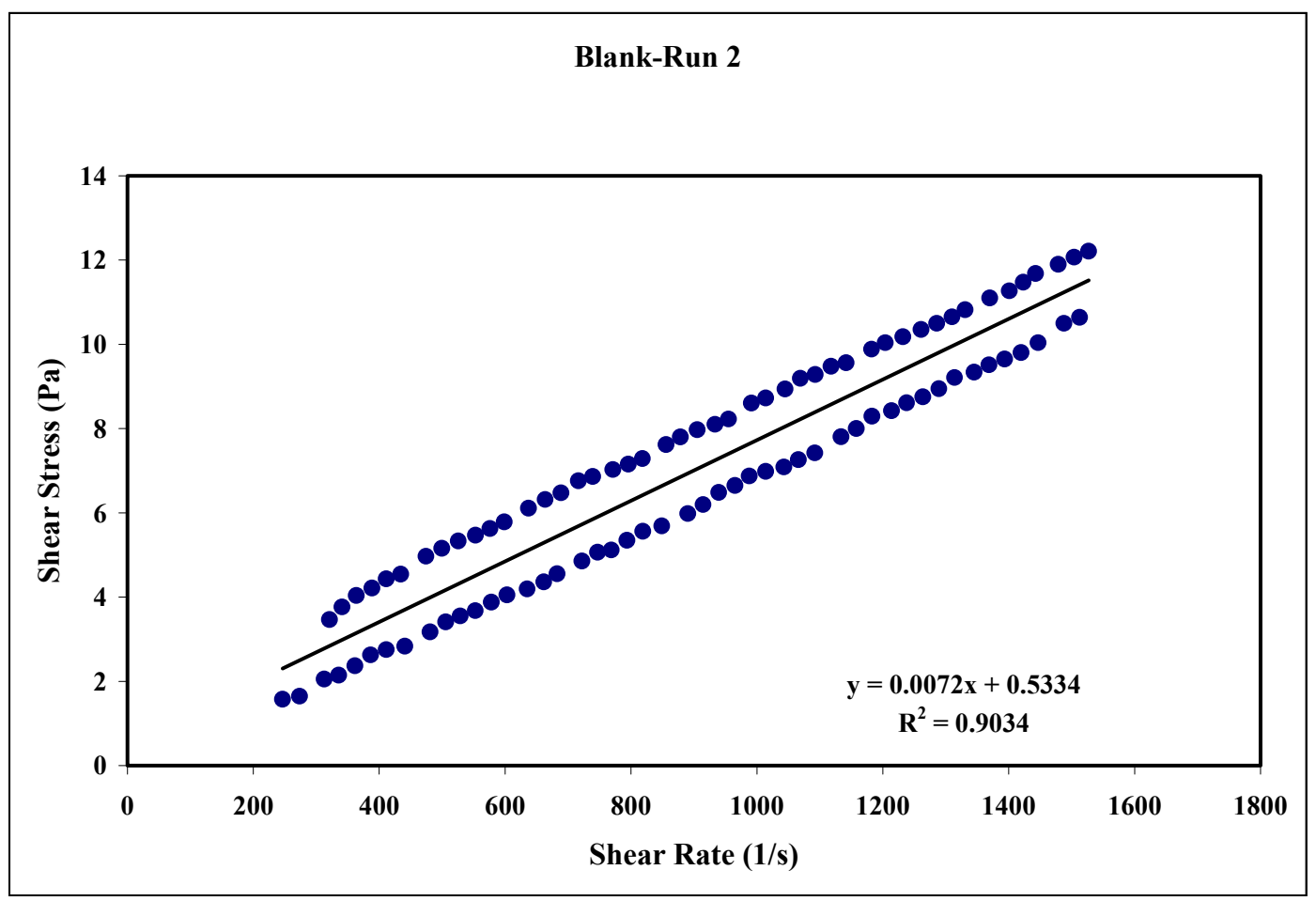

Figure 2. Blank Pretreated Run 2 
$\begin{array}{ll}\text { APPENDIX H - PART } 2 & \text { WSRC-TR-2000-00352 }\end{array}$

ENVELOPE C RHEOGRAMS

SRT-RPP-2000-00026

Page 332 of 514

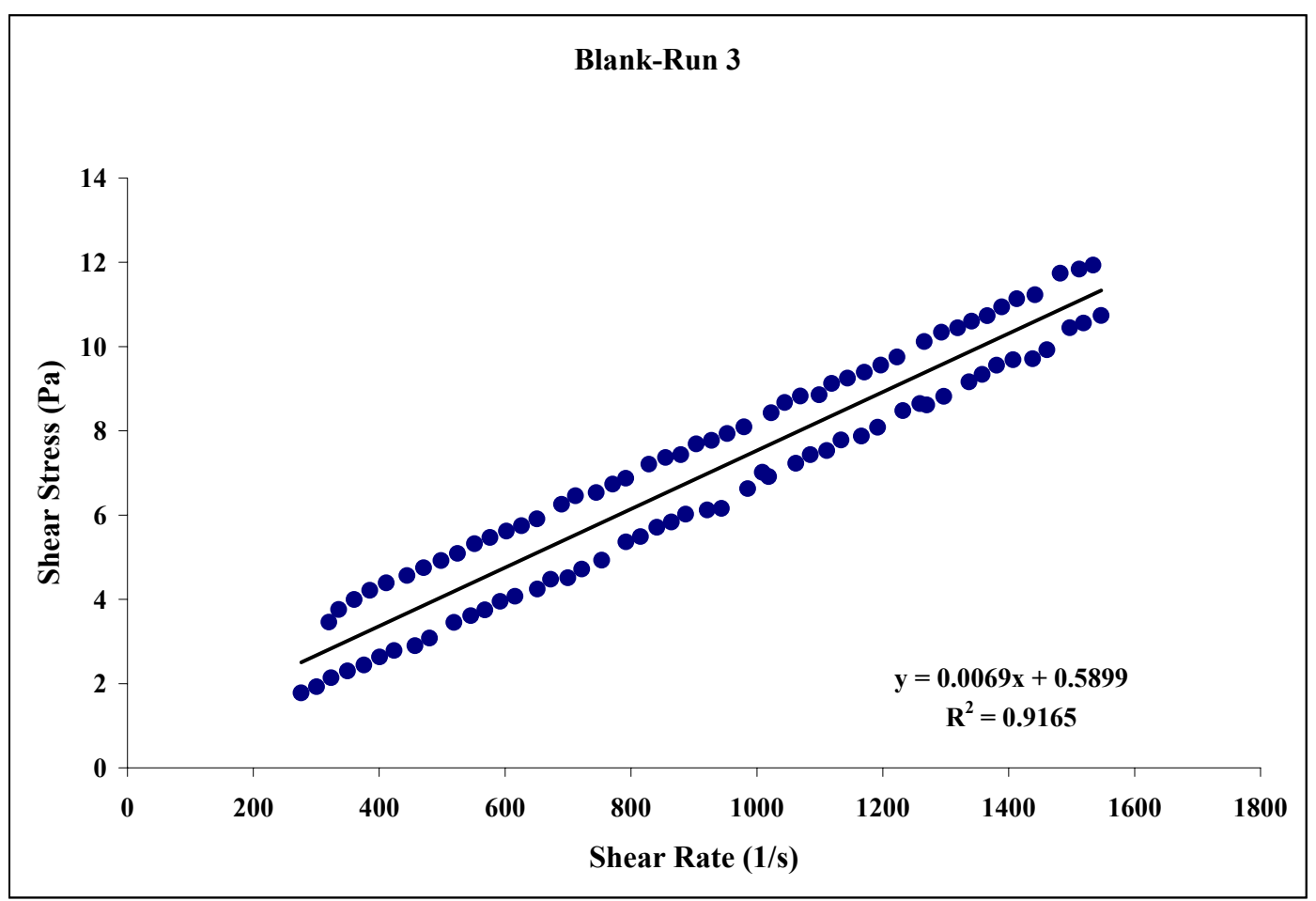

Figure 3. Blank Pretreated Run 3

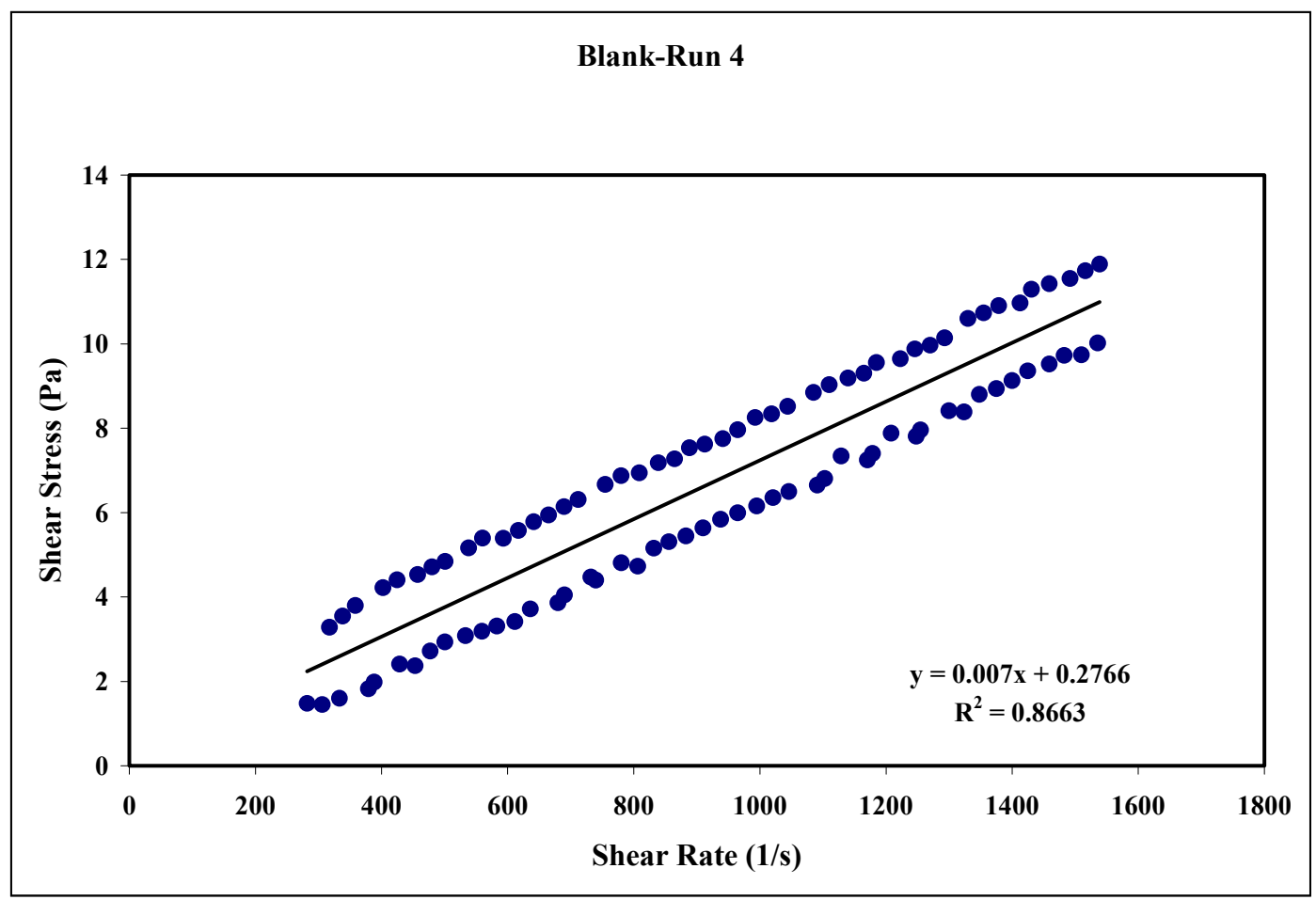

Figure 4. Blank Pretreated Run 4 
$\begin{array}{ll}\text { APPENDIX H - PART } 2 & \text { WSRC-TR-2000-00352 }\end{array}$

ENVELOPE C RHEOGRAMS

SRT-RPP-2000-00026

Page 333 of 514

\section{Blank-Run 5}

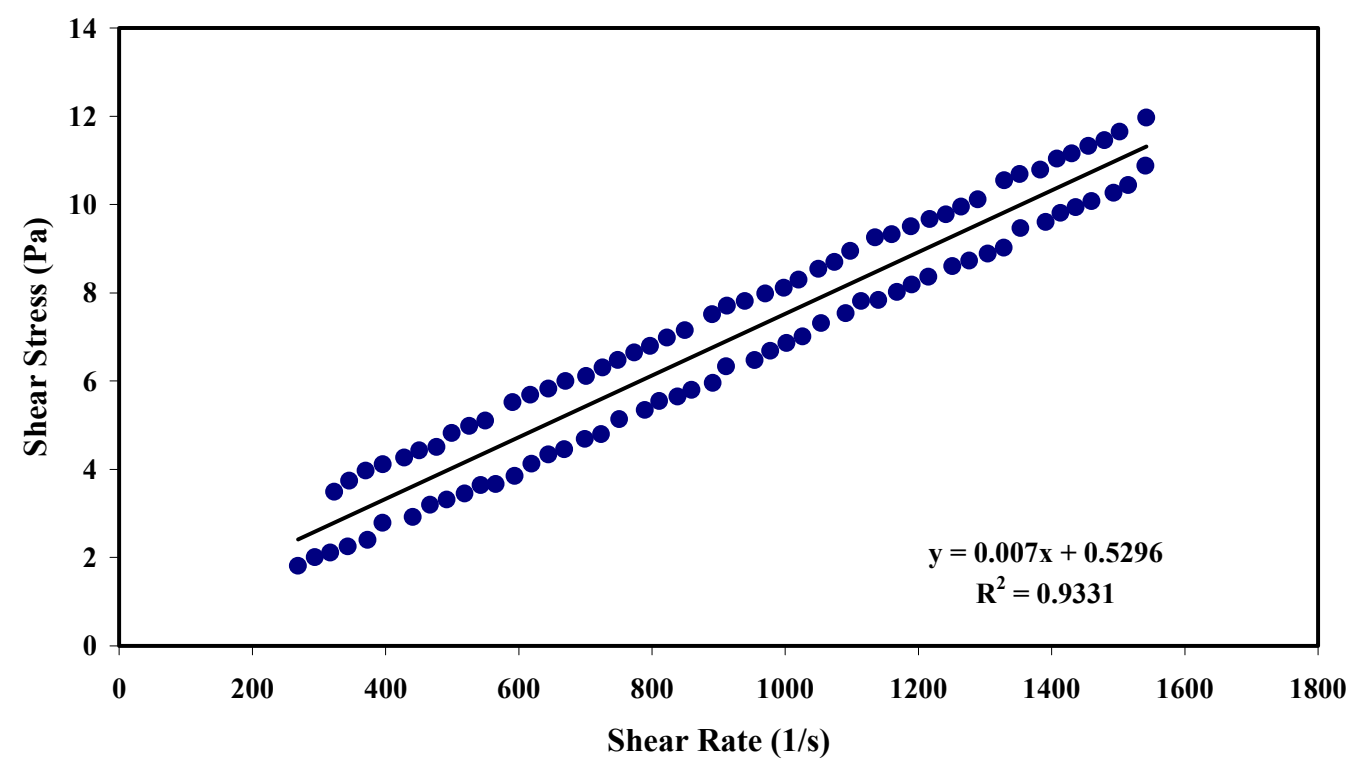

Figure 5. Blank Pretreated Run 5

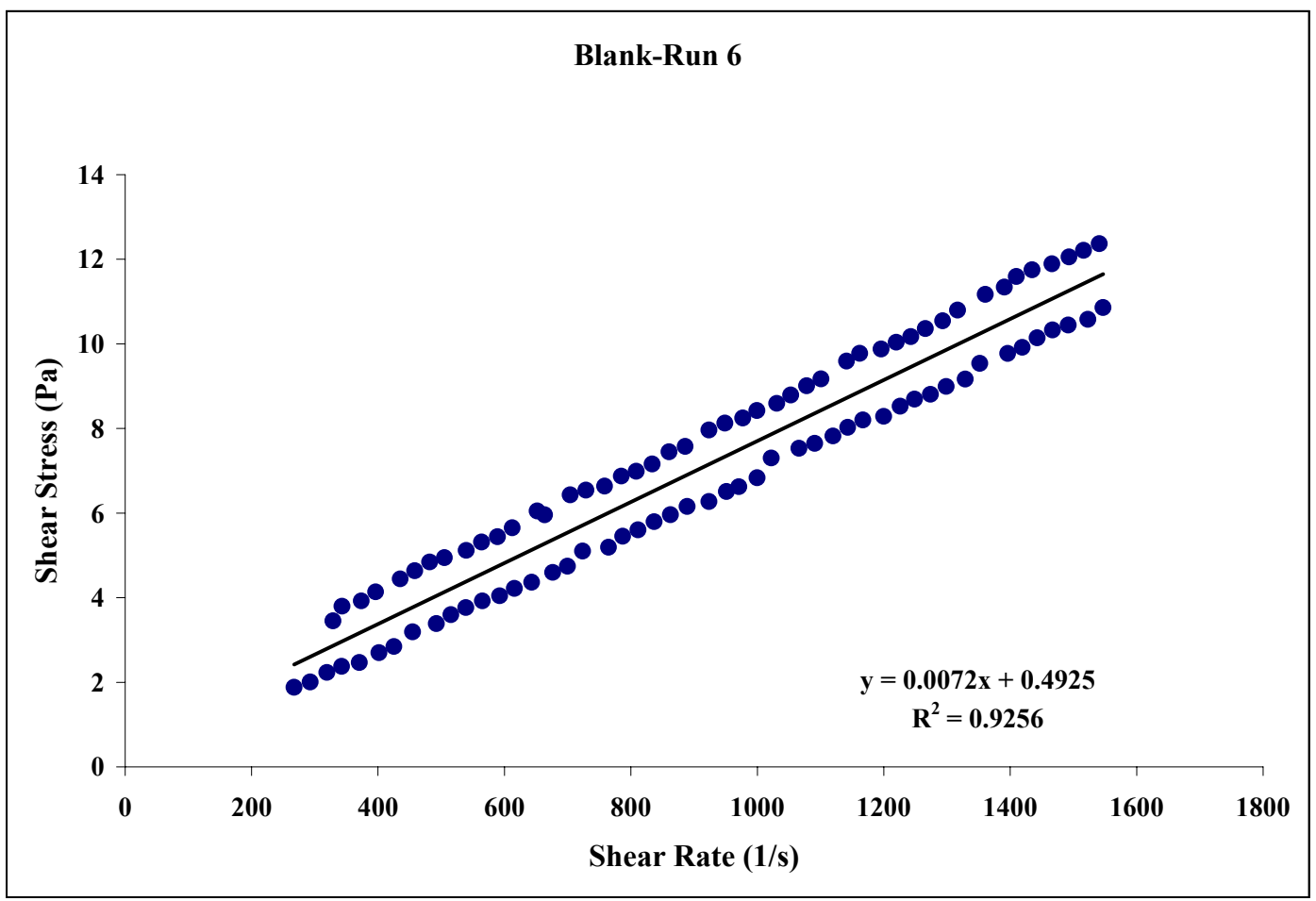

Figure 6. Blank Pretreated Run 6 
$\begin{array}{ll}\text { APPENDIX H - PART } 2 & \text { WSRC-TR-2000-00352 }\end{array}$

ENVELOPE C RHEOGRAMS

SRT-RPP-2000-00026

Page 334 of 514

\section{Blank-Run 7}

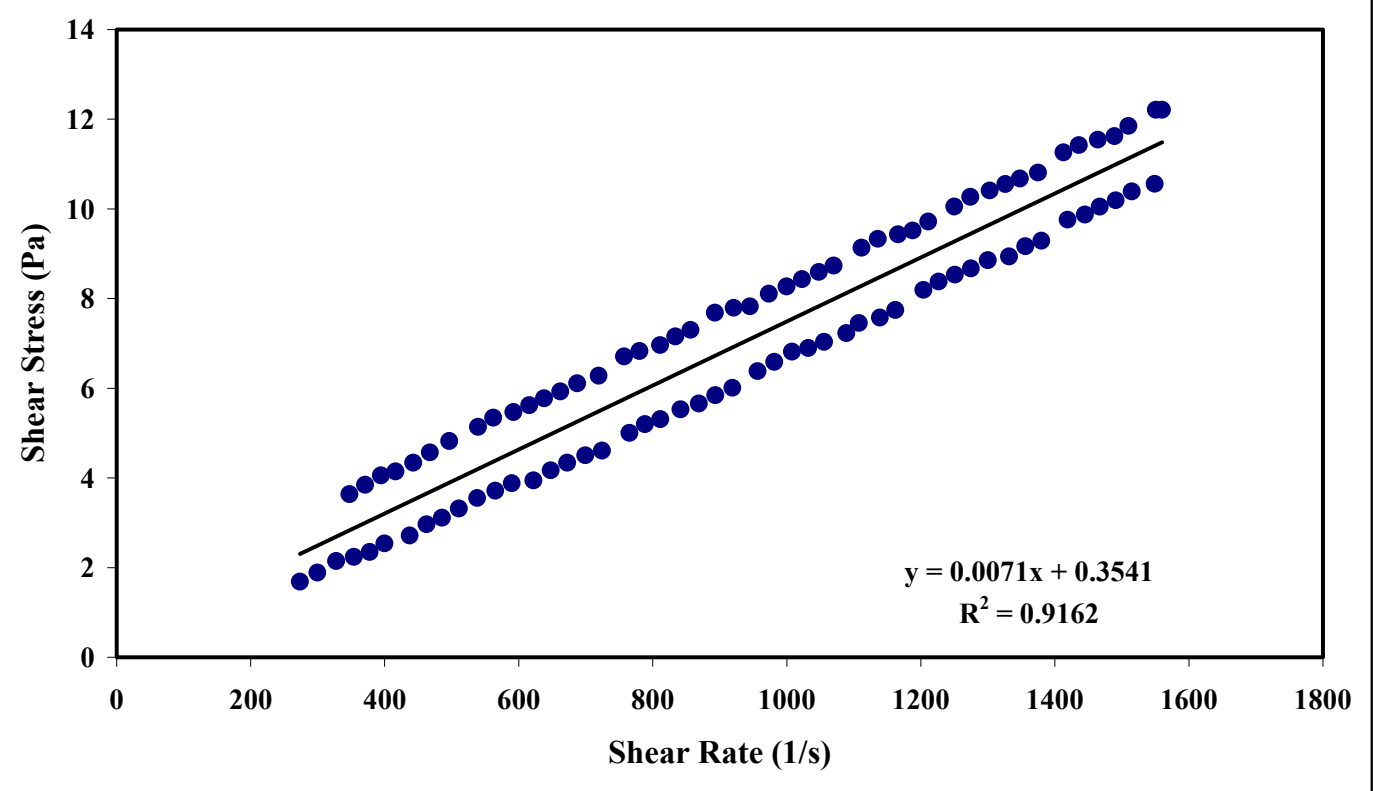

Figure 7. Blank Pretreated Run 7

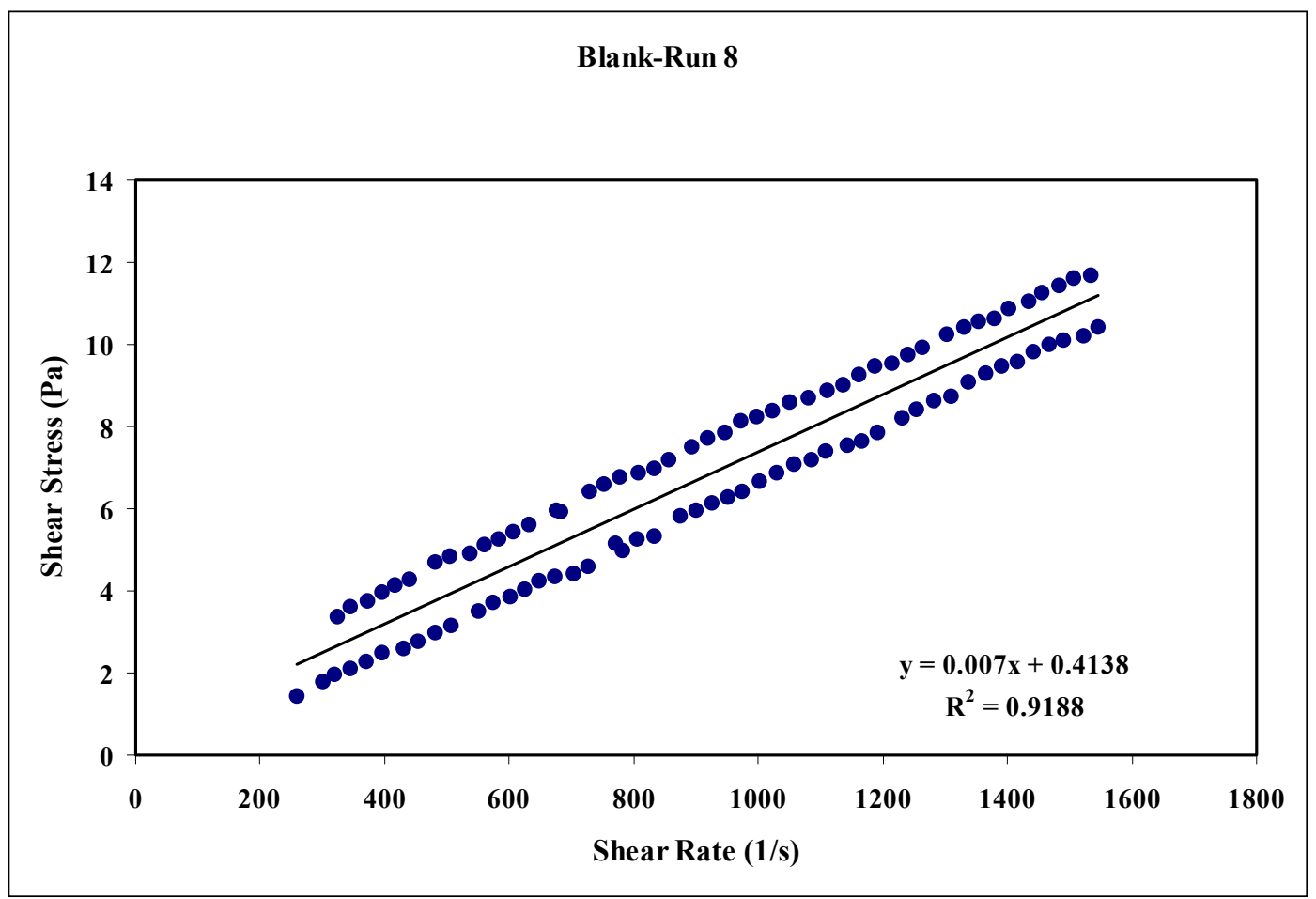

Figure 8. Blank Pretreated Run 8 
$\begin{array}{ll}\text { APPENDIX H - PART } 2 & \text { WSRC-TR-2000-00352 }\end{array}$

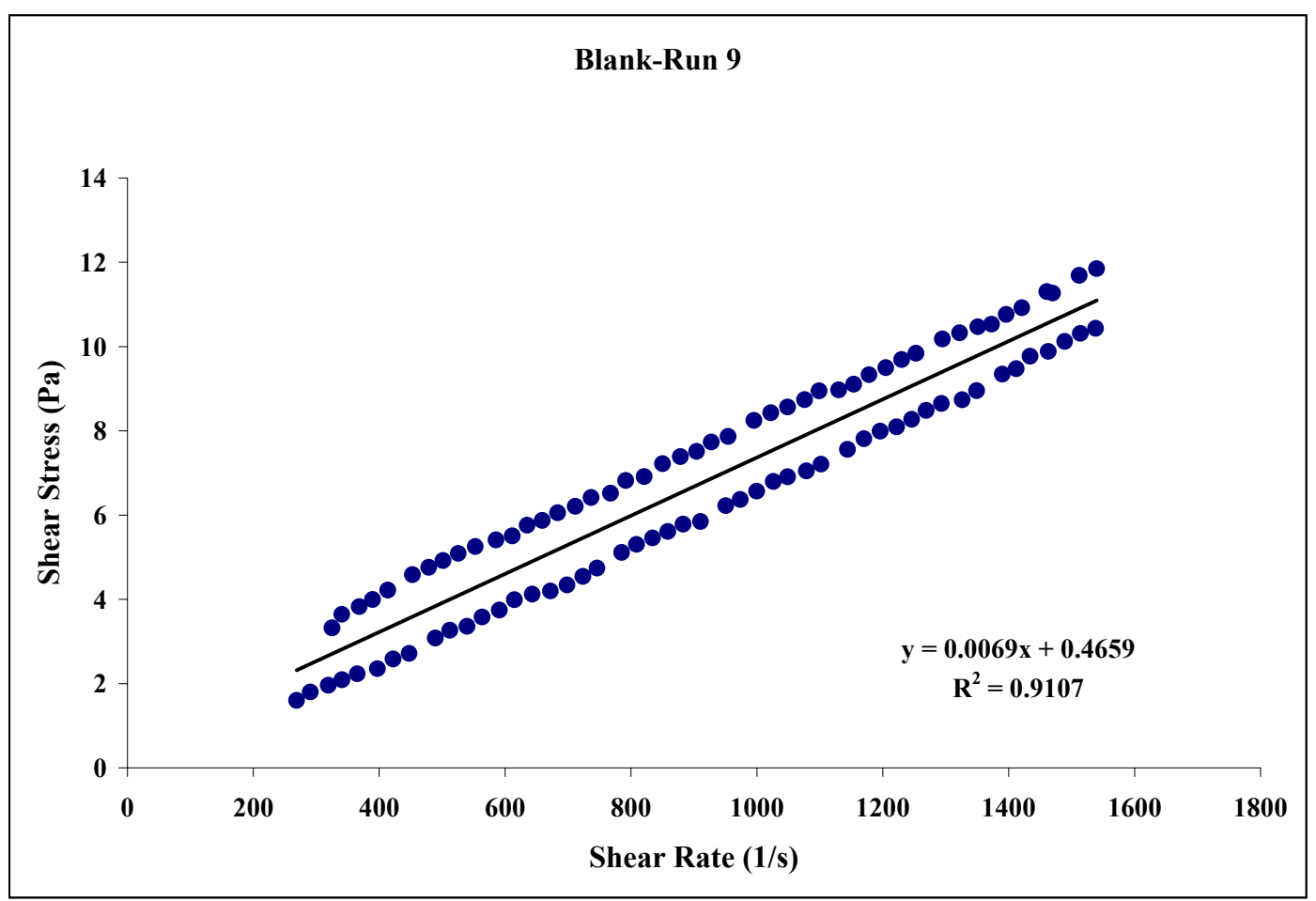

Figure 9. Blank Pretreated Run 9

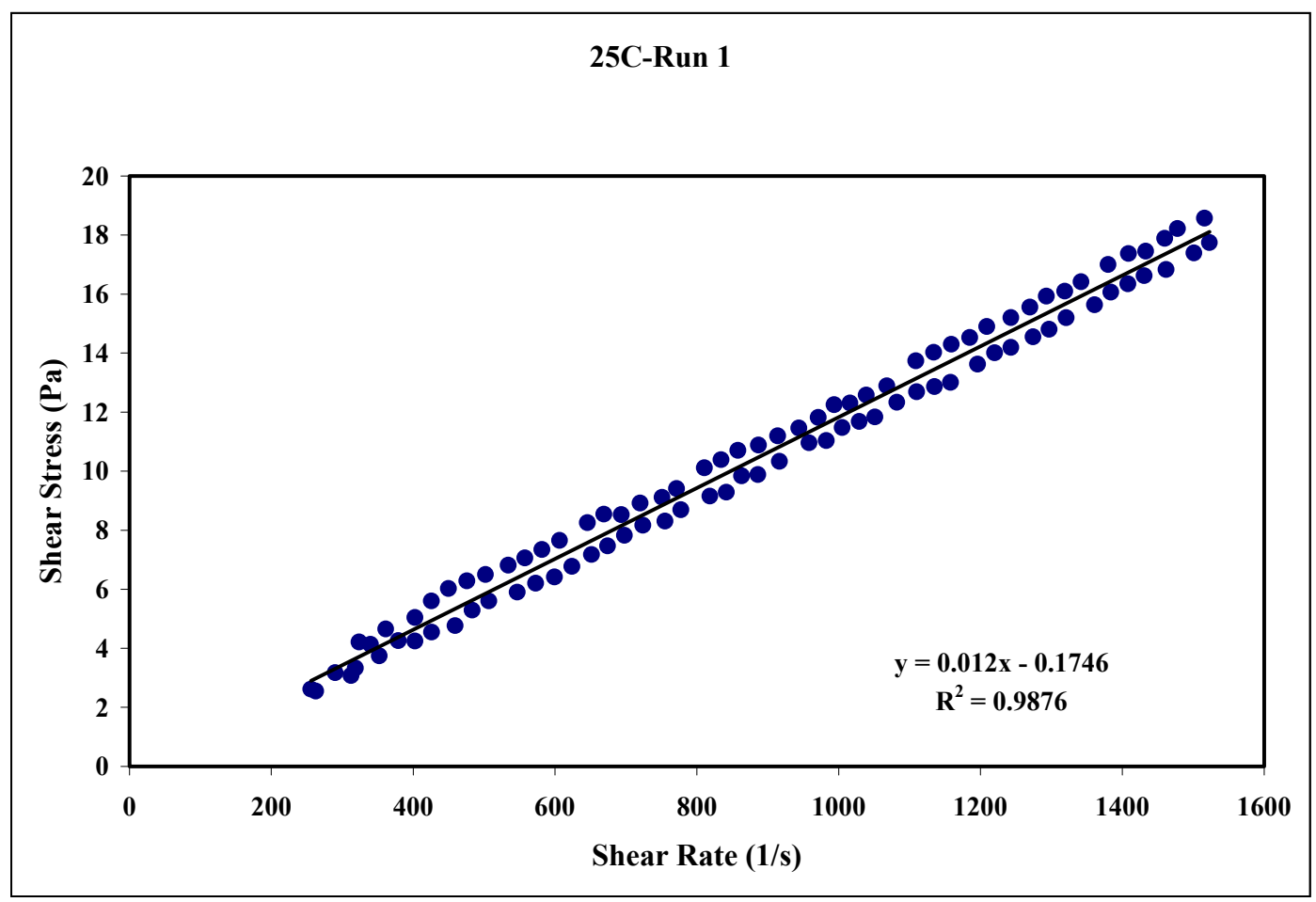

Figure 10. $25{ }^{\circ} \mathrm{C}$ Pretreated Run 1 
$\begin{array}{ll}\text { APPENDIX H - PART } 2 & \text { WSRC-TR-2000-00352 }\end{array}$

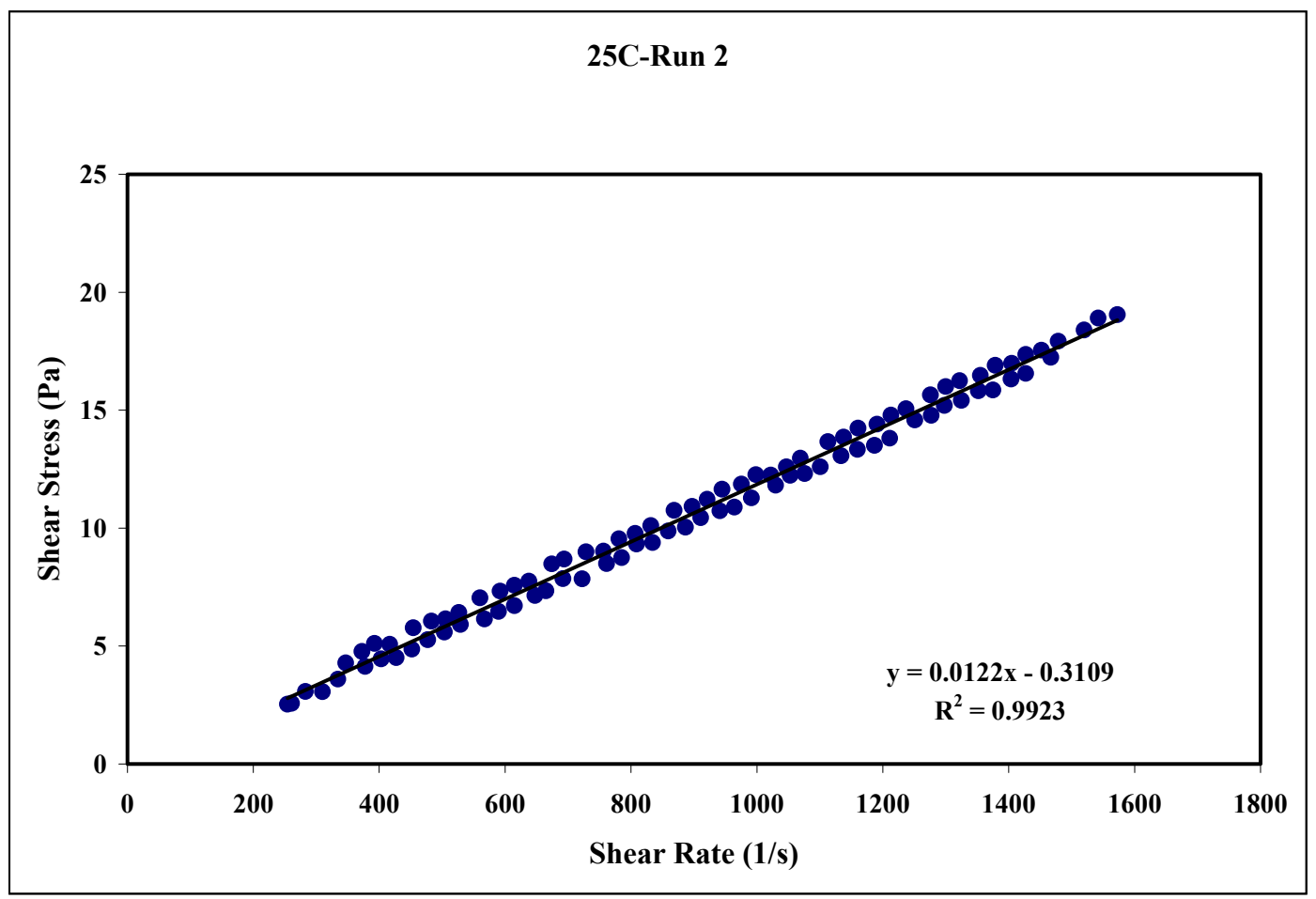

Figure 11. $25^{\circ} \mathrm{C}$ Pretreated Run 2

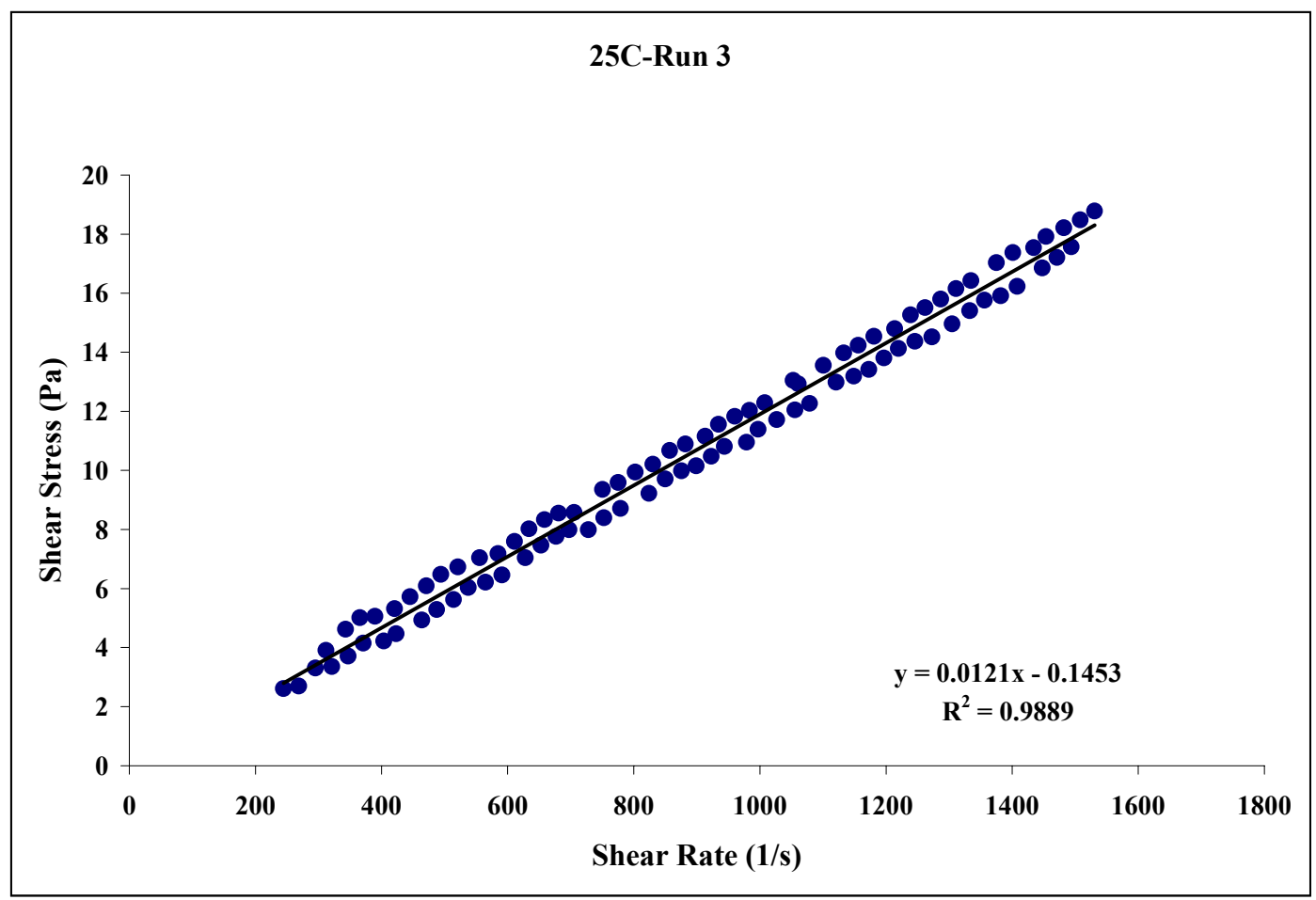

Figure 12. $25^{\circ} \mathrm{C}$ Pretreated Run 3 
$\begin{array}{ll}\text { APPENDIX H - PART } 2 & \text { WSRC-TR-2000-00352 }\end{array}$

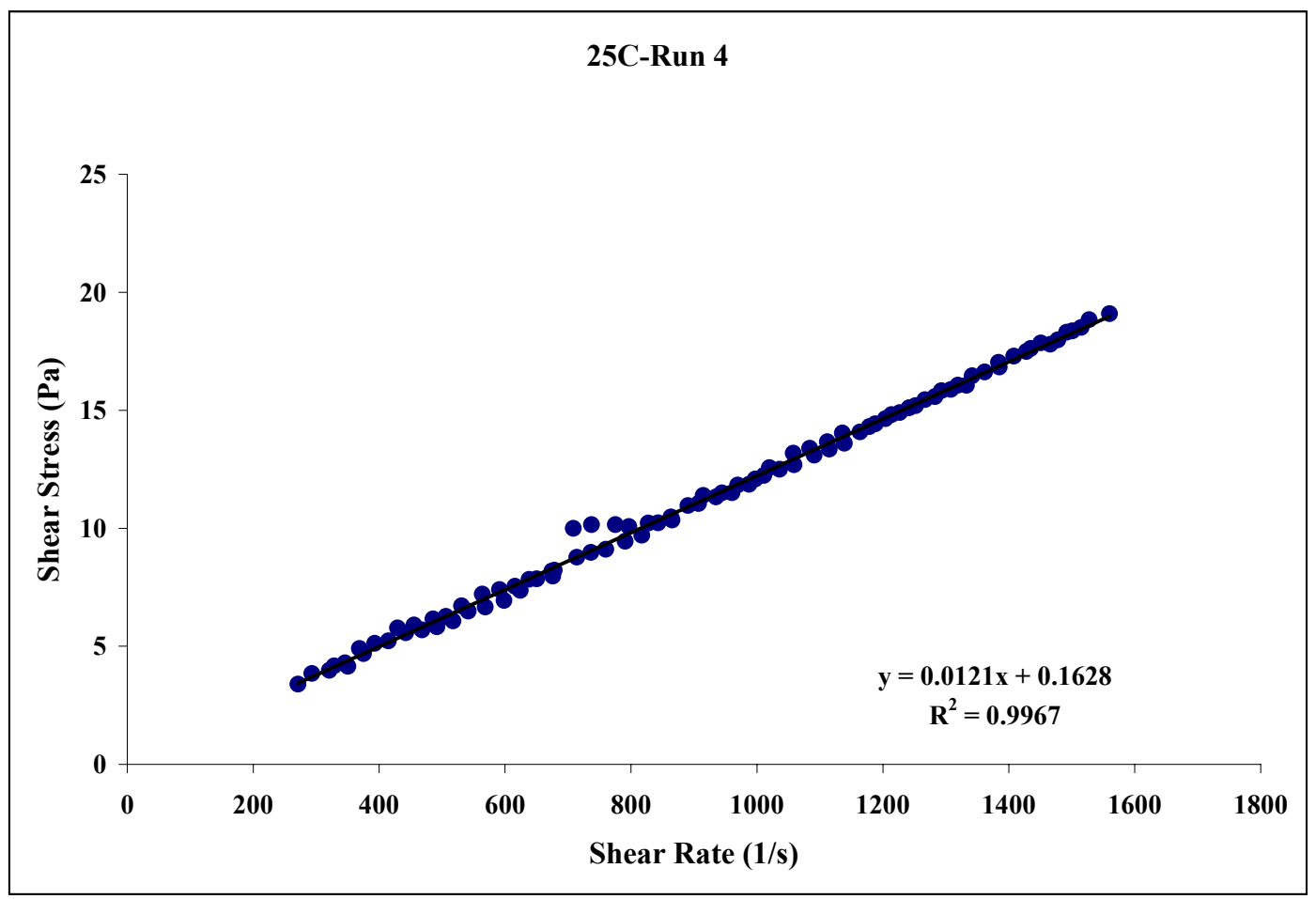

Figure 13. $25^{\circ} \mathrm{C}$ Pretreated Run 4

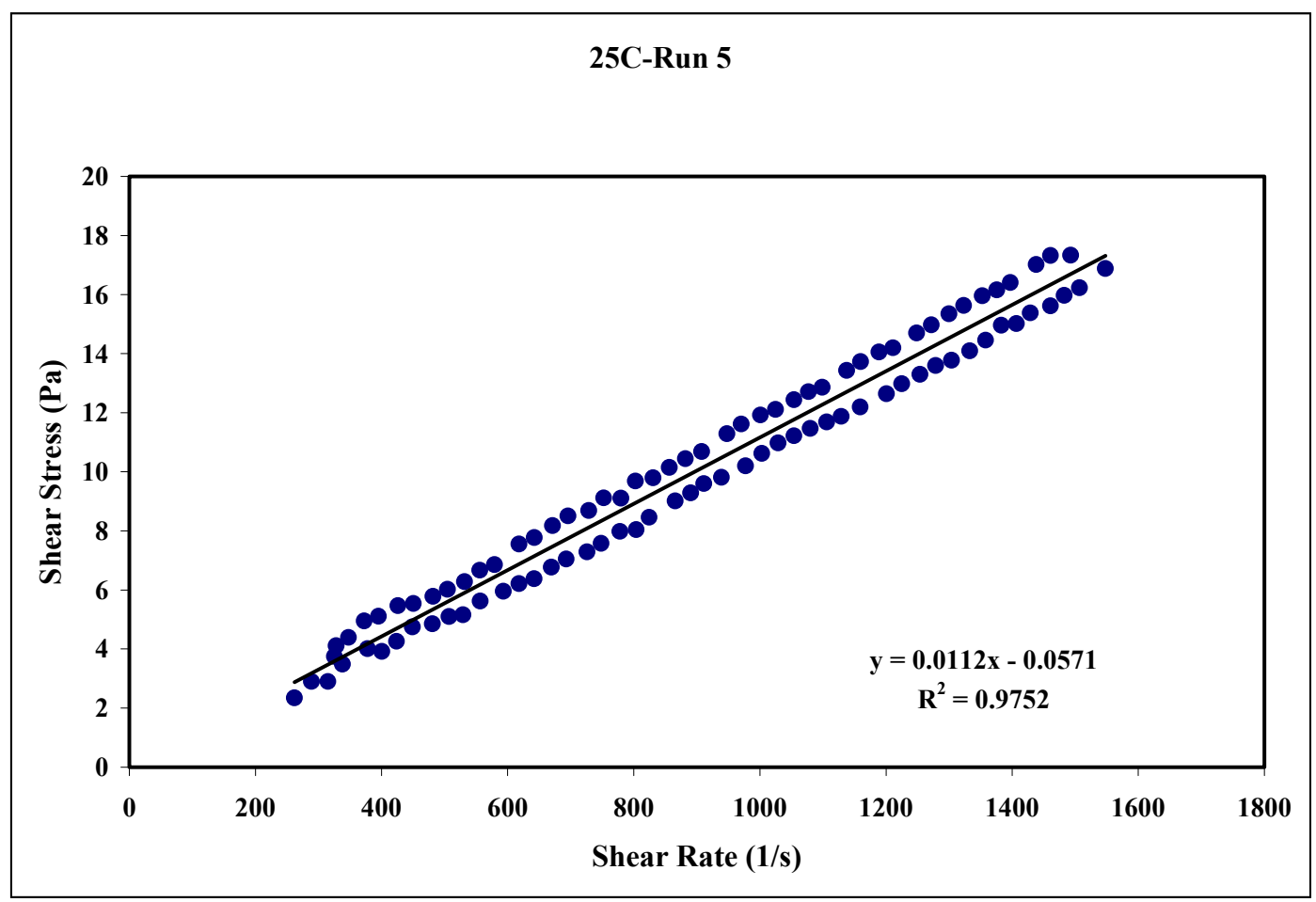

Figure 14. $25^{\circ} \mathrm{C}$ Pretreated Run 5 
$\begin{array}{ll}\text { APPENDIX H - PART } 2 & \text { WSRC-TR-2000-00352 }\end{array}$

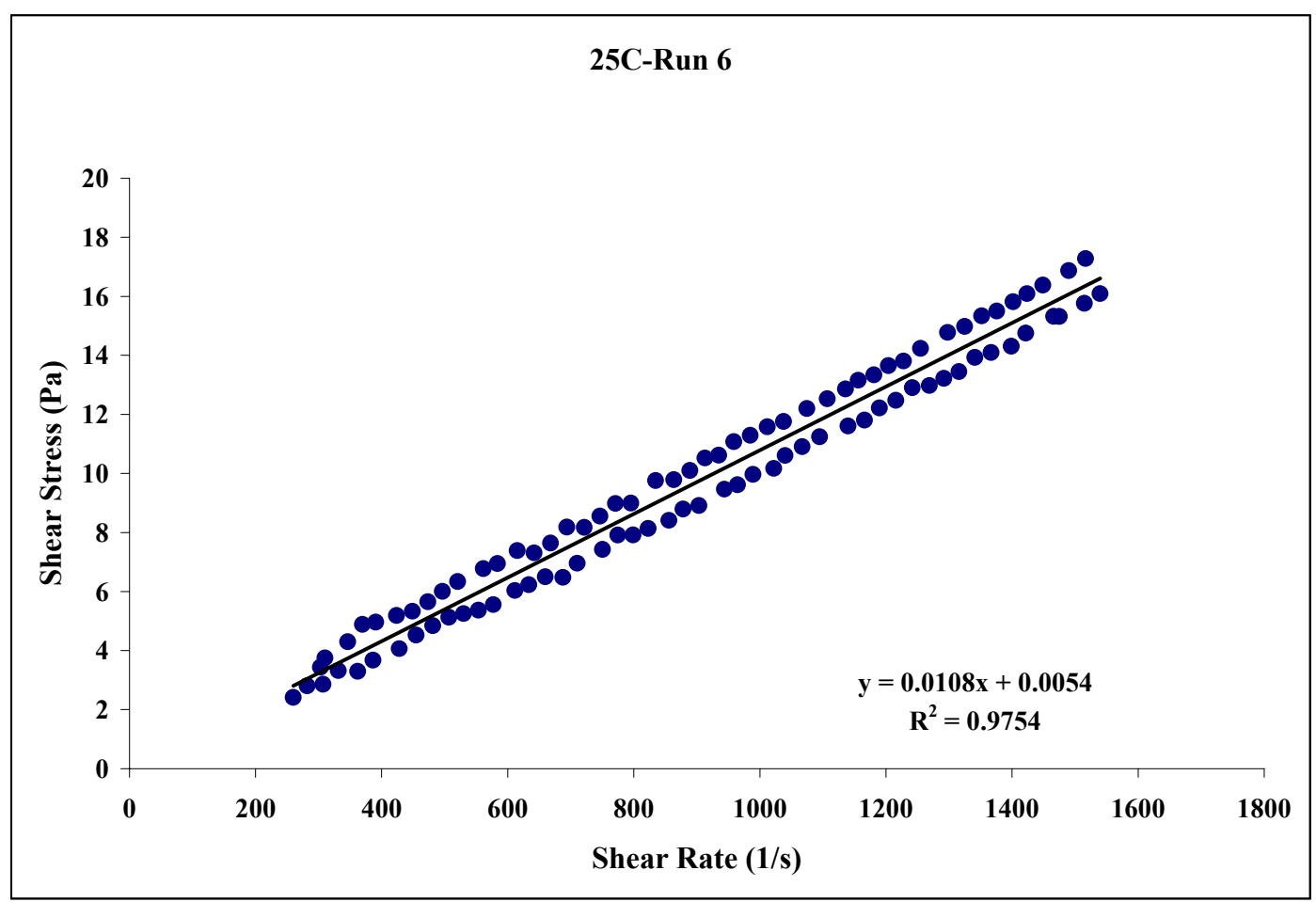

Figure 15. $25^{\circ} \mathrm{C}$ Pretreated Run 6

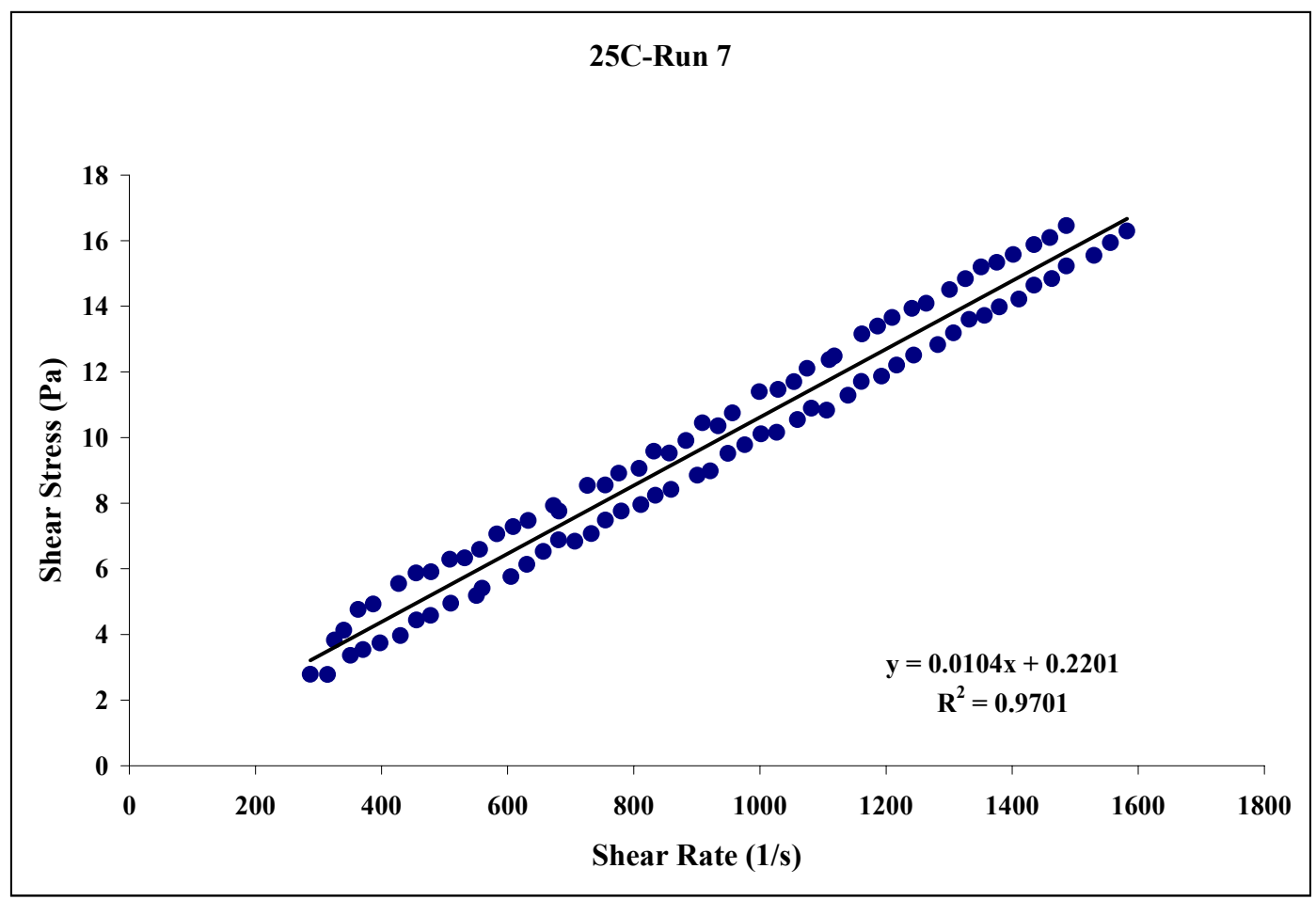

Figure 16. $25^{\circ} \mathrm{C}$ Pretreated Run 7 
$\begin{array}{ll}\text { APPENDIX H - PART } 2 & \text { WSRC-TR-2000-00352 }\end{array}$

ENVELOPE C RHEOGRAMS

SRT-RPP-2000-00026

Page 339 of 514

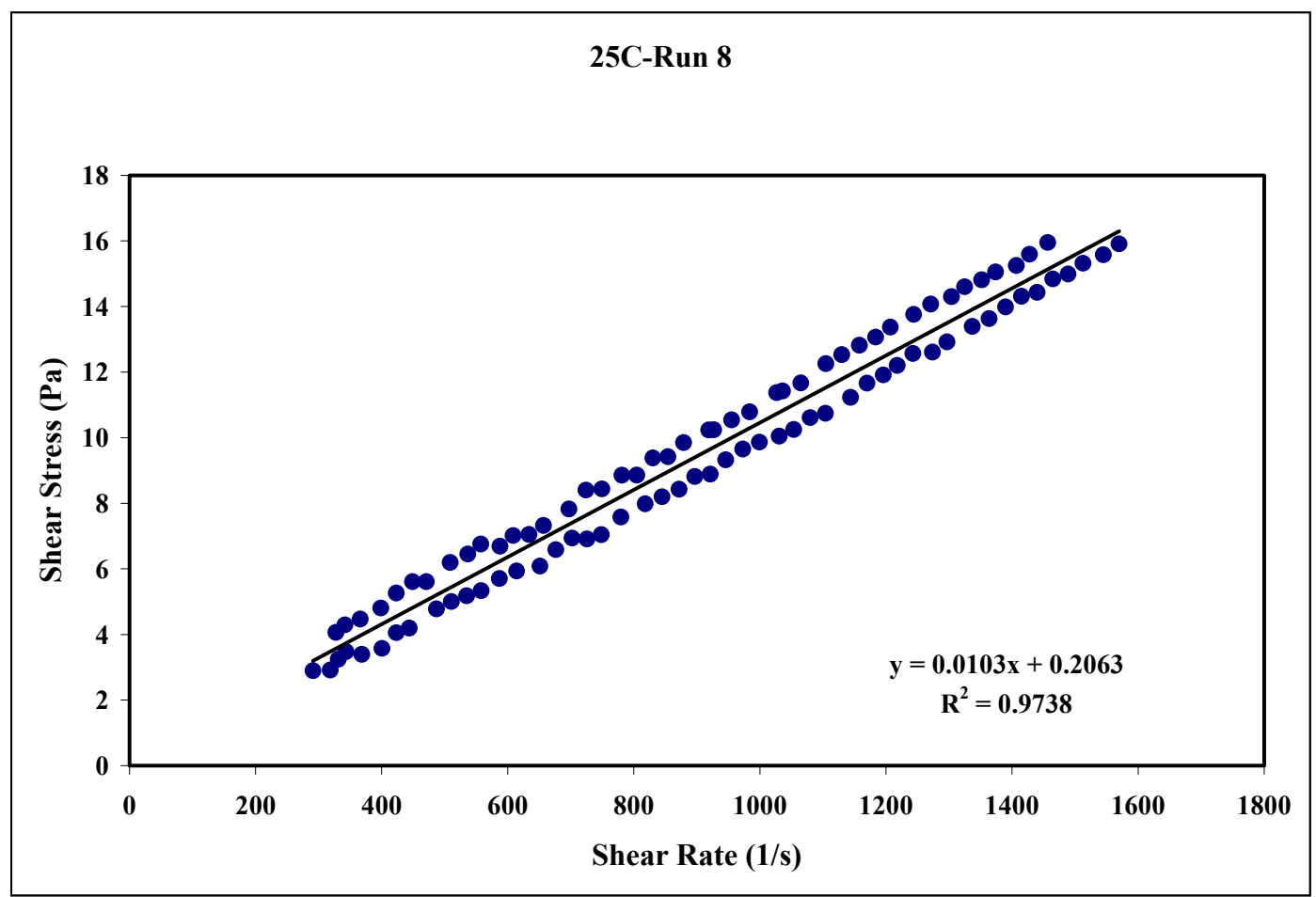

Figure 17. $25^{\circ} \mathrm{C}$ Pretreated Run 8

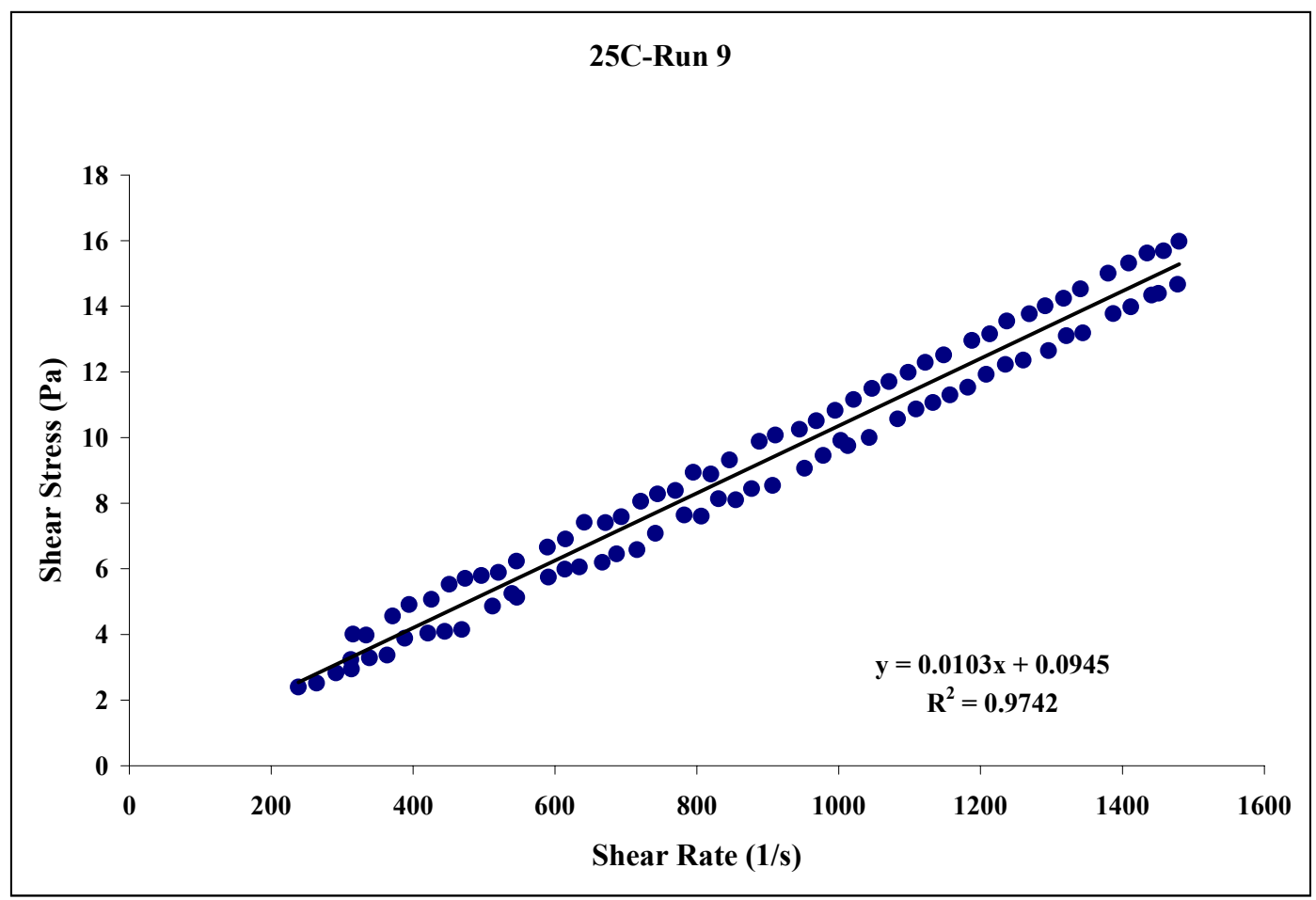

Figure 18. $25^{\circ} \mathrm{C}$ Pretreated Run 9 
$\begin{array}{ll}\text { APPENDIX H - PART } 2 & \text { WSRC-TR-2000-00352 }\end{array}$

ENVELOPE C RHEOGRAMS

SRT-RPP-2000-00026

Page 340 of 514

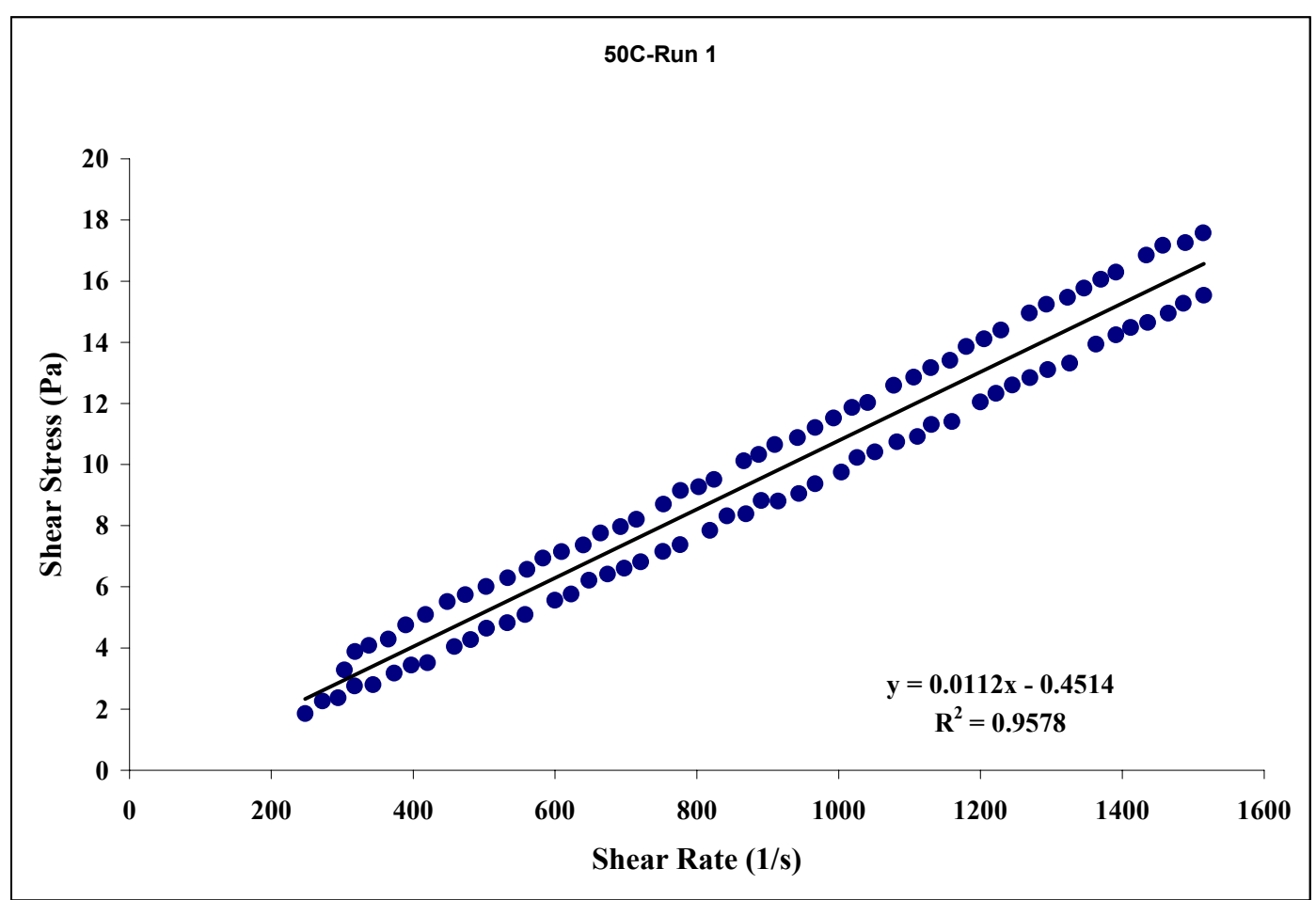

Figure 19. $50{ }^{\circ} \mathrm{C}$ Pretreated Run 1

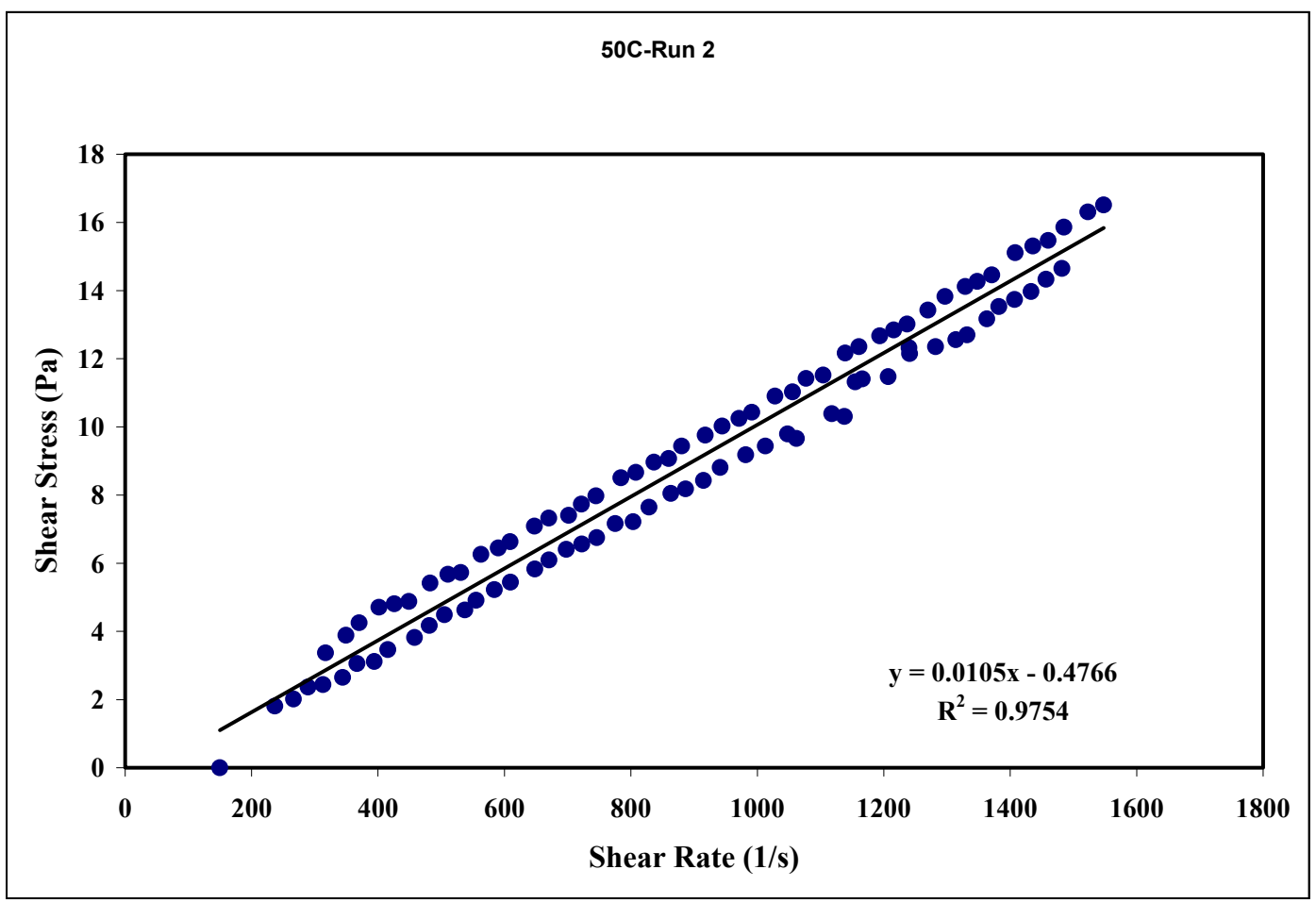

Figure 20. $50{ }^{\circ} \mathrm{C}$ Pretreated Run 2 
$\begin{array}{ll}\text { APPENDIX H - PART } 2 & \text { WSRC-TR-2000-00352 }\end{array}$

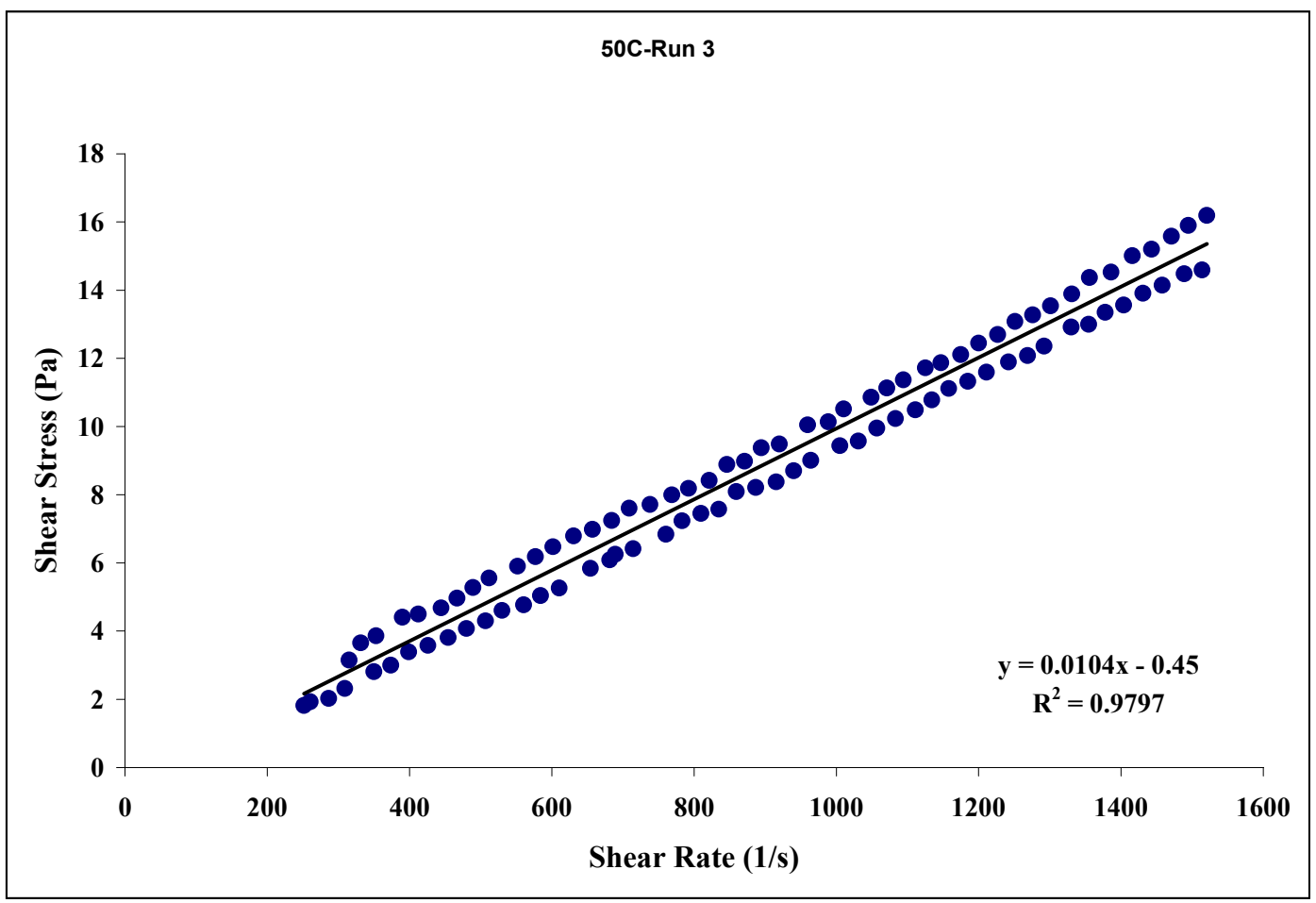

Figure 21. $50{ }^{\circ} \mathrm{C}$ Pretreated Run 3

Figure 22. $50{ }^{\circ} \mathrm{C}$ Pretreated Run 4 
$\begin{array}{ll}\text { APPENDIX H - PART } 2 & \text { WSRC-TR-2000-00352 }\end{array}$

ENVELOPE C RHEOGRAMS

SRT-RPP-2000-00026

Page 342 of 514

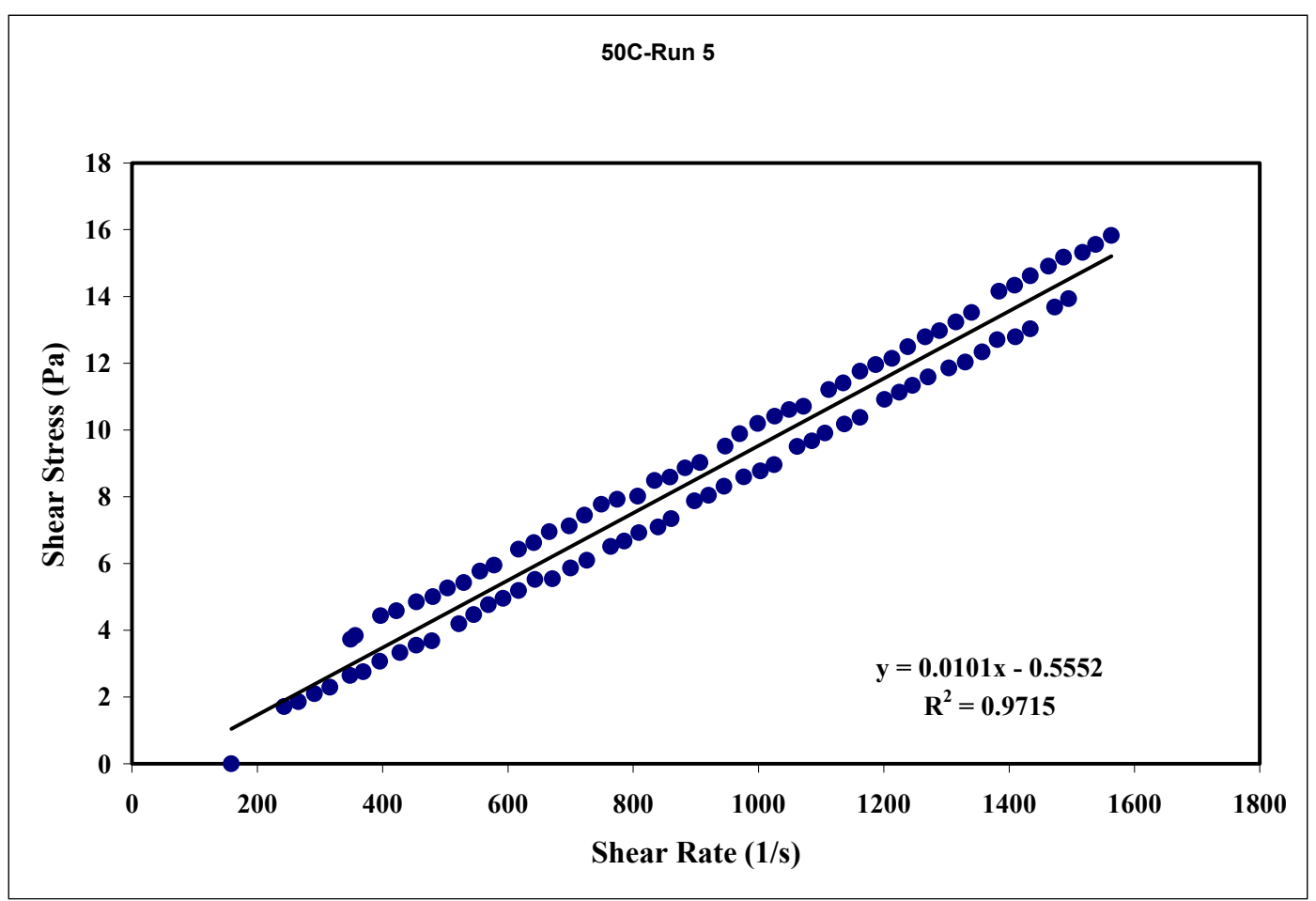

Figure 23. $50{ }^{\circ} \mathrm{C}$ Pretreated Run 5

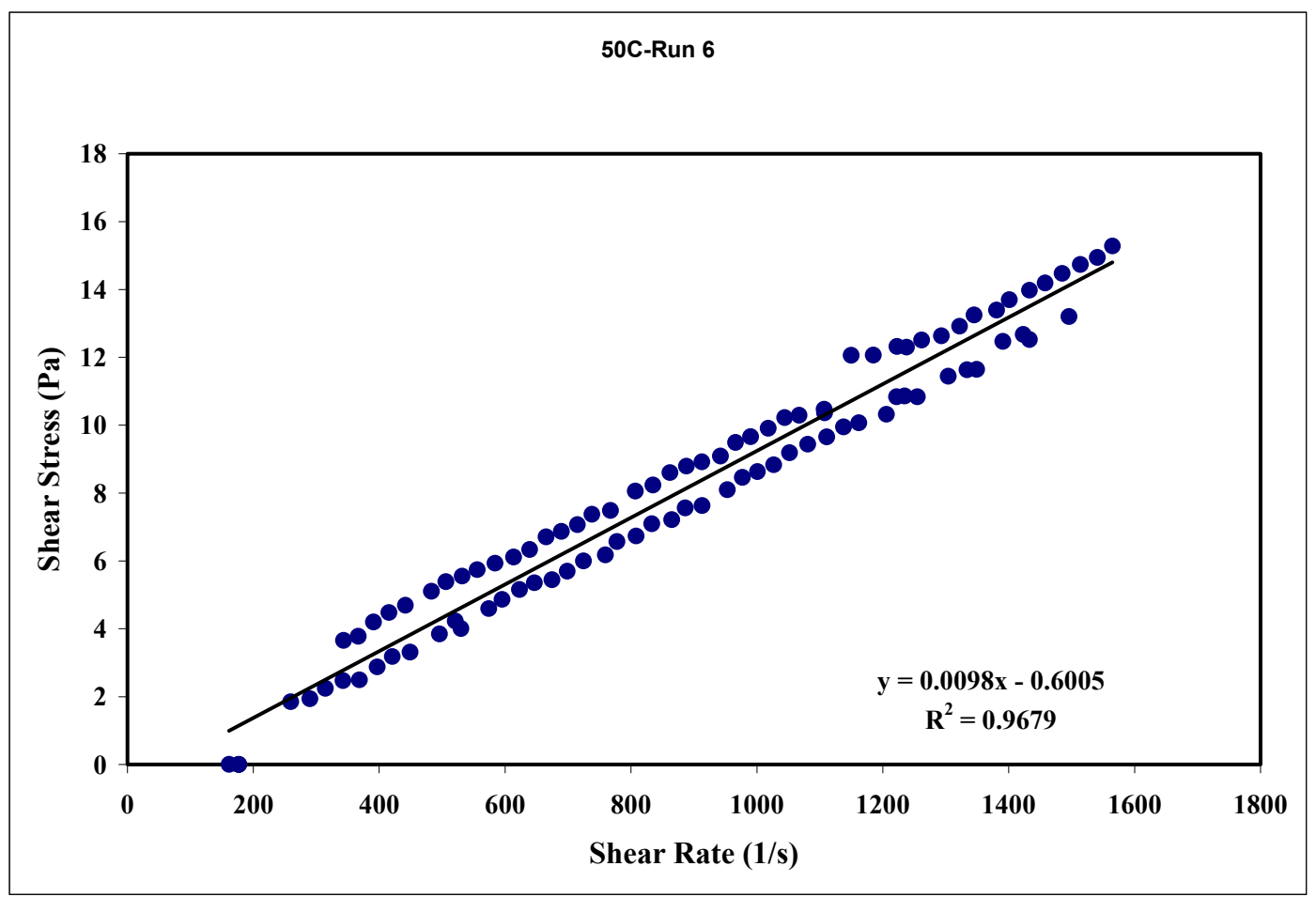

Figure 24. $50{ }^{\circ} \mathrm{C}$ Pretreated Run 6 
$\begin{array}{ll}\text { APPENDIX H - PART } 2 & \text { WSRC-TR-2000-00352 }\end{array}$

ENVELOPE C RHEOGRAMS

SRT-RPP-2000-00026

Page 343 of 514

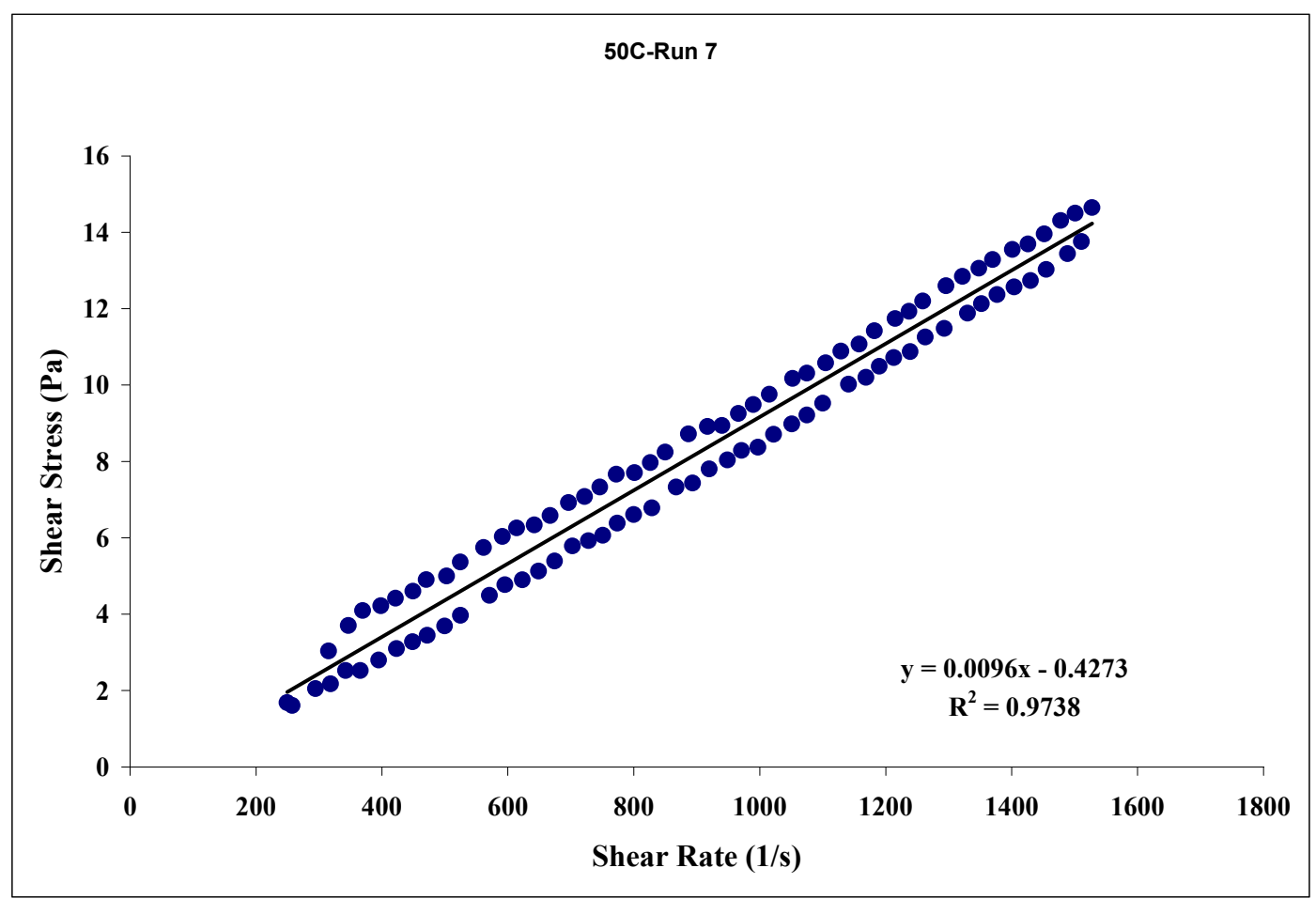

Figure 25. $50{ }^{\circ} \mathrm{C}$ Pretreated Run 7

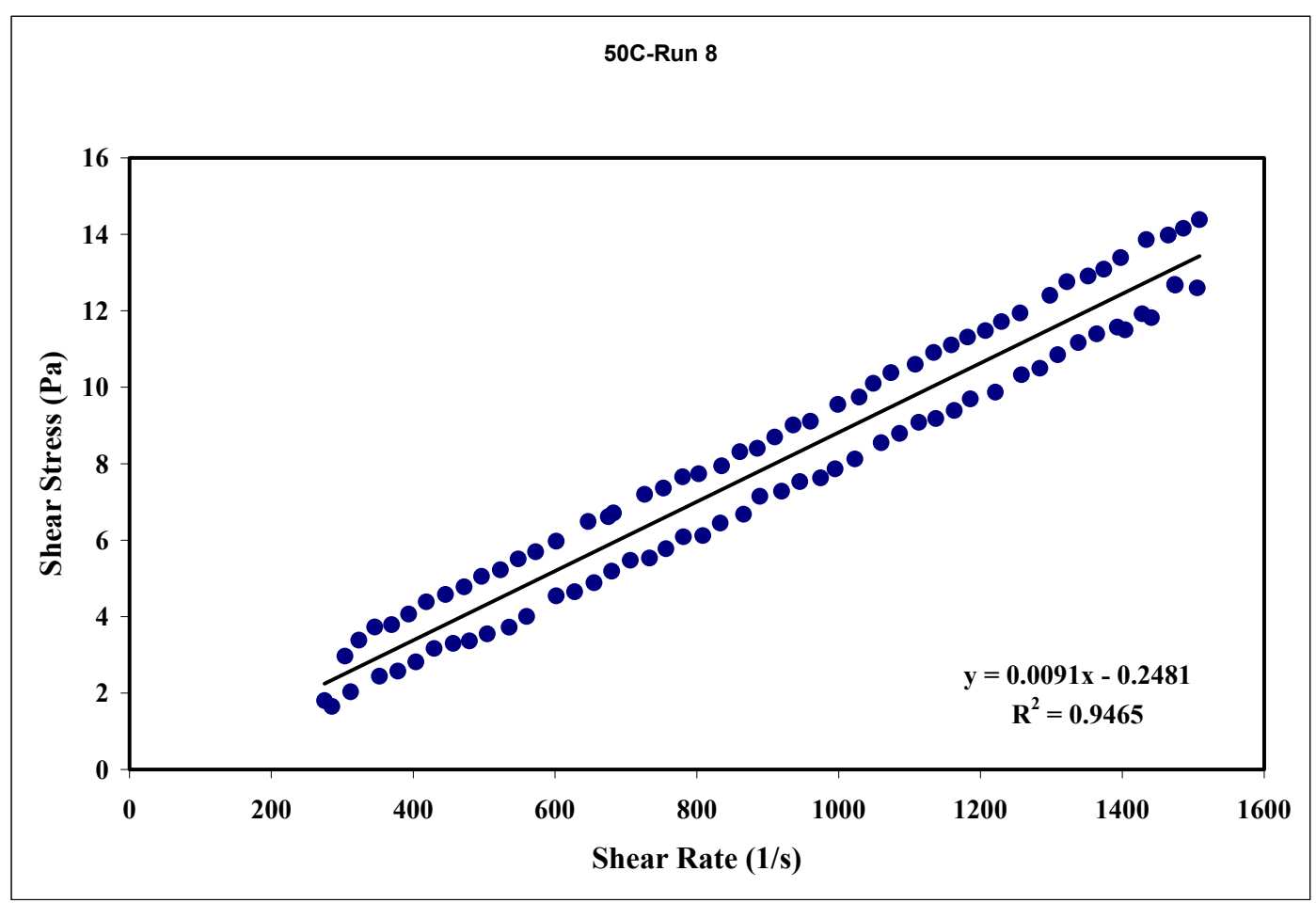

Figure 26. $50{ }^{\circ} \mathrm{C}$ Pretreated Run 8 
$\begin{array}{ll}\text { APPENDIX H - PART } 2 & \text { WSRC-TR-2000-00352 }\end{array}$

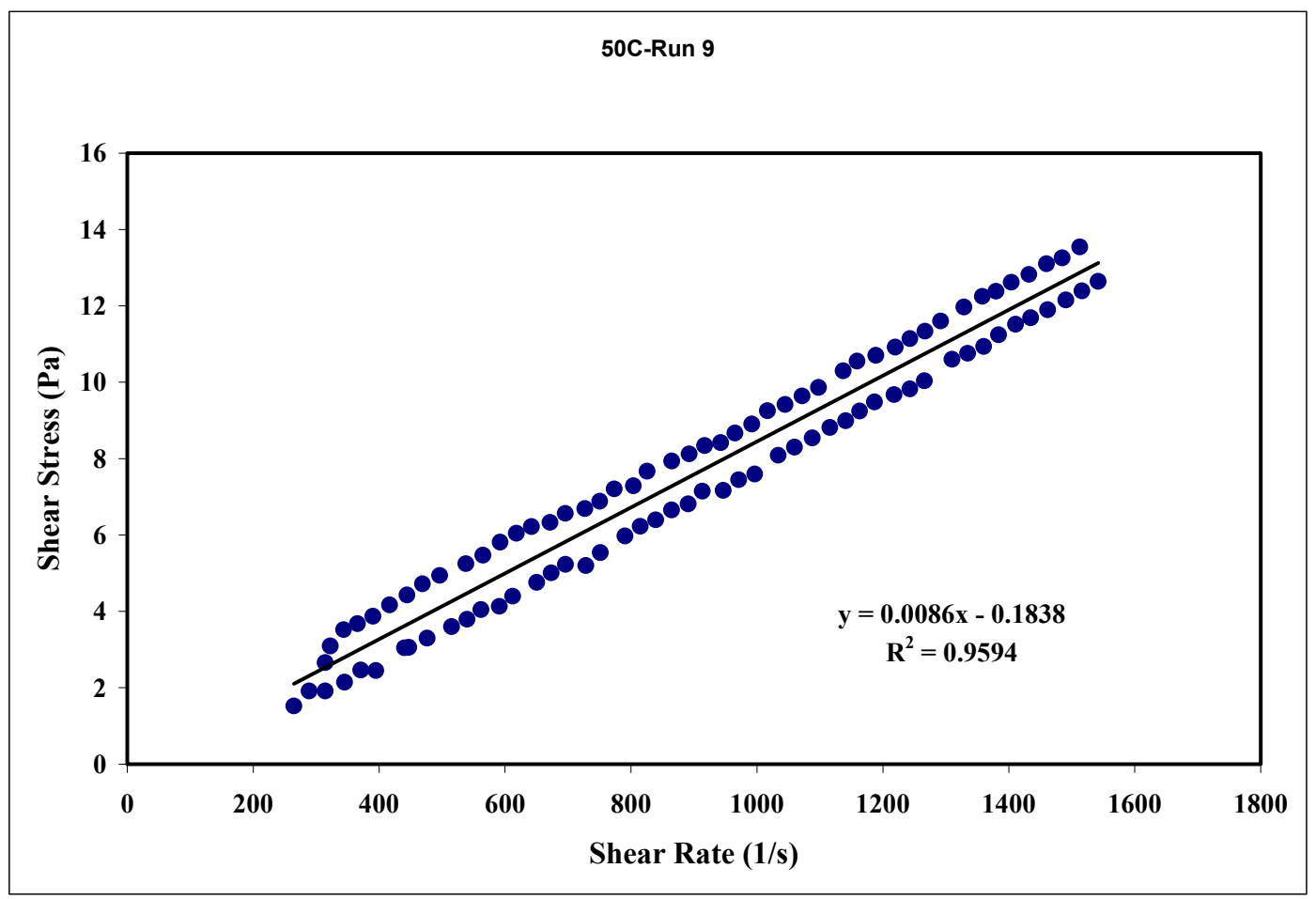

Figure 27. $50{ }^{\circ} \mathrm{C}$ Pretreated Run 9 


\section{APPENDIX I}

PART $1 \quad$ Page 347

SR/TRU PRECIPITATE SLURRY RHEOGRAMS AT 2 WT \%

PART 2 Page 371

SR/TRU PRECIPITATE SLURRY RHEOGRAMS AT 10 WT \%

PART $3 \quad$ Page 387

SR/TRU PRECIPITATE SLURRY RHEOGRAMS AT 13 WT \%

\section{PART $4 \quad$ Page 417}

SR/TRU PRECIPITATE SLURRY RHEOGRAMS AT 16 WT \% 
This page intentionally left blank. 


\section{APPENDIX I - PART 1}

\section{SR/TRU PRECIPITATE SLURRY RHEOGRAMS AT 2 WT \%}

Figure 1. Blank at $2 \mathrm{wt} \%$ Run 1

Figure 2. Blank at 2 wt $\%$ Run 2

Figure 3. Blank at 2 wt $\%$ Run 3

Figure 4. Blank at 2 wt \% Run 4

Figure 5. Blank at 2 wt $\%$ Run 5

Figure 6. Blank at 2 wt \% Run 6

Figure 7. Blank at 2 wt \% Run 7

Figure 8. Blank at 2 wt \% Run 8

Figure 9. Blank at $2 \mathrm{wt} \%$ Run 9

Figure $10 . \quad 10^{\circ} \mathrm{C}$ at $2 \mathrm{wt} \%$ Run 1

Figure $11.10{ }^{\circ} \mathrm{C}$ at $2 \mathrm{wt} \%$ Run 2

Figure $12.10^{\circ} \mathrm{C}$ at $2 \mathrm{wt} \%$ Run 3

Figure $13.10{ }^{\circ} \mathrm{C}$ at $2 \mathrm{wt} \%$ Run 4

Figure $14 . \quad 10^{\circ} \mathrm{C}$ at 2 wt \% Run 5

Figure $15.10^{\circ} \mathrm{C}$ at $2 \mathrm{wt} \%$ Run 6

Figure $16.10^{\circ} \mathrm{C}$ at 2 wt \% Run 7

Figure $17 . \quad 15^{\circ} \mathrm{C}$ at 2 wt $\%$ Run

Figure $18 . \quad 15^{\circ} \mathrm{C}$ at $2 \mathrm{wt} \%$ Run 2

Figure $19.15^{\circ} \mathrm{C}$ at $2 \mathrm{wt} \%$ Run 3

Figure $20.15^{\circ} \mathrm{C}$ at $2 \mathrm{wt} \%$ Run 4

Figure $21.15^{\circ} \mathrm{C}$ at $2 \mathrm{wt} \%$ Run 5

Figure 22. $15^{\circ} \mathrm{C}$ at $2 \mathrm{wt} \%$ Run 6

Figure $23.15^{\circ} \mathrm{C}$ at $2 \mathrm{wt} \%$ Run 7

Figure $24.15^{\circ} \mathrm{C}$ at $2 \mathrm{wt} \%$ Run 8

Figure $25.15^{\circ} \mathrm{C}$ at $2 \mathrm{wt} \%$ Run 9

Figure 26. $25^{\circ} \mathrm{C}$ at $2 \mathrm{wt} \%$ Run 1

Figure 27. $25^{\circ} \mathrm{C}$ at $2 \mathrm{wt} \%$ Run 2

Figure $29 . \quad 25^{\circ} \mathrm{C}$ at 2 wt \% Run 4

Figure $30.25{ }^{\circ} \mathrm{C}$ at $2 \mathrm{wt} \%$ Run 5

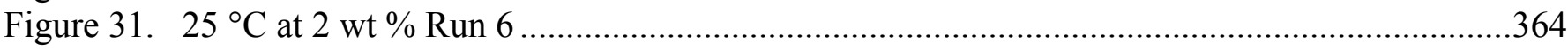

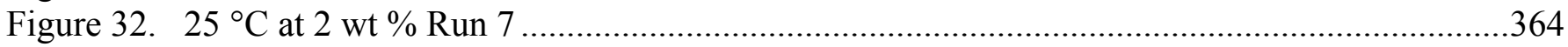

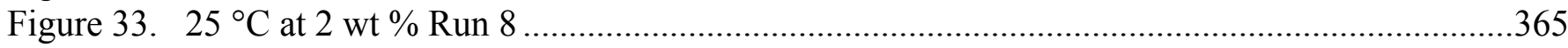

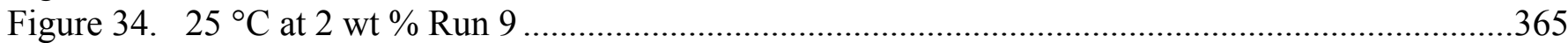

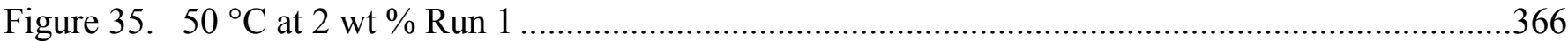

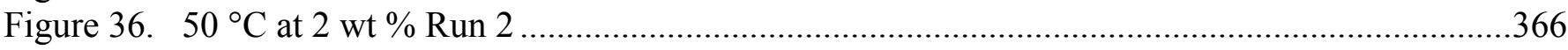

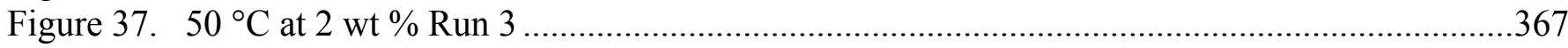

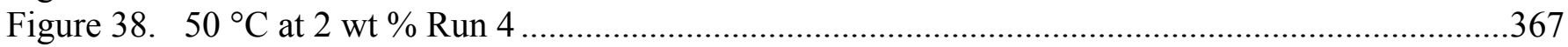




\begin{tabular}{|ll|}
\hline APPENDIX I - PART 1 & WSRC-TR-2000-00352 \\
SR/TRU PRECIPITATE SLURRY RHEOGRAMS & SRT-RPP-2000-00026 \\
\hline
\end{tabular}

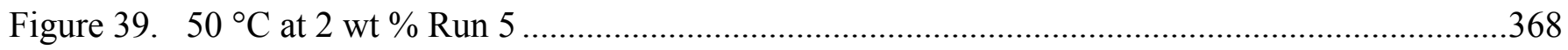

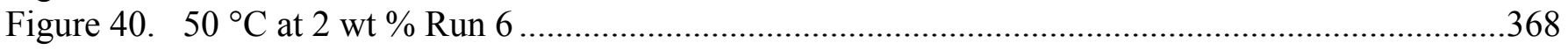

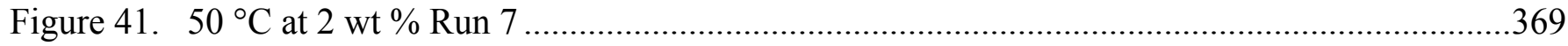

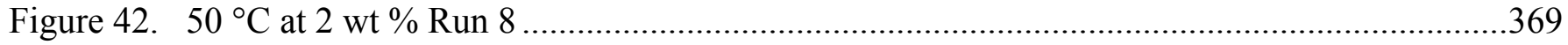

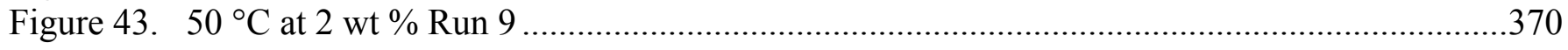




\begin{tabular}{|ll|}
\hline APPENDIX I - PART 1 & WSRC-TR-2000-00352 \\
SR/TRU PRECIPITATE SLURRY RHEOGRAMS & SRT-RPP-2000-00026 \\
\hline
\end{tabular}

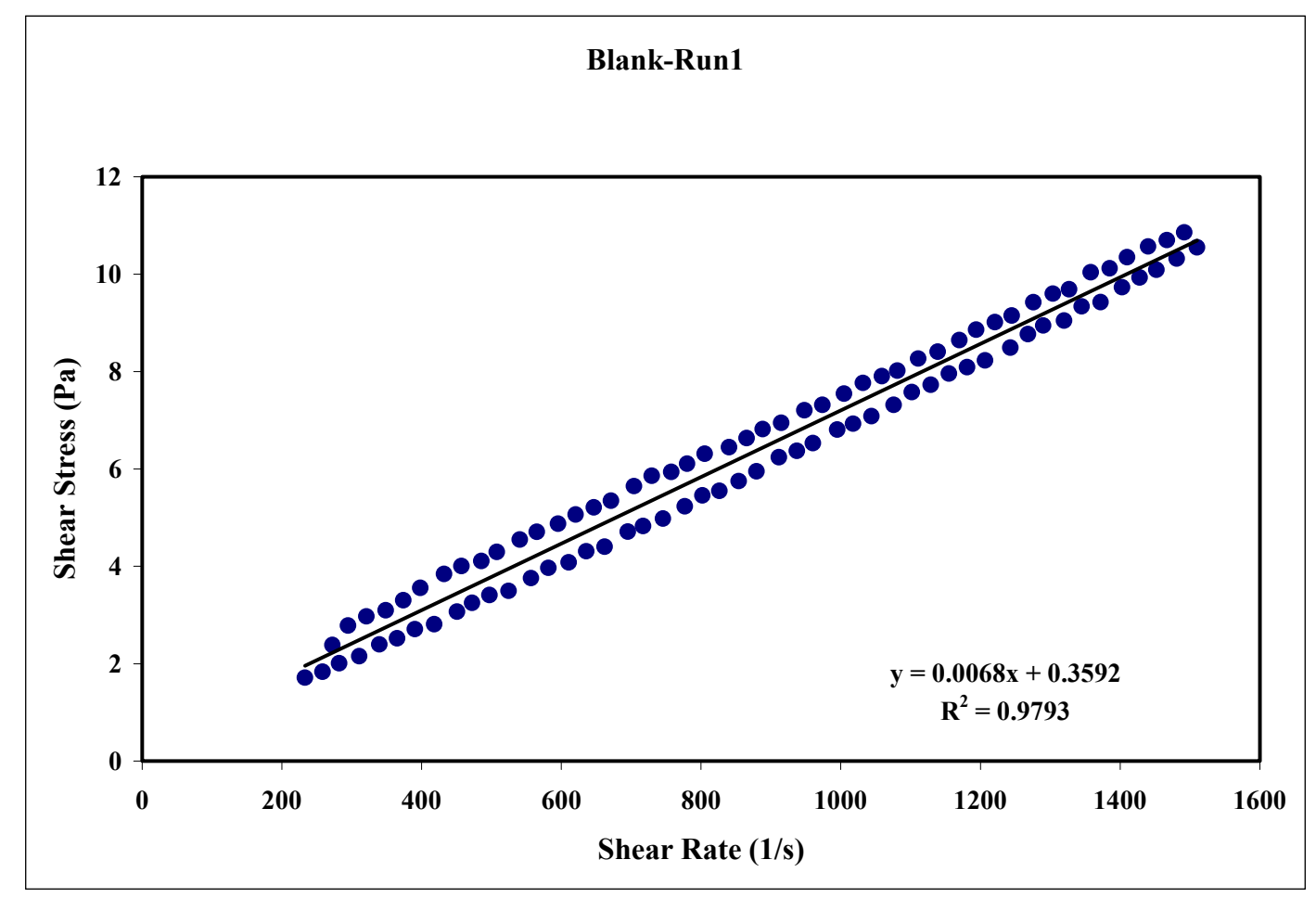

Figure 1. Blank at 2 wt \% Run 1

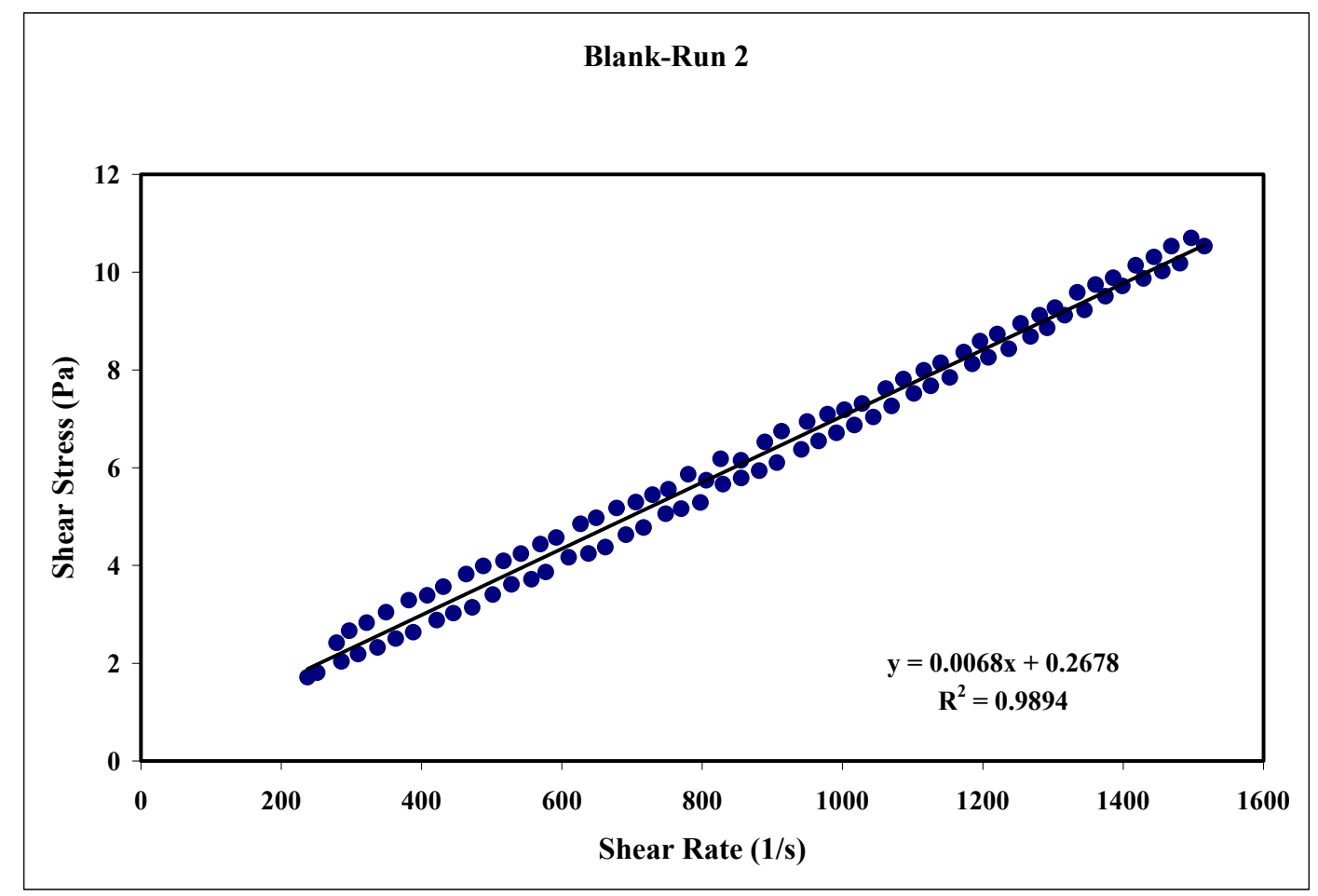

Figure 2. Blank at 2 wt \% Run 2 


\begin{tabular}{|ll|}
\hline APPENDIX I - PART 1 & WSRC-TR-2000-00352 \\
SR/TRU PRECIPITATE SLURRY RHEOGRAMS & SRT-RPP-2000-00026 \\
\hline
\end{tabular}

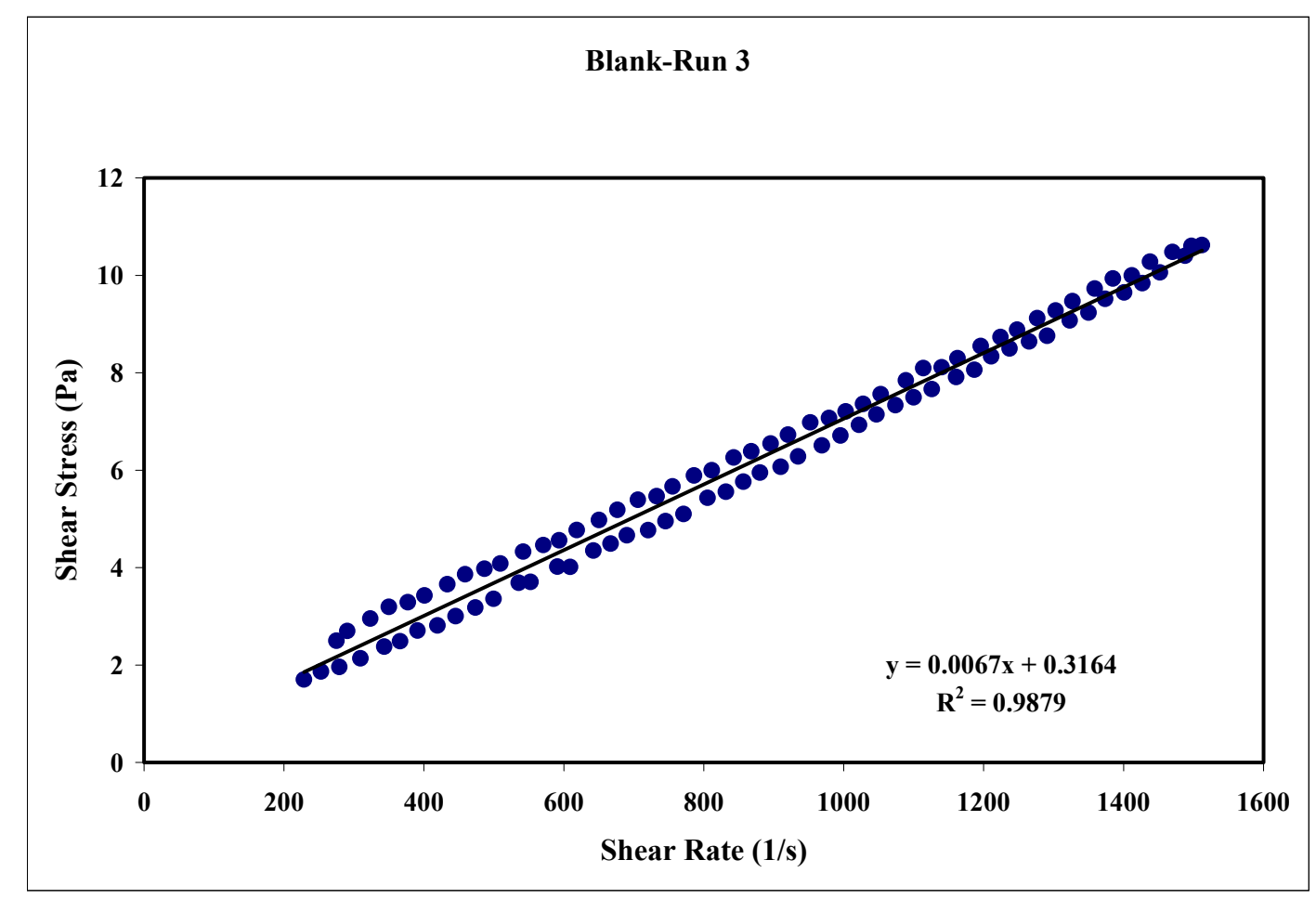

Figure 3. Blank at 2 wt \% Run 3

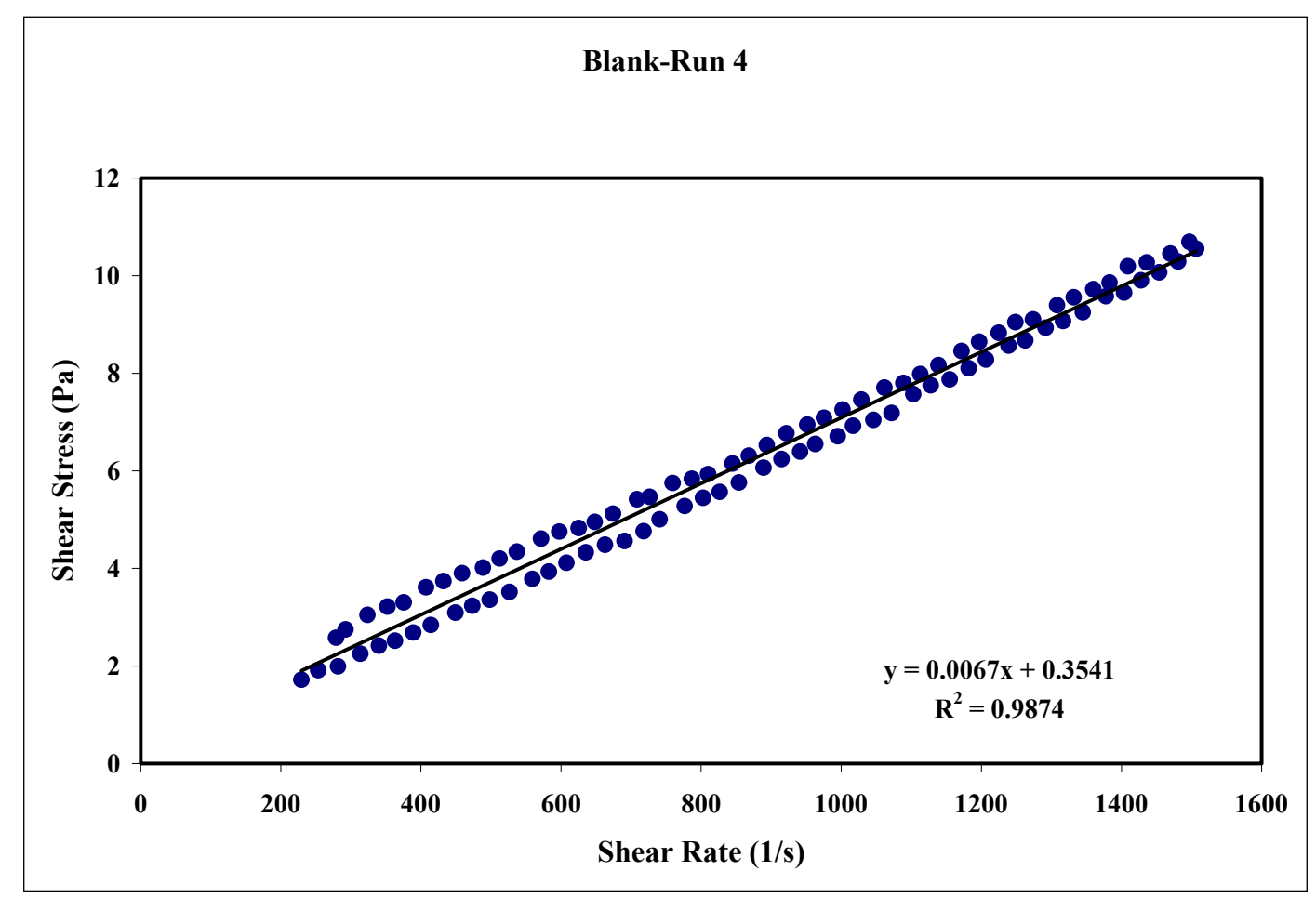

Figure 4. Blank at 2 wt \% Run 4 


\begin{tabular}{|ll|}
\hline APPENDIX I - PART 1 & WSRC-TR-2000-00352 \\
SR/TRU PRECIPITATE SLURRY RHEOGRAMS & SRT-RPP-2000-00026 \\
\hline
\end{tabular}

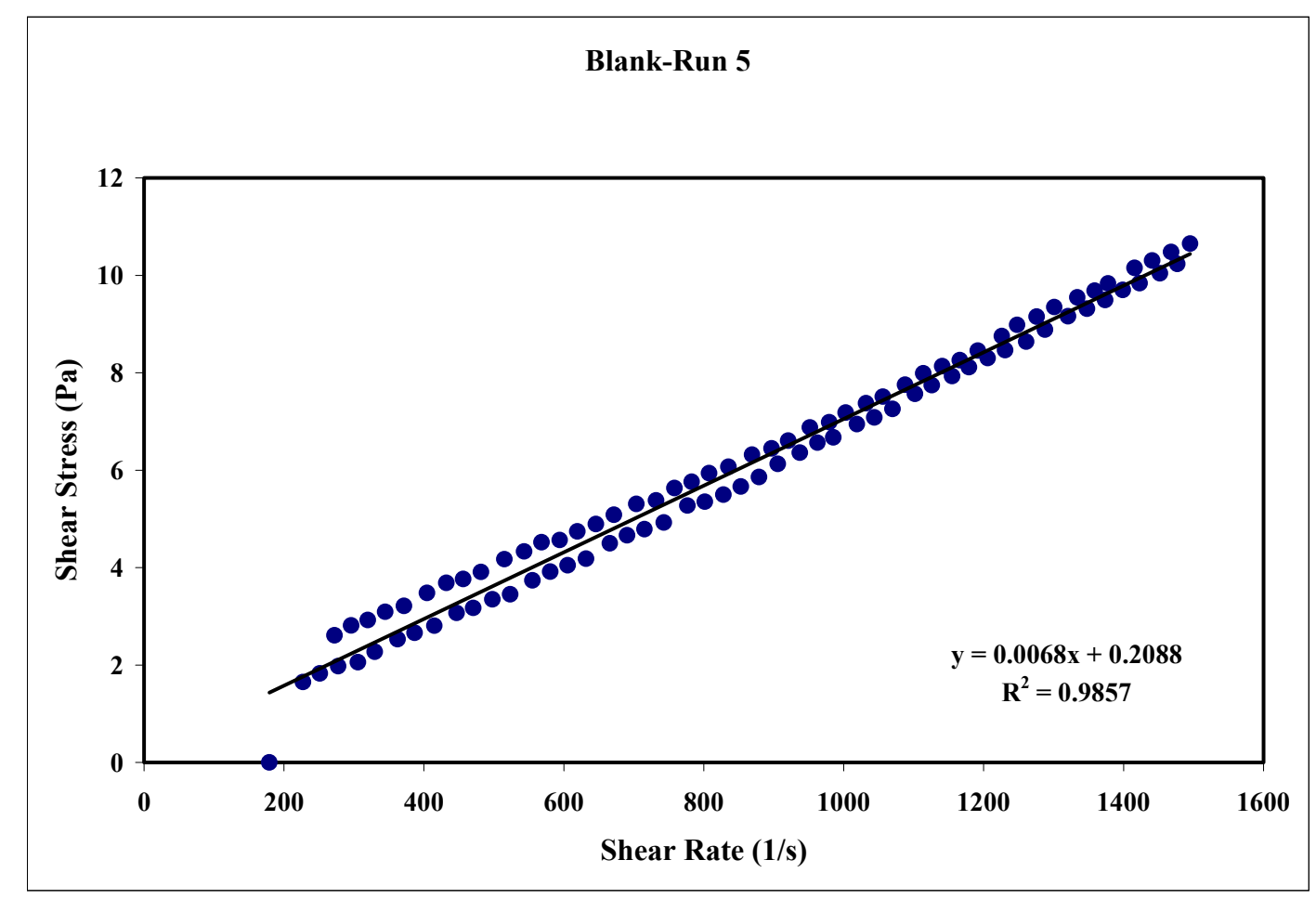

Figure 5. Blank at 2 wt \% Run 5

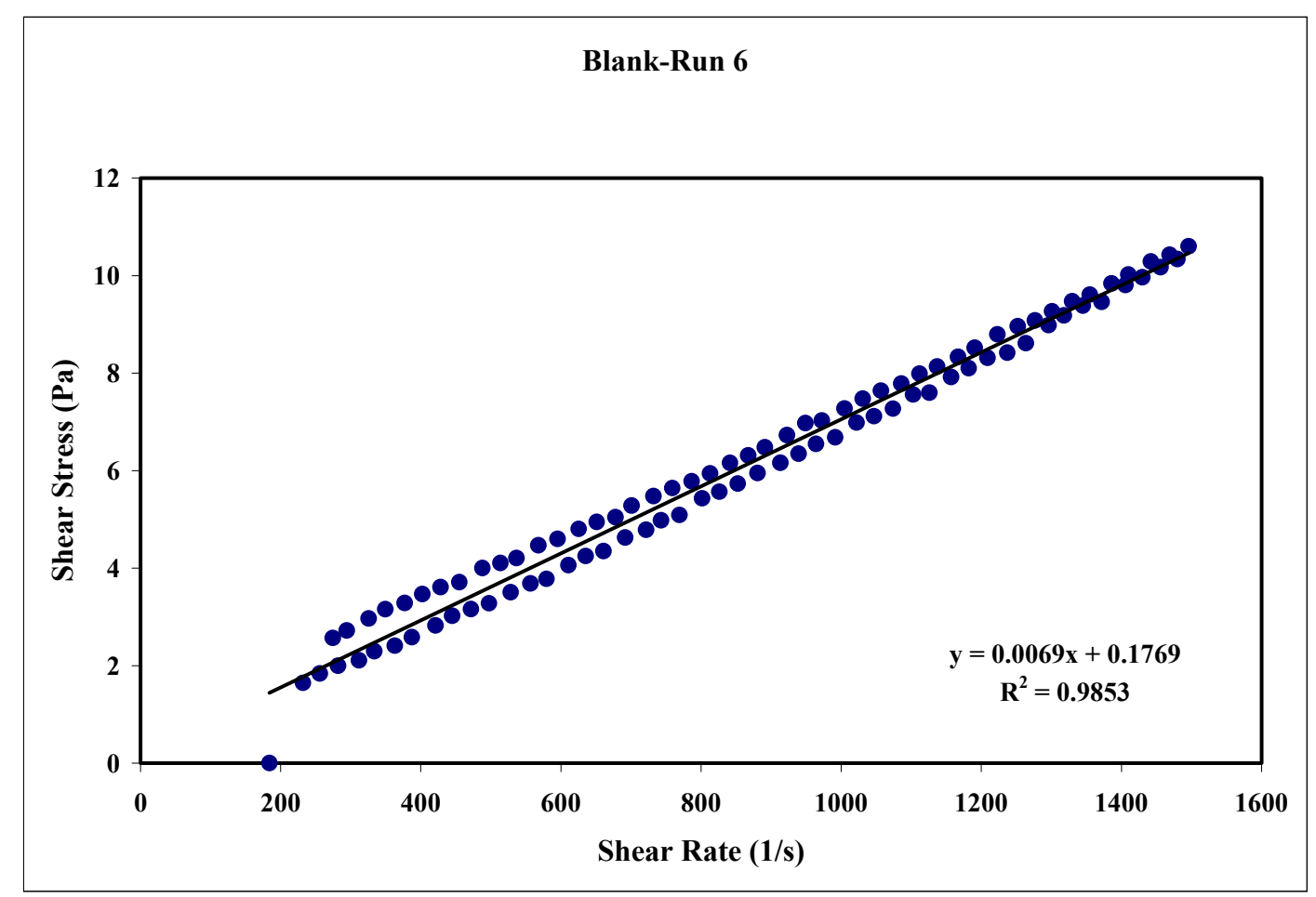

Figure 6. Blank at 2 wt \% Run 6 


\begin{tabular}{|ll|}
\hline APPENDIX I - PART 1 & WSRC-TR-2000-00352 \\
SR/TRU PRECIPITATE SLURRY RHEOGRAMS & SRT-RPP-2000-00026 \\
\hline
\end{tabular}

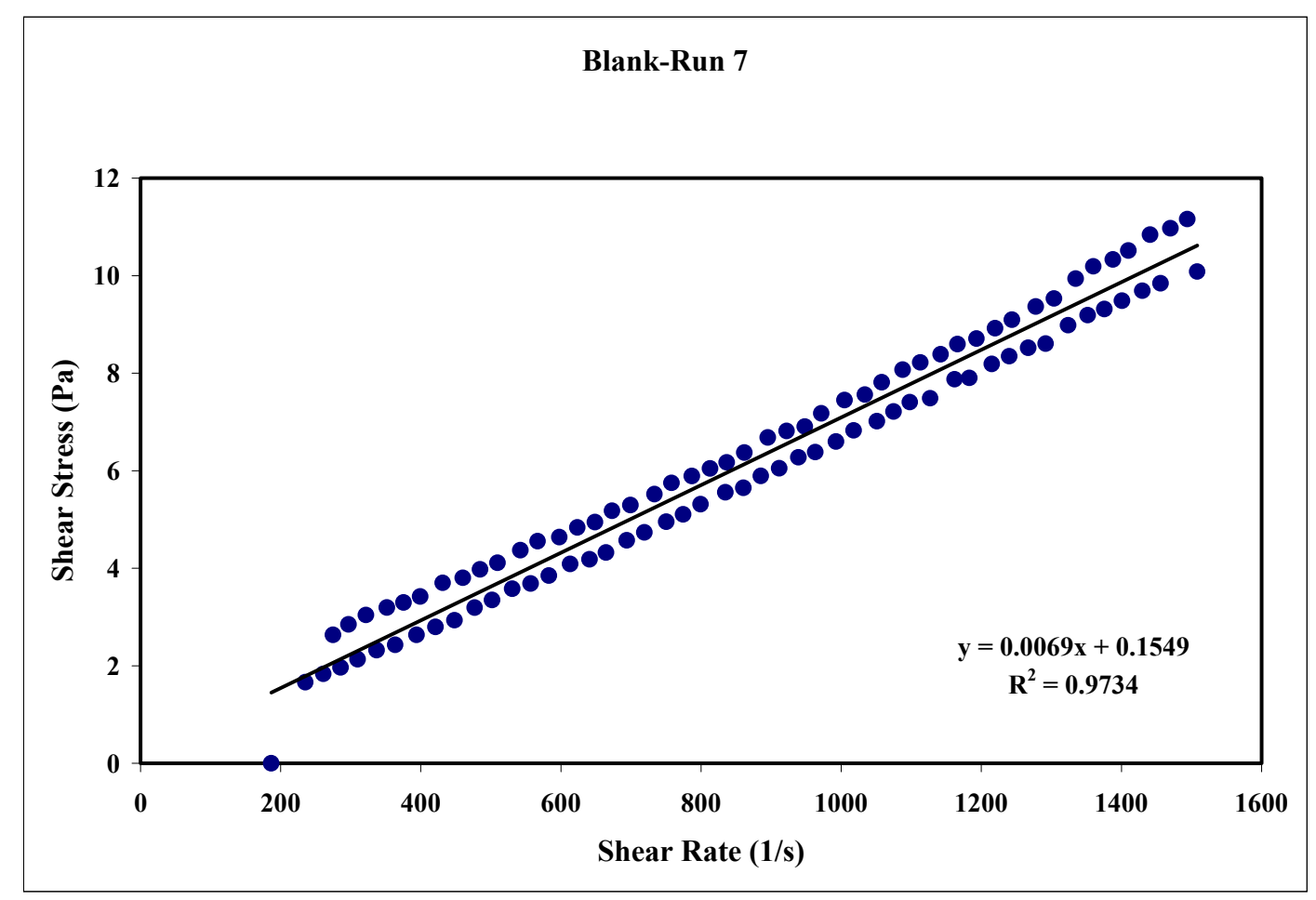

Figure 7. Blank at 2 wt \% Run 7

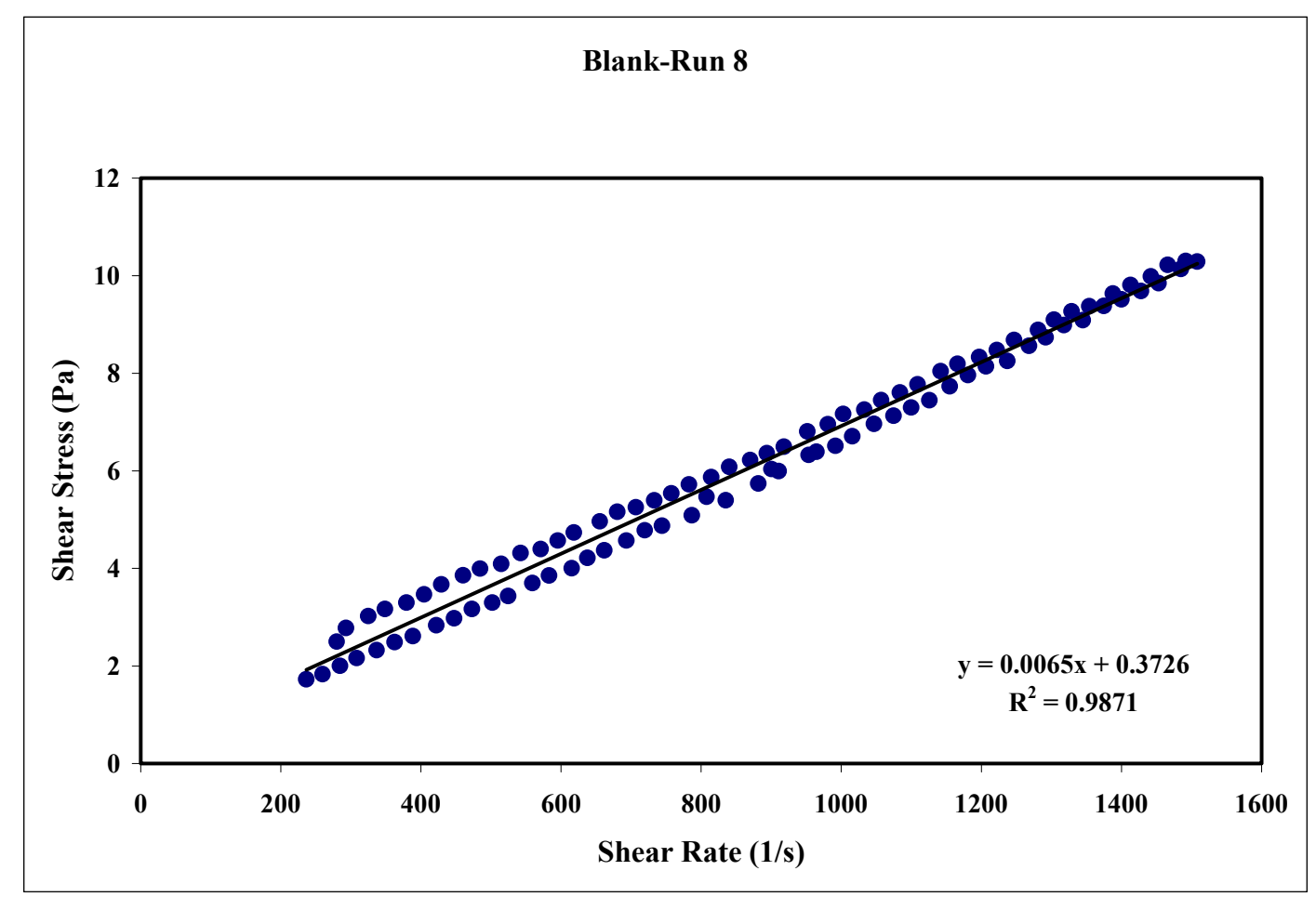

Figure 8. Blank at 2 wt \% Run 8 


\begin{tabular}{|ll|}
\hline APPENDIX I - PART 1 & WSRC-TR-2000-00352 \\
SR/TRU PRECIPITATE SLURRY RHEOGRAMS & SRT-RPP-2000-00026 \\
\hline
\end{tabular}

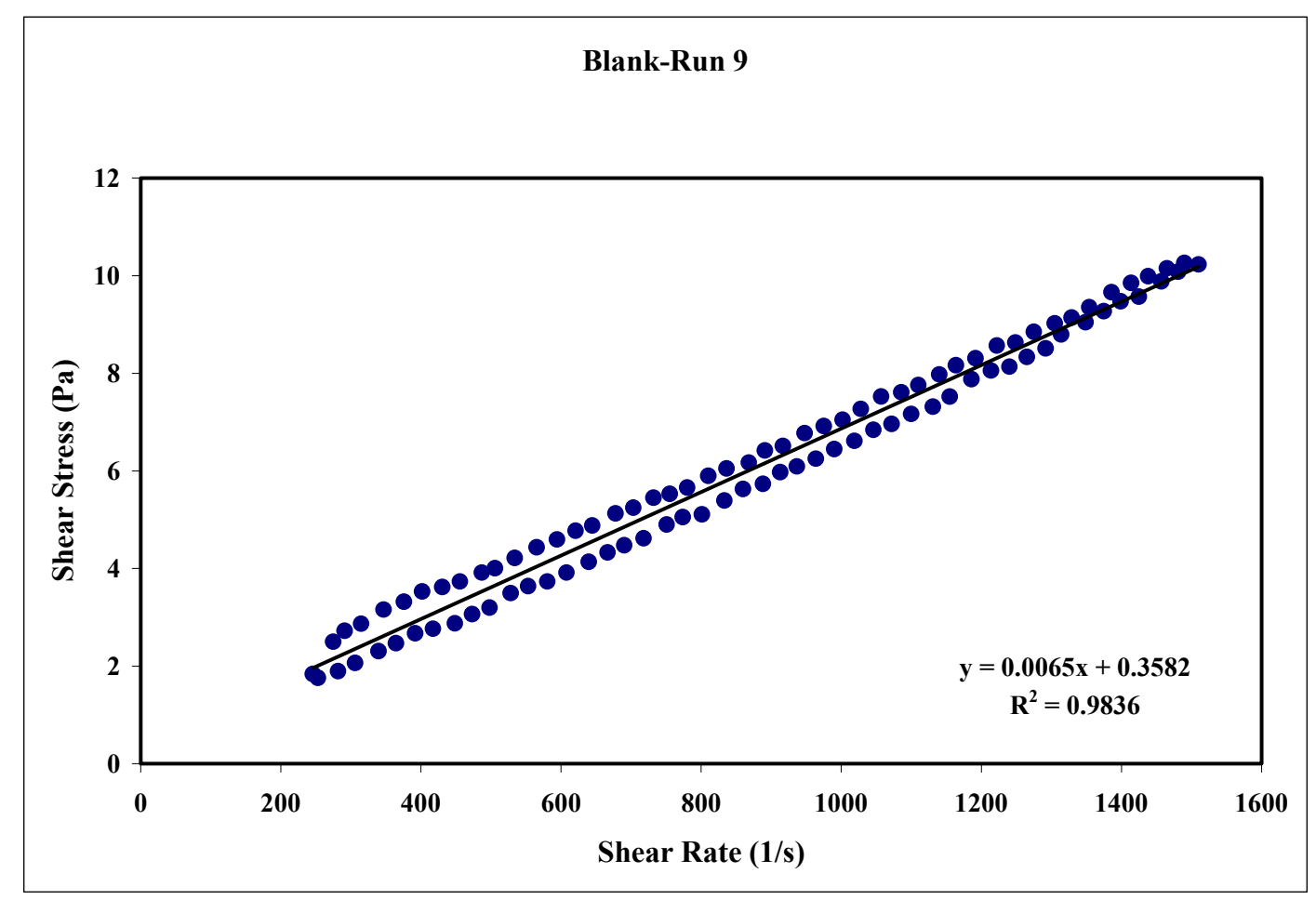

Figure 9. Blank at 2 wt \% Run 9

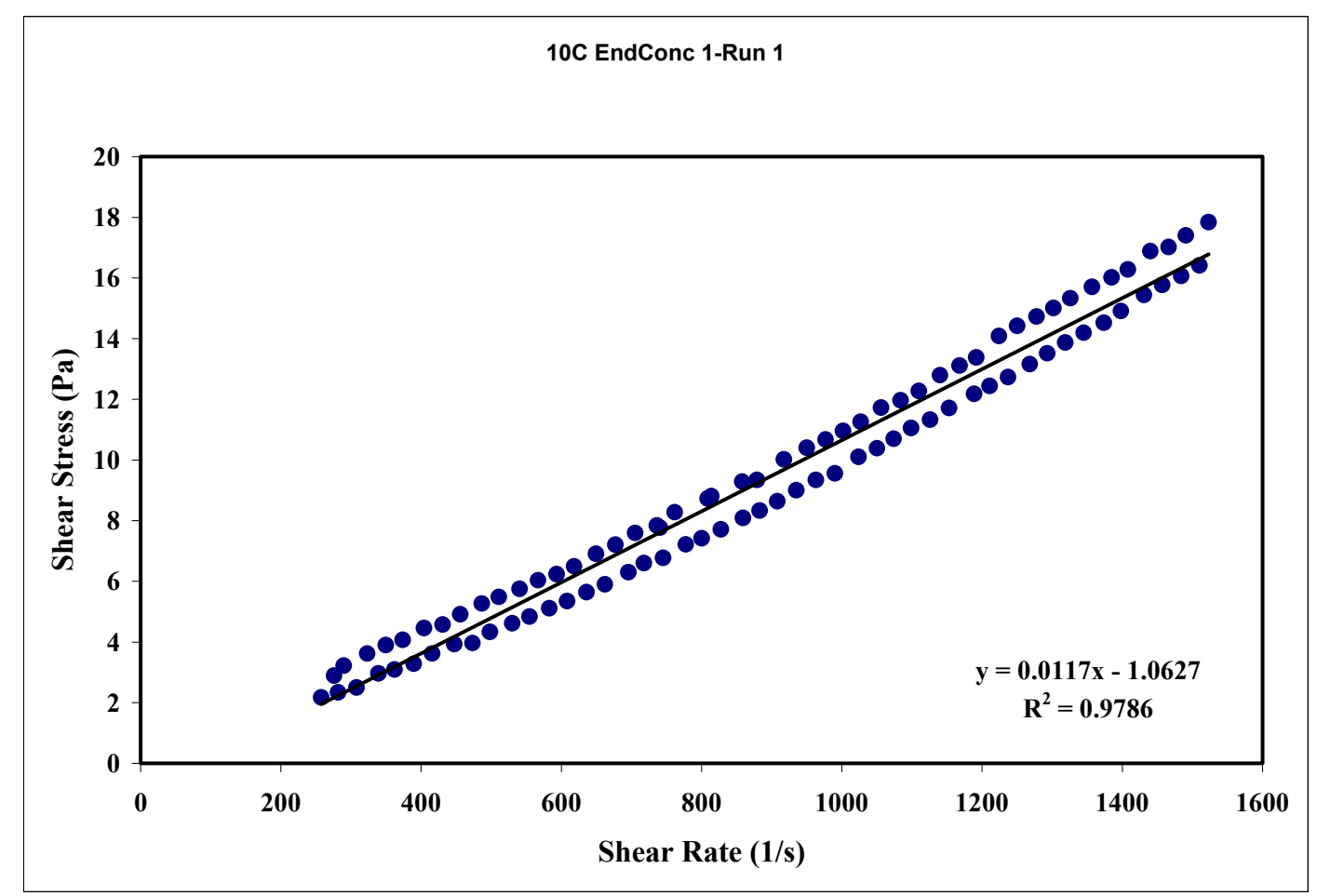

Figure $10.10{ }^{\circ} \mathrm{C}$ at 2 wt \% Run 1 


\begin{tabular}{|ll|}
\hline APPENDIX I - PART 1 & WSRC-TR-2000-00352 \\
SR/TRU PRECIPITATE SLURRY RHEOGRAMS & SRT-RPP-2000-00026 \\
\hline
\end{tabular}

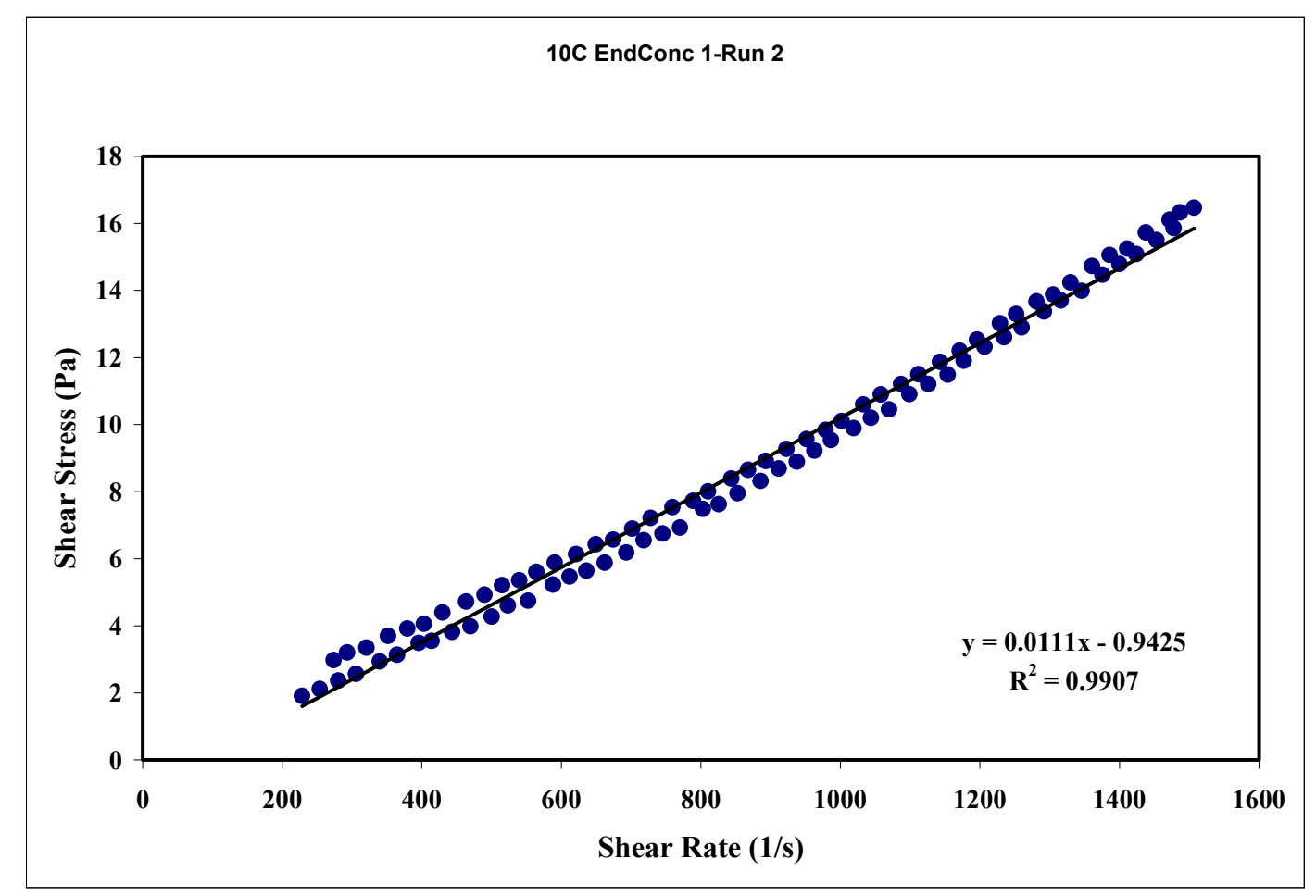

Figure 11. $10{ }^{\circ} \mathrm{C}$ at 2 wt \% Run 2

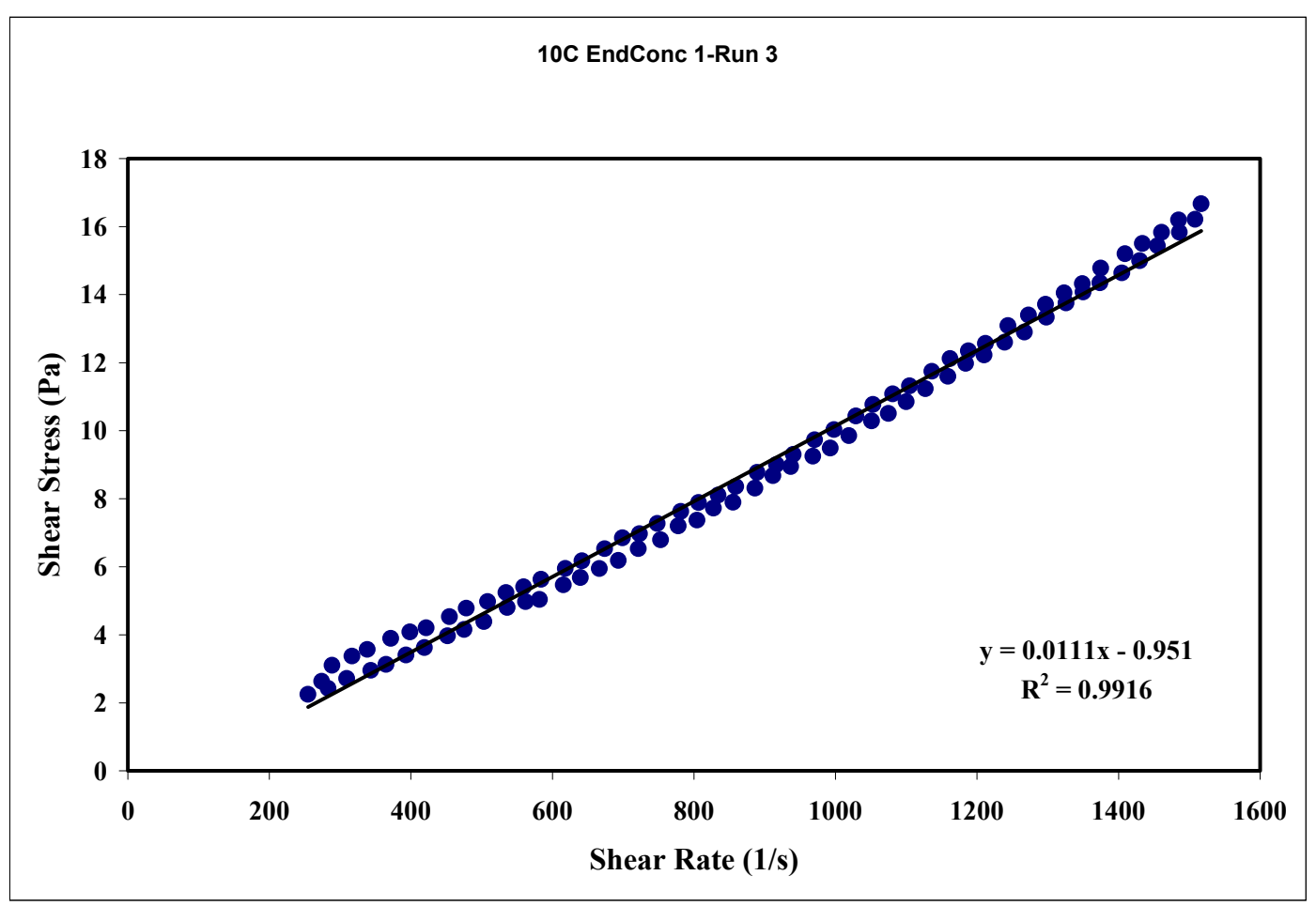

Figure 12. $10{ }^{\circ} \mathrm{C}$ at 2 wt $\%$ Run 3 


\begin{tabular}{|ll|}
\hline APPENDIX I - PART 1 & WSRC-TR-2000-00352 \\
SR/TRU PRECIPITATE SLURRY RHEOGRAMS & SRT-RPP-2000-00026 \\
\hline
\end{tabular}

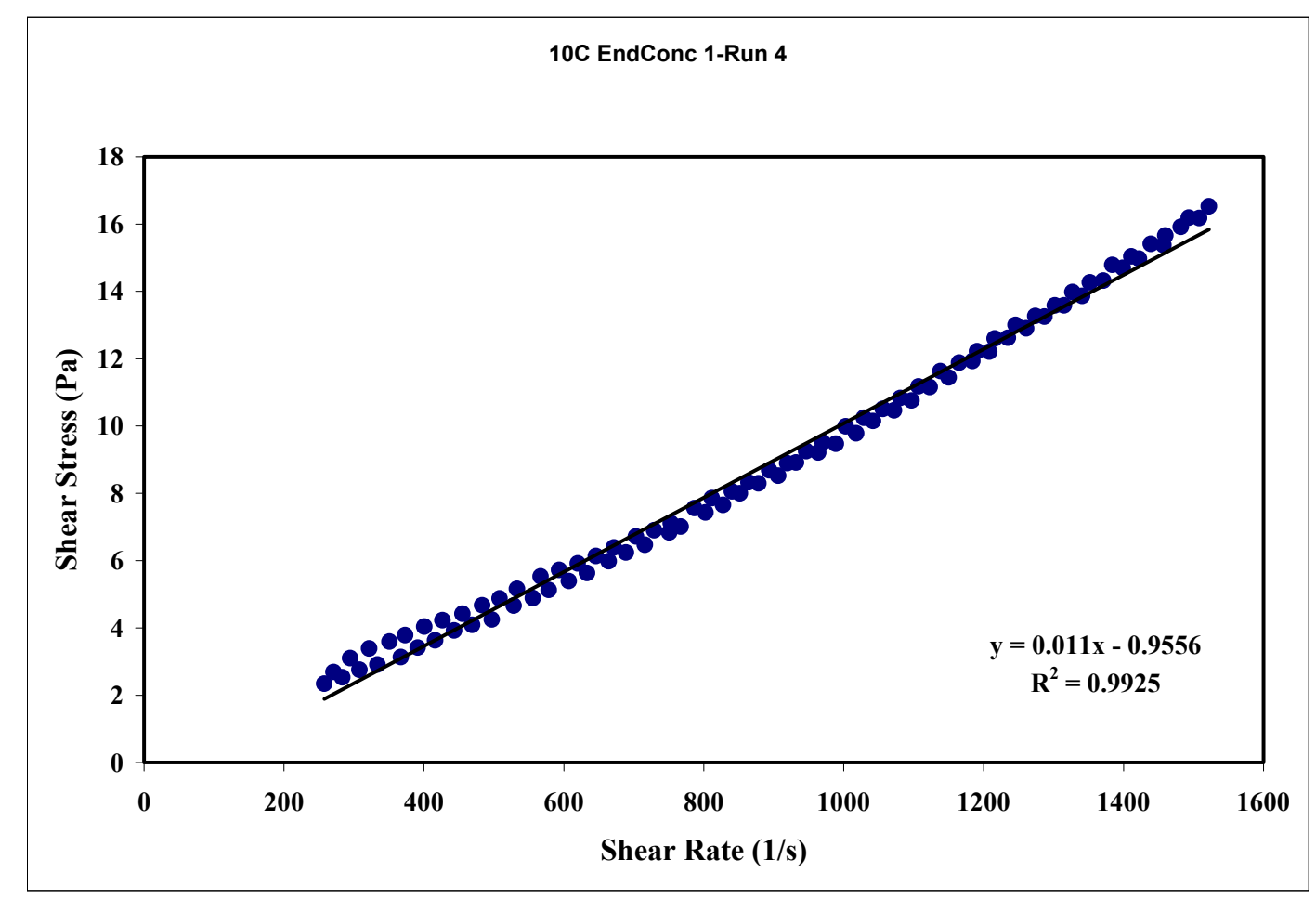

Figure 13. $10^{\circ} \mathrm{C}$ at 2 wt $\%$ Run 4

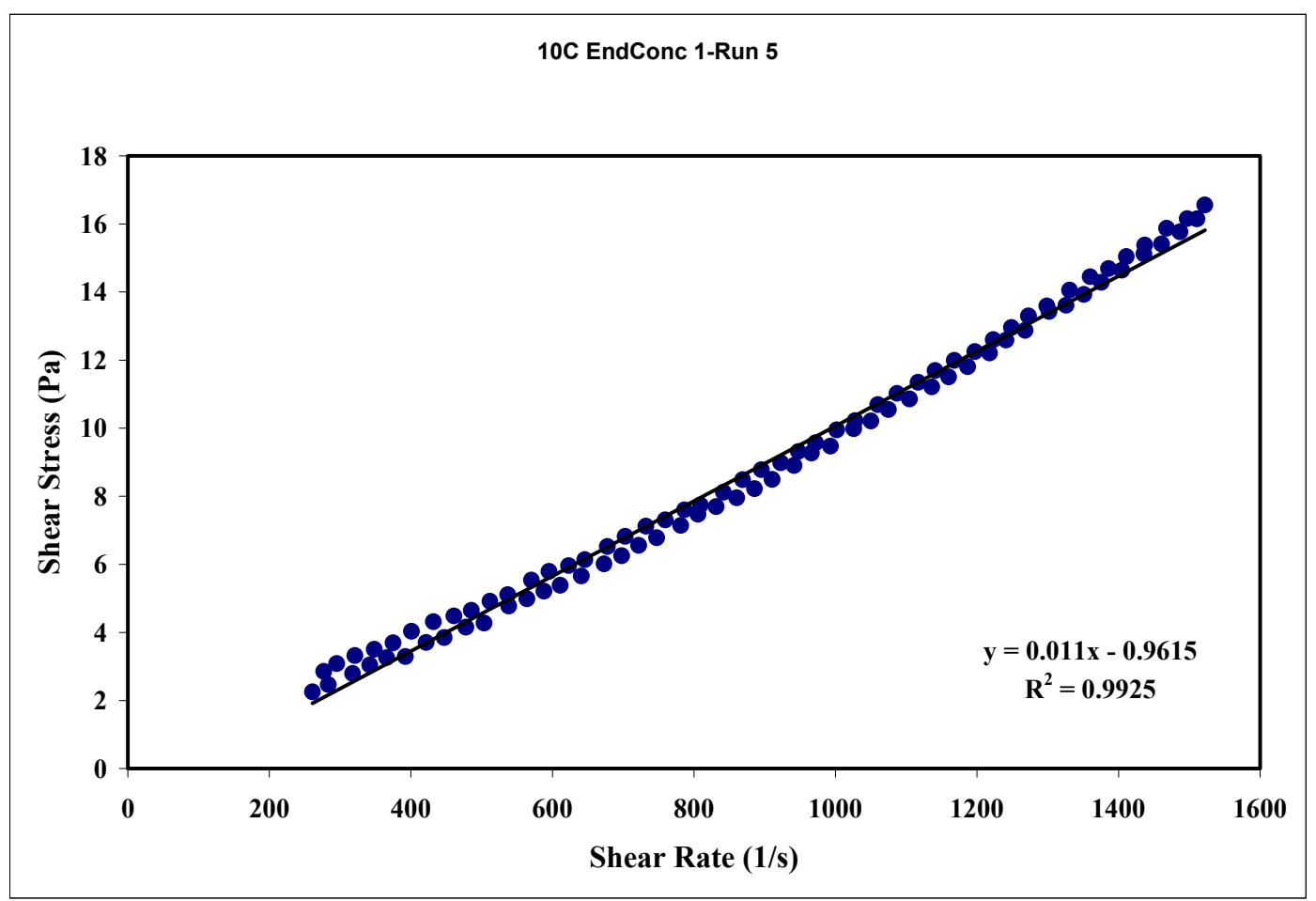

Figure 14. $10{ }^{\circ} \mathrm{C}$ at 2 wt $\%$ Run 5 


\begin{tabular}{|ll|}
\hline APPENDIX I - PART 1 & WSRC-TR-2000-00352 \\
& SRT-RPP-2000-00026 \\
SR/TRU PRECIPITATE SLURRY RHEOGRAMS & Page 356 of 514 \\
\hline
\end{tabular}

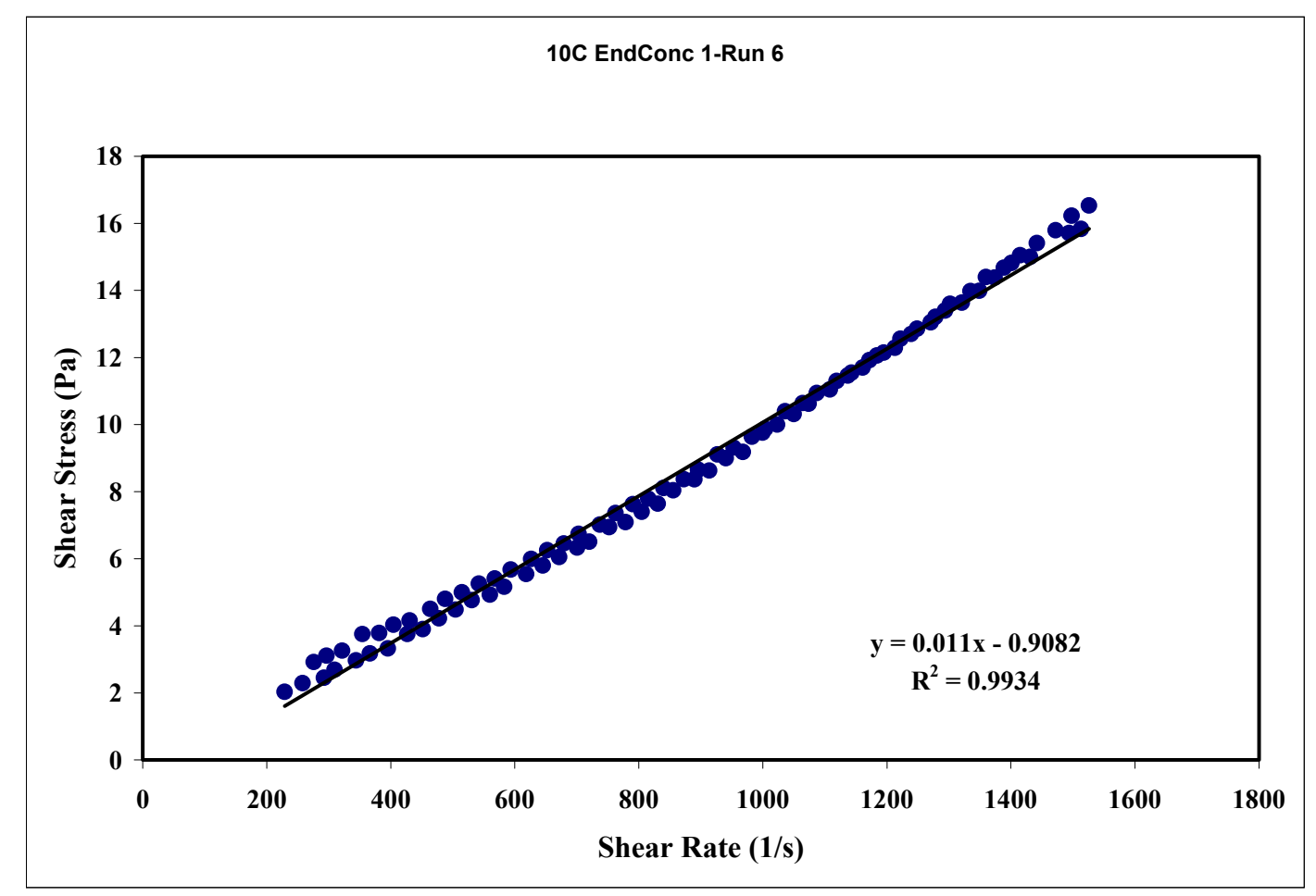

Figure 15. $10{ }^{\circ} \mathrm{C}$ at 2 wt \% Run 6

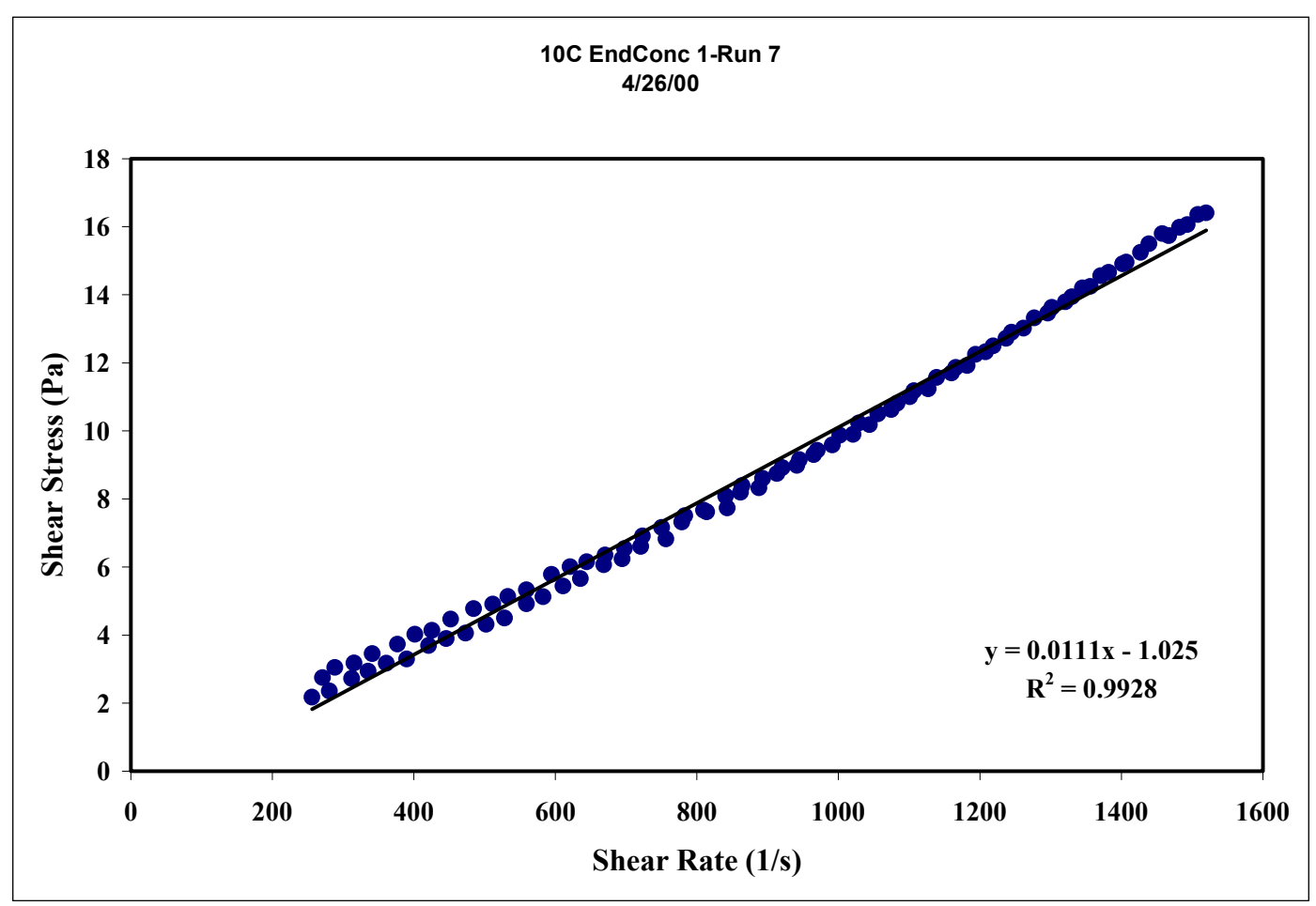

Figure 16. $10{ }^{\circ} \mathrm{C}$ at 2 wt $\%$ Run 7 


\begin{tabular}{|ll|}
\hline APPENDIX I - PART 1 & WSRC-TR-2000-00352 \\
SR/TRU PRECIPITATE SLURRY RHEOGRAMS & SRT-RPP-2000-00026 \\
\hline
\end{tabular}

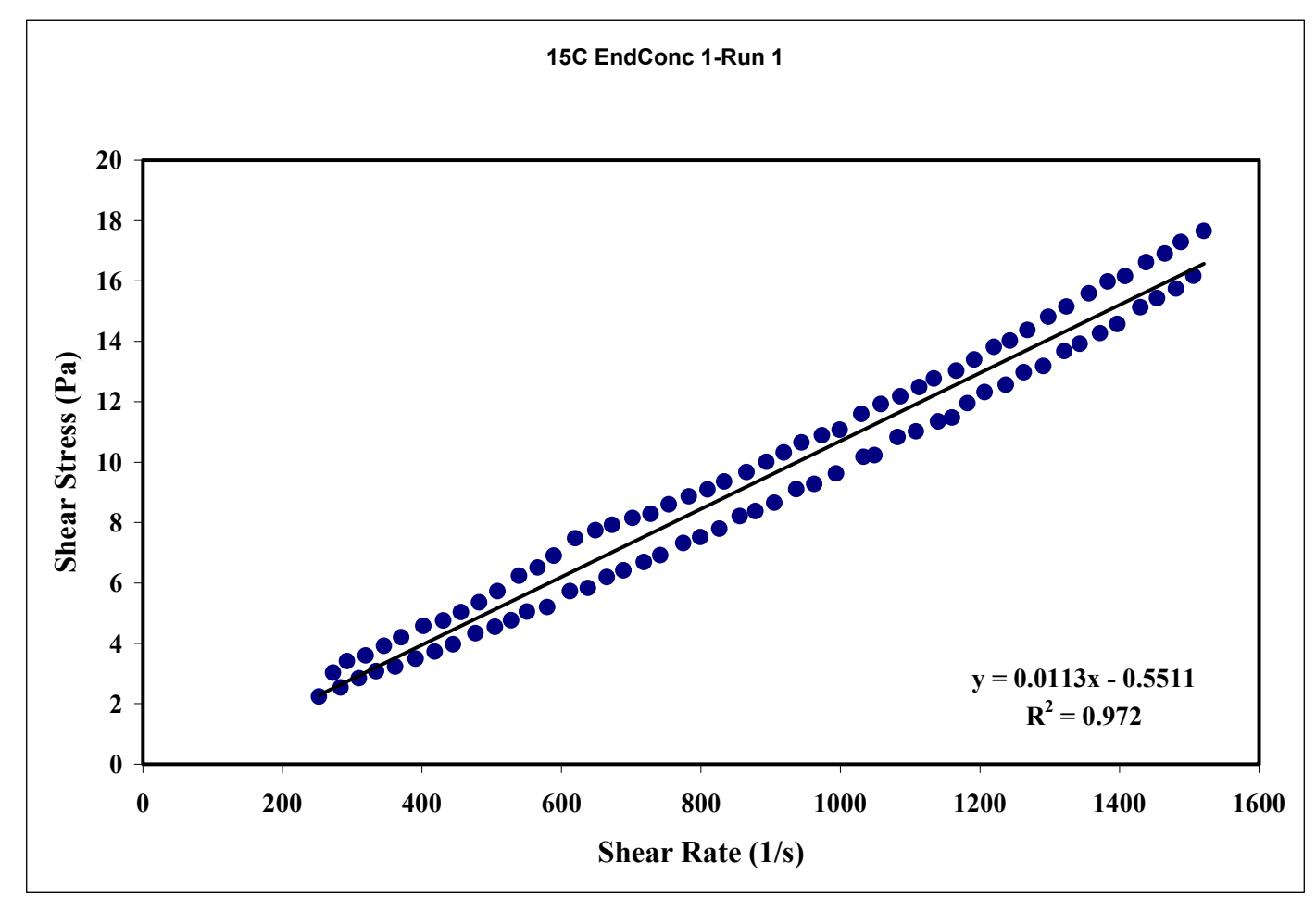

Figure $17.15^{\circ} \mathrm{C}$ at 2 wt \% Run 1

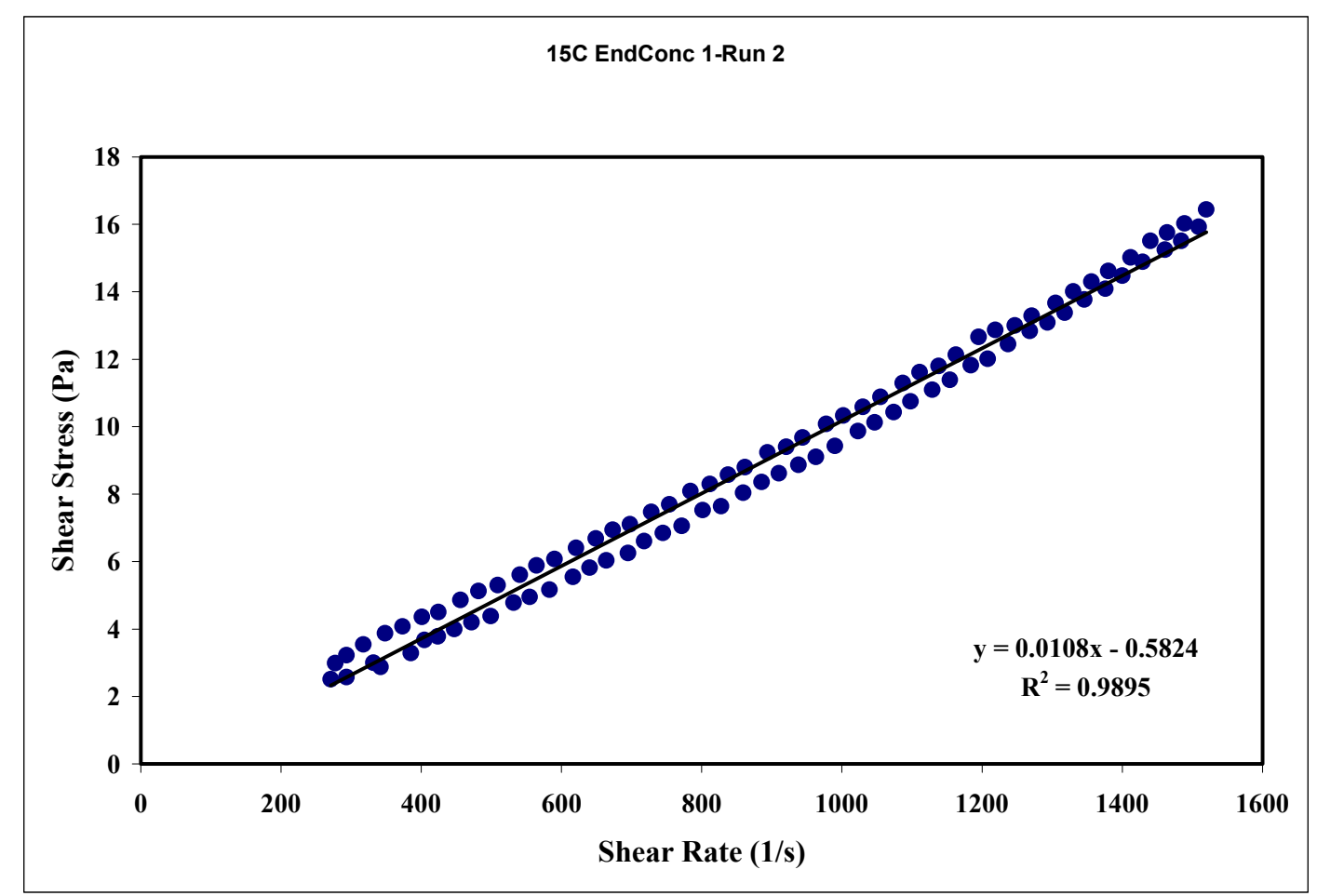

Figure 18. $15^{\circ} \mathrm{C}$ at 2 wt \% Run 2 


\begin{tabular}{|ll|}
\hline APPENDIX I - PART 1 & WSRC-TR-2000-00352 \\
SR/TRU PRECIPITATE SLURRY RHEOGRAMS & SRT-RPP-2000-00026 \\
\hline
\end{tabular}

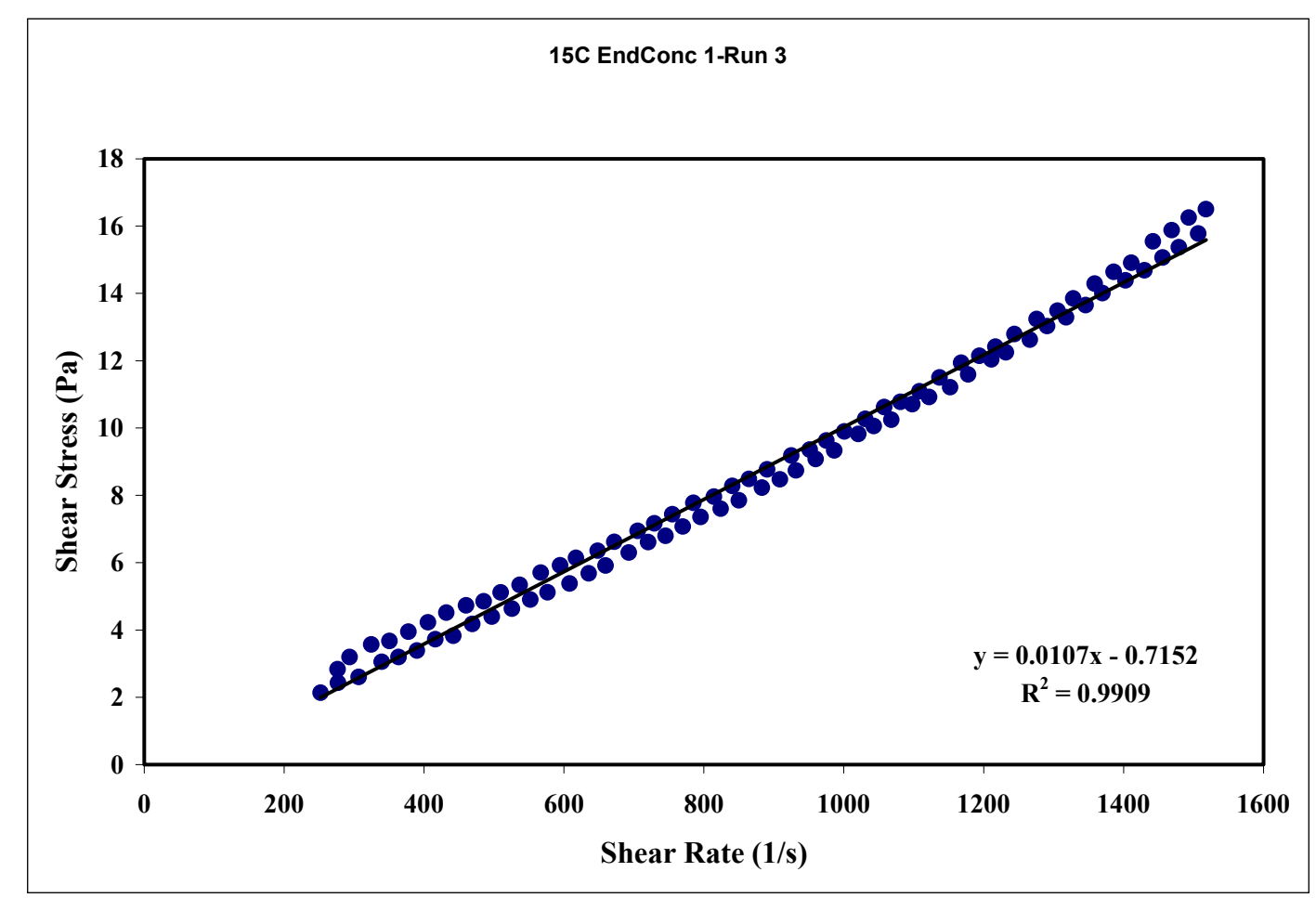

Figure 19. $15^{\circ} \mathrm{C}$ at 2 wt \% Run 3

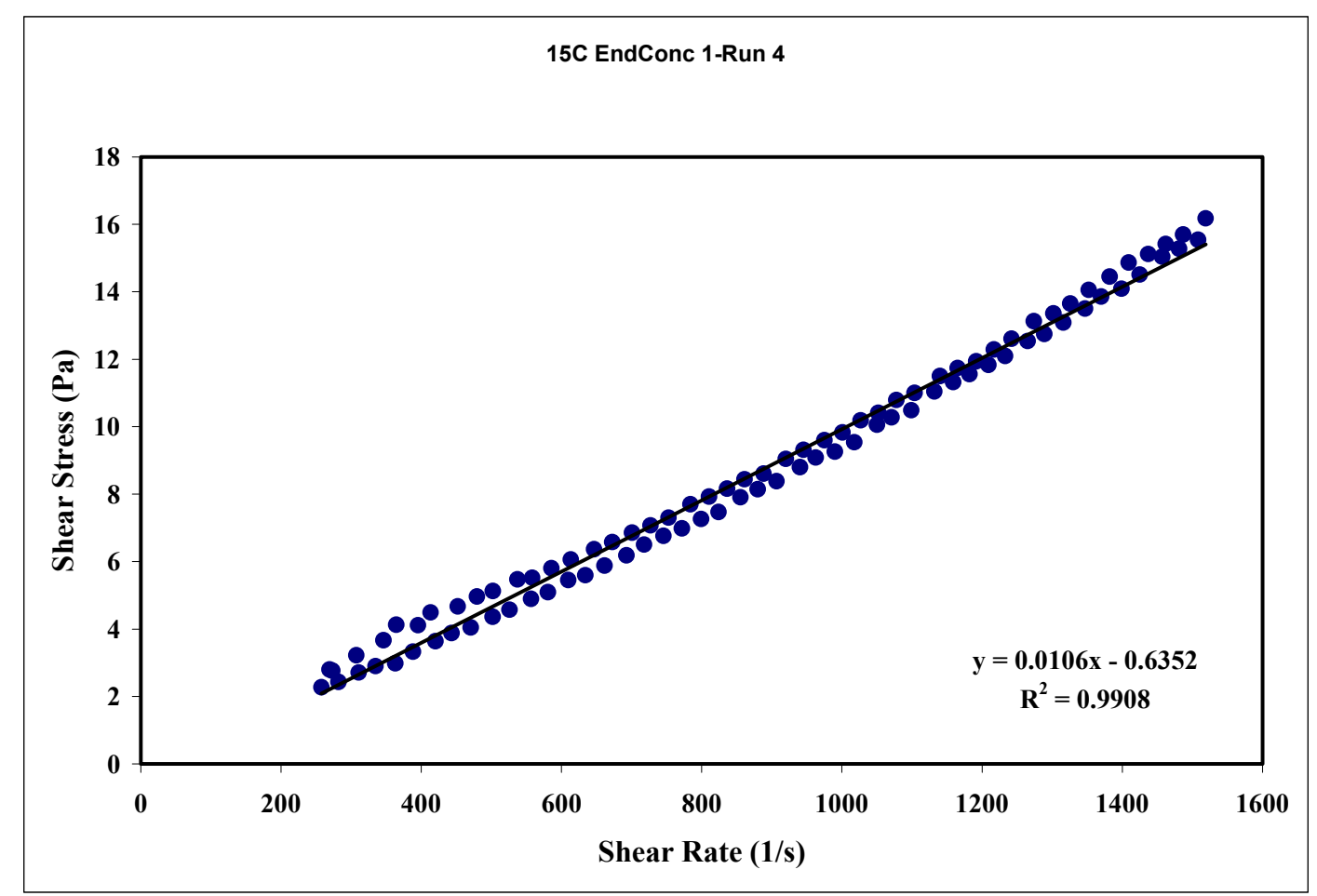

Figure 20. $15^{\circ} \mathrm{C}$ at 2 wt \% Run 4 


\begin{tabular}{|ll|}
\hline APPENDIX I - PART 1 & WSRC-TR-2000-00352 \\
SR/TRU PRECIPITATE SLURRY RHEOGRAMS & SRT-RPP-2000-00026 \\
\hline
\end{tabular}

15C EndConc 1-Run 5

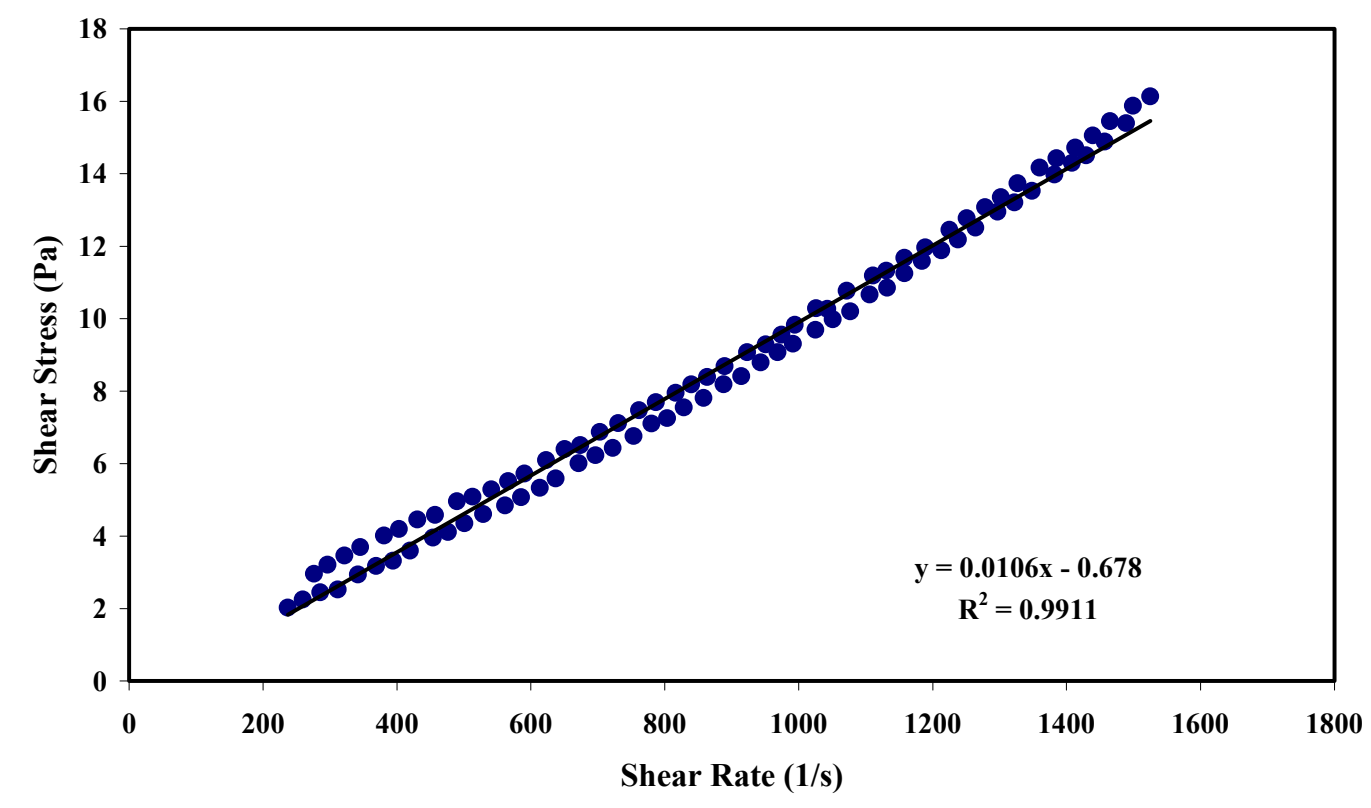

Figure 21. $15^{\circ} \mathrm{C}$ at 2 wt \% Run 5

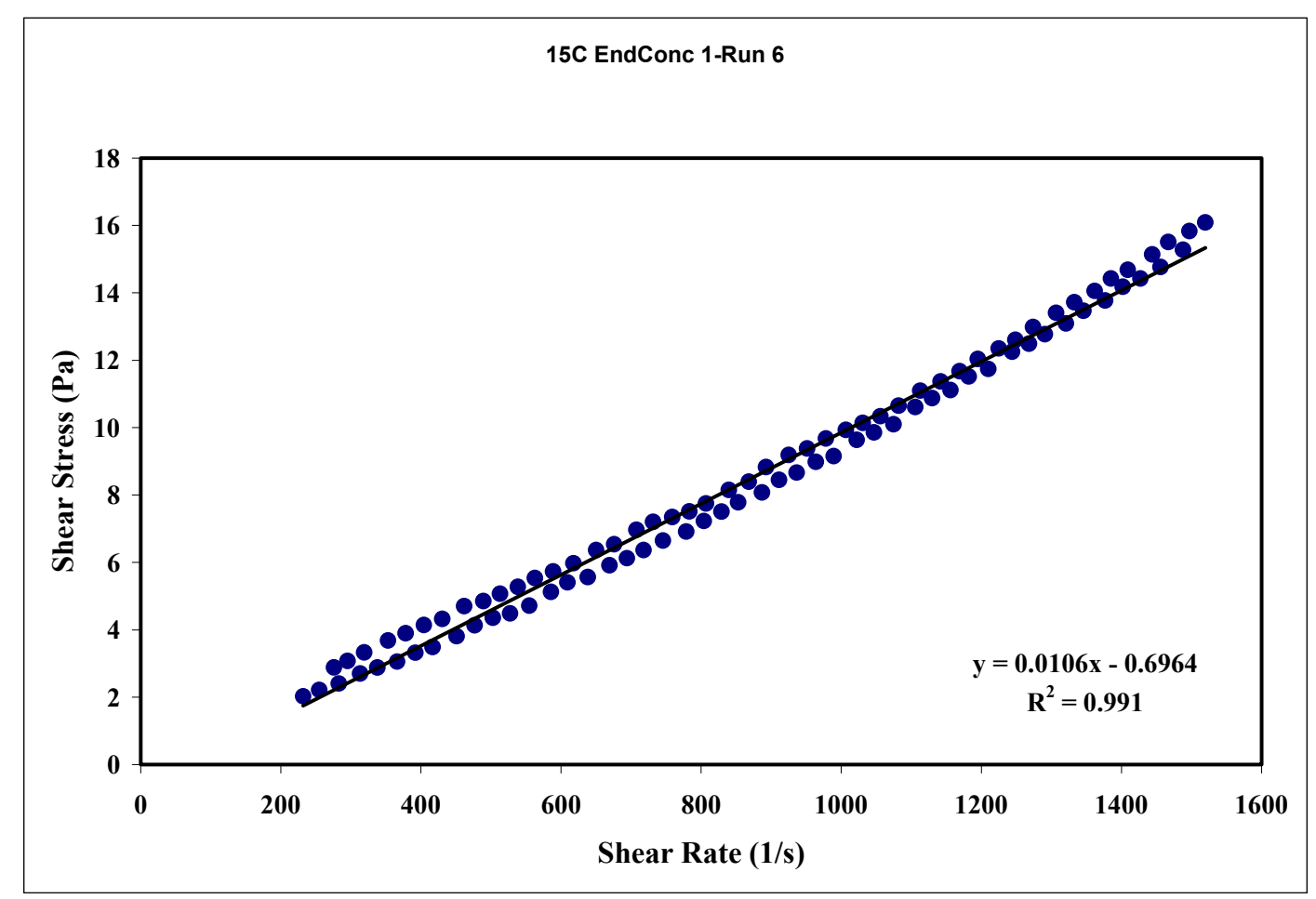

Figure 22. $15^{\circ} \mathrm{C}$ at 2 wt \% Run 6 


\begin{tabular}{|ll|}
\hline APPENDIX I - PART 1 & WSRC-TR-2000-00352 \\
SR/TRU PRECIPITATE SLURRY RHEOGRAMS & SRT-RPP-2000-00026 \\
\hline
\end{tabular}

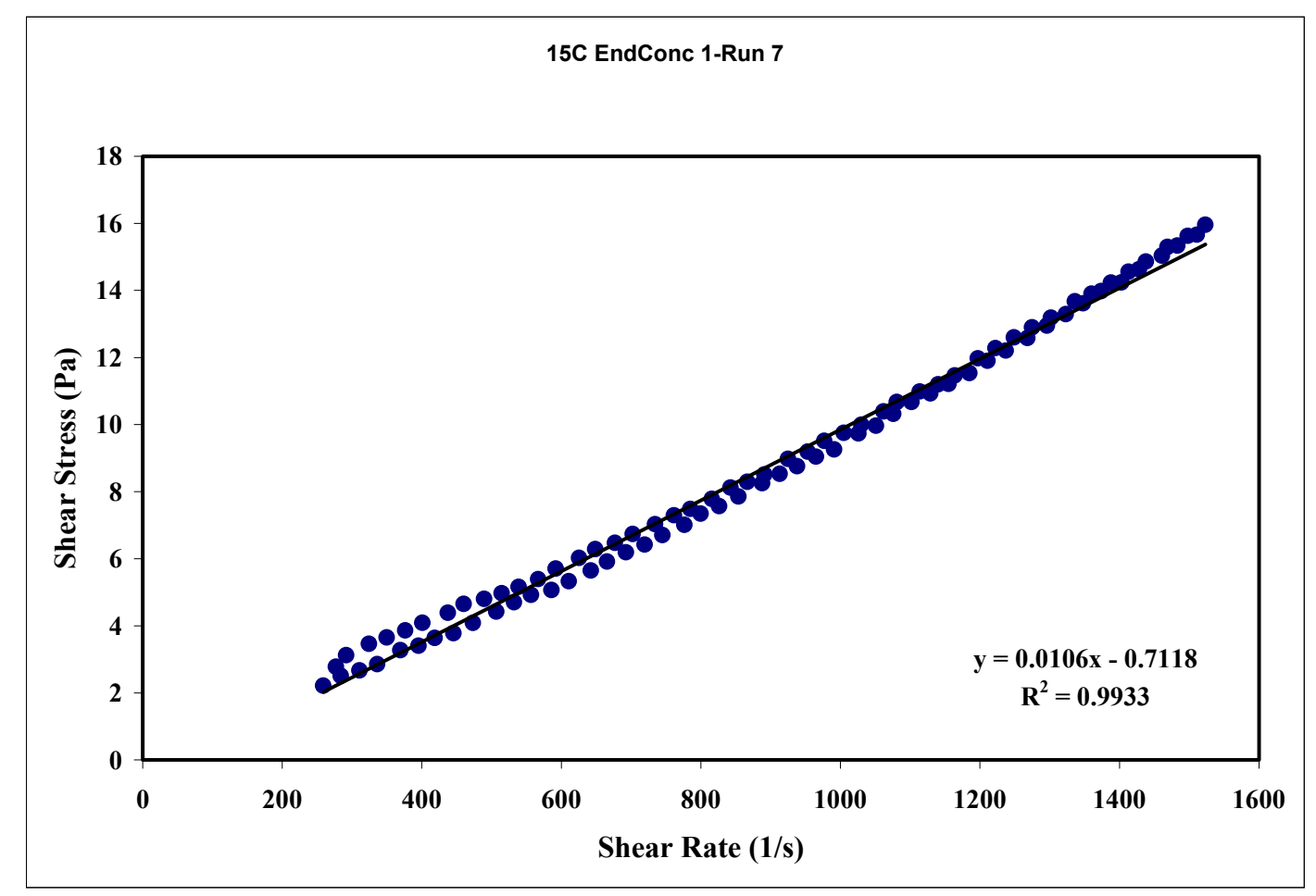

Figure 23. $15^{\circ} \mathrm{C}$ at 2 wt $\%$ Run 7

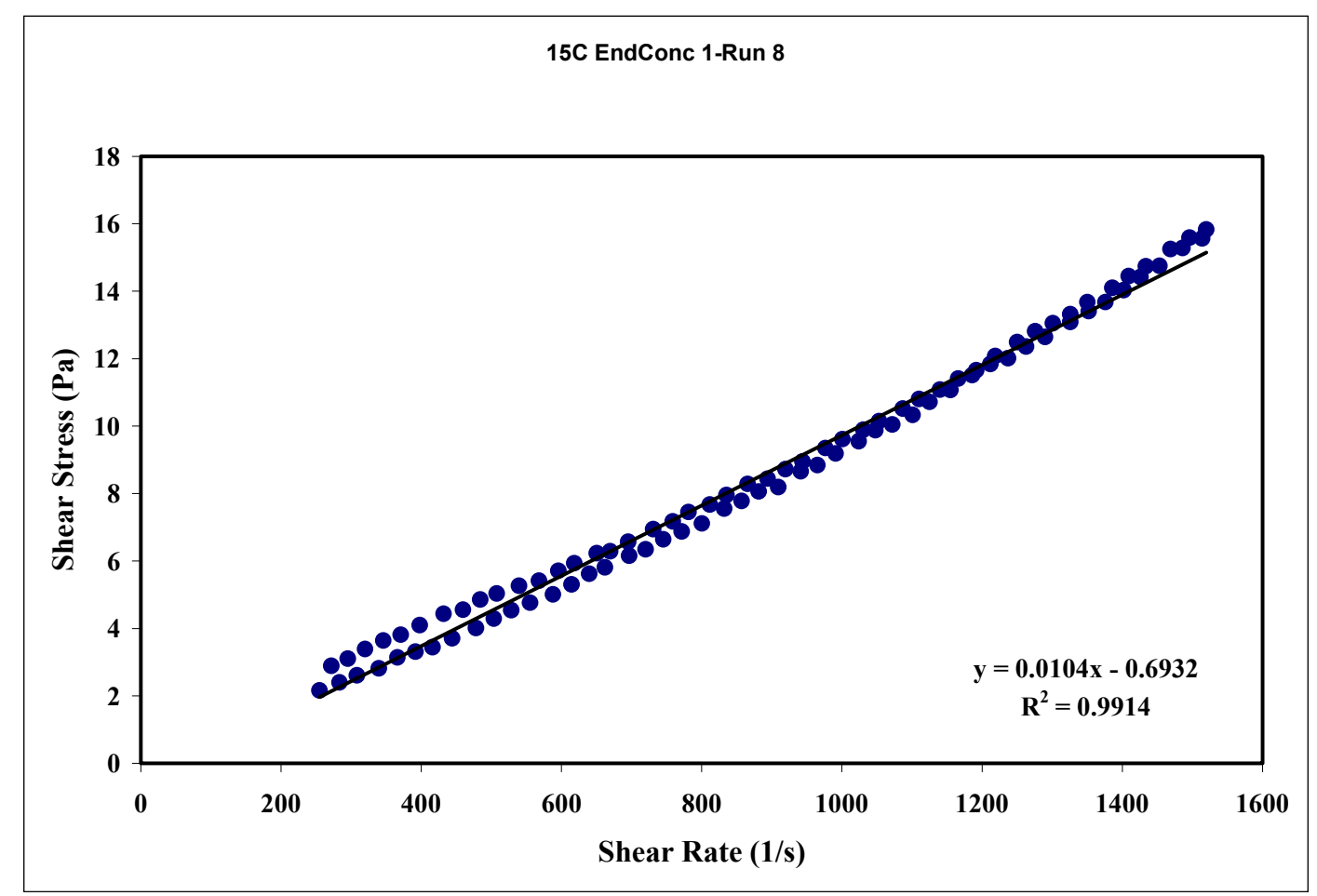

Figure 24. $15^{\circ} \mathrm{C}$ at 2 wt \% Run 8 


\begin{tabular}{|ll|}
\hline APPENDIX I - PART 1 & WSRC-TR-2000-00352 \\
SR/TRU PRECIPITATE SLURRY RHEOGRAMS & SRT-RPP-2000-00026 \\
\hline
\end{tabular}

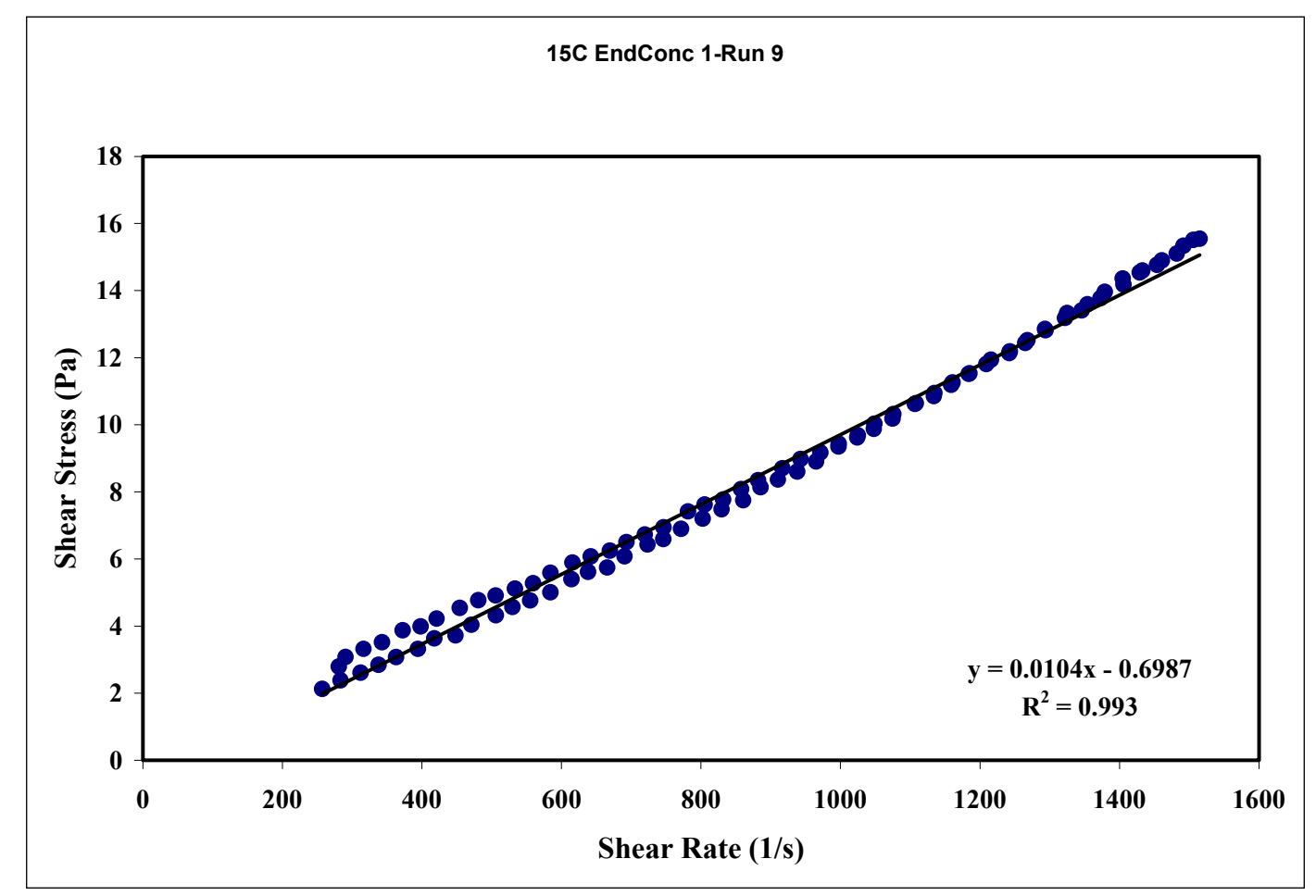

Figure $25.15^{\circ} \mathrm{C}$ at 2 wt \% Run 9

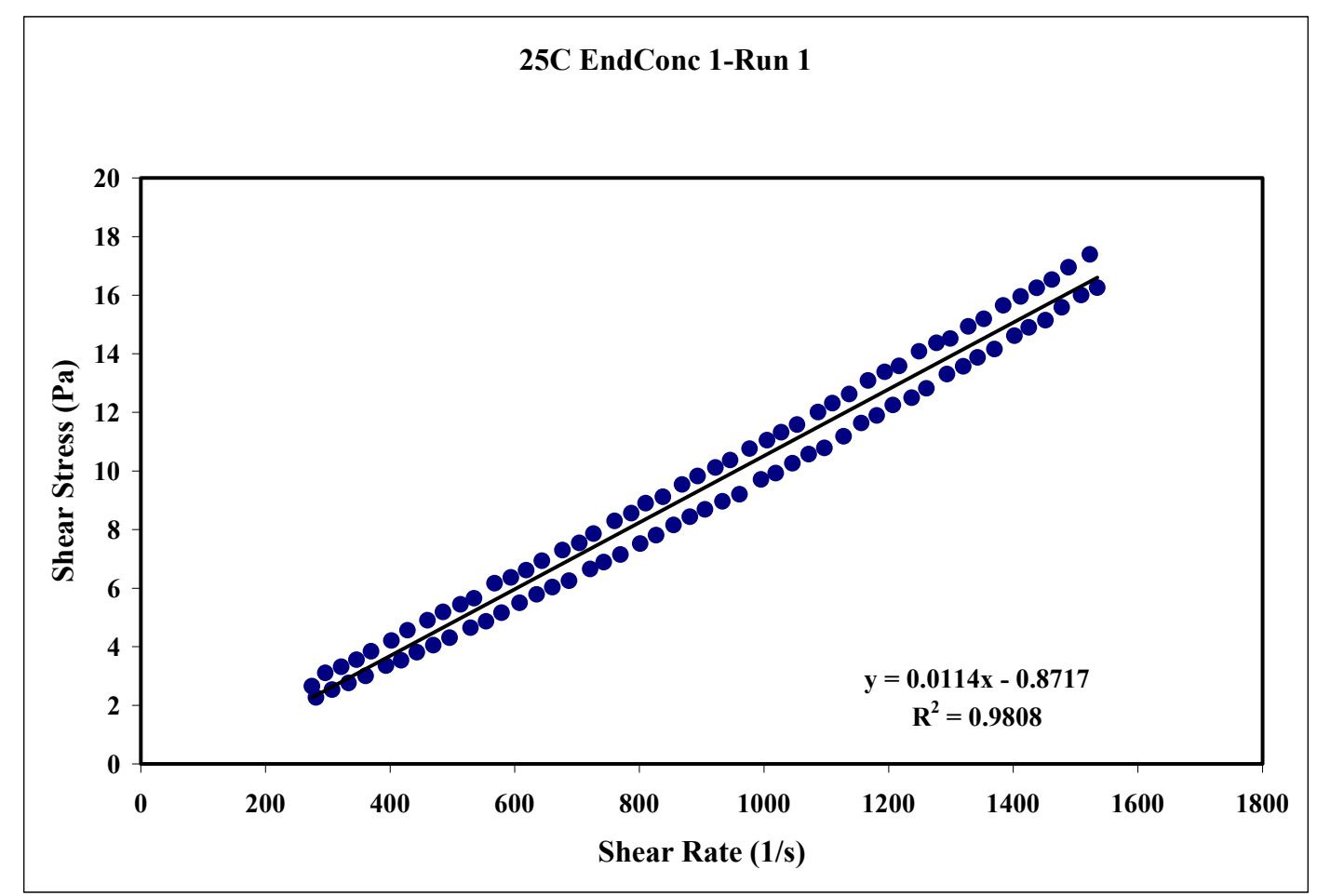

Figure $26.25^{\circ} \mathrm{C}$ at 2 wt \% Run 1 


\begin{tabular}{|ll|}
\hline APPENDIX I - PART 1 & WSRC-TR-2000-00352 \\
SR/TRU PRECIPITATE SLURRY RHEOGRAMS & SRT-RPP-2000-00026 \\
\hline
\end{tabular}

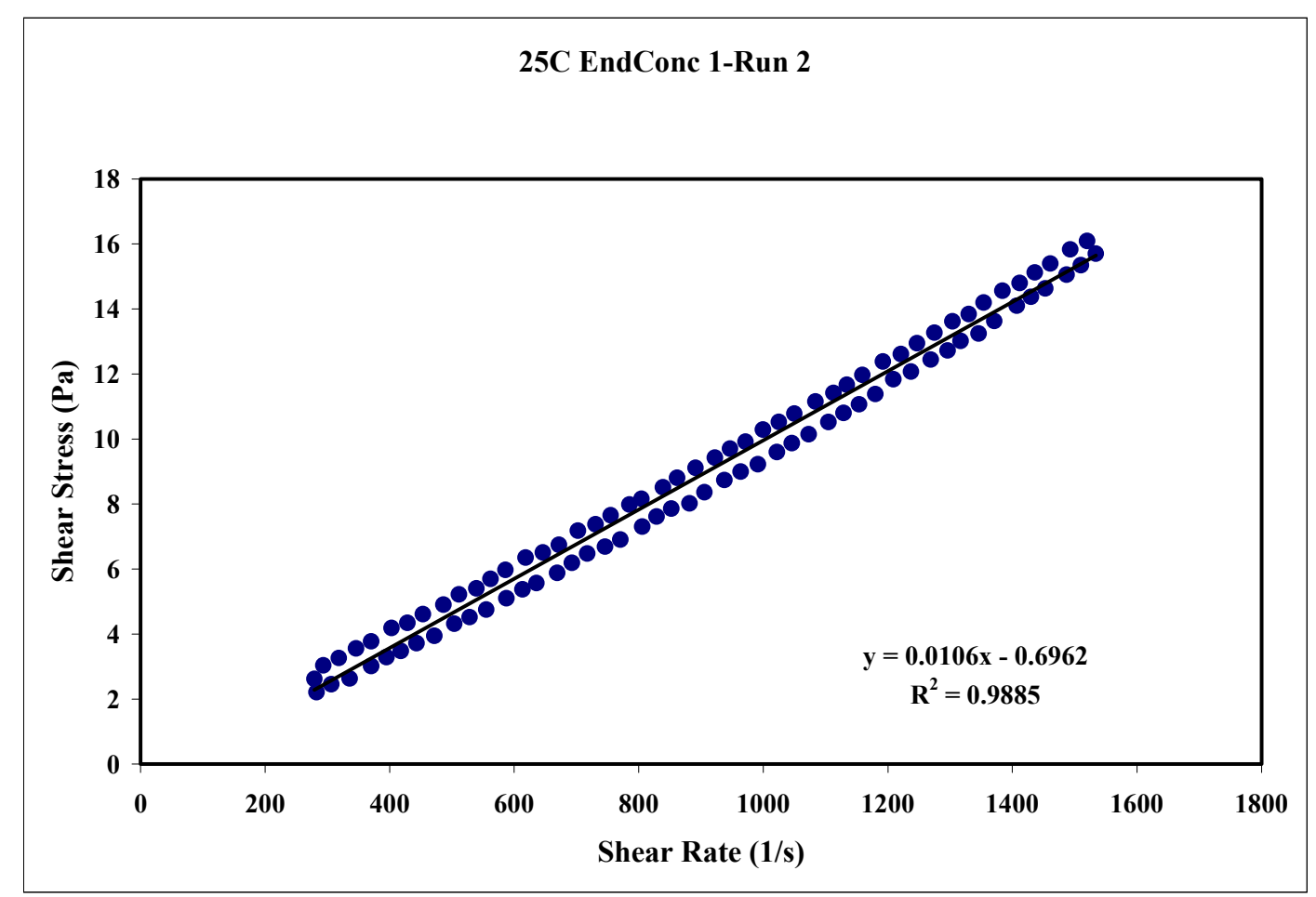

Figure $27.25^{\circ} \mathrm{C}$ at 2 wt \% Run 2

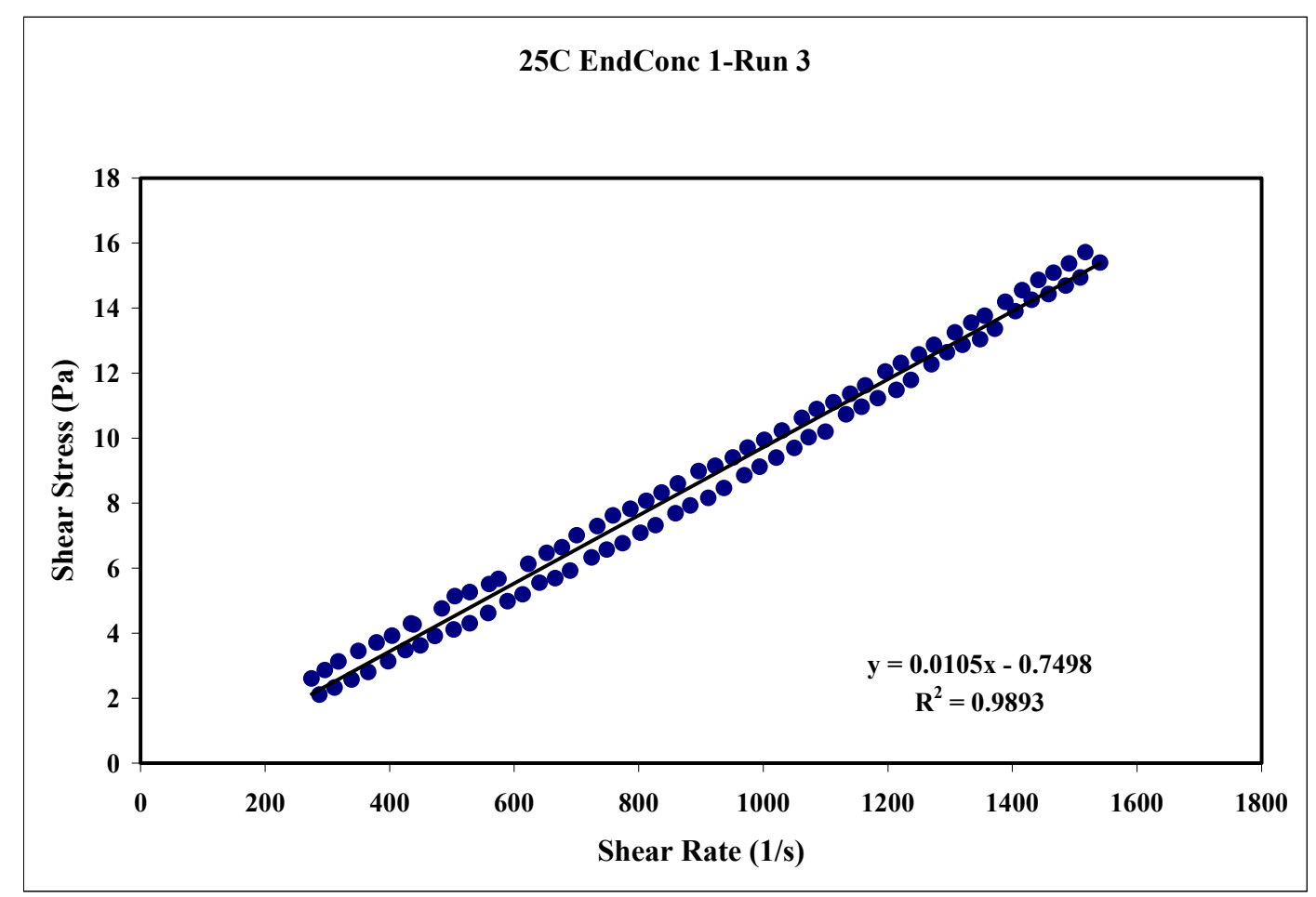

Figure 28. $25^{\circ} \mathrm{C}$ at 2 wt $\%$ Run 3 


\begin{tabular}{|ll|}
\hline APPENDIX I - PART 1 & WSRC-TR-2000-00352 \\
SR/TRU PRECIPITATE SLURRY RHEOGRAMS & SRT-RPP-2000-00026 \\
\hline
\end{tabular}

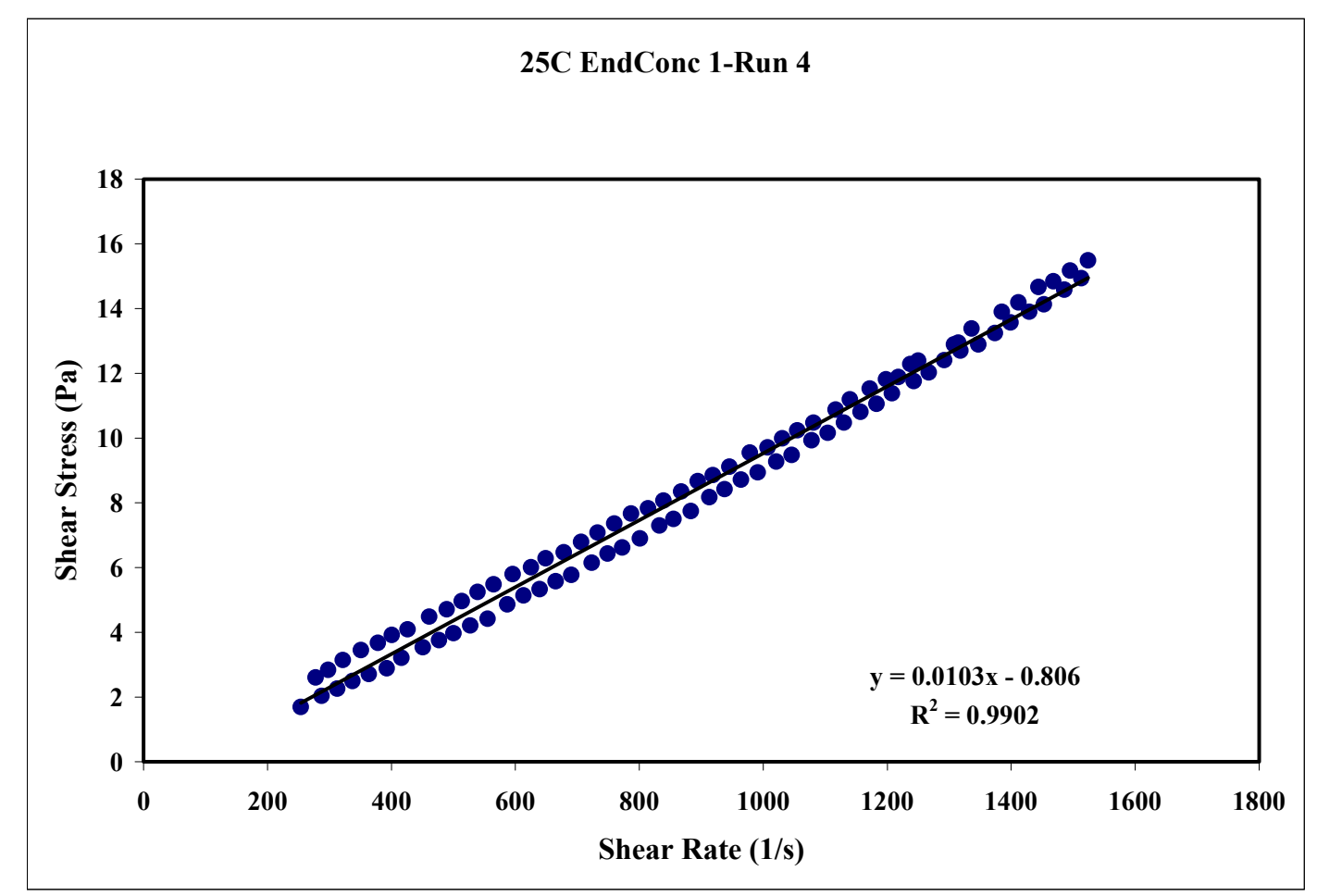

Figure 29. $25^{\circ} \mathrm{C}$ at 2 wt \% Run 4

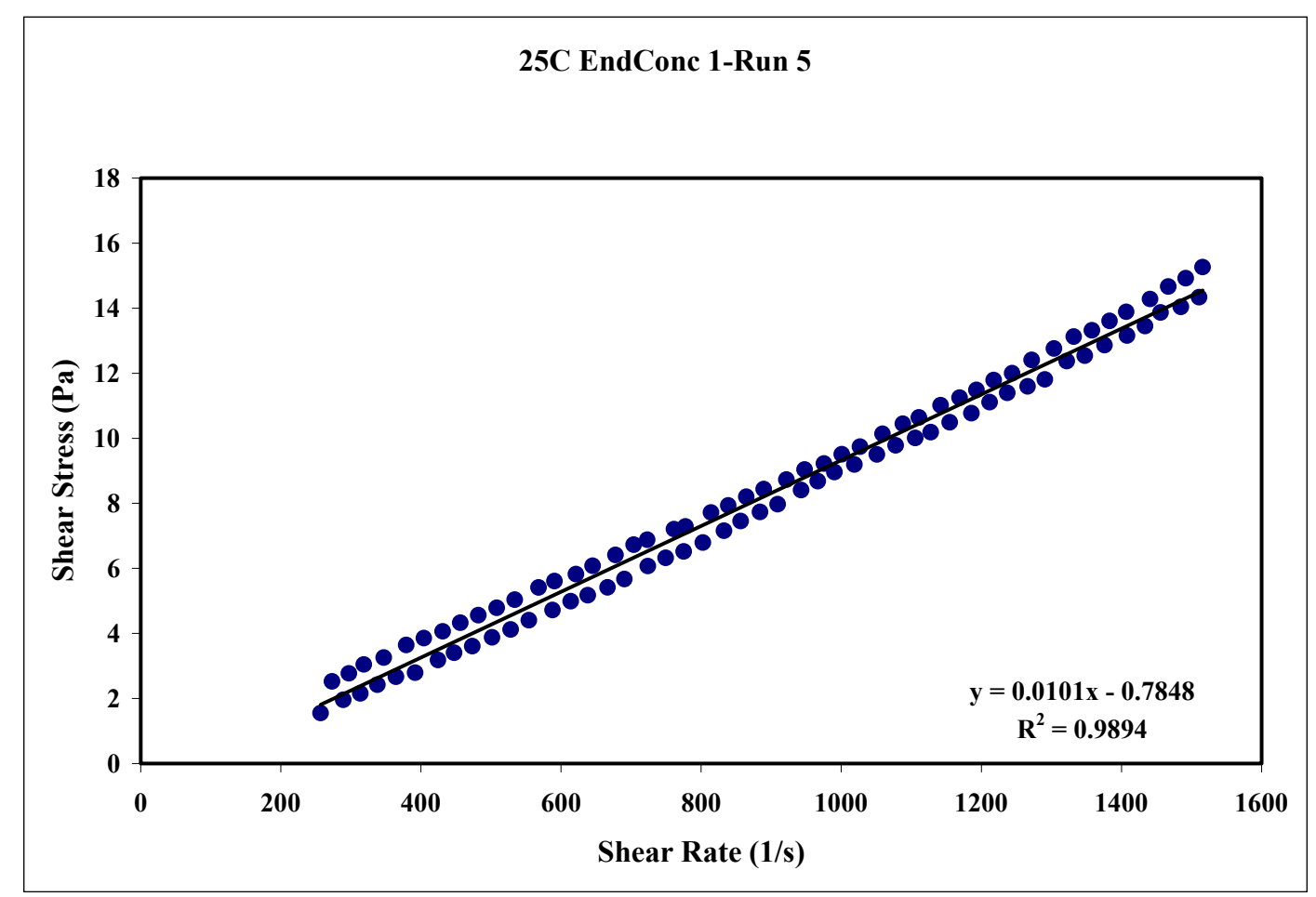

Figure 30. $25^{\circ} \mathrm{C}$ at 2 wt $\%$ Run 5 


\begin{tabular}{|ll|}
\hline APPENDIX I - PART 1 & WSRC-TR-2000-00352 \\
SR/TRU PRECIPITATE SLURRY RHEOGRAMS & SRT-RPP-2000-00026 \\
\hline
\end{tabular}

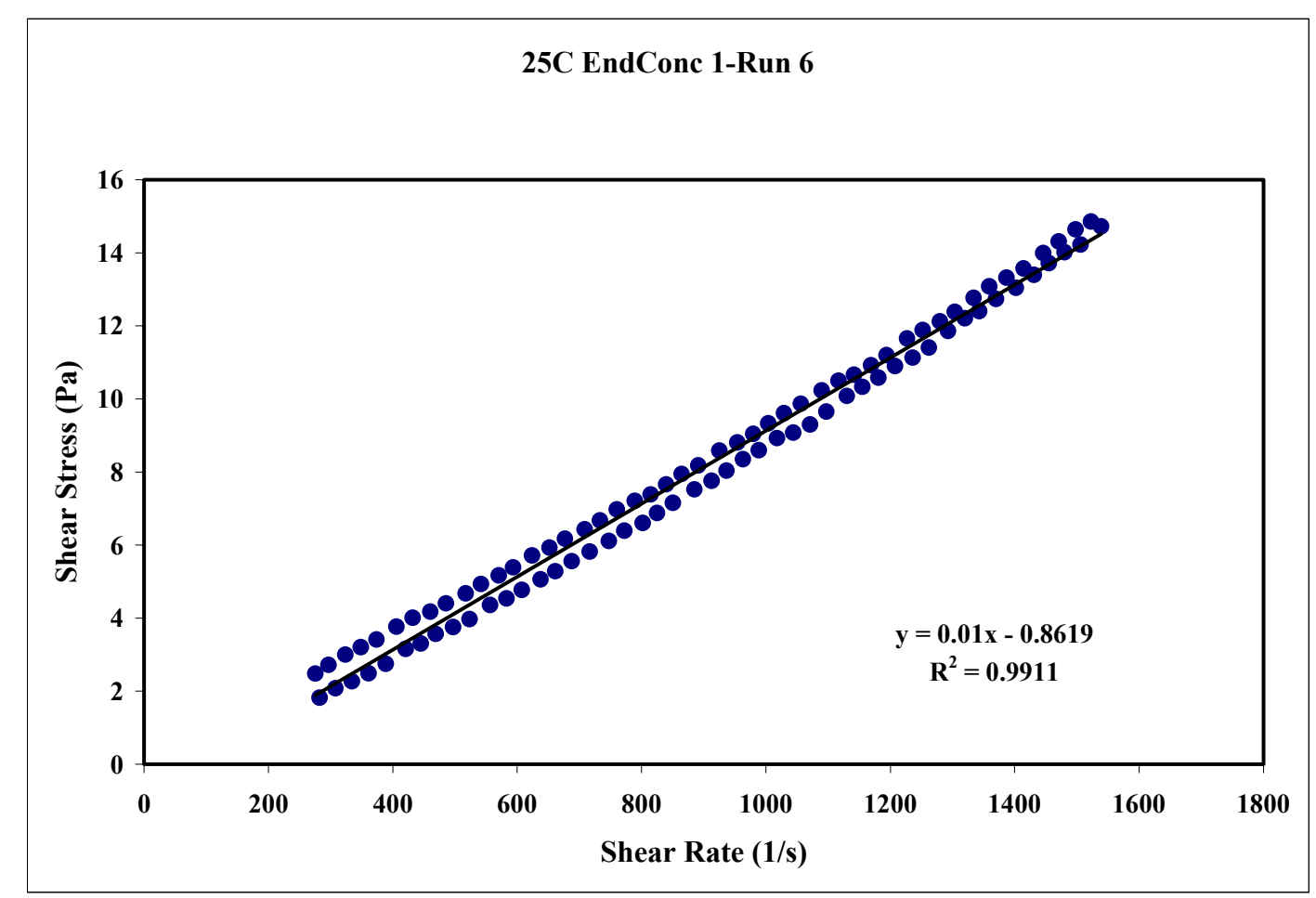

Figure 31. $25^{\circ} \mathrm{C}$ at 2 wt \% Run 6

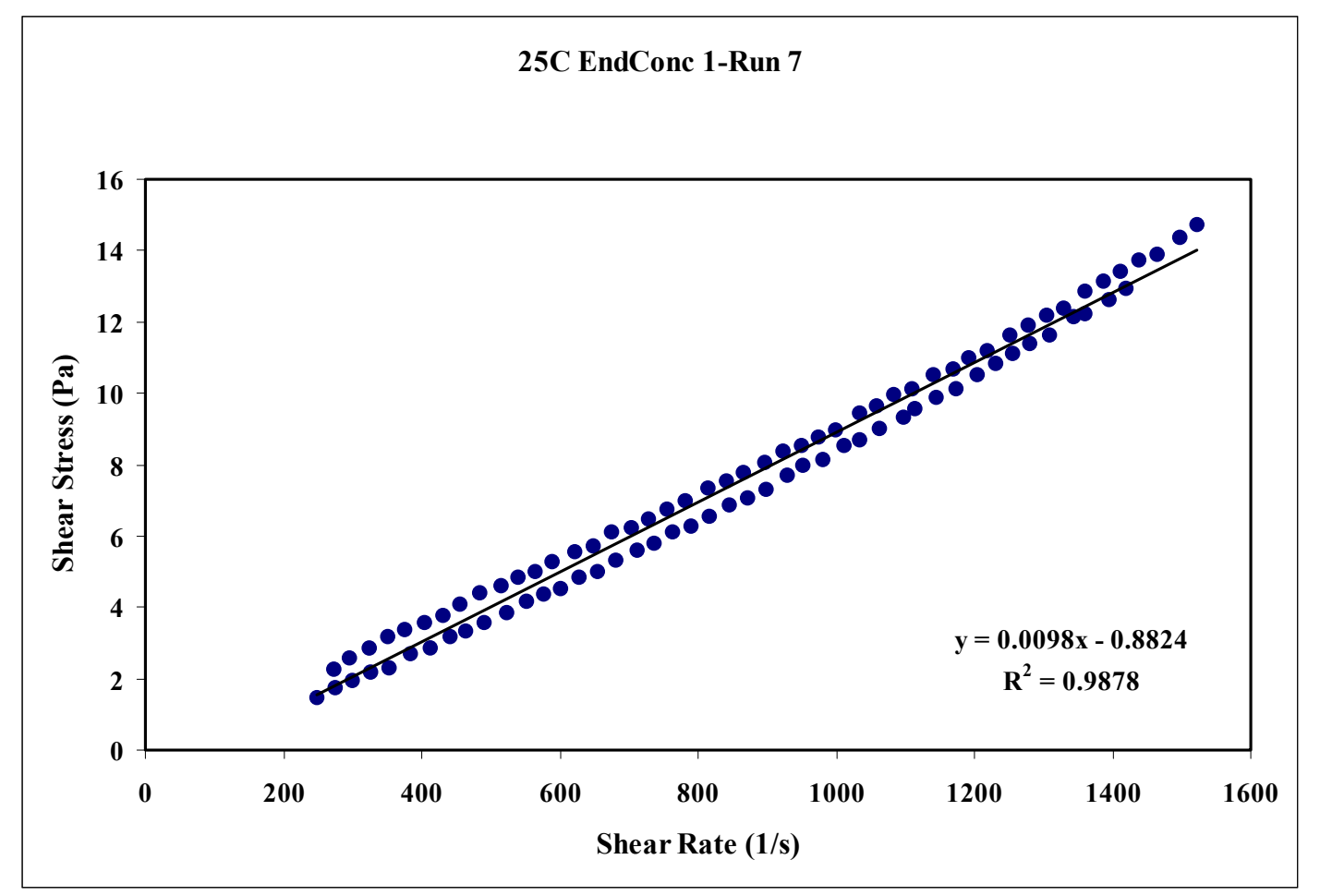

Figure 32. $25^{\circ} \mathrm{C}$ at 2 wt $\%$ Run 7 


\begin{tabular}{|ll|}
\hline APPENDIX I - PART 1 & WSRC-TR-2000-00352 \\
SR/TRU PRECIPITATE SLURRY RHEOGRAMS & SRT-RPP-2000-00026 \\
\hline
\end{tabular}

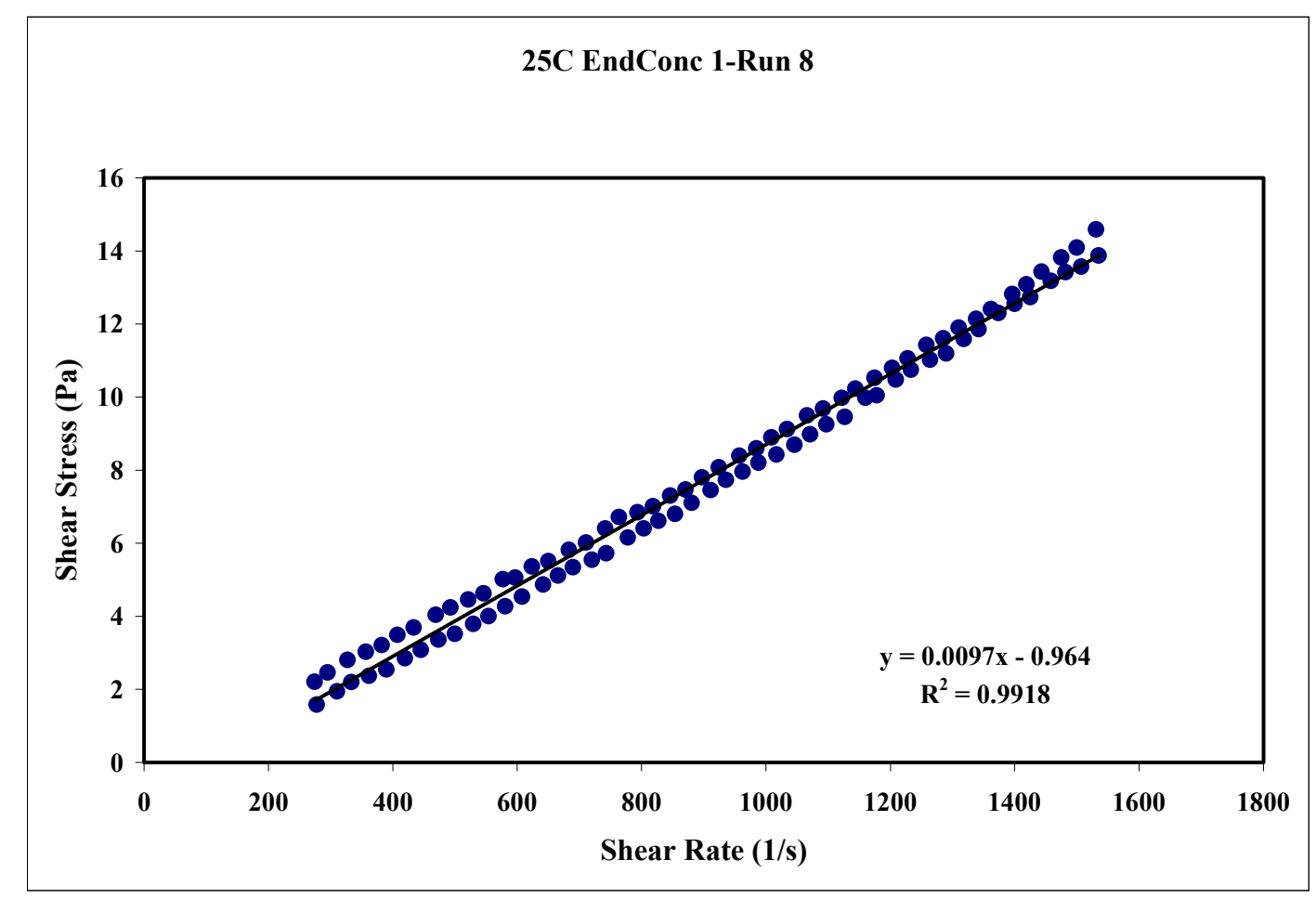

Figure 33. $25^{\circ} \mathrm{C}$ at 2 wt $\%$ Run 8

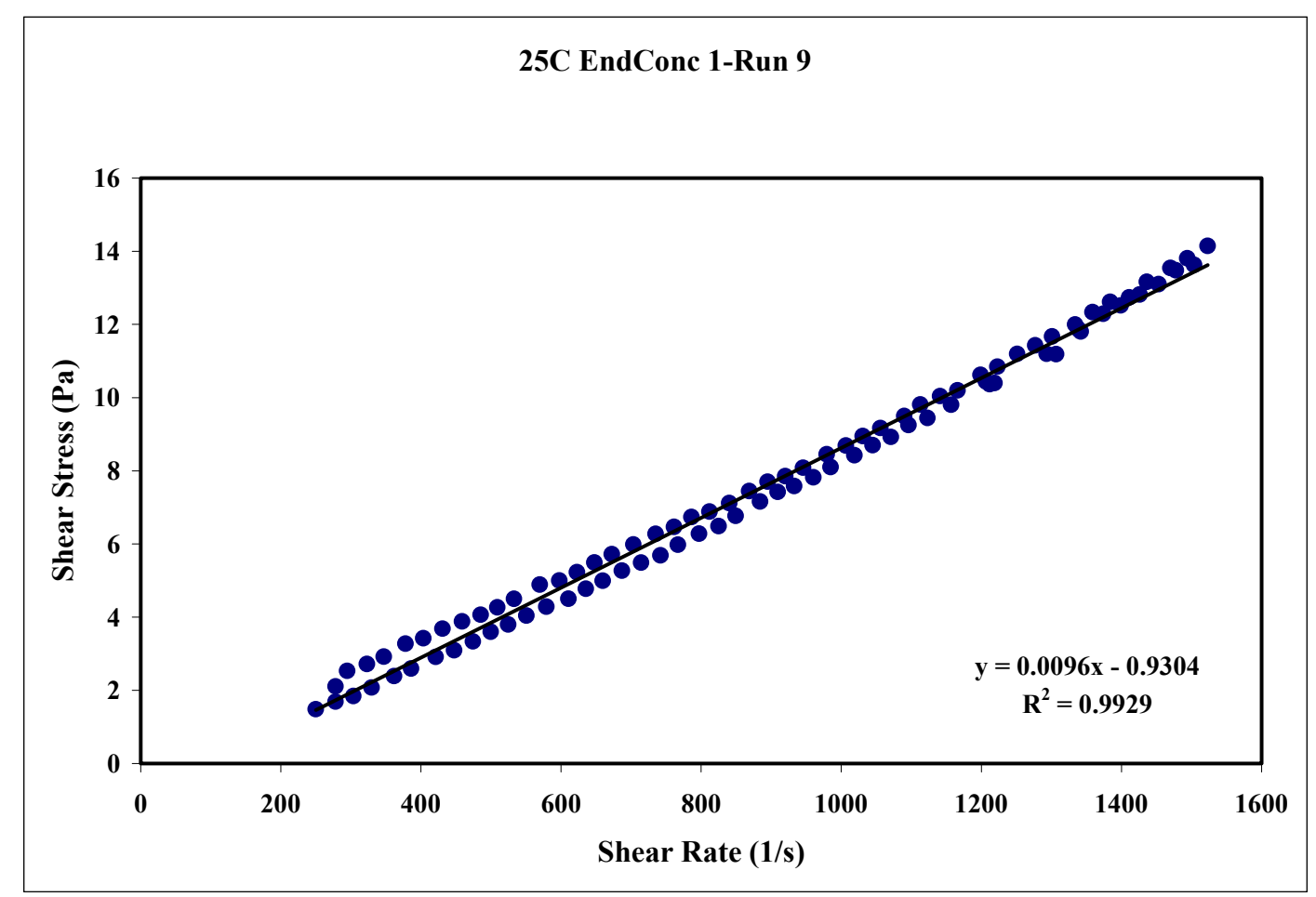

Figure 34. $25^{\circ} \mathrm{C}$ at 2 wt $\%$ Run 9 


\begin{tabular}{|ll|}
\hline APPENDIX I - PART 1 & WSRC-TR-2000-00352 \\
SR/TRU PRECIPITATE SLURRY RHEOGRAMS & SRT-RPP-2000-00026 \\
\hline
\end{tabular}

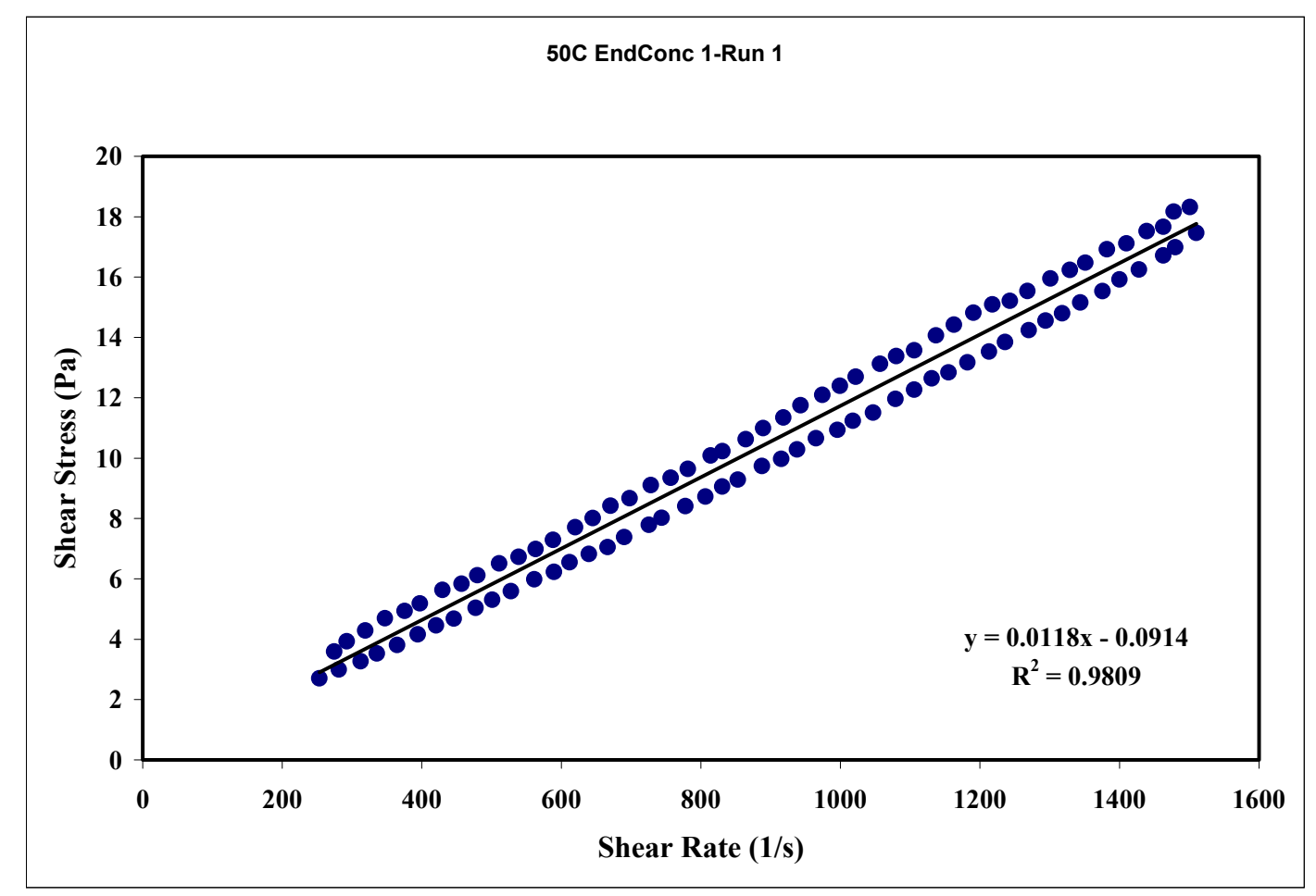

Figure $35.50{ }^{\circ} \mathrm{C}$ at 2 wt \% Run 1

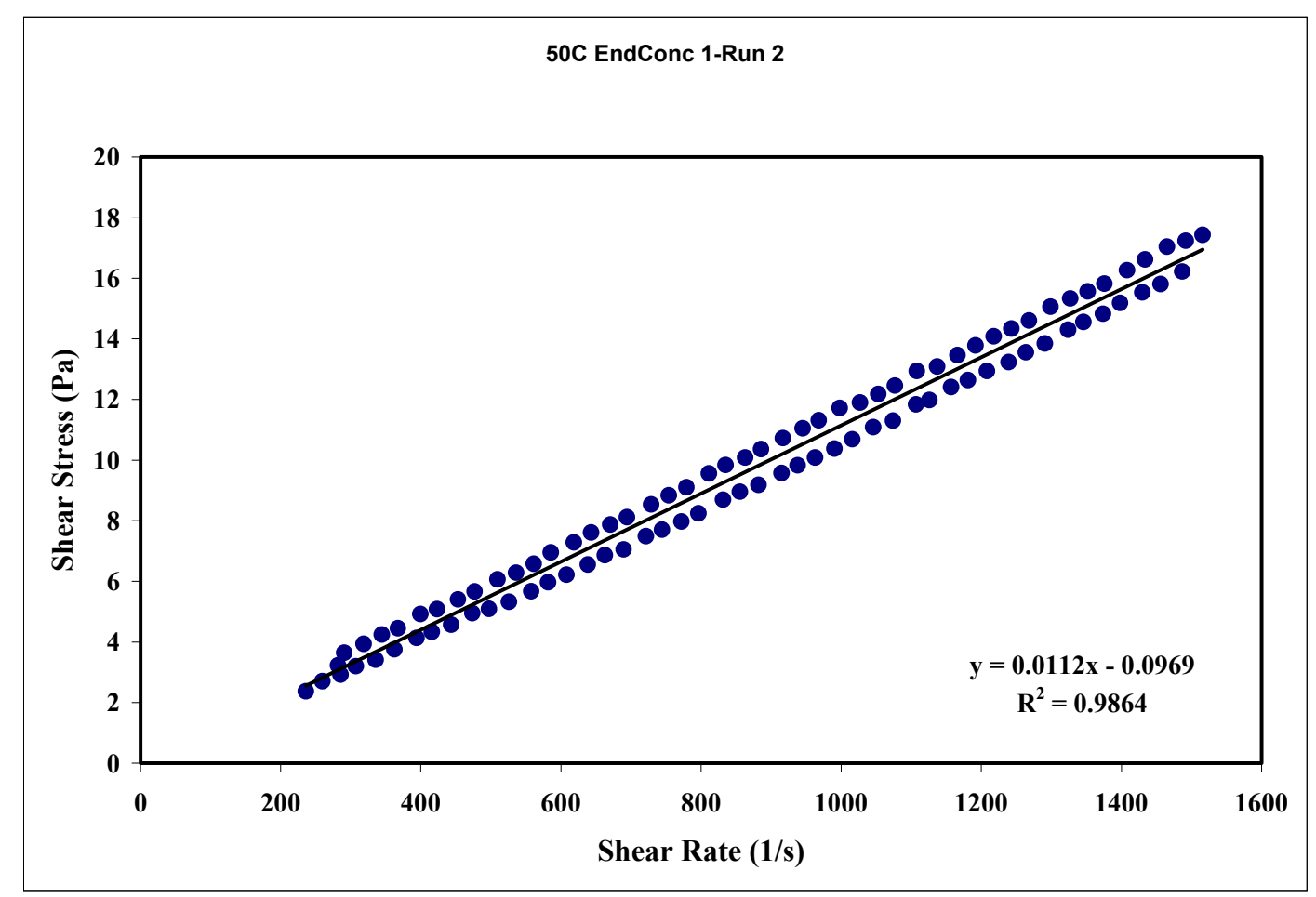

Figure 36. $50{ }^{\circ} \mathrm{C}$ at 2 wt $\%$ Run 2 


\begin{tabular}{|ll|}
\hline APPENDIX I - PART 1 & WSRC-TR-2000-00352 \\
SR/TRU PRECIPITATE SLURRY RHEOGRAMS & SRT-RPP-2000-00026 \\
\hline
\end{tabular}

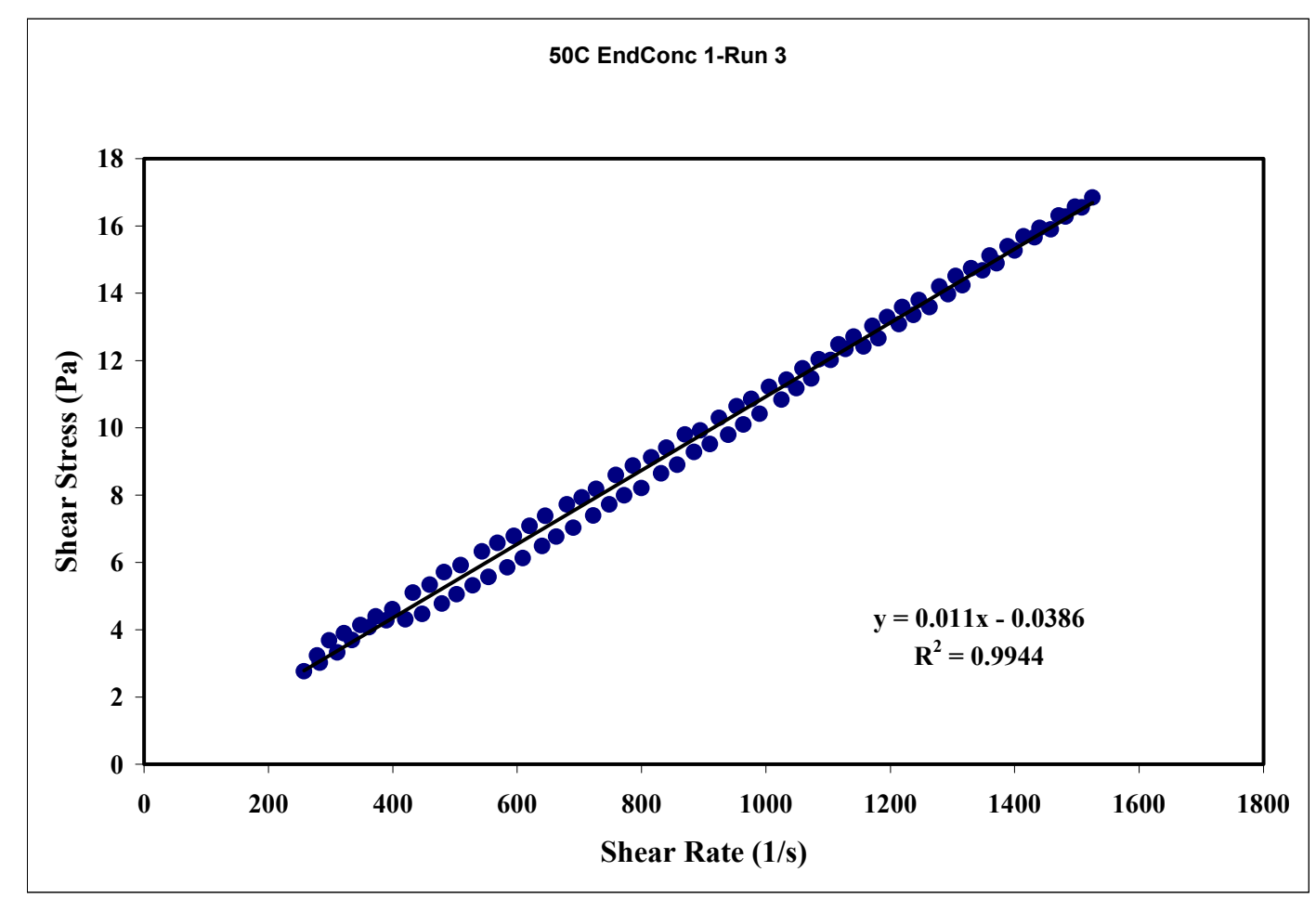

Figure $37.50{ }^{\circ} \mathrm{C}$ at 2 wt \% Run 3

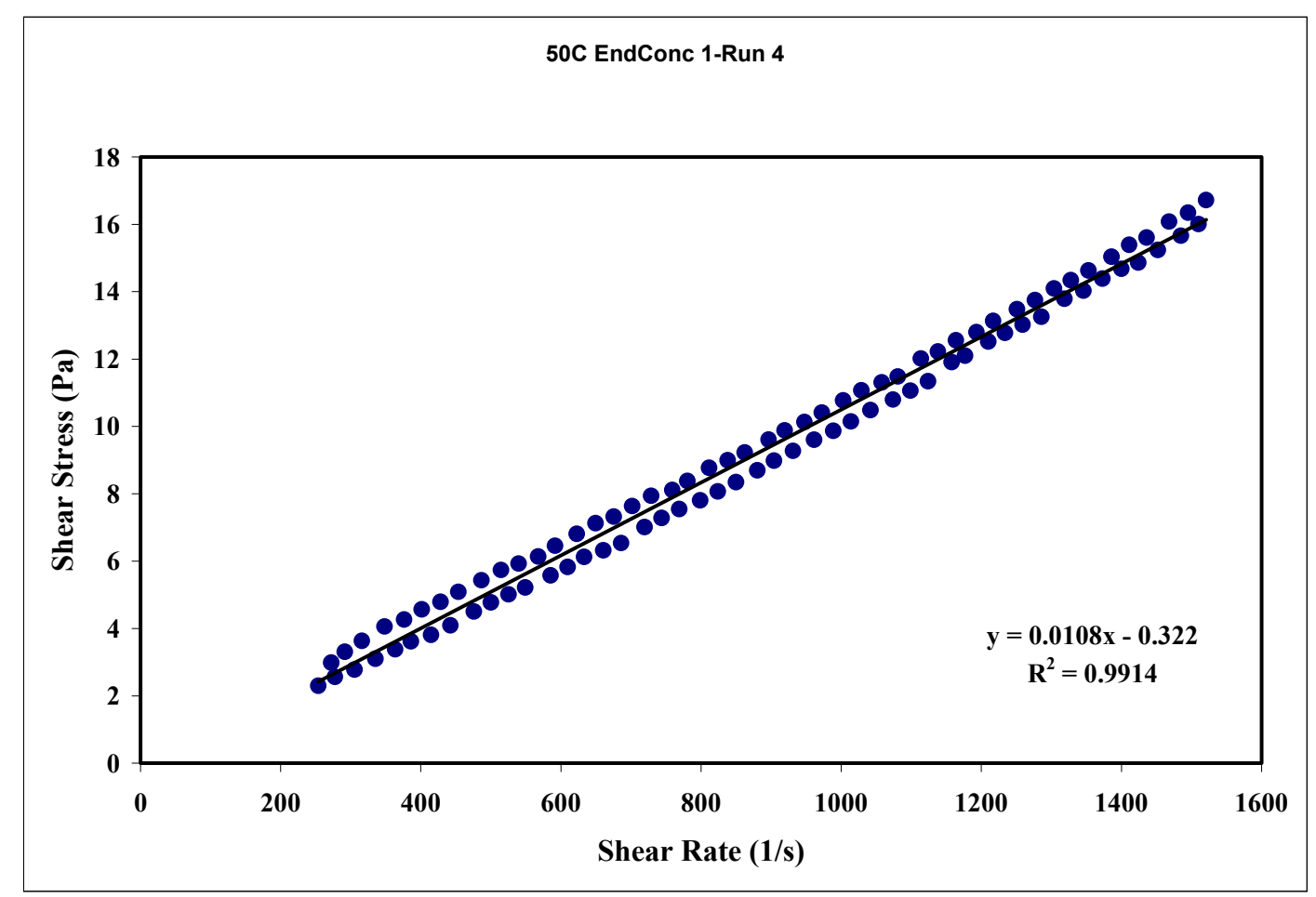

Figure 38. $50{ }^{\circ} \mathrm{C}$ at 2 wt $\%$ Run 4 


\begin{tabular}{|ll|}
\hline APPENDIX I - PART 1 & WSRC-TR-2000-00352 \\
SR/TRU PRECIPITATE SLURRY RHEOGRAMS & SRT-RPP-2000-00026 \\
\hline
\end{tabular}

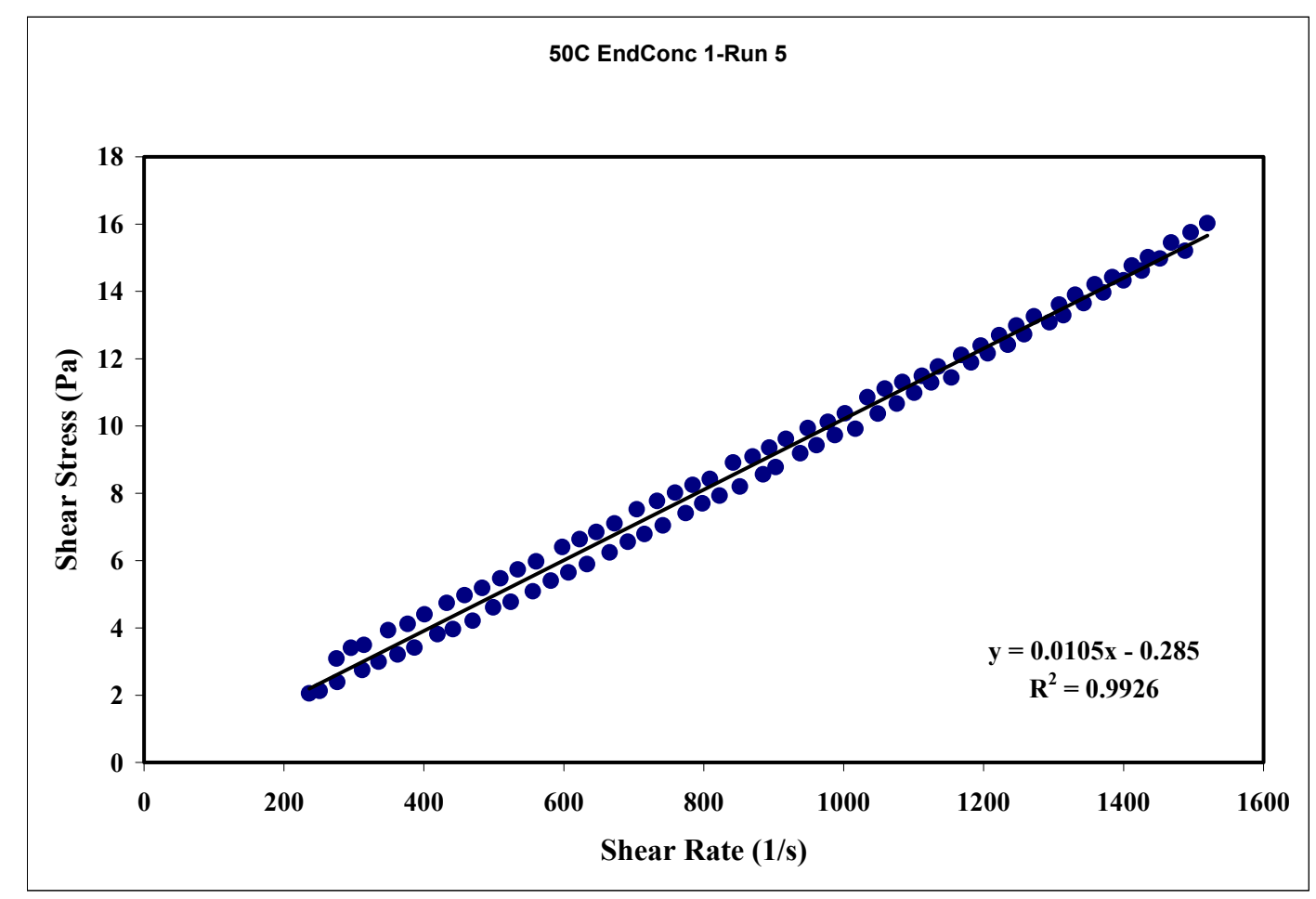

Figure 39. $50{ }^{\circ} \mathrm{C}$ at 2 wt $\%$ Run 5

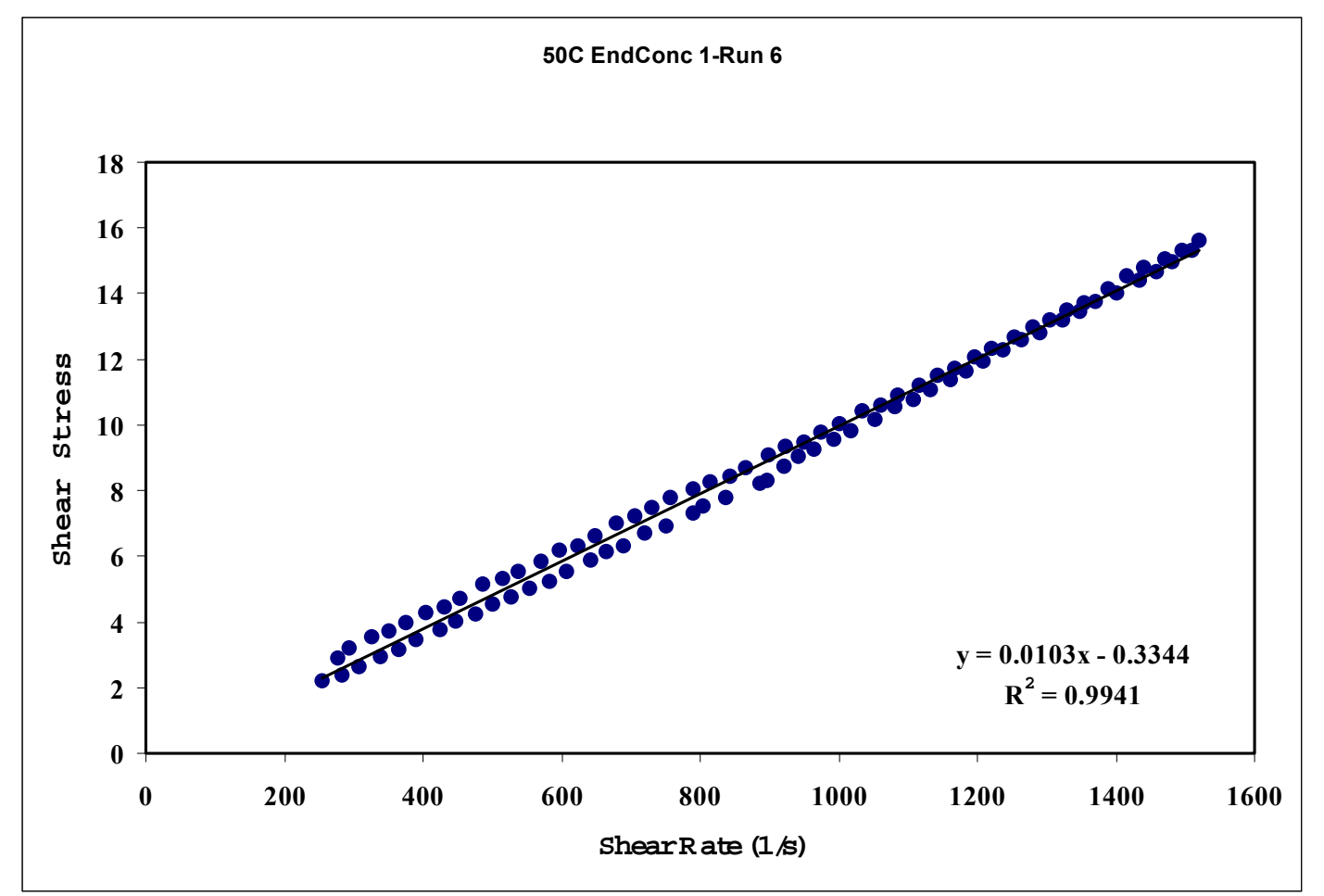

Figure 40. $50{ }^{\circ} \mathrm{C}$ at 2 wt \% Run 6 


\begin{tabular}{|ll|}
\hline APPENDIX I - PART 1 & WSRC-TR-2000-00352 \\
SR/TRU PRECIPITATE SLURRY RHEOGRAMS & SRT-RPP-2000-00026 \\
\hline
\end{tabular}

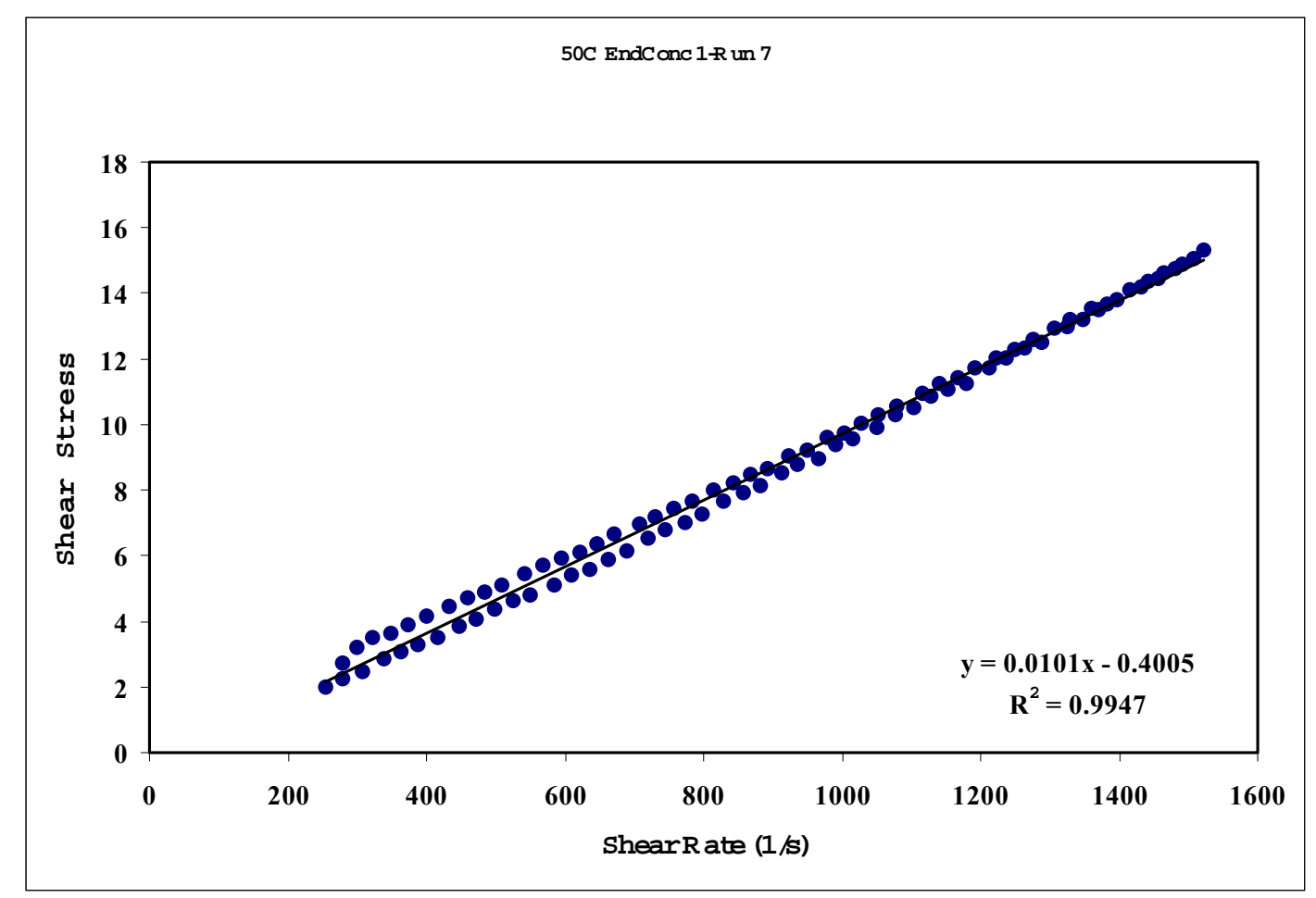

Figure 41. $50{ }^{\circ} \mathrm{C}$ at 2 wt \% Run 7

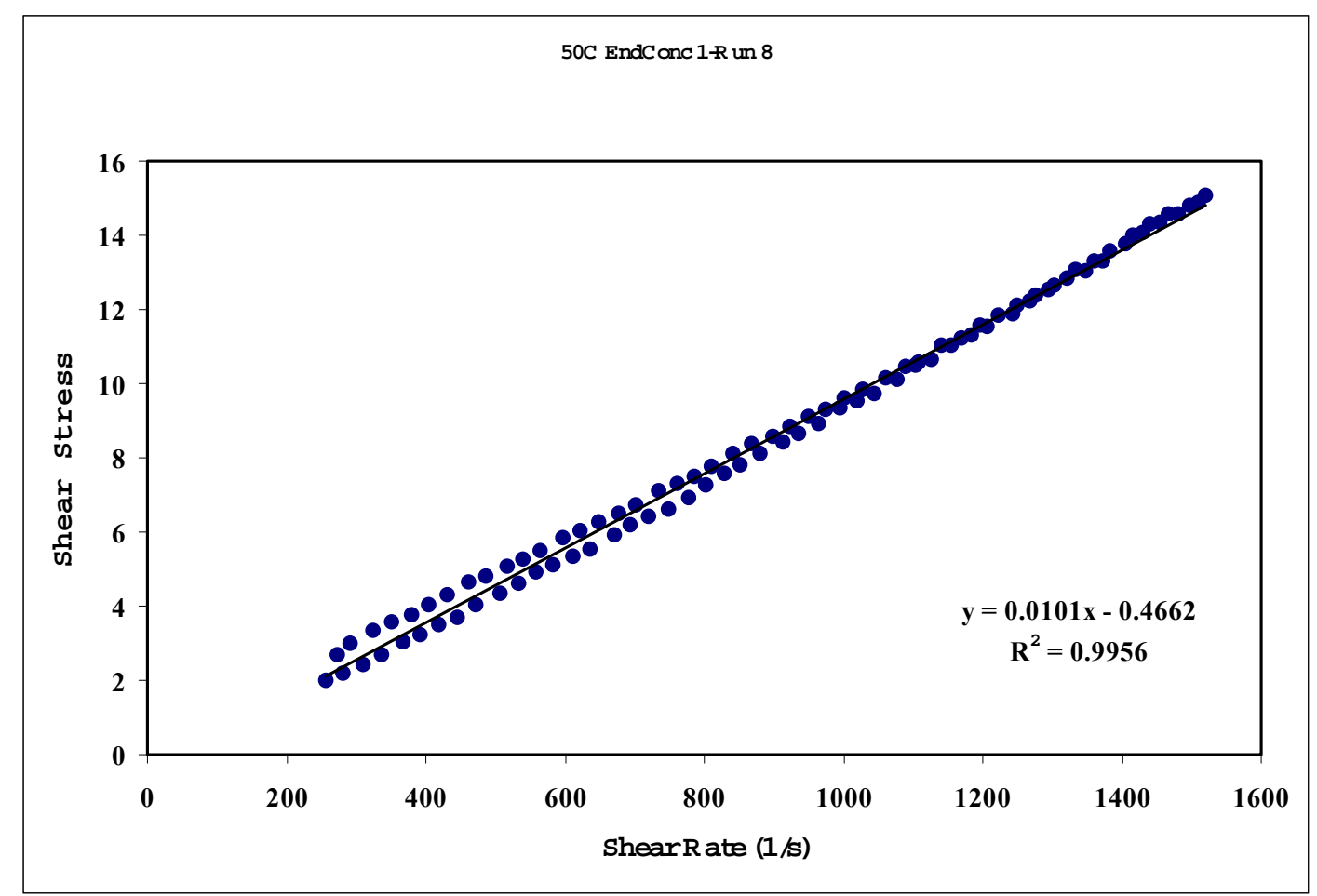

Figure $42.50{ }^{\circ} \mathrm{C}$ at 2 wt $\%$ Run 8 


\begin{tabular}{|ll|}
\hline APPENDIX I - PART 1 & WSRC-TR-2000-00352 \\
SR/TRU PRECIPITATE SLURRY RHEOGRAMS & SRT-RPP-2000-00026 \\
\hline
\end{tabular}

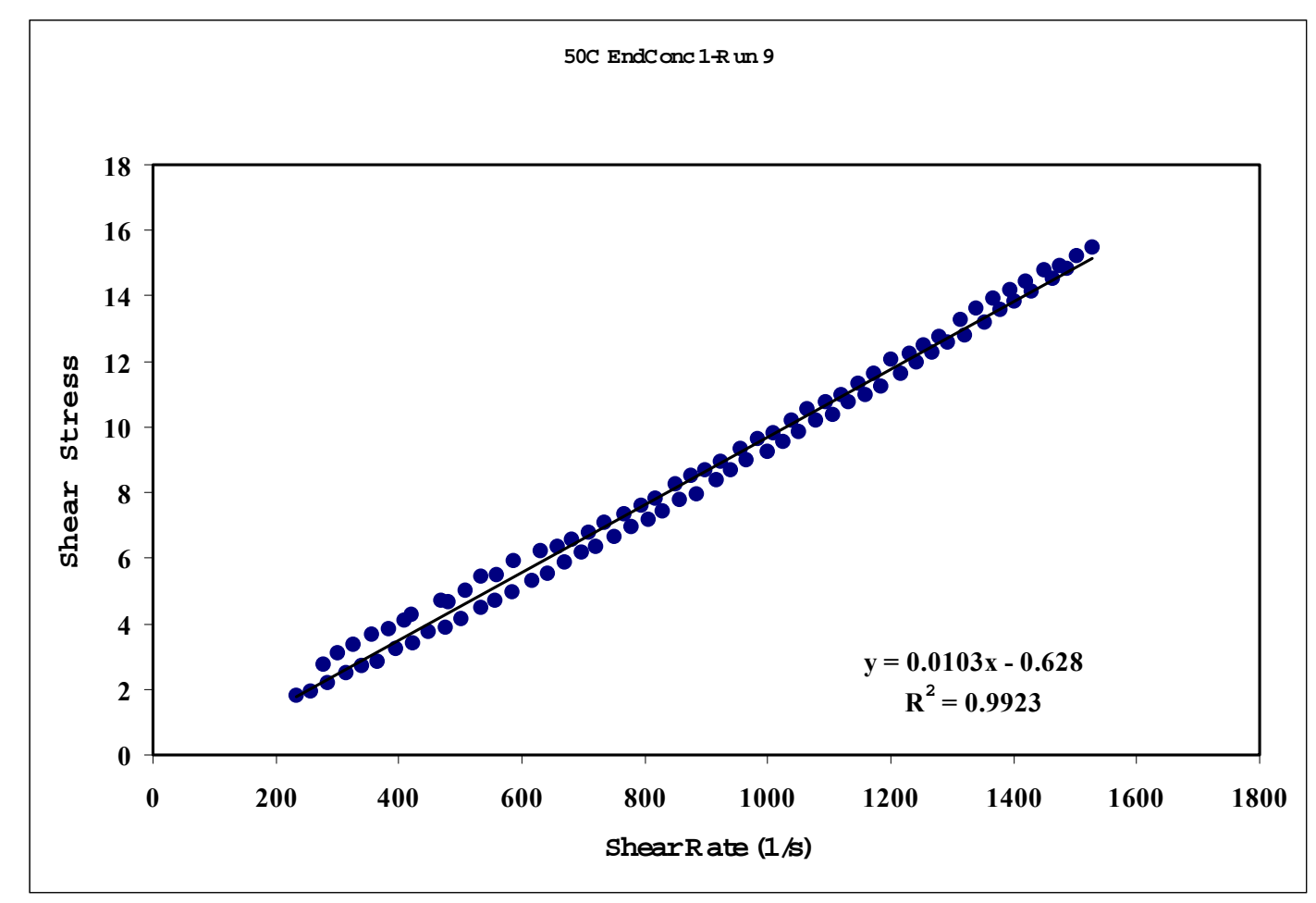

Figure $43.50{ }^{\circ} \mathrm{C}$ at 2 wt $\%$ Run 9 


\section{APPENDIX I - PART 2}

\section{SR/TRU PRECIPITATE SLURRY RHEOGRAMS AT 10 WT \%}

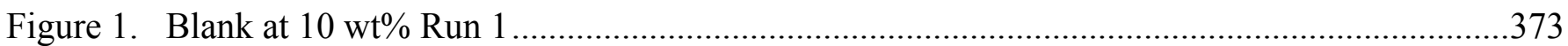

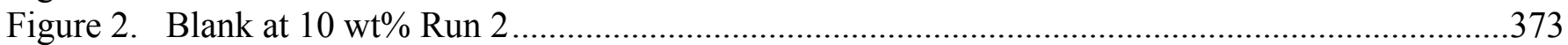

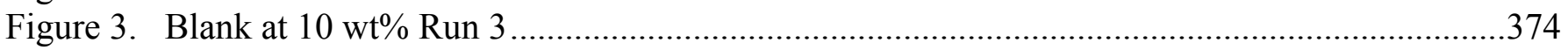

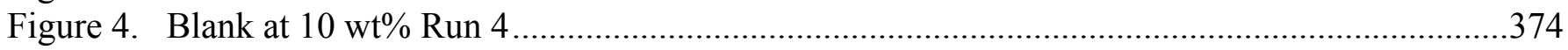

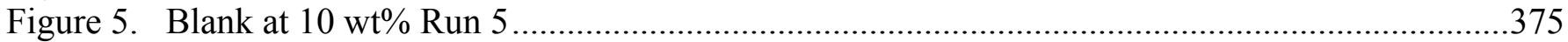

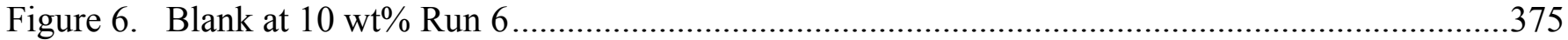

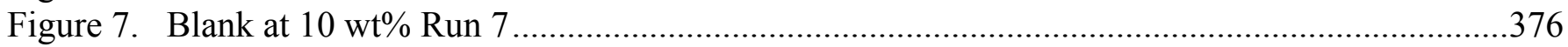

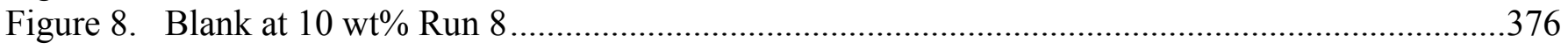

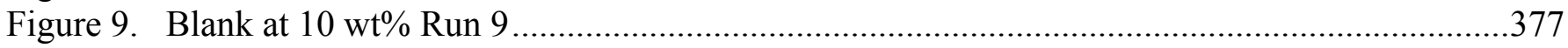

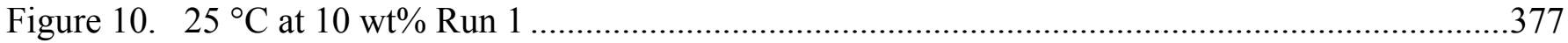

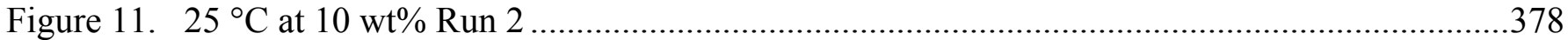

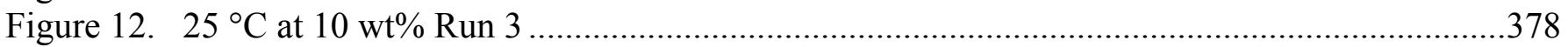

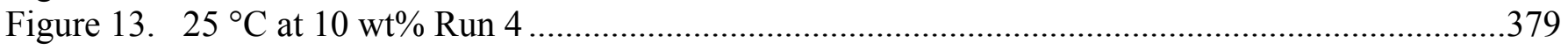

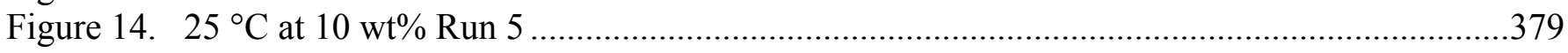

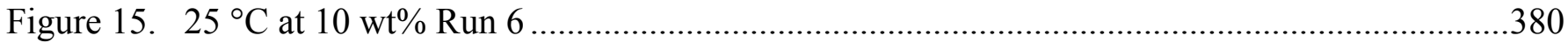

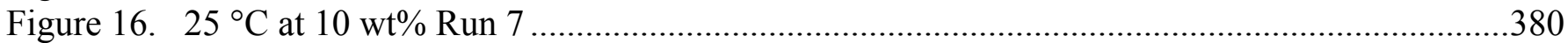

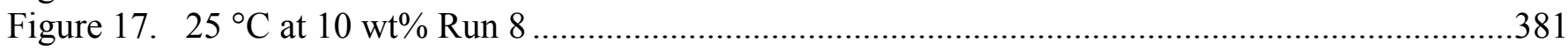

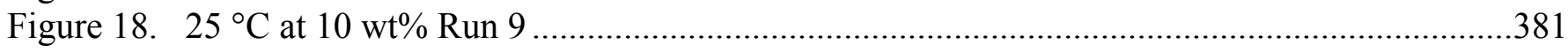

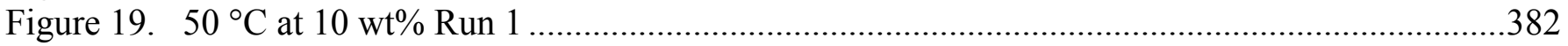

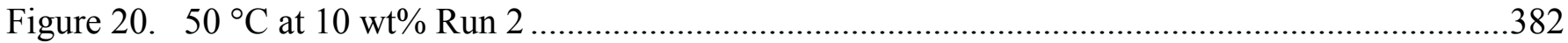

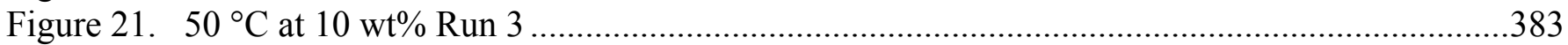

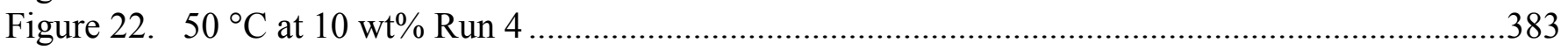

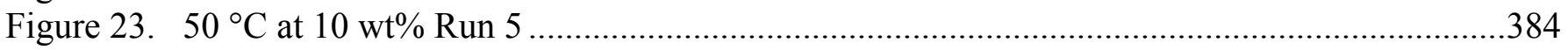

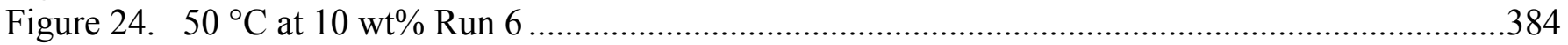

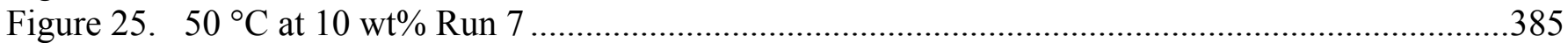

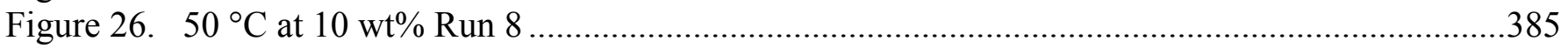

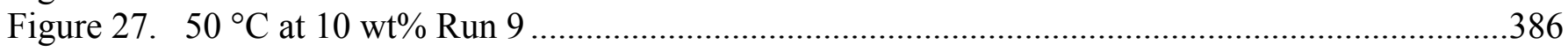




\begin{tabular}{|ll|}
\hline APPENDIX I - PART 2 & WSRC-TR-2000-00352 \\
& SRT-RPP-2000-00026 \\
SR/TRU PRECIPITATE SLURRY RHEOGRAMS AT 10 WT \% & Page 372 of 514 \\
\hline
\end{tabular}

This page intentionally left blank. 


\begin{tabular}{|ll|}
\hline APPENDIX I - PART 2 & WSRC-TR-2000-00352 \\
& SRT-RPP-2000-00026 \\
SR/TRU PRECIPITATE SLURRY RHEOGRAMS AT 10 WT \% & Page 373 of 514 \\
\hline
\end{tabular}

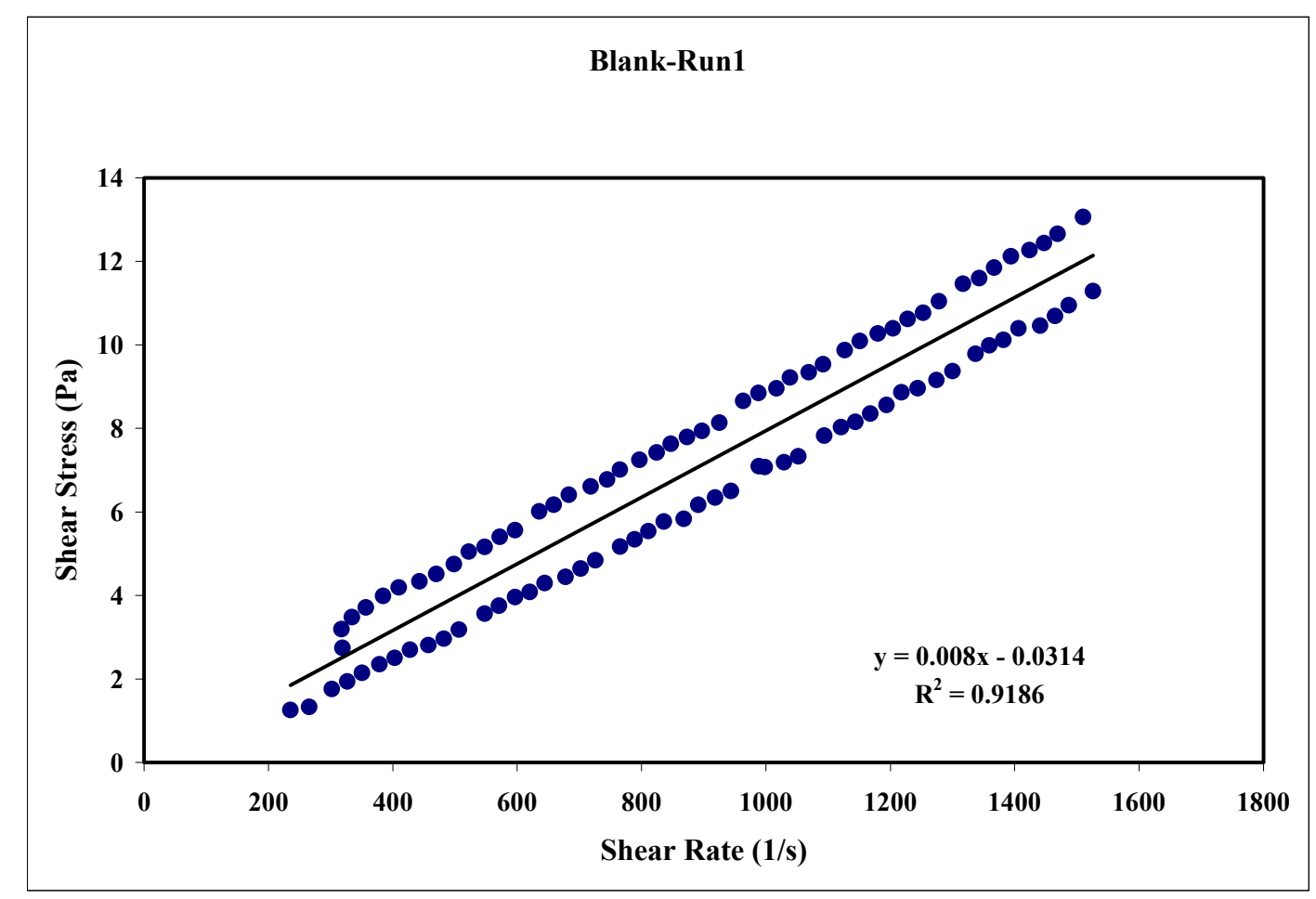

Figure 1. Blank at 10 wt\% Run 1

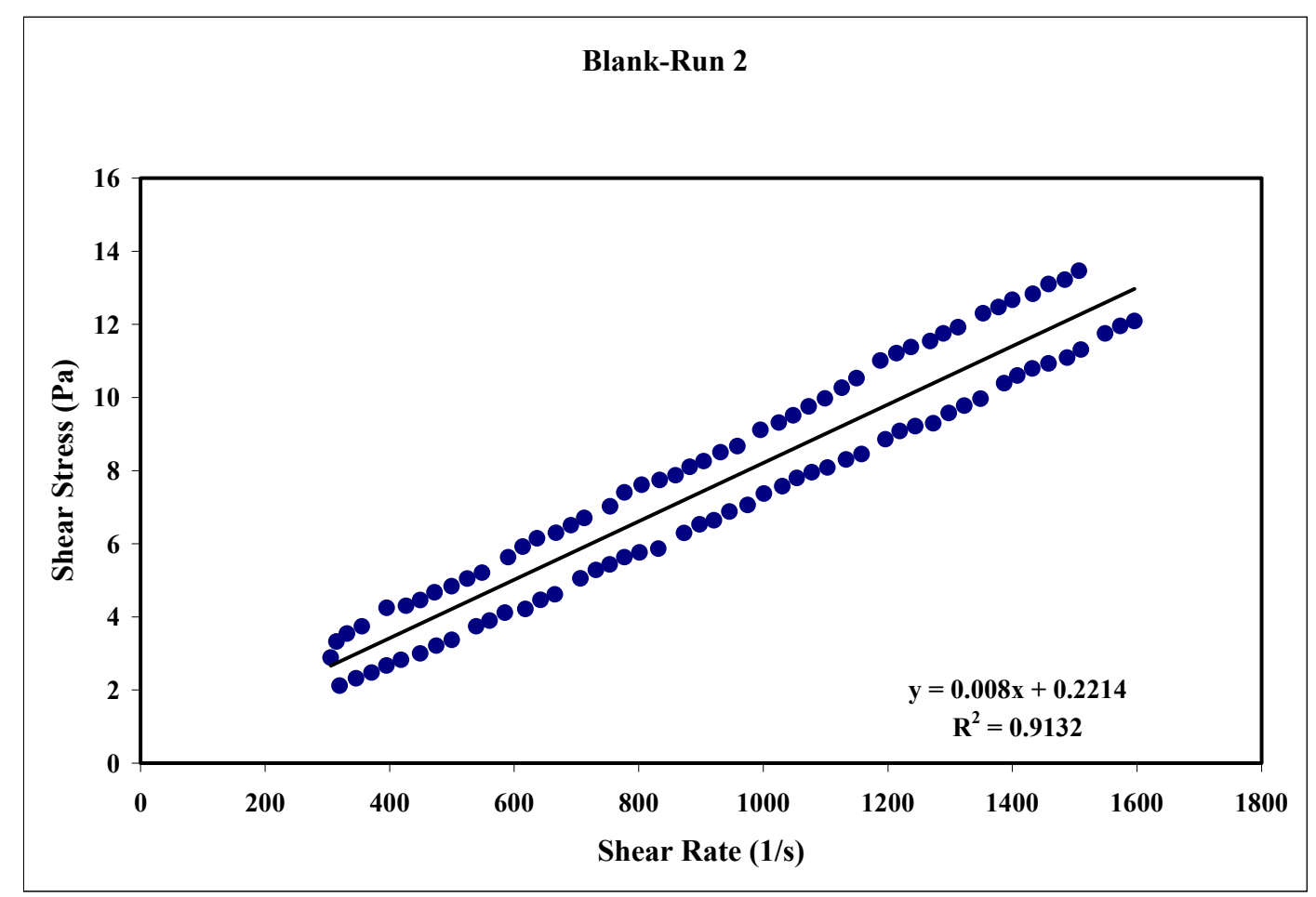

Figure 2. Blank at 10 wt\% Run 2 


\begin{tabular}{|ll|}
\hline APPENDIX I - PART 2 & WSRC-TR-2000-00352 \\
& SRT-RPP-2000-00026 \\
SR/TRU PRECIPITATE SLURRY RHEOGRAMS AT 10 WT \% & Page 374 of 514 \\
\hline
\end{tabular}

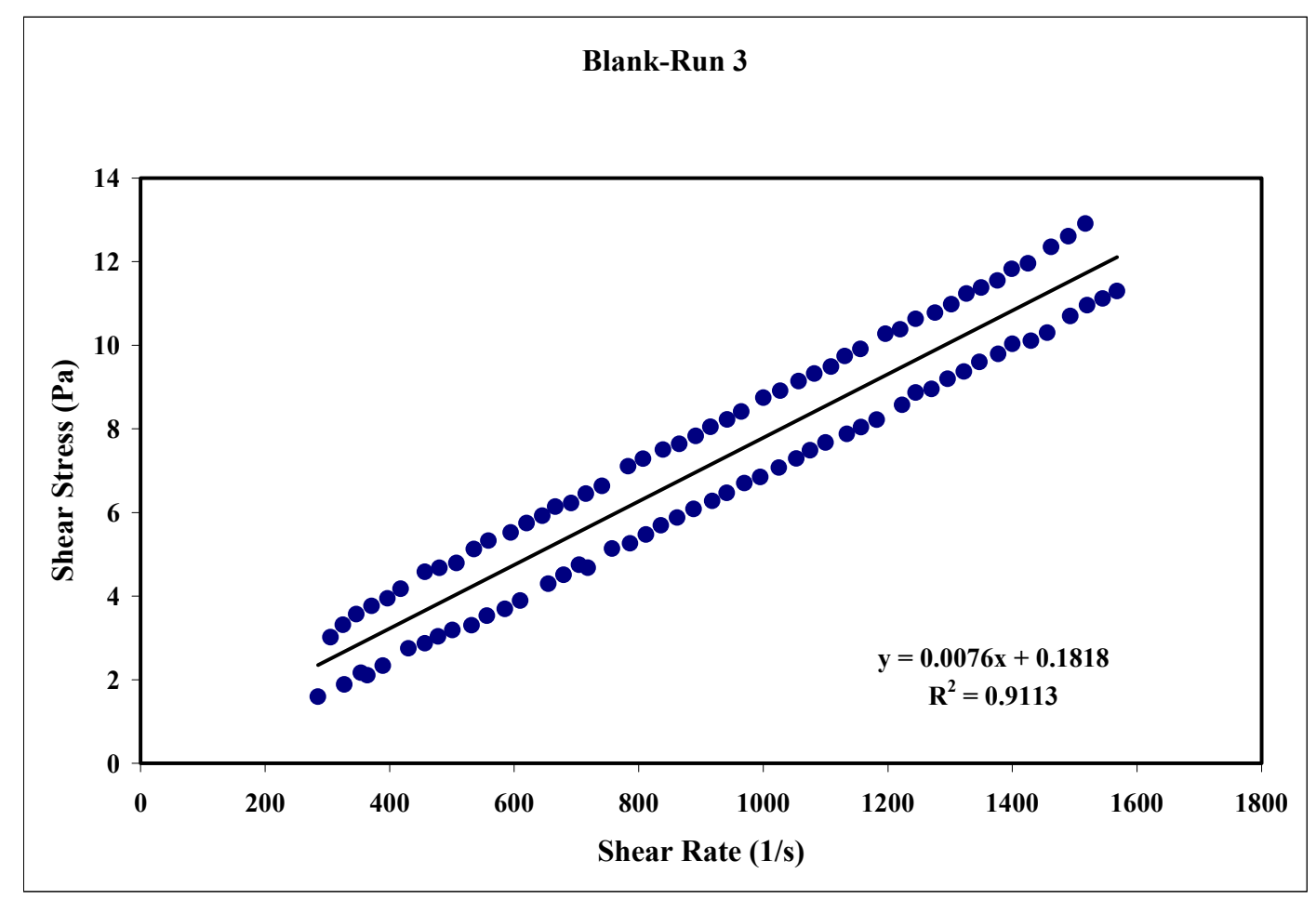

Figure 3. Blank at 10 wt\% Run 3

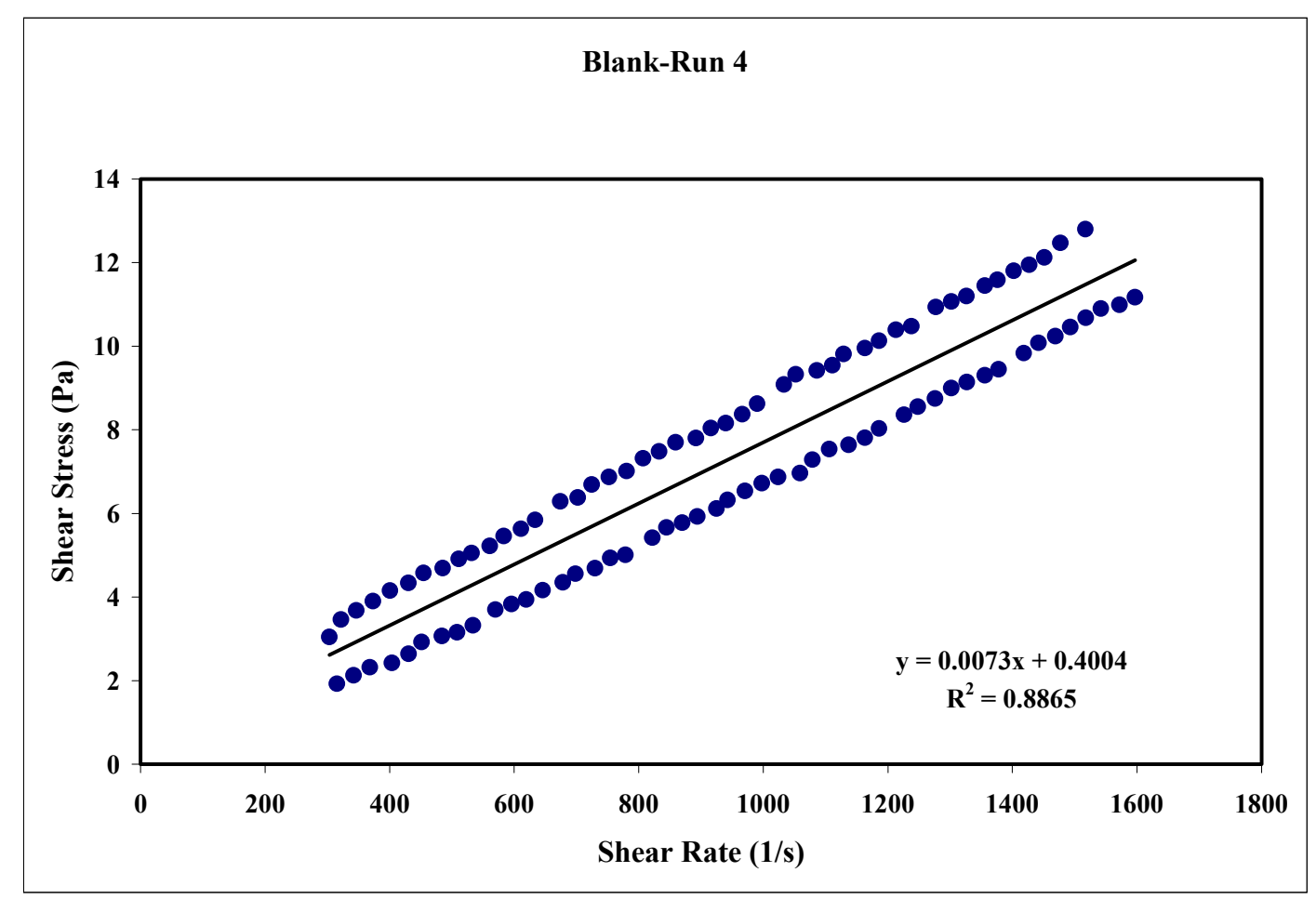

Figure 4. Blank at 10 wt\% Run 4 


\begin{tabular}{|ll|}
\hline APPENDIX I - PART 2 & WSRC-TR-2000-00352 \\
SR/TRU PRECIPITATE SLURRY RHEOGRAMS AT 10 WT \% & SRT-RPP-2000-00026 \\
\hline
\end{tabular}

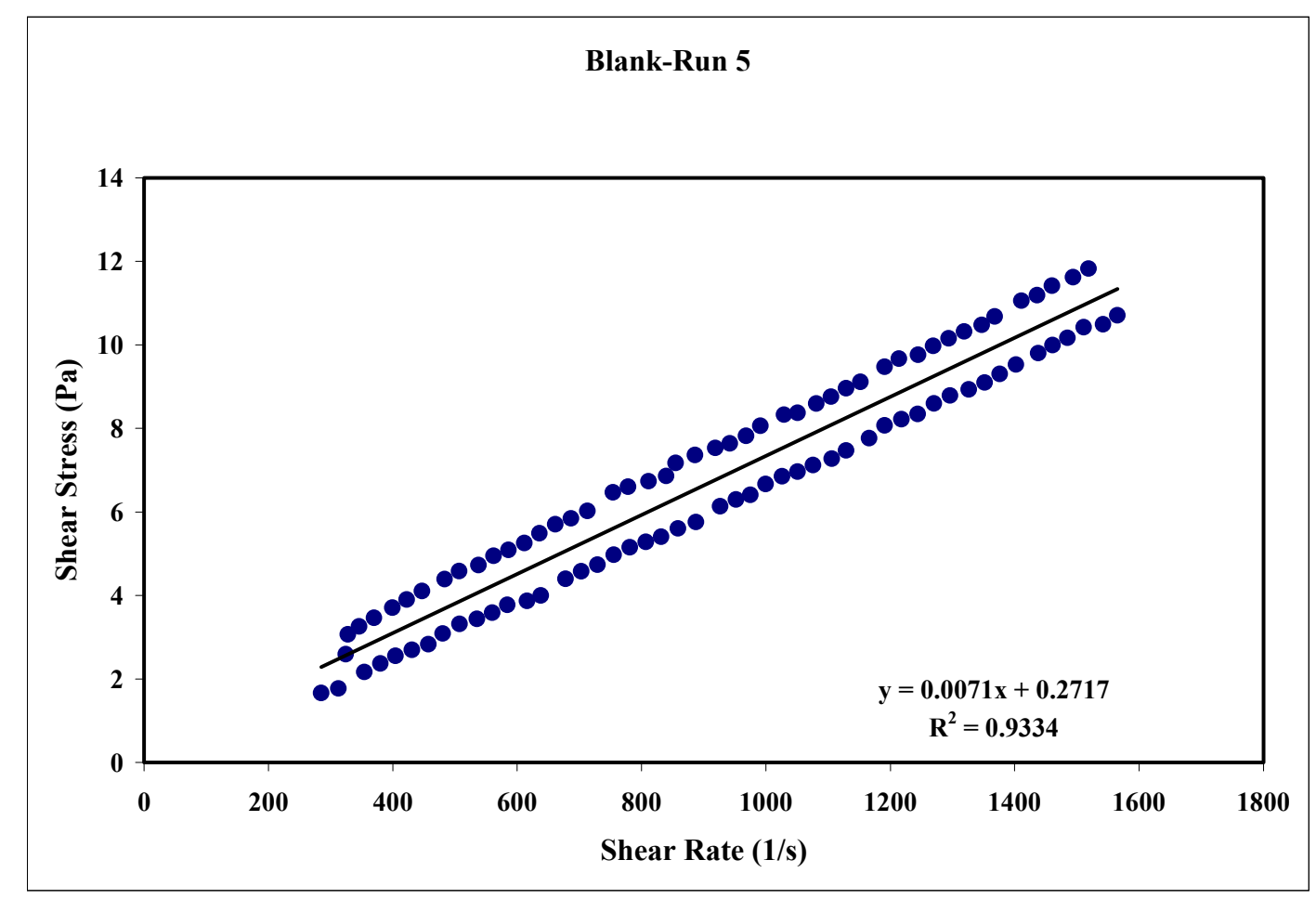

Figure 5. Blank at 10 wt\% Run 5

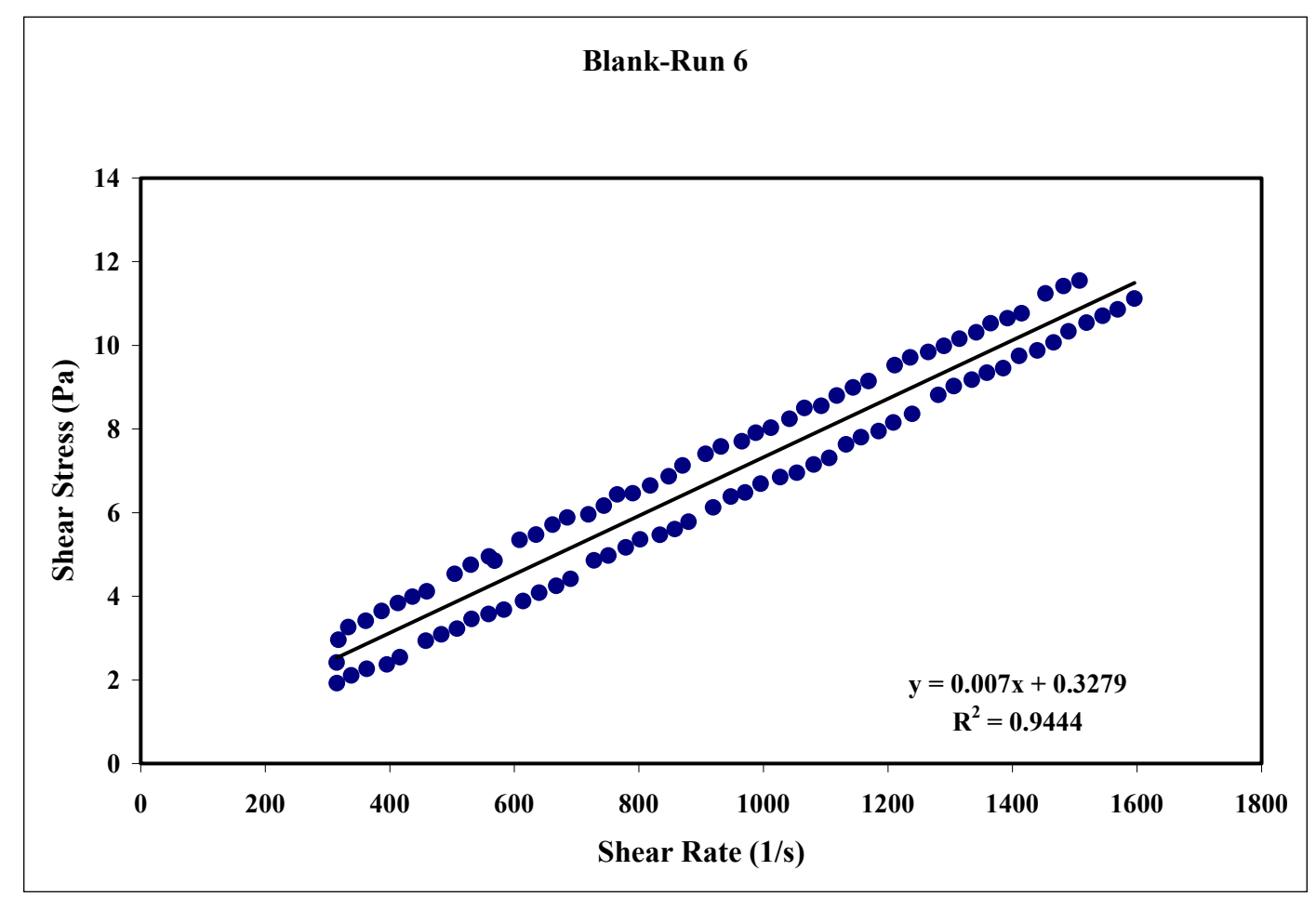

Figure 6. Blank at 10 wt\% Run 6 


\begin{tabular}{|ll|}
\hline APPENDIX I - PART 2 & WSRC-TR-2000-00352 \\
SR/TRU PRECIPITATE SLURRY RHEOGRAMS AT 10 WT \% & SRT-RPP-2000-00026 \\
\hline
\end{tabular}

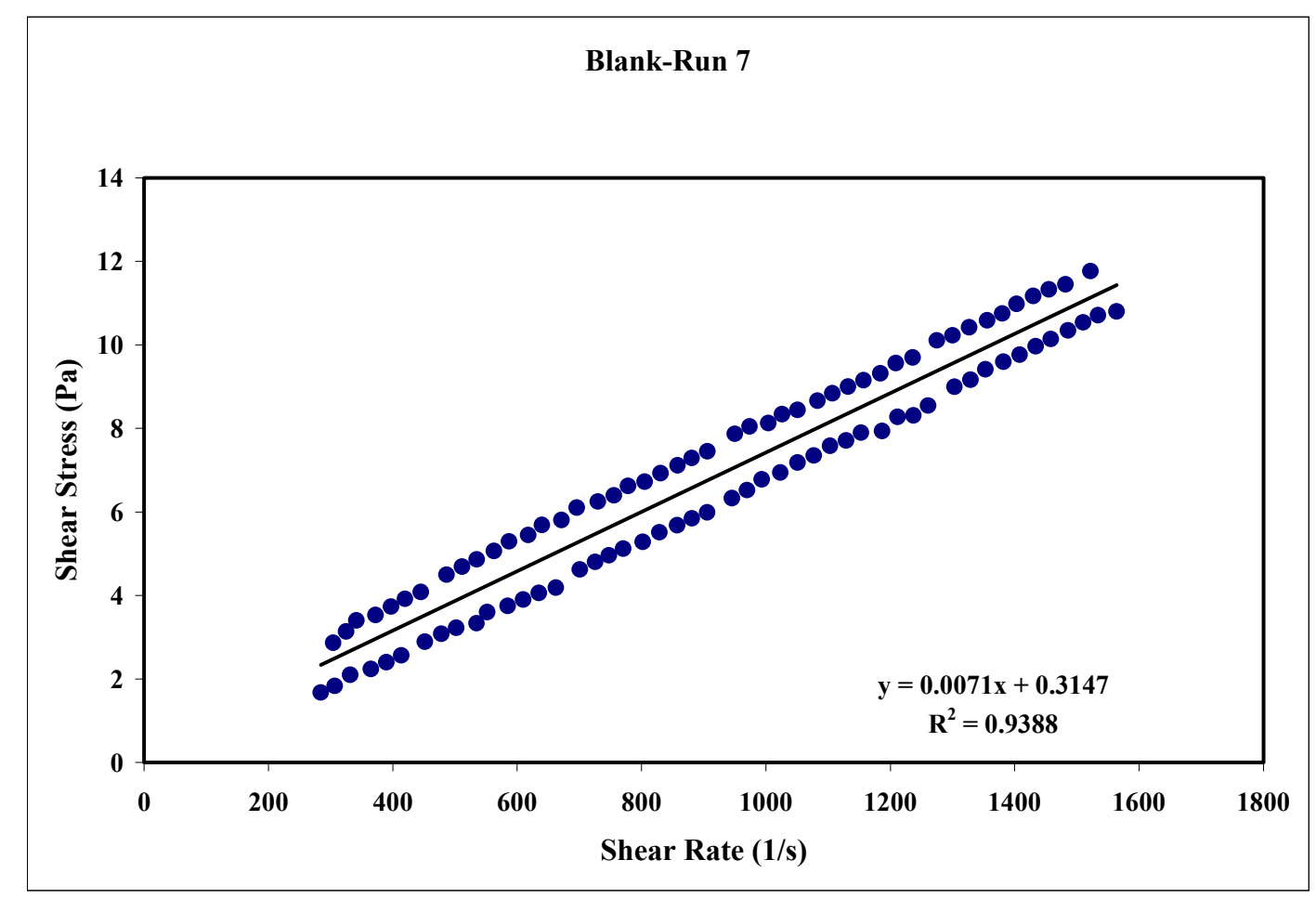

Figure 7. Blank at 10 wt\% Run 7

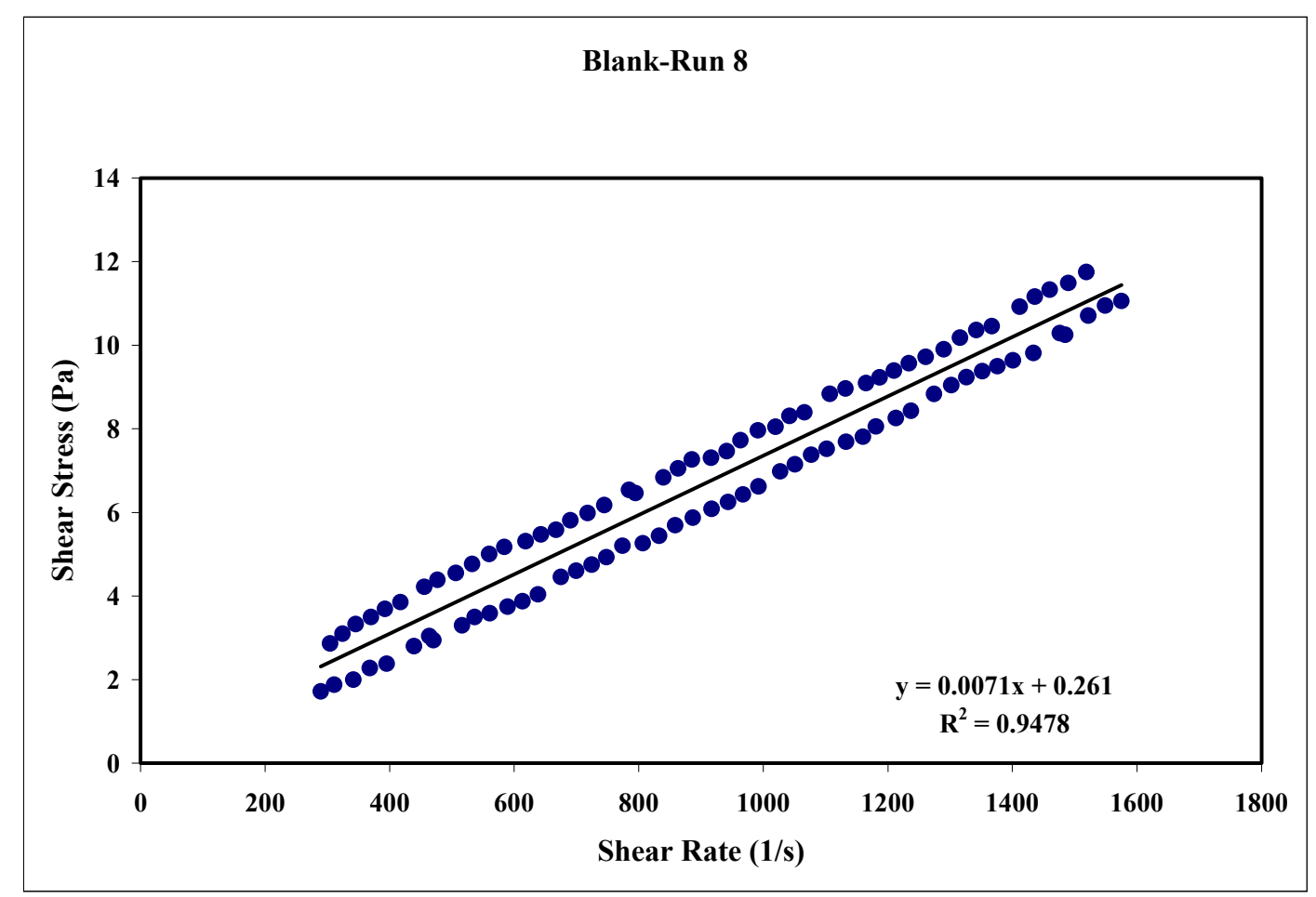

Figure 8. Blank at 10 wt\% Run 8 


\begin{tabular}{|ll|}
\hline APPENDIX I - PART 2 & WSRC-TR-2000-00352 \\
SR/TRU PRECIPITATE SLURRY RHEOGRAMS AT 10 WT \% & SRT-RPP-2000-00026 \\
\hline
\end{tabular}

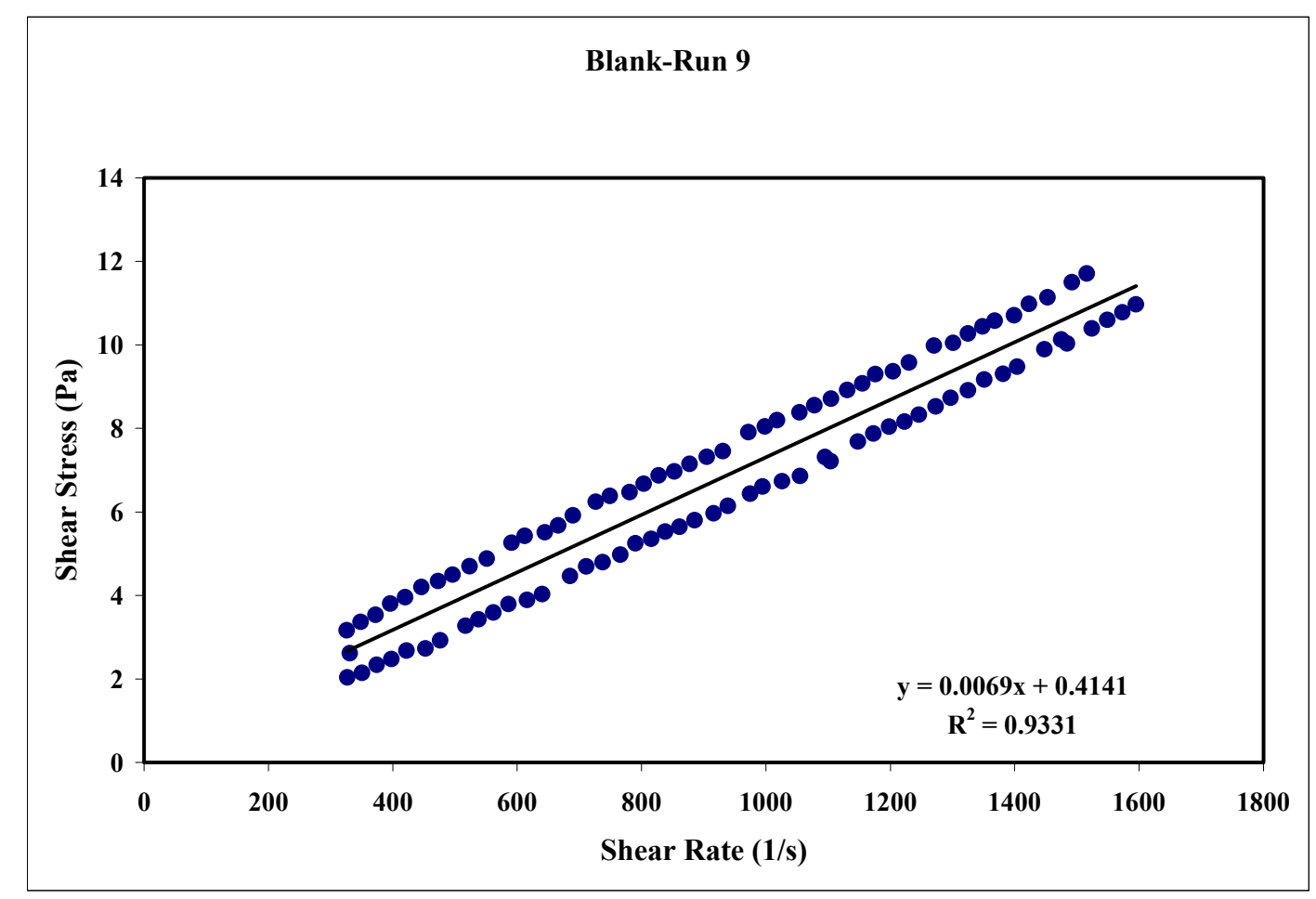

Figure 9. Blank at 10 wt\% Run 9

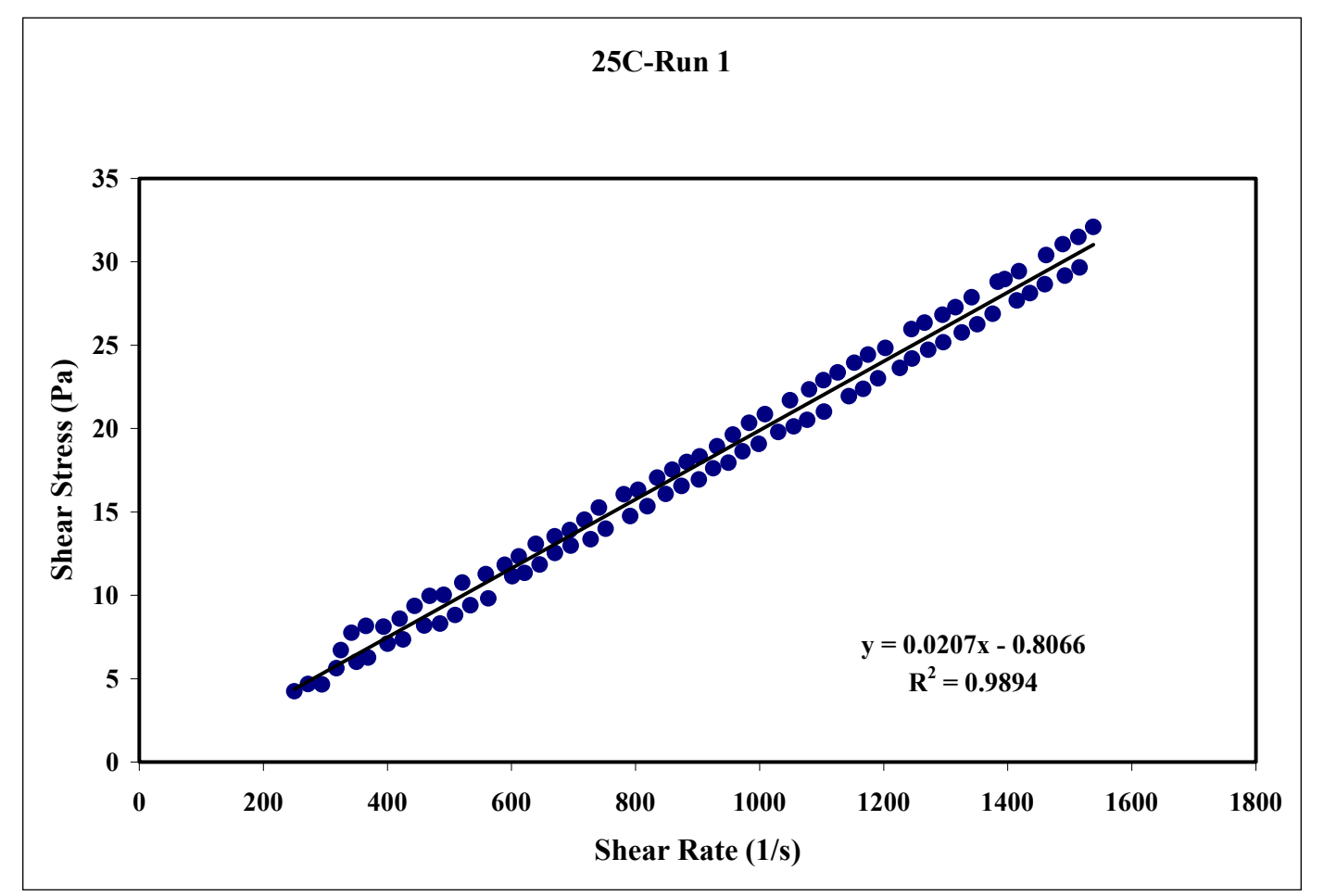

Figure 10. $25^{\circ} \mathrm{C}$ at $10 \mathrm{wt} \%$ Run 1 


\begin{tabular}{|ll|}
\hline APPENDIX I - PART 2 & WSRC-TR-2000-00352 \\
& SRT-RPP-2000-00026 \\
SR/TRU PRECIPITATE SLURRY RHEOGRAMS AT 10 WT \% & Page 378 of 514 \\
\hline
\end{tabular}

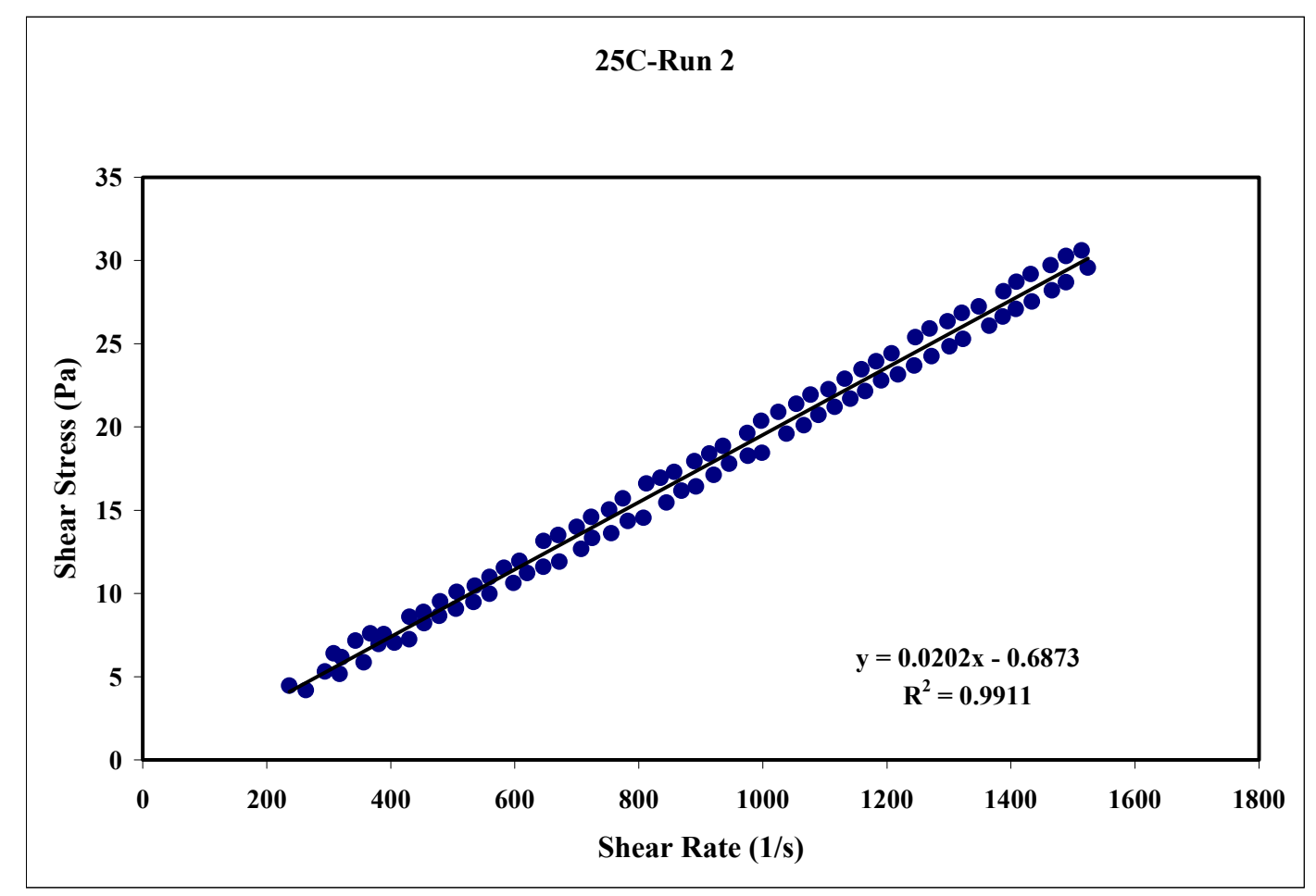

Figure 11. $25^{\circ} \mathrm{C}$ at 10 wt\% Run 2

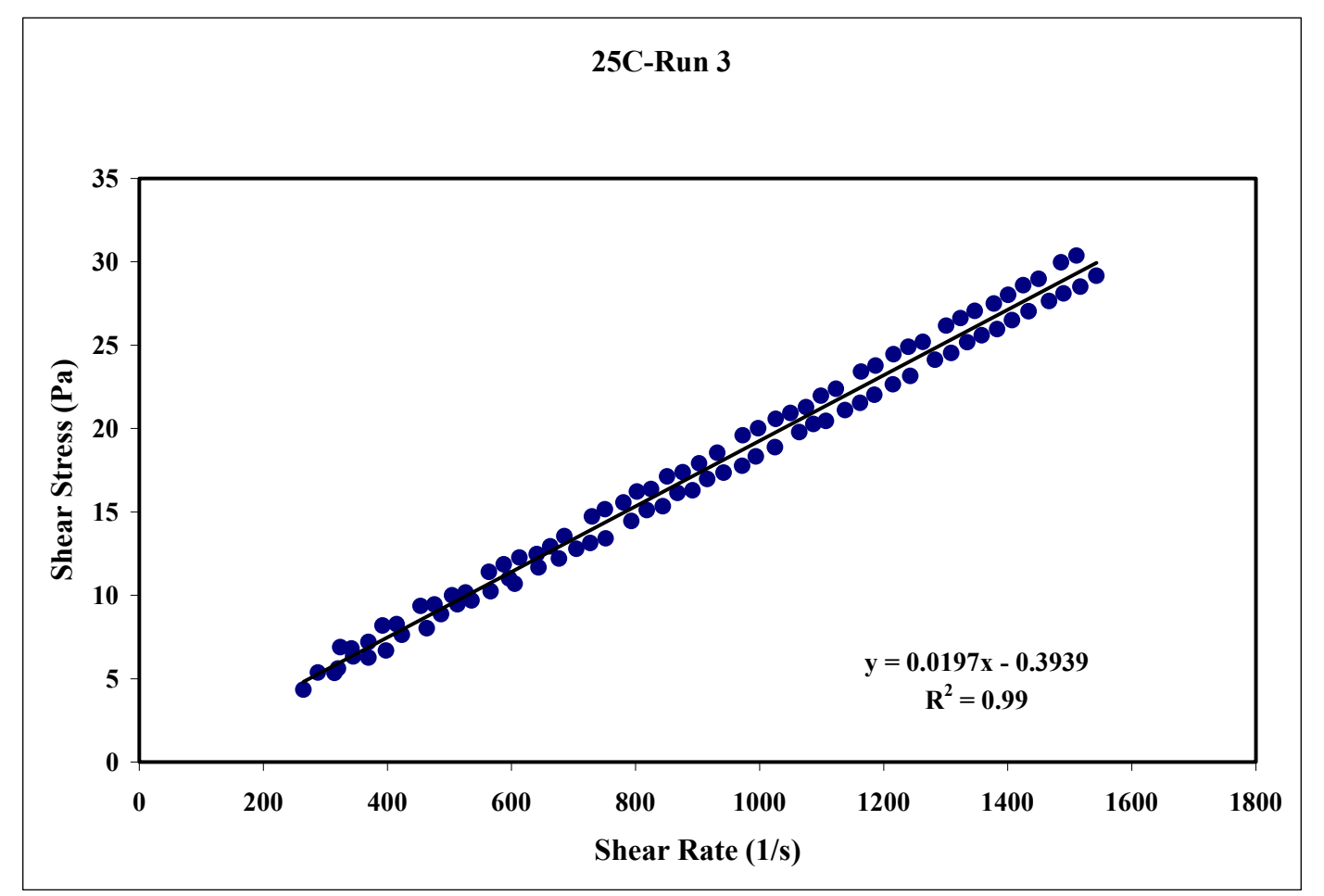

Figure 12. $25{ }^{\circ} \mathrm{C}$ at 10 wt\% Run 3 


\begin{tabular}{|ll|}
\hline APPENDIX I - PART 2 & WSRC-TR-2000-00352 \\
& SRT-RPP-2000-00026 \\
SR/TRU PRECIPITATE SLURRY RHEOGRAMS AT 10 WT \% & Page 379 of 514 \\
\hline
\end{tabular}

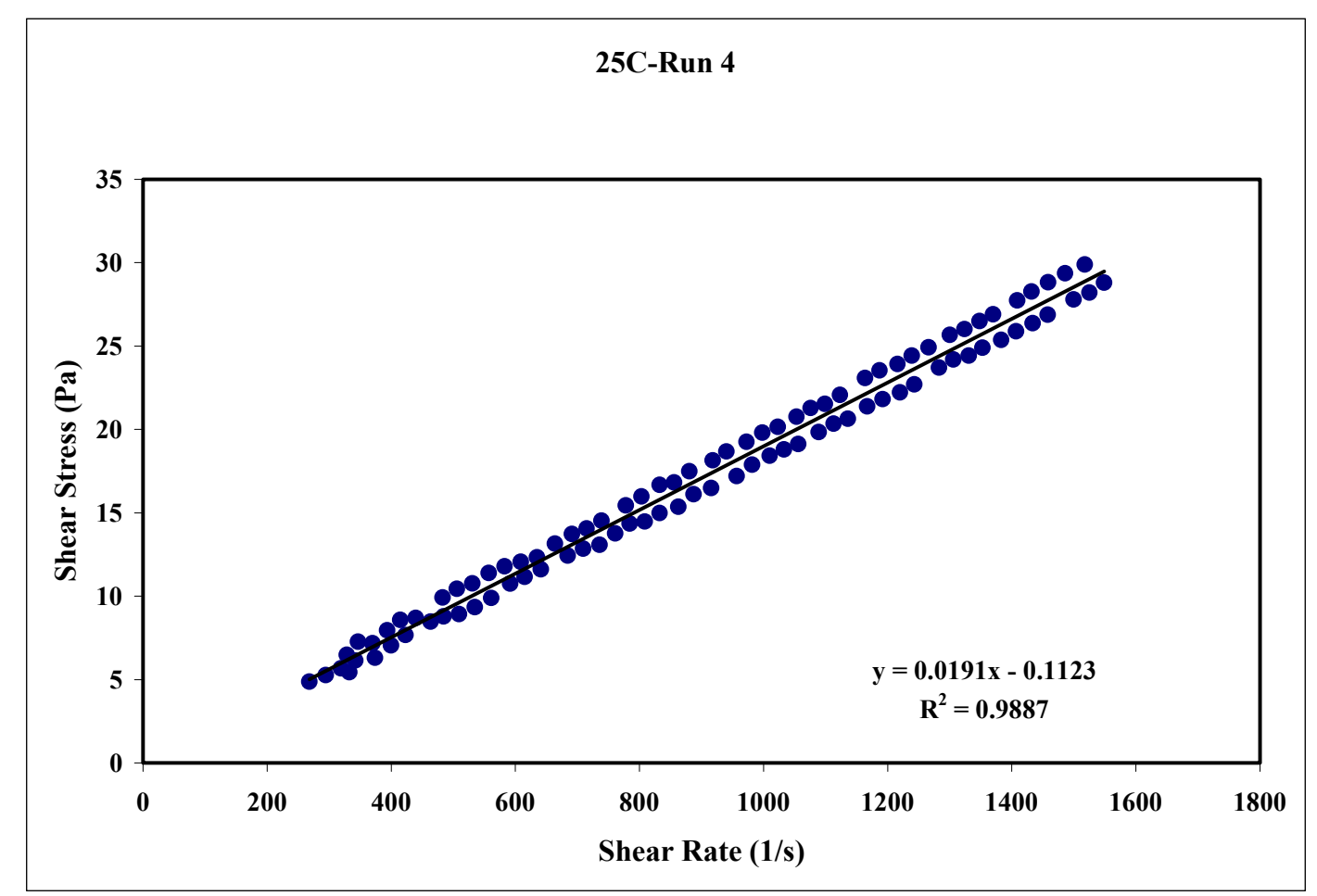

Figure 13. $25^{\circ} \mathrm{C}$ at 10 wt\% Run 4

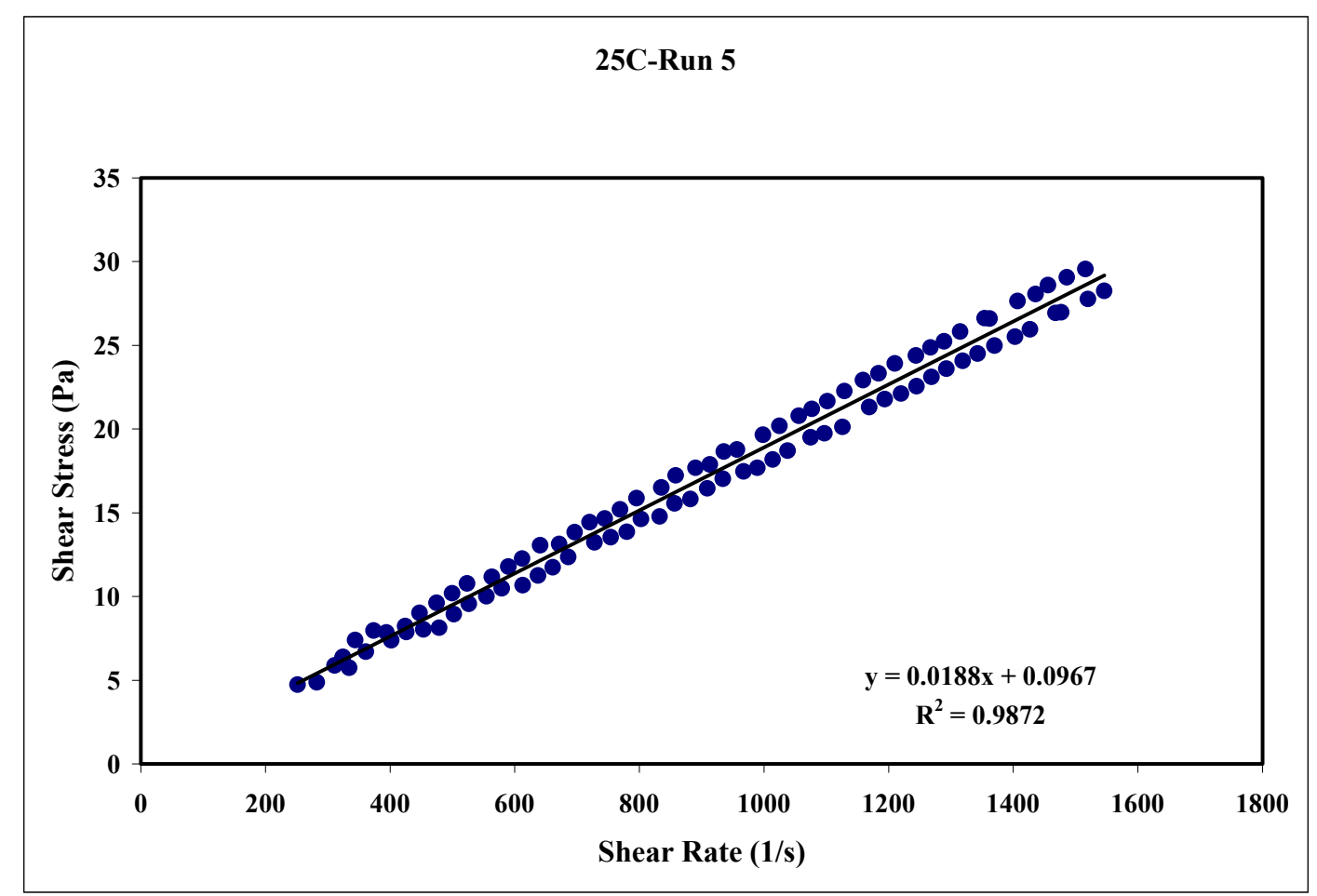

Figure 14. $25^{\circ} \mathrm{C}$ at 10 wt\% Run 5 


\begin{tabular}{|ll|}
\hline APPENDIX I - PART 2 & WSRC-TR-2000-00352 \\
& SRT-RPP-2000-00026 \\
SR/TRU PRECIPITATE SLURRY RHEOGRAMS AT 10 WT \% & Page 380 of 514 \\
\hline
\end{tabular}

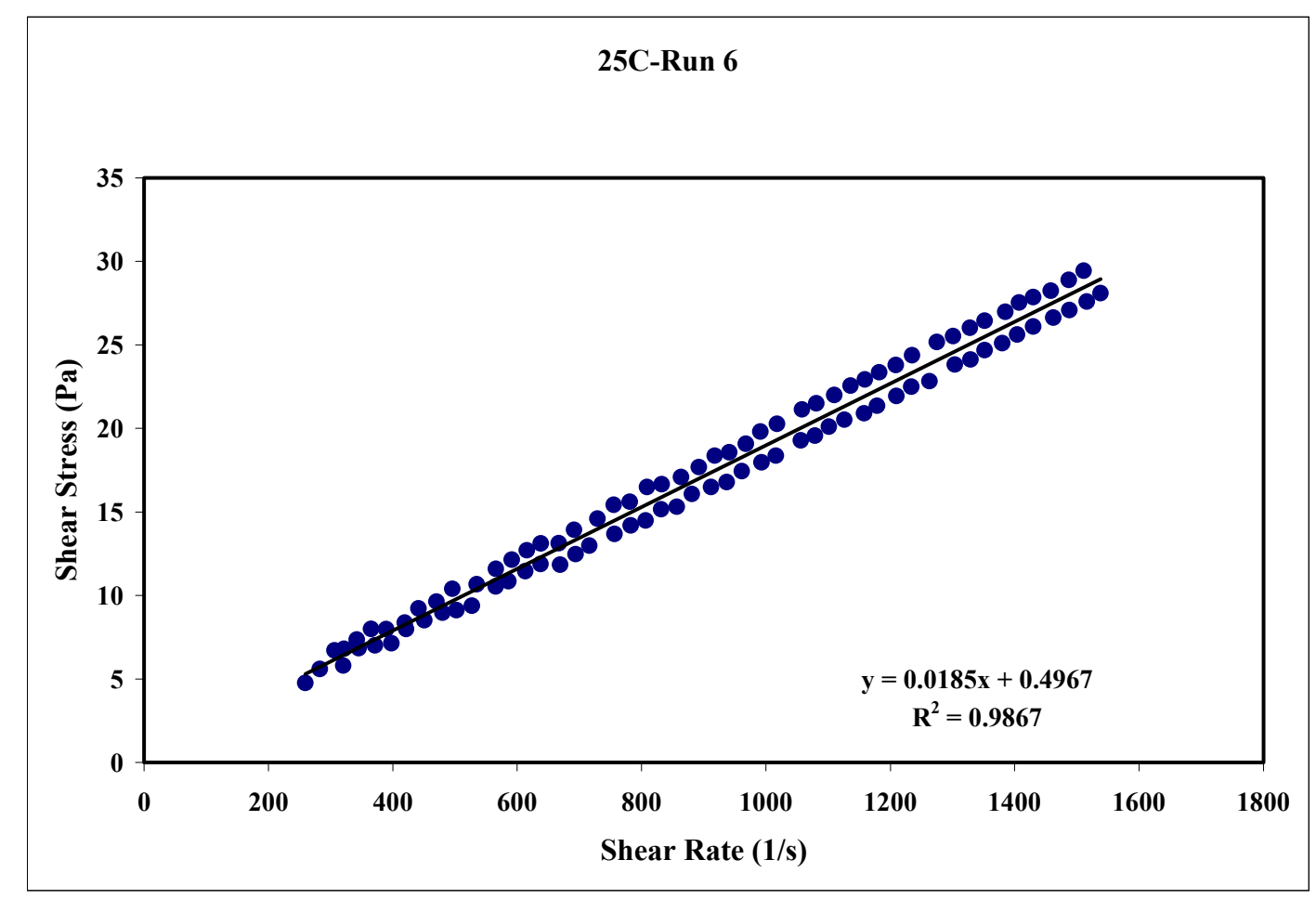

Figure 15. $25^{\circ} \mathrm{C}$ at 10 wt\% Run 6

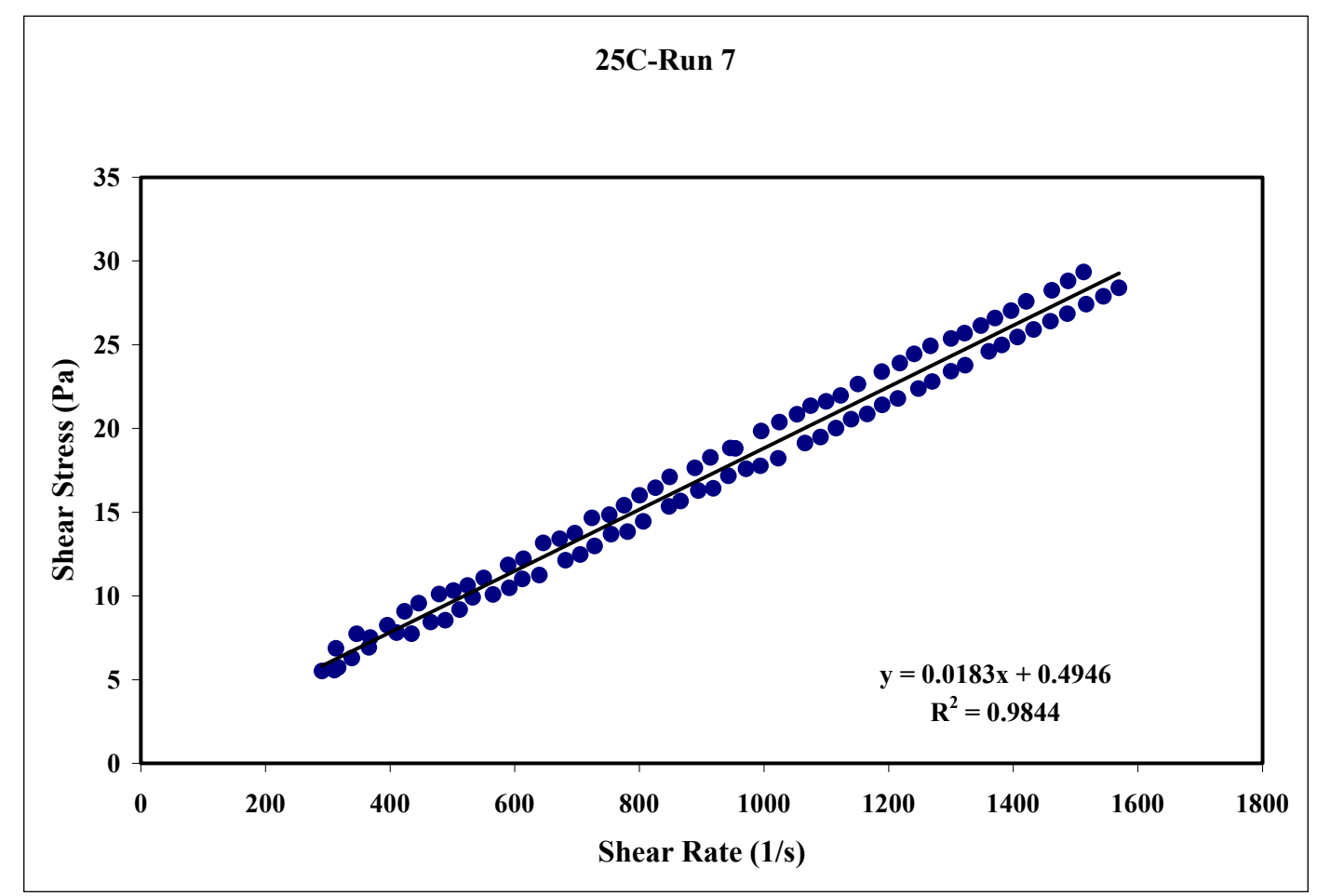

Figure 16. $25{ }^{\circ} \mathrm{C}$ at $10 \mathrm{wt} \%$ Run 7 


\begin{tabular}{|ll|}
\hline APPENDIX I - PART 2 & WSRC-TR-2000-00352 \\
& SRT-RPP-2000-00026 \\
SR/TRU PRECIPITATE SLURRY RHEOGRAMS AT 10 WT \% & Page 381 of 514 \\
\hline
\end{tabular}

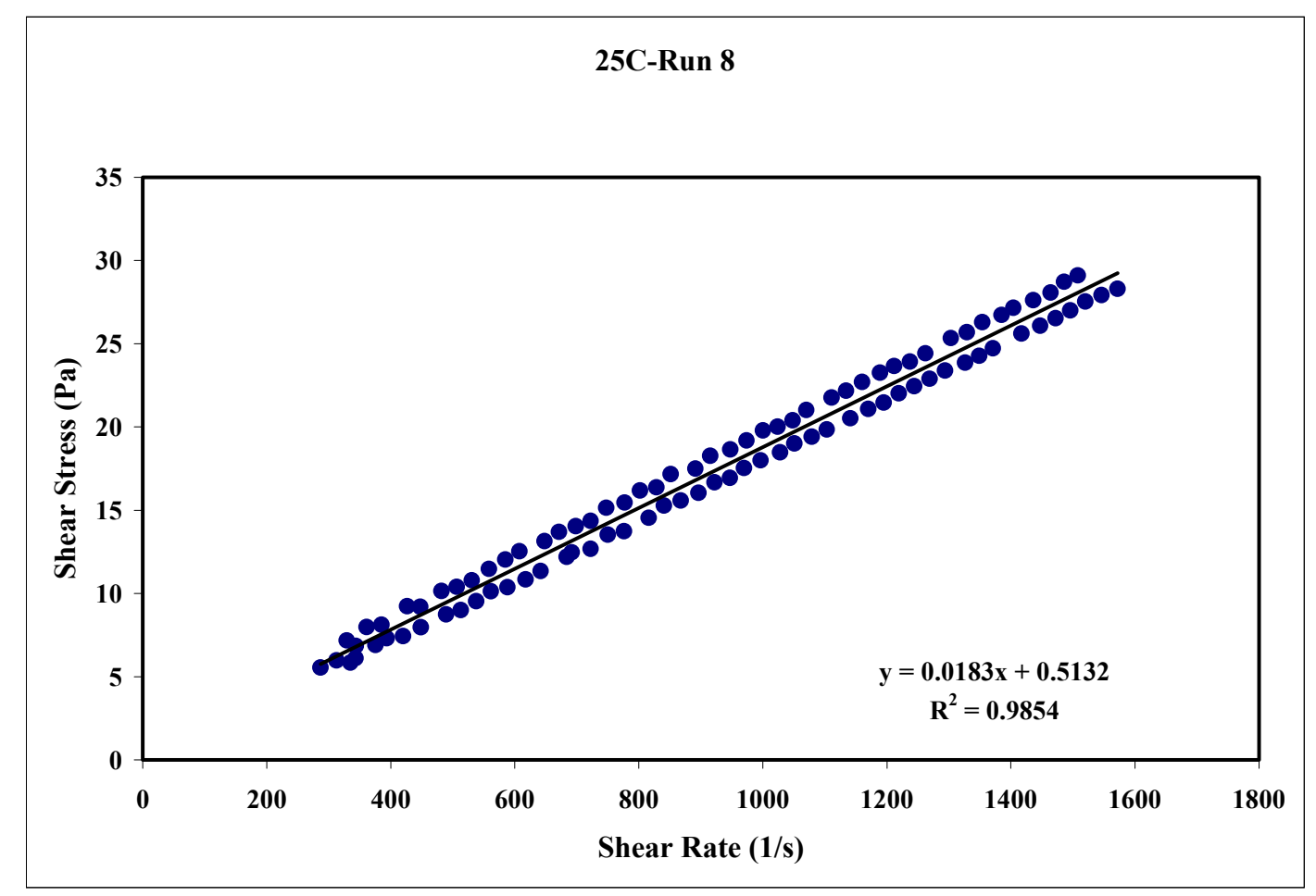

Figure 17. $25^{\circ} \mathrm{C}$ at 10 wt\% Run 8

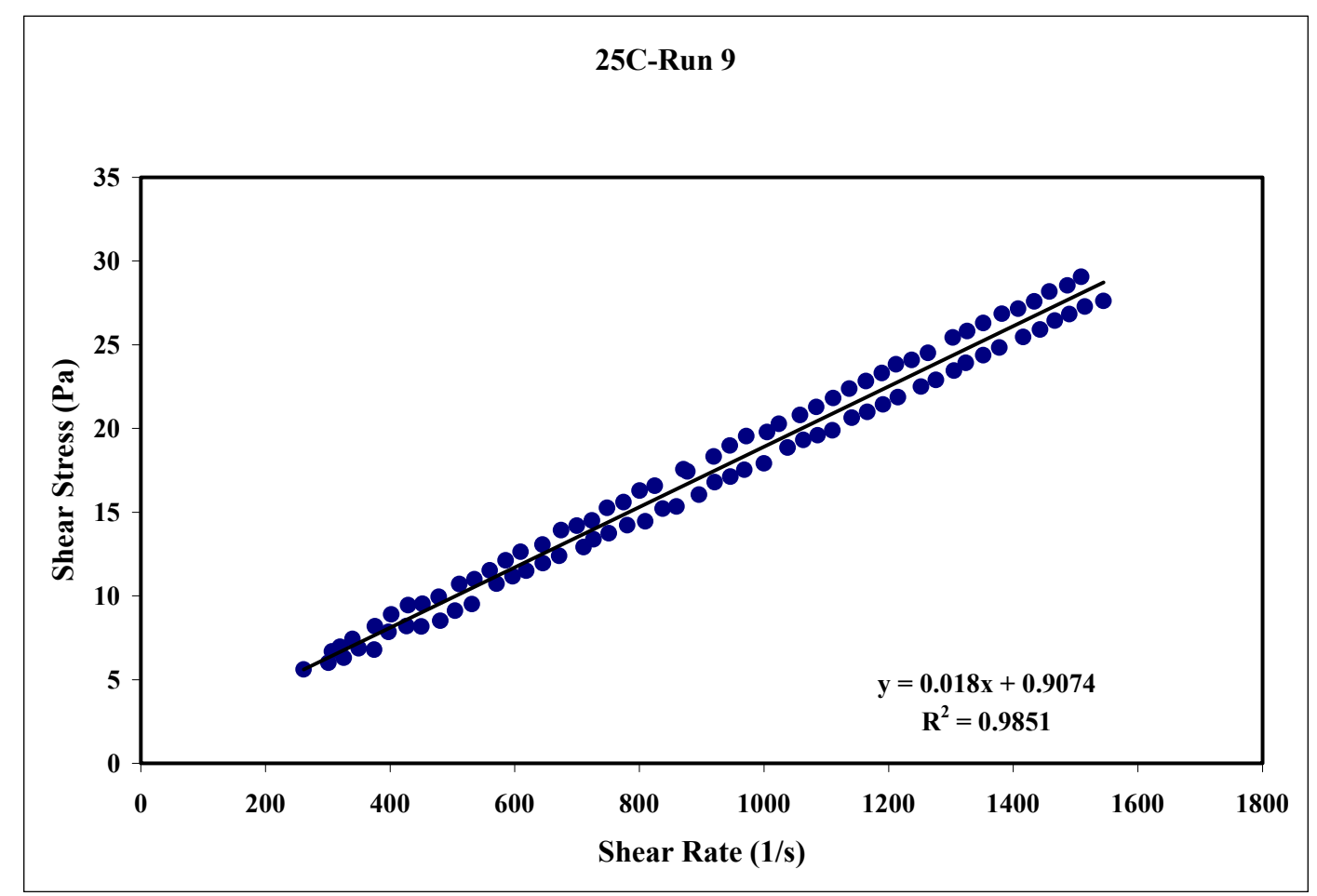

Figure 18. $25{ }^{\circ} \mathrm{C}$ at 10 wt\% Run 9 


\begin{tabular}{|ll|}
\hline APPENDIX I - PART 2 & WSRC-TR-2000-00352 \\
& SRT-RPP-2000-00026 \\
SR/TRU PRECIPITATE SLURRY RHEOGRAMS AT 10 WT \% & Page 382 of 514 \\
\hline
\end{tabular}

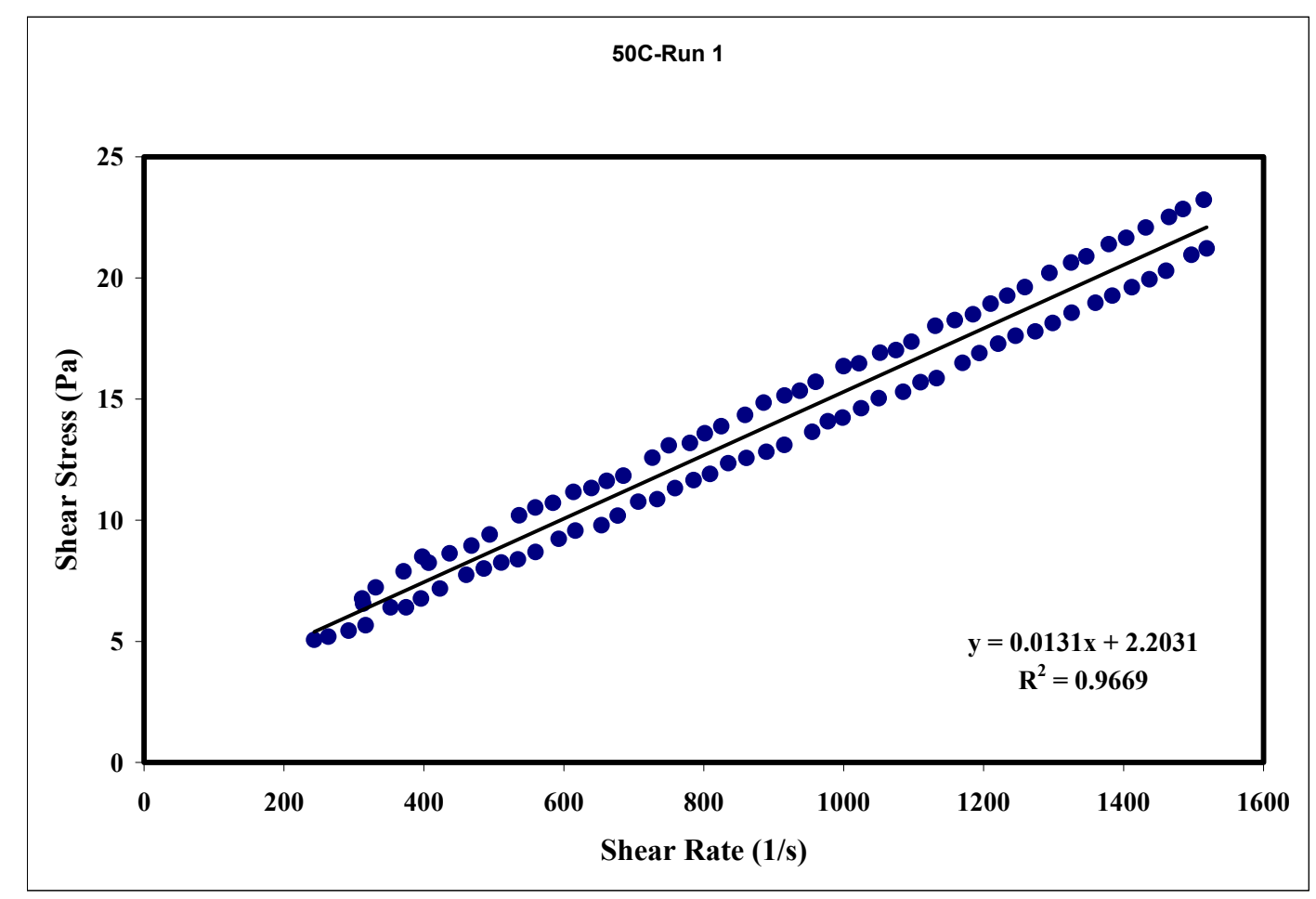

Figure 19. $50{ }^{\circ} \mathrm{C}$ at $10 \mathrm{wt} \%$ Run 1

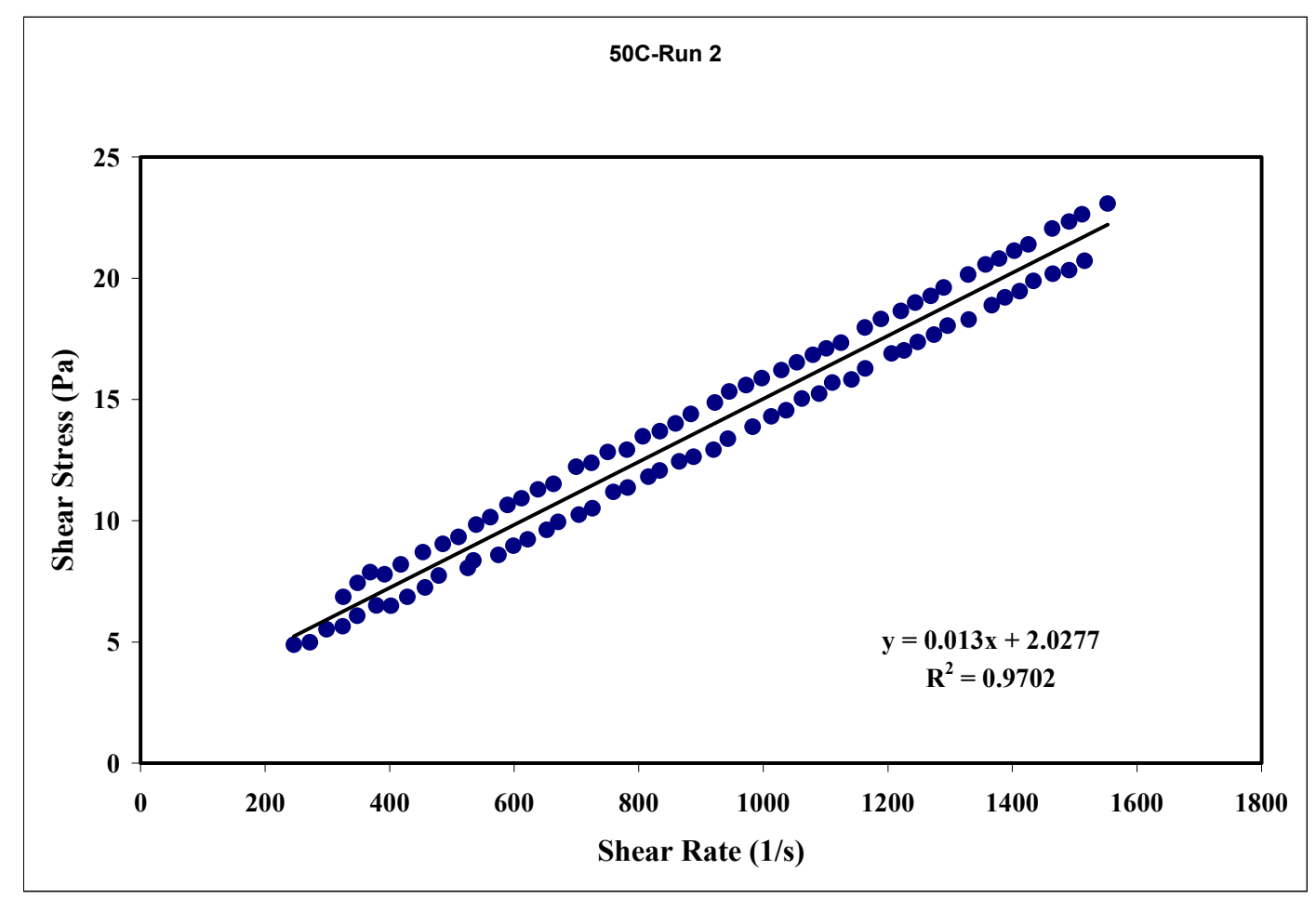

Figure 20. $50{ }^{\circ} \mathrm{C}$ at $10 \mathrm{wt} \%$ Run 2 


\begin{tabular}{|ll|}
\hline APPENDIX I - PART 2 & WSRC-TR-2000-00352 \\
& SRT-RPP-2000-00026 \\
SR/TRU PRECIPITATE SLURRY RHEOGRAMS AT 10 WT \% & Page 383 of 514 \\
\hline
\end{tabular}

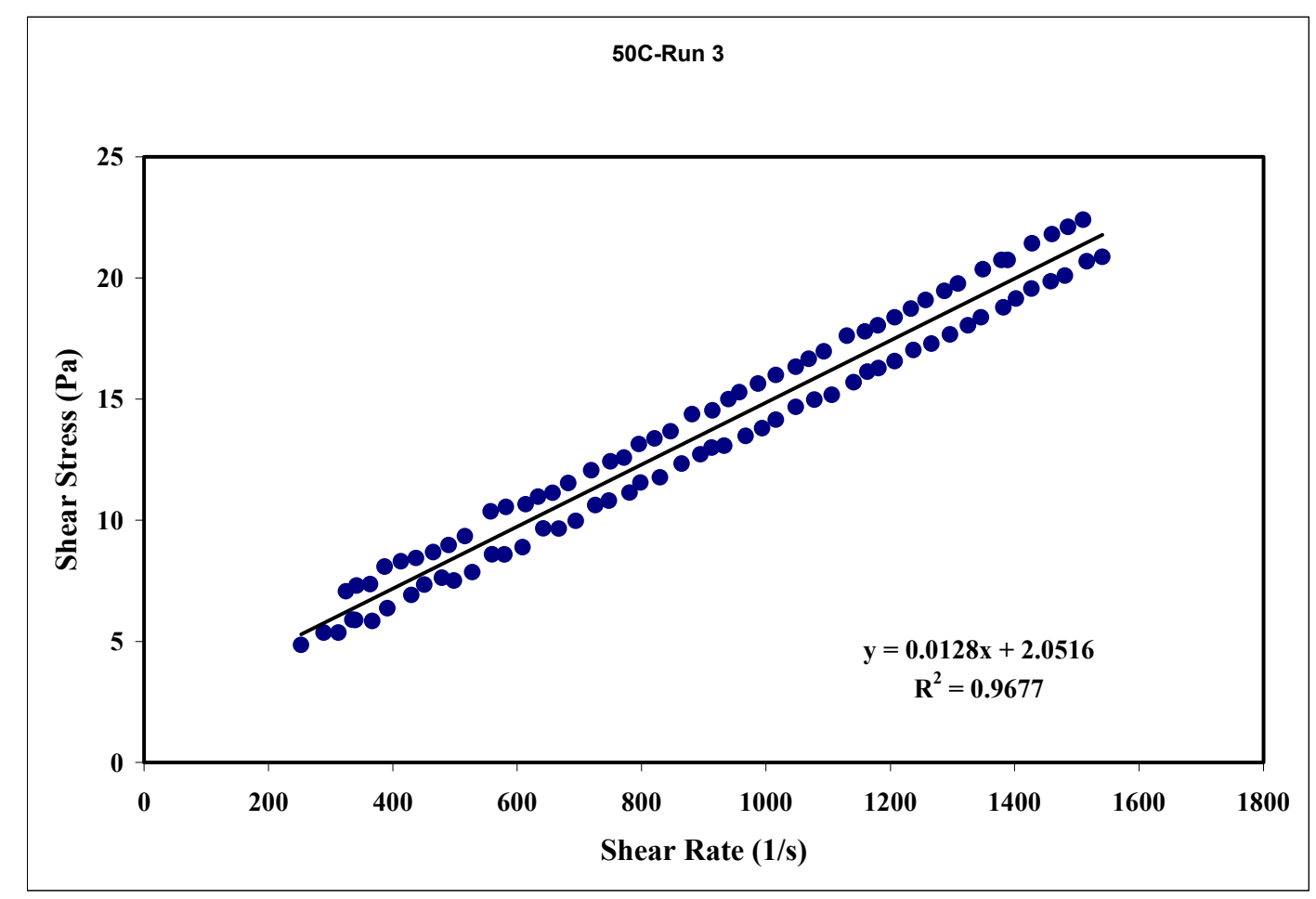

Figure 21. $50{ }^{\circ} \mathrm{C}$ at 10 wt $\%$ Run 3

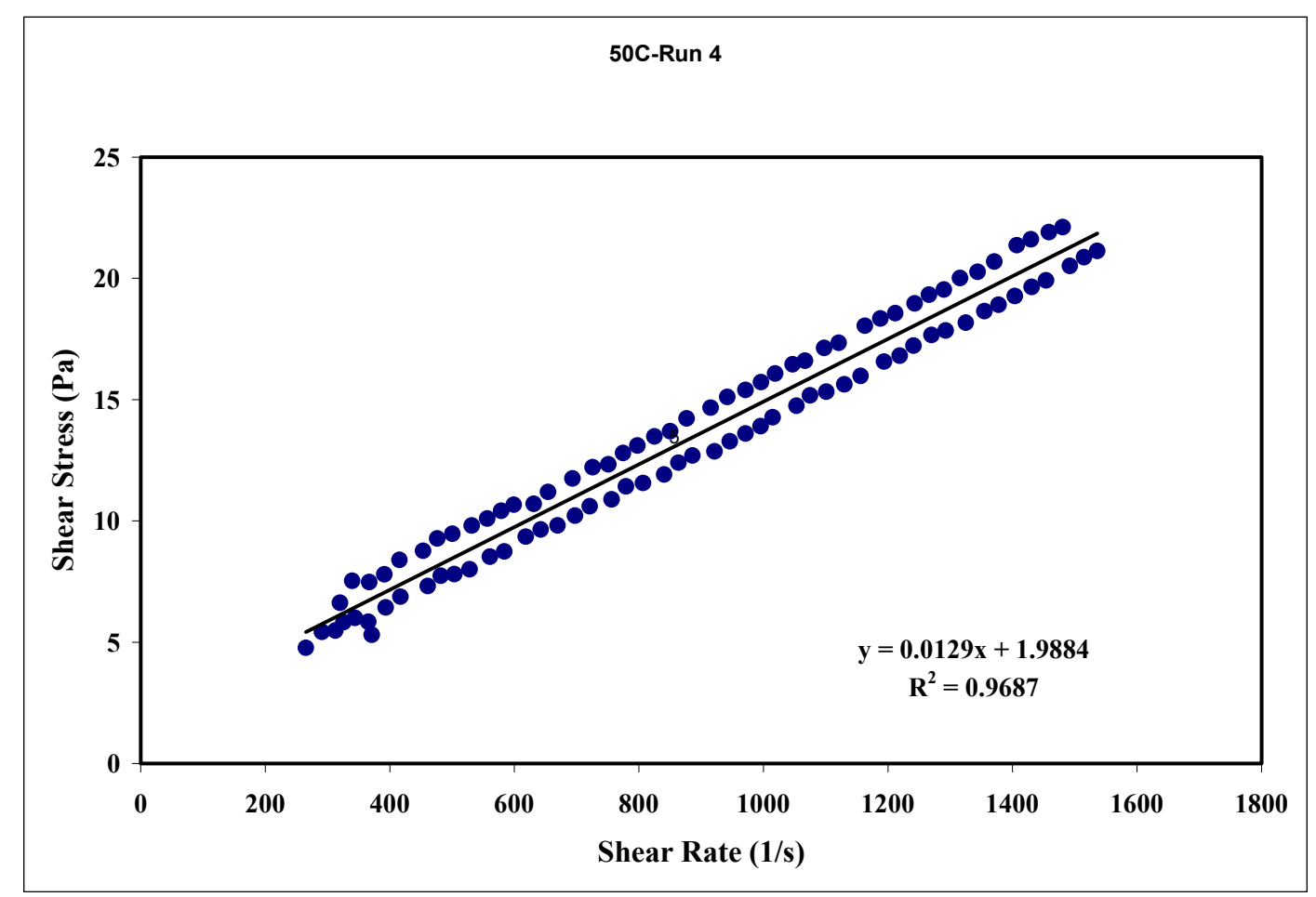

Figure 22. $50{ }^{\circ} \mathrm{C}$ at $10 \mathrm{wt} \%$ Run 4 


\begin{tabular}{|ll|}
\hline APPENDIX I - PART 2 & WSRC-TR-2000-00352 \\
& SRT-RPP-2000-00026 \\
SR/TRU PRECIPITATE SLURRY RHEOGRAMS AT 10 WT \% & Page 384 of 514 \\
\hline
\end{tabular}

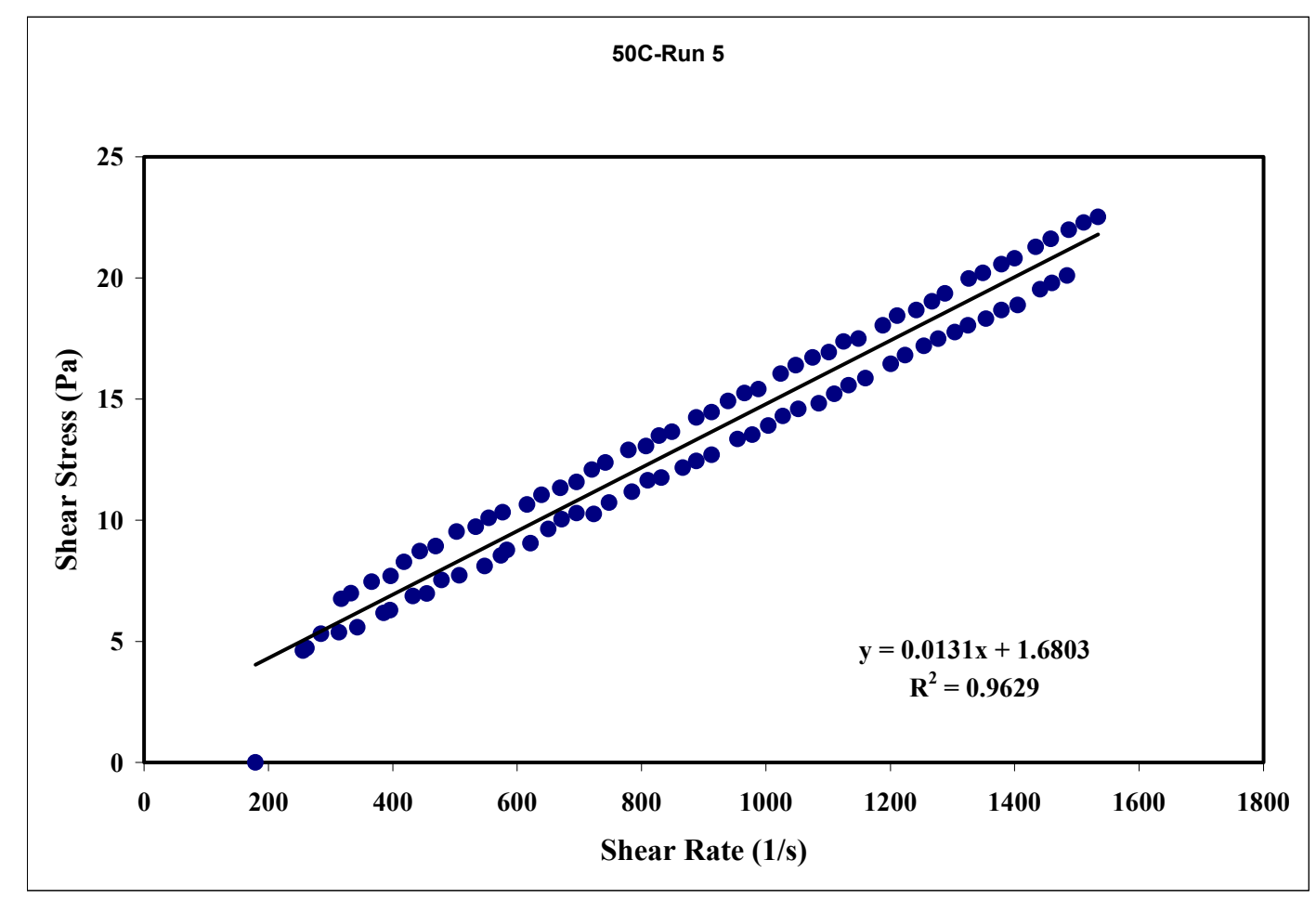

Figure 23. $50{ }^{\circ} \mathrm{C}$ at 10 wt\% Run 5

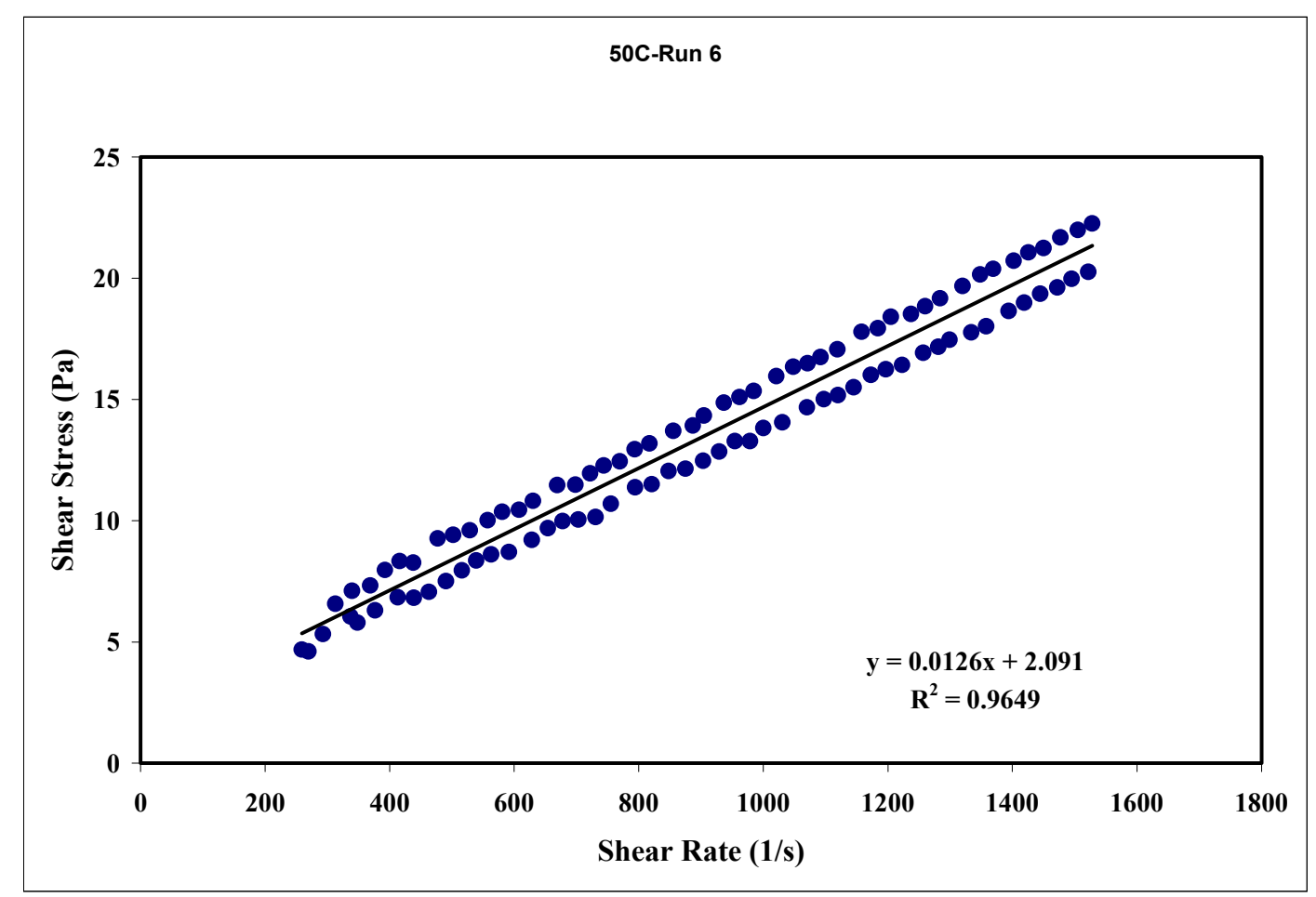

Figure 24. $50{ }^{\circ} \mathrm{C}$ at $10 \mathrm{wt} \%$ Run 6 


\begin{tabular}{|ll|}
\hline APPENDIX I - PART 2 & WSRC-TR-2000-00352 \\
& SRT-RPP-2000-00026 \\
SR/TRU PRECIPITATE SLURRY RHEOGRAMS AT 10 WT \% & Page 385 of 514 \\
\hline
\end{tabular}

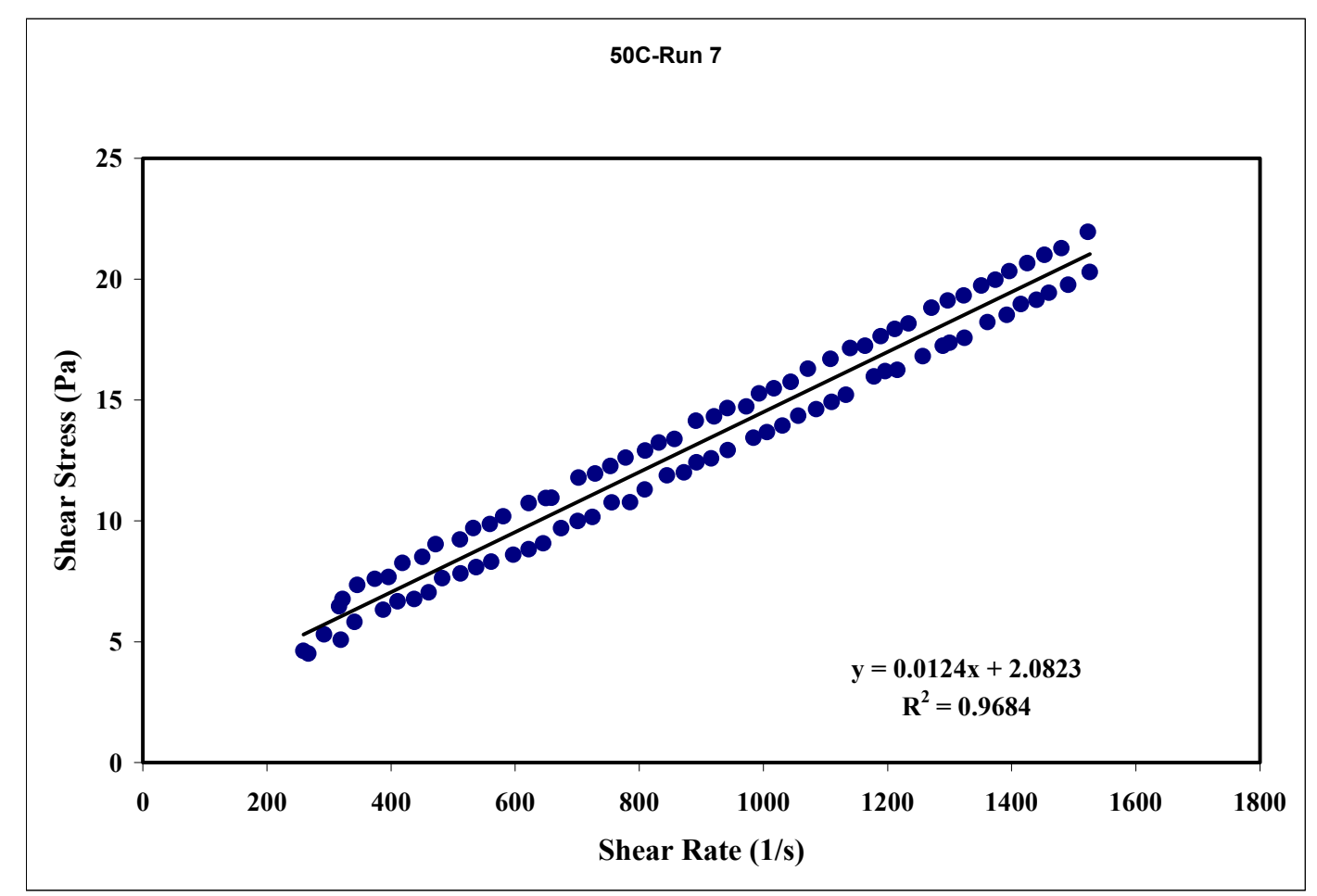

Figure 25. $50{ }^{\circ} \mathrm{C}$ at $10 \mathrm{wt} \%$ Run 7

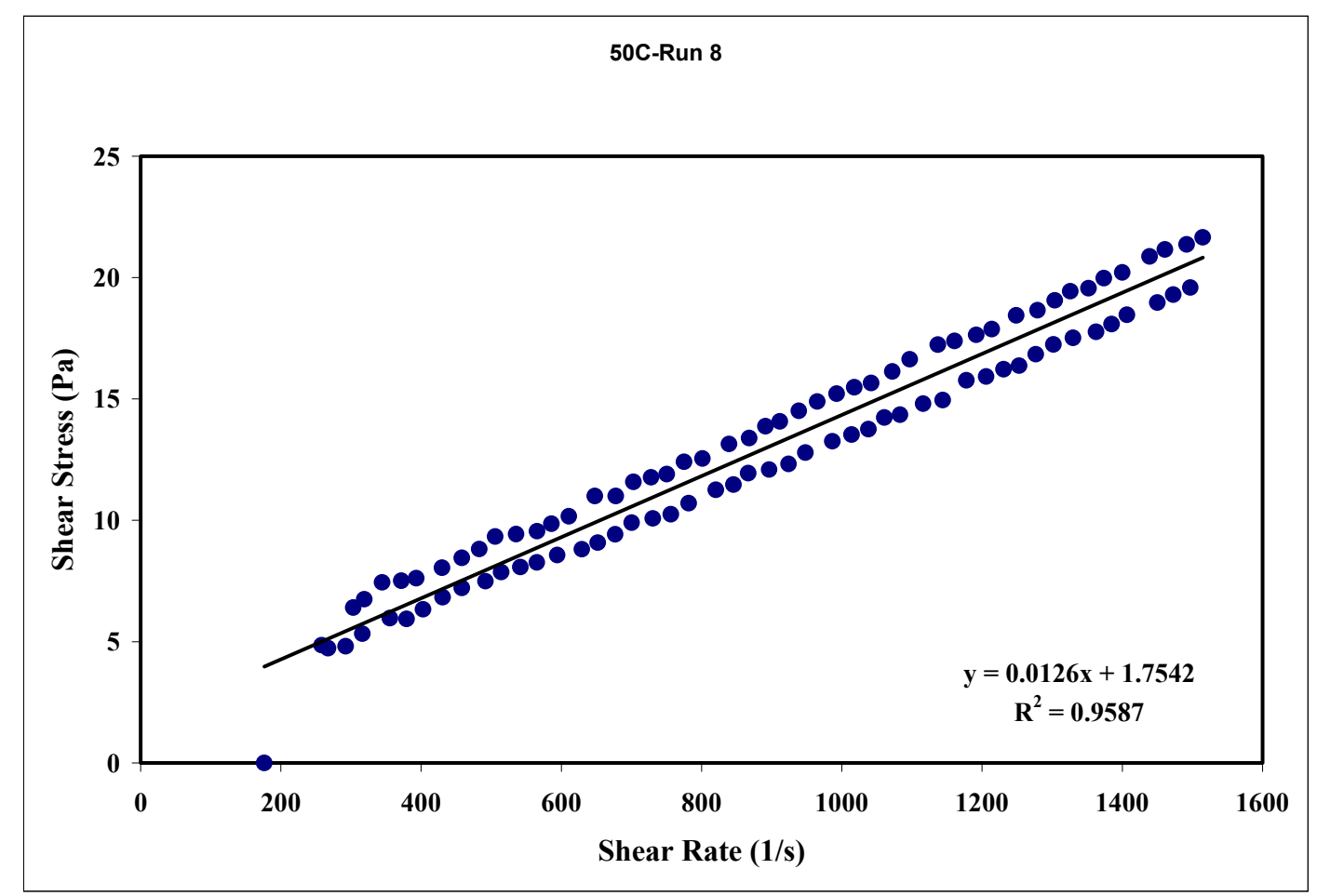

Figure 26. $50{ }^{\circ} \mathrm{C}$ at $10 \mathrm{wt} \%$ Run 8 


\begin{tabular}{|ll|}
\hline APPENDIX I - PART 2 & WSRC-TR-2000-00352 \\
& SRT-RPP-2000-00026 \\
SR/TRU PRECIPITATE SLURRY RHEOGRAMS AT 10 WT \% & Page 386 of 514 \\
\hline
\end{tabular}

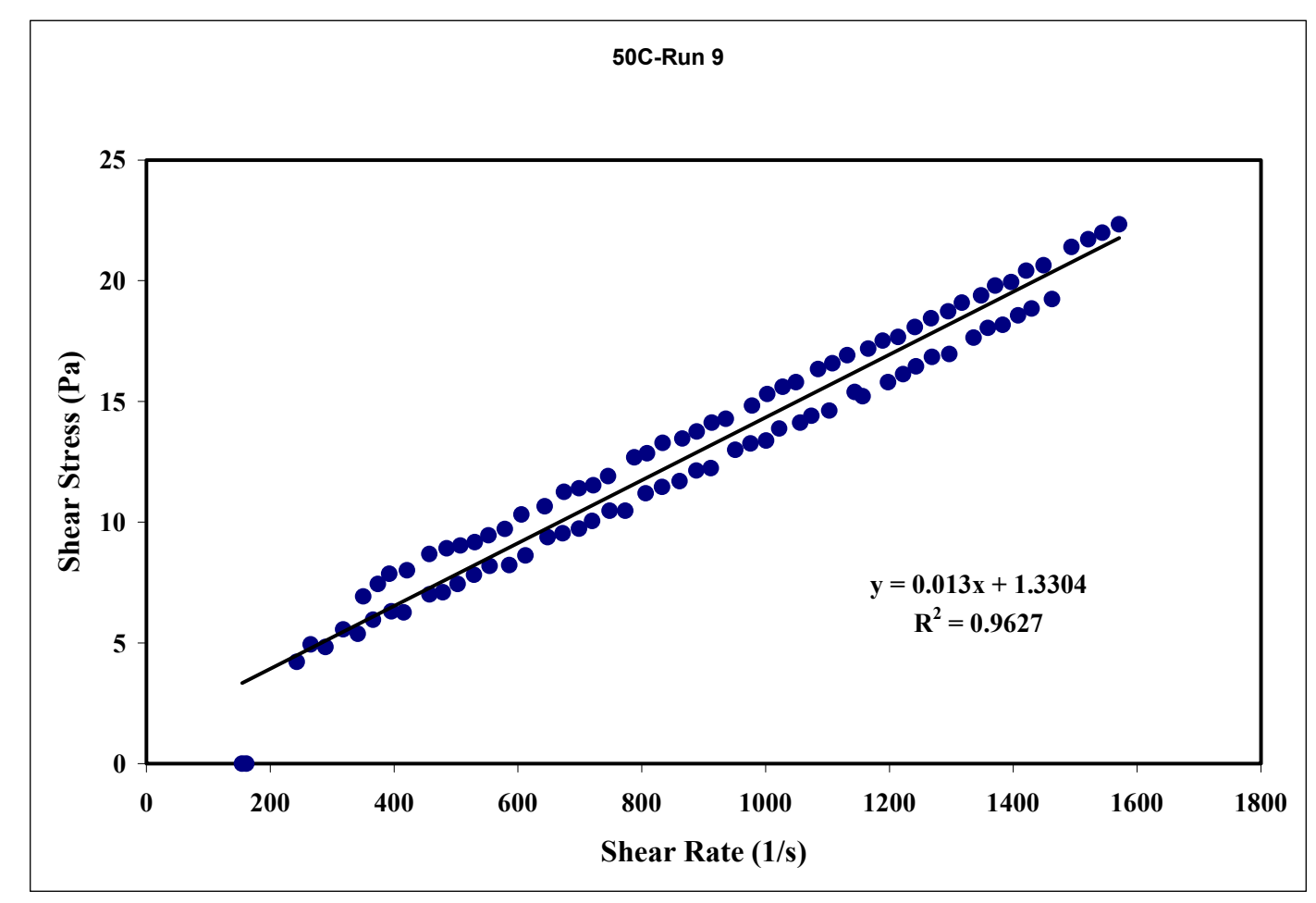

Figure 27. $50{ }^{\circ} \mathrm{C}$ at $10 \mathrm{wt} \%$ Run 9 


\section{APPENDIX I - PART 3}

\section{SR/TRU PRECIPITATE SLURRY RHEOGRAMS AT 13 WT \%}

Figure 1. Blank at $13 \mathrm{wt} \%$ Run 1

Figure 2. Blank at $13 \mathrm{wt} \%$ Run 2

Figure 3. Blank at $13 \mathrm{wt} \%$ Run 3

Figure 4. Blank at $13 \mathrm{wt} \%$ Run 4

Figure 5. Blank at $13 \mathrm{wt} \%$ Run 5

Figure 6. Blank at $13 \mathrm{wt} \%$ Run 6.

Figure 7. Blank at $13 \mathrm{wt} \%$ Run 7

Figure 8. Blank at $13 \mathrm{wt} \%$ Run 8

Figure 9. Blank at $13 \mathrm{wt} \%$ Run 9

Figure $10 . \quad 10{ }^{\circ} \mathrm{C}$ at $13 \mathrm{wt} \%$ Run 1

Figure $11.10{ }^{\circ} \mathrm{C}$ at $13 \mathrm{wt} \%$ Run 2

Figure $12 . \quad 10^{\circ} \mathrm{C}$ at $13 \mathrm{wt} \%$ Run 3

Figure $13.10^{\circ} \mathrm{C}$ at $13 \mathrm{wt} \%$ Run 4

Figure $14 . \quad 10^{\circ} \mathrm{C}$ at $13 \mathrm{wt} \%$ Run 5

Figure $15 . \quad 10^{\circ} \mathrm{C}$ at $13 \mathrm{wt} \%$ Run 6

Figure $16 . \quad 10^{\circ} \mathrm{C}$ at $13 \mathrm{wt} \%$ Run 7

Figure $17.10^{\circ} \mathrm{C}$ at $13 \mathrm{wt} \%$ Run 8

Figure $18 . \quad 10{ }^{\circ} \mathrm{C}$ at $13 \mathrm{wt} \%$ Run 9

Figure $19 . \quad 15^{\circ} \mathrm{C}$ at $13 \mathrm{wt} \%$ Run 1

Figure $20.15^{\circ} \mathrm{C}$ at $13 \mathrm{wt} \%$ Run 2

Figure $21.15^{\circ} \mathrm{C}$ at $13 \mathrm{wt} \%$ Run 3

Figure $22.15^{\circ} \mathrm{C}$ at $13 \mathrm{wt} \%$ Run 4

Figure $23.15^{\circ} \mathrm{C}$ at $13 \mathrm{wt} \%$ Run 5

Figure $24 . \quad 15^{\circ} \mathrm{C}$ at $13 \mathrm{wt} \%$ Run 6

Figure $25.15^{\circ} \mathrm{C}$ at $13 \mathrm{wt} \%$ Run 7

Figure 26. $15^{\circ} \mathrm{C}$ at $13 \mathrm{wt} \%$ Run 8

Figure $27.15^{\circ} \mathrm{C}$ at $13 \mathrm{wt} \%$ Run 9

Figure $28 . \quad 25^{\circ} \mathrm{C}$ at $13 \mathrm{wt} \%$ Run 1

Figure $29.25^{\circ} \mathrm{C}$ at $13 \mathrm{wt} \%$ Run 2

Figure $30 . \quad 25^{\circ} \mathrm{C}$ at $13 \mathrm{wt} \%$ Run 3

Figure $31.25^{\circ} \mathrm{C}$ at $13 \mathrm{wt} \%$ Run 4

Figure $32.25^{\circ} \mathrm{C}$ at $13 \mathrm{wt} \%$ Run 5

Figure 33. $25^{\circ} \mathrm{C}$ at $13 \mathrm{wt} \%$ Run 6

Figure $34.25^{\circ} \mathrm{C}$ at $13 \mathrm{wt} \%$ Run 7

Figure $35.25^{\circ} \mathrm{C}$ at $13 \mathrm{wt} \%$ Run 8

Figure $36.25^{\circ} \mathrm{C}$ at $13 \mathrm{wt} \%$ Run 9 


\begin{tabular}{|ll|}
\hline APPENDIX I - PART 3 & WSRC-TR-2000-00352 \\
& SRT-RPP-2000-00026 \\
SR/TRU PRECIPITATE SLURRY RHEOGRAMS AT 13 WT \% & Page 388 of 514 \\
\hline
\end{tabular}

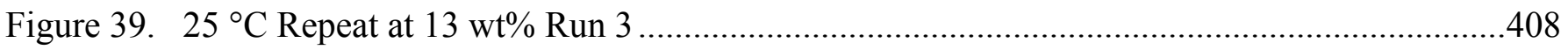

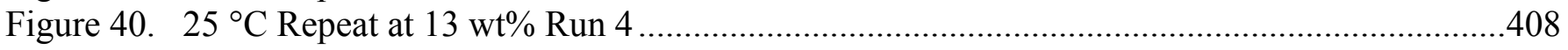

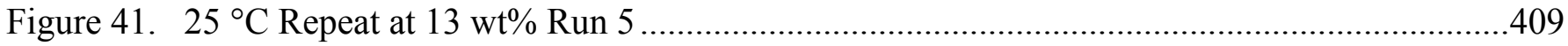

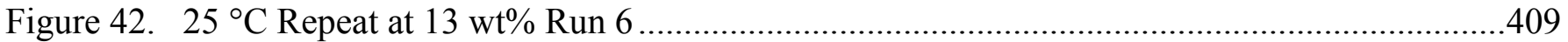

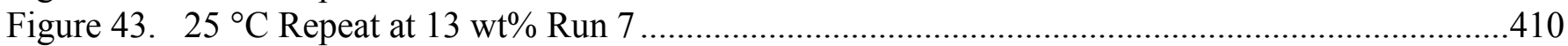

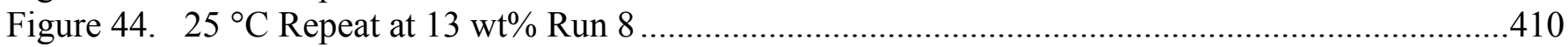

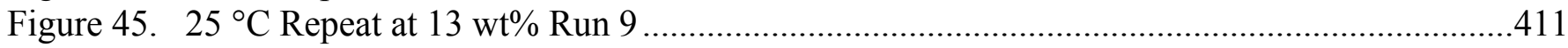

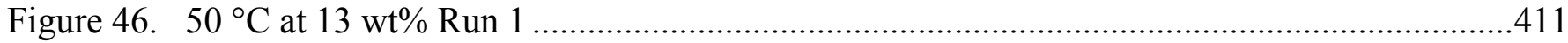

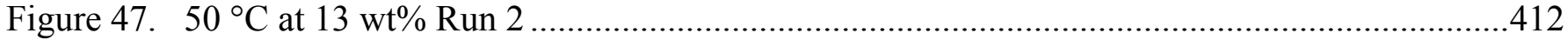

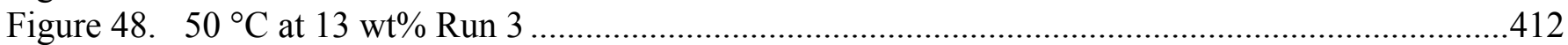

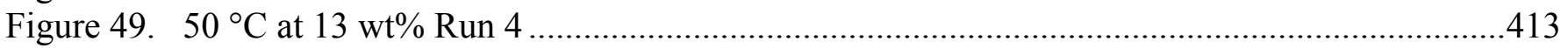

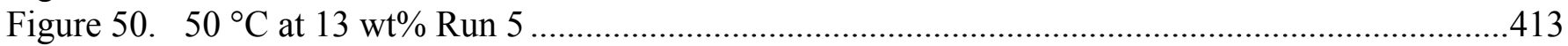

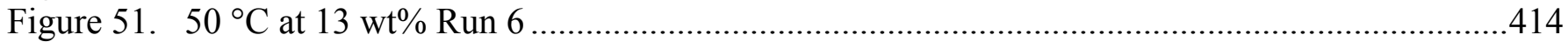

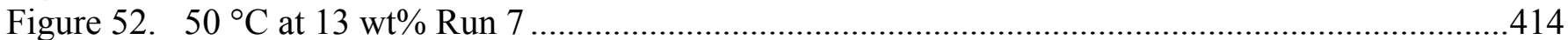

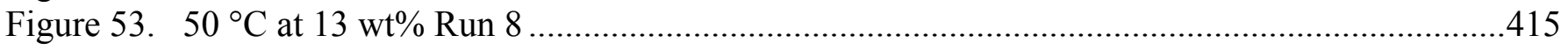

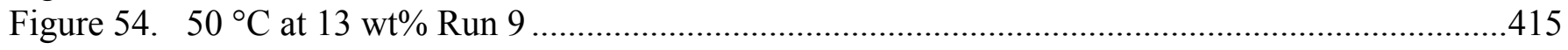




\begin{tabular}{|ll|}
\hline APPENDIX I - PART 3 & WSRC-TR-2000-00352 \\
& SRT-RPP-2000-00026 \\
SR/TRU PRECIPITATE SLURRY RHEOGRAMS AT 13 WT \% & Page 389 of 514 \\
\hline
\end{tabular}

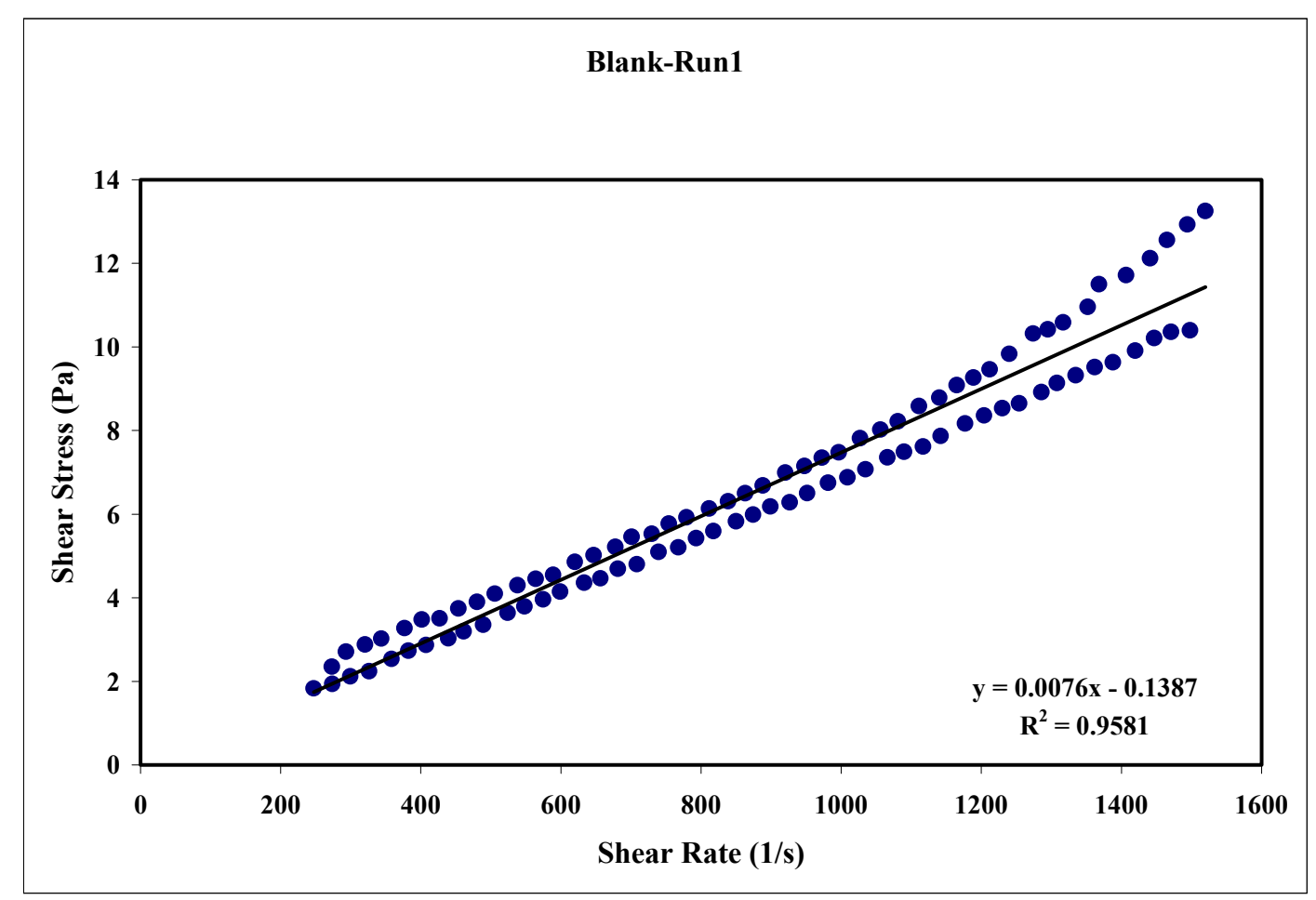

Figure 1. Blank at 13 wt\% Run 1

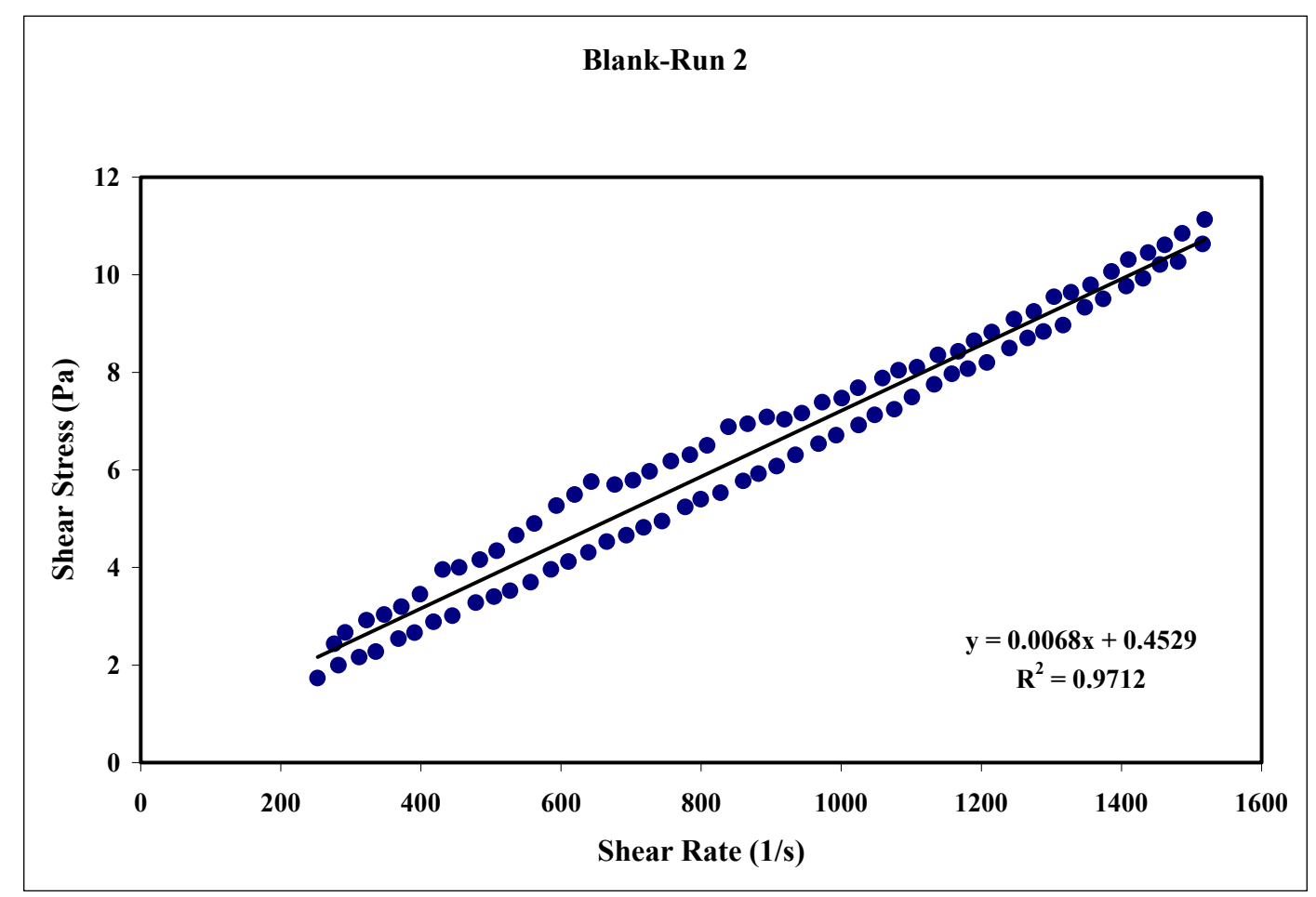

Figure 2. Blank at 13 wt\% Run 2 


\begin{tabular}{|ll|}
\hline APPENDIX I - PART 3 & WSRC-TR-2000-00352 \\
SR/TRU PRECIPITATE SLURRY RHEOGRAMS AT 13 WT \% & SRT-RPP-2000-00026 \\
\hline
\end{tabular}

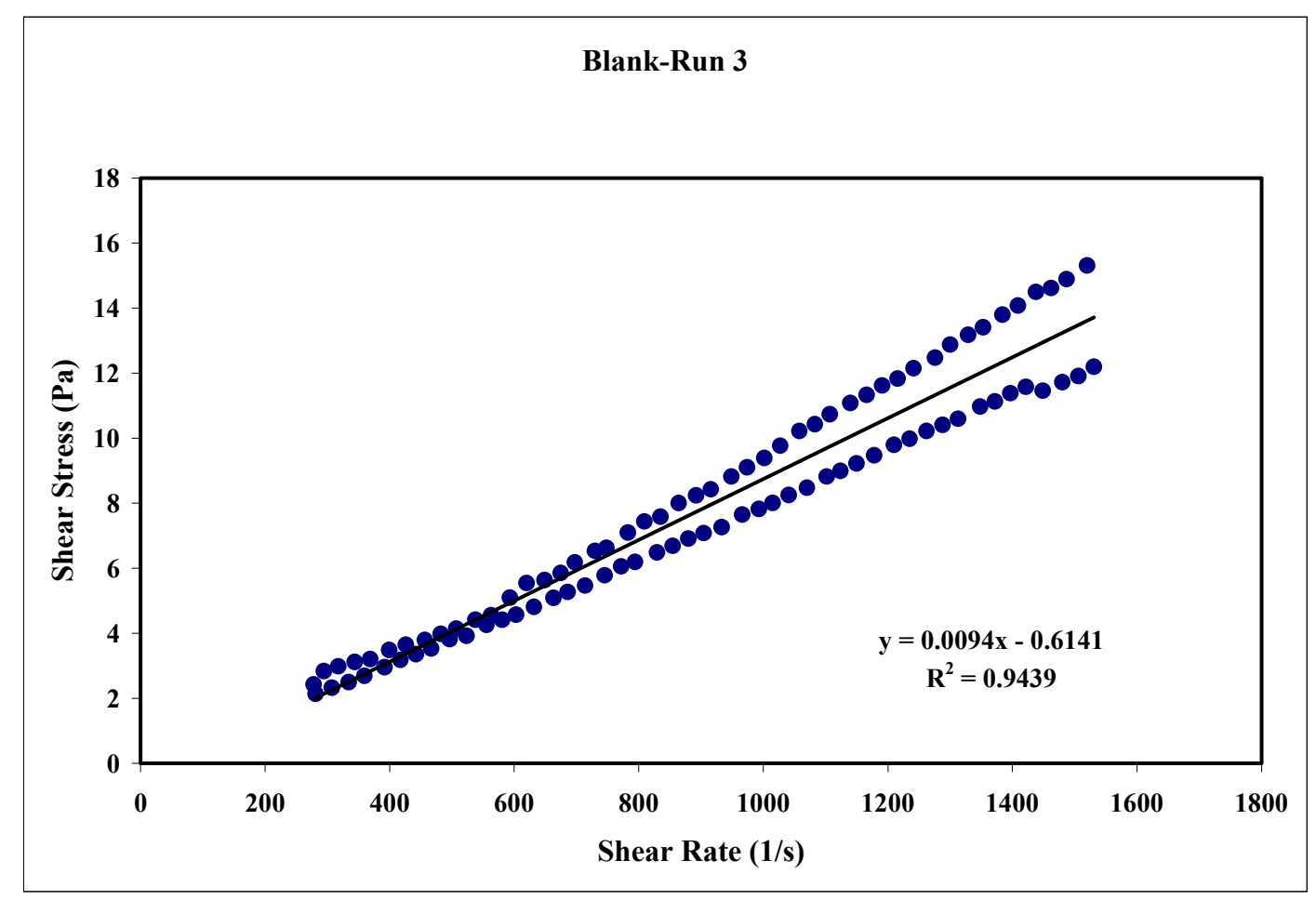

Figure 3. Blank at 13 wt\% Run 3

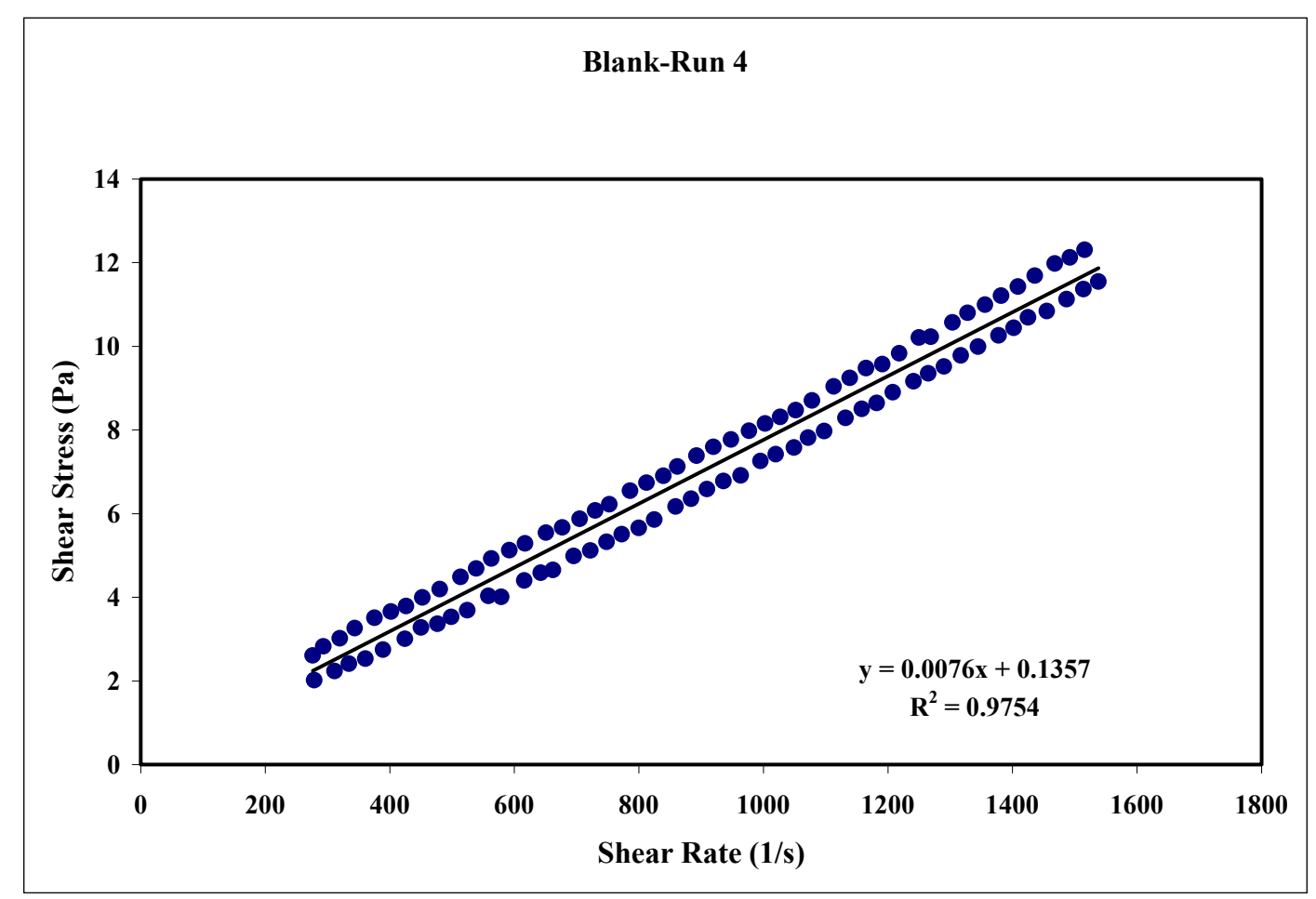

Figure 4. Blank at 13 wt\% Run 4 


\begin{tabular}{|ll|}
\hline APPENDIX I - PART 3 & WSRC-TR-2000-00352 \\
& SRT-RPP-2000-00026 \\
SR/TRU PRECIPITATE SLURRY RHEOGRAMS AT 13 WT \% & Page 391 of 514 \\
\hline
\end{tabular}

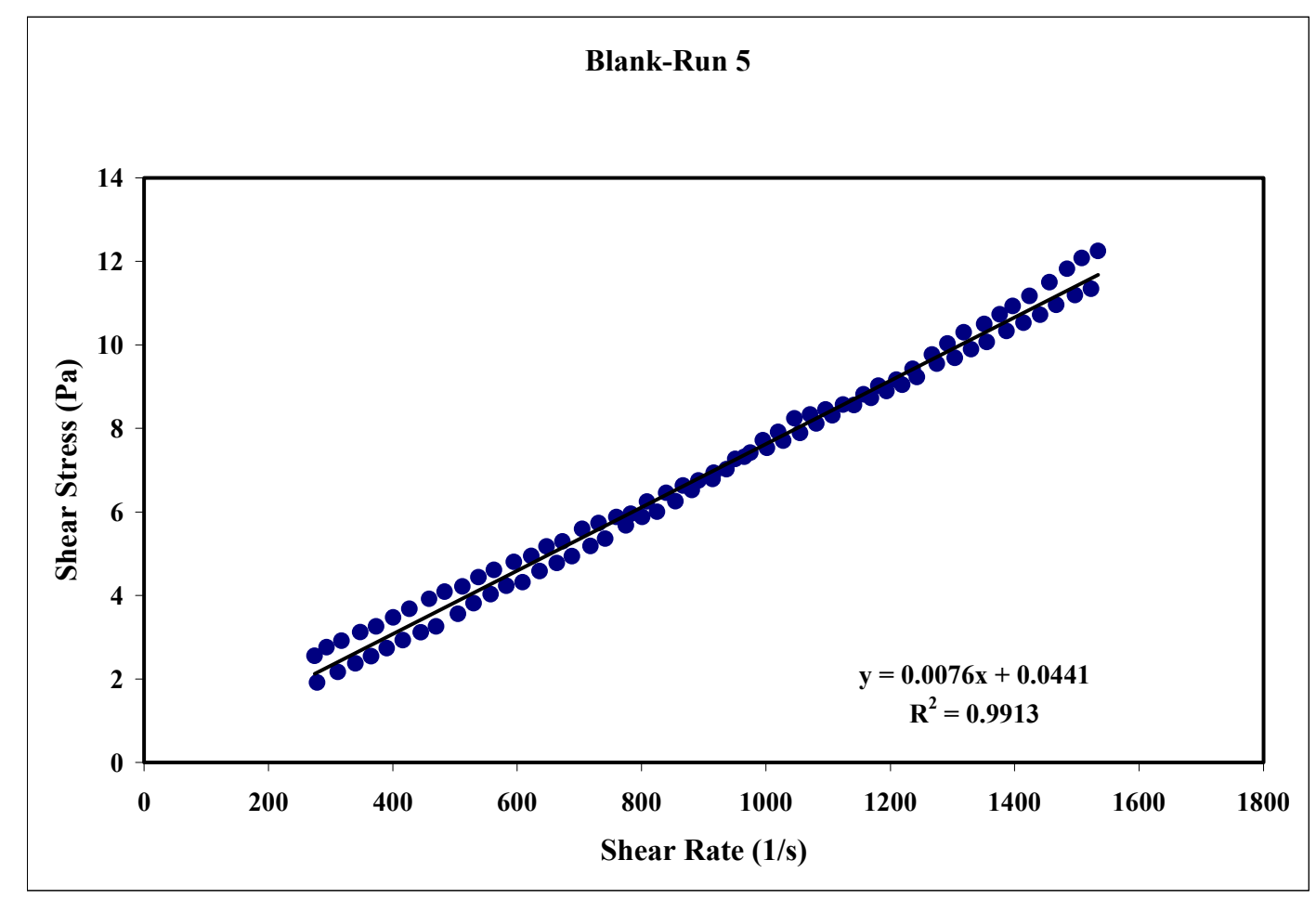

Figure 5. Blank at 13 wt\% Run 5

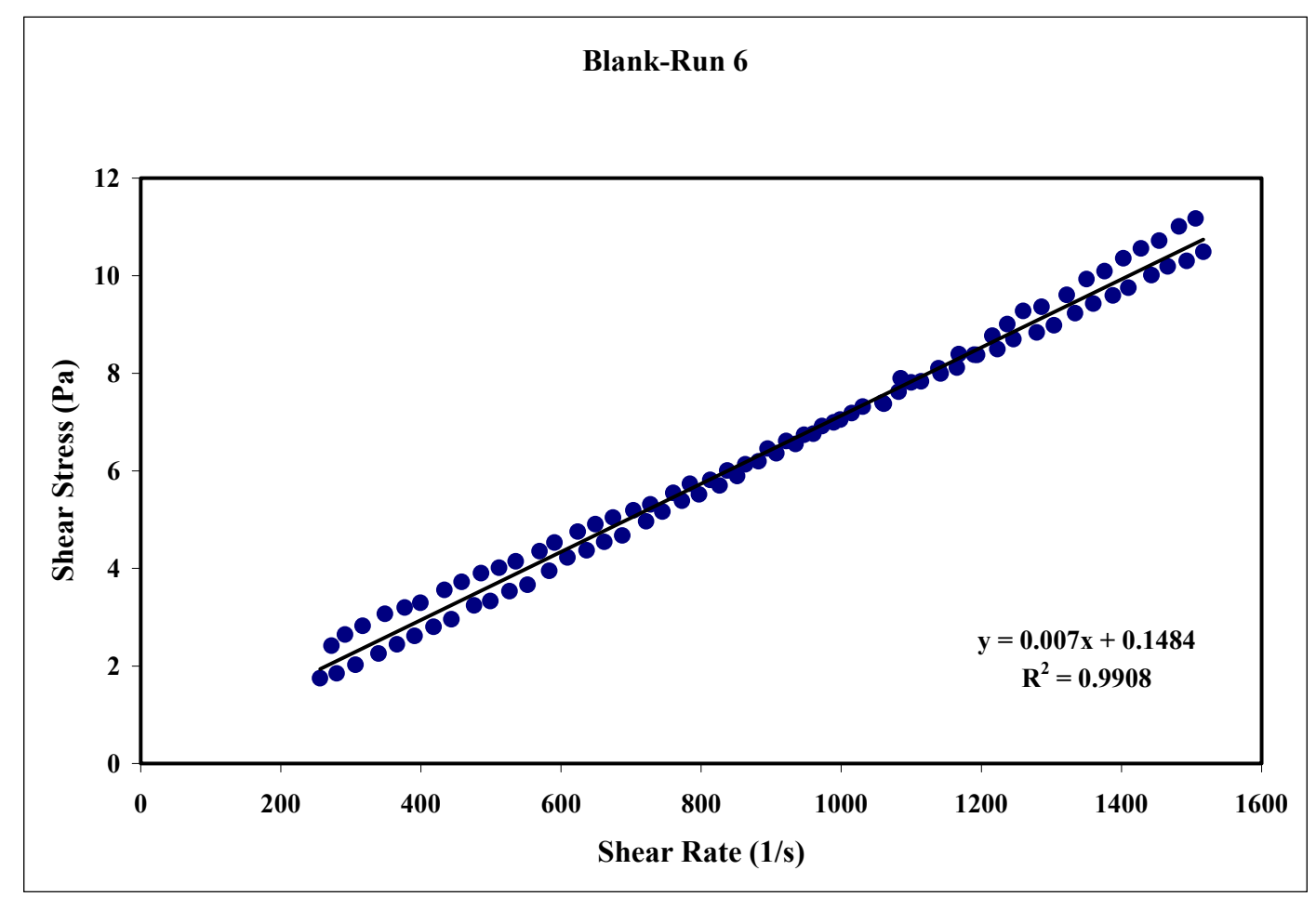

Figure 6. Blank at 13 wt\% Run 6 


\begin{tabular}{|ll|}
\hline APPENDIX I - PART 3 & WSRC-TR-2000-00352 \\
& SRT-RPP-2000-00026 \\
SR/TRU PRECIPITATE SLURRY RHEOGRAMS AT 13 WT \% & Page 392 of 514 \\
\hline
\end{tabular}

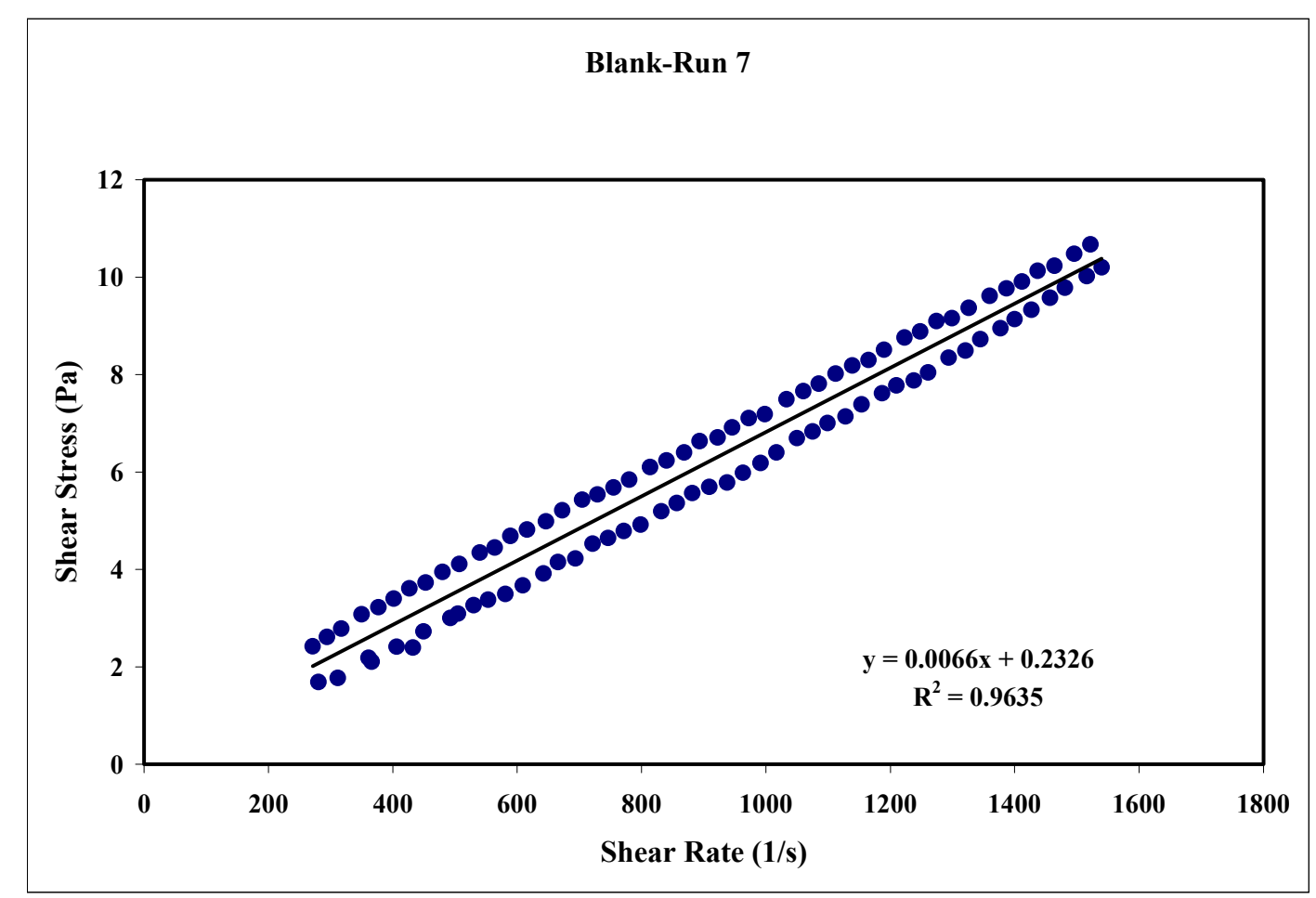

Figure 7. Blank at 13 wt\% Run 7

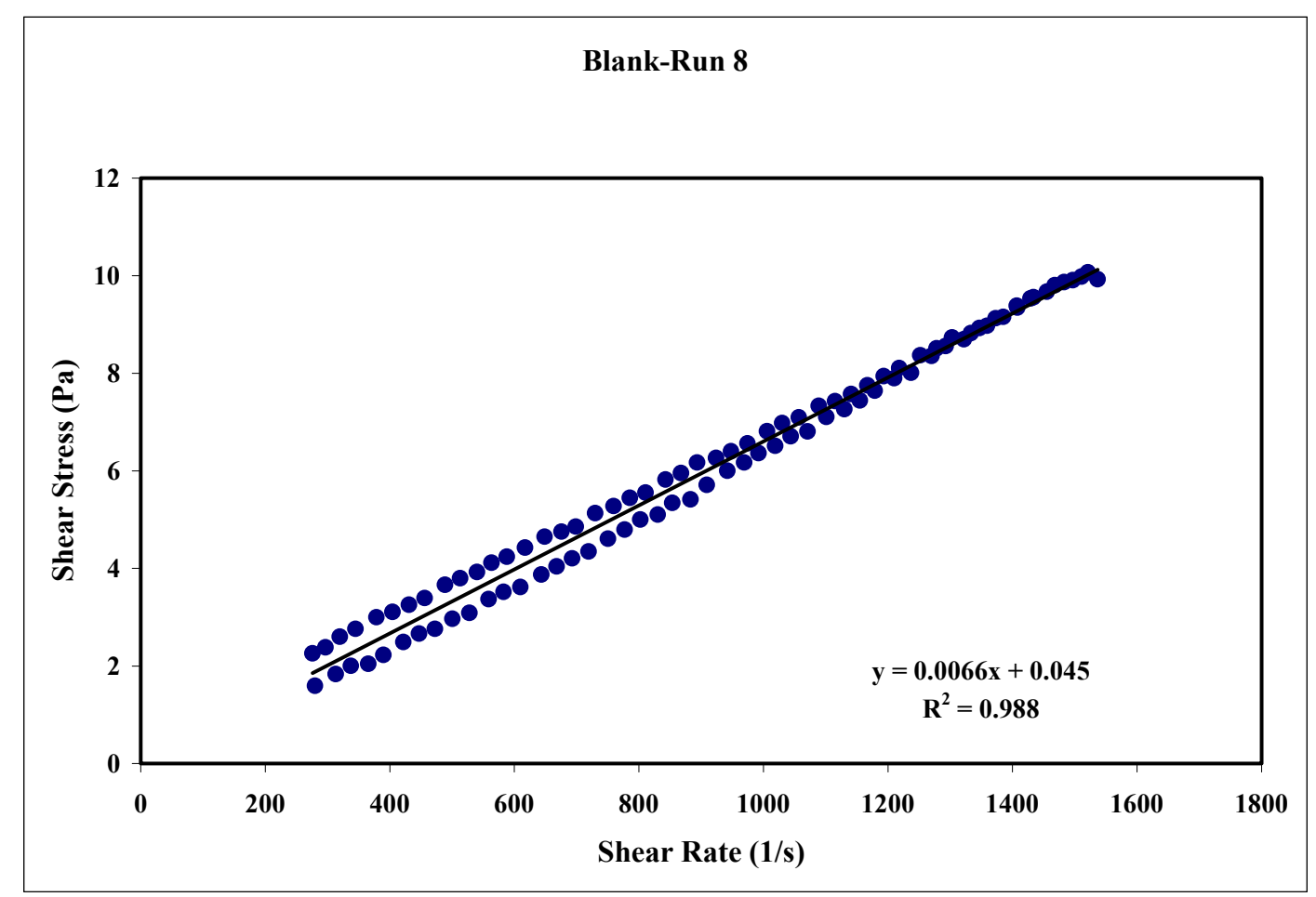

Figure 8. Blank at 13 wt\% Run 8 


\begin{tabular}{|ll|}
\hline APPENDIX I - PART 3 & WSRC-TR-2000-00352 \\
& SRT-RPP-2000-00026 \\
SR/TRU PRECIPITATE SLURRY RHEOGRAMS AT 13 WT \% & Page 393 of 514 \\
\hline
\end{tabular}

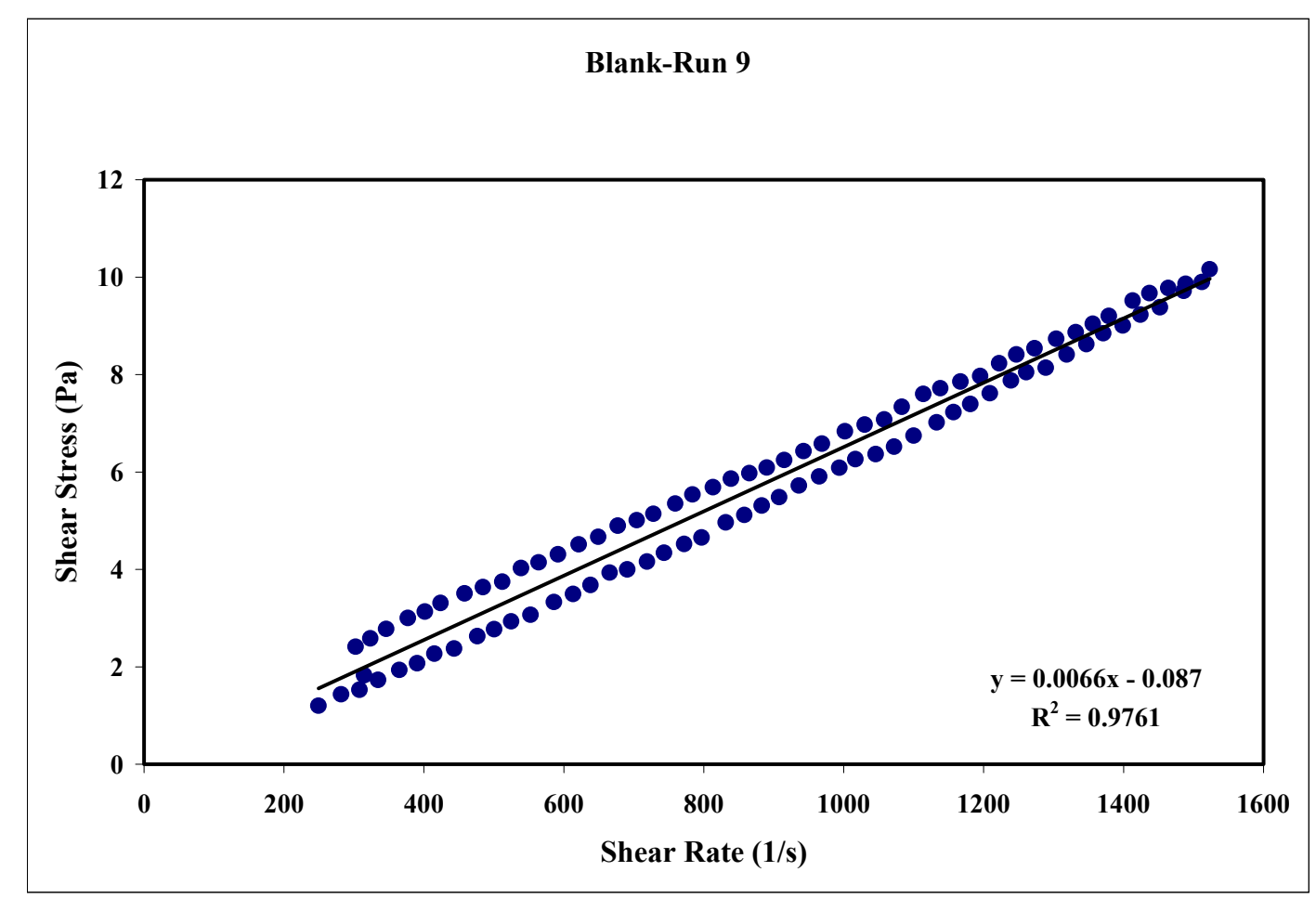

Figure 9. Blank at 13 wt\% Run 9

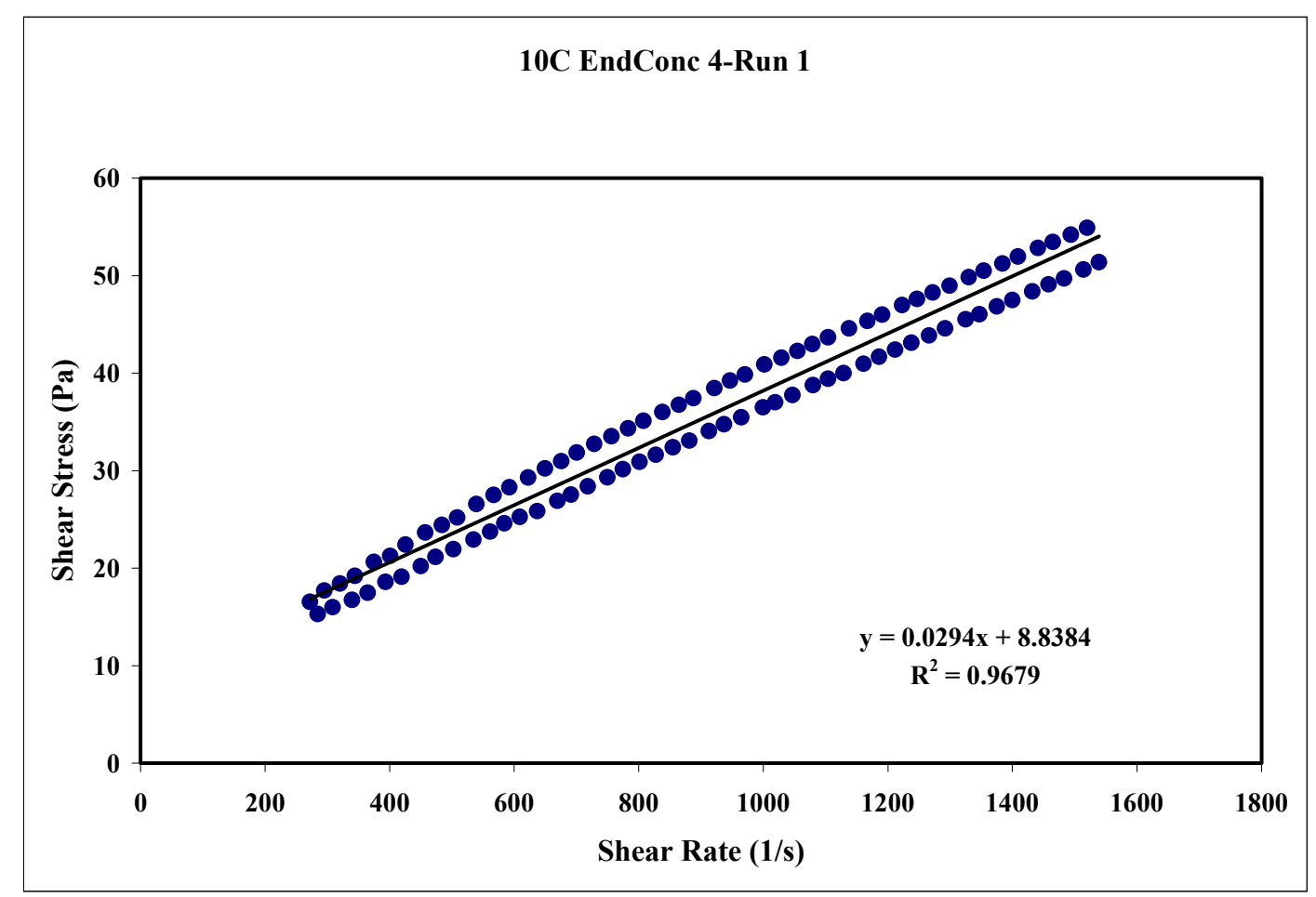

Figure $10.10{ }^{\circ} \mathrm{C}$ at $13 \mathrm{wt} \%$ Run 1 


\begin{tabular}{|ll|}
\hline APPENDIX I - PART 3 & WSRC-TR-2000-00352 \\
& SRT-RPP-2000-00026 \\
SR/TRU PRECIPITATE SLURRY RHEOGRAMS AT 13 WT \% & Page 394 of 514 \\
\hline
\end{tabular}

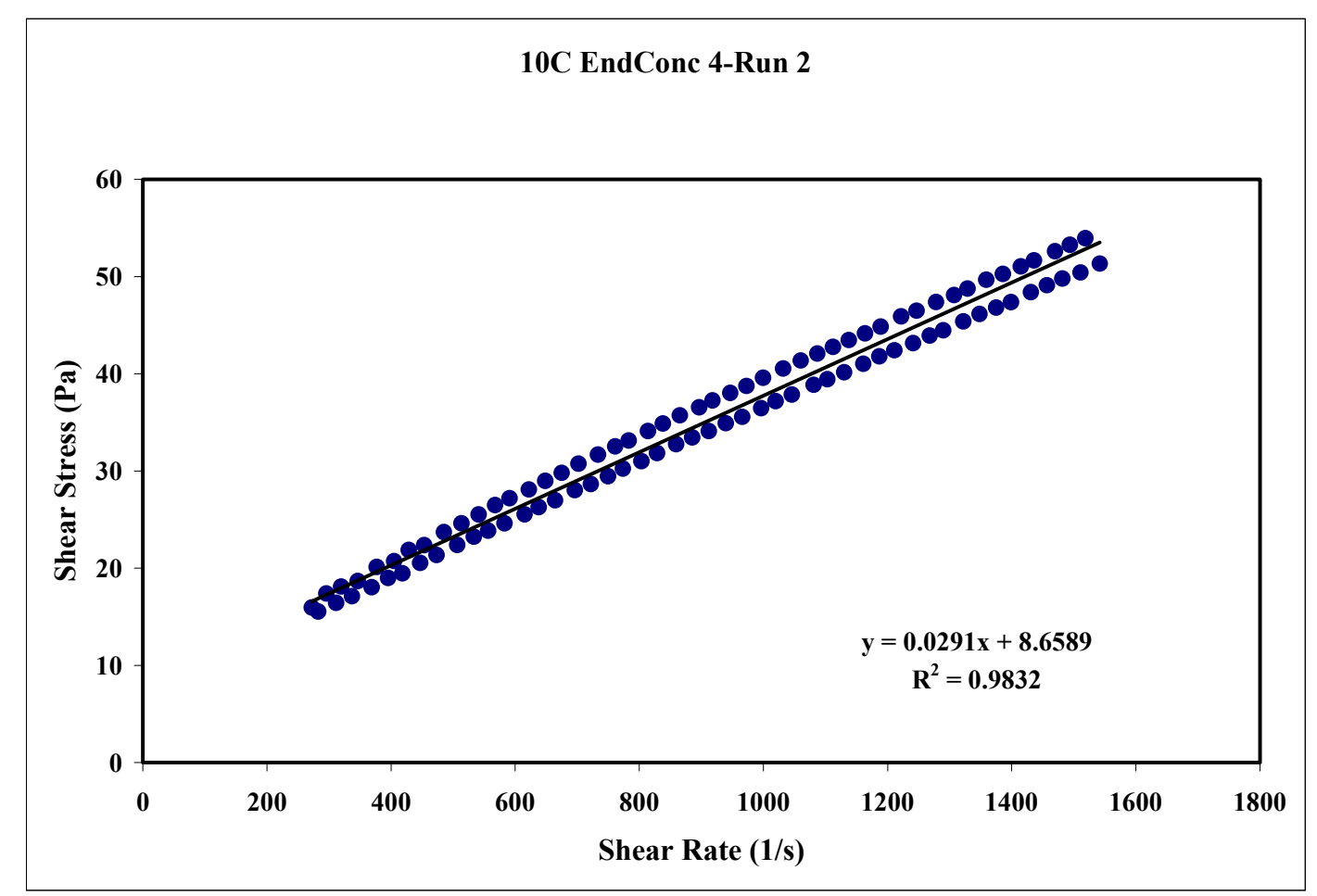

Figure 11. $10{ }^{\circ} \mathrm{C}$ at $13 \mathrm{wt} \%$ Run 2

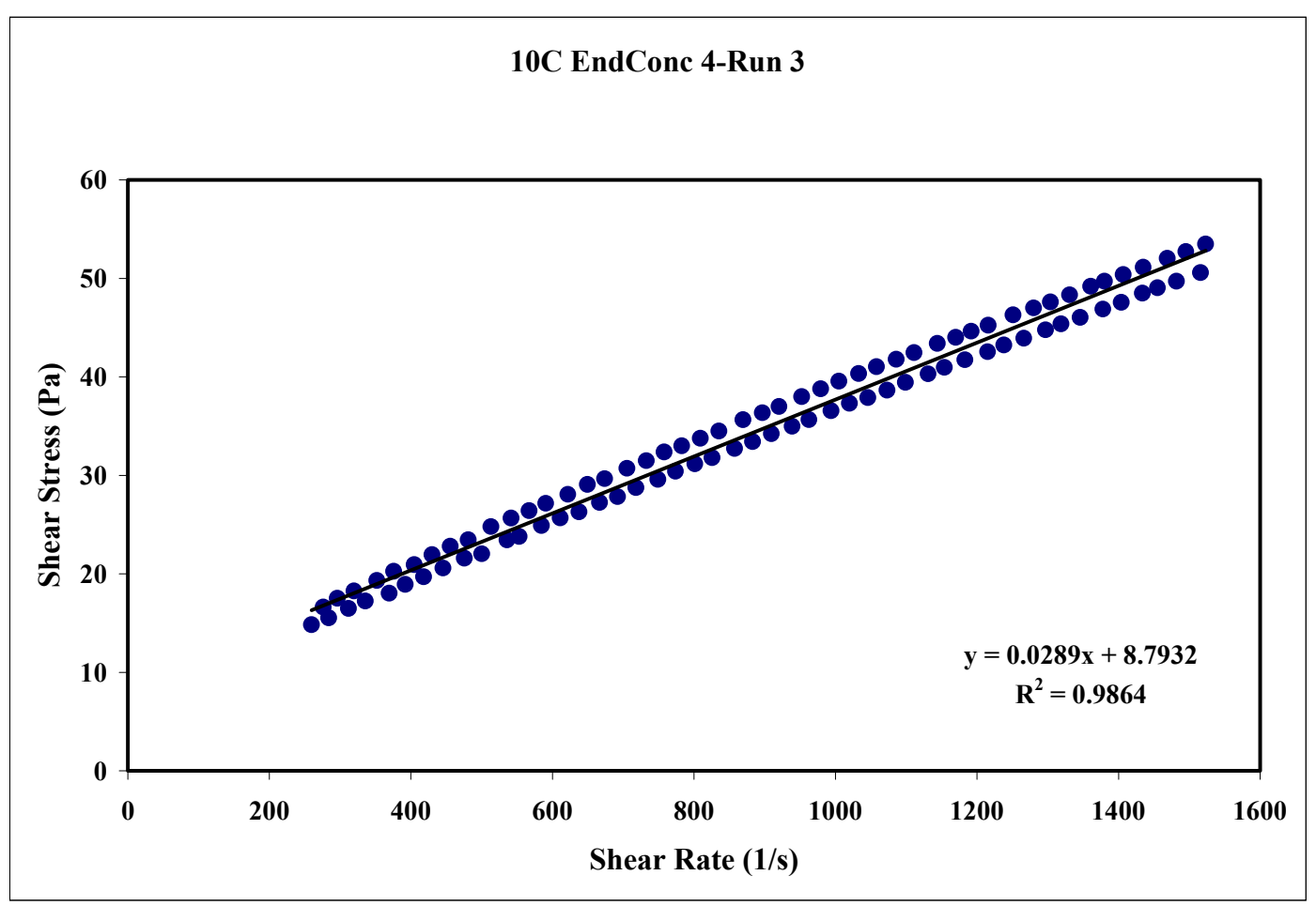

Figure 12. $10{ }^{\circ} \mathrm{C}$ at 13 wt $\%$ Run 3 


\begin{tabular}{|ll|}
\hline APPENDIX I - PART 3 & WSRC-TR-2000-00352 \\
& SRT-RPP-2000-00026 \\
SR/TRU PRECIPITATE SLURRY RHEOGRAMS AT 13 WT \% & Page 395 of 514 \\
\hline
\end{tabular}

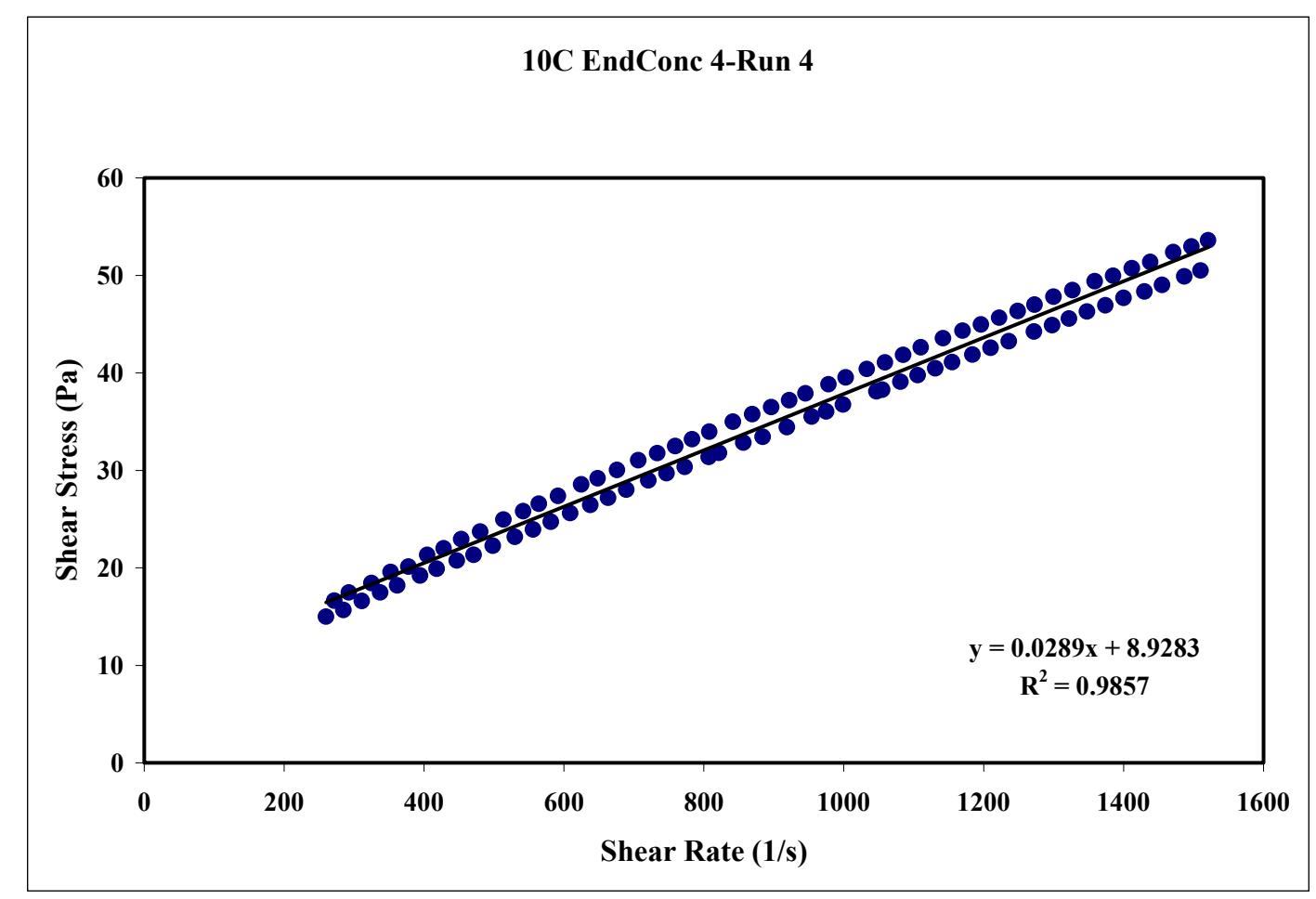

Figure 13. $10{ }^{\circ} \mathrm{C}$ at 13 wt\% Run 4

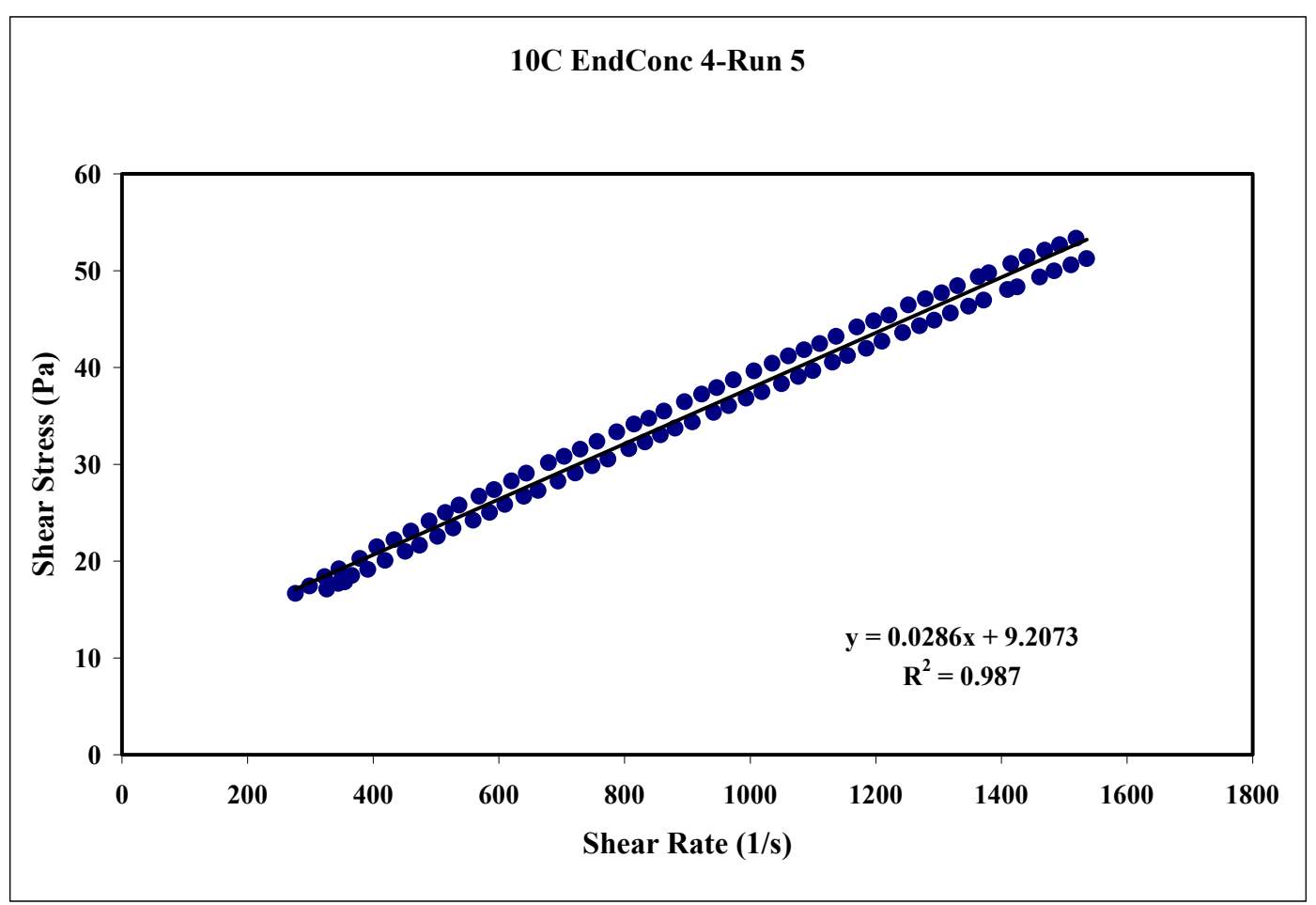

Figure 14. $10{ }^{\circ} \mathrm{C}$ at 13 wt\% Run 5 


\begin{tabular}{|ll|}
\hline APPENDIX I - PART 3 & WSRC-TR-2000-00352 \\
& SRT-RPP-2000-00026 \\
SR/TRU PRECIPITATE SLURRY RHEOGRAMS AT 13 WT \% & Page 396 of 514 \\
\hline
\end{tabular}

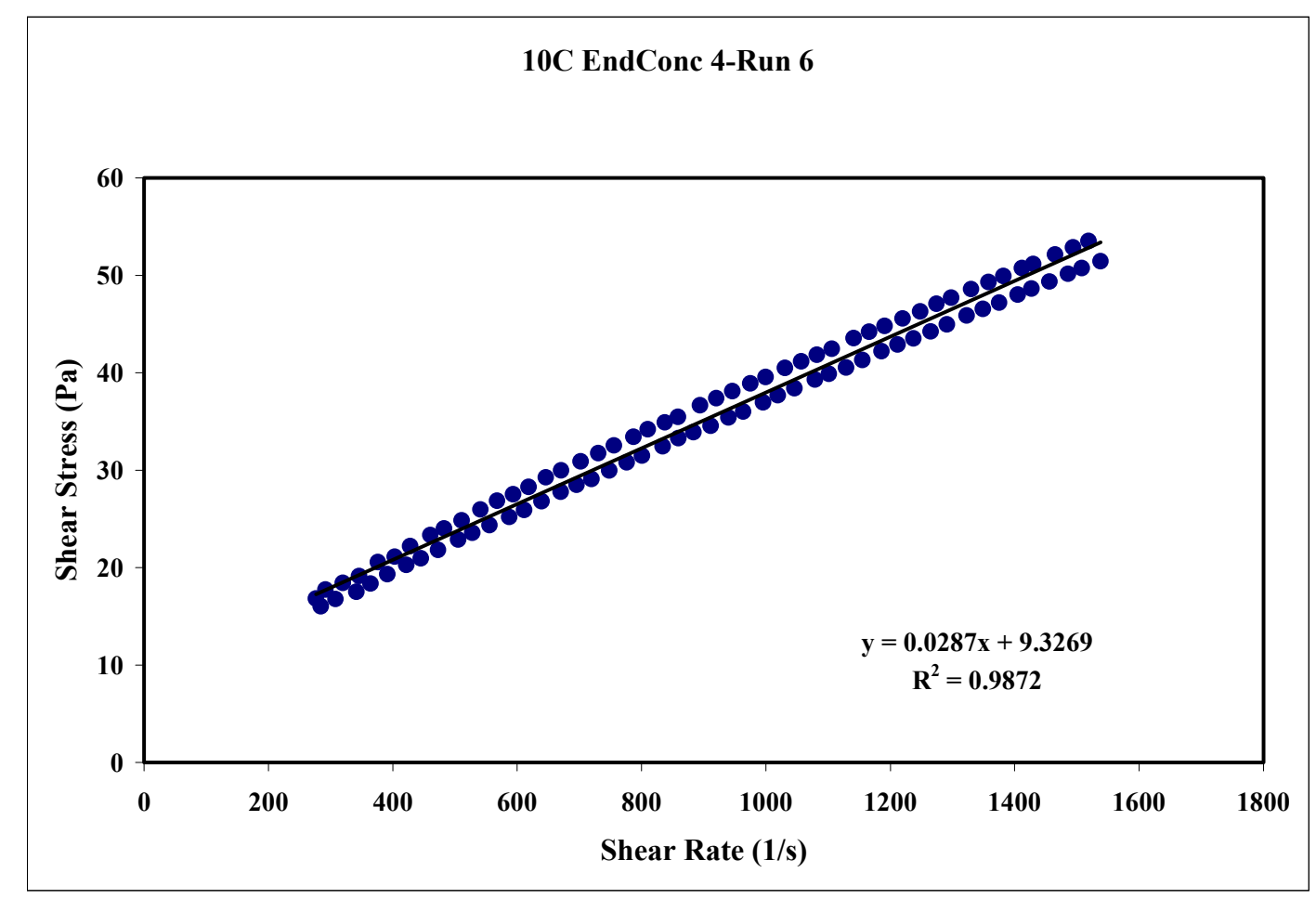

Figure $15.10{ }^{\circ} \mathrm{C}$ at 13 wt\% Run 6

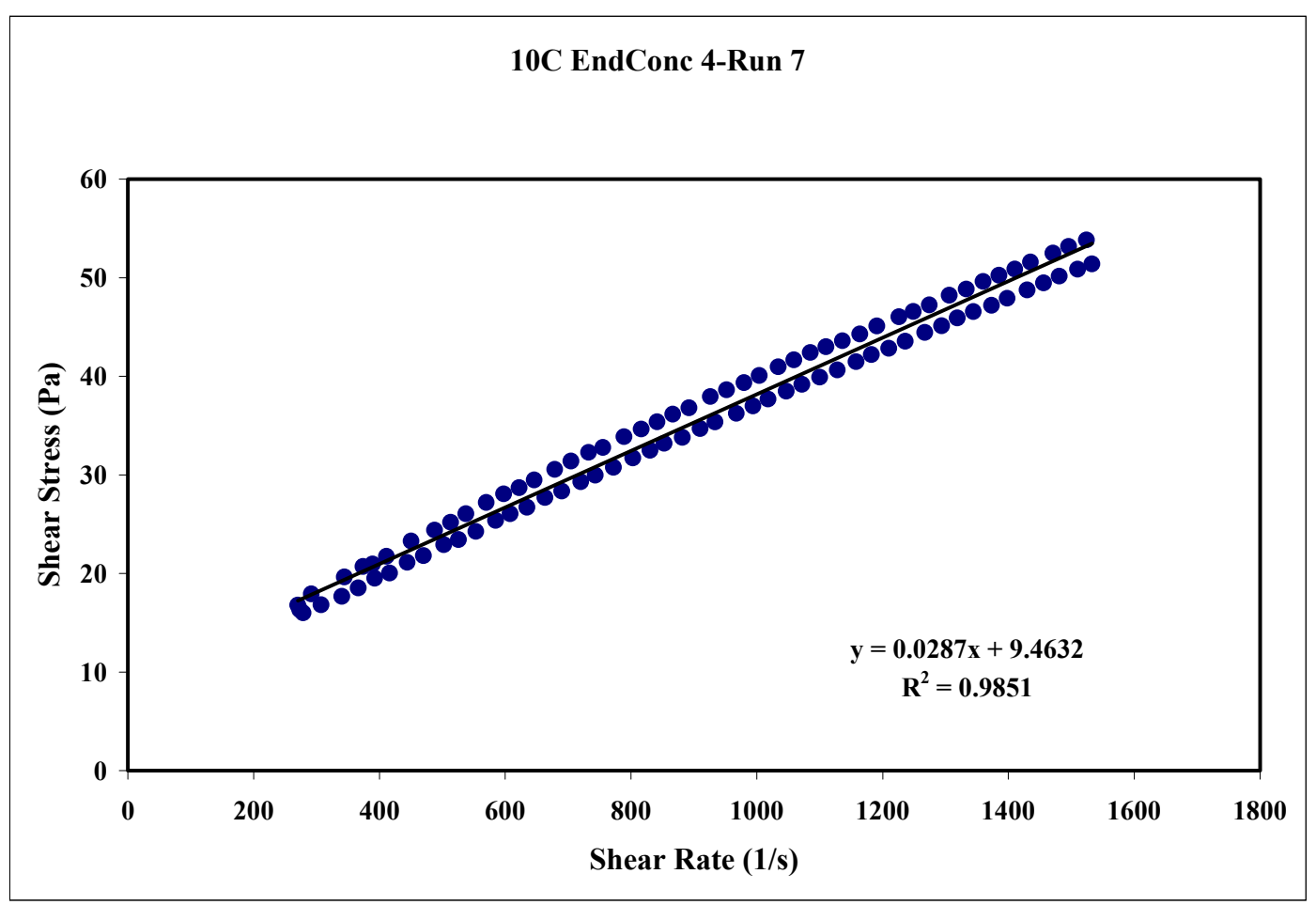

Figure 16. $10{ }^{\circ} \mathrm{C}$ at 13 wt\% Run 7 


\begin{tabular}{|ll|}
\hline APPENDIX I - PART 3 & WSRC-TR-2000-00352 \\
& SRT-RPP-2000-00026 \\
SR/TRU PRECIPITATE SLURRY RHEOGRAMS AT 13 WT \% & Page 397 of 514 \\
\hline
\end{tabular}

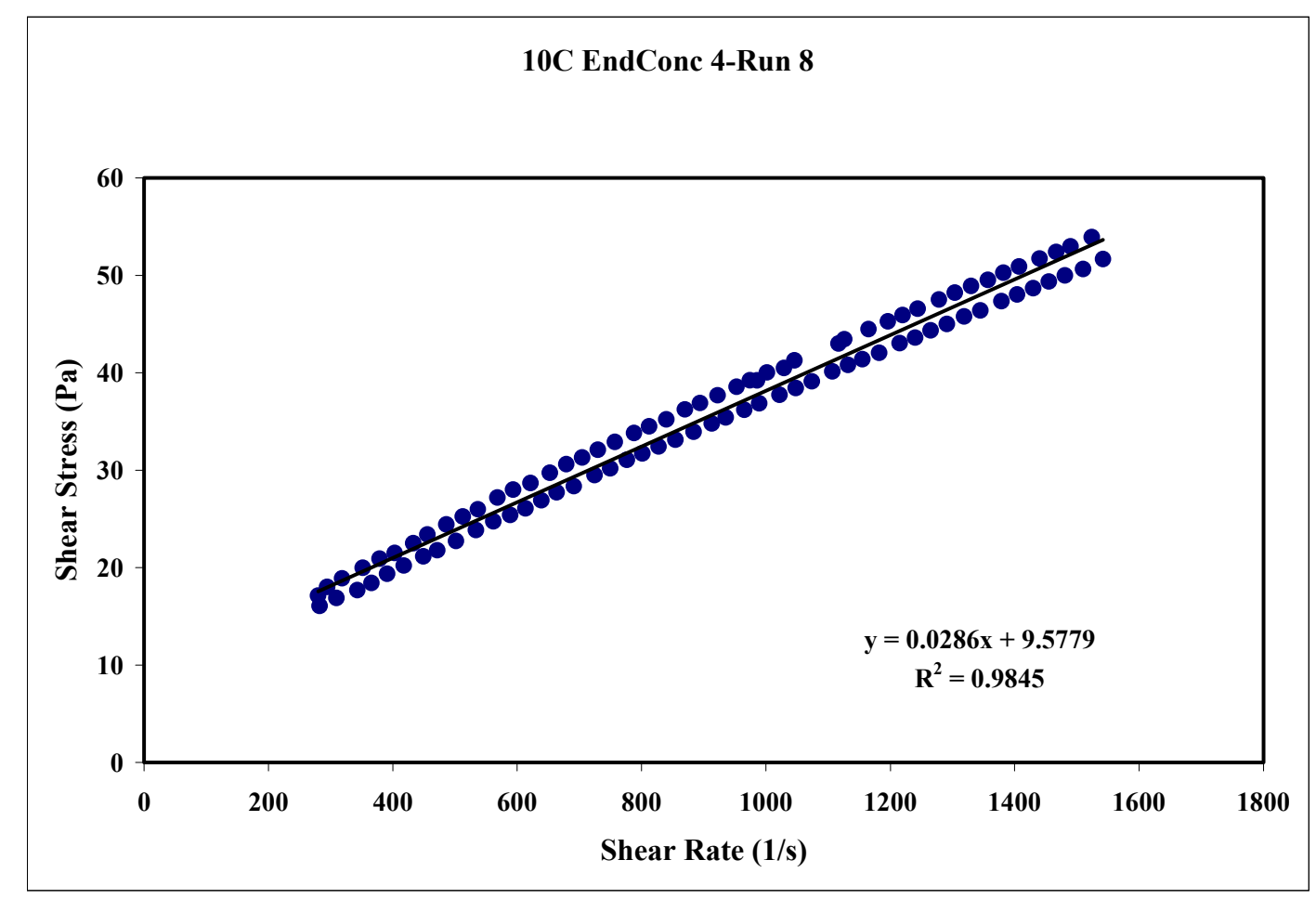

Figure $17.10{ }^{\circ} \mathrm{C}$ at 13 wt\% Run 8

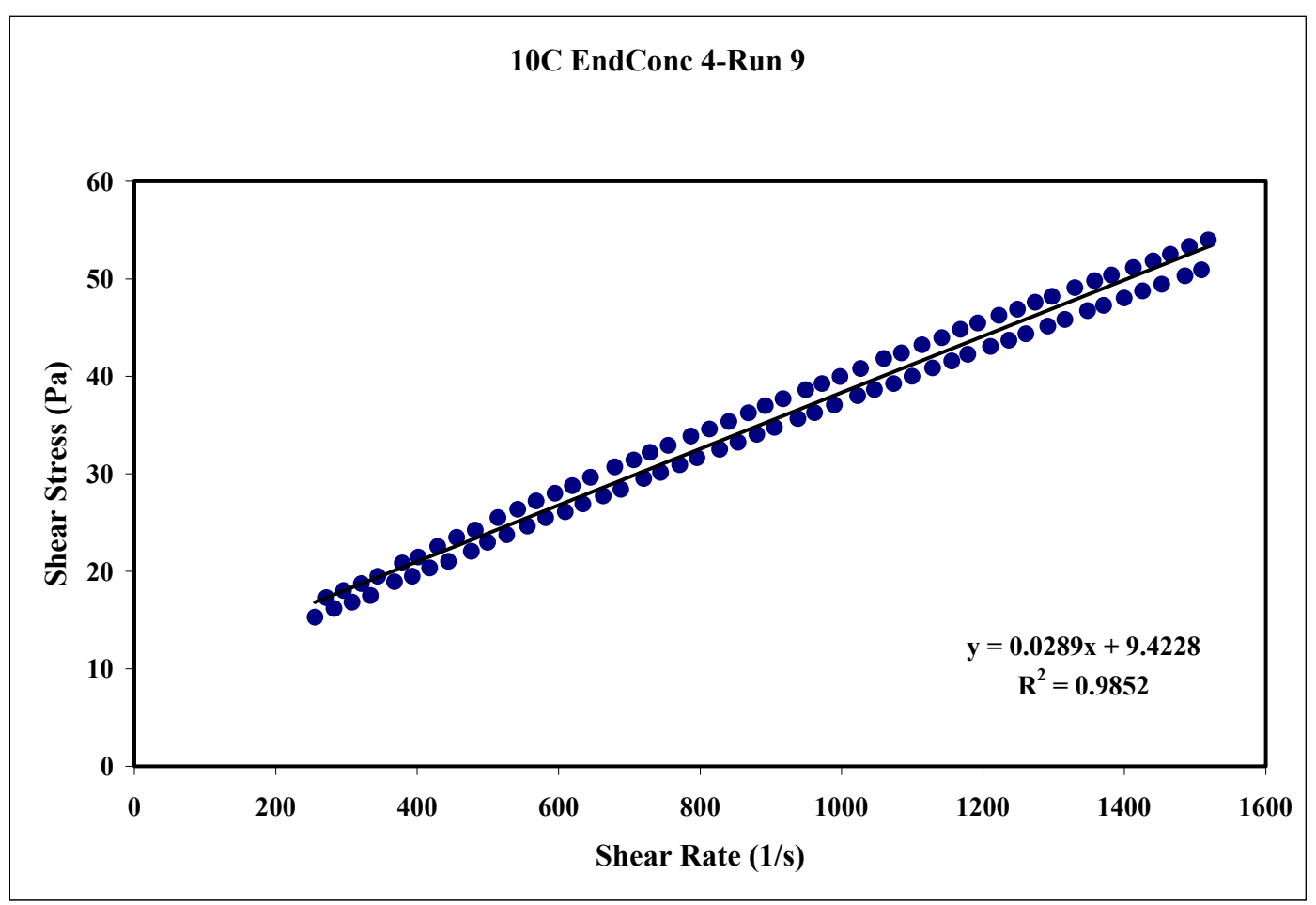

Figure 18. $10{ }^{\circ} \mathrm{C}$ at 13 wt\% Run 9 


\begin{tabular}{|ll|}
\hline APPENDIX I - PART 3 & WSRC-TR-2000-00352 \\
& SRT-RPP-2000-00026 \\
SR/TRU PRECIPITATE SLURRY RHEOGRAMS AT 13 WT \% & Page 398 of 514 \\
\hline
\end{tabular}

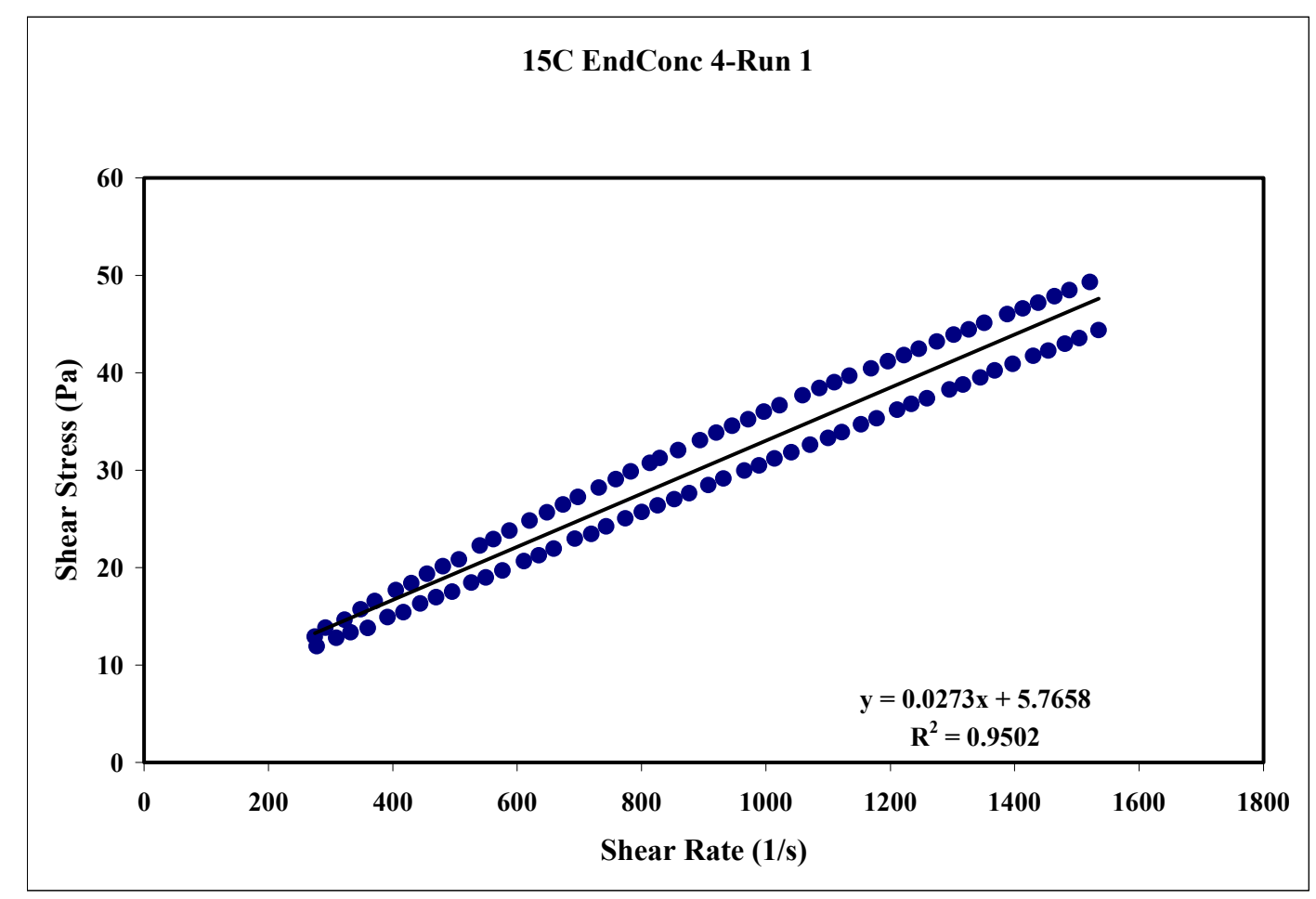

Figure 19. $15^{\circ} \mathrm{C}$ at $13 w^{\circ} \%$ Run 1

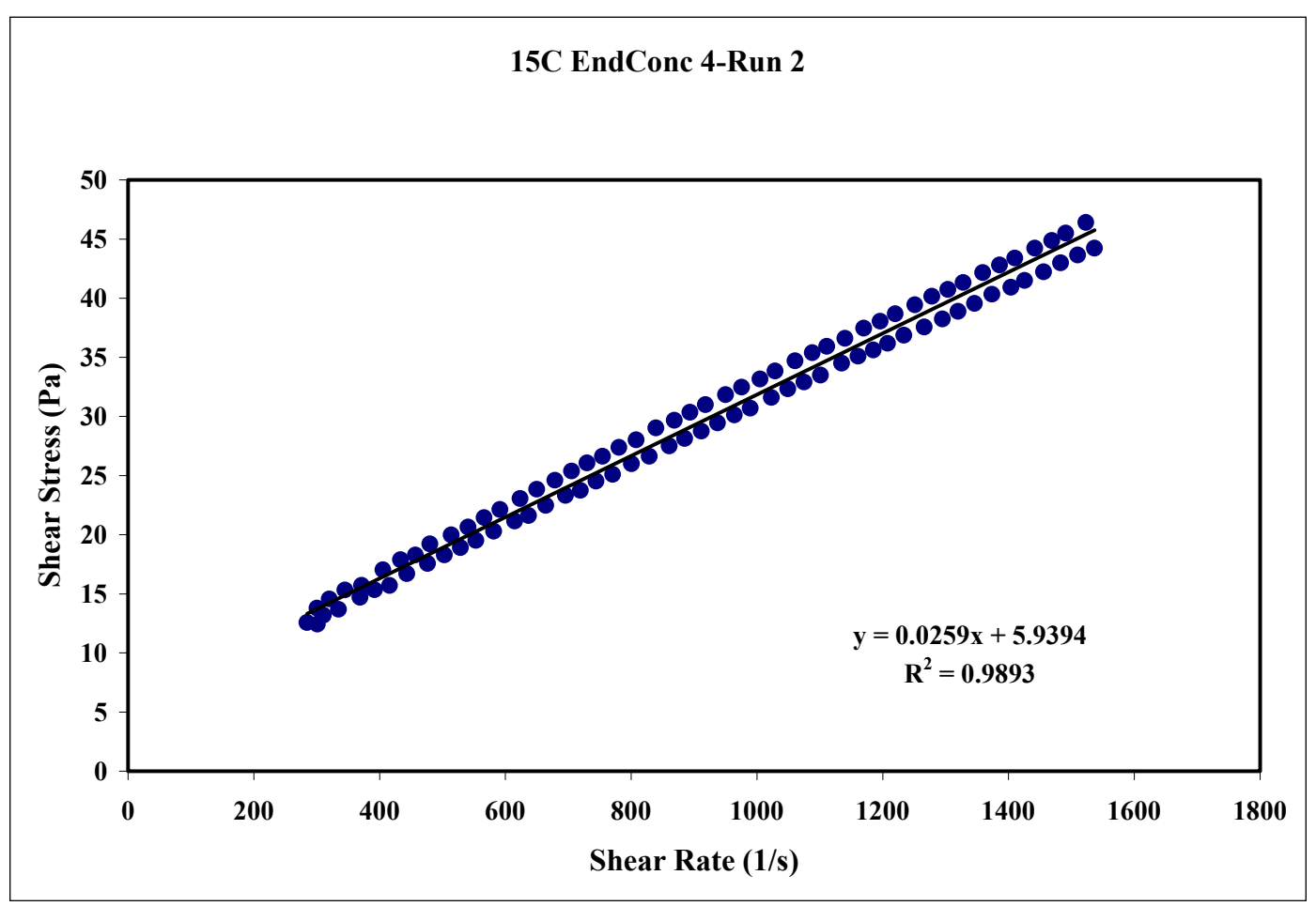

Figure 20. $15^{\circ} \mathrm{C}$ at 13 wt $\%$ Run 2 


\begin{tabular}{|ll|}
\hline APPENDIX I - PART 3 & WSRC-TR-2000-00352 \\
& SRT-RPP-2000-00026 \\
SR/TRU PRECIPITATE SLURRY RHEOGRAMS AT 13 WT \% & Page 399 of 514 \\
\hline
\end{tabular}

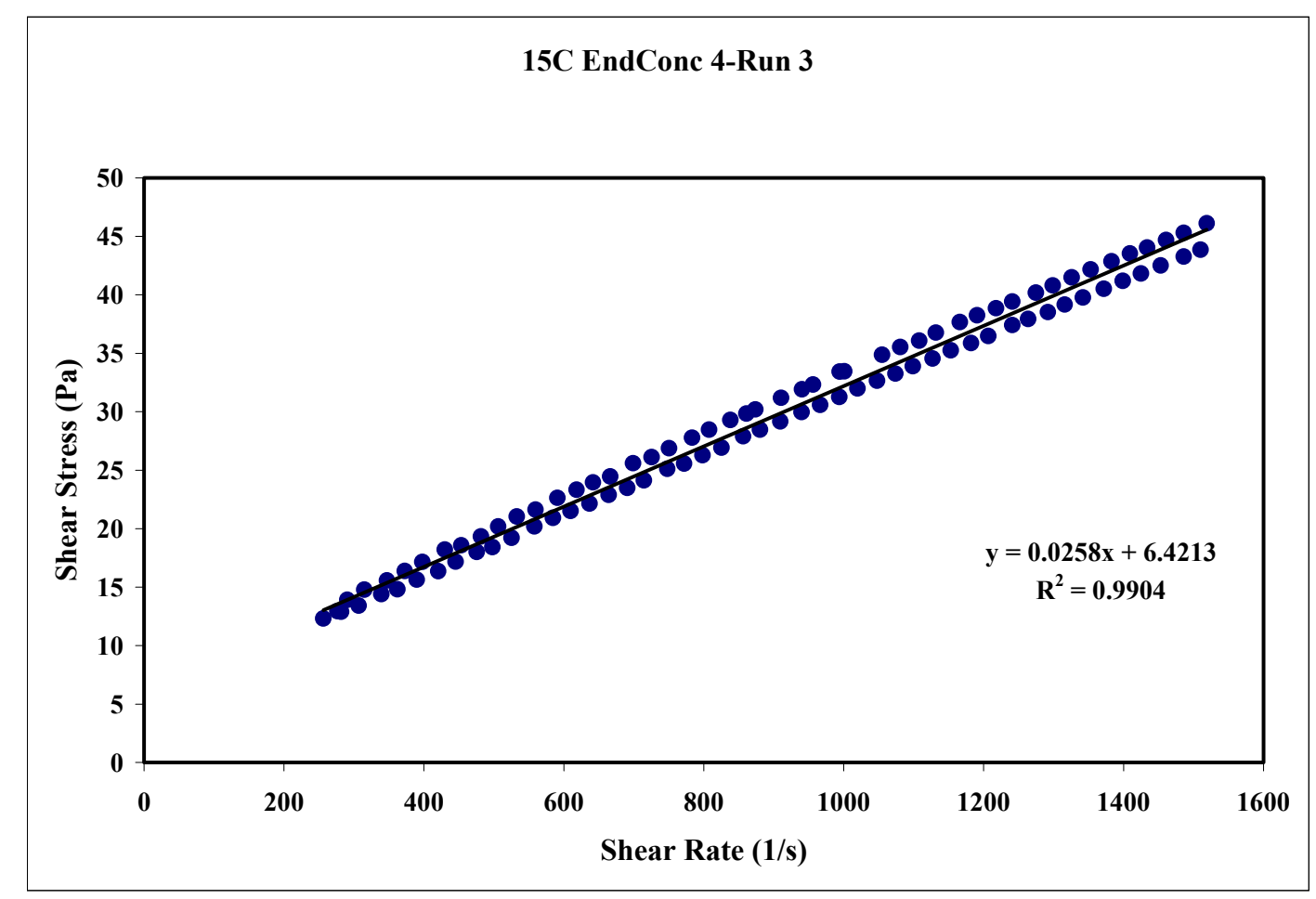

Figure 21. $15^{\circ} \mathrm{C}$ at 13 wt $\%$ Run 3

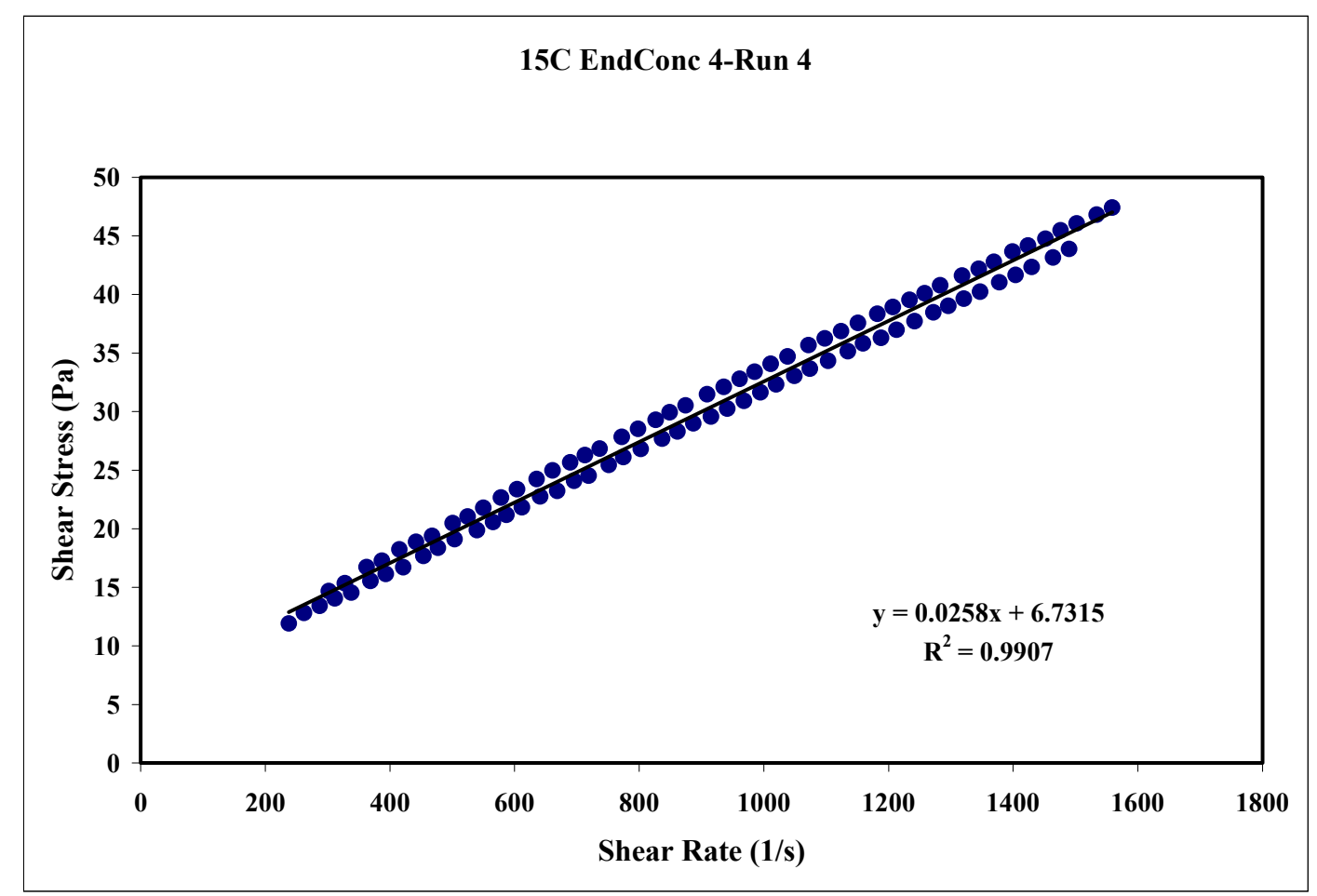

Figure 22. $15^{\circ} \mathrm{C}$ at 13 wt $\%$ Run 4 


\begin{tabular}{|ll|}
\hline APPENDIX I - PART 3 & WSRC-TR-2000-00352 \\
& SRT-RPP-2000-00026 \\
SR/TRU PRECIPITATE SLURRY RHEOGRAMS AT 13 WT \% & Page 400 of 514 \\
\hline
\end{tabular}

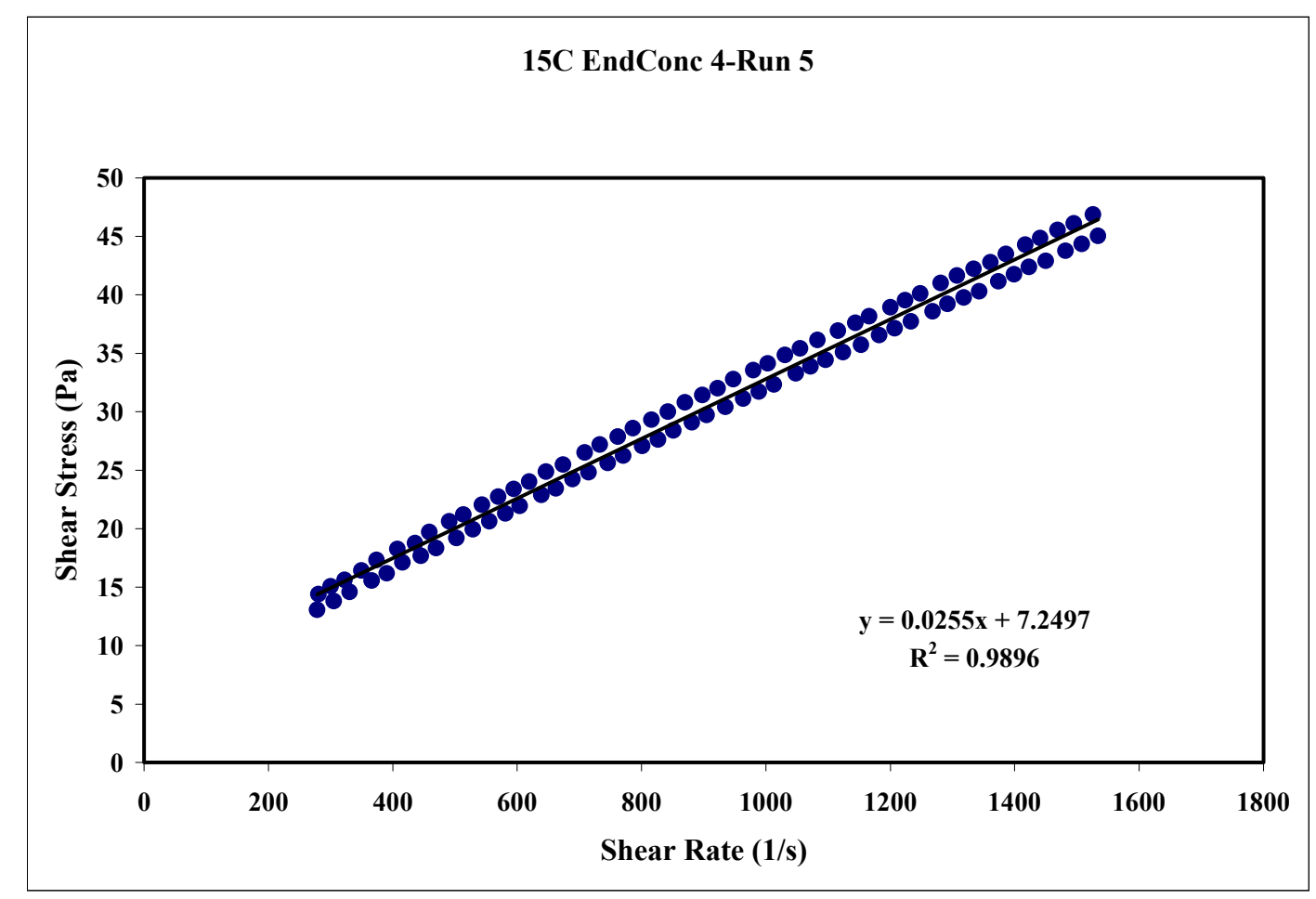

Figure 23. $15^{\circ} \mathrm{C}$ at 13 wt\% Run 5

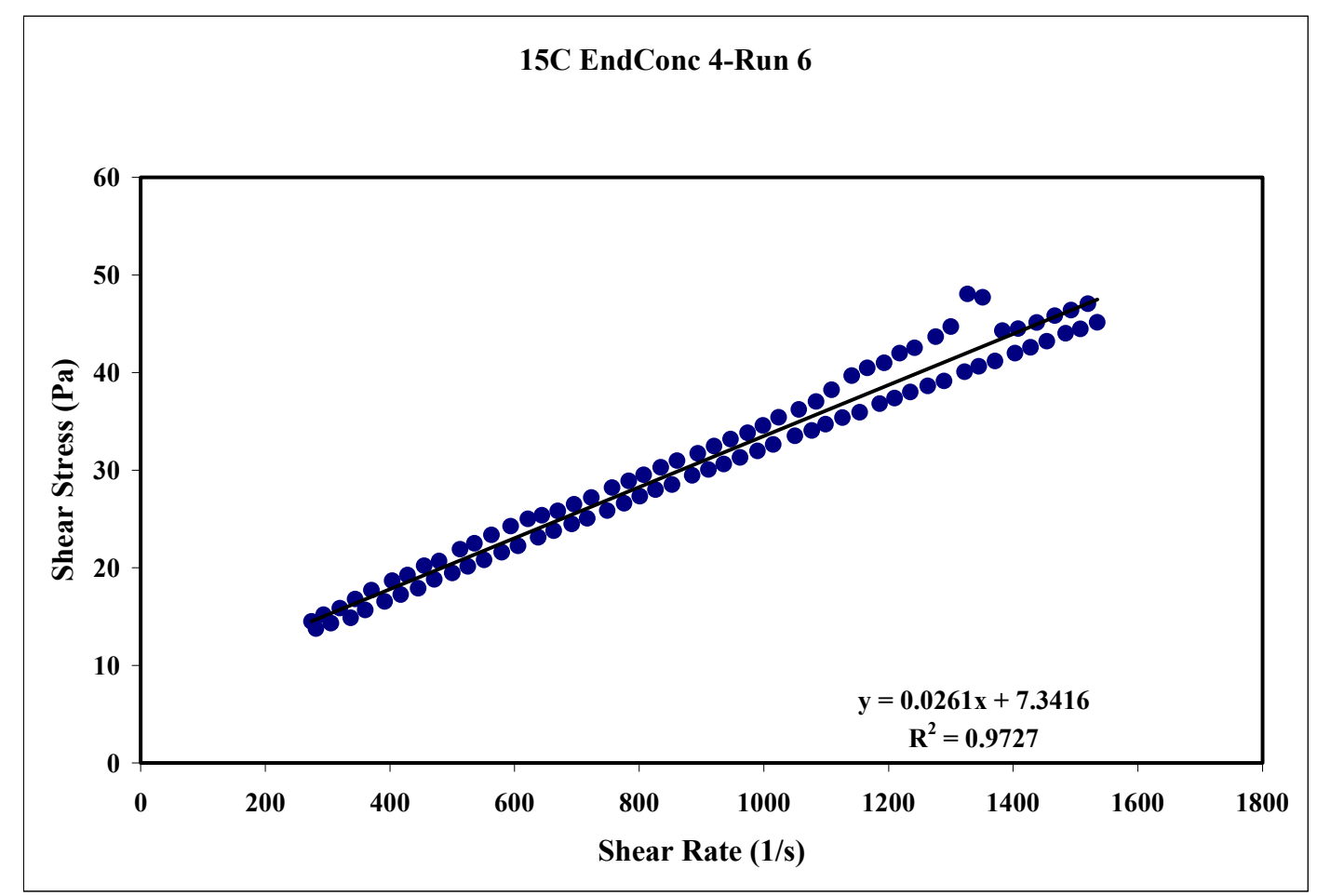

Figure 24. $15^{\circ} \mathrm{C}$ at 13 wt $\%$ Run 6 


\begin{tabular}{|ll|}
\hline APPENDIX I - PART 3 & WSRC-TR-2000-00352 \\
& SRT-RPP-2000-00026 \\
SR/TRU PRECIPITATE SLURRY RHEOGRAMS AT 13 WT \% & Page 401 of 514 \\
\hline
\end{tabular}

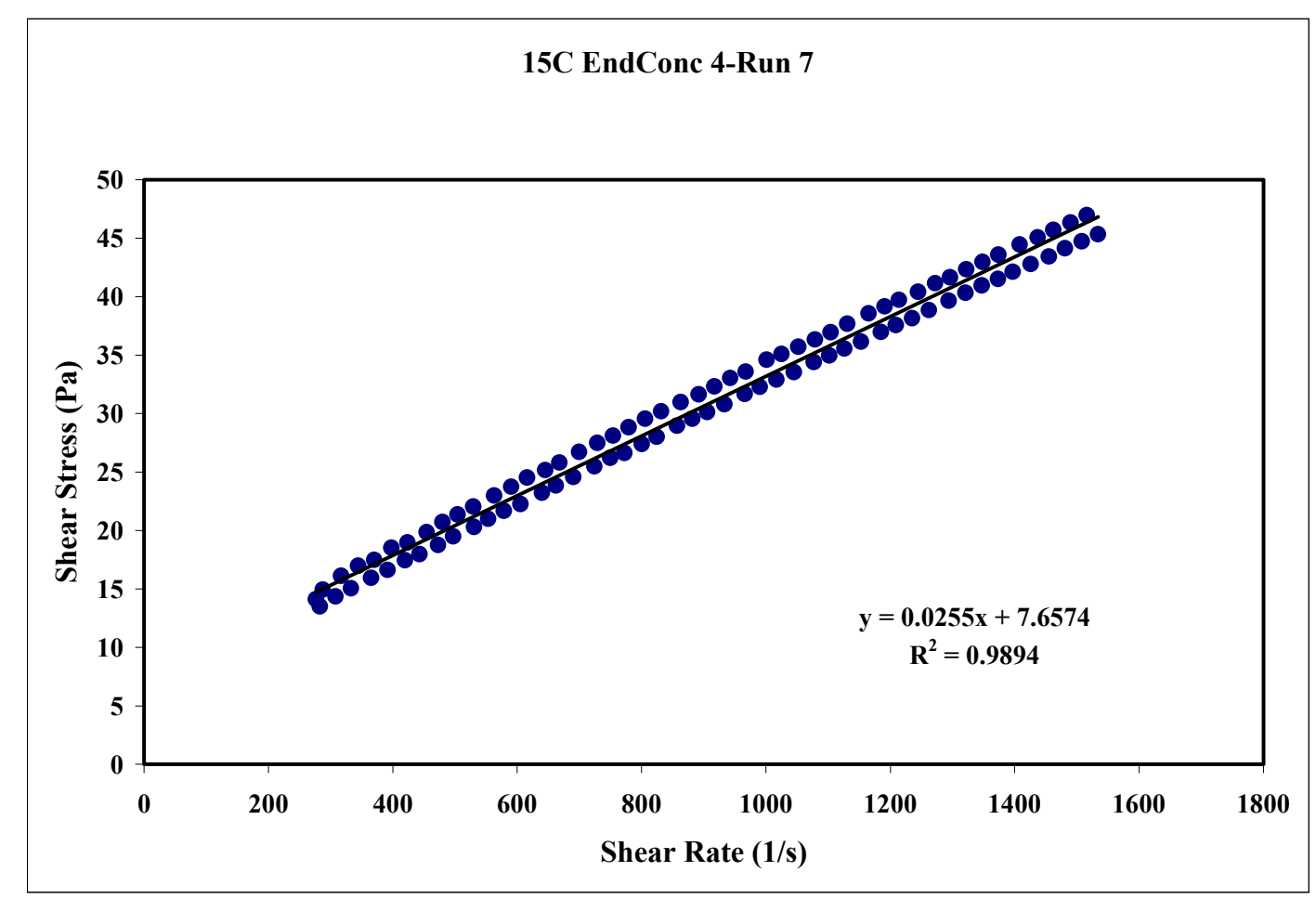

Figure 25. $15^{\circ} \mathrm{C}$ at 13 wt\% Run 7

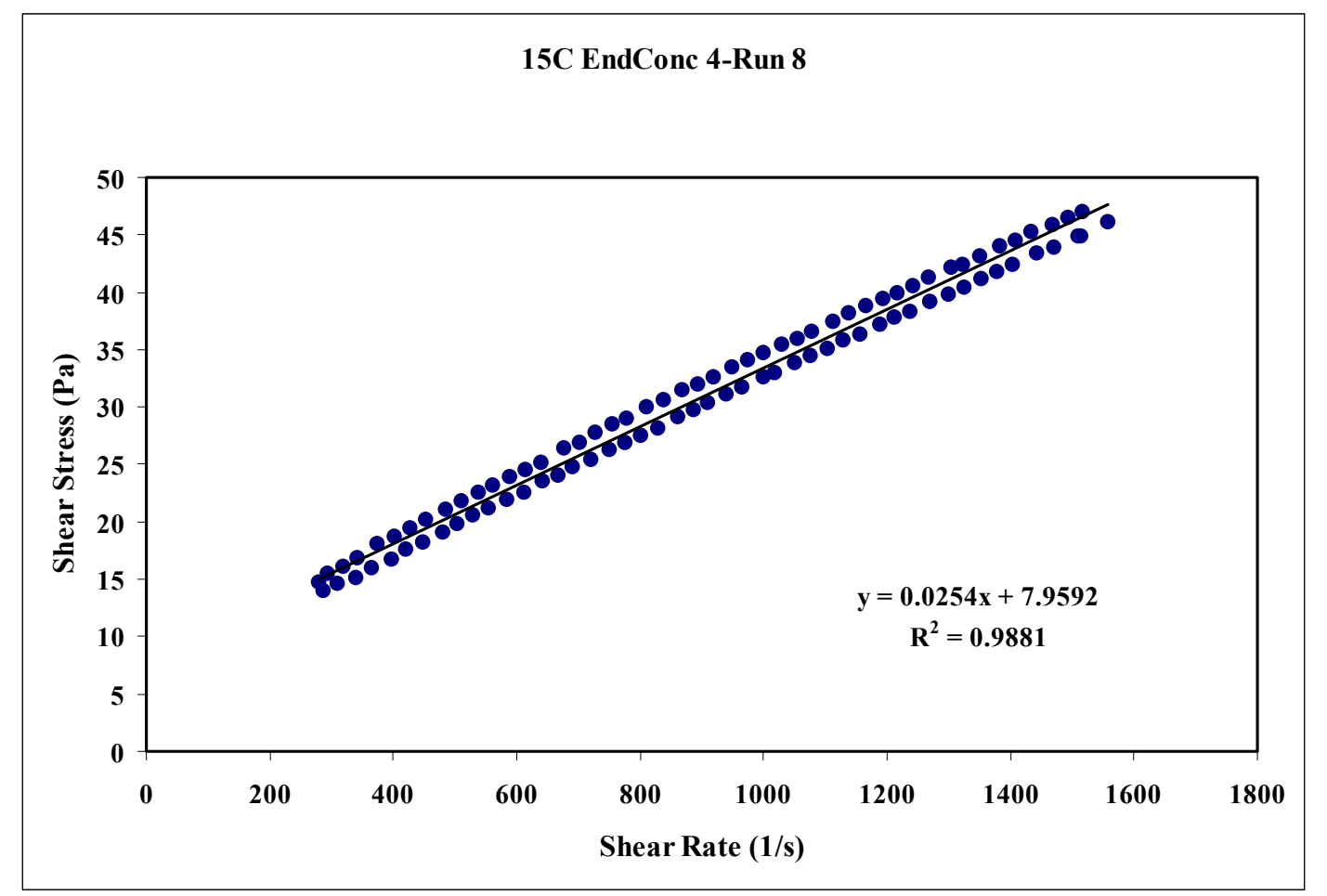

Figure 26. $15^{\circ} \mathrm{C}$ at 13 wt\% Run 8 


\begin{tabular}{|ll|}
\hline APPENDIX I - PART 3 & WSRC-TR-2000-00352 \\
& SRT-RPP-2000-00026 \\
SR/TRU PRECIPITATE SLURRY RHEOGRAMS AT 13 WT \% & Page 402 of 514 \\
\hline
\end{tabular}

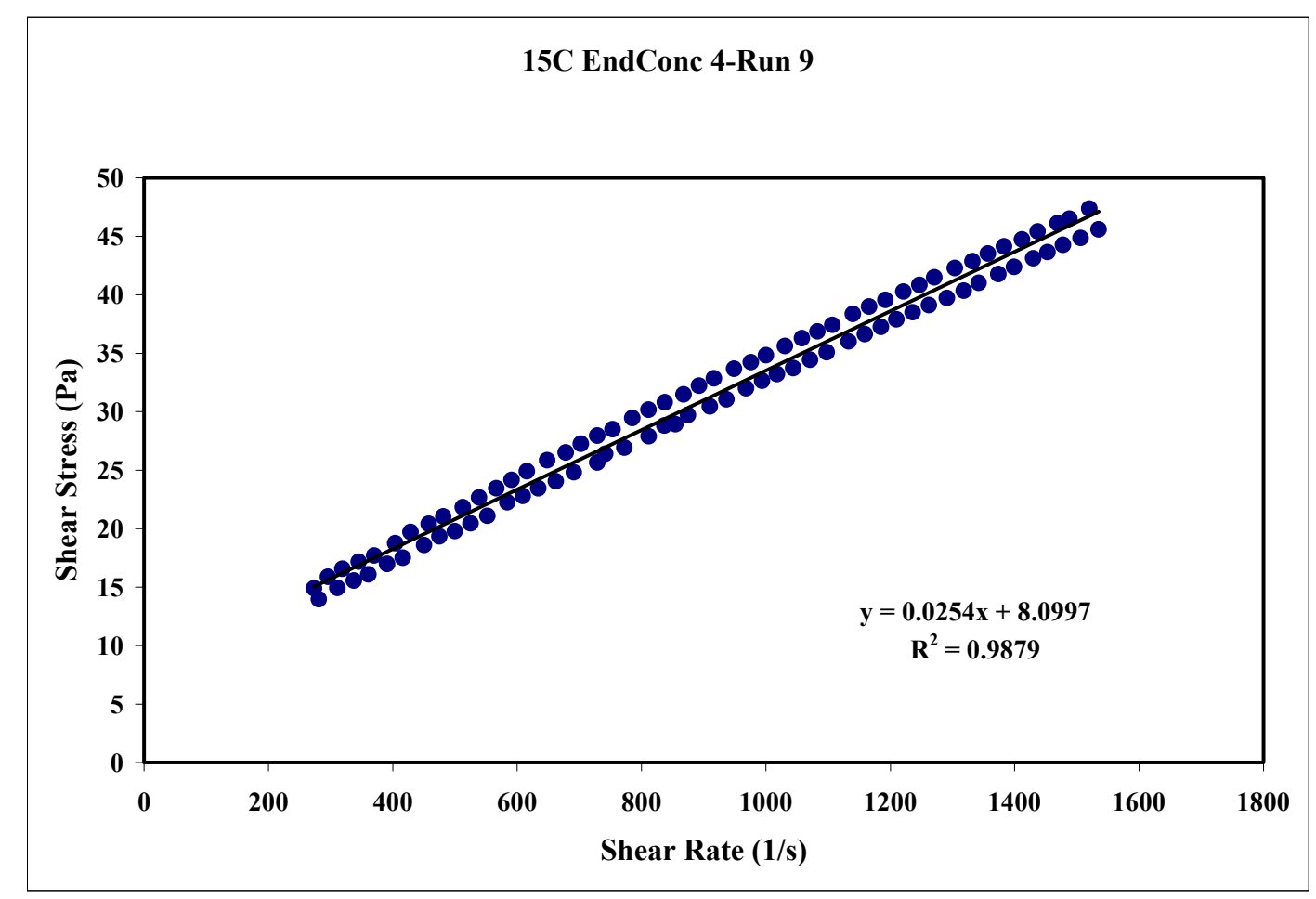

Figure 27. $15^{\circ} \mathrm{C}$ at 13 wt\% Run 9

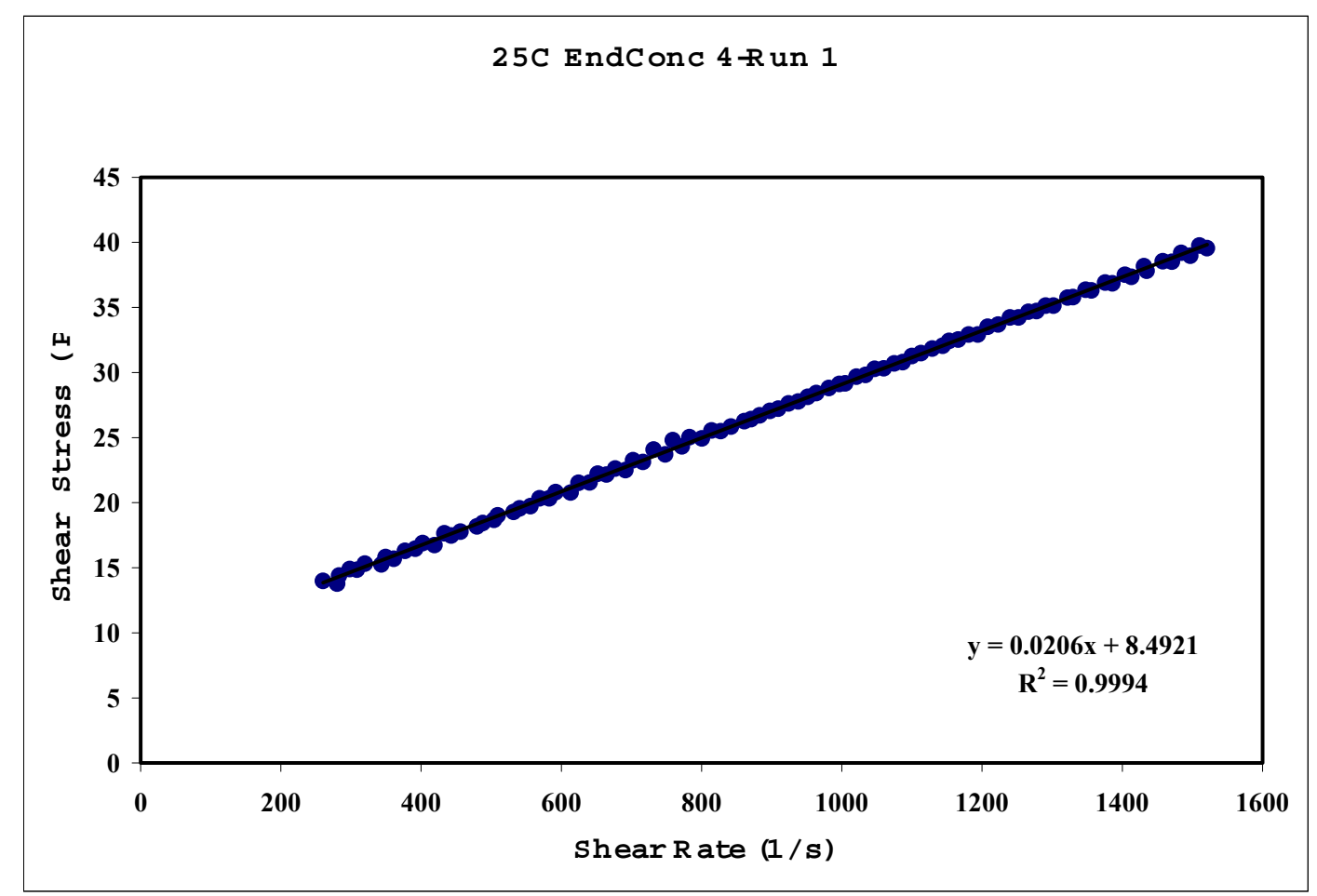

Figure 28. $25^{\circ} \mathrm{C}$ at 13 wt $\%$ Run 1 


\begin{tabular}{|ll|}
\hline APPENDIX I - PART 3 & WSRC-TR-2000-00352 \\
& SRT-RPP-2000-00026 \\
SR/TRU PRECIPITATE SLURRY RHEOGRAMS AT 13 WT \% & Page 403 of 514 \\
\hline
\end{tabular}

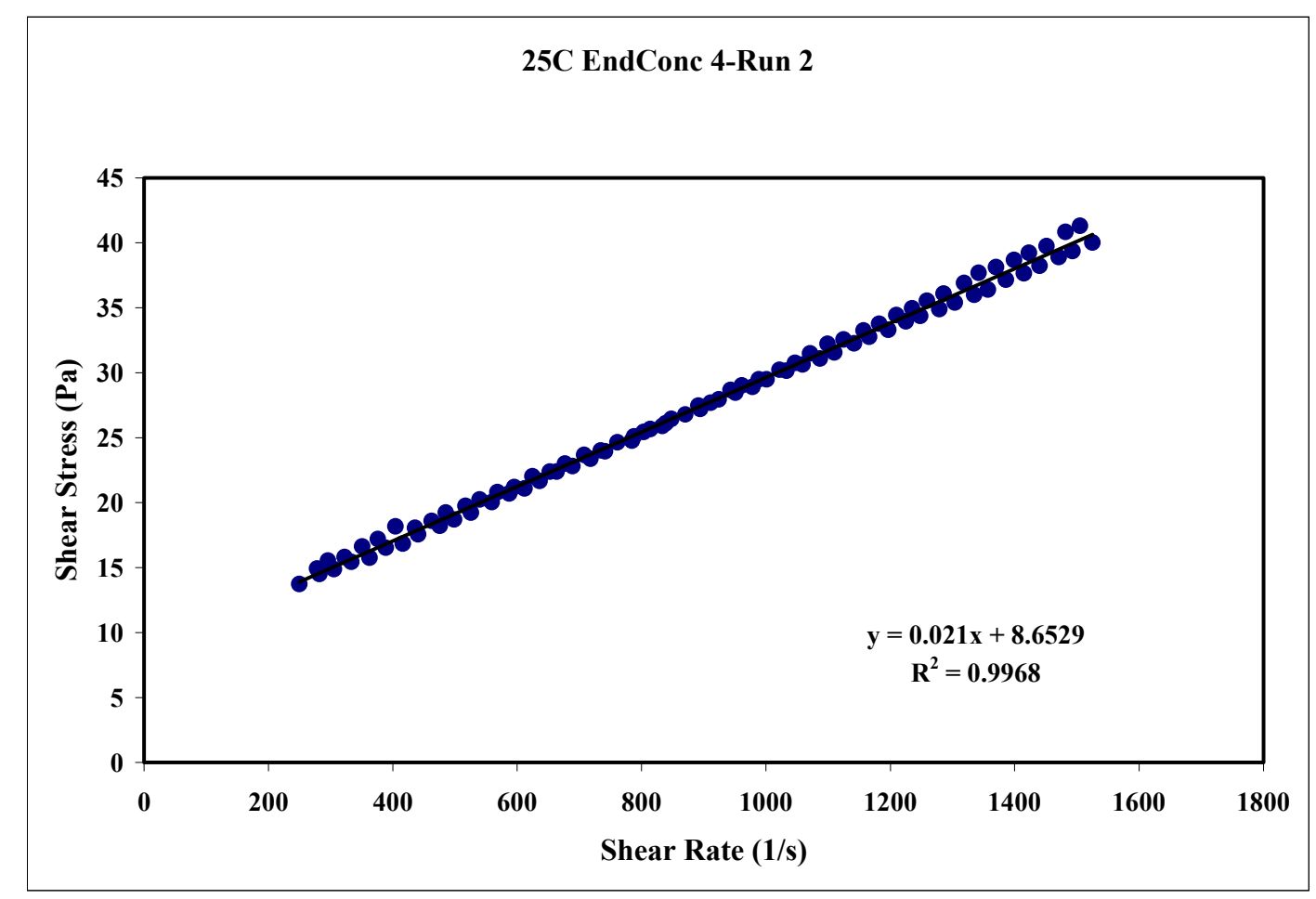

Figure 29. $25^{\circ} \mathrm{C}$ at $13 \mathrm{wt} \%$ Run 2

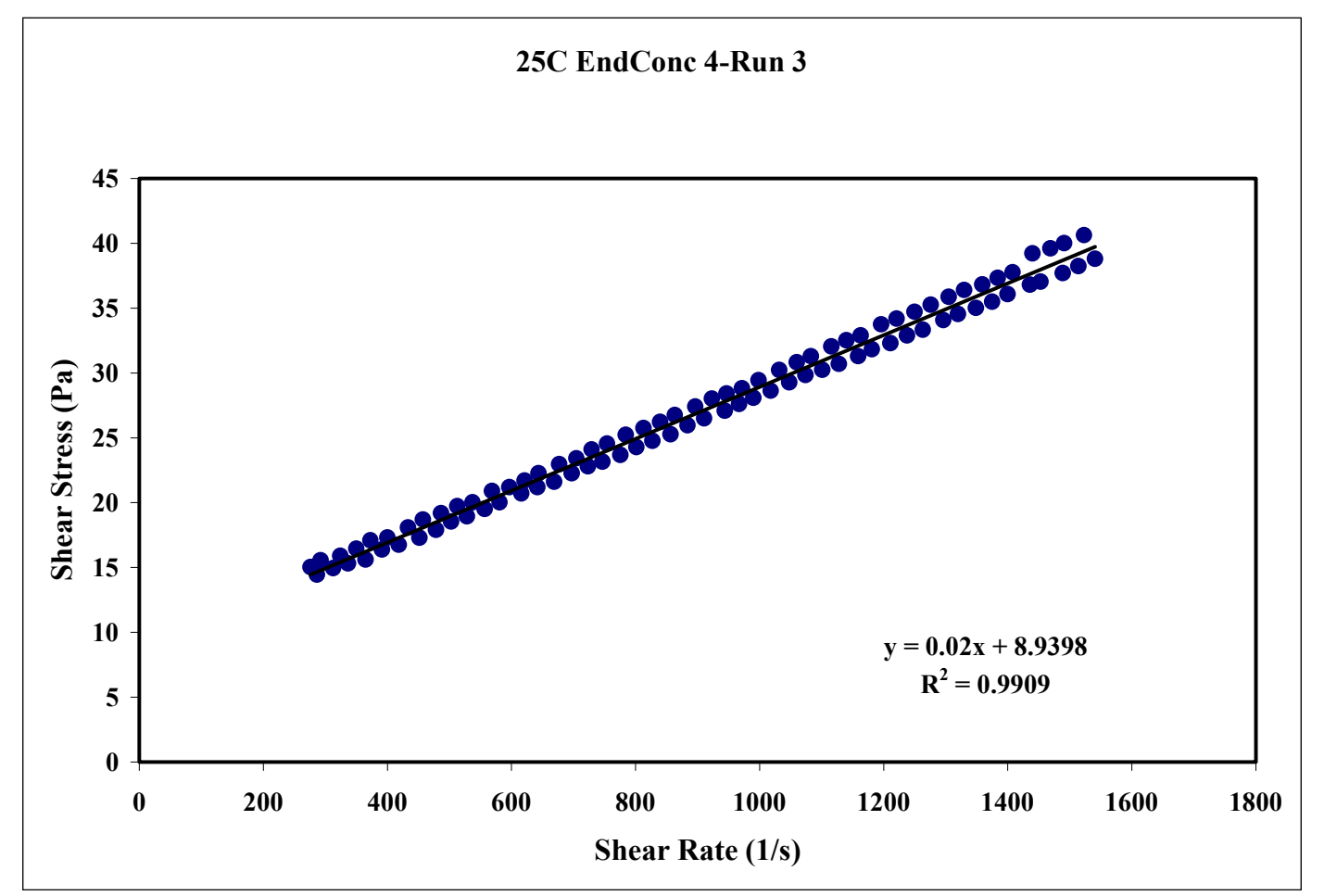

Figure 30. $25^{\circ} \mathrm{C}$ at 13 wt $\%$ Run 3 


\begin{tabular}{|ll|}
\hline APPENDIX I - PART 3 & WSRC-TR-2000-00352 \\
SR/TRU PRECIPITATE SLURRY RHEOGRAMS AT 13 WT \% & SRT-RPP-2000-00026 \\
\hline
\end{tabular}

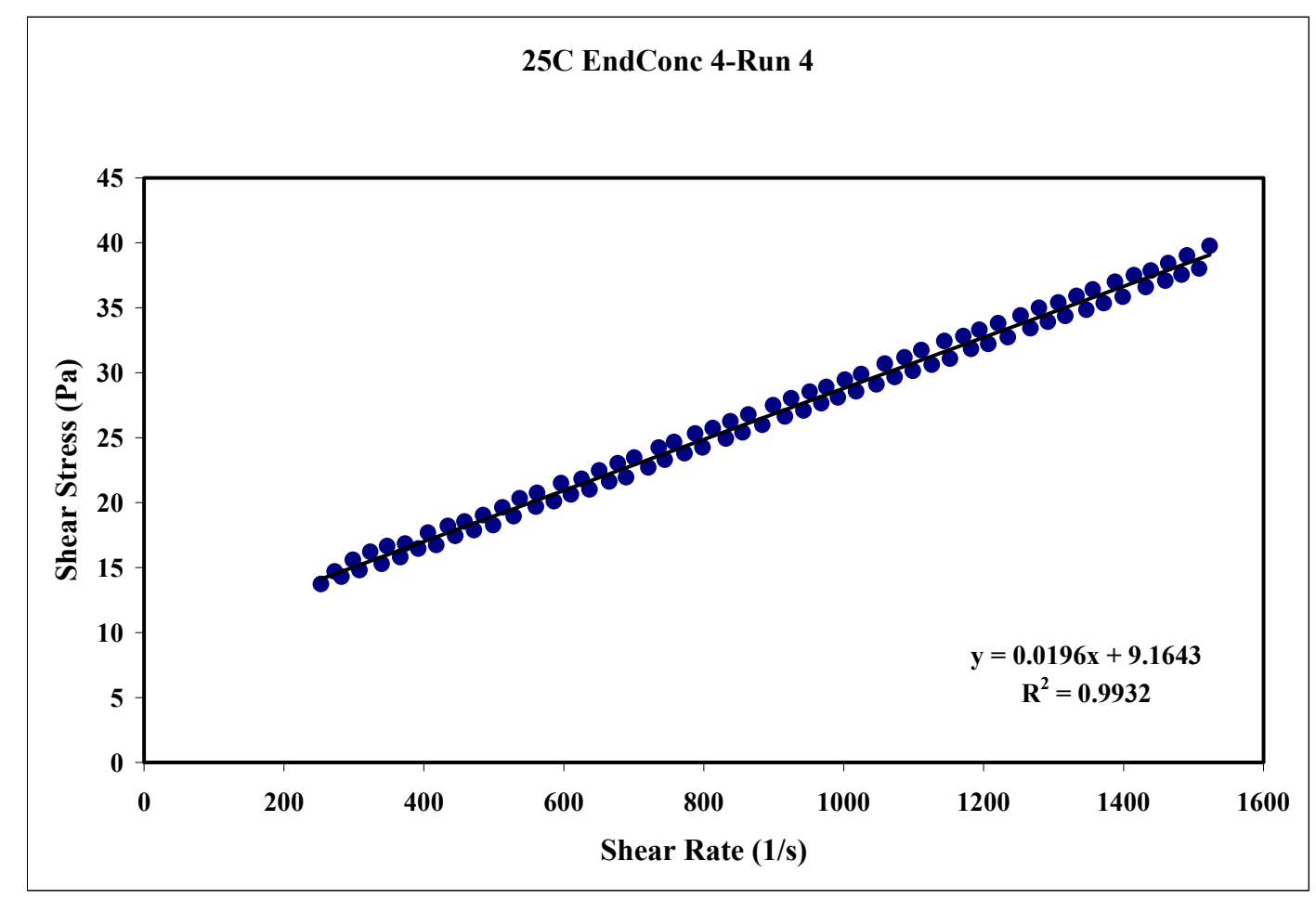

Figure 31. $25^{\circ} \mathrm{C}$ at 13 wt\% Run 4

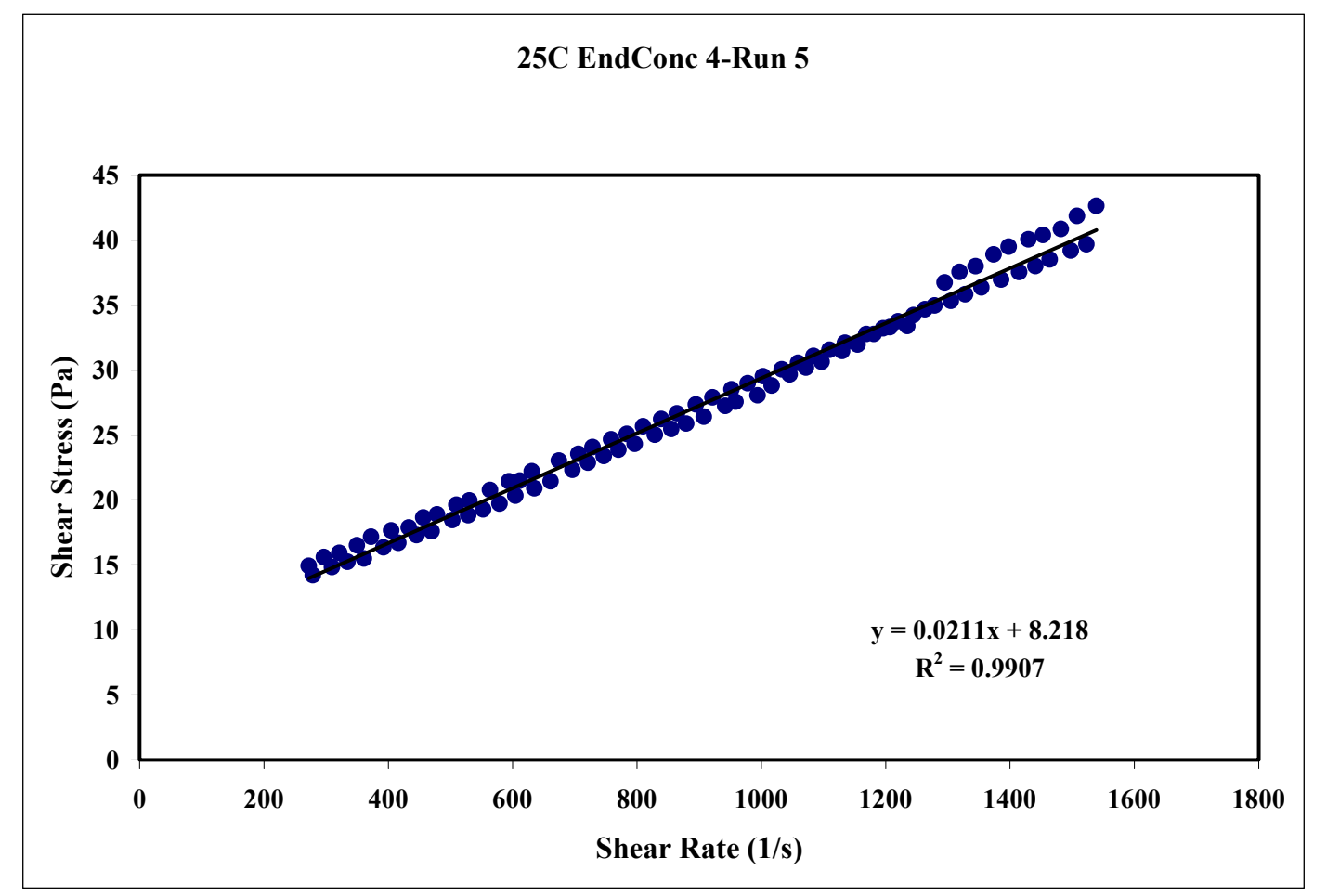

Figure 32. $25{ }^{\circ} \mathrm{C}$ at 13 wt\% Run 5 


\begin{tabular}{|ll|}
\hline APPENDIX I - PART 3 & WSRC-TR-2000-00352 \\
& SRT-RPP-2000-00026 \\
SR/TRU PRECIPITATE SLURRY RHEOGRAMS AT 13 WT \% & Page 405 of 514 \\
\hline
\end{tabular}

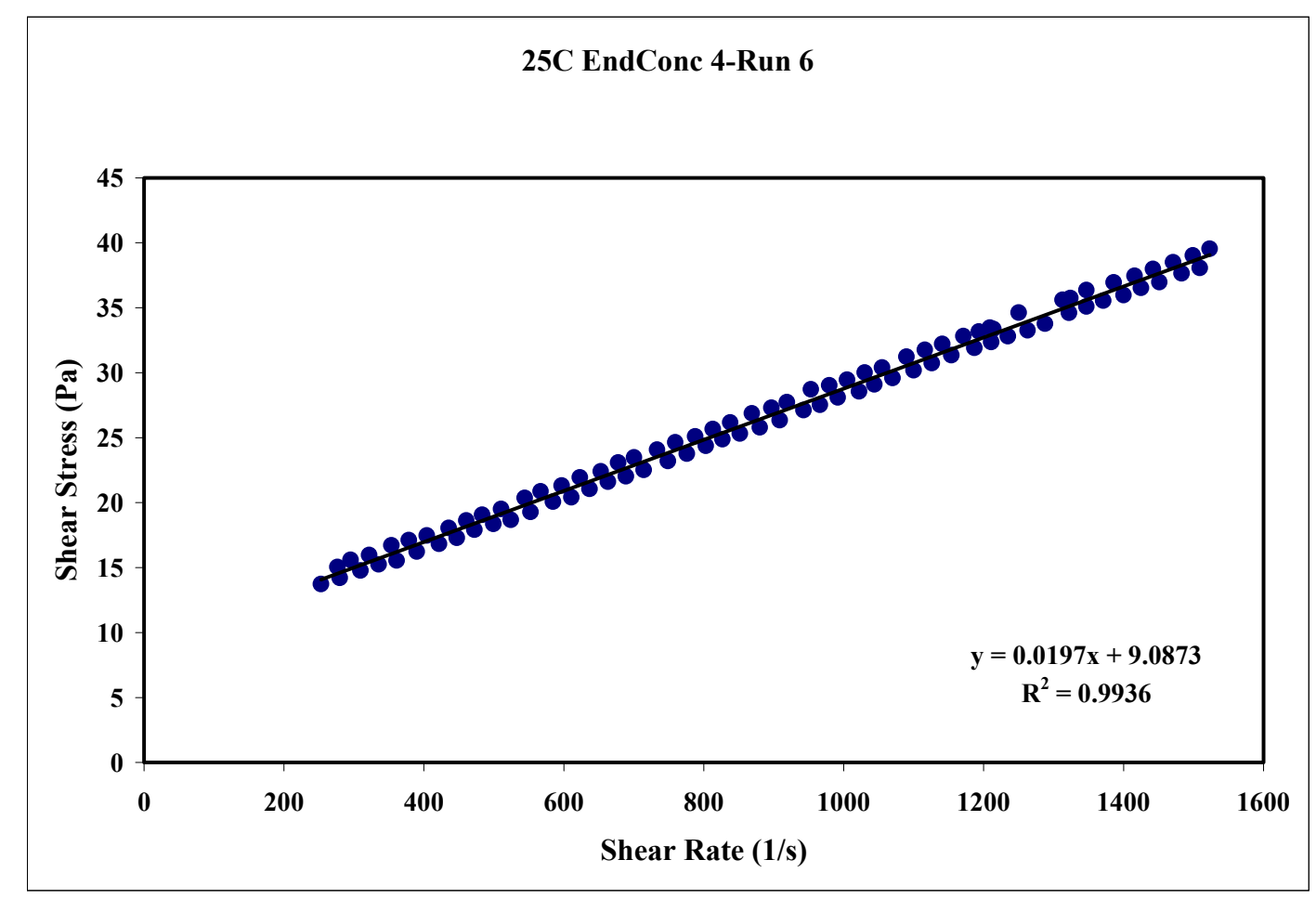

Figure 33. $25^{\circ} \mathrm{C}$ at 13 wt\% Run 6

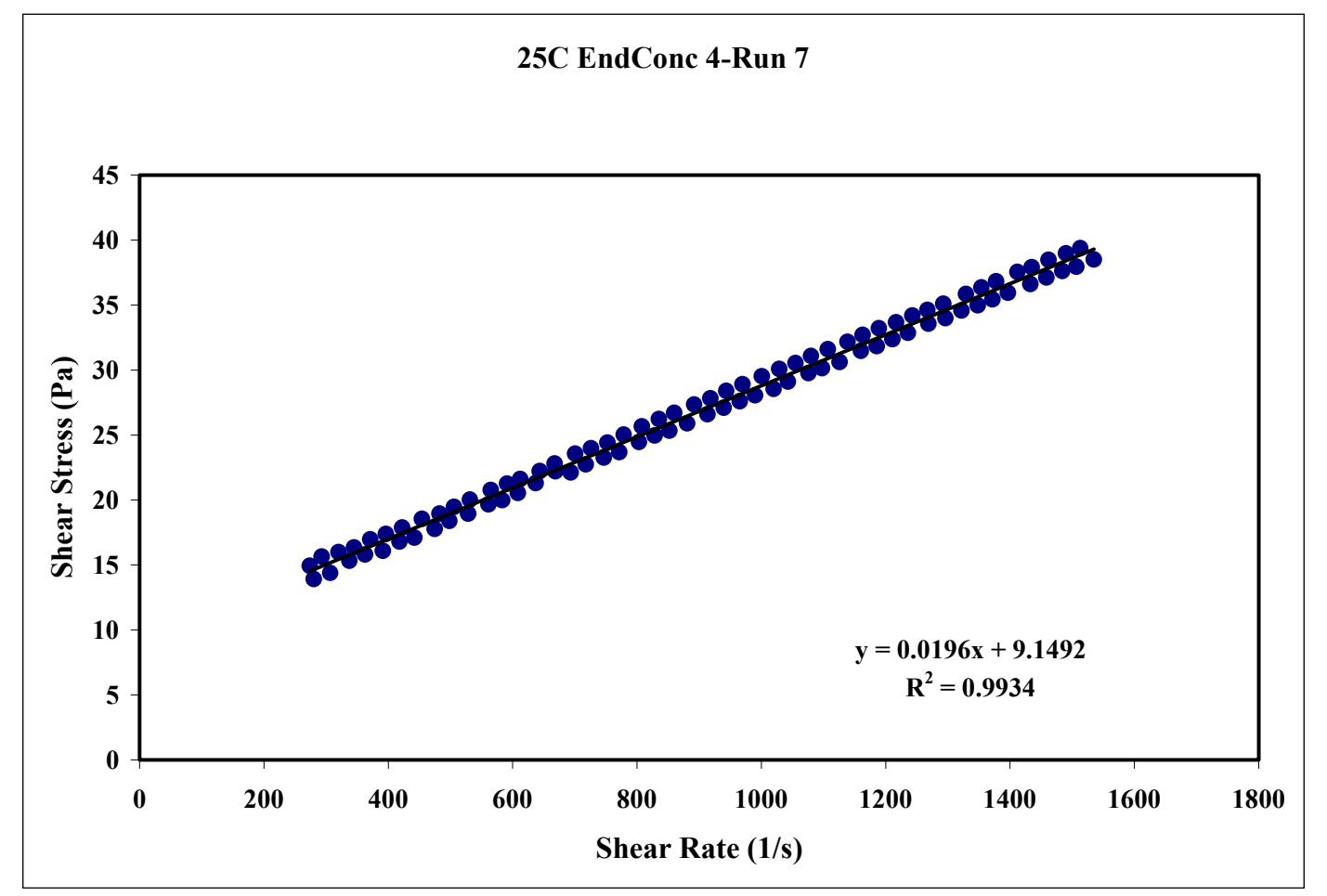

Figure 34. $25{ }^{\circ} \mathrm{C}$ at 13 wt\% Run 7 


\begin{tabular}{|ll|}
\hline APPENDIX I - PART 3 & WSRC-TR-2000-00352 \\
& SRT-RPP-2000-00026 \\
SR/TRU PRECIPITATE SLURRY RHEOGRAMS AT 13 WT \% & Page 406 of 514 \\
\hline
\end{tabular}

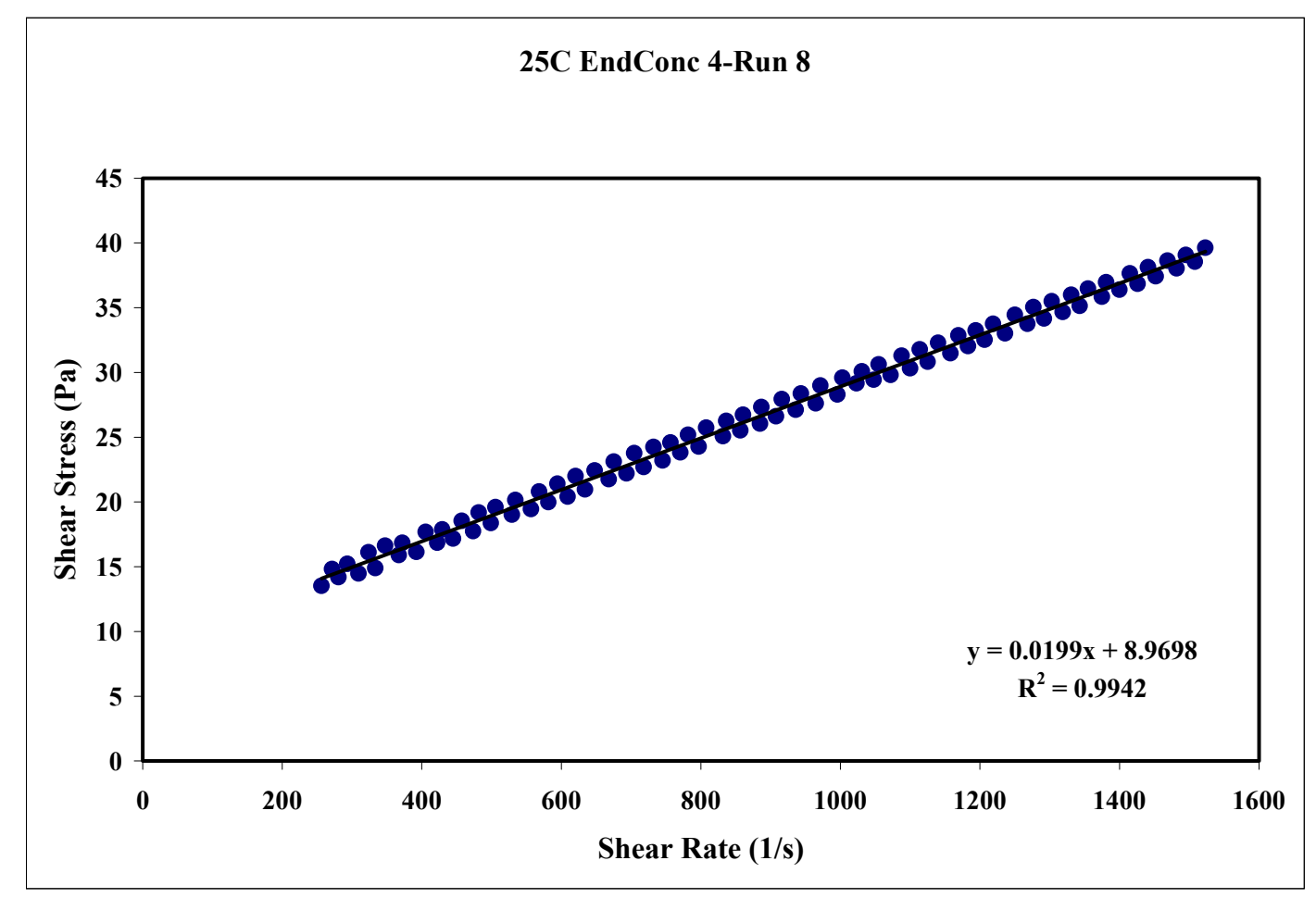

Figure 35. $25^{\circ} \mathrm{C}$ at 13 wt\% Run 8

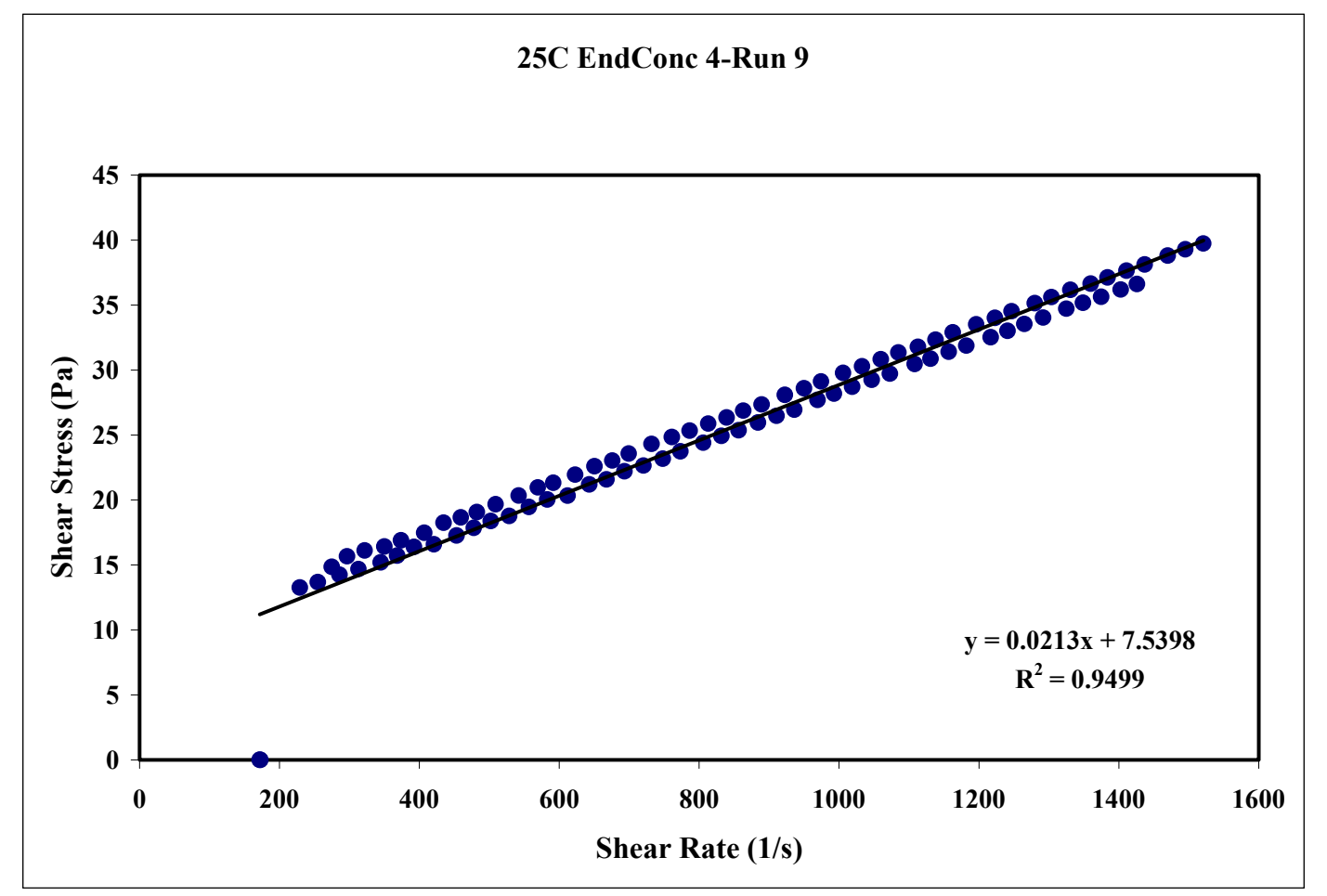

Figure 36. $25^{\circ} \mathrm{C}$ at 13 wt $\%$ Run 9 


\begin{tabular}{|ll|}
\hline APPENDIX I - PART 3 & WSRC-TR-2000-00352 \\
& SRT-RPP-2000-00026 \\
SR/TRU PRECIPITATE SLURRY RHEOGRAMS AT 13 WT \% & Page 407 of 514 \\
\hline
\end{tabular}

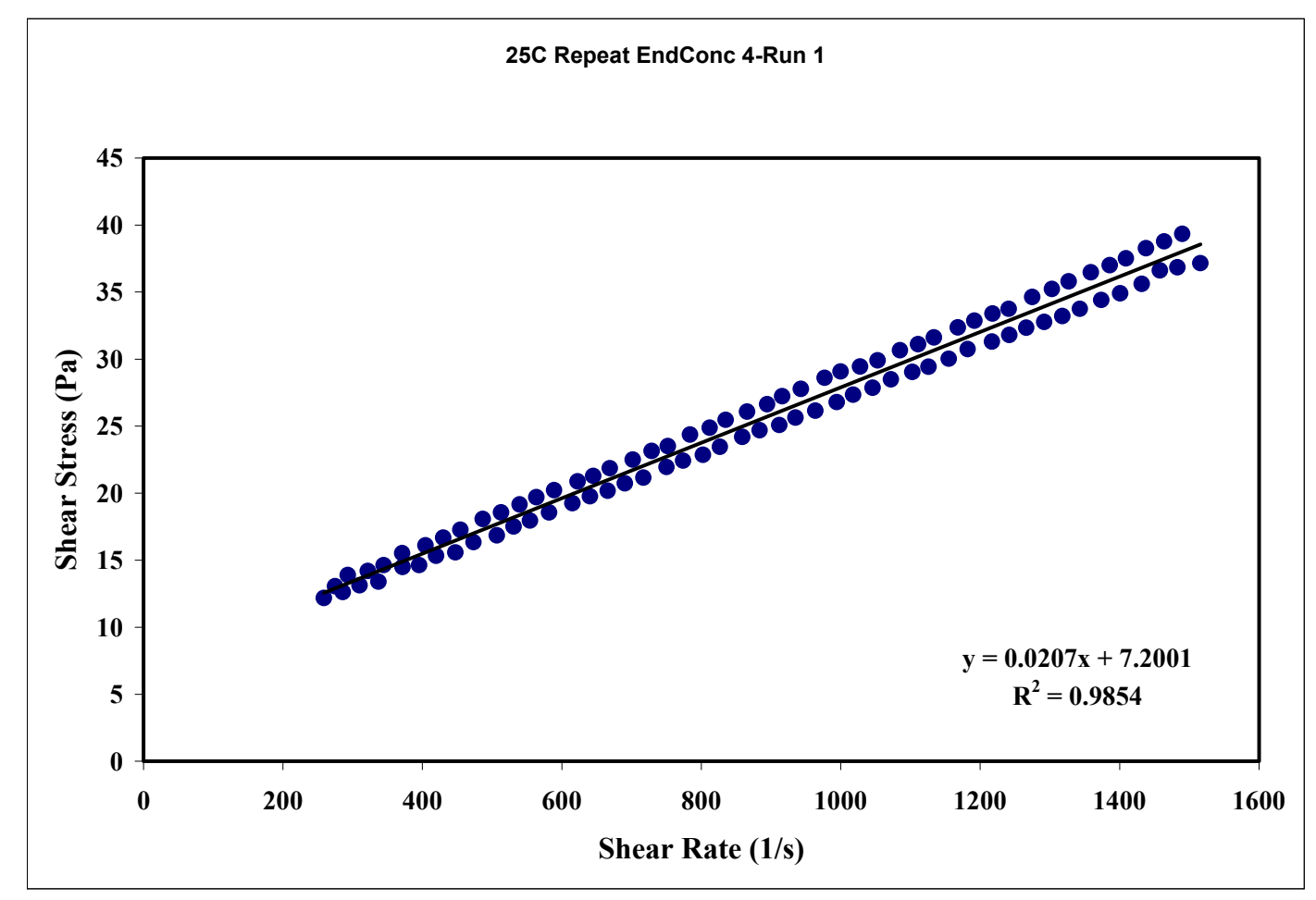

Figure 37. $25{ }^{\circ} \mathrm{C}$ Repeat at 13 wt\% Run 1

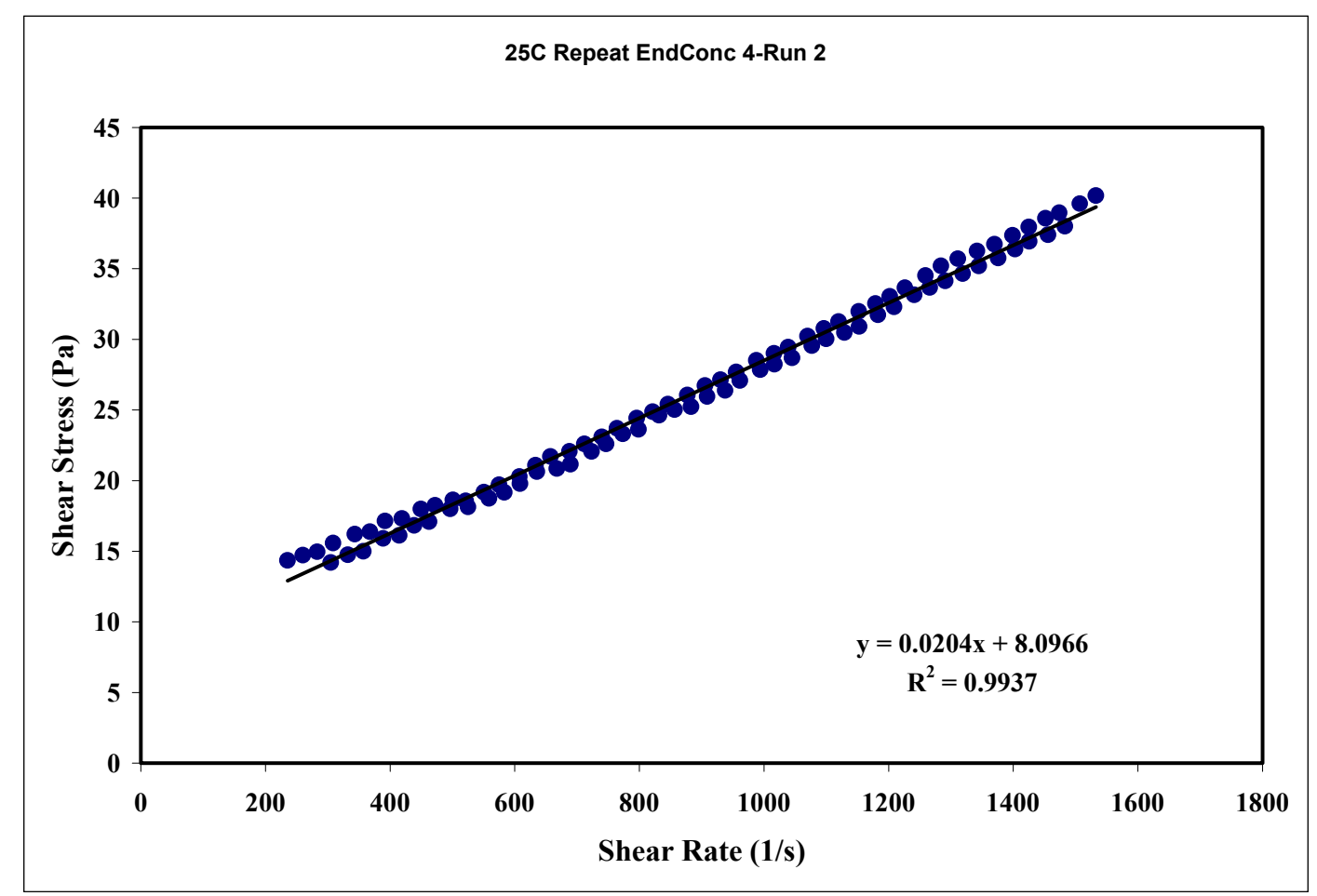

Figure 38. $25{ }^{\circ} \mathrm{C}$ Repeat at 13 wt\% Run 1 


\begin{tabular}{|ll|}
\hline APPENDIX I - PART 3 & WSRC-TR-2000-00352 \\
SR/TRU PRECIPITATE SLURRY RHEOGRAMS AT 13 WT \% & SRT-RPP-2000-00026 \\
\hline
\end{tabular}

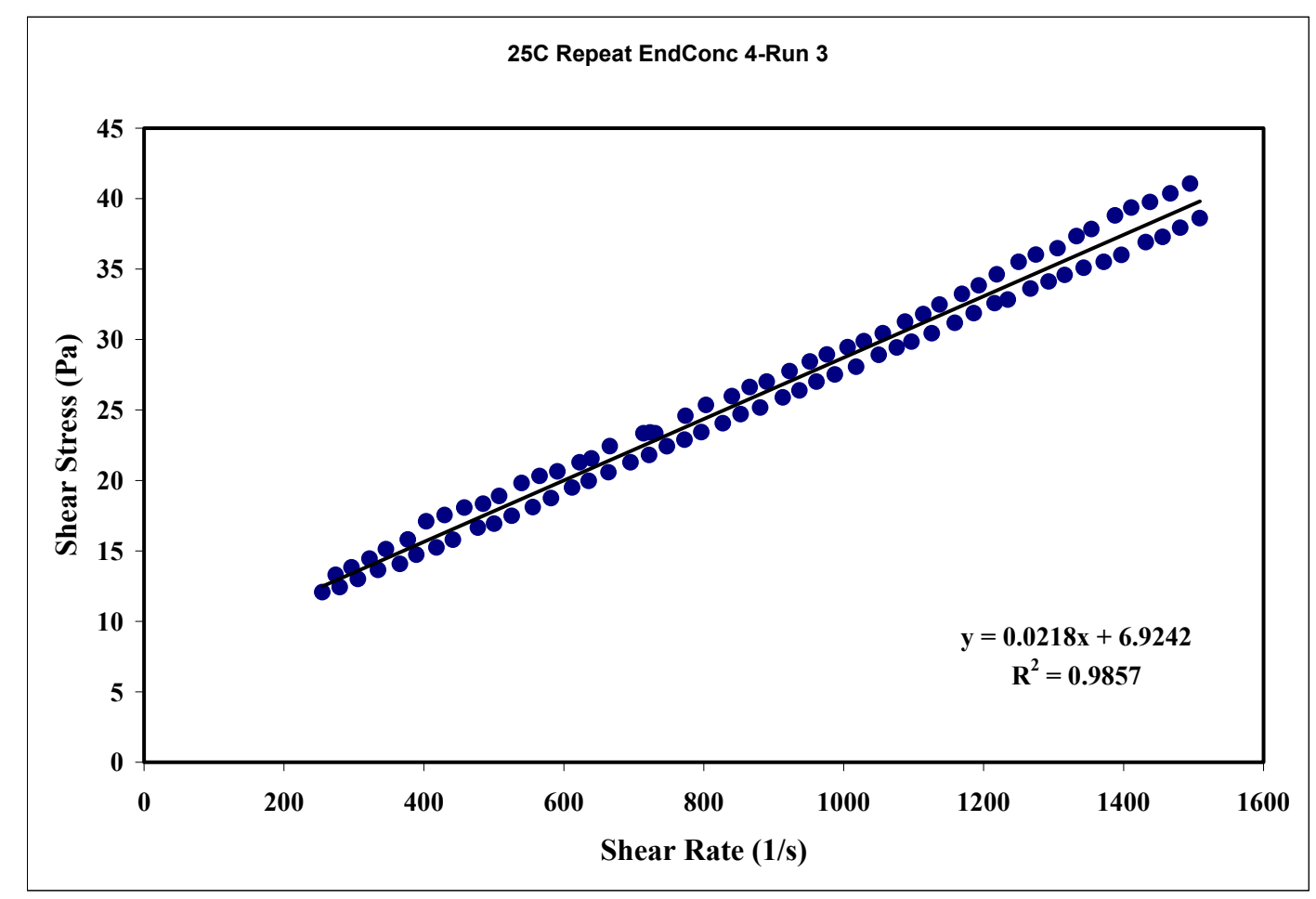

Figure 39. $25{ }^{\circ} \mathrm{C}$ Repeat at 13 wt\% Run 3

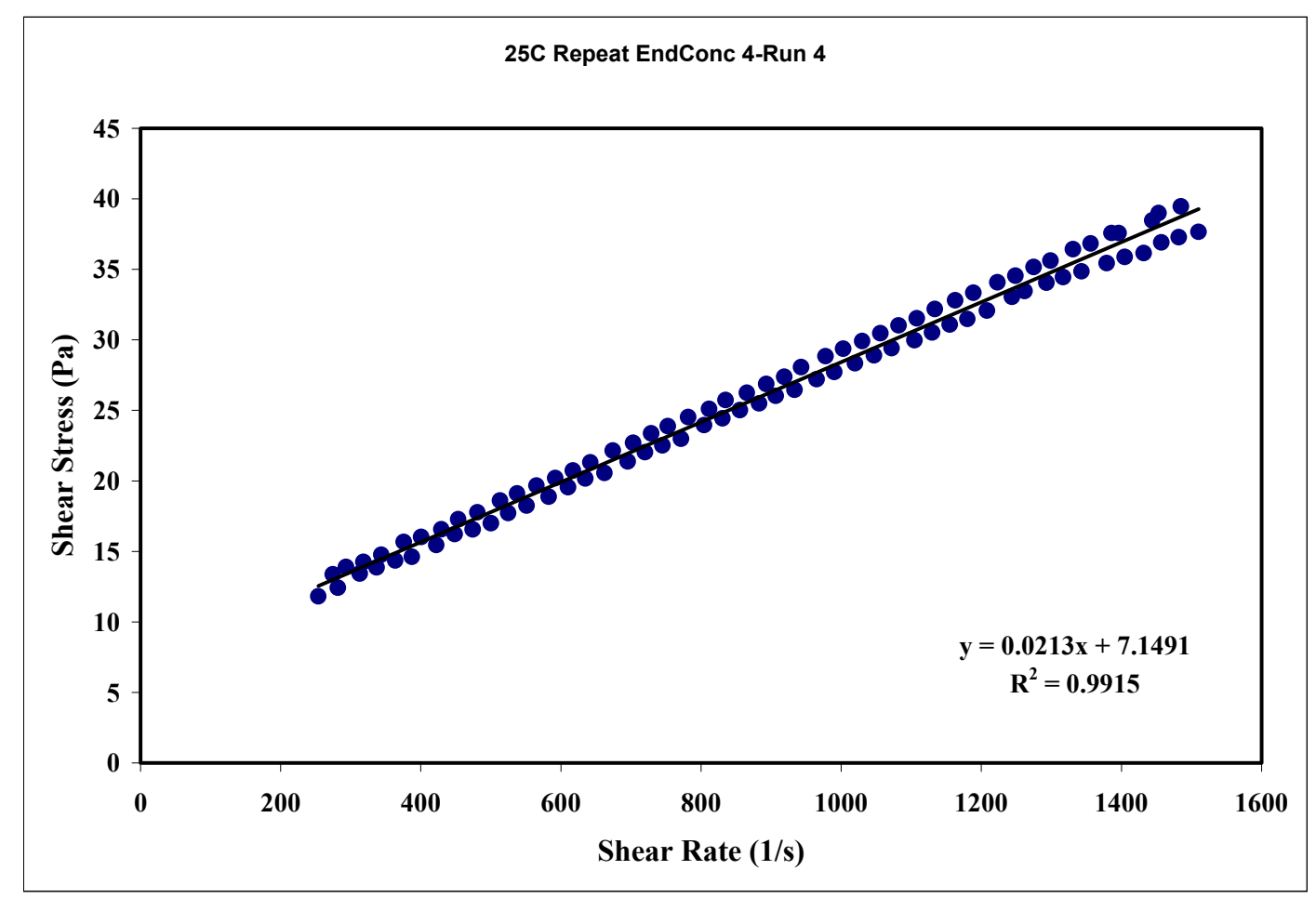

Figure 40. $25{ }^{\circ} \mathrm{C}$ Repeat at 13 wt\% Run 4 


\begin{tabular}{|ll|}
\hline APPENDIX I - PART 3 & WSRC-TR-2000-00352 \\
SR/TRU PRECIPITATE SLURRY RHEOGRAMS AT 13 WT \% & SRT-RPP-2000-00026 \\
\hline
\end{tabular}

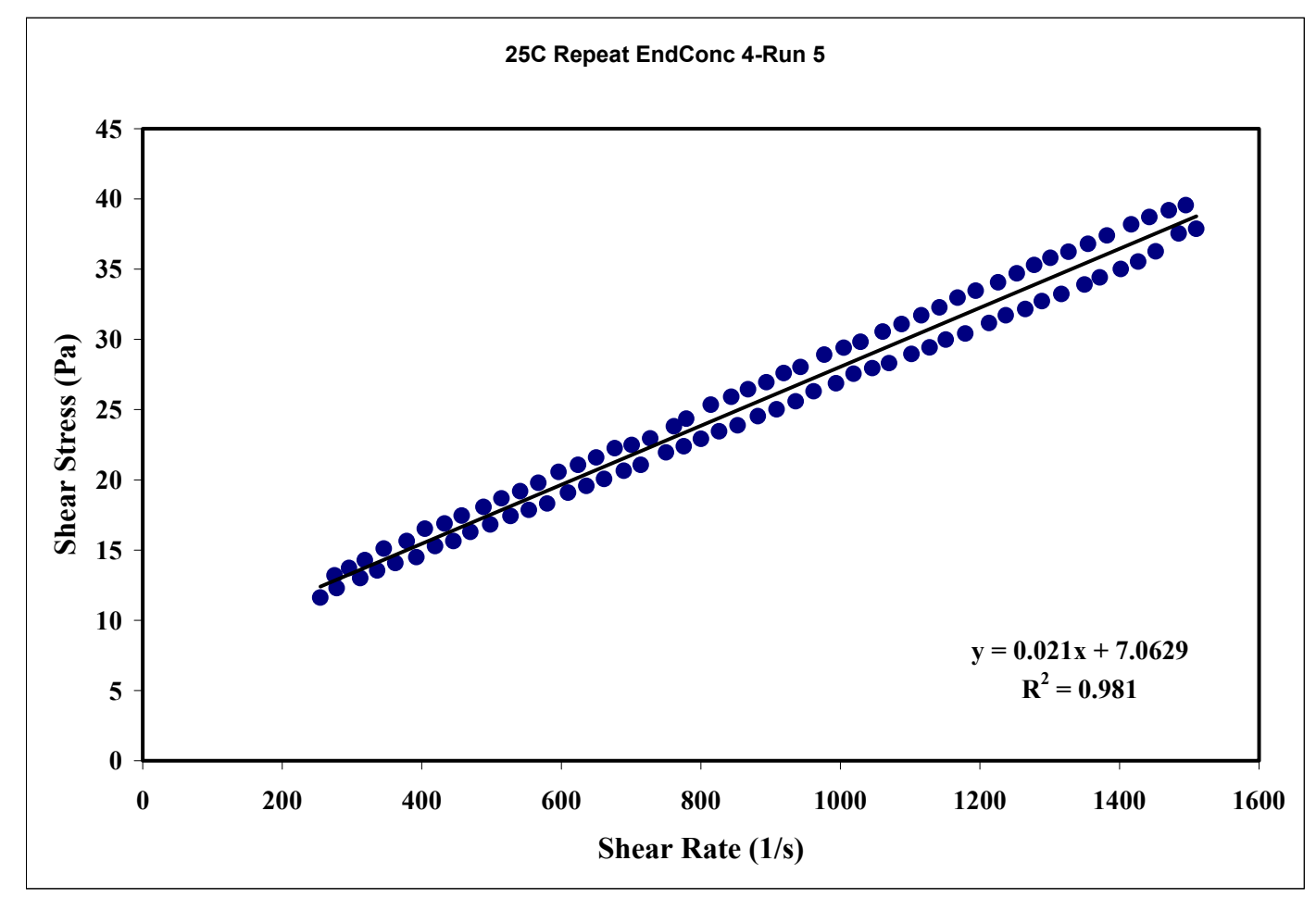

Figure 41. $25{ }^{\circ} \mathrm{C}$ Repeat at 13 wt\% Run 5

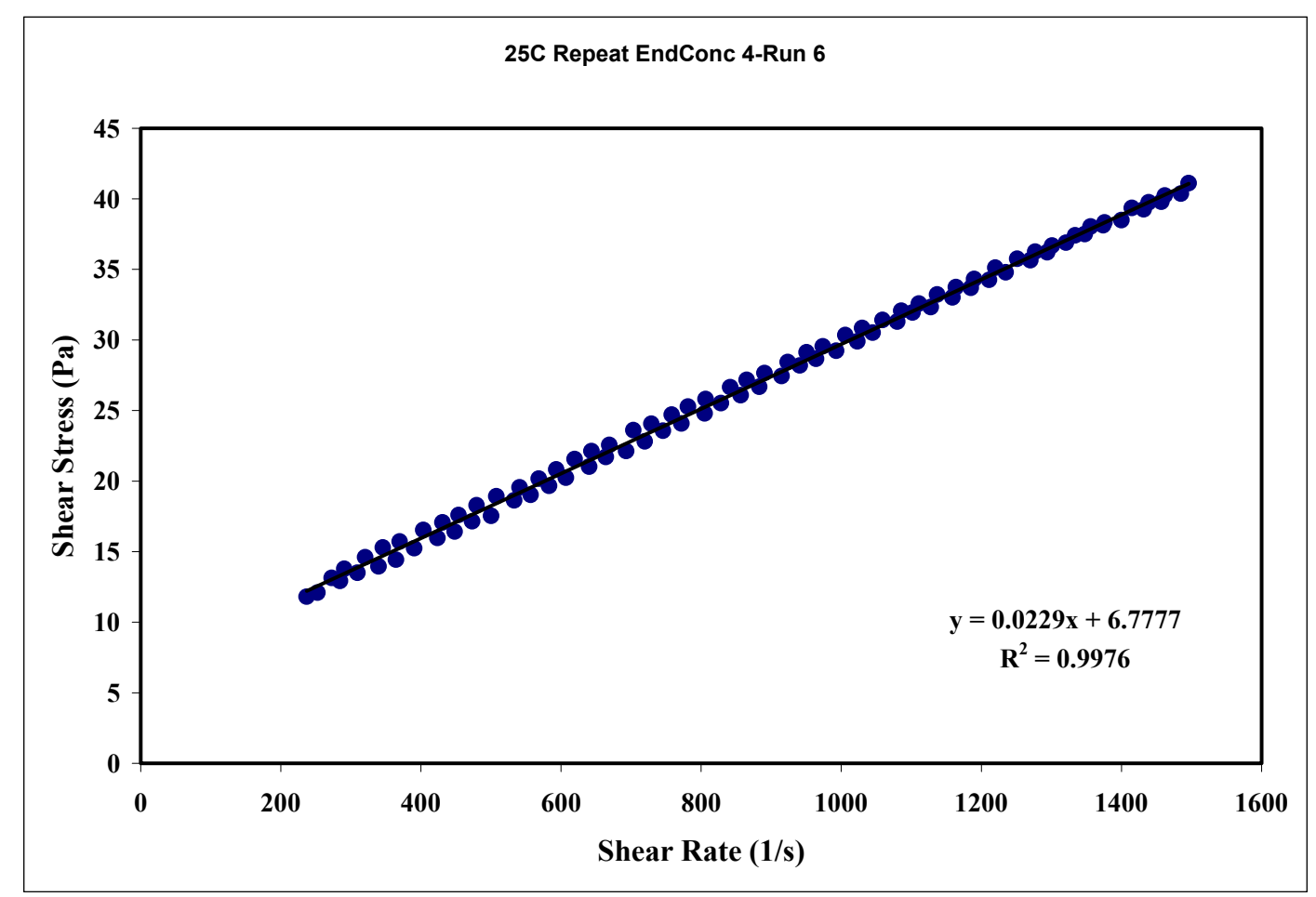

Figure 42. $25{ }^{\circ} \mathrm{C}$ Repeat at 13 wt\% Run 6 


\begin{tabular}{|ll|}
\hline APPENDIX I - PART 3 & WSRC-TR-2000-00352 \\
& SRT-RPP-2000-00026 \\
SR/TRU PRECIPITATE SLURRY RHEOGRAMS AT 13 WT \% & Page 410 of 514 \\
\hline
\end{tabular}

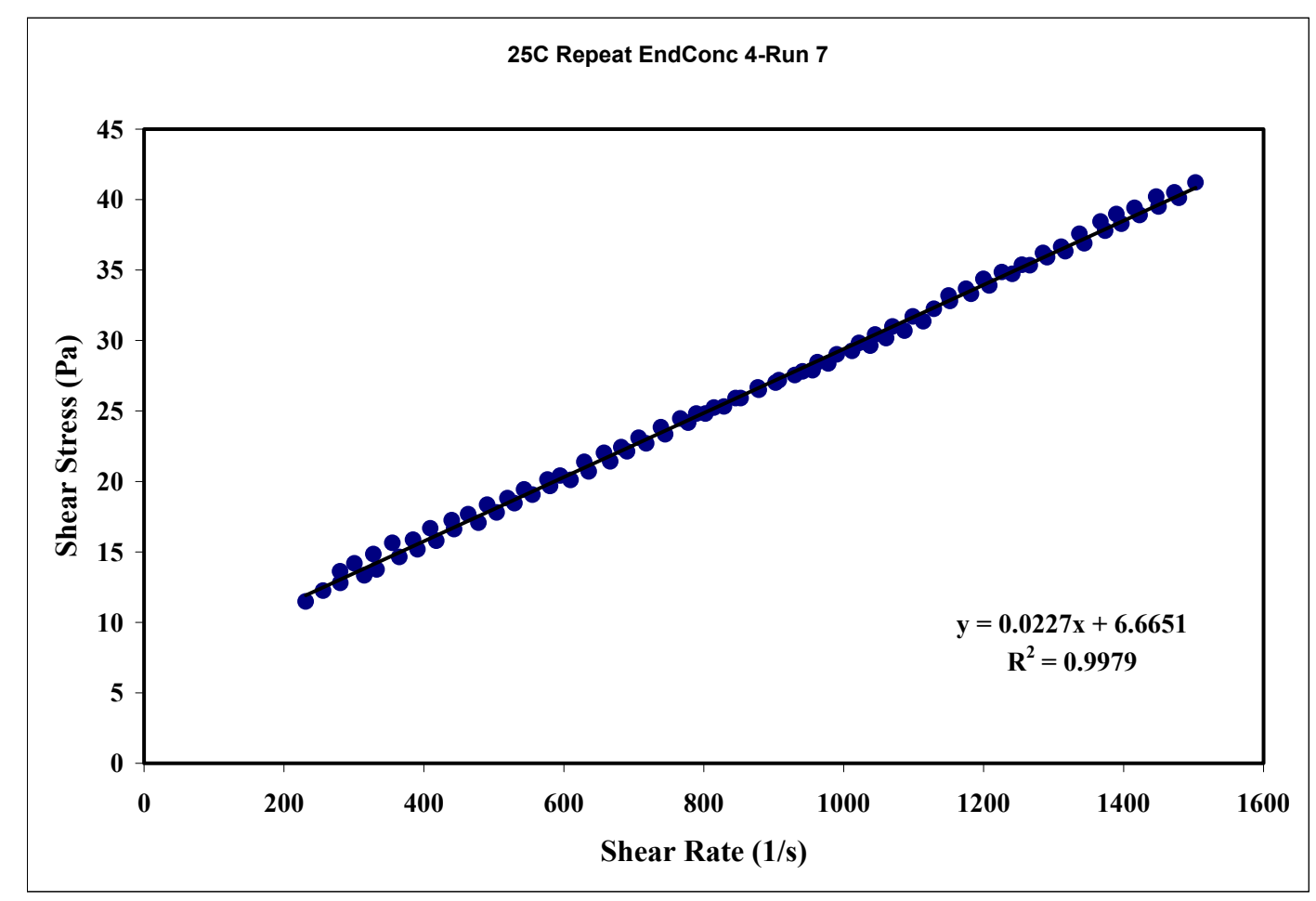

Figure 43. $25{ }^{\circ} \mathrm{C}$ Repeat at 13 wt\% Run 7

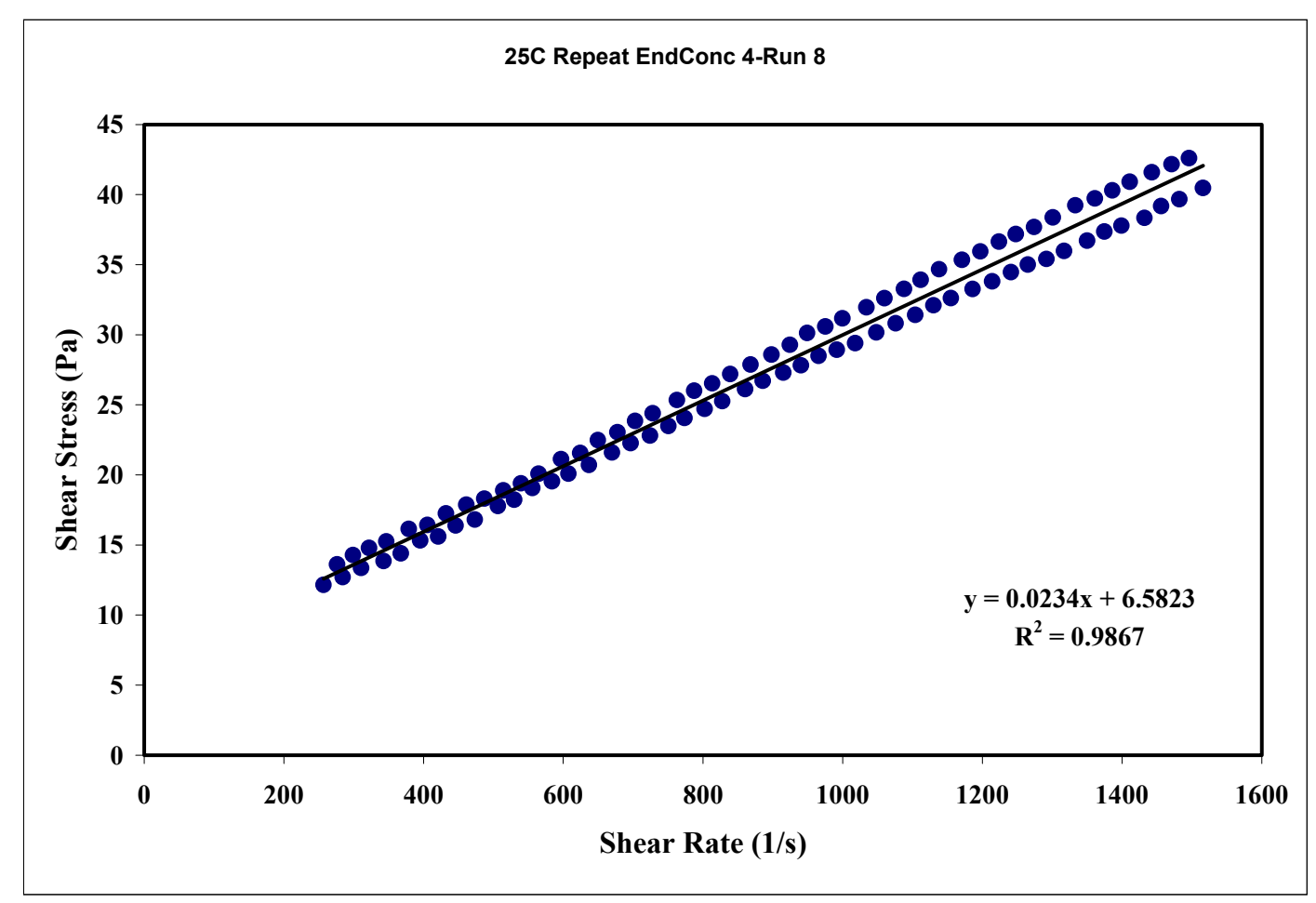

Figure 44. $25{ }^{\circ} \mathrm{C}$ Repeat at $13 \mathrm{wt} \%$ Run 8 


\begin{tabular}{|ll|}
\hline APPENDIX I - PART 3 & WSRC-TR-2000-00352 \\
SR/TRU PRECIPITATE SLURRY RHEOGRAMS AT 13 WT \% & SRT-RPP-2000-00026 \\
\hline
\end{tabular}

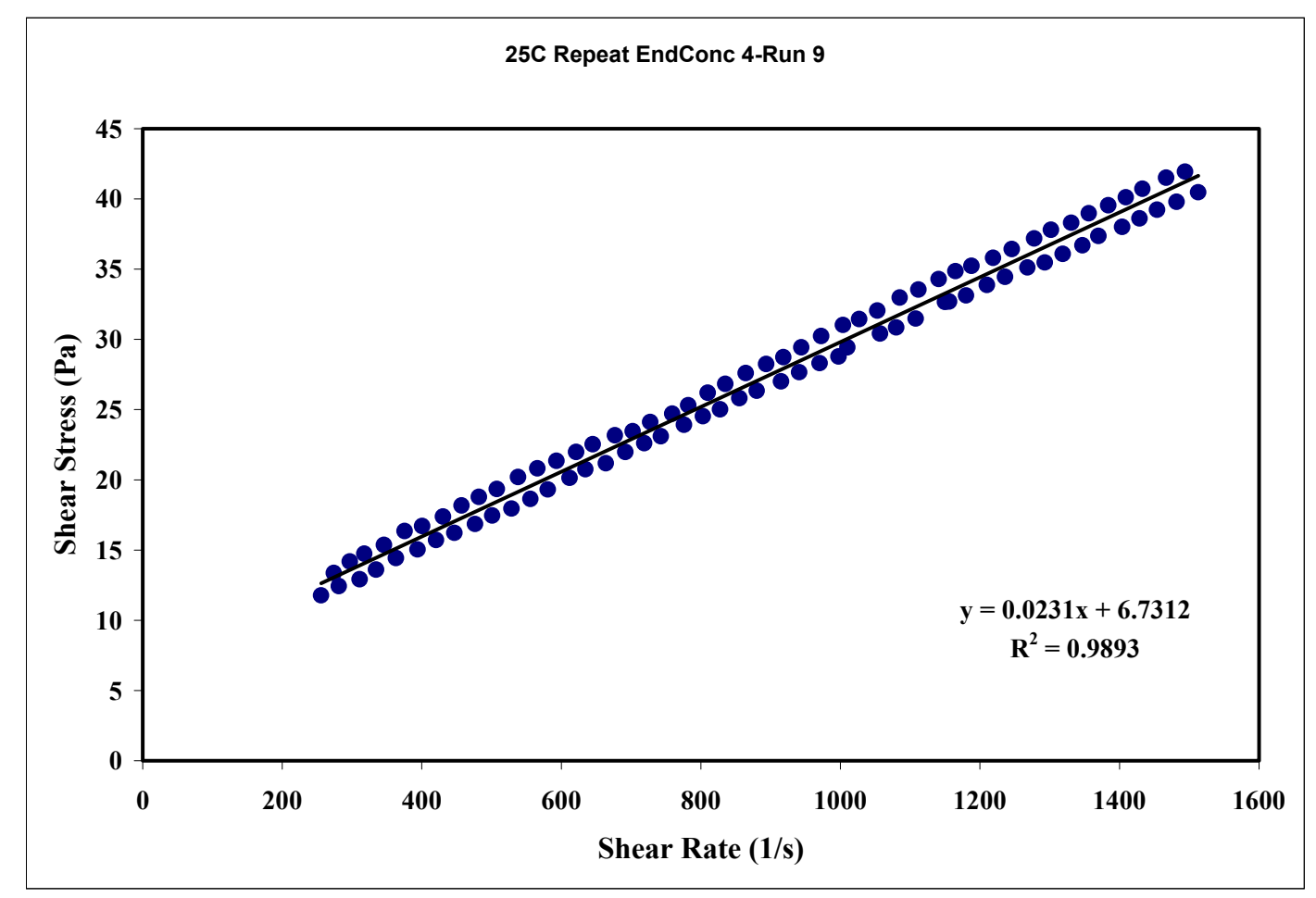

Figure 45. $25{ }^{\circ} \mathrm{C}$ Repeat at 13 wt\% Run 9

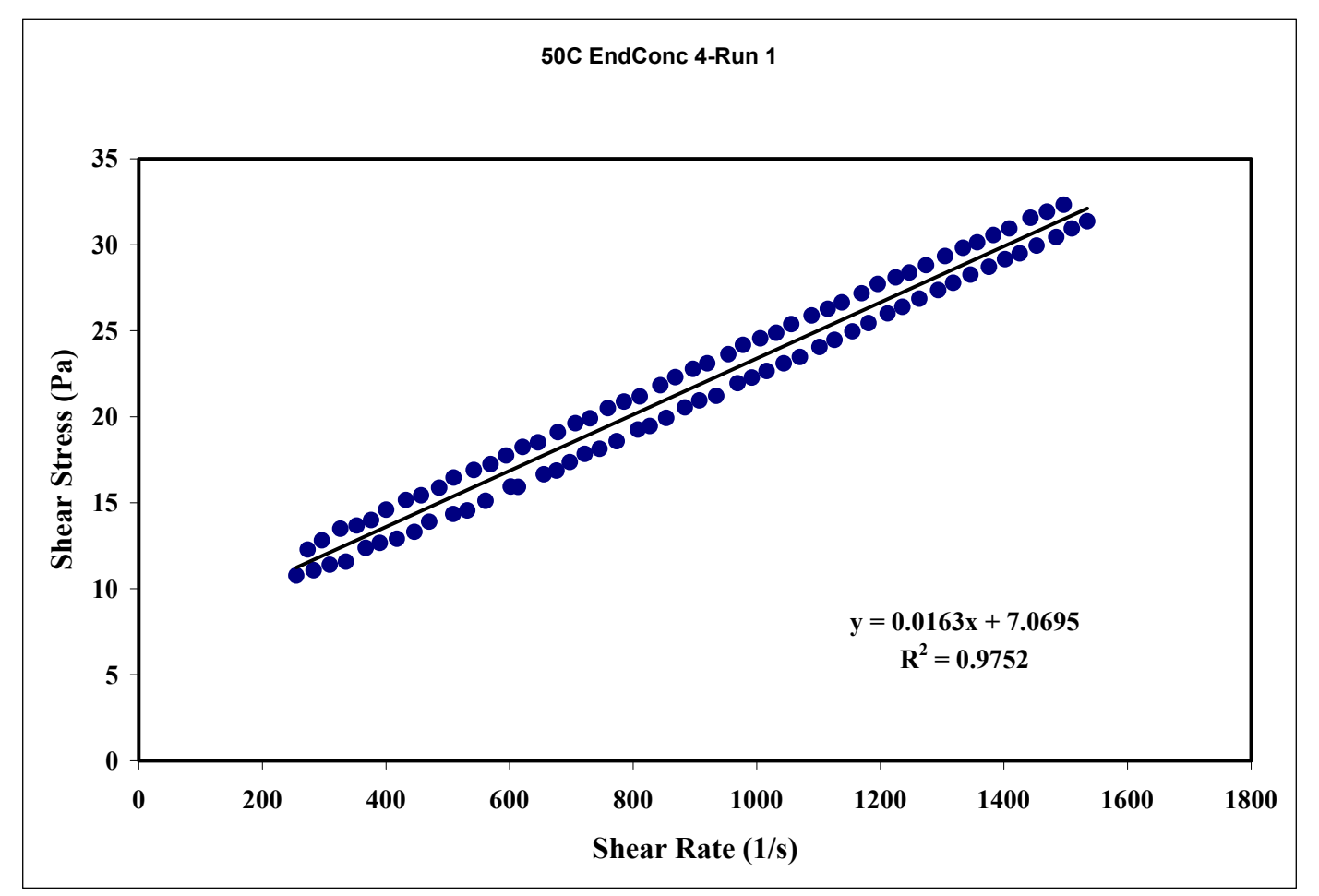

Figure $46.50{ }^{\circ} \mathrm{C}$ at $13 \mathrm{wt} \%$ Run 1 


\begin{tabular}{|ll|}
\hline APPENDIX I - PART 3 & WSRC-TR-2000-00352 \\
& SRT-RPP-2000-00026 \\
SR/TRU PRECIPITATE SLURRY RHEOGRAMS AT 13 WT \% & Page 412 of 514 \\
\hline
\end{tabular}

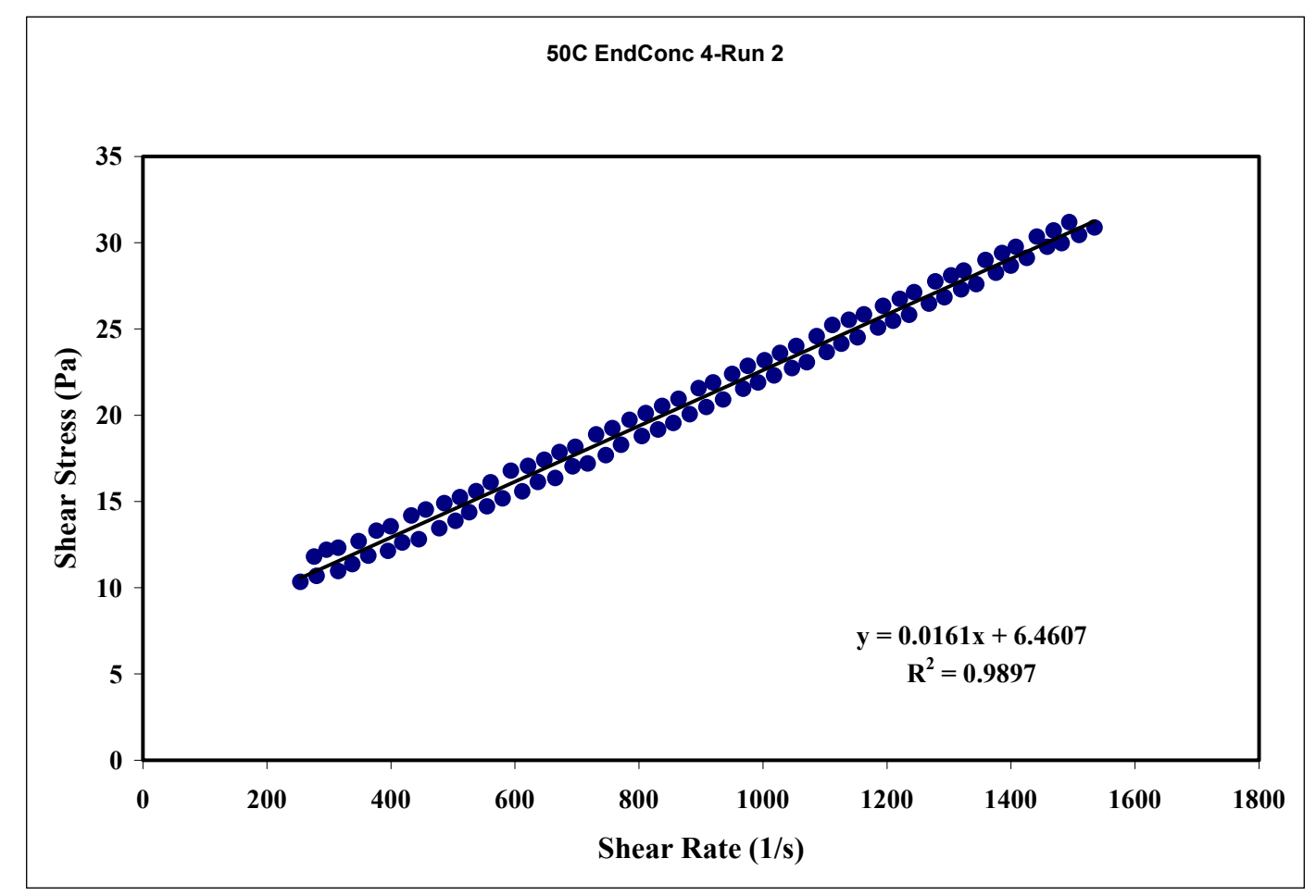

Figure $47.50{ }^{\circ} \mathrm{C}$ at $13 \mathrm{wt} \%$ Run 2

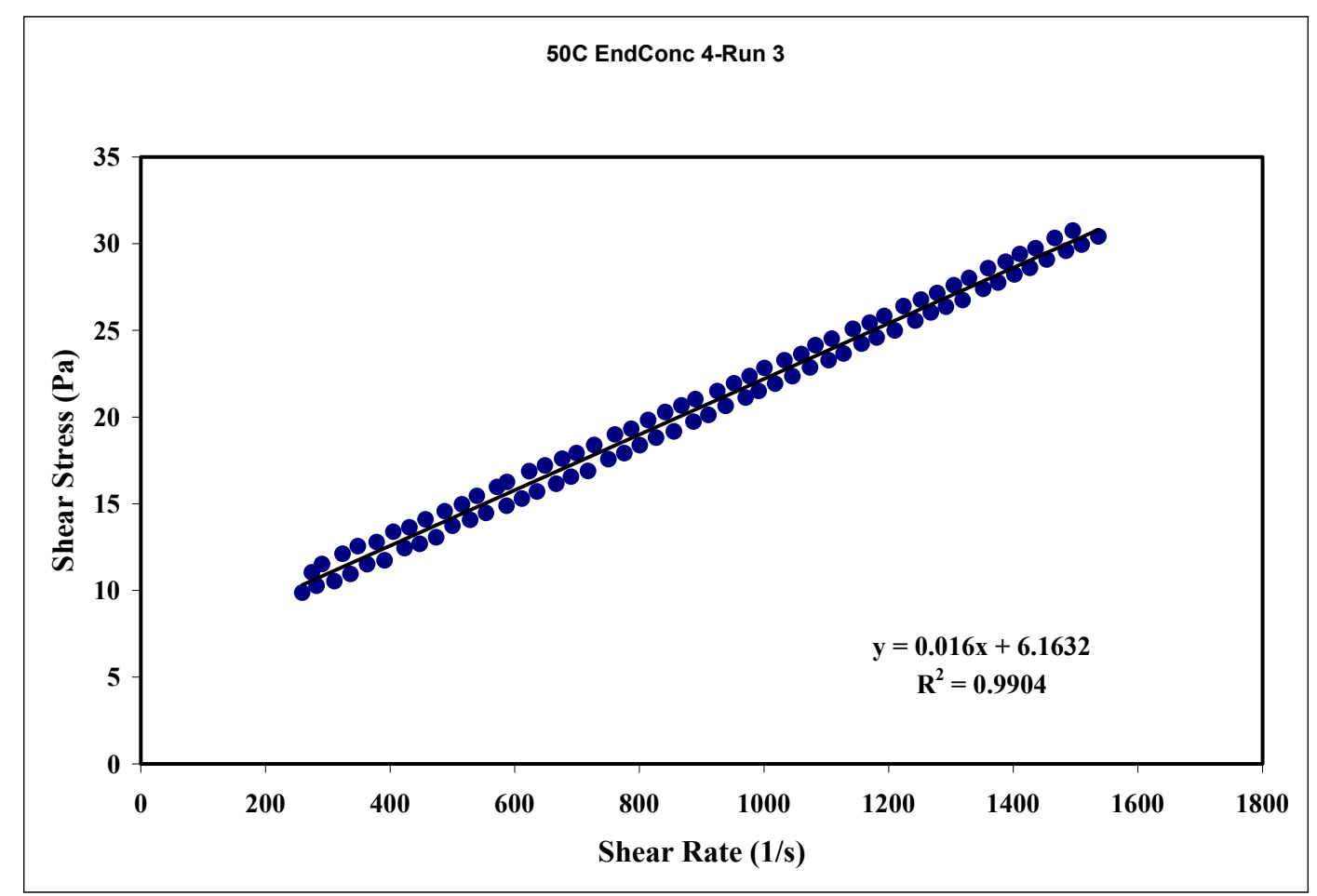

Figure $48.50{ }^{\circ} \mathrm{C}$ at 13 wt $\%$ Run 3 


\begin{tabular}{|ll|}
\hline APPENDIX I - PART 3 & WSRC-TR-2000-00352 \\
& SRT-RPP-2000-00026 \\
SR/TRU PRECIPITATE SLURRY RHEOGRAMS AT 13 WT \% & Page 413 of 514 \\
\hline
\end{tabular}

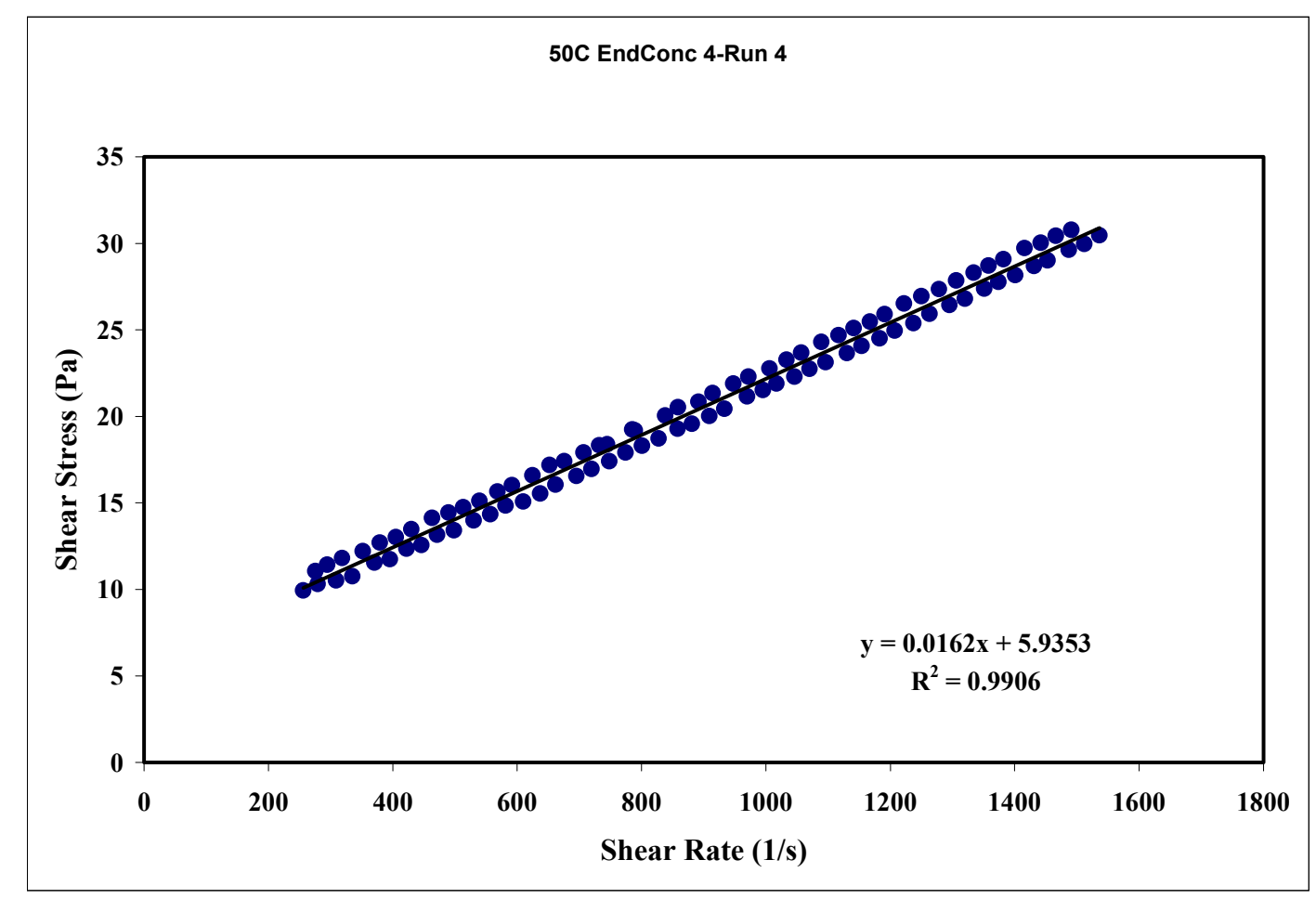

Figure 49. $50{ }^{\circ} \mathrm{C}$ at 13 wt\% Run 4

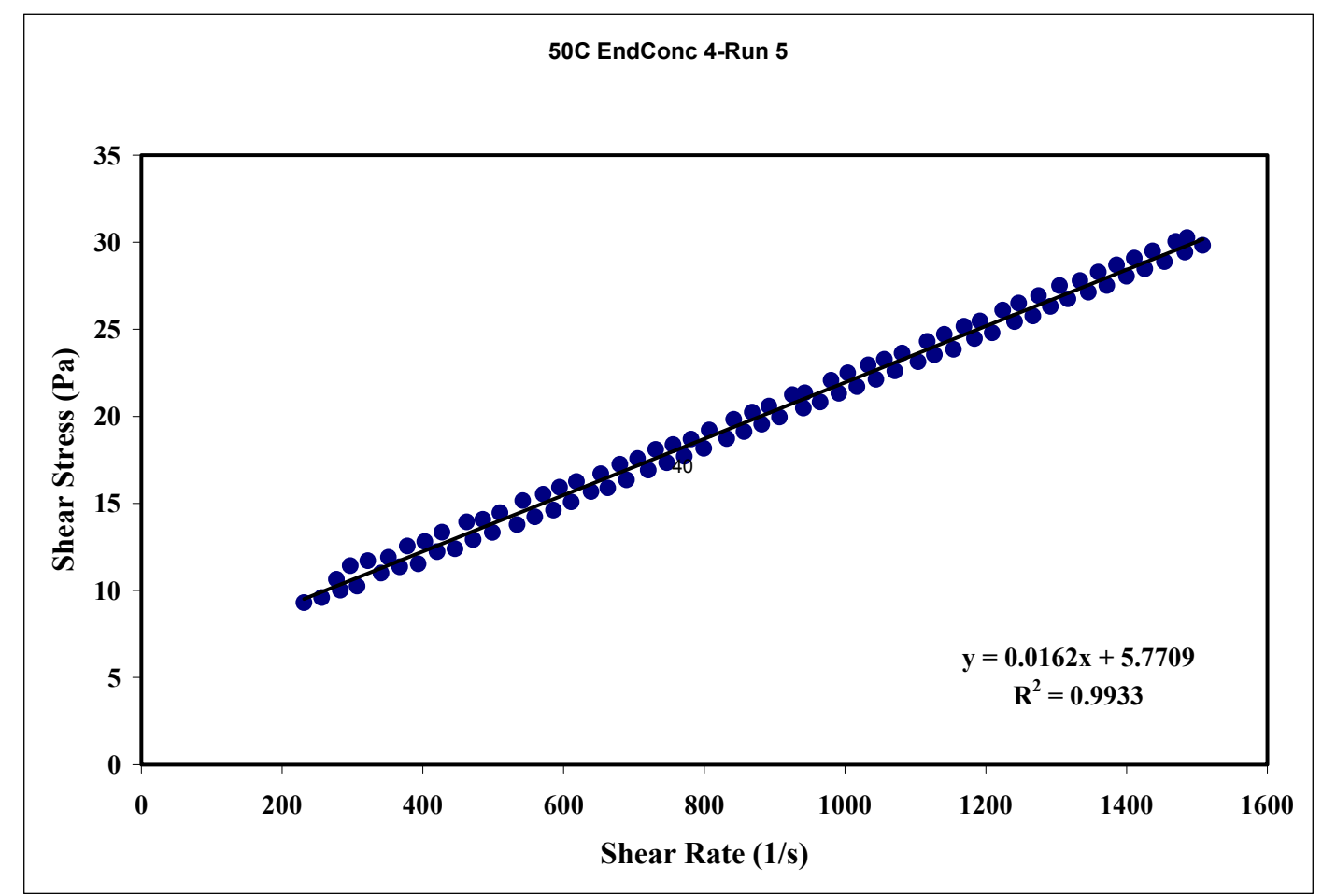

Figure 50. $50{ }^{\circ} \mathrm{C}$ at 13 wt\% Run 5 


\begin{tabular}{|ll|}
\hline APPENDIX I - PART 3 & WSRC-TR-2000-00352 \\
& SRT-RPP-2000-00026 \\
SR/TRU PRECIPITATE SLURRY RHEOGRAMS AT 13 WT \% & Page 414 of 514 \\
\hline
\end{tabular}

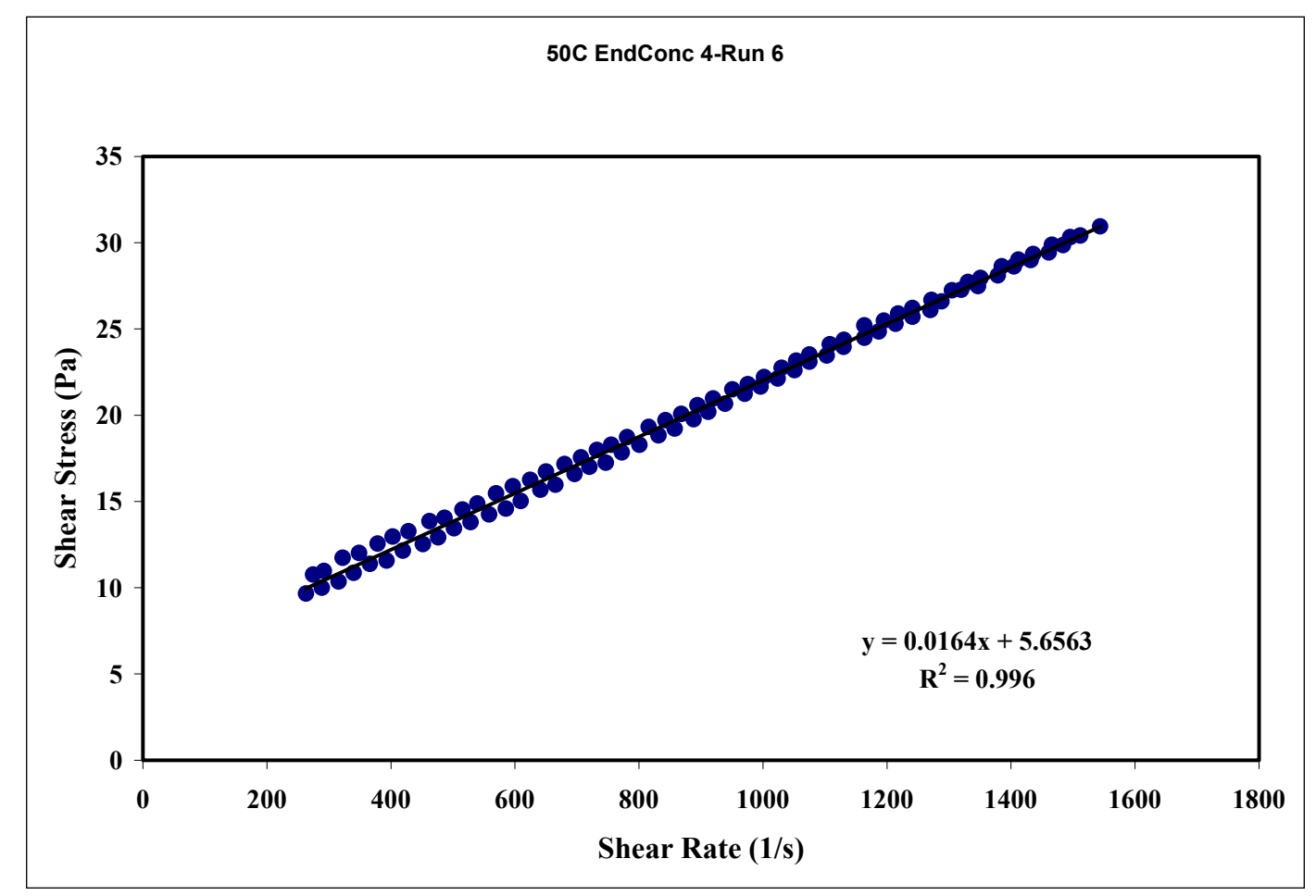

Figure 51. $50{ }^{\circ} \mathrm{C}$ at 13 wt\% Run 6

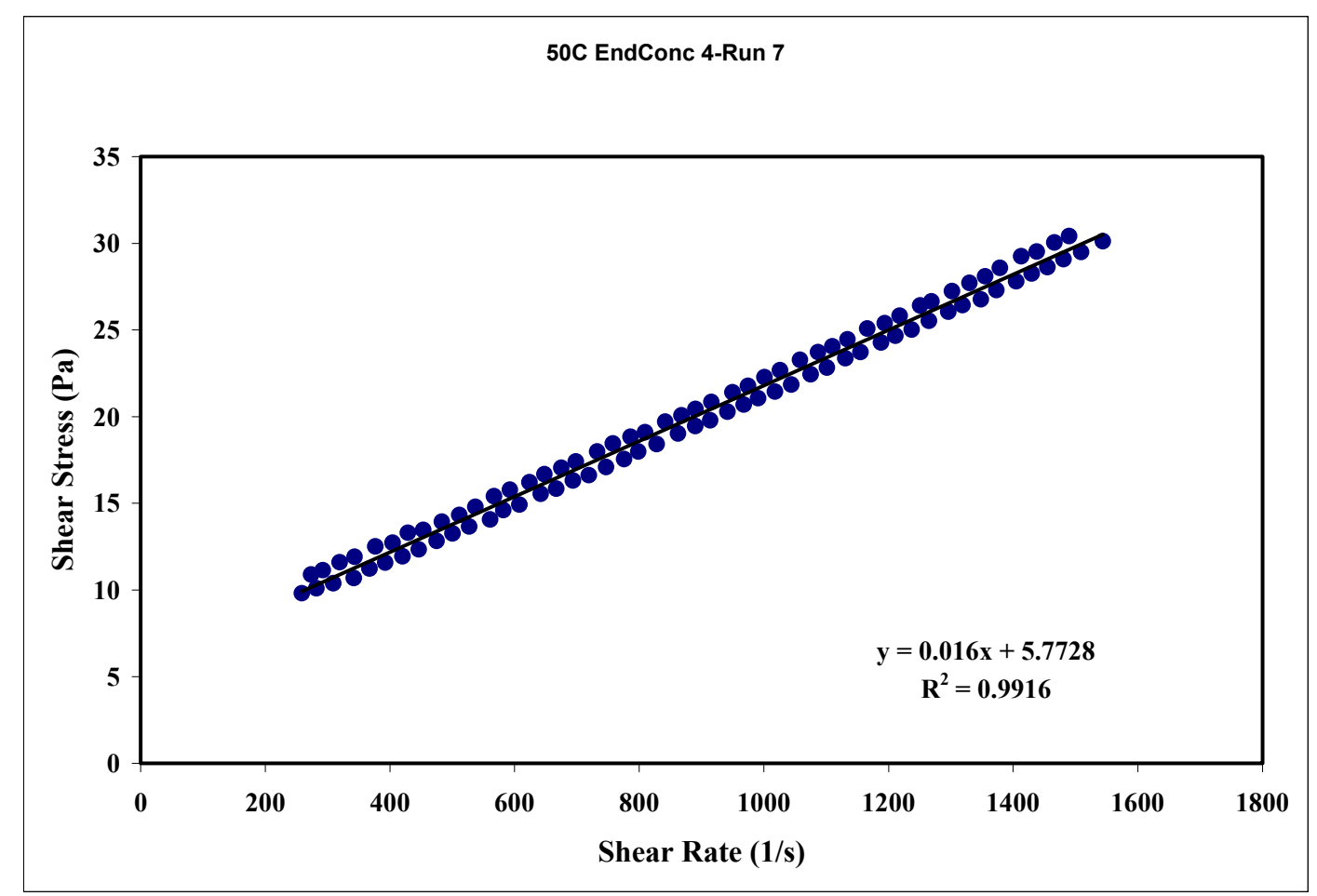

Figure 52. $50{ }^{\circ} \mathrm{C}$ at 13 wt\% Run 7 


\begin{tabular}{|ll|}
\hline APPENDIX I - PART 3 & WSRC-TR-2000-00352 \\
SR/TRU PRECIPITATE SLURRY RHEOGRAMS AT 13 WT \% & SRT-RPP-2000-00026 \\
\hline
\end{tabular}

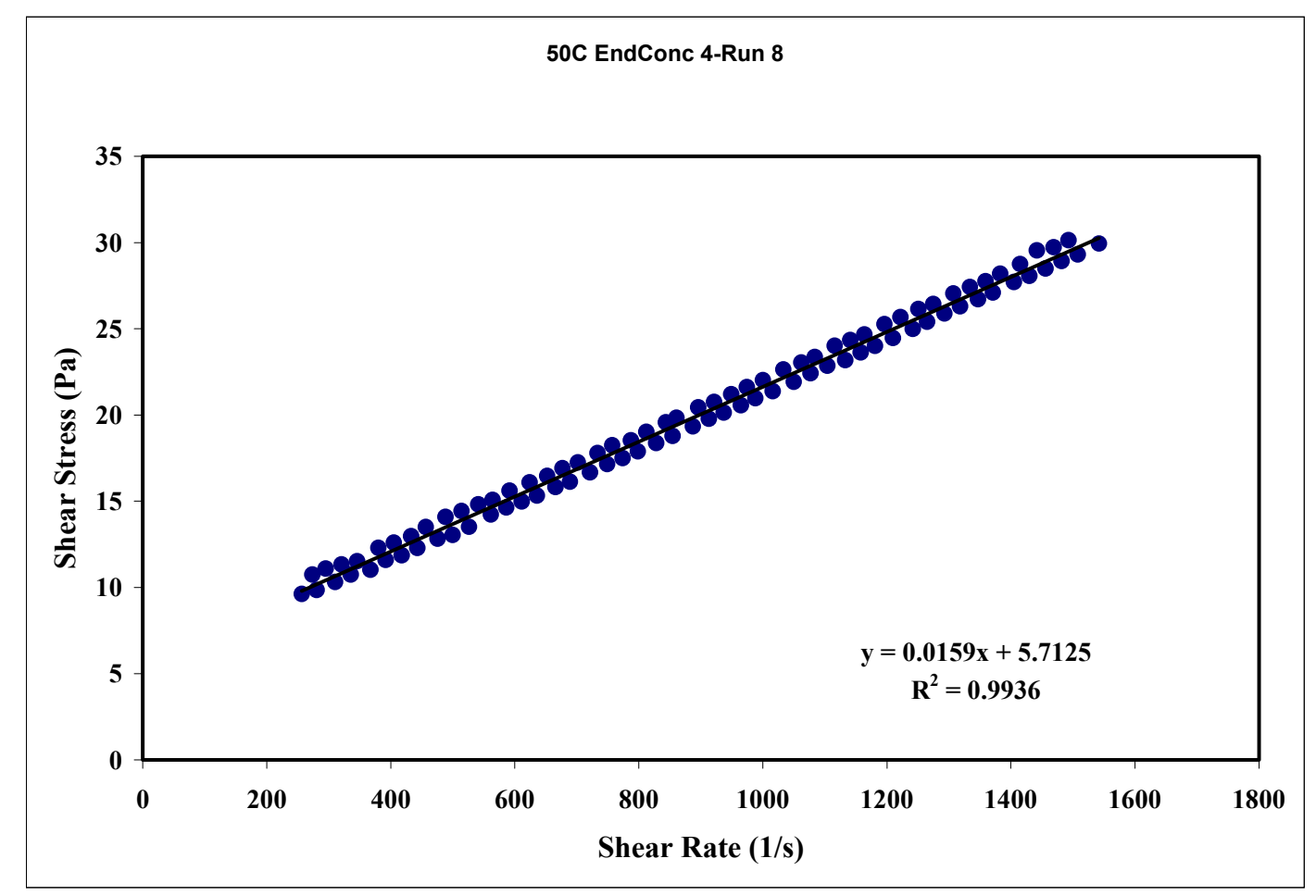

Figure 53. $50{ }^{\circ} \mathrm{C}$ at 13 wt\% Run 8

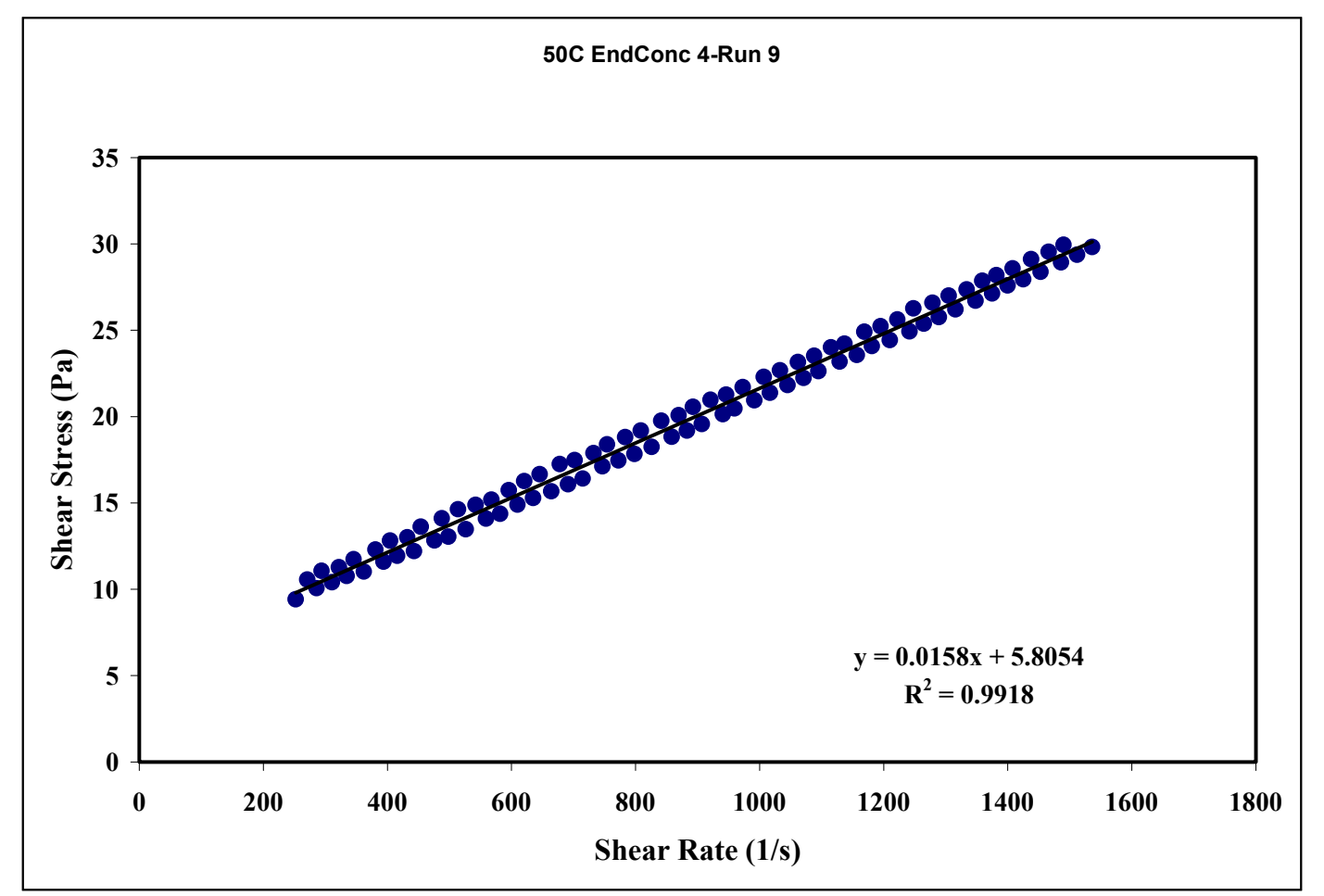

Figure 54. $50{ }^{\circ} \mathrm{C}$ at 13 wt $\%$ Run 9 


\begin{tabular}{|ll|}
\hline APPENDIX I - PART 3 & WSRC-TR-2000-00352 \\
& SRT-RPP-2000-00026 \\
SR/TRU PRECIPITATE SLURRY RHEOGRAMS AT 13 WT \% & Page 416 of 514 \\
\hline
\end{tabular}

This page intentionally left blank. 


\section{APPENDIX I - PART 4}

\section{SR/TRU PRECIPITATE SLURRY RHEOGRAMS AT 16 WT \%}

Figure 1. Blank at $16 \mathrm{wt} \%$ Run 1

Figure 2. Blank at $16 \mathrm{wt} \%$ Run 2

Figure 3. Blank at $16 \mathrm{wt} \%$ Run 3

Figure 4. Blank at $16 \mathrm{wt} \%$ Run 4

Figure 5. Blank at $16 \mathrm{wt} \%$ Run 5

Figure 6. Blank at $16 \mathrm{wt} \%$ Run 6

Figure 7. Blank at $16 \mathrm{wt} \%$ Run 7

Figure 8. Blank at $16 \mathrm{wt} \%$ Run 8

Figure 9. Blank at $16 \mathrm{wt} \%$ Run 9

Figure $10.10^{\circ} \mathrm{C}$ at $16 \mathrm{wt} \%$ Run 1

Figure $11.10{ }^{\circ} \mathrm{C}$ at $16 \mathrm{wt} \%$ Run 2

Figure $12 . \quad 10^{\circ} \mathrm{C}$ at $16 \mathrm{wt} \%$ Run 3

Figure $13.10^{\circ} \mathrm{C}$ at $16 \mathrm{wt} \%$ Run 4

Figure $14 . \quad 10^{\circ} \mathrm{C}$ at $16 \mathrm{wt} \%$ Run 5

Figure $15.10^{\circ} \mathrm{C}$ at $16 \mathrm{wt} \%$ Run 6

Figure $16.10^{\circ} \mathrm{C}$ at $16 \mathrm{wt} \%$ Run 7

Figure $17.10^{\circ} \mathrm{C}$ at $16 \mathrm{wt} \%$ Run 8

Figure $18 . \quad 10{ }^{\circ} \mathrm{C}$ at 16 wt \% Run 9

Figure $19.15^{\circ} \mathrm{C}$ at $16 \mathrm{wt} \%$ Run 1

Figure $20.15^{\circ} \mathrm{C}$ at $16 \mathrm{wt} \%$ Run 2

Figure $21.15^{\circ} \mathrm{C}$ at $16 \mathrm{wt} \%$ Run 3

Figure $22.15^{\circ} \mathrm{C}$ at $16 \mathrm{wt} \%$ Run 4

Figure $23.15^{\circ} \mathrm{C}$ at $16 \mathrm{wt} \%$ Run 5

Figure 24. $15^{\circ} \mathrm{C}$ at $16 \mathrm{wt} \%$ Run 6

Figure $25.15^{\circ} \mathrm{C}$ at $16 \mathrm{wt} \%$ Run 7

Figure $26.15^{\circ} \mathrm{C}$ at $16 \mathrm{wt} \%$ Run 8

Figure $27.15^{\circ} \mathrm{C}$ at $16 \mathrm{wt} \%$ Run 9

Figure 28. $25^{\circ} \mathrm{C}$ at $16 \mathrm{wt} \%$ Run 1

Figure 29. $25^{\circ} \mathrm{C}$ at $16 \mathrm{wt} \%$ Run 2

Figure $30.25^{\circ} \mathrm{C}$ at $16 \mathrm{wt} \%$ Run 3

Figure $31.25^{\circ} \mathrm{C}$ at $16 \mathrm{wt} \%$ Run 4

Figure $32.25^{\circ} \mathrm{C}$ at $16 \mathrm{wt} \%$ Run 5

Figure $33.25^{\circ} \mathrm{C}$ at $16 \mathrm{wt} \%$ Run 6

Figure $34.25^{\circ} \mathrm{C}$ at $16 \mathrm{wt} \%$ Run 7

Figure $35.25^{\circ} \mathrm{C}$ at $16 \mathrm{wt} \%$ Run 8

Figure $36.25^{\circ} \mathrm{C}$ at 16 wt \% Run 9 


\begin{tabular}{|ll|}
\hline APPENDIX I - PART 4 & WSRC-TR-2000-00352 \\
SR/TRU PRECIPITATE SLURRY RHEOGRAMS AT 16 WT \% & SRT-RPP-2000-00026 \\
\hline
\end{tabular}

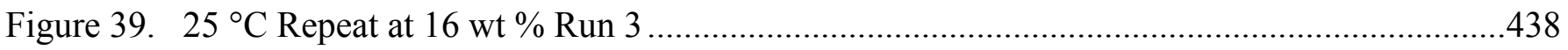

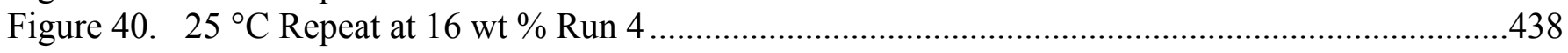

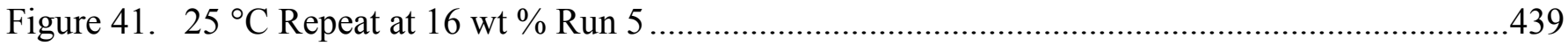

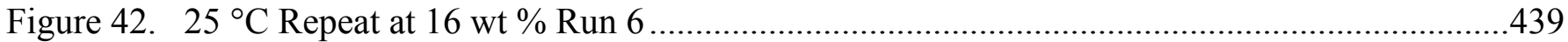

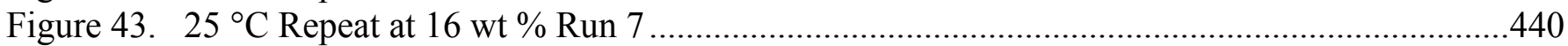

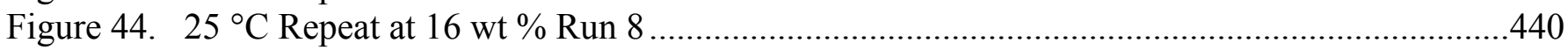

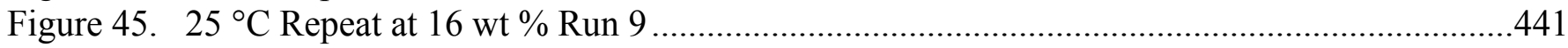

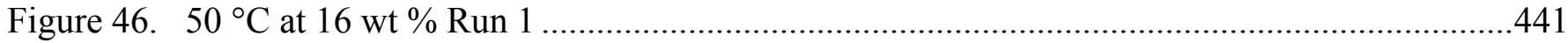

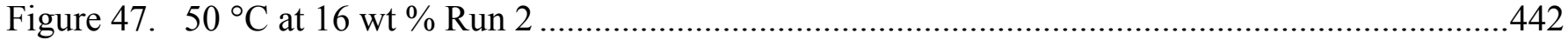

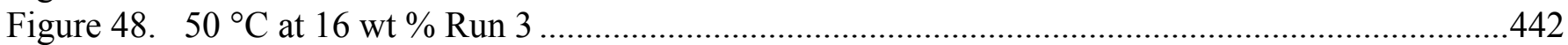

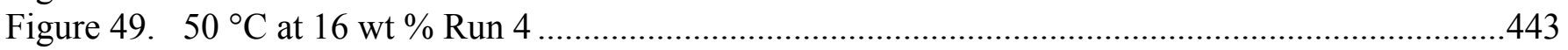

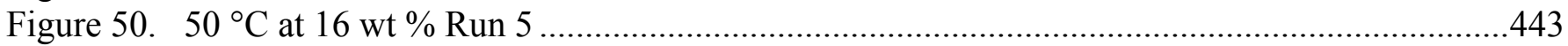

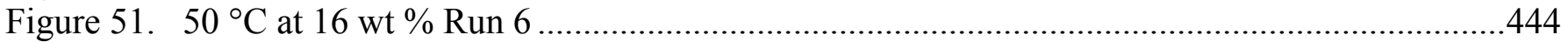

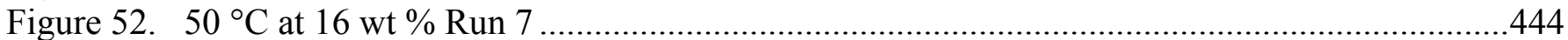

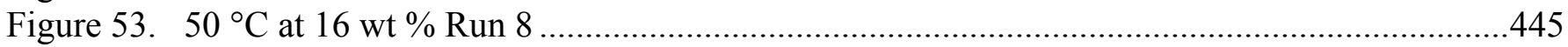

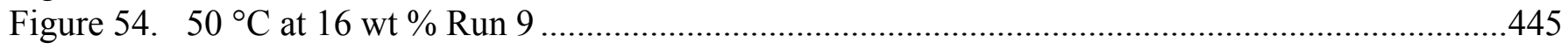




\begin{tabular}{|ll|}
\hline APPENDIX I - PART 4 & WSRC-TR-2000-00352 \\
& SRT-RPP-2000-00026 \\
SR/TRU PRECIPITATE SLURRY RHEOGRAMS AT 16 WT \% & Page 419 of 514 \\
\hline
\end{tabular}

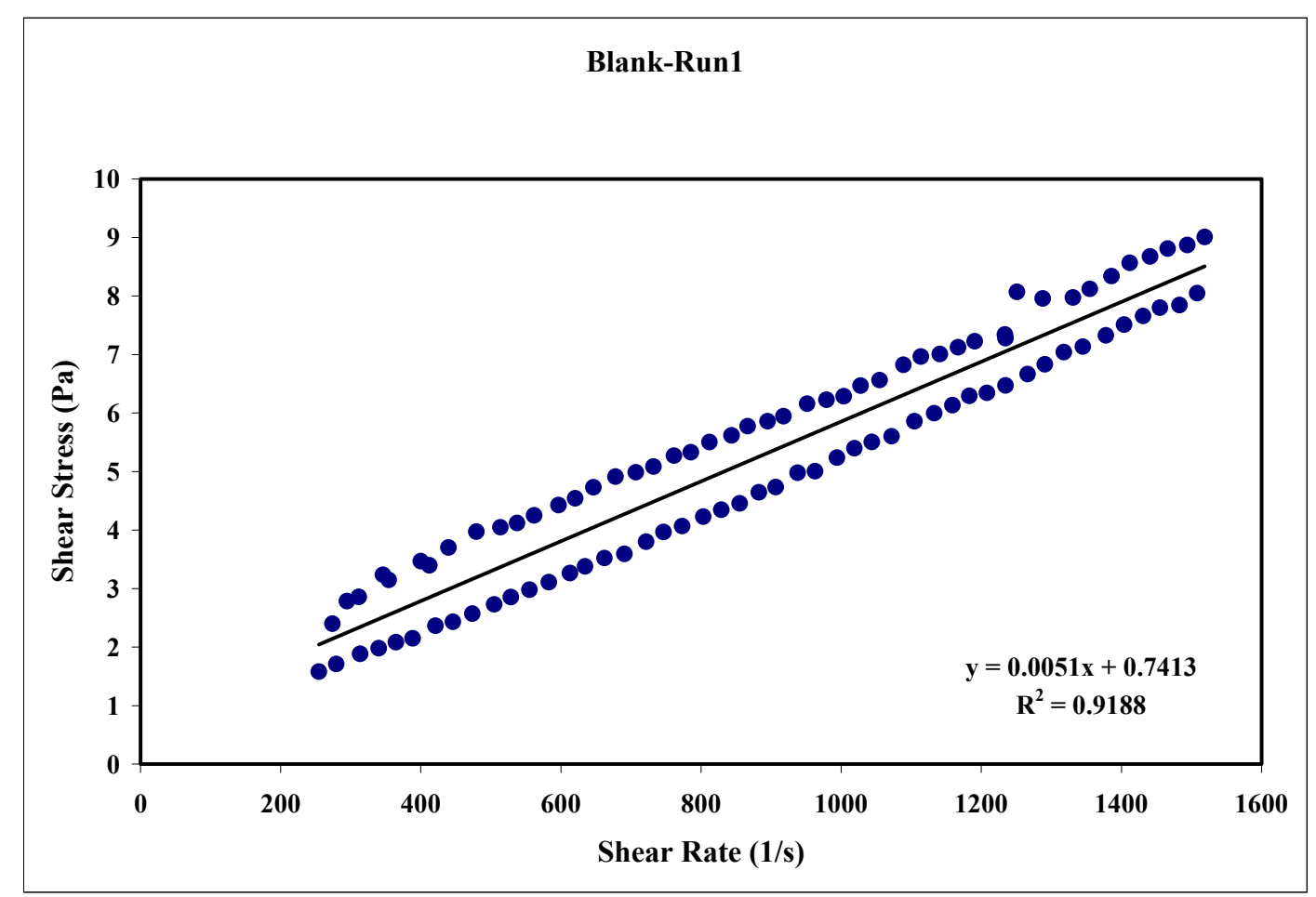

Figure 1. Blank at 16 wt \% Run 1

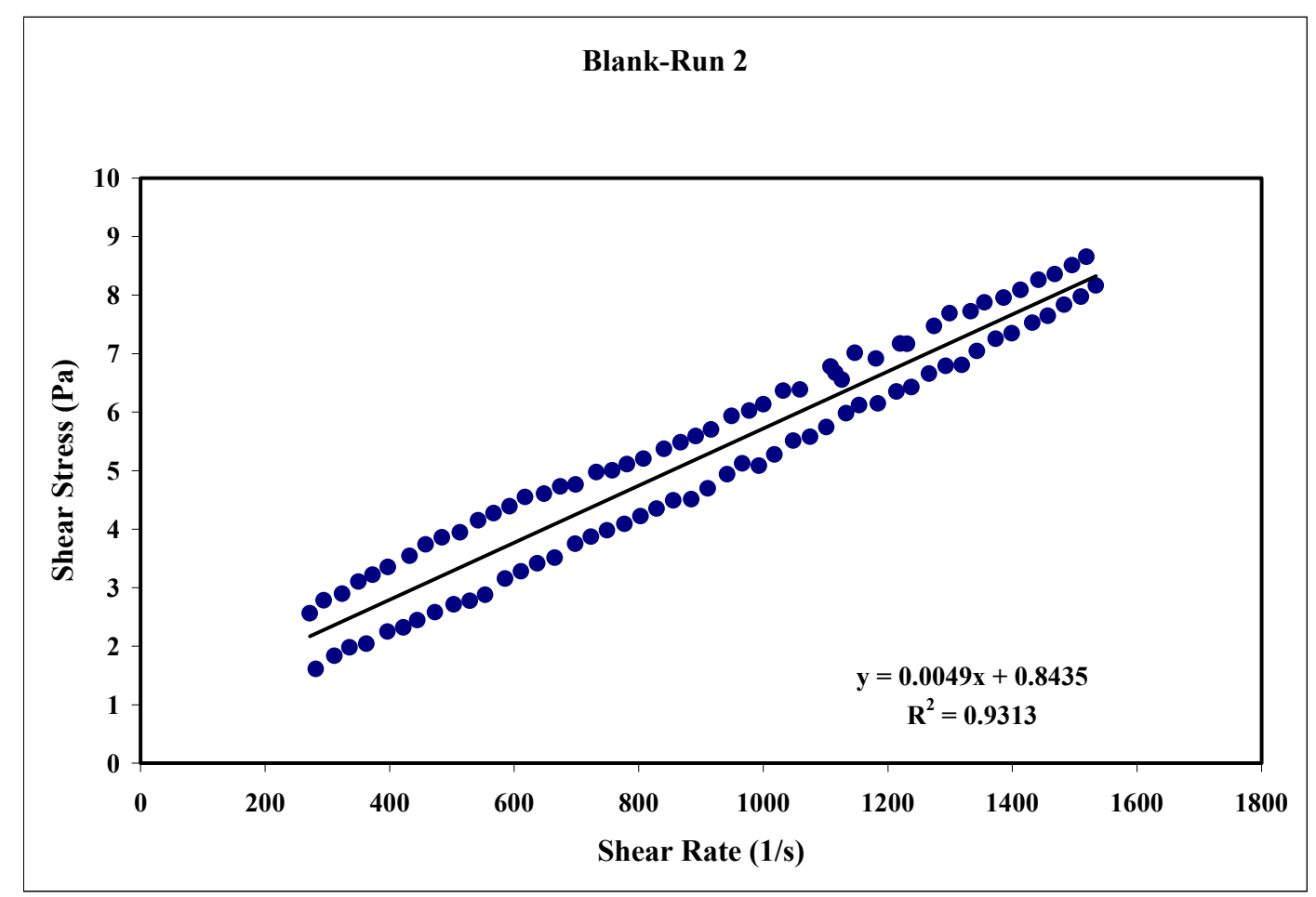

Figure 2. Blank at 16 wt \% Run 2 
\begin{tabular}{ll}
\hline APPENDIX I - PART 4 & WSRC-TR-2000-00352
\end{tabular}

SR/TRU PRECIPITATE SLURRY RHEOGRAMS AT 16 WT \%

SRT-RPP-2000-00026

Page 420 of 514

\section{Blank-Run 3}

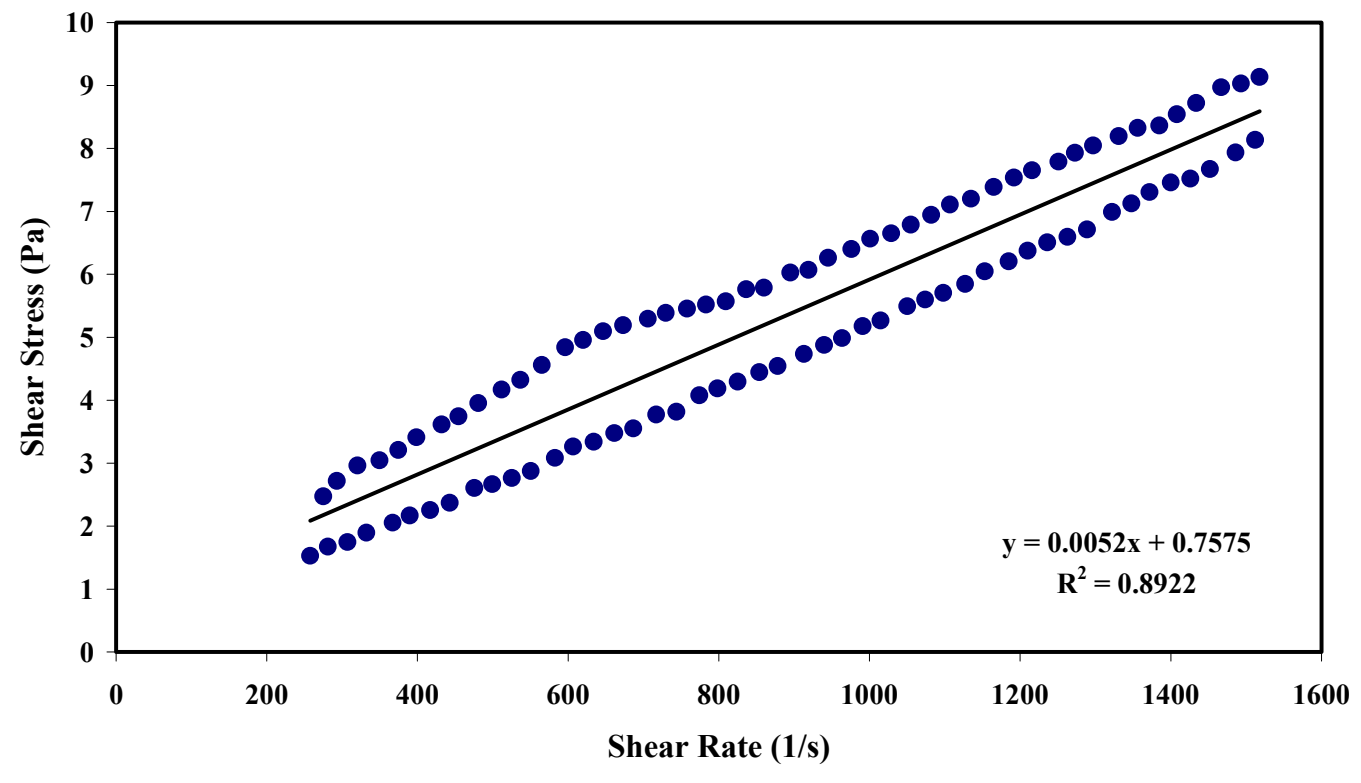

Figure 3. Blank at 16 wt \% Run 3

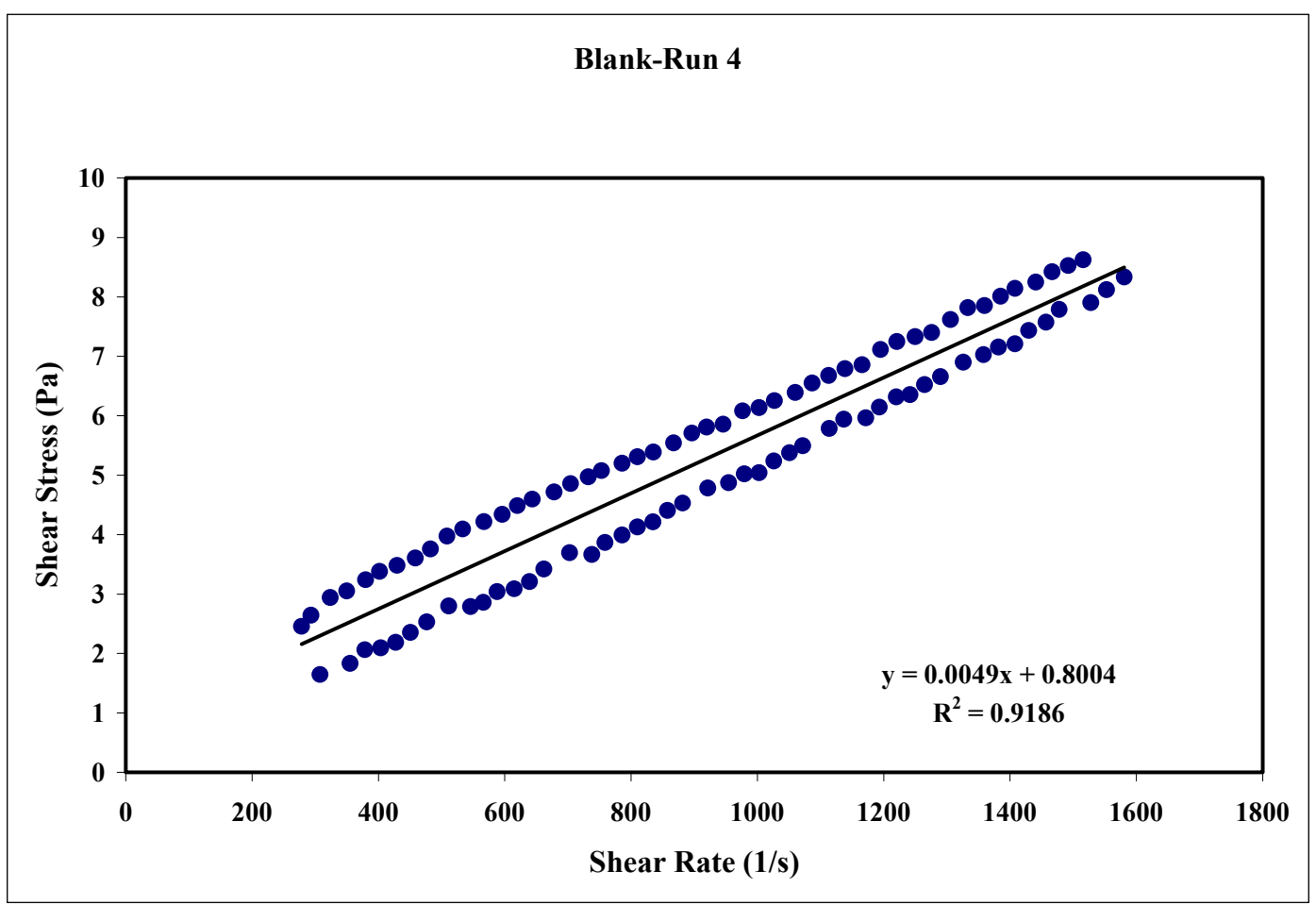

Figure 4. Blank at 16 wt \% Run 4 


\begin{tabular}{|ll|}
\hline APPENDIX I - PART 4 & WSRC-TR-2000-00352 \\
SR/TRU PRECIPITATE SLURRY RHEOGRAMS AT 16 WT \% & SRT-RPP-2000-00026 \\
\hline
\end{tabular}

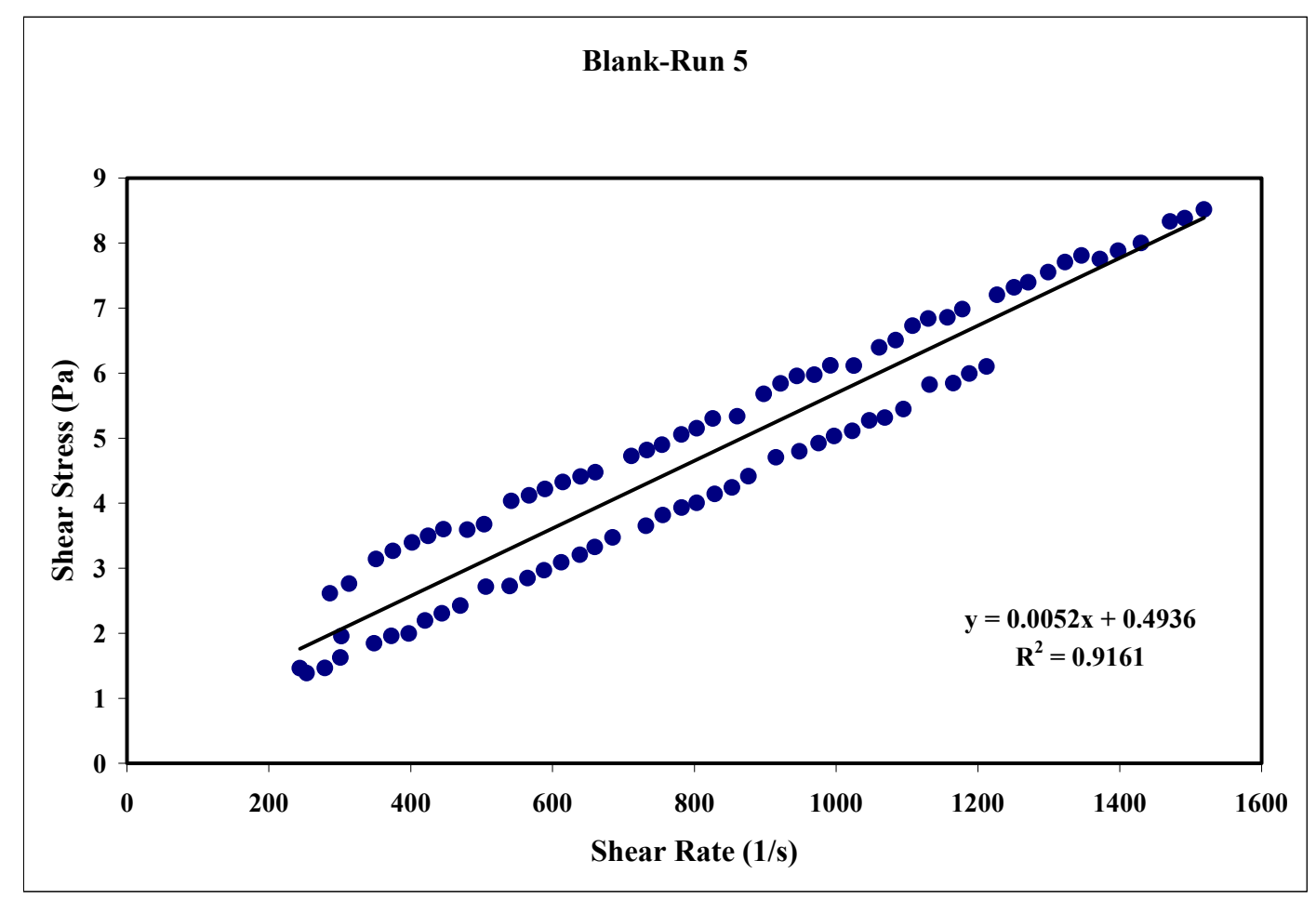

Figure 5. Blank at 16 wt \% Run 5

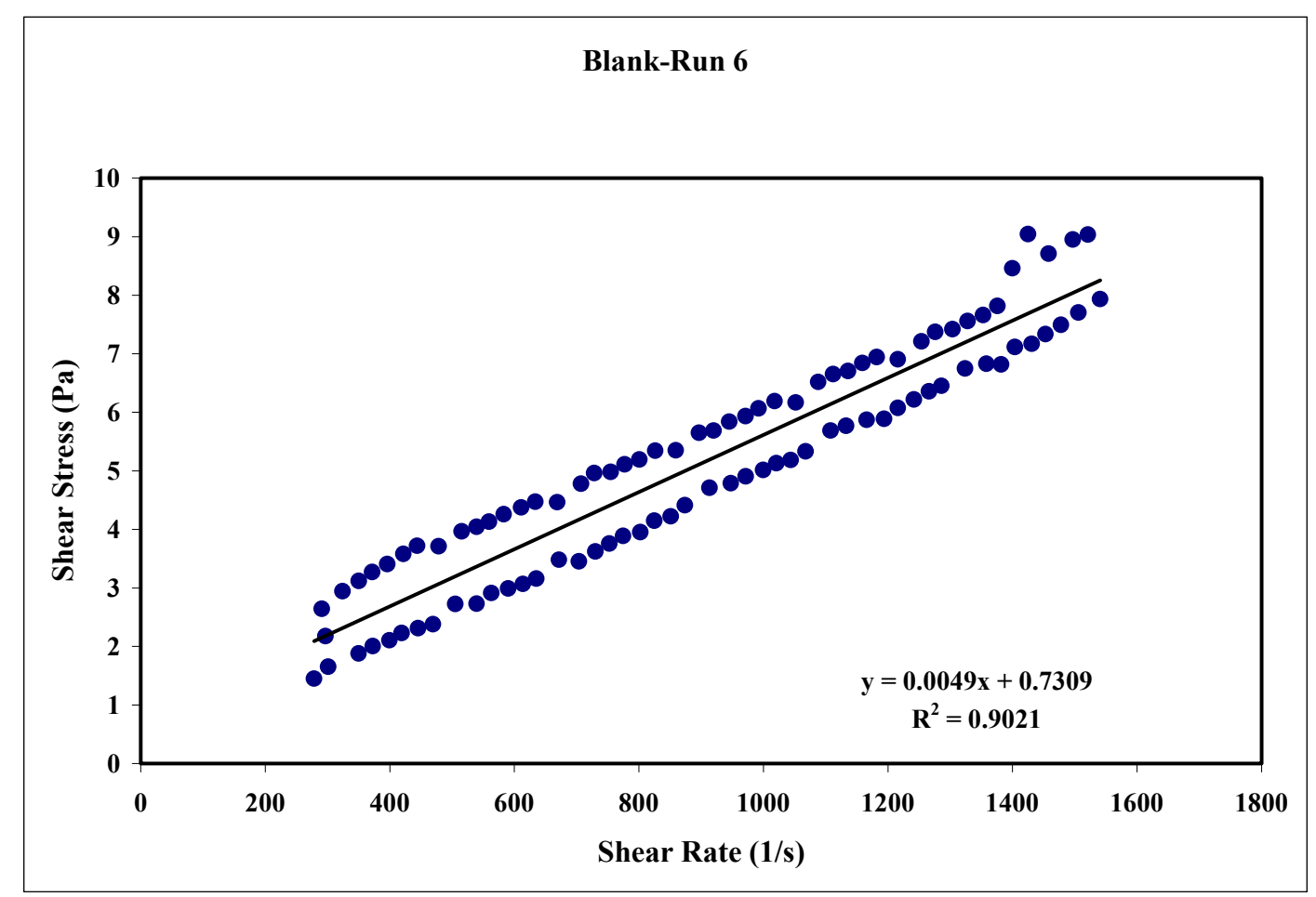

Figure 6. Blank at 16 wt \% Run 6 


\begin{tabular}{|ll|}
\hline APPENDIX I - PART 4 & WSRC-TR-2000-00352 \\
SR/TRU PRECIPITATE SLURRY RHEOGRAMS AT 16 WT \% & SRT-RPP-2000-00026 \\
\hline
\end{tabular}

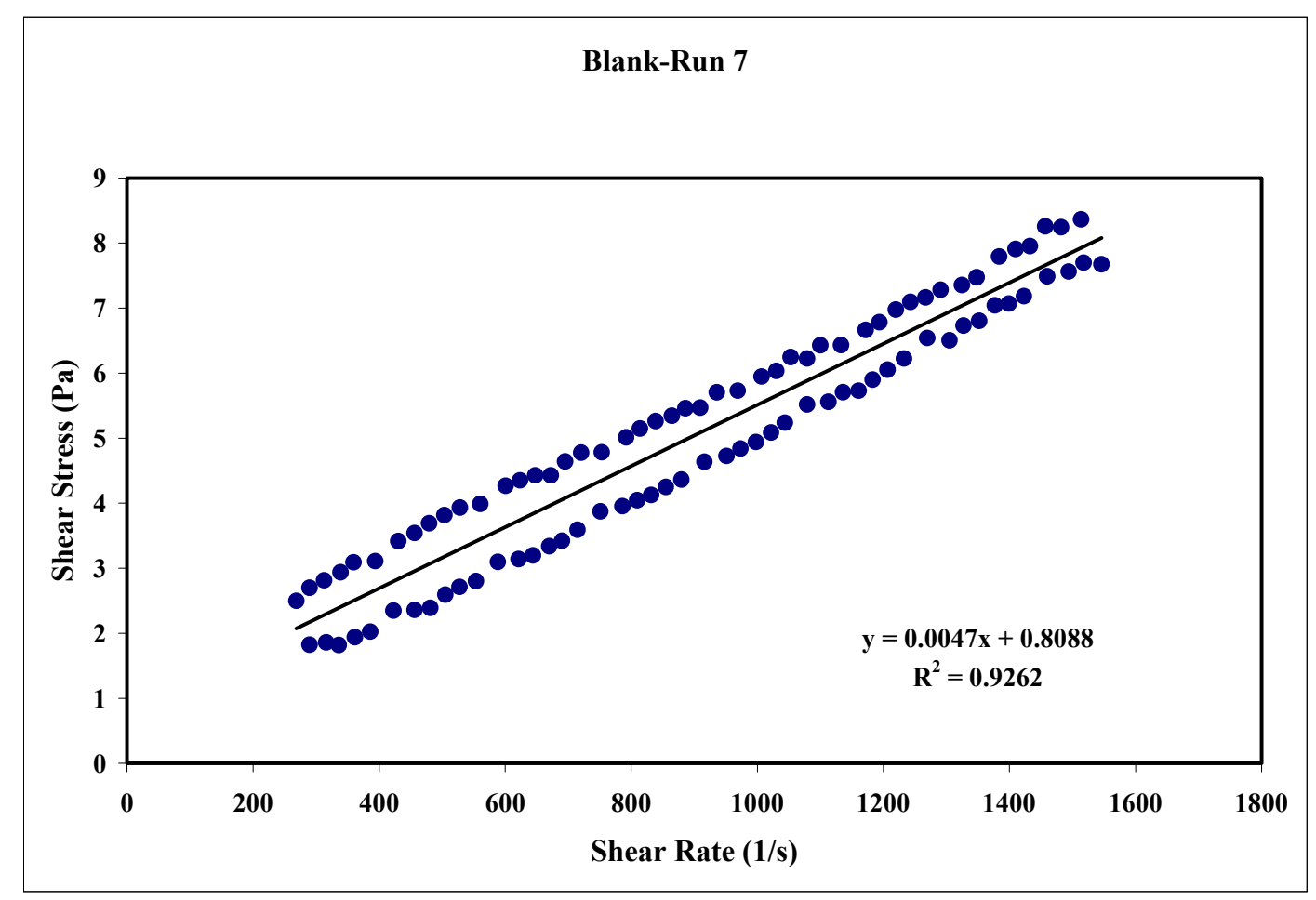

Figure 7. Blank at 16 wt \% Run 7

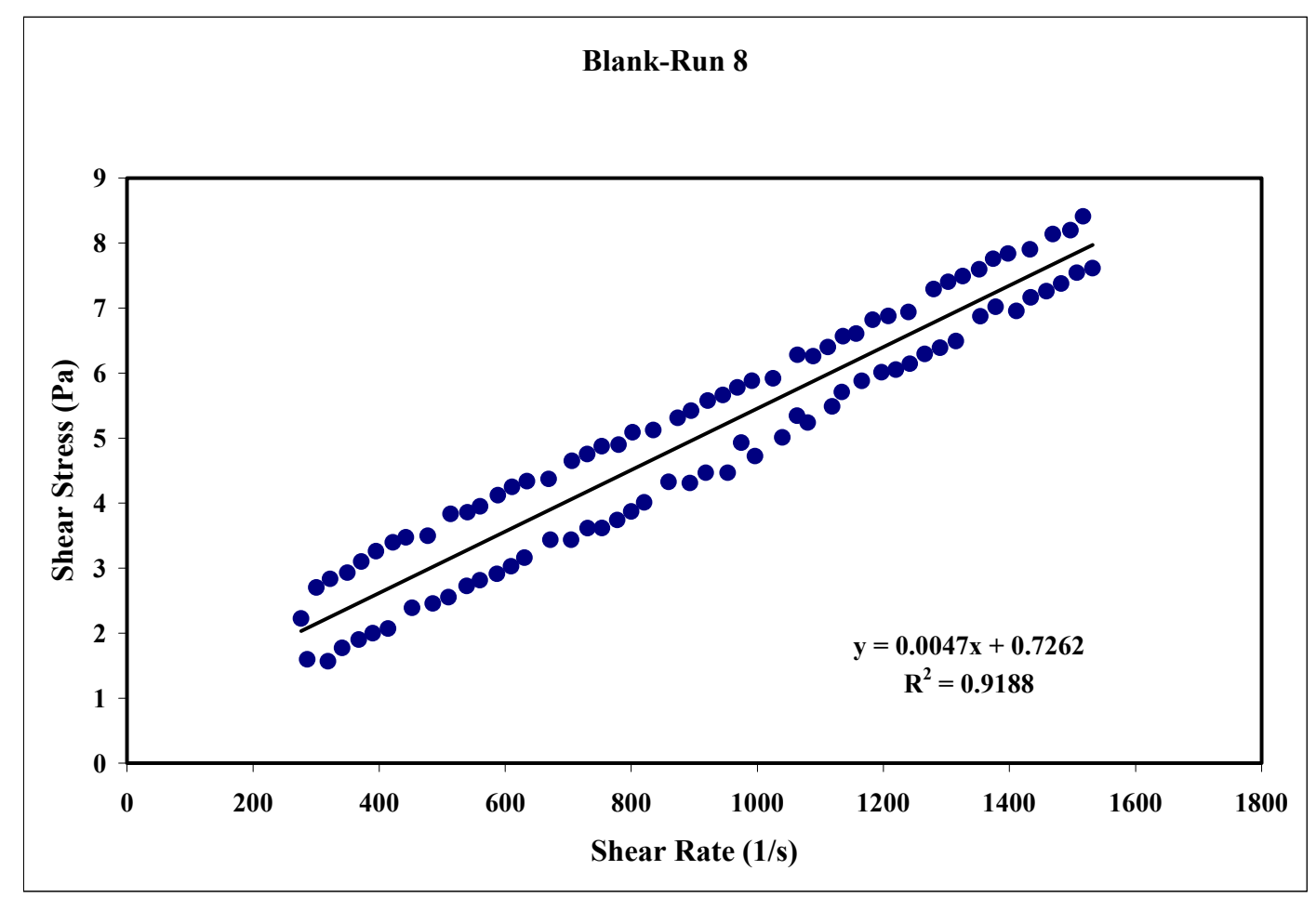

Figure 8. Blank at 16 wt \% Run 8 


\begin{tabular}{|ll|}
\hline APPENDIX I - PART 4 & WSRC-TR-2000-00352 \\
SR/TRU PRECIPITATE SLURRY RHEOGRAMS AT 16 WT \% & SRT-RPP-2000-00026 \\
\hline
\end{tabular}

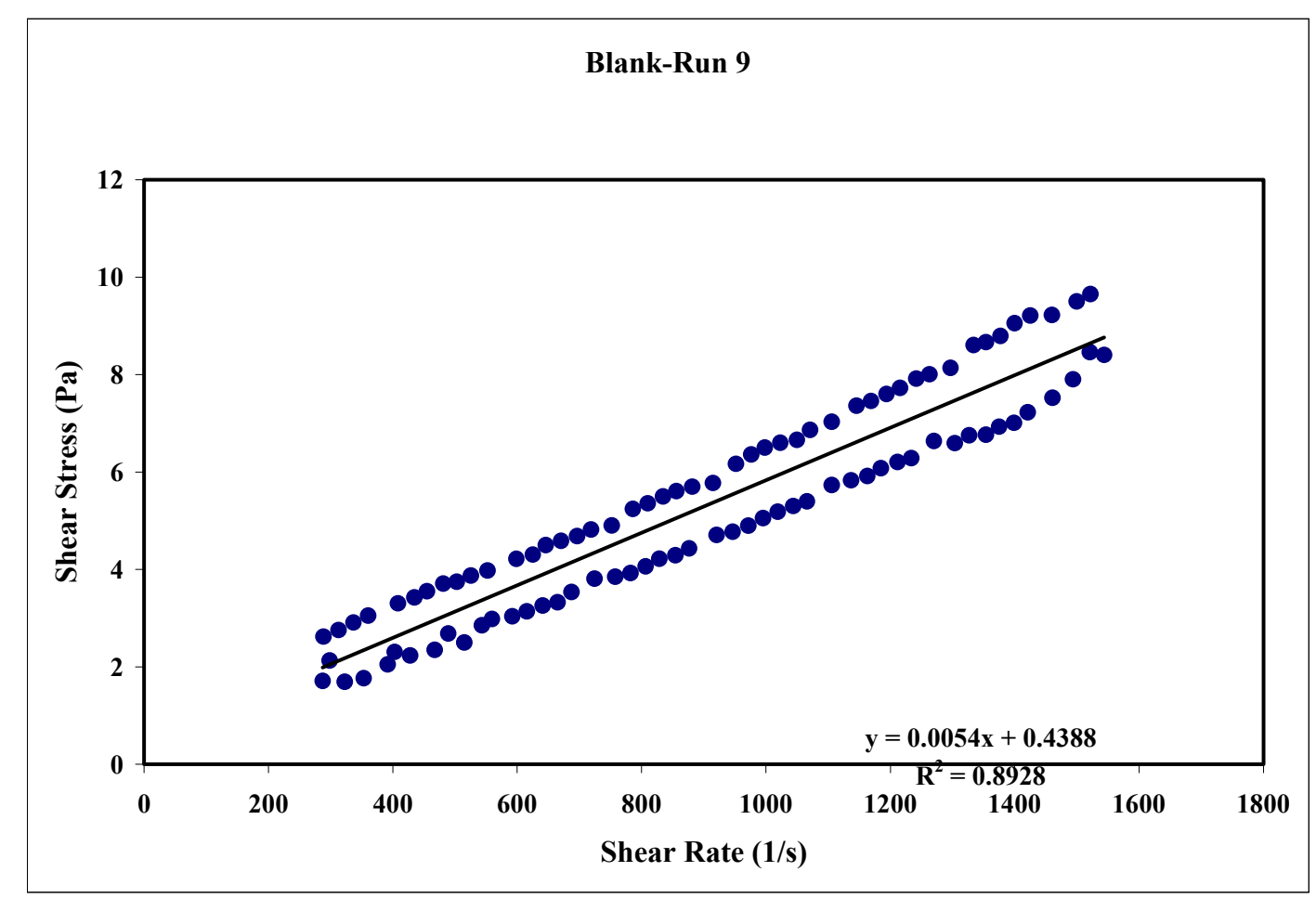

Figure 9. Blank at 16 wt \% Run 9

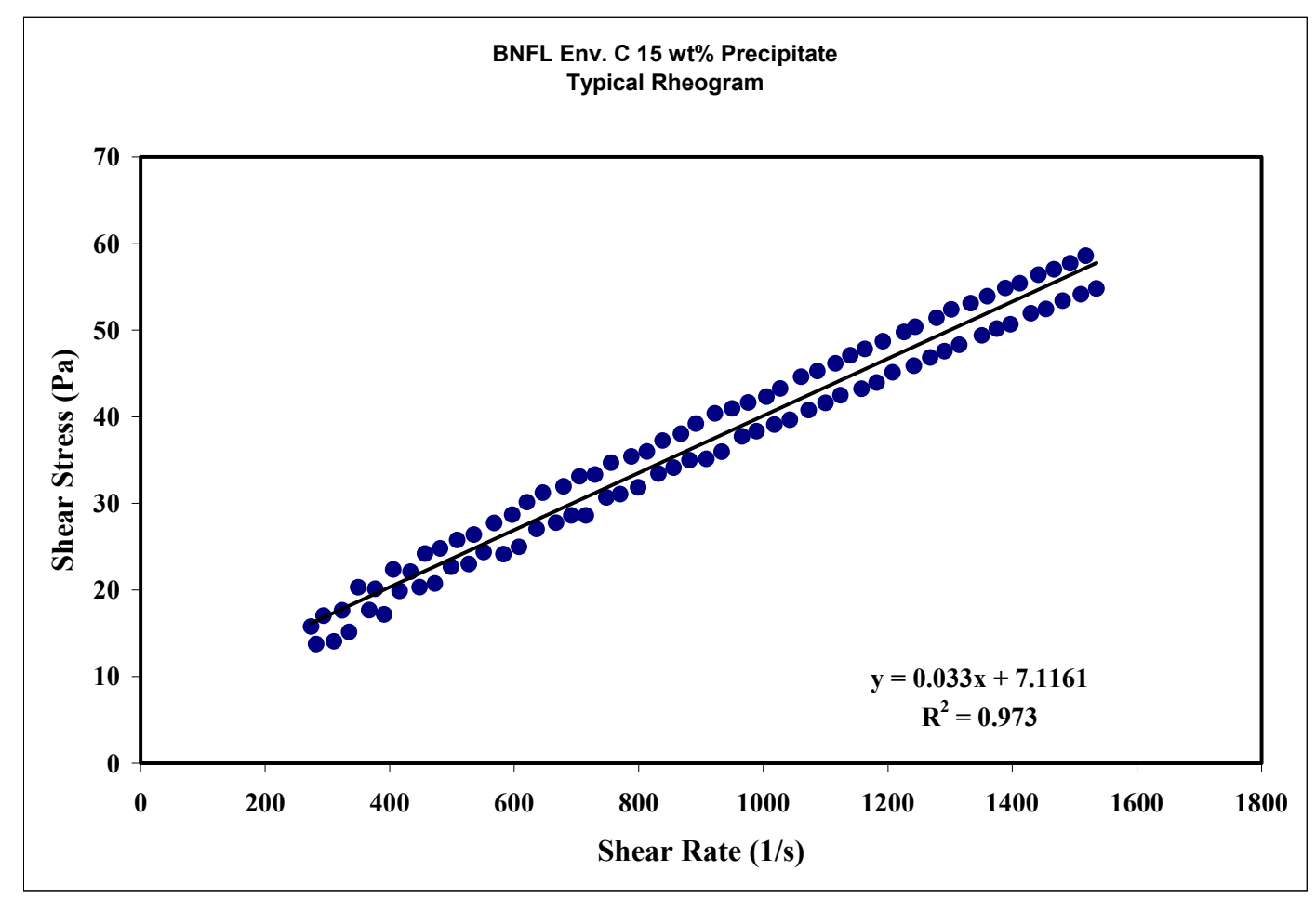

Figure $10.10{ }^{\circ} \mathrm{C}$ at 16 wt \% Run 1 


\begin{tabular}{|ll|}
\hline APPENDIX I - PART 4 & WSRC-TR-2000-00352 \\
SR/TRU PRECIPITATE SLURRY RHEOGRAMS AT 16 WT \% & SRT-RPP-2000-00026 \\
\hline
\end{tabular}

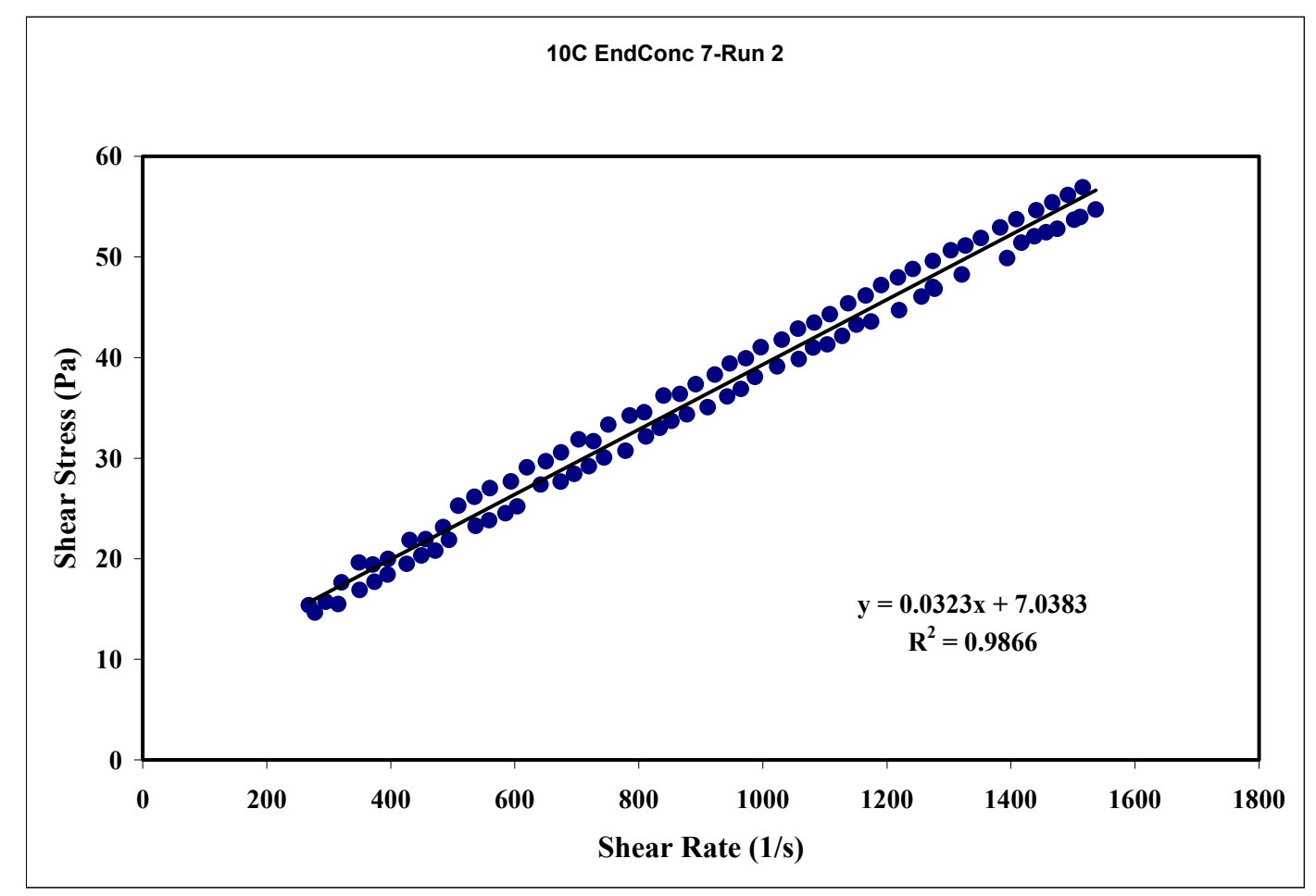

Figure 11. $10{ }^{\circ} \mathrm{C}$ at 16 wt \% Run 2

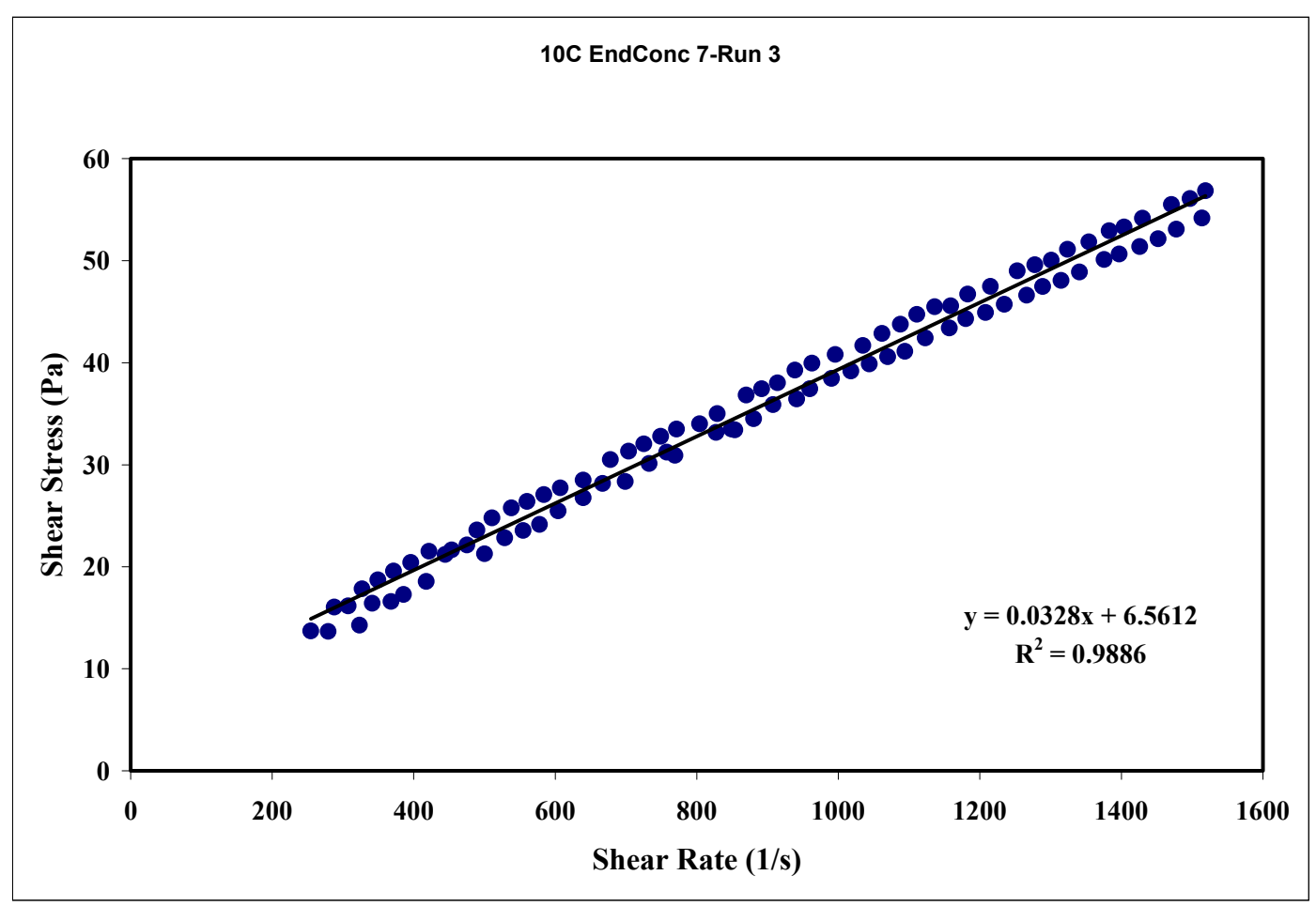

Figure 12. $10{ }^{\circ} \mathrm{C}$ at 16 wt $\%$ Run 3 


\begin{tabular}{|ll|}
\hline APPENDIX I - PART 4 & WSRC-TR-2000-00352 \\
& SRT-RPP-2000-00026 \\
SR/TRU PRECIPITATE SLURRY RHEOGRAMS AT 16 WT \% & Page 425 of 514 \\
\hline
\end{tabular}

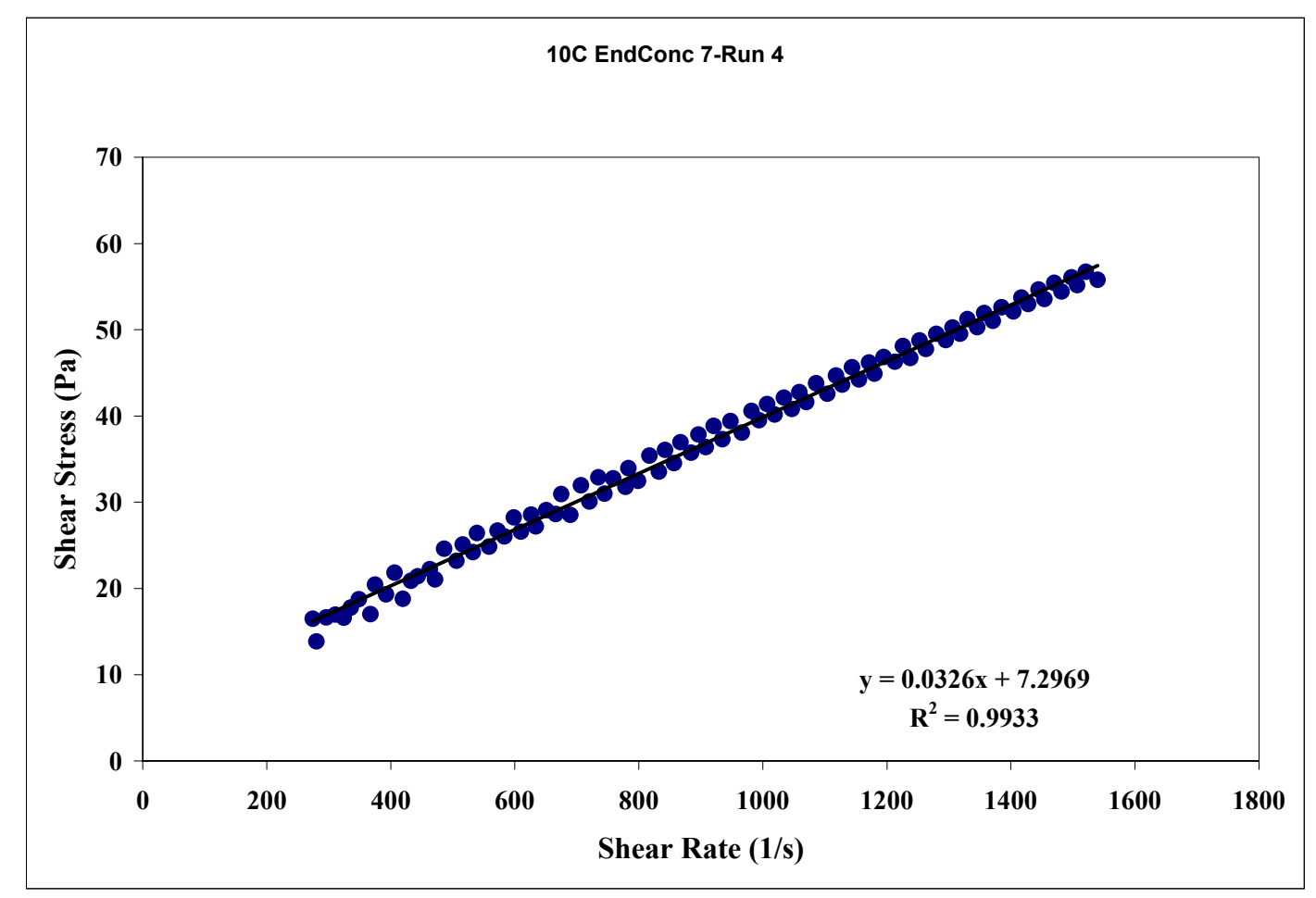

Figure 13. $10{ }^{\circ} \mathrm{C}$ at 16 wt \% Run 4

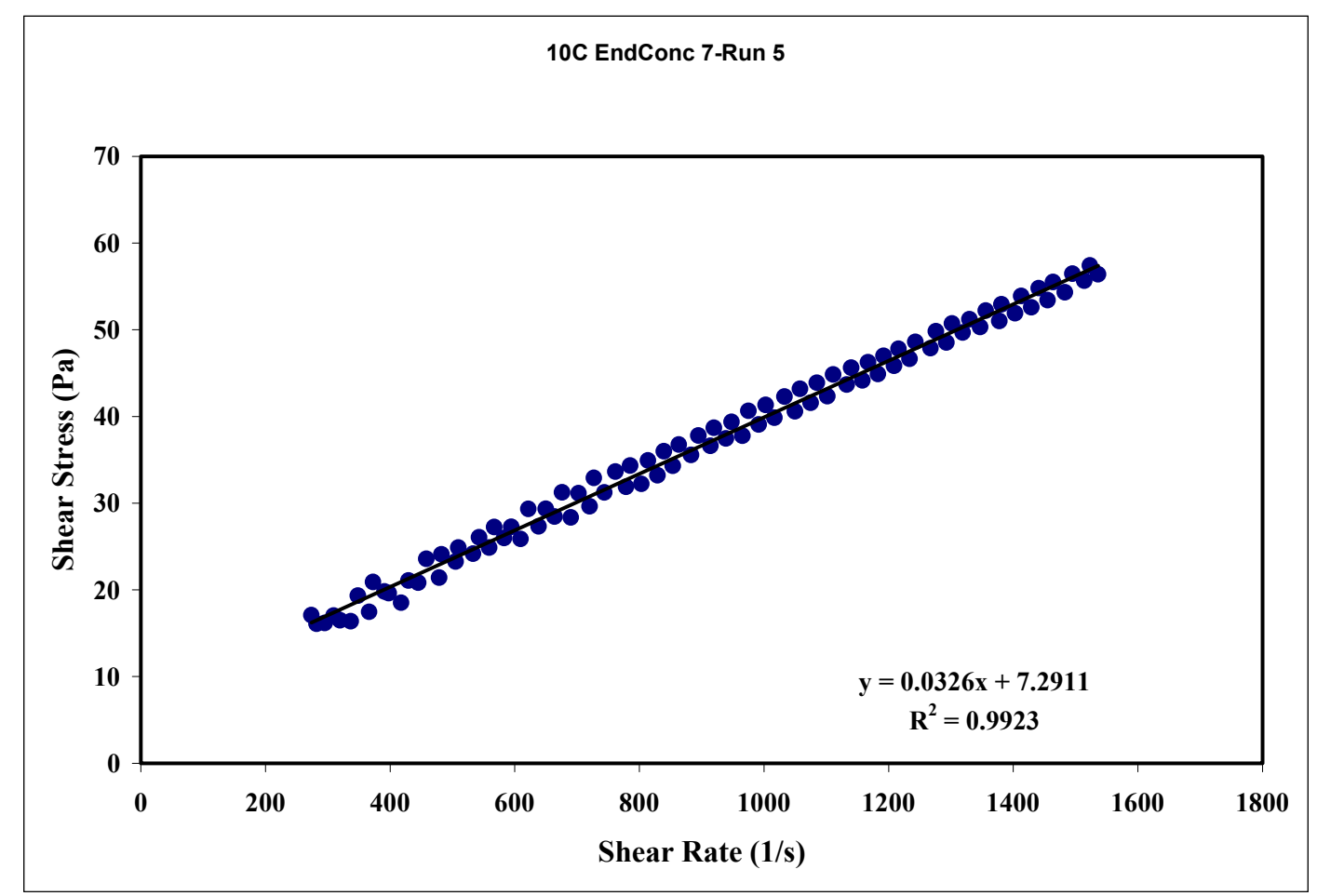

Figure 14. $10{ }^{\circ} \mathrm{C}$ at 16 wt \% Run 5 


\begin{tabular}{|ll|}
\hline APPENDIX I - PART 4 & WSRC-TR-2000-00352 \\
SR/TRU PRECIPITATE SLURRY RHEOGRAMS AT 16 WT \% & SRT-RPP-2000-00026 \\
\hline
\end{tabular}

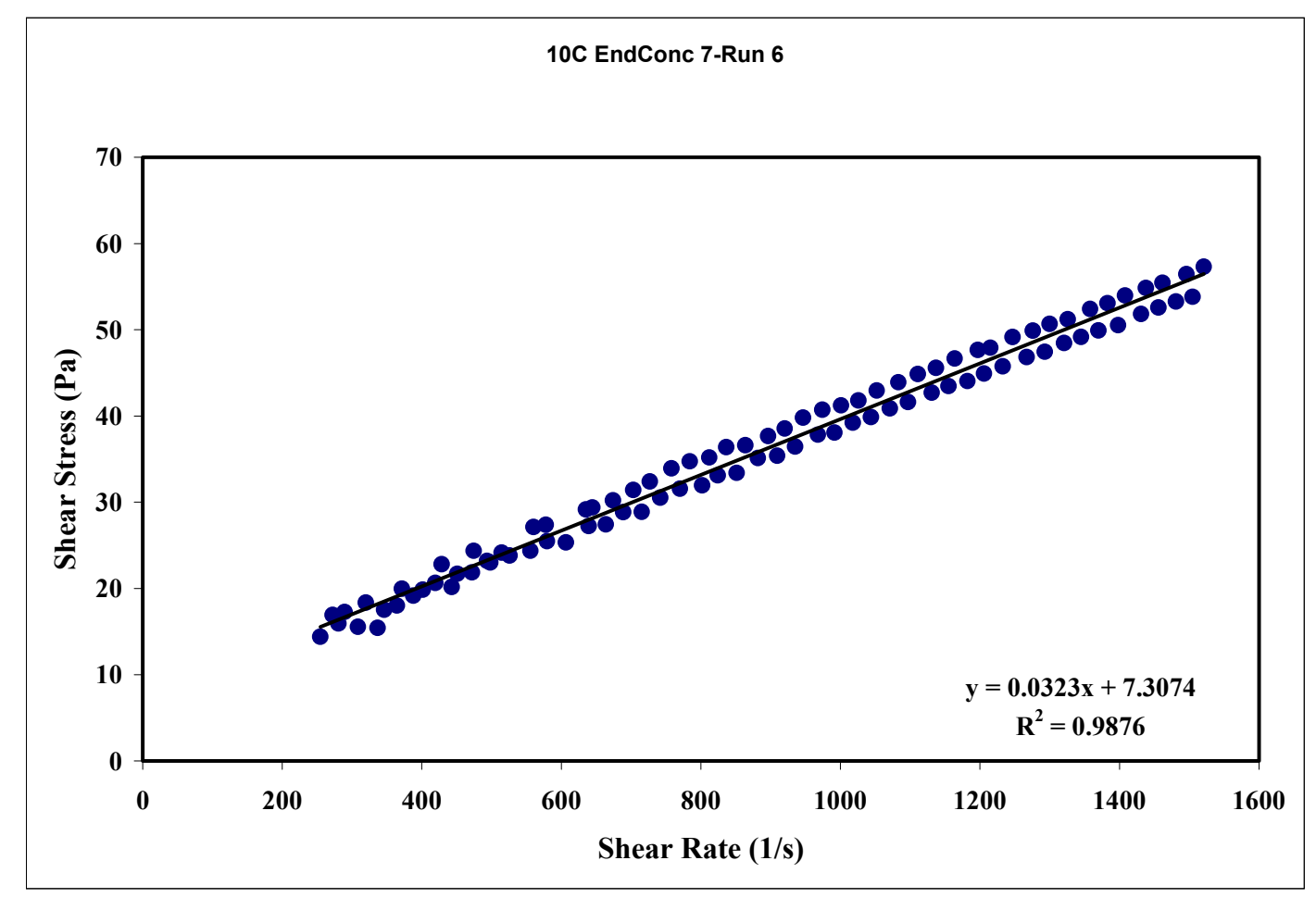

Figure $15.10{ }^{\circ} \mathrm{C}$ at 16 wt \% Run 6

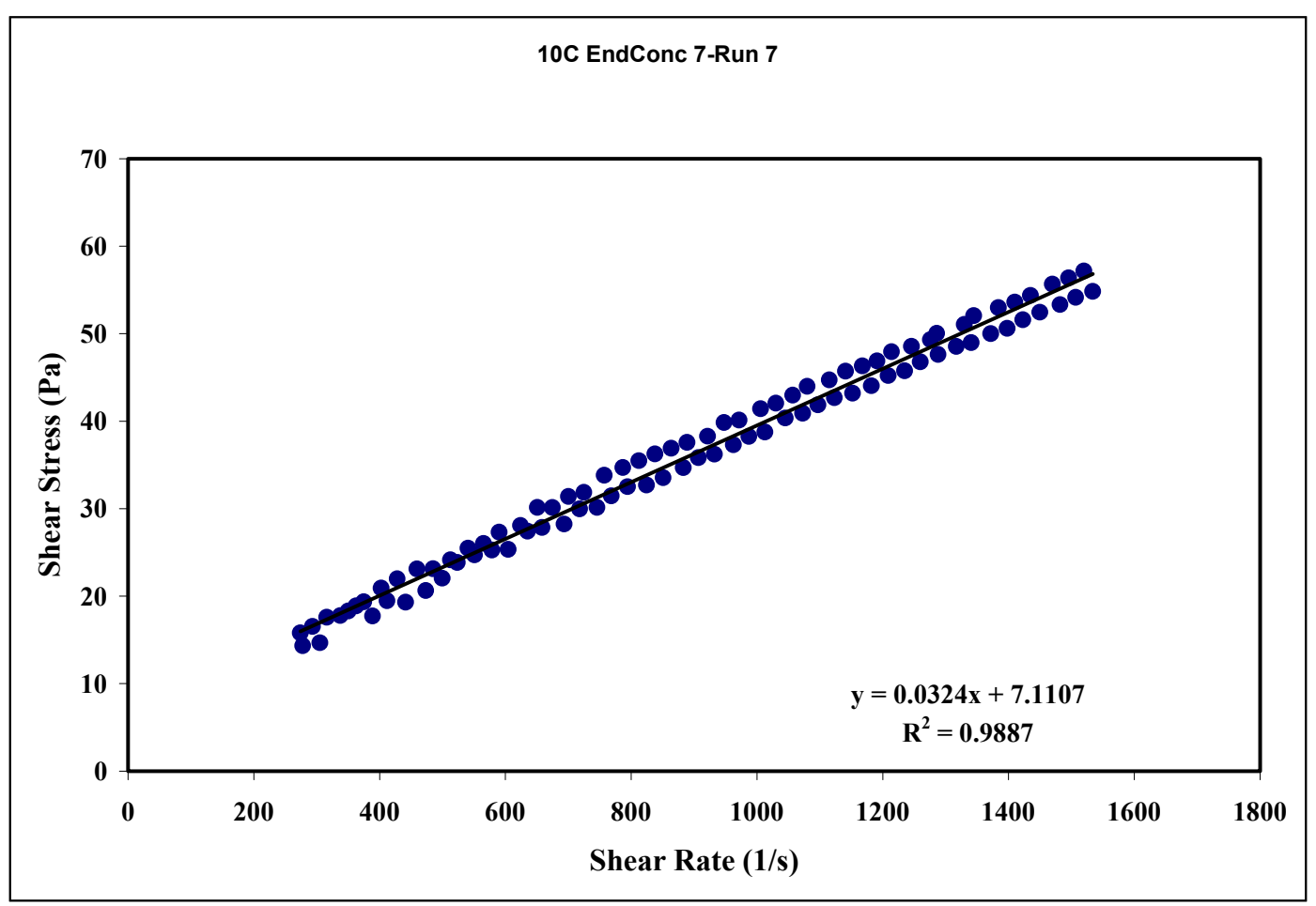

Figure 16. $10{ }^{\circ} \mathrm{C}$ at 16 wt \% Run 7 


\begin{tabular}{|ll|}
\hline APPENDIX I - PART 4 & WSRC-TR-2000-00352 \\
& SRT-RPP-2000-00026 \\
SR/TRU PRECIPITATE SLURRY RHEOGRAMS AT 16 WT \% & Page 427 of 514 \\
\hline
\end{tabular}

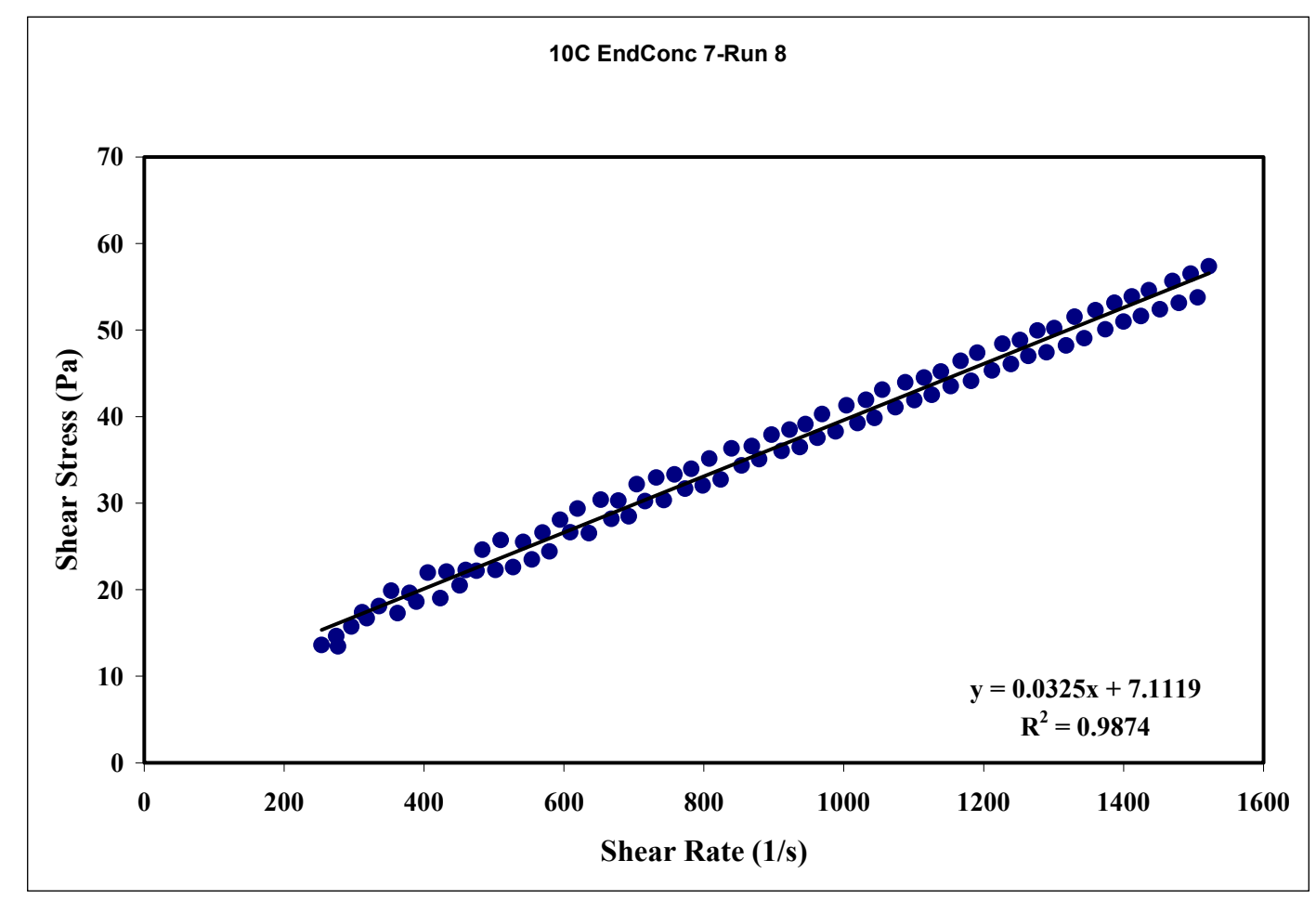

Figure $17.10{ }^{\circ} \mathrm{C}$ at 16 wt \% Run 8

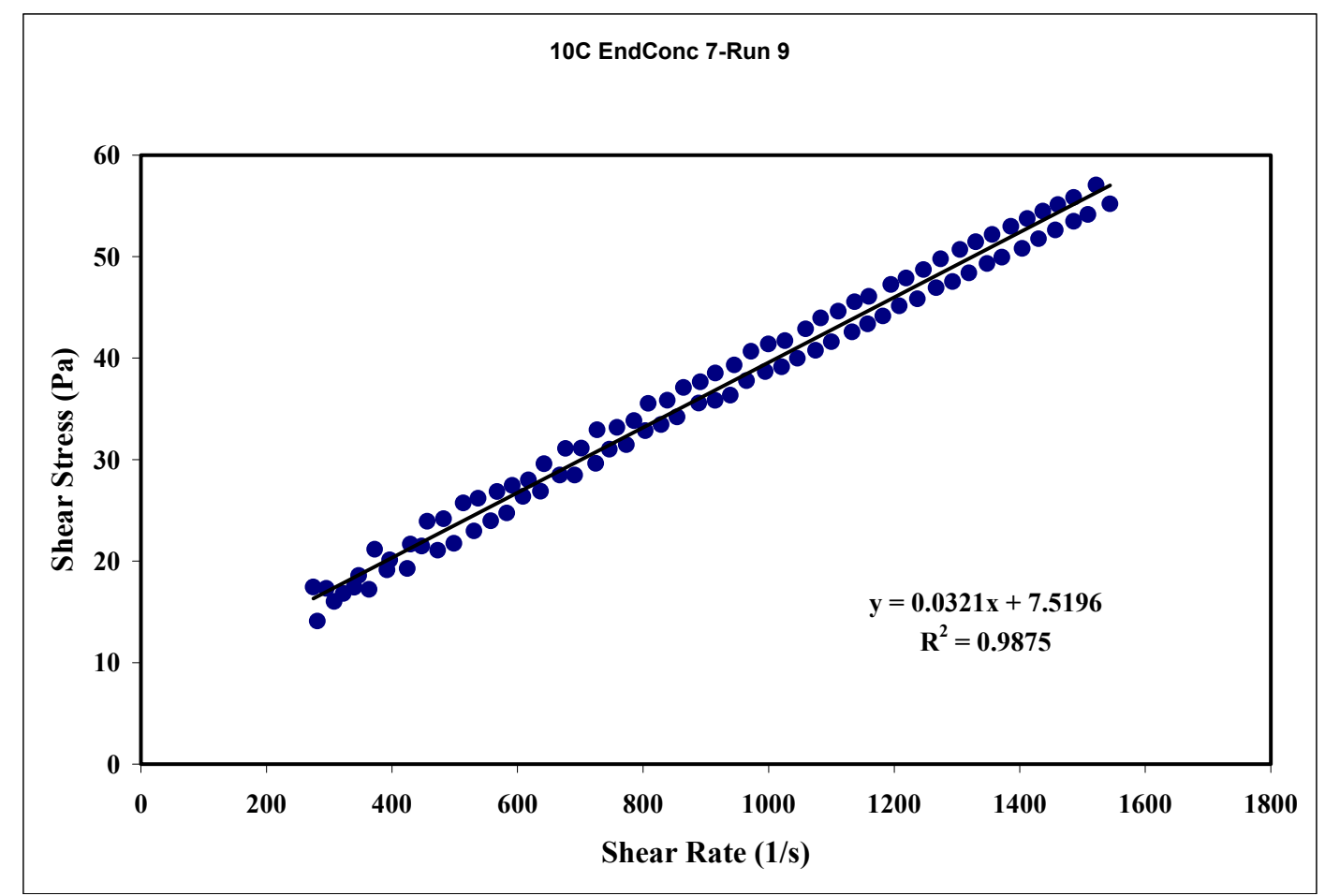

Figure 18. $10{ }^{\circ} \mathrm{C}$ at 16 wt \% Run 9 


\begin{tabular}{|ll|}
\hline APPENDIX I - PART 4 & WSRC-TR-2000-00352 \\
& SRT-RPP-2000-00026 \\
SR/TRU PRECIPITATE SLURRY RHEOGRAMS AT 16 WT \% & Page 428 of 514 \\
\hline
\end{tabular}

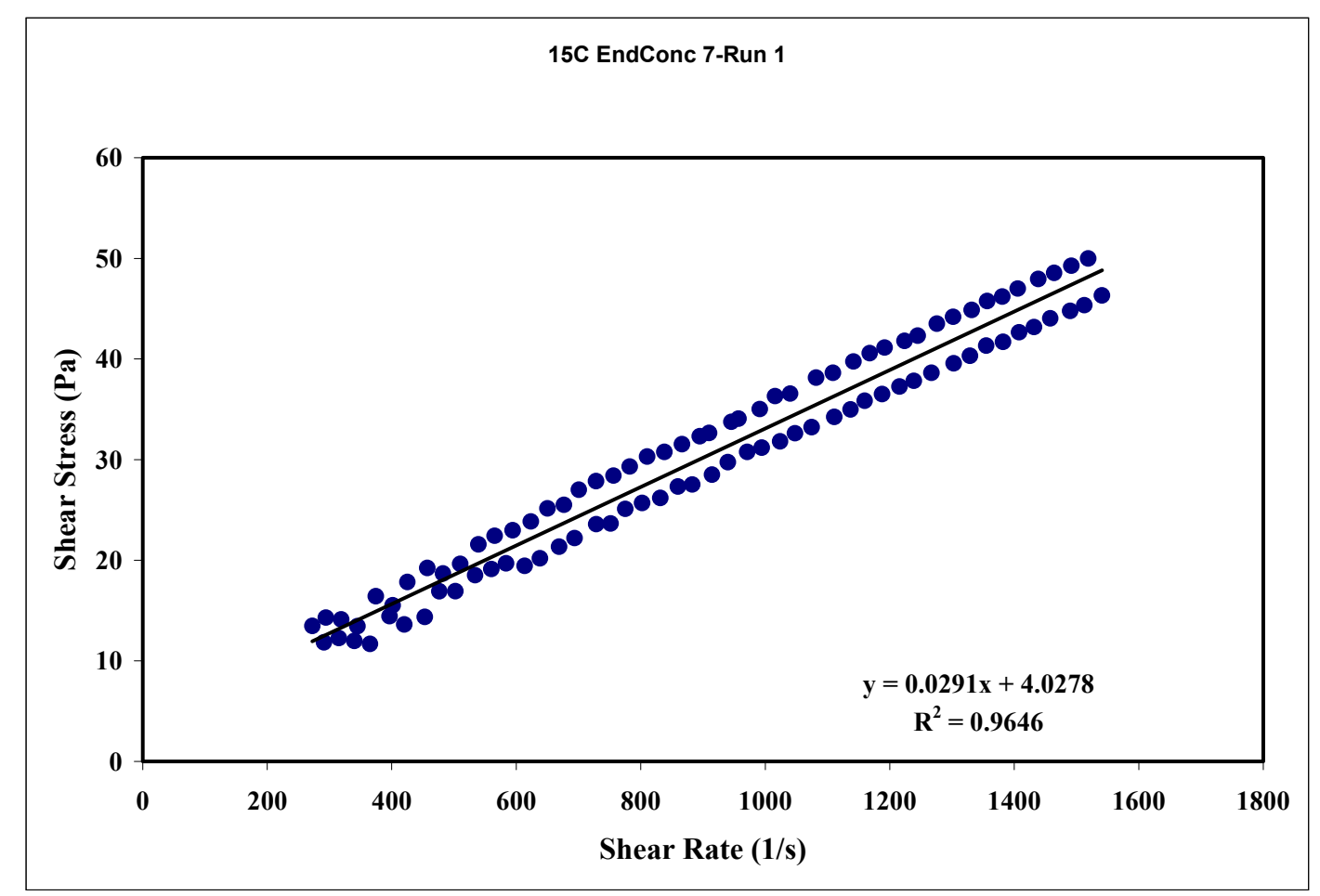

Figure 19. $15{ }^{\circ} \mathrm{C}$ at 16 wt \% Run 1

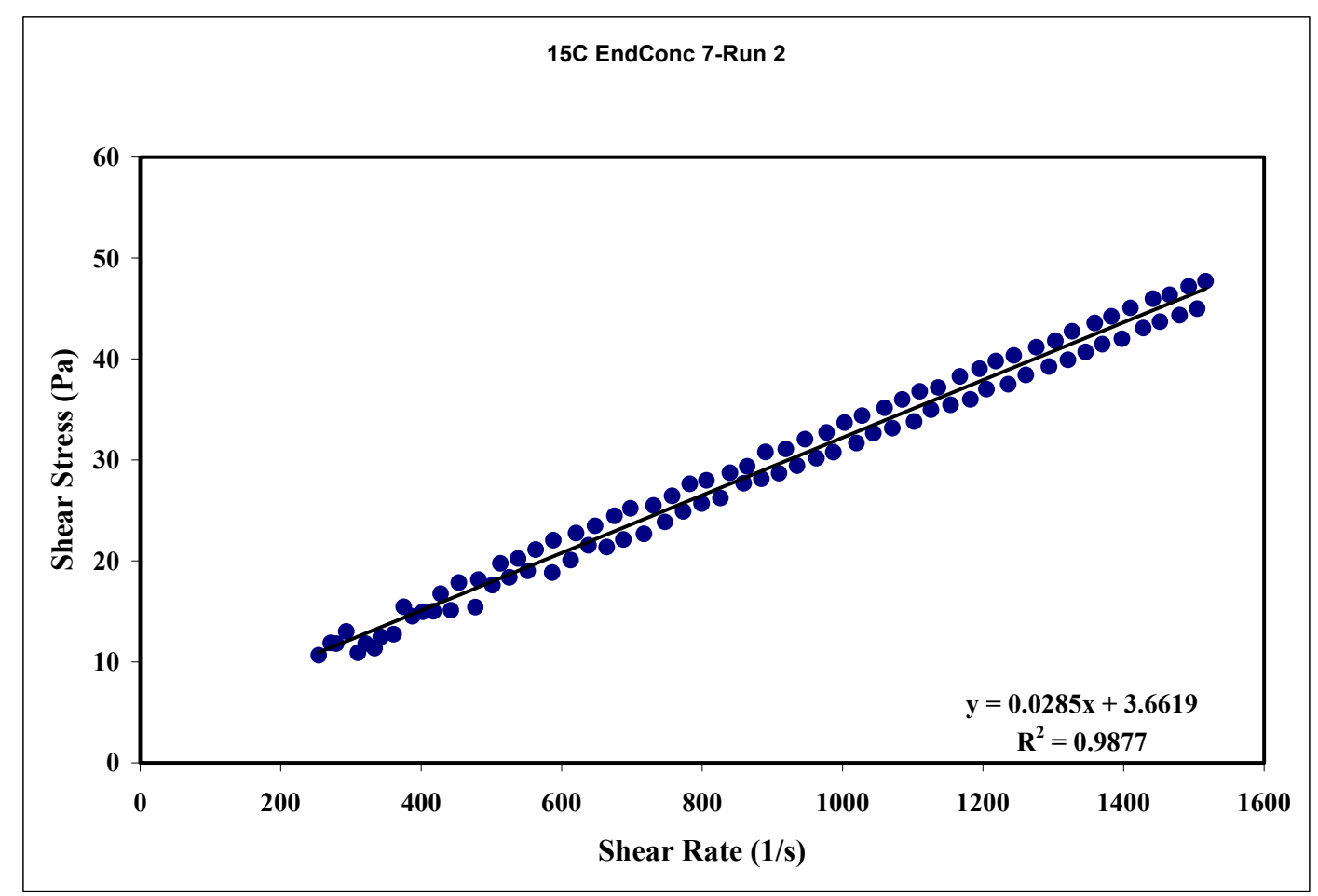

Figure 20. $15{ }^{\circ} \mathrm{C}$ at 16 wt \% Run 2 


\begin{tabular}{|ll|}
\hline APPENDIX I - PART 4 & WSRC-TR-2000-00352 \\
& SRT-RPP-2000-00026 \\
SR/TRU PRECIPITATE SLURRY RHEOGRAMS AT 16 WT \% & Page 429 of 514 \\
\hline
\end{tabular}

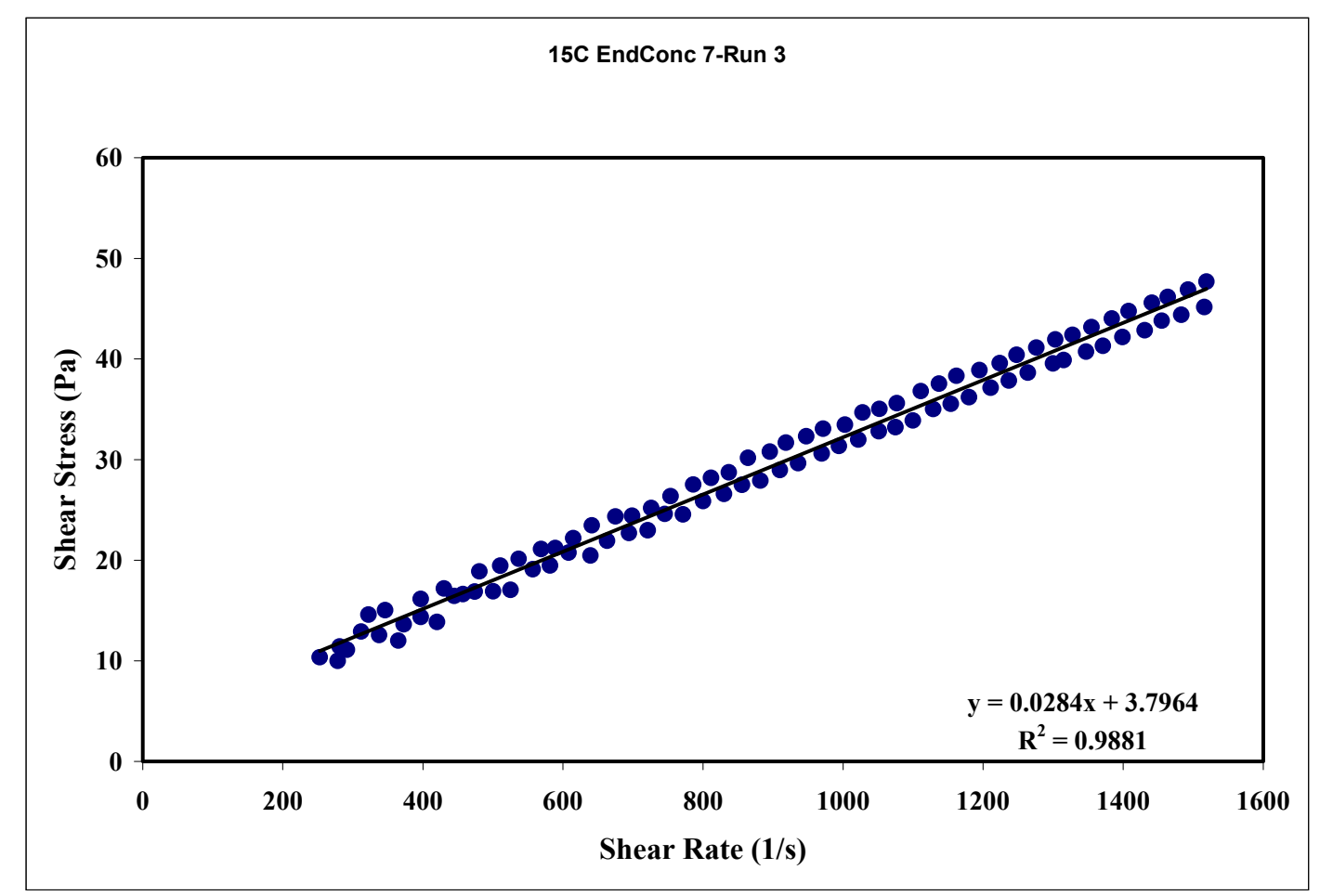

Figure 21. $15^{\circ} \mathrm{C}$ at 16 wt \% Run 3

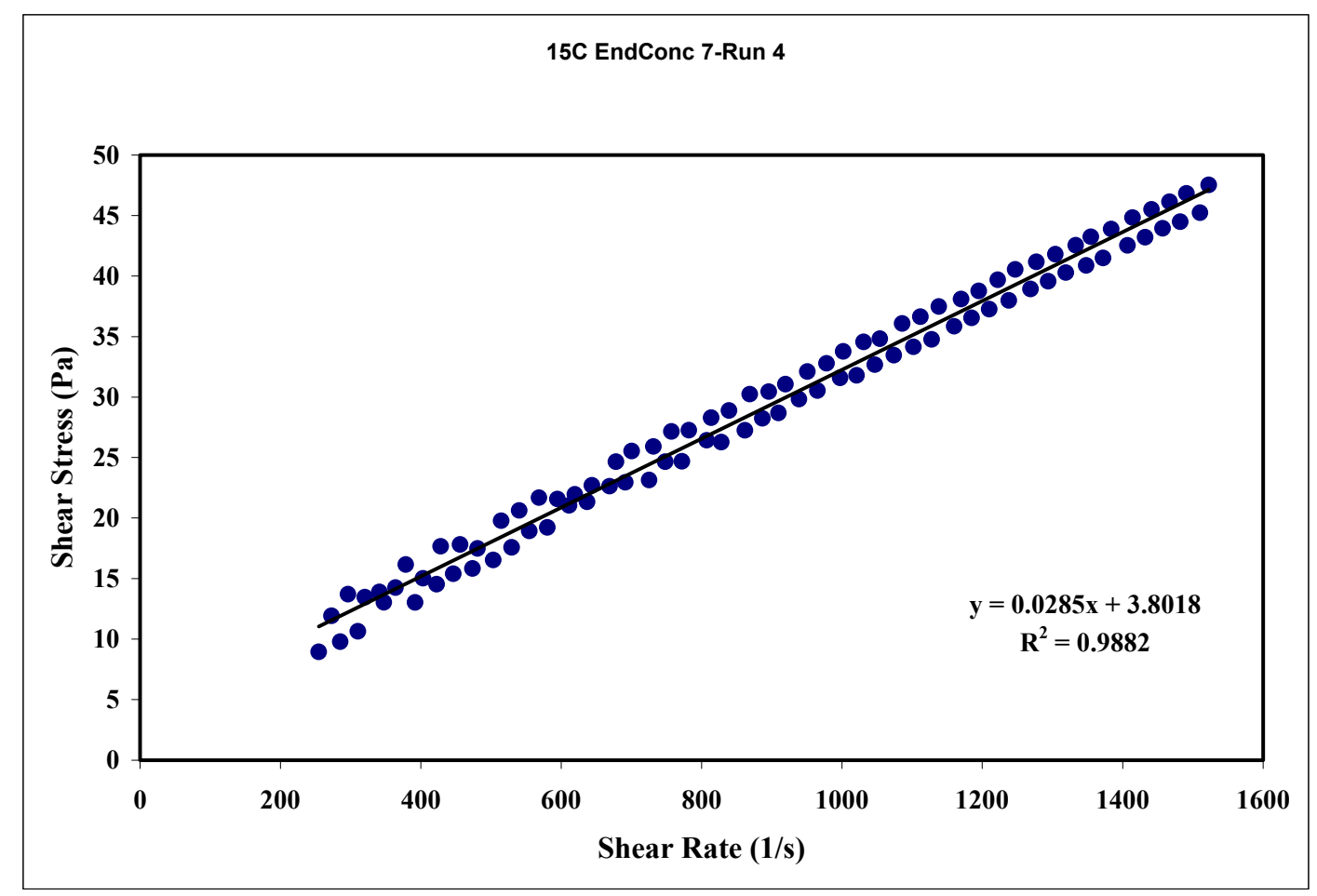

Figure $22.15^{\circ} \mathrm{C}$ at 16 wt \% Run 4 


\begin{tabular}{|ll|}
\hline APPENDIX I - PART 4 & WSRC-TR-2000-00352 \\
& SRT-RPP-2000-00026 \\
SR/TRU PRECIPITATE SLURRY RHEOGRAMS AT 16 WT \% & Page 430 of 514 \\
\hline
\end{tabular}

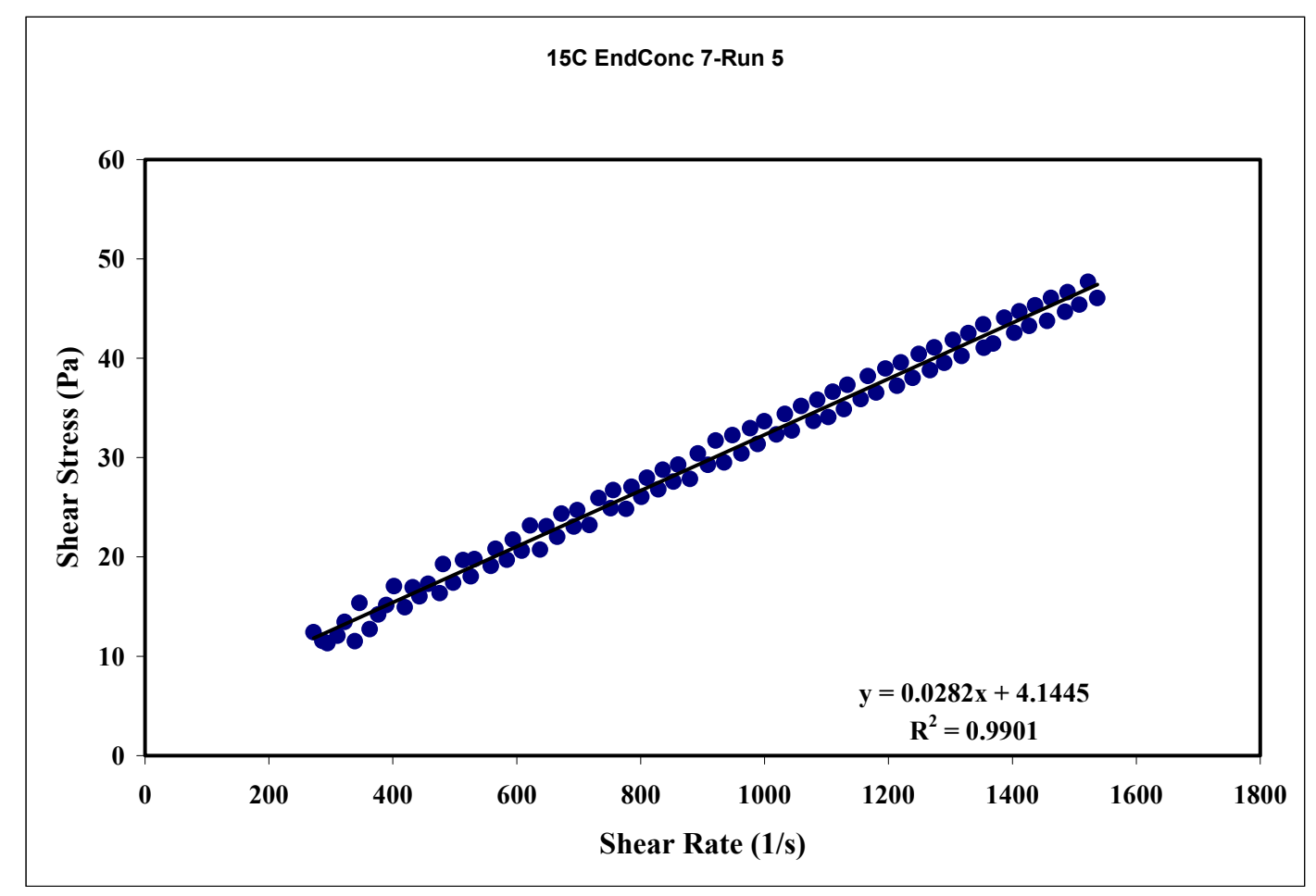

Figure 23. $15^{\circ} \mathrm{C}$ at 16 wt \% Run 5

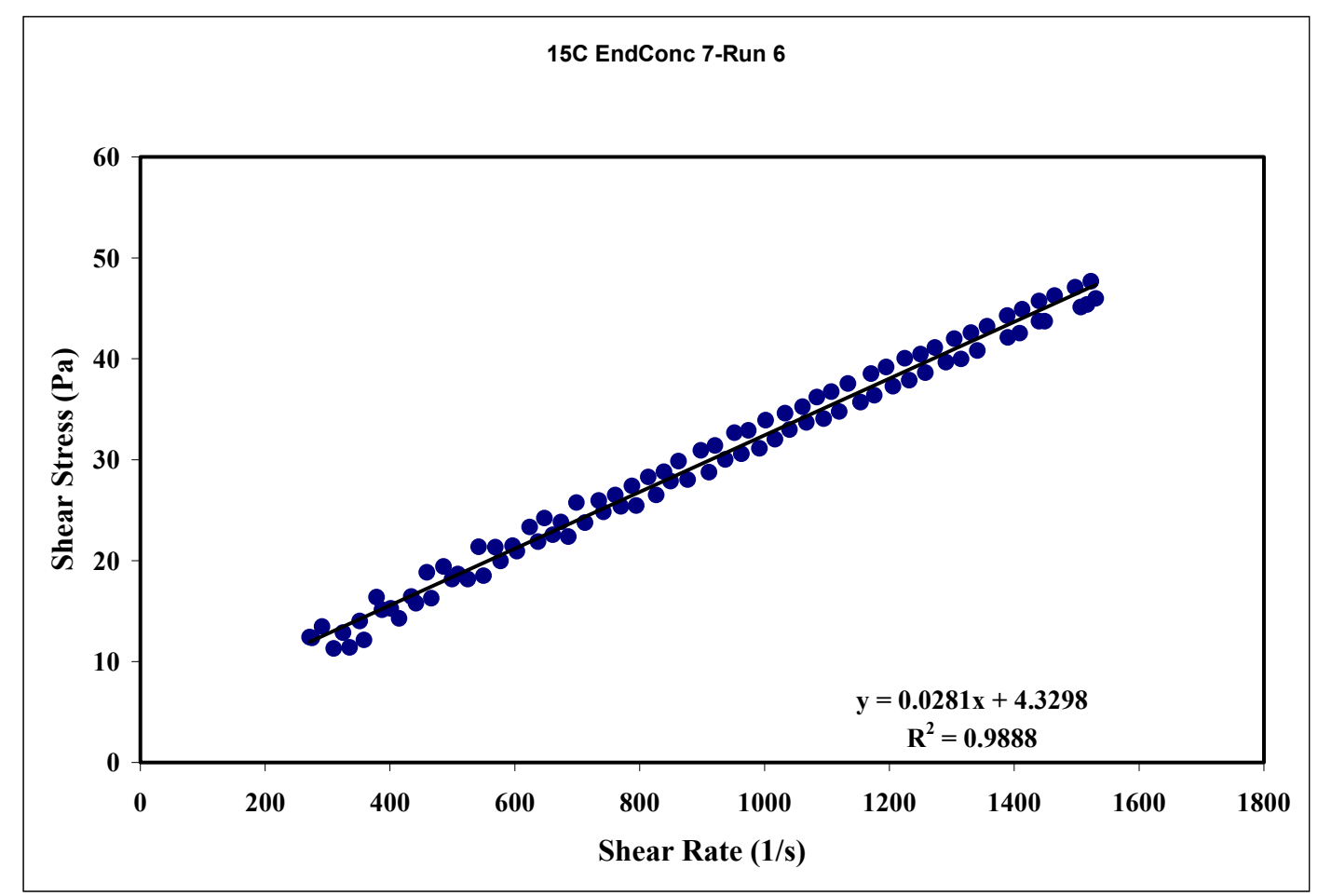

Figure 24. $15^{\circ} \mathrm{C}$ at 16 wt \% Run 6 


\begin{tabular}{|ll|}
\hline APPENDIX I - PART 4 & WSRC-TR-2000-00352 \\
& SRT-RPP-2000-00026 \\
SR/TRU PRECIPITATE SLURRY RHEOGRAMS AT 16 WT \% & Page 431 of 514 \\
\hline
\end{tabular}

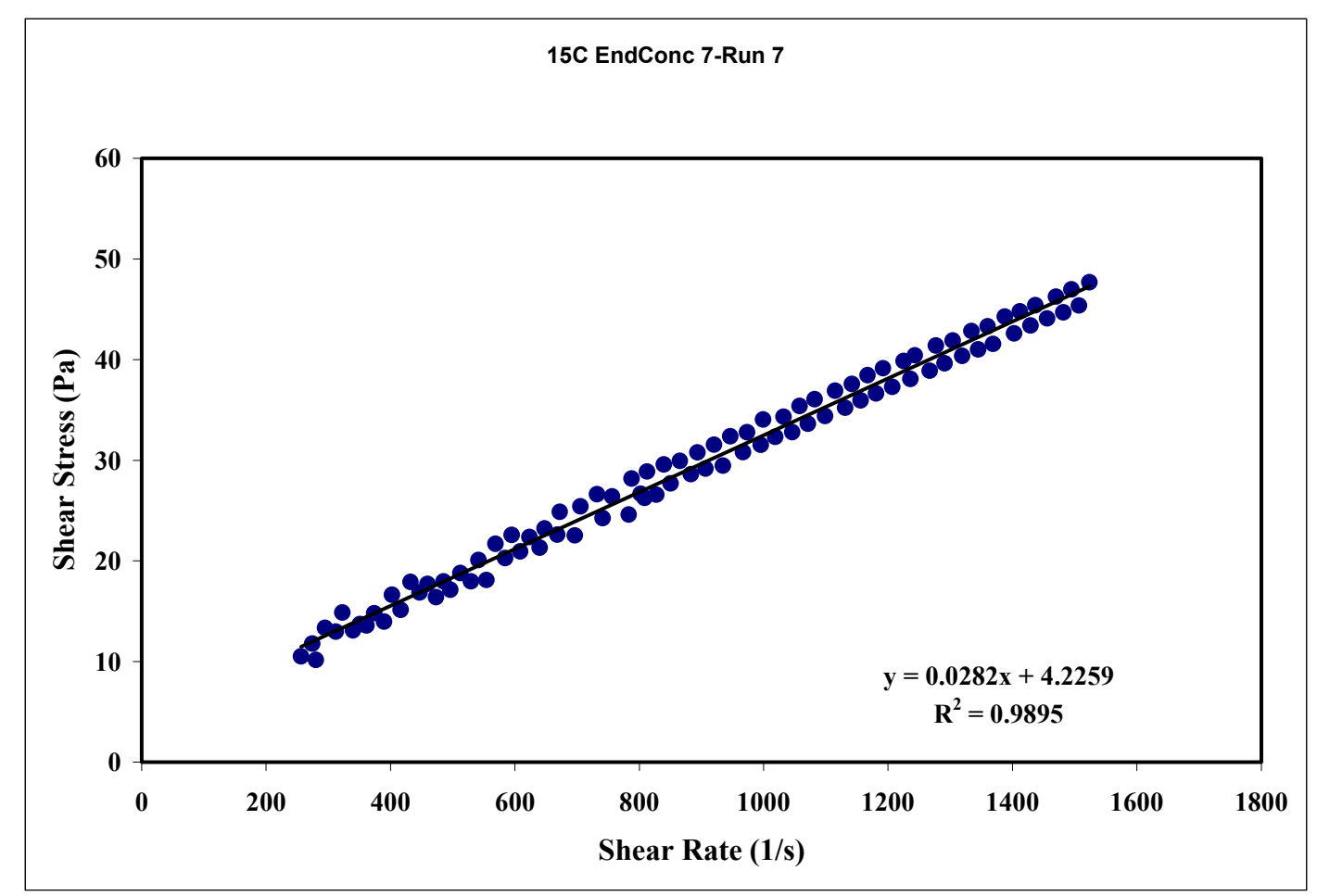

Figure 25. $15{ }^{\circ} \mathrm{C}$ at 16 wt \% Run 7

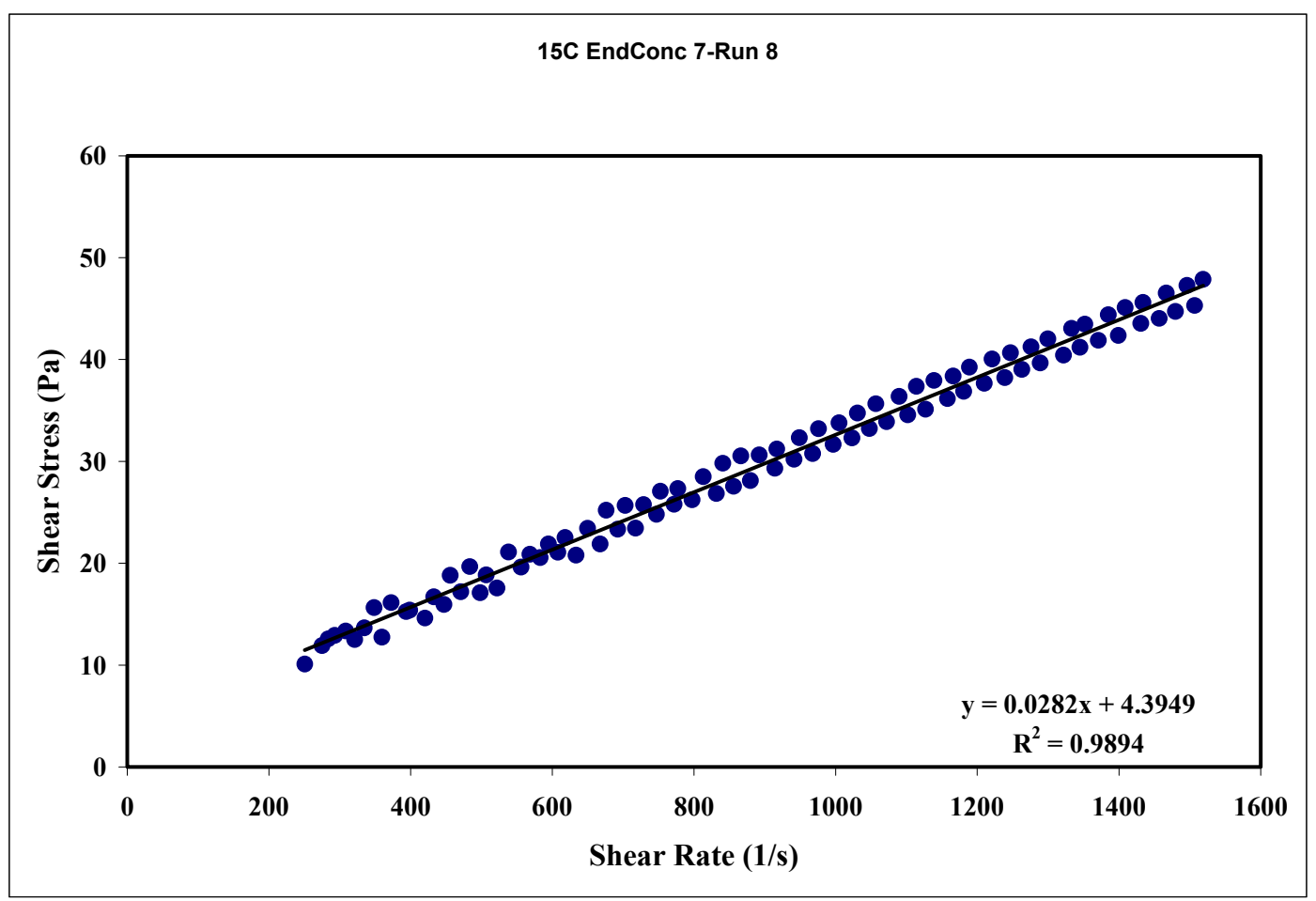

Figure 26. $15^{\circ} \mathrm{C}$ at 16 wt \% Run 8 


\begin{tabular}{|ll|}
\hline APPENDIX I - PART 4 & WSRC-TR-2000-00352 \\
SR/TRU PRECIPITATE SLURRY RHEOGRAMS AT 16 WT \% & SRT-RPP-2000-00026 \\
\hline
\end{tabular}

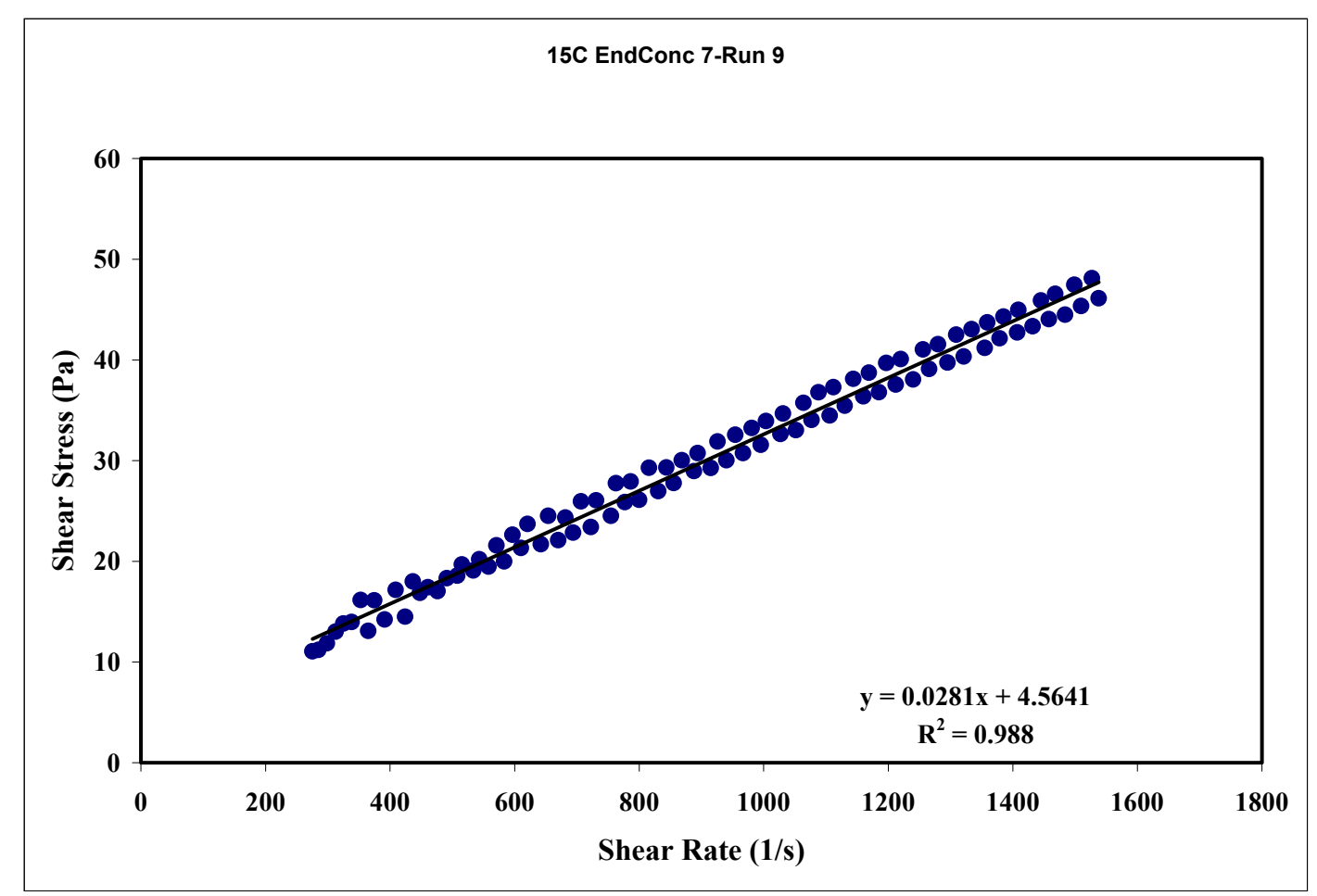

Figure 27. $15{ }^{\circ} \mathrm{C}$ at 16 wt \% Run 9

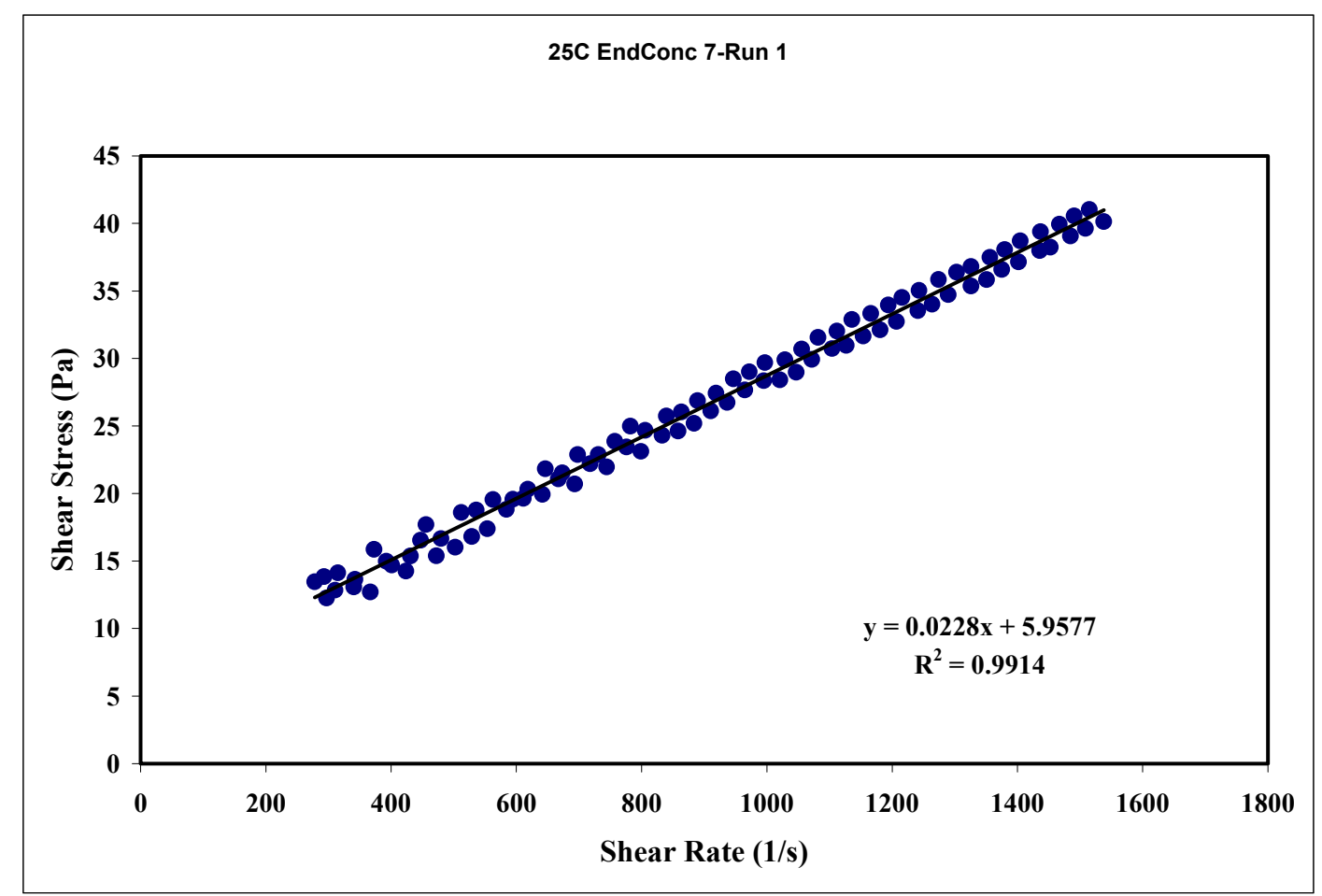

Figure 28. $25{ }^{\circ} \mathrm{C}$ at 16 wt \% Run 1 


\begin{tabular}{|ll|}
\hline APPENDIX I - PART 4 & WSRC-TR-2000-00352 \\
SR/TRU PRECIPITATE SLURRY RHEOGRAMS AT 16 WT \% & SRT-RPP-2000-00026 \\
\hline
\end{tabular}

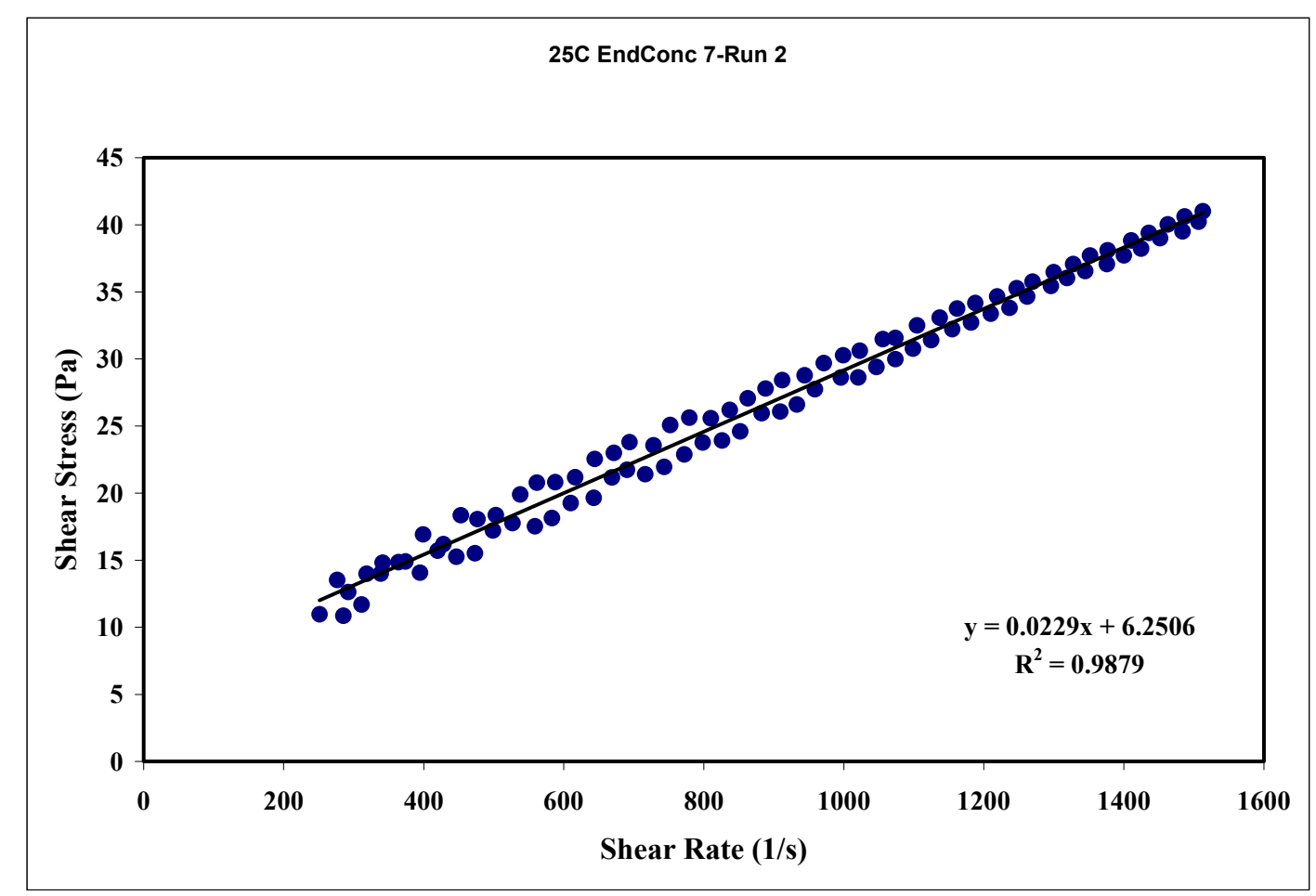

Figure 29. $25{ }^{\circ} \mathrm{C}$ at 16 wt \% Run 2

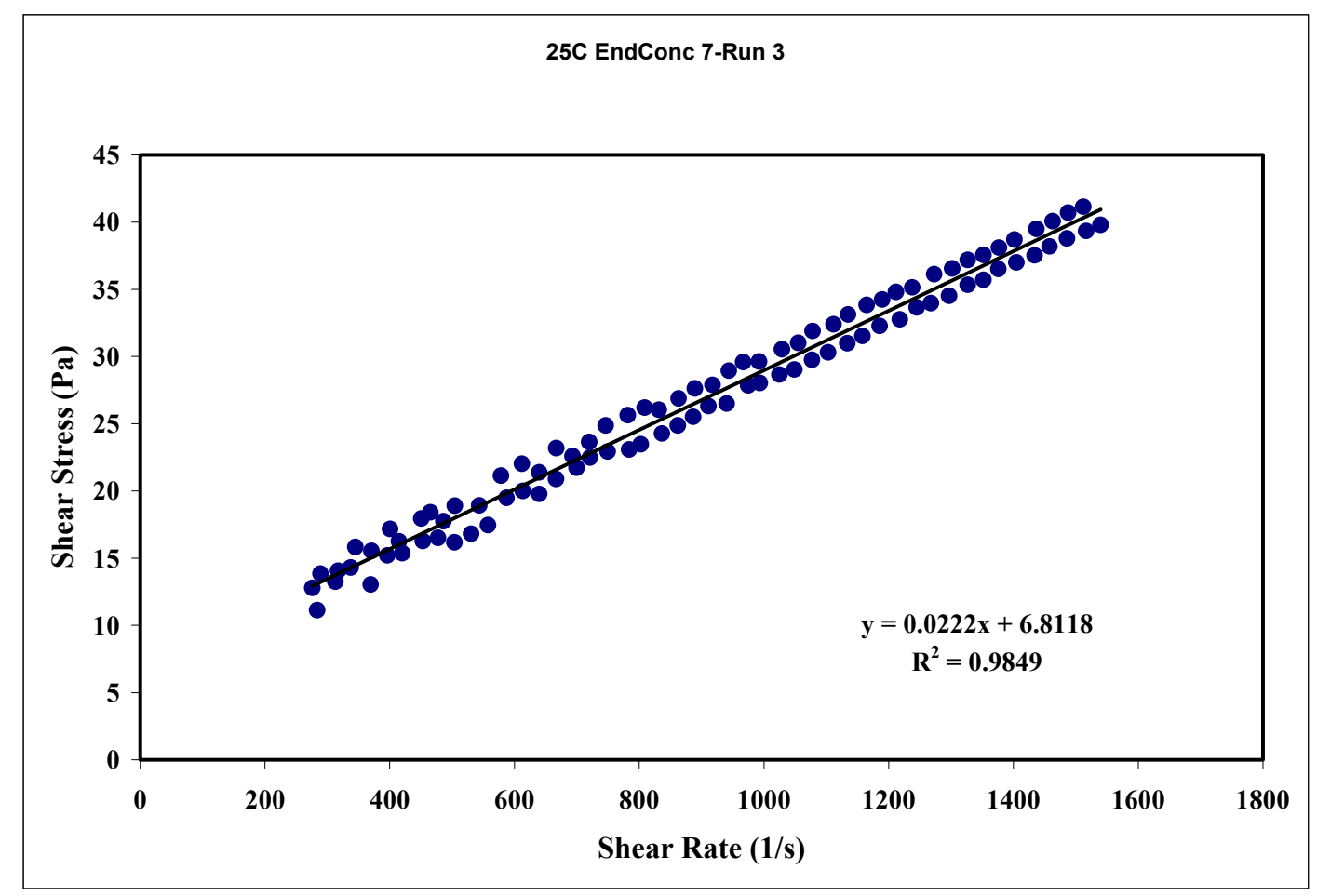

Figure 30. $25{ }^{\circ} \mathrm{C}$ at 16 wt \% Run 3 


\begin{tabular}{|ll|}
\hline APPENDIX I - PART 4 & WSRC-TR-2000-00352 \\
SR/TRU PRECIPITATE SLURRY RHEOGRAMS AT 16 WT \% & SRT-RPP-2000-00026 \\
\hline
\end{tabular}

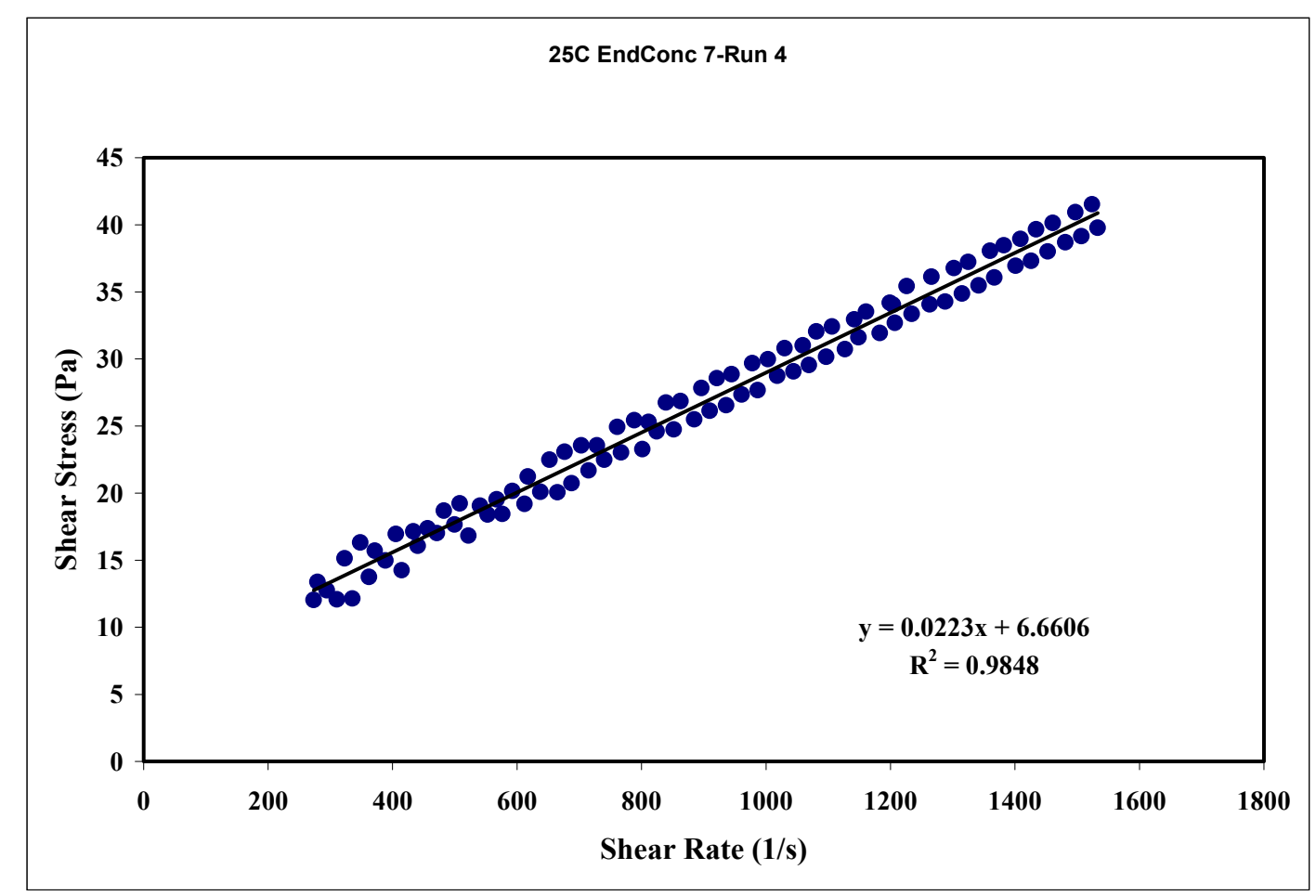

Figure 31. $25^{\circ} \mathrm{C}$ at 16 wt \% Run 4

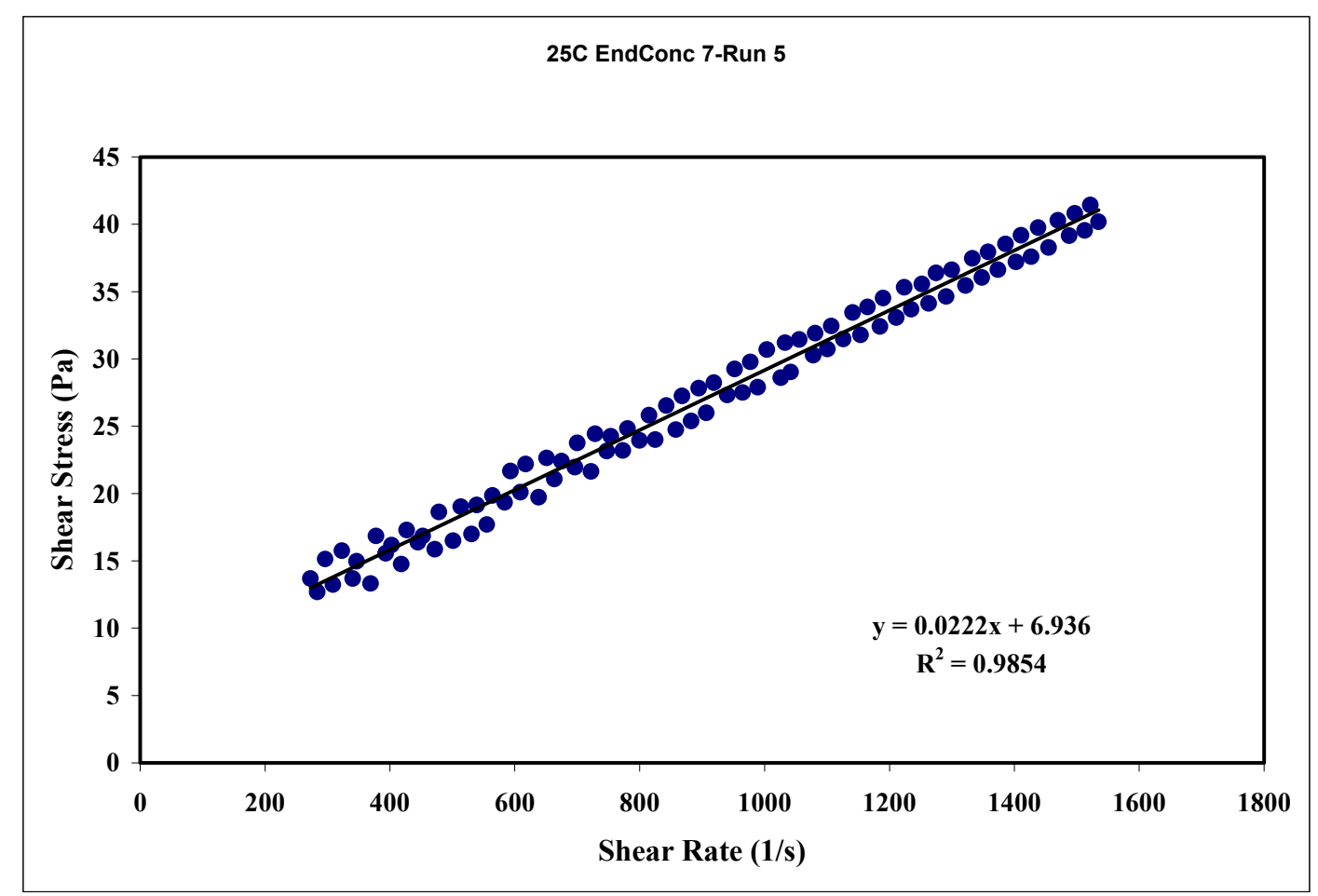

Figure 32. $25^{\circ} \mathrm{C}$ at 16 wt \% Run 5 


\begin{tabular}{|ll|}
\hline APPENDIX I - PART 4 & WSRC-TR-2000-00352 \\
& SRT-RPP-2000-00026 \\
SR/TRU PRECIPITATE SLURRY RHEOGRAMS AT 16 WT \% & Page 435 of 514 \\
\hline
\end{tabular}

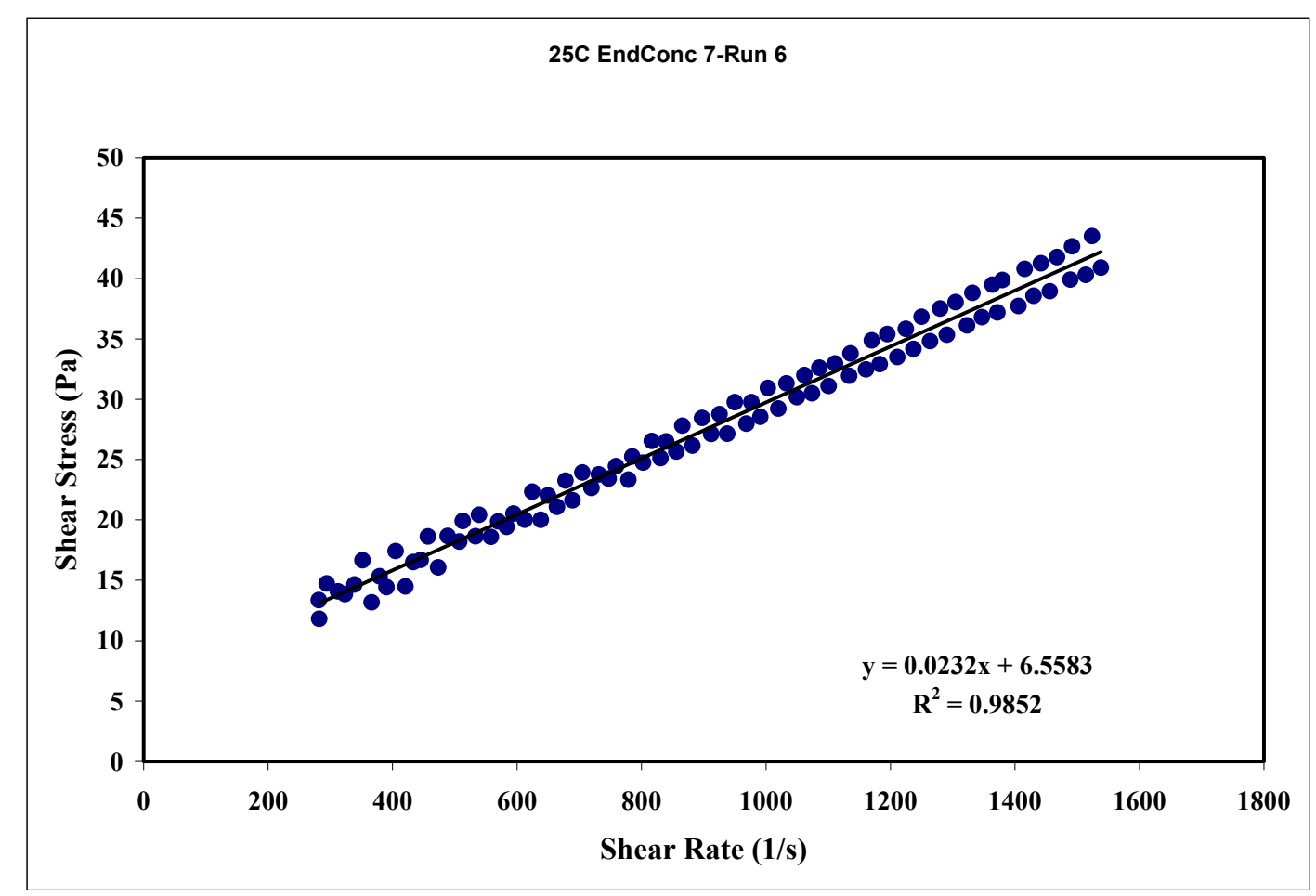

Figure 33. $25^{\circ} \mathrm{C}$ at 16 wt \% Run 6

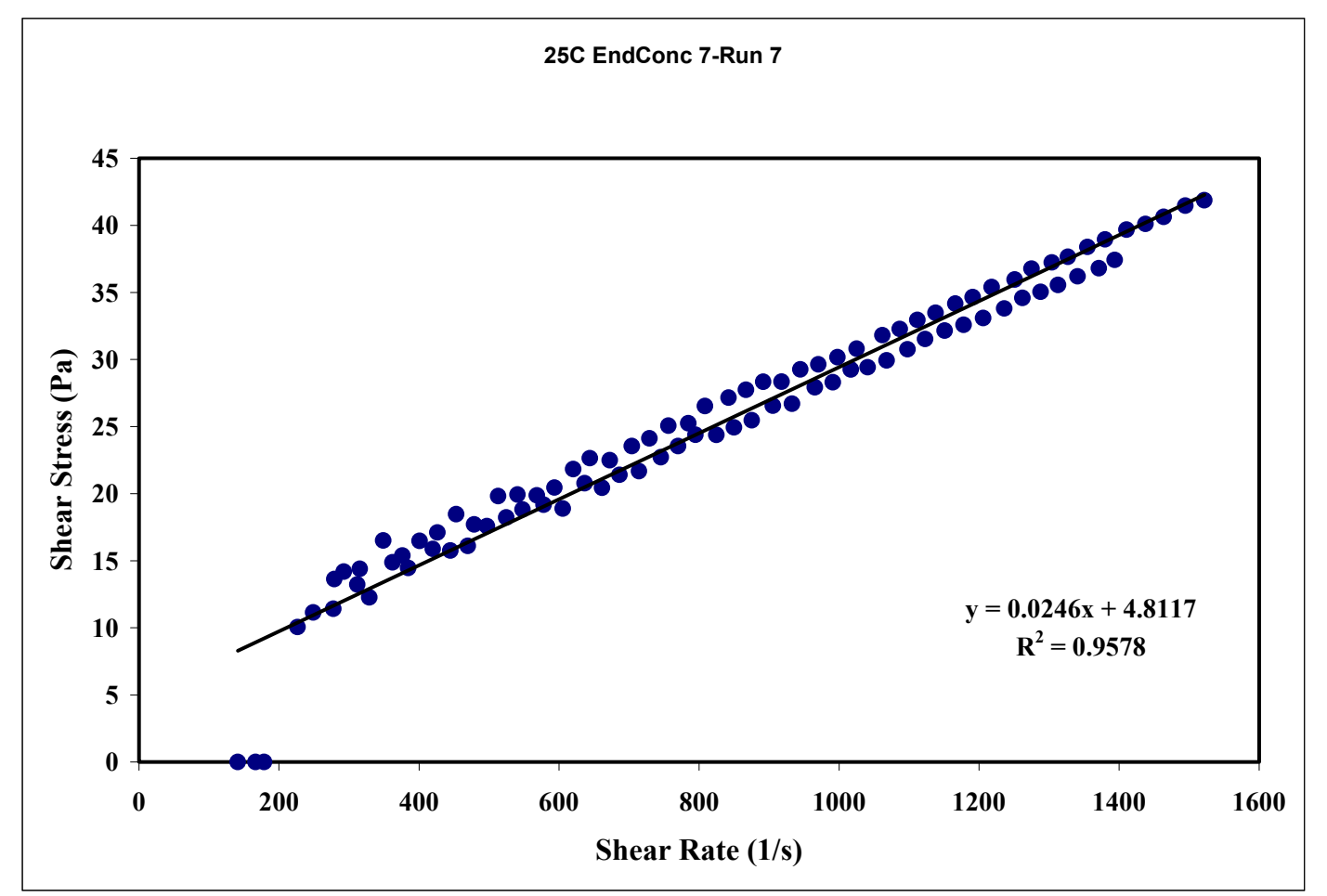

Figure 34. $25{ }^{\circ} \mathrm{C}$ at 16 wt \% Run 7 


\begin{tabular}{|ll|}
\hline APPENDIX I - PART 4 & WSRC-TR-2000-00352 \\
SR/TRU PRECIPITATE SLURRY RHEOGRAMS AT 16 WT \% & SRT-RPP-2000-00026 \\
\hline
\end{tabular}

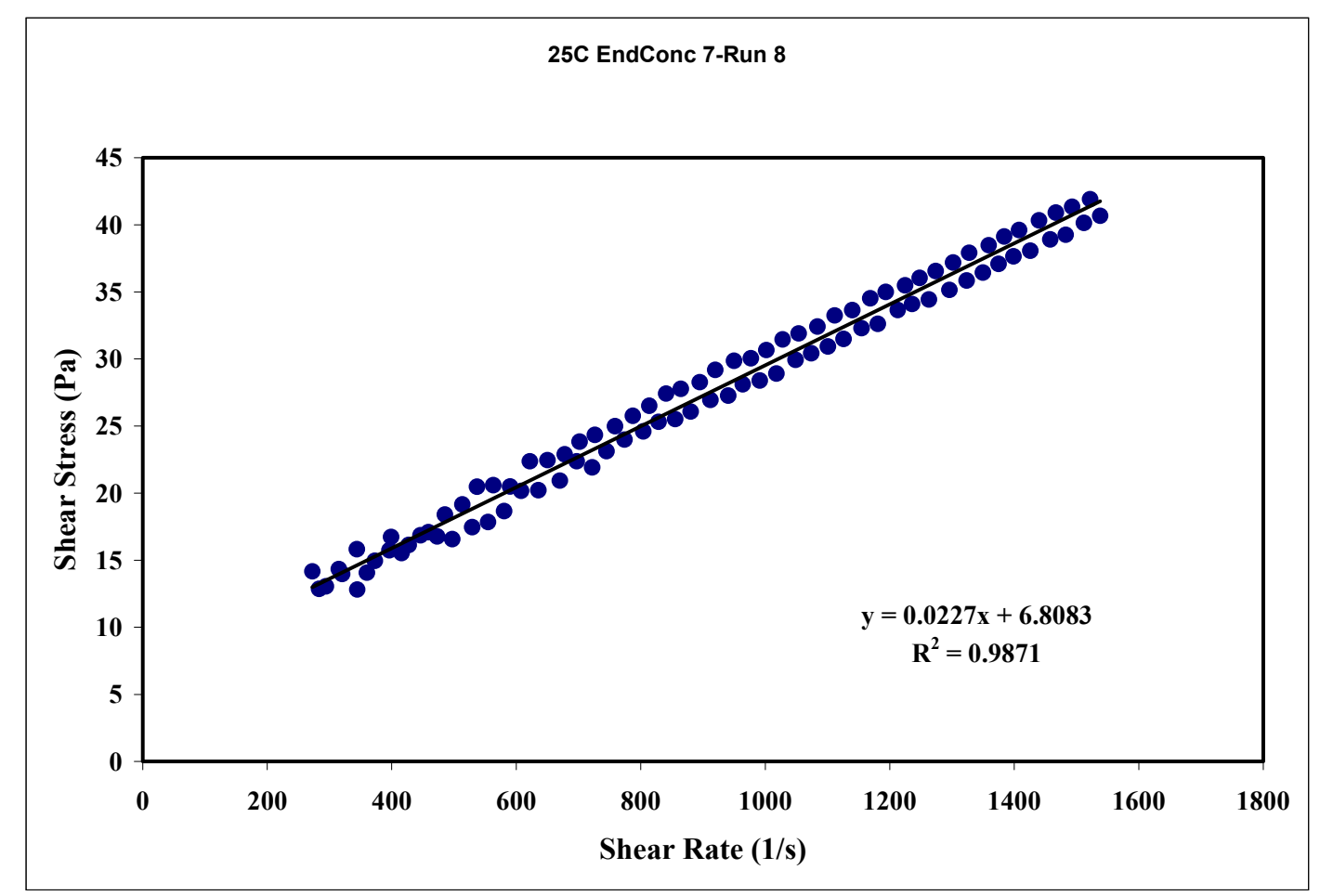

Figure 35. $25^{\circ} \mathrm{C}$ at 16 wt \% Run 8

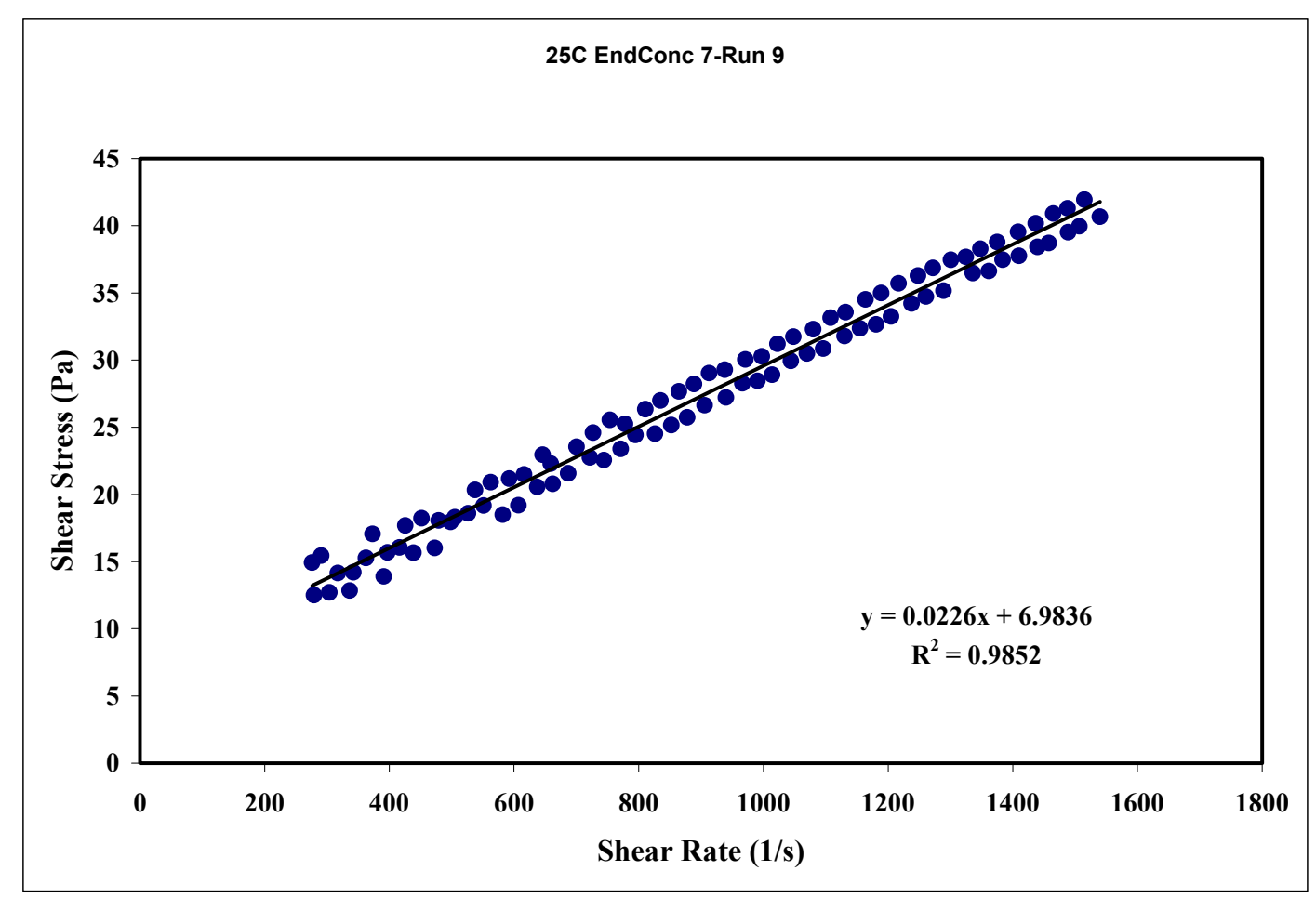

Figure 36. $25{ }^{\circ} \mathrm{C}$ at 16 wt \% Run 9 


\begin{tabular}{|ll|}
\hline APPENDIX I - PART 4 & WSRC-TR-2000-00352 \\
SR/TRU PRECIPITATE SLURRY RHEOGRAMS AT 16 WT \% & SRT-RPP-2000-00026 \\
\hline
\end{tabular}

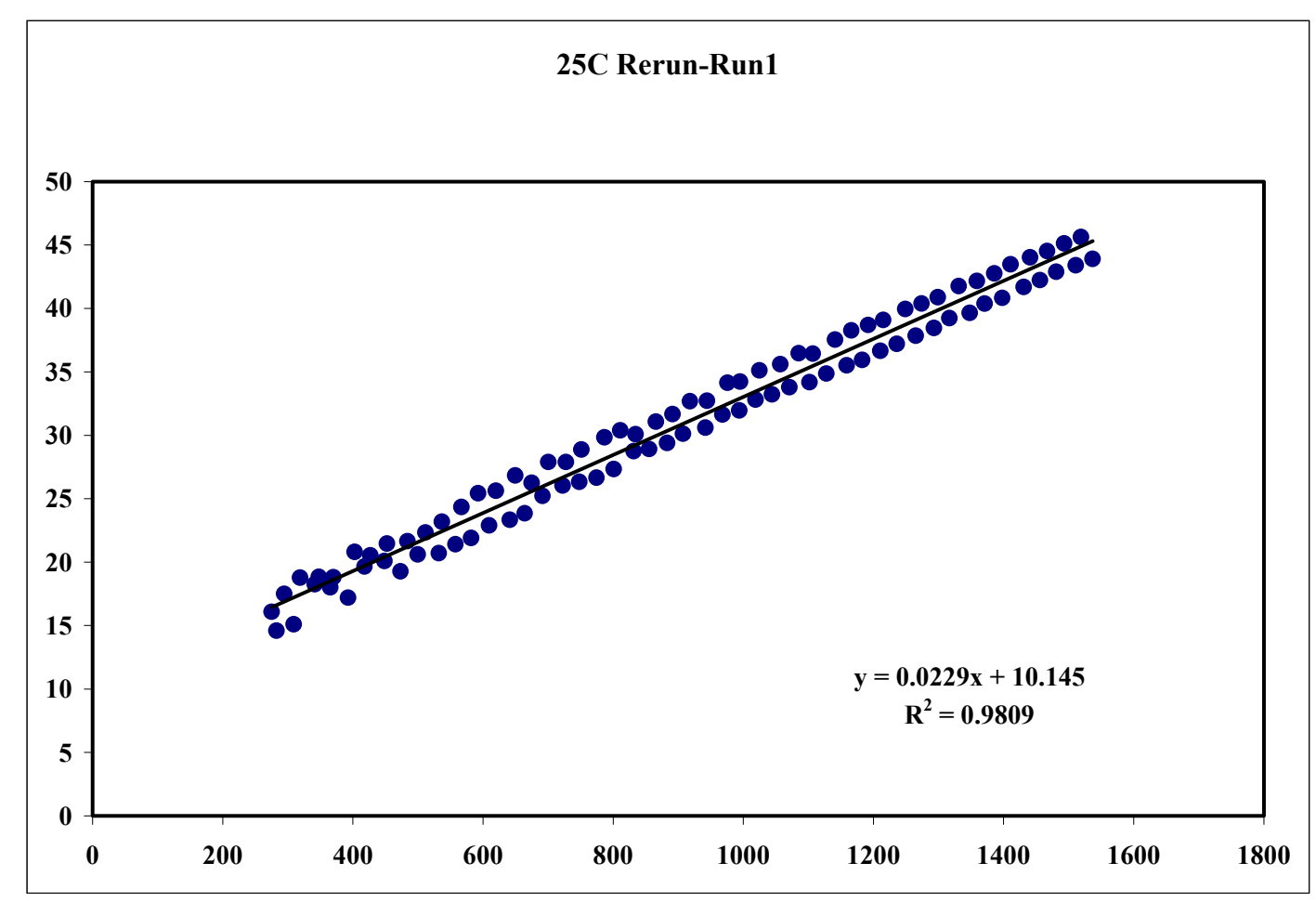

Figure 37. $25{ }^{\circ} \mathrm{C}$ Repeat at 16 wt \% Run 1

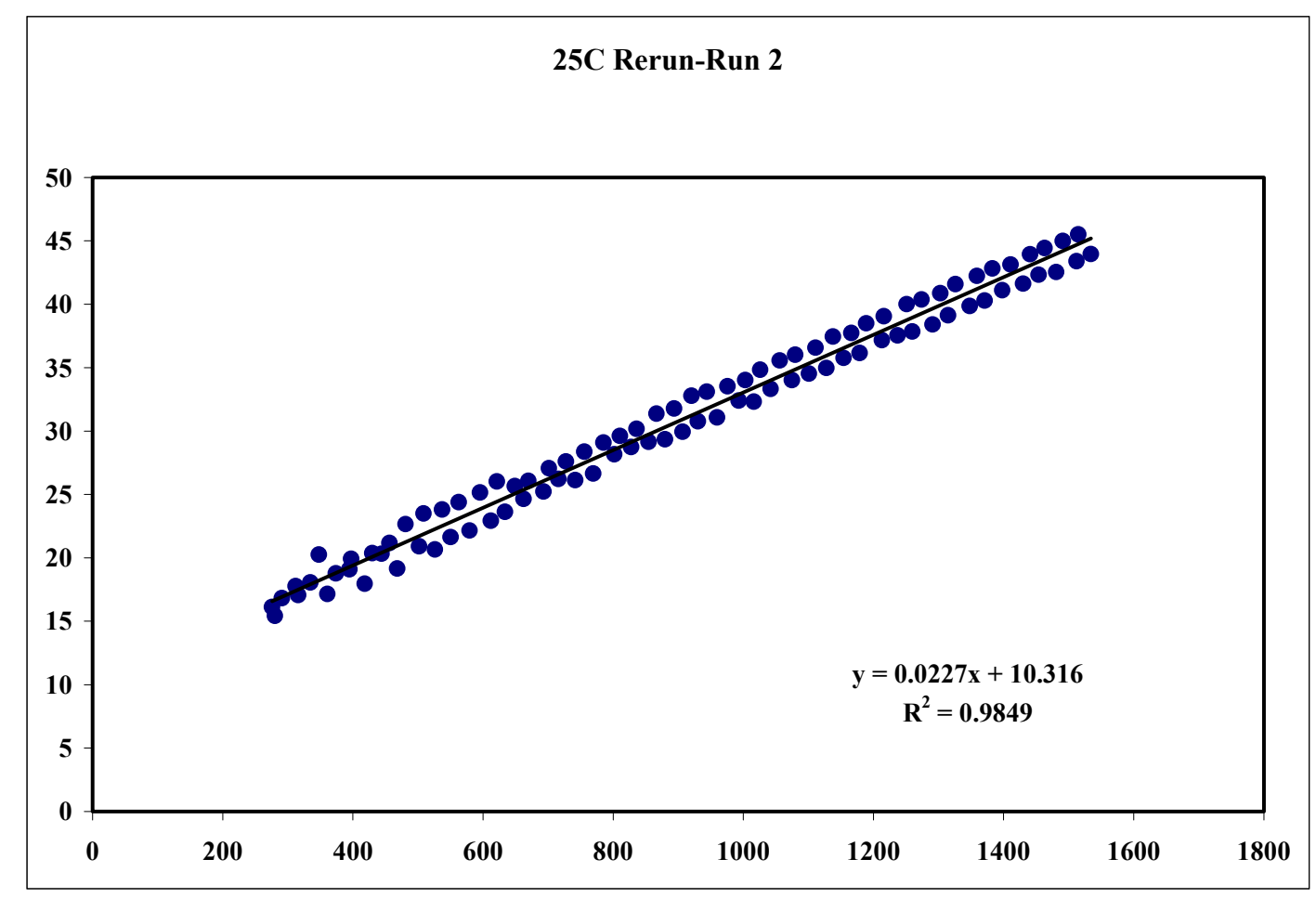

Figure 38. $25{ }^{\circ} \mathrm{C}$ Repeat at 16 wt \% Run 2 


\begin{tabular}{|ll|}
\hline APPENDIX I - PART 4 & WSRC-TR-2000-00352 \\
& SRT-RPP-2000-00026 \\
SR/TRU PRECIPITATE SLURRY RHEOGRAMS AT 16 WT \% & Page 438 of 514 \\
\hline
\end{tabular}

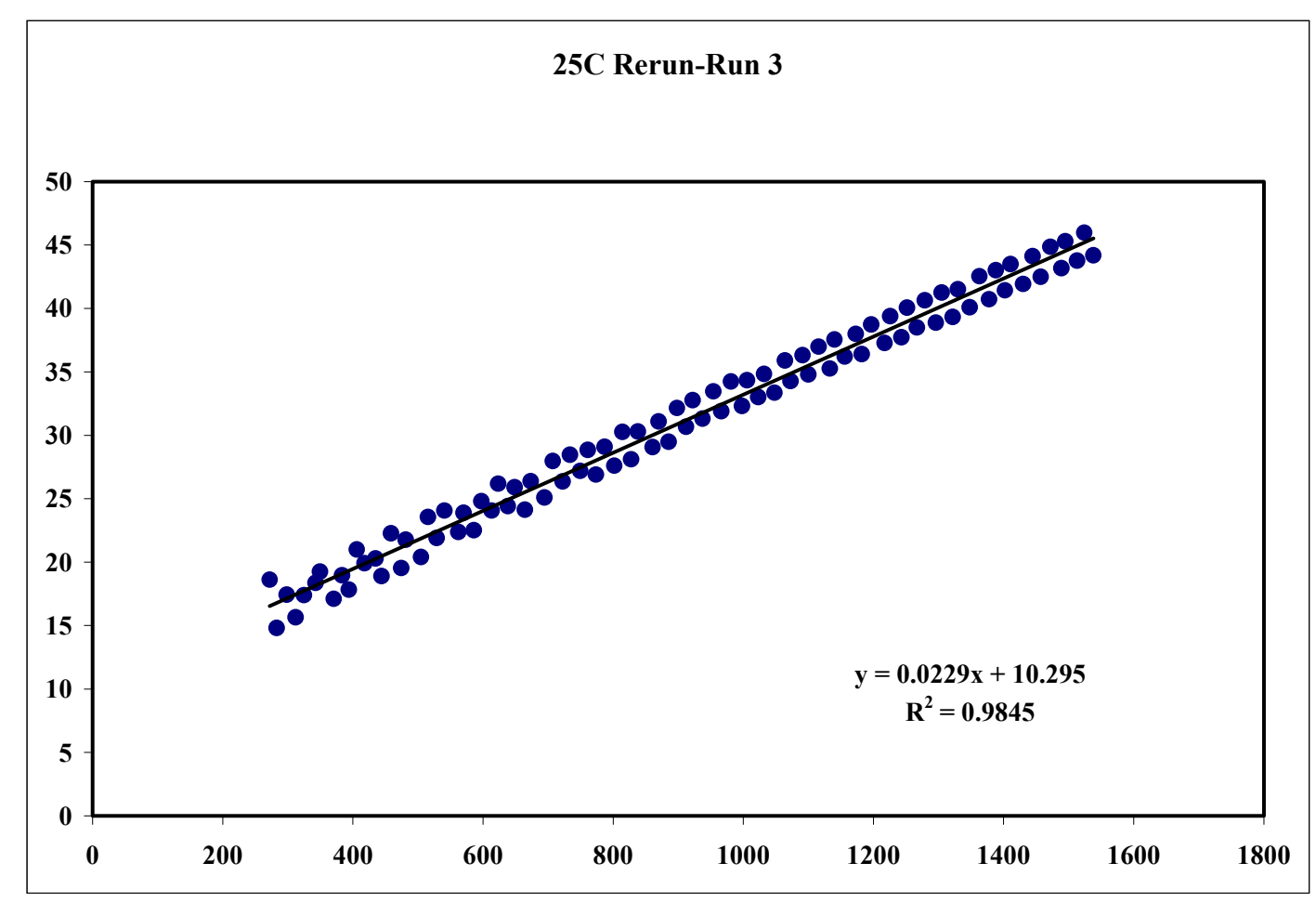

Figure 39. $25^{\circ} \mathrm{C}$ Repeat at 16 wt \% Run 3

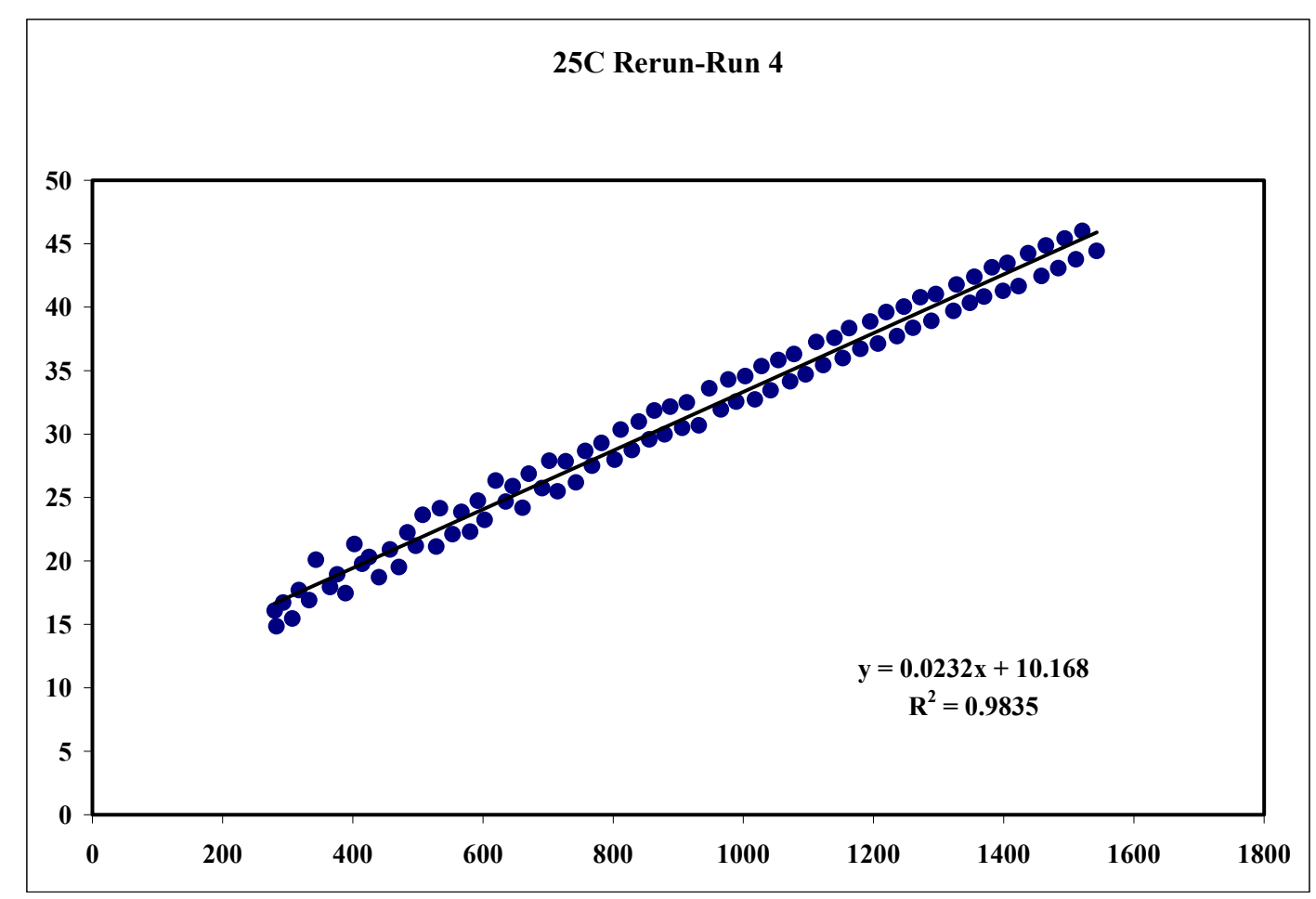

Figure 40. $25{ }^{\circ} \mathrm{C}$ Repeat at 16 wt \% Run 4 


\begin{tabular}{|ll|}
\hline APPENDIX I - PART 4 & WSRC-TR-2000-00352 \\
& SRT-RPP-2000-00026 \\
SR/TRU PRECIPITATE SLURRY RHEOGRAMS AT 16 WT \% & Page 439 of 514 \\
\hline
\end{tabular}

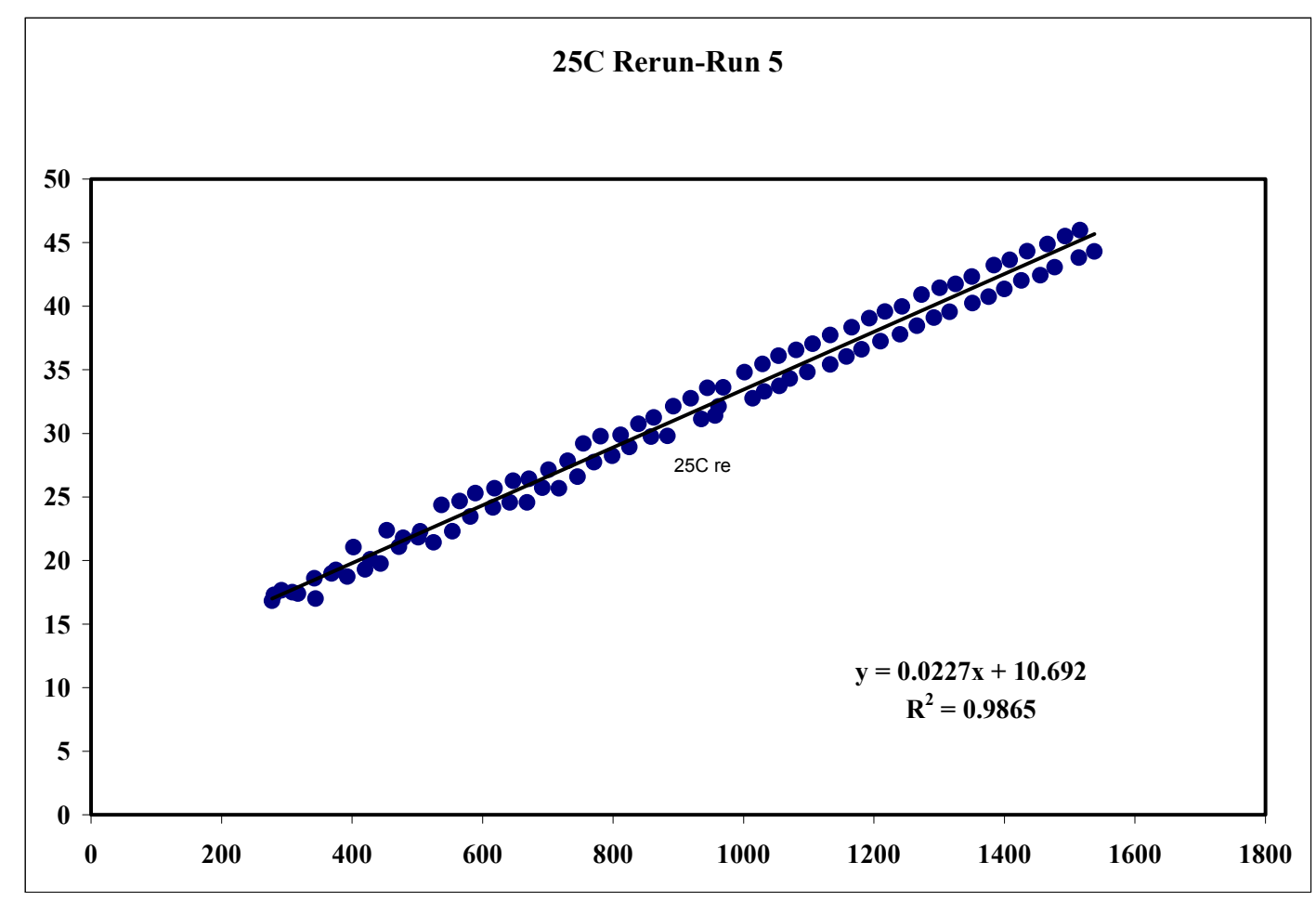

Figure 41. $25^{\circ} \mathrm{C}$ Repeat at 16 wt \% Run 5

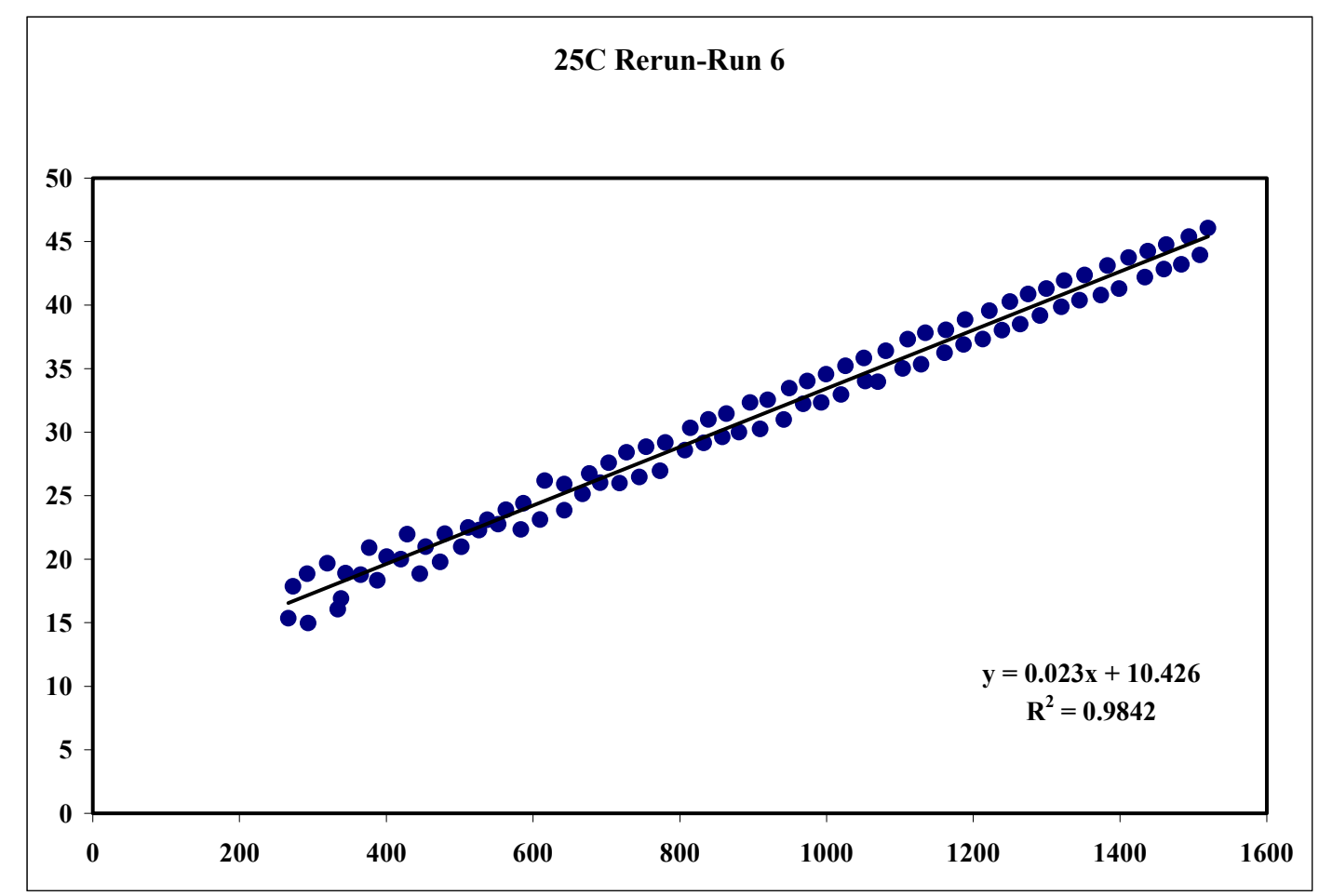

Figure 42. $25^{\circ} \mathrm{C}$ Repeat at 16 wt \% Run 6 


\begin{tabular}{|ll|}
\hline APPENDIX I - PART 4 & WSRC-TR-2000-00352 \\
SR/TRU PRECIPITATE SLURRY RHEOGRAMS AT 16 WT \% & SRT-RPP-2000-00026 \\
\hline
\end{tabular}

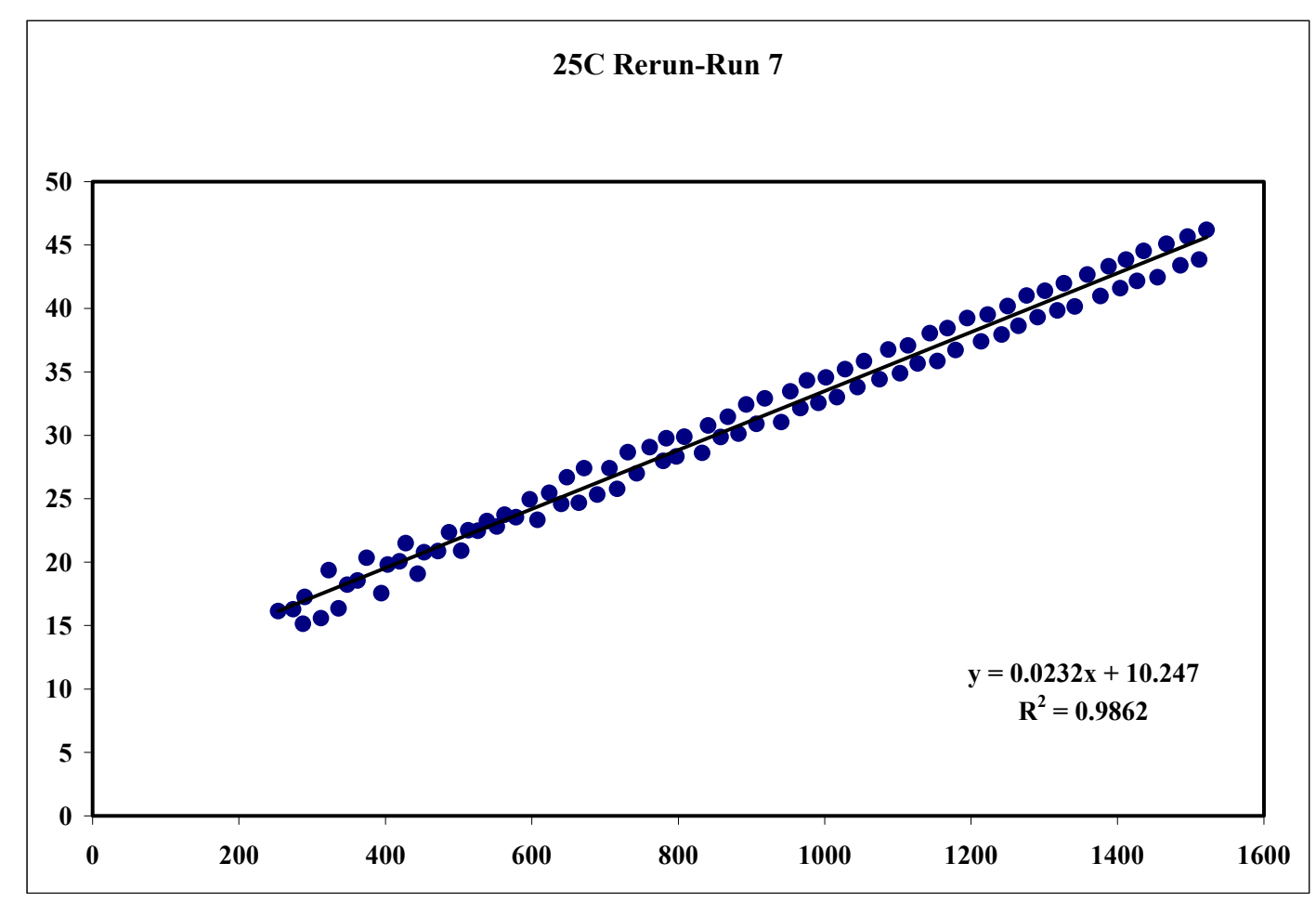

Figure 43. $25{ }^{\circ} \mathrm{C}$ Repeat at 16 wt \% Run 7

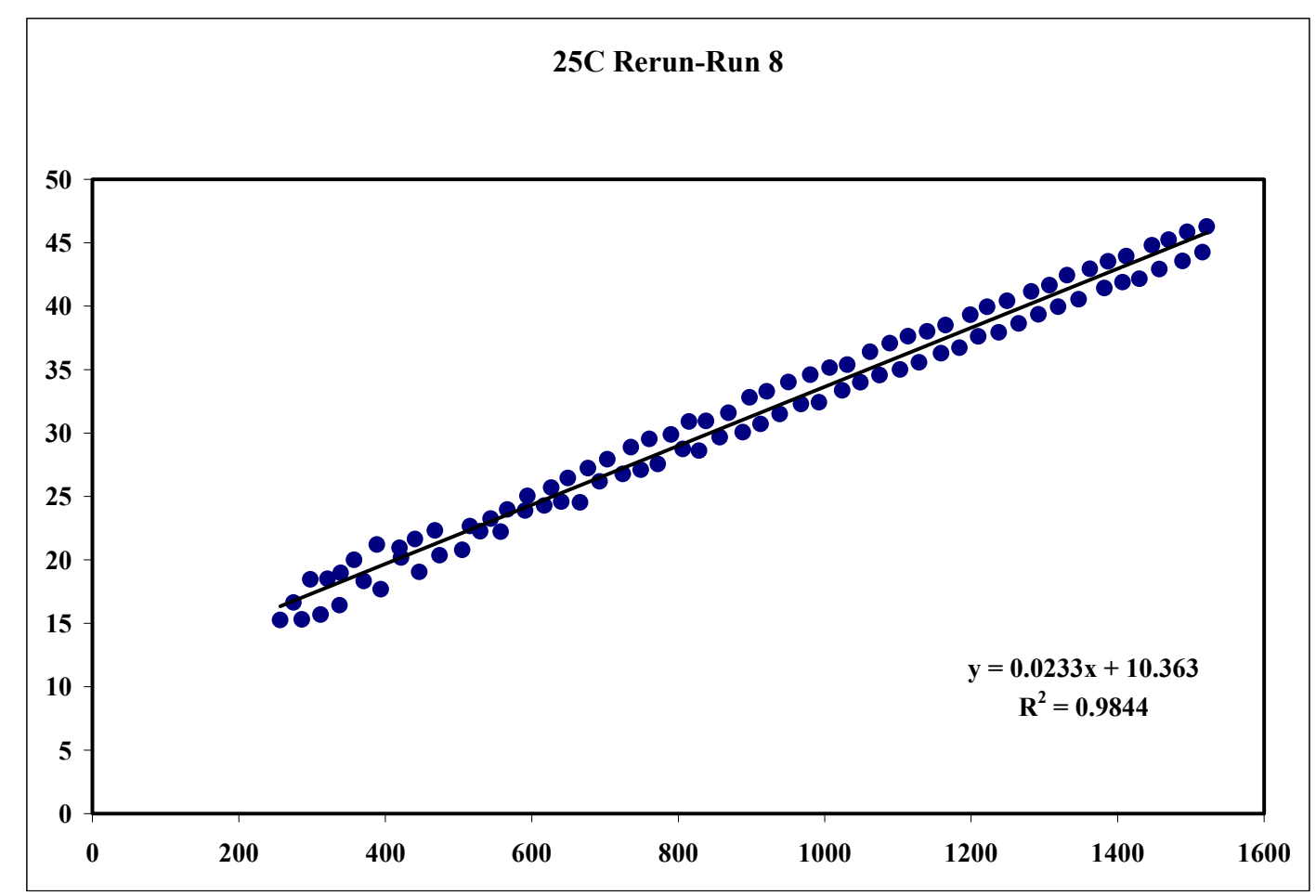

Figure 44. $25{ }^{\circ} \mathrm{C}$ Repeat at 16 wt \% Run 8 


\begin{tabular}{|ll|}
\hline APPENDIX I - PART 4 & WSRC-TR-2000-00352 \\
SR/TRU PRECIPITATE SLURRY RHEOGRAMS AT 16 WT \% & SRT-RPP-2000-00026 \\
\hline
\end{tabular}

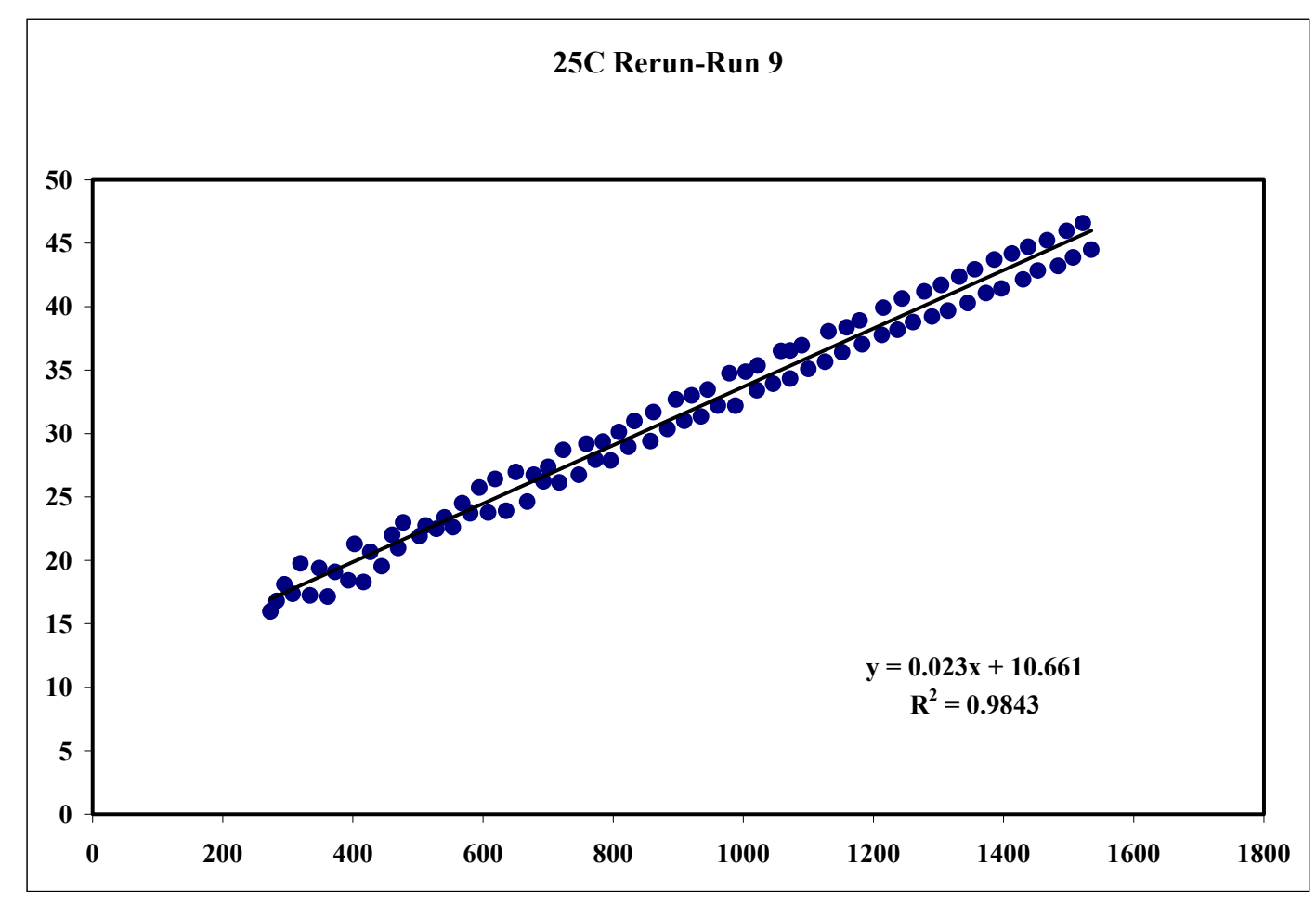

Figure 45. $25{ }^{\circ} \mathrm{C}$ Repeat at 16 wt \% Run 9

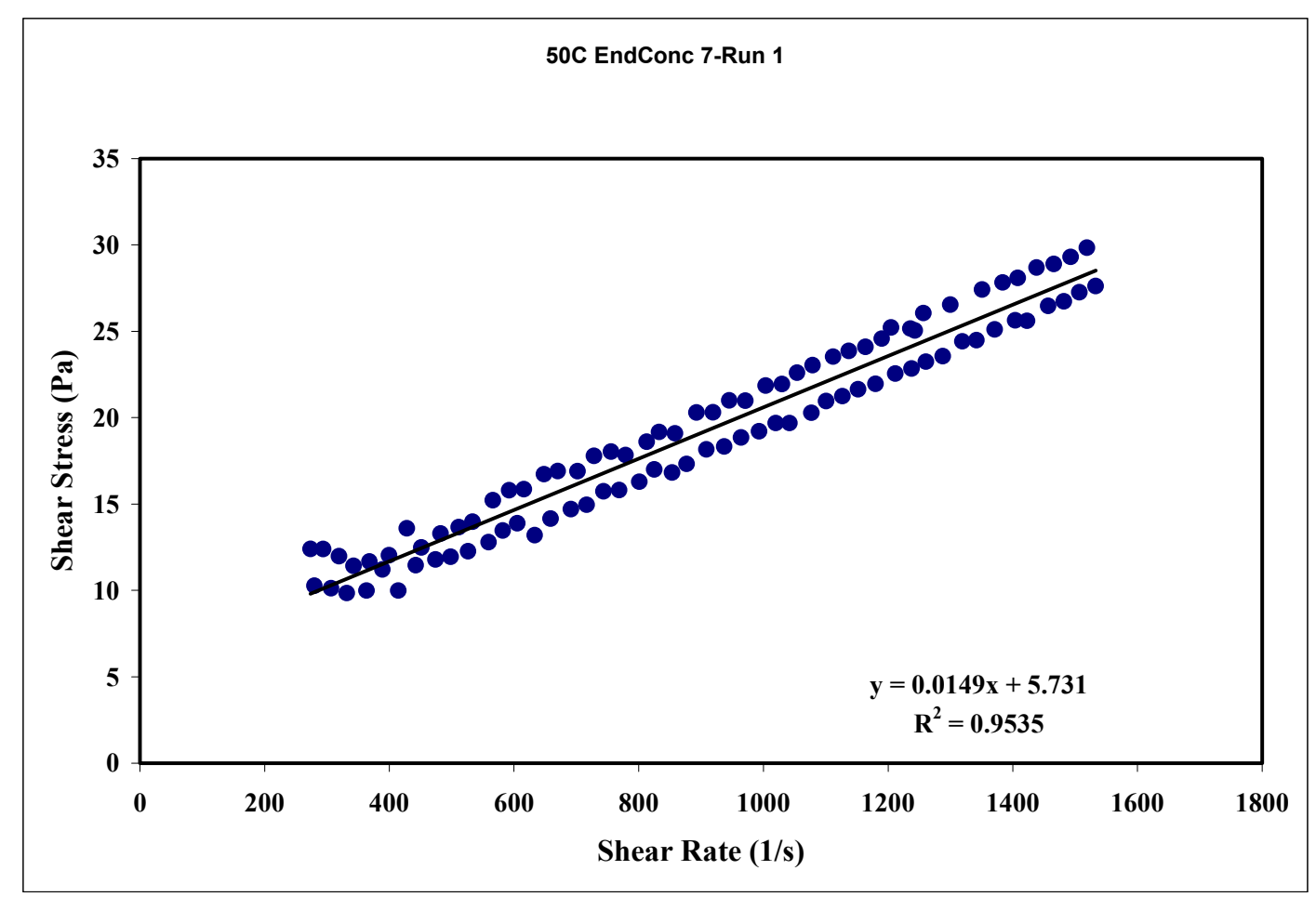

Figure 46. $50{ }^{\circ} \mathrm{C}$ at 16 wt $\%$ Run 1 


\begin{tabular}{|ll|}
\hline APPENDIX I - PART 4 & WSRC-TR-2000-00352 \\
& SRT-RPP-2000-00026 \\
SR/TRU PRECIPITATE SLURRY RHEOGRAMS AT 16 WT \% & Page 442 of 514 \\
\hline
\end{tabular}

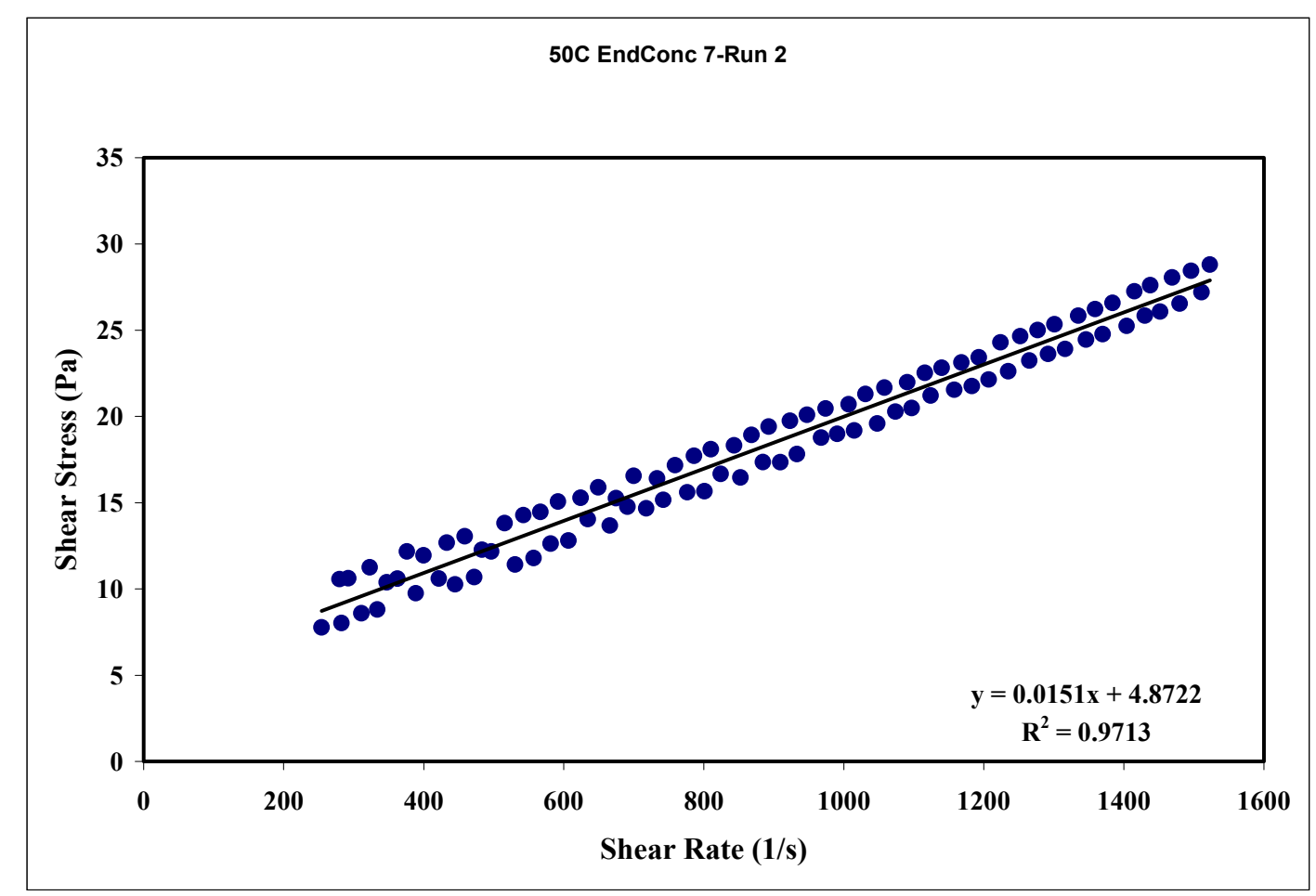

Figure $47.50{ }^{\circ} \mathrm{C}$ at 16 wt $\%$ Run 2

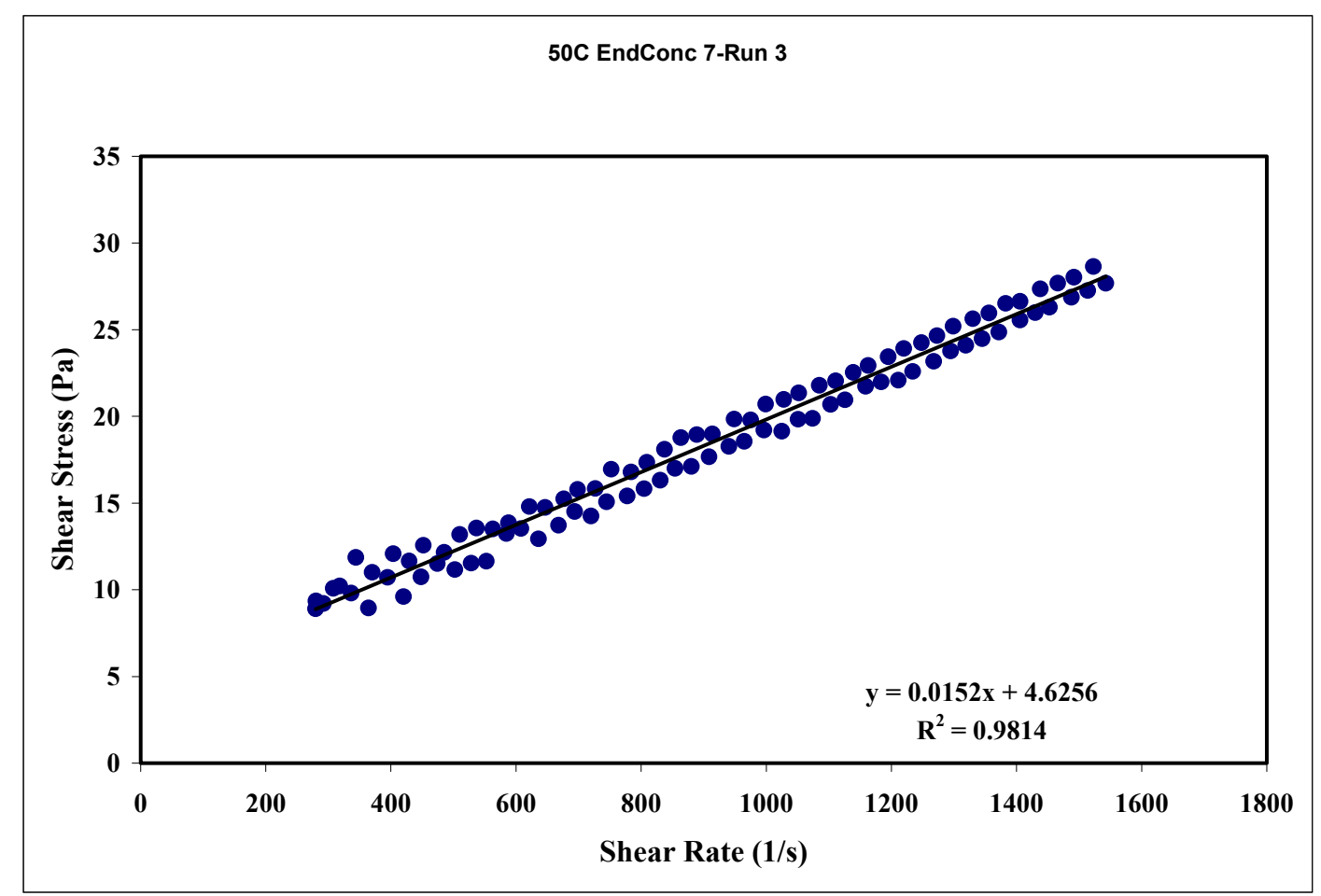

Figure $48.50{ }^{\circ} \mathrm{C}$ at 16 wt $\%$ Run 3 


\begin{tabular}{|ll|}
\hline APPENDIX I - PART 4 & WSRC-TR-2000-00352 \\
& SRT-RPP-2000-00026 \\
SR/TRU PRECIPITATE SLURRY RHEOGRAMS AT 16 WT \% & Page 443 of 514 \\
\hline
\end{tabular}

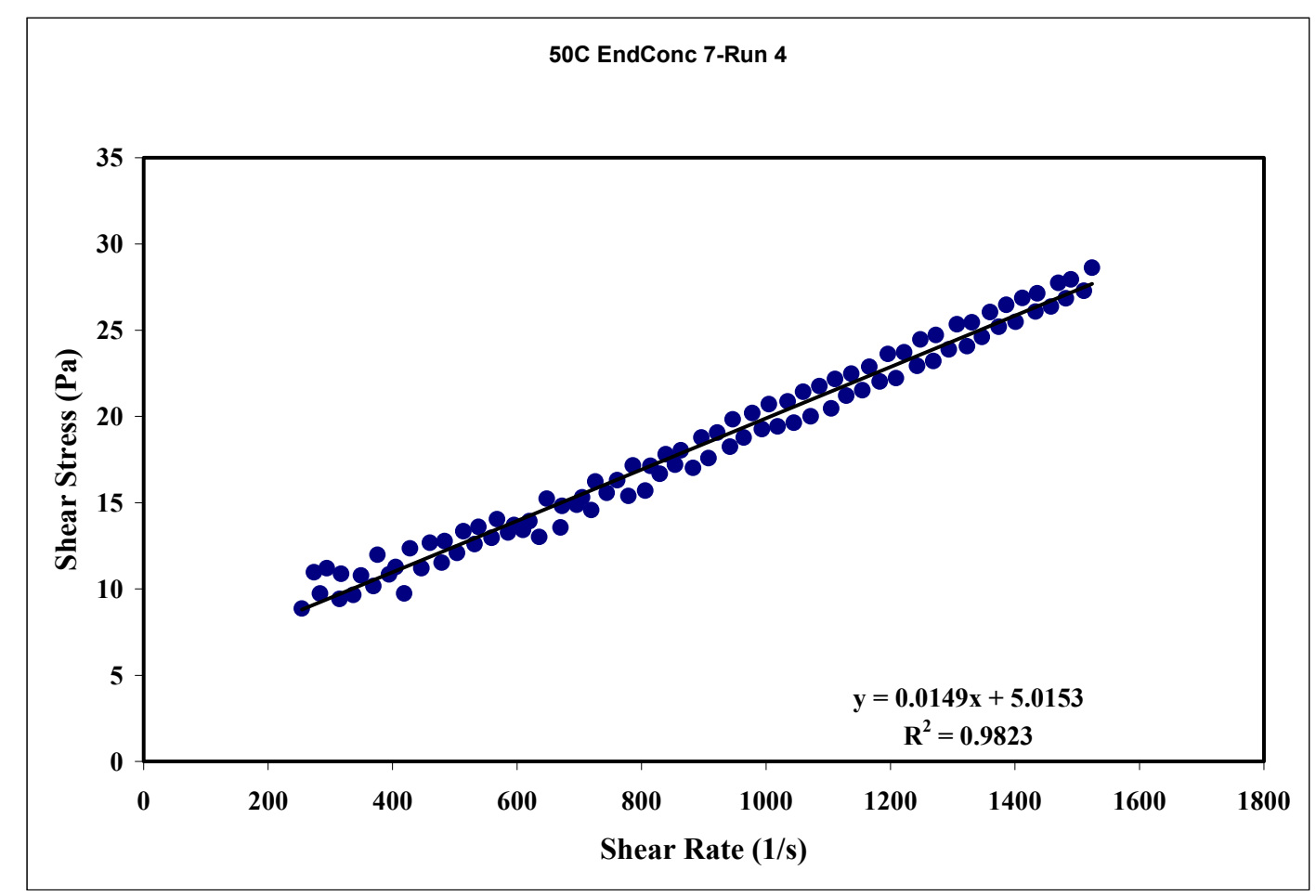

Figure 49. $50{ }^{\circ} \mathrm{C}$ at 16 wt \% Run 4

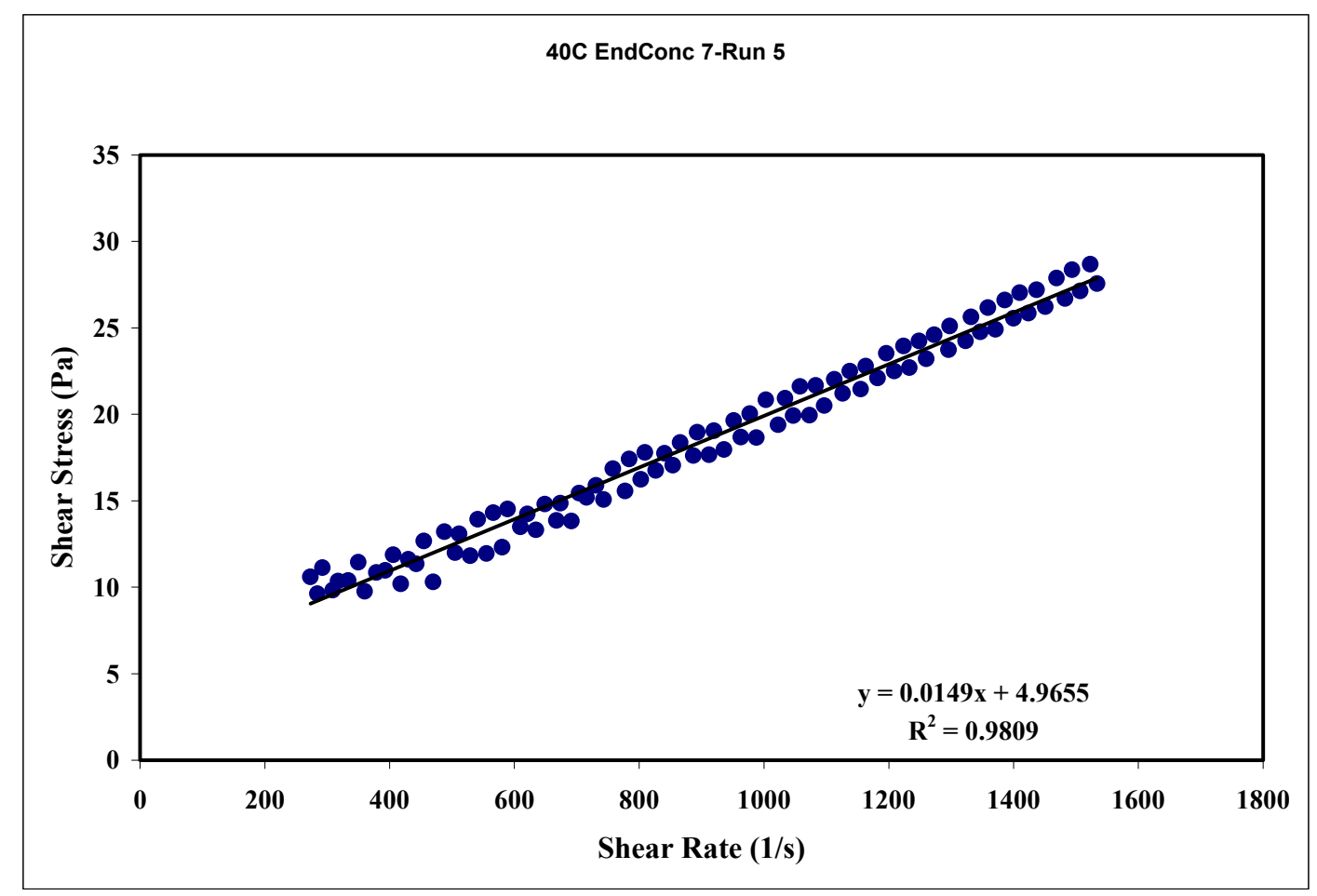

Figure 50. $50{ }^{\circ} \mathrm{C}$ at 16 wt \% Run 5 


\begin{tabular}{|ll|}
\hline APPENDIX I - PART 4 & WSRC-TR-2000-00352 \\
SR/TRU PRECIPITATE SLURRY RHEOGRAMS AT 16 WT \% & SRT-RPP-2000-00026 \\
\hline
\end{tabular}

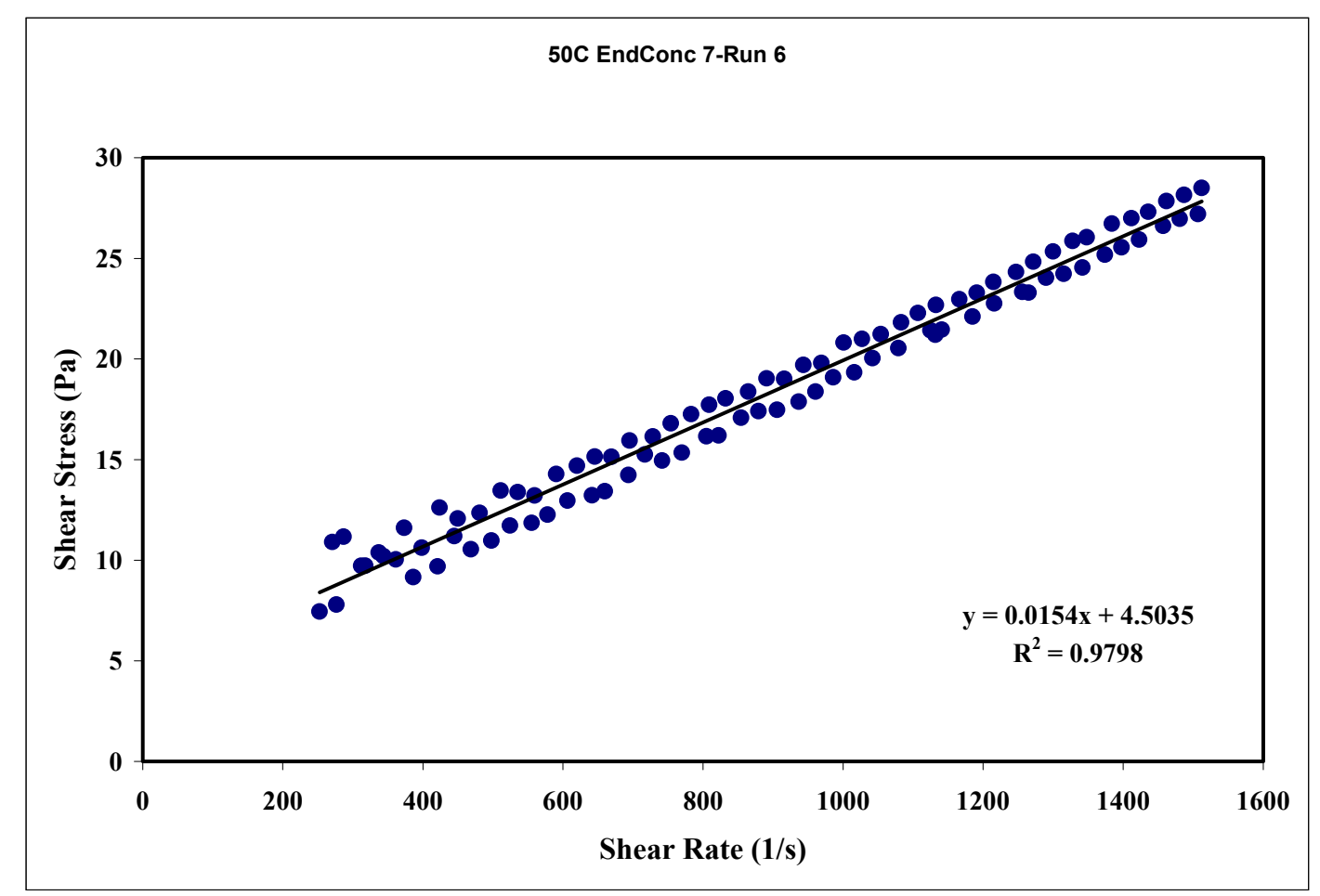

Figure 51. $50{ }^{\circ} \mathrm{C}$ at 16 wt \% Run 6

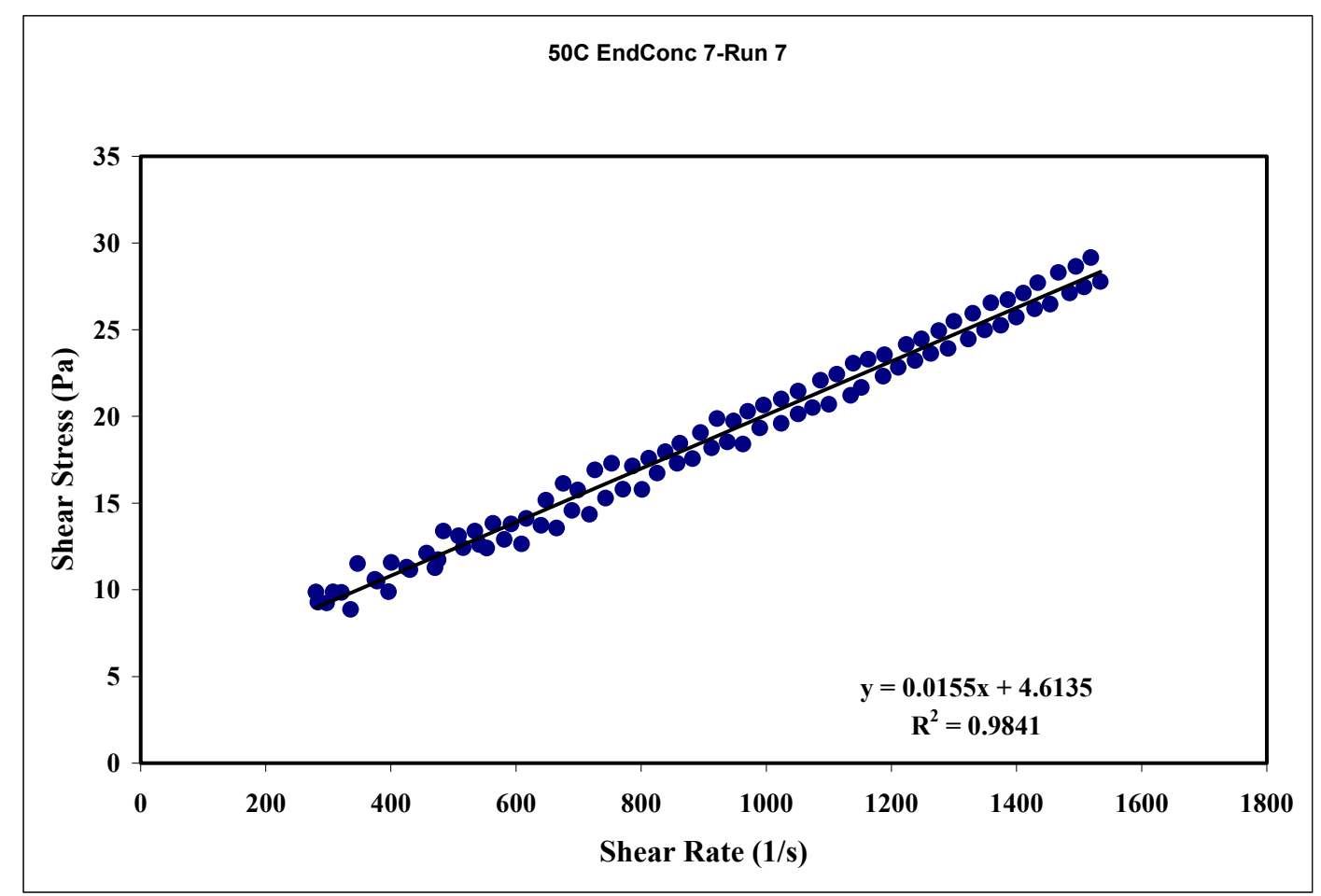

Figure 52. $50{ }^{\circ} \mathrm{C}$ at 16 wt $\%$ Run 7 


\begin{tabular}{|ll|}
\hline APPENDIX I - PART 4 & WSRC-TR-2000-00352 \\
& SRT-RPP-2000-00026 \\
SR/TRU PRECIPITATE SLURRY RHEOGRAMS AT 16 WT \% & Page 445 of 514 \\
\hline
\end{tabular}

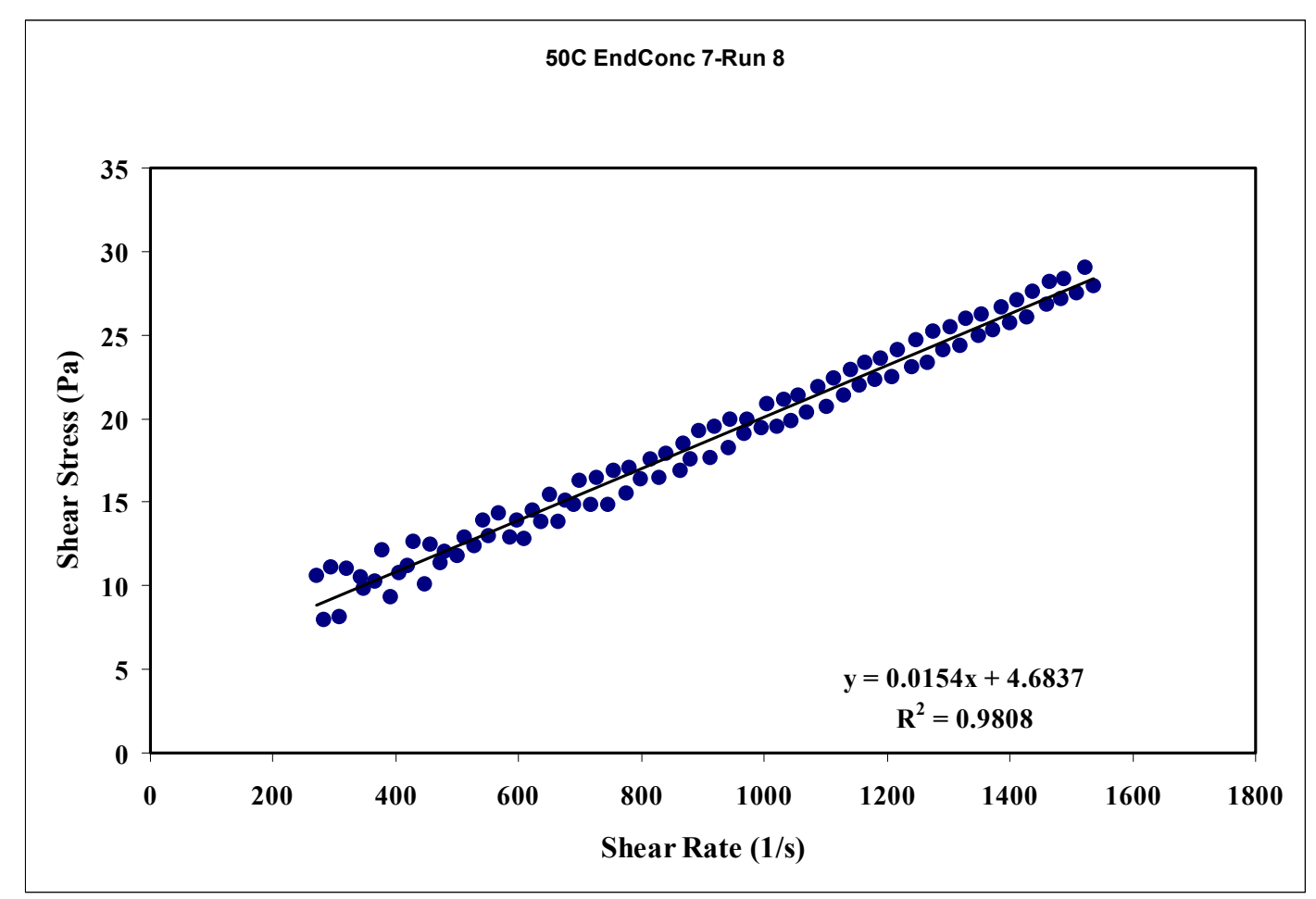

Figure 53. $50{ }^{\circ} \mathrm{C}$ at 16 wt \% Run 8

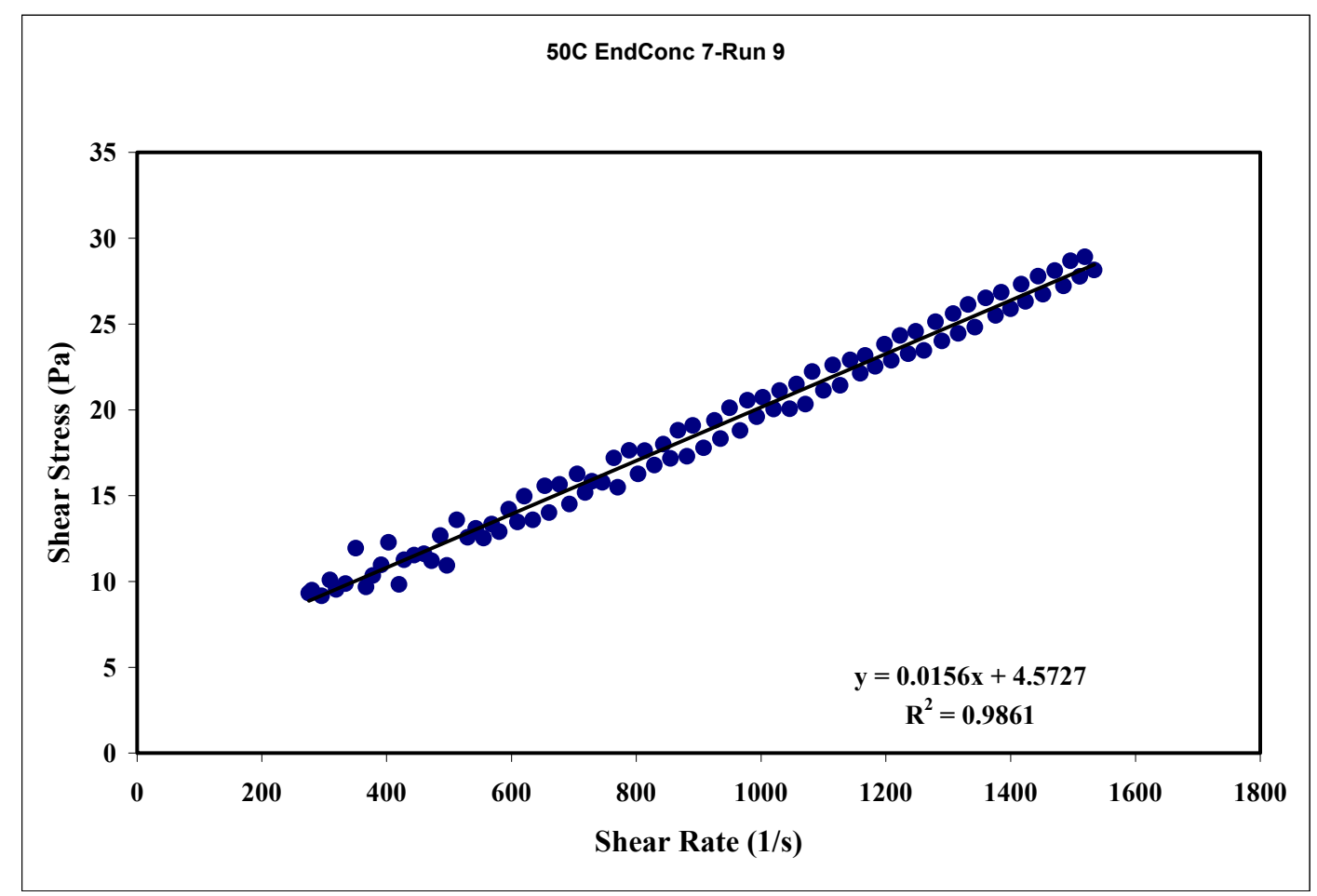

Figure 54. $50{ }^{\circ} \mathrm{C}$ at 16 wt \% Run 9 


\begin{tabular}{|ll|}
\hline APPENDIX I - PART 4 & WSRC-TR-2000-00352 \\
& SRT-RPP-2000-00026 \\
SR/TRU PRECIPITATE SLURRY RHEOGRAMS AT 16 WT \% & Page 446 of 514 \\
\hline
\end{tabular}

This page intentionally left blank. 


\begin{tabular}{|ll|}
\hline APPENDIX J & WSRC-TR-2000-00352 \\
ENVELOPE A RHEOLOGY SUMMARIES & SRT-RPP-2000-00026 \\
Page 447 of 514
\end{tabular}

\section{APPENDIX J}

\section{ENVELOPE A RHEOLOGY SUMMARIES}

Table 1. Rheometer Response for Blank - As Received ................................................... 449

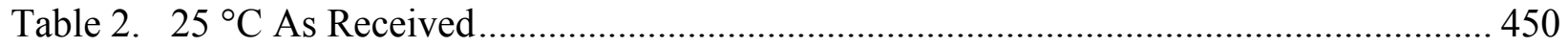

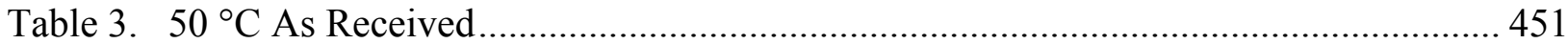

Table 4. Rheometer Response for Blank - Diluted ........................................................... 452

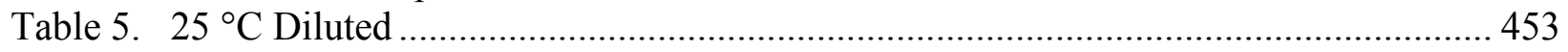

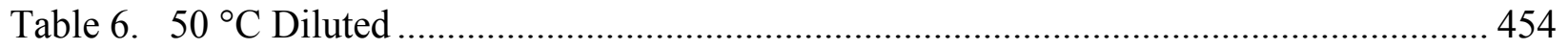


This page intentionally left blank. 
Table 1. Rheometer Response for Blank - As Received

\begin{tabular}{|c|c|c|c|}
\hline Run \# & $\begin{array}{c}\mathbf{M}^{* \mathbf{1 0 0 0}} \\
\mathbf{( P a - s )}\end{array}$ & $\begin{array}{c}\mathbf{B} \\
\mathbf{( P a )}\end{array}$ & $\mathbf{R}^{\mathbf{2}}$ \\
\hline 1 & 7.3 & -0.0499 & 0.8353 \\
\hline 2 & 7.1 & -0.4077 & 0.8807 \\
\hline 3 & 6.6 & -0.1097 & 0.9012 \\
\hline 4 & 6.9 & -0.4566 & 0.9316 \\
\hline 5 & 6.6 & -0.2415 & 0.9139 \\
\hline 6 & 6.7 & -0.4058 & 0.9258 \\
\hline 7 & 6.5 & -0.2474 & 0.9233 \\
\hline 8 & 6.5 & -0.1745 & 0.9382 \\
\hline 9 & 6.5 & -0.0789 & 0.9248 \\
\hline & & & $\mathrm{N}=9$ \\
\hline & $\mathrm{DF}=8$ & & \\
\hline Statistics for M & Mean $=6.7$ & Sigma $=0.3$ & $95 \% \mathrm{CL}= \pm 0.2$ \\
\hline Statistics for B & Mean $=0.2413$ & Sigma $=0.1524$ & $95 \% \mathrm{CL}= \pm 0.1171$ \\
\hline
\end{tabular}

$\mathrm{M}, \mathrm{B}$, and $\mathrm{R}^{2}$ are the slope, intercept, and residual explanation, respectively, derived from the least squares fit of the stress/strain curve. DF and $\mathrm{N}$ correspond to the degrees of freedom and number of observations (replicates), respectively, used in the calculation of the $95 \%$ confidence limit (CL). 
Table 2. $25^{\circ} \mathrm{C}$ As Received

\begin{tabular}{|c|c|c|c|c|}
\hline Run \# & $\begin{array}{c}\mathbf{M} * \mathbf{1 0 0 0} \\
\mathbf{( P a - s )}\end{array}$ & $\begin{array}{c}\mathbf{B} \\
\mathbf{P a})\end{array}$ & $\begin{array}{c}\mathbf{R}^{\mathbf{2}} \\
\mathbf{( \mathbf { c } )}\end{array}$ \\
\hline 1 & 31.0 & -2.5636 & 0.9099 & 24.3 \\
\hline 2 & 30.0 & -2.5118 & 0.9936 & 23.3 \\
\hline 3 & 29.8 & -2.6132 & 0.9971 & 23.1 \\
\hline 4 & 29.8 & -2.5783 & 0.9973 & 23.1 \\
\hline 5 & 29.6 & -2.4162 & 0.9969 & 22.9 \\
\hline 6 & 29.7 & -2.4736 & 0.9968 & 23.0 \\
\hline 7 & 29.5 & -2.3872 & 0.9864 & 22.8 \\
\hline 8 & 29.4 & -2.4243 & 0.9965 & 22.7 \\
\hline 9 & 29.3 & -2.4926 & 0.9968 & 22.6 \\
\hline & & & & \\
\hline & $\mathrm{DF}=8$ & $\mathrm{~N}=9$ & & \\
\hline & & & & \\
\hline Statistics for M & Mean $=29.8$ & Sigma $=0.5$ & $95 \% \mathrm{CL}= \pm 0.4$ & Avg $=23.1 \pm 0.6$ \\
\hline Statistics for B & Mean $=2.4956$ & Sigma $=0.0784$ & $95 \% \mathrm{CL}= \pm 0.0603$ & \\
\hline
\end{tabular}

NOTE: The instrument was not properly zeroed for the above experiment. 
Table 3. $50^{\circ} \mathrm{C}$ As Received

\begin{tabular}{|c|c|c|c|c|}
\hline Run \# & $\begin{array}{c}\mathbf{M}^{* 1000} \\
(\mathbf{P a}-\mathbf{s})\end{array}$ & $\begin{array}{c}\mathbf{B} \\
\mathbf{( P a})\end{array}$ & $\mathbf{R}^{\mathbf{2}}$ & $\begin{array}{c}\text { Viscosity } \\
(\mathbf{c P})\end{array}$ \\
\hline 1 & 13.4 & -0.7783 & 0.9754 & 6.7 \\
\hline 2 & 13.1 & -0.7119 & 0.9844 & 6.4 \\
\hline 3 & 13.1 & -0.8085 & 0.9819 & 6.4 \\
\hline 4 & 12.9 & -0.7185 & 0.9806 & 6.2 \\
\hline 5 & 12.8 & -0.8017 & 0.9841 & 6.1 \\
\hline 6 & 12.8 & -0.7750 & 0.9854 & 6.1 \\
\hline 7 & 12.7 & -0.8496 & 0.9851 & 6.0 \\
\hline 8 & 12.7 & -0.9759 & 0.9839 & 6.0 \\
\hline 9 & 12.6 & -0.8889 & 0.9813 & 5.9 \\
\hline & & & & \\
\hline & $\mathrm{DF}=8$ & $\mathrm{~N}=9$ & & \\
\hline & & & & \\
\hline Statistics for M & Mean $=12.9$ & Sigma $=0.3$ & $95 \% \mathrm{CL}= \pm 0.2$ & Avg $=6.2 \pm 0.4$ \\
\hline Statistics for B & Mean $=0.8120$ & Sigma $=0.0833$ & $95 \% \mathrm{CL}= \pm 0.0641$ & \\
\hline
\end{tabular}


Table 4. Rheometer Response for Blank - Diluted

\begin{tabular}{|c|c|c|c|}
\hline Run \# & $\begin{array}{c}\text { M*1000 } \\
(\mathbf{P a}-\mathbf{s})\end{array}$ & $\begin{array}{c}\mathbf{B} \\
\mathbf{( P a )}\end{array}$ & $\mathbf{R}^{\mathbf{2}}$ \\
\hline 1 & 3.9 & 0.3501 & 0.6731 \\
\hline 2 & 3.5 & 0.4374 & 0.7340 \\
\hline 3 & 3.2 & 0.6725 & 0.6725 \\
\hline 4 & 3.1 & 0.6169 & 0.6169 \\
\hline 5 & 3.4 & 0.3385 & 0.6923 \\
\hline 6 & 3.3 & 0.4129 & 0.6953 \\
\hline 7 & 2.9 & 0.6778 & 0.6582 \\
\hline 8 & 2.7 & 0.8153 & 0.6241 \\
\hline 9 & 2.7 & 0.7568 & 0.6573 \\
\hline & & & $\mathrm{N}=9$ \\
\hline & $\mathrm{DF}=8$ & & \\
\hline Statistics for M & Mean $=3.2$ & Sigma $=0.4$ & $95 \% \mathrm{CL}= \pm 0.3$ \\
\hline Statistics for B & Mean $=0.5642$ & Sigma $=0.1814$ & $95 \% \mathrm{CL}= \pm 0.1394$ \\
\hline
\end{tabular}


Table 5. $25^{\circ} \mathrm{C}$ Diluted

\begin{tabular}{|c|c|c|c|c|}
\hline Run \# & $\begin{array}{c}\mathbf{M}^{*} \mathbf{1 0 0 0} \\
\mathbf{( P a - s )}\end{array}$ & $\begin{array}{c}\mathbf{B} \\
\mathbf{P a})\end{array}$ & $\begin{array}{c}\text { Viscosity } \\
\mathbf{( c P )}\end{array}$ \\
\hline 1 & 7.0 & 0.8073 & 0.9231 & 3.8 \\
\hline 2 & 6.5 & 1.0328 & 0.9030 & 3.3 \\
\hline 3 & 6.4 & 1.2279 & 0.9159 & 3.2 \\
\hline 4 & 6.2 & 1.1533 & 0.9226 & 3.0 \\
\hline 5 & 6.5 & 0.9696 & 0.9313 & 3.3 \\
\hline 6 & 6.4 & 1.0246 & 0.9200 & 3.2 \\
\hline 7 & 6.1 & 1.3167 & 0.9131 & 2.9 \\
\hline 8 & 6.1 & 1.2704 & 0.9197 & 2.9 \\
\hline 9 & 6.0 & 1.2604 & 0.9331 & 2.8 \\
\hline & & & & \\
\hline & $\mathrm{DF}=8$ & $\mathrm{~N}=9$ & & \\
\hline & & & & Avg $=3.2 \pm 0.5$ \\
\hline Statistics for M & Mean $=6.4$ & Sigma $=0.3$ & $95 \% \mathrm{CL}= \pm 0.4$ & \\
\hline Statistics for B & Mean $=1.118$ & Sigma $=0.1696$ & $95 \% \mathrm{CL}= \pm 0.1304$ & \\
\hline
\end{tabular}


Table 6. $50{ }^{\circ} \mathrm{C}$ Diluted

\begin{tabular}{|c|c|c|c|c|}
\hline Run \# & $\begin{array}{c}\text { M*1000 } \\
(\mathbf{P a}-\mathbf{s})\end{array}$ & $\begin{array}{c}\text { B } \\
(\mathbf{P a})\end{array}$ & $\begin{array}{c}\text { Viscosity } \\
\mathbf{( c P )}\end{array}$ \\
\hline 1 & 5.9 & 0.5424 & 0.8894 & 2.7 \\
\hline 2 & 6.1 & 0.3905 & 0.8850 & 2.9 \\
\hline 3 & 5.9 & 0.4042 & 0.8856 & 2.7 \\
\hline 4 & 5.8 & 0.4541 & 0.8797 & 2.6 \\
\hline 5 & 5.7 & 0.4080 & 0.8960 & 2.5 \\
\hline 6 & 5.5 & 0.4523 & 0.9177 & 2.3 \\
\hline 7 & 5.5 & 0.6326 & 0.8633 & 2.3 \\
\hline 8 & 5.8 & 0.2338 & 0.8930 & 2.6 \\
\hline 9 & 5.4 & 0.6096 & 0.9015 & 2.2 \\
\hline & & & & \\
\hline Statistics for M & Mean $=5.7$ & Sigma $=0.3$ & $95 \% \mathrm{CL}= \pm 0.2$ & Avg $=2.5 \pm 0.5$ \\
\hline Statistics for B & Mean $=0.4586$ & Sigma $=0.1229$ & $95 \% \mathrm{CL}= \pm 0.0945$ & \\
\hline
\end{tabular}


$\begin{array}{ll}\text { APPENDIX K } & \text { WSRC-TR-2000-00352 }\end{array}$

ENVELOPE B RHEOLOGY SUMMARIES

SRT-RPP-2000-00026

Page 455 of 514

\section{APPENDIX K}

\section{ENVELOPE B RHEOLOGY SUMMARIES}

Table 1. Rheometer Response for Blank - As Received ................................................ 457

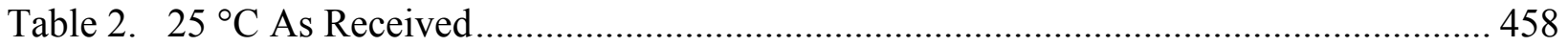

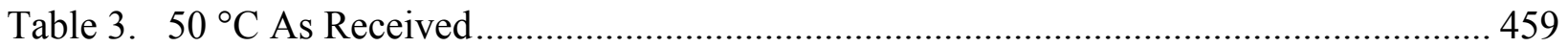

Table 4. Rheometer Response for Blank - Pretreated ....................................................... 460

Table 5. $25{ }^{\circ} \mathrm{C}$ Pretreated ............................................................................................... 461

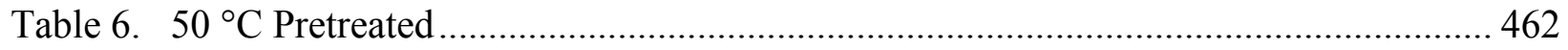


This page intentionally left blank. 
Table 1. Rheometer Response for Blank - As Received

\begin{tabular}{|c|c|c|c|}
\hline Run \# & $\begin{array}{c}\text { M*1000 } \\
(\mathbf{P a}-\mathbf{s})\end{array}$ & $\begin{array}{c}\mathbf{B} \\
\mathbf{( P a )}\end{array}$ & $\mathbf{R}^{\mathbf{2}}$ \\
\hline 1 & 6.7 & 0.3030 & 0.9318 \\
\hline 2 & 6.0 & 0.1452 & 0.8745 \\
\hline 3 & 5.8 & 0.3504 & 0.7860 \\
\hline 4 & 5.5 & 0.2068 & 0.9045 \\
\hline 5 & 6.1 & -0.4718 & 0.8468 \\
\hline 6 & 5.5 & -0.2120 & 0.8502 \\
\hline 7 & 5.7 & -0.1691 & 0.9586 \\
\hline 8 & 5.6 & -0.1516 & 0.9612 \\
\hline 9 & 4.8 & -0.4770 & 0.8541 \\
\hline & & & \\
\hline & $\mathrm{DF}=8$ & $\mathrm{~N}=9$ & \\
\hline Statistics for M & Mean $=5.7$ & Sigma $=0.5$ & $95 \% \mathrm{CL}= \pm 0.4$ \\
\hline Statistics for B & Mean $=0.0529$ & Sigma $=0.3162$ & $95 \% \mathrm{CL}= \pm 0.2431$ \\
\hline
\end{tabular}


Table 2. $25^{\circ} \mathrm{C}$ As Received

\begin{tabular}{|c|c|c|c|c|}
\hline Run \# & $\begin{array}{c}\mathbf{M} * \mathbf{1 0 0 0} \\
\mathbf{( P a - s )}\end{array}$ & $\begin{array}{c}\mathbf{B} \\
\mathbf{( P a})\end{array}$ & $\begin{array}{c}\mathbf{R}^{\mathbf{2}} \\
\mathbf{( c P )}\end{array}$ \\
\hline 1 & 9.9 & 1.0889 & 0.9358 & 4.2 \\
\hline 2 & 9.9 & 0.9688 & 0.9631 & 4.2 \\
\hline 3 & 9.7 & 1.0792 & 0.9540 & 4.0 \\
\hline 4 & 9.7 & 1.0694 & 0.9640 & 4.0 \\
\hline 5 & 9.3 & 1.0138 & 0.9489 & 3.6 \\
\hline 6 & 9.3 & 1.0207 & 0.9448 & 3.6 \\
\hline 7 & 9.0 & 1.2081 & 0.9418 & 3.3 \\
\hline 8 & 9.0 & 1.0144 & 0.9646 & 3.3 \\
\hline 9 & 8.6 & 1.1178 & 0.9094 & 2.9 \\
\hline & & & & \\
\hline & $\mathrm{DF}=8$ & $\mathrm{~N}=9$ & & \\
\hline & & & & Avg $=3.7 \pm 0.7$ \\
\hline Statistics for M & Mean $=9.4$ & Sigma $=0.5$ & $95 \% \mathrm{CL}= \pm 0.3$ & \\
\hline Statistics for B & Mean $=1.0670$ & Sigma $=0.0732$ & $95 \% \mathrm{CL}= \pm 0.0562$ & \\
\hline
\end{tabular}


Table 3. $50^{\circ} \mathrm{C}$ As Received

\begin{tabular}{|c|c|c|c|c|}
\hline Run \# & $\begin{array}{c}\text { M*1000 } \\
(\mathbf{P a - s})\end{array}$ & $\begin{array}{c}\mathbf{B} \\
(\mathbf{P a})\end{array}$ & $\begin{array}{c}\text { Viscosity } \\
(\mathbf{c P})\end{array}$ \\
\hline 1 & 8.4 & 0.9233 & 0.9396 & 2.7 \\
\hline 2 & 8.4 & 0.7050 & 0.9349 & 2.7 \\
\hline 3 & 8.3 & 0.7669 & 0.9373 & 2.6 \\
\hline 4 & 8.1 & 0.8321 & 0.9312 & 2.4 \\
\hline 5 & 8.2 & 0.7187 & 0.9435 & 2.5 \\
\hline 6 & 8.1 & 0.8240 & 0.9264 & 2.4 \\
\hline 7 & 7.9 & 0.8460 & 0.9308 & 2.2 \\
\hline 8 & 7.6 & 1.0909 & 0.9300 & 1.9 \\
\hline 9 & 7.6 & 1.0240 & 0.9352 & 1.9 \\
\hline & & & & \\
\hline & $\mathrm{DF}=8$ & $\mathrm{~N}=9$ & & \\
\hline & & & & Avg $=2.4 \pm 0.6$ \\
\hline Statistics for M & Mean $=8.1$ & Sigma $=0.3$ & $95 \% \mathrm{CL}= \pm 0.2$ & \\
\hline Statistics for B & Mean $=0.8589$ & Sigma $=0.1319$ & $95 \% \mathrm{CL}= \pm 0.1014$ & \\
\hline
\end{tabular}


Table 4. Rheometer Response for Blank - Pretreated

\begin{tabular}{|c|c|c|c|}
\hline Run \# & $\begin{array}{c}\text { M*1000 } \\
(\mathbf{P a}-\mathbf{s})\end{array}$ & $\begin{array}{c}\mathbf{B} \\
(\mathbf{P a})\end{array}$ & $\mathbf{R}^{\mathbf{2}}$ \\
\hline 1 & 6.4 & -0.0443 & 0.9621 \\
\hline 2 & 6.5 & -0.2189 & 0.9699 \\
\hline 3 & 6.7 & -0.4036 & 0.9627 \\
\hline 4 & 7.0 & -0.8120 & 0.9462 \\
\hline 5 & 6.8 & -1.2929 & 0.9242 \\
\hline & & & \\
\hline & $\mathrm{DF}=4$ & $\mathrm{~N}=5$ & \\
\hline Statistics for M & Mean $=6.7$ & Sigma $=0.3$ & $95 \% \mathrm{CL}= \pm 0.2$ \\
\hline Statistics for B & Mean $=0.5543$ & Sigma $=0.5018$ & $95 \% \mathrm{CL}= \pm 0.4643$ \\
\hline
\end{tabular}


Table 5. $25^{\circ} \mathrm{C}$ Pretreated

\begin{tabular}{|c|c|c|c|c|}
\hline Run \# & $\begin{array}{c}\mathbf{M} * \mathbf{1 0 0 0} \\
\mathbf{( P a - s )}\end{array}$ & $\begin{array}{c}\mathbf{B} \\
\mathbf{P a})\end{array}$ & $\begin{array}{c}\text { Viscosity } \\
\mathbf{( c P )}\end{array}$ \\
\hline 1 & 8.7 & -0.0464 & 0.9723 & 2.0 \\
\hline 2 & 8.8 & -0.2100 & 0.9722 & 2.1 \\
\hline 3 & 8.6 & -0.1743 & 0.9786 & 1.9 \\
\hline 4 & 8.8 & -0.2855 & 0.9730 & 2.1 \\
\hline 5 & 8.8 & -0.2805 & 0.9803 & 2.1 \\
\hline 6 & 8.9 & -0.3975 & 0.9465 & 2.2 \\
\hline 7 & 8.9 & -0.7086 & 0.9741 & 2.2 \\
\hline 8 & 8.9 & -0.7048 & 0.9666 & 2.2 \\
\hline 9 & 8.9 & -0.6590 & 0.9737 & 2.2 \\
\hline & & & & \\
\hline & $\mathrm{DF}=8$ & $\mathrm{~N}=9$ & & \\
\hline & & & & \\
\hline Statistics for M & Mean $=8.8$ & Sigma $=0.1$ & $95 \% \mathrm{CL}= \pm 0.1$ & Avg $=2.1 \pm 0.3$ \\
\hline Statistics for B & Mean $=0.3852$ & Sigma $=0.2482$ & $95 \% \mathrm{CL}= \pm 0.1908$ & \\
\hline
\end{tabular}


Table 6. $50{ }^{\circ} \mathrm{C}$ Pretreated

\begin{tabular}{|c|c|c|c|c|}
\hline Run \# & $\begin{array}{c}\mathbf{M}^{* 1000} \\
(\mathbf{P a - s})\end{array}$ & $\begin{array}{c}\mathbf{B} \\
(\mathbf{P a})\end{array}$ & $\begin{array}{c}\text { Viscosity } \\
(\mathbf{c P})\end{array}$ \\
\hline 1 & 8.0 & 0.2045 & 0.9678 & 1.3 \\
\hline 2 & 7.7 & 0.4721 & 0.9486 & 1.0 \\
\hline 3 & 7.7 & 0.3353 & 0.9124 & 1.0 \\
\hline 4 & 7.1 & 0.5021 & 0.9243 & 0.4 \\
\hline 5 & 7.1 & 0.4384 & 0.9284 & 0.4 \\
\hline 6 & 7.2 & 0.2370 & 0.9470 & 0.5 \\
\hline 7 & 7.1 & 0.3488 & 0.9317 & 0.4 \\
\hline 8 & 7.4 & 0.0981 & 0.9381 & 0.7 \\
\hline 9 & 7.2 & 0.3180 & 0.9494 & 0.5 \\
\hline & & & & \\
\hline & $\mathrm{DF}=8$ & $\mathrm{~N}=9$ & & \\
\hline & & & & Avg $=0.7 \pm 0.4$ \\
\hline Statistics for M & Mean $=7.4$ & Sigma $=0.3$ & $95 \% \mathrm{CL}= \pm 0.2$ & \\
\hline Statistics for B & Mean $=0.3283$ & Sigma $=0.1324$ & $95 \% \mathrm{CL}= \pm 0.1018$ & \\
\hline
\end{tabular}




\begin{tabular}{|ll|}
\hline APPENDIX L & WSRC-TR-2000-00352 \\
ENVELOPE C RHEOLOGY SUMMARIES & SRT-RPP-2000-00026 \\
\hline
\end{tabular}

\section{APPENDIX L}

\section{ENVELOPE C RHEOLOGY SUMMARIES}

Table 1. Rheometer Response for Blank - As Received .................................................... 465

Table 2. $25^{\circ} \mathrm{C}$ As Received............................................................................................ 466

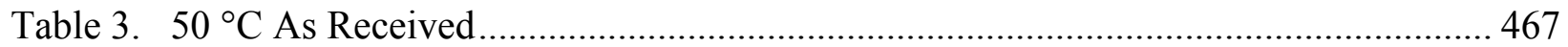

Table 4. Rheometer Response for Blank - Diluted ............................................................ 468

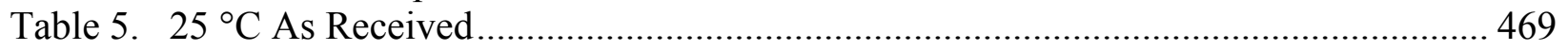

Table 6. $50^{\circ} \mathrm{C}$ As Received........................................................................................... 470

Table 7. Rheometer Response for Blank - Pretreated ...................................................... 471

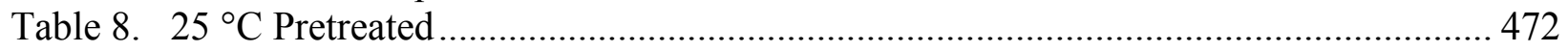

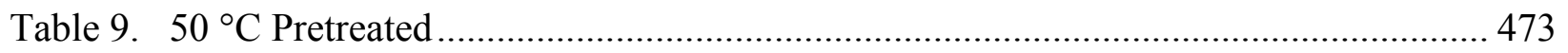


This page intentionally left blank. 
Table 1. Rheometer Response for Blank - As Received

\begin{tabular}{|c|c|c|c|}
\hline Run \# & $\begin{array}{c}\text { M*1000 } \\
(\mathbf{P a}-\mathbf{s})\end{array}$ & $\begin{array}{c}\mathbf{B} \\
\mathbf{( P a )}\end{array}$ & $\mathbf{R}^{\mathbf{2}}$ \\
\hline 1 & 6.2 & 0.4124 & 0.9625 \\
\hline 2 & 6.6 & 0.3122 & 0.8999 \\
\hline 3 & 6.3 & 0.5484 & 0.8818 \\
\hline 4 & 5.7 & 0.7628 & 0.9375 \\
\hline 5 & 6.0 & 0.7354 & 0.8935 \\
\hline 6 & 6.0 & 0.6518 & 0.8887 \\
\hline 7 & 5.7 & 0.9482 & 0.8843 \\
\hline 8 & 5.5 & 0.9709 & 0.8654 \\
\hline 9 & 5.4 & 0.9865 & 0.8632 \\
\hline & & & $\mathrm{N}=9$ \\
\hline & $\mathrm{DF}=8$ & & \\
\hline Statistics for M & Mean $=5.9$ & Sigma $=0.4$ & $95 \% \mathrm{CL}= \pm 0.3$ \\
\hline Statistics for B & Mean $=0.7032$ & Sigma $=0.2451$ & $95 \% \mathrm{CL}= \pm 0.1884$ \\
\hline
\end{tabular}


Table 2. $25^{\circ} \mathrm{C}$ As Received

\begin{tabular}{|c|c|c|c|c|}
\hline Run \# & $\begin{array}{c}\mathbf{M} * \mathbf{1 0 0 0} \\
\mathbf{( P a - s )}\end{array}$ & $\begin{array}{c}\mathbf{B} \\
\mathbf{P a})\end{array}$ & $\begin{array}{c}\text { Viscosity } \\
\mathbf{( c P )}\end{array}$ \\
\hline 1 & 13.2 & -0.1825 & 0.9836 & 7.3 \\
\hline 2 & 13.1 & -0.3579 & 0.9874 & 7.2 \\
\hline 3 & 13.0 & -0.2607 & 0.9887 & 7.1 \\
\hline 4 & 12.7 & -0.2784 & 0.9750 & 6.8 \\
\hline 5 & 12.7 & -0.3844 & 0.9831 & 6.8 \\
\hline 6 & 12.3 & -0.0898 & 0.9847 & 6.4 \\
\hline 7 & 12.4 & -0.1465 & 0.9864 & 6.5 \\
\hline 8 & 12.1 & 0.0824 & 0.9870 & 6.2 \\
\hline 9 & 12.2 & 0.0840 & 0.9892 & 6.3 \\
\hline & & & & \\
\hline & $\mathrm{DF}=8$ & $\mathrm{~N}=9$ & & \\
\hline & & & & \\
\hline Statistics for M & Mean $=12.6$ & Sigma $=0.4$ & $95 \% \mathrm{CL}= \pm 0.3$ & Avg $=6.7 \pm 0.6$ \\
\hline Statistics for B & Mean $=1.1704$ & Sigma $=0.1720$ & $95 \% \mathrm{CL}= \pm 0.1322$ & \\
\hline
\end{tabular}


Table 3. $50^{\circ} \mathrm{C}$ As Received

\begin{tabular}{|c|c|c|c|c|}
\hline Run \# & $\begin{array}{c}\mathbf{M}^{* 1000} \\
(\mathbf{P a}-\mathbf{s})\end{array}$ & $\begin{array}{c}\mathbf{B} \\
\mathbf{P a})\end{array}$ & $\mathbf{R}^{\mathbf{2}}$ & $\begin{array}{c}\text { Viscosity } \\
(\mathbf{c P})\end{array}$ \\
\hline 1 & 7.1 & 0.7650 & 0.9464 & 1.2 \\
\hline 2 & 7.1 & 0.7728 & 0.9062 & 1.2 \\
\hline 3 & 6.7 & 0.8619 & 0.8941 & 0.8 \\
\hline 4 & 6.8 & 0.7839 & 0.9195 & 0.9 \\
\hline 5 & 6.6 & 0.8300 & 0.8947 & 0.7 \\
\hline 6 & 6.6 & 0.9703 & 0.9066 & 0.7 \\
\hline 7 & 6.5 & 0.9186 & 0.9027 & 0.6 \\
\hline 8 & 6.5 & 0.9841 & 0.9108 & 0.6 \\
\hline 9 & 6.3 & 1.0034 & 0.8878 & 0.4 \\
\hline & & & & \\
\hline & $\mathrm{DF}=8$ & $\mathrm{~N}=9$ & & Avg $=0.8 \pm 0.5$ \\
\hline & & & & \\
\hline Statistics for M & Mean $=6.7$ & Sigma -0.3 & $95 \% \mathrm{CL}= \pm 0.2$ & \\
\hline Statistics for B & Mean $=0.8767$ & Sigma $=0.0951$ & $95 \% \mathrm{CL}= \pm 0.0731$ & \\
\hline
\end{tabular}


Table 4. Rheometer Response for Blank - Diluted

\begin{tabular}{|c|c|c|c|}
\hline Run \# & $\begin{array}{c}\text { M*1000 } \\
(\mathbf{P a}-\mathbf{s})\end{array}$ & $\begin{array}{c}\mathbf{B} \\
\mathbf{( P a )}\end{array}$ & $\mathbf{R}^{\mathbf{2}}$ \\
\hline 1 & 6.2 & 0.1133 & 0.8887 \\
\hline 2 & 6.2 & -0.1714 & 0.9289 \\
\hline 3 & 5.9 & -0.1188 & 0.9235 \\
\hline 4 & 5.9 & -0.1818 & 0.9046 \\
\hline 5 & 5.8 & -0.2633 & 0.8946 \\
\hline 6 & 5.8 & -0.3555 & 0.8987 \\
\hline 7 & 5.5 & -0.2247 & 0.8981 \\
\hline 8 & 5.3 & -0.1636 & 0.8791 \\
\hline 9 & 5.2 & -0.0930 & 0.8869 \\
\hline & & & \\
\hline & $\mathrm{DF}=8$ & $\mathrm{~N}=9$ & \\
\hline Statistics for M & Mean $=5.8$ & Sigma $=0.4$ & $95 \% \mathrm{CL}= \pm 0.3$ \\
\hline Statistics for B & Mean $=0.1620$ & Sigma $=0.1297$ & $95 \% \mathrm{CL}= \pm 0.0997$ \\
\hline
\end{tabular}


Table 5. $25^{\circ} \mathrm{C}$ As Received

\begin{tabular}{|c|c|c|c|c|}
\hline Run \# & $\begin{array}{c}\mathrm{M} * 1000 \\
(\mathrm{~Pa}-\mathrm{s})\end{array}$ & $\begin{array}{c}\mathrm{B} \\
(\mathbf{P a})\end{array}$ & $\mathbf{R}^{2}$ & $\begin{array}{l}\text { Viscosity } \\
\text { (cP) }\end{array}$ \\
\hline 1 & 8.8 & 2.5166 & 0.9533 & 3.0 \\
\hline 2 & 9.0 & 2.2253 & 0.9673 & 3.2 \\
\hline 3 & 8.4 & 2.7055 & 0.9441 & 2.6 \\
\hline 4 & 8.3 & 2.6255 & 0.9697 & 2.5 \\
\hline 5 & 8.5 & 2.5744 & 0.9452 & 2.7 \\
\hline 6 & 8.5 & 2.4945 & 0.9490 & 2.7 \\
\hline 7 & 8.4 & 2.5836 & 0.9389 & 2.6 \\
\hline 8 & 8.4 & 2.5087 & 0.9476 & 2.6 \\
\hline 9 & 8.5 & 2.2429 & 0.9483 & 2.7 \\
\hline & $\mathrm{DF}=8$ & $N=9$ & & \\
\hline Statistics for M & Mean $=8.5$ & Sigma $=0.3$ & $95 \% \mathrm{CL}= \pm 0.2$ & Avg $=2.7 \pm 0.5$ \\
\hline Statistics for B & Mean $=2.4974$ & Sigma $=0.1629$ & $95 \% \mathrm{CL}= \pm 0.1252$ & \\
\hline
\end{tabular}

NOTE: The instrument was not properly zeroed for these experiments. 
Table 6. $50^{\circ} \mathrm{C}$ As Received

\begin{tabular}{|c|c|c|c|c|}
\hline Run \# & $\begin{array}{c}\mathbf{M}^{* 1000} \\
(\mathbf{P a}-\mathbf{s})\end{array}$ & $\begin{array}{c}\mathbf{B} \\
\mathbf{( P a})\end{array}$ & $\begin{array}{c}\text { Viscosity } \\
(\mathbf{c P})\end{array}$ \\
\hline 1 & 8.3 & 1.5352 & 0.9256 & 2.5 \\
\hline 2 & 8.2 & 1.2949 & 0.9488 & 2.4 \\
\hline 3 & 7.9 & 1.5946 & 0.9189 & 2.1 \\
\hline 4 & 7.8 & 1.5969 & 0.9286 & 2.0 \\
\hline 5 & 7.9 & 1.4198 & 0.9261 & 2.1 \\
\hline 6 & 8.1 & 1.1546 & 0.9171 & 2.3 \\
\hline 7 & 7.4 & 1.6668 & 0.9242 & 1.6 \\
\hline 8 & 7.2 & 1.8475 & 0.8965 & 1.4 \\
\hline 9 & 7.1 & 1.8152 & 0.9115 & 1.3 \\
\hline & & & & \\
\hline & $\mathrm{DF}=8$ & $\mathrm{~N}=9$ & & \\
\hline & & & & Avg $=2.0 \pm 0.6$ \\
\hline Statistics for M & Mean $=7.8$ & Sigma -0.5 & $95 \% \mathrm{CL}= \pm 0.3$ & \\
\hline Statistics for B & Mean $=1.5473$ & Sigma $=0.2278$ & $95 \% \mathrm{CL}= \pm 0.1751$ & \\
\hline
\end{tabular}


Table 7. Rheometer Response for Blank - Pretreated

\begin{tabular}{|c|c|c|c|}
\hline Run \# & $\begin{array}{c}\mathbf{M}^{* \mathbf{1 0 0 0}} \\
\mathbf{( P a - s )}\end{array}$ & $\begin{array}{c}\mathbf{B} \\
\mathbf{( P a )}\end{array}$ & $\mathbf{R}^{\mathbf{2}}$ \\
\hline 1 & 7.8 & 0.5181 & 0.8700 \\
\hline 2 & 7.2 & 0.5334 & 0.9034 \\
\hline 3 & 6.9 & 0.5899 & 0.9165 \\
\hline 4 & 7.0 & 0.2766 & 0.8663 \\
\hline 5 & 7.0 & 0.5296 & 0.9331 \\
\hline 6 & 7.2 & 0.4925 & 0.9256 \\
\hline 7 & 7.1 & 0.3541 & 0.9162 \\
\hline 8 & 7.0 & 0.4138 & 0.9188 \\
\hline 9 & 6.9 & 0.4639 & 0.9107 \\
\hline & & & $\mathrm{N}=9$ \\
\hline & $\mathrm{DF}=8$ & & \\
\hline Statistics for M & Mean $=7.1$ & Sigma $=0.3$ & $95 \% \mathrm{CL}= \pm 0.2$ \\
\hline Statistics for B & Mean $=0.4635$ & Sigma $=0.0990$ & $95 \% \mathrm{CL}= \pm 0.0761$ \\
\hline
\end{tabular}


Table 8. $25^{\circ} \mathrm{C}$ Pretreated

\begin{tabular}{|c|c|c|c|c|}
\hline Run \# & $\begin{array}{c}\mathbf{M} * \mathbf{1 0 0 0} \\
\mathbf{( P a - s )}\end{array}$ & $\begin{array}{c}\mathbf{B} \\
\mathbf{P a})\end{array}$ & $\begin{array}{c}\text { Viscosity } \\
\mathbf{( c P )}\end{array}$ \\
\hline 1 & 12.0 & -0.1746 & 0.9876 & 4.9 \\
\hline 2 & 12.2 & -0.3109 & 0.9923 & 5.1 \\
\hline 3 & 12.1 & -0.1453 & 0.9889 & 5.0 \\
\hline 4 & 12.1 & 0.1628 & 0.9967 & 5.0 \\
\hline 5 & 11.2 & -0.0571 & 0.9752 & 4.1 \\
\hline 6 & 10.8 & 0.0054 & 0.9754 & 3.7 \\
\hline 7 & 10.4 & 0.2201 & 0.9701 & 3.3 \\
\hline 8 & 10.3 & 0.2063 & 0.9738 & 3.2 \\
\hline 9 & 10.3 & 0.0945 & 0.9742 & 3.2 \\
\hline & & & & \\
\hline & $\mathrm{DF}=8$ & $\mathrm{~N}=9$ & & \\
\hline & & & & Avg $=4.2 \pm 0.8$ \\
\hline Statistics for M & Mean $=11.3$ & Sigma $=0.9$ & $95 \% \mathrm{CL}= \pm 0.6$ & \\
\hline Statistics for B & Mean $=0.0364$ & Sigma $=0.1824$ & $95 \% \mathrm{CL}= \pm 0.1402$ & \\
\hline
\end{tabular}


Table 9. $50{ }^{\circ} \mathrm{C}$ Pretreated

\begin{tabular}{|c|c|c|c|c|}
\hline Run \# & $\begin{array}{c}\text { M*1000 } \\
(\mathbf{P a - s})\end{array}$ & $\begin{array}{c}\mathbf{B} \\
(\mathbf{P a})\end{array}$ & $\begin{array}{c}\text { Viscosity } \\
(\mathbf{c P})\end{array}$ \\
\hline 1 & 11.2 & -0.4514 & 0.9578 & 4.1 \\
\hline 2 & 10.5 & -0.4766 & 0.9754 & 3.4 \\
\hline 3 & 10.4 & -0.4500 & 0.9797 & 3.3 \\
\hline 4 & 10.1 & -0.3992 & 0.9649 & 3.0 \\
\hline 5 & 10.1 & -0.5552 & 0.9715 & 3.0 \\
\hline 6 & 9.8 & -0.6005 & 0.9679 & 2.7 \\
\hline 7 & 9.6 & -0.4273 & 0.9738 & 2.5 \\
\hline 8 & 9.1 & -0.2481 & 0.9465 & 2.0 \\
\hline 9 & 8.6 & -0.1838 & 0.9594 & 1.5 \\
\hline & & & & \\
\hline & DF $=8$ & $\mathrm{~N}=9$ & & \\
\hline & & & & Avg $=2.8 \pm 0.7$ \\
\hline Statistics for M & Mean $=9.9$ & Sigma $=0.8$ & $95 \% \mathrm{CL}= \pm 0.5$ & \\
\hline Statistics for B & Mean $=0.4213$ & Sigma $=0.1331$ & $95 \% \mathrm{CL}= \pm 0.1023$ & \\
\hline
\end{tabular}


This page intentionally left blank. 
This page intentionally left blank. 
This page intentionally left blank. 
This page intentionally left blank. 
This page intentionally left blank. 


\begin{tabular}{|ll|}
\hline APPENDIX M & WSRC-TR-2000-00352 \\
SR/TRU PRECIPITATE SLURRY RHEOLOGY SUMMARIES & SRT-RPP-2000-00026 \\
Page 479 of 514
\end{tabular}

\section{APPENDIX M}

\section{SR/TRU PRECIPITATE SLURRY RHEOLOGY SUMMARIES}

Table 1. Rheometer Response for Blank - 2 wt \% .......................................................... 481

Table 2. $10^{\circ} \mathrm{C}$ at $2 \mathrm{wt} \%$....................................................................................... 482

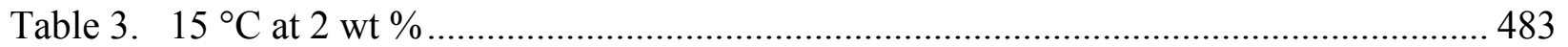

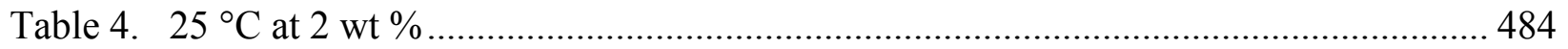

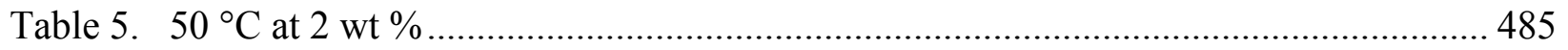

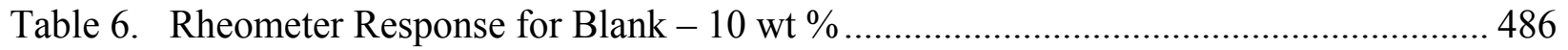

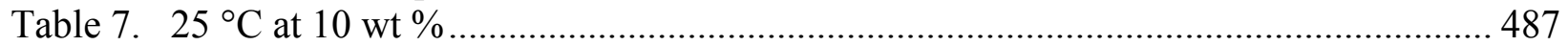

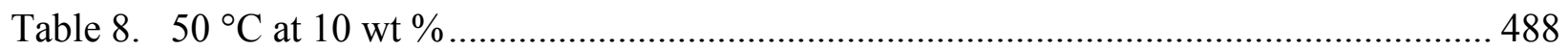

Table 9. Rheometer Response for Blank - 13 wt \% .......................................................... 489

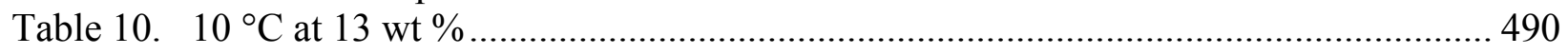

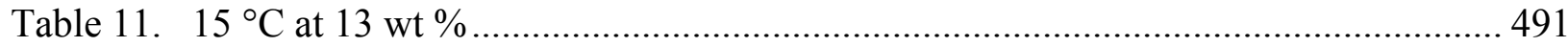

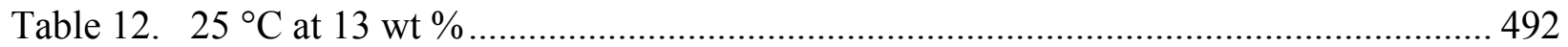

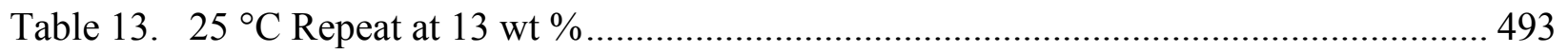

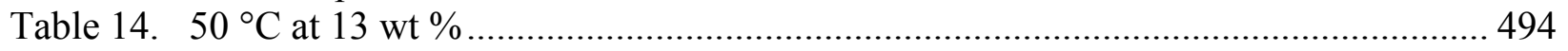

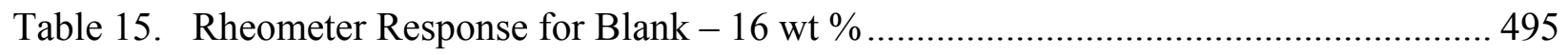

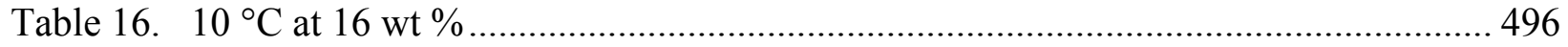

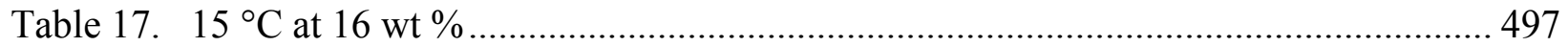

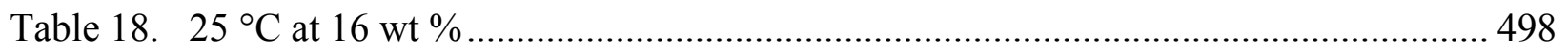

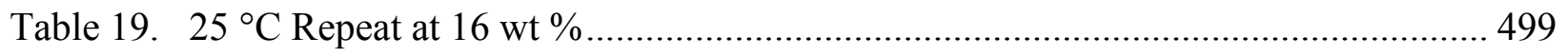

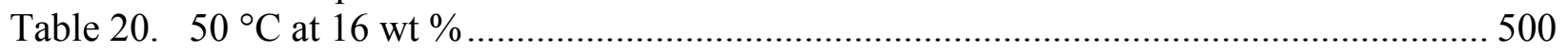




\begin{tabular}{|ll|}
\hline APPENDIX M & WSRC-TR-2000-00352 \\
SR/TRU PRECIPITATE SLURRY RHEOLOGY SUMMARIES & SRT-RPP-2000-00026 \\
Page 480 of 514
\end{tabular}

This page intentionally left blank. 
Table 1. Rheometer Response for Blank - 2 wt \%

\begin{tabular}{|c|c|c|c|}
\hline Run \# & $\begin{array}{c}\mathbf{M}^{* \mathbf{1 0 0 0}} \\
\mathbf{( P a - s )}\end{array}$ & $\begin{array}{c}\mathbf{B} \\
\mathbf{( P a )}\end{array}$ & $\mathbf{R}^{\mathbf{2}}$ \\
\hline 1 & 6.8 & 0.3592 & 0.9793 \\
\hline 2 & 6.8 & 0.2678 & 0.9894 \\
\hline 3 & 6.7 & 0.3164 & 0.9879 \\
\hline 4 & 6.7 & 0.3541 & 0.9874 \\
\hline 5 & 6.8 & 0.2088 & 0.9857 \\
\hline 6 & 6.9 & 0.1769 & 0.9853 \\
\hline 7 & 6.9 & 0.1549 & 0.9734 \\
\hline 8 & 6.5 & 0.3726 & 0.9871 \\
\hline 9 & 6.5 & 0.3582 & 0.9836 \\
\hline & & & $\mathrm{N}=9$ \\
\hline & $\mathrm{DF}=8$ & & \\
\hline Statistics for M & Mean $=6.7$ & Sigma $=0.15$ & $95 \% \mathrm{CL}= \pm 0.1$ \\
\hline Statistics for B & Mean $=0.2854$ & Sigma $=0.0859$ & $95 \% \mathrm{CL}= \pm 0.0660$ \\
\hline
\end{tabular}




\begin{tabular}{|ll|}
\hline APPENDIX M & WSRC-TR-2000-00352 \\
SR/TRU PRECIPITATE SLURRY RHEOLOGY SUMMARIES & SRT-RPP-2000-00026 \\
\hline
\end{tabular}

Table 2. $10{ }^{\circ} \mathrm{C}$ at $2 \mathrm{wt} \%$

\begin{tabular}{|c|c|c|c|c|}
\hline Run \# & $\begin{array}{c}\text { M*1000 } \\
(\mathbf{P a}-\mathbf{s})\end{array}$ & $\begin{array}{c}\mathbf{B} \\
\mathbf{( P a )}\end{array}$ & $\mathbf{R}^{\mathbf{2}}$ & $\begin{array}{c}\text { Viscosity } \\
\mathbf{( c P )}\end{array}$ \\
\hline 1 & 11.7 & -1.0627 & 0.9786 & 5.0 \\
\hline 2 & 11.1 & -0.9425 & 0.9907 & 4.4 \\
\hline 3 & 11.1 & -0.9510 & 0.9916 & 4.4 \\
\hline 4 & 11.0 & -0.9556 & 0.9925 & 4.3 \\
\hline 5 & 11.0 & -0.9615 & 0.9925 & 4.3 \\
\hline 6 & 11.0 & -0.9082 & 0.9934 & 4.3 \\
\hline 7 & 11.1 & -1.0250 & 0.9928 & 4.4 \\
\hline & & & & \\
\hline & $\mathrm{DF}=6$ & $\mathrm{~N}=7$ & & Avg $=4.4 \pm 0.3$ \\
\hline & & & & \\
\hline Statistics for M & Mean $=11.1$ & Sigma $=0.3$ & $95 \% \mathrm{CL}= \pm 0.2$ & \\
\hline
\end{tabular}


Table 3. $15^{\circ} \mathrm{C}$ at 2 wt $\%$

\begin{tabular}{|c|c|c|c|c|}
\hline Run \# & $\begin{array}{c}\mathbf{M} * \mathbf{1 0 0 0} \\
\mathbf{( P a - s )}\end{array}$ & $\begin{array}{c}\mathbf{B} \\
\mathbf{P a})\end{array}$ & $\begin{array}{c}\text { Viscosity } \\
\mathbf{( c P )}\end{array}$ \\
\hline 1 & 11.3 & -0.5511 & 0.9720 & 4.6 \\
\hline 2 & 10.8 & -0.5824 & 0.9895 & 4.1 \\
\hline 3 & 10.7 & -0.7152 & 0.9909 & 4.0 \\
\hline 4 & 10.6 & -0.6352 & 0.9908 & 3.9 \\
\hline 5 & 10.6 & -0.6780 & 0.9911 & 3.9 \\
\hline 6 & 10.6 & -0.6964 & 0.9910 & 3.9 \\
\hline 7 & 10.6 & -0.7118 & 0.9933 & 3.9 \\
\hline 8 & 10.4 & -0.6932 & 0.9914 & 3.7 \\
\hline 9 & 10.4 & -0.6987 & 0.9930 & 3.7 \\
\hline & & & & \\
\hline & $\mathrm{DF}=8$ & $\mathrm{~N}=9$ & & \\
\hline & & & & Avg $=4.0 \pm 0.3$ \\
\hline Statistics for M & Mean $=10.7$ & Sigma $=0.3$ & $95 \% \mathrm{CL}= \pm 0.2$ & \\
\hline Statistics for B & Mean $=0.6624$ & Sigma $=0.0596$ & $95 \% \mathrm{CL}= \pm 0.0458$ & \\
\hline
\end{tabular}


Table 4. $25^{\circ} \mathrm{C}$ at $2 \mathrm{wt} \%$

\begin{tabular}{|c|c|c|c|c|}
\hline Run \# & $\begin{array}{c}\text { M*1000 } \\
(\mathrm{Pa}-\mathrm{s})\end{array}$ & $\begin{array}{c}\mathrm{B} \\
(\mathrm{Pa})\end{array}$ & $\mathbf{R}^{2}$ & $\begin{array}{c}\text { Viscosity } \\
\text { (cP) }\end{array}$ \\
\hline 1 & 11.4 & -0.8717 & 0.9808 & 4.7 \\
\hline 2 & 10.6 & -0.6962 & 0.9885 & 3.9 \\
\hline 3 & 10.5 & -0.7498 & 0.9893 & 3.8 \\
\hline 4 & 10.3 & -0.8060 & 0.9902 & 3.6 \\
\hline 5 & 10.1 & -0.7848 & 0.9894 & 3.4 \\
\hline 6 & 10.0 & -0.8619 & 0.9911 & 3.3 \\
\hline 7 & 9.8 & -0.8824 & 0.9878 & 3.1 \\
\hline 8 & 9.7 & -0.9640 & 0.9918 & 3.0 \\
\hline 9 & 9.6 & -0.9304 & 0.9929 & 2.9 \\
\hline & $\mathrm{DF}=8$ & $N=9$ & & \\
\hline Statistics for M & Mean $=10.7$ & Sigma $=0.6$ & $95 \% \mathrm{CL}= \pm 0.4$ & $\mathrm{Avg}=4.0 \pm 0.5$ \\
\hline Statistics for B & Mean $=0.2352$ & Sigma $=0.2256$ & $95 \% \mathrm{CL}= \pm 0.1734$ & \\
\hline
\end{tabular}


Table 5. $50{ }^{\circ} \mathrm{C}$ at $2 \mathrm{wt} \%$

\begin{tabular}{|c|c|c|c|c|}
\hline Run \# & $\begin{array}{c}\text { M*1000 } \\
(\mathbf{P a}-\mathbf{s})\end{array}$ & $\begin{array}{c}\mathbf{B} \\
\mathbf{( P a )}\end{array}$ & $\begin{array}{c}\text { Viscosity } \\
\mathbf{( c P )}\end{array}$ \\
\hline 1 & 11.8 & -0.0914 & 0.9809 & 5.1 \\
\hline 2 & 11.2 & -0.0969 & 0.9864 & 4.5 \\
\hline 3 & 11.0 & -0.0386 & 0.9944 & 4.3 \\
\hline 4 & 10.8 & -0.0322 & 0.9914 & 4.1 \\
\hline 5 & 10.5 & -0.0285 & 0.9926 & 3.8 \\
\hline 6 & 10.3 & -0.3344 & 0.9941 & 3.6 \\
\hline 7 & 10.1 & -0.4005 & 0.9947 & 3.4 \\
\hline 8 & 10.1 & -0.4662 & 0.9956 & 3.4 \\
\hline 9 & 10.3 & -0.6280 & 0.9923 & 3.6 \\
\hline & & & & \\
\hline Statistics for M & Mean $=10.7$ & Sigma -0.6 & $95 \% \mathrm{CL}= \pm 0.4$ & Avg $=4.0 \pm 0.5$ \\
\hline Statistics for B & Mean $=0.2352$ & Sigma $=0.2256$ & $95 \% \mathrm{CL}= \pm 0.1734$ & \\
\hline
\end{tabular}


Table 6. Rheometer Response for Blank - $10 \mathrm{wt} \%$

\begin{tabular}{|c|c|c|c|}
\hline Run \# & $\begin{array}{c}\mathbf{M}^{* \mathbf{1 0 0 0}} \\
\mathbf{( P a - s )}\end{array}$ & $\begin{array}{c}\mathbf{B} \\
\mathbf{( P a )}\end{array}$ & $\mathbf{R}^{\mathbf{2}}$ \\
\hline 1 & 8.0 & -0.0314 & 0.9186 \\
\hline 2 & 8.0 & 0.2214 & 0.9132 \\
\hline 3 & 7.6 & 0.1818 & 0.9113 \\
\hline 4 & 7.3 & 0.4004 & 0.8865 \\
\hline 5 & 7.1 & 0.2717 & 0.9334 \\
\hline 6 & 7.0 & 0.3279 & 0.9444 \\
\hline 7 & 7.1 & 0.3147 & 0.9388 \\
\hline 8 & 7.1 & 0.2610 & 0.9478 \\
\hline 9 & 6.9 & 0.4141 & 0.9331 \\
\hline & & & $\mathrm{N}=9$ \\
\hline & $\mathrm{DF}=8$ & & \\
\hline Statistics for M & Mean $=7.3$ & Sigma $=0.4$ & $95 \% \mathrm{CL}= \pm 0.3$ \\
\hline Statistics for B & Mean $=0.2624$ & Sigma $=0.1340$ & $95 \% \mathrm{CL}= \pm 0.1030$ \\
\hline
\end{tabular}


Table 7. $25^{\circ} \mathrm{C}$ at $10 \mathrm{wt} \%$

\begin{tabular}{|c|c|c|c|c|}
\hline Run \# & $\begin{array}{c}\mathbf{M} * \mathbf{1 0 0 0} \\
\mathbf{( P a - s )}\end{array}$ & $\begin{array}{c}\mathbf{B} \\
\mathbf{P a})\end{array}$ & $\begin{array}{c}\text { Viscosity } \\
\mathbf{( c P )}\end{array}$ \\
\hline 1 & 20.7 & -0.8066 & 0.9894 & 13.4 \\
\hline 2 & 20.2 & -0.6873 & 0.9911 & 12.9 \\
\hline 3 & 19.7 & -0.3939 & 0.9900 & 12.4 \\
\hline 4 & 19.1 & -0.1123 & 0.9887 & 11.8 \\
\hline 5 & 18.8 & 0.0967 & 0.9872 & 11.5 \\
\hline 6 & 18.5 & 0.4967 & 0.9867 & 11.2 \\
\hline 7 & 18.3 & 0.4946 & 0.9844 & 11.0 \\
\hline 8 & 18.3 & 0.5132 & 0.9854 & 11.0 \\
\hline 9 & 18.0 & 0.9074 & 0.9851 & 10.7 \\
\hline & & & & \\
\hline & $\mathrm{DF}=8$ & $\mathrm{~N}=9$ & & \\
\hline & & & & \\
\hline Statistics for M & Mean $=19.1$ & Sigma $=0.3$ & $95 \% \mathrm{CL}= \pm 0.2$ & Avg $=11.8 \pm 0.5$ \\
\hline Statistics for B & Mean $=0.0565$ & Sigma $=0.5968$ & $95 \% \mathrm{CL}= \pm 0.4587$ & \\
\hline
\end{tabular}


Table 8. $50{ }^{\circ} \mathrm{C}$ at 10 wt $\%$

\begin{tabular}{|c|c|c|c|c|}
\hline Run \# & $\begin{array}{c}\text { M*1000 } \\
(\mathbf{P a}-\mathbf{s})\end{array}$ & $\begin{array}{c}\mathbf{B} \\
\mathbf{P a})\end{array}$ & $\begin{array}{c}\text { Viscosity } \\
\mathbf{( c P )}\end{array}$ \\
\hline 1 & 13.1 & 2.2031 & 0.9669 & 5.8 \\
\hline 2 & 13.0 & 2.0277 & 0.9702 & 5.7 \\
\hline 3 & 12.8 & 2.0516 & 0.9677 & 5.5 \\
\hline 4 & 12.9 & 1.9884 & 0.9687 & 5.6 \\
\hline 5 & 13.1 & 1.6803 & 0.9629 & 5.8 \\
\hline 6 & 12.6 & 2.0910 & 0.9649 & 5.3 \\
\hline 7 & 12.4 & 2.0823 & 0.9684 & 5.1 \\
\hline 8 & 12.6 & 1.7542 & 0.9587 & 5.3 \\
\hline 9 & 13.0 & 1.3304 & 0.9627 & 5.7 \\
\hline & & & & \\
\hline Statistics for M & Mean $=12.8$ & Sigma -0.3 & $95 \% \mathrm{CL}= \pm 0.2$ & Avg $=5.5 \pm 0.5$ \\
\hline Statistics for B & Mean $=1.9121$ & Sigma $=0.2742$ & $95 \% \mathrm{CL}= \pm 0.2107$ & \\
\hline
\end{tabular}

NOTE: The instrument was not properly zeroed for these experiments. 
Table 9. Rheometer Response for Blank - 13 wt \%

\begin{tabular}{|c|c|c|c|}
\hline Run \# & $\begin{array}{c}\mathbf{M}^{* \mathbf{1 0 0 0}} \\
\mathbf{( P a - s )}\end{array}$ & $\begin{array}{c}\mathbf{B} \\
\mathbf{( P a )}\end{array}$ & $\mathbf{R}^{\mathbf{2}}$ \\
\hline 1 & 7.6 & -0.1387 & 0.9581 \\
\hline 2 & 6.8 & 0.4529 & 0.9712 \\
\hline 3 & 9.4 & -0.6141 & 0.9439 \\
\hline 4 & 7.6 & 0.1357 & 0.9754 \\
\hline 5 & 7.6 & 0.0441 & 0.9913 \\
\hline 6 & 7.0 & 0.1484 & 0.9908 \\
\hline 7 & 6.6 & 0.2326 & 0.9635 \\
\hline 8 & 6.6 & 0.0450 & 0.988 \\
\hline 9 & 6.6 & -0.0870 & 0.9761 \\
\hline & & & \\
\hline & $\mathrm{DF}=8$ & $\mathrm{~N}=9$ & \\
\hline Statistics for M & Mean $=7.3$ & Sigma $=0.9$ & $95 \% \mathrm{CL}= \pm 0.6$ \\
\hline Statistics for B & Mean $=0.0243$ & Sigma $=0.2962$ & $95 \% \mathrm{CL}= \pm 0.2277$ \\
\hline
\end{tabular}


Table $10.10{ }^{\circ} \mathrm{C}$ at $13 \mathrm{wt} \%$

\begin{tabular}{|c|c|c|c|c|}
\hline Run \# & $\begin{array}{c}\mathbf{M} * \mathbf{1 0 0 0} \\
\mathbf{( P a - s )}\end{array}$ & $\begin{array}{c}\mathbf{B} \\
\mathbf{P a})\end{array}$ & $\begin{array}{c}\text { Viscosity } \\
\mathbf{( c P )}\end{array}$ \\
\hline 1 & 29.4 & 8.8384 & 0.9679 & 22.1 \\
\hline 2 & 29.1 & 8.6589 & 0.9832 & 21.8 \\
\hline 3 & 28.9 & 8.7932 & 0.9864 & 21.6 \\
\hline 4 & 28.9 & 8.9283 & 0.9857 & 21.6 \\
\hline 5 & 28.6 & 9.2073 & 0.9870 & 21.3 \\
\hline 6 & 28.7 & 9.3269 & 0.9872 & 21.4 \\
\hline 7 & 28.7 & 9.4632 & 0.9851 & 21.4 \\
\hline 8 & 28.6 & 9.5779 & 0.9845 & 21.3 \\
\hline 9 & 28.9 & 9.4228 & 0.9852 & 21.6 \\
\hline & & & & \\
\hline & $\mathrm{DF}=8$ & $\mathrm{~N}=9$ & & \\
\hline & & & & \\
\hline Statistics for M & Mean $=28.9$ & Sigma $=0.3$ & $95 \% \mathrm{CL}= \pm 0.2$ & Avg $=21.6 \pm 0.8$ \\
\hline Statistics for B & Mean $=9.1353$ & Sigma $=0.3359$ & $95 \% \mathrm{CL}= \pm 0.2582$ & \\
\hline
\end{tabular}


Table 11. $15^{\circ} \mathrm{C}$ at $13 \mathrm{wt} \%$

\begin{tabular}{|c|c|c|c|c|}
\hline Run \# & $\begin{array}{c}\mathbf{M} * \mathbf{1 0 0 0} \\
\mathbf{( P a - s )}\end{array}$ & $\begin{array}{c}\mathbf{B} \\
\mathbf{P a})\end{array}$ & $\begin{array}{c}\text { Viscosity } \\
\mathbf{( c P )}\end{array}$ \\
\hline 1 & 27.3 & 5.7658 & 0.9502 & 20.0 \\
\hline 2 & 25.9 & 5.9394 & 0.9893 & 18.6 \\
\hline 3 & 25.8 & 6.4213 & 0.9904 & 18.5 \\
\hline 4 & 25.8 & 6.7315 & 0.9907 & 18.5 \\
\hline 5 & 25.5 & 7.2497 & 0.9896 & 18.2 \\
\hline 6 & 26.1 & 7.3416 & 0.9727 & 18.8 \\
\hline 7 & 25.5 & 7.6574 & 0.9894 & 18.2 \\
\hline 8 & 25.4 & 7.9592 & 0.9881 & 18.1 \\
\hline 9 & 25.4 & 8.0997 & 0.9879 & 18.1 \\
\hline & & & & \\
\hline & $\mathrm{DF}=8$ & $\mathrm{~N}=9$ & & \\
\hline & & & & \\
\hline Statistics for M & Mean $=25.9$ & Sigma $=0.6$ & $95 \% \mathrm{CL}= \pm 0.4$ & Avg $=18.6 \pm 1.0$ \\
\hline Statistics for B & Mean $=7.0184$ & Sigma $=0.8509$ & $95 \% \mathrm{CL}= \pm 0.6541$ & \\
\hline
\end{tabular}


Table 12. $25^{\circ} \mathrm{C}$ at $13 \mathrm{wt} \%$

\begin{tabular}{|c|c|c|c|c|}
\hline Run \# & $\begin{array}{c}\mathbf{M} * \mathbf{1 0 0 0} \\
\mathbf{( P a - s )}\end{array}$ & $\begin{array}{c}\mathbf{B} \\
\mathbf{P a})\end{array}$ & $\begin{array}{c}\text { Viscosity } \\
\mathbf{( c P )}\end{array}$ \\
\hline 1 & 20.6 & 8.4921 & 0.9994 & 13.3 \\
\hline 2 & 21.0 & 8.6529 & 0.9968 & 13.7 \\
\hline 3 & 20.0 & 8.9398 & 0.9909 & 12.7 \\
\hline 4 & 19.6 & 9.1643 & 0.9932 & 12.3 \\
\hline 5 & 21.1 & 8.2180 & 0.9907 & 12.8 \\
\hline 6 & 19.7 & 9.0873 & 0.9936 & 12.4 \\
\hline 7 & 19.6 & 9.1492 & 0.9934 & 12.3 \\
\hline 8 & 19.9 & 8.9698 & 0.9942 & 12.6 \\
\hline 9 & 21.3 & 7.5398 & 0.9499 & 14.0 \\
\hline & & & & \\
\hline & $\mathrm{DF}=8$ & $\mathrm{~N}=9$ & & \\
\hline & & & & \\
\hline Statistics for M & Mean $=21.9$ & Sigma $=1.1$ & $95 \% \mathrm{CL}= \pm 0.7$ & Avg $=14.6 \pm 1.2$ \\
\hline Statistics for B & Mean $=7.0210$ & Sigma $=0.4581$ & $95 \% \mathrm{CL}= \pm 0.3521$ & \\
\hline
\end{tabular}


Table 13. $25^{\circ} \mathrm{C}$ Repeat at 13 wt $\%$

\begin{tabular}{|c|c|c|c|c|}
\hline Run \# & $\begin{array}{c}\mathbf{M}^{* 1000} \\
\mathbf{( P a - s )}\end{array}$ & $\begin{array}{c}\mathbf{B} \\
\mathbf{P a}\end{array}$ & $\mathbf{R}^{\mathbf{2}}$ & $\begin{array}{c}\text { Viscosity } \\
\mathbf{( c P )}\end{array}$ \\
\hline 1 & 20.7 & 7.2001 & 0.9854 & 13.4 \\
\hline 2 & 20.4 & 8.0966 & 0.9937 & 13.1 \\
\hline 3 & 21.8 & 6.9242 & 0.9857 & 14.5 \\
\hline 4 & 21.3 & 7.1491 & 0.9915 & 14.0 \\
\hline 5 & 21.0 & 7.0629 & 0.9810 & 13.7 \\
\hline 6 & 22.9 & 6.7777 & 0.9976 & 15.6 \\
\hline 7 & 22.7 & 6.6651 & 0.9979 & 15.4 \\
\hline 8 & 23.3 & 6.5823 & 0.9867 & 16.0 \\
\hline 9 & 23.1 & 6.7312 & 0.9893 & 15.8 \\
\hline & & & & \\
\hline & DF $=8$ & $\mathrm{~N}=9$ & & \\
\hline & & & & Avg. $=14.6 \pm 1.2$ \\
\hline Statistics for M & Mean $=21.9$ & Sigma $=1.1$ & $95 \% \mathrm{CL}= \pm 0.7$ & $(8 \%)$ \\
\hline Statistics for B & Mean $=7.0210$ & Sigma $=0.4581$ & $95 \% \mathrm{CL}= \pm 0.2993$ & \\
\hline
\end{tabular}


Table 14. $50{ }^{\circ} \mathrm{C}$ at $13 \mathrm{wt} \%$

\begin{tabular}{|c|c|c|c|c|}
\hline Run \# & $\begin{array}{c}\mathbf{M}^{*} \mathbf{1 0 0 0} \\
(\mathbf{P a - s})\end{array}$ & $\begin{array}{c}\mathbf{B} \\
\mathbf{P a})\end{array}$ & $\begin{array}{c}\text { Viscosity } \\
(\mathbf{c P})\end{array}$ \\
\hline 1 & 16.3 & 7.0695 & 0.9752 & 9.0 \\
\hline 2 & 16.1 & 6.4607 & 0.9897 & 8.8 \\
\hline 3 & 16.0 & 6.1632 & 0.9904 & 8.7 \\
\hline 4 & 16.2 & 5.9353 & 0.9906 & 8.9 \\
\hline 5 & 16.2 & 5.7709 & 0.9933 & 8.9 \\
\hline 6 & 16.4 & 5.6563 & 0.9960 & 9.1 \\
\hline 7 & 16.0 & 5.7728 & 0.9916 & 8.7 \\
\hline 8 & 15.9 & 5.7125 & 0.9936 & 8.6 \\
\hline 9 & 15.8 & 5.8054 & 0.9918 & 8.5 \\
\hline & & & & \\
\hline & $\mathrm{DF}=8$ & $\mathrm{~N}=9$ & & \\
\hline Statistics for M & Mean $=16.1$ & Sigma -0.2 & $95 \% \mathrm{CL}= \pm 0.1$ & Avg $=8.8 \pm 0.7$ \\
\hline Statistics for B & Mean $=6.0385$ & Sigma $=0.4632$ & $95 \% \mathrm{CL}= \pm 0.3560$ & \\
\hline
\end{tabular}


Table 15. Rheometer Response for Blank - $16 \mathrm{wt} \%$

\begin{tabular}{|c|c|c|c|}
\hline Run \# & $\begin{array}{c}\mathbf{M}^{* \mathbf{1 0 0 0}} \\
\mathbf{( P a - s )}\end{array}$ & $\begin{array}{c}\mathbf{B} \\
\mathbf{( P a})\end{array}$ & $\mathbf{R}^{\mathbf{2}}$ \\
\hline 1 & 5.1 & 0.7413 & 0.9188 \\
\hline 2 & 4.9 & 0.8435 & 0.9313 \\
\hline 3 & 5.2 & 0.7575 & 0.8922 \\
\hline 4 & 4.9 & 0.8004 & 0.9186 \\
\hline 5 & 5.2 & 0.4936 & 0.9161 \\
\hline 6 & 4.9 & 0.7309 & 0.9021 \\
\hline 7 & 4.7 & 0.8088 & 0.9262 \\
\hline 8 & 4.7 & 0.7262 & 0.9188 \\
\hline 9 & 5.4 & 0.4388 & 0.8928 \\
\hline & & & $\mathrm{N}=9$ \\
\hline & $\mathrm{DF}=8$ & & \\
\hline Statistics for M & Mean $=5.0$ & Sigma $=0.3$ & $95 \% \mathrm{CL}= \pm 0.2$ \\
\hline Statistics for B & Mean $=0.7045$ & Sigma $=0.1413$ & $95 \% \mathrm{CL}= \pm 0.1086$ \\
\hline
\end{tabular}


Table 16. $10{ }^{\circ} \mathrm{C}$ at $16 \mathrm{wt} \%$

\begin{tabular}{|c|c|c|c|c|}
\hline Run \# & $\begin{array}{c}\mathbf{M} * \mathbf{1 0 0 0} \\
\mathbf{( P a - s )}\end{array}$ & $\begin{array}{c}\mathbf{B} \\
\mathbf{P a})\end{array}$ & $\begin{array}{c}\text { Viscosity } \\
\mathbf{( c P )}\end{array}$ \\
\hline 1 & 33.0 & 7.1161 & 0.9730 & 28.0 \\
\hline 2 & 32.3 & 7.0383 & 0.9866 & 27.3 \\
\hline 3 & 32.8 & 6.5612 & 0.9886 & 27.8 \\
\hline 4 & 32.6 & 7.2969 & 0.9933 & 27.6 \\
\hline 5 & 32.6 & 7.2911 & 0.9923 & 27.6 \\
\hline 6 & 32.3 & 7.3074 & 0.9876 & 27.3 \\
\hline 7 & 32.4 & 7.1107 & 0.9887 & 27.4 \\
\hline 8 & 32.5 & 7.1119 & 0.9874 & 27.5 \\
\hline 9 & 32.1 & 7.5196 & 0.9875 & 27.1 \\
\hline & & & & \\
\hline & $\mathrm{DF}=8$ & $\mathrm{~N}=9$ & & \\
\hline & & & & \\
\hline Statistics for M & Mean $=32.5$ & Sigma $=0.3$ & $95 \% \mathrm{CL}= \pm 0.2$ & Avg $=27.5 \pm 0.4$ \\
\hline Statistics for B & Mean $=7.1504$ & Sigma $=0.2660$ & $95 \% \mathrm{CL}= \pm 0.2045$ & \\
\hline
\end{tabular}


Table 17. $15^{\circ} \mathrm{C}$ at $16 \mathrm{wt} \%$

\begin{tabular}{|c|c|c|c|c|}
\hline Run \# & $\begin{array}{c}\mathbf{M} * \mathbf{1 0 0 0} \\
\mathbf{( P a - s )}\end{array}$ & $\begin{array}{c}\mathbf{B} \\
\mathbf{P a})\end{array}$ & $\begin{array}{c}\text { Viscosity } \\
\mathbf{( c P )}\end{array}$ \\
\hline 1 & 29.1 & 4.0278 & 0.9646 & 24.1 \\
\hline 2 & 28.5 & 3.6619 & 0.9877 & 23.5 \\
\hline 3 & 28.4 & 3.7964 & 0.9881 & 23.4 \\
\hline 4 & 28.5 & 3.8018 & 0.9882 & 23.5 \\
\hline 5 & 28.2 & 4.1445 & 0.9901 & 23.2 \\
\hline 6 & 28.1 & 4.3298 & 0.9888 & 23.1 \\
\hline 7 & 28.2 & 4.2259 & 0.9895 & 23.2 \\
\hline 8 & 28.2 & 4.3949 & 0.9894 & 23.2 \\
\hline 9 & 28.1 & 4.5641 & 0.9880 & 23.1 \\
\hline & & & & \\
\hline & $\mathrm{DF}=8$ & $\mathrm{~N}=9$ & & \\
\hline & & & & \\
\hline Statistics for M & Mean $=28.4$ & Sigma $=0.3$ & $95 \% \mathrm{CL}= \pm 0.2$ & Avg $=23.4 \pm 0.4$ \\
\hline Statistics for B & Mean $=4.1052$ & Sigma $=0.3064$ & $95 \% \mathrm{CL}= \pm 0.2355$ & \\
\hline
\end{tabular}


Table 18. $25^{\circ} \mathrm{C}$ at $16 \mathrm{wt} \%$

\begin{tabular}{|c|c|c|c|c|}
\hline Run \# & $\begin{array}{c}\mathbf{M} * \mathbf{1 0 0 0} \\
\mathbf{( P a - s )}\end{array}$ & $\begin{array}{c}\mathbf{B} \\
\mathbf{P a})\end{array}$ & $\begin{array}{c}\text { Viscosity } \\
\mathbf{( c P )}\end{array}$ \\
\hline 1 & 22.8 & 5.9577 & 0.9914 & 17.8 \\
\hline 2 & 22.9 & 6.2506 & 0.9879 & 17.9 \\
\hline 3 & 22.2 & 6.8118 & 0.9849 & 17.2 \\
\hline 4 & 22.3 & 6.6606 & 0.9848 & 17.3 \\
\hline 5 & 22.2 & 6.9360 & 0.9854 & 17.2 \\
\hline 6 & 23.2 & 6.5583 & 0.9852 & 18.2 \\
\hline 7 & 24.6 & 4.8117 & 0.9578 & 19.6 \\
\hline 8 & 22.7 & 6.8083 & 0.9871 & 17.7 \\
\hline 9 & 22.6 & 6.9836 & 0.9852 & 17.6 \\
\hline & & & & \\
\hline & $\mathrm{DF}=8$ & $\mathrm{~N}=9$ & & \\
\hline & & & & \\
\hline Statistics for M & Mean $=23.0$ & Sigma $=0.2$ & $95 \% \mathrm{CL}= \pm 0.2$ & Avg $=18.0 \pm 0.4$ \\
\hline Statistics for B & Mean $=10.3681$ & Sigma $=0.1958$ & $95 \% \mathrm{CL}= \pm 0.1507$ & \\
\hline
\end{tabular}


Table 19. $25^{\circ} \mathrm{C}$ Repeat at $16 \mathrm{wt} \%$

\begin{tabular}{|c|c|c|c|c|}
\hline Run \# & $\begin{array}{c}\mathbf{M}^{* 1000} \\
\mathbf{( P a - s )}\end{array}$ & $\begin{array}{c}\mathbf{B} \\
\mathbf{P a})\end{array}$ & $\begin{array}{c}\mathbf{R}^{\mathbf{2}} \\
\mathbf{( c P )}\end{array}$ \\
\hline 1 & 22.9 & 10.145 & 0.9809 & 17.9 \\
\hline 2 & 22.7 & 10.316 & 0.9849 & 17.7 \\
\hline 3 & 22.9 & 10.295 & 0.9845 & 17.9 \\
\hline 4 & 23.2 & 10.168 & 0.9835 & 18.2 \\
\hline 5 & 22.7 & 10.692 & 0.9865 & 17.7 \\
\hline 6 & 23.0 & 10.426 & 0.9842 & 18.0 \\
\hline 7 & 23.2 & 10.247 & 0.9862 & 18.2 \\
\hline 8 & 23.3 & 10.363 & 0.9844 & 18.3 \\
\hline 9 & 23.0 & 10.661 & 0.9843 & 18.0 \\
\hline & & & & \\
\hline & DF $=8$ & $\mathrm{~N}=9$ & & \\
\hline & & & & Avg. $=18.0 \pm 0.4$ \\
\hline Statistics for M & Mean $=23.0$ & Sigma $=0.2$ & $95 \% \mathrm{CL}= \pm 0.2$ & $(3 \%)$ \\
\hline Statistics for B & Mean $=10.368$ & Sigma $=0.196$ & $95 \% \mathrm{CL}= \pm 0.128$ & \\
\hline
\end{tabular}


Table 20. $50{ }^{\circ} \mathrm{C}$ at $16 \mathrm{wt} \%$

\begin{tabular}{|c|c|c|c|c|}
\hline Run \# & $\begin{array}{c}\mathbf{M}^{* 1000} \\
(\mathbf{P a}-\mathbf{s})\end{array}$ & $\begin{array}{c}\mathbf{B} \\
\mathbf{( P a )}\end{array}$ & $\mathbf{R}^{\mathbf{2}}$ & $\begin{array}{c}\text { Viscosity } \\
\mathbf{( c P )}\end{array}$ \\
\hline 1 & 14.9 & 5.7310 & 0.9535 & 9.9 \\
\hline 2 & 15.1 & 4.8722 & 0.9713 & 10.1 \\
\hline 3 & 15.2 & 4.6256 & 0.9813 & 10.2 \\
\hline 4 & 14.9 & 5.0153 & 0.9823 & 9.9 \\
\hline 5 & 14.9 & 4.9655 & 0.9809 & 9.9 \\
\hline 6 & 15.4 & 4.5035 & 0.9798 & 10.4 \\
\hline 7 & 15.5 & 4.6135 & 0.9841 & 10.5 \\
\hline 8 & 15.4 & 4.6837 & 0.9808 & 10.4 \\
\hline 9 & 15.6 & 4.5727 & 0.9861 & 10.6 \\
\hline & & & & \\
\hline & $\mathrm{DF}=8$ & $\mathrm{~N}=9$ & & \\
\hline & & & & \\
\hline Statistics for M & Mean $=15.2$ & Sigma -0.3 & $95 \% \mathrm{CL}= \pm 0.2$ & Avg $=10.2 \pm 0.4$ \\
\hline Statistics for B & Mean $=4.8426$ & Sigma $=0.3788$ & $95 \% \mathrm{CL}= \pm 0.2912$ & \\
\hline
\end{tabular}




\begin{tabular}{|ll|}
\hline APPENDIX N & WSRC-TR-2000-00352 \\
ENVELOPE C AS-RECEIVED CALORIMETRY DATA & SRT-RPP-2000-00026 \\
\hline
\end{tabular}

\section{APPENDIX N}

\section{ENVELOPE C AS-RECEIVED CALORIMETRY DATA}

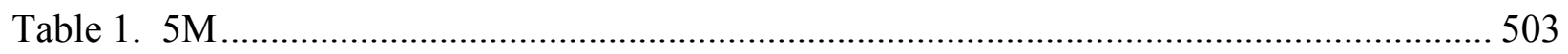

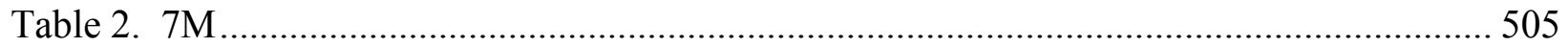

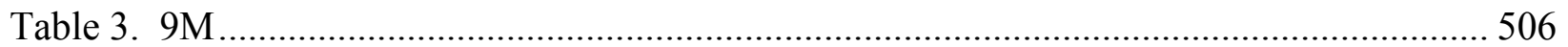


Table 1. 5M

\begin{tabular}{|c|c|c|c|c|c|c|c|c|}
\hline & Standard & Empty & Sample & Sample - Empty & $\begin{array}{l}\text { Sample Mass- } \\
\text { Corrected }\end{array}$ & $\begin{array}{l}\text { Standard - } \\
\text { Empty }\end{array}$ & Sapphire HC & Sample HC \\
\hline \multicolumn{9}{|l|}{$5 \mathrm{M} 1$} \\
\hline 40 & -13.044597 & -1.701997 & -32.09271 & -30.39071 & -44.52592908 & -11.34260017 & 0.1899 & 0.745461694 \\
\hline 50 & -13.209693 & -2.032199 & -32.17041 & -30.13821 & -44.15598429 & -11.17749483 & 0.1939 & 0.76598965 \\
\hline 60 & -13.478388 & -2.342977 & -32.00045 & -29.65747 & -43.45164649 & -11.13541133 & 0.1979 & 0.772228397 \\
\hline 70 & -13.468677 & -2.602768 & -31.82563 & -29.22286 & -42.81489084 & -10.86590867 & 0.2019 & 0.795545658 \\
\hline 80 & -13.612737 & -2.877127 & -31.53914 & -28.66201 & -41.99318184 & -10.73560967 & 0.2059 & 0.805394049 \\
\hline 90 & -13.7487 & -3.100499 & -31.19922 & -28.09872 & -41.16789429 & -10.6482015 & 0.2099 & 0.811511785 \\
\hline \multicolumn{9}{|l|}{$5 \mathrm{M} 2$} \\
\hline 40 & -13.044597 & -1.701997 & -30.79133 & -29.08933 & -42.61925606 & -11.34260017 & 0.1899 & 0.713539806 \\
\hline 50 & -13.209693 & -2.032199 & -30.61652 & -28.58432 & -41.87935476 & -11.17749483 & 0.1939 & 0.726496143 \\
\hline 60 & -13.478388 & -2.342977 & -30.36886 & -28.02588 & -41.06117742 & -11.13541133 & 0.1979 & 0.729744665 \\
\hline 70 & -13.468677 & -2.602768 & -30.1115 & -27.50873 & -40.30349107 & -10.86590867 & 0.2019 & 0.748881212 \\
\hline 80 & -13.612737 & -2.877127 & -29.7473 & -26.87017 & -39.36792788 & -10.73560967 & 0.2059 & 0.755043878 \\
\hline 90 & -13.7487 & -3.100499 & -29.21316 & -26.11266 & -38.25808545 & -10.6482015 & 0.2099 & 0.754152909 \\
\hline \multicolumn{9}{|l|}{$5 \mathrm{M} 3$} \\
\hline 40 & -13.044597 & -1.701997 & -29.15974 & -27.45774 & -40.22878699 & -11.34260017 & 0.1899 & 0.673518112 \\
\hline 50 & -13.209693 & -2.032199 & -28.83925 & -26.80705 & -39.27544755 & -11.17749483 & 0.1939 & 0.681325234 \\
\hline 60 & -13.478388 & -2.342977 & -28.53819 & -26.19521 & -38.379033 & -11.13541133 & 0.1979 & 0.68207724 \\
\hline 70 & -13.468677 & -2.602768 & -28.36337 & -25.7606 & -37.74227735 & -10.86590867 & 0.2019 & 0.70129117 \\
\hline 80 & -13.612737 & -2.877127 & -28.18856 & -25.31143 & -37.08419253 & -10.73560967 & 0.2059 & 0.711243747 \\
\hline 90 & -13.7487 & -3.100499 & -27.96033 & -24.85983 & -36.42254383 & -10.6482015 & 0.2099 & 0.717970255 \\
\hline
\end{tabular}




APPENDIX N
ENVELOPE C AS-RECEIVED CALORIMETRY DATA

Table 1. 5M - continued

\begin{tabular}{|c|c|c|c|c|c|c|c|c|}
\hline & Standard & Empty & Sample & Sample - Empty & $\begin{array}{c}\text { Sample Mass- } \\
\text { Corrected }\end{array}$ & $\begin{array}{c}\text { Standard - } \\
\text { Empty }\end{array}$ & Sapphire HC & Sample HC \\
\hline 5M4 & & & & & & & & \\
\hline 40 & -13.044597 & -1.701997 & -29.06262 & -27.36062 & -40.0864949 & -11.34260017 & 0.1899 & 0.67113583 \\
\hline 50 & -13.209693 & -2.032199 & -29.00435 & -26.97215 & -39.51733824 & -11.17749483 & 0.1939 & 0.685521398 \\
\hline 60 & -13.478388 & -2.342977 & -28.72756 & -26.38458 & -38.65648207 & -11.13541133 & 0.1979 & 0.687008102 \\
\hline 70 & -13.468677 & -2.602768 & -28.31967 & -25.7169 & -37.67825177 & -10.86590867 & 0.2019 & 0.700101507 \\
\hline 80 & -13.612737 & -2.877127 & -28.31481 & -25.43768 & -37.26916347 & -10.73560967 & 0.2059 & 0.714791334 \\
\hline 90 & -13.7487 & -3.100499 & -28.00889 & -24.90839 & -36.49368987 & -10.6482015 & 0.2099 & 0.719372704 \\
\hline & & & & & & & & \\
\hline
\end{tabular}


Table 2. $7 \mathrm{M}$

\begin{tabular}{|c|c|c|c|c|c|c|c|c|}
\hline & Standard & Empty & Sample & Sample - Empty & $\begin{array}{l}\text { Sample Mass- } \\
\text { Corrected }\end{array}$ & $\begin{array}{l}\text { Standard - } \\
\text { Empty }\end{array}$ & Sapphire HC & Sample HC \\
\hline \multicolumn{9}{|l|}{$7 \mathrm{M} 1$} \\
\hline 40 & -13.0446 & -1.701997 & -39.34258 & -37.6405835 & -39.52261268 & -11.34260017 & 0.1899 & 0.661695205 \\
\hline 50 & -13.20969 & -2.032199 & -38.74531 & -36.7131115 & -38.54876708 & -11.17749483 & 0.1939 & 0.668719248 \\
\hline 60 & -13.47839 & -2.342977 & -38.4151 & -36.072123 & -37.87572915 & -11.13541133 & 0.1979 & 0.673132458 \\
\hline 70 & -13.46868 & -2.602768 & -38.17231 & -35.569542 & -37.3480191 & -10.86590867 & 0.2019 & 0.693965437 \\
\hline 80 & -13.61274 & -2.877127 & -37.62359 & -34.746463 & -36.48378615 & -10.73560967 & 0.2059 & 0.699728455 \\
\hline 90 & -13.7487 & -3.100499 & -36.90977 & -33.8092715 & -35.49973508 & -10.6482015 & 0.2099 & 0.699779619 \\
\hline \multicolumn{9}{|l|}{$7 \mathrm{M} 2$} \\
\hline 40 & -13.0446 & -1.701997 & -37.94408 & -36.2420835 & -38.05418768 & -11.34260017 & 0.1899 & 0.637110551 \\
\hline 50 & -13.20969 & -2.032199 & -37.34195 & -35.3097515 & -37.07523908 & -11.17749483 & 0.1939 & 0.643157431 \\
\hline 60 & -13.47839 & -2.342977 & -36.61356 & -34.270583 & -35.98411215 & -11.13541133 & 0.1979 & 0.639514391 \\
\hline 70 & -13.46868 & -2.602768 & -36.0697 & -33.466932 & -35.1402786 & -10.86590867 & 0.2019 & 0.652943299 \\
\hline 80 & -13.61274 & -2.877127 & -35.54526 & -32.668133 & -34.30153965 & -10.73560967 & 0.2059 & 0.657874795 \\
\hline 90 & -13.7487 & -3.100499 & -35.18107 & -32.0805715 & -33.68460008 & -10.6482015 & 0.2099 & 0.663999226 \\
\hline \multicolumn{9}{|l|}{$7 \mathrm{M} 3$} \\
\hline 40 & -13.0446 & -1.701997 & -38.06548 & -36.3634835 & -38.18165768 & -11.34260017 & 0.1899 & 0.639244678 \\
\hline 50 & -13.20969 & -2.032199 & -37.43906 & -35.4068615 & -37.17720458 & -11.17749483 & 0.1939 & 0.644926263 \\
\hline 60 & -13.47839 & -2.342977 & -36.93891 & -34.595933 & -36.32572965 & -11.13541133 & 0.1979 & 0.645585662 \\
\hline 70 & -13.46868 & -2.602768 & -36.42418 & -33.821412 & -35.5124826 & -10.86590867 & 0.2019 & 0.65985924 \\
\hline 80 & -13.61274 & -2.877127 & -35.81234 & -32.935213 & -34.58197365 & -10.73560967 & 0.2059 & 0.663253285 \\
\hline 90 & -13.7487 & -3.100499 & -35.32675 & -32.2262515 & -33.83756408 & -10.6482015 & 0.2099 & 0.667014491 \\
\hline
\end{tabular}


Table 3. 9M

\begin{tabular}{|c|c|c|c|c|c|c|c|c|}
\hline & Standard & Empty & Sample & Sample - Empty & $\begin{array}{l}\text { Sample Mass- } \\
\text { Corrected }\end{array}$ & $\begin{array}{l}\text { Standard - } \\
\text { Empty }\end{array}$ & Sapphire HC & Sample HC \\
\hline \multicolumn{9}{|l|}{$9 M 1$} \\
\hline 40 & -13.0446 & -1.701997 & -37.26911 & -35.5671135 & -40.74051183 & -11.34260017 & 0.1899 & 0.682085508 \\
\hline 50 & -13.20969 & -2.032199 & -36.16196 & -34.1297615 & -39.09409045 & -11.17749483 & 0.1939 & 0.678179167 \\
\hline 60 & -13.47839 & -2.342977 & -34.92371 & -32.580733 & -37.31974871 & -11.13541133 & 0.1979 & 0.6632515 \\
\hline 70 & -13.46868 & -2.602768 & -33.95738 & -31.354612 & -35.91528284 & -10.86590867 & 0.2019 & 0.667343692 \\
\hline 80 & -13.61274 & -2.877127 & -33.08332 & -30.206193 & -34.59982107 & -10.73560967 & 0.2059 & 0.663595583 \\
\hline 90 & -13.7487 & -3.100499 & -31.99317 & -28.8926665 & -33.09523617 & -10.6482015 & 0.2099 & 0.652381538 \\
\hline \multicolumn{9}{|l|}{$9 \mathrm{M} 2$} \\
\hline 40 & -13.0446 & -1.701997 & -37.35166 & -35.6496635 & -40.8350691 & -11.34260017 & 0.1899 & 0.683668604 \\
\hline 50 & -13.20969 & -2.032199 & -36.83208 & -34.7998815 & -39.86168245 & -11.17749483 & 0.1939 & 0.69149486 \\
\hline 60 & -13.47839 & -2.342977 & -36.07456 & -33.731583 & -38.63799507 & -11.13541133 & 0.1979 & 0.686679548 \\
\hline 70 & -13.46868 & -2.602768 & -35.25633 & -32.653562 & -37.40317102 & -10.86590867 & 0.2019 & 0.694990218 \\
\hline 80 & -13.61274 & -2.877127 & -34.53038 & -31.653253 & -36.25736253 & -10.73560967 & 0.2059 & 0.69538584 \\
\hline 90 & -13.7487 & -3.100499 & -33.92825 & -30.8277515 & -35.31178808 & -10.6482015 & 0.2099 & 0.696074761 \\
\hline \multicolumn{9}{|l|}{$9 M 3$} \\
\hline 40 & -13.0446 & -1.701997 & -37.04088 & -35.3388835 & -40.47908474 & -11.34260017 & 0.1899 & 0.677708645 \\
\hline 50 & -13.20969 & -2.032199 & -36.48245 & -34.4502515 & -39.46119717 & -11.17749483 & 0.1939 & 0.684547499 \\
\hline 60 & -13.47839 & -2.342977 & -35.54041 & -33.197433 & -38.02615053 & -11.13541133 & 0.1979 & 0.675805766 \\
\hline 70 & -13.46868 & -2.602768 & -34.65663 & -32.053862 & -36.71624193 & -10.86590867 & 0.2019 & 0.682226353 \\
\hline 80 & -13.61274 & -2.877127 & -33.95738 & -31.080253 & -35.60101707 & -10.73560967 & 0.2059 & 0.682797684 \\
\hline 90 & -13.7487 & -3.100499 & -33.195 & -30.0945015 & -34.47188354 & -10.6482015 & 0.2099 & 0.679518354 \\
\hline
\end{tabular}




APPENDIX N
ENVELOPE C AS-RECEIVED CALORIMETRY DATA

Table 3. 9M - continued

\begin{tabular}{|c|c|c|c|c|c|c|c|c|}
\hline & Standard & Empty & Sample & Sample - Empty & $\begin{array}{c}\text { Sample Mass- } \\
\text { Corrected }\end{array}$ & $\begin{array}{c}\text { Standard - } \\
\text { Empty }\end{array}$ & Sapphire HC & Sample HC \\
\hline 9M4 & & & & & & & & \\
\hline 40 & -13.0446 & -1.701997 & -36.90006 & -35.1980635 & -40.31778183 & -11.34260017 & 0.1899 & 0.675008081 \\
\hline 50 & -13.20969 & -2.032199 & -36.44361 & -34.4114115 & -39.41670772 & -11.17749483 & 0.1939 & 0.683775724 \\
\hline 60 & -13.47839 & -2.342977 & -35.74921 & -33.406233 & -38.26532144 & -11.13541133 & 0.1979 & 0.680056343 \\
\hline 70 & -13.46868 & -2.602768 & -34.92856 & -32.325792 & -37.02772538 & -10.86590867 & 0.2019 & 0.688014043 \\
\hline 80 & -13.61274 & -2.877127 & -34.27787 & -31.400743 & -35.9681238 & -10.73560967 & 0.2059 & 0.689838483 \\
\hline 90 & -13.7487 & -3.100499 & -33.62232 & -30.5218215 & -34.96135917 & -10.6482015 & 0.2099 & 0.689167019 \\
\hline & & & & & & & & \\
\hline
\end{tabular}


This page intentionally left blank. 


\begin{tabular}{|ll|}
\hline APPENDIX O & WSRC-TR-2000-00352 \\
SR/TRU PRECIPITATE SLURRY CALORIMETRY DATA & SRT-RPP-2000-00026 \\
\hline
\end{tabular}

\section{APPENDIX 0}

\section{SR/TRU PRECIPITATE SLURRY CALORIMETRY DATA}

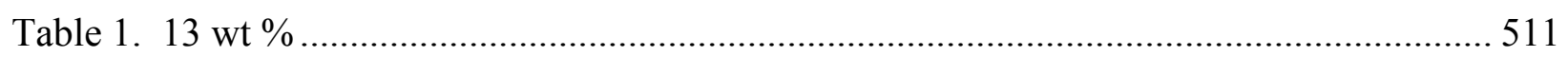

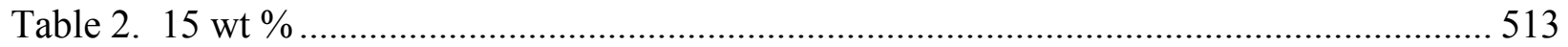


Table 1. 13 wt \%

\begin{tabular}{|c|c|c|c|c|c|c|c|c|}
\hline & Standard & Empty & Sample & Sample - Empty & $\begin{array}{l}\text { Sample Mass- } \\
\text { Corrected }\end{array}$ & $\begin{array}{l}\text { Standard - } \\
\text { Empty }\end{array}$ & Sapphire HC & Sample HC \\
\hline \multicolumn{9}{|l|}{ Run 1} \\
\hline 35 & -28.66608 & 7.27966 & -27.99695 & -35.27660967 & -96.62723517 & -35.94573967 & 0.1899 & 0.510478074 \\
\hline 45 & -29.02247 & 7.703121 & -27.25023 & -34.95335067 & -95.74178661 & -36.72558817 & 0.1939 & 0.505487682 \\
\hline 55 & -29.44431 & 8.094257 & -26.47443 & -34.568687 & -94.68814265 & -37.5385695 & 0.1979 & 0.499187467 \\
\hline 65 & -29.72797 & 8.401347 & -26.05743 & -34.45877733 & -94.38708574 & -38.12931733 & 0.2019 & 0.499792652 \\
\hline 75 & -29.79828 & 8.572672 & -26.00894 & -34.58161233 & -94.72354683 & -38.37094983 & 0.2059 & 0.508290214 \\
\hline 85 & -30.06739 & 8.701973 & -25.86348 & -34.56545333 & -94.67928522 & -38.76936083 & 0.2099 & 0.512600196 \\
\hline \multicolumn{9}{|l|}{ Run 2} \\
\hline 35 & -28.66608 & 7.27966 & -28.03574 & -35.31539967 & -96.73348604 & -35.94573967 & 0.1899 & 0.511039394 \\
\hline 45 & -29.02247 & 7.703121 & -27.44419 & -35.14731067 & -96.27306835 & -36.72558817 & 0.1939 & 0.508292689 \\
\hline 55 & -29.44431 & 8.094257 & -26.77505 & -34.869307 & -95.51158004 & -37.5385695 & 0.1979 & 0.503528556 \\
\hline 65 & -29.72797 & 8.401347 & -26.41624 & -34.81758733 & -95.36991313 & -38.12931733 & 0.2019 & 0.504996858 \\
\hline 75 & -29.79828 & 8.572672 & -26.29987 & -34.87254233 & -95.52044204 & -38.37094983 & 0.2059 & 0.512566384 \\
\hline 85 & -30.06739 & 8.701973 & -26.29987 & -35.00184333 & -95.87461435 & -38.76936083 & 0.2099 & 0.519071791 \\
\hline \multicolumn{9}{|l|}{ Run 3} \\
\hline 35 & -28.66608 & 7.27966 & -26.67807 & -33.95772967 & -93.01465083 & -35.94573967 & 0.1899 & 0.491392926 \\
\hline 45 & -29.02247 & 7.703121 & -27.01749 & -34.72061067 & -95.10428139 & -36.72558817 & 0.1939 & 0.502121847 \\
\hline 55 & -29.44431 & 8.094257 & -26.88173 & -34.975987 & -95.80379048 & -37.5385695 & 0.1979 & 0.505069063 \\
\hline 65 & -29.72797 & 8.401347 & -26.70717 & -35.10851733 & -96.16680835 & -38.12931733 & 0.2019 & 0.509216528 \\
\hline 75 & -29.79828 & 8.572672 & -26.50352 & -35.07619233 & -96.07826596 & -38.37094983 & 0.2059 & 0.515559689 \\
\hline 85 & -30.06739 & 8.701973 & -26.46473 & -35.16670333 & -96.32618739 & -38.76936083 & 0.2099 & 0.521516638 \\
\hline
\end{tabular}




APPENDIX O
SR/TRU PRECIPITATE SLURRY CALORIMETRY DATA

Table 1. 13 wt $\%$ - continued

\begin{tabular}{|c|r|r|r|r|r|c|c|c|}
\hline & Standard & Empty & Sample & Sample - Empty & $\begin{array}{c}\text { Sample Mass- } \\
\text { Corrected }\end{array}$ & $\begin{array}{c}\text { Standard - } \\
\text { Empty }\end{array}$ & Sapphire HC & Sample HC \\
\hline Run 4 & & & & & & & & \\
\hline 35 & -28.66608 & 7.27966 & -24.1858 & -31.46545967 & -86.18799822 & -35.94573967 & 0.1899 & 0.455327975 \\
\hline 45 & -29.02247 & 7.703121 & -24.03063 & -31.73375067 & -86.92288226 & -36.72558817 & 0.1939 & 0.458926534 \\
\hline 55 & -29.44431 & 8.094257 & -23.98215 & -32.076407 & -87.86146265 & -37.5385695 & 0.1979 & 0.463197817 \\
\hline 65 & -29.72797 & 8.401347 & -23.7688 & -32.17014733 & -88.11822965 & -38.12931733 & 0.2019 & 0.466598193 \\
\hline 75 & -29.79828 & 8.572672 & -23.67183 & -32.24450233 & -88.3218977 & -38.37094983 & 0.2059 & 0.473938717 \\
\hline 85 & -30.06739 & 8.701973 & -23.63303 & -32.33500333 & -88.56979174 & -38.76936083 & 0.2099 & 0.479522976 \\
\hline & & & & & & & & \\
\hline
\end{tabular}


Table 2. $15 \mathrm{wt} \%$

\begin{tabular}{|c|c|c|c|c|c|c|c|c|}
\hline & Standard & Empty & Sample & Sample - Empty & $\begin{array}{l}\text { Sample Mass- } \\
\text { Corrected }\end{array}$ & $\begin{array}{l}\text { Standard - } \\
\text { Empty }\end{array}$ & Sapphire HC & Sample HC \\
\hline \multicolumn{9}{|l|}{ Run 1} \\
\hline 35 & -28.66608 & 7.279659667 & -71.19003 & -78.46968967 & -126.7587295 & -35.94573967 & 0.1899 & 0.669661633 \\
\hline 45 & -29.0224675 & 7.703120667 & -70.80212 & -78.50524067 & -126.816158 & -36.72558817 & 0.1939 & 0.669550966 \\
\hline 55 & -29.4443125 & 8.094257 & -69.97783 & -78.072087 & -126.1164482 & -37.5385695 & 0.1979 & 0.664874699 \\
\hline 65 & -29.72797 & 8.401347333 & -69.58992 & -77.99126733 & -125.9858934 & -38.12931733 & 0.2019 & 0.667112701 \\
\hline 75 & -29.7982775 & 8.572672333 & -69.299 & -77.87167233 & -125.7927015 & -38.37094983 & 0.2059 & 0.675008498 \\
\hline 85 & -30.0673875 & 8.701973333 & -69.44446 & -78.14643333 & -126.2365462 & -38.76936083 & 0.2099 & 0.683453389 \\
\hline \multicolumn{9}{|l|}{ Run 2} \\
\hline 35 & -28.66608 & 7.279659667 & -73.61443 & -80.89408967 & -130.6750679 & -35.94573967 & 0.1899 & 0.690351503 \\
\hline 45 & -29.0224675 & 7.703120667 & -73.63382 & -81.33694067 & -131.3904426 & -36.72558817 & 0.1939 & 0.693701806 \\
\hline 55 & -29.4443125 & 8.094257 & -74.01202 & -82.106277 & -132.6332167 & -37.5385695 & 0.1979 & 0.699230523 \\
\hline 65 & -29.72797 & 8.401347333 & -73.75019 & -82.15153733 & -132.7063295 & -38.12931733 & 0.2019 & 0.702698338 \\
\hline 75 & -29.7982775 & 8.572672333 & -73.07136 & -81.64403233 & -131.8865138 & -38.37094983 & 0.2059 & 0.707708131 \\
\hline 85 & -30.0673875 & 8.701973333 & -74.08961 & -82.79158333 & -133.74025 & -38.76936083 & 0.2099 & 0.72407896 \\
\hline \multicolumn{9}{|l|}{ Run 3} \\
\hline 35 & -28.66608 & 7.279659667 & -76.07761 & -83.35726967 & -134.654051 & -35.94573967 & 0.1899 & 0.711372322 \\
\hline 45 & -29.0224675 & 7.703120667 & -75.6897 & -83.39282067 & -134.7114795 & -36.72558817 & 0.1939 & 0.711235876 \\
\hline 55 & -29.4443125 & 8.094257 & -74.76844 & -82.862697 & -133.8551259 & -37.5385695 & 0.1979 & 0.70567232 \\
\hline 65 & -29.72797 & 8.401347333 & -73.75019 & -82.15153733 & -132.7063295 & -38.12931733 & 0.2019 & 0.702698338 \\
\hline 75 & -29.7982775 & 8.572672333 & -73.32349 & -81.89616233 & -132.2938007 & -38.37094983 & 0.2059 & 0.709893648 \\
\hline 85 & -30.0673875 & 8.701973333 & -73.7114 & -82.41337333 & -133.1292954 & -38.76936083 & 0.2099 & 0.72077121 \\
\hline
\end{tabular}


Table 2. 15 wt $\%$ - continued

\begin{tabular}{|c|c|c|c|c|c|c|c|c|}
\hline & Standard & Empty & Sample & Sample - Empty & $\begin{array}{c}\text { Sample Mass- } \\
\text { Corrected }\end{array}$ & $\begin{array}{c}\text { Standard - } \\
\text { Empty }\end{array}$ & Sapphire HC & Sample HC \\
\hline Run 4 & & & & & & & & \\
\hline 35 & -28.66608 & 7.279659667 & -69.39597 & -76.67562967 & -123.8606325 & -35.94573967 & 0.1899 & 0.654351095 \\
\hline 45 & -29.0224675 & 7.703120667 & -68.00922 & -75.71234067 & -122.3045503 & -36.72558817 & 0.1939 & 0.645731042 \\
\hline 55 & -29.4443125 & 8.094257 & -67.2722 & -75.366457 & -121.7458152 & -37.5385695 & 0.1979 & 0.64183311 \\
\hline 65 & -29.72797 & 8.401347333 & -66.91338 & -75.31472733 & -121.6622518 & -38.12931733 & 0.2019 & 0.644218422 \\
\hline 75 & -29.7982775 & 8.572672333 & -66.78732 & -75.35999233 & -121.7353722 & -38.37094983 & 0.2059 & 0.653236713 \\
\hline 85 & -30.0673875 & 8.701973333 & -66.03091 & -74.73288333 & -120.72235 & -38.76936083 & 0.2099 & 0.653599149 \\
\hline & & & & & & & & \\
\hline
\end{tabular}

